\title{
○ิ
}

\section{The Routledge History of Death since 1800}

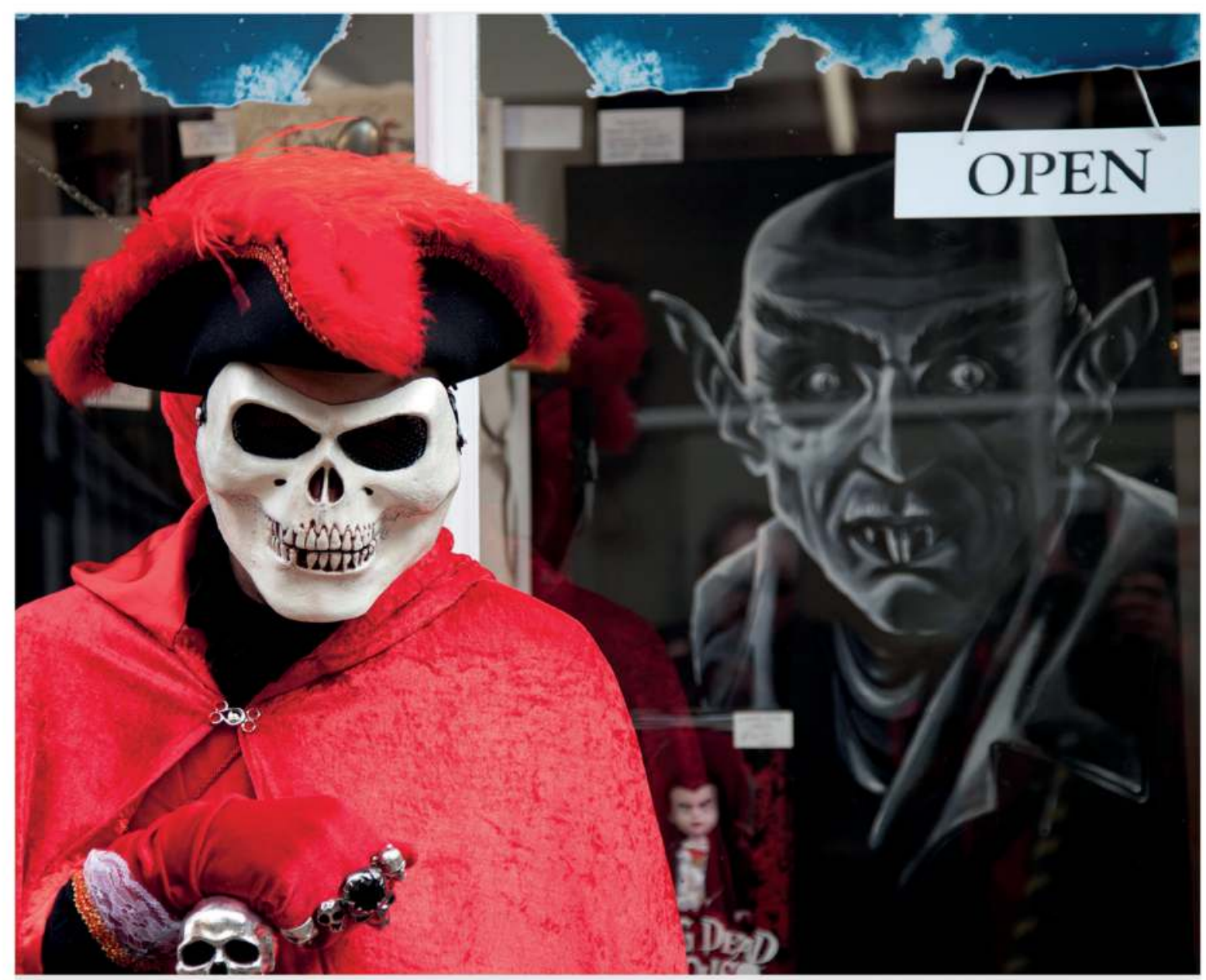

Edited by Peter N. Stearns 


\section{THE ROUTLEDGE HISTORY OF DEATH SINGE 1800}

The Routledge History of Death Since 1800 looks at how death has been treated and dealt with in modern history - the history of the past 250 years - in a global context, through a mix of definite, often quantifiable changes and a complex, qualitative assessment of the subject.

The book is divided into three parts, with the first considering major trends in death history and identifying widespread patterns of change and continuity in the material and cultural features of death since 1800. The second part turns to specifically regional experiences, and the third offers more specialized chapters on key topics in the modern history of death. Historical findings and debates feed directly into a current and prospective assessment of death, as many societies transition into patterns of ageing that will further alter the death experience and challenge modern reactions. Thus, a final chapter probes this topic, by way of introducing the links between historical experience and current trajectories, ensuring that the book gives the reader a framework for assessing the ongoing process, as well as an understanding of the past.

Global in focus and linking death to a variety of major developments in modern global history, the volume is ideal for all those interested in the multifaceted history of how death has been dealt with in different societies over time and who want access to the rich and growing historiography on the subject.

Peter N. Stearns is University Professor of History at George Mason University, USA. 


\section{THE ROUTLEDGE HISTORIES}

The Routledge Histories is a series of landmark books surveying some of the most important topics and themes in history today. Edited and written by an international team of world-renowned experts, they are the works against which all future books on their subjects will be judged.

THE ROUTLEDGE HISTORY OF GENDER, WAR, AND THE U.S. MILITARY

Edited by Kara Dixon Vuic

THE ROUTLEDGE HISTORY OF THE AMERICAN SOUTH

Edited by Maggi M. Morehouse

THE ROUTLEDGE HISTORY OF ITALIAN AMERICANS

Edited by William f. Connell \& Stanislao Pugliese

THE ROUTLEDGE HISTORY OF LATIN AMERICAN CULTURE

Edited by Carlos Manuel Salomon

THE ROUTLEDGE HISTORY OF GLOBAL WAR AND SOCIETY

Edited by Matthew S. Muehlbauer and David F. Ulbrich

THE ROUTLEDGE HISTORY OF TWENTIETH-CENTURY UNITED STATES

Edited by Ferald R. Podair and Darren Dochuk

THE ROUTLEDGE HISTORY OF WORLD PEAGE SINGE 1750

Edited by Christian Philip Peterson, William M. Knoblauch and Michael Loadenthal

THE ROUTLEDGE HISTORY OF MEDIEVAL MAGIC

Edited by Sophie Page and Catherine Rider

THE ROUTLEDGE HISTORY OF MONARGHY

Edited by Elena Woodacre, Lucinda H.S. Dean, Chris Jones, Russell E. Martin and Zita Eva Rohr

THE ROUTLEDGE HISTORY OF EMOTIONS IN EUROPE, 1100-1700

Edited by Andrew Lynch and Susan Broomhall

THE ROUTLEDGE HISTORY OF HUMAN RIGHTS

Edited by Jean $H$. Quataert and Lora Wildenthal

THE ROUTLEDGE HISTORY OF WOMEN IN EARLY MODERN EUROPE

Edited by Amanda L. Capern

THE ROUTLEDGE HISTORY OF WITCHCRAFT

Edited by Fohannes Dillinger

THE ROUTLEDGE HISTORY OF AMERICAN SEXUALITY

Edited by Kevin P. Murphy, Fason Ruiz and David Serlin

THE ROUTLEDGE HISTORY OF DEATH SINCE 1800

Edited by Peter $\mathcal{N}$. Stearns

For more information about this series, please visit:

https://www.routledge.com/Routledge-Histories/book-series/RHISTS 


\title{
THE ROUTLEDGE HISTORY OF DEATH SINGE 1800
}

\author{
Edited by \\ Peter $\mathcal{N}$. Stearns
}


First published 2021

by Routledge

2 Park Square, Milton Park, Abingdon, Oxon OX14 4RN

and by Routledge

52 Vanderbilt Avenue, New York, NY 10017

Routledge is an imprint of the Taylor \& Francis Group, an informa business

(C) 2021 selection and editorial matter, Peter N. Stearns; individual chapters, the contributors

The right of Peter N. Stearns to be identified as the author of the editorial material, and of the authors for their individual chapters, has been asserted in accordance with sections 77 and 78 of the Copyright, Designs and Patents Act 1988.

With the exception of Chapter 1, no part of this book may be reprinted or reproduced or utilized in any form or by any electronic, mechanical, or other means, now known or hereafter invented, including photocopying and recording, or in any information storage or retrieval system, without permission in writing from the publishers.

Chapter 1 of this book is available for free in PDF format as Open Access from the individual product page at www.routledge.com. It has been made available under a

Creative Commons Attribution-Non Commercial-No Derivatives 4.0 license.

Trademark notice: Product or corporate names may be trademarks or registered trademarks, and are used only for identification and explanation without intent to infringe.

British Library Cataloguing-in-Publication Data

A catalogue record for this book is available from the British Library

Library of Congress Cataloging-in-Publication Data

A catalog record has been requested for this book

ISBN: 978-0-367-13716-8 (hbk)

ISBN: 978-0-429-02827-4 (ebk)

Typeset in Baskerville by Swales \& Willis, Exeter, Devon, UK 


\section{GONTENTS}

List of images $\quad$ viii

List of figures $\quad$ ix

List of tables $\quad \mathrm{x}$

List of contributors $\quad$ xi

Introduction 1

PETER N. STEARNS

\section{PART 1}

General patterns and connections

1 Patterns of death, 1800-2020: global rates and causes

ROMOLA DAVENPORT

2 Mass death during modern epidemics: horrors and their consequences

SAMUEL GOHN

3 Violent death

PHILIP DWYER

4 Suicidology on the cusp of modernity: sociology and psychiatry in the nineteenth century

DAVID LEDERER

5 Death-seeking turns political: a historical template for terrorism ANNA GEIFMAN

6 Toward a world without the death penalty 
7 The cemetery 123 ERIN-MARIE LEGACEY

8 Death, commemoration, and the era of total war in Europe 136 JESSE KAUFFMAN

9 The transformation of death discourse: from 'taboo' to 'revival' at the threshold of the new millennium

PART II

Regional patterns

10 'Why may not man be one day immortal?': rethinking death in the age of enlightenment

JOSEPH CLARKE

11 "Now for the grand secret": a history of the post-mortem identity and Heavenly reunions, 1800-2000

JOHN G. WEAVER AND DOUG MUNRO

12 Death in modern North American history PETER N. STEARNS

13 Death in Mexico: image and reality

14 Death in modern Japan (1800-2020) TIMOTHY O. BENEDICT

15 Picturing the dead in early twentieth-century Ghina: bodies, burial, and the photography of the Chinese Red Cross

Burial Corps

CAROLINE REEVES

16 Remaking the Hindu pyre: cremation in India since the 1830s DAVID ARNOLD

17 Muslim beliefs about death: from classical formulations to modern applications 
19 Rituals of death in the Caribbean diaspora, 1970-: the immigrant dilemmas

20 Premature burial and the mysteries of death JOANNA BOURKE

21 Murdering mothers and dutiful daughters: infanticide in eighteenth- and nineteenth-century Mexico

NORA E. JAFFARY

22 "I wish we could have saved him for you": Australia's experience of death and bereavement in war, 1914-1918

JEN ROBERTS

23 Soviet cemeteries

SVETLANA MALYSHEVA

24 Death in modern film

THOMAS BRITT

25 Of presidential mausoleums and politics in neo-liberal Zambia, 2008 to 2018

WALIMA T. KALUSA AND KELVIN CHANDA

26 Gelebrating creation and commemorating life: ritualizing pet death in the U.S. and Japan

BARBARA R. AMBROS

27 Hospice: a way to die LUCY BREGMAN

28 "A profound shift in policy": the history of assisted suicide IAN DOWBIGGIN

29 Future trajectories of death: issues and possibilities 


\section{IMAGES}

0.1 Medicalization and military casualties: Florence Nightingale in a hospital during the Crimean War

2.1 Efforts to use smoke to dispel cholera in Granada, 1884

7.1 Père Lachaise cemetery in the middle of the nineteenth century 128

8.1 First World War Memorial in Lille, France 139

13.1 Day of the Dead altar, Oaxaca 250

14.1 Memorial tablet (ihai) for the deceased B29 Airmen 255

15.1 Chinese Red Cross Society Burial Corps 279

15.2 Zhijiang Red Cross Chapter burying the dead 281

15.3 Red Cross Burial Corps caring for the unclaimed dead 282

16.1 A Western view of cremation in Calcutta, c. $1886 \quad 290$

23.1 A common anonymous grave of victims of reprisals with homemade signs by relatives. Don Cemetery, Moscow

23.2 The combination of Orthodox crosses and Soviet stars on the tombstones. Arsk cemetery, Kazan

25.1 President Levy Patrick Mwanawasa's Mausoleum 449

25.2 President Frederick Titus Chiluba's mausoleum 451

25.3 President Michael Chilufya Sata's mausoleum 452

26.1 The Cemetery for All God's Creatures at St. Andrew's in Richmondtown, NY, is located adjacent to the church's historical human cemetery 466

26.2 Statue of St. Francis in the Cemetery for All God's Creatures 467

26.3 Pet columbarium at the Rengeji Tetsugakudō Animal Cemetery in Tokyo, Japan

26.4 Interment service at the Rengeji Tetsugakudō Animal Cemetery in Tokyo, Japan 


\section{FIGURES}

0.1 Frequency of the word "death" in English, 1800-2008

1.1 Distributions by age of survivors (a, c, e) and deaths $(b, d, f)$ in a synthetic cohort subject to the death rates prevailing in the population of England and Wales in 1841, 1950, and 2016

1.2 Long-run trends in life expectancy at birth 25

1.3 The distribution of the global population by life expectancy 26

1.4 Annual age-specific mortality rates, England and Wales, 1841-2011 32

1.5 Mortality by category of cause, ages 60-64, England and Wales, 1891-1967 33

1.6 Tuberculosis mortality, England and Wales, 1848-2000 34

1.7 'Preston curves'; national life expectancies in relation to income per capita 39

11.1 NGram Viewer depiction of frequency of appearance of "meet in Heaven" and "God will forgive me" in Google scanned books, 1780-2000 198

12.1 Frequency of the words "death" and "grief" in American English, 1700-2008 


\section{TABLES}

11.1 Mentions of "meet in Heaven" and "God will forgive me" in scanned newspapers: United Kingdom, Australia, and New Zealand, Raw Counts, $1820-2000$

11.2 Mentions of "meet in Heaven" in scanned newspapers: United Kingdom and Australia, 1820-1960. Controlled for variations in source material

11.3 Counts of "meeting in Heaven": poetry (1820-1940) and World War I family in Memoriam and honor roll references from UK and Australian newspapers

12.1 Table showing the relationship between life expectancy and infant mortality from 1860 through 1990. Michael Robert Haines and Richard H. Steckel, A Population History of North America (New York: Cambridge University Press, 2000), 308 


\section{GONTRIBUTORS}

Barbara Ambros is Professor of East Asian Religions in the Department of Religious Studies in the University of North Carolina at Charlotte. She works on various aspects of religions in Japan. She has cochaired the Animals and Religion group in the American Academy of Religion since 2014.

David Arnold is Professor Emeritus in the Department of History, University of Warwick. His published work ranges widely over the history of modern India, with particular emphasis on disease, medicine, science, technology and the environment. Books include Colonizing the Body: State Medicine and Epidemic Disease in Nineteenth-Century India (1993), Toxic Histories: Poison and Pollution in Modern India (2016) and Burning the Dead: India, Empire, and Cremation (2021).

Timothy O. Benedict is Assistant Professor in the School of Sociology at Kwansei Gakuin University in Japan. His research and teaching interests are centered on Japanese religion, death and dying, ethnography and medical anthropology. He is the author of "Practicing Spiritual Care in the Japanese Hospice," Fapanese Fournal of Religious Studies (2018).

Joanna Bourke is Professor of History at Birkbeck College, University of London, and Fellow of the British Academy. She is the Principal Investigator for a Welcome Trustfunded project entitled "Sexual Violence, Medicine, and Psychiatry" (October 2018 to September 2023). She is the prize-winning author of thirteen books, including histories on modern warfare, military medicine, psychology and psychiatry, the emotions and sexual violence, as well as over 100 articles in academic journals.

Stanley H. Brandes holds a doctorate in Anthropology from the University of California, Berkeley, where he taught for over forty years. He has conducted extensive fieldwork in Spain, Mexico and the USA and has lectured widely throughout Europe, Latin America and Asia. His research areas currently include animal-human relations, the history of anthropology in fascist Europe, ethnographic photography, popular Catholicism and the anthropology of food and drink. Brandes is the author of six books and numerous scholarly articles, book chapters, and short communications. His most recent monographs are Staying Sober in Mexico City (2002) and Skulls to the Living, Bread to the Dead: the Day of the Dead in Mexico and Beyond (2006). 
Lucy Bregman received her Ph.D. from the University of Chicago's Divinity School, and taught in the Religion Department of Temple University until her retirement in 2019. She has written widely on the subject of death and religion, including Preaching Death: The Transformation of American Protestant Funeral Sermons (2011).

Thomas Britt is Associate Professor in the Film and Video Studies Program at George Mason University. He oversees the screenwriting concentration and teaches the Ethics of Film and Video, among several other courses.

Kelvin Chanda holds B.Sc. and M.Sc. degrees from the University of Zambia and Greenwich University in the UK, respectively. He is currently the Chief Natural Heritage Officer at the National Heritage Conservation Commission in Lusaka, Zambia. His research interest is in environmental conservation and protection.

Joseph Clarke is Lecturer in History at Trinity College Dublin. He has written widely on cultural change and political violence in eighteenth-century France and the Mediterranean.

Samuel Cohn, Jr. is Professor of Medieval History at the University of Glasgow, Fellow of the Royal Society of Edinburgh, and Honorary Fellow of the Institute for Advanced Studies in the Humanities, Edinburgh. From the late 1990s, he has published and taught on two broad themes: popular insurrection in medieval and early modern Europe, and the history of plague and other diseases from antiquity to the present. His most recent books include Cultures of Plague: Medical Thinking at the End of the Renaissance (2010) and Epidemics: Hate $E$ Compassion from the Plague of Athens to AIDS (2018). He is currently drafting a monograph on popular protest and ideals of democracy in lateRenaissance Italy.

Romola Davenport is Senior Research Associate at the Cambridge Group for the History of Population and Social Structure (University of Cambridge). She has degrees in history, biology and demography, and held a Royal Society Dorothy Hodgkin fellowship in plant sciences before changing career to become a historical demographer. Her work combines historical, quantitative social science and evolutionary approaches to historical epidemiology and economic history.

Garrey Dennie is Associate Professor of History at St. Mary's College of Maryland. He obtained his Ph.D. in African History at the Johns Hopkins University, Baltimore, Maryland. Dr. Dennie is a former speech writer for Nelson Mandela and a founding member of Africa and African Diaspora Studies at St. Mary's College. At St. Mary's College he teaches courses in African history, Caribbean history, and American slave systems. His key publications include Flames of Race, Ashes of Death: Re-inventing Cremation in Johannesburg and The Standard of Dying: Race, Indigence, and the Disposal of the Dead Body in Johannesburg. He is currently completing a two-volume general history of St. Vincent and the Grenadines that will be published later this year. His coauthors on this project are Dr. Cleve Scott at the UWI and Dr. Adrian Fraser, Emeritus Professor of the UWI. He is one of three active professional historians born in St. Vincent and the Grenadines. 
Ian Dowbiggin is Professor of History at the University of Prince Edward Island in Canada. A Fellow of the Royal Society of Canada, he is the author of seven books on the history of medicine.

Philip Dwyer is Professor of History and the founding Director of the Centre for the Study of Violence at the University of Newcastle, Australia. He is editor of (with Lyndall Ryan) Theatres of Violence: Massacre, Mass Killing and Atrocity throughout History (2012); (with Amanda Nettelbeck) Violence, Colonialism and Empire in the Modern and World (2017); (with Mark Micale) On Violence in History (2019); and general editor (with Joy Damousi) of the four-volume Cambridge World History of Violence (2020). He is currently engaged in writing Violence: A Very Short Introduction for Oxford University Press and The Savage Heart: A History of Human Violence for Bloomsbury in London and Basic Books in New York.

Anna Geifman, Ph.D., is the author of Thou Shalt Kill: Revolutionary Terrorism in Russia, 1894-1917 (1993) and Entangled in Terror: The Azef Affair and the Russian Revolution (2000). She is the editor of Russia under the Last Tsar: Opposition and Subversion, 1894-1917 (1999). Among her other major publications are La mort sera votre dieu: du nihilisme russe au terrorisme islamiste (2005) and Death Orders: The Vanguard of Modern Terrorism in Revolutionary Russia (2010). Geifman is Professor of History Emerita at Boston University and a Principal Researcher in the Political Studies Department at Bar-Ilan University in Israel.

Cortney Hughes Rinker is Associate Professor in the Department of Sociology and Anthropology at George Mason University, where she is also a member of the Steering Committee of the Ali Vural Ak Center for Global Islamic Studies and a faculty affiliate of Women and Gender Studies. She is the author of Islam, Development, and Urban Women's Reproductive Practices (2013) and coeditor of Applied Anthropology: Unexpected Spaces, Topics, and Methods (2016).

Michael Hviid Jacobsen is Professor of Sociology and Social Work at Aalborg University. He publishes, both in English and Danish, on topics in social theory, the sociology of death and crime and violence. His coauthored book, What Use is Sociology?, has been translated into over ten languages.

Nora Jaffary is a Latin Americanist who teaches in the History Department at Concordia University in Montreal. Her research focuses on social and gender history in colonial and nineteenth-century Mexico. Her book, Reproduction and Its Discontents in Mexico: Childbirth and Contraception from 1750 to 1905 (2016), won the Canadian Historical Association's Wallace K. Ferguson Prize in 2017, and Honorable Mention for the Latin American Studies Association's Howard F. Cline Book Prize in Mexican History in 2018. She is currently at work on a book treating the history of abortion in nineteenth-century Mexico.

Walima T. Kalusa holds a doctoral degree in medical history from the Johns Hopkins in the USA, and he is currently teaching African history at the University of Eswatini. He has taught at Georgetown University, USA, and the University of Zambia. Besides being a Visiting Research Fellow at Cambridge University in 2006-2007 and 2017-2018, he worked there as Research Associate on the "Death in Africa" project 
from 2008 to 2010. His papers have appeared in several journals, including fournal of African History, Gournal of Southern African Studies and International Gournal of African Historical Studies. His present research project focuses on the history of women and clothes on the colonial Zambian Copperbelt.

Jesse Kauffman is Associate Professor of History at Eastern Michigan University, where he teaches courses on modern European political and military history. He is the author of Elusive Alliance: The German Occupation of Poland in World War I (2015).

David Lederer is Associate Professor at Maynooth University. A cultural and social historian, he has taught and researched extensively in Australia, China, the USA and across Europe. In extensive publications dedicated to the history of suicide and psychiatry, he explores the role of religion, medicine, statistics, economics and other social forces that change perceptions of self-killing across space and time. Currently, he is writing a global history on the relationship between suicide and culture.

Rebekah Lee is Senior Lecturer in History at Goldsmiths, University of London. She has published work on South African history and culture, including the book African Women and Apartheid: Migration and Settlement in Urban South Africa (2009). Her research interests span issues of health, gender, migration, mobility, urbanization, religion and identity. She is currently completing her second monograph, Death and Memory in Modern South Africa, and has begun research into road accidents and road safety. The research on death was part of a major collaboration with Professor Megan Vaughan funded by the Arts Humanities Research Council of the UK (www.gold.ac.uk/deathinafrica). Dr. Lee holds degrees from Harvard University (B.A.) and the University of Oxford (M.Phil and D.Phil), and has taught at universities in the UK, USA and South Africa.

Erin-Marie Legacey is an Associate Professor of History at Texas Tech University. She is a historian of modern France, with a special interest in the post-revolutionary period. She is the author of several articles and one book, Making Space for the Dead: Cemeteries, Catacombs, and the Reimagining of Paris, 1780-1830, which examines the origins and significance of some of Paris's most well-known burial sites.

Svetlana Malysheva is Professor of Russian history at the Institute of International Relations, History and Oriental Studies, Kazan Federal University. She has authored books on the history and historiography of the 1917 Russian Revolution and the Civil War; the history of everyday life in the nineteenth and twentieth centuries; Russian festive and leisure-time culture; and the history of death in Russia in the twentieth century.

Doug Munro is Adjunct Professor of History at the University of Queensland. He started as a historian of the Pacific Islands. As well as studies on suicide with John C. Weaver, his interests include history/culture wars and the telling of academic lives. He has written about historians as varied as G.R. Elton and George Rudé. His recent publications include Bearing Witness: Essays in Honour of Brij V. Lal (coedited with Jack Corbett) and Clio's Lives: Biographies and Autobiographies of Historians (coedited with John G. Reid). Both were published in 2017 by the ANU Press and are available as free downloads on https://press.anu.edu.au. 
Caroline Reeves is Associate in Research at Harvard University's Fairbank Center, specializing in the history of Chinese charity and philanthropy. She has taught at Harvard University, Williams College, Emmanuel College and on Semester at Sea. She was coorganizer of the collaborative project "The Social Lives of Dead Bodies in Modern China" and has published on charitable responses to burial in Republican China, the birth of humanitarian photography in China, the founding and development of China's national Red Cross Society and on China's charitable tradition as a model for the Global South. She is now working on a manuscript on the history of Chinese philanthropy and its import in the contemporary global arena.

Jen Roberts is Lecturer in History at the University of Wollongong, south of Sydney, Australia. She holds a Ph.D. and first-class honours degree from that institution, and her research interests include the social history of war and conflict, commemoration, psychiatry and mental illness, and war grave pilgrimage. Her work has been previously published in the Fournal of Australian Studies, Health and History, and in Martin Crotty and Marina Larsson's Anzac Legacies: Australians and the Aftermath of War.

Abdulaziz Sachedina, Ph.D., is Professor and IIIT Chair in Islamic Studies at George Mason University in Fairfax, Virginia. Dr. Sachedina has been conducting research and writing in the field of Islamic Law, Ethics, and Theology (Sunni and Shiite) for more than two decades. In the last ten years, he has concentrated on social and political ethics, including interfaith and intrafaith relations, Islamic biomedical ethics and Islam and human rights. Dr. Sachedina's publications include The Islamic Roots of Democratic Pluralism (2002), Islamic Biomedical Ethics: Theory and Application (2009) and Islam and the Challenge of Human Rights (2009).

Peter N. Stearns is University Professor of History at George Mason University. He has written widely on US social and cultural history and on world history. He has a number of publications on thematic approaches to world history. He has also been deeply engaged in the growing field of the history of emotions, with several studies on changing patterns of grief, fear and guilt, all relevant to the assessment of death.

Alice Storey is Lecturer in Law and Associate Director of the Centre for Human Rights at Birmingham City University, UK. Her research interests consider the intersection between international human rights, the UN's Universal Periodic Review and the death penalty.

Megan Vaughan joined UCL's Institute of Advanced Studies in 2015 as Professor of African History and Health. Her work, which crosses disciplinary boundaries, has focused on the history of medicine and psychiatry in Africa, on the history of famine, food supply and gender relations and on slavery in the Indian Ocean region. Most recently she held a major AHRG award on the history of death and death practices in Eastern and Southern Africa. She is now working on a Wellcome Trust-funded history of epidemiological change in Africa, focusing on "chronic" diseases. She began her career at the University of Malawi and maintains strong links there and elsewhere in the region. She is committed to working collaboratively with African scholars and institutions and is a past president of the African Studies Association of the UK. Her publications include 


\section{CONTRIBUTOR S}

(with Walima Kalusa) Death, Belief and Politics in Central Africa (2015); Creating the Creole Island: Slavery in Eighteenth-Century Mauritius (2005); (with Henrietta Moore) Cutting Down Trees: Gender, Nutrition and Agricultural Change in Northern Zambia (1995); Curing Their Ills: Colonial Power and African Illness (1991); The Story of an African Famine (1987).

John C. Weaver FRSG is Distinguished University Professor in the Department of History at McMaster University in Hamilton, Ontario. The 2010 recipient of the FrançoisXavier Garneau Medal, awarded by the Canadian Historical Association for The Great Land Rush and the Masking of the Modern World, his current interests are in economic restructuring as well as global trade logistics.

Jon Yorke is the Director of the Centre for Human Rights at the School of Law, Birmingham City University. He is an advisor to the UK government on death penalty matters. He has been a consultant on the death penalty to the United Nations, the European Union and the Council of Europe and has worked on death penalty cases in the USA, Sudan and the African Commission on Human and Peoples' Rights. He is a founding member of the International Academic Network for the Abolition of Capital Punishment, and has published widely on the death penalty. 


\title{
INTRODUGTION
}

\author{
Peter $\mathcal{N}$. Stearns
}

The modern history of death offers a fascinating and complex mixture of certainties and debates, their combination vital toward promoting better understanding of the modern condition. Few aspects of the human experience have changed as much as death has, over the past two and a half centuries - though of course our ultimate mortality has not. And few historical topics offer such a challenging combination of quantifiable change and complex shifts in meaning, both aspects essential in evaluating patterns of death over time.

The purpose of this volume centers on providing current summaries of findings about the many facets of death in modern history - the history of the past 250 years - in a global context. There is little question that significant changes in death began to emerge in the later 18th century, with some of the new scientific interests of the Enlightenment and, fairly soon, some crucial alterations in public health and living standards that would begin - very slowly at first - to affect traditional patterns of death, particularly among children. But this modern history was complicated by a number of factors - including huge regional differences in relevant living standards and cultural assumptions - that must also be embraced in any global approach.

Not surprisingly, historians have devoted considerable attention to death, particularly as the topical range in the discipline began to expand in the 1960s and 1970s to embrace more facets of people's lives in the past. At the same time, interest in death has fluctuated, and there are many opportunities for additional work. Some regions of the world have received more attention than others, while special topics invite further attention. This volume, laying out many of the key developments in death in modern times, captures core findings in the modern history of death, but also helps identify a number of opportunities for further inquiry.

\section{Measurable change: death rates and more}

The list of quantifiable changes, some beginning in the 18th century, others more recently, is truly impressive. ${ }^{1}$ Over two and a half centuries, death rates have dropped in almost every region of the world - though in some cases only fairly recently. Child mortality has plummeted, removing childhood as a common site of death for the first time in human history. But adults, as well, can expect greater longevity. Shifts of this magnitude invite careful analysis, and also set up additional issues, particularly around the extent to which attention to death declines as well.

Accompanying what might be called a death revolution, the most common causes of death shifted significantly: from primary concerns about communicable diseases (and digestive diseases in infancy), to degenerative diseases (heart attacks, cancer) plus a growing role for accidents. The nature of epidemics shifts in this process as well. 
While dramatic shifts in mortality rates and causes form the most obvious theme, a host of other changes enter in as well. For increasing numbers of people the location of death changes, from home to hospital. The role of doctors and nurses in dealing with death goes up (a process sometimes referred to as medicalization), the role of religious officials (on the whole) declines. ${ }^{2}$ While timing varied (depending among other things on rates of urbanization), the shift of death away from the home at some point in the 20th century - thanks to the growing use of hospitals but also the rise of death through road accidents plus increasing intervention by government authorities and medical personnel wherever death occurred - constitutes a major change that might also impinge on grief rituals and other reactions to mortality.

Beyond this, the location of cemeteries has changed, as has their appearance. In many regions, new professions linked to the rise of the funeral home reflect shifting approaches to death and prompt further adjustments in turn. ${ }^{3}$ Even governments get in on the act, actively generating new rules about how death must be determined and how dead bodies must be handled and disposed of. Many people also increasingly come to expect government intervention, in cases of natural or manmade catastrophe, to help limit the death toll.

The dead body itself has a modern history. In some regions, a revival of embalming and growing concern for recovering the remains of war dead signaled new approaches. Elsewhere, changes in cremation, or simply new acceptance of cremation, reflected major shifts in attitudes and conditions alike. More recently still, medical advances have challenged past definitions of how death can be determined, and the growing emphasis on brain death has

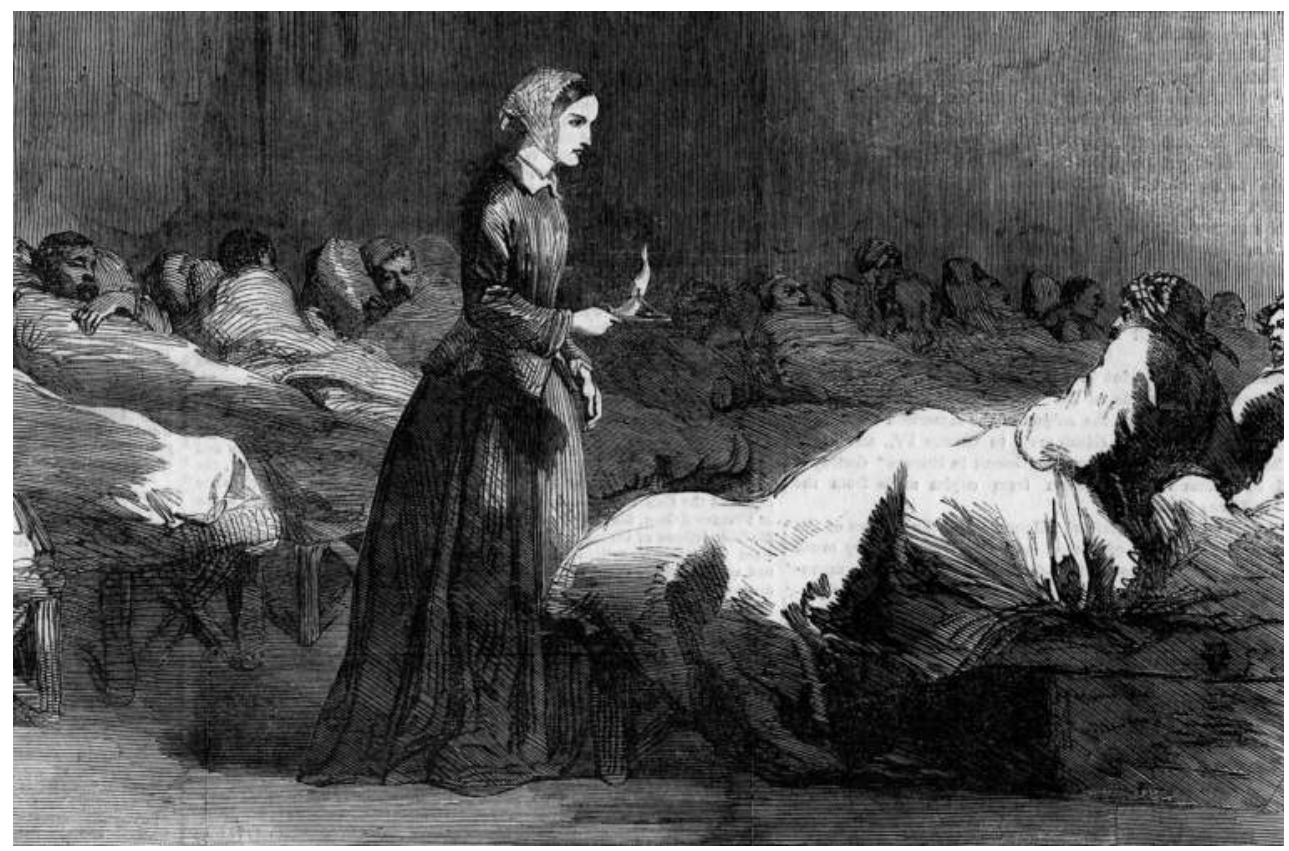

Image 0.1 Medicalization and military casualties: Florence Nightingale in a hospital during the Crimean War.

(C) INTERFOTO/Alamy Stock Photo. 
permitted unprecedented efforts to harvest organs before physical death is complete. The same medical developments often require explicit decisions about when complete death should be allowed, a new experience that has raised issues in many cultural traditions and, in more personal terms, often added to the emotional turmoil of death.

Other shifts deserve attention. Geographical mobility, but also improvements in transportation, create challenges to traditional funeral rituals, where more time must be arranged so that distant family members can be present. Death in war changes, with devastating new military technologies but also new medical interventions and, often, mounting public expectation that casualties should be limited and the bodies of the fallen returned home. ${ }^{4}$

Basic shifts of this sort - in death rates, the experience of children, death causes, government responsibilities and (increasingly) the process of medicalization - are ultimately global, though most of them first began in the West. (It was only in 2018, for example, that the African nation of Sierra Leone began to develop a small fleet of used vehicles that could serve as ambulances, thus increasing the role of hospitals.) Differences in timing and, still today, extent involve important complexities even in the most fundamental trends. And a few societies, beset by unusually extreme poverty or endemic warfare, do not really participate in the major developments at all or (as with contemporary Syria) slide back from previous gains.

And there are other complexities. The Taiwanese, though almost as prosperous as, say, Americans, rely less systematically on doctors to treat disease. ${ }^{5}$ They do share the recourse to physicians in cases of seemingly serious illness - to that extent, participating in one of the leading global trends; but they turn to more traditional remedies, sometimes even magic, in dealing with other ailments. While the general point about the rise of doctors holds true (with variations in timing and relevant standards of living), religion remains a much more vital force in dealing with death in some contemporary societies than in others. Attacks on contagious diseases have been generally successful - now directly encouraged by a key global agency, the World Health Organization - but, again, some societies stand apart in interesting ways. Resistance to polio vaccinations in Pakistan is an interesting contemporary variant; so is the strong though minority current against childhood inoculations that has gained ground in the contemporary United States. ${ }^{6}$

Increasing reliance on a medical approach in dealing with death leads to other issues, again complicating generalizations even about measurable trends. By the later 20th century, the emergence of sophisticated life support technology and changes in the definition of death provoked various reactions, from societies and individuals alike. Japan, for example, long hesitated about relying on measurements of brain death, compared to other advanced industrial countries. ${ }^{7}$ Similar hesitations have arisen in Islamic countries, where the issue of how to define death, in relation to traditional approaches based largely on cessation of heartbeat, has occasioned considerable theological debate. While Western countries have avoided such systematic discussion, individual families and medical practitioners routinely grapple with tensions between desirable death and new technical opportunities to prolong life - tensions that can include great emotional intensity.

Medicalization has also increasingly been applied to suicide, a form of death often treated separately but open to connection with the larger history of death as well. Many traditional societies regarded suicides as sinful or taboo. This prejudice began gradually to break in the West in the 18th and 19th century, though the act was now seen as a sign of societal maladjustment - though an interesting social science argument did 
emerge that connected high suicide rates to levels of progress and modernization. Medicalization more clearly set in during the 20th century, with increasing calls for therapeutic interventions. In the United States the first suicide prevention center was established in Los Angeles in 1958, and wider "hot line" services sprang up in the early 21st century. More extensive therapy in Western Europe and North America was credited with stabilizing the suicide rate by the 1980s, though there was slippage in some places after 2000. Regional variation persisted - in East Asia, therapy was shunned a shameful - and nowhere did the stigma against suicide entirely disappear. Still, the rise of medical intervention was an important development.

The overall point is clear: significant global themes, changing the physical nature of death in important ways and expanding medical services and evaluations, deserve serious attention, but they also have involved important disparities in timing and extent, and incorporate some other local variants as well. The story is not always straightforward.

\section{Qualitative and comparative themes}

Even more important, what may be seen as the physical history of death is accompanied by two other huge and often related issues. First, while changes in death rates and medical treatments can be fairly precisely measured, they also yield a vigorous debate over the qualitative features of death - the role of death in ritual, family life, and personal fears and experiences. This aspect of the modern history of death, arguably at least as significant as material change, has also altered significantly, sometimes indeed before the more basic physical changes clearly applied. But it is much harder to interpret, yielding a set of basic questions more than absolutely firm conclusions. ${ }^{8}$

Second, global trends must be seriously complicated by the ongoing regional variations, not just in the timing of basic changes or in policy disputes about the determination of death, but in the larger cultural apparatus that surrounds the ways death is experienced. Increasingly, historians and other social scientists are realizing the importance of regional continuities in death responses - over and above the more obvious theme of change. Here is a serious though intriguing challenge in dealing with the modern history of death from a global perspective, rather than through a strictly Western lens.

Evaluating the shifts in the meaning of modern death (in the Western experience but ultimately beyond) and addressing comparative distinctions and complex combinations of change and continuity must be combined with attention to the physical shifts. Ultimately, the various patterns interact in shaping how the death of self and the death of others are perceived and handled.

\section{The meaning of death in modern society}

Questions about what death has come to mean over the course of the last 250 years flow in part from the physical trends. For example, at least in many societies, preferences for what might be defined as a "good" death have changed, possibly in very revealing ways, partly because of the alterations in common causes of death and partly because of broader adjustments in popular expectations. A general hope that death would occur in later age did not yield, but an earlier interest in a somewhat lingering death, surrounded by relatives, gave way by the 20th century to a widespread interest in quick deaths, in 
which adjustments to the process of dying might be bypassed. This new preference had huge implications for the ways individuals thought about their own death prospects, but also for how a broader spectrum of people - friends and relatives, most obviously might be able to cope with deaths of others. ${ }^{9}$

The sheer frequency of discussions of death diminished, perhaps inevitably given longer life expectancies and the fact that the deaths of children were no longer a family inevitability. A Google-based search for the frequency of references to death, compared to other subjects, shows a marked decline - for example, in all the English-speaking societies (see Figure 0.1). ${ }^{10}$ In a sense, this was simply a predictable response to the facts that death was occurring less often (particularly thanks to the rapid decline of infant mortality) and that many of the deaths that did occur now happened away from full view, in emergency wards or terminal units of hospitals or nursing homes. But was less discussion of death also a reflection of new fears and anxieties? Death also declined, as a subject, in children's literature - still a common theme in the 19th century, it has become rare over the past hundred years; indeed, many traditional stories that had featured death have been reworked, as in the Disney versions of stories like Cinderella and her once-doomed stepsisters. $^{11}$

Did larger emotional reactions change as well? In some societies, at least, grief might be reconsidered. In the West, for example, new therapies emerged in the 20th century to help people "manage" grief, with the clear implication that too much grief could become a problem, and also a nuisance to others. ${ }^{12}$ Many mourning rituals were curtailed as well. At the same time, guilt over death might actually have increased: as the death of children became uncommon, for example, emotional reactions when a death did occur might have become far more overwhelming. A larger belief that death before old age should be preventable, and considerable hope that modern medicine could keep death at bay, might heighten guilt and confusion even when adults neared the end. ${ }^{13}$ Tension between the impulse to fight death, and emotional reconciliation, might increase. Other changes deserve consideration. In more urban societies, issues of inheritance, though still present, tend to decline, which may well contribute, along with other shifts in family relationships, to new attitudes toward the death of elderly relatives.

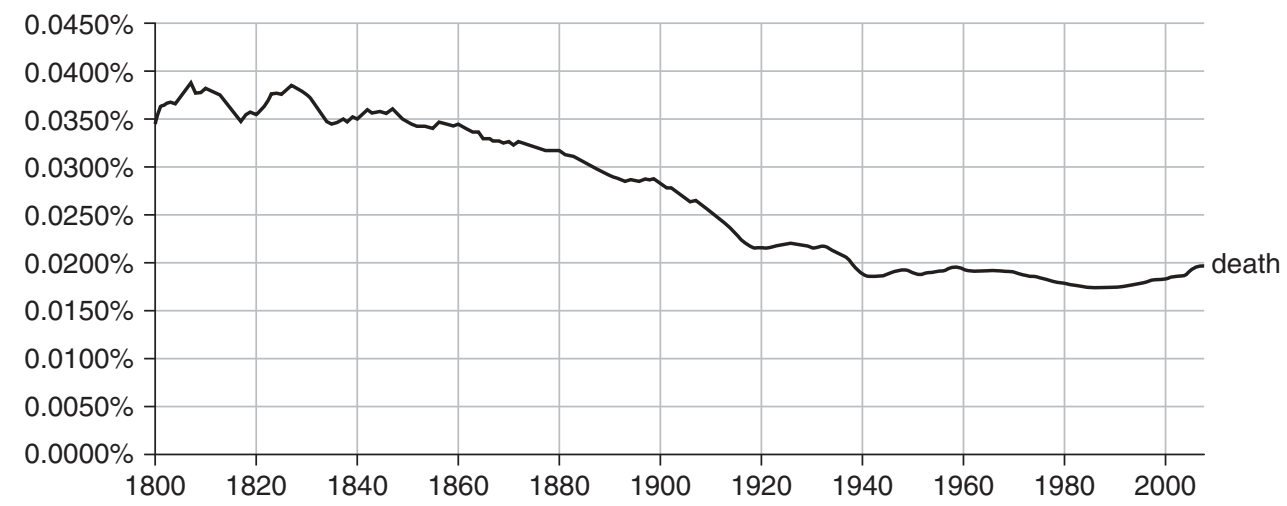

Figure 0.1 Frequency of the word "death" in English, 1800-2008.

Google Ngram Viewer. Accessed January 8, 2019. 
Does fear increase or decrease as direct contact with death declines? A fascinating episode from the late 18th to the mid-19th century involved a surprising anxiety about being buried alive. Elaborate new devices were introduced to make sure people could alert others if they found themselves prematurely interred; George Washington left specific instructions that he not be buried for at least two days after apparent death, reflecting this striking concern. ${ }^{14}$ On another front, by the 20th century, popular culture movies and then video games - often trivialized death with scenes of mindless violence, exposing many young people to casual portrayals of mass death at unprecedented levels; was this in any way connected to attitudes and concerns in "real" life?

For some observers, deeply critical of modern society and/or genuinely concerned about a decline in the human ability to anticipate and integrate death, the changes in attention to death constitute a deep flaw. For several decades many observers - some historians and even more sociologists and psychologists - have worried that death has become a "taboo" topic in modern society (or at least modern Western society), with the result that (since death ultimately remains inescapable) people are far less prepared to handle the experience than they should be. ${ }^{15}$ Life itself becomes more superficial, without intense awareness of human mortality, and a host of practical issues arise as well. In a death-denying culture, people put off making arrangements that will facilitate dealing with death (making wills, for example), but sometimes, perhaps ironically, face more challenging problems in letting go of others. Debatable prolongations of life, in intensive care units, arguably reflect not only new medical techniques but a widespread unwillingness to come to terms with death itself. Many people no longer die as they would wish because of the pressures (and new medical capacities) to keep life going regardless of quality.

Have modern people suffered or benefited from the wide range of changes that have altered the experience of death since the late 18th century? ${ }^{16}$ Here is the crucial debate, which cuts deeply into the interpretation of modern life, and it is not easy to resolve. The range of issues - from clear-cut changes in rates and treatment of death to more subjective debates over patterns of grief or cultural denial - sets an ambitious agenda for the chapters that follow, which sum up current historical understandings in both categories. Ultimately, the interactions between physical shifts in death and death arrangements, on the one hand, and the larger cultural role of death in modern life deserve particular attention, as we try to figure out the meaning of the changes in the role of death in the wider human experience over the past two centuries. And this involves attention to a number of subjects - such as emotion and ritual - that can be tackled historically but without the precision possible with subjects like death rates or killer disease.

One other issue warrants attention: the tension between an emphasis on "modern" changes and the fact that there have been a number of adjustments, even outright zigzags, within the modern period itself. Clearly, for example, Victorian reactions to death differed from those that emerged by the 1920s. But several dominant 20th-century trends also shifted later on, and some observers believe that a major transformation has taken shape in recent decades, among other things countering any sense that death was taboo. Keeping a more complex periodization in mind will be vital for a number of specific features of the modern history of death, as well as in any overall evaluation of cultural responses. 


\section{The global framework}

Modern death is at once a common and a culturally diverse experience around the world, and this tension has become increasingly significant in historical interpretations in recent decades. There is increasing awareness that different cultures continue to tackle death variously, even as they interact with some common modern patterns. Historical and anthropological work over the past two decades has reemphasized not only cultural distinctions but also the important continuities that aid societies in dealing with death.

Cultural differences around the world enter into the modern history of death from the late 18th century onward, and they continue to condition death responses into the $21 \mathrm{st}$ century. While there is a genuinely global history of death in one sense - particularly in the almost-universal reduction of death rates by the later 20th century - there is absolutely no global pattern overall: comparative issues, not sweeping trends, predominate.

In the West, for example, the 19th century saw the emergence of a more intense cultural framework for death - with heightened rituals and, probably, heightened emotions as well, including the fascinating fear of being buried alive, but also some new optimism about prospects after death - considerably before some of the most significant physical changes in death, including major alterations of the death rate, began to emerge. This Victorian death culture, which generated among other things some intriguing changes both in the nature of cemeteries and in the treatment of dead bodies, established several enduring features of the Western approach to death, but it would be substantially modified, even attacked outright, in the first half of the 20th century. A full modern history of death must probe this kind of cultural evolution, even though it does not mesh completely with other categories and trends, and it does not fully spill over into patterns in other regions.

The broad category of death culture looms even larger in dealing with many other regions, where Victorian death values for example had little impact and where different patterns of religion and ritual would affect actions throughout the past 250 years and into the present. In some cases, cultural continuities may have encouraged more constructive responses to death than (some have contended) emerged in the West. And, of course, other influences may enter the regional cultural mix, such as the innovations introduced by communism in key parts of eastern Europe and East Asia or the differential effects of modern war.

Emphasis on cultural differences and regional continuities directly affects the overall evaluation of death in modern societies. The issue of death as a modern taboo, for example, tends to recede - at least at a global level - in light of what we are learning about the variety of responses that have persisted through the modern centuries and into the present day.

\section{Basic questions}

Clearly, several overriding themes help frame the modern history of death - though they must not preclude attention to a number of more specific topics.

- First (and this is a standard question for modern history more generally): What is the balance between common themes and regional differences and persistences? How do some of the important global trends, such as the reduction of infant mortality, interact with more particular regional cultures and policies? 
- Second, and closely related: What is the balance between culture - beliefs and values and material realities in the modern history of death? This is not simply a tension between cultural persistence and shifts in living standards and medical practices, for cultures change as well - in part because of growing global contacts.

- And third: What, ultimately, is the ratio between gain and loss in the evolution of death over the past 250 years? And how much do answers vary according to different regional and group experience, or according to different chronological points even with a region?

\section{Other guidelines}

Four other preliminaries are vital in setting the framework for the sections that follow. The first returns us to the commitment to a global approach, but with several more specific concerns. To the greatest extent possible, this collection aims at global coverage, with a key section devoted to the experience of particular cultural regions and with global themes and comparisons embraced in thematic chapters as well.

But the fact is that, until recently, the most widely known histories of death have focused on western European and North American experience, and this is where much of the debate over death as a modern taboo has centered as well. And it is also true that, chronologically, some of the initial shifts in attitudes toward death, in medicalization, even in shifts in the nature of cemeteries began first in the West. The danger of a "West and the rest" approach to the history of death, with other regions simply following a Western lead, is quite real, particularly given ongoing disparities in levels of knowledge about regional particulars. The choice of the later 18th century as the point of departure for the modern history of death itself reflects some Western bias, not necessarily applicable to other key regions.

Complicating globalism still further is the fact that research on modern death history remains regionally imbalanced even outside the West. Work on East Asia, for example, has not been matched by comparable attention to patterns in India. Modern Mexico has won more attention that other parts of Latin America.

Yet the fact is that changes in death - particularly in the past hundred years - have touched virtually every society, and they involve far more than Western precedent or even the important differences in the timing of some key trends. For, as we have already suggested, different regions entered the modern period with very different specific cultures applied to death. Religious beliefs and practices varied widely. Different cultural baselines have easily combined with variations in timing to produce important distinctions in the ways death occurred and the ways death was managed, into the early $21 \mathrm{st}$ century. Capturing the mixture of variety and common experience and drawing comparative conclusions is no easy task. Awareness of the problems of Western overemphasis and regional imbalance, even in a book systematically committed to a genuinely global approach, offers a useful caution.

On a second key point, it is important to factor in as well the mixture of long-term trends and short-term adjustments that make up the modern history of death in any major region. There are some durable patterns, beginning in some cases in the later 18th century - even beyond key demographic themes like the decline of contagious disease. Thus, in the West, the 18th-century Enlightenment, deeply interested in prolonging life as part of human progress, kicked off a persistent interest in cutting death rates, and 
employing science in the process, that clearly lasts into the present day, and not only in the West. Recurrent interest in cryonics - preservation of life through freezing, in anticipation that in the future medical advances will further limit common causes of death - is on the one hand a very recent development (the first cryonics facility opened in the United States in 1976). On the other, it also reflects a longer-standing modern fascination with advancing longevity - an example of persistence over time, in other words.

But against important, sweeping trends, other developments have been more episodic, warning against oversimplifications about modern developments that will cut across the whole two-and-a-half-century span. It is particularly important not to rush to the most recent phases of the modern period - which feature, for example, the most striking advances in medical technology: an obvious corrective highlights attention to important and sometimes distinctive developments in the 19th and early 20th centuries, including the ongoing importance of epidemics. At the same time, current developments - for example, the growing concern about dealing with deaths of the elderly in contemporary Japan, or the growing individualism in funeral ceremonies in Europe - raise the possibility that we may be entering yet another crucial subperiod within the overall modern approach to death: an issue to which we will return.

There is a third key point, obvious but essential. In the history of death, as with other social phenomena, undeniably sweeping trends affect various groups in different ways, even within a single region. Poor people may experience death quite differently from the rich, despite the fact that, ultimately, both groups see a decline in traditional death rates. Different ethnic and religious groups deal with death variously in the modern period, particularly in multicultural societies like the United States. ${ }^{17}$ Gender patterns may vary in key cases as well. To take an obvious example, women traditionally faced particular fears about death in childbirth, though this gender distinction has clearly declined over time as maternal mortality rates have dropped.

The essays in this volume, collectively, aim at a balance between important chronological periods and the longer-term trends, and between general patterns and at least a number of specific group experiences - granting that many opportunities for even greater detail continue to emerge.

And the fourth and final preliminary: changes in the modern experience of death, whatever their chronological range, spill over into a wide variety of related topics. It is not easy to delimit what subtopics a modern history of death should include, without diluting a core framework.

For example, beginning with the United States Civil War, Americans, and soon many other people, evinced a new interest in trying to make sure that to the greatest extent possible the bodies of dead soldiers could be returned home for burial or at least assured a proper marker in organized cemeteries elsewhere. ${ }^{18}$ This was a new interest, facilitated of course by new methods of transportation and also by a revival of the practice of embalming. But it suggests that new concerns about death impinge significantly on military policies, with (on the whole) decreasing tolerance for faceless deaths in battle. On the other hand, changes in military technology steadily extended the capacity for causing death (including civilian death), another important conundrum in modern history.

Approaches to the death penalty changed, at least in some key regions. ${ }^{19}$ In the West, in the 19th century, traditional assumptions that some criminals should be put to death in public gave way to an intriguing preference for administering the death penalty in private a preference that predominates still today. Why did this change occur, and what does it say 
about larger attitudes toward death? Later, of course, in some regions but definitely not in all, public standards would further shift toward disapproval of the death penalty itself. Then there is the huge and intriguing issue of death and modern media. As attention to death has declined overall, modern media (beginning with movies) have made it possible to represent death, and particularly violent death, more widely than ever before. How do these developments relate to the more basic trends in dealing with death itself?

Other connections seem more direct but raise further interpretive issues. The modern history of suicide has its own dynamic, particularly as societies moved away from dealing with the issue as a sin and sought more psychological and sociological approaches and also strove to develop more explicit prevention efforts. ${ }^{20}$ Were there broader relationships between patterns and changes in suicide and other shifts in attitudes toward death? For example, some recent commentators, trying to explain the increase in United States suicide rates, have argued that suicide has lost some of its traditional stigma, making it easier to contemplate the act. This is not necessarily true (other Western societies are not experiencing a comparable rate increase), but it might relate to other ways in which references to death have become more casual.

On another front, what about deaths of domesticated animals? Modern societies, more urban, obviously encounter animal death less often than rural communities did and, increasingly, even manage to package meat in ways that pull people away from awareness of the deaths of the animals of origin. Were these changes significant, and again do they link to other developments in the shifting panorama of modern death. (And, of course, what about the deaths of pets, the one domain in which animal death has not been largely obscured? $)^{21}$

The chapters that follow treat many of these extensions in the modern history of death, but some connections surely deserve further attention in future. What, ultimately, is the range of impacts caused by the shifts in the nature and culture of death in modern history?

\section{Dealing with the history of death}

Death is not exactly a standard historical topic. Textbooks may briefly cover relevant changes in public health and medicine and offer a brief bow to death rates in the major wars, but they largely stay away from details and usually ignore key questions like the decline of death as a cultural focus. Modern aversion to death may make the subject less inviting, even for dispassionate historical scholars, than other subjects. This is a barrier that still warrants attention.

But the modern history of death has attracted considerable attention despite the barriers. And while the historiography on death initially retained an implicitly Western focus - again, an obvious caution here - the findings and basic questions involved have helped stimulate a wider comparative interest which increasingly (though not completely) modifies the dangers of a West-and-the-rest approach.

Most obviously, some scholars interested in celebrations of modern progress inevitably touched on changes in death as part of histories of public health or medical research. This optimistic approach has been revived recently by some of the commentary about social progress issued by nonhistorians such as Steven Pinker, who uses rising life expectancy levels and additional claims about declines in deaths by violence and war as central points in his hymn to modern life. ${ }^{22}$ 
In contrast, at key points, and most notably in the 1960s, the countercultural concerns, about the superficiality of consumer culture and the need to promote revisions in the modern approach to death, spurred historical efforts as well, beginning with a monumental effort by a French scholar, Philippe Ariès, who sought to use history to call attention to dangerous contemporary deficiencies. ${ }^{23}$ And the growing commitment of social and cultural historians to deal with all major aspects of the human experience inevitably encouraged interest in such topics as the rise of funeral homes or changes in the nature of cemeteries, even apart from any deeper involvement in debates over the meaning of modern life. The result of all this has been an impressive array of scholarship on the history of death, but also a somewhat episodic interest recent work on death history has not quite matched the range of work that emerged in the 1960s and 1970s.

Death is not, of course, an entirely new field for historians. Most obviously, histories of religion routinely discussed theological approaches to death, sometimes with attention to relevant rituals as well. The elaborate belief structures in the Abrahamic religions, and particularly Christianity and Islam, supported commentary on the ways death and divine judgments after death were imagined. Death may have been a slightly less central topic in Hinduism and Buddhism, where the emphasis on reincarnation offers a different kind of representation. However, the general point that major religions - particularly amid the decline of polytheism - emphasize attention to death and the nature of existence after death has long been a standard feature of religious history. ${ }^{24}$ This same applies, by extension, to efforts in art history that focused, for example, on the world's most famous representation of death, the crucifixion of Christ.

Demographic history - a more specialized interest - obviously has always dealt with death rates as a core part of its measurements of the past. ${ }^{25}$ Histories of disease inevitably treat the causes of death. This was particularly true for some groundbreaking treatments of epidemic disease, beginning with Hans Zinsser's pioneering and charmingly entitled work Rats, Lice and History and continuing with William McNeill's analysis of plagues. Medical history, similarly, had to deal with efforts to explain and treat causes of mortality. $^{26}$

Finally military history, including accounts of the great invasions and battles, has always touched on death. Efforts to calculate casualty rates have been a standard part of this genre. So too is the realization that, until very recent times, more military deaths resulted from disease than from actual combat. ${ }^{27}$

There is ample precedent, in sum, and wide-ranging data on the history of death from a variety of standard subject categories. This said, it remains true that an emphasis on death itself, and its social and cultural role, is a more recent interest that in turn has set the stage for a wider range of historical analysis and understanding.

More extensive inquiry could flow from several of the approaches that had already been established, as historians, under the general impulse of social history, began to probe the human experience more widely. Demographic historians explored further data sources concerning mortality rates and, in some cases, expanded from this to wider analysis of death in the past. ${ }^{28}$ By the 1980 s, an interest in the history of the life cycle emerged, following from sociological work on the same subject, and death obviously figured in this approach. ${ }^{29}$ Research on reactions to disease and epidemics expanded as well, with some particularly rich studies of the Renaissance period. ${ }^{30}$ 
Not surprisingly, some of the most striking breakthroughs reached out from religious history. In the United States, where work on colonial social history and family life became particularly rich by the 1960s, David Stannard explored American, and especially Puritan, death culture explicitly, in the belief that this was a particularly vital part of the whole Puritan experience. The result - two books in the 1970s - was among the first of what might be called the new histories of death, where the subject moved front and center. ${ }^{31}$

French scholarship, ultimately even more elaborate, similarly moved out from the premise that religious beliefs and practices must be examined in new ways, toward trying to figure out what they actually meant in the lives (and deaths) of ordinary people. Though focused more on fear of death than death itself, a series of works by Jean Delumeau explored the ways Catholicism built on anxieties about death in the popular mind, until the more secular culture of the Enlightenment promoted popular alternatives. ${ }^{32}$ Even more important was the work of Michel Vovelle, in the early 1980s, who focused more strongly on the results of secularization on beliefs and practices concerning death in early modern France. ${ }^{33}$ This kind of analysis - still significant today - easily inspired additional work on death in Europe and elsewhere: death became a standard part of the agenda of a number of social and cultural historians.

Historical work on death received a further boost - also by the 1970s - from the growing current of dismay over the inadequacies of contemporary death practices in the Western world. Already in the 1960s sociologist Geoffrey Gorer introduced the notion that death had become a modern taboo, with harmful consequences for the larger quality of life. This current was taken further in the famous work by Elisabeth Kubler-Ross, with her work on death and dying in 1969, and then Ivan Ilich, whose book, Medical Nemesis (1975) railed against the harmful control that doctors had assumed over the experience of death. ${ }^{34}$ These studies, broadly speaking part of the cultural challenge of the 1960s, prompted wide attention - including a rash of new college courses on death and dying - and inevitably spurred responses in a number of disciplines, including history. For, while the new perspective was not explicitly historical, it strongly implied that past practices and beliefs concerning death were preferable to those that had assumed ascendancy at some point in modern history - and historians might easily take up the challenge either to explore premodern experience more elaborately or to work on the evolution (and limitations) of more modern reactions.

Philippe Ariès provided the most powerful example of using history to critique modern approaches to death: his book. The Hour of Our Death, appeared in 1981, with a follow-up volume four years later. Ariès powerfully argued for the harmful effects of the decline of religion in dealing with death - noting, interestingly, that the United States suffered a bit less from this deterioration because of the continued commitment to religious practices. Aries' work had considerable public impact, and it too helped inspire additional historical study. ${ }^{35}$

With this base established, the 1980s and 1990s saw a proliferation of significant historical work on death. Continued interest attached to the impact of religious change and continuity, particularly within Protestantism. The role of the Enlightenment and secularization also inspired considerable study. Work on the United States expanded to include growing attention to cemeteries and funeral practices - including the rise of the funeral home as an American institution - with research on other topics as well, such as the preoccupation with recovering the bodies of dead soldiers after the Civil War. Studies of 
subgroup practices, and particularly the extraordinary history of the African-American funeral, also proliferated. American work was diverse, but some of it continued to explore the notion that the national approach toward death might continue to embrace some distinctive features, at least compared to developments in modern Europe. ${ }^{36}$

While it receded somewhat as an explicit theme, the need to confront claims about deterioration in the modern experience did not disappear. Most notably, a leading Swiss demographic historian offered a sweeping cultural interpretation in 1996, contrasting how our ancestors "coped" with an everyday life surrounded by death, with the stark claim that modern life had become impossibly hard. And a major contribution by Michael Lesy, dealing with the removal of death from daily life and the rise of professionals - not only doctors and embalmers, but coroners and even slaughterhouse workers - highlighted some related themes. ${ }^{37}$

Work on the history of death continued to diversify in the early 21 st century, as in an ambitious effort to deal with the treatment of human remains over a long span of time. ${ }^{38}$ Growing interest in the history of emotion, as a subfield, has supported more explicit studies on the history of grief and, to a lesser extent, guilt and fear as part of the experience of and reaction to death in modern history. ${ }^{39}$

Further, while not always systematically historical, a major literature has emerged in the past two decades around the question of whether a new, "postmodern" approach to death is taking shape, departing from the major themes of the 20th century. Arguments here embrace the growing individuality of many funerals, with innovations including the use of popular music; the rise of palliative care, modifying somewhat the dominant "medicalization" approach; new media attention to victims of war; and public mourning for the deaths of celebrities. The result adds to, and challenges, existing historical models.

On another front, and at least as significant, the expansion of world history has called attention to the importance of dealing with changes and continuities in the nature of death in societies in Africa, Asia and Latin America. Here, the past quarter-century has seen a variety of studies, though more around specific topics - like the impact of death on Mexican political culture or the modern Buddhist approach to death - than full regional surveys. ${ }^{40}$ While it might be an exaggeration to claim an explosion of interest in death in the major areas of the world outside the West, there is no question that scholarship has begun to expand significantly over the past 25 years - the latest, and vital, addition to existing historiography, and one that bears on several of the basic questions about the modern history of death while also enhancing the vital comparative dimension. The surge of work on death history in a variety of major societies, though distressingly uneven, has on the whole bypassed the interest in modern taboos - which may turn out to be a disproportionately Western issue. But it has raised other important connections, such as the role of representations of death in political life (a linkage actively explored both in Mexico and for parts of Africa).

Certainly, while a host of important topics remain to be covered in the history of death, a rich foundation has been established, particularly over the past half-century. Death is, almost by definition, an elusive topic. It involves beliefs and fears that are not always articulated. It expands into a variety of human practices - not just funerals, but wills, lessons to children and national memorials. There will always be opportunity for additional work and further analysis, even in topic areas that have been covered by some of the pioneering studies. Certainly the hovering question - about whether death is a cultural Achilles heel in contemporary society - legitimately inspires continued attention. 
And it seems probable that an interest in death and its history will gain additional momentum over the next decade or two, simply because the sheer quantity of death will almost certainly increase. As a growing number of societies, from Europe to China to the United States and even Latin America, experience unprecedented population ageing, questions about how to handle death, how to adjust to the growing importance of Alzheimer's and dementia in overall mortality, what level of resources to expend on treatment and ritual, will become increasingly pressing - and history, appropriately, must play a role in these inescapable assessments. Already in Japan, issues about the adequacy of attention to death have become an urgent part of the social agenda, with the discovery of growing numbers of elderly dying alone and untended. ${ }^{41}$ What we already know about the history of death must surely be applied to issues of this sort, and there will be a spur to further inquiry as well.

\section{Structure of the book}

After this first, introductory overview, the book is divided into three major parts, each with a brief orienting preface. Part I explores a number of general topics, where some overall patterns and issues can be identified - as in changes to the causes or locations of death. The chapters here do not always capture a fully global approach, if only because of limitations in existing literature, but the focus is on widespread patterns of change and continuity in both the material and the cultural features of death.

Part II offers regional histories, centered on developments over a considerable portion of the modern period if not always the whole span. Not every region is fully covered, and some are represented mainly by a particular country or subregion - again, reflecting disparities in the state of the field and the fact that some regions are more diverse and challenging than others.

Part III, finally, offers a mix of a number of more specific studies, expanding the range of regional and topical coverage and considering several recent developments as well. Here, there is no claim to comprehensive treatment. The goal is to represent some of the variety and vitality of the modern history of death as a field while inviting the identification of yet additional topics or the extension of some of the particular interests to additional regions and chronologies.

\section{Ongoing change}

A final chapter aims at extending a sense of the modern history of death into issues in the future. For modern death has involved continual adaptation, as groups and societies seek to adjust more fully to the dramatic changes of the past century or two. History, including very recent history, offers a vital means of identifying and evaluating the initiatives involved, even though we must await further experience to be able to distinguish the transient from the significant. Directions of change vary: many new initiatives work to extend the death-fighting interest, most obviously through new medical approaches or, for a handful of wealthy individuals, experiments in cryonics. But other innovations aim at enhancing rituals or improving awareness of death - working, in other words, to compensate for modern limitations.

Even a handful of contemporary examples can demonstrate the impressive vitality of contemporary approaches and their connection, or potential connection, to larger modern 
patterns of change. In 1995, for example, the phenomenon of funeral strippers first emerged in Taiwan. Having some young women take off all or part of their clothing during a burial procession reflected an old desire - to have as many people as possible participate in the funeral ceremony, in order to demonstrate appropriate respect to the dead - now complicated by the fact that so many relatives lived too far away to attend. Already professional mourners had been employed to swell the numbers, but now, though controversially, some additional inducements were sometimes brought to bear as well. ${ }^{42}$

Also in the mid-1990s a new phenomenon of "death tourism" began to be identified, whereby tourists (mainly though not exclusively Western) took trips to places like Auschwitz or the killing fields of Cambodia - sites of mass death in the past - primarily in search of a new way to contemplate their own death, to peel away from the more superficial concerns of contemporary culture. ${ }^{43}$ A further innovation sought to reduce the distance from death: a series of coffin clubs sprang up in New Zealand, aimed at crafting attractive, personalized caskets well in advance of demise. Yet another intriguing venture: early in 2018 a new online app became available, called WeCroak, that sought to implement a Bhutanese belief that a happy life is possible only through constant awareness of mortality - subscribers received five calls a day to remind them that they will die, spiced by a brief bit of accompanying wisdom. ${ }^{44}$

And these developments occurred on top of some more familiar recent trends, such as new kinds of public mourning at the deaths of certain groups or individuals, or the precedent-shattering rise of cremation in Western and East Asian nations, or the complex and again largely recent discussion of assisted suicide. ${ }^{45}$ Death inspires a host of innovations to fit the human constant into a rapidly changing historical context. The modern history of death offers a fundamental framework for assessing the ongoing process, as well as a vital window on the human past.

\section{In mid-crisis}

This book was conceived and the chapters completed before the 2020 Covid-19 crisis hit, and it would be rashly premature to predict where this epidemic will fit in the larger history of death. But there is equally no question about the importance of historical perspective even as people try to grasp what is happening around them, including the massive disruption to rituals for mourning and burial. There are obvious echoes from the past, applying studies like those of Samuel Cohn (Chapter 2): plagues commonly invite efforts at scapegoating and see the wealthy attempting to gain special advantage, features clearly on display early in the 2020 pandemic - along with acts of kindness and generosity. The groups that invoke divine protection, whether in Mississippi or Mexico or Pakistan, recall the past quite directly. But there is room as well to consider how the contemporary crisis reveals changes in the approach to death: the increasing reliance on medical redress and the hospital system, the political pressures that result from modern expectations about controlling death. The fascinating debate about trade-offs between death and economic normalcy has novel overtones as well. The crisis has even brought forth renewed claims about modern cultural deficiencies in the face of death, the refusal to contemplate it adequately - claims discussed in several of the essays that follow.

The point is that the modern history of death has much to offer as humanity faces an unexpected challenge and that there is a rich historiography available. Small wonder that 
the media have been making abundant use of historical example, both to help assess the magnitude of what is going on around us and to provide more specific policy guidelines (as in the effectiveness of social distancing in response to the 1918/19 influenza pandemic). It would be rash, of course, to expect a durable surge in historical interest: the influenza pandemic also reminds us how fleeting a crisis can be, how easily forgotten though the context, shaped by the massive deaths in World War I, may be different from our own. At the moment at least, the history of death has unexpected currency, providing an unwelcome relevance for many of the discussions that follow.

\section{Notes}

1 E.A. Wrigley, Population and History (London: Weidenfeld and Nicolson, 1969); Roger Schofield, The Decline of Mortality in Europe (New York: Oxford University Press, 1991); Ansley Coale, "Age Patterns and Time Sequence of Mortality in National Populations with the Highest Expectation of Life at Birth," Population and Development Review 22 (1) (1996): 127-135.

2 Peter Conrad, The Medicalization of Society: On the Transformation of Human Conditions into Treatable Disorders (Baltimore: Johns Hopkins Press, 2007); Donna Dickenson, Death, Dying, and Bereavement (Thousand Oaks, CA: Sage Publications, 2000); Peter N. Stearns, Revolutions in Sorrow: The American Experience of Death in Global Perspective (Boulder: Paradigm Publishers, 2007).

3 Alain Corbin, The Foul and the Fragrant: Odor and the French Social Imagination (Cambridge, MA: Harvard University, 1986); Gary Laderman, Rest in Peace: A Cultural History of Death and the Funeral Home in Twentieth-Century America (New York: Oxford University Press, 2003).

4 Jesse Kauffmann, Elusive Alliance: The German Occupation of Poland in World War I (Cambridge, MA: Harvard University Press, 2015); A.C. Grayling, Among the Dead Cities: The History and Moral Legacy of the WWII Bombing of Civilians in Germany and Fapan (New York: Walker \& Co., 2007); Matthew White, "Statistics of War, Oppressions and Atrocities of the NineteenthCentury," www.necrometrics.com/wars 19c.html (accessed January 7, 2019).

5 Arthur Kleinman, Patients and Healers in the Context of Culture: An Exploration of the Borderland Between Anthropology, Medicine, and Psychiatry (Berkeley: University of California Press, 1981).

6 Azhar Hussain, Syed Ali, Madiha Ahmed, and Sheharyar Hussain, "The Anti-Vaccination Movement: A Regression in Modern Medicine," Cureus 10 (7) (2018): e2919.

7 Margaret Brindle and Gerald Salancik, "The Social Construction of an Institution: The Case of Brain Death and Organ Transplant Industry," in John Wagner, ed., Advances in Qualitative Organization Research (Stamford, CT, 1999), 1-36; Kazumasa Hozhino, "Legal Status of Brain Death in Japan: Why Many Japanese do not Accept 'Brain Death' as a Definition of Death," Bioethics 7 (2/3) (1993): 234-238.

8 Jean Delumeau, Sin and Fear: The Emergence of a Western Guilt Culture, 13th-18th Centuries (New York: St. Martin's Press, 1990).

9 Ezekiel J. Emanuel and Linda L. Emanuel, "The Promise of a Good Death," Lancet 351 (May, 1998): SII21-SII29.

10 Google Ngram Viewer is a search application that allows one to measure the frequency of particular terms or words in the Google Books database. While in some ways problematic and obviously not a complete representation, the tool is a helpful way to assess cultural trends and changes. The decline in the rate of references to death in American English outstrips the change in English more generally - an interesting comparative challenge in a society where religion has remained particularly important.

11 Perry Nodelman, The Hidden Adult: Defining Children's Literature (Baltimore: Johns Hopkins University Press, 2008); Lois Rauch Gibson and Laura M. Zaidman, "Death in Children's Literature: Taboo or Not Taboo?" Children's Literature Association Quarterly 16 (4) (Winter 1991): 232-234; Dinah Seibert and Judy C. Drolet, "Death Themes in Literature for Children Ages 3-8," Fournal of School Health 63 (2) (1993): 86-90. The Seibert and Drolet study, for example, notes how rarely feelings about death are discussed even when the topic is mentioned at all. 
12 Cas Wouters, "The Quest for New Rituals in Dying and Mourning: Changes in the We-I Balance," Body \& Society 8 (1) (2002): 1-27.

13 Elisabeth Kübler-Ross and David Kessler, On Grief and Grieving: Finding the Meaning of Grief Through the Five Stages of Loss (New York: Scribner, 2005).

14 Joanna Bourke, Fear: A Cultural History (Emeryville, CA: Shoemaker \& Hoard, 2005).

15 Geoffrey Gorer, "The Pornography of Death," in Death, Grief and Mourning in Contemporary Britain (London: Cresset, 1965), 169-175.

16 Arthur Erwin Imhof, Lost Worlds: How Our European Ancestors Coped with Everyday Life and Why Life is so Hard Today (Charlottesville: University of Virginia Press, 1996).

17 Suzanne E. Smith, To Serve the Living: Funeral Directors and the African American Way of Death (Cambridge, MA: Harvard University Press, 2010); Julie-Marie Strange, "She Cried a Very Little': Death, Grief, and Mourning in Working-Class Culture, c. 1880-1914," Social History 27 (2) (2002): 143-161.

18 Drew Gilpin Faust, This Republic of Suffering: Death and the American Civil War (New York: Vintage Books, 2008).

19 David Jacobs and Jason T. Carmichael, "The Political Sociology of the Death Penalty," American Sociological Review 67 (2002): 109-131; Amnesty International, The Death Penalty in America; Study Guide (2006); Michael Radelet and Marian Borg, "The Changing Nature of Death Penalty Debates," Annual Review of Sociology 26 (2000): 43-61; Julia Eckert, "Death and the Nation: State Killing in India," in Austin Sarat and Christian Boulanger, eds., The Cultural Lives of Capital Punishment (Stanford, CA: Stanford University Press, 2005), 195-218; Pieter Spierenburg, Violence and Punishment: Civilizing the Body Through Time (Malden, MA: Polity Press, 2013); V.A.C. Gatrell, The Hanging Tree: Execution and the English People, 1770-1868 (New York: Oxford University Press, 1994).

20 Marzio Barbagli, Farewell to the World: A History of Suicide (New York: John Wiley \& Sons, 2015); Special Issue: The Politics of Suicide: Historical Perspectives on Suicidology before Durkheim, Fournal of Social History 46 (3) (Spring 2013): 607-816; George Minois, History of Suicide: Voluntary Death in Western Culture (Baltimore: Johns Hopkins University Press, 1999); Susan Morrissey, Suicide and the Body Politic in Imperial Russia (Cambridge, UK: Cambridge University Press, 2007); Howard Kushner, American Suicide (Brunswick, NJ: Rutgers University Press, 1991); Olive Anderson, Suicide in Victorian and Edwardian England (New York: Oxford University Press, 1987).

21 Keith Thomas, Man and the Natural World: Changing Attitudes in England, 1500-1800 (New York: Oxford University Press, 1996); Barbara R. Ambros, Bones of Contention: Animals and Religion in Contemporary Fapan (Honolulu: University of Hawai'i Press, 2012).

22 Steven Pinker, Enlightenment Now: The Case for Reason, Science, Humanism, and Progress (New York: Penguin Books, 2019).

23 Philippe Ariès, The Hour of Our Death: The Classic History of Western Attitudes Toward Death Over the Last One Thousand Years (New York: Vintage Books, 2008).

24 Harold G. Coward, Life after Death in World Religions (Maryknoll, NY: Obris Books, 1997).

25 Ansley Johnson Coale, Growth and Structure of Human Populations: A Mathematical Investigation (Princeton: Princeton University Press, 2015).

26 Hans Zinsser, Rats, Lice and History (New York: Routledge, 2007); William H. McNeill, Plagues and People (Garden City, NY: Anchor Books, 1977).

27 Michael Clodfelter, Warfare and Armed Conflicts: A Statistical Encyclopedia of Casualty and Other Figures, 1492-2015 (Jefferson, NC: McFarland \& Company, 2017). Various scholars have tried to compare modern war deaths to the more remote pasts; despite the devastation of the two world wars, some earlier conflicts apparently surpass them in sheer mortality - though most of the conflicts stretched over longer periods of time.

28 Maris Vinovskis, "Death," in Mary K. Clayton, Elliott J. Gorn, and Peter W. Williams, III, eds., Encyclopedia of American Social History, eds. (New York: Charles Scribner's Sons, 1993), 2063-2070.

29 Tamara K. Hareven, "Aging and Generational Relations: A Historical and Life Course Perspective," Annual Review of Sociology 20 (1994): 437-461.

30 Samuel Kline Cohn, The Black Death Transformed: Disease and Culture in Early Renaissance Europe (London: Bloomsbury Academic, 2003); Sharon T. Strocchia, Death and Ritual in Renaissance 
Florence (Baltimore: Johns Hopkins University Press, 1992); Richard J. Evans, Death in Hamburg: Society and Politics in the Cholera Years (New York: Penguin Books, 2005).

31 David E. Stannard, American Holocaust: The Conquest of the New World (New York: Oxford University Press, 1992); Stannard, Death in America (Philadelphia: University of Pennsylvania Press, 1975).

32 Delumeau, Sin and Fear, see also John McManners, Death and the Enlightenment: Changing Attitudes to Death Among Christians and Unbelievers in Eighteenth-Century France (New York: Oxford University Press, 1985).

33 Michel Vovelle, L'heure du Grand Passage: Chronique de la Mort (Paris: Gallimard, 1993); Vovelle, La Mort et l'Occident: de 1300 à Nos Jours (Paris: Gallimard, 200); see also Pierre Chanu, La Mort à Paris. More recent explorations include Joachim Whaley (ed.), Mirrors of Mortality: Studies in the Social History of Death (New York: Routledge, 1981); Peter Jupp and Glennys Howarth (eds.), The Changing Face of Death: Historical Accounts of Death and Disposal (London: MacMillan Press, 1997); Ralph Anthony Houlbrooke, Death, Religion, and the Family in England, 1480-1750 (New York: Oxford University Press, 1998); Mary J. Dobson, Contours of Death and Disease in Early Modern England (New York: Cambridge University Press, 2002); Ellen Badone, The Appointed Hour: Death, Worldview, and Social Change in Brittany (Berkeley: University of California Press, 1989).

34 Gorer, "The Pornography of Death"; Kübler-Ross and Kessler, On Grief and Grieving; Ivan Illich, Medical Nemesis: The Expropriation of Health (New York: Pantheon, 1982).

35 Ariès, The Hour of Our Death; Ariès, Western Attitudes toward Death: From the Middle Ages to the Present (Baltimore: Johns Hopkins University Press, 1975); Ariès, Images of Man and Death (Cambridge, MA: Harvard University Press, 1985).

36 See also a comparable effort on Australia: Pat Jalland, Changing Ways of Death in TwentiethCentury Australia: War, Medicine, and the Funeral Business (Sydney: UNSW Press, 2006).

37 Imhof, Lost Worlds; Michael Lesy, The Forbidden Zone (New York: Farrar, Strauss and Giroux, 1987).

38 Thomas W. Laqueur, The Work of the Dead: A Cultural History of Mortal Remains (Princeton: Princeton University Press, 2015).

39 Paul C. Rosenblatt, Bitter, Bitter Tears: Nineteenth-Century Diarists and Twentieth-Century Grief Theories (Minneapolis: University of Minnesota Press, 1983); Stearns, Revolutions in Sorrow; Barbara H. Rosenwein, Generations of Feeling: A History of Emotions, 600-1700 (New York: Cambridge University Press, 2016).

40 Michael Hviid, Jacobsen, “'Spectacular Death' - Proposing a New Fifth Phase to Philippe Aries' Admirable History of Death," Humanities (2016); Kate Beveridge, Vigor Mortis: The End of the Death Taboo (London: Profile, 2002); Anthony Elliott, The Mourning of Fohn Lennon (Berkeley: University of California Press, 1998); Tony Walter, Mourning for Diana (Oxford: Berg, 1999); Ruth McManus, Death in a Global Age (London: Palgrave MacMillan, 2013); Tony Walters, The Revival of Death (London: Routledge, 1994). See also Allan Kellehear, A Social History of Dying (New York: Reaktion Books, 2014); William Spellman, A Brief History of Death (Oxford: Blackwell, 2005); Claudio Lomnitz-Adler, Death and the Idea of Mexico (New York: Zone Books, 2008).

41 The problem of Kodokushi, or dying a lonely death, was first identified in the 1980s. Hikau Suzuki (ed.), Death and Dying in Contemporary Japan (New York: Routledge, 2013); Anne Allison, Precarious Fapan (Durham: Duke University Press, 2013).

42 Marc Moskowitz documentary film, Dancing for the Dead: Funeral Strippers in Taiwan, 2011.

43 Sometimes called "dark" or "thano" tourism, partly to distinguish it from travel in search of places where assisted suicide is legal. Other sites include the Nanjing massacre museum, Chernobyl, Ground Zero in New York and several European castles. See John Lennon and Malcolm Foley, Dark Tourism: The Attraction of Death and Disaster (London: Thomson, 2006).

44 Kelly Rossetto, P.J. Lannutti, and Elena C. Strauman, "Death on Facebook: Examining the Roles of Social Media Communication for the Bereaved," Fournal in Social and Personal Relationships 32 (7) (2014): 974-994, for exploration of how social media are affecting death reactions.

45 On new patterns of public grief, see Adrian Kear, Mourning Diana: Nation, Culture, and the Performance of Grief (New York: Routledge, 1999). 


\section{PART I}

\section{GENERAL PATTERNS AND GONNEGTIONS}

The chapters in this part deal with major patterns of death in modern world history that cut across geographic regions, addressing death as a physical and a cultural phenomenon alike. Topics range from basic material trends - shifts in death rates and causes and the modern evolution of epidemics - to connections with death in domains such as capital punishment or trends in murder and suicide, exploring the complex patterns of violence in the modern history of death, to key cultural and ritual features, such as the design of cemeteries or the impact of major war on commemoration. Several modern settings have generated distinctive attitudes toward death - of others and often of self - on the part of terrorists. A final chapter, focused on debates over death as a modern taboo, touches on fundamental cultural and emotional issues, inviting juxtaposition with the analyses of changes in the physical nature of death and aspects of modern death policies.

While global in implication, the chapters vary in their actual geographical coverage, reflecting different stages of research on the subject and in some cases, demonstrable regional variants - such as the unusual conditions that generate the terrorist approach to death.

Collectively the chapters also raise issues about connections. The initial essay on basic trends on mortality rates and causes centers on the huge physical changes in the nature of death over the past 250 years, but the patterns also reflect prior shifts and expectations and policies concerning death and its prevention, ultimately on a global basis. Cultural issues and outcomes, in other words, surround the modern demographic structures.

Other connections invite attention. Do change and variations in murder rates, for example, have much to do with other facets of death in the modern world, or are they mainly linked to factors such as judicial policy or cultures of honor? How do the marked shifts in the incidence of death penalties (and the ways such penalties are administered) relate to broader trends in attitudes toward death? Interpretations of suicide have obviously changed greatly since the eighteenth century; is this an isolated development or does it express other recalibrations in thinking about death and preventions of death? New commemorations of war reflect modern types of casualties but also wider cultural shifts toward the death of individuals. The subject of death in war deserves attention not only in its own right but for its implications for other aspects of the modern death 
experience (a theme picked up in several later chapters as well). How have changes in cemetery design reflected - or anticipated - shifts in the material realities of death?

Overall, the essays in this part particularly invite attention to the close and mutual links between changes in the physical features of death and the cultural and even emotional impacts of death as part of the modern condition. 


\title{
PATTERNS OF DEATH, 1800-2020 \\ Global rates and causes ${ }^{1}$
}

\author{
Romola Davenport
}

This chapter provides a brief introduction to mortality patterns over the last two centuries from a global perspective. The period witnessed enormous and ubiquitous rises in life expectancy at birth, from a global average of perhaps 30 years in 1800 to an estimated average of 72.6 years in $2019 .^{2}$ Remarkably, these changes took place despite a perhaps seven- to eight-fold increase of the world's population, from around 1 billion in 1800 to 7.7 billion in $2020,{ }^{3}$ and in the context of unprecedented increases in the speed and frequency of transmission of infectious diseases as a consequence of urbanization, colonization, technological change, and the globalization of trade. This chapter will outline the broad geography and chronology of changes in patterns of mortality and will introduce debates regarding the role of economic growth and those of medicine and states. It concludes with a discussion of recent and future trends.

The wide chronological and geographical reach of the chapter necessitates an immediate caveat. For much of the world, we know very little about mortality rates or levels for national populations before the mid-twentieth century at the very earliest. The United Nations began to publish estimates of mortality rates and data on causes of death for member states in 1950; however, these series are very incomplete and in many cases rely on estimates from rather fragmentary data, even now. For most of sub-Saharan Africa, scholars and public health systems remain reliant on epidemiological surveillance centers and international survey data, and even the largest states, India and China, still use sample registration and surveillance systems in lieu of complete registration of all deaths by age and cause. ${ }^{4}$ Given these limitations regarding national patterns and trends, we naturally know even less about health and mortality amongst subgroups within national populations, and about the heterogeneity of experiences in historical societies. This chapter therefore offers a rather impressionistic and selective view of the profound changes in mortality patterns that have occurred in the last two hundred years.

\section{Ages and stages}

In the broadest terms, what the economist and demographer Richard Easterlin dubbed the 'Mortality Revolution' was predominantly a function of enormous reductions in infectious disease mortality. ${ }^{5}$ These diseases had their greatest impacts on young children and young adults, and therefore mortality improved first and fastest in these age groups. 
Since 1800 death has been ever-increasingly confined to the extremes of life, in early infancy and old age, and infectious diseases have given way to non-communicable diseases, especially cancers, strokes and cardiovascular diseases, and, increasingly, dementia, as more and more people live to the ages where these diseases predominate. Latterly, improvements in the prevention and treatment of non-communicable diseases have improved survival even at the oldest ages, further extending life expectancy. These patterns have, however, unfolded with very different chronologies in different parts of the world, and with some notable reversals.

Figure 1.1 presents this mortality transition in stylized form for the population of England and Wales. Panels a and b represent the situation in 1841. By 1841, as discussed below, the English population was already free of famine, and major mortality crises were extremely rare. Nonetheless, life expectancy at birth was only 42 years, and infectious diseases accounted for nearly half of all deaths. Panel a illustrates the chance of survival to each age, using a hypothetical population of 1,000 people. We have to imagine that these people were all born at the same time and lived their lives with the mortality rates of the English and Welsh population in 1841 (this is not what actually happened to those born in 1841, most of whom were subject to substantial improvements in prevailing mortality rates during their lives). This type of plot is termed a 'survivorship curve', and shows the survivors of our hypothetical population, or cohort, at each age. Of the 1,000 babies born in 1841, 138 would have been dead before their first birthday. For those who survived infancy, the risk lessened; however, a quarter of all those born would not survive to age 5 . By age 47 , half would be dead.

Panel b shows the distribution of these deaths by age, in 1841. Deaths were heavily concentrated in infancy (the first year of life) and early childhood. However, deaths in adulthood were also relatively high at all adult ages. This contrasts markedly with the situation a century later. By 1950 almost everyone born into our hypothetical cohort could expect to live to adulthood, and half would survive to 74 . Deaths had become concentrated overwhelmingly in late adulthood. The absolute numbers of deaths were higher in late adulthood than in 1841, not because death rates were higher at these ages in 1950, but because a far higher proportion of the cohort now survived to these ages.

Comparison of panels a and c illustrates what has been termed the 'rectangularization of mortality'. This is the shift in the pattern of survival from a fairly constant downward curve by age, as in panel a, to a more rectangular form, where most people survive until late age, into their $70 \mathrm{~s}$ or $80 \mathrm{~s}$, and there is a compression or a concentration of mortality into these age groups. Panels e and f illustrate subsequent changes in the mortality patterns of affluent populations since the mid-twentieth century. Broadly, there has been a further 'rectangularization', and deaths are even more concentrated at the oldest ages. However there has also been a shift of the whole survival curve to older ages. That is, not only do most people survive to later ages (over half of our hypothetical cohort would live to 85, under the mortality rates of 2016), but the age at which most of the population has died has shifted to older ages.

Figure 1.1 also shows life expectancy measures for the population of England and Wales in 1841, 1950, and 2016. Critically, life expectancy at birth is not the age by which most of the population would be dead, as is sometimes assumed. Rather, it is the average age at death. This means that even in populations with relatively low life expectancies, a substantial proportion of adults lived into their sixties and seventies. Indeed, as 


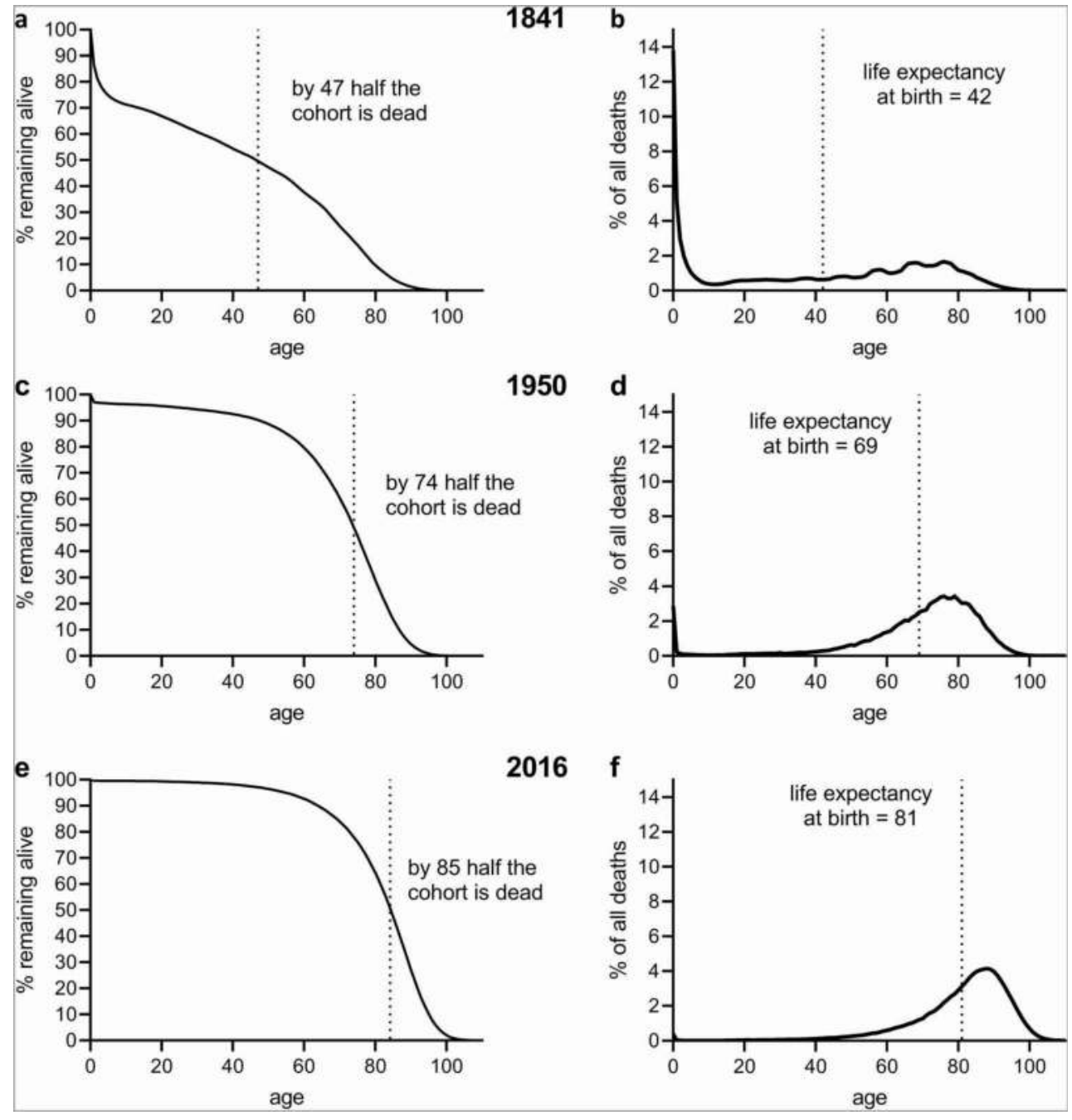

Figure 1.1 Distributions by age of survivors (a, c, e) and deaths (b, d, f) in a synthetic cohort subject to the death rates prevailing in the population of England and Wales in 1841, 1950, and 2016.

Source: Human Mortality Database (University of California, Berkeley), and Max Planck Institute for Demographic Research (Germany), www.humanmortality.de (downloaded on January 2, 2020).

can be seen in Figure 1.1, the average age at death is usually lower than the age to which half the population could expect to survive, because the former figure takes into account how many years people lived. High death rates in childhood lower the average age at death because they contribute only very small values to the sum of ages at death. Life expectancy is calculated using hypothetical populations, as in our example, instead of actual populations because the average age at death in real populations is affected by the age structure of the population. The age structures of populations, and therefore the average age of death, can vary very considerably over time and between populations for 
reasons other than prevailing mortality rates, including historical changes in fertility, mortality, and age-specific migration rates.

Figure 1.1 depicts the very profound changes in mortality patterns that occurred in the population of England and Wales over the last 170 years. The rest of the chapter considers the processes underlying these trends, and how widespread and uniform these processes were globally.

\section{Global patterns, an overview}

How ubiquitous has this 'mortality revolution' been? Figure 1.2a shows estimates of global average life expectancy since 1800, together with life expectancies for England and Sweden. England and Sweden were forerunners in mortality improvements and in the reporting of mortality statistics. The main pattern is clearly one of enormous improvements in survival. These started first in Europe and amongst the settler populations of North America, Australia and New Zealand in the nineteenth century (or even the mid-eighteenth century, in some cases) and extended to a small number of Latin American and Caribbean countries by the late nineteenth and early twentieth centuries. ${ }^{6}$ On the other hand, it is likely that mortality rose in the nineteenth century in some populations that had remained relatively isolated from global disease pools before this period, including Japan, the indigenous populations of North America, Australasia and the Pacific, and parts of tropical Africa. ${ }^{7}$

There is evidence for fairly widespread reductions in mortality globally after c.1920, and by the 1940s mortality declines were apparently underway in a wide variety of settings. The evidence for this is largely indirect and rests on the assumption that the very high rates of population growth witnessed in most of the world by this date (producing an estimated global rate of c. 1.5 per cent per annum by 1950) were driven at least in part by falling mortality, as well as rising fertility. ${ }^{8}$ These trends may have been interlinked. Where mortality declines were associated with improving adult and maternal health, then this may have resulted in increases in fecundity (the potential to conceive) and fertility.

Gains in life expectancy gathered speed in the second half of the twentieth century, especially in poorer countries. By the early 1950s, when (often rather fragile) statistics became available for many UN member states, the picture was one of enormous disparities in mortality. According to an elegant analysis by Chris Wilson, the world's population was divided (unequally) between poor countries with low life expectancies (averaging around 35-39 years) and rich or communist Eastern bloc countries with average life expectancies of 65-69 years (Figure 1.3a). ${ }^{9}$ That is, the distribution of life expectancies was fairly bimodal (two-peaked), with very little overlap between the two groups. Within the space of 25 years, however, the picture had changed enormously. By the late 1970 s only a very small percentage of the world's population lived in states with average life expectancies below 40 years, and nearly 60 per cent of the global population now lived in states with life expectancies of 60 years or more (Figure 1.3b). By the twenty-first century there had been an enormous and almost ubiquitous convergence of life expectancies in rich and poor countries (Figure 1.3c). The United Nations estimated that the average life expectancy of the global population in 2019 was 72.6 years, a higher figure than that attained by any national population in $1950 .^{10}$ 

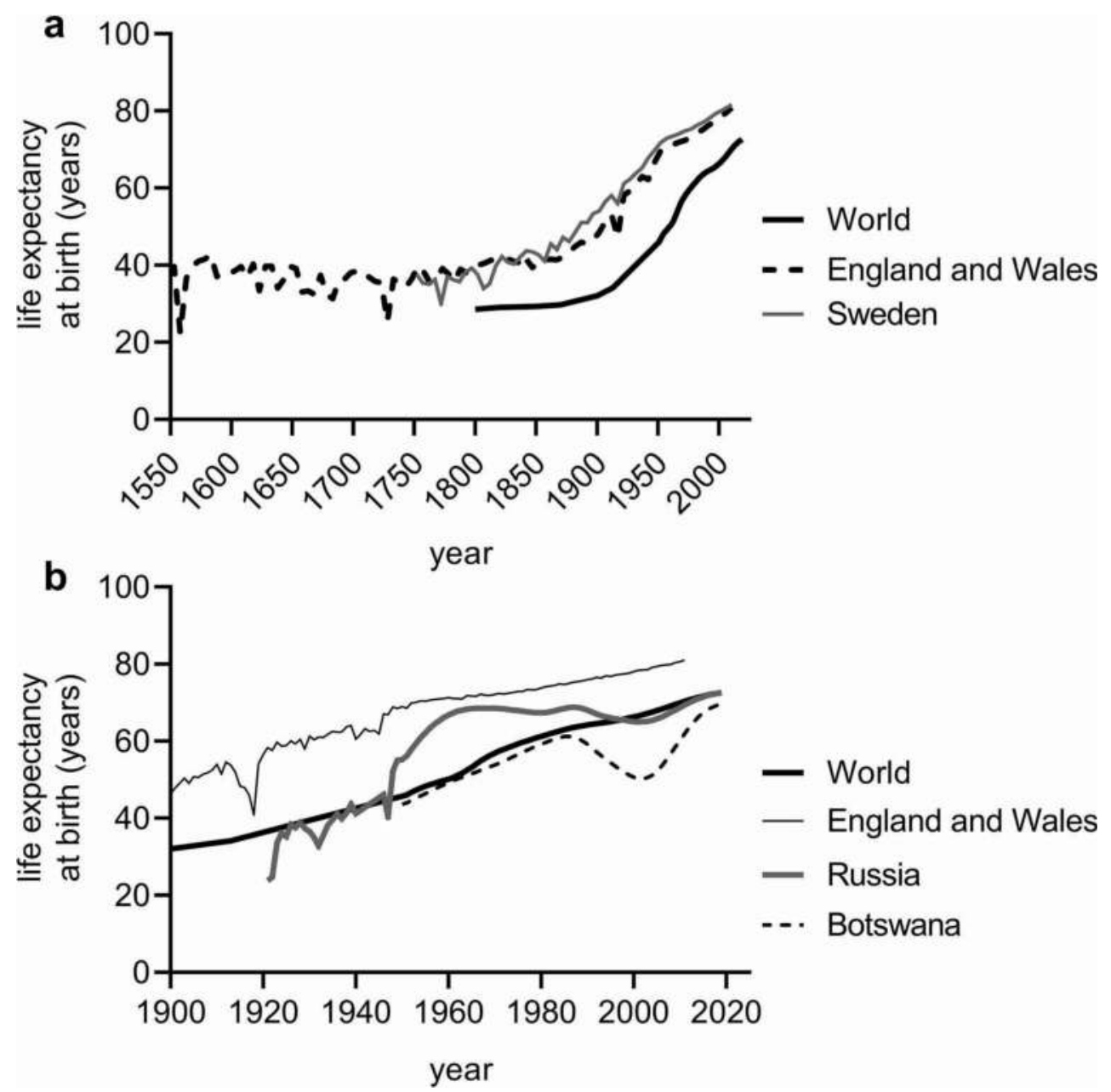

Figure 1.2 Long-run trends in life expectancy at birth.

Data are annual estimates except in the case of England and Wales in panel a, which are five-year averages. Sources: Human Mortality Database (University of California, Berkeley), and Max Planck Institute for Demographic Research (Germany), www.humanmortality.de (downloaded on January 2, 2020); M. Roser, E. OrtizOspina, and H. Ritchie, 'Life Expectancy', published online at OurWorldInData.org (2020), retrieved from https://ourworldindata.org/life-expectancy [Online Resource]; E.A. Wrigley, R. S. Davies, J. E. Oeppen and R. S. Schofield, English Population History From Family Reconstitution 1580-1837, (Cambridge: Cambridge University Press) (1997).

Within this general pattern of improvement and global convergence there have also been notable periods of stagnation and reversal. The most striking reversal was caused by the HIV/AIDS pandemic. The most-HIV affected countries in sub-Saharan Africa were in southern and eastern Africa and included some of those which had made the greatest prior gains in life expectancy (most notably Botswana, Figure 1.2b). Life expectancy is estimated to have fallen by 10 years in Botswana, South Africa, Namibia, and Zambia, and 20 years in Swaziland during the 1990s and early 2000s, before embarking 
a 950-55

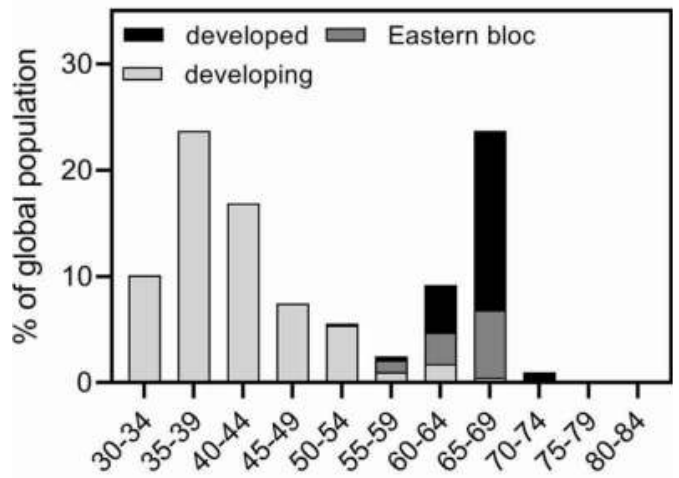

b $\quad 1975-80$

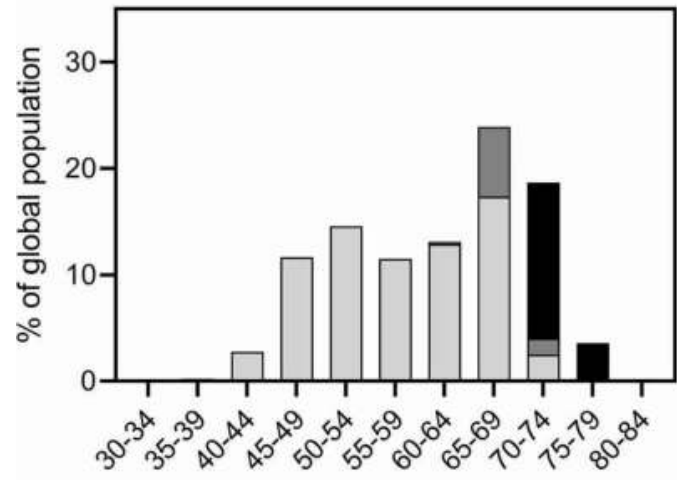

C $\quad 2000$

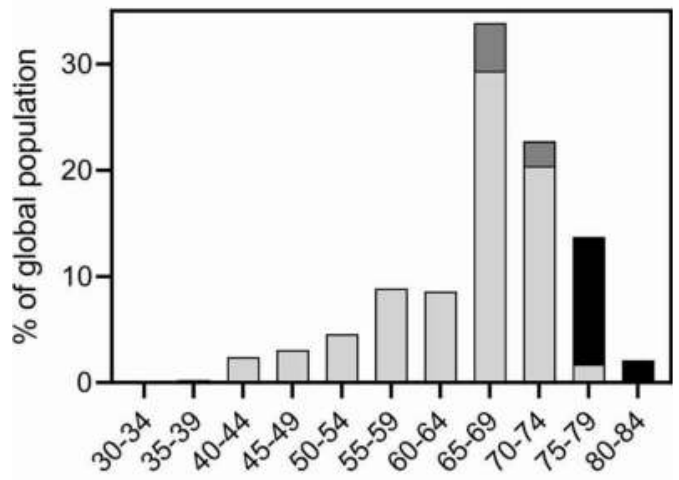

life expectancy at birth (years)

Figure 1.3 The distribution of the global population by life expectancy.

Populations were distributed according to the estimated average life expectancy of each national population (both sexes), except in the cases of India and China, where populations and life expectancies for states and provinces were used.

Source: C. Wilson, 'On the Scale of Global Demographic Convergence 1950-2000', Population and Development Review, 27 (2001), 155-171. 
upon a sustained recovery from around 2005, driven by the increasing availability of retroviral therapies, as well as public health initiatives and behavioral changes. ${ }^{11}$

Another very marked reversal is the fall in life expectancy in some parts of the former Soviet Union after the break-up of the union in 1991 (Figure 1.2b). This was preceded by a prolonged stagnation in life expectancy and infant mortality in the USSR in the decades of the 1960s-1980s. However, the collapse of communism in Europe precipitated marked increases in mortality especially of adult Russian males after 1991, a substantial proportion of which were alcohol-related. ${ }^{12}$ Another notable period of stagnation occurred amongst adult males in many high-income countries in the period c.1950-1980. The timing and extent of this slow-down varied by country and was closely related to life-time smoking patterns. In the UK and the USA, males born in the late 1890s and early 1900s had the highest lifetime smoking rates, perhaps reflecting WWI military policies to pay recruits partly in cigarettes. It was these men who experienced the largest absolute rises in lung cancers and cardiovascular diseases in the third quarter of the twentieth century, against a backdrop of steadily improving death rates for women (See Figure 1.5). ${ }^{13}$

\section{Epidemiological transitions}

Can these enormous improvements in survival since 1800 be understood within a unified model of epidemiological change? The most widely used descriptive model of mortality decline is Abdel Omran's 'epidemiological transition' framework. ${ }^{14}$ Omran described a three-stage process that started with the 'Age of Pestilence and Famine', which he regarded as the traditional state of large agrarian societies. In this state, life expectancy fluctuated between 20 and 40 years, and population growth was slow or negligible. The onset of secular mortality decline was associated with the 'Age of Receding Pandemics', and included reductions in the level and volatility of mortality, and a progressive shift of mortality from infectious to 'degenerative' diseases. This was followed by the 'Age of degenerative and man-made diseases', as infectious diseases diminished decisively, and most deaths occurred as a result of chronic non-communicable diseases (especially cancers and cardiovascular diseases) in late adulthood. Omran thought that gains in survival would slow or cease in this stage. However, life expectancy has continued to rise inexorably, driven largely by improvements in survival at advanced ages. This has led some scholars to advocate a fourth stage, the 'Age of delayed degenerative diseases'. ${ }^{15}$

Omran's model is widely used; however, it is largely descriptive rather than analytical and may be a poor representation of the historical processes at work in many societies. In this section, we provide a very brief overview of mortality trends in England and compare these with trends elsewhere. England is the only national population for which we have very long-run and robust mortality data for the last half millennium (from the 1540s). It was also a forerunner in terms of mortality declines. We divide the discussion into four periods: (1) c.1550 to 1750, when mortality crises abated and mortality stabilized, albeit at lower levels of life expectancy; (2) 1750-1870, when some of the most lethal infectious diseases including smallpox, typhus, malaria, and cholera were brought under control in the English population; (3) 1870-1940, when an impressive range of improvements in sanitation and hygiene, preventative medicine, and living standards profoundly reduced mortality from childhood diseases and tuberculosis in European and neo-European populations; and (4) the antibiotic era post-1940, when medical 
technologies including antibiotics and a range of vaccines acted in synergy with older strategies to confine infectious disease mortality largely to the oldest ages in developed countries. We conclude with a discussion of potential future trends.

\section{Receding pandemics $(1550-1750)$}

England was very precocious in escaping famine and major mortality crises. The last nation-wide subsistence crisis in England occurred in the 1590s, and mortality crises diminished progressively following the disappearance of plague after the $1660 \mathrm{~s} .{ }^{16}$ However, contrary to Omran's model, life expectancy actually fell as food security improved and epidemics subsided (Figure 1.2a). The most plausible explanation for this worsening of mortality in the period $1650-1750$ is that the urbanization and integration of the English economy that occurred in this period was accompanied by an epidemiological integration, that increased the circulation of infectious diseases. That is, the development of a functioning national grain market and improved transport infrastructure, which helped to reduce the impact of harvest failures, also promoted the spread of infections. ${ }^{17}$

Towns were also associated with high mortality, because they acted as hubs for disease importation and because poor sanitary conditions and dense populations facilitated the spread of gastrointestinal and airborne infections. As disease exposure increased, many of the diseases that had caused rare but large epidemics in the past became routine infections of childhood. This was the case for diseases that conferred lifelong immunity on survivors. ${ }^{18}$ Thus in historical societies, urbanization, trade, and economic growth tended to raise mortality rates and to concentrate some infectious diseases in childhood. ${ }^{19}$

\section{Receding famine and infectious diseases (1750-1870)}

This process of worsening mortality was reversed after around 1750, in England and in other populations of north-western Europe. The reasons for this reversal remain obscure. Most puzzling is the fact that the largest improvements occurred in urban populations. Before the late eighteenth century, cities in Europe appear to have been so lethal as to require a constant flow of immigrants simply to maintain their populations. In early eighteenth-century London, a third of infants died in the first year of life, and only about 60 per cent of those born in London survived to age $15 .{ }^{20}$ However, after 1750 urban mortality rates improved very markedly, and cities became capable of maintaining their own populations through natural increase, although mortality remained substantially higher than in rural areas until the twentieth century. ${ }^{21}$ As Simon Kuznets argued, these early improvements in urban mortality rates were an essential pre-requisite for the unprecedented rates of urbanization that followed. ${ }^{22}$ With the death rates prevailing in early modern cities, modern levels of urbanization would have caused the decline of national populations.

Famine also receded from western Europe in the late eighteenth century (notwithstanding the Great Famine in Ireland, 1845-1849, and the Finnish famine of 1866-1868). ${ }^{23}$ In the twentieth century, famine reappeared briefly in western Europe in association with the extreme stresses of the two world wars. However at the global level, the twentieth century witnessed some of the largest famines ever, including the Great Leap Forward famine in China in 1959-1961 that may have killed 20-30 million 
people. ${ }^{24}$ Nonetheless, by the late twentieth century, the global impact of famine had dwindled enormously, and even the most severe famines of recent decades (including Somalia in 1991-1992 and North Korea in 1995-2000) have been small by historical standards. $^{25}$

There is lively debate about the causes of famines and whether famines are primarily a function of declines in food availability or of institutional or market failures. Amartyr Sen famously argued that modern famines represented a 'failure of exchange entitlements', rather than an absolute shortfall in food supplies. ${ }^{26}$ That is, certain groups lose their capacity to exchange their primary entitlement (for example, their labor) for food. This may occur, for example, because the high price of food or other factors has reduced demand for the products or services which they supply. The price of food may also bear an ambiguous relationship to actual food availability. It may be driven up by demand elsewhere or by hoarding or requisitioning in the absence of any shortfall in production. Alternatively, as Malthus noted, prices may rise only modestly despite starvation, because those starving are too poor to enter the market and to influence prices. ${ }^{27}$

Explanations for the disappearance of famine often focus on advances in agriculture and food production, including the introduction of New World crops (especially potatoes and maize), and, in the twentieth century, the Green Revolution in crop productivity that started in the 1950s. However, in the English case, explanations for the early escape from famine acknowledge the importance of favorable ratios of population to food production, but also emphasize improvements in the operation of grain markets, on the one hand, and improvements in institutional responses on the other, especially the English poor laws. ${ }^{28}$ Recent work by Phil Slavin on the greatest famine in European history, that of 1315-1317, has revealed clear evidence of hoarding of grain. ${ }^{29}$ Other studies of credit have demonstrated how the impact of successive harvest failures in 1315-1317 was amplified in England by the actions of landlords and even family members who took advantage of the crisis to engross landholdings and other assets. ${ }^{30}$ Conversely, work by Richard Smith has demonstrated how these practices were replaced, by the sixteenth century, with a parish-based system of taxation and poor relief (before compulsory legislation). This system of communal welfare may have been sufficient to avert severe mortality in the English population during the harvest failures of the 1590s and, more assuredly, in the dearth years of the 1690s, when perhaps 10 per cent of the Scottish population died. ${ }^{31}$ These works raise the intriguing question of whether the early disappearance of famine in Western Europe owed more to improvements in famine relief measures and other institutional changes than to improvements in food production and market distribution.

Another important element in the disappearance of famine (and more generally of dearth-related mortality), is the control of epidemic diseases. It is likely that the immediate cause of most famine-related mortality in historical populations (except perhaps in very isolated and small-scale societies) was infectious diseases, rather than starvation. Typhus, or 'famine fever', was a common and lethal correlate of harvest failure, together with typhoid, dysentery, and other diseases including smallpox and malaria. ${ }^{32}$ Epidemics generally accompanied major harvest failures because these failures often prompted migration in search of work and food. Such migration brought rural populations into contact with novel diseases, especially when they moved into towns, and exposed them to over-crowded and insanitary conditions at a point when they were malnourished and probably immune-compromised. ${ }^{33}$ These epidemics also tended to become general, and therefore the mortality associated with past famines may have been fairly socially unselective. ${ }^{34}$ 
Reductions in epidemic disease mortality associated with famines probably occurred via several mechanisms. First, where famine relief measures were relatively local and effective (as in the English case from the late sixteenth or seventeenth century onwards), these would have reduced migration and associated disease exposure. Second, disease control measures played an increasingly important role over time. These started with measures to isolate infected individuals and to disinfect clothing and bedding and extended in the twentieth century to large-scale immunization and medical programs in concert with often international famine relief measures. A grim testament to the effectiveness of such measures is the famine associated with the siege of Leningrad. The city was blockaded for nearly two years (1941-1943), and 800,000 civilians died, mainly of starvation. However sanitary measures were so strictly adhered to that there was no major outbreak of epidemic disease. ${ }^{35}$ It is a peculiar achievement of the twentieth century that so many famine victims escaped infectious diseases for long enough to starve to death.

Warfare too became less disease-prone in the eighteenth century, although it was not until the early twentieth century that battle deaths exceeded those from disease during military conflicts. Indeed eighteenth-century efforts to contain and prevent major disease outbreaks in the navy; in prisons and workhouses; and on slave, convict, and immigrant ships, probably led the way in developing effective techniques for reducing the transmission of contagious (person-to-person) and vector-borne diseases. ${ }^{36}$ These techniques included epidemiological surveillance (first adopted in Italy against plague, and entrenched in England in the form of the London bills of mortality), the isolation of individuals suspected of infection, contact tracing, surveillance, improved hygiene and diet, and reductions in crowding. While many of these measures, such as obsessive ventilation, were based on erroneous models of disease, others, such as isolation of the infected or the issuing of new clothes to sailors, were of clear benefit regardless of their theoretical justification. ${ }^{37}$ Perhaps more importantly, the publication and dissemination of these ideas subjected them to wide scrutiny and replication.

Pre-eminent amongst the diseases that declined before the mid-nineteenth century was smallpox. Smallpox was probably the single most lethal infectious disease in eighteenthcentury Europe and was also a major cause of death in the Americas and parts of Asia and Africa. Although smallpox is usually considered an ancient disease of humans, its origins remain obscure. Recent breakthroughs in the sequencing of ancient DNA have produced evidence that a new variant of smallpox may have spread through Europe and then globally in the seventeenth century. ${ }^{38}$ By the second half of the eighteenth century, smallpox was responsible for 8 per cent of all deaths in Sweden and northern England, and 20 per cent in Manchester. ${ }^{39}$ Attempts to control smallpox through isolation of victims and inoculation with small doses of the virus were very widespread. ${ }^{40}$ However, the advent of immunization with cowpox (vaccination), discovered by Edward Jenner in 1796, and published in 1798, proved a decisive turning point. Vaccination was immediately recognized as a godsend, and it spread rapidly through Europe and into European colonies and ex-colonies, although its immediate effects varied enormously. ${ }^{41}$ It is difficult to estimate the true impact of smallpox, and of vaccination, on global mortality trends. This is because, first, vaccination was introduced well before most states introduced the systematic measurement of cases and deaths, and second, smallpox appears to have evolved into a number of subtypes with very different rates of mortality in the course of the nineteenth century. Therefore, although smallpox is celebrated as the first disease to 
be eradicated by human efforts (declared in 1980), the true impact of smallpox on historical populations probably remains under-appreciated.

By 1820 then, mortality patterns in north-western Europe were already profoundly different from previous centuries. Famine had receded, and the most lethal epidemic diseases (plague, typhus, smallpox, and malaria) had waned decisively in impact. The mortality associated with these diseases had been fairly indiscriminate, affecting wealthy and well-nourished individuals as well as the poor. Indeed, there is little evidence for a survival advantage to wealth before the nineteenth century. ${ }^{42}$ This is very surprising, since it might be thought that the advantages of better diet, clothing, and housing would have been even more important in the past, when living standards were lower. However, as Stephen Kunitz and Stanley Engerman have argued, the disease environment may have been sufficient to overwhelm these advantages before the nineteenth century. ${ }^{43}$ As these more lethal diseases receded, then, mortality became concentrated amongst the very young, the very old, and the poor. Because we know less about mortality patterns before the nineteenth century, it is common to think of these patterns as traditional; however, they probably represented a new and 'modern' pattern compared with previous centuries. $^{44}$

In Britain further improvements in longevity were impeded after c.1830 by unprecedented rates of urbanization, and possibly by scarlet fever, which underwent an apparently autonomous increase in virulence. ${ }^{45}$ However, in Sweden, where urbanization remained very modest before the late nineteenth century, life expectancy improved fairly continuously from 1750 to the present (Figure 1.2a). Woods has argued that similar underlying improvements in health in Britain were masked by the rapid redistribution of population from relatively healthy but poor rural areas to wealthier but unhealthier urban ones. ${ }^{46}$

\section{The eclipse of infectious diseases (1870-1940)}

Rapid secular mortality decline resumed in England from c.1870, interrupted only by the major wars and the 1918/19 influenza pandemic (Figure 1.2b). Most of the decline in mortality between 1870 and 1940 was driven by declines in infection-related mortality in children and young adults, with the most rapid improvements occurring in the period 1900-1940 (Figure 1.4).

Scarlet fever and respiratory tuberculosis mortality began to decline from the 1870 s. The other major 'childhood' diseases (measles, diphtheria, and pertussis) all underwent very substantial and poorly understood declines in the period after 1900. In the case of scarlet fever there is reason to think that the pathogen (Streptoccocus pyogenes) declined in virulence, but no similar arguments have been made for other infections in this period, with the partial exception of tuberculosis ${ }^{47}$ and streptococcal puerperal fever. ${ }^{48}$ These childhood diseases are transmitted person to person and are highly infectious, and it appears that reductions in mortality resulted from declines in case-fatality rates rather than in incidence of infection. In the case of measles, Peter Aaby and colleagues have made a strong case for the importance of crowding and dosage of infection to mortality from measles. ${ }^{49}$ Improvements in nutrition associated with rising incomes doubtless acted to increase host resistance. In addition, improvements in housing conditions and the progressive and substantial declines in fertility and family size from the 1870s would also have acted to reduce infection dosage and exposure to opportunistic infections. ${ }^{50}$ The 


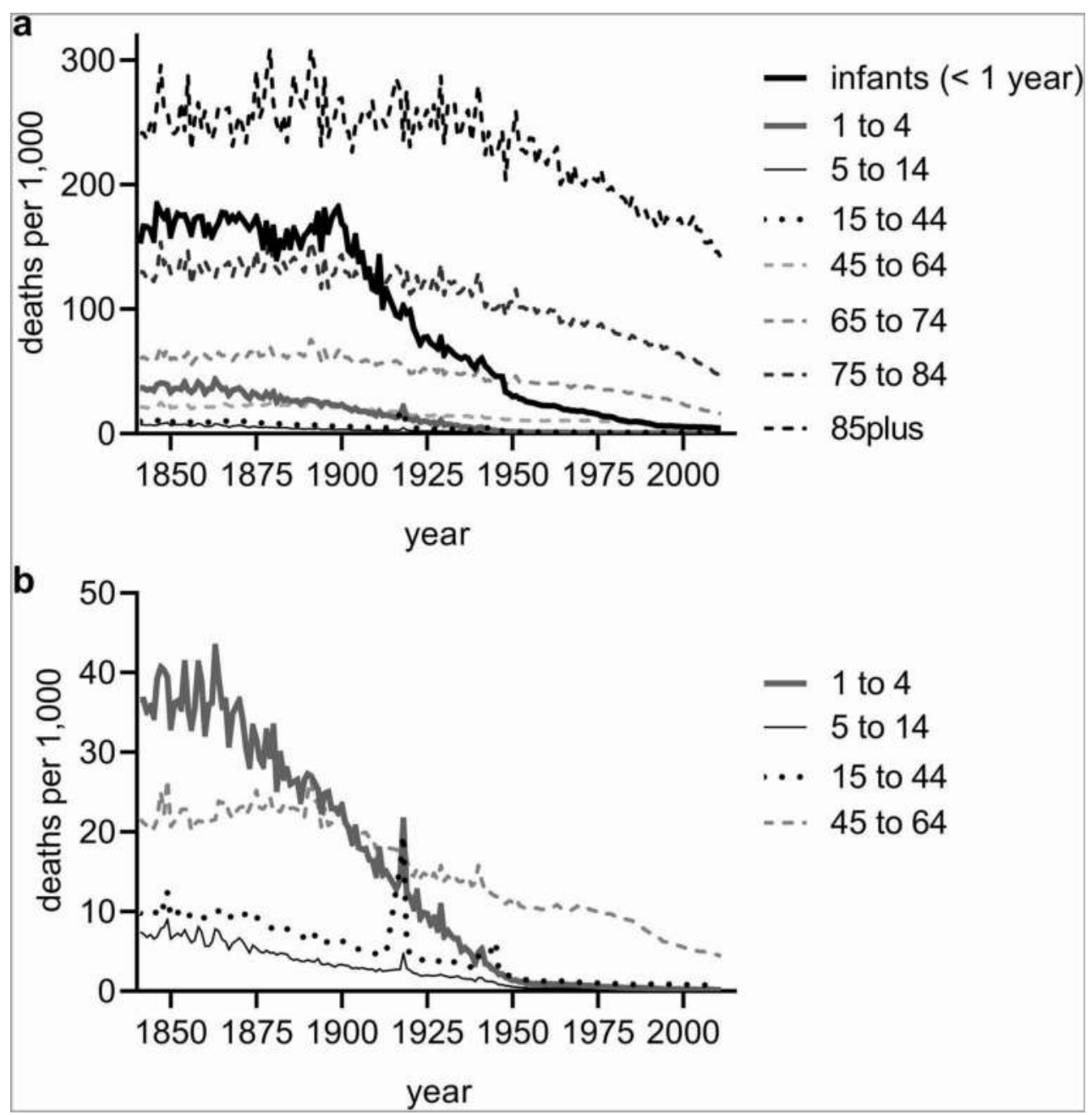

Figure 1.4 Annual age-specific mortality rates, England and Wales, 1841-2011.

Panel a includes all age groups, panel b displays ages where mortality rates were comparatively low (and declined early).

Source: Human Mortality Database (University of California, Berkeley), and Max Planck Institute for Demographic Research (Germany), www.humanmortality.de (downloaded on January 2, 2020).

same constellation of factors probably contributed substantially to improvements in mortality from tuberculosis (Figure 1.6), although in this case the extent to which incidence of infection was also reduced via preventative measures such as confinement of sufferers to sanitoria remains unknown. ${ }^{51}$

The most lethal waterborne diseases (cholera, typhoid, and dysentery) declined from the 1860s. These trends imply early improvements in the quality of drinking water. However, diarrhoeal diseases displayed a more complicated pattern, declining from the 1870s amongst adults and older children but remaining stubbornly high, and indeed rising in 


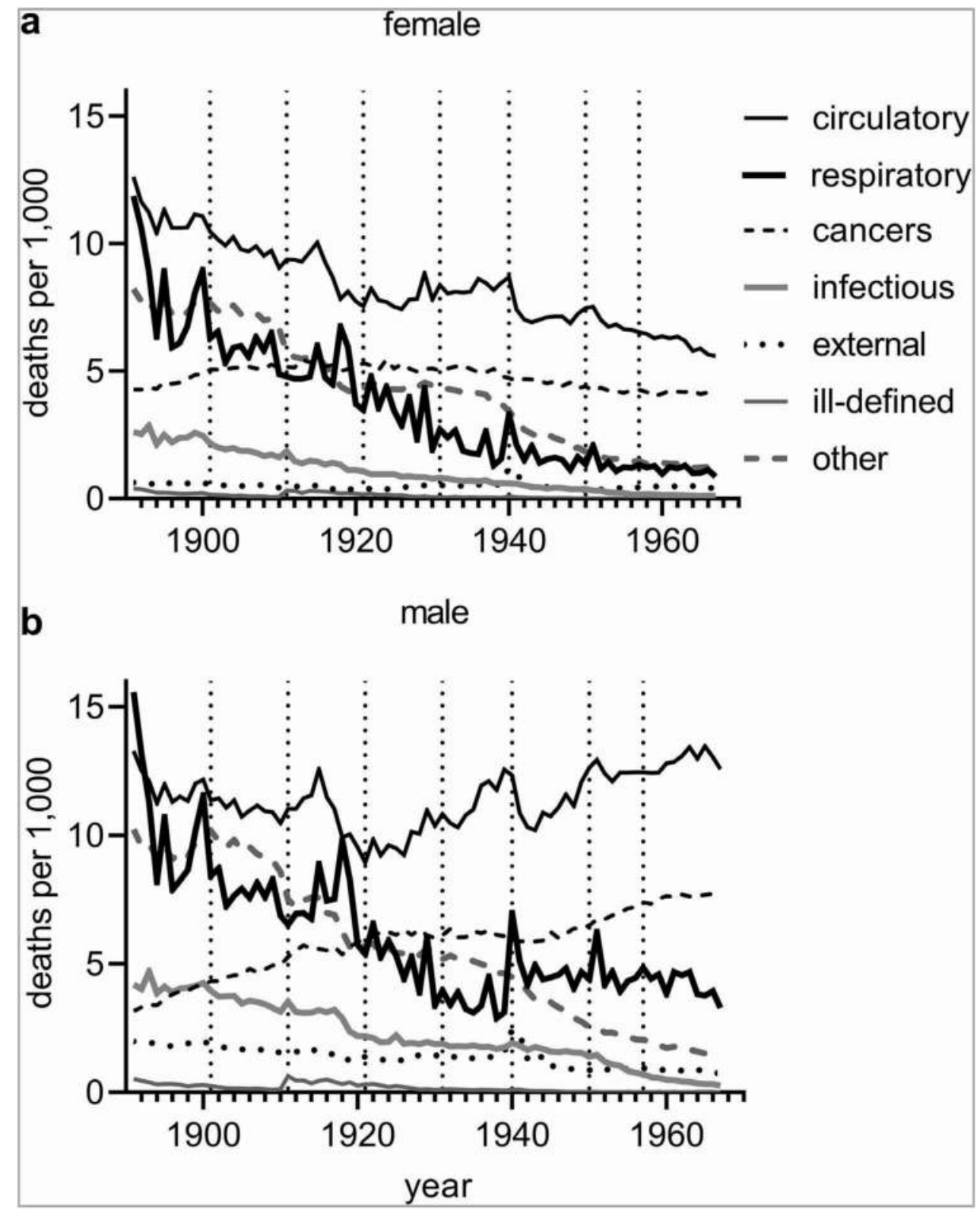

Figure 1.5 Mortality by category of cause, ages 60-64, England and Wales, 1891-1967.

Dashed vertical lines indicate dates of changes in cause of death coding schemes. 'Respiratory' includes respiratory tuberculosis, influenza, and pneumonia; 'circulatory' includes strokes; 'external' includes all accidental and violent deaths.

Source: R.J. Davenport, Annual Deaths By Cause, Age and Sex in England and Wales, 1848-1900 [dataset] (2007), Economic and Social Data Services (SN5705); Office of National Statistics, 'Twentieth Century Mortality: 100 Years of Mortality Data in England and Wales By Age, Sex, Year and Underlying Cause' (2003). 


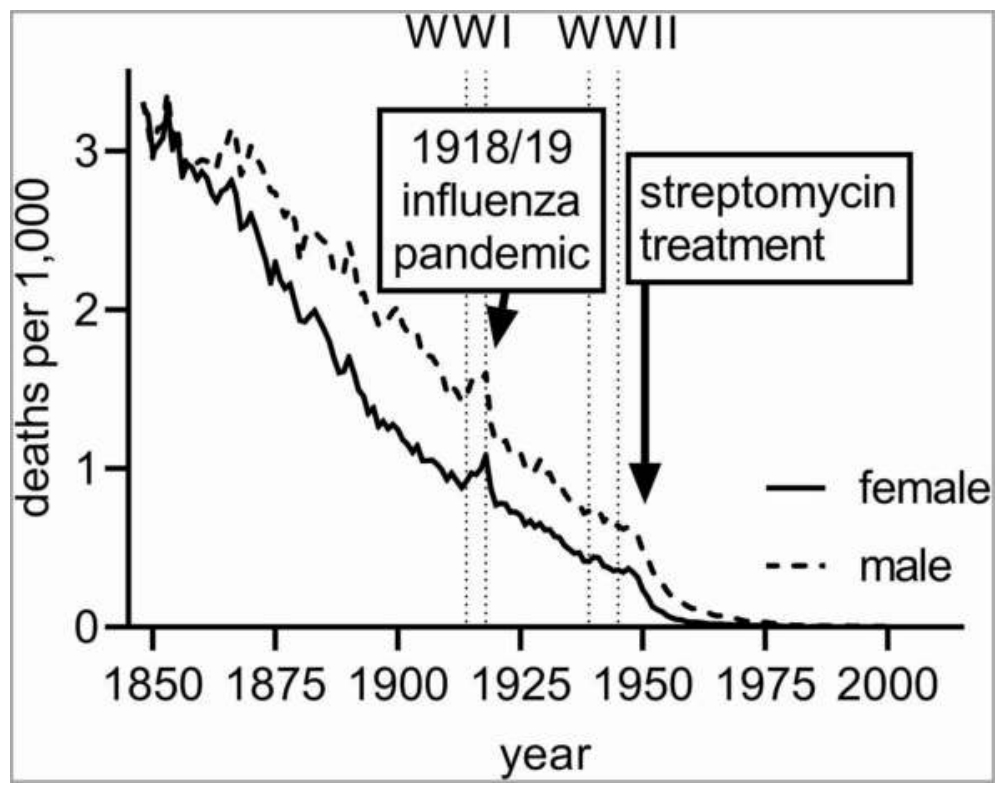

Figure 1.6 Tuberculosis mortality, England and Wales, 1848-2000.

Death rates are directly age-standardized using the 2012 population of England and Wales. Source: R.J. Davenport, Annual Deaths By Cause, Age and Sex in England and Wales, 1848-1900 [dataset] (2007), Economic and Social Data Services (SN5705).

the 1890s, amongst infants. ${ }^{52}$ The late decline of infant diarrhoeal mortality may reflect differences in disease transmission pathways between infants and other age groups. Infants appear to have been relatively insensitive to water quality, in England, and diarrhoeal mortality in this age group was probably more closely related to sanitary conditions (including sewerage and fecal disposal) and domestic practices (breastfeeding, handwashing, and food preparation). Conversely, diarrhoeal diseases in older children and adults were probably more closely related to the quality of drinking water. ${ }^{53}$

These patterns of declines in gastrointestinal (or 'fecal-oral') diseases present a puzzle, because they fit very poorly with the traditional chronology of improvements in water and sanitation in England, which were especially marked after 1870 (when urban authorities began to make very major investments in water provision and sewerage). ${ }^{54}$ The precocious declines of the most water-dependent diseases (cholera, dysentery, and typhoid) pre-dated large-scale investments in water supplies and suggest that relatively cheap or incomplete attempts by towns to source clean water may have been sufficient to reduce transmission of these diseases, in many cases before investments in water purification or sewerage. Conversely, infant diarrhoeal mortality was apparently unaffected by very major investments in water supplies. ${ }^{55}$ However the installation of comprehensive urban sewerage systems and sewage treatment lagged well behind investments in clean water supplies, and the continued retention of large volumes of human excrement in many British towns until the early twentieth century may account for the continued high levels of infant diarrhoea against a backdrop of major declines in waterborne diseases. 
This pattern, of precocious improvements in the most lethal waterborne diseases and slower improvements in diarrhoeal diseases of early childhood was also evident globally in the twentieth century. The more lethal 'Asiatic' cholera (which killed perhaps 15 per 100 of those infected) has been largely displaced since the 1960s by the 'El Tor' cholera subtype, which has a case-fatality rate of around 1 death per 100 infections. ${ }^{56}$ Typhoid and dysentery have also declined enormously as causes of death. Paul Ewald argued that these changes in water-borne diseases reflected widespread improvements in water quality, which have dramatically reduced water-based disease transmission. ${ }^{57}$ The WHO and UNICEF estimated in 2019 that 90 per cent of the global population now had access to 'improved' water sources (a clean source within 30 minutes' round walk). ${ }^{58}$ Nonetheless, infant diarrhoeal diseases still constitute some of the most common causes of death in young children globally. ${ }^{59}$ If, as was the case in England, most diarrhoeal diseases in young children are more commonly caused by poor hygiene and fly-borne contamination of food than by contaminated water, then much more investment in fecal disposal will be required to reduce diarrhoeal mortality further. In affluent countries it proved much harder to remove excrement from cities and to treat sewage than to provide uncontaminated water.

Mortality began to improve in older adults after c.1900 in England (with the exception of the oldest ages, 85 years and over, where substantial improvements occurred only in the second half of the twentieth century) (Figure 1.4b). However, here the patterns were more complex. Figure 1.5 shows death rates by cause for 60-64-year-old men and women in England between 1891 and 1967. Whereas improvements at younger ages reflected mainly falls in infectious disease mortality, the main gains in life expectancy at ages 40 and over from 1900 were a consequence of reductions in deaths attributed to tuberculosis, other respiratory infections (especially pneumonia and influenza), cardiovascular diseases (strokes and heart disease), and 'other' causes (Figure 1.5). The latter category included mainly diseases related to the stomach, liver, and kidneys and was supposed by Samuel Preston to contain a substantial cardiovascular component, ${ }^{60}$ but also included diseases of infectious origins (such as acute nephritis caused by streptococcal infection).

Some of the patterns in Figure 1.5 reflect changes in diagnostic accuracy (and especially the transfer of deaths from ill-defined categories into specific categories, including cancers). However, similar age-specific and cause-specific patterns of mortality decline have been observed in a variety of populations at different stages of economic development. ${ }^{61}$ Preston demonstrated, using long-run cause of death data for 42 countries, that falls in cardiovascular disease made a major contribution to the early stages of mortality decline, and he attributed this both to the tendency of respiratory infections in particular to trigger heart attacks, and to longer term effects of infections on susceptibility to chronic non-communicable conditions.

Infectious diseases may influence non-communicable diseases in a variety of ways, and on a variety of time scales. However, the magnitude of effects of early-life infections on health in adulthood remains hotly debated. ${ }^{62}$ There are clear examples of specific pathways from childhood infection to adult disease, as in the case of rheumatic fever and valvular heart disease or Helicobacter pylori infection and stomach cancer. ${ }^{63}$ However, at the population level, the influences of early-life disease environments are less obvious. Life expectancy at older ages bears little relationship to levels of infection amongst the same cohorts during childhood (as gauged by childhood 
mortality rates in these cohorts). Elderly cohorts in countries such as Japan and Italy with relatively high levels of historic childhood mortality now enjoy some of the highest life expectancies in the world. ${ }^{64}$ The synergies between different causes of death remain an under-researched area.

\section{The age of degenerative diseases (1940-present)}

We have divided the discussion of English mortality patterns at 1940 because the development of antibiotics in the late 1930s and early 1940s marked a turning point in the capacity to cure disease. Most health innovations up to this point were preventative they reduced exposure to disease. Antibiotics, on the other hand, could be used to cure infections. ${ }^{65}$

The discovery of the bactericidal properties of protonsil in 1931 led to the proliferation of related 'sulfa drugs' that came into wide use in Europe and the USA by the late 1930s. Sulfa drugs were effective against a narrow but important range of bacterial infections, including the streptococcal strains responsible for puerperal fever in recently delivered women and streptococcal wound infections, but not the streptococcal strains that cause scarlet fever. Their impact on maternal mortality was dramatic. ${ }^{66}$ Neonatal mortality, particularly deaths in the first week of life, also declined precipitously with the introduction of sulfa drugs. Anne Løkke has argued persuasively that antibiotics were key in making caesarean deliveries safe and that this meant that difficult deliveries ended more frequently in a successful live birth, rather than stillbirth and maternal infection. ${ }^{67}$ Sulfa drugs also proved effective in treating pneumonia but had no impact on tuberculosis.

Penicillin was developed in the 1930s and widely used from 1944. It proved effective against wound infections and was widely deployed in the closing stages of WWII by the American and British armies. Of even greater significance was the discovery in 1946 that streptomycin cured tuberculosis. Nonetheless, the immediate impact of penicillin-type drugs on life expectancy trends was slight (Figure 1.2b). Even in the case of tuberculosis, the impact in Britain was much smaller than it would have been had penicillin been discovered several decades earlier. Tuberculosis mortality declined enormously in the late nineteenth century and the first half of the twentieth century, before penicillin (Figure 1.6). Nonetheless, the advent of penicillin marked a decisive break, and, within ten years of its use, most sanatoria for the treatment of tuberculosis sufferers had been converted to ski resorts, psychiatric hospitals, and for other uses.

Importantly, the apparently small contribution of the discovery of antibiotics to life expectancy trends masks their enormous contribution over the last 70 years to the treatment of non-communicable diseases. Before 1950, surgery remained a very dangerous procedure, despite very significant developments in sterile procedures and wound treatment. Many of the gains in survival from heart disease and cancers in the last 50 years depended and continue to depend on surgical interventions that would have involved substantial risk before the advent of penicillin. ${ }^{68}$ Chemotherapy, which reduces the patient's resistance to infection, also relies on the prophylactic use of antibiotics to reduce the risk of opportunistic infections, as does organ transplant technology.

In European populations, infectious diseases had been controlled to a great extent by 1940 using methods that prevented transmission or raised population resistance (rather than cured infection per se). These older methods of disease prevention were further enhanced in the second half of the twentieth century by the development or wider 
implementation of new vaccines against a range of infectious diseases, including measles, pertussis, yellow fever, tuberculosis, hepatitis, typhus, typhoid, polio, pneumonia, and chickenpox.

An example of this mix of preventive and curative approaches is tuberculosis. Tuberculosis was (and remains) a major cause of mortality globally. The BCG vaccine against tuberculosis was developed in the 1920s but was only widely used after WWII. Antibiotics, BCG, and surveillance were then used in a three-pronged strategy to identify and treat those infected, to reduce transmission, and to immunize the population against infection. In addition, some of the declines in tuberculosis over the latter half of the twentieth century reflect the same factors in operation in affluent countries before the advent of antibiotics and BCG vaccine, including better nutrition. However, tuberculosis resurged in the 1990s as an opportunistic infection in HIV-affected individuals and because of the development of strains resistant to multiple types of antibiotic treatment. BCG immunization does not face the same problem of evolved resistance, and it remains routine in most low- and middle-income countries. ${ }^{69}$ However, its efficacy varies. BCG is protective against tuberculosis infection in infants, but the efficacy of BCG against adult respiratory forms of the disease varies with latitude and age at immunization, with efficacy ranging from zero at low latitudes to $60-80$ per cent protection at latitudes above 40 degrees. $^{70}$ Therefore, the relative contributions of antibiotics, immunization, and improvements in living conditions to global declines in tuberculosis are very geographically as well as chronologically heterogeneous.

\section{The globalization of mortality decline, 1950 onwards}

Famines and major epidemic outbreaks began, slowly, to recede in much of the world in the late nineteenth century and the first half of the twentieth century so that, by the time the world emerged into the spotlight of global statistics in the 1950s, profound changes had already taken place in much of the world's population. In India, for instance, large upswings in mortality caused by famine, bubonic plague, and smallpox diminished substantially after 1920. Tim Dyson and Monica Das Gupta attributed this stabilization of mortality to colonial policies that improved food distribution, monitored plague outbreaks, and increased smallpox vaccination coverage. ${ }^{71}$ A similar stabilization of mortality after 1920 is evident in Jamaica, where a precocious public health administration, education, and registration system facilitated rapid gains in life expectancy from this date, with the fastest gains in the period 1925-1940. ${ }^{72}$ In many African societies, the progressive eradication of smallpox, culminating in the WHO global campaign from 1950 to 1980, must have had an effect on populations with hitherto low rates of vaccination as profound as that in early nineteenth-century Europe.

While the early gains in life expectancy in affluent countries had increased global inequalities in health by the mid-twentieth century, the period after 1950 witnessed very substantial convergence, at the national level (Figure 1.3). In poor countries, the process of infectious disease control, so drawn out in England, was enormously accelerated in the second half of the twentieth century by the rapid availability of medical and public health technologies and knowledge. These included immunization, antibiotics, germ theory and sanitation technologies, and insecticides. The rapidity of global improvements in mortality is all the more astonishing when one considers the challenging disease environments in which some of these changes occurred. England's slow mortality decline took 
place in a temperate island setting, with a low burden of arthropod-borne infections and relatively easy means of controlling disease importation through quarantine. Populations in low-altitude tropical areas faced much higher disease burdens as a consequence of climatic and ecological conditions, especially in Africa where co-evolution between pathogens and humans and other primates was very longstanding. Moreover, these rapid mortality declines have occurred in the context of ever-increasing globalization and urbanization.

Some of the very rapid gains made in the decade after WWII must be attributed to the widespread use of DDT to destroy the insect vectors of malaria, trypanosomiasis, yellow fever, and typhus. ${ }^{73}$ Malaria in particular was a major cause of mortality and morbidity across tropical Africa, Central and South America, and South and South-East Asia (including southern China). Malarial infection induces only short-lived immunity, but, where malarial exposure is most intense, the burden of infection falls mainly on very young children and immigrants (because these groups lack immunity). Malarial mortality was dramatically reduced by DDT spraying of mosquito breeding sites and, more latterly, by anti-malarial drugs and the use of bed nets. However, the complexity of the malarial life cycle, the evolution of drug resistance, and regulation of insecticide use has repeatedly threatened these gains.

The huge gains in global life expectancy since 1950 were accompanied by very substantial economic development. The extent of these changes over the last 70 years remains very under-appreciated. Today over 90 per cent of the world's population lives in 'middleincome' or 'high-income' countries, and half lives in states with below-replacement levels of fertility (that is, with total fertility rates below 2.1 children per woman). ${ }^{74}$ Economic growth has coincided with rapid increases in education, medical technologies, and falls in fertility, as well as enormous social and cultural changes. Given the rapidity of change, is it possible to identify the key distal drivers of mortality improvements?

The difficulties involved in teasing out the relationships between economic growth and demographic outcomes can be illustrated using so-called 'Preston curves'. In a landmark paper in 1975, Samuel Preston produced a series of plots of national life expectancy by national income per capita (adjusted for change in nominal value of GDP over time), for 1900, 1930, and $1960 .^{75}$ Figure 1.7 plots similar relationships for 1960 and 2018, and for the United States in 1900. As Figure 1.7 demonstrates, there is a strong relationship in any given year between the per capita income of a country and its average life expectancy at low levels of income. Conversely, above a certain threshold, income ceases to be associated with further gains in life expectancy. Crucially, however, although this relationship between income and life expectancy appears strong in any given year, the life expectancy that could be achieved for a given income has risen consistently over time. In 1900 the United States was the richest country in the world; however, it was unable to procure an average life expectancy for its citizens of more than 50 years. By 1960 countries with the same average income as the United States in 1900 had an average life expectancy of more than 60 years. By 2018 for the same income the average life expectancy was over 70 years.

Preston's interpretation of these patterns was that rising national incomes could only account for a small fraction (less than a quarter) of improvements in life expectancy over time. ${ }^{76} \mathrm{He}$ argued that health had in fact grown cheaper over time so that technologies that were not available at any price in 1900 (such as antibiotics) have become widely available since. 


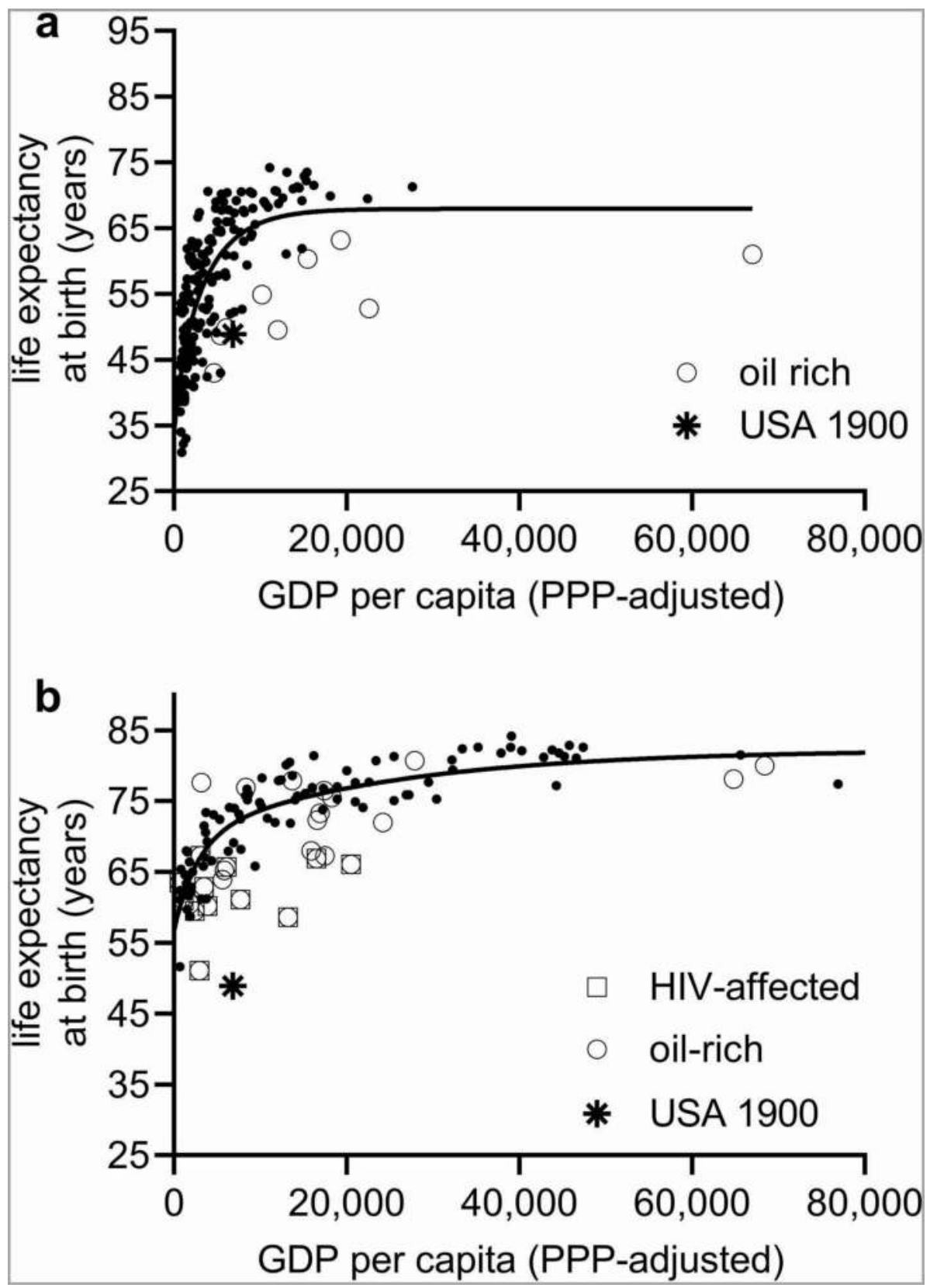

Figure 1.7 'Preston curves'; national life expectancies in relation to income per capita.

GDP per capita measures are expressed in international dollars (fixed 2011 prices). 'Oil-rich' states are defined as those which derive 10 per cent or more of GDP from oil rents. HIV-affected states are those with an estimated HIV prevalence of 5 per cent or more of adults aged 15-49.

Sources: HIV prevalence: CIA, www.cia.gov/library/publications/the-world-factbook/rankorder/raw data_2155.txt; GDP per capita and oil rents: World Bank, compiled by www.gapminder.org (downloaded February 10, 2020); M. Roser, E. Ortiz-Ospina, and H. Ritchie, 'Life Expectancy', published online at OurWorldInData.org (2020), retrieved from https://ourworldindata.org/life-expectancy. 
Other scholars have argued that state interventions or cultural factors were more important than economic growth to improving longevity. Jack Caldwell drew on examples of 'high-performing' poor countries or states that managed to achieve very high levels of life expectancy before they achieved rapid rates of economic growth. These included Sri Lanka, Costa Rica, Jamaica, Kerala (in India), China, Cuba, and Vietnam. ${ }^{77}$ Analyzing the noncommunist states, Caldwell concluded that these 'high-performers' were characterized by cultural traits including relatively high female status (and therefore relatively high levels of female education), institutional factors that included fairly egalitarian access to healthcare (such as immunization, rural clinics, and 'barefoot doctor' services), and either relatively egalitarian access to food or a social safety net that operated in times of dearth. ${ }^{78} \mathrm{He}$ also drew attention to the poor performance of oil-rich states, which had relatively high incomes but unimpressive life expectancies (as is evident in Figure 1.7a).

More recently James Riley analyzed a wider set of early-achieving 'high performers' and concluded that there were in fact many pathways to rapid health improvements. ${ }^{79}$ Riley's high performing states (relative to GDP) included a number of oil-rich populations (in particular, Oman and Venezuala), although it is only in recent decades that the generally poor performance of oil-rich countries has diminished (Figure 1.7b). Riley's work emphasizes the heterogeneity of possible routes to low mortality.

Improvements in mortality since 1800 were associated with very profound economic, technological, and social changes and defy monocausal explanations. Explanations fall largely into two camps, those invoking economic development and rising living standards, especially improved nutrition, ${ }^{80}$ and those which attribute the bulk of improvement to public health measures. ${ }^{81}$ Predictably, the evidence is ambiguous. The ubiquity of mortality improvements in global populations at very different levels of income, development, and life expectancy points to the potential multiplicity of pathways and perhaps to the importance of fertility decline and education. ${ }^{82}$ Additionally, there may be bi-directional influences between health and income. Robert Fogel described a 'techno-physio revolution', where improvements in diet increased human productivity, which then further improved output and economic development in a virtuous circle of improving health and wealth. ${ }^{83}$ Easterlin, on the other hand, considered that both economic growth and mortality improvements derived from the growth of scientific knowledge but were not themselves strongly causally related. ${ }^{84}$ The relative importance of household income, medical technologies, public health interventions, and wider changes in fertility and education remains controversial, and this may be because their influence varied widely. This is almost inevitably the case, because local cultures, histories, and ecological conditions varied so widely.

\section{The future}

What can the past tell us about potential future trends? Perhaps the most important pattern to note is that life expectancy has generally continued to rise, despite the enormous challenges posed by rapid population growth and urbanization. These factors would have caused unsustainable increases in mortality in traditional societies, and yet now famines are rare, and most urban populations have higher life expectancies than their rural counterparts. Remarkably, the trend in 'best case' life expectancy (for the national population with the highest life expectancy in any given year) has continued to rise fairly linearly (at a rate of approximately three years of extra life per decade) since $1840 .{ }^{85}$ This suggests 
that any biological limits to human life expectancy, if they exist, are yet to be encountered. Therefore, future trends will probably continue to depend on human capacity to overcome old threats and new challenges to human health.

These threats are very substantial, potentially even larger than the factors that drove the numerous setbacks to longevity gains over the last two centuries. Reasons why survival gains could falter or even reverse include the emergence of novel pathogens (through inter-species transfers and autonomous changes in pathogen properties), the multifarious effects of climate change (on environmental conditions, migration, and disease vector ranges), antibiotic resistance, and obesity. On the positive side, a concerted move towards clean energy will make cities substantially healthier, and this is where the majority of the world's population now lives. Urban populations now generally enjoy higher life expectancies than rural populations, a striking reversal of historic norms. However, this advantage reflects, in part, the higher wealth of cities and superior access to public health measures (sewerage, clean water, immunization programs) and to clinics and hospitals, which outweigh the disamenities of crowding and poor air quality. This balance will change as air pollution reduces but also as rural populations gain greater access to health facilities. The balance of these and other trends will help to determine whether individual and global inequalities in health and longevity widen or narrow in the twenty-first century.

This chapter is Open Access with funding from Wellcome Trust Award 103322.

\section{Notes}

1 This work was funded by Wellcome Trust award 103322 (to Prof. Richard Smith, University of Cambridge). The author gratefully acknowledges this funding.

2 M. Roser, E. Ortiz-Ospina, and H. Ritchie, "Life Expectancy," published online at OurWorldInData.org (2020). Retrieved from: https://ourworldindata.org/life-expectancy [Online Resource].

3 M. Roser, H. Ritchie, and E. Ortiz-Ospina, "World Population Growth," published online at OurWorldInData.org (2020). Retrieved from: https://ourworldindata.org/world-populationgrowth [Online Resource].

4 Global Burden of Disease (GBD) 2015 Mortality and Causes of Death Collaborators, "Global, Regional, and National Life Expectancy, All-Cause Mortality, and Cause-Specific Mortality for 249 Causes of Death, 1980-2015: A Systematic Analysis for the Global Burden of Disease Study 2015," Lancet, 388 (2016), 1459-1544.

5 R.A. Easterlin, Growth Triumphant: The Twenty-First Century in Historical Perspective (Ann Arbor, MI: The University of Michigan Press, 1996).

6 J.-C. Chesnais, The Demographic Transition: Stages, Patterns, and Economic Implications (Oxford: Clarendon Press, 1992); J.G. Riley, Rising Life Expectancy: A Global History (Cambridge: Cambridge University Press, 2001) and Poverty and Life Expectancy: The Famaica Paradox (Cambridge: Cambridge University Press, 2005).

7 A. Crosby, Ecological Imperialism: The Biological Expansion of Europe, 900-1900 (Cambridge: Cambridge University Press, 2015); S. Doyle, Before HIV: Sexuality, Fertility and Mortality in East Africa 1900-1980 (Oxford: Oxford University Press, 2013); and A.B. Jannetta, Epidemics and Mortality in Early Modern Fapan (Princeton, NJ: Princeton University Press, 1987).

8 T. Dyson and M. Murphy, "The Onset of Fertility Transition," Population and Development Review, 11 (1985), 399-440; and Roser et al., "World Population Growth."

9 C. Wilson, "On the Scale of Global Demographic Convergence 1950-2000," Population and Development Review, 27 (2001), 155-171.

10 Roser et al., "Life Expectancy."

11 Global Burden of Disease [GBD] 2017 HIV collaborators, "Global, Regional, and National Incidence, Prevalence, and Mortality of HIV, 1980-2017, and Forecasts to 2030, for 195 


\section{ROMOLA DAVENPORT}

Countries and Territories: A Systematic Analysis for the Global Burden of Diseases, Injuries, and Risk Factors Study 2017," Lancet HIV, 6 (2019), e831-e859; and M. Roser and H. Ritchie, "HIV/AIDS," published online at OurWorldInData.org (2020). Retrieved from: https://our worldindata.org/hiv-aids [Online Resource].

12 P. Grigoriev, F. Meslé, V. Shkolnikov, E. Andreev, A. Fihel, M. Pechholdova, and J. Vallin, "The Recent Mortality Decline in Russia: Beginning of the Cardiovascular Revolution?" Population and Development Review, 40 (1) (2014), 107-129.

13 S. Preston and H. Wang, "Sex Mortality Differences in the United States: The Role of Cohort Smoking Patterns," Demography, 43 (4) (2006), 631-646; and I. Waldron, "Contributions of Changing Gender Differences in Behavior and Social Roles to Changing Gender Differences in Mortality," in D.F. Sabo and D.F. Gordon eds., Research on Men and Masculinities Series, Vol. 8 Men's Health and Illness: Gender, Power, and the Body (London: Sage, 1995), pp. 22-45.

14 A. R. Omran, "The Epidemiologic Transition: A Theory of the Epidemiology of Population Change," Milbank Memorial Fund Quarterly, 49 (4) (1971), 509-538.

15 S.J. Olshansky, M.A. Rudberg, B.A. Carnes, B.A. Cassel, and J.A. Brady, "Trading Off Longer Life for Worsening Health: The Expansion of Morbidity Hypothesis," Fournal of Aging and Health, 3 (1991), 194-216.

16 E.A. Wrigley and R. Schofield, The Population History of England 1541-1871: A Reconstruction (Cambridge: Cambridge University Press, 1989).

17 J. Walter and R. Schofield, "Famine, Disease and Crisis Mortality in Early Modern Society," in J. Walter and R. Schofield eds., Famine, Disease and the Social Order in Early Modern Society (Cambridge: Cambridge University Press, 1989), pp. 65-73.

18 W.H. McNeill, Plagues and Peoples (New York: Anchor, 1976).

19 Ibid; W.H. McNeill, "Migration Patterns and Infection in Traditional Societies," in N.F. Stanley and R.A. Joske eds., Changing Disease Patterns and Human Behaviour (London: Academic Press, 1980), pp. 28-36.

20 J. Landers, Death and the Metropolis: Studies in the Demographic History of London, 1670-1830 (Cambridge: Cambridge University Press, 1993), p. 172.

21 R.J. Davenport, "Urbanisation and Mortality in Britain c.1800-1850," Economic History Review, 73 (2), (2020), 455-485.

22 J. De Vries, European Urbanization, 1500-1800 (London: Methuen, 1984); and S. Kuznets, Modern Economic Growth: Rate, Structure and Spread (New Haven, CT: Yale University Press, 1996), p. 60 .

23 G. Alfani and C. Ó Gráda eds., Famine in European History (Cambridge: Cambridge University Press, 2017).

24 C. Ó Gráda, Eating People Is Wrong, and Other Essays on Famine, Its Past, and Its Future (Princeton, NJ: Princeton University Press, 2015).

25 G. Ó Gráda, Famine: A Short History (Princeton, NJ: Princeton University Press, 2009), pp. 23-24.

26 A. Sen, Poverty and Famines: An Essay on Entitlement and Deprivation (Oxford: Clarendon Press, 1981).

27 E.A. Wrigley, Poverty, Progress, and Population (Cambridge: Cambridge University Press, 2004), pp. 206-208.

28 R. Hoyle, "Britain," in G. Alfani and G. Ó Gráda eds., Famine in European History (Cambridge: Cambridge University Press, 2017), pp. 166-184; M. Kelly and C. Ó Gráda, "Living Standards and Mortality Since the Middle Ages," Economic History Review 67 (2) (2014), 358-381; and J. Walter, "The Social Economy of Death," in J. Walter and R. Schofield eds., Famine, Disease and the Social Order in Early Modern Society (Cambridge: Cambridge University Press, 1989), pp. 75-128.

29 P. Slavin, "Market Failure During the Great Famine in England and Wales (1315-1317)," Past and Present, 222 (2014), 9-49.

30 R.M. Smith, "Dearth and Local Political Responses: 1280-1325 and 1580-1596/7 Compared," in M. Kowaleski, J. Langdon, and P.R. Schofield eds., Peasants and Lords in the Medieval English Economy: Essays in Honour of Bruce M. S. Campbell (Turnhout: Brepols, 2015), pp. 387-391.

31 Hoyle, "Britain"; Smith, "Dearth and Local."

32 T. Dyson, "On the Demography of South Asian Famines: Part I", Population Studies, 45 (1) (1991), 5-25; and Walter and Schofield, "Famine, Disease."

33 Walter and Schofield, "Famine, Disease." 
34 Kelly and Ó Gráda, "Living Standards"; P.R. Galloway, "Differentials in Demographic Responses to Annual Price Variations in Pre-Revolutionary France," European Fournal of Population, 2 (1987), 269-305.

35 Ó Gráda, Famine, pp. 108-121.

36 S.J. Kunitz, "Speculations on the European Mortality Decline," Economic History Review, 36 (3) (1983), 349-364; and J.C. Riley, The Eighteenth-Century Campaign to Avoid Disease (New York: St. Martin's Press, 1987).

37 Riley, The Eighteenth-Century.

38 A.T. Duggan, M.F. Perdomo, D. Piombino-Mascall, S. Marciniak, D. Poinar, M.V. Emery, et al., "17th Century Variola Virus Reveals the Recent History of Smallpox," Current Biology, 26 (2016), 3407-3412.

39 R.J. Davenport, M. Satchell, and L.M.W. Shaw-Taylor, "The Geography of Smallpox in England Before Vaccination: a Conundrum Resolved', Social Science and Medicine, 206 (2018), 75-85.

40 D.R. Hopkins, Princes and Peasants: Smallpox in History (Chicago, IL: University of Chicago Press, 1983).

41 J. Banthia and T. Dyson, "Smallpox in Nineteenth-Century India," Population and Development Review, 25 (4) (1999), 649-680; and E.J. Edwardes, A Concise History of Small-Pox and Vaccination in Europe (London: Lewis, 1902).

42 M. Livi-Bacci, Population and Nutrition: An Essay on European Demographic History (Cambridge: Cambridge University Press, 1991); and T. Bengtsson and F. van Poppel, "Socioeconomic Inequalities in Death from Past to Present: An Introduction," Explorations in Economic History, 48 (2011), 343-356.

43 S.J. Kunitz and S.L. Engerman, "The Ranks of Death: Secular Trends in Income and Mortality," Health Transition Reviewe, 2 (Supp.) (1992), 29-42.

44 J. Landers, "Historical Epidemiology and the Structural Analysis of Mortality," Health Transition Reviewe, 2 (Supp.) (1992), 47-75.

45 Davenport, "Urbanisation."

46 R. Woods, "The Effects of Population Redistribution on the Level of Mortality in NineteenthCentury England and Wales," The Fournal of Economic History, 45 (3) (1985), 645-651; see also C. Torres, V. Canudas-Romo, and J. Oeppen, "The Contribution of Urbanization to Changes in Life Expectancy in Scotland, 1861-1910," Population Studies, 73 (2019), 387-404.

47 R. Woods, The Demography of Victorian England and Wales (Cambridge: Cambridge University Press, 2000).

48 I. Loudon, Death in Childbirth: An International Study of Maternal Care and Maternal Mortality 1800-1950 (Oxford: Clarendon Press, 1992).

49 P. Aaby, J. Bukh, I.M. Lisse, and M.C. da Silva, "Decline in Measles Mortality: Nutrition, Age at Infection, or Exposure?" British Medical Journal (Clinical Research Edition), 296 (6631) (1988), $1225-1228$.

50 R. Reves, "Declining Fertility in England and Wales as a Major Cause of the Twentieth Century Decline in Mortality," American Fournal of Epidemiology, 122 (1) (1985), 112-126.

51 E. Vynnycky and P.E.M. Fine, "The Natural History of Tuberculosis: The Implications of Age-Dependent Risks of Disease and the Role of Reinfection," Epidemiology and Infection, 119 (1987), 183-201.

52 R.J. Davenport, M. Satchell, and L.M.W. Shaw-Taylor, "Cholera as a 'Sanitary Test' of British Cities, 1831-1866," History of the Family, 24 (2019), 404-438.

53 Ibid.

54 B. Harris and A. Hinde, "Sanitary Investment and the Decline of Urban Mortality in England and Wales, 1817-1914," The History of the Family, 24 (2019), 339-376.

55 Davenport et al., "Cholera."

56 P. Ewald, Evolution of Infectious Disease (Oxford: Oxford University Press, 1993).

57 Ibid, 77-86.

58 Progress on Household Drinking Water, Sanitation and Hygiene 2000-2017. Special Focus on Inequalities (New York: United Nations Children's Fund (UNICEF) and World Health Organization, 2019).

59 H. Ritchie and M. Roser, "Causes of Death," published online at OurWorldInData.org. (2020). Retrieved from: https://ourworldindata.org/causes-of-death [Online Resource]. 
60 S.H. Preston, Mortality Patterns in National Populations (London: Academic Press, 1976).

61 Ibid.

62 G.E. Finch and E.M. Crimmins, "Inflammatory Exposure and Historical Changes in Human Life-Spans," Science, 305 (5691) (2004), 1736-1739.

63 I.T. Elo and S.H. Preston, "Effects of Early Life Conditions on Adult Mortality: A Review," Population Index, 58 (1992), 186-212.

64 E. Barbi and J. Vaupel, "Comment on Inflammatory Exposure and Historical Changes in Human Life-Spans," Science, 308 (2005), 16.

65 There were some notable curative antecedents, such as quinine, which was used against malaria from at least the sixteenth century in Bolivia and Peru; disinfectants that killed pathogens and were deployed in the treatment of wounds; anti-toxins (antibodies) against diphtheria and tetanus, developed in 1890; and salvarsan against spirochaete infections, notably syphilis (discovered in 1909).

66 Loudon, Death.

67 A. Løkke, "The Antibiotic Transformation of Danish Obstetrics. The Hidden Links between the Decline in Perinatal Mortality and Maternal Mortality in the Mid-Twentieth Century," Annales de Démographique, 123 (2012), 205-224.

68 R. Smith and J. Coast, "The True Cost of Antibacterial Resistance," British Medical Fournal, 346 (2013), f1493.

69 BCG World Atlas, 2nd edition (2017). Retrieved from: www.bcgatlas.org/index.php [Online Resource].

70 P. Mangtani, I. Abubakar, C. Ariti, P. Mangtani, I. Abubakar, C. Ariti, et al., "Protection By BCG Against Tuberculosis: A Systematic Review of Randomised Controlled Trials," Clinical Infectious Diseases, 58 (2014), 470-480.

71 T. Dyson and M. Das Gupta, "Demographic Trends in Ludhiana District, Punjab, 1881-1981: An Exploration of Vital Registration Data in Colonial India," in T-J. Liu, J. Lee, D. S. Reher, O. Saito, and W. Feng eds., Asian Population History (Oxford: Oxford University Press, 2001), pp. 79-104.

72 Riley, Poverty.

73 M. Livi-Bacci, A Concise History of World Population, 3rd edition (Oxford: Blackwell, 2001).

74 M. Roser, "Fertility Rate," Published online at OurWorldInData.org (2020). Retrieved from: https://ourworldindata.org/fertility-rate [Online Resource]; and H. Rosling, O. Rosling, and A.R. Rönnlund, Factfulness (New York: Flatiron Books, 2018), pp. 32-38.

75 S.H. Preston, "The Changing Relation between Mortality and Level of Economic Development," Population Studies, 29 (2) (1975), 231-248.

76 Ibid.

77 J.C. Caldwell, "Routes to Low Mortality in Poor Countries," Population and Development Revierw, 12 (2) (1986), 171-220.

78 Ibid.

79 J.C. Riley, Social Growth and Good Health: A History of Twelve Countries (Berkeley, CA: University of California Press, 2008).

80 T. McKeown, The Modern Rise of Population (London: Edward Arnold, 1976); and R. Floud, R. W. Fogel, B. Harris, and S.C. Hong, The Changing Body: Health, Nutrition, and Human Development in the Western World Since 1700 (Cambridge: Cambridge University Press, 2011).

81 S. Szreter, "Economic Growth, Disruption, Deprivation, Disease, and Death: On the Importance of the Politics of Public Health for Development," Population and Development Reviere, 23 (1997), 693-728; A. Hardy, The Epidemic Streets (Oxford: Clarendon Press, 1993); and A. Deaton, The Great Escape (Princeton, NJ: Princeton University Press, 2013).

82 Riley, Rising Life; Woods, The Demography.

83 Floud et al., The Changing Body.

84 Easterlin, Growth.

85 J. Oeppen and J.W. Vaupel, "Broken Limits to Life Expectancy," Science, 296 (5570) (2002), 1029-1031. 


\title{
MASS DEATH DURING MODERN EPIDEMICS
}

\author{
Horrors and their consequences
}

\author{
Samuel Cohn
}

Mortality rates from epidemic diseases have declined considerably over the past century, thanks to improvements in medicine and, especially, public health, including new levels of coordination associated with globalization. Epidemics have not disappeared, however, and they were a major element in the history of death in the nineteenth and early twentieth centuries. This chapter takes a new look at epidemics and the reactions they generated, on a global basis, with primary attention to nineteenth- and early twentieth-century experience but with reference as well to the new kinds of epidemics that have cropped up more recently. The result offers important if unexpectedly complex perspectives on modern attitudes to death and attendant features such as medicalization, with links as well to the premodern past.

Concerning the emotional consequences of big epidemics across time and the globe, a general consensus persists: the horrors of epidemic mass death feed the worse side of human nature - and this characterization applies in contemporary experience as in times past. In the words of the Enlightenment Danish-German statesman and scholar of antiquity, Barthold Georg Niebuhr: "Times of plague are always those in which the bestial and diabolical side of human nature gains the upper hand." ${ }^{1}$ Shortly after World War II, the annalist historian, René Baehrel agreed: epidemics induced "class hatred" and these reactions were a part of our "structures mentales ... constantes psychologiques". ${ }^{2}$ These negative perceptions of epidemics' psychological effects became more frequent and louder with the mysteries of HIV/AIDS in the 1980s and have continued into the twenty-first century. According to Carlo Ginzburg, "the prodigious trauma of great pestilences intensified the search for a scapegoat on which fears, hatreds and tension ... could be discharged." "For Dorothy Nelkin and Sander Gilman claimed, "Blaming has always been a means to make mysterious and devastating diseases comprehensible." "Roy Porter concurred with Susan Sontag: "deadly diseases" especially when "there is no cure to hand" and the "aetiology ... is obscure ... spawn sinister connotations." From earthquake-wrecked, cholera-stricken Haiti, the physician, anthropologist and "University Professor" at Harvard, Paul Farmer, concluded: "Blame was, after all, a calling card of all transnational epidemics." 6

Yet, several years before the AIDS crisis, two remarkable and seminal works were published on the global Influenza Pandemic from 1918, which lasted in places such as Australia and China into the 1920s-Alfred Crosby's America's Forgotten Pandemic, first 
published in 1976 as Epidemic and Peace, 1918, ${ }^{7}$ and Richard Collier's more global analysis, The Plague of the Spanish Lady. ${ }^{8}$ Both presented evidence of a diametrically opposed view of human nature in the face of horrific mortalities. In this case, they amounted to the highest numbers ever estimated for a pandemic in world history, presently climbing to as high as 100 million. $^{9}$ Most of these, moreover, occurred within a two-month period, October and November 1918. Neither author revealed any incidents of individual or collective violence against victims of the disease or its supposed carriers. Instead, the picture is overwhelmingly one of compassion, volunteerism, and martyrdom. In Crosby's words: "People of all kinds poured into Emergency Aid Headquarters ... thrusting themselves into the presence of lethal disease."10 Despite this influenza's lightning contagion, the authors' evidence shows the pandemic uniting communities across neighborhoods, religions, ethnicity, and class lines.

However, neither author placed the 1918 experience in a broader historic context of epidemics' socio-psychological reactions: was this explosion of good will unique to 1918-20? Furthermore, those decrying the early reactions to HIV/AIDS, principally in the United States, and who then generalized these reactions as common to epidemics across history, did not pause to consider the evidence from 1918, published only five years earlier. Instead, they repeated a one-dimensional view of human nature. Several even compared the homophobic attitudes of far-right preachers and publicists such as Pat Robertson, Pat Buchanan, Jerry Falwell, and William F. Buckley to the mass slaughter of Jews in Europe during the Black Death, 1348-50. ${ }^{11}$ No one to date has catalogued the violence against the infected or homosexual communities directly connected to the rise of HIV/AIDS. Certainly, in Africa collective violence against those associated with HIV/AIDS has appeared in the press and in scholarly analyses, ${ }^{12}$ but I know of few acts of collective violence against AIDS victims or those thought to be carriers outside of Africa. Had the Ray family been at home when neighbors torched their house in Arcadia, Florida, on 28 August 1987, because three of their children were haemophiliacs, this might have been a case of murder caused by the fear of AIDS. Instead, the community, which had banned these children from school, then offered the family charity (although it was refused). ${ }^{13}$ Nonetheless, the blame and hatred against HIV/AIDS victims in the 1980s, despite its deep and crippling discrimination, cannot not compare with the atrocities of 1348 to 1350 that changed the shape of Jewry in Europe by exterminating entire communities down the Rhineland, across German-speaking regions, and into France and Spain.

We might then hypothesize that a "civilizing process" transformed reactions to big epidemics from antiquity and the Middle Ages to the more enlightened nineteenth century and thereafter. This supposition, however, would be convincing only if we possessed one point of comparison to cover the entirety of the pre-nineteenth-century world - the Black Death of 1348. Instead, epidemics provoking blame or violent persecution of the victims of disease or any others are almost completely absent from the pre-nineteenth-century past - which means, as we will see, that the modern story itself is more complex.

In his account of the fifth-century BCE "Plague of Athens", Thucydides reported that some may have "even said" that this plague had been spread at Piraeus by Peloponnesians poisoning cisterns. ${ }^{14}$ However, when the plague became full-blown at Upper Athens, decimating almost a third of the population, Thucydides' description changed. Reactions quickly transformed from self-preservation and refusal to visit the sick to compassion: 
More often those who had recovered ... had pity for the dying and the sick, because they had learnt what it meant and were themselves by this time confident of immunity; for the disease never attacked the same man a second time, at least not with fatal results. ${ }^{15}$

Although only a fourth of his history of Rome has survived, Livy mentions at least fifty-seven plagues from the sixth to first century BCE but describes none provoking mass violence, hatred, or blame. Instead, the epidemics often spawned sympathy that ended warfare and internal strife between the plebes and the senatorial classes as with a plague in 433-2 BCE and another two decades later. ${ }^{16}$ With a particularly severe and mysterious plague in $399 \mathrm{BCE}$, for which the priests could find no remedies, Livy describes the Romans doing just the opposite of what scholars from the perspective of AIDS have assumed as the natural reaction to diseases like AIDS with no immediate cures at hand. The Romans' solution was the invention of a new eight-day holiday of banqueting in which

the front gates of the houses were thrown open and all sorts of things placed for general use in the open courts, all comers, whether acquaintances or strangers, being brought in to share the hospitality. Men who had been enemies held friendly and sociable conversations with each other and abstained from all litigation, the manacles even were removed from prisoners. ${ }^{17}$

After the Plague of Athens, the best-known epidemic in Antiquity - the Antonine Plague of 165-180 CE, during the reign of Marcus Aurelius - was new, mysterious, and originated beyond the Empire's borders. Moreover, on the eve of the epidemic, Marcus was engaged in wars with Germanic tribes and persecuting Christians. Yet no blame or collective violence ensued. Instead, he created new opportunities for "barbarian" outsiders, and from his estates gave charity to the afflicted, including funerals for the poor at public expense. ${ }^{18}$ The next pandemic to sweep across Europe and that originated beyond the borders of the Roman Empire - the Plague of Justinian - struck Constantinople soon after 541 CE. Plague deaths here mounted from a trickle to "five thousand ... even ten thousand a day", creating "confusion and disorder everywhere". Yet no source reports this plague provoking waves of blame or persecution. Instead, its principal historian, Procopius, paid tribute to the emperor and his administration. Justinian sent the military to assist civilians and to distribute money to them, and his chief administrator coordinated the relief, allocating public funds for assistance as well as dipping into his own purse to ensure the burial of the plague dead. ${ }^{19}$ Procopius' praise was all the more remarkable given his previous disposition towards the emperor as revealed in his Secret History, which berated Justinian for his greed, corruption, and support of the circus of the Blues ${ }^{20}$ that had previously fanned division throughout the city. Moreover, the pandemic's charitable response was not just top down. The plague ended, at least temporarily, the circuses' perennial divisions. Those who before the plague despised one another now united to honor the dead.

There is, however, no denying the Black Death's reactions of blame, cruel abandonment of family members, and the slaughter of hundreds of communities of Jews, even if this brutal destruction was not universal across Europe and the Middle East. Yet the magnitude of the Black Death's blame and social violence was an exception in world 
history, and even before the plague ended in the late summer of 1348, contemporaries in Tuscany detected changes in attitudes. The Florentine chronicler Matteo Villani and the Pisan Ranieri Sardo reported people recovering their humanity, realizing that flight and abandonment of their duties and loved ones were counterproductive: charity and abnegation were more efficacious for self and community preservation. ${ }^{21}$ For the frequent occurrences of plague into the sixteenth century, reports of collective violence against Jews or other minorities or cruel abandonment were almost unheard of. ${ }^{22}$ Now occurrences of plague could spawn the very opposite as with the rise in plague mortalities in 1399-1400 that gave birth to a new flagellant movement. This one was the polar opposite of the Black Death's flagellant movement in 1349. Instead of re-enacting Black Death division and hate, in 1400 "the Bianchi" was a peace movement with processions that cut across class, crossed city walls into the countryside, and brought men, women, and children together to end social conflict from everyday litigation to factional strife among aristocratic clans and warfare between territorial states.

To be sure, plague did not remain innocent of casting blame and provoking violence. As early as 1530 and into the seventeenth century, depending on the place, accusations and trials against supposed plague spreaders (untori, engraisseurs) by commoners and elites and aided by magistrates and even the medical profession blighted cities such as Geneva, Palermo, Toulouse, Lyon, and Milan. The scale of these trials, however, proved to be no return to Black Death atrocities. In the best-documented of these persecutions - that of Milan in 1630 - the trial transcripts reveal only five executions, with fewer than ten more coming from other sources. ${ }^{23}$

\section{Cholera}

Let us return to "modernity", more specifically, the nineteenth century. Had advances in medical science, the spread of ideas of the Enlightenment, and democratic movements dampened the epidemic-hate connection? The answer is a resounding no. The next near-global epidemic was cholera that in 1817 spread from Bengal into parts of eastern Europe and Asia. Its second wave of the 1820s and 1830s, however, was more dramatic in its spread across Russia, Europe, and the oceans to Japan and the Americas. It was also more dramatic for its socio-psychological consequences. With this disease, an overriding mythology about cholera's cause triggered social violence to levels not witnessed with any disease since 1348. Most remarkable was the duration and consistency of these deadly fantasies. Across strikingly different cultures, economies, and regimes - East, West, and South in Europe and on the east coast of the US - class conflicts arose with the poor as the perpetrators of violence and elites as their targets. ${ }^{24}$ Without obvious communication among rioters from New York to Asiatic Russia or evidence that the protesters (often illiterate) were aware of these riots occurring simultaneously across long distances, the cholera conspiracies repeated stories of elites masterminding a Malthusian cull of the poor, with health boards, doctors, pharmacists, nurses, and government officials as the agents of the planned class mass murder. In places in Europe, these same myths recurred into the twentieth century, igniting widespread deadly rioting, underpinned by similar stories as far east as Java ${ }^{25}$ and Japan. ${ }^{26}$ Cholera's high lethality rates often meant that few patients returned alive from hospitals, triggering suspicions against doctors and authorities that ignited collective violence. ${ }^{27}$ In the British Isles alone, I have discovered seventy-two cholera riots in fifteen months alone, November 1831 to January 1833, 


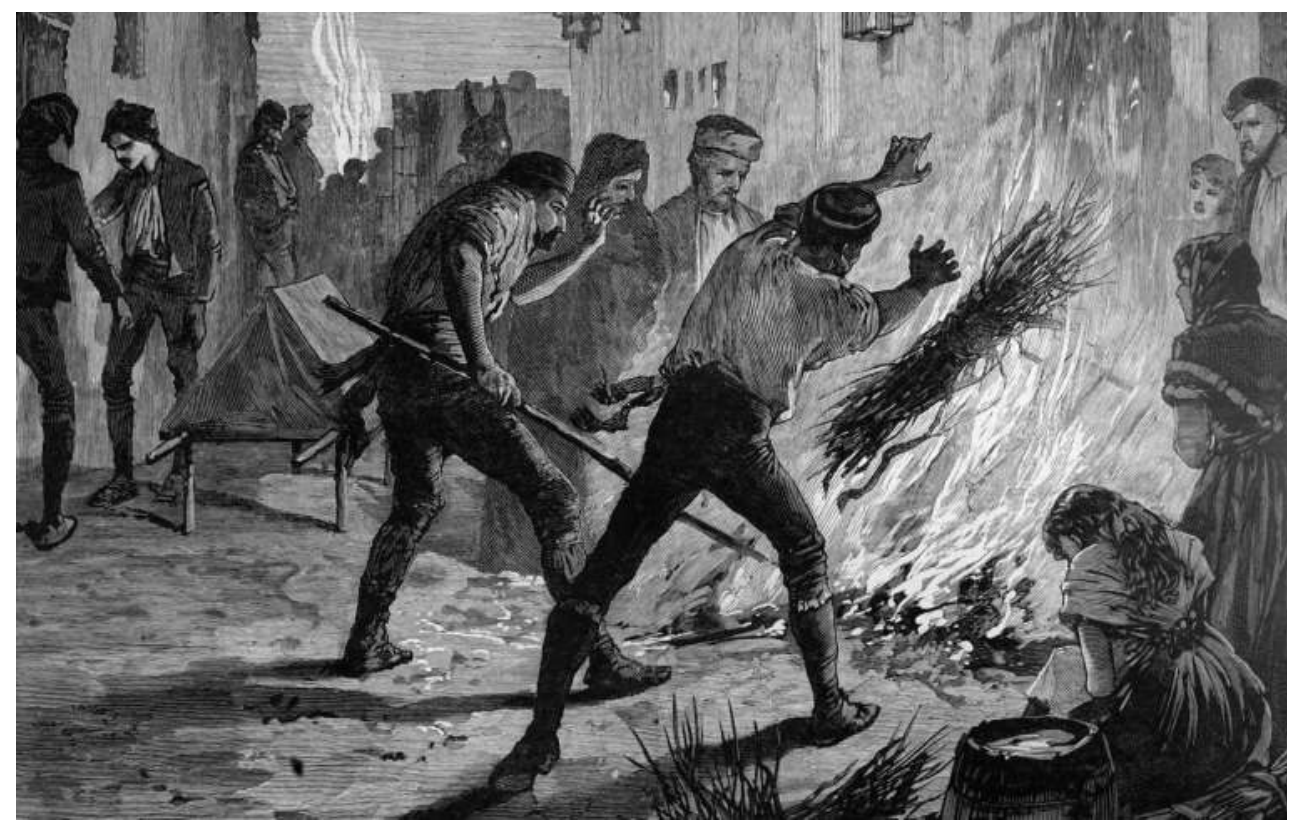

Image 2.1 Efforts to use smoke to dispel cholera in Granada, 1884.

(C) World History Archive/Alamy Stock Photo.

several of which amassed crowds of more than $2,000 .^{28}$ These destroyed hospitals, ceremoniously ripped cholera hearses to pieces, and attacked doctors, nurses, and police. Here, an ingredient rarely found elsewhere added to the fears of the poor: to satisfy the growing appetite of anatomy colleges to acquire cadavers for teaching, body snatching and even murder for these purposes had become well publicized before cholera had struck Britain and Ireland. These fears were not entirely matters of popular superstitions, as seen in a cholera riot in Paisley (Scotland) at the end of March 1832. Boys discovered two small shovels and a hook in an empty coffin buried in the town cemetery. Crowds assembled to inspect the evidence and then processed through the town attacking the cholera hospital and destroying everything inside it along with the homes of doctors.

In these riots, perpetrators and targets were not in the main interfaced in the ways we have come to expect with epidemic blame and violence. First, cholera rioters rarely blamed or attacked those infected or imagined as carrying the disease. Instead, the rioters imagined themselves "liberating" their friends and families, who, they believed had been seized by medical corps and police to be killed in death chambers called hospitals. Second, across the British Isles, the poor, often recently arrived Irish Catholics, poor women, and adolescent "lads" were the perpetrators of violence. ${ }^{29}$ The same was true in other regions, as with the immigrant Sarts in Russian cities such as Saratov or impoverished fig growers and women in Italian towns such as Barletta. As I have argued previously, the cholera riot was a class struggle, but one which we may not wish to romanticize. ${ }^{30}$ 
Despite strikingly different political cultures, cholera riots in Russia, Eastern Germany, Hungary, and Italy assumed the same patterns as seen in Britain or places such as New York City. There were, however, three major differences: the crowd sizes of protesters were often larger, the damages to property and especially to persons were more brutal, and especially in Russia and Italy, these riots did not diminish in frequency or size after the cholera's European debut in the 1830s. Even in provincial towns such as Serdobsk, cholera rioters in 1892 burnt 300 houses, causing "great distress" and homelessness. ${ }^{31}$ Much greater was the destruction the same year at Hughesofka (now Donetsk in the Ukraine). Serious rioting began on 1 August at Hughes' steelworks and collieries. A "mob" stoned the police, while they were removing a woman to the hospital. The rioters then attacked Cossacks dispatched to defend the police. Next, the crowd sacked the hospital and tried to set the town ablaze. According to an Odessa correspondent, 10,000 rioters excited by drink began the day by killing several Jews, but no evidence shows that the Jews were blamed for the disease or that they were more than incidental targets of residual anti-Semitism. Instead, the rioters turned their destruction against the town itself and on a second day of rioting burnt the town down, "every house reduced to ashes, including even the church". They also invaded the factories, "taking everything not nailed down" with the Welsh owners running for their lives. ${ }^{32}$ Restoring order took three days and only after two regiments and a battery of artillery had arrived. ${ }^{33}$ According to Le Temps, on the first day of fighting alone, the rioters killed or gravely injured twenty-five Cossacks, while 200 rioters were "put out of combat". Yet by the second day, "the Cossacks still were unable to defend themselves against the thousands of workers and were mostly killed." 34

The longevity of cholera's social violence was also marked in Italy and not only in the south. For the first cholera wave, which did not reach the peninsula and islands until 1836, cholera riots were confined almost entirely to Sicily. ${ }^{35}$ For successive waves from the $1860 \mathrm{~s}$ to Italy's last major cholera wave in 1910-11, they spread through the mainland, reaching towns around Rome, near Milan, ${ }^{36}$ and in Venice itself. ${ }^{37}$ For instance, at the end of August, 1911, in the isolated Calabrian town of Verbicaro, a "mob" of between 500 and 1,200 (depending on the newspaper) from a population of around 6,000, armed with spades, knives, sticks, and agricultural implements, paraded through the town, shouting "Long live the king!" and "Death to the Mayor!". The rebels besieged the town hall, wrecked the furniture, burnt the archives, then the building, along with the praetorial court, the telegraph office, and the mayor's house, and released prisoners from the local jail. The mayor, town clerk, and judge fled. A group of eleven, including three women, caught the clerk and "hacked [him] to pieces", and the judge "died of fright" as he reached the train station. The police wounded several and killed a woman. Troops arrived the following day and arrested eleven ringleaders. Corpses were left unburied, and cholera lethality rates (the percentage of those afflicted with the disease who died) exceeded 50 percent. Verbicaro's mayor escaped, but not for long: two days later, when threatened with dismissal, he returned and was murdered, tragically repeating his grandfather's fate fifty-five years earlier in another cholera revolt. $^{38}$

On first inspection, this riot may not seem to fit previous examples, where doctors and hospitals were the major targets. Fantasies of authorities inventing the disease to cull the poor, however, is expressed here as emphatically as in any cholera riot. Its opening scene was a town meeting. An employee, late for the meeting, was discovered to have been involved with the town's census. A woman struck his head with a knotty stick. 
Another shot him with a revolver, and while on the ground, a third slashed his head off with a pruning knife. The horrific scene had not happened by chance. The employee's role in the census was viewed with contempt. Harking back to cholera's central story line, across time and space, the peasants believed that the compilation of the census was the method for choosing who to eliminate to resolve overpopulation: "certain ones" had been selected in advance "for the sacrifice". ${ }^{39}$ With the arrival of carabinieri, as many as 4,000 - two-thirds of Verbicaro's population - fled, "disappearing into the gorges and ravines between the bramble and vineyards". According to Corriere della Sera, the reason for their exodus was not fear of reprisals for rioting but the belief that the government planned to poison them. ${ }^{40}$

Even as late as 1911, these ideas about cholera's origins and assaults on governments and the medical profession were not a monopoly of the Mezzagiorno. Space allows only one illustration, this one from a town in Rome's shadow, Segni, in October 1911. Because of the Giolitti censorship on cholera and its internationally embarrassing side effects, I have not been able to find reports of this riot in the Italian press, but thanks to the telephone, Reuter's news agency picked it up and the Scotsman, The Times, and several Australian papers published the story. Here, the size of the crowd, estimated at 3,000, was considerably larger than Verbicaro's and greater even than those in the vastly larger cities of London, Manchester, Edinburgh, and Glasgow in 1832. According to the papers, a new cholera hospital, which had admitted only five cholera patients, was the riot's spark:

The idea spread among ignorant people that the municipal authorities, the Government, and the Carabineers were in league to poison them [the poor of Segni], and had organized a slaughter of the innocents. A mob of three thousand marched on the Town Hall, shouting for the release from the hospital of their relations and friends. Soon the whole military force of the town, consisting of only five Carabineers, was overwhelmed ... the rioters ... battered down the Town Hall's door, intending to sack and destroy it and murder any they imagined responsible for the cholera ... The women were particularly ferocious ... The mob broke into the Town Hall, and threw all they could lay their hands upon out of the windows. A woman seized the municipal flag and shouted, "To the hospital". Her cry was taken up immediately, and the mob surged through the town, crying "Death to the doctors and nurses." 41

We have seen the commonalities in cholera reactions, but we should also examine their differences. First, far fewer episodes of cholera riots have been reported outside of Europe, both in the scholarly literature and by contemporary online newspapers. Even with Readex's "World Newspaper Archive collections of Caribbean Newspapers, 1718-1876" and "Latin American Newspapers, 1805-1922", I have found only two cholera riots south of the US border (before the seventh cholera wave in the $1990 \mathrm{~s}^{42}$ ), and these did not appear in a newspaper. In her biography of her husband, Sir Richard F. Burton, Lady Burton briefly describes cholera riots in Monte Video and Buenos Aires in 1869, which followed the same patterns as those in Europe: "mobs" gathered in market squares, murdered doctors, and accused the government of poisoning. ${ }^{43}$ Similarly, for Japan, I know of only one cholera riot, and it too came during a later cholera wave - not with the initial shock of the new disease's first performance. The Japanese one also followed the accustomed European plot, even if it possessed its own ghoulish 
embellishments. In 1897, when cholera hit Tokyo and few returned alive from hospitals, rumors spread of doctors draining blood from patients, plucking out their eyes, and sending them alive to the incinerator. As in Europe, such stories sparked "violent riots" against local authorities and required military intervention. ${ }^{44}$ But was Europe the exception in the size and frequency of cholera riots? Further research beyond Europe is needed. However, for the US with thousands of online newspapers available and Charles Rosenberg's classic work, ${ }^{45}$ we can speak with some confidence. Cholera riots here certainly were much rarer than in Europe and on a much smaller scale. Yet not even Rosenberg drew this comparison, and no one has yet to ask why most US cities, especially ones west of the eastern seaboard, even those severely devastated by cholera such as New Orleans and St Louis, ${ }^{46}$ were mostly spared from social violence.

Moreover, as we have seen, cholera reactions also varied across different cities and regions within Europe. An uplifting case study comes from Berlin. With the usual cholera-inspired rumors spreading and cholera riots swirling nearby at Königsberg, Posen, Memel, and Stettin in 1831, the king of Prussia intervened and modified Berlin's strict quarantine regulations. The city's middle classes also played their part by organizing soup kitchens for the unemployed and providing for orphans. ${ }^{47}$ Not only did Berlin escape cholera rioting in 1831, cholera social violence appears never to have invaded the city, despite its occurrence in other German cities until 1892.

Berlin's experience contrasts sharply with the history of cholera in the small cathedral city of Ostuni in Puglia. From the first cholera wave in Italy in 1836-7 to the last major one in 1910-11, local authorities prohibited non-elites from performing their traditional burial rites, visiting afflicted friends and relations, and viewing the bodies of loved ones before burial, while city's elites were allowed to bury theirs in traditional church grounds. Such class-based impositions supported fears that doctors and the state were conspiring to murder the poor. Seeing their relatives unceremoniously "thrown into ditches" of newly created cholera grounds, the "popolo" of Ostuni rioted in $1837 .{ }^{48}$ In 1910, the state's class-based burial restrictions remained in place, and fears of poisoning and burials alive resurfaced; in mid-November collective violence exploded beyond any of its previous incidents: 3,000 in a town of 18,500 wrecked the cholera hospital, "liberated the patients", burnt down the town hall and health department, took possession of the town square, attacked health workers, stoned carabinieri, and destroyed doctors' homes. ${ }^{49}$

Not all of Italy's cholera stories were so bleak. Yet, as with Ostuni's, its history of cholera reaction fails to plot a simple positive progressive. The worst cholera catastrophe as portrayed in literature ${ }^{50}$ and newspaper reports occurred in 1884, with Naples as the epicenter. The response of the young nation was unique in the history of cholera; however, as we will see, it resembled the US response during its worst yellow-fever pandemics. Newspapers such as Milan's La Perseveranza organized charitable subscriptions for the impoverished southerners; Verona's provincial government, the Giunta comunale of Livorno, and the Venetians collected funds to buy sheets for afflicted southerners; Florence, Piacenza, "and other places" organized public walks ("passeggiati") to benefit the Neapolitans. Homeopathic doctors offered care to Naples free of charge. Workers' groups such as Livorno's firemen and Venice's gondoliers (la Società Bucintoro) sent goods and money to Naples; across Italy carabinieri pledged funds. Clubs, such as the rowers of Rome (La Società del canottieri) raised money. Florentine workers, La Confederazione Operaia, formed their own "Comitato di soccorso" to care for Naples's "colerosi", and young northern "squadristi", such as those of la Squadra dei garibaldini livornesi, tied to the anti-clerical, democratic politics of the new republic, 
traveled south and risked their lives to care for their southern "fratelli". As a result (or at least a correlation), no serious cholera rioting was reported in Naples that year, despite experiencing its worst bout ever with cholera. With Italy's last major wave, 1910-11, the abnegation and unity no longer survived. Northerners now viewed cholera-stricken southerners not as "fratelli" but as the uneducated, superstitious, and exploited. Instead of bedsheets, monies, or volunteers, their assistance was words alone that explained the roots of southern backwardness. ${ }^{51}$

\section{Smallpox violence}

Cholera was certainly not the only epidemic disease to create civil strife and violence. Little known to American historians, the disease that provoked the most violent reactions in US history was smallpox. Moreover, its potential for sparking violence came late in day in smallpox history, with the epidemic in 1881-2, and lasted into the 1910s, that is, long after it had become a familiar disease both endemically and epidemically. Furthermore, smallpox had been less a mysterious disease than most others; for almost a century before the laboratory revolution, it possessed sound preventive procedures, first with inoculation, then with perfected versions of vaccination. In addition, the mortality rates of the 1881-2 epidemic were below other smallpox epidemics in 1836-8, 1852-4, and 1871-2. On the other hand, in epidemic form, with a strain contemporaries called "black smallpox", its lethality rates could be high, 30 percent or more, that is, higher than cholera's by the end of the nineteenth century. ${ }^{52}$

For the US alone, I have uncovered 72 major incidents from newspaper reports of collective violence provoked by the threat of smallpox invading their communities. These clustered in twenty-five years from 1881 to 1905 and differ from cholera riots in several important respects. ${ }^{53}$ First, the crowds varied in number from gangs of ten to ones in thousands as with the well-studied Milwaukee revolts of 6-29 August 1894 and the presently unnoticed smallpox revolts that occurred in Chicago at the same time. ${ }^{54}$ On the whole, the smallpox ones tended to be smaller than the cholera riots. More fundamentally, the social character of the perpetuators of violence and their targets was the opposite of those involved in cholera riots. They had switched sides. The social confrontation of most smallpox riots fulfils our present expectations coming from the age of AIDS: generally, those from the propertied classes formed the "mobs", and those attacked and often murdered were the victims of the disease, who also almost always were the poor and, in the South, overwhelmingly blacks. In small towns such as Topeka, Kansas, business elites threatened that any attempt to transport smallpox victims into their town would be met with force. In January 1882, the mayor bowed to the demands of Topeka's elites and cancelled any further plans to establish a pesthouse to care for the mounting numbers of smallpox patients. ${ }^{55}$ At Saint Croix, New Brunswick, that straddles the USCanadian border, with a hundred laborers on the Canadian side infected with smallpox, the townsmen placed armed guards on all its bridges to prevent any suspected of carrying smallpox from crossing. ${ }^{56}$

Far worse was the torching of pesthouses, often with the inmates inside. Outside Bessemer, Alabama, a pesthouse was built to accommodate thirty new cases of smallpox, "nine-tenths of whom were negroes". With the arrival of its first patient - a "negro"-on 27 July 1897, "a mob", comprised of neighboring white farmers came at night and "riddled" the pesthouse with bullets, sending the guards, nurses, and the patients fleeing. 
The mob, not fearing arrest, justified their actions "as the best and quickest means of ridding themselves of [smallpox's] presence". ${ }^{57}$ Such incidents were not confined to the South. In 1896, at Grand View, a suburb of Kansas City, Missouri, a "mob" of 200 men, armed with guns and "urged on by a number of women", destroyed a pesthouse and scattered all its lumber over a ten-acre site. The builders left the scene; no arrests were made, ${ }^{58}$ illustrating another difference between cholera and smallpox violence. For the former, where the crowds were the poor, arrests were common, and severe punishments with lashings and executions could follow, while with smallpox's propertied "mobs", few faced any reprisals.

In addition, smallpox social violence draws another parallel with HIV/AIDS, displaying the same assault by the media on the victims of the disease. Doctors, journalists, and intellectuals used the press and medical conventions to blame minorities and the poor for the disease - "negroes" in the South, "Bohemians, Germans, and the lowest of the Italians" in New York City, Chicago, and St Louis, and the "tramp" almost everywhere. They were blamed for their supposed susceptibility to the disease, their poverty, slovenly habits, ignorance, and superstition in allegedly refusing to be vaccinated. The largest group, however, to be pilloried were "Chinamen", especially those entering US ports on immigrant vessels. Along with their supposed "ignorant" and "dishonest" Chinese doctors, who the papers accused of hiding cases of smallpox while in transit, Chinese laborers were blamed for spreading smallpox to epidemic levels during the last two decades of the nineteenth century. ${ }^{59}$ Yet, even the same newspapers in which editorials lambasted the absence of controls on this scourge on America, occasionally presented statistics from ports of entry and hospitals that showed little evidence of Chinese passengers infected with smallpox on these ships. In addition, hospital records evinced the opposite of editors' claims: smallpox morbidity and mortality rates of the Chinese were much lower than those of the indigenous white populations in places such as New York City; Milwaukee; Baltimore; Port Huron, Michigan; and Honolulu. ${ }^{60}$

Smallpox riots and the prejudices of elites against the victims of the disease can be seen outside the US, most prominently in Canada. At Montreal the longest period of smallpox rioting, comprised of crowds larger than any I have found in the US, erupted between 28 September and 31 December. These included at least eight different confrontations, four of which amassed crowds over a thousand. ${ }^{61}$ Moreover, on the eve of the riots and continuing through these months of conflict, Montreal's English-language press, along with reports from England and the US, targeted the largely working class of French-Canadian Catholics. Montreal's Herald in a long editorial blamed the disease on them because of their "filthy customs", "ignorance of vaccination", and the "venality of their priests". ${ }^{62}$ New York's Sun followed, describing Montreal's two conflicting classes as different races, and, before any rioting erupted, charged the French community with "harboring and breeding the smallpox", without considering its effects "on the English population". It asserted that the French speakers fought the disease only "by pious observations and ceremonies"; its "backward" clergy preached that "the plague" was sent as punishment for neglecting religious duties and concluded, there was no surprise, "the believers in St. Roch ... and those in St. Vaccination ... soon came to blows." "63 When the rioting erupted, however, the configuration of forces resembled more cholera riots in Europe than the majority of smallpox riots in the US. Those on the barricades were impoverished French Canadians, and their targets were government officials and health officers implementing compulsory vaccination. The rioters attacked city hall, the central 
police station, and the Herald newspaper's office. They blocked the sanitary police from removing smallpox cases from tenements but, unlike during the cholera riots, doctors and nurses were not targeted. ${ }^{64}$

England also shows some evidence of smallpox riots, and similar to the US, they too came late in the disease's history, either during the epidemic of 1881 or afterwards. However, I have spotted only three, and only one assumed any traits of US smallpox riots. The earliest of these was mainly a riot of rumors that circulated through small towns and villages in the Black Country outside Birmingham: "three black doctors" had been sent "ostensibly to carry out the Vaccination Acts". "In reality", their mission was to murder "all the infant children"; and in the village of Holly Hall, the "black doctors" were rumored "to have kidnapped and eaten a child". Although covered by at least twentyfive papers in England, the most violent incidents were of mothers taking their children from school and breaking some school-house windows. ${ }^{65}$

The other two received less attention. In May 1884, a wagon carting away smallpox casualties in Hackney caused a crowd to form, which "threatened violence". 66 The police, however, intervened, and no more was heard of any rioting. A third incident at Bisley, near Gloucester, in 1886, conformed more closely to the US lines of rioting, with the respectable as the mobsters. At midnight, men from Bisley barred an ambulance carrying the first smallpox patient to its hospital and forced the patient to return to Stroud. The following night, the men "besieged, wrecked, and burnt down" the hospital. ${ }^{67}$ More significant than these small incidents, however, were the actions of elites from some of the wealthiest boroughs of London-Highgate, Hampstead and Fulhamwho used the courts, even Parliament, to close smallpox hospitals and disallow the transport of smallpox victims to wealthier districts for care as mandated by the Metropolitan Asylums Board. The elites' victories left the victims of smallpox either in desperately over-crowded facilities in poorer boroughs such as Hackney or without care at all. ${ }^{68}$ These actions through the press and tribunals may have resulted in more pain and deaths from smallpox than all the riots staged by businessmen militias or shotgun-toting white farmers in the US. As in the US, London's perpetrators of this subtler form of violence were elites, and their targets were the marginal and impoverished, turned into double victims, first of the disease, then of blame and discrimination.

Surprisingly, across the globe, few other examples of smallpox-induced social violence appear from the thousands of newspapers I have searched, and the few to appear do not conform to the US patterns. The best known of these were Rio de Janiero's riots of 1904. Similar to Montreal's, they were provoked by a bill instituting compulsory vaccination. Barricades were erected, tramcars burnt, water mains cut, and some were killed in street fighting. The violence continued to the end of January and ended with military law and a climbdown by the municipal government to "seriously" enforce the compulsory ordinance. ${ }^{69}$ The other major smallpox riot comes later still, in October 1935 at Calcutta, and involved thousands. Not only did it not conform with smallpox riots in the US, it differed from any disease-fueled violence seen in the Americas or Europe for any epidemic. Again, a government ordinance was the trigger; this one, however, did not concern vaccination but a prohibition against self-torture as penance when worshipping Mariamman, the goddess of smallpox. The participants, 10,000 to 15,000, at a ceremony to engage in self-torture defied the ordinance by piercing themselves with large hooks. The worshipers then pelted the police, chased and beat magistrates, and killed the chief constable. $^{70}$ 


\section{Plague}

We turn now to a third big killer of the late nineteenth and early twentieth centuries that was culpable of provoking mass violence-bubonic and pneumonic plague, whose agent was Yersinia pestis. These reactions of collective violence assembled several of the largest crowds of rebels and demonstrations seen for any protest for any epidemic in any period of history. Crowds in Calcutta over several days rioted in early July 1897 against military searches and other intrusive plague measures and were estimated at 5,000. ${ }^{71}$ In November 1898, the much smaller town of Seringapatam in Mysore amassed crowds of over 10,000 over three days. Armed with guns, swords, and axes and joined by villagers from the hinterland, they freed prisoners, captured the city's fortress, and took control of the city. ${ }^{72}$ However, the largest and most significant riot began with Mumbai's Julai weavers, after a young woman misdiagnosed with plague was forcibly confined to quarantine. The riot took over a quarter of the city and was reinforced by a general strike that included the closure of piece-goods markets and larger city bazaars, and 15,000 dockers, laborers, and cartmen employed at railway stations, which totally closed Mumbai's commerce. ${ }^{73}$

As with cholera and smallpox riots, plague riots also had a specific geographic focus. Although they occurred in Europe, the Middle East, China, and Russia, their theatre of action overwhelmingly was in India. Moreover, here they clustered into a narrow sevenyear period, 1896 to 1902, in contrast to cholera riots, which in Italy and Russia continued for ninety years, and to smallpox riots in the United States, which lasted for over thirty years. What were the principal patterns of the riots provoked by plague? Did they resemble those of European cholera, smallpox in the US, or assume a different pattern? Several reports from the international and Indian press, plus conclusions drawn from one or two riots by modern historians pictured plague violence in India as reproducing fantasies similar to those dominating cholera. ${ }^{74}$ But the evidence in support of such claims is thin. Through hundreds of newspaper reports from around the world and from India itself with the online Indian Times and hundreds of indigenous papers extracted and translated biweekly for British surveillance, ${ }^{75} \mathrm{I}$ have found sixty-three plague riots in India alone. Yet allusions to beliefs of intentional poisoning or doctors and governments conniving to murder the poor enter less than a handful of reports, most notably concerning two of India's earliest plague riots. Both were attacks against Mumbai's hospital on Arthur Road, first on 10 October, then on 5 November, 1896. Enraged by the forceful removal of a "healthy and innocent" female worker to the hospital, millworkers attacked the building. Reportedly, rumors spread of patients not returning from the hospital alive; something "diabolic" about it, therefore, had "claimed so many victims", and blood was being drained from patients' feet. ${ }^{76}$

In fact, segregation or quarantine camps, but not hospitals, were the prime targets of plague rioters across the subcontinent. Their protests, however, were not propelled by fantasies of doctors killing off the poor but often turned on the opposite - the lack of medical care and documented conditions of malnourishment in the camps with infants not even supplied milk. ${ }^{77}$ As for doctors, the protesters often complained of their absence and especially of the paucity of women doctors. ${ }^{78}$ Moreover, Indian papers called on their municipal governments and the British to engage in scientific studies of the character of the plague's spread and for its patterns of mortality to be based on the systematic collection of data on housing, people's habits, diet, climate, and other factors that anticipated the large international plague reports beginning in $1906 .^{79}$ 
Instead of myths, Indian plague protesters pointed to concrete reasons for distrust, centered on a wide range of humiliating and damaging practices. In addition to of forcible removal of the healthy to plague camps, protesters presented demands, something almost never seen in cholera or smallpox riots. These included petitions to end harassment of passengers at ports and train stations; indifference or hostility to religious customs, the destruction of shrines, and violation of sacred spaces; abuse from police and military "search parties", pre-dawn military strip examinations especially of women in public places; destruction of homes, especially of the poor; and a general milieu of arrogance, corruption, and blackmail. ${ }^{80}$ Finally, the protesters could include the Indian intelligentsia and galvanized support from newspaper editors. Backed by new evidence from European and Indian biological scientists, proving that this plague was not behaving according to patterns of rapid contagion known from Europe's medieval and early modern past, these newspapers attacked entrenched theories and policies of European stringent quarantine controls for plague prevention as unnecessary, even counterproductive. ${ }^{81}$

As this cross-class melding of protest suggests, plague protest in India differed crucially from those of cholera and smallpox, which had sharply divided communities by class, religion, and race. Moreover, concerns over plague prevention and combatting British offenses to religious sensibilities fused the sympathies of the subcontinent's two major religions, as at Hyderabad in April 1897. "A monster meeting" united Hindus and Muslims, "high and low", to oppose compulsory segregation - a practice that violated the religious rites and social customs of both religions. ${ }^{82}$ The same union of faiths produced the crowds of 10,000 at Seringapatam. By contrast, few cholera riots or protests possessed any hint of a united front; they failed even to unite various strata and occupations within the working class, unlike plague demonstrations in India that could rely on strikes from workers and middle-class shopkeepers. ${ }^{83}$ This unity may in part explain much of the success of India's plague riots - why, unlike cholera or smallpox riots, India's plague protests ended well before plague mortalities reached their climax in the Presidency of Bombay in 1907 or in the Punjab a decade later. Once governments modified their policies, the riots declined. Breakthroughs in medical science and alterations in public health policies proved a less plausible antidote against fears based on imaginary forces: discovery of cholera's epidemiology and pathology, along with better preventive measures, failed to dampen class hatred and violence against doctors and the state, in Italy, into the twentieth century and, in Russia, as late as 1921. At Astrakhan, a hub of cholera riots through the nineteenth century, cholera violence exploded again with the "maltreatment" of doctors and attacks now on Communist Commissaries, ending with 200 people killed. ${ }^{84}$

\section{Plagues of compassion}

To end on this note would do modernity an injustice. The emotional consequences of large waves of epidemics in the nineteenth century did not always or even generally produce social violence, either because of suspicion and blame or for political ends. We have already mentioned the Great Influenza of 1918, called in places (but not everywhere) the Spanish flu and by similar variants. Yet this most global of pandemics nowhere provoked collective violence against Spaniards or anyone else. The confrontation of mass death with one of history's most contagious diseases, instead, provoked individual and collective charity and self-sacrifice and unified communities in strikingly 
different social, cultural, and economic milieus, from hard-hit and impoverished miningtowns such as Kimberley in South Africa to cosmopolitan cities such as Philadelphia and Melbourne. One aspect of this charitable spirit, labeled by the US Surgeon General in 1918 as "the thousands of deeds of heroism", was the central role women played as organizers of nursing, household care, and as the volunteers risking their lives for utter strangers. Curiously, this gender dimension of the Great Influenza is missing from the two classic studies of the pandemic mentioned above. Yet in thousands of newspaper articles in the US, Canada, Australia, and South Africa, women were at the forefront as the nurses, founders of soup kitchens, poor relief, orphanages, and as ambulance drivers. Such sacrifice and devotion even cut across enemy lines as at Zolingue (Zolingen), a German-speaking village in the Swiss Agrovienne allied with Germany in 1918. When "a great number" of enemy French-speaking soldiers became stricken with "la grippe", "a large cohort of local ladies and girls", on their own initiative and with "exceptional zeal and perseverance", converted their school house into a hospital for these soldiers. We know of the incident only because the captain of the French troops soon after the Armistice wrote a column in his local newspaper, conveying the gratitude of his troops and thanking their "dear confederates". 85

The Great Influenza was certainly exceptional in its mass outpouring of charity and compassion globally and did not fit the patterns described above for cholera, smallpox, and plague in that the Great Influenza's reactions were global and not concentrated in a specific region or continent. Yet, the pandemic of 1918 was not the only pandemic to produce mass volunteerism and martyrdom in facing the horrors of epidemic mass casualty. The history of yellow fever, and not just one wave of it, presents similar reactions from at least the 1794 outbreak at Philadelphia to this disease's finale as a pandemic across the Deep South in 1905, even if, initially, panic and fear prompted distrust of authorities and abandonment of the ill. ${ }^{86}$ Still, incidents of destroying hospitals or town halls, killing doctors or police, and attacks against the victims of the disease are unknown for this disease's long history in the US and elsewhere. Nonetheless, the outpouring of charity and abnegation, especially once the disease became focused on the Deep South, differed from the 1918 influenza response. For places such as New Orleans in 1853, Memphis in 1878-9, and Pensacola and Jacksonville, Florida; Mena, Arkansas; Atlanta, Georgia; and many other cities and towns in 1905, young men, not women, were the mainstay of this volunteerism. Moreover, the relief efforts were organized from previously established men's organizations such as the Masonic Relief Board; the Shoulder-ToShoulder Club; the Southern Yacht Club at Lake Pontchartrain, north of New Orleans; the Negro Chauffeurs' Association; and even a Little League baseball team in New Orleans. These clubs organized the flow of monies and vital supplies to communities across the Deep South, devastated by yellow fever, and they came principally from northern states at times when North-South relations were dangerously strained as during the epidemic of 1853, on the eve of the Civil War, or in 1878, in the midst of Reconstruction, the passage of Jim Crow laws, and a crescendo of lynchings through the South. As Keating's 454-page chronicle of the Memphis experience in 1878 reveals, the North sent south more than supplies. Men such as the Reverend Louis Schuyler, a priest from Hoboken, New Jersey, raced to Memphis to volunteer his services and, by winter, had paid for it with his life. Moreover, during this epidemic, probably the worst in US history in mortality before 1918, the Howard Association made use of its interregional networks and took to the rails to break the rigid, but legal, "shotgun" quarantines that had 
reduced towns across Mississippi to starvation. In addition to supplies, its special relief train carried two physicians and fifteen nurses into this hecatomb of disease and established "a public kitchen" to feed, nurse, and clothe the afflicted and abandoned. ${ }^{87}$

\section{Conclusion}

The varied modern responses raise new comparative questions about reactions to mass slaughter caused by epidemic disease. First, their social and psychological reactions cannot be read one-dimensionally, as in the citations at the outset of this essay. It is simply not true that no matter what the disease, epidemics bring out human nature's "diabolical side", yielding to prejudice, blame, and social violence. Nor can we argue that epidemics were more likely to spawn violence and blame when they were mysterious without cures at hand, as argued for the discrimination initially unleashed by HIV/ AIDS. If this were the case, then epidemics of the distant past would have been the ones most potent in spurring hate and violence, and they were not. Thirdly, disease mattered. Not only did the worst pandemic in human history, as far as global mortality goes, fail to draw any acts of collective violence against victims of the disease or others blamed for it, no epidemics of influenza that I know of have ever sparked collective social violence. Fourthly, even with those epidemic diseases such as cholera, smallpox, and plague with histories of social violence and with crowds numbering in the thousands, the character of that blame and violence varied radically depending on the disease. Fifthly, in the age of AIDS, compassion and self-sacrifice are two reactions to horrific death by epidemics that historians, social scientists, and public intellectuals have dramatically underplayed. Given the mass and global volunteerism and political action unleashed by HIV/AIDS over the past four decades, that silence is certainly without justification. ${ }^{88}$

Finally, I would hypothesize that one feature determining an epidemic's potentiality to provoke violence centers on its rates of lethality, rather than its levels of mortality. As we have seen, in places like Segni (Lazio), only five cases of cholera were enough to send 3,000 from this small town rioting, destroying their town hall, hospital, and other government buildings, attacking carabinieri and government officials, and marching through their streets, chanting "Death to the doctors and nurses". A mantra of these diseases of high lethality was often, "Here, if the people come into the hospital, they don't leave alive," as was heard in cholera riots of the 1830s and still with Ebola in West Africa in 2014. ${ }^{89}$

Specific characteristics of epidemic diseases such as whether they generate signs and symptoms of disgust, kill quickly in a week or less, disproportionately involve adults, etc. need also to be considered. At the same time, variables outside the disease must also be evaluated, as seen with Berlin's escape from violence and blame in 1831, when mythologies of hate and blame were stirring and neighboring cities were rioting. Many enigmas remain and cry out for new comparative research: Why was cholera violence less frequent and ferocious in the US than in Europe but with smallpox it was the opposite? Were cholera riots as prevalent in places beyond Europe's borders as within Europe, and if not, as I would hypothesize, what were the reasons? How did these countries quell the anxieties and suspicions? Or did their social peace endure because of previous cultural contexts? Finally, what have been the underlying factors that have created plagues of compassion? In short, the emotional histories of epidemics, modern and premodern alike, are in their infancy. 


\section{SAMUEL GOHN}

\section{Notes}

1 Cited in Johannes Nohl, The Black Death: A Chronicle of the Plague Compiled from Contemporary Sources, trans. G.H. Clarke (London, 1926; originally, Potsdam, 1924), preface.

2 Baehrel, "La haine de classe en temps d'épidémie", Annales: E.S.C., 7 (1952): 351-60.

3 Ginzburg, "Deciphering the Sabbath", in B. Ankarloo and G. Henningsen (eds), Early Modern European Witchcraft: Centres and Peripheries (Oxford, 1990), 121-38.

4 Nelkin and Gilman, "Placing Blame for Devastating Disease", Social Research, 55 (1988): 362-78.

5 Porter, "The Case of Consumption", in J. Bourriau (ed.), Understanding Catastrophe (Cambridge, 1992), 179-203, 179.

6 Farmer with J. S. Mukherjee, Haiti after the Earthquake (New York, 2011), 191.

7 Crosby, America's Forgotten Pandemic: The Influenza of 1918, 2nd edn (Cambridge, 2003).

8 Collier, The Plague of the Spanish Lady: The Influenza Pandemic of 1918-1919 (London, 1974).

9 Niall Johnson and Jürgen Mueller. "Updating the Accounts: Global Mortality of the 1918-1920 'Spanish' Influenza Pandemic", Bulletin of the History of Medicine, 76 (2002): 105-15.

10 Crosby, America's Forgotten Pandemic, 82.

11 William Eamon, "Cannibalism and Contagion: Framing Syphilis in Counter-Reformation Italy", Early Science and Medicine, 3 (1998): 1-31, 1; Robert Swenson, "Plagues, History, and AIDS", American Scholar, 57 (1988): 183-200, 198; William Rushing, The AIDS Epidemic: Social Dimensions of an Infectious Disease (Boulder, 1995), 157; Irwin Sherman, The Power of Plagues (Washington, DG, 2006), 112-13. For other similar pronouncements, see Samuel Cohn, Jr., Epidemics: Hate and Compassion from the Plague of Athens to AIDS, 559, note 58.

12 See John Iliffe, The African AIDS Epidemic (Oxford, 2006); Mandisa Mbali, South African AIDS, Activism and Global Health Politics (Basingstoke, 2013); and Samuel K. Cohn, Jr.,Epidemics: Hate and Compassion from the Plague of Athens to AIDS (Oxford, 2018), for more references.

13 "AIDS Panic Rocks Florida Town", www.fact.com, 4 September 1987; and William Foege, "Plague: Perceptions of Risk and Social Responses", in Arien Mack (ed.) Time of Plague: The History and Social Consequences of Lethal Epidemic Disease, (New York, 1991), 9-20, 14.

14 Thucydides, History of the Peloponnesian War, 2.48.

15 Ibid., 2.47.

16 Livy, History of Rome, 4.25 and 4.52.

17 Ibid, 5.13.

18 Historia Augusta, 4.13 and 4.21.

19 Procopius, History of the Wars, 2.22.

20 On circus factions, see Alan Cameron, Circus Factions: Blues and Greens at Rome and Byzantium (Oxford, 1976), esp. ch. 1.

21 Cronaca di Pisa di Ranieri Sardo, ed. Ottavio Banti, Fonti per la Storia d'Italia 99 (Rome, 1963), 96-7; and Matteo Villani, Cronica con la continuazione di Filippo Villani, ed. Giuseppe Porta, 2 vols (Parma, 1995), I, 11.

22 See Cohn, Epidemics, 53-64.

23 Ibid., 132-41.

24 Ibid., 180.

25 Here, I have found two both during the sixth cholera wave, 1909-10. The first at Batavia (Jakarta) resulted from rumors of the government killing the "people" with cholera to place their heads in the foundation stones of bridges. Cholera mobs attacked disinfectant parties, injuring several and killing two; The Straits Times, 4 October 1909, 7. The second occurred at Bandung (West Java): "the populace" brutally attacked supposed poisoners, beating two to death; troops were summoned; "many were arrested"; ibid., 6 January 1910, 6.

26 Ruth Rogaski, Hygenic Modernity: Meanings of Health and Disease in Treaty-Port China (Berkeley, 2004), 152.

27 For a lethality rate of 59 percent from Edinburgh's cholera hospital in 1832, see Charles Creighton, History of Epidemics in Britain, ed. D.E.C. Eversley, 2 vols (London, 1965; original, Cambridge, 1891-4), II, 812.

28 Cohn, Epidemics, 175.

29 Cohn, Epidemics, ch. 7. 
30 Cohn, "Cholera Revolts: A Class Struggle We May Not Like", Social History, 42 (2017): $162-80$.

31 Evening Bulletin, 19 August 1892.

32 London Daily News, 9 August 1892, 5; and New York Herald, 10 August 1892, 6.

33 Scotsman, 27 August 1892, 7.

34 Le Temps, 28 August 1892, 2; Scotsman, 27 August 1892, 7.

35 Alfonso Sansone, Gli Avvenimenti del 1837 in Sicilia (Palermo, 1890) remains the best source for these revolts in Sicily. For several small ones in Cilento region of Puglia, see Franco Della Perutta, Mazzini e i revoluzionari italiani: Il "partito d'azione" 1830-1845 (Milan, 1974), 265.

36 At Crescenzano; Scotsman, 29 August 1867, 4.

37 La Nazione, 5 September 1867, 2; La Riforma (Florence), 10 September 1867, 4; Ibid., 18 September $1867,4$.

38 The Scotsman, 17 October 1911, 8: from Reuters; The Times, 17 October 1911, 5; La Stampa, 17 October 1911, 4: "Una ripetizione dei fatti di Verbicaro"; Corriere della Sera, 17 September 1911, front page. Also a number of Australian papers covered it, such as The Maitland Daily Mercury, 17 October 1911, 5.

39 Corriere della Sera, 31 August 1911, 2: "La folla paura degli "untori"”

40 Ibid., 4 September 1911, front page.

41 Scotsman, 17 October 1911, 8

42 See Marco Cueto, "Stigma and Blame during an Epidemic: Cholera in Peru, 1991", in D. Armus (ed.) Disease in the History of Modern Latin America: From Malaria to AIDS, (Durham, NC, 2003), 268-89, 281-3; and Charles Briggs, "Theorizing Modernity Conspiratorially: Science, Scale, and the Political Economy of Public Discourse in Explanations of a Cholera Epidemic", American Ethnologist, 31 (2004): 164-87, 169-70.

43 Lady Isabel Burton, The Life of Sir Richard F. Burton (London, 1898), 30.

44 Rogaski, Hygenic Modernity, 152.

45 Rosenberg, The Cholera Years: The United States in 1832, 1849, and 1866 (Chicago, 1962).

46 See Charles Roth, "The Western Cholera Trail: Studies in the Urban Response to Epidemic Disease in the Trans-Mississippi West, 1848-1850", PhD dissertation, University of California at Santa Barbara, 1993, who mentions no cholera violence.

47 Richard Ross, III, Contagion in Prussia, 1831: The Cholera Epidemic and the Threat of the Polish Uprising (Jefferson, NC, 2015), 249.

48 Della Perutta, Mazzini, 265.

49 Corriere della Sera, 15 November 1910 and ibid., 16 November 1910, 2; based on Il Giornale d'Italia, 14 November 1910; La Stampa, 15 November 1910, 1; Advertiser, 17 November 1910, 9.

50 See Matilde Serao, Il Ventre di Napoli (Naples, 1884).

51 For the newspaper references to these charitable events and others, see Cohn, Epidemics, 252-4.

52 S.L. Kotar and J.E. Gessler, Smallpox: A History (Jefferson, NC, 2013), 387; and Michael Willrich, Pox: An American History (New York, 2011 ), 31.

53 Cohn, Epidemics, ch. 12; several of these occurred in the colonial period and are discussed on p. 278.

54 Ibid., 287-9.

55 Iola Register, 27 January 1882, 6.

56 National Republican, 2 March 1882.

57 Birmingham State Herald, 27 July 1897; New York-Tribune, 28 July 1897, front page.

58 San Francisco Chronicle, 30 March 1896, 2.

59 For proclamations at a medical convention at Chicago in 1881, see Baltimore Sun, 25 June 1881, 4. These slurs against minorities appear in numerous newspapers, see Cohn, Epidemics, ch. 11.

60 Baltimore Sun, 25 June 1881, 4; Chicago Daily Tribune, 30 June 1881, 9; Hawaïan Gazette, 16 February 1881, 2; The Saturday Press, 22 October 1881, 2; Sun, 30 April 1881, front page; and New Tork Times, 14 May 1882.

61 Michael Bliss, Plague: A Story of Smallpox in Montreal (Toronto, 1991); Cohn, Epidemics, 293-5.

62 Bliss, Plague, 120.

63 Sun, 27 September 1885, 9. 
64 Cohn, Epidemics, 293-5.

65 Northern Echo, 16 May 1881; Bucks Herald, 28 May 1881; Reynold's Newespaper, 29 May 1881.

66 Trenton Evening Times, 29 May 1884, 2.

67 American Practitioner and News, 21-2 (1896): 141.

68 Cohn, Epidemics, ch. 13.

69 R. Bruce Low, "The Incidence of Smallpox throughout the World in Recent Years", Reports to the Local Government Board on Public Health and Medical Subjects no. 117 (1918): 85-6.

70 Straits Times, 15 October 1935, 19.

71 Guardian, 3 July 1897.

72 Scotsman, 24 November 1898, 5; and The Times, 24 November 1898, 5.

73 Guardian, 10 March 1898, 11 March 1898, and 12 March 1898; The Times, 10 March 1898, 5; Scotsman, 10 March 1898; and many others.

74 Ira Klein, "Plague, Policy and Popular Unrest in British India", Modern Asian Studies, 22 (1988): 723-55, 749; and Ian Catanach, "Fatalism? Indian Responses to Plague and Other Crises", Asian Profile, 12 (1984): 183-92, 190.

75 These are the Indian Office Records and Private Papers, the L/R/5 section now housed in the British Library and organized by district. I have consulted the extracts and translations from the Bombay Presidency, the Punjab, Bengal, Madras, the North-Western Provinces and Oudh.

76 L/R/5/150: week ending 7 November 1896, no. 12; and Mumbai's Anglo-Gujaráti Kaisere-Hind, 1 November 1896.

77 For the evidence from numerous India papers along with the Guardian and others, see Cohn, Epidemics, chs. 14 and 16.

78 See for instance, L/R/5/152, week ending 3 April 1897, no. 14, Muslim Herald; L/R/5/157, week ending 13 March 1897, no. 23, Kesari; and L/R/5/156, week ending 25 May 1898, no. 41, Sudárak.

79 L/R/5/153, week ending 16 April 1898, no. 23, Mahrátta; L/R/5/156, week ending 26 October 1901, Indian Spectator, and especially, L/R/5/80, week ending 16 May 1902, no. 11, Advocate.

80 See, for example, L/R/5/151, week ending 7 November 1896, no. 19, Champion; ibid., 153, week ending 19 March 1898, no. 21, Kaiser-e-Hind; and The Times, 14 April 1900, 3; L/R/5/ 153, week ending 19 March 1898, no. 21, Indian Spectator, and many others.

81 See the editorials of Bal Gangadhar Tilak in his paper, the Mahratta, for instance L/R/5/153, week ending 16 April 1898, no. 23.

82 L/R/5/152, week ending 10 April 1897, no. 34; and Cohn, Epidemics, ch. 10.

83 See Cohn, Epidemics, ch. 10; and Aditya Sarkar, "The Tie that Snapped: Bubonic Plague and Mill Labour in Bombay,1896-1898", International Revue of Social History, 59 (2014): 181-214, 186-7, 190, 193, 202, 206.

84 Guardian, 23 July 1921, front page. Moreover, cholera riots were "raging on a vast scale ... in many cities and villages", which were not named, and the Red Army dispatched "in a desperate effort to restore order"; New-York Tribune, 24 July 1921; and Evening Telegraph, 25 July 1921, 4.

85 Gazette de Lausanne, 28 December 1918, 2.

86 For these initial reactions, see the contemporary accounts of Mathew Carey, A Short Account of the Malignant Fever, Lately Prevalent in Philadelphia, 4th edn (Philadelphia, 1794); Richard Allen and Absalom Jones. A Narrative of the Proceedings of the Black People, during the Late Awful Calamity in Philadelphia, in the Year 1793 (Philadelphia, 1794); and J.M. Keating, History of the Yellow Fever: The Yellow Fever Epidemic of 1878, in Memphis, Tenn. (Memphis, 1879).

87 For these organizations and individuals, see Waco Daily Examiner, 23 October 1878, but principally Keating, History of the Yellow Fever, and for New Orleans during the epidemic of 1853, John Duffy, Sword of Pestilence: The New Orleans Yellow Fever Epidemic of 1853 (Baton Rouge, 1966); and Jo Ann Carrigan, The Saffron Scourge: A History of Yellow Fever in Louisiana, 1796-1905 (Lafayette, LA, 1994) for yellow fever epidemics after 1879.

88 See especially Dennis Altman, Power and Community: Organizational and Cultural Responses to AIDS (London, 1994); and Cohn, Epidemics, ch. 25

89 Adam Nossiter, "Fear of Ebola Breeds a Terror of Physicians", New York Times, 28 July 2014, from Kolo Bengou, Guinea. 


\title{
VIOLENT DEATH
}

\author{
Philip Dwyer
}

\section{"Murder is the king of crimes"}

The scholarship on the history of homicide has grown substantially over the last few decades. Criminologists, historical sociologists and historians look to homicide because it is often the easiest way to document violence, although there are enormous methodological problems involved. Homicide trends are plotted by estimating how many murders per 100,000 of the population occurred in any given year, but the extant sources can vary enormously from one country to another, and no two countries ever kept identical records. What counts as homicide can also vary. Are we to include deaths from infanticide, for instance? In Scotland, infanticide - generally considered to be the killing of a new-born shortly after birth - was deemed a separate crime by a 1690 act of parliament and is not counted in the homicide rate. ${ }^{2}$ In Ireland, one study that includes infanticide concludes that there were consistently higher rates of homicide from the eighteenth century to the 1930s in comparison to the rest of the United Kingdom, while another study that excludes it finds that homicide in Ireland was only marginally higher than in England and Wales. ${ }^{3}$ Many scholars, however, argue that infanticide is a separate category.

Should citizens who are killed overseas be counted in the national statistics, as is the case in the Netherlands? ${ }^{4}$ Even the nature of the inquest and the criminal justice system, as well as more mundane matters such as the unwillingness of court or state officials to spend money on suspicious deaths, could have an impact on the number of homicides that are reported (or underreported as the case may be). ${ }^{5}$ Manslaughter, or the accidental killing of a person, and killing a person in self-defence are not now considered homicide in many parts of the world, but once were. In nineteenth-century Chicago, for example, in an attempt to protect the public from "dangerous behavior", a whole array of new charges were brought against people for causing death that might otherwise have been considered manslaughter or where previously the death would have been dismissed as an accident, including death by car accident, "midwives whose patients died as a result of abortions, ... women who committed infanticide, ... physicians whose patients died as a result of poor care, and ... drug dealers whose customers died" from overdoses. ${ }^{6}$ We have seen a revision of this interpretation of the law in some parts of the world in recent years. In 2019, for example, the state of Queensland in Australia expanded its definition of homicide to include "reckless indifference to human life". In November of that year, the mother of two little girls who died in a hot car parked outside her home was the first person to be charged with two counts of murder under the new law. 
The story is complicated even further by the fact that some homicides result in no punishment. In the past, many homicides went unpunished for all sorts of reasons. As one recent work has shown, legal codes around homicide are often based on a society's sense of moral outrage. ${ }^{7}$ In France, what were considered "crimes of passion" (crimes passionnels) were dealt with far more leniently than premeditated murders. A similar phenomenon existed in the United States, where men who killed their spouse's lover sometimes escaped punishment, at least during the nineteenth century. ${ }^{8}$ It can also be seen in the use of a "gay panic defense" in countries like the United States, the United Kingdom, Australia and New Zealand. ${ }^{9}$ In many parts of the Middle East and South Asia today, honour killings often go unpunished. In the same vein, infanticide in earlier centuries was often treated leniently or overlooked altogether. ${ }^{10}$ Similarly, mercy killings and assisted suicides pose real moral dilemmas for many around the world today as legislation is slowly being introduced to allow the state to take control of euthanasia, to take it out of the hands of individuals and to decriminalize it.

\section{Death by numbers: statistics and homicide rates}

Homicide rates may provide an indication of the degree to which violence was (or was not) kept within certain limits in any given society at any given time, but they do not necessarily offer an indication of overall levels of violence in a given society. ${ }^{11}$ Much of the interpersonal levels of violence that historians talk about - sexual assault, domestic violence, child abuse, as well as examples of cruelty such as animal baiting - cannot be measured in any accurate way. Think too of deaths during war, normally excluded from homicide rates, or indeed the levels of violence that existed in twentieth-century totalitarian societies with relatively low rates of homicide but high levels of death through incarceration in concentration camps and gulags.

The debates around homicide rates began in the early 1980s with the publication of two articles, one by Alfred Soman, the other by Ted Gurr, that postulated a decline in homicide rates from relatively high levels in 1300 to low levels in $1800 .{ }^{12}$ Gurr's article in particular was a synthesis of about thirty studies that had been done up until then. Since Soman and Gurr, hundreds more studies have been carried out, mostly on towns or regions in western Europe, but also in the United States, with a few books and articles attempting to extrapolate national and even transnational trends from these local and regional studies. The evidence points to a demonstrable decline in homicide in the Western world, which has led some scholars to conclude that the past was violent, even dramatically more violent, and that the present has never been more peaceful.

Such a conclusion is appealing but also a little simplistic. Some of the earlier studies in particular were fraught with methodological problems, the statistics were and remain incomplete, the data sets varied enormously, the decline was neither uniform nor consistent and they tell us little about contemporary attitudes towards murder. The statistics can, in short, considerably skew the reality of life in past centuries. Very little is said, for example, about the crucial period that saw the Wars of Religion and the Thirty Years War, and the enormous loss of life that accompanied them. Given the problems with these statistics, scholarly claims for the decline are much more circumspect than they used to be. James Sharpe, for example, concludes that the generally accepted levels of homicide probably averaged about 20 in 100,000 in England in the Middle Ages, which dropped to around 1 in 100,000 by $1800 .{ }^{13}$ Randolph Roth argues that the actual 
homicide rate in England in the early 1600 s was probably around 15 in $100,000 .{ }^{14}$ Some scholars go so far as to argue that between the late Middle Ages and the early modern period the figures do not point to a "consistent pattern of decline". ${ }^{15}$ It is not really until the eighteenth century that we can begin to make accurate generalizations about homicide rates in Europe, by which time the rate is around the same as it is today, but even then, the calculations are fraught with difficulties. What we can say with a degree of certainty is that the real decline occurred from the seventeenth to the eighteenth century in a core number of western European countries - England, the Netherlands, Germany and possibly France. That said, homicide rates remained relatively high in some Mediterranean countries - Italy, Spain, Greece - until later in the nineteenth century. Athens, for example, was the murder capital of the world at the end of the nineteenth century, at a time when there was a migrant influx from the countryside, but by 1920 , it had fallen to one of the lowest levels in the world and indeed remains one of the least violent cities to this day. ${ }^{16}$ In China in the eighteenth century, on the other hand, we can observe the reverse trend, where homicide rates increased, largely as a result of the loss of central control by the Qing state, millenarian rebellions, piracy and banditry. ${ }^{17}$ That trend was reversed in the twentieth century so that today countries such as China, Japan, Korea and Singapore have some of the lowest homicide rates in the world. It is possible that the economic growth Japan, Korea and Singapore have experienced without accompanying concentrations of poverty may have a role to play, as well as the strong social stigma attached to arrest for crime in those societies. In other words, national and regional variations have to be taken into consideration in any assessment of homicide rates, but, just as importantly, those discrepancies have to be accounted for.

So, what do we know? It is possible to make some generalizations about homicide that appear to be consistent across time and across cultures. First, there appears to have been little change in the sex and age structure of the vast majority of murderers over the centuries. The vast majority of homicides are committed by younger men (or at least men in their reproductive prime, so from their teens to around the age of 40), usually against other young men, often of similar social status, often single and, for the last two hundred years or so, often belonging to lower socio-economic groups. Although there are slight variations, this breakdown is consistent through the ages.

There has been one change in the last two hundred years or so; there appears to be an increase in the number of women who are the victims of homicide, although this too can vary from country to country. In Japan, South Korea and Hong Kong, for example, which have very low homicide rates (between 0.3 and 0.4 in 100,000 in 2011-12), women make up around 53 per cent of homicide victims. ${ }^{18}$ The largest number of murders of women takes place in Asia, and in India in particular. In India, around 40 per cent of homicide victims are women, but the figure is also high in countries such as Austria, Germany, Norway and Switzerland, again countries with very low homicide rates but where, respectively, around 40, 47, 47 and 50 per cent of homicide victims are women. ${ }^{19}$ However, women and girls are at greatest risk in Africa. In South Africa, about 3,000 women were murdered in the course of 2018 - one woman every three hours. That's about the same number for the whole of Europe, which is more than five times higher than the global average. (Sexual assaults in South Africa are much higher, with a woman raped roughly every 30 seconds.) Women and girls still account for a smaller share of total homicides, around 20 per cent. That is, men are still killing other 
men in greater numbers, although women and girls continue to bear the greatest burden of intimate partner and family related homicides.

Women killers have made up around 5-10 per cent of the number convicted of murderers, depending on the region of the world, at least since 1900. Before that date, they could be involved in a higher percentage of killings. ${ }^{20}$ In early eighteenth-century Stockholm, for example, women made up about 45 per cent of murder and manslaughter offenders, although that high rate is the exception to the rule and occurred within a specific cultural context. ${ }^{21}$ Women mostly kill within their own domestic circle, but they were far more likely to hang than men, generally because their crimes were seen as a greater threat to the social order, even though there is in reality little difference in the character of male and female violence. In England, between 1351 and 1826, females committing homicide were seen as having committed "petty treason", which would nominally be punished by burning at the stake. Men and women, in other words, were treated differently, with men more likely to get off than women. ${ }^{22}$ This trend appears to have changed by the second half of the nineteenth century when women who killed their violent husbands, although not treated leniently, were less likely to hang, as an ideology of "female innocence and weakness" emerged that required men to treat women more "chivalrously". ${ }^{23}$ An example in belle époque France is the case of Henriette Caillaux, who shot the French Minister of Finance, Gaston Calmette, in July $1914 .^{24}$ She was acquitted; her lawyer argued that her act was a "tragic result of unbridled female passions". Sometimes women killed (their children, for example) rather than transgress their religious beliefs around suicide so that they could face the death penalty. ${ }^{25}$

\section{Explaining decline and disparity}

The two crucial problems facing historians are, first of all, how to explain the relative decline in most European countries from the seventeenth into the eighteenth centuries and, second, how to explain the disparity in rates of homicide across the world. In the West, the explanations put forward for this decline are often tied to the German sociologist, Norbert Elias and his idea of the civilizing process and the rise of the state. Historians of crime have been especially seduced by Elias because his theory neatly matches what they were finding in their research, at least as long as a decline in the homicide rate is regarded as equivalent to a decline in rates of violence overall. Let me examine these ideas before going on to look at some of the reasons advanced to explain why the United States has a particularly high homicide rate.

\section{The civilizing process}

It hardly does justice to the complexity of the theory to summarize it in a paragraph or two, but essentially Norbert Elias' "civilizing process" involved a complex change over long periods of time that led to, in the western European example he focused on, greater degrees of state control in parallel with individual self-control. It was once accepted among historians that people in the Middle Ages were characterized by their impulsiveness, that they had less control over their emotions, that they were more spontaneous and were therefore quicker to anger and violence than people in contemporary society. In one study done by Barbara Hanawalt of England in the first half of the fourteenth century, she found that around 60 per cent of rural and 84 per cent of urban homicides 
occurred as the result of an argument. ${ }^{26}$ It reinforces the notion that violence in earlier centuries was the result of an inability on the part of people to control their emotions. The "civilizing" of behavior brought about by a complex process that involved the internal regulation of emotions, first by the nobility in court society, and subsequently by the bourgeoisie, meant that individuals had to moderate their "spontaneous emotions".

There are problems with that approach, especially since it implies a greater control of affect in modern Europeans in contrast to their "spontaneous" forbears. ${ }^{27}$ The idea that people in past societies were quicker to react to anger, to kill and were therefore more prone to violence is a misrepresentation of both history and our own times. As a cursory glance at the homicides committed in the contemporary Western world will show, homicides are also committed in the heat of the moment over unimportant issues. Then as now, an insult, verbal abuse or an argument can quickly degenerate into violence, especially if the consumption of alcohol is involved. Studies reveal a willingness to use violence to resolve petty disputes in eighteenth-century Scotland, nineteenth-century Ireland, in the South of the United States in the eighteenth and nineteenth centuries and in Sri Lanka in the late nineteenth and early twentieth centuries. ${ }^{28}$ In Amsterdam in the century between 1651 and 1750, "impulsive violence was overwhelmingly dominant" with "three-fifths of killings" resulting from conflicts in taverns or streets, even if that kind of impulsiveness declined to between one third and a half over the next fifty years. $^{29}$ According to the British Home Office Statistics for the years 2003 to 2004, 54 per cent of the homicides that took place were committed in a violent rage. ${ }^{30}$ This is not to say that homicide is "irrational" - it is often a response to an insult or a perceived slight or wrong - but contrary to what proponents of Norbert Elias and the civilizing process argue, there still appears to be a high level of impulsivity surrounding many homicides. It raises questions about the degree to which modern man is any more in control of his emotions than his forebears. It is possible that the overwhelming majority of murders committed, not only in the past but also in the modern era, involve an element of masculine honor, that is, a conflict over perceived slights to that honor, however defined. $^{31}$ Thus, one criminologist argues that about two-thirds of male-on-male homicides in Britain can be "characterized as spontaneous honour contests.",32

\section{The role of the state and the marginalization of violence}

Most scholars have simply assumed that a correlation exists between growing state intervention and declining homicide rates. The argument is based on a flawed premise that most scholars have come to accept and that few have questioned since it was first posited by Max Weber in the nineteenth century, reiterated by Elias in the 1930s: namely, the idea that the monopolization of violence by the state has led to a diminution in interpersonal violence. In fact, there is little research on the impact of the state on interpersonal violence. ${ }^{33}$ We know, for example, that homicide rates dropped to contemporary levels in western Europe before 1800, that is, well before the development of modern-day police forces, which raises all sorts of questions about the supposed role of the state in controlling violence. ${ }^{34}$ There does not appear to be any clear correlation between levels of policing and homicide rates. ${ }^{35}$ In fact, policing is generally reactive rather than preemptive in nature. There does, however, appear to be a correlation between a willingness to use local courts in an attempt to resolve disputes and a decline in interpersonal violence. ${ }^{36}$ That is, standards are not imposed from above by some centralizing 
agency but rather are a reflection of a willingness to use existing structures to negotiate outcomes rather than use force or violence. To this extent, the participation of ordinary men and women in the court system reveals a desire to prosecute, contain and control various forms of violence. ${ }^{37}$

Up until the sixteenth century, authorities tended to look upon homicides that resulted from issues of honor with a great deal of leniency. ${ }^{38}$ Only extreme cases of premeditated murder resulted in the death penalty. That began to shift between the sixteenth and seventeenth centuries, when the settlement of a homicide was taken out of the hands of the families concerned and fell increasingly into the hands of judges and sovereigns. This was the case in France, for example. It was during this time that homicide began to be seen as a crime, and people who committed homicide began to be seen as criminals. ${ }^{39}$ This is what Pieter Spierenburg refers to as the "marginalization of homicide", which goes hand in hand with an increasing social control that redefined the relationship between the state and its citizens. That is, declining homicide rates often occurred at times when there was a shift towards a broader acceptance of the state, which was seen as operating on behalf of its citizens and capable of delivering justice. The marginalization of murder also corresponds with the argument advanced by the sociologist, Donald Black, that crime is a form of aggressive conflict management largely confined to lowstatus people who find themselves outside the law. In fact, they often consider the law removed from their daily experiences and repressive. They are consequently more likely to use aggression, including killing, to resolve their conflicts. ${ }^{40}$

Of course, this is difficult to prove in reality. The decline in homicide does not necessarily correlate with the rise of the state and its monopolization of violence; rather, it appears to have more to do with the relationship between the state and its citizens. In northern Europe, for example, the state was generally considered to be a legitimate institution that was designed to protect its citizens. In southern Europe, which had higher rates of homicide than the European core, quite the opposite was happening, a deep distrust was emerging between the people and state institutions. ${ }^{41}$ The same phenomenon can be observed in the United States. Randolph Roth argues that there have been four correlates of low rates of homicide in North America and western Europe over the past 450 years: the belief that government is stable and that its legal and judicial institutions will redress wrongs and protect lives and property; a feeling of trust in government and the officials who run it; empathy and fellow feeling arising from racial, religious or political solidarity; and the belief that the social hierarchy is legitimate, that one's position in society is or can command the respect of others without resorting to violence. ${ }^{42}$ Roth's argument in its broadest sense is about nation-building, which is not only about the establishment of government that is considered "legitimate" but also about "comity among elites, strong institutions, security, inspired leadership, and a sense of community that transcends differences over religion, gender, class, race, ethnicity."43

Why would trust in government lead to a decline in violence? This is not, I would argue, the same thing as the state's monopolization of violence, force and coercive power. Trust and coercive power are not necessarily correlates. Roth argues that when:

people believe that their government shares their values, speaks for them and acts on their behalf, they feel empowered, have greater self-respect and gain confidence in their dealings with people outside their families. When people feel that the government is antagonistic toward them and they question its legitimacy, especially on the national level, they can feel frustrated, alienated and 
dishonoured. And those feelings, in turn, can stimulate the hostile, defensive and predatory feelings that lead to violence against friends, acquaintances and strangers. $^{44}$

Some might argue that this is reductionist, but could this then have something to do with the fact that, in the West at least, homicide has largely become associated with lower status groups? ${ }^{45}$ The argument is that the poor, the unemployed, the young, migrant groups and marginalized ethnic or indigenous groups have an antagonistic relationship with the law and are obliged therefore to seek "justice" outside of the law, to take things into their own hands. This is true only up to a point of course. In countries such as India and Indonesia, where poverty is widespread, the homicide rate in 2012 was respectively around the 3.8 and 0.6 in 100,000. ${ }^{46}$ In the Australian state of New South Wales, for example, the most populous of the six states, unskilled workers make up about 15 per cent of the Australian population aged 15 and over, but they represent about 56 per cent of homicide offenders. Conversely, less than 0.5 per cent of killers come from the professional classes. ${ }^{47}$ Again in Australia, people of Aboriginal descent are thirteen times more likely to kill than white people. We see similar figures for the United States, where African Americans are about five times more likely, and Native Americans about twice as likely, to commit homicide than white Americans. Of course, these groups of people are often dispossessed, often on the margins of society, and often alienated as a result. There are exceptions to this rule of course. In the case of lynching in the United States, local elites assumed leadership roles, helping to organize and lead the lynching parties, even if they did not necessarily do the actual killing. ${ }^{48}$

\section{The American exception}

The United States is an interesting case because it is a pluralistic democracy with a relatively stable political system. And yet, it is two and a half to eight times more homicidal than any other affluent democracy, even if this was not always the case. According to Randolph Roth, homicide rates in the early to mid-eighteenth century were relatively low, on a par with its European counterparts. It was only from the mid- to late nineteenth century that there was a radical departure in rates between America and western Europe. This is the point at which American exceptionalism appears to have taken hold, as homicide rates "exploded" and parts of the country went from some of the least to some of the most homicidal in the Western world. ${ }^{49}$ Despite a decline in homicide rates in America between 1939 and 1954 (so from the Great Depression through to the aftermath of the Second World War) and again between 1994 and 2004, two-thirds of the world live in countries that are less homicidal than America. A number of other regions in the world have higher homicide rates - Russia, the Baltic states, sub-Saharan Africa, Central and Latin America, and the Caribbean. The Central and Latin American region is possibly "the most dangerous place on earth", with 33 per cent of the world's homicides (and only 9 per cent of its population). ${ }^{50}$ Mexico, Honduras, El Salvador, Venezuela and Brazil were among the most violent countries in 2016. ${ }^{51}$ Among affluent nations, however, America has the highest homicide rates.

The distinction between American and European rates of violence has been recognized since at least 1932, and since then scholars have been trying to understand the divergence. Before we attempt to explain American homicide (and the issue that is often 
tied with it - American gun violence), we can have a look at a few figures. In the five years between the shooting at Sandy Hook Elementary School in Connecticut in December 2012, which shockingly took the lives of 26 people, including 20 children aged between 6 and 7, and the school shooting in Parkland, Florida, in February 2018, in which 17 people were killed, America experienced 99 mass shootings, at least 239 school shootings, and at least 188,000 gun related deaths (of which 8,000 were children). ${ }^{52}$ Every day, 100 Americans are shot to death and hundreds more are wounded in gun violence.

Those figures have to be broken down. Two-thirds of those gun related deaths are a result of suicide. Just as people are up to eight times more likely to be murdered in America, so too are people up to ten times more likely to commit suicide than in most other affluent countries. ${ }^{53}$ Of the homicides, black Americans represent the majority of homicide victims. In fact, black Americans are ten times more likely to die from homicide by gun than a white American. The black American homicide rate surpassed those of whites in the late nineteenth century and has remained higher to this day. ${ }^{54}$ Political and economic discrimination that led to structural racism have played a role in marginalizing black Americans from mainstream society, undermining their faith in government and pushing some into criminal occupations.

Some of those killings involve police shootings. Although only a small proportion of the overall number of killings, American police kill more people than in any other Western country. In 2018, a total of 992 people were shot by American law-enforcement agents. ${ }^{55}$ Roughly half of the victims of those police shootings were white, one quarter were made up of various ethnicities and one quarter were made up of black Americans, an over-represented proportion of the population brought into the public consciousness through the Black Lives Matter movement. Police shootings are not included in homicide rates by the FBI. Compare the number of people shot by police in America with England and Wales where the number of people shot by police between 2006 and 2016 came to a grand total of twenty-three. ${ }^{56}$ Even if we adjust that figure for population size, the death rate in the US is 64 times higher than in the UK.

Attempts to explain the marked difference in homicide rates between America and the rest of the affluent world range from the historian Richard Slotkin's attempt to map the American national character and what he has dubbed the "myth of regeneration through violence", 57 to the role of slavery and violence in the south, ${ }^{58}$ to the hypothesis of Dutch criminologist Pieter Spierenburg that democracy came too early to the United States (he is referring of course to the American Revolution), to arguments around gender, masculinity and honour. ${ }^{59}$

According to Spierenburg, the early American state was not able to gain a monopoly over violence in the same way that European states were able. In Europe, the monopolization of force by different states took centuries to complete, but eventually the vast majority of people accepted being disarmed in day-to-day affairs. On the American continent, on the other hand, democracy was introduced before the people had got accustomed to being disarmed - so the argument goes - so that an ethic of self-help and distrust in state agencies remains widespread among Americans. Of course, the first part of that argument is only valid if we accept that there is a correlation between gun ownership and a high homicide rate, a point to which I shall return below.

In a similar vein, some scholars argue that notions of honor may be at the heart of a good deal of the violence. American men, it is argued, have not yet learned to 
internalize honor in the same way that European men purportedly learned to do. Of course, this doesn't necessarily explain why some honor societies are less violent than others. Honor, Roth believes, is "a proximate cause of violence rather than the ultimate cause". 60 There is also the additional complication of "modernization", which took place at different times on the American continent as it was colonized, beginning anew each time a region was occupied. ${ }^{61}$ Erich Monkkonen argues that America's "tolerance for violence" may have originated in a demographic imbalance - a preponderance of young men in certain regions at certain times of American history - that was exacerbated by a whole raft of "daily practices", such as mass-produced guns from the mid-nineteenth century. ${ }^{62}$ There is also an apparent extraordinary tolerance for violence in the judicial system. ${ }^{63}$ In the United States, for various reasons, around three-fifths of killers, escape punishment. ${ }^{64}$ This was also the case, for example, of vigilante justice in the United States, where as many as 4,700 people (mostly African Americans, but also Mexicans, Italians and some whites) were lynched between 1882 and 1968. Only a very small percentage of the perpetrators ever appeared before the courts, and an even smaller percentage were convicted. It reinforced the idea among sections of the American population that the state was not there to protect them.

It is difficult to talk about homicide rates in America without mentioning gun violence, gun ownership and what some have dubbed its "gun culture". 65 America has a far larger proportion of households that own a gun (nearly half of all households) than any other country in the world, with the possible exceptions of Israel and Switzerland, where conscription requires military personnel to have a gun. ${ }^{66}$ Of course, there are two different things going on here. In Israel and Switzerland, the state requires gun ownership, and with it come certain duties and responsibilities. This is not the case in the United States, where about 30 per cent of Americans own a gun and where gun ownership is an individual choice. ${ }^{67}$ Of those, two-thirds own more than one gun. This is twice the rate of the next highest countries, France and Canada, not exactly known for their high homicide rates. Not surprisingly, there appears to be a correlation between gun ownership and the propensity to use firearms, especially when it comes to domestic violence and suicide. ${ }^{68}$ As well, given the high proportion of Americans who own a gun, it also impossible to know whether the prevalence of gun ownership has a kind of heightening effect, undermining trust on any number of levels - citizens towards each other, law enforcement agents towards citizens and vice versa. It is impossible to know the extent to which some gun homicides might occur because people assume the other person might have a gun.

The question is whether gun ownership offers a material or a cultural explanation for America's high homicide rate. A good deal has been written about the Second Amendment and the "right of the people to keep and bear arms". ${ }^{69}$ To some extent at least, the arguments around gun ownership - the subject of intense and often highly emotive and politically charged debate - are beside the point, which is that the prevalence of gun ownership does not always translate into a high homicide rate (as we can see with Israel and Switzerland). If you eliminate homicide by shooting in the United States, the murder rate is still higher than in any other Western country. ${ }^{70}$ In other words, widespread gun ownership is not the primary cause of America's high homicide rate. ${ }^{71}$ That does not mean to say that guns are not a problem; easy access to guns facilitates gun violence.

The issue, I would argue, lies deeper than simple gun possession and is tied to both social and to distinct cultural-ideological traits. It has more to do with attitudes towards 
the state and the role of the individual in society. In asking what the difference is between the United States and the other advanced industrialized economies that might help explain the divergence in rates of homicide and interpersonal violence, one factor stands out above all else - in America, the rights of the individual (often) take precedence over the rights of the collective. Individual rights were enshrined in the Constitution of the United States. Indeed, it was the first document that enabled individual rights to be protected through binding constitutional law. Within the Constitution, government and its institutions were meant to protect individual rights; government and its institutions exist by virtue of their obligations to the individual. This is somewhat different from the theory of "social contract", which dominated western European political thinking. That has implications for government policy, attitudes toward government and the nature of American society. The apparent emphasis on individual (masculine) honor, the tolerance for violence and the notion of a culture of self-help may, in part at least, explain both higher homicide rates and higher levels of interpersonal violence in the United States than in the rest of the Western world.

\section{Conclusion}

Outside of warfare, homicide is one of the largest contributors towards violent deaths globally. Roughly 560,000 people died violent deaths in 2016 (excluding suicides). Fewer than 20 per cent of those individuals died in armed conflict, while around 68 per cent were the victims of intentional homicides. ${ }^{72}$ The number of homicides globally has been on the rise for the past three decades, from 362,000 deaths in 1990 to 464,000 deaths in $2017 .^{73}$ The focus of this chapter has been largely on the Western world for the simple reason that the historical homicide rates for most other parts of the globe are not available. We do not know, then, whether there has been a global long-term decline in homicide (without further research being done) or whether the high rates in many parts of the rest of the world are linked to historical fluctuations and are contingent upon the social, economic and political circumstances of those regions.

What does not appear to have much of an impact on lethal violence is urbanization, state intervention (including policing) or the criminalization of violence. The most important factor appears to be the strength of community or civil society. In communities where there is a sense of cohesiveness and coherence, where there is a willingness to confront and contain violence, both inside and outside of the judicial system, homicide rates, and probably overall rates of violence, appear to be low. Communities that are fractured, on the other hand, where the social, civil and religious fabric is fraying, tend to display higher rates of homicide (and higher rates of violence overall). This is the idea of "civil society", one built on a relationship of trust between the state and its citizens (and not just on the coercive power of the state). ${ }^{74}$

There is, then, always the possibility of reversing trends in countries where homicide rates are high, although how one achieves that reversal will no doubt be hotly debated. The social, religious and cultural divisions that continue to separate people from one another and that supposedly drive people to commit murder are more entrenched in some societies than in others. The kinds of platforms that politicians like to espouse - getting tougher on crime, more and better policing, more incarceration - all simple answers to complex problems, will not prevent people from committing murder. On the other hand, public officials can help shape attitudes by both 
example and with policy. Social cohesion and mutual respect need to be constantly asserted by our political leaders (they are often sadly lacking on that front) and continually worked on. They may not be able to stop hatred and distrust, but they can at least try to create an environment in which they are considered unacceptable. We have seen the opposite happening in a number of Western and, indeed, some developing countries where populists and budding dictators have been using incitement to hatred as a means of garnering electoral support. Their rhetoric has been provoking murderous impulses. Even in democratic societies, hateful extremism is openly promulgated. How has that come to pass? Homicide rates may decline, as they have in the past, but, as we have seen, rates are never permanent and insoluble, and under the right circumstances they can just as easily rise.

\section{Notes}

1 Lawrence Friedman, Crime without Punishment: Aspects of the History of Homicide (Cambridge: Cambridge University Press, 2018), p. 1.

2 William J. Knox with L. Thomas, "Homicide in Eighteenth-Century Scotland: Numbers and Theories," Scottish Historical Review, 94:1 (2015), 53, 54.

3 Kyle Hughes and Donald M. MacRaild, "Introduction: Crime, Violence, and the Irish in the Nineteenth Century; Themes and Perspectives," in Kyle Hughes and Donald M. MacRaild (eds), Crime, Violence, and the Irish in the Nineteenth Century (Liverpool: Liverpool University Press, 2017), pp. 2-3.

4 Pieter Spierenburg, A History of Murder: Personal Violence in Europe from the Middle Ages to the Present (Cambridge: Polity, 2008), pp. 208-9.

5 Mary Beth Emmerichs, "Getting away with Murder?: Homicide and the Coroners in NineteenthCentury London," Social Science History, 25:1 (2001), 93-100; Sharpe, A Fiery \& Furious People: A History of Violence in England (London: Random House, 2016), p. 30; Howard Taylor, "The Politics of the Rising Crime Statistics of England and Wales, 1914-1960," Crime, Histoire \& Sociétés/Crime, History \& Societies, 2:1 (1998), 5-28. This view has been contested by Robert M. Morris, "Lies, Damned Lies and Criminal Statistics': Reinterpreting the Criminal Statistics in England and Wales," Crime, Histoire EO Sociétés/Crime, History \& Societies, 5:1 (2001), 111-27.

6 Jeffrey S. Adler, "Halting the Slaughter of the Innocents': The Civilizing Process and the Surge in Violence in Turn-of-the-Century Chicago," Social Science History, 25:1 (2001), 31, 36-37.

7 The argument put forward by Friedman, Crime without Punishment, pp. 1-3, 133-35.

8 Peter Stearns, Jealousy: The Evolution of an Emotion in American History (New York: New York University Press, 1989), pp. 28-30, 154-58.

9 See Christina Pei-Lin Chen, "Provocation's Privileged Desire: The Provocation Doctrine, Homosexual Panic, and the Non-Violent Unwanted Sexual Advance Defense," Cornell Fournal of Law and Public Policy, 10:1 (2000). Available at: https://scholarship.law.cornell.edu/cjlpp/ vollo/iss $1 / 8$

10 See, for example, Dana Rabin, "Bodies of Evidence, States of Mind: Infanticide, Emotion, and Sensibility in Eighteenth-Century England," in Infanticide: Historical Perspectives, 1550-2000 (London: Ashgate 2002), pp. 73-92.

11 Richard McMahon, "Histories of Interpersonal Violence in Europe and North America, 1700 Present," in Paul Knepper and Anja Johansen (eds), The Oxford Handbook on the History of Crime and Criminal Fustice (Oxford: Oxford University Press, 2016), p. 120.

12 Alfred Soman, "Deviance and Criminal Justice in Western Europe, 1300-1800: An Essay in Structure," Criminal Fustice History: An International Annual, 1 (1980), 1-28; and Robert Ted Gurr, "Historical Trends in Violent Crimes: A Critical Review of the Evidence," Crime and Fustice: An Annual Review of Research, 3 (1981), 295-353. Their findings engendered a debate in the journal, Past \& Present. Lawrence Stone, "Interpersonal Violence in English Society 1300-1980," Past \& Present, 101 (1983), 22-33; James A. Sharpe, "The History of Violence in England: Some Observations," 108 (1985), 206-15, and Lawrence Stone, "A rejoinder," Past \& Present, 108 


\section{PHILIP DW YER}

(1985), 216-24, in which Stone pointed to some of the problems of evidence and interpretation, but asserted that both Gurr and he were "broadly correct". The debate is discussed in J. S. Cockburn, "Patterns of Violence in English Society: Homicide in Kent, 1560-1985," Past \& Present, 130 (1991), 70-106; and Robert Shoemaker, "Male Honour and the Decline of Public Violence in Eighteenth-Century London," Social History, 26:2 (2001), 190-208.

13 James Sharpe, A Fiery \& Furious People, p. 29.

14 Randolph Roth, "Homicide in Early Modern England 1549-1800: The Need for a Quantitative Synthesis," Crime, Histoire E Sociétés/Crime, History \& Societies, 5:2 (2001), 45-46.

15 Richard McMahon, Joachim Eibach and Randolph Roth, "Making Sense of Violence? Reflections on the History of Interpersonal Violence in Europe," Crime, Histoire E̊ Sociétés/Crime, History E Societies, 17:2 (2013), 8-9.

16 Jeffrey S. Adler and Thomas W. Gallant, "What Do Historians Have to Say About Violence?," The Harry Guggenheim Foundation Review of Research, 4 (2000), www.hfg.org/hfg_review/4/adler-gal lant-3.htm

17 Thomas Buoye, "Homicide and Punishment in Eighteenth-Century China," in Robert Antony, Stuart Carroll, and Caroline Dodds Pennock, (eds), The Cambridge World History of Violence, 4 vols (Cambridge: Cambridge University Press, 2020), iii. pp. 350-69.

18 Global Study on Homicide, 2013: Trends, Contexts, Data (Vienna: United Nations Publications, 2014), pp. 54-55.

19 Global Study on Homicide, p. 137, 138, 139.

20 Malcolm Feeley and Deborah L. Little, "The Vanishing Female: The Decline of Women in the Criminal Process, 1687-1912," Law and Society Review, 25:4 (1991), 719-57.

21 Manuel Eisner, "Long-Term Historical Trends in Violent Crime," Crime and Fustice, 30 (2003), 83-142, here 112.

22 Garthine Walker, Crime, Gender, and Social Order in Early Modern England (Cambridge: Cambridge University Press, 2003), p. 135-36.

23 Martin J. Wiener, Men of Blood: Violence, Manliness and Criminal Justice in Victorian England (Cambridge: Cambridge University Press, 2003), pp. 123-24, 127, 131, 185.

24 Edward Berenson, The Trial of Madame Caillaux (Berkeley: University of California Press, 1992).

25 Arne Jansson, From Swords to Sorrow: Homicide and Suicide in Early Modern Stockholm (Stockholm: Almqvist \& Wiksell, 1998).

26 Barbara A. Hanawalt, Crime and Conflict in English Communities, 1300-1348 (Cambridge: Harvard University Press, 1979), pp. 171-72; David Nicholas, The Later Medieval City, 1300-1500 (London: Longman, 1997), pp. 312-13.

27 For a discussion of Spierenburg's ideas on honour see, McMahon, Eibach and Roth, "Making Sense of Violence?," 11-15.

28 Knox, "Homicide in Eighteenth-Century Scotland," 64, 70-71; Richard McMahon, Homicide in Pre-Famine and Famine Ireland (Liverpool: Liverpool University Press, 2013), pp. 47-73; Randolph Roth, American Homicide (Cambridge, MA: Belknap Press, 2009), pp. 227, 312; and John D. Rogers, Crime, Justice and Society in Colonial Sri Lanka (London: Curzon, 1987), pp. 135, 153.

29 Pieter Spierenburg, "Faces of Violence: Homicide Trends and Cultural Meanings: Amsterdam, 1431-1816," Journal of Social History, 27:4 (1994), 701-16, here 711-12.

30 The Law Commission Consultation Paper No 177, A New Homicide Act for England and Wales? A Consultation Paper, 325, www.law.upenn.edu/cf/faculty/cfinkels/workingpapers/Report\% 20 for \%20British\%20Law\%20Commission\%20cp177.pdf

31 Spierenburg, A History of Murder, pp. 7-10.

32 Fiona Brookman, Understanding Homicide (London: Sage, 2005), pp. 123-24.

33 McMahon, "Histories of Interpersonal Violence," pp. 121-22.

34 Joseph F. King, The Development of Modern Police History in the United Kingdom and the United States (Lewiston, NY: Edwin Mellen Press, 2004). Some figures in Spierenburg, A History of Murder, pp. $167-69$.

35 McMahon, "Histories of Interpersonal Violence," p. 122; Roth, American Homicide, pp. 9-10.

36 Stuart Carroll, "Thinking with Violence," History and Theory, 56:4 (2017), 23-43, here 33-34.

37 McMahon, Eibach and Roth, "Making Sense of Violence?," 17-18. 
38 Xavier Rousseaux, "From Case to Crime: Homicide Regulation in Medieval and Modern Europe," in Dietmar Willoweit (ed.), Die Entstehung des öffentlichen Strafrechts: Bestandsaufnahme eines europäischen Forschungsproblems (Cologne: Böhlau, 1999), pp. 143-75; and Manuel Eisner, "From Swords to Words: Does Macro-Level Change in Self-Control Predict Long-Term Variation in Levels of Homicide?," Crime and Fustice, 43:1 (2014), 65-134.

39 Rousseaux, "From Case to Crime," p. 154; Eisner, "Long-Term Historical Trends in Violent Crime," 126-27.

40 For a summary of Black's arguments see, Mark Cooney, "The Decline of Elite Homicide," Criminology, 35:3 (1997), 381-407, here 393-97.

41 Eisner, "Long-Term Historical Trends in Violent Crime," 128-29.

42 From Randolph Roth, "Biology and the Deep History of Homicide," British Journal of Criminology, 51 (2011), 544.

43 Randolph Roth, "Yes We Can: Working Together toward a History of Homicide that is Empirically, Mathematically, and Theoretically Sound," Crime, Histoire E' Sociétés/Crime, History ES Societies, 15:2 (2011), 131-45, here 131.

44 Roth, "Biology and the Deep History of Homicide," 547.

45 Cooney, "The Decline of Elite Homicide," 381-407; idem., Warriors and Peacemakers: How Third Parties Shape Violence (New York: New York University Press, 1998), pp. 2, 24-26.

46 Global Study on Homicide, pp. 128, 129.

47 Cooney, "The Decline of Elite Homicide," 383, 385, 386.

48 Cooney, "The Decline of Elite Homicide," 391-92; W. Fitzhue Brundage, Lynching in the New South: Georgia and Virginia, 1880-1930 (Urbana: University of Illinois Press, 1993), pp. 37-38.

49 Roth, American Homicide, p. 299.

50 Robert Holden, "Violence, the State and Revolution in Latin America," in Louise Edwards, Nigel Penn and Jay Winter (eds), The Cambridge World History of Violence, 4 vols (Cambridge: Cambridge University Press, 2020), iv. p. 506.

51 Claire McEvoy and Gergely Hideg, Global Violent Deaths 2017: Time to Decide (Geneva: Small Arms Survey, Graduate Institute of International and Development Studies, 2017), pp. 18, 19.

52 Jeneen Interlandi, “Amid the Tears, She Took Action,” New Fork Times, 23 September 2019, A26.

53 Erin Grinshteyn and David Hemenway, "Violent Death Rates in the US Compared to Those of the Other High-Income Countries, 2015," Preventive Medicine, 123 (June 2019), 20-26.

54 Roth, American Homicide, pp. 387, 410-11. See also James W. Clarke, "Black-on-Black Violence," Society, 33:5 (July/August 1996), 46-50.

55 www.washingtonpost.com/graphics/2019/national/police-shootings-2019/

56 www.independent.co.uk/news/uk/crime/m62-huddersfield-us-police-shooting-chicago-ukcharts-compare-difference-killings-officers-a7507051.html

57 Richard Slotkin, Regeneration Through Violence: The Mythology of the American Frontier, 1600-1860 (Middletown, CT: Wesleyan University Press, 1973); The Fatal Environment: The Myth of the Frontier in the Age of Industrialization, 1800-1890 (New York: Atheneum, 1985); and Gunfighter Nation: The Myth of the Frontier in Twentieth-Century America (New York: Atheneum, 1992).

58 James W. Clarke, The Lineaments of Wrath: Race, Violent Crime, and American Culture (New Brunswick, NJ: Transaction Publishers, 1998), pp. 209-25.

59 Pieter Spierenburg, "Democracy Came Too Early: A Tentative Explanation for the Problem of American Homicide," American Historical Review, 111:1 (February 2006), 104-14. Stephen Mennell, The American Civilizing Process (Cambridge: Polity, 2007), pp. 143-44, adopts a similar argument.

60 Roth, American Homicide, p. 12.

61 Pieter Spierenburg, "Violence and Culture: Bloodshed in Two or Three Worlds," in idem., Violence and Punishment: Civilizing the Body through Time (Cambridge: Polity, 2013), p. 58, 62-68.

62 We know the American West was particularly violent as transient populations with easy access to guns and alcohol led to frequent violent encounters, often over petty disputes. See, Clare V. McKanna, Homicide, Race, and Justice in the American West, 1880-1920 (Tucson: University of Arizona Press, 1997).

63 Erich Monkkonen, "Searching for the Origins of American and European Violence Differences," in idem., Crime, Justice, History (Columbus: Ohio State University Press, 2002), pp. 61-71.

64 Roth, American Homicide, p. 10. 


\section{PHILIP D W Y E R}

65 The term obviously poses conceptual difficulties, which space doesn't permit me to go into here. See the discussion in James T. Lindgren, "Fall from Grace: Arming America and the Bellesiles Scandal," Tale Law Fournal, 111 (2002), 2195-250, here 2201-02.

66 Mennell, The American Civilizing Process, pp. 140-43.

67 www.pewsocialtrends.org/2017/06/22/the-demographics-of-gun-ownership/

68 See the forum "Historians and Guns," William and Mary Quarterly, 59:1 (2002), and, in particular, Randolph Roth, "Guns, Gun Culture, and Homicide: The Relationship between Firearms, the Uses of Firearms, and Interpersonal Violence," 223-40.

69 A good introduction to the debates around the Second Amendment can be found in Jack N. Rakove, "Words, Deeds, and Guns: 'Arming America' and the Second Amendment," The William and Mary Quarterly, 59:1 (2002), 205-10; and idem., "The Second Amendment: The Highest Stage of Originalism," Chicago-Kent Law Review, 6 (2000), 103-66.

70 Monkkonen, Crime, Justice, History, p. 62.

71 Roth, "Guns, Gun Culture, and Homicide," 224.

72 These are no more than rough estimates. Many states still do not produce data on violent deaths. McEvoy and Hideg, Global Violent Deaths 2017, pp. 10, 11, 15, 20, 91.

73 Global Study on Homicide 2019 (Vienna: United Nations Office on Drugs and Crime, 2019), p. 11.

74 Manuel Eisner, "What Causes Large-Scale Variation in Homicide Rates?," in Hans-Henning Kortüm and Jürgen Heinze, Aggression in Humans and Other Primates: Biology, Psychology, Sociology (Berlin: de Gruyter, 2013), p. 155. 


\title{
SUIGIDOLOGY ON THE GUSP OF MODERNITY
}

\author{
Sociology and psychiatry in the nineteenth century
}

\author{
David Lederer
}

\section{Introduction}

In thanatology, the history of self-inflicted death is a relative newcomer. Since the early 1990s, however, historical suicide studies began to indicate how the genesis of suicidology influenced our social understanding of death more generally. For example, the dechristianization of self-murder (an act previously laden with harsh religious censure) into decriminalized suicide (a secularized neologism) occurred over the course of the Enlightenment. Suicide, a concept alien to the ancient Romans, had only come into common usage during the eighteenth century, when apologetic treatises by philosophers such as David Hume argued against legal sanctions. The adoption of suicide over the term self-murder in many European languages reflected an intellectual trend toward benign toleration and decriminalization. Nonetheless, the etymological metamorphosis notwithstanding, a whiff of criminality hovered over the new word still related to the perpetration of homicide, because one committed suicide. Indeed, despite the efforts of luminaries, the act retained actual criminal status in several major jurisdictions; in England until 1961, in Ireland until 1994, and, in Hume's own Scotland, laws against self-killing technically remain on the books to this day. This is but one example of how the emergence of historical suicide studies as an ancillary of suicidology has enhanced our critical understanding.

For despite decriminalization, pejorative explanations for self-killing lived on in pathologized psychiatric explanations for suicidal motivation as a form of insanity. The redirection of prejudice from a religious to a medical diagnosis is a by-product of nineteenth-century heroic psychiatry. Analogously, early sociologists actively promoted criminological methodology in the study of suicide as a social problem of great consequence to the state. Early sociologists elaborated suicidality alternately as a measurable symptom of social deviance, a Malthusian check on population control, a benchmark in the civilizing process or as a modality of natural selection. As a potentially damaging but manageable phenomenon, suicide mattered to the state. It underscored the value of moral statistics as determinants of the morale and health of the body politic. Early suicidologists thereby proved their essential professional relevance. The analysis and interpretation of suicide by early moral statisticians ultimately occasioned the first case study in history, Émile Durkheim's Le Suicide (1897), a foundational text of sociology.

As ascertainable patterns of suicidal behavior emerged, they took on the law-like characteristics of quantifiable physics: Suicide rates proved constant, if always on the increase; Protestants killed themselves more often than Catholics; men killed themselves more 
often than women. Patterns became interwoven with master narratives of material progress, essentialist national character, psychological and criminological typologies of degeneracy, indicators of social dis/integration or moral panics and conjunctural crises.

Western methods spread across the world with the bureaucracies of the nineteenthand twentieth-century colonial empires. After the Second World War, a galvanized international strategy targeted the amelioration, if not downright eradication, of suicide as preventable mortality. The World Health Organization continues to support the uniform collection and analysis of data globally with that goal in mind. Today, suicidology attracts the attention of governments, insurance actuaries, NGOs and teams of university researchers across the planet. Strategies for monitoring and prevention take regional variations into account, yet overall recorded rates of suicide remain considerable. Recent historical study gives us pause to reflect: Is suicide a soluble problem or even a problem at all, or simply another natural cause of death in need of reclassification?

For suicidology too is a Western neologism. Like genocide (conceived of by Raphael Lemkin in 1943/4 to describe Nazi war crimes), it is a Greco-Latin cognate (sui + cidium = self-killing $+\lambda o ́$ yo $=$ reason) meaning "loosely the scientific study of self-destructive behaviors." "From its invention in the twentieth century as an umbrella term created to envelope an ever-broadening spectrum of research and prevention strategies, suicidology had its origins in the nineteenth century. Historically, it emerged within the context of nationalism and emergent social sciences after the Napoleonic Wars. Methodologies associated with early suicide studies generated actionable data intended for strategic consumption by governments, offered up by the newly founded disciplines of psychiatry and sociology. They deployed statistics nationally and comparatively in the public, civil and criminological service to establish suicide as an irrefutable social fact, rather than an individual aberration.

Originally, by reconfiguring self-killing as a social fact rather than an individual act, suicidology highlighted consistent patterns of human behavior over time and contributed to government policies measuring and regulating social normalcy. Not surprisingly, in historical context, parallels arose with the modernity thesis. Suicide statistics were projected onto unrelated arenas of thought - religion, social Darwinism, technological progress, etc. Above all, the twin modern forces of political nationalism and professionalization (foremost in sociology and psychiatry) drove suicidology onward during the nineteenth century into what it has become today.

Therefore, a reflective history of scientific suicidology is timely. It well illustrates how the field initially suffered from unreflective presuppositions, how it contained politicized elements and how, despite the vaunted secularization of suicide during the Enlightenment, suicidology continued to stigmatize self-killing. In order to better comprehend the origins of suicidology, however, we first need to familiarize ourselves with previous modes of thought which developed into the scientific study of suicide and suicidal behaviours.

\section{Pre-modern perceptions}

Historians regularly trace the Western prohibition against self-killing to the fifth-century patristic writings of North African theologian, Augustine of Hippo (354-430). Previously, the Romans exhibited a more nuanced, if not entirely tolerant attitude toward self-killing, dependent upon circumstances. ${ }^{2}$ However, in his City of God, Augustine blatantly charged self-killers with a sinful violation of the fifth Mosaic commandment ("thou shalt not kill"), as well as cowardice, vain self-indulgence and the desperate loss of hope in their own eternal salvation. ${ }^{3}$ Some historians critique Augustine's blanket condemnation of self-killing in light 
of his vocal opposition to the Donatist movement during the later Roman persecutions, since willing martyrdom endangered the vitality of early Christianity by robbing the nascent sect of adherents. ${ }^{4}$

Gradually, the conflation of self-killing with self-murder influenced theology, although it took many centuries to impact upon quotidian practices. All the while, it is important to note that an insanity defence (non compos mentis) was always an admissible ground for leniency. The attribution of intentional self-killing to despair and demonic agency gained widespread popularity through literature and art depicting the orthodox Christian catalogue of vices/virtues. For example, in the allegorical Psychomachia by another fifthcentury author, Prudentius, despair (de spes or desperatio, the loss of hope) represented the antithesis of hope (spes) in eternal salvation. A prominent illustration of this paired relationship figures in Giotto's fourteenth-century frescoes adorning the Arena Chapel in Padua. In that image, Despair is portrayed as a self-hanged man goaded on by an impish demon. ${ }^{5}$

By the twelfth century, criminal codes introduced laws against self-murder as a felonious offense (felo de se). Subsequent cases first appeared in the records of English courts, followed by those in France and the Holy Roman Empire, respectively. Penalties included confiscation of property and the dishonorable disposal of corpses in waste places, in barrels jettisoned into nearby rivers or through burial under the local gibbet. ${ }^{6}$ By the fifteenth century, the free-imperial city of Nuremberg routinely undertook cautious criminal investigations to establish cause of death either as self-murder, murder or death by misadventure. ${ }^{7}$ Judicial authorities across Europe conducted similar investigations well into the eighteenth century, when continental states began the decriminalization of self-murder as part of Enlightened reforms. ${ }^{8}$ However, in the British Isles, laws against suicide continued to threaten self-murderers with sanctions, even if largely unenforced, until the latter half of the twentieth century.

Apart from official sanctions, unofficial popular rituals also aimed at punishing selfkillers. In Flanders and parts of the Holy Roman Empire, these could include dragging a corpse behind an ass, "executing" the corpse by hanging it upside down from a pitchfork (furca) and, in addition to judicial confiscations of goods, the manic pillaging and burning of the self-killer's property (ravage) by local communities. ${ }^{9}$ Physical contact with the body of a self-killer caused legal dishonor, in turn resulting in exclusion from guilds or marriage to honorable persons. For that reason, dishonorable tradespersons were employed for removals, and executioners claimed the right to all property within the circumference of a sword's swing of the body. ${ }^{10}$ Not infrequently, the executioner sold the body parts of dead criminals as a medicinal remedy popularly referred to as "mummy." 11

Dishonorable burials ingrained popular fears of divine retribution meted out against communities allowing the profanation of their cemeteries by the interment of a self-killer. During the height of the climatic shift known as the Little Ice Age (c.1580-1630), communities especially feared weather-related disasters such as hailstorms, culminating in crop failures, famine and disease. ${ }^{12}$ Reports of suicide increased as famine brought pestilence in its wake, and the infirm killed themselves in the throes of fever-related illnesses like typhus. ${ }^{13}$ Here, popular beliefs inverted cause and effect when communities collectively blamed famine and plague on persons who actually killed themselves to end their terrible suffering. Judicial records confirm outbreaks of violence, so-called suicide revolts, 
when communities rose up to prevent the interment of self-killers in hallowed ground after they had been legally judged non compos mentis by the authorities. ${ }^{14}$

At the same time, there was a move among Renaissance authors such as Thomas Moore and Michel de Montaigne to re-introduce the classical interpretation of justifiable self-killing as personal sacrifice, in defence of personal honor or in cases of terminal illness. In doing so, however, they never questioned the existence of damnable despair nor the legality of sanctions against self-murderers. Like apologists for accused witches (e.g. the physician Johann Weyer and father-confessor Friedrich Spee, SJ), they never denied the possibility of demonic agency in the realm of human affairs. When the English clergyman John Donne devoted a religious tract to a comparison of the passion of Christ as self-sacrifice to potentially allowable forms of self-killing in 1608, he forbade its publication during his lifetime, and it only appeared posthumously in 1647. ${ }^{15}$ Similarly, when the seminal treatise by Thomas Browne, English physician and author of the Religio Medici (1643), introduced the word "suicide" into our vocabulary (in reference to those who heroically imitated Cato), no universal justification was offered.

Nonetheless, the incremental steps of notable Renaissance and early Enlightenment authors emboldened others to advocate openly for the secularization and decriminalization of suicide outright. Typically, the unabashed Venetian libertine Giacomo Casanova argued energetically against Augustinian prudery in the treatment of self-killers, citing classical cases such as Socrates in his justifications of suicide as an act of free-will. ${ }^{16}$ The famous Italian jurist Ceasare Beccaria decried laws ordering the punishment of suicide as redundant and anachronistic. ${ }^{17}$ In a philosophical reflection (also published posthumously), Essays on Suicide and the Immortality of the Soul (1799), John Hume famously pointed out, "No man ever threw away life while it was worth keeping", echoing current medical opinion that terminal illness or mental torment both provide adequate individual justification for ending one's own life. Nonetheless, as late as 1790, the luminary Dr. Christian Gruner expressed despondency over the unenlightened attitude of common people toward suicide, bemoaning the custom of refusing obviously disturbed individuals a Christian burial, attacking persistent prejudices against dishonorable persons who removed the bodies of self-killers (legally abolished throughout Germany since 1731) and condemning weather-related superstitions. ${ }^{18}$ Unfortunately, popular superstitions and prejudices permeated all levels of society. Long after suicide had been decriminalized in places such as Prussia, police officials consciously punished the corpses of destitute self-killers by consigning them to anatomical theatres for medical dissection into the nineteenth century. ${ }^{19}$

\section{The tradition of suicidology: discovery or invention?}

On 9 February 1929, the Psychiatric Juridical Society of Amsterdam invited Willem Adriaan Bonger to give an annual address celebrating the twenty-second year of their foundation. The first professor of Sociology and Criminology in the Netherlands and the country's self-professed first Marxist criminologist, Bonger spent his career unraveling connections between economic circumstance and criminal activity. Fatefully, he chose the topic of "Suicide as a Social Phenomenon" for his talk. ${ }^{20}$ An early advocate for abortion rights, improved race-relations, a separation of religion and state and the decriminalization of homosexuality, as well as an opponent of Lambrosian psychiatric-criminology, Bonger was a notable champion of social reform. He belonged to Amsterdam society and his sister, Johanna, was married to Theo van Gogh. As a vocal opponent of National 
Socialism, he could not reconcile himself to the capitulation of Holland to the Germans in 1940, and, one day later, he took his own life together with his wife. Today, the Bonger Institute of Criminology, a multidisciplinary research group within the Faculty of Law at the University of Amsterdam, is dedicated to the study of the dynamic relationship between criminology and practical law enforcement, in his name.

The address, recorded by a stenographer, later appeared in the journal Man and Society, official organ of the Dutch National Bureau of Anthropology, with a few alterations. $^{21}$ Bonger initially counted self-murder ${ }^{22}$ among other socio-pathological abnormalities (e.g. crime, alcoholism and prostitution). However, even though selfmurder too represented an abnormality against human nature (self-preservation) and the nature of society, it occupied a special place in that category. Certainly, attitudes differed from place to place, and approaches in Japan were very different from those in the West, where religious condemnation was the sharpest and where, in certain states like England, the act remained a crime (such that a person who merely attempted suicide could be punished). Significantly, he noted that attitudes were changing over time and highlighted how the scientific study of self-murder was changing attitudes. Bolger went on to name that novel scientific discipline and located its origins among the earliest alienists:

The science of self-murder, suicidology, as one might call it, is more than a century old. At the end of the 18th century, physicians (psychiatrists) undertook scientific observations of self-murder for the first time. In that period, they viewed self-murder as a consequence of insanity. Because of Esquirol, one of the first great psychiatrists, they reckoned it among the monomanias. ${ }^{23}$

The Dutch criminologist then shifted his attention from medicine to criminal statistics. When, in 1826, French police produced their first crime statistics, they classified self-murder as a legally constituted category for criminal analysis. Here, Bonger undoubtedly referred to the collection of data on crime (first in Paris and subsequently extended to the rest of the country) initiated by the Ministry of Justice under the direction of the jurist André-Michel Guerry in 1825. One might add that, at the time, suicide was already in common usage in France, where it was not a crime, having already been decriminalized in $1791 .^{24}$

Bonger went on to laud the Belgian polymath Adolphe Quetelet, the first to demonstrate that self-murder was a mass social phenomenon which, like crime, recurred with great regularity. He also noted the important contributions of Italian psychiatrist Enrico Morselli and Tomáš Garrigue Masaryk, who went on to become the first president of independent Czechoslovakia. Needless to say, his 1929 address to the Psychiatric Juridical Society contained a veritable list of "firsts" in suicidology. And yet, Bonger's historical attribution of suicidology to these early pioneers long remained unexplored. Today, even his own contribution is overshadowed by the reputation of clinical psychologist Edwin S. Shneidman, founder of the American Association of Suicidology in 1968, oft cited as the field's father figure. Here, let us recall the early precursors of suicidology, who set the field in motion. We now turn our attention to their story and its historical context.

\section{Morality, morale and statistics}

Recent historical attention to the Foucauldian concept of biopower has drawn scholarly gaze onto the population management strategies undertaken by emerging modern states 
to control and direct human resources. ${ }^{25}$ After the French Revolution, moral statistics played a central role in the development of legislative policy. In emergent bourgeois society, state representatives sought bureaucrats with university qualifications and promoted meritocracy, rather than privileges accorded to the ecclesiastical and noble estates of the ancien régime. Alienists and social physicists joined the ranks of national bureaucracies in service of both national and professional interests. Psychiatrists envisioned themselves the secular healers of the soul, superseding the spiritual physic once dispensed by the clergy. ${ }^{26}$ Statisticians, who offered policymakers concrete measurements, came to replace cameral advisors to the prince. ${ }^{27}$

The collation of modern demographic statistics became practicable after the introduction of parish registers during the sixteenth century in the wake of religious reforms. In 1662, John Graunt compiled his Natural and Political Observations upon the Bills of Mortality to help predict outbreaks of plague in London, based upon "weekly Bills of Mortality extant at the Parish-Clerks Hall." ${ }^{28}$ By the eighteenth century, his thanatological breakthrough was being applied across many areas of statecraft. In 1749, Gottfried Achenwall wrote that statistics are "remarkable facts about the state," derived from the Italian for statesman (statista). ${ }^{29}$ Compilers targeted subjects associated with national economy (births, deaths, livestock, industry, etc.).

After the Napoleonic Wars, a peculiar branch of statistics, the science of moral statistics, began to develop. An exact definition of moral statistics is elusive, not least because early practitioners were amateur polymaths concerned less with terminological specificity than with tendering patriotic recommendations for government policy. By way of an example, consider Hans Järta, a Swedish revolutionary who helped overthrow King Gustav IV Adolf in 1809 and draft a new constitution. In 1823, he complained bitterly of statistics focusing solely on economic materialism (crops, populations, livestock, etc.). ${ }^{30}$ Järta subscribed to the Romantic theory of genius. Above all, statisticians had to conduct moral inquiries into spiritual values of any particular people and their times (quite literally, Hegel's Zeitgeist) to be of any real scientific worth.

In what is generally recognized as the first work on moral statistics, André-Michel Guerry's Essai sur la statistique morale de France, published in 1833, defined moral statistics in an all-encompassing fashion:

Moral statistics, having as its object of investigation the mind of man, studies his capabilities, his morals and customs, his feelings and sentiments, and his passions. Thus it encompasses at once the whole of moral philosophy, politics, religion, legislation, history, literature and the arts. ${ }^{31}$

According to Guerry, "Among the subjects included in moral statistics, suicide is one of those which has attracted the most lively attention and about which there has been the most discussion." 32 Although he conducted the first social content analysis of suicide notes for the Paris police, Guerry nevertheless emphasized strategic generalizations over individual concerns. One of the main patterns he discerned was an inverse relationship of suicide to homicide; whereas the north of France registered higher reports of suicides and lower rates of murder, the south exhibited contrary traits. He also addressed the issue of recidivism in the relationship of criminal to suicidal behavior.

The Belgian Adolphe Quetelet concurred with his colleague's criminological findings while conducting his own statistical investigations into the regularity of human behavior. 
His massive behavioral analysis of human faculties, subtitled an Essay in Social Physics, established a verifiable social abstraction, the average man. ${ }^{33}$ Over four books, he compared a wide variety of social variables from birth to death, statistically assessing their average probability of a normal distribution. In book three, he considered the development of intellectual and moral qualities, including the alienation of the mind, suicide and dueling, and finally a lengthy discourse on crime. Book four was dedicated entirely to the properties of the average man and the utility of social systems.

Four years later, Auguste Comte established sociology as the historical teleology of modern progress. ${ }^{34}$ Comte had only resorted to sociology as a moniker for his positivist system after Quételet appropriated his initial title, social physics. Unlike Guerry, Quetelet and Comte were system builders. Comte's positive philosophy concentrated chiefly on the contributions of natural science to human advancement. However, he proposed that social physics, "may perhaps convince men worthy of the name of statesmen that there is a real and eminent utility in labours of this kind." The alarming revolutionary reconstitution of modern societies led him to argue the utility of social physics in political terms:

The Ancients used to suppose Order and Progress to be irreconcilable: but both are indispensible conditions in a state of modern society; and their combination is at once the grand difficulty and the main resource of every genuine political system. ${ }^{35}$

Unlike Quetelet, Comte showed little concerned for moral statistics, limiting his observations to scientific progress. However, Comte had an early personal experience of suicide, having attempted to take his own life in 1827. He had just recovered from a severe nervous condition the year before, when he was admitted to the clinic of the alienist JeanÉtienne Dominique Esquirol, where he had been treated for mania and paranoia. ${ }^{36}$ After his release, Comte suffered a relapse and threw himself into the Seine from the Pont des Arts. Had it not been for the chance intervention of a Royal Guard, he may well have succeeded. ${ }^{37}$ Esquirol, an avid participant in the broad psychiatric debate on suicide in nineteenth-century France, treated Comte's nervous disorder once again. ${ }^{38}$

In Germany, another significant contribution to moral statistics treated suicide at length as well. National economist Adolf Wagner had a notoriously choleric temperament (once challenged by an aristocratic member of the Reichstag to a duel) and, initially, was not offered an academic chair in Germany. ${ }^{39}$ Instead, he took a post at the German-speaking Czarist University of Dorpat, until he was summoned back to a chair in political science (Staatswissenschaft, another term attributed to Gottfried Achenwall) at the University of Berlin in 1870 and became a principle architect of Bismarck's social welfare program. He is remembered for his Law of Increase, which posited the unceasing expansion of public expenditure and bureaucratization by the state.

Wagner began his career as a statistician and, in 1864, he published a monumental study on The Laws of Regularity in Apparently Arbitrary Human Behavior from the Viewpoint of Statistics. ${ }^{40} \mathrm{He}$ statistically demonstrated how individual behaviors conform to patterns that are generally constant but have a tendency toward increase. Wagner focused overwhelmingly on suicide statistics. In that context, he laid out what was to become sociology's "One Law": Protestants kill themselves more frequently than Catholics. Wagner briefly engaged in a discussion of a Protestant spirit which might contribute to such a condition, but he refused to elaborate because he had not conducted an adequate multi-variate analysis. 


\section{Religion, nationalism and race}

The function of religion intrigued alienists and social physicists. In one case, a squabble between British and French psychiatrists arose in the last years of the Napoleonic Wars. ${ }^{41}$ In 1815, alienist George Burrows opened debate with his French colleagues, alluding to revolutionary irreligiosity as the probable cause behind high rates of suicide in Paris:

Whether this deplorable propensity by the consequence only of recent political events which, having annihilated religion have deprived the wretched of its resources and consolations in affliction, and by their demoralizing effects dissolved the social compact that alone makes life a blessing, is not easy to determine... periods of great scarcity and distress, and sudden revolutions, political or religious, are always active and universal agents in originating insanity. ${ }^{42}$

In response, Esquirol "took up cudgels against the egregious Burrows who had dared to suggest that Parisians are more suicidal than Londoners." ${ }^{43}$ On the one hand, both instrumentalized suicide for political purposes, seeing this form of death as a reflection on the nation. On the other, they tacitly agreed that alienists and physicians were qualified to discuss it transnationally. Twenty-five years later, an ethnographic treatise by Englishwoman Harriet Martineau compared the French to the Irish, noting the abundance of suicide among the former. ${ }^{44}$ In Ireland, however, religious beliefs mitigated higher rates of suicide since:

This profusion of self-murders could not have taken place amidst a serious belief of an immediate entrance upon purgatory, such as is held by the majority of the Irish. Only in a state of vague speculation as to another life could the future have operated as so slight a check upon the rash impulses of the present. The Irish, an impetuous race, like the French, and with a good share of vanity, of sympathy, and of sentiment, are probably deterred from throwing away life by those religious convictions and sentiments which the French once held in an equal degree, but from which they are now passing over into another state. ${ }^{45}$

Like Burrows, her paternalistic anti-republicanism implicated revolutionary irreligiosity as the chief cause behind greater numbers of suicides in France, though there is no evidence in either case to suggest this was in fact true. ${ }^{46}$

In 1821, when Karl Theodor Hermann (an Achenwall student from Germany) began publishing on suicide statistics in Russia, he and three colleagues were accused of spreading atheism. Like Guerry's north-south divide in France, Hermann's moral map of Russia linked higher rates of suicide to civilized areas and lower rates to more traditional ones. ${ }^{47}$ The subject was contested; the Orthodox Church stilled viewed suicide as both a sin and a crime. In mid-nineteenth century Russia, suicide preoccupied scientists, writers and slavophiles. Dostoevsky deployed it as a literary trope against Westernizing influences. ${ }^{48}$ In his condemnation of the immorality exerted by modernizing influences on Russian society, Dostoevsky concluded that religious and patriotic values (Eastern Orthodoxy and Mother Russia) provided the only moral bar on the door to many unthinkable crimes, such as suicide, permitted by socialist nihilism imported from the West. 
At the University of Dorpat, Adolf Wagner's former colleague Alexander von Oettingen published his Moralstatistics in 1868 and a second book, On Acute and Chronic Suicide, in 1881, specifically devoted to the subject. He developed an explanation for higher rates of Protestant suicide based upon the premise that Protestants represented the spirit of modernity. Suicide among modern Protestants was likened to an infectious disease, with the evangelical religion representing the proper antiseptic, like some carbolic acid. ${ }^{49}$ As for their duties, Oettingen couldn't deny that Protestantism demanded greater sacrifices of its followers than Catholicism, "with their priestly life-insurance tendencies" (e.g. the sacrament of penance), or for the Orthodox "orientalists." He argued that their superstitious religiosity differentiated them from Protestants:

Therefore it is understandable that the German, with his high culture and deep inner affective life, with his tendency to self-reflection and self-critique, carries with him a greater danger of suicide than the easy-living, sanguine Roman or the even less developed, less-civilized Slav, who only tends toward suicide, when licked by half-culture or if infected with nihilism. ${ }^{50}$

Oettingen lived in the Baltic states of the Russian Empire, where most ethnic Germans were Evangelical Lutherans and where Dostoevsky's Demons, published in 1872 (nine years earlier), had already sparked controversy regarding Westernization, backwardness, nihilism and anarchism in Russia. Thomas Masaryk's Suicide and the Meaning of Civilization appeared in the same year, but it is unclear whether Oettingen's mention of halfeducation was a conscious reference to the Czech author. ${ }^{51}$ Appearing as it did at the tail end of the Kulturkampf, Oettingen's study of suicide manifested the deeply rooted longings of many ultra-nationalist Baltic Germans, who supported German unification under the Prussian state in 1871 and a hoped-for reintegration into a united German Fatherland.

A similar north-south divide later reported for Italy by Morselli was instrumentalized in the national debate over Italian unification to argue for the ostensible backwardness of the south. ${ }^{52}$ Along with fellow scholars like his mentor, the psychiatrist Carlo Livi and the eminent criminologist Caesare Lombroso, Morselli represented positivist rationalization against the conservative forces of particularism. All of them also had a professional agenda: Together, they promoted an evolutionary model based on phrenology to suggest that southern Italians suffered from [physical] anthropological atavisms in order to promote a policy of penal reform which would grant psychiatrists a much larger scope in the care and treatment of prisoners.

Furthermore, Morselli turned our presumption that high rates of suicide represented an unfavorable condition on its head by developing an evolutionary view of civilization, modernity and progress. Counter-intuitively, he claimed that high rates of suicide actually mirrored higher levels of civilization in the advance toward modernity. He first published interim findings from a phreniatric study of the prison population in southern Italy in 1875 and then as his award-winning masterpiece, Suicide: A Study of Comparative Moral Statistics, which appeared in 1879 to international acclaim and was quickly translated into German and English. ${ }^{53}$ Certainly, it was no coincidence that Durkheim chose the same title for his own Le Suicide eighteen years later. As a psychiatrist, Morselli viewed the pressures of modernity in terms of an internalization of violence. Whereas in less civilized areas (e.g. southern Italy) violence was liable to be externalized as murder, in more 
civilized areas, the internalization of social pressures moved weaker members of society to take their own lives; regrettable and preventable, suicide ultimately represented a positive and natural Darwinian check on deviance in populations, a "fatal tendency of civilized society." In this regard, suicide was a perverse badge of modernity.

\section{Conclusions: the victory of suicidology}

The modernity thesis and its attendant teleology is largely discredited today, owing in no small part to the labors of subaltern historians and their critiques of Euro-centrist master narratives. ${ }^{54}$ However, if the Western concept of modernity continued to shape global policy at institutions such as the United Nations throughout the twentieth century, ${ }^{55}$ its political and social implications categorically obsessed most nineteenth-century European thinkers.

Indeed, in nineteenth-century sociology, the proposed relationship between suicide and religion assumed near law-like status as the so-called "One Law of Sociology," i.e. Protestants ostensibly kill themselves more often than Catholics and, to a less clear extent, Jews. This theorem is widely attributed to the arch-system-builder Émile Durkheim, first academic chair of sociology and considered by many to be the founder of the discipline. His influential Le Suicide (1897) represents the first sociological case study in history. As suggested in its subtitle (quite bold at the time), the monograph was actually a "Study of Sociology" (Étude de sociologie); that is, a demonstration of his sociological method. Durkheim employed suicide as the most viable vehicle of his age to demonstrate and confirm the scientific credentials of the quantitative method for measuring and establishing the existence of social phenomena through demographic analysis. Armed with the scientific positivism of Comte, Durkheim set out to prove the existence of coercive social forces transcending individuals and controlling human behavior at the strategic level of shared beliefs and moral values. His analyses of collective consciousness rendered sociology quite distinct from psychology and psychiatry, which dealt with the individual rather than the group.

The history of nineteenth-century suicidology evidences the pervasiveness of both nationalism and bureaucratization, an awareness of republicanism and self-assertive professionalization in contemporary thought. Less expected is the continued importance of religion inherent in the interpretation of moral statistics, which was ubiquitous, from Italy, France, Britain, Russia and even Scandanavia, whereby suicide continued to be viewed pejoratively. ${ }^{56}$ Richard Bell has recently demonstrated how the topic of suicide was intricately woven into the broader cultural tapestry of the United States as part of political fear-mongering, generational conflict, campaigns for public altruism and criminal reform and the abolitionist debate from the end of the Revolution until the Civil War. ${ }^{57}$ Obviously, there is far more at stake in the topic for historians than a simple empirical analysis along the lines of the Durkheimian quantitative method.

Indeed, the present consideration may give pause to reflect on the development and derivation of this method, coming as it did from within the particular context of a nineteenth-century debate on the nature of the state and modern society and their relationship to the nascent disciplines of sociology and psychiatry. Studies like Durkheim's represented the culmination of an intellectual process. The present overview demonstrates how suicide caught the attention of the early suicidologists - psychiatrists, moral statisticians and, subsequently, sociologists. 
If the growth in interest in suicide was a ubiquitous European phenomenon that was gradually exported throughout the globe along with the master narrative of modernity, then its practitioners nonetheless found reason to quarrel over the nature of their findings. The French (e.g. Comte, Durkheim, Mauss) and the Italian (e.g. Morselli, Lombroso, Levi) modernists supported a positivist and scientific approach. Their theories of material progress tended to be mechanistic and, more often than not, fatalistic. The German idealists, on the other hand, were more prone to anti-positivism. They developed an interpretation of Hegel's spirit of the times (Zeitgeist) as the social dynamic behind progress which found its apotheosis in Weber's Protestant ethic and spirit of capitalism. Werner Sombart, a student of Adolph Wagner who also supported an interpretation of the Renaissance and Reformation as dynamic spiritual forces behind modern processes, once famously derided the pseudo-science of French positivism as "physics envy." In the end however, the seminal input to moral statistics by Germans such as Wagner, Oettingen and Drobisch disappeared from modern suicidology, while Morselli is only slightly better recognized, and the theories of Durkheim have been replaced by the bland empiricism of biometrics. Or, as Theodore Porter suggests:

From our perspective, this German tradition has become almost invisible, and the more mathematical approach developed by English biometricians has become almost obligatory. Within that kind of statistics, the primacy of elemental social facts has, to a degree, been restored. Durkheim was no party to the biometricians' scheme of social explanation. ${ }^{58}$

Apart from that, the history of nineteenth-century suicidology implies the importance of historicizing that process, which continues to have lasting and immediate significance. Perhaps, in the history of the relationship between suicide, sociology and the state, it is as Andreas Bähr so aptly points out, more a question of semantics than numbers. ${ }^{59}$

In a vague definition of moral statistics, Alexander von Oettingen expressed dissatisfaction with the French practice of statistique morale, preferring instead to adhere closely to the original French meaning, describing morals as customs (Sitten), though not in an ethnographic sense. ${ }^{60}$ In reference to suicide, the Italian moral statistician Melchiorre Gioia suggested that "Suicides should be considered an integral part of costumi [habits of a population] as, in their majority, they depend on moral causes.",61

Ironically, however, since much of the actionable data available was limited to deviant behavior coming to the attention of criminal authorities, "One might sooner speak of immoral rather than moral statistics." ${ }^{22}$ Early suicidologists harmonized their methods and theories with contemporary ideologies on the body politic, modernity and social Darwinism, simultaneously imbuing suicide with both socially positive and individually negative qualities, while maintaining a focus on the act as a distinctive and troubling form of death. One dictionary of sociology defines moral statistics as measurements of "suicide, divorce, mental health, illegitimacy, and abortion" as indicators of social pathology, defined, in turn, as "An early form of deviance theory, no longer in wide use, which drew upon the organic metaphor to suggest that parts of societies, like parts of bodies, could suffer breakdown and disease."63 As Ursula Baumann is quick to point out, moral statististicians and psychiatrists tended to focus - far more often than not - on types of behavior generally recognized as immoral. ${ }^{64}$ The values measured "silently presumed their negative evaluation." ${ }^{65}$ A vital question, extending to our own day, even amid the 
emergence (since the 1950s) of new kinds of preventative efforts, is the extent to which attitudes toward suicide continue to reflect the kind of stigma attached to the act by the secularized science of the nineteenth century.

\section{Notes}

1 Ronald W. Maris, Alan L. Berman \& Morton M. Silverman, The Comprehensive Textbook of Suicidology (New York and London, 2000), 62.

2 Indeed, the word "suicide" was alien to the ancient Romans; see Anton van Hoof, From Autothanasia to Suicide. Self-Killing in Classical Antiquity (London, 1990).

3 On the evolution of the Western prohibition of suicide during the Middle Ages, see Georges Minois, History of Suicide. Voluntary Death in Western Culture (Baltimore and London, 1999), 27-41; Mario Barbagli, Farewell to the World. A History of Suicide (Cambridge, 2015), 40-46.

4 Though, in effect, this represents a modern demographic rationalization for Augustine's singularly religious arguments.

5 For a detailed explanation of the vices/virtues portrayed in Prudentius' Psychomachia and the Scrovegni Chapel, see David Lederer, "Religion and Spirituality," in: Susan Broomhall \& Philippa Maddern (eds.), A Cultural History of the Emotions in the Late-Medieval, Reformation and Renaissance Age (London, 2019), 31-48.

6 Alexander Murray, Suicide in the Middle Ages, Vol. I: The Violent against Themselves (Oxford, 1998), 120-250.

7 Jürgen Diestelhorst, "Die Bestrafung der Selbstmörder im Territorium der Reichstadt Nürnberg," in: Mitteilung des Vereins für Geschichte der Stadt Nürnberg 44 (1953), 8-230.

8 Briefly, to cite some examples of the growing number of studies relating to criminal procedure against suicides during the early modern period: Michael MacDonald \& Terence Murphy, Sleepless Souls: Suicide in Early Modern England (Oxford, 1990); Markus Schär, Seelennöte der Untertanen. Selbstmord, Melancholie und Religion im Alten Zürich (Zürich, 1985); Vera Lind, Selbstmord in der frühen Neuzeit (Göttingen, 1999); Michael Watt, Choosing Death: Suicide and Calvinism in Early Modern Geneva (Kirksville, 2001); Andreas Bähr, Der Richter im Ich. Die Semantik der Selbsttöttung in der Aufklärung (Göttingen, 2002); David Lederer, Madness, Religion and the State in Early Modern Europe: A Bavarian Beacon (Cambridge, 2006); Alexander Kästner, Tödliche Geschichte(n). Selbsttötungen in Kursachsen im Spannungsfeld von Normen und Praktiken (1547-1815) (Konstanz, 2012); Florian Kühnel, Kranke Ehre? Adlige Selbsttötung im Übergang zur Moderne (Munich, 2013); Rikka Miettinen, Suicide, Law and Community in early Modern Sweden (Cham, 2019).

9 Lieven Vandekerckhove, On Punishment. The Confrontation of Suicide in Old-Europe (Leuven, 2000); Mary Lindemann, "Armen und Eselsbegäbnis in der europäischen Früheneuzeit, eine Methode sozialer Kontrolle," in: Paul Richard Blum (ed.), Studien zur Thematik des Todes im 16. Fahrhundert (Wolfenbüttel, 1983), 125-139.

10 David Lederer, "The Dishonourable Dead: Perceptions of Suicide in Early Modern Germany," in: Sibylle Backman, Hans-Jörg Künast, Sabine Ullmann \& B. Ann Tlusty (eds.), Ehrenkonzepte in der Frühen Neuzeit. Identitäten und Abgrenzungen (Berlin, 1998), 349-365.

11 On these macabre practices, see Kathy Stuart, Defiled Trades and Social Outcasts Honor and Ritual Pollution in Early Modern Germany (Cambridge, 2009), 149-186.

12 Hanns Bächtold-Stäubli (ed.), Handwörterbuch des deutschen Aberglaubens, Vol. VII (Berlin/Leipzig, 1935/36), 1629 .

13 On incidents in hospitals in the Free-Imperial city of Augsburg, see David Lederer, "Wieder ein Fass aus Augsburg...': Suizid in der frühneuzeitlichen Lechmetropole," in: Mitteilungen des Instituts für Europäische Kulturgeschichte (2005), 47-72.

14 David Lederer, "Verzweiflung im Alten Reich. Selbstmord während der 'Kleinen Eiszeit'," in: Wolfgang Behringer, Hartmut Lehman \& Christian Pfister (eds.), Kulturelle Konsequenzen der "Kleinen Eiszeit"/Cultural Consequences of the Little Ice Age (Göttingen, 2005), 255-280.

15 David Lederer, "Suicide in the Early Modern World," in: Robert Antony, Stuart Carroll \& Dr Caroline Dodds Pennock (eds.), The Cambridge World History of Violence Volume III: The Early

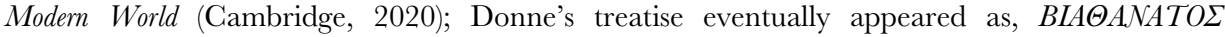




\section{SUICIDOLOGY ON THE GUSP OF MODERNITY}

[Biathanatos, orSelf-Killing]: A Declaration of that Paradoxe, or Thesis, that Selfe Homicide is not so Naturally Sinne.

16 Giacomo Casanova, Über den Selbstmord und die Philosophen (Frankfurt and New York, 1999).

17 Dei delitti e delle pene (Harlem, 1764).

18 Christian Gottfried D. Gruner, "Unehrlichkeit und unehrliches Begräbnis. Der Selbstmord," in: Almanach für Ärtze und Nichtärtze auf das Jahr 1790 (Jena 1790), 112-121.

19 On the judicial consignment of the corpses of suicides to medical facilities for anatomical study in Prussia, see Julia Schreiner, "Zwischen Aufgeklärtheit und Moral, Zweckmäßigkeit und Pathologie," in: Eckhart Hellmuth, Immo Meenken \& Michael Trauth (eds.), Zeitwende? Preußen um 1800 (Stuttgart, 1999), 207-228; Ruth Richardson, Death, Dissection and the Destitute (Chicago, IL, 2001). According to Olive Anderson, Suicide in Victorian and Edwardian England (Oxford, 1987), 221, this punishment was suggested, but never practiced in Britain.

20 W.A. Bonger, "Zelfmoord als Maatschappelijk Verschijnsel," in: Mens en Maatschappij 5/4 (1929), 281-303. Bonger originally gave the talk in Amsterdam in 1929 and the Society circulated it internally as a stenogram but no longer possesses the original version. outThis version appeared later that same year in the Amsterdam journal cited here.

21 Ibid., 281-303.

22 Bonger employed the idiomatic Dutch term, zelfmoord, when referring to suicide, as is still colloquially common.

23 Ibid., 282.

24 On the depenalization of suicide in France, see Dominique Godineau, S'abréger les jours. Le suicide en France au XVIIIe siècle (Paris, 2012).

25 For a recent overview of the concept and its historical applicability, see Majia Holmer Nadesan, Governmentality, Biopower, and Everyday Life (New York and London, 2008).

26 On the practice of spiritual physic, see David Lederer, Madness, Religion and the State in Early Modern Europe: A Bavarian Beacon (Cambridge, 2006).

27 See, for example, Lars Behrisch, Vermessen, Zählen, Berechnen. Die Politische Ordnung des Raums im 18. Fahrhundert (Frankfurt am Main, 2006); I. Bernard Cohen, The Triumph of Numbers: How Counting Shaped Modern Life (New York, 2006).

28 Natural and political observations mentioned in a following index and made upon the bills of mortality ... with reference to the government, religion, trade, growth, ayre, diseases, and the several changes of the said city (London, 1662), 4.

29 Ian Hacking, The Taming of Change (Cambridge, 1990), 24; Mary Poovey, A History of the Modern Fact: Problems of Knowledge in the Sciences of Wealth and Society (Chicago, 1998), 308. On the Italian derivation of the word "statistics," see Alexander von Oettingen, Die Moralstatistik in ihrer Bedeutung für eine Socialethik (Erlangen, $\left.1882^{3}\right), 7$.

30 Evelyne Luef, "Low Morals at a High Altitude? Suicide in Nineteenth-Century Scandinavia," in: Journal of Social History 46/3 (2013), 668-683, esp. 669f.

31 "La Statistique morale (1), ayant pour objet l'homme intellectual, elle étudie ses faculties, ses moeurs, ses sentimens, ses passions; elle embrasse ainsi à-la-fois dans son ensemble, la philosophie morale, la politique, le culte, la legislation, l'histoire, la literature et les art': André-Michel Guerry, Essai sur la statistique morale de la France (Chez Crochard, Paris 1833), 69; translation from Hugh P. Whitt \& Victor W. Reinking, eds. \& trans., A Translation of André-Michel Guerry's Essay on the Moral Statistics of France (1833): A Sociological Report to the French Academy of Science (Lewiston, 2002), 136. Promoted to Director of Criminal Statistics shortly after the Revolution of 1830, Guerry had been commissioned to compose a massive compilation of criminal data from 1825, the Compte général de l'administration de la justice criminelle en France, summarized in the Essai.

32 "Parmi les sujets qu'embrasse la statistique morale, le suicide est un de ceux qui ont le plus vivement attiré l'attention, et sur lesquels on a le plus dissertê': André-Michel Guerry, Essai, 61; Whitt \& Reinking, Translation, 121 .

33 Most significantly, his Sur l'homme et le développement de ses facultés, ou Essai de physique sociale (Paris, 1835).

34 Harriet Martineau (trans.), The Positive Philosophy of Auguste Comte, 2 vols. (London, 1853). Comte's interpretation of historical stages is introduced from the onset (vol. 1, p. 2), while the full explication of "the historical question," i.e. each stage and its characteristics appears later (vol. 2, pp. 181-483).

35 Ibid, vol. 2, p. 2 f. 
36 Mary Pickering, Auguste Comte: An Intellectual Biography (Cambridge, 1993), 2 vols., vol. 1, 371-392.

37 Ibid., 394.

38 For an overview of the psychiatric discourse in France concerning suicide, see the recent $\mathrm{PhD}$ dissertation of Eva Yampolsky, La folie du suicide: Une histoire de la mort volontaire comme objet médical en France de la fin du XVIIIe siècle au années 1870 (University of Lausanne, 2019).

39 David Lederer, "Sociology's 'One Law': Moral Statistics, Modernity, Religion and German Nationalism in the Suicide Studies of Adolf Wagner and Alexander von Oettingen," in: Fournal of Social History 46/3 (2013), 684-699.

40 Adolph Wagner, Die Gesetzmässigkeit in den scheinbar willkührlichen menschlichen Handlungen vom Standpunkte der Statistik (Hamburg, 1864).

41 The incident is described by Ian Hacking as, "the beginning of numerical sociology because (a) there were numbers and (b) the numbers of suicides were seen as a moral indicator of the quality of life": Taming of Chance, 64.

42 George Burrows, Commentaries on the Causes, Forms, Symptoms, and Treatment, Moral and Medical, of Insanity (London, 1828), 442.

43 Hacking, Taming of Chance, $65 \mathrm{ff}$.

44 Harriet Martineau, How to Observe Morals and Manners (London, 1838).

45 Martineau, Morals and Manners, 98. Martineau's visit to Ireland is recounted in her Letters from Ireland (London, 1852; republished Dublin, 2001).

46 On suicide in revolutionary France, see Jeffrey Merrick, "Death and Life in the Archives: Patterns of and Attitudes to Suicide in Eighteenth-Century Paris," in: David Wright \& John Weaver (eds.), Histories of Suicide: International Perspectives on Self-Destruction in the Modern World (Toronto, 2009), 73-90; Merrick, "Suicide and Politics in Pre-Revolutionary France," in: Eighteenth-Century Life 30 (2006), 32-47; Merrick, "Suicide in Paris, 1775," in: Jeffrey Watt (ed.), From Sin to Insanity: Suicide in Early Modern Europe (Ithaca, 2004), 158-174.

47 Susan K. Morrissey, "Mapping Civilisation: The Cultural Geography of Suicide Statistics in Russia," in: Fournal of Social History 43/3 (2013), 651-667, esp. 656.

48 On this aspect of Russian literature, see Irina Paperno, Suicide as a Cultural Institution in Dostoevsky's Russia (Ithaca, 1997), esp. 123-161. On suicide in nineteenth-century Russia more broadly, see Susan Morrissey, Suicide and the Body Politic in Imperial Russia (Cambridge, 2006).

49 Alexander von Oettingen, Über die Akuten und Chronischen Selbstmord (Dorpat, 1881), 28.

50 "So erklärt es sich, daß er Germane mit seiner Hochkultur und seinem tief innerlichen Gemüthsleben, mit seiner Neigung zur Selbstbeobachtung und Selbstkritik, auch eine gröre Selbstmordgefahr in sich trägt, als der leichtlebige, sanguinische Romane oder der noch unentwickelte, naturwüchsige Slave, der nur dort zum Selbstmord neigt, wo ihn die Halbkultur beleckt oder der Nihilismus angekränkelt hat": Ibid., 31.

51 Though it seems likely that it probably was, and, occasionally, the theory is also attributed to Adolph Wagner. The habilitation of Tomáš G. Masaryk is Der Selbstmord als sociale Massenerscheinung der modernen Civilisation (Vienna, 1881). Masaryk later became the first President of Czechoslovakia.

52 Maria Teresa Brancaccio, “The Fatal Tendency of Civilized Society': Enrico Morselli’s Suicide, Moral Statistics and Positivism in Italy," in: Fournal of Social History 46 (2013), 700-715.

53 Enrico Morselli, Il suicidio: saggio di statistica morale comparata (Milan, 1879).

54 The inadequacies of the modernity thesis were, most recently, a subject of an extensive forum in the American Historical Review 116 (2011); the concluding piece by Richard Wolin, "Modernity': The Peregrinations of a Contested Historiographical Concept," 741-751, provides a useful overview.

55 For example, see Mark Mazower's critical history of the United Nations presented in his 2008 Lawrence Stone lectures at Princeton University and published as No Enchanted Palace: The End of Empire and the Ideological Origins of the United Nations (Princeton, NJ, 2009).

56 Andreas Bähr, "Between 'Self-Murder' and 'Suicide': The Modern Etymology of Self-Killing," in: Fournal of Social History 46 (2013), 620-632.

57 Richard Bell, We Shall Be No More: Suicide and Self-Government in the Newly United States (Cambridge, MA, 2012).

58 Porter, "Statistical and Social Facts," 25. 


\section{SUICIDOLOGY ON THE CUSP OF MODERNITY}

59 The central thesis of his prize-nominated monograph on self-killing from the Enlightenment to Romanticism; Andreas Bähr, Der Richter im Ich, Die Semantik der Selbsttötung in der Aufklärung (Vandenhoeck and Ruprecht, Göttingen, 2002).

60 Oettingen, Moralstatistik, 11.

61 Melchiorre Gioia, "Nota dei suicidj avvenuti nelle Provincie Lombarde negli anni 1817 al 1827," in: Annali universali di statistica, economia pubblica, storia, viaggi e commercio 17 (July 1828): $67-68$.

62 "Sodann aber umfassen die zählbaren und registrirbaren Daten fast ausschliesslich böse, unmoralische Handlungen. Man könnte eher von einer Immoralitäts-, als von einer Moralstatistik reden": Oettingen, Moralstatistik, 12.

63 Gordon Marshall, "Moral Statistics," in: A Dictionary of Sociology (1998), Encyclopedia.com. (15 January 2020). www.encyclopedia.com/doc/1O88-moralstatistics.html.

64 Ursula Baumann, Vom Recht auf den eigenen Tod. Die Geschichte des Suizids vom 18. bis 20. Jahrhundert (Weimar, 2001), 220.

65 Ibid., 220. 


\title{
DEATH-SEEKING TURNS POLITICAL
}

\author{
A historical template for terrorism
}

\author{
Anna Geifman \\ "We shall die! Oh, how gloriously we shall die!" \\ A. I. Odoevskii on the day of the \\ 14 December 1825 uprising in St. Petersburg
}

\section{Introduction}

For the first time in modern history, extremists routinely resorted to ideologically justified suicidal violence in late-imperial Russia at the turn of the 20th century, and new attitudes toward death were central to their approach. The upsurge of politicized self-destructiveness coincided with the country's shattering sociopsychological ordealan abrupt breakdown of the traditional value system, of ethical and aesthetic conventions, and of connections people had felt with their culture, centering around family, community, and religion. Fragmentation of shared meanings and bonds that had hitherto sustained individual emotional stability and social cohesion, known as "historical dislocation," was patent. With the old values no longer valid and the new ones not yet integrated, ${ }^{1}$ the cultural crisis manifested a motley of challenges and perils, including zealous nihilism, violence, and suicide. Death-seeking often camouflaged itself as suicidal terrorism.

Reliable data about incentives and drives of Russian terrorists in the early 20th century are inevitably scant, as is the case concerning the psychology of ideological extremists today. Methodologically, the article analyzes scarce historical materials related to terrorists' motivations and decision-making through the prism of and within a larger psychosocial context, linking perpetrators of jihadist suicide attacks to their Russian forerunners a century ago. Self-proclaimed atheists, the Russian suicide terrorists did not aspire to an afterlife, nor did most of them struggle against "occupation" or "colonialism." Yet, a comparative analysis indicates a paradigm that crosses temporal, geographic, and ethnic boundaries and renders the "situation-dependent psychological differences between suicide bombers" in the 20th and 21st centuries less significant than has been assumed. ${ }^{2}$ What follows is a template which connects socioeconomic and political causes of suicidal political violence with deeper psychological stimuli. 


\section{The trauma of "historical dislocation": a comparative view}

In various parts of Europe in the second half of the 19th century, rapid mobility and urbanization created "an extraordinary number of persons uprooted from ancestral soil and local allegiances. Experiencing grave economic insecurity and psychological maladjustment, these were very susceptible to demagogic propaganda, socialist or nationalist or both." 3 Less ready for the advent of modernization, the Russians were especially vulnerable and prone to releasing a bottled-up rage when external conditions stimulated aggression-venting. The difficult renovation process in Europe, in Russia turned into a crisis. It surfaced around 1900 and spiraled swiftly into a political calamity.

A similar situation has developed over the past decades in parts of the Muslim world, which has undergone a

transformation not unlike that of Europe in the late 19th century. Large numbers of villagers and tribesmen have moved to the vast urban slums of Cairo, Algiers, and Amman, leaving behind the often preliterate Islam of the countryside. Islamism has filled the void ${ }^{4}$

the way radical socialism and anarchism had filled the cultural vacuum in Russia in the early 1900s.

Islamism too is bred by "trauma of uprootedness." Rapid modernization correlates strongly with emergence of ideological extremism, especially "in countries where sudden wealth (e.g. from oil) has precipitated a change from tribal to high-tech societies in one generation or less." As norms crumble or "seem irrelevant, new radical ideologies ... may become attractive" to the psychologically "displaced." Islamism thus "emerges from peoples' reactions to the modern, Western-shaped world" and "the anger and confusion of that reaction." Estranged from communal routines and conventions of the mainstream Islam, numerous individuals suffer "exile" or alienation. "Old ways of life are disrupted; new ones are not yet established." The burden of pluralism, with its conflicting and confusing options, is arduous. Religious commitment is undermined, along with familial hierarchies. Self-perception as a member in a group comes under threat. Habitual meanings, crucial "to furnishing human beings with a stable concept of reality, are torn apart." Primary "needs - security, identity, dignity, belonging" - are frustrated, contributing to a developing mindset of a humiliated victim, "besieged, thwarted, filled with real and invented grievances." ${ }^{\prime 7}$ Akin to the uprooted Russian extremists, the Islamists - most of whom are "not poor or ignorant but well-off and educated" - are part of "a lost generation," unmoored from traditional cultures. ${ }^{9}$

Victims of historical dislocation in Russia ascribed their misery to detrimental economic conditions in their country and to its autocratic leadership. Their Muslim successors pair the sting of injured pride with most pronounced traits of modernity, such as the "cult of economic growth, hedonism and permissiveness" which undercut communal life and family - the shrine of Muslim culture. Other foes include more abstract notions of democratic liberalism, individualism, globalization, and the general "Westoxication," or perceived conspiracy against Islam. ${ }^{10}$

In Russia, the breakdown of civic values led to widespread disappointment and an array of life-undermining symptoms, concomitant with alienation: emptiness, apathy, hopelessness, disengagement, anxiety, a sense of "as if" living. The "not staunch" and the 
"half-cracked"11 sought refuge in "the new"- from the hitherto unthinkable "uninhibited sexuality," to toying with exotic cultures, to occult, satanism, and death-seeking. ${ }^{12}$ A hodgepodge of fads fluctuated at whim during the "Silver Age," the era of intellectual and aesthetic turmoil and rebellion against the convention. "Everything that declared itself as a protest was received with sympathy and curiosity." 13

The new trend yielded its own archetype, a "beau ideal" to be admired and emulated - a complicated individual, burdened by inner contradictions, "the split, bizarre type."14 People held melancholy and neurasthenia as "an attribute of refinement" and even "invented vices and perversions for themselves," not to appear banal. ${ }^{15}$ They applauded everything "abnormal," "odd," "sick," antisocial, ${ }^{16}$ including perversions, addictions, satanism, blood-rites, and death-worship. ${ }^{17}$

"These were the days when love and all sane and kindly emotions were regarded as commonplace and old-fashioned." On the other hand, "destructiveness was considered a sign of good taste," 18 and its champions sought venues to express it as action: "Some different higher principle is needed. Since there is none, rebellion and violence of all sorts take its place ... self-destruction - debauch, drinking, all the forms of suicide." 19 As part of a cycle of emotional investments and disappointments in "alternative lifestyles," sooner or later one stumbled on political extremism.

\section{The appeal of political nihilism}

The revolt against Russian traditional culture was intrinsically nihilistic. Admittedly, "the banner that united us was the denial of life that had formed us," recaptured novelist Andrei Bely. ${ }^{20}$ In accordance with their new role as revolutionaries, seeking to annihilate their environment, self-destructive behaviors acquired overt political coloring. Writer Aleksei Remizov eyed in disbelief the multitudes of self-destroyers "who fanatically direct their extraordinary capacities against themselves!" His famous partner in trade Maksim Gorkii went a step further: "nowhere do people wreck themselves with such headlong, senseless speed, as in our Russia." 21 Their objectives were often ambiguous, articulated feverishly:

to look for the ultimate courage in the ultimate despair; to finish with all the old ... If Russia today is a dry forest, ready for fire, then the Russian decadents are the driest top branches of these woods; when the lightening will strike, they will ignite first, setting fire to the whole forest. ${ }^{22}$

"I cannot live peacefully," declared a revolutionary; "I like danger, so as to feel the thrill." ${ }^{, 23}$ Participation in radical politics was analogous to narcotics or cheap, overstimulating entertainment - a means to escape from apathy and debilitating boredom, as some extremists admitted. ${ }^{24}$ The "enterprises and adventures," others confessed, enticed and spurred them on along the radical path. ${ }^{25}$

Subversive action had one essential advantage over all other forms of marginal behaviors. Unlike individuals engaged in diversions requiring them to cope with cheerless nadirs between peaks of excitement, the radical retained elation in the perpetual pursuit of visionary ideals. The forever-distant dream substantiated erratic life and gave it a quality of transcendence. For the emotionally and often physically exhausted, depleted by experimentation with various addictive fads, political extremism provided new meaning and fierce energy to go on. "Revolution was becoming the fashion," 26 acknowledged one of its leaders, Viktor Chernov. 
Tom Wolfe would dub it "radical chic" that gripped society. Almost no occupation carried "more prestige among educated Russians" than terrorism. ${ }^{27}$

In the next century it would become "a career as much as a passion" or "addictive" infatuation. ${ }^{28}$ It is a modern "psychosocial disease" among proponents of Islamism, whose personal circumstances combine with generalized disaffection in "a boiling fury." They are sought after and become easy prey for extremist recruiters, who offer the dislocated marginals new values and ideology, "a narrative - an actual story with a logical flow from glorious past through decadent present to reglorified future," as well as prototypes of heroes (revolutionaries) and villains (the persecuting local and global enemies). ${ }^{29}$

\section{Sociocultural and group climate as triggers for suicide}

Russian cultural climate was akin to that in turn-of-the-century Vienna, where "deviance increased, and society was pervaded by ... tensions, mistrust, and hostility." Ezra Pound's "antennae of the world," artists "sensed the atmosphere of dissolution and decease and depicted it in their works." "30 Likewise, a Russian heroine of Leonid Andreev's famous story, "Girl in Black" symbolized the "noblest instincts" of her generation when hoping "to meet death dancing." 31 Among the new-age aesthetes gripped by "the dark side," many obsessed about the notion of the "nonbeing." Fascinated with a symbiosis of artistic ecstasy and destructive energy, they sought "poetry in death.",32 A reputable public intellectual grieved that love of death was the chief motivation of Russian intellectual youth. ${ }^{33}$

One "gazed into the abyss of sorrow and despair," with "nothing but confusion, madness, death," 34 and perceived life as a vulgar play. "The entire world seems to me like some kind of masquerade," complained a contemporary of the cultural crisis: everyone, "each without exception is in a mask." With despondency being a mainstream attitude, many asked themselves whether it would not be better "at once to end [it all]." 35 And many felt that their pseudo-existence was not worth drawing out.

In the 19th century, Russia had had the lowest suicide rate in Europe - three cases in a population of $100,000 .^{36}$ By 1905 , self-inflicted death figures had multiplied ten times, reaching 370 per million, ${ }^{37}$ and were continuing to increase. There was "a massive rise in suicides and attempted suicides" from 1906 to a peak in $1910 .{ }^{38}$ In the capital, the rate of suicide went up by 25 percent. $^{39}$

Physicians and journalists declared a real epidemic of self-caused death in St. Petersburg, the epicenter of Russia's "historical dislocation," where the "clash between ... tradition and modernity" 40 was particularly glaring. Amidst the city's conflicting imperial solidity and nihilistic restlessness of the new times, the capital was in many eyes an urbanistic monster "in the grip of nervous expectation, cataclysmic foreboding and a fatal urge to destruction." 41 Its citizens' craving for self-annihilation was such that the rate of suicide tripled in half-a-decade: from 500 cases per million residents to a colossal 1,640 per million in 1910 , to fall only slightly to $1,550 \mathrm{in} 1911$ - "easily the highest rate in Europe." 42

Most Russians who took their own lives in the early 20th century blamed not the economic poverty but the "poverty of values" or "life" as a whole, which, they decided, was "not worth it to live." ${ }^{43}$ In the disintegrating social environment, death by suicide became a new vogue, a trend distinct from isolated instances of individuals taking their own lives due to strictly personal suffering or private dramas. Like their 
apolitical contemporaries, the radical socialists and other political subversives submitted themselves to the "suicidal urge," 44 having failed to self-repudiate in the radical movement.

To escape inner anguish, many sought to situate their vulnerable selves in the revolutionary milieu, which advertised a new worldview and offered a renewed sense of purpose. This also provided a designated enemy to those who strove to relocate the experience of estrangement, inner depletion, worthlessness and the resultant self-denigration to the hated other. ${ }^{45}$ The recruited received a set of group values and a vocabulary to delineate them. In their inimitable lexicon, collective dependence translated as "comradery" and self-destruction for the revolution's sake as "symbolic immortality." 46

In practice, the sought-after refuge and self-abandonment among the extremists was tantamount to "the life of a hunted wolf," recapped a former member of a terrorist organization. A conspirator "must be prepared to perish" every second; for him, any serious attachment is "a genuine misfortune ... Apart from five to ten like-minded persons, one must deceive from morning to night literally everyone; one must hide from everyone, suspect in everyone an enemy." 47 A writer close to the extremist milieu dispelled the myth of a radical brotherhood, noting that often the revolutionaries "hated their opponents as much as they hated one another." 48 On occasion, scions of the "revolutionary family" recognized that, in truth, it was severely dysfunctional. ${ }^{49}$ It maintained cohesiveness, despite the inner discord among the unsettled, antagonistic personalities, largely due to their anxiety-provoking isolation from the outside world.

In the disharmony of the self-imposed radical exile, desocialization from the rest of humanity was prevalent. It simply "did not exist for me," as terrorist Mariia Shkol'nik put it in a nutshell. ${ }^{50}$ Her SR colleague Dora Brilliant has severed all connections not only with society at large, but also with her party comrades who were not terrorists: "her entire world was confined to the Combat Organization." Its other member, Aleksei Pokotilov, confirmed: "For me, the entire revolution is terror." 51

To find oneself outside the underground setting entailed facing a fragmented self; for many, it was worse than death. A radical who had developed a heart condition disqualifying him as "either a terrorist, or a rebel," killed himself, "unable to live without the cause." 52 Peaceful life abroad was meaningless and drove some ex-terrorists to suicidethe fate of the 24-year old SR Rashel' Lur'e. ${ }^{53}$

For numerous terrorists, apprehension of the allegedly hostile outside world turned into a catalyst for self-destructive acts. Some killed themselves rather than risk falling into the hands of the police and living through the process of an investigation, trial, and imprisonment. Suicide episodes during arrests were numerous: one anarchist exploded a hand grenade under his own feet; another placed dynamite in his mouth and blew himself up. ${ }^{54}$ SR Evgenii Kudriavtsev shot himself immediately after he had killed the St. Petersburg governor V. F. von der Launits. "What if we fail?" had pondered SR Ivan Kaliaev before, bomb in hand, as he walked out to kill the governor-general of Moscow the Grand Duke Sergei Aleksandrovich; "then I think we should do it the Japanese way ... the hara-kiri." "Against all my efforts, I did not die" in the explosion on 4 February 1905, he lamented in a follow-up letter to comrades. "Happy to hear," he replied to his judges, who had sentenced him to hanging. ${ }^{55}$

Until 1907, when conditions in most penal institutions noticeably toughened, political prisoners rarely complained of mistreatment; life behind the bars was "merrier than any wedding," some reminisced. ${ }^{56}$ Nonetheless, for numerous political inmates, imprisonment 
proved intolerable; scores of them suffered breakdowns, ended their lives in mental asylums, and killed themselves. Many memoirs underscore their urgency to die: a female extremist managed to strangle herself with her long braid; another set herself on fire in her cell. The two suicide attempts that Bolshevik terrorist Simon Ter-Petrosian (Kamo) made in prison were consistent with his recurrent compulsion to end his life at times of forced passivity. ${ }^{57}$ Occasionally the radicals succeeded in giving the long-awaited death political significance: SR Sergei Sazonov, who "wished to avoid captivity by suicide," failed to kill himself right after he had assassinated interior minister Viacheslav von Plehve but subsequently used another chance to do away with his life - in protest against repressive conditions in his hard-labor prison. ${ }^{58}$

Group leaders classified peaceful life as apostasy, and most would-be-dissenters conformed. One young man confessed in his suicide note that his inability to be a true fighter, his repulsion for revolutionary activity, and an "unconquerable wish for an ordinary life" had tormented him. We have "sentenced this completely innocent young human soul to death by our moral coercion" and "merciless demand for revolutionary service," lamented his former comrade. ${ }^{59}$ The belief that "sacred is only the determination to sacrifice oneself - that's the tragedy of the Russian intelligentsia," confirmed another ex-socialist: "All that ends with death is sanctified; all is permitted to a person who is ready to die." 60

The deleterious underground climate undermined the radical's life even more than the distressing routine from which he had sought to flee. A beautiful woman in her twenties, Brilliant "spent her days in silence," in "concentrated suffering," in inner torment that filled "her saddened soul." Gloomy, "she did not know the joy of life." "61 Dejected, the radicals welcomed the culturally commended "nonbeing" and rationalized deathwishing with the rhetoric of revolutionary martyrdom - an approach that has recurred with the Islamicists. "I didn't tell him [the suicide bomber] to kill himself. He wanted to and I helped him," explained a Palestinian recruiter a century later, ${ }^{62}$ when terrorism became a preferred form of politicized self-destruction. "i do not have a friend, i have no one to speak too, no one to consult, no one to support me and i feel depressed and lonely. i do not know what to do." Internet user "Farouk 1986" was no other than a 23year old Umar Farouk Abdulmutallab, whose desperate postings leave no doubt why his al-Qaeda recruiters considered him be a great candidate for the 2009 Christmas-day plane explosion attempt. ${ }^{63}$

On 16 June 2002, Jihadist recruiters dispatched a young man infected with the HIV virus to detonate himself among the Israeli police officers, ${ }^{64}$ a century earlier, an SR leader Mikhail Gots sought to persuade a student suffering from an advanced stage of tuberculosis to die the beautiful death of a political assassin. ${ }^{65}$ The head-hunters did not (and do not) consider these conscription methods cynical, assuming that suicide, shrouded with the rhetoric of political altruism, was a choice superior to "commonplace dying." A recruiter might have even seen himself as giving a helping hand when, admittedly, he specifically sought out for suicide operations "guys who were desperate and sad."66

The Russian terrorists repeatedly explained their longing to destroy themselves as a need to atone for the spilt blood of their enemies. "I must die"; bomb-maker in the Combat Organization, Brilliant begged her superiors to appoint her as a bomb-thrower. "One life appeared as payment for another," in their eyes, and both were sacrifices for some future ideals, wrote Albert Camus in the Rebel. ${ }^{67}$ Yet, the fact that numerous radicals had contemplated suicide and had made one or more attempts on their own lives 
prior to their involvement in terrorism undermined any rationalizations of their search for death. "So many times in my youth I thought of killing myself," wrote Boris Vnorovskii to his parents before his attack against governor-general of Moscow Fedor Dubasov, but "I continued living ... for you." 68 By joining the Combat Organization, Vnorovskii gave himself a mandate to die.

While jeopardizing their lives in terrorist operations, the radicals sought indirect ways of doing away with themselves - slowly, usually by undermining their health and mental agility. SR Boris Savinkov first sought to "alleviate his tension by increasing consumption of alcohol and opiates"; then, with opium no longer providing sufficient sedation, by injecting morphine. ${ }^{69}$ Pokotolov, Savinkov's subordinate in the Combat Organization, was a heavy drinker. A tense and jittery type, nervous, pale, with "feverishly dilated pupils," Pokotilov pursued death constantly, insisting on "the honor" of blowing to pieces designated targets and seeking every chance to provoke a violent encounter with the police. ${ }^{70}$

When Russian anarchists blew themselves up with dynamite inside police headquarters, they chose "the means of political assassination in order to end their lives," explained a contemporary psychologist. " If presented with an opportunity for a terrorism venture, many young men and women "would enthusiastically go for a heroic deed, rather than end their lives by suicide," acknowledged revolutionaries in the early 1900s. ${ }^{72}$ A "terrorist act was often a suicide mission." ${ }^{73}$ SR Lidiia Sture liked to repeat that if she had not been able to enter a terrorist group, she would have killed herself. ${ }^{74}$

\section{Death-seeking employed for a political cause}

Cesare Lombroso considered Russian radicals to be "odd homicides," who lacked the courage to kill themselves directly. ${ }^{75}$ On the contrary, contends criminologist Moshe Hazani, these "suicides indirects" did not fear but courted death- "only on condition that it be in an appropriate value-driven context," $" 76$ a tribute to a lofty cause. Other experts confirm that suicide terrorists across the world have not only been prepared to die but in fact desire death. ${ }^{77}$ A Russian extremist shared his motives for joining a terrorist group: "I am terribly fed up with my life."78 Pilot Ziad Jarrah had revealed prior to the 9/11 attack that he was "dissatisfied with his life" and "didn't want to leave Earth in a natural way.,"79

A man of his epoch, 24-year-old anarchist-individualist Dmitrii Bogrov scorned both the conventional and the revolutionary ethics; to support his passion for gambling, in 1907 he offered his services as a secret informer to the Kiev secret police. In 1911, convinced of his police ties, his anarchist comrades made Bogrov a proposition common among the extremists: he would either commit a terrorist act against one of his official superiors or face the shameful death of a traitor. His only other alternative was to try to escape, but the "depressed, bored and lonely" Bogrov, the "internally dismal and autumnal" cynic, who perceived life as "nothing but an endless number of meat cutlets," chose a subliminal death of a "revolutionary martyr." 80 On 1 September 1911, he fired two lethal shots at Prime Minister Petr Stolypin in a crowded theater. Shooting from very close range, the assassin left himself no chance of escape. Prior to his act of homicidal suicide, Bogrov had asked a prominent SR representative if the party would take responsivity for the attack: "I want to make sure that after my death there will be people ... who will interpret my behavior correctly, explaining it by social and not personal motives." ${ }^{81}$ No other terrorist better elucidated the quest for symbolic immortality. 
Nearly a third of the SR Combat Organization, women came to comprise approximately one quarter of all Russian terrorists in the $1900 \mathrm{~s}^{82}$ and were particularly susceptible to suicidal behavior. Their idol was Sof'ia Perovskaia, a leader of the terrorist organization "People's Will" and the first woman in Russia to be executed for a political crime in 1881. The Russian government's rigid policies, limiting higher education and career opportunities for females, drove women into the revolutionary milieu. The leaders gave them ample opportunity to assert themselves in the male-dominated radical camp and to validate their lives by taking part in dangerous operations. This they did with utter fanaticism and willingness for self-sacrifice. Without those "wonderful ... few years, my life would have had no real meaning," affirmed a former terrorist, speaking also for many of her comrades. ${ }^{83}$

In an environment conducive to hostility, an acrid brew of personal motives drove many to self-directed violence camouflaged as sociopolitical goals. "My soul was restless and in pain," complained SR Ekaterina Breshko-Breshkovskaia: "the monotony of my purposeless life became unbearable." ${ }^{24}$ Her party comrade Lidiia Ezerskaia despised herself for triviality, for the lack of a special talent, and agonized that at the age of 38 "time was slipping away." Her attempted murder of Mogilev governor Klingenberg was a desperate last chance to give purpose to an otherwise empty life. ${ }^{85}$

For Fruma Frumkina, terrorist motives likewise "stemmed from a deep feeling of inadequacy and a desire to confirm her own importance as an individual." Here a strong impetus for self-devaluation was that Frumkina was exceptionally unattractive, to the point of being physically deformed. "I have longed, and still long" only for terror; "I have thought, and still think only of that ... I cannot control myself," she raged and, according to her comrades, flung herself against literally every jailer at her hard labor prison. ${ }^{86}$ She provoked reciprocal brutality, as if wishing to be killed. "I want them to kill me; I cannot imagine the grief ... if they do not kill me ... My death now will have a tremendous revolutionary significance," wrote 19-year-old Mariia Spiridonova, who in 1906 had assassinated a high-ranking official in Tambov, from prison. ${ }^{87}$ Hysterical and impulsive, clinging to the image of a revolutionary martyr, she was emotionally undeveloped to the point that even a decade later, already a leader of the Left SR Party, she would have "the mental attitude of a high-school girl." 88 Her act was suicidal; she could not hope - and did not try - to escape from the site of the terrorist attack.

For his sacrifice for Allah, a male shahid is promised seventy-two virgins in heaven, where he would also secure a place for his seventy relatives. ${ }^{89}$ And what would be the reward if the martyr happened to be a married mother of four Kahira Sa'adi from AlRam, who on 21 March 2002 facilitated a suicide attack in the center of Jerusalem? ${ }^{90}$ Or a 57-year old grandmother Fatma Najar, who on 23 November 2006 set a new age record for suicide bombers. ${ }^{91}$ The jihadist media market the image of female suicide terrorists as "independent and determined women with strongly held opinions" and "noble personal qualities," who "take their fate into their hands ... like a modern version of Joan of Arc." The image "fades away," affirm those who have interviewed perpetrators of abortive attacks. ${ }^{92}$

Their incentives vary from the desire to avenge a personal loss to ideology-inspired outrage at perceived injustice, ${ }^{93}$ but most often recruiters select their cadres from among the "damaged goods": seduced, raped, unmarried girls at a relatively advanced age, or divorced women. They may be barren, like the Chechen terrorists who brought down two Russian jets in August 2004 or like Wafa Idris, the first Palestinian female suicide 
bomber, a 27-year old divorcee, who had no children after nine years of marriage; "she was ready for any kind of escape. In traditional Palestinian society, a divorced, barren woman is a burden on the family and has no chance of ever remarrying." A male variant may be a homosexual, threatened that unless he enlists for a fatal terrorist act, he will become a disgrace to his family when "his sexual deviance will be publicized." "94 Among the stigmatized, a promising candidate for a suicide attack may also be a woman discovered to have had children out of wedlock.

Traditional Muslim communities are "shame societies," and should an unmarried girl become pregnant, a wife commit adultery, or a daughter refuse an arranged marriage, the only way for a "sharmouta" (whore) to cleanse the public dishonor from herself and her family is to become a martyr for Allah which cancels all sins. ${ }^{95}$ Martyrdom is also the only escape for families suspected of enemy collaboration. The father of a brilliant high-school student Ayat al-Akhras had been accused of treason because of his work with an Israeli construction firm; he faced a lynching. "There was only one chance to save her family from disgrace" and violence - to become a martyr, Ayat's friend explained, after al-Akhras had been dispatched to explode in a Jerusalem supermarket in March 2002. ${ }^{96}$ "It was always my wish to turn my body into deadly shrapnel ... and to knock on Heaven's doors with the skulls of Zionists," 97 declared Reem Riashi from Gaza, a mother of two toddlers, who killed herself and four Israelis on 14 January 2004. Poetic as the terrorist's farewell statement might have been, if truth be told, Riashi volunteered to be a martyr only after she had been discovered to have an illicit love affair. ${ }^{98}$

The primacy of terrorists' desire to destroy themselves overshadows any post factum ideological justification of suicide. Death-seeking Palestinian student Dareen Abu Aisha threatened that unless she were assigned a martyr's task, "she would buy a knife, go kill [Israeli] soldiers at a roadblock, and die that way." "99 Black sheep of her family, Thouria Khamour from the Jenin areas was arrested a day before her planned suicide attack in Jerusalem. "I was 25 years old, unmarried," she revealed, outlining the hopelessness behind the decision to do away with her life. "I already tried to harm myself twice, but they stopped me." 100 Life was worthless anyway because "my father wouldn't let me marry the boy I wanted," complained another would-be martyr; "so, I ... volunteered, to get back at my father." 101

An emblematic female candidate for self-sacrifice is a denigrated social outcast, dejected, and "dead-in-life." 102 In Chechnya, female rape victims who customarily killed themselves not to bring shame upon their families have been "funneled into the Black Widows"103 to explode among the Russians. The dispatchers offer "the defective," the "not whole," dehumanized women, a chance to gain personal meaning by becoming "a flag bearer" for any ideological cause. They encourage, push, threaten, and coerce their confused and miserable victim to carry out the violent act as the only opportunity to uphold her worthless existence. "They used me," admitted a failed suicide terrorist. ${ }^{104}$

Fascination with terrorism as veiled suicide was particularly widespread among the young - a phenomenon consistent with research findings about the intensified adolescents' delinquency during periods of the "historical dislocation."105 In Russia, where party leaders "created a cult of dynamite and the revolver, and crowned the terrorist with a halo ... murder and the scaffold acquired a magnetic charm and attraction for the youth." ${ }^{106}$ Under the influence of the extremist ethos, familiar teenage rebellion against parental-and overall adult-authority often took radical forms. Ample 
opportunities for heroic self-sacrifice provided the youngsters with an outlet for their mundane, age-related drives.

Recklessness and adolescent adventurism, nourished by boundless energy, drove them to take part in life-threatening enterprises alongside the adult extremists. Like them, the minors "talked with enthusiasm about dying." Many showed strong self-destructive tendencies, their death-wishing rationalized as a "heroic deed." Some adolescents were quick to adopt the adult vernacular to justify suicidal behavior; for example, asserting that the true beauty of life was in "death for death's sake." 107

\section{Constructed death culture}

"For the Palestinian people death has become an industry,"108 boast engineers of a sociocultural climate of glorified brutality, or "death culture." In fact, it is rife across much of the Middle East, from Iran to Gaza, where the Jihadists seeks to concoct and maintain a giant enterprise of indoctrination in destruction and self-destruction. Its primary victims are children brainwashed for homicidal suicide. Posters in kindergartens scream: "The children are holy martyrs of tomorrow." 109 Books for the young are filled with similar messages. ${ }^{110}$ "Blessings to those who wage [fihad] with their body," children hear in sermons ${ }^{111}$ and learn that when a hero "turns into torn organs that spread all over," it is not "a loss" because those who die as martyrs feel no pain and receive rewards in the afterlife. ${ }^{112}$

"We don't encourage our children to hate the Jews," explains an imprisoned Palestinian terrorist; "We just tell them ... that the Jews killed their families, and they reach the conclusion to hate the Jews on their own." "113 Jews also killed their "dear friends," and kindergarten teachers smear children's palms with red paint to represent the spilt blood. ${ }^{114}$ Older children are taught that Israelis deliberately poison air and water, distribute food that contains cancer-causing ingredients, and sell sports shoes that "cause the wearer to become paralyzed" and perfumes that lead to drug addiction. They disseminate AIDS, "sexually stimulating drops and chewing gum" to "weaken and destroy" Palestinian youth. Israelis are said to perform Nazi-like experiments on Arab prisoners. Reenacted for the official PA TV are episodes of rape by Israeli soldiers, ${ }^{115}$ who are also accused of kidnaping Palestinian children for body parts. ${ }^{116}$

Instruction in jihad is a segment of a regular military summer camp curriculum, in which professional combatants coach boys and girls and indoctrinate them in hatred for Israel and the West. ${ }^{117}$ Official eulogies, television broadcasts, and naming football tournaments after suicide terrorists, monument inaugurations and school ceremonies, writing essays and poems in class, as well as religious sermons, have the common denominator of depicting a suicide mission as an act of ultimate virtue. The Islamic University in Gaza has held a competition for the best martyr's farewell testament. ${ }^{118}$

"What is your most lofty aspiration?" six-year olds in Hamas attire are asked during a kindergarten graduation ceremony; they are trained to scream in unison: "Death for the sake of Allah!" 119 In the environment where 70 percent of the Palestinians approve of suicide bombings, ${ }^{120}$ between 72 and 80 percent of their children yearn to die as martyrs. ${ }^{121}$ "We don't want this world," affirms an 11-year old; "Every Palestinian child ... says, O Lord, I would like to become a shahid."122

In 1906, 16-year old Leibish Rapoport, angry at his mother, ran away from home and contemplated suicide but then swapped it for a nicer idea of a "political act," 
claiming responsibility for the recent assassination of Ekaterinoslav governor. Against his hope to be executed immediately, Leibish spent months under investigation and psychiatric observation. He was sentenced to twelve years in prison and served three - thanks to his mother's tireless efforts to prove her son's innocence. ${ }^{123}$

In more recent decades, so many times the parents have intervened too late to save their children. A 13-year-old Palestinian girl was killed attempting "to stab a civilian security guard at the entrance to the settlement of Anathoth," Israel, ran a press statement on 23 January 2016. According to the investigation, Raqiya Abu Eid had left home in the nearby village after a fight with her family; she carried a knife in her bag. Her father went after her but arrived at the scene shortly after her bid of homicidal suicide. $^{124}$

\section{Search for immortality}

On 7 February 1908, the St. Petersburg police surrounded SR Vsevolod Lebedintsev on his way to kill Minister of Justice I. G. Shcheglovitov. "Be careful. I am wrapped around with dynamite. If I explode, the entire street will be destroyed," he shouted before the officers took him and his accomplices into custody. Seven terrorists were sentenced to death. "How these people died," recalled a police official; "no sighs, no remorse, no pleas, no signs of weakness. ... a smile on their faces." 125 According to another witness, SR Zinaida Konopliannikova "went to her execution as one would go to a holiday festivity." 126 For her part, Brilliant wept upon hearing that the SRs would discontinue terrorist operations; the decision of the party leadership deprived her of a much soughtafter meaningful demise.

The elation of a "trance-like state," a "kind of serene joy, often coupled with an other-worldly smile" is "sometimes visible on the faces of suicidal homicides prior to their final acts." 127 The depleted "dead men on leave," ${ }^{128}$ terrorists can barely suffer their existence. They seek death as a reprieve.

Paradoxically, they also aspire to overcome death by sacrificing their lives. The struggle "is never just about territory, sovereignty, geography, or democracy," writes Louis René Beres, in reference to Jihadists. In fact, "true believers" of various persuasions seek "something much more compelling and personal," hiding behind lofty slogans - namely "life everlasting.","129

And here, aspects of the terrorists' death culture spill over into other political movements, at least in the desire to transcend mortality. Hazani reflects on the universal "semi-religious quality" of the radicals' desire to regulate the natural laws - a tradition leading back to the French Revolution. Its visionaries sought to control infinity by defining "September 22, 1792 - the start of the first year - as the zero point of time." Romme's "décréter l'éternitê" aspired "to arrest the temporal flux - i.e. to conquer death."130

Radical socialism in Russia revealed its messianic nature after the 1917 Bolshevik takeover. For all their atheism, the Communists' deeper goal that lay in the realm of secular metaphysics was discernible: to find the ultimate answer to a pivotal quandary of being - its transience and finality, to overcome the inevitability of demise. Nathan Leites considers the extremist mindset in the context of a distinctively "Russian horror of death against which Bolshevism reacts."131 Bolshevik goals for socialist paradise was indeed a rejoinder to mortality. Death was to be overpowered via a brilliant paradox, entailing the elimination of the individual - the source of the predicament. 
"Alone - free - the human being is always defeated. It must be so, because every human being is doomed to die, which is the greatest of all failures." Yet, George Orwell outlines the totalitarian alternative in 1984, if the man "can make complete, utter submission ... if he can merge himself in the Party so that he is the Party," then "the death of the individual is not death." As long as the individual is fully assimilated into the collective, "he is indestructible and immortal" because he "has no purpose, worth and destiny apart from his collective body," to which he has relinquished his life. ${ }^{132}$ The issue of mortality would simply be extraneous as one's identity ceased to exist, his corporeal "I" fused with "a common destiny," and dissolved in the eternal "group mind.",133 The Bolsheviks sought to expand infinitely the concept of "a group," to encompass millions, transforming each person into a selfless cell in a gigantic and everlasting state organism. Collective eternity was to be sustained at a dire price for the individual, "a particle" of the Soviet power. ${ }^{134}$

Prominent Bolshevik intellectual Anatolii Lunacharskii considered Marxism to be a form of religion, whose adherents could "overcome death and coffin" because their values were connected "with that which is higher than the body and can pass from generation to generation without perishing." 135 For Bolshevik leader Aleksandr Bogdanov, "collectivism was a religion, and even promised a triumph over death." "Almost mystical" was Bogdanov's reverence for the Communist commune, in which "workers will lose their sense of an individual ' $\mathrm{I}$ ' in favor of an all-encompassing 'we' that will some day triumph over nature and achieve collective immortality." "136 His party colleague Martin Liadov envisaged that in the future communist society each person "will feel pain ... if his personal interests in any way contradicted the interests of the collective." 137

The Bolshevik expert on terrorism Leonid Krasin was fixated on immortality. In 1920 he predicted that the time "will come when science will become all-powerful, that it will be able to recreate a deceased organism" and even "to resurrect great historical figures." Lenin's death in January 1924 presented "an obvious choice"; in February, Krasin insisted that the significance of Lenin's grave would surpass that of Mecca and Jerusalem and urged the construction of a mausoleum. ${ }^{138}$ There the incarnation of Bolshevism would be preserved-presumably, until his resurrection. For his part, in an ostensibly half-conscious statement, Lenin expressed a conviction that "those who really merit the name of a political personality do not die in politics when they die physically.", 139

Illustrative indeed was Trotsky's belief in the impending victory over physical decay in the soon-to-be-built social paradise. He concluded his Literature and Revolution (1924) with "a rhapsodic vision of the new man," who

will become immeasurably stronger, wiser and subtler; his body will become more harmonized, his movements more rhythmic, his voice more musical. ... The average human type will rise to the heights of an Aristotle, a Goethe, or a Marx. And above this ridge new peaks will rise. ${ }^{140}$

There could be no tolerance for anyone slowing down the triumphant marching towards the paradise. To get there was to eradicate "the average human type," who held on to his petty, if obstinate "I" and refused to merge it with the everlasting "we."

The "salvationist religion"141 of the Communists featured everlasting collective life on earth, and there were and are analogues elsewhere. The Nazi version of repudiated death presupposed a form of mystic fusion with the racially pure "body of Germany" 
and secular salvation via self-negation within a sacred community - the German Volk. Under the green banner of radical Islam, zealots aspiring to eternity seek death again. "As much as you love life, the Muslim loves death and martyrdom," Jihadists broadcast to the West. ${ }^{142}$ "The Americans are fighting so they can live ... but we are fighting so we can die in the cause of God." 143 "We desire Death, as you desire Life." 144

Death-wishing, justified ideologically and realized via extremist acts, is not limited to a specific cultural or national origin. A broader set of modern contexts, repeated now in several regions and over a considerable stretch of time, underlies choices made by certain individuals to commit to death of self and others. The distinctive feature of "the Internationale of thanatophiles" is the love of mortality and ruin, with death-seeking often taking the form of martyrdom. ${ }^{145}$

\section{Notes}

1 Robert J. Lifton, Boundaries: Psychological Man in Revolution (New York: Random House, 1970), 43.

2 Ariel Merari, Ilan Diamant, Arie Bibi, Yoav Broshi, and Giora Zakin, "Personality Characteristics of 'Self Martyrs'/'Suicide Bombers' and Organizers of Suicide Attacks," Terrorism and Political Violence 22 (2010): 96.

3 Carlton J. H. Hayes, A Generation of Materialism (New York: Harper \& Brothers, 1941), 254.

4 Fukuyama and Samin, "Can Any Good Come of Radical Islam?," www.commentarymaga zine.com/viewarticle.cfm/can-any-good-come-of-radical-islam

5 Sayyid (Syed) Qutb, a leading ideologue of Islamism in the 20th century, cited in Michael J. Mazarr, Unmodern Men in the Modern World: Radical Islam, Terrorism, and the War on Modernity (New York: Cambridge University Press, 2007), 44.

6 Randy Borum, et al., "Psychology of Terrorism," University of Southern Florida, October 2003, www.ncjrs.gov/pdffiles1/nij/grants/208551.pdf

7 Mazarr, Unmodern Men in the Modern World, 9, 65, 17.

8 Counterterrorist expert Bruce Hoffman quoted in Bobby Ghosh, "Most Domestic 'Jihadists' Are Educated, Well-Off," Time (14 December 2009), www.time.com/time/nation/article/ 0,8599,1947703,00.html

9 Oliver Roy, "Why Do They Hate Us? Not Because of Iraq," New York Times (22 July 2005), www.nytimes.com/2005/07/22/opinion/22roy.html?_r=1

10 Mazarr, Unmodern Men in the Modern World, 172, 9, 7.

11 Andrei Belyi, Na rubezhe doukh stoletii (Chicago, IL: Russian Language Specialties, 1966), 5.

12 Laura Engelstein, The Keys to Happiness: Sex and the Search for Modernity in Fin-de-Siècle Russia (Ithaca, NY: Cornell University Press, 1992), 216, 1; Bernice Glatzer Rosenthal, ed., The Occult in Russian and Soviet Culture (Ithaca, NY: Cornell University Press, 1997), 1.

13 Iurii Karabchievskii, Voskresenie Maiakovskogo (“Strana i mir:” Munich, 1985), 86.

14 Andrei Belyi, Nachalo veka (Gosudarstvennoe izdatel'stvo khudozhestvennoi literatury: MoscowLeningrad, 1933), 3; Belyi, Na rubezhe doukh stoletii, 3.

15 Aleksei Tolstoi, Ordeal (Moscow: Progress Publishers, 1953), 29.

16 Belyi, Nachalo veka, 3.

17 Kirk Rodby, The Dark Heart of Utopia: Sexuality, Ideology, and the Totalitarian Movement (New York and Bloomington, IN: iUniverse, Inc, 2009), 216; Aleksandr Etkin, Khlyst (Sekty, literature i revoliutsiia) (Moscow: Novoe literaturnoe obozrenie, 1998), 8-9.

18 Tolstoi, Ordeal, 30.

19 Alexander Blok, "The People and the Intelligentsia," in Marc Raeff, ed., Russian Intellectual History: An Anthology (NJ: Rutgers University Press, 1978), 362.

20 Belyi, Na rubezhe doukh stoletii, 4.

21 Gited in Nathan Leites, A Study of Bolshevism (Glencoe, IL: The Free Press Publishers, 1953), 345.

22 Dmitrii Merezhkovskii, "Revoliutsiia i religiia," Russkaia mysl', No. 2-3 (1907).

23 Cited in Nestroev, Iz dnevnika maksimalista (Paris, 1910), 218. 
24 "Materialy o provokatorakh," Arkhiv Partii Sotsialistov-Revoliutsionerov, International Institute of Social History, Amsterdam [cited hereafter as PSR, followed by "box" and "folder" numbers], 5-518.

25 Report of 28 June (11 July) 1913, Arkhiv Zagranichnoi Agentury Departamental Politsii (Okhrana Collection), Hoover Institution Archives, Stanford University, Stanford, CA [cited hereafter as Okhrana, followed by "box" and "folder" numbers], XXII-1B.

26 V. M. Chernov, Pered burei (New York: Izdatel'stvo imeni Chekhova, 1953), 169.

27 Cited in Garry Saul Morson, "Dagger and Swagger," The Weekly Standard (October 2018), www.weeklystandard.com/gary-saul-morson/the-history-of-russian-terrorism-dagger-andswagger

28 Stern, "The Protean Enemy," www.cfr.org/pub6146/jessica_stern/the_protean_enemy.php

29 Mazarr, Unmodern Men in the Modern World, $27,12$.

30 Moshe Hazani, "Sacrificial Immortality: Towards a Theory of Suicidal Terrorism and Related Phenomena," in L. Bryce Boyer, Alan Dundes, Stephen M. Sonnenberg, eds., The Psychoanalytic Study of Society, vol. 18 (London: Routledge, 1993), 425.

31 Eugene Kayden, "Leonid Andreyev: 1871-1919," http://worldlibrary.net/eBooks/Wordthe que/en/aaabnxe.txt

32 Cited in Viacheslav Venozhinskii, Smertnaia kazn' i terror (St. Petersburg, 1908), 28.

33 A. S. Izgoev, "Ob intelligentnoi molodezhi," Vekhi, 1909, www.vehi.net/vehi/izgoev.html; Valter Laqueur, Terrorism (Boston and Toronto, 1977), 130, 127.

34 Kayden, "Leonid Andreyev," http://worldlibrary.net/eBooks/Wordtheque/en/aaabnxe.txt

35 Cited in Susan K. Morrissey, Heralds of Revolution: Russian Students and the Mythologies of Radicalism (New York: Oxford University Press, 1998), 185.

36 V. B. Bezgin and D. P. Zherebchikov, "Suitsid v gorode i derevne Rossiiskoi provintsii kontsa XIX — nachala XX vekov," Vestnik TGTU 18 (4) (2012): 1122, http://vestnik.tstu.ru/rus/ t_18/pdf/18_4_051.pdf

37 Roberta Ann Kaplan, “A Total Negation of Russia': Russian Intellectual Perception of Suicide, 1900-1914" (unpublished paper, Harvard University, 1988), 32.

38 Susan K. Morrissey, Suicide and Body Politic in Imperial Russia (New York: Cambridge University Press, 2006), 315.

39 Bezgin and Zherebchikov, "Suitsid v gorode i derevne," 1122. http://vestnik.tstu.ru/rus/ t_18/pdf/18_4_051.pdf

40 "Land and Sea in the Traditional Russian World View," http://enspire.syr.edu/nevaproject/ river\&city/land\&sea.html

41 George Reavey, "Foreword," in Andrey Biely, ed., St. Petersburg (New York: Grove Press Inc., 1959), vii.

42 Morrissey, Suicide and Body Politic, 316. Fifteen hundred Peterburgers killed themselves in 1908 alone (W. Bruce Lincoln, Sunlight at Midnight: St. Petersburg and the Rise of Modern Russia (New York: Basic Books, 2000), 208).

43 Morrissey, Heralds of Revolution, 197.

44 Laqueur, Terrorism, 130.

45 Vamik Volkan, The Need to Have Enemies and Allies: From Clinical Practice to International Relationships (Northvale, NJ and London: Jason Aronson, 1988), 33.

46 The term "symbolic immortality," referring to those aspects of one's life that live on after his death, is discussed in Robert J. Lifton's Broken Connection: On Death and the Continuity of Life (1979).

47 Lev Tikhomirov cited in Richard Pipes, The Degaev Affair. Terror and Treason in Tsarist Russia (New Haven: Yale University Press, 2003), 17-18.

48 Mark Aldanov, "Azef," Poslednie novosti (1924), Boris Nicolaevsky Collection, Hoover Institution Archives, Stanford University, Stanford, CA, box 205, folder 19.

49 An atmosphere of acrimony and disparagement among the terrorists, prototypes of the SR Combat Organization, is depicted in Boris Savinkov (V. Ropshin), Pale Horse: A Novel of Revolutionary Russia (Pittsburgh: University of Pittsburgh Press, 2019).

50 Amy Knight, "Female Terrorists in the Russian Socialist Revolutionary Party," Russian Review 38 (2) (April 1979): 152. 
51 Boris Savinkov, Vospominanïa terrorista (Khar'kov, 1926), 40; Anna Geifman, Thou Shalt Kill: Revolutionary Terrorism in Russia, 1894-1917 (Princeton, NJ: Princeton University Press, 1993), 53.

52 Undated letter from P. Polivanov to his comrades, PSR 1-63.

53 Knight, "Female Terrorists," 149-150; Savinkov, Vospominaniia terrorista, 186.

54 Geifman, Thou Shalt Kill, 308.

55 Cited in Savinkov, Vospominanïa terrorista, 49, 93, 101, 111.

56 Cited in Ivan Bunin, Okaiannye dni (Leningrad, 1991): 65.

57 Medvedeva Ter-Petrosian, "Tovarishch Kamo," Poletarskaia revoliutsiia 8-9 (31-32) (1924): 141.

58 Savinkov, Vospominaniia terrorista, 61.

59 Semen Frank, Sochinenia (Moscow: "Pravda:", 1990), 153.

60 Izgoev, "Ob intelligentnoi molodezhi," www.vehi.net/vehi/izgoev.html

61 Savinkov, Vospominaniza terrorista, 40, 117.

62 Cited in Anat Berko, The Path to Paradise: The Inner World of Suicide Bombers and Their Dispatchers (Westport, CT: Praeger Security International, 2007), 1.

63 Douglas C. Lovelace, Jr., Terrorism. Commentary on Security Documents, vol. 148: Lone Wolf Terrorists (Oxford University Press, 2018), 130.

64 Ami Pedahzur, Suicide Terrorism (Cambridge, UK: Polity Press, 2005), 141.

65 Report of 4 [17] December 1904, Okhrana XVIIh-2G.

66 Cited in Berko, The Path to Paradise, 1.

67 Cited in O. V. Budnitskii, "'Krov' po sovesti': terrorizm v Rossii (vtoraia polovina XIXnachalov XX v.," Otechestvennaia istoriia 6 (1994): 205.

68 Cited in Savinkov, Vospominaniia terrorista, 218.

69 Gited in Geifman, Thou Shalt Kill, 164.

70 V. O. Levitskii, "A. D. Pokotilov," Katogra i ssylka 3 (1922): 159, 171; Savinkov, Vospominaniia terrorista, 26-27; Richard B. Spence, Boris Savinkov: Renegade on the Left (New York, 1991), 47; Geifman, Thou Shalt Kill, 53.

71 Kaplan, "A Total Negation of Russia," 42.

72 Report of 22 December 1901, Okhrana, XVIIIb[1]-1, Outgoing dispatches [1901], doc. 11.

73 Knight, "Female Terrorists," 150.

74 I. Zhukovskii-Zhuk, "Pamiati Lidii Petrovny Sture," Katogra i ssylka 19 (1925): 253.

75 Cesare Lombroso, Les anarchistes (Paris: Flammarion, n.d.), 93-99.

76 Moshe Hazani, "Red Carpet, White Lilies: Love of Death in the Poetry of the Jewish Underground Leader Avraham Stern," The Psychoanalytic Review 89 (2002): 35.

77 Ariel Merari, "The Readiness to Kill and Die: Suicidal Terrorism in the Middle East," in Walter Reich, ed., Origins of Terrorism (Cambridge, England, 1990), 193.

78 Copy of 14 March 1907 letter from a revolutionary from St. Petersburg to Dmitriev in Geneva, Okhrana, XXIVi-1B.

79 Gited in Adam Lankford, "Précis of The Myth of Martyrdom: What Really Drives Suicide Bombers, Rampage Shooters, and Other Self-Destructive Killers," Behavioral and Brain Sciences (2014): 356, https://sci-hub.tw/10.1017/S0140525X13001581.

80 Cited in A. Serebrennikov, ed., Ubiistvo Stolypina. Svidetel'stva $i$ dokumenty (New York: Teleks, 1986), 79, 84, 105, 130, 139.

81 Ibid., 146-147.

82 Norman M. Naimark, "Terrorism and the Fall of Imperial Russia," published lecture, Boston University (April 14, 1986): 5; Laqueur, Terrorism, 121.

83 Cited in Geifman, Thou Shalt Kill, 316n15.

84 Daniel Field, "Breshkovskaia and Her Memoirs: Problems of Retrospection and Identity," unpublished manuscript, n.p., n.d.

85 Letter from Lidia Pavlovna Ezerskaia, PSR 1-2.

86 Knight, "Female Terrorists," 151, 153.

87 Undated letter from M. A. Spiridonova, PSR, 4-351.

88 Oliver Radkey cited in Knight, "Female Terrorists," 159.

89 For example, Sheikh Isma'il Aal Radhwan cited in "Blessings for whoever has saved a bullet in order to stick it in a Jew's head," 27 December 2003, Jihad Watch, www.jihadwatch.org/ archives/000501.php 
90 "The Role of Palestinian Women in Suicide Terrorism," 30 January 2003, Israel Ministry of Foreign Affairs, www.mfa.gov.il/MFA/MFAArchive/2000_2009/2003/1/The\%20Role\%20of $\% 20$ Palestinian $\% 20$ Women $\% 20$ in $\% 20$ Suicide $\% 20$ Terrorism

91 Yoram Schweitzer, "Palestinian Female Suicide Bombers: Virtuous Heroines or Damaged Goods?," 16, www.labat.co.il/articles/palestinian \%20female\%20suicide\%20bombers.pdf

92 Ibid., 39-40. See also Berko, The Path to Paradise, 5-7, 96.

93 Shaul Kimhi and Shemuel Even, "Who Are the Palestinian Suicide Bombers?" Terrorism and Political Violence 16 (2004): 827.

94 Ibid., 825-826; Berko, The Path to Paradise, 2; Mia Bloom, Dying to Kill: The Allure of Suicide Terror (New York: Columbia University Press, 2005), 163.

95 Denis MacEoin, "Suicide Bombing as Worship," Middle East Quarterly 16 (4) (Fall 2009), www. meforum.org/2478/suicide-bombing-as-worship

96 For examples and discussion, see Schweitzer, "Palestinian Female Suicide Bombers," 5, 29, 32,38 .

97 Rim Riashi (Reem Riyashi) cited in Itamar Marcus and Barbara Crook, "Kill a Jew-Go to Heaven. A Study of the Palestinian Authority's Promotion of Genocide," Palestinian Media Watch, http://palwatch.org/STORAGE/special\%20reports/Kill_Jew_go_to_heaven.pdf

98 MacEoin, "Suicide Bombing as Worship," http://www.meforum.org/2478/suicide-bombingas-worship

99 Schweitzer, "Palestinian Female Suicide Bombers," 28.

100 Ibid., 31-32.

101 Cited in Berko, The Path to Paradise, 1.

102 Term coined by Robert J. Lifton, cited in Anna Geifman, Death Orders: The Vanguard of Modern Terrorism in Revolutionary Russia (Santa Barbara, CA: Praeger Security International, 2010), 17.

103 Bloom, Dying to Kill, 163.

104 Schweitzer, "Palestinian Female Suicide Bombers?," 3, 3133.

105 Moshe Hazani, "The Breakdown of Meaning and Adolescent Problem Behavior," International Fournal of Adolescent Medical Health 15 (3) (2003): 207-218.

106 Cited in James Frank McDaniel, "Political Assassination and Mass Execution: Terrorism in Revolutionary Russia, 1878-1938” (Ph.D. dissertation, University of Michigan, 1976), 97.

107 Nestroev, Iz dneonika Maksimalista, 78.

108 Public speech of Member of the Palestinian Legislative Council member Fathi Hamad, AlAqsa TV, 29 February 2008, www.youtube.com/watch?v=RTu-AUE9ycs

109 Paul Berman, Terror and Liberalism (New York: Norton, 2003), 112.

110 For examples of textbooks and child readings filled with incitement for violence see www.mfa. gov.il/MFA/MFAArchive/2000_2009/2001/11/SPOTLIGHT-+Incitement-+Antisemitism +and+Hatred+of.htm

111 Sermon by Sheikh Ibrahim Mudeiris at Sheikh Ijlin Mosque, Gaza City, 15 August 2003 and 22 August 2003, Broadcasts on Palestinian Television, Palestinian Authority Friday Sermons, MEMRI, 16 September 2003, www.memri.org/bin/articles.cgi?Page=archives\&Area=s d\&ID=SP57403; Steven Stalinsky, "Palestinian Authority Sermons 2000-2003," MEMRI, 26 December 2003, www.memri.org/bin/articles.cgi?Area=sr\&ID=SR2403

11217 August 2001 sermon broadcast live on Palestinian TV from the Sheik "Ijlin mosque in Gaza," PA TV Friday Sermon Calls for Jihad and Martyrdom, MEMRI, 24 August 2001, www.memri.org $/$ bin/articles.cgi? Page $=$ subjects\&Area $=$ conflict\&ID $=$ SP26101

113 Cited in Berko, The Path to Paradise, 110-112.

114 "Finish Celebration of a Summer Camp," www.israel-wat.com/pics1_eng.htm\#a5

115 Robert S. Wistrich, Muslim Anti-Semitism. A Clear and Present Danger (The American Jewish Committee, 2002), 34, www.ajc.org/atf/cf/\%7B42D75369-D582-4380-8395-D25925B85EAF \%7D/WistrichAntisemitism.pdf; Palestinian news agency FPNP cited in Itamar Marcus and Barbara Crook, "Hamas police official: Israel smuggles 'sexual stimulants' to destroy Palestinian youth" (Bulletin July 17, 2009), PMW, http://col17w.coll17.mail.live.com/default.aspx? n=1309909505; and Al-Hayat Al-Jadida, 4 July 2008, "Canada and the Palestinian Authority" (Special Report, 25 March 2009), PMW, http://pmw.org.il/Bulletins_Apr2009. htm\#b050409 
116 MEMRI TV Project Special Report: Iranian TV Drama Series about Israeli Government Stealing Palestinian Children's Eyes, 22 December 2004, www.memri.org/bin/latestnews.cgi? $\mathrm{ID}=\mathrm{SD} 83304$

117 See, for example, "Gaza Summer Camps Train Teens for Jihad," The Times of Israel, 23 July 2016, www.timesofisrael.com/gaza-summer-camps-train-teens-for-jihad/

118 Christoph Reuter, My Life Is a Weapon: A Modern History of Suicide Bombing (Princeton, NJ: Princeton University Press, 2002), 89-90.

119 "Kindergarteners Choose Death for the Sake of Allah," WND, 6 January 2007, www.wnd. com/2007/06/41878/

120 Peter W. Singer, "The New Children of Terror," in James Forest, ed., The Making of a Terrorist (Prager, 2005), 115, www.brookings.edu/wp-content/uploads/2016/06/chap ter8_20051215.pdf

121 U.S. Senate Committee Hearing on Palestinian Education (2003), cited in Geifman, Death Orders, 148.

122 Cited in "Young Children Convinced of Death as a Shahid as Ideal," [PA TV June 2002], TV Archives - Video Library, www.pmw.org.il/tv\%20part1.html

123 G. Novopolin, "Delo ob ubiistve general-gubernatora V. P. Zheltonovskogo," Katogra i ssylka 31 (1927): 26-39.

124 Ynetnets, 23 January 2016, www.ynetnews.com/articles/0,7340,L-4756579,00.html

125 Cited in Geifman, Thou Shalt Kill, 64.

126 Cited in Knight, "Female Terrorists," 150.

127 Berko, The Path to Paradise, 10; Hazani, "Sacrificial Immortality," 436.

128 Eugen Leviné cited in Neal Ascherson, "A Terror Campaign of Love and Hate," The Observer, 28 September 2008, www.guardian.co.uk/world/2008/sep/28/germany.terrorism.

129 Louis René Beres, "Terrorism as Power over Death, BESA Center Perspectives Paper No. 1,053," 1 January 2019.

130 "Ce Romme est un métaphysicien obscur," remarked an opponent of this effort to rule over time (Edmond Biré, Fournal d'un bourgeois de Paris pendant la Terreur, vol. 4 (Perrin: Paris, 1794), 51, http://books.google.co.il/books?id=jsLSAAAAMAAJ\&pg=PA51\&dq=decreter $+1 \%$ E2 $\%$ $80 \% 99$ eternite $\% \mathrm{E} 2 \% 80 \% 9 \mathrm{D} \% 2 \mathrm{BRomme} \& \mathrm{hl}=\mathrm{en} \# \mathrm{v}=$ onepage $\& \mathrm{q}=\& \mathrm{f}=$ false

131 Leites, A Study of Bolshevism, 351.

132 George Orwell, 1984 (New York: Harcourt Brace Jovanovich, 1977), 267. Eric Hoffer, The True Believer: Thoughts on the Nature of Mass Movements (New York: Harper \& Row Publishers, 1951), 124, 61 .

133 Reich, ed., Origins of Terrorism, 31, 33.

134 Karabcheevskii, Voskresenie Maiakovskogo, 183-184.

135 A. V. Sarapul'tseva, "Religioznye iskaniia A. V. Lunacharskogo: bogostroitel'stvo," Sofia: Rukopisnyi zhurnal Obshchestva revnitelei russkoi filosofi 9 (2006): 32, http://lunacharsky.newgod.su/ issledovania/religioznye-iskania-a-v-lunacharskogo-bogostroitelstvo

136 Cited in Eliot Borenstein, Man without Women: Masculinity and Revolution in Russian Fiction, 1917-1929 (Durham, NC: Duke University Press: 2001), 29-30.

137 Cited in Rodby, The Dark Heart of Utopia, 203.

138 Bernice Glatzer Rosenthal, New Myth, New World: From Nietzsche to Stalinism (College Park: Pennsylvania State University Press, 2002), 188.

139 Cited in Leites, A Study of Bolshevism, 137.

140 Leon Trotsky, Literature and Revolution (New York: Russell \& Russell, 1957), 256.

141 Donald D. MacRae, "Bolshevik Ideology: The Intellectual and Emotional Factors in Communist Affiliation," Cambridge Fournal 5 (1951): 167.

142 Cited in Steven Stalinsky, "Dealing in Death," The National Review on Line, 24 May 2004, www.nationalreview.com/comment/stalinsky200405240846.asp

143 Cited in Steven Brockerman, "The Moral Thing to Do," Capitalism Magazine, 11 November 2001, www.capitalismmagazine.com/2001/11/the-moral-thing-to-do/

144 Fathi Hamad, Al-Aqsa TV, 29 February 2008, www.youtube.com/watch?v=RTu-AUE9ycs

145 Hazani, "Red Carpet, White Lilies," 45, 43. 


\title{
TOWARD A WORLD WITHOUT THE DEATH PENALTY
}

\author{
Fon Torke and Alice Storey
}

\section{Introduction}

The sovereign's relationship with the death penalty is in entropy. ${ }^{1}$ A once venerable attribute of legitimate state coercion and retribution has been demonstrated globally to be inhumane and arbitrary, with the few governments that still impose the punishment increasingly viewed as pariah states. ${ }^{2}$ A crucial moment in the synthesis of the new international standards created since 1948 - when the Universal Declaration of Human Rights marked the starting point for modern-day human rights - occurred in 1994 when the Italian government introduced the opportunity for a UN General Assembly resolution to further a wider political agreement. Subsequently the increase in member state denunciations of the punishment resulted in the passing of the first General Assembly "Resolution on the moratorium on the use of the death penalty," in 2007, with 104 states calling for a global suspension of the punishment. ${ }^{3}$ Still more recently, biennial votes have recorded a steady increase in state affirmations, most recently on 17 December 2018 in the General Assembly with 121 endorsements. ${ }^{4}$

This chapter emphasizes the importance of history in assessing the present global perspective on the death penalty, ${ }^{5}$ both in understanding the early origins of the state's reliance on the penalty, beginning at least with Mesopotamian legal codes from $2100 \mathrm{BCE}{ }^{6}$ and in appreciating the re-evaluation of the penalty in more recent times. Changes in the religious base for the penalty, already complicated by the commitment of major faiths to protect life, began to emerge with the Treaty of Westphalia. ${ }^{7}$ Then the rise of new kinds of humanitarian sentiment began to foster a wider political movement toward the fuller appreciation of the death penalty's cruelty. ${ }^{8}$

Already by the late 18th century, principled arguments on the subject began to be matched by legal reform. The first examples of sovereigns abandoning the death penalty occurred in 1767 with Catherine II and Leopold II of Tuscany, and then the removal of the capital sanction from the corpus juris criminalis by Joseph II of Austria in 1787. ${ }^{9}$ However, it took a post-World War II human rights cosmopolitanism to fully underscore the power of abolitionism and to translate tentative steps into wider action.

\section{Anthropological beginnings and the monotheistic impetus for the death penalty}

The cultural anthropologist, Christopher Boehm, argues that, "we humans surely have been resorting to capital punishment for at least 45,000 to 80,000 years or more, 
depending on when you believe we became behaviorally modern, and hence, morally modern." Perhaps the Neolithic period cave painting at Cueva Remigia, Castellon, Spain, provides the first depiction of this behavioral modernism in its execution scene, with leaping warriors having shot arrows into the condemned. ${ }^{10}$

The advent of more sophisticated religions added divine force to the more primitive motives for capital punishment: now as Yuval Harari observes, "[h] umans died because God decreed it, and their moment of death was a sacred metaphysical experience exploding with meaning." 11 The sacred capital judicial system is found in the Judaic texts of the Tanakh, as the death penalty was applied for various capital crimes, for example, murder, forms of theft, and sexual offenses. A similar articulation later occurred in Islam, as in Sharia law, which provides a theological mandate for the Muslim capital judicial process. There is an increasing body of opinion that the death penalty contradicts the merciful spirit of Islam, as well as violating international human rights, complicates the picture, but the traditional sanction goes well back in Islamic history. ${ }^{12}$

\section{The eisegesis for the Christian death penalty}

In Christianity, the punishment began to gain approval over 300 years after the death of Christ, through the affiliation of the Church with the Roman government. Christians who still attempt to legitimize the death penalty have often utilized the Tanakh, or what they call the "Old Testament." Common is the use of the lex talionic within Genesis 9:6, "[w] hoever sheds the blood of man, by man shall his blood be shed," and in Leviticus 24:17, "[i]f anyone takes the life of a human being he must be put to death." The Christian thanatologists who project this "eye for an eye" retribution attempt to get around the exegetical difficulty that God himself did not kill Cain and also gave him protection in his moment of clemency, or even that the Jewish death penalty itself was theologically discontinued after $70 \mathrm{CE}$ by referring to the Apostle Paul's endorsement in Romans 13 that:

[e]veryone must submit himself to the governing authorities for there is no authority except that which God has established ... if you do wrong, be afraid, for he does not bear the sword for nothing. He is God's servant, an angel of wrath to bring punishment on the wrongdoer. ${ }^{13}$

The execution of Bishop Priscillian of Avila in 385 CE, marked the beginning of the Church's formal promotion of the use of the death penalty on one of their brethren, for heresy, but there was tension from the outset. Glenn Stassen argues that in the sanguinary use of the death penalty, Christian thanatologists have drowned out the fact that, "[e]very instance of the death penalty mentioned in the New Testament is clearly presented as an injustice."

\section{Beginnings of change}

The Treaty of Westphalia, in 1648, somewhat inadvertently, launched developments within which the death penalty could be reconsidered. The key point was the reduction of religious justifications for the acts of European states, as the authority of the Church was constrained. Now it was simply the sovereign, and not God, who imposed death, a point made in the social contract theories of Thomas Hobbes. Even John Locke, 
though more restrictive, allowed that a properly-constituted government had the right to make laws "with the penalty of death," a conclusion also allowed by Rousseau in his discussions of the General Will. ${ }^{15}$ Immanuel Kant even later agreed that all murderers deserve death.

Other Enlightenment thinkers, however, reflected further on the problems of capital punishment and argued that the transfer of sovereignty did not in fact justify the continuation of the death penalty. A significant blow for the maintenance of the punishment as part of the succession thesis was provided in 1764 when the Italian humanist Cesare Beccaria published his tract On Crimes and Punishment, arguing that the death penalty was "[c]ertainly not a right which is the foundation of sovereignty and laws" and that:

the death penalty is not a matter of right ... but is an act of war on the part of society against the citizen that comes about when it is deemed necessary or useful to destroy his existence. But if I can go on to prove that such a death is neither necessary nor useful, I shall have won the cause of humanity. ${ }^{16}$

In 1793, Thomas Paine took up the charge to argue against this act of war in the capital case against Louis Capet, Louis XVI, because, "our eyes are now open, and the veil of delusion at length withdrawn." Further, "this very circumstance affords to the French nation a blessed occasion of extricating itself from the yoke of kings, without defiling itself in the impurities of their blood," and, as "[m]onarchy, in France, was a system pregnant with crime and murders," it is "our duty as legislators not to spill a drop of blood when our purpose may be effectually accomplished without it." This extrication from tradition was a recognition that sovereign power can change and that, in the future, past mistakes can be identified and explicit decisions can guard against their repetition. Punishment must change to become effective and humane, and only impose the necessary infringement of a criminal's liberty. ${ }^{17}$ The imposition of needless suffering and pain in punishment should now be confined to the annals of penological history.

In 1837 Jeremy Bentham published a pamphlet entitled To his Fellow Citizens of France, on Death Punishments in which he provided his utilitarian thesis against the death penalty that would rupture the succession right of sovereignty and argued:

$[w]$ hatever may have been the case antecedently to this experience - subsequently to the demonstration thus afforded of the needlessness and uselessness of this so highly objectionable mode of punishment, the leaving it unabolished, was everywhere without excuse. ${ }^{18}$

This was because the death penalty was nothing more than a "sinister interest" of the sovereign, which had been found percolating into public opinion, and this occurs when the population "shut $[\mathrm{s}]$ their eyes against the light." François Pierre Guillaume Guizot, in A Treatise on Death Punishments (1848), ${ }^{19}$ echoed Bentham in dispelling the political reasons for the death penalty and demonstrated its ineffectiveness to act as a deterrent against the worst offenders; sovereigns must now learn this because in continuing to use the death penalty, "[w]e live in a society recently overturned, where legitimate and illegitimate interests, honourable and blameable sentiments, just and false ideas, are so mingled, that it is very difficult to strike hard without striking wrong," and therefore, "let us dispense with killing," for we, "have no more need of it; less terrible remedies will suffice." 
Clearly, at the level of Western political theory, the momentum for change was building steadily. The Enlightenment had introduced a decisive shift in traditional thinking about the use of death.

In reality, of course, most Western states continued to insist on their right to inflict capital punishment, but even here there were significant new steps. During the 18th century the British, in absence of effective policing, had actually expanded the number of crimes subject to the death penalty, but during the 19th century this trend was steadily reversed. Most Western governments dramatically reduced the number of capital crimes, focusing mainly on murder and treason; this trend even spread to Russia during its reform period later in the century. Many states also sought what they regarded as more humane methods when death was inflicted; this was the source, famously, of the introduction of the guillotine in France. Growing efforts also sought to limit the imposition of death penalties on juveniles.

Perhaps most revealingly, Western countries also decisively reversed the traditional habit of administering executions in public, now implementing the death penalty in isolated prison complexes. Charles Dickens was one of many mid-century writers to argue that public executions simply stimulated base instincts. Death was now too gruesome to inflict on a wide audience, whose sympathies might in any event be roused for the criminal. This was an intriguing compromise between a sense that death was still an essential deterrent, and a growing public revulsion against exposure to the fact of death.

Finally, haltingly, a number of countries, and American states, began to abolish the death penalty altogether, though there was frequent oscillation between periods of reform and reassertion. In the United States, the state of Michigan abolished the death penalty in 1846, without subsequent reversal. A number of Latin American countries joined the movement in the later 19th century, including Venezuela in 1863 and, on a de facto basis, Brazil not too long afterward. Portugal also took this decisive step in 1867. These actions remained exceptional, to be sure, but they reflected growing momentum. An emerging sense of basic human rights, along with an increasing awareness that capital punishment was not infrequently inflicted on people later to be found innocent, fueled an increasing debate over this old. ${ }^{20}$ Further, public arguments against the death penalty surfaced in parliaments throughout the Western world - as in a passionate denunciation by the Marquis de Lafayette in France in 1830 or in a number of British commissions on the subject - even when no action was taken.

\section{The crucial turning point}

It was not, however, until the violence of the World War era and the jolts to European sovereignty imposed by World War II that definitive and durable change took shape, first in Europe but then more widely, as more and more discussion focused on the death penalty as an "irreparable doom." Thus in the drafting debates of the European Convention on Human Rights from 1949-1950, the need for a new manifestation of sovereignty was recognized. As Henri-Pierre Teitgen, rapporteur to the Committee on Legal and Administrative Affairs, argued, "[i]t is necessary ... to create in advance a conscience in Europe which will sound the alarm," not only because, "the reason of state [is a] permanent temptation," but also because, "Fascism and Hitlerism have unfortunately tainted European public opinion. These doctrines of death have infiltrated into our countries."21 Between 1949 and 1953, the British Royal Commission on Capital Punishment (RCCP) 
conducted a thorough review on the death penalty, with the collation of evidence from across the world, including from Professor Thorsten Sellin from the University of Pennsylvania, who stated, "it is impossible to arrive confidently at firm conclusions about the deterrent effect of the death penalty." Lord Templewood further reviewed the evidence presented to the RCCP from foreign governments and stated that the, "conclusion seems to be inescapable that, whatever may be argued to the contrary, the existence of the death penalty makes little or no difference to the security of life." 22 By this point, the abolitionist argument was firmly established: death penalties were cruel; they had no demonstrable impact on crime rates; and they frequently and irreparably targeted innocent people.

Even with this new momentum, it was only in 1981 that the death penalty began to be abandoned generally in western Europe, a trend that would spread to central and eastern Europe following the fall of communism and the expansion of the Council of Europe during the 1990 s to 2000s. While concerns persisted that the reform might yet someday be reversed, many authorities argued that "The abolition is now inscribed into European law. It has become, in a way ... out of the reach of law, since it falls under a higher order, that of international treaties." Also noteworthy was the increasing concern over the death penalty by the leadership of the Catholic Church, here too reversing a longstanding tradition, with full opposition becoming papal policy from 2011 onward. And it was at the point, indeed, toward the end of the 20th century, that abolitionist discourse clearly entered the realm of international human rights doctrines. ${ }^{23}$

\section{Human rights and the political regions}

\section{The United Nations}

Following the enumeration of the right to life in Article 3 of the Universal Declaration of Human Rights in 1948, it was necessary for the death penalty to receive closer attention. In 1959 the General Assembly recommended that UN Economic and Social Council investigate the punishment. ${ }^{24}$ Since then the UN General Assembly has noted the global shift away from the imposition of capital punishment. In 1968 it stated in a resolution on the death penalty that,

if one looked at the whole problem of capital punishment in a historical perspective, it became clear that there was a world-wide tendency towards a considerable reduction in the number and categories of offences for which capital punishment might be imposed. ${ }^{25}$

Furthermore, in 1971, the General Assembly affirmed, "the main objective to be pursued is that of progressively restricting the number of offences for which capital punishment may be imposed, with a view to the desirability of abolishing the punishment in all countries." 26

Over time, the United Nations has engendered a sophisticated framework for increased legislative scrutiny under the International Covenant on Civil and Political Rights (ICCPR) Article 6, which protects the right to life, ${ }^{27}$ and Article 7, which prohibits torture and inhumane punishment. The Second Optional Protocol to the ICCPR, aiming at the abolition of the death penalty, rendered an opportunity to galvanize state 
abolition, and there are currently 88 member state parties. ${ }^{28}$ This corpus of international law directed toward advancing global abolition offers a significant mandate for the multilateral and bilateral political initiatives in the General Assembly, the quinquennial reporting to the Secretary General, the Human Rights Committee decisions, and the Universal Periodic Review of every member state of the United Nations. ${ }^{29}$

This intricate UN machinery was reflected in the Human Rights Council on 4 March 2015, in the high-level panel which, "exchange[d] views on the questions of the death penalty, and [addressed] regional efforts aiming at ... abolition." Mr. Joachim Rücker, President of the Human Rights Council, drew attention to the "major achievement" represented by the world's progress from 1948 when only 14 countries had abolished the death penalty, to there now being 142 countries around the world that had "either abolished the death penalty, introduced a moratorium or did not practice it." Ms. Ruth Dreifuss, former President of the Swiss Confederation, affirmed that "humanity had made considerable advances towards the universal abolition of the death penalty." The panel concluded that "it was the international community's responsibility to move towards universal abolition" and that, considering each of the human rights regions, it is "possible to move gradually towards abolition through dialogue and advocacy" - because the death penalty is "not about any particular culture or any religion." Abolition of the death penalty has become a universal ideal. ${ }^{30}$

\section{The European regions: the Gouncil of Europe and the European Union}

In his influential, Reflections on the Guillotine (1949), Albert Camus stated, "in the unified Europe of the future the solemn abolition of the death penalty ought to be the first article of the European Code we all hope for." 31 There has been a distinct symbiosis of the Council of Europe and the European Union in promoting the abolition of the death penalty. The elevation of the right to life, the prohibition against inhumane punishments, and insistence on the inherent dignity of the person have now been applied to confine the punishment to the annals of European history. With the exception of Belarus (which is not a member of either European regional body), this aspirational future has become a present reality and a norm for European values.

\section{Council of Europe}

By 1981, all member states of the Council of Europe, without any overall regional mandate, had individually decided not to impose executions for ordinary crimes, ${ }^{32}$ which led to the adoption of Protocol No. 6 in 1983 for the abolition of the death penalty in times of peace. It provided the platform in 1996 for the Parliamentary Assembly to propose abolition as a "prerequisite for membership of the Council of Europe." Following the expansion of the region into central and eastern Europe, the European Commission for Democracy through Law (commonly known as the "Venice Commission") observed in 1999 that:

[i]t can therefore be asserted, and with confidence, that the national and international dimensions of European law tend both independently and together towards the abolition of capital punishment. The evolution in this direction is clear and is becoming a cornerstone of European public order. ${ }^{33}$ 
This confidence was put to the test following the London tube and bus bombings, the ETA attacks in Spain, and the Beslan school siege in South Ossetia. However, in 2002, Protocol No. 13 was adopted which provides for abolition of the death penalty in all circumstances, ${ }^{34}$ and it has held strong, demonstrating that European sovereignty has turned its back upon the use of the punishment even in terrorist circumstances. The Council of Europe has become a death-penalty-free region, and it now calls for all countries of the world to abolish the punishment.

\section{The European Union}

The anti-death penalty position "lies at the heart of the EU,"35 and the European Union's Action Plan on Human Rights and Democracy 2015-2019, ${ }^{36}$ affirmed the EU commitment to contribute to global abolition. The opening lines to the EU Guidelines on the Death Penalty are:

[t]he European Union has a strong and unequivocal opposition to the death penalty in all times and in all circumstances. Therefore, and encouraged by the growing momentum towards abolition of the death penalty worldwide, the EU will continue its long-standing campaign against the death penalty. ${ }^{37}$

These words were echoed in the EU's statement, "Towards a World United Against the Death Penalty" on 10 October 2016, and had been foreshadowed in the human rights standards within the EU Treaties beginning with the 1992 Treaty of Maastricht. ${ }^{38}$ All of this facilitated the explicit prohibition of the death penalty within the Charter of Fundamental Rights of the European Union in 2000, as Article 2(2) mandates, "[n]o one shall be condemned to the death penalty, or executed."39 This legal requirement for abolition then became a prerequisite for membership of the European Union in 2001, causing even aspirant applicants, like Turkey, to abolish capital punishment (in 2004, though this was reversed in 2018). ${ }^{40}$

To mirror its internal abolitionist policy, the EU now seeks to promote abolition of the death penalty globally through the work of the European External Action Service (EEAS). The EEAS coordinates the EU abolitionist policies in bilateral communications and at the multilateral fora, including at the UN General Assembly, and in individual capital punishment cases in third countries through filing amicus curiae briefs, and it also authors general and specific démarches. ${ }^{41}$

\section{The Organization of American States}

The Organization of American States (OAS) has a long tradition of abolitionism, with Venezuela standing as the first country in the world to abolish the death penalty for peacetime offences, and Costa Rica joining the revolution in 1877. Since then abolition has been achieved in South America and Canada, with the most recent abolitionist country being Suriname in 2015. Of the 35 independent states of the Americas, for the tenth year running, the United States of America is the only country that has imposed executions, although the death penalty remains a viable option in a number of Caribbean islands, and in 2016 Barbados, Guyana, and Trinidad and Tobago did impose death sentences. ${ }^{42}$ Article 1 of the American Declaration on the Rights and Duties of 
Man states, "[e]very human being has the right to life," and the American Convention on Human Rights, Article 4, provides for the right to life with section 2 limiting the death penalty to the, "most serious crimes." However, a particular provision for promoting abolition, is found in Article 4(3), which states, "[ $\mathrm{t}]$ he death penalty shall not be reinstated in states that have abolished it." The removal of the death penalty in the OAS is further promoted in the 1990 Protocol to the American Convention on Human Rights on the abolition of the death penalty, which affirmed that the OAS considers abolition to be a "progressive development of the American Convention."

The institutional promotion of this abolitionist ideology was accepted in 1983 when the Inter-American Commission on Human Rights requested the Inter-American Court of Human Rights provide an Advisory Opinion on the "Restrictions to the death penalty."44 Leaving no room for doubt, the court ruled that the Convention forbids extending the application of the death penalty, while the re-establishment of the death penalty for any crime was prohibited. In 2011, the Commission published a detailed report on the death penalty, "The Death Penalty in the Inter-American Human Rights System: From Restrictions to Abolition", and it called upon "OAS Member States that still have the death penalty to abolish it or, at least, to impose a moratorium to its application." In the conclusion of the report, it recommended that the states, "[i]mpose a moratorium on executions as a step toward the gradual disappearance of this penalty," and for all states to ratify the 1990 Protocol to bring about legal abolition in the region. ${ }^{45}$

Over the past two decades, the Commission has also heard individual death penalty cases, ruling upon the merits and handing down rulings for the members to follow. Many of these cases originate in the USA, given that it is the only major country in the OAS to have imposed the punishment over the past ten years. For example, in 2009, the Commission heard the joint case of José Ernesto Medellín, Ramírez Cardenas, and Leal García, three men who were facing execution in Texas. The case alleged a number of constitutional and international law violations, such as breaches of the Vienna Convention on Consular Relations, concerns over lethal injection, conditions on death row, and the performance of their US-state-appointed attorneys. The Commission specifically commented upon the Texas capital public defender system, noting that it:

[had] no [S]tate-wide agency responsible for providing specialized representation in capital cases [and that a] great majority of lawyers who handle death penalty cases in Texas are sole practitioners lacking the expertise and resources necessary to properly defend their clients, and as a result, capital defendants frequently receive deficient legal representation. ${ }^{46}$

The Commission concluded that because there had been violations of the fair trial guarantees, the State of Texas should, "[v]acate the death sentences imposed and provide the victims with an effective remedy, which includes a new trial in accordance with the equality, due process and fair trial protections." 47

While the United States stands out as a troubling American exception, it is important to note that a number of additional states have abolished the death penalty, particularly since the 1990s. As of March 23, 2020, 22 states had ended the death penalty for any crime, most recently Colorado, and several others are no longer implementing it. Public opinion polls, at odds with European and other American trends in the 1970s and 
1980s, amid a growing concern about crime rates, have trended in a different direction more recently. Debates over the cruelty of death penalty methods, particularly lethal injections, have added to what is a significant internal controversy, linked to other issues in this now-polarized nation. Current United States exceptionalism over the death penalty does call for careful comparative analysis, for in the 19th century American opinion and policy were squarely aligned with the growing though still halting critiques of capital punishment common in western Europe and the Americas.

\section{The African Union}

Article 4 of the African Charter on Human and Peoples' Rights, states, "[h] uman beings are inviolable. Every human being shall be entitled to respect for his life and the integrity of his person. No one may be arbitrarily deprived of his life." Since the adoption of the Charter, the African Union has increasingly examined the exception included in the third sentence of Article 4, with an aim of nullifying its applicability. ${ }^{48}$ In 1999 the African Commission on Human and Peoples' Rights adopted the Resolution Urging States to Envisage a Moratorium on the Death Penalty, and it called upon retentionist states to "limit the imposition of the death penalty to the most serious crimes," to "consider establishing a moratorium on executions," and to "reflect on the possibility of abolishing the death penalty." 49 In 2008 the aspiration evolved from an "urging to envisage" to a "calling to observe a moratorium." 50 This strengthened language exhorted states to comply with the minimum standards for a capital judicial process and urged "State Parties that still retain the death penalty to observe a moratorium on the execution of death sentences with a view to abolishing the death penalty."51

In 2009, the African Commission organized a conference for participants from Central, Eastern and Southern Africa on "The Question of the Death Penalty in Africa," and adopted the "Kigali Framework Document on the Abolition of the Death Penalty in Africa," which stipulated that in endorsing a strategy for the African Union, the African Commission should, "continue its campaign to abolish the death penalty by adopting Resolutions, its Promotion Missions, its Special Mechanisms, examination of State Reports and Communication Procedures." 52

Then in 2010, a conference was organized for North and West Africa by the African Commission, from which was adopted the "Cotonou Framework Document on the Abolition of the Death Penalty in Africa." It recommended that the African Commission enhance its "human rights education programmes, including the adoption of a media strategy to create public awareness on the need to abolish the death penalty." The Cotonou Framework identified the need for engaging with "different religious groups as well as the different faiths and traditions on the abolition and moratorium on the death penalty."53

In May 2017, at the 60th Ordinary Session for the African Commission, a panel discussion was convened to "reflect on the reluctance of certain States to abolish the death penalty with the aim of identifying possible strategies that could persuade those States to abolish the death penalty." 54 In their statement on the World Day Against the Death Penalty (occurring annually on 10 October), the Working Group stated that it "reaffirms its commitment to continue to work with its partners in its efforts to raise awareness on the abolition of the death penalty in Africa." 55 The need to collaborate with different religious groups "expressed in the Cotonou Framework," is a vital component in dealing with questions on the death penalty given the different religions and indigenous beliefs systems on the subcontinent. 


\section{Asian states}

The movement against the death penalty has made less progress in Asia than in most of the other world regions, even including the United States. This includes democratic countries like Japan, with low crime rates, and authoritarian states as well.

China is regularly reported to be the country that imposes the most executions, but in 2018, Japan carried out 15 executions, Singapore 13, and Vietnam 85+ executions, according to Amnesty International. Several Middle Eastern countries maintained high rates as well. The International Federation for Human Rights has noted the "significant setbacks with regard to the abolition of the death penalty," and for East Asia these have been the focus of the Asia Pacific Forum of National Human Rights Institutions. ${ }^{56}$ The Forum has produced an educational program to engage with Asia Pacific NHRIs in their roles as human rights bodies in their national governments and in providing reports to the UN's Universal Periodic Review. The Forum has been particularly active in Malaysia, Myanmar, and the Philippines, and organized a conference with the National Human Rights Commission of Myanmar to call for the country to shift from de facto abolition to drafting explicit national law to provide for formal abolition. ${ }^{57}$ This region remains a focus of the World Coalition Against the Death Penalty, which is attempting to coordinate civil society to contribute to the capital defense and abolitionist efforts in the region. ${ }^{58}$

\section{Conclusion}

In his research on human progress, Steven Pinker argues that the globalization of abolitionism means that, "today the death penalty is on death row," as the, "uncanny assemblage of scientific, institutional, legal, and social forces all [push] to strip government of its power to kill." ${ }^{59}$ Human rights efforts around the world are pressing against the death penalty. What has already been achieved, reflecting a complicated and checkered historical evolution since the 18th century, represents a dramatic attack not only on government traditions but on larger beliefs about the nature of death in punishment. Obviously, debate still continues. The regional differences in dealing with this issue reflect fascinating, if troubling, distinctions in cultural and political traditions, even in this global age. The arguments for a single global norm are powerful, and they have unquestionably gained ground over the past 30 years. It is vital to rely on the implications of the human rights movement to extend and maintain this contribution to a more civilized society. Promoting understanding of the force of international human rights applied to what has become an archaic punishment is the beating heart of the story.

\section{Notes}

1 For the theory of entropy in time and space, see Carol Rovelli, The Order of Time (London: Allen Lane, 2018), pp. 23-33; and for a discussion of the use of time in the death penalty, see Jon Yorke, "An Experience of Time in the Capital Judicial Process," Texas Fournal of Civil Liberties \& Civil Rights 24 (2) (2018), 1-30.

2 William Schabas, The Abolition of the Death penalty in International Law, 3rd ed. (Cambridge: Cambridge University Press, 2002), p. 363.

3 See Hands off Cain, "Initiative for a UN Moratorium on Death Penalty," Summer 2007, www.handsoffcain.info/chisiamo/index.php?idtema=9319441

4 UNGA Resolution on the moratorium on the use of the death penalty, 62/149 of 18 December 2007; UNGA Resolution on the moratorium on the use of the death penalty, 63/168 of 
18 December 2008; UNGA Resolution on the moratorium on the use of the death penalty, 65/206 of 21 December 2010; UNGA Resolution on the moratorium on the use of the death penalty, 67/176 of 20 December 2012; UNGA Resolution on the moratorium on the use of the death penalty, 69/186 of 18 December 2014; and UNGA Resolution on the moratorium on the use of the death penalty, 71/187 of 19 December 2016.

5 See Marc Ancel, "The Problem of the Death Penalty," in Thorsten Sellin ed., Capital Punishment (New York: Harper and Row, 1967), p. 6.

6 David Ibbetson, "Wrongs and Responsibilities in Pre-Roman Law," The Fournal of Legal History 25 (2) (August 2004), 99-127.

7 For a wider discussion of the development of sovereignty post-Westphalia, see Daniel Philpott, Revolutions in Sovereignty: How Ideas Shaped Modern International Relations (Princeton: Princeton University Press, 2001) and Gene M. Lyons and Michael Mastanduno eds., Beyond Westphalia? State Sovereignty and International Relations (Baltimore, MD: Johns Hopkins University Press, 2001).

8 Jon Yorke, "Sovereignty and the Unnecessary Penalty of Death: European and United States Perspectives," in Austin Sarat and Jurgen Martschukat eds., Is the Death Penalty Dying? European and American Perspectives (Cambridge University Press, 2011), pp. 236-268.

9 See Ancel, "The Problem of the Death Penalty," p. 6.

10 Christopher Boehm, "Prehistoric Capital Punishment and Parallel Evolutionary Effects," Minding Nature 10 (2) (2017), 24; and Austin Sarat ed., The Killing State: Capital Punishment in Law, Politics, and Culture (Oxford University Press, 1999). The extension of this theory is that sovereignty can attempt to use the death penalty as an expression of legitimate violence to affirm itself. This chapter argues that this proposition of power is now anachronistic and is refuted through the human rights discourse.

11 Yuval Harari, Homo Deus: A Brief History of Tomorrow (London: Vintage, 2017).

12 Tariq Ramadan, An International Call for Moratorium on Corporal Punishment, Stoning, and the Death Penalty in the Islamic World, Tariq Ramadan (Apr. 5, 2005), https://tariqramadan.com/an-inter national-call-for-moratorium-on-corporal-punishment-stoning-and-the-death-penalty-in-theislamic-world/. Note, there is an increasing body of opinion that the death penalty contradicts Islamic values.

13 Romans 13:1, 4.

14 Stassen, "Biblical Teaching on Capital Punishment," Review and Expositor 93 (1996), 485-496. For example the trial and stoning of Stephen in Acts 7.

15 Locke, Second Treatise on Government (Hackett Publishing, 1989), p. 8; Jean-Jacques Rousseau, The Social Contract and Discourses (Everyman, 1993), p. 209.

16 Richard Bellamy, Beccaria: On Crimes and Punishment and Other Writings (Cambridge: Cambridge University Press, 1995), p. 66.

17 Thomas Payne, "Reasons for Preserving the Life of Louis Capet," in Michel Foot and Isaac Krammick eds., Thomas Payne Reader (London: Penguin Books, 1987), pp. 395, 397.

18 Jeremy Bentham, To His Fellow Citizens of France, on Death Punishments (London: Robert Howard, 1831), p. 12.

19 François Guizot, "A Treatise on Death Punishments," in General History of Civilisation in Europe: From the Fall of the Roman Empire Till the French Revolution (London: William and Robert Chambers Press, 1848).

20 A. Hammel, Ending the Death Penalty: The European Experience in Global Perspective (2014); David Brion Davis, "The Movement to Abolish Capital Punishment in the United States, 1787-1861," American Historical Review 63 (1957), 23-46.

21 Council of Europe, Collected Edition of the "Travaux Preparatories," of the European Convention on Human Rights, v. 2 (Martinus Nijhoff, 1975), pp. 36, 174.

22 Viscount Templewood, The Shadow of the Gallows: The Case Against Capital Punishment (London: Victor Gollancz, 1951), p. 85.

23 Jacques Derrida and Elizabeth Roudinesco, For What Tomorrow ... A Dialogue (Stanford University Press: 2004), p. 137.

24 Marc Ancel, "Capital Punishment," UN Doc. ST/SOA/SD/9, Sales No. 62.IV.2.; Norval Morris, “Capital Punishment: Developments 1961-1965," Sales No. 62.IV.15. 
25 Capital Punishment, UNGA Res. 2393 (XXIII) Preamble, 1727th Plenary Meeting, 26 November 1968. This was affirmed in the Resolution on capital punishment in southern Africa, 2394 (XXIII), 1727th Plenary Meeting (26 November 1968).

26 Capital Punishment, 2856 (XXVI), 2027th plenary meeting (20 December 1971) para. 3.

27 International Covenant on Civil and Political Rights (adopted 16 December 1966, entered into force 23 March 1976) 999 UNTS 171, Article 6.

28 The Second Optional Protocol to the International Covenant on Civil and Political Rights, aiming at the abolition of the death penalty, GA Res. 44/128, 15 December 1989. See also Safeguards Guaranteeing Protection of the Rights of Those Facing the Death Penalty, Economic and Social Council Resolution, 1984/50; Additions to the Safeguards Guaranteeing Protection of the Rights of Those Facing the Death Penalty as Agreed by the Economic and Social Council Resolution 1989/64; and the Strengthening of the Safeguards Guaranteeing Protection of the Rights of Those Facing the Death Penalty as Agreed by the Economic and Social Council Resolution 1996/15.

29 For example, in the Independent Expert on the human rights situation in Sudan www.ohchr. $\mathrm{org} / \mathrm{EN} /$ Countries/AfricaRegion/Pages/SDIndex.aspx (accessed 22 January 2020); in the Special Rapporteur on Extrajudicial, Summary and Arbitrary Executions www.ohchr.org/EN/ Issues/Executions/Pages/SRExecutionsIndex.aspx (accessed 22 January 2020); in the Special Rapporteur on Torture and other Cruel, Inhuman, or Degrading Treatment or Punishment www.ohchr.org/EN/Issues/Torture/SRTorture/Pages/SRTortureIndex.aspx (accessed 22 January 2020); and Judge v. Canada, Communication No. 829/1998, U.N. Doc. CGPR/C/78/ D/829/1998 (2003); UPR Info, "Database: Death Penalty," www.upr-info.org/database (accessed 22 January 2020).

30 High-level panel discussion on the question of the death penalty, Report of the United Nations High Commissioner for Human Rights, UNGA, A/HRC/30/21 (16 July 2015), pp. 2, 3, 12.

31 Albert Camus, Reflections on the Guillotine (Fridtjof-Karla Publications, 1959) p. 230.

32 The country that still applied to the death penalty but did not execute anyone was the Republic of Ireland. Michael Manning was the last person to be executed in Ireland, in 1954. The death penalty remained on the statute books until it was abolished in 1990, and then the Twenty First Amendment of the Constitutional Act 2001, Article 15.5.2, explicitly prohibited the death penalty. See Twenty-First Amendment of the Constitution Act, 2001, available at www.irishstatutebook.ie/2001/en/act/cam/0021/sched.html\#sched-parti

33 Protocol No. 6 to the Convention for the Protection of Human Rights and Fundamental Freedoms concerning the abolition of the death penalty (28 April 1983) C.E.T.S. No. 114; Resolution 1097 on the abolition of the death penalty in Europe, Parliamentary Assembly (24th Sitting, 28 June 1996), para. 6; and European Commission for Democracy through Law, Activities of the Venice Commission relating to the suppression of the death penalty by $\mathrm{Mr}$ J Klucka presented to the Colloquy on "The Impact of the Case-Law of the European Court of Human Rights on the Activity of the Constitutional Courts of Central and Eastern Europe," (University of Clermont-Ferrand, 15-16 November 2002), CDL-JU (2002) 38, Strasbourg 20 November 2002, s. 2.

34 Protocol No. 13 to the Convention for the Protection of Human Rights and Fundamental Freedoms concerning the abolition of the death penalty in all circumstances, CETS. 187 (3 May 2002).

35 Statement by Steven Vanackere, Vice Prime Minister and Minister of Foreign Affairs of Belgium, Opening Session, at the 12th Annual EU-NGO Forum of Human Rights: EU Human Rights Instruments and the Lisbon Treaty: State of Play and Way Forward (12 July 2010).

36 European Union Action Plan on Human Rights and Democracy (2015-2019), https://ec. europa.eu/anti-trafficking/eu-policy/action-plan-human-rights-and-democracy-2015-2019_en (accessed 22 January 2020). See also Christian Behrmann and Jon Yorke, "The European Union and the Abolition of the Death Penalty," Pace International Law Review Online Companion 4 (1) (2013), 1-79, http://digitalcommons.pace.edu/pilronline/39/.

37 European External Action Service, EU Guidelines on the Death Penalty: Revised and Updated Version (2013), http://data.consilium.europa.eu/doc/document/ST-8416-2013-INIT/en/pdf (accessed 2 June 2020). 
38 European External Action Service, "Towards a World United Against the Death Penalty," A Joint EU Ambassador Statement (10 October 2016), https://eeas.europa.eu/headquarters/ headquarters-homepage/11355/towards-world-united-against-death-penalty_en (accessed 22 January 2020). The World Day Against the Death Penalty occurs every 10 October; Treaty on European Union (Treaty of Maastricht), 7 February 1992, 1992 O.J. (C 191), Article $\mathrm{F}(2)$. Treaty on European Union (Treaty of Maastricht), 7 February 1992, 1992 O.J. (C 191), Article F(2).

39 Charter of Fundamental Rights of the European Union, 18 December 2000, 2000 O.J. (C 364/1).

40 See Commission Communication to the Council and the Parliament, the European Union's Role in Promoting Human Rights and Democratisation in Third Countries, COM (2001) 252 Final (May 2001), which stated that the "EU's commitment to the abolition of the death penalty was reaffirmed in Article 2 of the EU Charter. It is a requirement for countries seeking EU membership," 16.

41 The European External Action Service, https://eeas.europa.eu/headquarters/headquar ters-homepage_en (accessed 22 January 2020); see EU Guidelines on the Death Penalty, https://eeas.europa.eu/sites/eeas/files/guidelines_death_penalty_st08416_en.pdf (accessed 22 January 2020).

42 Amnesty International, The Death Penalty in 2018: Facts and Figures; See generally, Richard J. Wilson, "The United States' Position on the Death Penalty in the Inter-American Human Rights System," Santa Clara Law Review 42 (4) (2002), 1159-1190.

43 Protocol to the American Convention on Human Rights to Abolish the Death Penalty, OAS Treaty Ser. No. 73 (6 August 1990).

44 I/A Court H.R., Restrictions to the Death Penalty (Arts. 4(2) and 4(4) American Convention on Human Rights). Advisory Opinion OC-3/83 of 8 September 1983. Series A No. 3, para. 57.

45 Inter-American Commission on Human Rights, The Death Penalty in the Inter-American Human Rights System: From Restrictions to Abolition, OEA/Ser. L/V/II Doc. 68 (31 December 2011), pp. 2, 188.

46 Inter-American Commission on Human Rights, Report No 90/09, Case 12.744 (7 August 2009).

47 Ibid.

48 For a detailed review of the death penalty in Africa, see Lilian Chenwi, Towards the Abolition of the Death Penalty in Africa: A Human Rights Perspective (Cape Town: Pretoria University Law Press, 2007); Andrew Nowak, The Death Penalty in Africa: Foundations and Future Prospects (London: Palgrave Macmillan 2014); and Andrew Nowak, The African Challenge to Global Death Penalty Abolition: International Human Rights Norms in Local Perspective (Cambridge: Cambridge University Press, 2016).

49 Resolution on the Moratorium on the Death Penalty, ACHPR/Res.42 (XXVI) 9.

50 Resolution on the Moratorium on the Death Penalty, ACHPR/Res.136 (XXXXIII) 8.

51 Ibid., para. 2, 5.

52 Kigali Framework Document on the Abolition of the Death penalty in Africa, Kigali, Rwanda (25 September 2009).

53 Cotonou Framework Document on the Abolition of the Death Penalty in Africa, Cotonou, Benin (15 April 2010).

54 Commissioner Kayitesi Zainabo Sylvie, Intersession Activity Report (November 2016April 2017), 60th Ordinary Session, ACHPR, Niamey, Niger (8-22 May 2017).

55 "Statement by the Chairperson of the Working Group on Death Penalty and Extrajudicial, Summary or Arbitrary Killings in Africa of the African Commission on Human and People" on the World Day Against the Death Penalty, Rights Banjul (10 October 2016).

56 International Federation for Human Rights, "Going Backwards: The Death Penalty in Southeast Asia" (2016), www.fidh.org/IMG/pdf/asie682apdmweb.pdf; and Asia Pacific Forum of National Human Rights Institutions, www.asiapacificforum.net

57 See generally for the Universal Periodic Review, www.ohchr.org/EN/HRBodies/UPR/Pages/ CyclesUPR.aspx; "Outcome Statement on the Workshop for the Consideration of a Moratorium on the Death Penalty Pending Abolition," The Global New Light of Myanmar, 


\section{JON YORKE AND ALICE STOREY}

2 November 2017, www.globalnewlightofmyanmar.com/outcome-statement-workshop-consider ation-moratorium-application-death-penalty-pending-abolition/

58 See The World Coalition Against the Death Penalty, "Moving Away from the Death Penalty in Asia," 13 October 2013, www.worldcoalition.org/thailand-china-asia-death-penalty-aboli tion-laos-brunei-myanmar-un-ohchr-adpan.html

59 Steven Pinker, Enlightenment Now: The Case for Reason, Science, Humanism and Progress (London: Allen Lane, 2018), pp. 209, 213. 


\title{
7 \\ THE GEMETERY
}

\author{
Erin-Marie Legacey
}

Writing about the history of cemeteries in a global context is a difficult and daunting task. Humans have buried or contained their dead in some form or another dating back to the Paleolithic era. " However, the first modern cemeteries-large, public, pastoral burial sites like Père Lachaise Cemetery in Paris or Mount Auburn Cemetery near Boston-only appeared at the beginning of the nineteenth century. For the sake of clarity and focus, this chapter will examine the modern cemetery as a distinct phenomenon - one of a great many forms of burial across the globe. ${ }^{2}$ One scholar has defined cemeteries concisely as "specially demarcated sites of burial, with internal layout that is sufficiently well-ordered to allow families to claim and exercise control over their particular grave space." ${ }^{3}$ The cemeteries that appeared in nineteenth-century Europe did not appear out of nowhere; they were both an outgrowth of, and reaction against, the centuries' old practice of burying the dead in small community graveyards attached to neighborhood churches (i.e., churchyards). As one guidebook to London's Kensal Green Cemetery described the difference between the two:

In the metropolitan churchyards, we seldom found anything but loathsome relics of mortality and festering disease. In a cemetery we find ourselves surrounded with such harmonious minglings of nature and art, that while our senses are gratified with the fragrance of beautiful flowers, the merry chirping of birds, and the graceful productions of the sculptor's genius, our minds are elevated by the many peaceful associations of the scene, and those great truths so silently yet eloquently asserted in the garden of the dead. ${ }^{4}$

As this enthusiastic endorsement makes clear, nineteenth-century cemeteries did significantly more than safely contain the dead; they were unique social spaces that catered to the needs and tastes of the living. But these tastes extended far beyond aesthetic preferences. As spaces designed for the living to use, cemeteries provided a kind of social cohesion, bringing the living together through affective experiences, confirming community values, and creating a tangible connection to a shared past.

Underpinning this interpretation is the understanding that cemeteries are quintessentially "other" spaces, what Foucault called "heterotopias" in his brief 1984 essay, "Of Other Spaces." Unlike their better-known cousins, utopias, heterotopias are actually existing places but are often in marginal or peripheral locations. And rather than serving 
as a thought experiment in the form of a perfect vision of society, heterotopias provide a real platform for testing out alternative or experimental relationships or modes of social organization. At the level of the individual, this recalls a married Percy Shelley meeting the young Mary Wollstonecraft Godwin at her parents' gravesite to carry on an illicit affair. At a bigger societal level, this appears in the way early nineteenth-century cemeteries featured an uncannily precise visual and spatial demarcation between the poor, middling, and wealthy. Foucault reflected on this feature of heterotopias when he described their ability to function as "another real space, as perfect, as meticulous, as well arranged as ours is messy, ill constructed, and jumbled.",6

This chapter will examine how and why the historic churchyard gave way to the cemetery at the beginning of the nineteenth century. In doing so, it will highlight continuities as well as differences. Most notably, it will focus on the social significance of the cemetery, which drew from the churchyard's historic place at the (metaphorical and geographical) heart of the community. It will also examine the larger global impact of this predominantly European revolution in burial practices and explore more recent alternatives that have challenged the dominance of the cemetery.

\section{The churchyard}

Before there were cemeteries there were churchyards: fields adjacent to churches that held the community's dead. Although there had been a Roman ban on civic interment since antiquity, Pope Gregory the Great (540-604) first consented to the practice of burying particularly important clerics and monastics on church grounds for a fee. Over the next few hundred years, this practice expanded, and, during the ninth and tenth centuries, churches across Europe began cultivating the yards adjacent to parish churches to contain more members of the ecclesiastical community. By the eleventh century, this practice had expanded to include lay members of the community, and a new cultural expectation took hold: the faithful in good standing with the church would be buried on sacred ground, close to the prayers of the parishioners, and in a relatively orderly and ritualized fashion. By the twelfth century, many churchyards were enclosed by walls to protect them from the dangers of the secular world, including wild animals, graverobbers, and illicit social and sexual practices. ${ }^{7}$

Although they shared certain obvious similarities with the cemeteries that would eventually replace them, churchyards differed in several key ways. First, they were almost always understood to be temporary resting places, by both sacred and secular metrics. In terms of sacred time, the bodies of the faithful were simply short-term occupants of the churchyard awaiting the return of Christ. This is why the dead lay in an east-west position, so that they would arise facing the Holy Land, to facilitate their resurrection. Indeed, the Latin term coemeterium (a cognate for the greek koimhthrion) refers to a sleeping place or dormitory, which for early Christians implied a special place where the dead could rest while they awaited the Second Coming. In secular terms, churchyards were temporary because most of the dead only remained buried in them for a few years while their corporeal matter decomposed, after which point their bones would be disinterred and transferred for storage in a nearby charnel house. 
Churchyards also differed from cemeteries in that the vast majority of churchyard burials and exhumations took place in communal-rather than individual-graves. A deep pit would usually be dug and gradually filled with corpses over the course of a season, before being filled in with earth and left to sit for a fixed number of years. Thus, the modern practice of entering a cemetery to visit the grave of a beloved family member, friend, or celebrity would generally have been impossible and unthinkable in a medieval churchyard, for both logistical and conceptual reasons. As Thomas Laqueur described it in The Work of the Dead, "The churchyard was and looked to be a place for remembering a bounded community of the dead who belonged there rather than a place for individual commemoration and mourning." 8

Laqueur's reference to a "bounded community of the dead" reminds us that while pre-modern churchyards may have had a different social function from that of the modern cemetery, they were, nevertheless profoundly important and powerful social spaces of both cohesion and control. In order to be buried in the churchyard, one had to theoretically meet certain requirements that reinforced community and religious values. Suicides, the unbaptized, criminals, and religious minorities were either excluded from churchyards altogether, or, when permitted they were deliberately marginalized by being buried carelessly, along the wrong axis, or on the less-desirable north side of the church. As Anthony Perron has noted, exclusion from the consecrated soil of the churchyard "mattered a great deal" in the medieval period, because by the twelfth century "the cemetery came to be understood as a place where the living and the dead formed one society bound together by ties of mutual obligation."9 Similarly, Vanessa Harding has argued in her detailed study of burial culture in latemedieval London and Paris, burial spaces revealed "a widely shared vision of society as ascertainably differentiated and hierarchical. The spatial order reflected the social, in a paradigm of centrality and marginality, inclusion and exclusion." 10 While the dead only rested in peace temporarily in churchyards, the brief time that they spent there mattered.

Churchyards also functioned as useful social spaces for the living. As scholars such as Vanessa Harding and Christine Métayer have shown, communities regularly used churchyards as sites to arrange meetings, sell wares, and procure services (both legal and illicit). When describing Paris's oldest and largest burial space, the Cimetière des Saints Innocents, Métayer points to the many people who lived and worked within that sprawling cemetery's walls before the eighteenth century, blurring the line between private and public space, and creating a veritable "micro society" of cemetery dwellers at the "frontier of the sacred and the profane, the public and private." This particular burial site was by the eighteenth century, she explains, "a veritable space of integration." "11 This early modern French burial space recalls twentieth- and twenty-first-century stories about Cairo's famous "City of the Dead"-in which multistory family tombs serve as permanent (if unauthorized) housing for hundreds of thousands of Egyptians, discussed further, below. Churchyards were thus clearly more than simply places to temporarily store the dead in the pre-modern era. They were at the very heart of European communities and worked to reinforce community standards, social hierarchies, and religious authority. While churchyards only offered temporary refuge for most of the individual dead while they decomposed, the burial sites themselves remained as permanent sites in cities and villages across Europe, blurring the boundaries between private and public, sacred and secular. This elemental cultural practice had evolved over centuries, but, by the 
eighteenth century, churchyards were firmly entrenched and familiar social spaces in virtually every European community.

\section{The decline of the churchyard}

It was only during eighteenth-century Enlightenment that this custom began to falter. After nearly a millennium of slow growth, the institution of the churchyard came under sustained and powerful attack. It ultimately gave way beneath the weight of a new and powerful discourse of science, hygiene, and individualism, as discussed by Joseph Clarke in Chapter 10 of this volume. By the first decade of the nineteenth century, the age of the cemetery had arrived.

The first churchyard to fall was one of the oldest, largest, and most well-known burial sites in Europe, the previously mentioned Cimetière des Saints Innocents in Paris. Innocents had stood at the heart of the city in roughly the same form since at least the twelfth century, when King Philippe II (Philippe Auguste) had a wall built to enclose terrain where Parisians had been burying bodies since antiquity. Innocents was famous across Europe for its age, its size, and for the sheer quantity of bodies it contained, both as corpses decomposing in communal graves and as disinterred bones, stacked in galleries around the perimeter of the space. For centuries, critics had complained about overcrowding in Innocents, which became particularly evident and intolerable each summer when the temperature spiked and the scent of death hovered around central Paris. But by the middle of the eighteenth century, these complaints gained traction when physicians and urban reformers began framing the debate in the language of public health. Simply put, the smell of death was shifting in its meaning from being unpleasant and inconvenient to being dangerous and lethal. A series of studies conducted at the request of the French state at the middle of the eighteenth century concluded that Paris's cemeteries presented a clear and present threat to the living. A 1763 report described mass graves that remained open for months, cemeteries so filled with bodies that the level of the soil was seven feet above street level, and church charnel houses bursting at the seams. More urgently, the report noted that the burials taking place throughout Paris were making the air unbreathable. "The cadavers buried beneath our feet" and the mephitic vapors they exuded, it explained, were the most likely source of otherwise-mysterious illnesses in the capital. ${ }^{12}$ As cemetery historian Blanche M. G. Linden described it, "reputed to be the most notorious and fearsome of all European burial places, the Innocents was ... a walled wilderness in the heart of the city's most active quarter." 13

Critics of churchyard burial relied on miasmic theory to explain why this particular mode of burial was unsustainable. Miasmic theory had dominated the European understanding of illness for centuries: its proponents maintained that all manner of physical ailments, from the plague to headaches, were caused by harmful airs that emanated from rotting animal matter. Alain Corbin explains this particular strain of scientific thinking:

The stench of corpses seems to have been the first to arouse nearly universal intolerance, as the early date of complaints by people living next to cemeteries attests ... To separate the abode of the dead from that of the living became an incessant demand. ${ }^{14}$ 
Despite widespread concerns and proposals for reform throughout the eighteenth century, it took a sensory calamity to finally bring about lasting change. In 1780, the occupant of a house bordering the Cemetery of the Innocents reported that vapors from decomposing human remains were seeping up from his basement. Once a small dispatch of inspectors confirmed this nightmarish breech, the French state ordered Innocentsand ultimately all cemeteries within Paris's walls - to be closed.

However, one must be careful about placing too much weight on the power of decomposing corpses. The shift from churchyard to cemetery was not simply a product of determined Enlightenment-era urban reformers. As Thomas Laqueur argued, the public health argument for burial reform can also be interpreted as a thinly veiled disguise for anticlericals looking to wrest control of this crucial social practice (of caring for the dead) from the Catholic Church. ${ }^{15}$ Just as significant, for the purposes of this essay, is what the closing of Innocents signified. For Paris and Parisians, the closure of the immense and centrally located Innocents was a tremendous disruption, which was only exacerbated by the events of the French Revolution less than a decade later. ${ }^{16}$ However, in the longer history of the cemetery, it is a watershed moment signaling the end of a specific way of thinking about the place and role of the dead in the city. Simply put, the dead were no longer needed, desired, or welcome in the heart of urban spaces. Instead of cementing bonds of Christian community, the dead in churchyards now threatened their fellow parishioners with their potentially lethal mephitic vapors. The intimate and sacred relationship between the dead, the living, and their respective spaces was changing.

In the decades that followed the closing of Innocents, many French reformers tried to envision a new style of burial space to replace the churchyard. They sought an urban space that would somehow meet all of the needs of the community without endangering them (or shoring up the power of the church). This new-style burial space needed to perform the same social functions as churchyards had for centuries: it needed to reinforce communal values through rituals of inclusion and exclusion, it needed to create and strengthen new - preferably secular - ties between the dead and the living, and it had to do this all while remaining a safe distance from the bustling city and its inhabitants. The most extreme versions of these imaginary cemeteries of the future included elaborate rituals for judging the dead before burial (in so-called "Egyptian death tribunals"), separate cemeteries for good and bad citizens, and even a chemical system for turning the bones of the dead into inspiring glass sculptures. ${ }^{17}$ What ultimately succeeded, however, was far less eccentric: an unprecedentedly huge stand-alone cemetery just beyond city walls, designed with lush and fragrant landscaping, and an impressive array of permanent monuments for the dead. When it opened in 1804 it was known as the Cemetery of the East, but it soon became famous by its now well-known moniker, Père Lachaise Cemetery (see Image 7.1).

\section{The rise of the cemetery}

Père Lachaise broke with many of the longstanding practices of European burial. Most significantly, it was separated, both geographically and administratively from the Catholic Church. Indeed, the Napoleonic decree that accompanied the opening of Père Lachaise announced that no burials were permitted inside of cities or villages, especially not within any building of worship. ${ }^{18}$ Specifically, cemeteries needed to be a minimum of 30 meters outside of any urban setting. Rather than being a corpse-stuffed appendage to a parish 


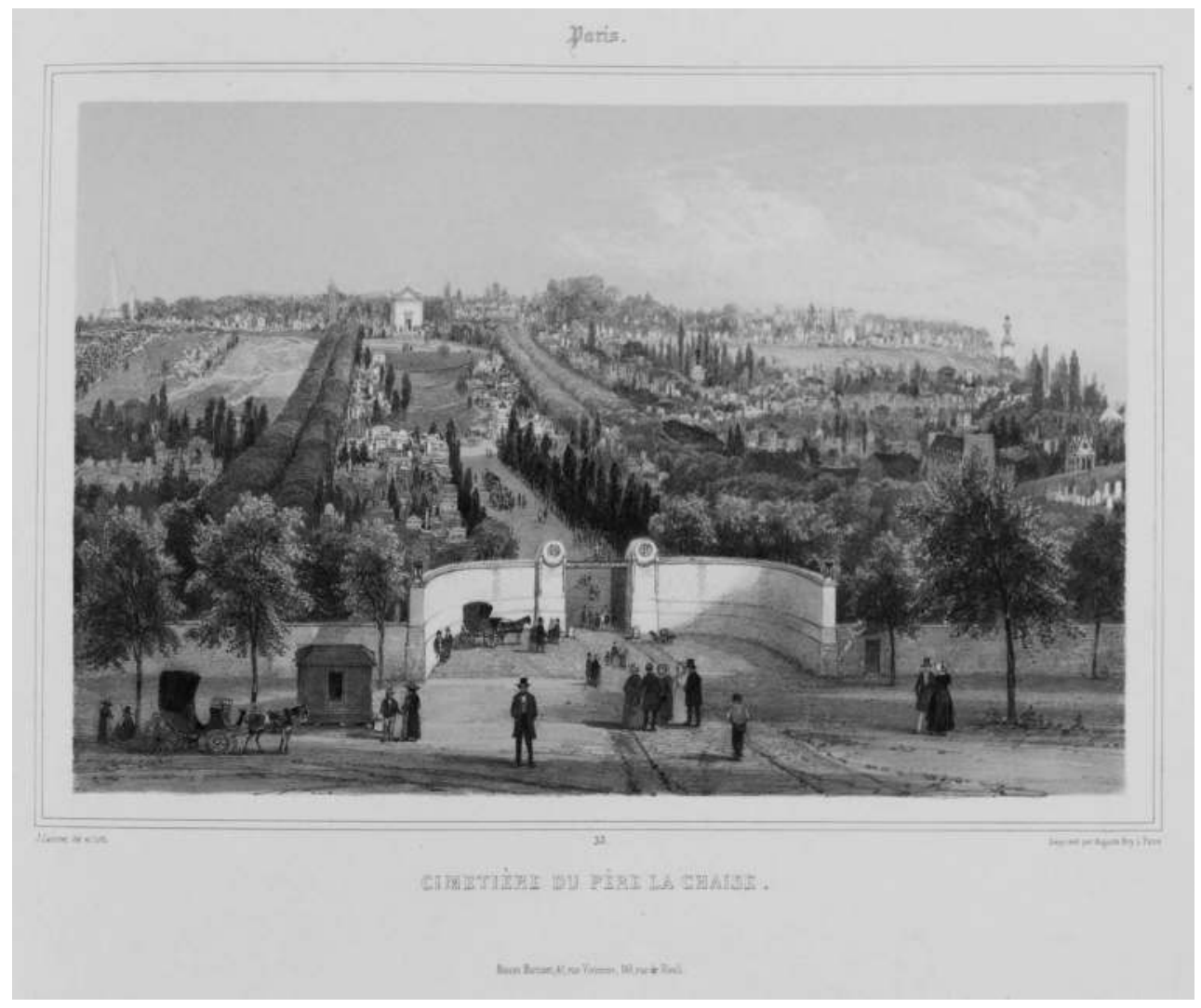

Image 7.1 Père Lachaise cemetery in the middle of the nineteenth century.

J. Jacoltet, Paris et ses environs (Paris, 1856). The Newberry Library, Chicago (folio G 397.66)

church, Père Lachaise was a stand-alone and intentionally landscaped space. "This cemetery," one French visitor explained in 1817, "is nothing like the terrible and terrifying cemeteries that we used to see." Visitors to it need not fear any "putrid miasmas" or lethal exhalations. Instead, they would find "a vast picturesque garden" filled with winding paths, bucolic scenes, and the scent of violets, lilacs, and honeysuckle. ${ }^{19}$ On a sensory level, the difference between the new modern cemetery and the old churchyard was stark.

Other than sweet flowers and charming paths, visitors to Père Lachaise would have witnessed an unprecedented number of tombstones, from simple wooden crosses to elaborate family mausolea. This reflected the other major shift in burial practices since the eighteenth century: the modern cemetery prioritized individual, rather than communal burial. According to the Napoleonic Decree, each burial was to take place in a specially dug individual grave, of approximately 2 meters deep and 80 centimeters wide. Graves were to be separated by a gap of at least 30 centimeters and to remain closed for at least five years. Although this sounds like a remarkably egalitarian plan-befitting an age of democratic revolution - in practice, burial culture at Père Lachaise was remarkably 
hierarchical. This stratification was owed primarily to the invention of "concessions" burial plots that could be purchased or rented by those seeking "a distinct and separate space" within the cemetery. ${ }^{20}$ Unlike churchyard burial, which was largely anonymous, collective, and temporary, burial in Père Lachaise offered an individualized and permanent place for the dead. This valorization of individuality and the "rights" of the dead was a clear reflection of the massive political and social changes that swept through Europe during the age of Enlightenment and Revolution.

Although concessions only accounted for a small percentage of annual burials at Père Lachaise, they quickly became the cemetery's main attraction. Tour guide books, directing potential visitors to the sights and routes of Père Lachaise began appearing shortly after the cemetery opened its gates in the first decade of the nineteenth century. With names including Promenade to the Cemeteries of Paris, Serious Promenade to the Cemetery of Père Lachaise, and Religious and Sentimental Travels to the Four Cemeteries of Paris, macabre travel writers of the early nineteenth century created and catered to a budding industry of cemetery tourism in the French capital. ${ }^{21}$ For their readers, these authors described and illustrated some of the most notable graves at Père Lachaise, either because they contained famous inhabitants, such as the ill-fated medieval lovers Abélard and Héloïse, or particularly effective burial monuments, remarkable for their artistic design and/or evocative epitaphs. By the 1820s most general guidebooks to Paris included substantial entries for Père Lachaise, indicating that it had become as much of a draw to visitors as an established historic site like Notre Dame Cathedral.

Many of descriptive guides to Parisian cemeteries were also quite prescriptive, in both the epitaphs they transcribed and the commentary they offered. This reflects both the epitaph culture that flourished in the nineteenth century and a widespread desire to turn cemeteries into "schools for the living." 22 Accordingly, epitaphs constantly reaffirmed useful social values: they commended the deceased for being excellent spouses, parents, and friends. For example, Antoine Albaret's tomb (1750-1813) proclaimed that anyone who took his life as a model ("good since childhood, he never wavered, he was a good husband and father") would find happiness. ${ }^{23}$ It was very common to describe a woman as "an example of virtue ... the best of wives and the most sincere of friends" a man as "the best of husbands, the tenderest of fathers, and the most sincere of friends." 25 Other epitaphs included a tremendous amount of biographical detail about the lives of the deceased. For example, Regnault de Saint-Jean-D'Angely commissioned a cenotaph and a cinerary urn to commemorate his death. His epitaph provided a detailed retelling of his major life events. One learned not only about his birth and death but also about his election to the constituent assembly (1789), his proscription (1794), his various promotions in the Napoleonic State (1799-1815), and the exile (1815) that may have eventually led to his death (1819). As one author who drew and described the tomb noted, "What more could we add to the story of M. le compte Regnault de Saint-Jean d'Angely's life? Everything is written out on his tomb."26

In an 1825 guide to the city of Paris, Jacques-Antoine Dulaure commended the civicmindedness of so many of the city's epitaphs: "Previously," he explained, "we would have thought it better to commend the dead not for his virtues, but for his titles, his dignities, and the signs of his power, but such feudal pride is in short supply at Père Lachaise." 27 Feudal pride may have been in short supply, but civic pride and private accomplishments were clearly on full display. 


\section{A global cemetery revolution}

The cemetery revolution that started in Paris quickly spread, or had counterparts, across the world. Most obviously this included other European rural cemeteries, but the French cemetery revolution also influenced urban policy across the Atlantic in the new American Republic, as well as in Egypt, which Napoleon attempted to conquer in 1798. When the French invaded Egypt at the end of the nineteenth century, they encountered a very different burial culture that fascinated and outraged their Enlightenment sensibilities in equal measure. As one scholar has recently described it, "Historically, Egyptians refuse to see the graveyard as a dark, evil, and forbidden place. No atmosphere of fear or danger surround the cemetery. The cemetery has always been an active part of the community." ${ }^{28}$ In stark contrast to their European invaders, Egyptian populations did not exhibit the same aversion to living in close proximity to the dead. Quite to the contrary, Egyptian mourning and burial culture was structured to facilitate regular and sustained contact with the dead: cemeteries inside and outside of city limits had long featured multi,story house-like burial structures for families featuring subterranean chambers for the dead and above-ground sitting and sleeping rooms for the living who visited them for extended periods of time. These "villes des tombeaux" (cities of the dead) were allegedly so well-known in Europe by the time of the French invasion, that the authors of the Napoleonic text Description de l'Egypte did not think they warranted much explanation. Known locally as Al Qarafa, these cemeteries appeared as a dense network of funerary dwellings, connected by streets and courtyards, which looked like a veritable a city unto itself. ${ }^{29}$

As a consequence of the brief French occupation of Egypt in 1798-1799, all cemeteries located within Cairo's walls were destroyed and their remains relocated to the large and centuries old "City of the Dead" to the East of the City. However even after the French were gone, the European influence remained, and similar policies continued to be enacted throughout the nineteenth century. Most notably, this included a new policy under Muhammad Ali that attempted to limit activities in the City of the Dead to things directly related to burial and commemoration. In practice, this prohibition was supposed to prevent people from spending extended periods of time in the cemetery dwellings. While it may have been successful in the short term, this prohibition was ultimately unsuccessful, and the cemeteries were gradually "repopulated" by the living in the late nineteenth century. By the twenty-first century there were at least half a million people living in Cairo's City of the Dead. However, it is worth noting that the European moralizing stigma remained, and the presence of the living in the City of the Dead has been interpreted as a problem since the nineteenth century. As the authors of one of the few scholarly works about Cairo's City of the Dead explained it, "Only in the past few centuries, after fourteen hundred years of being fixated on their final resting places, have the residents of Cairo seen those places as an obstacle to their city's development." 30 The expectation that special spaces for the dead, like cemeteries, need to be separate and distinct from living communities has been, since the nineteenth century, embedded in Western cultural expectations.

While cemeteries kept the dead a safe distance from the living, they nevertheless expected, invited, and catered to visitors, as the previous discussion of Père Lachaise Cemetery made clear. Rural cemeteries in the model of Père Lachaise gradually took over European and North American burial culture throughout the nineteenth century. 
Perhaps the most well-known American cemetery of this era was Cambridge, Massachusetts's Mount Auburn Cemetery, which opened to the public in 1831 and was modeled explicitly on Père Lachaise. ${ }^{31}$ It in turn inspired many other famous "suburban cemeteries," including Brooklyn, New York's Greenwood Cemetery, which opened in 1838; London's "Magnificent Seven" cemeteries, which all opened between 1833 and 1841; and Ohlsdorf Cemetery in Hamburg, which opened several decades later in 1877 and still enjoys the distinction of being the largest rural cemetery in the world. An early twentieth-century guide to the city for English-language visitors described the "Burial-grounds at Ohlsdorf" in what should by now be familiar terms: "The grounds ... rather resemble a beautiful park than a burial-ground ... A rich vegetation, comprising limes, beeches, oaks, and flowers from various climes, abounds, and the effect of the scenery is augmented by the splendid monuments." Sensitive tourists were further directed to "picturesque bridges" a world-class rose-garden, and-lest one forget that this was after all a space of the dead-one of the oldest crematoriums in Germany. ${ }^{32}$

As an abundance of guidebooks for local and foreign visitors attest, much like Père Lachaise, these nineteenth-century rural cemeteries became famous attractions in the cities in which they were located, and one could purchase any number of books or maps to guide a real or textual visit through their tomb-lined alleys. And, as the many nineteenth-century guidebooks dedicated to these sites illustrate, these cemeteries all shared a similar bucolic aesthetic and strongly appealed to their audiences' Romantic sensibilities. For example, an early guidebook by Nathaniel Dearborn to Mount Auburn cemetery described "the grounds" as "mostly overshadowed by foliage of large forest trees, the whole combining to affiliate the spot, as a suitable place for the living to visit and there ponder on the ever changing state of man's mortality." 33

\section{Cemeteries and social cohesion}

As Nathaniel Dearborn clearly illustrates, for many who visited them, cemeteries provided opportunities to "ponder" and contemplate sublime thoughts related mortality. This initially sounds like a fundamentally private and isolating experience. However, this same macabre sensibility could also function as a kind of social cohesive, tying community members together with powerful affective bonds. These bonds had multiple strands, but the strongest were empathy and history - both of which make perfect sense given the romantic and nationalist ethos of the nineteenth century. To illustrate how effectively cemeteries evoked and facilitated empathy, one need look no further than the first ever guidebook to Parisian cemeteries, Antoine Caillot's heavy-handed moral tract, Voyage religieux et sentimentale aux quatre cimetières de Paris, first published in 1808. Caillot's book is an extended rumination on his emotional turmoil as he passes through each of Paris's extant cemeteries, but he also makes several concerted attempts to connect with his fellow Parisians, strangers to him. The most extreme example of this occurred in the midst of Caillot's journey to Père Lachaise, when he came across the tombstone of a 25-year-old woman named Anne-Josephine François Bontemps. After translating her epitaph, which described a dearly missed daughter, sister, friend, wife and mother, Caillot described his reaction: he sat down on the bench behind her grave overcome with "sympathy" 34 and began to imagine that he had personally suffered the "deplorable loss" of this young woman. Tears fell from his eyes and his head dropped to his chest, overwhelmed by grief. $^{35}$ 
On a grander scale, cemeteries can also act as cohesive spaces by providing a sense of continuity across time. Despite the many changes that the cemetery has undergone over the last few centuries, several key features remain constant. Most notably, the cemetery (and before it, the churchyard) has always been an important liminal space, bridging the gap between the living and the dead, and providing a special protected space where the two can remain linked. Many have reflected on this function using similar terminology. Michel Ragon referred to cemeteries as "that space of passage between the space of the living and that of the dead." "36 While Lloyd Warner imagined cemeteries as a metaphorical bridge, linking generations across time, “Today's dead are yesterday's living and today's living are tomorrow's dead. Each is identified with each other's fate." "37 Using similar language, one early nineteenth-century visitor to the Paris Catacombs (a subterranean "cemetery" par excellence) often described being surrounded by the city's past, present, and future. ${ }^{38}$ At the beginning of his Guide to Kensal Green Cemetery, H.J. Croft expounded at length about the important liminal space that the cemetery occupied, linking the past with the present and future. "Places of human sepulture," he explained, "are silent records of the past and stern tokens of the future. The solemn breath of history lingers about their sacred precincts, and the evidence of mortal doom is graven on their every stone." This type of memento mori was always associated with burial places, but Croft quickly moves beyond this point and gets to the material value of cemeteries: "How many a missing link in the narrative of bygone ages have the tombs of the ancient worlds supplied!" But even closer to home, he explained, "Every village graveyard is a little history." 39

Of course, this connection between the past, present, and future is not simply a reference to the material remains of the dead but also to the carefully managed relationship between the past and the present that occurs in the unique space of the cemetery. As Elizabethada Wright has argued, the cemetery can easily and usefully be "read" as a "lieu de mémoire," precisely because of the combined stories cemeteries can tell about the past and the present. "Reading the cemetery, we think we see the past, but what is restored, what is seen, and what is saved is as much a product of the present as it is a product of the past." ${ }^{40}$ Cemeteries are never just places to contain and commemorate the dead; they are also valuable social junctions that bring the living into contact with each other, the dead, and their shared past. Cemeteries are spaces of cohesion that reinforce a given society's accepted values, customs, and practices, while excluding others. Cemeteries are unique and powerful sites that construct, reflect, and challenge the communities that they serve.

\section{Conclusion: new directions in cemetery culture}

If the rural cemetery movement reflected the transforming needs, values, and interests of a post-Enlightenment era, it should come as no surprise that burial practices have continued to evolve and change in reaction to broader political, social, and cultural changes. The first big challenge to the rural cemetery came in the twentieth-century United States. As spaces such as Mount Auburn or Greenwood indicate, the rural cemetery certainly flourished in the nineteenth century; however, by the twentieth century, Americans had spearheaded another shift in burial culture that reimagined large-scale spaces for the dead yet again. As a consequence of an increasingly business- and entrepreneurialoriented American cemetery culture, American cemeteries in the twentieth century 
transformed into what scholars have called lawn-park cemeteries. These new cemeteries emphasized sparsely landscaped rolling hills of sod, rather than the crowded and lushly verdant rural landscapes of the nineteenth century. Memorial parks took this a step further by eliminating many upright grave markers altogether, opting instead for simple bronze or granite plates flush with the earth. These memorial parks replaced cluttered eclecticism with simple uniformity and made regular maintenance of cemeteries much easier and more efficient: it was much easier to mow the grass around a memorial plate set into the ground than around an elaborate obelisk and flowering garden. ${ }^{41}$

More recently, in the twenty-first century, there has been a renewed interest in alternative modes of burial that substantially reduce the amount of waste and pollution caused by chemically treated bodies and the receptacles that contain them. The increased use of cremation obviously challenges cemetery burials, but there are other innovations beyond this. For example, when the American actor Luke Perry died in 2019, his family honored his request to be buried in a "mushroom suit," part of a process called mycoremediation, in which the suit slowly consumes the corpse, leaving behind only a plot of mushrooms where the body is buried. ${ }^{42}$ This innovation came out of a media-savvy tech start-up, whose fusion of futuristic science and eco-friendly practices seems to be as much a reflection of our contemporary era as tree-lined alleys and individual burial plots did in 1804 when Père Lachaise Cemetery opened to the public.

\section{Notes}

1 The oldest known grave is in Taforalt cave in Morocco. It contains the remains of at least 34 individuals and is over 15,000 years old.

2 An excellent collection that describes and analyzes many of these forms of burial is The Oxford Handbook of the Archaeology of Death and Burial, eds. Sarah Tarlow and Liv Nilsson Stutz (Oxford: Oxford University Press, 2013).

3 Julie Rugg, "Defining the Place of Burial: What Makes a Cemetery a Cemetery?" Mortality 5: 3 (2000), 264.

4 H.J. Croft, Guide to Kensal Green Cemetery (London: Howell, 1881), 10.

5 The first published version of Foucault's essay appeared as "Des espaces autres" in the October 1984 issue of the French journal Architecture-Mouvement-Continuité, based on a public lecture Foucault gave in March 1967. The English translation appeared two years later as "Of Other Spaces."

6 Michel Foucault, "Of Other Spaces," trans. Jay Miskowiec Diacritics 16: 1 (Spring 1986), 27.

7 Philippe Ariès makes much of the rumored prostitutes who circulated around Paris's Cemetery of the Innocents in the twelfth century. The Hour of Our Death, trans. Helen Weaver (New York: Knopf, 1981), 70.

8 Thomas Laqueur, The Work of the Dead: A History of Mortal Remains (Princeton, NJ: Princeton University Press, 2015), 138.

9 Anthony Perron, "The Medieval Cemetery as Ecclesiastical Community: Regulation, Conflict, and Expulsion, 1000-1215," in Dealing with the Dead: Mortality and Community in Medieval and Early Modern Europe (Leiden: Brill, 2018), 273.

10 Vanessa Harding, The Dead and the Living in Paris and London, 1500-1670 (Cambridge: Cambridge University Press, 2002), 11.

11 Christine Métayer, "Une espace de vie: les charniers du cimetière des SS. Innocents à Paris sous l'Ancien Régime," Fournal of the Canadian Historical Association 4: 1 (2003), 187.

12 Bibliothèque National de France, Collection Joly de Fleury 1209, "Avis des officiers du Châtelet au sujet des cimetières," f.14v.

13 Blanche Linden-Ward, Silent City on a Hill: Landscapes of Memory and Boston's Mount Auburn Cemetery (Ohio State University Press, 1989), 29. 
14 Alain Corbin, The Foul and the Fragrant: Odor and the French Social Imagination (Cambridge, MA: Harvard University Press, 1986), 58.

15 Thomas Laqueur first made this argument in "The Place of the Dead in Modernity," in The Age of Cultural Revolutions in Britain and France, 1750-1820, eds. Colin Jones and Drohr Wahrman (Berkeley, CA: University of California Press, 2002), 17-32.

16 For more on how Parisians responded to the closing of the Cimetière des Innocents, see ErinMarie Legacey, Making Space for the Dead: Cemeteries, Catacombs, and the Reimagining of Paris, 1780-1830 (Ithaca, NY: Cornell University Press, 2019).

17 I describe and analyze all of these proposals for new burial practices in chapter two of Making Space for the Dead. They are also addressed by Pascal Hintermeyer in Politiques de la mort: Tirées du concours de l'Institute Germinal an VIII-Vendémiaire an IX (Paris: Pavot, 1980).

18 Napoleon Bonaparte, "Décret impérial sur les Sépultures," 23 prairial an XII, Collection des lois, senatus-consultes, décrets impériaux et avis du conseil d'état relatifs aux cultes; publiés depuis le concordat jusqu'au 1er janiver 1813 (Paris: Rivals, 1813), 180-184.

19 C.-P. Arnaud, Receuil de tombeaux des quatre cimetières de Paris, avec leurs epitaphs et leurs inscriptions, vol. I (Paris, 1817), 81, 85.

20 Napoleon Bonaparte, "Décret impérial sur les Sépultures."

21 M.P. St.-A [Pierre Pietresson de Saint-Aubin], Promenade aux cimetières de Paris, aux sépultures royales de Saint-Denis, et aux Catacombes (Paris, 1816); François-Marie Marchant de Beaumont, Vues Pittoresques, historiques, et morales du cimetière du P. La Chaise Desinée par MM. Vigneront et Duplat, et gravées a l'aqua-tinta par M. Jazet et les meilleurs artistes (Paris, 1821); Antoine Caillot, Voyage religieux et sentimentale aux quatre cimetières de Paris (Paris, 1809).

22 This concept was first articulated by the French political writer Pierre-Louis Roederer in 1795. Pierre-Louis Roederer, Des institutions funéraires convenables à une république qui permet tous les Cultes, et n'en adopte aucun: mémoire lu par Roederer dans le séance publique de l'Institut national des Sciences et des Arts, le 25 Messidor, l'an IV (Paris: B. Mathey, 1795), 11.

23 M.M. Roger, père et fils, Le Champ du repos, ou le cimetière Mont-Louis dit du Père Lachaise, ouvrage orné avec de planches, représentant plus de 2000 mausolées érigés dans ce cimetière, depuis sa création jusqu'au 1er janvier, 1816, avec leurs épitaphes; son plan topographique, tel qu'il existait du temps du père delachaise, et tel qu'il existe aujourd'hui; précédé du portrait de ce jésuite, d’un abrégé de sa vie; et suivi de quelques remarques sur la manière dont différents peuples honorent les défunts (Paris, September 1816), vol 2, 121.

24 Ibid., vol 2, 25.

25 Ibid., vol 2, 370.

26 F.-G.-T. de Jolimont, Les Mausolées français: recueil des tombeaux les plus remarquables par leur structure, leurs épitaphes ou les cendres qu'ils contiennent, érigés dans les nouveaux cimetières de Paris (Paris: FirminDidot, 1821). There are no page numbers in this book.

27 Jacques-Antoine Dulaure, Histoire physique, civile, et morale de Paris: depuis les premiers temps, tome quatrième (Bruxelles: Baudouin, 1825), 234-236.

28 Jeffrey Nedoroscik, The City of the Dead: A History of Cairo's Cemetery Communities (Westport, CT: Bergin and Garvey, 1997), 6.

29 Description de l'Egypte, ou recueil des observations et des recherches qui ont été faites en Égypte pendant l'expédition de l'armée Française 2nd ed. vol. 18 (Paris: Panckoucke, 1829), 347.

30 Galila El Kadi and Alain Bonnamy, Architecture for the Dead: Cairo's Medieval Necropolis (Cairo: The American University in Cairo Press, 2007), 36.

31 Stanley French, "The Cemetery as Cultural Institution: The Establishment of Mount Auburn and the 'Rural Cemetery' Movement," American Quarterly 26: 1 (March 1974), 37-59.

32 Hamburg and its Environs: Practical Guide, 7th ed. (Hamburg: Verlagsanstalt und DruckerelGesellschaft, 1913), 54-55.

33 Nathaniel Dearborn, A Concise History Of, and Guide Through Mount Auburn (Boston, MA, 1843$), 5$.

34 As Lynn Hunt has pointed out "sympathy" is this context is analogous to "empathy" as we understand it. "Empathy" didn't enter into common usage until the twentieth century. Lynn Hunt, Inventing Human Rights: A History (New York: W.W. Norton \& Co., 2007), 65.

35 Antoine Caillot, Voyage religieux et sentimentale aux quatre cimetières de Paris (Paris, 1809), 225.

36 Michel Ragon, The Space of Death: A Study of Funerary Architecture, Decoration, and Urbanism, trans. Alan Sheridan (Charlottesville, VA: University Press of Virginia, 1983), 65.

37 Loyd W. Warner, The Living and the Dead (New Haven, CT: Yale University Press, 1959), 286. 


\section{THE GEMETER Y}

38 For more on the Paris Catacombs as a space of the dead see: Erin-Marie Legacey, "The Paris Catacombs: Remains and Reunion Beneath the Post-Revolutionary City," French Historical Studies 40: 3 (August 2017), 509-536.

39 H.J. Croft, Guide to Kensal Green Cemetery, 5.

40 Elizabethada Wright, "Reading the Cemetery, 'Lieu de Mémoire par Excellence," Rhetoric Society Quarterly 33: 2 (Spring 2003), 29.

41 For more on American cemeteries in the contemporary era, see David Charles Sloane, The Last Great Necessity: Cemeteries in American History (Baltimore, MD: Johns Hopkins University Press, 1991).

42 For more on the process of mycoremediation and green burial practices more generally, see Katie Rogers, "Mushroom Suits, Biodegradable Urns, and Death's Green Frontier," The New Tork Times (April 22, 2016), https://nyti.ms/1WKob31 


\title{
DEATH, GOMMEMORATION, AND THE ERA OF TOTAL WAR IN EUROPE
}

\author{
Jesse Kauffman
}

The modern history of war intertwines with death in many ways. There is, first of all, the periodization of war itself: major conflagration around the French Revolution and Napoleon, then for a century a number of smaller wars including imperialist struggles, then the surge of the great world wars, followed by a return to more limited conflicts. Regional variation matters: some areas have been much more war-prone than others. The steady increase in the lethality of weapons, from Napoleon's mobile field artillery to the machine guns and then aerial bombardments of more recent conflicts, intensifies the level of death, including, from the 1930s onward, deaths of civilians. Major modern wars are not the deadliest in history, per capita, but they can concentrate massive casualty rates in relatively short spans of time. ${ }^{1}$

Modern war has also seen steady advances in military medicine, from the growing roles of doctors and nurses in the Crimean War and American Civil War onward, both reflecting and causing new approaches to military death. Other movements have sought to limit certain types of military violence, as in the efforts of Geneva Conventions (from 1864 onward) to improve treatment of the wounded and those taken prisoner. Even fictional representations of modern war, including toy soldiers, films, and recent video games, deserve consideration, often providing the most frequent contact with images of death for many young people and generating fervent if inconclusive debates about desensitization.

Finally, the impact of modern war has deeply affected broader attitudes to death and grief, leading in turn to distinctive forms of commemoration and efforts to guide or manipulate public memories of those who fell in combat. This essay, focused on European reactions in the crisis decades of the twentieth century, deals with the changes involved in this evolving connection between war and death.

$* * * * * * *$

In the first decade of the twentieth century, the French sociologist Robert Hertz distinguished between two distinct processes to which the dead are subjected, the first biological and the second cultural. The first - the decay of the deceased's mortal remains - is a relatively straightforward matter of nature taking its course and, as such, does not vary substantially from one society to another. But the cultural processes which set to work on the dead are in many ways more complex. They are fitted into memories, both individual and collective, and are thereby made part of a story to which they give meaning, and which, in 
turn, gives their memory meaning. This aspect of death, the aspect of memory and meaning, is always embedded within a larger culture and therefore subject to considerable variation across time and space. ${ }^{2}$

For those societies caught up in the cataclysmic violence that swept Europe between 1914 and 1945, attending to both aspects of the aftermath of death-the physical and the cultural-proved overwhelming. The wars killed millions of soldiers (including Robert Hertz, who died defending France in 1915) whose remains had to be collected, identified, and interred. ${ }^{3}$ In addition, the sheer scale of the slaughter fueled an oftenanguished search for meaning in both the public and private realms. This search continues, with varying intensity according to circumstances and place, in Europe today.

\section{The First World War}

In Europe, the First World War marked a major turning point in the history of death and its commemoration. This was due in part to the appalling level of human loss, and in part to the historical moment in which these losses occurred. Military deaths amounted to some 10 million in total. This number, when broken down into percentages of land forces killed for some of the belligerents, gives a sense of what the toll meant for each army: Russia and Great Britain each lost over $11 \%$ of their soldiers, while $15 \%$ of German soldiers and a staggering $17 \%$ of French soldiers were killed. ${ }^{4}$ The French historian Antoine Prost has recently illustrated how the grim practicalities of wartime death rapidly overwhelmed the wholly inadequate systems set up to deal with them. From the first weeks of the war, belligerents had to grapple with questions such as where the swiftly multiplying dead were to be buried and how families were to be notified. ${ }^{5}$ At the same time, the political and cultural context in which these deaths occurred led to an unprecedented culture of remembrance and mourning. These wartime deaths occurred in an age of widespread literacy and popular political participation, and so translating them into a meaningful sacrifice for a greater cause became a political necessity for states before and after the war. More broadly, within the history of death, these mass casualties occurred during the expanding age of what Walter Laqueur has recently called "necronominalism," that is, the belief that all dead should be recorded and remembered by name - partly as a result of the increasing sweep of public and private institutions concerned with maintaining such records, and partly as a result of changing views on the inherent worth of every individual life, no matter how obscure or lowly. Names, Laqueur argues, came to do the cultural work that human bodies once did, creating meaning as well as a sense of community that transcends time. ${ }^{6}$ The collision of these two forces - of the staggering physical realities of mass death and the cultural forces of grief and the search for meaning - produced a rich and charged culture of commemoration and memorialization in Europe after the First World War, giving rise to sites where public and private grief, rage, pride, anger, or despair could all mingle and amplify each other. ${ }^{7}$

\section{Commemoration and military cemeteries}

One of the most significant shifts in the way that wartime death was treated during and after the Great War was the attention paid, both physical and cultural, to individual sacrifice. George Mosse, one of the most influential scholars of war, death, and commemoration in Europe, has noted that in the nineteenth century, when fallen soldiers were 
commemorated, it was usually with some sort of collective monument, such as the one erected in Berlin for those who had died in the Napoleonic Wars. But even if a handful of monuments to individual dead existed, this did not reflect any sort of actual care for their physical remains. In the wake of the much-mythologized Battle of the Nations in Leipzig, for example, a visitor "came upon the naked corpses of the so-called fallen heroes lying in a schoolyard to be eaten by ravens and dogs." ${ }^{\text {A }}$ Abandonment, anonymity, and mass graves were the norm for dead soldiers in the nineteenth century, as they had been for centuries. This changed in the First World War, when both the material and cultural commemoration of the individual became the norm. However, even as the First World War drew attention to individual sacrifice, it also subsumed those individual sacrifices within a wider interpretive framework of collective sacrifice for a greater good - the nation. Few sites of commemoration exemplified this fusion of the individual with the collective as vividly and tangibly as Europe's new military cemeteries.

The park-like, carefully tended military cemeteries that are such a pronounced feature of the western European cultural landscape are a product of both the Great War and changes in cemetery construction. At the outset of the war, the dead were often buried, in great haste, in makeshift graves near where they fell. Still, as Antoine Prost has pointed out - confirming Laqueur's arguments about the age of necronominalism - pains were usually taken to leave some sort of marker of individual identity, such as a rifle jammed into the ground as a kind of makeshift headstone, a hastily constructed memorial or sign of some sort, or personal belongings. ${ }^{9}$ As the war continued, however, armies and the states that directed them began to regularize their processes of identifying and burying their dead, with individual markers, in specially designed cemeteries. With their long rows of identical gravestones, these cemeteries blended military order and unity with the preservation of individual identity, creating a kind of vast monument to the ferocious appetites of twentieth-century warfare. ${ }^{10}$ In addition, new institutions were established to provide official oversight and maintenance of these graveyards, the best known of which is probably the Imperial War Graves Commission, now the Commonwealth War Graves Commission. ${ }^{11}$

The novelty of these cemeteries did not lie only in their purpose. They also incorporated changes in in the landscaping and geography of cemeteries - which in turn reflected deeper cultural changes in the history of death - that had been ongoing since the eighteenth century. One shift had come about as a result of hygiene concerns; graveyards within cities had become malodorous, and so Europeans had become accustomed to burying their dead in large cemeteries removed from the confines of the urban landscape, as the war cemeteries came to be. Cemeteries also became secularized in a way they had not been before. Drawing on the Enlightenment and, even more so, Romantic ideals, their design came to replace explicitly sacred symbolism with landscaping that emphasized the connection between human life and the deeper rhythms of nature. This connection could be emphasized in different ways. Paris's Père Lachaise, for example, was carefully sculpted, while Munich's Waldfriedhof adopted a wilder, less obviously planned approach to the integration of graves and landscape. Both would exercise a strong influence on the design of military cemeteries in and after the First World War. ${ }^{12}$

\section{Other forms of commemoration}

Cemeteries, however, were, in a peculiar sense, for the fortunate: for those whose remains could be recovered, identified, and buried. The sheer destructive power brought 
to bear on human bodies during the Great War, however, meant that many who went off to war simply disappeared, their bodies pulverized and pounded into the mud by artillery, or their makeshift graves destroyed by waves of successive fighting. Even when remains were found, however, they were often in such a state that they could not be identified. From the tension between the need to acknowledge each individual loss and the impossibility of doing so under the conditions of the First World War arose one of the war's bestknown and enduring innovations: the figure of the Unknown Soldier. In the early 1920s, Great Britain and then France each selected an unidentified corpse to represent the sacrifice of all the dead and to provide a kind of national focal point for the intense emotions left in the wake of the war's human devastation. ${ }^{13}$ The practice soon spread, and to this day Tombs of the Unknown Soldier remain sites of intense emotional attachment as well as solemn political and military ceremonies in places as diverse as the United States, Poland, the Dominican Republic, and India. ${ }^{14}$

The aftermath of the First World War also gave rise to massive memorials, primarily in Belgium and Northern France, that were something of a cross between a monument to the unknown and a cemetery. These were the massive monuments to the missing. Often listing tens of thousands of names, these memorials were established near the sites of battles and gave the names of men believed to have died fighting in or around that region. These names belonged to both the thousands of unidentified soldiers who were buried nearby in graves without names on the headstones, as well as those who had disappeared. ${ }^{15}$ One of the most well-known of these monuments is the Menin Gate, which lists the names of over 50,000 missing men, nearly all of whom almost certainly

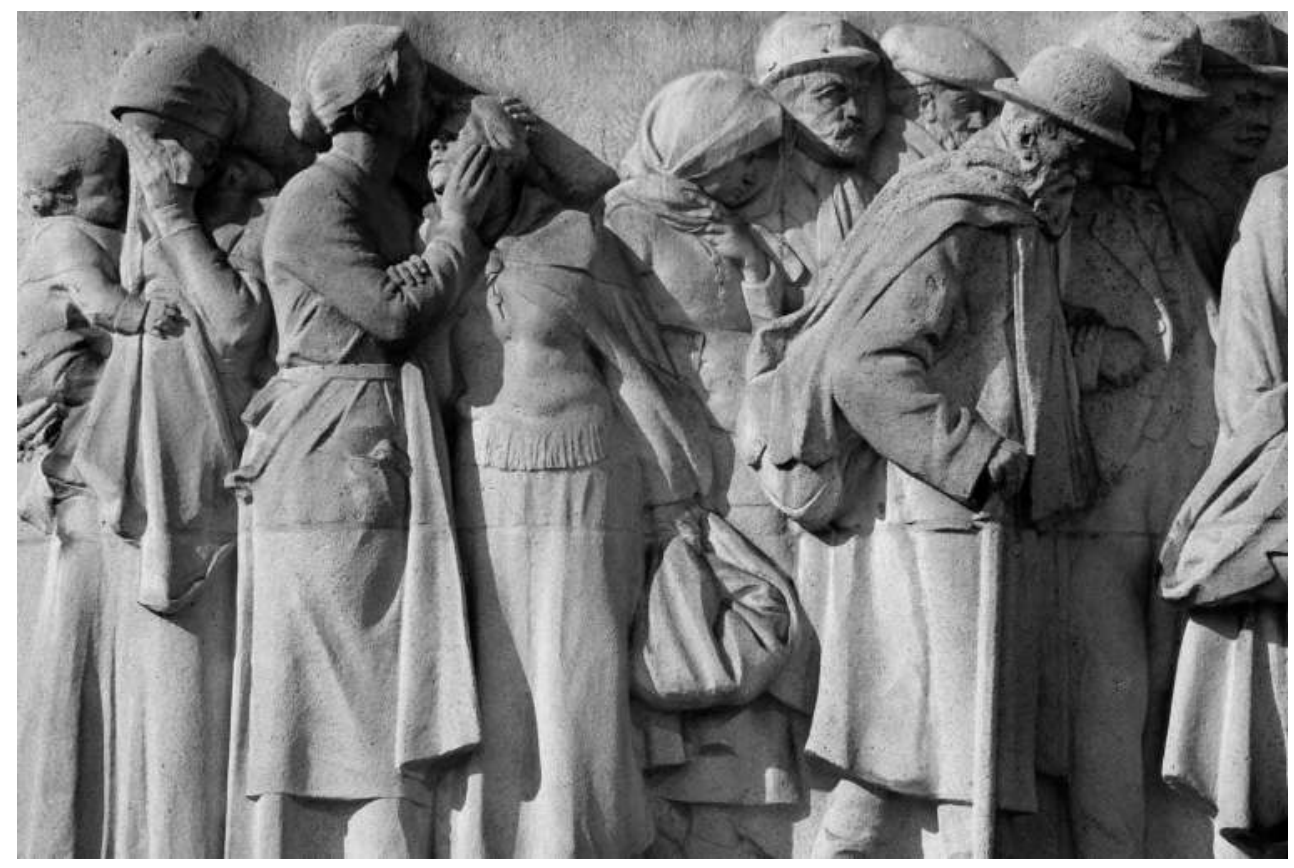

Image 8.1 First World War Memorial in Lille, France.

(C) robertharding/Alamy Stock Photo. 
died in the horrific killing fields around Ypres. In his recent book The Work of the Dead, eminent cultural historian Thomas Laqueur provides an engrossing account of what he calls not only the "emotional infrastructure" that went into the creation of monuments such as these but also of the concrete practicalities that had to be sorted out. His evocation of the skilled craftsman trying to calculate what size to make each name, so that all could eventually fit, is both powerfully illuminating and deeply disturbing. ${ }^{16}$ Not everyone was impressed by the result. "Here was the world's worst wound," wrote the veteran and poet Siegfried Sassoon. ${ }^{17}$

Even Sassoon, however, was at least ambiguous in his feelings towards the most successful of the postwar memorials, the Cenotaph in London. Designed by Sir Edward Lutyens, the Cenotaph - "empty tomb"-is based on ancient Greek models, and has no obvious connection to any religious or national tradition. A stone sarcophagus sits atop a long, narrow, tiered tower; simple stone wreaths on top of the sarcophagus as well as on each end of the tower are the only decorative elements. The inscriptions are simple: the dates of the war and the single phrase THE GLORIOUS DEAD. It was not meant to be a permanent memorial. A temporary version was first erected in 1919, to serve as a kind of prop for a victory parade. The overwhelming public response to it, however, led authorities to have a permanent monument built. In the astute analysis of Jay Winter, it was precisely the simplicity of the Cenotaph's design that was the key to its emotional power, since it provided a focal point into which people could read whatever mix of grief and pride they felt. "It is a form," Winter writes, "on which anyone could inscribe his or her on thoughts, reveries, sadnesses. It became a place of pilgrimage, and managed to transform the commemorative landscape by making all of 'official' London [it is on Whitehall] into an imagined cemetery.", In "At the Cenotaph," Sassoon imagines Satan saying an infernal prayer at the monument, asking God to "make them forget ... what this Memorial/Means; their discredited ideas revive." And so along with millions of others in Great Britain, Sassoon was able to imagine an essential message within the Cenotaph, in his case one that war is an unmitigated horror.

In Germany prior to the rise of the Nazis, no central memorial to the dead of the Great War ever emerged, despite numerous attempts to create one. The reasons were numerous, including the intense political and emotional animosities stirred by the lost war and the Treaty of Versailles. Regionalism and the federal structure of the German state also played a role. The memorial created in Berlin in the neoclassical Neue Wache-originally a guardhouse monument to the victory over Napoleon - was called the "Prussian Memorial." A bleak stone chamber in which a skylight directed the sun onto a single symbolic square block topped by a wreath, the memorial never gained much of a foothold in the public mind. ${ }^{19}$ Nor did another of postwar Germany's memorials command much public attention. This was Johannes and Walter Krüger's monument to the Battle of Tannenberg, a victory won by the Germans over the Russians in August 1914. Inaugurated in 1927, the memorial took the form of an octagonal fortress with eight massive stone towers. Twenty unknown German soldiers were buried within it. However, the monument was more readily associated with Paul von Hindenburg, the general who had commanded German forces at the battle and whose name was thereafter permanently linked with its glory. When Hindenburg died in 1934, the Nazis buried him (against his wishes) in the monument, accompanied by an elaborate, carefully choreographed public spectacle. The Nazis thereby laid claim to the legacy of the Great War and to Hindenburg's renown. ${ }^{20}$ Enhancing the legitimacy of the National Socialist regime was the "work" to which Hindenburg's mortal remains were put after his death. 


\section{Memorials and meaning}

As Jay Winter notes, the broad public outpouring of feeling that transformed the Cenotaph from a temporary to a permanent memorial highlights the important role played by war memorials, especially national memorials, after the war. They became places where communities engaged in collective mourning for their loved ones, but they also became lightning rods, and perhaps amplifiers, for the unfathomable depths of grief, rage, bitterness, and resentment that many Europeans felt in the wake of the First World War. "For many Europeans," James Sheehan has noted, "the private and public legacies of the war were fused, their personal sorrow seamlessly joined to their political frustrations and discontents." This contributed to the general mood of anger, resentment, and disillusionment that beset the continent in the 1920s and 30s and helped to fuel the turn towards radical politics. ${ }^{21}$ While most readily associated with turns towards right-wing extremism in places like Germany and Italy, this mixture of public and private despair found other outlets as well, as the case of Vera Brittain shows. A young woman from a comfortable household when the war broke out, she volunteered as a nurse. Brittain not only saw firsthand the horrors of the war but also suffered horrible personal loss as a result: her brother, fiancé, and two close friends were all killed in the fighting. After the war, Brittain tried to transform her horror and grief into something positive by dedicating herself to various radical causes, including pacifism. ${ }^{22}$ However, the link between war monuments and right-wing extremism has justifiably received the bulk of analytical and scholarly attention. It is, once again, Mosse whose arguments have proven to be the most enduringly influential. ${ }^{23}$

Writing under the shadow of National Socialism-whose hateful brutality forced Mosse and his family to flee their native Germany in 1933-Mosse's central argument in Fallen Soldiers is that that the various monuments to the dead erected in the wake of the war were instrumental in cementing and spreading what he calls the "Myth of the War Experience" [sic]. According to Mosse, this quasi-religious Myth held that heroic, idealistic, enthusiastic soldiers had willingly gone to their deaths so that the nation might live. These individual deaths thus became subsumed into a salvific purpose that transcended not only the individuals themselves but also the bonds of time and space. Mosse included cemeteries in his roster of sites where "the fallen were transformed into symbols which people could see and touch and which made their cult come alive." ${ }^{24}$ While not necessarily disputing Mosse's claims, other historians have noted the way that the public meaning ascribed to war memorials was shaped by particular national, regional, and ideological contexts. Antoine Prost, for example, notes that some memorials of the Great War became the foundation stones on which a distinct Australian national identity was erected, while others became lingering reminders of American influence in Europe. ${ }^{25}$ In this way, the dead continued to do their "work" for the living long after the guns had fallen silent.

The primacy of the nation in postwar commemorations made commemoration difficult in the newly created states of central and eastern Europe, whose citizens had primarily fought in the armies of multinational empires. The ways in which commemoration of the war became assimilated into narratives of national rebirth and regeneration provides a compelling contrast to the memorialization of the dead in other European countries, as does the way this transposition of the Myth of the War Experience into a new key required the almost wholescale elision of the realities of the war in this region. For these reasons, this remains an area rich in potential for future research. Poland, for example, 
was reborn as an independent state after the war for the first time since its neighbors had annexed it in the eighteenth century. The vast majority of Polish soldiers who fought in the First World War had served in the ranks of their Russian, German, and Habsburg imperial overlords. After the war, however, the millions of veterans of these armies found themselves simply left out of a national narrative that focused on the sacrifices and exploits of the very small number of soldiers who had waged armed struggle in the name of Polish independence, especially the "Legionaries" who had fought under nationalist leader Jozef Pilsudski. Similarly, the Tomb of the Unknown Soldier that was unveiled in Warsaw in the 1920s was primarily dedicated to the dead of the border wars that Poland fought after November 1918, especially the Polish-Ukrainian and Polish-Soviet War. ${ }^{26}$

Russia provides the most complex and difficult but also compelling points of comparison for transnational histories of the work of the dead after the Great War. While the Russian Empire was one of the key belligerents, its millions of dead were consigned to cultural (and physical) oblivion due to the 1917 Bolshevik Revolution and ensuing establishment of a Communist regime. As Aaron Cohen notes in his indispensable introduction to the topic of the memory of the war in Russia, various public and private bodies in the empire were actively at work in planning monuments and memorials to the dead. ${ }^{27}$ In the first months of the war, such initiatives were undertaken primarily by local associations and institutions. In the spring of 1915, however, the Russians established the Society for Remembrance, a central body that was administered by a board of directors drawn from the ranks of the imperial elite and that counted the Tsar as its official patron. The society had numerous tasks, including overseeing and coordinating the creation of monuments and memorials. Intriguingly, it was also committed to ensuring that every individual soldier was identified and given a proper burial. ${ }^{28}$ Given that the Russian Empire was an autocracy, this could make for a compelling point of comparison between Russia and the other belligerents, particularly as it relates to the culture of death in wartime (historians of Russia, such as Melissa Stockdale, have tended to relate this phenomenon to the creation of a unifying, patriotic civic culture during the war). What new light can be shed, for example, on the cultural forces shaping the commemoration of individual war dead in the twentieth century by drawing pre-Revolutionary Russia more fully into the trans-national, comparative framework that has been established by scholars such as Laqueur and Mosse? Certainly a common Christian heritage was important, as the society's own materials proclaimed that "by their blood [i.e., the blood of the fallen] we must consolidate for all time" the feelings of patriotic unity created by the war. ${ }^{29}$

Another major wartime project, the Moscow City Fraternal Cemetery, provides another illuminating glimpse into the emerging Russian culture of commemoration and how it was both like and unlike the commemorative cultures of the dead in the other warring nations. The initiative for the establishment of the cemetery came from the Grand Duchess Elizaveta Federovna (who would later be murdered by the Bolsheviks), while organizational oversight and direction was provided by a physician, Sergei Puchkov.

On August 6, 1915, the cornerstone for the cemetery church, the Church of the Transfiguration of the Savior, was laid by two families whose sons had died in the war exactly one year before - a reminder that the combination of public and private mourning was a pan-European phenomenon. Over the next few years, some 17,000 people came to be buried there. Unlike cemeteries elsewhere in Europe, which were almost exclusively organized along national lines, the Tsar's loyal subjects were all interred without regard to ethnicity, mother tongue, or religion. Even civilians who had been killed, 
including women, were buried here. There was no cemetery quite like it in the rest of Europe, a difference worth exploring, in order to mine what it has to tell us about the varieties of death and commemoration in the twentieth century and the deeper cultural and political currents that shape them. Plans for the cemetery's future also give a fascinating glimpse of what might have been. There were hopes that the cemetery could one day become just one part of a vast complex including a park, a museum, and military training facilities. ${ }^{30}$ Here the living and the dead, civilian and military, would mingle and co-exist - would do their respective "work" together - as in no other place in Russia, and perhaps the world.

Unfortunately, such plans would never be realized, and even the Fraternal Cemetery itself eventually disappeared, because of the Bolshevik revolution. This was partially because of the sheer scale of the rupture with the past that the Revolution represented, as well as the horrific death and suffering that accompanied the years-long civil war that followed the Bolshevik coup. The world-historical character of both events cast a long historical shadow that obscured - even if it did not quite obliterate - the memory of what had come before, not only to those who lived through it, but in the subsequent century of historical writing. ${ }^{31}$ There were other reasons, more specific to the Communist regime and its ideology, that also mitigated against the establishment of a culture of commemoration after the war. As Karen Petrone notes in her book The Great War in Russian Memory, Communism, with its explicitly materialist rejection of any transcendent spiritual reality, strongly discouraged any kind of commemorative culture of death, redolent as it was of Christianity and its saints and martyrs. (With the important exception, noted below, of Lenin.) In addition, Communism was resolutely forward-looking, consciously separating itself from the past and flinging the world, so it imagined, headlong into a world made wholly new. ${ }^{32}$ This left the memory of the war and its dead to be cultivated primarily by Russian émigrés who had fled the Revolution. ${ }^{33}$

Finally, the long-standing scholarly focus on memorials intended to serve as central, national sites of collective mourning leaves out the vast majority of the monuments to the dead erected after the war: the innumerable local sites of commemoration that are to be found in nearly every belligerent country. ${ }^{34}$ These local memorials take different forms and are located in different places. Many parish churches in England, for example, have a tablet listing the names of all parishioners who died in the war. In addition, companies would commission memorials for their fallen employees, such as the monument to the thousands of workers of the Great Western Railway erected at Paddington Station. A bronze statue depicts, in realist fashion, a soldier reading a letter. The names of the dead are written on a scroll that is embedded within the monument rather than carved on the monument itself. ${ }^{35}$ Similar memorials can be found throughout Europe, from the former borderlands of the Austrian empire to Northern Ireland. Whether a simple memorial to "fallen comrades" erected by a city's postal workers or the local pharmacists union; or a plaque in a high school listing the names of teachers and students killed in the fighting; or a tablet in a town square listing the names of locals who died; or a cluster of graves in a rural cemetery, in which the dead of the Great War have been lain to rest near one another-these memorials represent a promising site for further research. How were they shaped by local and regional traditions and interests, for example? How did they vary across and within national boundaries? What were the emotional and political links binding together, or not binding together, the local and the national memories of the dead? Stefan Goebel, for example, has shown one path forward 
in his 2004 article "Re-Membered and Re-Mobilized: The 'Sleeping Dead' in Interwar Germany and Britain." Moving away from a focus on the national, and charting a methodological course that reaffirms that war memorials were about both private grief and political mobilization (the combination of which, as noted above, proved so combustible), Goebel shows that the trope of enchanted sleep was a part of both German and British memorials. Arguing that the figure of a person suspended in a death-like sleep, from which they may be awakened, was deeply rooted in folk and fairy tales, Goebels illustrates the way figures in repose, with messages of their potential resurrection someday, sent an ambiguous message about the war and whether it might safely be considered as fully over. One of the monuments analyzed by Goebel, for example, is the memorial to the dead in Munich. The memorial consists of a crypt within which is a sculpture of a dead soldier who lies peacefully on a plinth. (Of course, the soldier does not bear much resemblance to an actual dead soldier, given that both his body and his uniform are intact and unblemished by unsightly wounds, such as a missing jaw or limb). THEY SHALL RISE AGAIN, the memorial proclaimed to Müncheners suffering from crushing private grief as well as public resentment over the terms of the Treaty of Versailles. ${ }^{36}$ The example of the Munich memorial is a particularly important reminder of the importance of factoring regionalism into any analysis of the commemoration of the dead in postwar Germany, since it was, after all in Munich, not Berlin, that the Nazis arose.

\section{The interwar period}

As both Goebel's relatively recent work as well as the enduring resonance of George Mosse's arguments suggest, the way the monuments and memorials of the Great War became intertwined, in the eyes of both contemporaries and subsequent historians, with the political upheavals of the interwar period makes it difficult to distinguish between the memorialization of the dead of the Great War and the postwar political uses to which these monuments were put as distinctive subjects. Nonetheless, a distinction can and should be made between the first attempts to come to private and public grips with the unprecedented slaughter of the war and later efforts to instrumentalize those deaths in the service of a political cause, most notoriously in the case of Italian Fascism and German National Socialism. Both political movements glorified militarism and harbored aggressive foreign policy plans, and sought to enshrine the dead of the war in their particular ideological pantheons. In so doing, they drew on currents and trends that emerged after the war but also added new modes of expression and layers of meaning.

In Italy, the dead of the war were physically assembled and deployed for political purposes. Italian war cemeteries created in the immediate aftermath of the conflict tended to be rather small and unassuming places. When the Fascists came to power, however, they disinterred the soldiers from their graves and reburied them in massive cemeteries, whose layout and architecture are intended to evoke the putative glories of military sacrifice on behalf of the state. The largest of these is at Redipuglia. Officially opened in 1938, it holds the remains of some 70,000 soldiers killed in the fighting on and around the Isonzo front. Of these, about 40,000 were identifiable. They are buried under a series of terraced grave sites that climb a hill, each site resembling a step on a massive staircase that is flanked by trees. The names of the dead are, in keeping with the age of necronominalism, listed on the steps, with the Italian word PRESENTE inscribed above them. Another 30,000 unidentified remains are buried in two mass graves at the top of the hill. ${ }^{37}$ 
The Nazis also sought to enlist the dead of the Great War in the service of their vision of national regeneration through conquest; they were regularly invoked in Nazi propaganda both before and after the 1933 "Seizure of Power." One particularly vivid example of this can be seen in the famous film Triumph of the Will (Triumph des Willens). Directed by Leni Riefenstahl, the film documents the elaborate ceremonies, rituals, and displays at the September 1934 Nazi Party rally. At one point in the film, Hitler emerges to review and address the men of the Reich Arbeitsdienst (RAD/Reich Labor Service), a civilian but highly militarized compulsory labor service. Over 50,000 RAD men stand in perfect military formation before the Führer, all wearing identical uniforms. They carry shovels, which they hold like rifles and which they use in choreographed militarystyle drills. Much of the display is accompanied by march-style music, but at one point a sudden boom of a drum signals a change in tone. At its sound, one of the men's leaders, addressing Hitler directly, solemnly declares that, while the workers assembled there did not fight in the Great War, they are nonetheless "soldiers." The leader then begins intoning a secular litany of First World War battlefields, such as Langemarck, Tannenberg, and Verdun. As the litany is chanted, flag bearers slowly dip the points of their flagstaffs downward, until both the flag and the tip of the staff are on the ground. Another sharp drumbeat signals yet another change: at its sound, the flags are simultaneously whipped straight upright again, while the RAD leader proclaims: "You are not dead! You live in Germany!" 38 The ceremony has something of the air of a séance, with the rising of the flags making visible not only the resurrection of the Great War's dead but their presence at the rally. (Though the scene should not be construed as transmitting a purely ideological and quasi-spiritual point-given that the post-First World War German army was limited by the Treaty of Versailles to 100,000 men - the sight of half that many uniformed and marching "labor service men" was meant to convey a very serious threat to the regime's enemies both within and without.)

The memory of the dead did not only nurture fascism, however. In Weimar Germany, for example, the legacy of the war-and, by extension, the right to speak for the dead - was fiercely contested. While there was no shortage of aggressive nationalists who, like the Nazis, sought to enlist the dead in their cause of violent revenge, other individuals and groups, including some veterans of the war, insisted that the message of the dead was perfectly clear: never again must such violence be allowed to happen. ${ }^{39}$ Likewise, Antoine Prost, in a direct challenge to George Mosse, has argued that war memorials and the commemoration of the dead could be, and was, used to strengthen the bonds in France between the republican political system and its citizens. Prost concedes that there were many types and forms of memorials erected in France after the war, and that those that went too far in the glorification of violence or of the nation transmitted potentially anti-democratic and aggressive cultural messages. Others, however, were well suited to nurturing the sense of interlocking obligations and protections that are at the heart of the idea of citizenship. To give one example, Prost identifies the memorial in the town of Maisons-Alfort, near Paris. The memorial consists of a simple wall with columns set into it. On tables between the columns are listed the names of the dead from the town. A NOS SOLDATS MORTS POUR LA FRANCE, the inscription reads, with the beginning and ending years of the war bookending it. The memorial wall is guarded by a realistic statue of a French soldier of the era, holding his rifle and looking watchful but not particularly violent or aggressive. ${ }^{40}$ At such sober places of reflection, Prost argues, civic values such as equality and the need for voluntary sacrifice were 
reinforced. These values were also transmitted in the many Armistice Day ceremonies that took place at local First World War memorials throughout the country. Some were explicitly religious and involved the local clergy in blessings and prayers; others were secularized but maintained the aura of sacral activity, with the names of the dead solemnly intoned and silences held. ${ }^{41}$ Armistice Day was uniquely powerful, Prost believes, because it solemnly celebrated "not abstract principles but palpable citizens." By remembering each individual who had fallen, Armistice Day "honoured those who had done their duty, and the survivors, who had also done their duty, took up their place alongside the dead." ${ }^{2}$ The work of the dead here, then, (to be carried out with assistance from the living) was to provide moral and civic instruction and thereby ensure the continuing health of the republic for which they had died.

The Soviet Union, meanwhile, continued to venture into uncharted waters as it sought new ways to deal with death. As noted above, the Soviet regime saw itself as highly modern; it also wanted to detach Russia and the other Socialist "republics" as thoroughly as possible from the ideas, social structures, and ways of life of Imperial Russia. This included the rituals surrounding death, which were particularly elaborate and rich in Russia, heir as it was to overlapping and mutually reinforcing folk practices and Orthodox Christian traditions. Bolshevik attempts to fashion ideologically acceptable replacements for traditional funerary practices began even before the 1917 coup, when fallen Marxists were honored with military-style processions escorted by workers carrying banners with political slogans. ${ }^{43}$ After the seizure of power, the Bolsheviks wasted no time in turning funerals into the service of the new regime they were fighting to establish. One of the first was the memorial held for the more than 200 Bolsheviks who had died fighting to take control of Moscow. Their open coffins were paraded through the streets to their resting places to the accompaniment of music, marching soldiers, and political sloganeering. The purposes of such spectacles were manifold: they anointed the new regime's heroes and martyrs; they transmuted their martyrdom into legitimacy for the new state; they signaled a clear break with the past; and, not least, they were meant to play a role in the formation of new identities among the peopleto create a Soviet citizenry. ${ }^{44}$ The most elaborate display of Bolshevik funerary culture occurred when Lenin died in 1924. An elaborate funeral took place, after which Lenin's embalmed body was put on display in a mausoleum in Red Square. Like a saint, his incorruptible remains could be viewed through a glass casket. ${ }^{45}$ Lenin's funeral and the subsequent display of his corpse may have played the role in Russia that the Tombs of the Unknown Soldiers did in other countries, providing a focal point where people could gather and communally express public and private grief, though only they knew who and what, deep in their hearts, they were really mourning. ${ }^{46}$ The corpse of Lenin also played a regenerative role similar to that played by Western monuments that transformed individual death into collective survival for the nation. Lenin was in life, and remained in death, the font from which flowed the Bolshevik experiment. Throughout the life of the Soviet Union, Lenin's mausoleum was used as a stage on which the ruling elite were placed for various political events, a tradition which continues to this day. ${ }^{47}$

\section{The Second World War and the Cold War}

The war that the Nazi regime unleashed in Europe in 1939 caused death on a scale that managed to surpass, by a considerable degree, the carnage of the First World War. While a precise number is difficult to establish, reasonable estimates reach into the tens 
of millions. ${ }^{48}$ And yet, the scholarly literature on death and its commemoration after the Second World War is much thinner than that on the First, for the simple reason that the mass mortality of the 1939-1945 war resulted in neither major institutional changes in how death on this scale was handled nor a particularly rich or deep culture of remembrance. The reasons for this are manifold. One is that the civil and military institutions charged with handling war deaths were more extensive and experienced than they had been in 1914. "The problems facing the [British War Graves] commission after the 1939-1945 war were perhaps less daunting than in 1919: it was now an experienced organization," one history notes, laconically summarizing a terrible truth: mass violent death had become a familiar part of the European political and cultural landscape. ${ }^{49}$ In practice, this meant that the commission, for example, could simply follow the principles and practices established after the last war, such as listing the missing by name. The commemorative culture of the last war was built on in other ways as well; in some battlefield cemeteries, new sections were added to accommodate the new arrivals, while memorial tablets and walls had new dates and new lists of names added to them. ${ }^{50}$

The stark differences between the scope and scale of the commemoration devoted to the dead in each World War, and to the disparate impacts on the wider cultures within which commemorative practices were formed, are topics that are ripe for further exploration. Jay Winter, among others, has suggested that the sheer scale of barbarism exhibited in the Second World War both overwhelmed and undermined pre-war cultural values so thoroughly that no register, no language was left appropriate to it. ${ }^{51}$ Winter also argues that the extent of collaboration with the Nazi occupiers in much of Europe made a simple "ally and enemy" dichotomy harder to assert, and therefore complicated the commemorative process. (France, for example, which has a complex, and perhaps even tortured relationship with its Second World War past, has relatively few monuments to the 1939-1945 war, compared to the ubiquitous memorials to $1914-1918) .{ }^{52}$ Another painfully important difference Winter highlights is that the period after the First World War was marked by a sincere hope that a better world would emerge from the terrible slaughter, a world in which such slaughters were unthinkable. This hope, which proved to be so sadly mistaken, helped to fuel the broad scope and scale of the memorializing process. It was an illusion that bitter experience denied to those who had to sift through the emotional, physical, and cultural wreckage of the Second World War. ${ }^{53}$ Finally, there is the fact that so many of those who died in the Second World War were civilians; and civilian deaths have always occupied an uncertain place in the commemorative culture of war, to the point that they are rarely commemorated at all. Exceptions tend to be modest and local, as in the memorial to civilians killed in Saint-Lô in Normandy. ${ }^{54}$

Still, the Cold War did provide the impetus for assigning the dead and their monuments to new kinds of "work," as they became conscripted in the drive to legitimize and strengthen the post-1945 political order. In France, the American war cemeteries played a key role in postwar diplomacy. These beautiful, immaculately tended and meticulously designed and laid out cemeteries were built immediately after the war, when the numerous temporary cemeteries built by the Americans during the fighting were consolidated. The cemetery near the D-Day landing beaches is probably the best known; and in the decades that followed the war, American political leaders displayed solidarity with their western European counterparts by participating in solemn, emotionally charged commemoration ceremonies there. (The site could also be put to the opposite purpose, as Charles de Gaulle demonstrated by absenting himself from the 20th anniversary 
ceremonies). ${ }^{55}$ The Soviets were meant to take note of such displays, as well as the cemetery's more subtle message: that Americans had fought and died to combat tyranny in Europe before, and therefore could be counted on to do so again. ${ }^{56}$ The dead could also strain international alliances when they caused the past to intrude too forcefully, and too clearly, into the present. This was demonstrated by Ronald Reagan's ill-received visit in 1985 to a German military cemetery near Bitburg. Conceived as a gesture meant to demonstrate that the past had been overcome and that the US and West Germany's alliance was firmly grounded in shared values and interests, it instead provoked a furious backlash in the United States when journalists discovered that the cemetery contained the graves of a number of SS men. ${ }^{57}$

For its part, the Soviet Union, who had lost millions of its citizens to the Nazis' murderous fury, also transmuted the sacrifices of its dead into symbols to be deployed in the postwar world. Immediately after the war, the most important of these was the combination cemetery and war memorial established in Treptower Park in East Berlin, the soon-to-be capital of the Soviet satellite state of East Germany. The memorial is the burial site of about 5,000 Soviet soldiers, who lie in unmarked mass graves. (The center of the memorial complex has several large squares laid out like mass graves and crowned with wreaths, but these are symbolic; the actual bodies are buried elsewhere within the memorial's boundaries.). The memorial is dominated by a massive statue (designed, it was been argued, with personal input from Josef Stalin) of a Soviet soldier standing atop a mausoleum, which is itself atop a kurgan, an ancient Russian burial mound. The soldier carries a child in one arm; the hand of the other holds a sword. Under the soldier's boot is a broken swastika. Along the central path that leads visitors past the grave squares and toward the towering statue are stone sarcophagi carved with reliefs telling, from a Soviet point of view, the story of the war: the Germans had invaded, committed terrible atrocities, and finally been driven out by the valiant Soviet people, who had arrived in central Europe as liberators. The monument was intended in part to reinforce the staggering sacrifice made by the Soviets to defeat the Nazis, but other aspects of the memorial's message were vague and open to shifting interpretations. Most important among them was how the defeated and occupied Germans should be seen; were they Nazis, crushed under the Soviet boot, or were they to be included in the ranks of those "liberated" from Nazi rule? As Soviet rule over East Germany was solidified and the Cold War set in, the tone of the ceremonies held every year to mark the end of the war began to shift noticeably from the former to the latter. ${ }^{58}$

\section{Conclusion}

Although the Cold War has ended and the psychological scars of the Second World War are no longer fresh and raw, the monuments and memorials connected with both continue to be sites around which political passions crystallize. The Treptower Park memorial, for example, still attracts thousands of participants to annual "Liberation Day" ceremonies, though these have, as of late, begun to attract small but vocal groups of political extremists from both the right and the left, seeking to use the occasion to attract attention to their causes. The monument, along with other Soviet memorials in Berlin, has also been attacked by vandals. ${ }^{59}$ Memorials commemorating the Soviet war dead in other parts of the former eastern bloc have also remained sites of potential conflict. In 2007, for example, a Soviet-era statue in Tallinn, Estonia, was removed from its location 
in the center of town, while the Soviet soldiers who had been buried near it were exhumed and moved to a cemetery. This caused fierce riots in the city, resulting in the death of one person, and severely strained Russian-Estonian relations. ${ }^{60}$ This, in turn, points to a larger issue in the commemoration of Europe's many war dead in the present day. Driven by the project of creating a European Union, European institutions are attempting to fuse its member states' individual histories into a shared "European" past. It remains to be seen, however, what sort of work the dead can reasonably be asked to do in this attempt at top-down historical revisionism. But there are far too many of them to simply ignore, and it is unlikely that they will pass quietly into a hazy dream of European solidarity.

\section{Notes}

1 Charles Townshend, ed., Oxford History of Modern War (New York: Oxford University Press, 2006).

2 Thomas W. Laqueur, The Work of the Dead (Princeton, NJ: Princeton University Press, 2015), 10.

3 This essay will focus primarily on military deaths, even though, in the Second World War, civilian deaths were far higher. The problematic relationship between civilian suffering and public commemoration of wartime sacrifice will be discussed below. For a brief interdisciplinary overview of some of the key issues and themes in the history of death in war, see Claude Javeau, "War Deaths," in Encyclopedia of Death and the Human Experience, ed. Clifton Bryant and Dennis Peck (Thousand Oaks, CA: Sage Publications, 2009), 985-987. For a discussion focused on Europe in the era of the World Wars, see Jay Winter, "Commemorating War, 1914-1945," in Cambridge History of War, Volume 4: War and the Modern World, ed. Roger Chickering (Cambridge: Cambridge University Press, 2012).

4 James Sheehan, Where Have All the Soldiers Gone?: The Transformation of Modern Europe (Boston, MA: Houghton Mifflin, 2008), 100. For a careful accounting of the dead from all belligerents, see the figures in Antoine Prost, "The Dead," in The Cambridge History of the First World War, Volume 3: Civil Society, ed. Jay Winter (Cambridge: Cambridge University Press, 2014), 587-588, which are based on a rigorous and thorough comparison of a variety of sources. Prost's essay provides an excellent introduction to death in the Great War.

5 Ibid.

6 Laqueur, The Work of the Dead, 387. For a thorough discussion of "necronominalism," see chapters 6-9; for the First World War, see pp. 447-488. Laqueur's book is the most recent work on the cultural history of death in the West, and may displace Philippe Ariès, The Hour of Our Death: The Classic History of Western Attitudes toward Death Over the Last One Thousand Years, 2nd Vintage books ed. (New York: Vintage Books, 2008) as the standard. The key work on culture, death, and commemoration after the First World War remains Jay Winter, Sites of Memory, Sites of Mourning: The Great War in European Cultural History (Canto Classics; Cambridge University Press, 2014). Within the broader cultural history of death, Winter is sharply critical of the enduring influence of Ariès and calls for a vigorous and critical engagement with this model.

7 In the United States, the Civil War had a similar impact to that of the Great War in Europe, both in the way that it spurred changes in how the remains of the dead were handled as well as in the culture of mourning and commemoration. However, just as the military aspects of the Civil War seem to have made little impression on Europe, so too with the practices of dealing with mass death in wartime. See the now-classic account, Drew Gilpin Faust, This Republic of Suffering (Vintage, 2009).

8 George L. Mosse, Fallen Soldiers (Oxford University Press, 1990), 43-47, quote on p. 45

9 Prost, "The Dead," 569-570.

10 For more on both the logistical-institutional background of First World War cemeteries, as well as their varying cultural and symbolic significance, see ibid., 576-585. For a poignant reminder that the attempt to recover and identify the remains of the dead of the Great War continues to this day, see Louise Loe, "Remember Me to All": The Archaeological Recovery and 
Identification of Soldiers Who Fought and Died in the Battle of Fromelles, 1916 (Oxford: Oxford Archaeological, 2014), a book on excavations at a burial site in Fromelles, France. Previously undiscovered remains were found at Verdun as late as 2013.

11 Sheehan, Where Have All the Soldiers Gone?, 101. For an introduction to the institution and its history, see Philip Longworth, The Unending Vigil: A History of the Commonwealth War Graves Commission (Barnsley, UK: Pen \& Sword Military, 2010) and Edwin Gibson, Courage Remembered: The Story behind the Construction and Maintenance of the Commonwealth's Military Cemeteries and Memorials of the Wars of 1914-1918 and 1939-1945, 2. impression. ed. (London: HMSO, 1995).

12 Mosse, Fallen Soldiers, 39-43.

13 Sheehan, Where Have All the Soldiers Gone?, 101-103.

14 For a wide-ranging, readable survey of how, when, and why the cult of the Unknown Soldier emerged, see Neil Hanson, Unknown Soldiers: The Story of the Missing of the First World War (Knopf, 2006); recent scholarly trends on the commemoration of unknown soldiers after the Great War may be explored by consulting François Cochet and Jean-Noël Grandhomme, Les soldats inconnus de la Grande Guerre (2012), a collection of papers from a 2010 conference on the subject.

15 Laqueur, The Work of the Dead, 449

16 Ibid., 401, 418-419, 447-449. For a first-hand account by one of the chief designers of Britain's war memorials on the Western Front, including the Menin Gate, see Reginald Blomfield, Memoirs of an Architect (London: MacMillan, 1932).

17 "On Passing the New Menin Gate," Siegfried Sassoon, Collected Poems of Siegfried Sassoon (New York: Viking, 1949), 188.

18 Winter, Sites of Memory, Sites of Mourning, 104-105.

19 Rudy Koshar, From Monuments to Traces (University of California Press, 2000), 107-108. For a photo of the memorial, see the Bundesarchiv's photo Bild 102-11786, accessible online at www.bundesarchiv.de/DE/Navigation/Finden/Bilder/bilder.html

20 Koshar, From Monuments to Traces, 106-107.

21 Sheehan, Where Have All the Soldiers Gone?, 103-105.

22 Vera Brittain, Testament of Youth: An Autobiographical Study of the Years 1900-1925 (Virago, 2018).

23 Although Winter, Sites of Memory, Sites of Mourning, is now regarded as the definitive work on commemoration after the Great War, his argument, while not ignoring politics, is targeted at scholars of the broader cultural history of the war. Winter is primarily interested in challenging Paul Fussell's enormously influential The Great War and Modern Memory (Oxford University Press, USA, 2000) by asserting, contra Fussell, that the war's impact was culturally mediated through deeply traditional forms of artistic expression.

24 Mosse, Fallen Soldiers, 80. Mosse, who draws on a number of obscure writers to fortify his position, argues that the Myth developed directly from Christian language and imagery.

25 Prost, "The Dead," 579-585.

26 https://encyclopedia.1914-1918-online.net/article/tomb_of_the_unknown_soldier_warsaw

27 Aaron J. Cohen, "Commemoration, Cult of the Fallen (Russian Empire)," in 1914-1918-online. International Encyclopedia of the First World War, ed. Ute Daniel, Peter Gatrell, Oliver Janz, Heather Jones, Jennifer Keene, Alan Kramer, and Bill Nasson, issued by Freie Universität Berlin, Berlin 2014-10-08. DOI: 10.15463/ie1418.10421. See also Melissa Stockdale, "United in Gratitude: Honoring Soldiers and Defining the Nation in Russia's Great War," Kritika 7, no. 3 (2006), 459-486.

28 Ibid., 466. Still, anonymous burial in mass graves remained the norm for Russian soldiers.

29 Quoted in Ibid., 467

30 Kristiane Janeke, "Suppressed Remembrances," in The Forgotten Front (The University Press of Kentucky), 288-290.

31 For a good overview of the way the Revolution has dominated historical thinking about Russia in the twentieth century, at the expense of the First World War, see the introduction to Sean McMeekin, The Russian Origins of the First World War (Cambridge, MA: Harvard University Press, 2011).

32 Karen Petrone, The Great War in Russian Memory (Bloomington, IN: Indiana University Press, 2011), and Aaron Cohen, "Commemoration, Cult of the Fallen (Russian Empire)," https:// encyclopedia.1914-1918-online.net/article/commemoration_cult_of_the_fallen_russian_empire. 
33 Aaron J. Cohen, "Oh, That! Myth, Memory, and the First World War in the Russian Emigration and the Soviet Union," Slavic Review 62, no. 1 (2003), 69-86.

34 Laqueur, The Work of the Dead, 448-449.

35 For a description of this memorial as well as a guide to further war memorials in the United Kingdom, see www.iwm.org.uk/memorials.

36 Stefan Goebel, "Re-Membered and Re-Mobilized: The 'Sleeping Dead' in Interwar Germany and Britain," Fournal of Contemporary History 39, no. 4 (2004), 492-493.

37 Prost, "The Dead," 583-584. For photos and more background on the cemetery's architects, see the website of Great Britain's Twentieth Century Society, https://c20society.org.uk/warmemorials/italy-il-sacrario-di-redipuglia/.

38 Leni Riefenstahl, "Triumph of the Will," (2006).

39 Benjamin Ziemann, Contested Commemorations (Cambridge University Press, 2013).

40 Antoint Prost, "War Memorials of the Great War: Monuments to the Fallen," in Republican Identities in War and Peace: Representations of France in the Nineteenth and Twentieth Centuries (Oxford: Berg, 2002), 20.

41 Ibid., 28-31.

42 Ibid., 32.

43 For more on pre-Soviet Union funerals, see N.S. Polishchuk, "Obryad kak sotsial'noe yavlenie," Sovetskaya etnografiya 6, (1991), 25-39.

44 Janeke, "Suppressed Remembrances," 292; Catherine Merridale, in War and Remembrance in the Twentieth Century, eds. Emmanuel Sivan and J.M. Winter (Cambridge: Cambridge University Press, 1999), 67.

45 Ibid., 68. Merridale notes that the choice of music for the funeral presented the organizers with something of a problem. The revolutionary anthem "l'Internationale" was the usual song for such ceremonies, but some among those in charge thought it inappropriate to the gravity of the occasion. In the end it was used but so was much from the decidedly non-revolutionary canon of Western music, such as Beethoven and Chopin.

46 Janeke, "Suppressed Remembrances," 292-293.

47 In the ensuing decades, the Soviet regime intensified its attempts to replace completely preRevolutionary funeral practices. To this end, it placed a lot of pressure on Soviet citizens to opt for a "modern" and "scientific" cremation. Given the importance placed on both body and soul in the Orthodox tradition, cremation was virtually unheard of in Imperial Russia. Merridale, War and Remembrance in the Twentieth Century, 66, 71-72.

48 Ian Dear and Michael Richard Daniell Foot, The Oxford Companion to World War II (Oxford University Press, USA, 1995), 290.

49 Gibson, Courage Remembered, 58.

50 Ibid., 58-63; Winter, "Commemorating War, 1914-1945," 324.

51 Winter, Sites of Memory, Sites of Mourning, 8-11, 228-229.

52 Kate Lemay, Triumph of the Dead: American World War II Cemeteries, Monuments, and Diplomacy in France (Tuscaloosa: University of Alabama Press 2018), 12.

53 Winter, "Commemorating War, 1914-1945," 326.

54 Lemay, Triumph of the Dead, 14-16.

55 Ibid., 13.

56 Ibid., $24-43$.

57 David B. Morris, "Bitburg Revisited: Germany's Search for Normalcy," German Politics \& Society 13, no. 4 (37) (1995), 92-109.

58 Paul Stangl, "The Soviet War Memorial in Treptow, Berlin," Geographical Review 93, no. 2 (2003), 213-236. For more on how the Second World War fundamentally altered the place of the dead in Soviet culture, politics, and society see Michael Ignatieff, "Soviet War Memorials," History Workshop no. 17 (1984), 157-163.

59 "Kampf ums Gedenken an das Kriegsende," Der Tagesspiegel, May 5, 2019.

60 Inge Melchior and Oane Visser, "Voicing Past and Present Uncertainties," Focaal 2011, no. 59 (2011), 33-50. 


\title{
THE TRANSFORMATION OF DEATH DISGOURSE
}

\author{
From 'taboo' to 'revival' at the threshold of the new \\ millennium
}

Michael Hvïd Jacobsen

\section{Introduction}

According to German philosopher G.W.F. Hegel, history is a record of what man does with death. ${ }^{1}$ The history of human life is therefore also the history of death. Death has been here all along, ever since the beginning of mankind. A crude estimate of human deaths since the beginning of humanity will tally at more than 100 billion people - and still counting. Every year generates over 55 million global deaths, and each day averages approximately 150,000 deaths around the world. Death is indeed an integral and irremovable part of human existence - it is a prerequisite for life as well as a predictable outcome of it. Even though according to demographic statistics populations are growing older every year, the ultimate mortality rate itself has not changed a bit since the Stone Ages, Middle Ages or early modernity. It is still firmly fixed at $100 \%$, and it is unlikely that this number will ever change. Therefore, it is important to recognize the allimportant role played by death in the way we live and organize our lives.

Death, however, does not stand still. Like most other things in life, it has a past or, as it were, a social and cultural history ${ }^{2}$. What this means is that our conception of, approach to and handling of death and dying in society have undergone some quite remarkable changes throughout human history. In fact, death has many different histories depending on who is writing them, how, when, with what material and for what purposes. ${ }^{3}$ Overall, however, and despite some minor or major internal disagreements, the history of death, at least in the Western world, is often described as one of decreasing familiarity and a gradually evolving estrangement towards this aspect of human life.

This chapter will explore how understandings of death in modern society have changed quite considerably since some of the early pioneers of so-called 'Death Studies' began to publish their work throughout the 20th century. First, we will briefly discuss the background of the notion of the 'death taboo' previously so prominent in the literature on death and dying. Following this, we will explore a number of the most-cited writings by the so-called 'tabooists' of the past century concerning our attitude towards death. This leads us into more contemporary research claiming that we have witnessed a 'revival' of death within recent decades that raises doubts over the validity, veracity or accuracy of the death taboo thesis. Finally, we will discuss how we might narrow down 
our society's perhaps increasingly complicated relationship with death, without ending up fully in either the 'tabooist' or the 'revivalist' camp. The main argument will be that our culture or society is always caught in a complex position somewhere between the extreme poles of either acceptance or rejection of death.

\section{Death as 'taboo'}

For a long time, in fact for almost an entire century, it was not only fashionable but also expected for scholars and researchers of death to claim that death is taboo in modern society. Death was seemingly such an unmentionable and unspeakable topic that we went to great lengths in order to avoid it altogether - in thought, in talk and in physical contact. Death was to be kept at an arm's length - preferably as far away as possible. Death marked the end of polite conversation, an obscenity and abomination to anyone confronted with the terrifying five-letter word 'death'. Perhaps it really was like this perhaps it was not. This is indeed difficult to determine with any measure of accuracy. We may consult codes of law or etiquette books about how people were expected to behave in order to live up to social, conversational and behavioral standards, but how they really did behave (having perhaps never read the etiquette books) in actual social intercourse is quite another matter. So we cannot be sure that people really did not talk about death, that they whispered the word whenever required to do so - e.g., in front of the children or other tender souls - or that they almost at all costs avoided any contact with the dying or the dead.

What we can be sure about, however, is that many scholars and researchers, who worked within different disciplines such as psychology, sociology, anthropology and history (as pioneers of the as-yet non-existent field of 'Death Studies') and who throughout the 20th century took a keen interest in describing our changing social and cultural attitude toward death, began insisting that death was tabooed, suppressed, moved to the margins of modern society. Let us look a little into the background of the taboo concept that - together with the notions of denial and repression - became so important in the scientific discourse on death and dying in the past century.

The origin of the notion of 'taboo' is often accredited to English seafarer Captain James Cook who upon his visits to Polynesian island cultures in the early 1770s (particularly to Tonga) witnessed how certain types of food by the natives were considered tabu or tapu - that is, taboo. This meant that it was forbidden to touch, eat or make use of certain kinds of food. To them, 'taboo' was a word that meant many things at the same time: dangerous, sacred, unclean, cursed, inviolable, forbidden, disallowed, restricted, prohibited, only to be handled by a few selected tribal members. It was also believed that any breaching of the taboo would entail punishment for the culprit. Even though Cook neither invented the concept of taboo nor applied it to death - and even though it has later been shown how the Polynesian tabu/tapu and the Westernized version of 'taboo' differ in content ${ }^{4}$ - he nevertheless brought it back with him in his logbooks and journals, testifying to the fact that certain things in a given culture were regarded as taboo. ${ }^{5}$

Later, many prominent functionalist anthropologists - such as for example Bronislaw Malinowski and Alfred Radcliffe-Brown - used the notion of 'taboo' in order to describe those practices, places, items or relationships that were generally regarded as forbidden in a given culture. Within a more modern context, Sigmund Freud in his Totem and 
Taboo ${ }^{6}$ explored in more detail the psychoanalytic dimensions of taboos and, by studying the existing religious and early anthropological literature, drew an interesting parallel between the behaviors of tribal 'savages' and modern-day neurotics in the way they treated taboos. But even though Freud did develop ideas relating to death in the book, he did not specifically or systematically make use of the notion of taboo to describe our modern relationship to death. Rather, he was more concerned with the taboos concerning such topics as incest and patricide - and thus the fear of death was for Freud always a secondary fear of something else. ${ }^{7}$

The notion of 'taboo' also inspired other scholars working particularly within disciplines such as sociology, psychology and anthropology, but it was more often related to the rituals and beliefs of primitive tribal cultures than to a modern enlightened, industrialized and increasingly secular context. Moreover, it was seldom that death was the main concern in the work of these scholars. Despite this, some of those writing in the wake of Freud, as we shall see, began using 'taboo' as a descriptive term for our attitude toward death and the dead. In their view, particularly at the threshold of the 20th century a period often described as one in which so-called 'modern society' was consolidated death and with it also grief seemed to become increasingly problematic experiences, and with this we began to see the contours of the so-called 'death taboo'.

\section{The 'tabooists'}

British sociologist Tony Walter, to whom we return later, once proclaimed that if it came to a vote, the tabooists would probably win handsomely'. ${ }^{8}$ By this he meant that looking at the history and content of the research conducted on the topic of death and dying within the social sciences and humanities throughout most parts of the 20th century, the notions of 'taboo' and 'denial' loomed large. In fact, most of those who took an interest in the study of death attitudes - and this group of scholars constituted an overwhelming minority as the topic of death itself for many years was a marginalized academic preoccupation - would somehow resort to notions of suppression, repression, denial or taboo when describing the society around them.

\section{The birth of the death taboo: Sigmund Freud}

Where to start when trying to understand why death seemingly became a taboo during the foregoing century? Sigmund Freud is a good place to begin as he was one of the first specifically - based on his psychoanalytic perspective - to suggest that death was increasingly becoming a problem for modern man. Obviously, Freud was neither the first nor the last to point out that our experience with death was problematic, but he became one of the key references for many subsequent scholars and researchers trying to tease out the attitude towards death in modern civilization. Even though the topic of death in itself was not a main concern in Freud's writings, there are many scattered references to death throughout his work on, for example, 'castration anxiety', 'the uncanny', 'mourning and melancholia', 'Eros/life instinct and Thanatos/death instinct', 'death wish' and so on.

However, in his essayistic (and at times admittedly somewhat confusing and contradictory) reflections in the piece titled 'Thoughts for the Times on War and Death', ${ }^{9}$ written some months into World War I, Freud - besides describing his disillusionment with the causes and consequences of the current drift toward war - specifically dealt with the 
changing attitude toward death in modern society. According to Freud, even though we seemingly appear to live by the conviction that 'death [is] the necessary outcome of life, that everyone owes nature a death and must expect to pay the debt', in reality, however, we show 'an unmistakable tendency to put death on one side, to eliminate it from life. We tried to hush it up'. ${ }^{10} \mathrm{He}$ thus went on to famously suggest that it is 'indeed impossible to imagine our own death ... or, to put the same thing in another way, that in the unconscious every one of us in convinced of his own immorality'. ${ }^{11}$ In Freud's psychoanalytic perspective, there is no equivalent to our own death in our encounter with the real or external world, and we therefore unconsciously think that we cannot and will not die. As he wrote, "What we call our "unconscious" - the deepest strata of our minds, made up of instinctual impulses - knows nothing that is negative, and no negation ... There is nothing instinctual in us which responds to a belief in death. ${ }^{12}$ This allows us to live, in our unconsciousness, as if we are in fact immortal.

Compared to primeval man (Freud does not specify when this 'man' lived), modern man has not been as accustomed to confronting the harsh reality of death. To primeval man, seeing death and dying and participating actively in killing was part of daily life. In modern society, death is no longer - at least in times of peace - an everyday occurrence or a real risk factor. However, both our prehistoric ancestors and ourselves, according to Freud, seem to share the same inconsistent and even contradictory attitude towards death. On the one hand, death is denied - especially one's own death and to some extent also the death of loved ones - but, on the other hand, the death of strangers or enemies is accepted, at times even desired, as Freud captured with the notion of the 'death wish'. He also insisted that 'civilised man' will avoid talking openly about the topic of death, especially if someone close to dying is present, and that only children are seemingly prone to 'disregard this restriction'. ${ }^{13}$

One of the main consequences of this taboo on/denial of death, although Freud neither specifically invokes nor systematically uses these notions in his piece, is that life is impoverished, it loses in interest, when the highest stake in the game of living, life itself, may not be risked'. The outcome of taboo/denial is a certain cautiousness, robbing life of some of the capricious excitement and danger, which, in his words, are then instead pursued in the surrogate worlds of fiction, literature and theatre. Freud believed, however, that this comforting bubble of unconscious immortality with the advent of World War I and the knowledge of countless of deaths on the battlefield was bound to burst, which meant that modern man would have to come out of his shell, as it were, and confront the reality of death. In his view, we would be unable to keep denying it. Freud's advice, ending his reflections on war and death, was that we need to let death back into life instead of trying to condemn it to a shadowy existence or to live a life of unconscious but also unreal immortality. ${ }^{14}$

\section{Death as pornography: Geoffrey Gorer}

The idea that death replaced sex as the greatest taboo of modern society became a recurrent feature of many of the taboo theories from the 20th century. These theories suggested that whereas sex was gradually - not least due to the so-called 'sexual revolution' of the 1960s - becoming more socially acceptable and publicly visible, death instead became something unspeakable and disgusting, to be kept away from public sight and polite conversation. Death and grief, so prominent among the Victorians of the 
preceding century, would sink into shameful oblivion. One of those who most forcibly promoted this line of argument was English social anthropologist and writer Geoffrey Gorer. In a small yet thought-provoking piece titled 'The Pornography of Death' from 1955, Gorer claimed that death was increasingly becoming shrouded in a new sense of prudery that was previously reserved for the topic of sex. It was Gorer's contention that death was now becoming a pornographic and tabooed topic whereas sex was seemingly about to come out of the closet and lose the aura of obscenity previously so strongly associated with it. In Victorian times, according to Gorer, copulation and birth had been the two 'unmentionables' - now their place was taken by the topic of death. Due to a low average life expectancy and not least a high infant mortality rate, death was already known to children at an early age during the 18th and 19th centuries. With the coming of the 20th century, this situation changed drastically and "death has become more and more "unmentionable" as a natural process ... The natural processes of corruption and decay have become disgusting, as disgusting as the processes of birth and copulation were a century ago'. ${ }^{15}$ However, just as sex - due to its illicit and immoral connotations has always stirred public and private imagination, so death - now tabooed - continues to fascinate and attract attention through the representations made publicly available in pulp magazines, science fiction books and horror comics. Gorer thus insisted - in an almost Freudian fashion - that 'while natural death became more and more smothered in prudery, violent death has played an ever growing part in the fantasies offered to mass audiences'. 16

In a book published a decade later, Death, Grief and Mourning (in which the pornography piece was republished), Gorer elaborated on how this situation had come about. Reflecting on his own memories and experiences of death, he reproduced three personal stories that illustrated the gradual transformation of our attitude towards death over half a century. From his own childhood, when he was five years old, Gorer recalled the extended ceremonials and rituals played out in the wake of the death of King Edward VII in 1910. At the time, public displays of mourning were customary and commonplace, which often showed in slowly moving and outstretched funeral processions and in the wearing of black mourning outfits for men and women, making Gorer note how 'this mass of black-garbed humanity struck me as a most depressing sight'. ${ }^{17}$ In 1915, his own father drowned when the Lusitania was sunk by the Germans during World War I. He recalled how he was made to wear a black tie and bands of crêpe and how he was treated at school with kindness but also some distance, almost 'like an invalid'. During those years, due to the enormity of losses of human life in the war, widows in full mourning in the streets were a frequent sight. This changed gradually in the following decades, during which Gorer observed how mourning in his close circles was increasingly becoming a lonely experience without 'any social ritual to ease it'. ${ }^{18}$ Nowhere was this more evident than in 1961, when his younger brother Peter was diagnosed with incurable lung cancer and passed away at the age of 54. Until the very end, he was kept in the dark about the severity of his predicament. Gorer had discussed his brother's situation with a friend and doctor, and they decided that only Peter's wife Elizabeth was to be informed about her husband's situation. After the brother's death, his widowed sister-in-law suffered from social isolation, despair and depression, and, even though she did not publicly show off her status as recently bereaved, she was nevertheless treated by those around her as if she was a social leper. Gorer remarked that 'only if she [Elizabeth] acted as though nothing of consequence had happened was she again socially acceptable', ${ }^{19}$ and as he noted 
in his conclusion, summarizing the status of grief and mourning rituals in modern society, 'giving way to grief is stigmatized as morbid, unhealthy, demoralizing'. ${ }^{20}$

These personal testimonies from his own life were used by Gorer to illustrate how death and grief had gradually but decisively been pushed to the margins of social life during the 20th century, fast becoming the new source of obscenity, embarrassment and loneliness. Death, previously so prominent and omnipresent, was now reduced either to empty entertainment or was surrounded by 'complete denial'. ${ }^{21}$

\section{The denial of death: Ernest Becker}

The claim that death is universally feared by humans is no more prominently proposed than in the Pulitzer-winning book The Denial of Death (1973/1997) by American cultural anthropologist Ernest Becker. In this somewhat obscure treatise, Becker advanced the idea that the fear of death is a universal feature of human life - that death, deep down, is what motivates people to live their lives the way they do. Becker regarded his own work, which relied on psychoanalytic and existentialist ideas coupled with insights from the Frankfurt School of critical theory, as an ambitious attempt at 'creating a merger of science and the religious perspective'. ${ }^{22}$ The basis of Becker's work was his anthropological understanding of man as first and foremost a creature, an animal not all that different from other animals in the fact that he lives and dies. However, man is also a selfpreserving symbol-maker capable of creativeness and self-awareness (the symbolic self). This duality of human nature informs the way humans confront death. On the one hand, life - individual and social - is full of numerous, smaller or bigger, repressions and denials of death. On the other hand, by way of what Becker calls 'heroics', humans also strive to confront the inevitability of death through meaningful human activity. As he noted in an interview conducted from his hospital bed shortly before his own death: 'It is natural for man to be a crazy animal; he must live a crazy life because of his knowledge of death.'23

In his book on death denial, Becker distinguished between two types of arguments regarding the role and function of death in life. On the one hand is what he termed the 'healthy-minded argument', which maintained that 'the fear of death is not a natural thing for man, that we are not born with it'. ${ }^{24}$ This argument thereby claims that specific circumstances and experiences (especially in childhood) - and thus only specific individuals - will promote a fear of death. On the other hand, is the so-called 'morbidlyminded argument', which insists that the 'fear of death is natural and is present in everyone, that it is the basic fear that influences all others, a fear from which no one is immune, no matter how disguised it may be'. ${ }^{25}$ Becker's own position was firmly located within this latter perspective, and his entire book therefore contained an extended argument for the fact that man fears death and so invents a variety of different denial and survival mechanisms or 'heroic' strategies in order to transmute and transcend death.

In Becker's understanding, the fundamental creatureliness of human life means that humans will go a long way to deny and repress the reality of death. Death to such a selfaware and self-conscious animal as the human being is seen as a terrible affront - the sum of all his/her fears. Hence, denial and repression of death constitute a characteristic of the human way-of-being-in-the-world, and, as Becker in a Freudian line of argument stated, 'there are many ways that repression works to calm the anxious human animal, so that he need not be anxious at all'. ${ }^{26}$ So according to Becker, humans, as the only 
creatures on earth knowing that they will die, are destined to live a life with this painful and gnawing awareness:

What does it mean to be a self-conscious animal? The idea is ludicrous, if it is not monstrous. It means to know that one is food for worms. This is the terror: to have emerged from nothing, to have a name, consciousness of self, deep inner feelings, an excruciating inner yearning for life and self-expression - and with all this yet to die. ${ }^{27}$

But despite strenuous and continuous efforts at hiding the ugly fact of death (the fact that we are nothing but 'food for worms') as an inevitable part of life through repression and denial, deep down death is still there, serving as the basis for all human activity. As Becker observed:

The idea of death, the fear of it, haunts the human animal like nothing else; it is a mainspring of human activity - activity designed largely to avoid the fatality of death, to overcome it by denying in some way that this is the final destiny for man. $^{28}$

Denial of death thus works in many different ways. In Becker's view, culture or society must allow its members to find meaningful or 'heroic' ways of living with the painful reality and knowledge of death. As he stated: '[E]verything that man does in his symbolic world is an attempt to deny and overcome his grotesque fate. ${ }^{29}$ There are many different ways, many 'illusions', through which this can be achieved, such as entertaining ideas that one may outlive death through personal immortality or by obtaining eternal life. ${ }^{30}$ In the book, Becker specifically mentions the so-called 'causa sui project' (the idea that one is superior to physical reality and hence to death) and 'cultural worldviews' or 'herosystems' (e.g. investments in collective ideas of immortality such as notions of 'the chosen ones', national identity or belonging to a powerful in-group) as common ways in which humans try symbolically and heroically to live a meaningful and fear-free life in the face of death. Previously, religion served as the main cultural worldview, inspiring hope in a life beyond death, but Becker points out how this worldview has come under increasing pressure due to the rise of reason, secularism and science.

Becker's perspective on death denial was later advanced, developed further and experimentally tested and verified by proponents of the so-called 'terror management theory' (TMT), that proposed to empirically test his theoretical universe in a number of studies intended to show how people practically confront and deal with living a life in the shadow of death. ${ }^{31}$ Thus, the spirit of Becker - his insistence on the importance of the denial of death as a natural element of human life - is today still very much alive within this particular research tradition.

\section{The forbidden and hidden death: Philippe Ariès}

Perhaps the most prominent among the 'tabooists', and certainly the one with the clearest historical perspective, was French historian Philippe Ariès, who, from the late 1960s until his death in the mid-1980s, published some of the most-referenced pieces of work on our changing understanding of death in the Western world. Ariès was, according to 
his own testimony in his memoirs, for many years a 'Sunday historian', 32 who for thirty years before he started working professionally as a historian had served as a director for a documentation center for international commerce in tropical fruits, and who on the side wrote about historical changes in the Western world. This interest culminated in a history of family and private life, a history of childhood and, not least, a history of death. Overall, what characterized Ariès' writings was an ability to capture the sweeping changes of historical development as well as his keen interest in the minutest of details of how history was lived and experienced by people.

Ariès's contribution to the taboo theories is obvious in his magisterial work on the changing historical 'death mentalities' in the West from the Middle Ages to the end of the 20th century, earning him the epithet of the 'doyen of the historians of death'. ${ }^{33}$ According to him, it was possible to distinguish between four (at other times he mentioned five) phases of development of death mentality that each in their way shaped the way death was experienced, understood and practiced. ${ }^{34}$ In Ariès's view, death was a social construction, which - besides its obvious biological facts - was very much influenced by the values, ideas and norms (the 'mentality') of the society in which it occurs. In his work, Ariès drew on a rich variety of sources such as historical testimony, architecture, poetry, iconography, theology, official documents, sociological research and so on. Moreover, it was important to him that historical understanding relied on a combined diachronic and synchronic perspective, taking into consideration the long historical haul behind contemporary practices as well as the many minor snapshots making up the present.

As a medieval historian, Ariès's starting point was the Middle Ages (moreover, medieval times provided a diametrical opposition to contemporary practices, which was an important analytical point for him to hammer home). Thus, the first phase of 'death mentality' identified by him was the 'tamed death', which captures a period marked by an omnipresent and omnipotent death in a medieval society with an average life expectancy of around 30-40 years due to epidemic diseases, wars and poor sanitation. Despite the unavoidable presence of death, people managed to take the sting out of it through religious beliefs and practices, thereby taming death. The second phase was called the 'death of the self' and was historically located around the time of the late Middle Ages and the Renaissance period. This was a time of dawning individualization, humanism and in some parts of Europe also a Reformation, changing the religious landscape with new ideas about liturgy, eschatology and different ways of depicting death in religious iconography. Death - and immortality - now became a concern for the individual, who needed to prepare for and plan his own death, rather than it being something to be organized by the community. The third phase coincided with the 19th century (the Victoria Era) and was titled the 'death of the other'. During this phase, the perspective shifted from a concern with one's own death to the experience of losing someone loved, culminating in 'a new intolerance of separation'. 35 Hence mourning culture became much more elaborate and elegiac, etiquettes for prescribed mourning periods were enforced and behavioral and ceremonial codes were directed toward showing the deep sadness and despair associated with the loss of a beloved. This period, at times also called 'The Victorian Cult of the Dead' (coinciding as it did with the reign of Queen Victoria), was thus one in which a romantic culture of mourning and grief surged within literature, poetry and psalms, funerary rites and even the physical organization and appearance of the cemetery. The final phase was called by Ariès either 'forbidden death' 
or 'hidden death', coinciding with what we conventionally call 'modern society'. This is a time when the average life expectancy rises rapidly to around 70-80 years, not least due to medical advances and a successful battle with infant mortality. This is the time of two devastating world wars, of the rise of the welfare state and of the threat of mutual destruction through nuclear warfare, shaping the thoughts, imagery and emotions of people about death and dying. This is also the time when the hospital becomes a place where the majority of people will eventually die. It is a time when the traditions, ceremonies and rituals associated with death become less publicly visible and more minimalistic, at least as compared to the preceding century, and when secularization begins to leave its mark.

Ariès's main point was to show that we had witnessed a 'reversal of death' during the millennium from the Middle Ages to modern society. This 'reversal' was particularly evident in three overall developments. First, the 'medicalization of death', leading to a decline in the role of religion - which had previously provided the primary explanatory framework for meaning-making vis-à-vis death, dying and hopes for immortality - giving way to medicine and medical technology as the all-important means for controlling and making sense of death. Second, the 'privatization and banning of mourning' from public life as part of the reversal process of death and dying, meaning that grief - which was previously a very public phenomenon - increasingly became something to be hidden from public sight. Third, the increasing 'deritualization of death and mourning' evident, for example, in the use of time-saving and minimalistic ceremonial practices and rituals such as cremation as the preferred mode of disposal instead of burial. Ariès, however, also noted the way funerals in America were increasingly becoming commercially exploited, leading to a more staged and ritualistic expression of death and grief than in western Europe. It was Ariès's main argument that death in modern society had become wild, whereas it was once, in medieval times, tamed. ${ }^{36}$ A major manifestation of this 'wildness' of death was the fact that dying was no longer an event taking place at home with the dying person at the centre of attention deciding the course of the final events. The dying were now removed to the hospital facility where their deaths became inscribed in a medicalized and professionalized setting, and the individual thereby lost control of the hour of his own death:

Death in the hospital is no longer the occasion of a ritual ceremony, over which the dying person presides amidst his assembled relatives and friends ... Death has been dissected, cut to bits by a series of little steps, which finally makes it impossible to know which step was the real death. ${ }^{37}$

Moreover, Ariès believed that modern man - not least due to the aforementioned notion of 'medicalization' and the rise of a scientific worldview at the expense of religion, was unable to accept his own mortal destiny and thus entertained the idea - as we also saw in Sigmund Freud earlier - of his own immortality:

Everything ... goes on as if neither I nor those who are dear to me are any longer mortal. Technically, we admit that we might die; we take out insurance on our lives to protect our families from poverty. But really, at heart we feel we are non-mortals. ${ }^{38}$ 
Ariès documented and adorned his written history of death with a rich and impressive photographic archive of the changing ars moriendi across a thousand years. ${ }^{39}$ Yet despite the originality and depth of his work, several scholars subsequently criticized Ariès for being excessively normative and nostalgic, selective and at times even sloppy with his sources of data, supportive of an almost unilinear or deterministic understanding of historical development. Notwithstanding such criticisms, however, Ariès was without the shadow of a doubt capable of convincingly showing how death had become increasingly forbidden and tabooed during the 20th century.

\section{The loneliness of the dying: Norbert Elias}

Besides medicalization, another and accompanying manifestation of Philippe Ariès's 'forbidden death' was the fact that old, sick and dying people were increasingly abandoned by the living. This development became the topic of a small book written by GermanEnglish sociologist Norbert Elias, who is perhaps best known in sociology circles as the inventor of the notion of the 'civilizing process'. In many ways, his book on death was a testimony of how the civilizing process in the West with its rationalization, individualization and institutionalization tendencies affected our experiences of death and dying. The book is about how death, previously a familiar feature of social and family life, gradually became something alien. Elias started out - in a vein similar to that of Ernest Becker - by capturing our human difficulties with death as such:

Death is the problem of the living. Dead people have no problems. Of the many creatures on this earth that die, it is human beings alone for whom dying is a problem ... They alone of all living beings know that they shall die; they alone can anticipate their own end ... It is not actually death, but the knowledge of death, that creates problems for human beings. ${ }^{40}$

Living as the only creatures on earth with this knowledge of death is indeed a daunting prospect, which fosters many attempts at evasion and the development of many hopeful imageries of immortality. According to Elias, people have entertained fantasies of immortality. Previously, these were mostly collectively entertained and rooted in religion as some sort of blissful eternal existence waiting after earthly life, but nowadays, due to what Elias called the 'spurt of individualization', these fantasies have increasingly become more personal and private. ${ }^{41}$ Consequently, our bids for immortality have become as individualized as everything else. Many different factors have contributed to this development: the significant extension of the average life expectancy throughout the past century; the fact that most deaths now take place in later life; the fact of living in relatively more secure and safer circumstances than our predecessors; the gradual transformation of the social role of the figuration of the family; the rise of more impersonal, informal and individualized ways of dealing with most aspects of life; and, last but not least, changing patterns of socialization, which have also had an impact on our relationship and experience with death. For instance, Elias noted how there was now a widespread aversion towards 'teaching children about the biological facts of death', which was previously an important part of socialization. ${ }^{42}$

In line with Geoffrey Gorer and Philippe Ariès, Elias commented specifically on the intricate connection between our previously constrained attitude towards the topic of sex and 
modern society's still more estranged attitude towards death: 'The defensive attitudes and the embarrassment with which, today, people often react to encounters with dying and death fully bear comparison with the reaction of people to overt encounters with aspects of sexual life in the Victorian age. ${ }^{43}$ Lifting the taboo on sex meant that a taboo on death instead took its place, leading to a distancing towards those who seem to personify death, such as the old, the sick and the dying. As Elias stated, there is now 'a peculiar embarrassment felt by the living in the presence of the dying. They often do not know what to say. ${ }^{, 44}$ Seeking to avoid the unpleasantness of such embarrassment results in what social researchers have termed 'social death' - the abandonment of the dying in institutions such as hospitals or nursing homes, and Elias observed how 'many old people's homes are therefore deserts of loneliness'. ${ }^{45}$ In his words, there has been a 'withdrawal from the moribund' with a silence spreading around them. ${ }^{46}$ The individualization of death and the isolation of the dying that follows from it was in his view closely connected to the severing of traditional communal ties and family bonds, and Elias speculated how

the idea of having to die alone is characteristic of a comparatively late stage of individualization and self-awareness [and] the special accent taken on in the modern period by the idea that one dies alone matches the accentuation in the period of the feeling that one lives alone. ${ }^{47}$

Consequently, we have, in a historically unparalleled manner, gradually removed ourselves from the previously familiar sight and smell of death:

Never before in the history of humanity have the dying been removed so hygienically behind the scenes of social life; never before have human corpses been expedited so odourlessly and with such technical perfection from the deathbed to the grave ... Never before have people died as noiselessly and hygienically as today ... and never in social conditions so much fostering solitude. ${ }^{48}$

Elias's point, then, was that death had gradually - as part of the ongoing civilizing and individualizing process - been moved 'behind the scenes of social life'. The reason for this was the fact that death and dying increasingly serve as unbearable and grim reminders of our own inescapable mortality, and thus 'the sight of a dying person shakes the defensive fantasies that people are apt to build like a wall against their own death' ${ }^{49}$

$* * * * * *$

A number of other scholars contributed actively to the 'tabooist' approach, well into the late 20th century. Jessica Mitford's powerful attack on the cost of American funerals assumed a public susceptibility to commercialism as a means of countering the growing fear of death. Ivan Illich, in a widely cited study, emphasized the role of medicalization in distorting the modern approach to death. The Polish sociologist Zygmunt Bauman, writing in 1992, in many ways revived Becker's argument about fear and the effort to conceal, with particular emphasis on the illusory modern emphasis on efforts to fight or conquer death. ${ }^{50}$ 


\section{The 'revivalists'}

Toward the end of the 20th century, a new wave of writings gradually emerged, insisting that the landscape of death was in fact changing, just as it had been, according to writers such as Freud, Gorer and Ariès, around a century before. Although there are many different voices in this new choir of death researchers, who have become prominent figures in the emerging field of 'Death Studies', a common concern is to show how we now 'do' death differently than was the case a generation or two ago. Below, we will look at the ideas of some of the most prominent voices within the camp of what is here called the 'revivalists'.

\section{The revival of death: Tony Walter}

British sociologist Tony Walter was one of the first substantially to reconsider our changing relationship to death on the eve of the 21 st century. In his book The Revival of Death (1994), Walter showed how death was no longer a tabooed topic as claimed by so many of the previously mentioned social thinkers, and he developed the notion of 'neo-modern' death, which was distinguished from both 'traditional' and 'modern' death. Whereas traditional death was described as quick and frequent death taking place within the social context of the local community and with religion as the guiding authority, the latter modern type of death was equivalent to hidden death, where the public and private dimensions were often clearly separated, and where medicine is the main authority consulted. ${ }^{51}$ Contrary to but also building on these previous forms, the idea of 'neo-modern death' describes a prolonged death where what was previously private now becomes public and where the self is regarded as the main authority in life and in death.

Neo-modern death, however, is not a one-dimensional or internally coherent phenomenon as it contains several different strands. According to Walter, relying on British sociologist Anthony Giddens's ideas of 'expert systems' and 'institutional reflexivity', 'late modern death' 'replaces control through medical understanding with control through psychological understanding, ${ }^{52}$ We now know so much about dying stages and grieving processes from so many different disciplines that a new kind of expert knowledge now supports the encounter with death, dying and bereavement, modifying purely medical control. 'Postmodern death instead places private feelings firmly onto the public agenda - in medical training, hospital routines and the media. Public discourse is thus fragmented and the authority of experts challenged. ${ }^{53}$ The postmodern revival of death draws on ideas of individualism and romanticism in order to advance the authority of the (consumer) subject in order to recognize that there are many different but equally meaningful ways of living, dying and grieving. Instead of expert knowledge, postmodern revivalists place the experiences of the ordinary human being as the primary decisionmaker in matters of death. Walter summarized the perspectives of the revivalist camps in the following way:

The expert ('late-modern') revivalist asserts the right of the individual to know they are dying and to express how they feel; but the expert goes further and insists that individuals need to express their feelings or at least talk about them, and that carers need to create an 'open awareness context' with those who are 
dying. The more radical ('postmodern') revivalist points to the wide diversity in how human beings encounter death; some individuals for example do not want to know they are dying or choose not to express their feelings of grief - this may work for them and must be respected. ${ }^{54}$

According to Walter, the contemporary revival of death (postulated in the mid-1990s) was thus one characterized by a 'double-coding' situation in which there was a mixture of often disparate elements of the traditional and the modern in a new neo-modern constellation (itself pregnant with both late modern and postmodern elements), which was, for example, visible within contemporary funeral practices, palliative and cancer care, outpatient chemotherapy, hospital architecture, etc. ${ }^{55}$

In another piece, Walter went a long way to show how the seemingly self-explanatory notion of the 'universal death taboo' needs to be rearranged and deconstructed if we are to understand what is meant - or what can be meant - when we claim that death is taboo in contemporary society. ${ }^{56}$ Walter (1991) differentiated among six types of taboo on death (depending on the range and character of the proposed taboo) that each in their way qualify and modify the commonsense view that death is and continues to be taboo. First, death was once taboo, but its taboo is now dissolving, especially among the intelligentsia/well-educated groups, whereas the working class may still be stuck in taboo. Second, death, dying and grief are not taboos as such but are hidden away in institutions, and those afflicted by death - the dying and the bereaved - are treated as lepers. Third, the taboo on death is not spread throughout all of society but is primarily reserved for specific groups such as those working within the healthcare system and the media. Fourth, death is not taboo, but we are lacking a language with which to communicate meaningfully about death with each other. Fifth, since all societies must in varying degrees simultaneously deny and accept death, it is up to the individual interpreter to determine whether and to what extent a taboo exists or not. Sixth, it is the modern individual rather than modern society as such that taboos death, as it is the individual who dies whereas society survives. These different qualifications of the notion of 'death taboo' each in their way illustrate how we need to be cautious when confidently claiming that death is, or was, taboo. All in all, according to Walter, who revisited the thesis of 'death revival' two decades after his initial work on it, the days of the dominance of the death taboo are definitively over, and we are now engaging with death in ways that cannot accurately be captured by the notion of taboo. ${ }^{57}$

\section{The end of the death taboo: Kate Berridge}

Claiming that death is no longer taboo - at least as compared to a generation or two ago - has also caught the cultural imagination in the new millennium. In her book Vigor Mortis: The End of the Death Taboo (2002), British journalist Kate Berridge asked the relevant question: can we still claim that death is taboo? Her answer, however, was not as straightforward as might perhaps be expected from the sub-title of her book. In the beginning of the book, Berridge discussed how the latter part of the 20th century was a time when death was locked up, put away, sealed off. The 21 st century, however, seems to approach death differently - 'new attitudes to death are emerging', as she states. Berridge is thus suggesting that 'by moving from the margins of society to the mainstream, and hence becoming social not anti-social, public not private, fashionable 
not fearful, death is the focus of a new permissiveness. Death is in. ${ }^{58}$ Throughout the book, she provides ample evidence for the fact that death is now in many respects coming out of the taboo closet and becoming fashionable, visible and permissible.

The 20th century was definitely, according to Berridge, a time when death was taboo, at least as compared to the preceding century. She testified to how death throughout most parts of the 20th century was hidden from children, who were also excluded from the deathbed and funeral ceremony; how the preparation and education for death, which was such a normal part of Victorian family life in the 19th century, was replaced by silence and denial; how extensive death and mourning rites were reduced to a bare minimum; and how public mourning dress as well as mourning jewellery signaling one's loss to others was abandoned. In less than a century, death simply disappeared from everyday life. In the subsequent chapters of the book, Berridge illustrates how death is seemingly re-appearing in many different contexts such as remembrance festivals, public mourning and commemoration ceremonies for dead royals and celebrities; in the ingenuity and visibility of funeral services at grand exhibitions and on internet portals; in more aesthetic and personally meaningful burial rites; in art exhibitions; in new ecological modes of disposing of the dead body and so on. There is now a vivid imagination and renewed interest surrounding death incomparable to the $20^{\text {th }}$-century taboo.

So is death still taboo then? The final chapter of Vigor Mortis captures the complexity of the matter very well. With a chapter heading titled 'The New Pornography of Death' as a direct reference to the work of Geoffrey Gorer, Berridge showed how death now seems to be everywhere, but at the same time it is often treated in a superficial manner. By invoking the notion of the 'pornography of death', Berridge - like Gorer - insists that the topic of death is often reduced to empty entertainment and public spectacle for attracting attention. She showed how death is a recurrent feature in provocative art installations, in photographic exhibitions, in theatre productions, in box office movies, in popular fiction, on the television screens and the internet, and, as she stated, 'we have more opportunity than ever before to experience death vicariously'. ${ }^{59}$ But the symbolism, significance and meaning of death on display are often shallow and have no deeper meaning beyond themselves. In many ways, we seem to be more culturally and individually estranged from death than ever before despite this new permissiveness. The question Berridge implicitly raised at the beginning of her book was whether death had in fact been liberated from its previous shackles of taboo or whether the new openness and permissiveness merely serve as a smokescreen for new forms of repression and denial. Her conclusion seems to point in both directions.

\section{The contemporary 'cult of death': Dina Khapaeva}

As many of the aforementioned 'revivalists' so far have claimed, a new social and cultural transformation in our understanding of and approach to death has been underway for the past few decades, some of the changes pertaining to the realm of thought, some to the realm of practice and yet others to the realm of representation. This last realm is the focus for Russian-American sociologist and language scholar Dina Khapaeva, who in her book The Celebration of Death in Contemporary Culture (2017) provides an in-depth analysis of some of the main processes behind and main expressions of a new present-day 'cult of death'. She starts out by suggesting that 
three decades ago, Halloween did not rival Christmas around the world; dark tourism was not a rapidly growing industry; death studies were not part of the school curriculum; funerals were incomparably more traditional and uniform; and Santa Muerte, 'Saint Death', was a marginal observance, not an international cult. In those days, 'corpse chic' and 'skull style' did not represent mainstream fashion; Gothic, horror, torture porn and slasher movies were not conventional entertainments; and vampires, zombies, cannibals and serial killers did not appeal broadly to audiences of all ages. ${ }^{60}$

Khapaeva thus claims that we in the Western world since the 1980s and 1990s have witnessed the rise of a 'cult of death' that engages with death in new and thought-provoking ways, which are particularly evident within popular cultural expressions of death. Regarding the new cult of death, Khapaeva insists that 'it is crucial to clearly distinguish it from traditional cults of the dead ... as a system of belief that unites the deceased and the living in a single community'. ${ }^{61}$ Contrary to these conventional communities creating symbolic bonds between the deceased and the living, 'as a popular cultural movement, the cult of death has no articulated sacral or religious meaning, although worship of violent death or vampires as sacred beings can be regarded as one of its manifestations'. ${ }^{62}$ So the new cult of death is not about worshipping the dead ancestors or linking the living with the already dead as in the old cults of the dead, but rather it is aimed at providing images, imageries and experiences that attract the attention and shape the consciousness of those participating in and consuming the cultural as well as popular cultural manifestations of death. Violence and death have now become some of the primary sources of entertainment rather than tabooed topics, and in this way there is a connection between the Victorians' obsession with death and mourning throughout the 19th century and our contemporary cult of the dead. One of the main trends in our present cultural celebration of death is how death has increasingly been subjected to processes of commodification that are evident in the ways in which death is continuously represented within literary fiction and movies, as Freud, Gorer and Berridge also mentioned earlier. One of Khapaeva's main contributions is to suggest that this 'cult' or 'cultural celebration' of death is no longer only evident in the West; by also drawing on examples from Russia, she shows how this cult is increasingly becoming a global phenomenon.

In her book, Khapaeva also outlines some of the aesthetic, intellectual and historical sources behind the rise of the new death cult. According to her, the new cult of death has been promoted and propagated by various movements within aesthetic, intellectual and historical development throughout the latter part of the 20th century. She particularly pays attention to what is called the 'Gothic Aesthetic', which is a notion capturing the deeply rooted fascination with the nightmare, horror and monsters in Western culture. This gothic imagination is a way not only of dealing with death but also with what it means to be a human being - it is an expression of a widespread disillusionment with rationalism and religion. In more recent times, this 'Gothic Aesthetic' is particularly evident in many of the popular novels, movies and television series devoted to monsters, vampires, zombies, robots, machines, cyborgs and non-humans.

Moreover, the rise of post-structural and postmodern social theory (perhaps particularly as it has been advanced by towering French intellectual figures such as Roland Barthes, Michel Foucault, Jean-François Lyotard, Jacques Derrida and Jean Baudrillard) 
since the 1960s has also challenged the philosophical prominence of the transcendental subject known from the Italian humanists and the Enlightenment period by famously proposing, for example, the 'death of the author'. In the wake of this literary, philosophical and sociological tradition, we have seen the rise of posthumanism and transhumanism as well as the animal rights movements, all of which seriously question the ontological, epistemological and moral exceptionalism and superiority of humans vis-à-vis other mortal creatures.

Besides pointing out some of the main intellectual motors behind the contemporary 'cult of death', Khapaeva also provides numerous examples of how the new 'cult of death' is being expressed within popular culture. According to her, the narrative script behind the popular cultural celebration of death is the integral motif or plot of the nightmare - on the one hand, as something that is experienced by the fictional characters but also, on the other, as something 'attempting to bring its audience as close as possible to a living nightmare'. ${ }^{63}$ Throughout the book, Khapaeva presents numerous examples of the new cultural celebration of death from recent literary bestsellers and movie blockbusters such as Harry Potter, Lord of the Rings, Tanya Grotter (a Russian equivalent of Harry Potter), The Walking Dead, The Vampire Diaries, The Twilight Saga and similar fantasy/mystery/horror productions. As she points out, throughout the Harry Potter coming-of-age novels/movies and all the other books, films and television series, 'the celebration of death emerges as a new strategy in entertainment and a prominent element in the culture of nightmare consumption, which presents violent virtual death as fun for the whole family'. ${ }^{64}$ Even though Khapaeva privileges popular culture in her book, it is important for her to stress that the new cult of death cannot be reduced only to a popular cultural phenomenon, but is also constituted by concrete practices carried out by real people at various moments such as at Halloween, theDay of the Dead, dark tourism or when creating roadside memorials for those killed in traffic accidents. As she states: "The cult of death is not a phantom of the imaginary world limited to philosophical ideas, novels and movies. It is a social and cultural phenomenon that manifests itself in various social practices"; however, Khapaeva still insists that the meaning of these practices "is best revealed and interpreted through the analysis of popular culture."65

The Cultural Celebration of Death is an important and impressive contribution to the recent development of 'revival' studies of death. The book explains how the new cult of death is not really about death as such but about how we as humans understand our own role in life and in the universe. It is obvious from this that Khapaeva provides a critical reading of some of the current trends and tendencies in our relationship with death, particularly as it is expressed and manifested within the world of contemporary popular culture.

\section{The age of spectacular death: Michael Hviid Jacobsen}

My own contribution to the diagnosis of our contemporary death culture began in 2001 when I published a small book, my first, titled Dødens mosaik (The Mosaic of Death). In this book I tried, in my own admittedly precocious way, to capture the 'spirit of the time' when it came to our attitude toward death. By that point, I had read and re-read the work of Freud, Ariès, Gorer, Walter and all the rest and devoured their ideas. I came up with the perhaps neither surprising nor convincing conclusion that something new was happening in our understanding of and relationship with death here at the threshold of 
a new millennium. Some years later, I began to think more critically about how to update the insights of Ariès to a new type of society beyond the grip of 'forbidden death', and I began toying with the idea of 'spectacular death'. ${ }^{66}$ This search for new terminology later culminated in an article in which it was boldly suggested that we have entered a new phase of 'death mentality' in the wake of Ariès's 'forbidden death', deeming this notion no longer accurate in attempting to capture contemporary death culture in the 21 st century. ${ }^{67}$ This is, I claim, the age of 'spectacular death', 68 and there are, I suggest, five dimensions of 'spectacular death' that even though they do not capture either the breadth or the depth of our cultural attitude toward death nevertheless neatly summarize some important aspects of what has taken place since the age of 'forbidden death'.

First, the mediation/mediatization of death: whereas death was previously something most people experienced and handled as a hands-on phenomenon, nowadays death is fast becoming something seen at a distance through the media or processed by professionals specially trained to take care of the dying and the dead on our behalf. This means that our comprehension of death - and our lack of experience with its actual look, smell and feel - have been gradually diminished.

Second, the commercialization of death: making money from death is not a new thing; however, with the rise of the modern-day 'funeral industry', with death being used to market items not even remotely associated with it, with funeral directors advertising on television, with the development of flat screens and mini-bars inserted into coffins or $\mathrm{QR}$ codes and video monitors on gravestones, with artificial diamonds made from the ashes of the deceased, with artists and branding experts using diamond-plated craniums, fish in blenders, headstones, skulls and bones and all other sorts of death imagery in order to attract attention, provoke responses or increase sales, it makes sense to suggest that something new has indeed happened.

Third, the re-ritualization of death: modern society, as also Tony Walter insisted above, was bent on de-traditionalizing death as well as almost all other aspects of life. This development was exacerbated by processes of urbanization, secularization and the advancement of medicine and technology. Time-honored ritual practices were replaced with minimalistic and seemingly more time-efficient ways of dealing with the great transitions of life. Rites of passage became less elaborate, time-consuming and removed from the orthodoxy of religion. In recent years, however, the 'repressed has returned' with the reappearance of many seemingly forgotten rituals in connection to death, dying and bereavement and with the invention of new ceremonies, rituals and practices aimed at making our encounter with death more meaningful on the personal and communal level. Just think of 'memorial walks', lighting candles by the bedside of the dying, singing songs or psalms for deceased residents leaving nursing homes or hospices, the spreading of the ashes of the deceased at sea, 'natural burial' practices, creating commemorative homepages for the dead, taking photographs of the dying and the dead (especially in neonatal wards), 'dining-with-death' events, 'spontaneous memorials', 'death awareness weeks', 'grief awareness weeks', 'good grief networks', 'compassionate communities' and so on.

Fourth, the humanization of death (also called 'the palliative care revolution'): according to most of the theories proposed by the 'tabooists', death was thoroughly medicalized and institutionalized during the modern era. With the rise of the hospice movement in the 1960s and later with the expansion of palliative care into many regions and corners of the healthcare system, a new way of understanding and dealing more dignifiedly with 
death gained momentum. Today, we have hospices in most parts of the Western world, we have palliative care doctors and nurses, we have palliative care wards and palliative medicine, all of which testify to the ambition of humanizing death and making it something we must learn to live with instead of something we incessantly and, in the end, unnecessarily seek to combat.

Finally, the specialization of death: meaning that the topic of death, dying and bereavement, which were for many years regarded as an obscure preoccupation among a small group of (often largely marginal) scholars, has now become a subject to be taught and studied at many different levels of the educational system, sometimes dealt with in its own right under the official heading of 'death education', at other times as a specialized part of other teaching modules and programs. Within disciplines such as sociology, anthropology, psychology, philosophy, nursing research and related fields of science, death is no longer shunned. Moreover, as an area of increased academic specialization, the topics of death, dying and bereavement now have their own journals, book series, seminars, conferences, research units, funding agencies, immortal texts, international glitterati, tricky terminology and so on, which all goes to show just how much has changed since the time when death was routinely tabooed.

According to this perspective, there is no doubt that something new and quite striking has happened in recent years in regard to our cultural attitude towards death - not least as the media and communications technologies have developed tremendously since the 1980s. But, as suggested, there are also many other reasons why death is imagined, managed, perceived and experienced differently nowadays as compared to when Ariès closed his eyes for the last time on a cold February day in 1984. Some are indeed technological, others more related to shifts in values, beliefs, mores, morality, behavioral codes and so on. Even though the notion of 'spectacular death' in itself has not yet caught on - and perhaps never will - as the most accurate or useful way of describing our contemporary development in 'death mentality', it seems as if at least some researchers agree that death in many ways today is more spectacular than previously, thus warranting a new descriptive label. ${ }^{69}$

\section{Death, indeterminacy and ambivalence}

This chapter has addressed the two principal ways that our attitude toward death in the modern West has been described within some important pieces of work on death from the social sciences and humanities over the past 150 years or so. The easy and obvious conclusion would be to claim that there once - particularly during the 20th century (it is always convenient when social phenomena fit so neatly into centennial frames) - was a 'death taboo' but that this has now (in the early 21 st century) imploded or disintegrated. However, this would be jumping to conclusions. There is considerable complexity when dealing with the presence, persistence or dissolution of taboos in general, and the death taboo is no exception to this rule. For example, when asked the question so popular among journalists with an interest in existential and philosophical issues, 'What is our attitude towards death today?', the researcher is often left to his or her own devices. An answer to this question is in fact made difficult by almost all the elements contained within the sentence: What is meant by 'our' (who is the 'we' referring to, is it Westeners, Brits, Americans, Danes)? What is meant by 'attitude' (is this something we can in fact measure and provide percentages of, or is it rather something less tangible and more 
fluffy)? What is meant by 'death' (are we talking about the event of death, the process of dying, the thought of death, is it our own death, that of people whom we know and love or the death of anonymous strangers)? And finally, what is meant by the word 'today' (are we talking about this year, this decade or something even more encompassing)? Such a question is equivalent to asking a researcher a similarly absurd one: What is our attitude towards life today? Surely, this would lead to a lot of frustration, tergiversation and evasiveness on the part of the recipient of the question, and most likely also on the part of the person processing the response. A similarly frequently asked question is: 'Is death still a taboo in our society?' Here too we immediately encounter several problems. First of all, what is meant by 'taboo'? As we saw above, there is a long history of taboo/ death taboo research, but there are also many problems involved in defining, delimiting, testing and proving the existence of a taboo, not least because taboos may seem to have different meanings and forms of expression in different cultural contexts. Second, the word 'still' seems to suggest that death was definitely a 'taboo' at some previous period in time, which within studies of death, as we have seen, is often dated particularly to the 20th century. However, are we really sure that death was in fact a taboo back then? Did people not talk about death at all? Did they always whisper when doing so? Was death banned from sight? Were children always kept away from the deathbed and funerals? Did people cross the road in order to avoid contact with a widowed person? Were the dying routinely hidden from sight? And so on. After all, death has never been officially codified as something entirely forbidden to be mentioned or dealt with, at least not in any of the social and cultural contexts described by the aforementioned studies. Even though there is little doubt that people in the 20th century managed death markedly differently if compared to the rituals and traditions of the preceding centuries, it is difficult to determine that this different way necessarily amounts to a 'taboo'. To be sure, this does not mean that death was not a taboo, but because the notion of 'taboo' has often been used in an under-theorized manner or is merely assumed, we are unable to tell in any definitive or incontrovertible way if and how this was indeed the case. Thus, as it has been rightly observed:

The 'death taboo' still appears to be a problematical term, vague and unspecific as to what it covers in terms of behaviours and by whom it is observed, deliberately trivialized by a former colonial society and used as catch-phrase for valueless and unfounded fears. ${ }^{70}$

Moreover, if death was in fact such a solid taboo during the 20th century, something entirely objectionable and unmentionable, it is somewhat surprising that so much research literature was in fact capable of dealing with the topic. This paradoxical situation made American psychiatrist Michael A. Simpson muse during the heyday of the 'death taboo', 'Death is a very badly kept secret; such an unmentionable topic that there are more than 650 books now in print asserting that we are ignoring the subject. ${ }^{, 71}$ Since then, thousands of new titles have been added to this list. Almost at the same time, French historian Michel Vovelle observed how 'the taboo on death is the latest fashion and everyone believes he has invented it'. ${ }^{72}$ It may seem as if many death researchers during most parts of the 20th century were perhaps being drawn to or seduced by the idea that death was a taboo - often without being able to provide any substantial empirical backing for the claim or any conceptual clarification of how, when and why death 
was indeed such a 'tabooed topic'. Perhaps the same goes for death researchers writing at the threshold of the 21 st century, who now seem adamantly to claim that death has been 'revived' and that the days of the death taboo are now definitively over.

As should be obvious from this, questions about the existence of a 'death taboo' or the question of how people actually feel deep down about death are extremely difficult to answer in any definitive manner. Obviously, we may consult the treasure troves of historical material, some available but not always convincingly produced statistics or idiographic case studies, popular cultural productions, media stories or simply inferences from our own everyday experiences. However, believing that we will eventually be able to get to the bottom of how people (individuals and collectives alike) really think about perhaps the most difficult thing in the world - death - would be misguided. At the end of the day, this does not mean that we should stop reflecting upon or writing about 'death mentalities', 'attitudes toward death' or changes in 'death cultures', but it does mean that we need to understand that we are often dealing with our own ideas - cognitive reflections and rationalizations - about death rather than about death itself or the role of death in society. I started out by claiming that death is a constant in human life the fact is that everybody dies. I also insisted, however, that death does not stand still, because humans continuously - through thought, emotions and actions - relate to and engage with death differently from their ancestors. This is the reason why it makes good sense to investigate the way our perceptions of death change - but perhaps also in many ways remains the same - throughout history.

From the previous presentation of theories on and studies of death in society, it is evident that there has been an unmistakable change of perspective from the 'tabooists' to the 'revivalists', as they have here been called - but also that there are important continuities as well. Even though the 'revivalists' claim that death today is no longer as hidden or forbidden as among the generations of the 20th century, they still believe that our relationship to death - especially when filtered through the media or popular culture - is one marked by a certain distance and not least some discomfort. We nowadays seem primarily to experience and view death from afar as a secondhand observation through screens that distort the experience of real or actual death. So, the fact that death has become, once again, a more public phenomenon neither means that it is now finally embraced nor that we have developed a 'natural attitude' (whatever that means) toward it. Rather, if we accept the perspectives of Kate Berridge and Dina Khapaeva, it seems as if the 'new pornography of death' and 'cult of death' remove us even further from appreciating what actual death really is. There is no doubt that denial and distance continue to constitute a characteristic feature of our contemporary approach to or attitude toward death. It thus seems as if our contemporary relationship to or attitude toward death cannot be boiled down (if it ever could) to a seductively neat one-liner or a simple formula - death, it seems, is at the same time revived, celebrated and accepted as well as tabooed, denied and hidden away. Perhaps this is not so surprising after all. Since it is impossible to either completely embrace or completely deny or hide death, all societies must simultaneously accept and deny death. ${ }^{73}$ This is the crux of the ambivalence of death. This acceptance-denial continuum is therefore always a matter of degree, and the way such an acceptance or denial is enforced and practiced clearly differs from society to society, from culture to culture, and from epoch to epoch. Just as several researchers in recent times have discussed and questioned whether we are in fact a so-called 'deathdenying' society, ${ }^{74}$ so we also need to discuss and question if and/or to what degree 
death is in fact a 'taboo'. However, the extent to which the notion of 'death taboo' is an accurate and fair description has within recent research not received as much attention as that of 'death denial'. ${ }^{75}$ This situation should thus be taken as a golden opportunity to investigate this matter further and to support and qualify any conclusions with the use of comprehensive data.

\section{Conclusion}

As this chapter has shown, death is and remains a continuous presence and a mystery in human life. It is also a continuous concern for those scholars and researchers trying to understand our human and social ways of dealing with the unpleasant awareness and reality that we are mortal creatures. Based on a chronological review of literature, the chapter has attempted, at risk of oversimplification, to describe two main camps within so-called 'Death Studies', namely one primarily popular in the 20th century, consisting of writings on 'death taboo' and another emerging around the threshold of the 21 st century concerned with a 'death revival'. The chapter has provided a compact introduction to the main ideas of some prominent proponents of these two respective factions, focusing on how each has contributed to the development of a terminology and an understanding of the changing role of death in society. Each of the theories or perspectives presented provides a snapshot of death in society - in many cases, however, a snapshot embedded within a more encompassing historical understanding - that captures certain aspects and changes but simultaneously leaves out or neglects others.

Despite their interpretative limitations, the theories and perspectives presented here have had an enormous impact on the way in which 'Death Studies' has influenced and, at times, monopolized our culture's view on death. It is important to note that the viewpoints of these two 'discursive camps' are not diametrically opposed: thus many of the so-called 'tabooists' did in fact insist that death became more visible (at least within popular culture) throughout the 20th century, while many of the 'revivalists' were keen to stress that, even though death was perhaps becoming more visible today than half a century ago, many of the new forms of visibility were accompanied or even caused by more subtle forms of concealment and alienation.

All in all, this chapter has aspired to show how the way those of us within the world of science in general and the social sciences and humanities in particular think, write or talk about death, our discursive and interpretative practices, as it were, is not an entirely innocent or innocuous activity. In fact, the way we construct a discourse about a given topic - be it death, sex or any other, as Michel Foucault already observed - is always part of a reconstructive effort that reveals something, while simultaneously leaving other truths unsaid and undiscovered.

\section{Notes}

1 Joachim Whaley, Mirrors of Mortality: Studies in the Social History of Death (London: Macmillan, 1981), pp. 1-14.

2 Hannah Malone, "New Life in Modern Cultural History of Death," The Historical Fournal 62 (2018), 833-852.

3 See, e.g., Philippe Ariès, The Hour of Our Death (London: Allen Lane, 1981); Allan Kellehear, A Social History of Dying (Cambridge: Cambridge University Press, 2007); Michael Kerrigan, The History of Death: Burial Customs and Funeral Rites from the Ancient World to Modern Times (Guilford, 
CT: The Lyons Press, 2007); Edgar Morin, L'homme et la mort (Paris: Seuil, 1970); Peter N. Stearns, Revolutions in Sorrow (Boulder, CO: Paradigm Publishers, 2007).

4 Helen Gilmore, Cyril Schafer and Siân Halcrow, "Tapu and the Invention of the "Death Taboo' - An Analysis of the Transformation of a Polynesian Concept," Fournal of Social Archaeology 13, no. 3 (2013), 331-349.

5 James Cook, The Fournals of Captain James Cook, Volume III: The Voyage of the Resolution and Discovery 1776-1780, ed. J.G. Beaglehole (Cambridge: Cambridge University Press for the Hakluyt Society, 1967).

6 Sigmund Freud, Totem and Taboo: Resemblances between the Mental Lives of Savages and Neurotics (New York: Cosimo Classics, 1918/2009).

7 Jerry S. Piven, Death and Delusion: A Freudian Analysis of Mortal Terror (Greenwich, CT: Information Age Publishing, 2004).

8 Tony Walter, "Modern Death: Taboo or Not Taboo?," Sociology 25, no. 2 (1991), 293-310.

9 Sigmund Freud, "Thoughts for the Times on War and Death," The Standard Edition of the Complete Psychological Works of Sigmund Freud (Volume 14) ed. James Strachey (London: Hogarth Press, 1915/1957), pp. 275-300.

10 Ibid., 289.

11 Ibid., 289.

12 Ibid., 296.

13 Ibid., 289, 292.

14 Ibid., 290-291, 299-300.

15 Geoffrey Gorer, "The Pornography of Death," Encounter October (1955), 50-51.

16 Ibid., 51.

17 Geoffrey Gorer, Death, Grief and Mourning (Garden City, NY: Doubleday, 1965), p. xv.

18 Ibid., xx.

19 Ibid., xxxii.

20 Ibid., 130.

21 Gorer, The Pornography of Death, 50.

22 Ernest Becker and Sam Keen, "A Conversation with Ernest Becker," Psychology Today 7 (1974), $71-80$.

23 Ibid., 74.

24 Ernest Becker, The Denial of Death (New York: Free Press, 1973/1997), p. 13.

25 Ibid., 15.

26 Ibid., 24.

27 Ibid., 27.

28 Ibid., xvii.

29 Ibid., 27.

30 Ibid., 24.

31 See, e.g., Sheldon Solomon, Jeff Greenberg and Tom Pyszczynski, The Worm at the Core: On the Role of Death in Life (New York: Random House, 2015).

32 Philippe Ariès, Un Historien du Dimanche (Paris: Seuil, 1980).

33 Roy Porter, "The Hour of Philippe Ariès," Mortality 4, no. 1 (1999), 83-90.

34 Philippe Ariès, Western Attitudes Toward Death from the Middle Ages to the Present (Baltimore: Johns Hopkins University Press, 1974a); Ariès, The Hour of Our Death.

35 Ariès, Western Attitudes toward Death, 59.

36 Ibid., 14.

37 Ibid., 88.

38 Ibid, 106.

39 Philippe Ariès, Images of Man and Death (Cambridge, MA: Harvard University Press, 1985).

40 Norbert Elias, The Loneliness of the Dying (London: Continuum, 1985/2001), pp. 3-5.

41 Ibid., 35.

42 Ibid., $18-19$.

43 Ibid., 43-44.

44 Ibid., 23.

45 Ibid., 74.

46 Ibid., 29. 
47 Ibid., 59-60.

48 Ibid., 23, 85.

49 Ibid., 10.

50 Zygmunt Bauman, Mortality, Immortality and Other Life Strategies (Cambridge: Polity Press, 1992); Ivan Illich, Limits to Medicine: Medical Nemesis: The Expropriation of Health (London: Penguin Books, 1976); Jessica Mitford, The American Way of Death (New York: Simon \& Schuster, 1963).

51 Tony Walter, The Revival of Death (London: Routledge, 1994), p. 47.

52 Ibid., 39.

53 Ibid., 41.

54 Ibid., 186.

55 Ibid., $42-43$.

56 Walter, "Modern Death: Taboo or Not Taboo?" 293-310.

57 Tony Walter, What Death Means Now: Thinking Critically About Dying and Grieving (Bristol: Policy Press, 2017), pp. 11-12.

58 Kate Berridge, Vigor Mortis: The End of the Death Taboo (London: Profile Books, 2002), p. 26.

59 Ibid., 247.

60 Dina Khapaeva, The Celebration of Death in Contemporary Culture (Ann Arbor, MI: University of Michigan Press, 2017), 1.

61 Ibid., 15.

62 Ibid., 15.

63 Ibid., 20.

64 Ibid., 174.

65 Ibid., 47. For another take on the decline of taboo, but with New Age thinking, see Raymond L.M. Lee, "The Deconstruction of Death: Postmodernism and Near-Death," Journal of NearDeath Studies 22, no. 3 (2004), 179-194; and Raymond L.M. Lee, "Mortality and ReEnchantment: Conscious Dying as Indiviualized Spirituality," Journal of Contemporary Religion 22, no. 2 (2007), 221-234.

66 Michael Hviid Jacobsen, "Den femte fase - med Philippe Aries ind I det 21. Arhundrede," Doden speglad $i$ aktuell kulturforskning ed. Anders Gustavsson (Upsala: Gustav Adolf Akademien, 2009).

67 Michael Hviid Jacobsen, "Individualised Imortality in Liquid-Modern Times: Teasing Out the Topic of Symbolic Immortality in the Sociology of Zygmunt Bauman," Postmortal Society Towards a Sociology of Immortality, ed. Michael Hviid Jacobsen (London: Routledge, 2017).

68 Michael Hviid Jacobsen, ed., The Age of Spectacular Death (London: Routledge, 2020).

69 See, e.g., Jacobsen, ed., The Age of Spectacular Death.

70 Gilmore, Schafer and Halcrow, "Tapu and the Invention of the 'Death Taboo," 341.

71 Michael A. Simpson, Dying, Death and Grief: A Critical Bibliography (Philadelphia, PA: University of Philadelphia Press, 1979), p. vii.

72 Michel Vovelle, "Rediscovery of Death Since 1960," Annals of the American Academy of Political and Social Science 447 (1980), 90.

73 Richard G. Dumont and Dennis C. Foss, The American View of Death: Acceptance or Denial? (Cambridge, MA: Schenkman Publishing Company, 1972).

74 See e.g., Allan Kellehear, "Are We a 'Death-Denying' Society? A Sociological Review," Social Science and Medicine 18, no. 9 (1984), 713-723; Carol Zimmerman, "Death Denial: Obstacle or Instrument for Palliative Care? An Analysis of Clinical Literature," Sociology of Health E Illness 29, no. 2 (2007), 297-314.

75 For some notable exceptions, see Walter, "Modern Death: Taboo or Not Taboo?"; Duncan Sayer, "Who's Afraid of the Dead? Archaeology, Modernity and the Death Taboo," World Archaeology 42, no. 3 (2010), 481-491. 


\section{PART II}

\section{REGIONAL PATTERNS}

Essays in Part II deal with major regional patterns in the modern experience of death, with particular emphasis on cultural, ritual and emotional features but attention is given too to basic material parameters. The regional approach stands in deliberate tension with the more global dimensions traced in Part I.

Not all regions are represented, which in some cases reflects different levels of available research. Factors highlighted in some regions are not always picked up in others another invitation to further work in the future to improve opportunities for comparative analysis.

This part begins with discussion of the implications of the Enlightenment, a Western cultural development that might gain some influence on other parts of the world over time. This is followed by analysis of changing patterns in popular religious outlook in three English-speaking countries. Other essays pick up on some interactions between Western patterns and regional traditions, with varying results. But the exploration of trends in places such as China and Japan also suggests the limited impact of external influences. The final chapter on recent developments in the Caribbean explores a clearly complicated tension between local traditions and United States patterns as mediated by migrants.

Collectively, essays in this section invite consideration of the relationship between the regional and the global, with considerable emphasis on the staying power of key cultural and religious variants. Modern death unquestionably involves some global features often linked to medicalization, but also to new ideas such as the challenges to the death penalty - and a certain number of mutual influences. (The essay on Mexico, for example, explicitly deals with the tension between common trends in the physical experience of death as opposed to regional beliefs and rituals.) But people also find comfort and guidance in regional continuities. In contrast to some other features of the human experience, it may be misleading to think in terms of a globalization of death. Essays on India, Islam and Africa raise the question of traditional continuities most explicitly, but the theme informs the whole part to some degree.

This means in turn that the modern history of death invites efforts at comparison, at least as a next step in analysis. Is there a counterpart, in other religions, to the effort to soften the Christian approach to death in the later nineteenth century? How much do the material changes in death, plus some shared cultural influences, alter death rituals in different societies? How have different traditions reacted to recent shifts in the medical definition of death? There is ample fodder for further juxtapositions. 


\section{$\because$ Taylor \& Francis Taylor \& Francis Group http://taylorandfrancis.com}




\title{
'WHY MAY NOT MAN BE ONE DAY IMMORTAL?'
}

\author{
Rethinking death in the age of enlightenment
}

\author{
Joseph Clarke
}

There are many reasons why William Godwin's Enquiry Concerning Political fustice attracted attention when it was first published in London in February 1793. A few weeks after Louis XVI had gone to the guillotine in Paris and with Britain on the brink of war with the regicide French Republic, any book that advocated change on the scale Godwin envisioned - an end to inequality, religion, wedlock, war, even to the state itself - was bound to raise eyebrows. However, it was Godwin's closing 'conjecture' concerning man's capacity for perfectibility that shocked many of his contemporaries most. Reflecting on 'the omnipotence of mind' - its ability, with time, to gain mastery over all matter - he posed the provocative question: 'Why may not man be one day immortal?' ${ }^{1}$ By the time Political Justice appeared, Godwin had been thinking about immortality for a long time. References to it appear repeatedly, if laconically, in his diary throughout the early 1790s, and, in Political fustice, the answer to that question was an optimistic yes. ${ }^{2}$ As Godwin saw it, disease, debility and even death were essentially psychosomatic disorders, and under the right conditions and with the appropriate mental effort - he recommended 'cheerfulness' and the suppression of both sleep and sexual urges especially - they would eventually disappear. Once the mind had overcome the frailty of flesh, the time would arrive when 'men will perhaps be immortal. The world will be a people of men, and not of children. Generation will not succeed generation ... There will be no war, no crimes, no administration of justice as it is called, and no government. ${ }^{3}$ Through a combination of rational reform and moral improvement, death's dominion would be undone.

Even by the standards of the revolutionary 1790s, this was heady stuff. And yet, Godwin was far being from the first eighteenth-century writer to imagine that man might achieve immortality in the here-and-now rather than in the hereafter of conventional Christian eschatology. The great Irish satirist, Jonathan Swift, had arguably anticipated this ambition almost seventy years before when he cast an acerbic eye over what immortality might mean in Gulliver's Travels, although Swift's aging but undying Struldbruggs were scarcely the stuff that utopian dreams were made of. ${ }^{4}$ A generation later, the editor of the Encyclopédie, Denis Diderot, was almost as sceptical as Swift. Writing in 1769, he dismissed the idea of extending man's life, even to 'only twice his natural span', as not even worth discussing in his philosophical dialogue, D'Alembert's Dream, although privately he also wondered whether lovers might ever attain a kind of immortality through the mingling of their matter in the grave. ${ }^{5}$ 
Diderot's dreams of molecular intimacy aside, attitudes were already starting to change, and the idea of achieving immortality on earth began to appear almost respectable in the 1770s. The English polymath and political radical Joseph Priestley hinted at the possibility in 1771 when he predicted a 'glorious and paradisiacal' future for mankind as it marched 'towards perfection', and a couple of years later, his friend and fellow scientist, the American revolutionary Benjamin Franklin was even more explicit about his expectations for the future. ${ }^{6}$ In an exchange with the French doctor Jacques BarbeuDubourg on the still uncertain 'distinction between death and the extinction of life' in April 1773, Franklin pondered the possibility that the dead might eventually be 'recalled to life' by advances in medical science, and he returned to the theme a few years later in a letter to Priestley in February $1780 .{ }^{7}$ Reflecting on 'the rapid progress true science now makes', Franklin looked forward to a future when 'all Diseases may by sure means be prevented or cured, not excepting even that of Old Age, and our Lives lengthened at pleasure even beyond the antediluvian Standard'. ${ }^{8}$ Both exchanges were already in the public domain by the time Godwin published his own take on this theme in 1793, and this kind of thinking was current in continental Europe too. Writing shortly after Political Fustice appeared, the French mathematician, philosopher and (once again) revolutionary Nicolas de Condorcet predicted a time would come when 'progress in medical care, nutrition and healthier accommodation', along with political reform, would lead, if not precisely to immortality, then to the next best thing: the 'indefinite' prolongation of life. ${ }^{9}$ Enlightenment science had already unleashed an extraordinary sense of anticipation in both Europe and America by the last quarter of the century, but with the ancien régime apparently disintegrating in both Old and New Worlds by the 1790s, humanity's potential for progress began to appear unbounded. The enlightened reading public had already been energized by Luigi Galvani's experiments in electrical re-animation in Bologna in the early 1780s, and the implications of revolutionary change in Paris at the end of the decade appeared equally exciting. ${ }^{10}$ When even medical adventurers like James Graham peddled their cures with the promise of 'prolonging Life to the very longest possible Period', the possibility that death might be denied seemed, in progressive circles at least, an idea whose time had come. ${ }^{11}$

Obviously, these were dreams and, as John McManners wryly noted, 'elitist ones at that', and they were never very widely shared. ${ }^{12}$ While Political fustice was generally well received, even Godwin's most sympathetic critics dismissed his views on immortality as 'fanciful', while the Anglican clergyman and economist Thomas Malthus was prompted to write his Essay on the Principle of Population to refute Godwin and Condorcet's 'conjectures', although significantly he based his case on economic rather than religious grounds. ${ }^{13}$ And yet, the fact remains: a world without death had started to seem 'thinkable' at the end of the eighteenth century. In America, Britain and France, the three epicentres of cultural and political change in the enlightened eighteenth century, serious thinkers, scientists, statesmen and philosophers (along with self-publicizing charlatans like Graham) had begun to think very seriously indeed about the possibility that death might be defeated. Admittedly, men like Franklin, Godwin and Condorcet were hardly typical of their times. However, their dreams of a world without death do embody, in admittedly extravagant relief, the convergence, possibly even the logical conclusion, of some of the eighteenth-century Enlightenment's most radical assumptions: its conviction that everything should be open to question; its confidence in man's capacity to improve his lot through scientific progress and social reform; its affirmation of the individual, and his 
(less often her) autonomy; its faith in the intellect, and the intellectual, as a force for emancipatory change. In an already revolutionary age, Franklin, Godwin and Condorcet's belief that the mind might finally free itself of its mortal coil represents the most enlightened ambition of all.

This dream of transcending death represents the eighteenth-century Enlightenment at its most audacious - or conversely at its most absurd - but there was more to this dream than that. This willingness to radically rethink humanity's understanding of its own mortality also suggests why so many historians have identified the late eighteenth century as a watershed moment in attitudes towards death throughout the Western world. From Michel Vovelle and Philippe Ariès's pioneering histories of death in the 1970s to Thomas Laqueur's magisterial The Work of the Dead more recently, the methods, inflections and even interpretations differ, but the essential point remains. Between the mid-eighteenth and early nineteenth century, far-reaching social, cultural and political changes fundamentally transformed how many among the educated elite thought about death and the relationship between the living and the dead. ${ }^{14}$ That transformation was both intensive and extensive; it affected individual beliefs and behaviors, modified social norms and repeatedly involved the state in the implementation of radical reform. Ultimately, this revolution ended in the 'overthrow', and the term is widely used in the scholarship, of 'a regime of the dead' that had survived in the West for over a thousand years. ${ }^{15}$

The vocabulary historians have employed to define this process varies, but the changes they discuss are dramatic nonetheless. Michel Vovelle, for example, has described the emergence of an emphatically agnostic 'discourse of death' during the Enlightenment, a new language that sought to 'demystify', even 'dechristianize', death by redefining it in scientific, as opposed to spiritual, terms. ${ }^{16}$ Vovelle's evidence is predominantly French, but few figures illustrate this new outlook more clearly than the aristocratic Italian atheist Alberto Radicati. His Philosophical Dissertation upon Death, published while he was in exile in London in 1732, sought to strip death of its religious terrors by defining it as merely a 'dissolution' of bodily matter, an entirely natural process and accordingly nothing to be feared. ${ }^{17}$ In 1732 , this argument was enough to have Radicati, his translator and publisher arrested, even in supposedly tolerant England, but in the decades that followed, the vision of death as a 'dissolution' was widely repeated in some of the radical Enlightenment's most celebrated works, bestsellers like the comte de Buffon's Histoire naturelle de l'homme in the 1740s, Diderot and d'Alembert's Encyclopédie in the 1750s and the baron d'Holbach's infamous Système de la nature of $1770 .{ }^{18}$ These were all important texts, but for all their notoriety, this kind of unapologetic (and very aristocratic) atheism was never the norm, even within the exceptionally anticlerical French Enlightenment. ${ }^{19}$ Vovelle's account of a 'demystifying offensive' embodies his vision of an emancipatory Enlightenment, a revolution in ideas that paved the way for the revolution in politics at the end of the century, but other scholars have been less sanguine. For Philippe Ariès and Arthur Imhof, these changes evoke disenchantment rather than demystification, and alienation more often than emancipation; in Imhof's wistful account, the eighteenth century marks the demise of a world where 'death came more easily' than it does today. ${ }^{20} \mathrm{By}$ contrast, Thomas Laqueur's many works on the transition between what he describes as the old and new regimes of the dead have balanced a shrewd assessment of the critical role that capitalism, class and consumer culture played in constructing 'the new regime of the dead' with an acute awareness that disenchantment and 'the reinvention of enchantment' were connected and complementary processes throughout this period. ${ }^{21}$ 


\section{Implementing the new ideas}

However we choose to interpret these seismic changes in the culture of death, their practical implications were obvious by the early nineteenth century. As Roy Porter and Ruth Richardson have argued, the period witnessed a simultaneous 'medicalization' and 'commodification' of death as a new cast of characters and institutions - doctors, undertakers, administrators and cemetery companies - supplanted the clerics and churches that had monopolized the business of dying for more than a millennium. ${ }^{22}$ In their hands, death became an ever more secular, and increasingly commercial, concern and both of these trends converged in the most critical change of all: 'the displacement of the dead' from the spaces they had occupied since late antiquity. ${ }^{23}$ Since the establishment of the Latin Church, the dead (unless they died in impenitent disgrace) had always been buried in close proximity to the sacred, and that placed them at the centre of the community and very emphatically 'among the living'. ${ }^{24}$ The dead had rights in this regard, quite literally in common and customary law, and that meant they were entitled to interment in either a church (ideally their own) or in the consecrated ground that surrounded it. ${ }^{25}$ It was generally understood that these rights should be observed according to clearly defined rules and in return, as Vanessa Harding makes clear, the dead continued to serve society long after their demise. ${ }^{26}$ Their presence in churchyards in the heart of towns and cities throughout Europe was seen as an essential element of the community's sense of identity and a key to understanding its own history. Admittedly, the Reformation had disrupted many of these conventions in the sixteenth century, but even as radically different theologies of salvation emerged across a confessionally divided Europe, much remained unchanged. ${ }^{27}$ While some German reformers established cemeteries beyond city walls to separate the dead from the living, Scotland's Calvinists frequently worked around official bans on 'kirk-burial' to ensure that they were interred alongside their less orthodox ancestors, while their co-religionists in Holland continued to bury their dead in their churches' vaults much as they had before the Reformation. ${ }^{28}$ The Reformation may have begun in revolt against the Catholic cult of the dead, but continuity rather than change remained the chief characteristic of the early modern topography of death across much of western Christendom.

After centuries of stability, change, when it came, arrived abruptly in the eighteenth century. From the middle of the century onwards, the crypts, churchyards and charnel houses that contained the remains of past generations of Parisians, Londoners and Viennese suddenly came to be seen as stinking sources of contagion rather than centres of collective identity, and crusading campaigners began to call for their closure. Some of those crusaders were clerics, ministers like Thomas Lewis in London or priests like Scipione Piattoli in Modena, and they were anxious to reform religious practice not replace it. ${ }^{29}$ But more often they were men of science like the Welsh naturalist Thomas Pennant or the French chemist Antoine Cadet de Vaux. Above all, they were engagé intellectuals like the ubiquitous Voltaire and the indefatigable Louis-Sébastien Mercier, writers who translated hard science, or at least what passed for it in the eighteenth century, into a damning critique of 'the sepulchral exhalations that continue to poison the faithful' in French churches. ${ }^{30}$ From the late 1770 s onward, this campaign began to bear fruit with the introduction of legislation banning burials either within churches or cities themselves; in the Duchy of Modena in 1774, in Prussia the following year and in France the year after that, in Tuscany, Lombardy and across the Habsburg empire in the early 1780s. ${ }^{31}$ As the Enlightenment progressed, educated opinion was no longer willing to tolerate the presence 
of the dead in its midst. Perhaps more importantly, absolutist rulers from Francesco III of Modena and Frederick II of Prussia to the emperors Joseph II and Napoleon I were also more than ready to avail themselves of an opportunity to trim the sails of their troublesome clergies by stripping them of the authority, and income, they derived from the business of death. When the dead were finally despatched to their new abodes on the outskirts of cities like Milan in 1786, Paris in 1804 and finally London in 1832, the sanitization, secularization and ultimately 'suburbanization' of death appeared complete. ${ }^{32}$ After centuries at the center of the Christian community, the dead were now merely matter, and malodorous matter at that: a subject for scientific study rather than religious reflection, something fit only, in the end, to be 'exiled' from view. ${ }^{33}$

The eighteenth century's debate on death was wide-ranging and all-encompassing. At its most ambitious and innovative, it aspired to re-orientate human endeavor away from the early modern preoccupation with preparing for the next world to focus on living in this one instead. At its most humane, it encouraged contemporaries to think compassionately, frequently for the first time, about how best to alleviate the suffering of those facing death, and it inspired an even more unprecedented investment of empathy and understanding in the question of suicide. ${ }^{34}$ However, the repercussions of this debate could also be unsettling, intrusive and, particularly during the French Revolution's radical assault on customary religious culture during the Terror, exceptionally violent. ${ }^{35}$ The enlightened eighteenth century laid the foundations, both intellectually and institutionally, for the modern 'regime of the dead' that emerged in cemeteries like Père Lachaise, Kensal Green and Mount Auburn, but that modernity was complex, often contradictory and frequently contested. It also engendered anxieties that could be just as disquieting as those it had been designed to alleviate.

In attempting to assuage 'the profound sentiment of fear in the face of death' that afflicted early modern Catholics or the 'intense and unremitting anxiety' that gripped their Reformed contemporaries, the Enlightenment gave rise to new and equally insistent forms of unease. ${ }^{36}$ We began with William Godwin, and he will remain a guide of sorts through the rest of this chapter. This is not simply a matter of convenience, although Godwin's long and very prolific life (1756-1836) does coincide remarkably closely with the chronology of this period's most decisive changes in the culture of death. This is not to imply that any one individual could ever embody a movement of ideas and opinions as multifaceted as the eighteenth-century Enlightenment, let alone seem to speak for an entire century - although Godwin did address his age at interminable length across many genres. Rather it is to suggest that the personal and philosophical journey that carried a boy from a profoundly religious household in mid-century Norfolk to that Promethean vision of a world without death in 1793 mirrors many of the hopes and fears that had inspired enlightened attempts to rethink death in the eighteenth century. And just as revealingly, his later Romantic musings on memory reflect some of the uncertainties that radical rethinking had been unable to allay. Godwin matters in this sense, not because he incarnates some imaginary spirit of his age but because he exemplifies the strange synthesis of expectation and anxiety that defined the Enlightenment's lengthy dialogue with death.

\section{Godwin's trajectory}

Although few realized it at the time, Godwin's birth in 1756 coincided with the onset of 'a new demographic era' in Europe, a period of sustained population growth that saw 
England's population, like that of the continent's, first stabilize after the last of the early modern era's major mortality crises in the 1720 s and then take off rapidly from around mid-century. ${ }^{37}$ By 1800, Europe's population was twice what it had been a century before, although the pace of this increase varied enormously from place to place. Hungary's population, for example, soared from 1.5 to 8.5 million over the century while Ireland's more than doubled, but France and England experienced more modest growth, from 22.6 to 29 million in the former and from approximately 5 to just over 9 million in the latter. ${ }^{38}$ If the overall pattern now appears clear, Europe's 'vital revolution' remained remarkably uneven and was regularly interrupted, especially on the continent's periphery, by ongoing mortality crises. Famine may have claimed as much as one fifth of the Irish population in the early 1740s, and the Seven Years War decimated Pomerania's population at mid-century, and, though the plague disappeared from England after 1666, episodic outbreaks of disease could still devastate entire localities. ${ }^{39}$ The old demographic order was changing, but death remained an unpredictable and acquisitive adversary; it could arrive at any moment and it still took a terrifying toll among the very young. For these reasons, it remained, as Lawrence Stone observed, 'at the centre of life, as the cemetery was at the centre of the village' in pre-industrial England, and Godwin's family history bears that dictum out in all its grim reality. ${ }^{40}$ By the time William was sixteen, his father and five of his thirteen siblings were already dead, a normal enough attrition rate in a period when barely two out of three children ever reached their teens and when those fortunate enough to survive infancy rarely saw their fiftieth year. ${ }^{41}$ As the Book of Common Prayer had counselled in 1662, 'in the midst of life we are in death', and that remained as true in the $1760 \mathrm{~s}$ as it had been a century before. ${ }^{42}$

Life was lived in the shadow of death in mid-eighteenth-century England and religious families, like Godwin's, raised their offspring accordingly. As a young boy, he later recalled, he went to bed every night with instructions to 'compose myself to sleep ... as if I were never to wake again' and, from an early age, he aspired to emulate the 'pious deaths' he read about in the devotional works that passed for children's books in godly English households. ${ }^{43}$ While the young William was clearly precocious, this kind of upbringing remained common enough in the mid-eighteenth century. Devotional works remained the stock-in-trade of popular book sellers throughout the period and while few new titles appeared over the eighteenth century, the fact was they did not need to. The eighteenth-century book trade was saturated with reprints of late seventeenth-century texts with catchy titles like John Hayward's Hell's Everlasting Flames Avoided or William Sherlock's perennially popular Practical Discourse Concerning Death, and the advice these works offered the faithful was always to be ready for death. ${ }^{44}$ As Sherlock directed his readers: 'it must be the Business of our whole lives to prepare for Death; our Accounts must be ready, because we know not how soon we may be called to given an Account of our stewardship', and young Godwin clearly took this kind of counsel to heart. ${ }^{45}$ As the dutiful son of a minister raised in the rigorous traditions of the Puritan 'saints', he could hardly have done otherwise. On leaving home, his schooling continued in similar vein. Although he read widely as a boy, his education was shaped by an unforgiving Sandemanian theology, a 'supra-Calvinist' certainty that the vast majority of mankind was eternally and irredeemably damned, and he carried that conviction with him into a short-lived, and stunningly unsuccessful, career as a dissenting minister in the early 1780s. ${ }^{46}$ By then, however, Godwin's views had already begun to change. 
In 1781, Godwin appears to have experienced an epiphany. He discovered radical politics and even more radical French philosophy, and the religious doubts he was already experiencing intensified when he encountered Jean-Jacques Rousseau's deism and, more scandalous still, the atheism of d'Holbach's notorious System of Nature (1770). By 1783, Godwin had abandoned the ministry and moved to London to make his way as a writer, and by the end of the decade, he had taken his 'last farewell of the Christian faith'. ${ }^{47}$ In many respects, Godwin's story up to this point reads like an English edition of a very familiar eighteenth-century narrative. Robert Darnton sketched out a very similar story in a French context decades ago, but this particular bildungsroman is about more than the young intellectual's ascent from provincial obscurity to radical renown. ${ }^{48}$ The goal is to understand the implications of Godwin's upbringing and to set his later belief in man's capacity to acquire immortality through his own efforts in the context of a life lived in the constant presence of death. It is, admittedly, impossible to say whether Godwin's hope of a worldly paradise was an attempt to square the circle between a youth lived in expectation of eternal reward (and in fear of eternal damnation) and d'Holbach's insistence that the soul, being indistinguishable from the body, 'perishes with it' and, as a result, 'the idea of its immortality is only an illusion'. ${ }^{49}$ Malthus, certainly thought so, and he mocked Godwin's 'conjectures' as 'a very curious instance of the longing of the soul after immortality' on the part of one 'who has rejected the light of revelation'. ${ }^{5}$ Godwin later conceded that 'the Calvinist system, which had been so deeply wrought into my mind in early life' still influenced his thinking when he was writing Political fustice, and the admission is intriguing. ${ }^{51}$ Over a decade after he had abandoned any semblance of religious orthodoxy, the residue of Godwin's godly upbringing could still be read in one of the Enlightenment's most radical reflections on mortality.

\section{Death and remembrance}

On Sunday, 10 September 1797, Mary Wollstonecraft died, ten days after giving birth to her second daughter, Mary. Godwin recorded his wife's death in his diary with a devastated '20 minutes before 8' and three strokes of his pen. Words had finally failed William, and after Mary's funeral five days later, he moved John Opie's portrait of her into his study, where it remained until his own death almost forty years later, and set about writing a candid - contemporaries thought too candid - memoir of her life. ${ }^{52}$ It would be simplistic to say that this shattering experience extinguished Godwin's earlier optimism that death might be denied, he had already begun to retreat from this when revising Political Fustice in 1795, but it did redefine it. A few months after Mary's death, he began drafting his Gothic fantasy, St. Leon: A Tale of the Sixteenth Century, in which the eponymous hero acquires the elixir of life only to suffer isolation and persecution as he wanders a Europe ravaged by war following the disintegration of his family and the death of his beloved wife - in childbirth. ${ }^{53}$ The autobiographical elements of this fable are striking, but so too is Godwin's newfound emphasis on feeling, the emotional bonds that bound individuals, families and communities together, and this stress on sentiment occupies center stage in his final attempt, if not to defeat death, at least to defy it: his Essay on Sepulchres of 1809.

Where Political Justice had concluded by anticipating an end to death, the Essay began by admitting its inevitability. Physical death, Godwin now accepted, was an inexorable law of nature', but it also afflicted those the dead left behind more than those who die, 
and so he proposed to 'paralyse the hand of oblivion' with the only means left at his disposal: memory. ${ }^{54}$ Where Godwin had once imagined that death could be denied by reason, remembrance was all that remained to him now, and his 'visionary' scheme sought to sustain a kind of 'intercourse', a sentimental and spiritual communion, between the living and the dead through the raising of memorials wherever the latter lay. ${ }^{55}$ These memorials were not meant to be grandiose affairs; it was enough, Godwin argued, to erect a simple 'shrine to their memory' because, he believed, something of the dead would still survive in these 'hallowed' places and in the memories they inspired: memories that would let the dead 'live on as my friends, my philosophers ... and my guides'. ${ }^{56}$ In this more disillusioned post-Revolutionary age, memory might yet confer something of the immortality that enlightened progress had once seemed to promise. The emotional intensity of this appeal to memory, its insistence that the dead are not dead - 'They are still with us in their stories, in their works, in their writings', like the portrait that hung on his study wall - all speak of Godwin's desolation at the loss of a loved one. ${ }^{57}$ But the sense of loss that imbues the Essay on Sepulchres runs deeper than this, because its use of religiously resonant terms like 'shrine' and 'hallowed' to describe the spaces the dead occupy also reflects a wider desire to re-sacralize what had been desacralized, to reconstitute a community of the living and the dead around the tomb. It might be too much to suggest that the Essay on Sepulchres sought to establish a cult of the saints for a secular age, although Godwin did feel obliged to insist that there was 'no danger ... of falling into idolatry' around these shrines, but it did aim to re-invest death with something of the sentimental and spiritual significance it had previously possessed. ${ }^{58}$

Godwin claimed that this proposal was 'visionary', but it was not. It built instead upon the Enlightenment's long-standing assumption that well-designed memorials and eulogies to the dead would inspire a spirit of virtuous emulation among the living, an assumption that had already inspired the establishment of a Revolutionary Panthéon in Paris in 1791 and a number of imitators across Europe in the decades that followed. ${ }^{59}$ Godwin's Essay also drew much of its emotional impact from the already well-established tradition of poetic meditations among the tombs that stretched from the mid-century 'graveyard poets', Thomas Gray, Robert Blair and Edward Young, by way of their continental imitators Salomon Gessner and the abbé Delille, to the tear-soaked visits to the tomb that did so much to define sentimental fiction in the wake of Rousseau's La Nouvelle Héloïse and Goethe's Sorrows of Young Werther in the 1760 s and 70s. ${ }^{60}$ From Bernardin de Saint-Pierre's phenomenally successful Études de la nature of 1784 to the Romantic poets, the generation of writers, and readers, that came of age with Godwin was a generation steeped in the 'voluptuous melancholy' of the tomb. ${ }^{61}$ In many ways, the torrents of tears, both real and imagined, that were shed over tombs during the period bears out Philippe Ariès's suggestion that these years signified a shift from the early modern obsession with preparing for one's own death to a preoccupation with 'the death of the other'. ${ }^{62}$ Godwin's Essay embodies that transition in many respects too, but it also exemplifies the complexity of that process, because the Enlightenment never really displaced the fear of one's own extinction and the need to be remembered, as well as to remember. Even as these authors promised that the community of the living and the dead could endure around the tomb, the anxiety of erasure remained.

Death, even if it was merely the 'dissolution' of matter that radical thinkers had maintained throughout the century, was not easily faced down, and however often the 
likes of Radicati or David Hume insisted that death was nothing to be feared, it seems unlikely that this conviction was widely shared. ${ }^{63}$ Famously, a few freethinkers anticipated death with scandalously studied resignation. The unbelieving French priest Jean Meslier awaited his end with a laconic 'soon I shall be nothing' in 1729, and Hume memorably staged his own demise with elaborately Epicurean insouciance in 1776, but their notoriety is a mark of how rare such resolution remained. ${ }^{64}$ In the end, even Radicati succumbed and when he died, he died repentant, in the bosom of the Reformed Church. ${ }^{65}$ Between Hume and Radicati, Denis Diderot's response to this dilemma was probably more typical. Despite the Encyclopédie's insistence that 'death is not as terrible as we think', its editor had always struggled with the implications of his own unbelief. ${ }^{66}$ Having abandoned any faith in a Christian afterlife early on in his career, Diderot had toyed, as we have seen, with the thought that his remains might retain a trace of the emotional affinities he had once enjoyed, but he admitted this was never anything more than a 'chimera', albeit a 'sweet' one. ${ }^{67}$ Elsewhere, he affected a more matter-of-fact approach to his own mortality. In the novel, Jacques le fataliste, written over the last twenty years of his life, his hero Jacques's, or perhaps his own, response to the question 'do you believe in the life to come?' was defiantly indecisive: 'I neither believe nor disbelieve. I just don't think about it. I do my best to enjoy this one. ${ }^{98}$ In place of the clergy's counsel to keep death constantly in mind, Jacques's refusal to even think about it was an act of revolt his readers would have well understood, but indecision was no answer.

Diderot ultimately sought his solution, as Godwin would more than forty years later, in remembrance, and the hope that he would live on in the memory of others. ${ }^{69}$ For Diderot, the appeal of posterity, 'holy and sacred posterity', was that it offered both consolation, the equivalent of 'the afterlife for the religious man', and a moral compass because the desire to be remembered well would always inspire men to righteousness. ${ }^{70}$ Posterity, however, was just as exclusive as the most austere Christian heaven, and in this account it remained the preserve of the enlightened elect. Just as Godwin's Essay on Sepulchres would later revolve around the graves of 'the illustrious dead', men like Milton, Bacon and Sidney, so Diderot's posterity seems only to have been open to the century's 'great souls'; everyone else, he admitted eventually, 'would perish like beasts'. ${ }^{11}$ The baron d'Holbach was, admittedly, less callous in his conclusions. Although accepting the essence of Diderot's argument, he granted 'the common man' a more limited afterlife in the reminiscences of his family, but for all that posterity was still reserved for the elite. ${ }^{72}$ For the eminent, the illustrious and the educated, the Enlightened discourse of memory held out the prospect of posthumous renown, a well-crafted eulogy, a place in a Panthéon, a cenotaph in a patrician park. For the less enlightened majority, the alternative was less appealing. At their most radical, the authors of the Enlightenment consigned most of their contemporaries to an amnesiac abyss. It is hardly surprising then that many of them rejected this new regime of the dead outright.

\section{Reform and resistance}

Goethe's Elective Affinities appeared a few months after Godwin's Essay on Sepulchres was published in 1809. Death looms large throughout the novel, and as in so many contemporary accounts of insalubrious graves and mouldering tombs, the churchyard initially 
seems a suspect space, somewhere best avoided by the novel's bien-pensant protagonists. Or rather, it remains so until one of the principal characters, the aristocratic Charlotte, takes matters in hand and oversees the transformation of this ramshackle assortment of scattered tombstones and 'bumpy graves' into a 'beautifully verdant' garden. Under her direction, the grounds are levelled and sown with flowers, and the tombstones dug up and despatched to the churchyard's perimeter wall. The end result is exactly what enlightened campaigners had been proposing for decades: a tasteful, well-ordered space where death, and the dead, are discretely hidden from view. ${ }^{73}$ Charlotte's remaking of the churchyard seems so emblematic of enlightened attitudes to death as to be almost a caricature, but in Goethe's telling, it is the local community's reaction to these reforms that seems most revealing. Some of the villagers complain about Charlotte's changes because they were imposed without consultation; others object that the removal of their ancestors' tombstones meant that 'the memory of them was thereby, as it were, extinguished', one family even hires an attorney to cancel the endowment they had paid the church to maintain their kinfolk's grave as they could no longer be sure of 'one day resting with them side by side'. ${ }^{74}$ 'The matter is not so important', Charlotte replied before convincing the lawyer that she, and not his 'selfish and stubborn' clients, had been right all along. The entire episode only occupies a few pages in Elective Affinities, but it also captures why so much of the Enlightenment's agenda proved so contentious. Historians of death have always tended to focus on the forces of change, the social, cultural and political factors and actors that converged to create a new regime of the dead at the end of the eighteenth century. They have, in short, preferred to think about the Charlottes of this world rather than those stubborn, surly villagers, and this chapter has been no different. And yet, Goethe's Elective Affinities reminds us that the attitudes and assumptions that had underpinned the old regime of the dead endured, and that revolutionary change invariably encounters resistance, especially when it touches upon something as sensitive as the relationship between the living and the dead.

In Elective Affinities, that resistance amounted to little more than grumbling on the part of a few disgruntled villagers. In reality, resistance was widespread, wide-ranging and often ferocious. At its most radical, in Revolutionary France, the Republican campaign to dechristianize churchyards and secularize funeral ceremonies provoked widespread despair in the mid-1790s, and that despair frequently turned to violence as communities rallied in defense of the rites they owed their dead. ${ }^{75}$ The events of the year II are certainly an extreme example, but even when funeral reforms did not arrive accompanied by Terror, they still encountered opposition. As Peter Brown noted of the early Christian church: 'burial customs are among the most notoriously stable aspects of most cultures', and challenging that stability proved just as contentious in the eighteenth century as it had in late antiquity. ${ }^{76}$ Long before the excesses of the Terror, the French monarchy's campaign to close city-center churchyards had been a constant source of conflict. It proceeded smoothly enough in Paris once the Catholic clergy had been brought on board in 1775, and few appear to have mourned the closure of the infamous Cemetery of the Holy Innocents in 1780 , but reactions in the provinces were far less predictable. ${ }^{77} \mathrm{New}$ cemeteries were set down in cities like Marseille and Aix in the 1780s, but in both places old churchyards remained in use decades later, and, elsewhere, attempts to close parish graveyards provoked a mix of petitioning, litigation and even rioting in the cities of Lille and Cambrai. ${ }^{78}$ Opposition to the closure of traditional burial grounds assumed any number of forms in late eighteenth- and early nineteenth-century France, but the most 
effective form of obstruction was often financial. New cemeteries were not just socially disruptive but expensive. They required new land, and plenty of it, and this was not always available or affordable in rapidly expanding urban centres, especially when it was up to the local authorities to pay. Accordingly, Angers only opened its new Cemetery of the East in 1848, over forty years after the Napoleonic legislation that had first mandated its creation, while Tours did not acquire its new burial ground until $1858 .^{79}$ If major urban centers were often unwilling to adopt the Père Lachaise prototype, the countryside proved even more quarrelsome. The sight of Monseigneur Bourret, bishop of Rodez, fleeing through fields to escape the rioting he had set off in Verrières when he announced the closure of its churchyard is a measure of the outrage outside interference in a community's relationship with its dead could provoke. But the fact that this happened in May 1876 is a mark of how tenacious these attachments were. ${ }^{80}$ The opening of Père Lachaise in 1804 was undoubtedly revolutionary, but across the rest of France, the new regime of the dead only emerged in fits and starts, and it frequently faltered in the face of violent resistance.

Resistance to change could be just as intense and as protracted elsewhere. It was the former in the Habsburg domains, where Joseph II's sweeping reform of the conduct of funerals in August 1784 provoked immediate opposition across the Empire. As in Elective Affinities, these measures seem to have succeeded in offending almost every section of society. The outlawing of tombstones over individual graves offended the elites who aspired to mark the place, and the social status, of their ancestors with some form of monument, while the provision that everybody should be buried economically, in linen sacks rather than coffins, appears to have upset everyone. By January 1785, as reports of unrest and even a threatened insurrection in Silesia reached Vienna, the Emperor was forced into an uncharacteristic climb-down on the coffin question, although the rest of his reforms remained in place. That concession was delivered via a conspicuously graceless decree:

the attitudes of the living are unfortunately still so materialistic that they attach immense importance to their bodies rotting slowly after death and remaining stinking carcasses for longer, and since it is of no great consequence to me how people wish to be buried ... I do not wish to compel anyone who is not rationally persuaded and so anyone may do what he likes with his coffin and make whatever arrangements he thinks most acceptable for his dead body. ${ }^{81}$

While opposition to reform arose instantly across the Empire in the 1780s, the process was more protracted in Portugal where successive regimes' attempts to modernize an exceptionally Baroque burial culture simply never got off the ground. In the absence of the muscular authoritarianism that a Joseph II or a Napoleon embodied, the spirit may have been willing under the Marquês de Pombal in the 1770s or the Costa Cabral ministry seventy years later, but the state was weak. Repeated attempts to discourage and later prohibit church burials remained dead letters in a society where all but the very poor viewed interment inside a church as their right. In 1805 and 1806, and again in 1835, liberal laws condemning church burials as insanitary and 'superstitious' failed to have any effect, while attempts to enforce new legislation in 1844 only succeeded in igniting a popular uprising, the Maria da Fonte rebellion, and bringing the Costa Cabral ministry down in $1846 .{ }^{82}$

This might have been the first time that a failed funeral reform brought down a government although there was already a precedent for this kind of resistance to change in 
the Lusophone world by this stage. In October 1836, liberal plans 'to bring civilization to' Salvador in Brazil by way of a French-style cemetery on the city's outskirts provoked 'the biggest street demonstration Salvador had ever seen', and the unrest only ended after the new Campo Santo cemetery had been thoroughly sacked by a vast crowd of men and women. ${ }^{83}$ The circumstances that inspired this unrest were certainly exceptional, not least because the French-educated director of Salvador's School of Medicine and the city's archbishop were in accord on the need to bring Bahia's Catholics kicking and screaming into the modern age, but the connection reformers drew between civilization and cemetery reform is telling nonetheless. It suggests the extent to which the educated elites, both secular and ecclesiastical, increasingly looked on most of their charges, from eighteenth-century France and Austria to nineteenth-century Brazil and Portugal, as boorish and backward, 'insanitary' and 'superstitious': in essence uncivilized. Those rioters paid the compliment back in full. They wanted little to do with this sort of civilization because they looked on the new, enlightened regime of the dead that successive waves of absolutists, revolutionaries and liberals sought to impose from Paris, Vienna or some other distant capital as an unwanted intrusion into spaces and customs they still cherished, not so much the advent of a civilizing process as the arrival of something entirely alien.

It did not help that these reforms were often perceived as not simply alien but foreign too. Across much of western Europe from the late eighteenth century onwards, new regulations concerning the closure of churchyards, the establishment of cemeteries and the rationalization of funeral rites often arrived as a result of foreign invasion, occupation or annexation, and that frequently colored how these reforms were received. The example of French military engineers driving roads through Cairo's main cemetery or tearing down tombs that got in the way of their plans for urban renewal in 1798 is an extreme one, but it is not entirely exceptional. ${ }^{84}$ Austrian rule in Tuscany and Lombardy had seen the Habsburg restriction on mourning and church burials extended south of the Alps in the 1780s, and this kind of cultural imperialism only intensified when the French took the Austrians' place from the 1790s on. As Radicati's example suggests, the Italian Enlightenment had conducted its own very animated debate on death and dying throughout the eighteenth century, and the educated classes across Italy, both clerical and lay, had been just as engaged as their counterparts elsewhere in considering how best to reform funerary culture according to local needs. However, with the application, word for word, of Napoleon's legislation on cemeteries throughout the peninsula between 1806 and 1809, all of those local ideas and initiatives were made to count for nothing. ${ }^{85}$ Small wonder then that the formerly pro-French poet Ugo Foscolo lamented the arrival of the French legislation that 'today places sepulchres / beyond the gaze of pity and denies / the dead a name' in Dei Sepolcri in $1807 .{ }^{86}$ In its universalizing ambition, that law's application in Italy extended its status as a conquered colony into the grave itself, and the situation was the same at the other end of the Empire too. In prosperous, Lutheran Hamburg, church burials came to an end on New Year's Eve in 1812, not because the locals had been considering reform for nearly two decades but because the city had been absorbed into the French Empire the year before and the new Napoleonic regulations took effect the next day. ${ }^{87}$

It is significant, too, that women were frequently to the fore in resisting the new regime's arrival. During, and after, the Terror in France, for example, women had regularly led the way in ensuring the dead were buried with religious rites, while in one village in the Loire, Belmont, up to a hundred women defied the authorities' attempts to dechristianize death by staging a sit-in in their parish's churchyard. ${ }^{88}$ It lasted three days 
before a detachment of National Guards arrived, and, even then, they were only dispersed with gunfire. As the name suggests, women also provided the backbone of the Maria da Fonte rebellion in Portugal, and eyewitnesses reported that it was women, lay sisters, who started the stone-throwing that ended in the sack of Salvador's Campo Santo cemetery too. ${ }^{89}$ This prominence may be a reflection of the fact that women's presence in the affairs of the dying had been steadily undermined by this new regime's innovations. In traditional societies, it was commonly women's work to care for the dying and prepare the dead for their final journey, but as these tasks gradually fell to (invariably male) professionals, the doctor and the undertaker, women's role in the business of death was increasingly reduced. ${ }^{90}$ In this respect, the gendered dimension of these protests may have owed much to women's marginalization in the social order this increasingly commercialized funerary culture represented, but it seems likely that these women took up arms, or encouraged their men to do so, in defense of something more deep-seated too: a sense that the dead remained, even in death, members of the community. If the new, enlightened regime of the dead appeared alien and inspired resistance, it was because its attempts to efface death by displacing the dead from the center of communal life to distant cemeteries threatened this sense of community beyond repair. In societies where the memory of the dead still retained 'a profound religious significance', where the ceremonies associated with death endured even as other religious rites were abandoned, the new regime of the dead appeared disorientating and divisive, a rent in the fabric of the social order and an affront to identity. ${ }^{91}$

If there is a common thread that connects Goethe's fictional villagers, Cambrai's very real rioters, Silesia's incipient rebels and Portugal's actual ones, it is the cultural chasm that had opened up over the eighteenth century between the elites and those they administered or, in Edward Thompson's classic terms, between patrician society and plebeian culture. ${ }^{92}$ The insistent individualism of the Enlightenment, its condemnation of the old regime of the dead as disorderly and indecorous, insanitary and superstitious, an embarrassing anachronism in an enlightened age, was a reflection of the distance that had developed during this period between a metropolitan - and cosmopolitan - elite that looked to science, secularity and social engineering to reshape the world in its own image and a customary culture that remained rooted in tradition and respect for the past, that still clung to what Thomas Laqueur has described as 'the Gemeinschaft of the dead'. ${ }^{33}$ Goethe realized this in Elective Affinities, and Wordsworth sensed it too. In his poem We are Seven, published in 1798, he dramatized this clash of cultures in an imagined dialogue between 'a little cottage girl' and her educated, and implicitly male, questioner. The girl's insistence that 'we are seven' even though 'two of us in the church-yard lie' provokes at first incredulity and then indignation on the part of her inquisitor. At the poem's close, his frustration finally erupts in an infuriated, but ineffectual, 'But they are dead: those two are dead!', although he admitted this was just 'throwing words away'. ${ }^{94}$ The girl and her inquisitor may have spoken the same language, but they belonged to different worlds. Their mutual incomprehension is a measure of the intense social, cultural and emotional dislocation that the attempt to rethink death in the age of Enlightenment initiated.

\section{Notes}

1 William Godwin, An Enquiry Concerning Political fustice (Oxford: Oxford University Press, 2013), 453.

2 William Godwin, The Diary of William Godwin, Victoria Myers, David O'Shaughnessy and Mark Philp, eds. (Oxford: Oxford Digital Library, 2010) http://godwindiary.bodleian.ox.ac.uk. 
3 Godwin, Political fustice, 458.

4 Jonathan Swift, Travels into Several Remote Nations of the World by Lemuel Gulliver, 2 vols. (London, 1726) vol. ii, 139-46.

5 Denis Diderot, Le Rêve de d'Alembert (Hermann: Paris, 1987), 113, and Correspondance de Denis Diderot, Georges Roth, ed., 14 vols (Paris: Éditions de Minuit, 1956), vol. ii, 284.

6 Joseph Priestley, An Essay on the First Principles of Government ... (London, 1771), 5.

7 Benjamin Franklin, The Papers of Benjamin Franklin, William Wilcox et al., eds. (New Haven, CT: Yale University Press, 1976), vol. xx, 161-5 and 189-90.

8 Franklin, The Papers of Benjamin Franklin, vol. xxxi, 455-6.

9 Jean-Antoine-Nicolas de Caritat, marquis de Condorcet, Esquisse d'un tableau historique des progrès de l'esprit humain (Paris, 1794), 379-80.

10 Marcello Pera, The Ambiguous Frog: The Galvani-Volta Controversy on Animal Electricity (Princeton, NJ: Princeton University Press, 2014); I. R. Morus, "Radicals, Romantics and Electrical Showmen: Placing Galvanism at the End of the English Enlightenment," Notes and Records of the Royal Society, 63 (2009), 263-75.

11 James Graham, A Short Treatise on the All-Cleansing, All-Healing and All-Invigorating Qualities of the Simple Earth (Newcastle, 1790). On enlightened medicine's pursuit of immortality, Mary Mulvey Roberts, "'A Physic Against Death': Eternal Life and the Enlightenment," in Mary Mulvey Roberts and Roy Porter, eds., Literature and Medicine During the Eighteenth Century (Routledge: London, 1993), 151-67.

12 John McManners, Death and the Enlightenment: Changing Attitudes to Death in Eighteenth-Century France (Oxford: Oxford University Press, 1981), 119.

13 Analytical Review (Aug. 1793), 403. The conservative Critical Review was less restrained about this 'absurdity', (Oct. 1793), 154. Thomas Malthus, An Essay on the Principle of Population (London, 1798), 241.

14 Across an immense and constantly expanding scholarship, see, in particular, Philippe Ariès, Western Attitudes toward Death from the Middle Ages to the Present (Baltimore: John Hopkins University Press, 1974), and L'homme devant la mort, 2 vols. (Paris: Seuil, 1977), translated as The Hour of Our Death (New York: Alfred Knopf, 1981); Pierre Chaunu, La mort à Paris, 16e, 17e et 18e siècles (Paris: Fayard, 1978); Margaret Cox, ed., Grave Concerns: Death and Burial in England, 1700 to 1850 (York: Council for British Archaeology, 1998); R. A. Etlin, The Architecture of Death: the Transformation of the Cemetery in Eighteenth-Century Paris (Cambridge, MA: MIT Press, 1984); Robert Favre, La mort dans la littérature et la pensée françaises au siècle des lumières (Lyon, Presses Universitaires de Lyon, 1978); Ralph Houlbrooke, Death, Religion and the Family in England, 1480-1750 (Oxford: Oxford University Press, 1998); Arthur E. Imhof, Lost Worlds: How Our European Ancestors Coped with Everyday Life and Why Life Is So Hard Today, translated by Thomas Robisheaux (Charlottesville, VA: University of Virginia Press, 1996); Graig Koslofsky, The Reformation of the Dead: Death and Ritual in Early Modern Germany, c. 1450-1700 (London: MacMillan, 2000); Thomas Laqueur, The Work of the Dead: A Cultural History of Mortal Remains (Princeton, NJ: Princeton University Press, 2015); F. Lebrun, Les hommes et la mort en Anjou aux XVIIe et XVIII siècles: essai de démographie et de psychologie historique (Paris: Flammarion, 1975); McManners, Death and the Enlightenment; David Stannard, The Puritan Way of Death: A Study of Religion, Culture and Social Change (New York: Oxford University Press, 1977); Peter N. Stearns, Revolutions in Sorrow: The American Experience of Death in Global Perspective (Boulder, CO: Paradigm, 2007); Michel Vovelle, La mort et l'Occident de 1300 à nos jours (Paris: Gallimard, 1983).

15 Laqueur, The Work of the Dead, 93; Michel Vovelle, Piété baroque et déchristianisation en Provence au XVIIIe siècle (Paris: Seuil, 1973), 274.

16 Vovelle, La mort et l'Occident, 394-411, and Piété baroque et déchristianisation en Provence.

17 Alberto Radicati, A Philosophical Dissertation upon Death, Composed for the Consolation of the Unhappy by a Friend of Truth (London, 1732), 11-13, 22.

18 For example, Georges-Louis Leclerc, comte de Buffon, Histoire naturelle de l'homme, in Oeuvres complètes de Buffon (Brussels, 1829), vol. v, 61; Louis de Jaucourt, "Mort, in Denis Diderot and Jean le Rond d'Alembert, eds. Encyclopédie ou dictionnaire raisonné des sciences, des arts et des métiers (Paris, 1751-72), vol. x, 716-18. Paul Thiry, baron d'Holbch, Système de la nature ou des loux du monde physique et du monde morale (London, 1774 ed.), passim. 
19 On the limited appeal of the eighteenth century's most radical religious beliefs, see S. J. Barnett, The Enlightenment and Religion: The Myths of Modernity, (Manchester: Manchester University Press, 2003), 32.

20 Imhof, Lost Worlds, 173. Italics in the original.

21 Thomas Laqueur, "Cemeteries, Religion and the Culture of Capitalism," in Jane Garnett et al., eds. Revival and Religion since 1700 (London: A\&C Walsh, 1993), 183-200, and The Work of the Dead, 14.

22 On these themes, see James Riley, The Eighteenth-Century Campaign to Avoid Disease (London: MacMillan, 1987); Roy Porter, Flesh in the Age of Reason (London: Penguin, 2004); Julie Rugg, "A New Burial Form and Its Meanings: Cemetery Establishment in the First Half of the Nineteenth Century," in Cox, ed. Grave Concerns, 44-53; Ruth Richardson, Death, Dissection and the Destitute (London: Penguin, 1988).

23 Ariès, L'Homme, 200-5; Thomas Kselman, Death and the Afterlife in Modern France (Princeton, NJ: Princeton University Press, 1993), 170.

24 Peter Brown, The Cult of the Saints: Its Rise and Function in Latin Christianity (Chicago, IL: Chicago University Press), 9; Vanessa Harding, The Dead and the Living in Paris and London (Cambridge: Cambridge University Press, 2002), 46.

25 On the legal dimensions of this obligation, see Laqueur, The Work of the Dead, 151-5, and McManners, Death and the Enlightenment, 277-84.

26 Harding, The Dead and The Living, 85.

27 The essays in Bruce Gordon and Peter Marshall, eds., The Place of the Dead: Death and Remembrance in Late Medieval and Early Modern Europe (Cambridge: Cambridge University Press, 2000) are a good introduction to some of these issues.

28 Koslofsky, The Reformation of the Dead, 41-76; Andrew Spicer, "“Rest of their Bones": Fear of Death and Reformed Burial Practices," in William Naphy and Penny Roberts, eds. Fear in Early Modern Society (Manchester: Manchester University Press, 1997), 167-83.

29 Mark Jenner, "Death, Decomposition and Dechristianization? Public Health and ChurchBurial in Eighteenth-Century England," English Historical Review, 120 (2005), 615-32.

30 Voltaire, "Enterrement," in Voltaire, Questions sur l'Encyclopédie, 9 vols (London, 1772), vol. v, 181; Louis-Sébastien Mercier, Le tableau de Paris, 12 vols (Neuchâtel, 1781), vol. i, 59.

31 Riley, The Eighteenth-Century Campaign, 108-9; Hannah Malone, Architecture, Death and Nationhood: Monumental Cemeteries in Nineteenth-Century Italy (New York: Routledge, 2017), 17-84; Derek Beales, Joseph II, vol. ii (Cambridge: Cambridge University Press, 2009), 320; McManners, Death and the Enlightenment, 310-19; M. Foisil, "Les attitudes devant la mort au XVIIIe siècle: sépultures et suppressions de sépultures dans le cimetière parisien des Saints-Innocents," Revue Historique, 251 (1974) 303-30.

32 On the 'suburbanization of death', see Peter Stearns, Satisfaction Not Guaranteed: Dilemmas of Progress in Modern Society (New York: New York University Press, 2012), 135. For Milan and Père Lachaise, see Malone, Architecture, Death and Nationhood, 20, and Erin-Marie Legacey, Making Space for the Dead: Catacombs, Cemeteries and the Reimagining of Paris, 1780-1830 (Ithaca, NY: Cornell University Press, 2019). For London's Kensal Green, see James Stevens Curl, ed. Kensal Green Cemetery: The Origins and Development of the General Cemetery of All Souls, Kensal Green, 1824-2001 (Chichester: Phillimore, 2001).

33 Alain Corbin, The Foul and the Fragrant: Odour and the Social Imagination (London, MacMillan, 1996), 58-9. Vovelle, La mort et l'Occident, 431.

34 On the Enlightenment's sometimes ambivalent attitude towards suicide, see Lester G. Crocker, "The Discussion of Suicide in the Eighteenth Century," Fournal of the History of Ideas, 13 (1959), 47-72, and Roísín Healy, "Suicide in Early Modern and Modern Europe," Historical fournal, 49 (2006), 903-19.

35 On the Terror, see Michel Vovelle, The Revolution Against the Church: From Reason to the Supreme Being (Cambridge: Polity Press, 1991), and Joseph Clarke, "The Dechristianization of Death in Revolutionary France," in James Kelly and Mary Ann Lyons, eds. Death and Dying in Ireland, Britain and Europe (Dublin: Irish Academic Press, 2013), 143-70.

36 Lebrun, Les hommes et la mort, 321, and Stannard, The Puritan Way of Death, 79. See also Vovelle, La mort et l'Occident, 384, and Houlbrooke, Death, Religion and the Family, 46, 75. 
37 James L. Sheehan, German History, 1770-1866 (Oxford: Oxford University Press, 1989), 75. Also Vovelle, La mort et l'Occident, 368-82, and Michael Flinn, The European Demographic System 1500-1820 (Baltimore, MD: John Hopkins University Press, 1981).

38 Vovelle, La mort et l'Occident, 368-70; David Dickson et al., "Hearth Tax, Household Size and Population Change 1672-1821," Proceedings of the Royal Irish Academy, 82c (1982), 156. Jack Goldstone, "Demography," in William Doyle, ed. The Oxford Handbook of the Ancien Régime (Oxford: Oxford University Press, 2012), 205; E. A. Wrigley and R. S. Schofield, The Population History of England, 1541-1871 (Cambridge: Cambridge University Press, 1989); and Paul Langford, A Polite and Commercial People: England 1727-1783 (Oxford: Oxford University Press, 1998), 146. The situation in colonial America was more complicated, but by the time of its first census in 1790, the United States was starting to undergo the same population explosion that had begun in Europe a generation earlier. Stephen J. Kunitz, "Mortality Change in America, 1620-1920," Human Biology, 56 (1984), 559-82.

39 Lebrun, Les hommes et la mort, 113-19.

40 Lawrence Stone, The Family, Sex and Marriage in England, 1500-1800 (London: Penguin, 1979), 54.

41 Peter Marshall, William Godwin (New Haven, CT: Yale University Press, 1984), 13.

42 The Book of Common Prayer and Administration of the Sacraments ... (Cambridge, 1762 edition), n.p.

43 William Godwin, Collected Novels and Memoirs of William Godwin, vol. i, Autobiography, Autobiographical Fragments and Reflections, Mark Philp, ed. (London: Pickering \& Chatto, 1992), 12.

44 Houlbrooke, Death, Religion and the Family, 66.

45 William Sherlock, A Practical Discourse Concerning Death, 16th ed. (London, 1715$), 307$.

46 Godwin, Autobiography, Autobiographical Fragments and Reflection. 52. On his education, Marshall, William Godwin, 22-8.

47 Godwin, Autobiography, Autobiographical Fragments and Reflections, 53.

48 Robert Darnton, "The High Enlightenment and the Low-Life of Literature in Prerevolutionary France," Past and Present, 51 (1971), 81-115.

49 D'Holbach, Système de la nature, vol. i, 257 and 261.

50 Malthus, An Essay on the Principle of Population, 241.

51 Godwin, Autobiography, Autobiographical Fragments and Reflections, 53.

52 Marhsall, William Godwin, 190-1.

53 William Godwin, St. Leon: A Tale of the Sixteenth Century (London, 1799).

54 William Godwin, Essay on Sepulchres: Or a Proposal for Erecting Some Memorial of the Illustrious Dead of All Ages on the Spot Where Their Remains Have Been Interred (London, 1809), 8, 28.

55 Godwin, Essay on Sepulchres, 75, 28, 77.

56 Ibid., 78.

57 Ibid., 83.

58 Godwin, Essay on Sepulchres, 28.

59 Joseph Clarke, Commemorating the Dead in Revolutionary France: Revolution and Remembrance, 1789-1799 (Cambridge: Cambridge University Press, 2007); and Eveline Bouwers, Public Pantheons in Revolutionary Europe: Comparing Cultures of Remembrance, c. 1790-1840 (Basingstoke: Palgrave, 2012).

60 James Stephen Curl, "Young's Night Thoughts and the Origins of the Garden Cemetery," Fournal of Garden History, 14 (1994), 92-118; and Roland Mortier, La poétique des ruines en France: ses origines, ses variations de la Renaissance à Victor Hugo, (Geneva: Droz, 1974).

61 Jacques-Henri Bernardin de Saint-Pierre, Studies of Nature (London, 1798 ed.), 329.

62 Ariès, Western Attitudes towards Death, 56.

63 Radicati, A Philosophical Dissertation, 11-13, 22. David Hume echoed the point in his essay "On the Immortality of the Soul," in Hume, Essays on Suicide and the Immortality of the Soul (London, 1783), 28.

64 Paul Hazard, La pensée européenne au XVIIIe siècle (Paris: Boivin, 1946), 74; Laqueur, The Work of the Dead, 203-8.

65 Margaret Jacob, The Secular Enlightenment (Princeton, NJ: Princeton University Press, 2019), 213.

66 Louis de Jaucourt, "Mort," in Diderot et al., Encyclopédie, vol. x, 716-18.

67 Diderot, Correspondance, vol. ii, 284. 
68 Denis Diderot, Jacques the Fatalist and his Master, translated by David Coward (Oxford: Oxford University Press, 1999), 164.

69 Diderot, "Immortalité," in Diderot, ed., Encyclopédie, vol. viii, 577.

70 Diderot, "Le Pour et le Contre ou Lettres sur la Postérité," in Diderot, Euvres complètes, H. Dieckmann and J. Varloot, eds., vol. xv, (Paris, 1986), 33.

71 Godwin, Essay on Sepulchres, 75; Diderot, "Immortalité," 577, and "Le Pour et le Contre," 11.

72 D'Holbach, Système de la Nature, 315.

73 Johann Wolfgang von Goethe, Elective Affinities, translated by David Constantine (Oxford: Oxford University Press, 1994), 14, 118.

74 Ibid., 118-19.

75 Clarke, "The Dechristianization of Death in Revolutionary France."

76 Brown, The Cult of the Saints, 24.

77 Foisil, "Les attitudes devant la mort," and Owen and Caroline Hannaway, "La fermeture du cimetière des Innocents," Dix-huitième siècle, 9 (1977), 181-92.

78 McManners, Death and the Enlightenment, 313; Alain Lottin, "Les Morts chassés de la cité; 'lumières et préjugés' les émeutes à Lille et à Cambrai lors du transfert des cimetières," Revue du Nord, 60 (1978), 73-117.

79 Kselman, Death and the Afterlife, 176; Madeleine Lasserre, "La loi et les morts: la difficile création du cimetière général de Tours au XIXe siècle," Annales de Bretagne et des pays de l'Ouest, 98 (1991), 303-13.

80 Peter M. Jones, Politics and Rural Society: The Southern Massif Central, c. 1750-1880 (Cambridge: Cambridge University Press, 1985), 138.

81 Decree of 17 Jan. 1785, in Beales, Foseph II, vol. ii, 325.

82 Rui Feijó and João de Pina-Cabral, "Conflicting Attitudes to Death in Modern Portugal: The Question of Cemeteries," in Rui Feijó, Herminio Martins and João de Pina-Cabral, eds. Death in Portugal: Studies in Portuguese Anthropology and Modern History (Oxford: JASO, 1983), 25; Francisco Queiroz and Julie Rugg, "The Development of Cemeteries in Portugal, c. 1755-1870," Mortality, 8 (2003), 113-28

83 J. J. Reis, "Death to the Cemetery': Funerary Reform and Rebellion in Brazil, 1836," History Workshop, 34 (1992), 34 and 42.

84 Juan Cole, Napoleon's Egypt: Invading the Middle East (New York: Palgrave, 2007), 185, 201.

85 Malone, Architecture, Death and Nationhood, 35-7.

86 Ugo Foscolo, Dei Sepolcri, translated by John Lindon, in The Italianist, 28 (2008), 171.

87 Joachim Whaley, "Symbolism for the Survivors: The Disposal of the Dead in Hamburg in the late Seventeenth and Eighteenth Centuries," in Joachim Whaley, ed. Mirrors of Mortality: Studies in the Social History of Death (London: Routledge, 2011), 104.

88 Olwen Hufton, Women and the Limits of Citizenship in the French Revolution (Toronto: University of Toronto Press, 1992), 120-2. Edouard Herriot, Lyon n'est plus, 4 vols. (Paris, 1937-40), vol. iii, 229.

89 David Birmingham, A History of Portugal (Cambridge: Cambridge University Press, 2003), 125; Reis, "Death to the Cemetery," 34.

90 Marie-France Gueusquin, "Gardiennes et portefaix: de la prééminence de la femme dans le travail funéraire d'une commune rurale de la Nièvre," Ethnologie Française, 13 (1983), 129-38.

91 Lebrun, Les hommes et la mort, 359.

92 E. P. Thompson, "Patrician Society, Plebeian Culture," Fournal of Social History, 7 (1974), 382-405.

93 Laqueur, "Cemeteries, Religion and the Culture of Capitalism," 192.

94 William Wordsworth, "We are Seven," in Lyrical Ballads with a Few Other Poems (London, 1798), $110-14$. 


\title{
"NOW FOR THE GRAND SEGRET"

\author{
A history of the post-mortem identity and Heavenly \\ reunions, $1800-2000$
}

\author{
Fohn C. Weaver and Doug Munro
}

Triumphant, the soul trembles with new hope in heavenly increasing ardour.

Tosca, Act III

\section{Introduction: researching the post-mortem identity}

This chapter dwells on people's conceptions of life after death, delving into the constructions of popular culture rather than impressions derived from either literary and artistic representation or the imaginings propagated by organized religion. ${ }^{1}$ In recent years, research has suggested an underestimation of "folk religion - the popular belief in God by many people who rarely attend church but still consider themselves Christian." We concur with and build on this observation by Patricia Jalland, as well as her follow-up that it has been common for the bereaved to experience the presence of the dead. ${ }^{2} \mathrm{We}$ acknowledge too the leads from prior histories of death and the afterlife, most notably the long-term perspective. ${ }^{3}$ However, the ensuing discussion is an outlier since it reaches into the Beyond. Our investigation of the post-mortem identity picks up the subject of death before the main event, at a point when individuals declared a future destination in preparation for their own death. Some "informants," though not on the death bed, were in equivalent situations, contemplating suicide or heading to the gallows. The "Grand Secret" also pertains to a time after the well-studied sequence of rituals that follow death, because images of Heaven and its populace are completely detached from the activities of the survivors dealing with terrestrial death management.

The subject highlights important adjustments in cultural approaches to death, capturing some changes - but also some continuities - in three English-speaking countries, mainly in the nineteenth century but with important extensions beyond. The findings emphasize the ongoing role of religion, at a time when some historians may overemphasize secularization, but also modifications in popular beliefs that, among other things, reflect the impact of Romanticism and familial emotion. The result should be compared to changes and continuities in the religious treatment of death in other regions.

In a landmark exploration of death and Western culture, The Hour of Our Death, Philippe Ariès set some of the agenda for this study; although, in common with Ralph Houlbrooke, writing in Death, Ritual, and Bereavement, we find that his "idealized picture of 
traditional death is unconvincing and rests on a few widely scattered examples." was right, however, about the importance of the long view: "the historian of death must not be afraid to embrace the centuries." Concentrating on popular notions, the current inquiry covers nearly two centuries, a period of inexpensive books and newspapers, an immensely important consideration for both the propagation of popular culture and research method. The digitization of the popular press in the United Kingdom, Australia, and New Zealand facilitated a quest for materials other than "widely scattered examples." Still, examples do feature here, drawn from several databases to illustrate the trends we seek.

The nature of the publications that support the inquiry - cheap and often local - directs attention away from debates over elaborate Victorian mourning customs and whether or not they helped the bereaved or were "a bonanza of commercial exploitation." ${ }^{6}$ While commercial initiatives preyed on, or perhaps served, hoped-for transitions to a better place, notions about the soul and Heaven took death from its standing as a great leveller to the next stage, namely a better life in the Beyond. Particular background situations point to the rebelliousness of people who declared an upcoming meeting in Heaven or announced that God would forgive them. Authorities responded critically to some self-declared grants of immortality. Moreover, in a modified form, disputes over the right to enter Heaven have continued into recent times as a problem in moral philosophy, a topic left for the chapter's concluding remarks.

The popular press is not where our interest in these subjects originated. We first encountered individuals imagining the Grand Secret during a study of suicide involving about 12,000 inquests in Queensland and New Zealand from 1890 to 2000. They documented life's troubles. ${ }^{7}$ Here and there they intimated a belief in a future. Although the inquest files contribute to the current study, especially in the suicide notes, the sheer abundance of accessible material in print enables a more extensive examination of people's handling of the Grand Secret. The permutations on the post-mortem identity and the fate of that core concept over the course of many decades present intricacies that caution against uncritical acceptance of over-theorizing and against the practice of tying together disparate events and practices. ${ }^{8}$ Given the subject, we can claim that the angel is in the details.

The analysis of suicides centred on the troubles that people were leaving, not on what lay ahead. Some definitely deliberated over what was in store for them after death. Others did not give the future a thought. Silences were prevalent. When an alcoholic sheep shearer predicted "I will die like a fucking Dingo," he surely was not dwelling on the mysteries of death but on the pointlessness of a life spent shuffling aimlessly between pubs and lockups. ${ }^{9}$ Other individuals, rational, concerned, and forthright, did fret. As one explained, "I am so worried as you might easily imagine." ${ }^{10}$ Men and women left evidence that they weighed a possible future in the Beyond against a desperately unhappy past. A distressed schoolteacher was seen "by a witness to walk towards the river then change his mind and walk back towards [town] before walking again toward the river." 11 Before a clerk ended his life, he "went down in the backyard and considered it." 12 "I wonder," another asked, "what I am going to do in one hour's time."13 A distressed carpenter wrote that he had "made up his mind to go out of this world.", A cattle drover left a note advising his boarding house keeper that he was leaving tonight "for another world." 15 These men had thought things over. "Still here," wrote another, "but expect to go tonight."16 Gestures, including kissing children farewell, saying 
goodbye instead of good night, and asking if others could manage the farm, signalled deliberation. The signs just cited appeared in documents from the turn of the century, as it happens, a turning point in popular culture references to Heavenly reunions and God's forgiveness.

The inquests afforded glimpses of individuals framing their own afterlife, not their funerals. Nor did they describe Heavenly "landscapes," cite Revelations, or mention John Milton's poetic variations on Revelations. Remarks about the Resurrection were rarely seen. A devout Roman Catholic contemplating suicide wrote to his Bishop that he believed "in the resurrection of the body and life everlasting" and got to his knees to "say the Act of Contrition." 17 Far more often, there were short statements, appeals to "pray to God for me" and "may God forgive me." 18 And presumptuous assertions that "God will forgive me." But a defining part of Christian theology, the Resurrection, was not very prominent even in published romantic characterizations of the Beyond. Popular culture ideas of the Beyond, expressed in print and documents, wandered away from the base of organized religion that it drew upon. Individuals concentrated on meeting loved ones soon, not waiting for Judgment Day. Select people, not the presence of angels, were what counted. ${ }^{19}$ "See you in Heaven." "Hope to meet in Heaven soon."21 "We may meet beyond the grave again."22 "Gertie. Trusting we will meet in the next world."23 A lovelorn young man wrote to his mother, "I am going to Dad." ${ }^{24}$ Another in a similar state of disappointment, wrote, "I shall have gone to join my sister Mary." Written in 1946, the writer of this suicide letter concluded with an admission of doubt about exactly what we should have expected from this time period: "I am just about to start on a long journey of which and where it will end I do not know." ${ }^{, 25}$ We are getting ahead of the chronology, but it is worthwhile calling attention to a cultural drift away from Heaven as the declared post-mortem destination.

Other statements affirmed trust in divine justice. Occasionally, there were hints of a rebellion against the oppression of the current world. "God will have mercy on my soul." 26 "May I be forgiven when I appear before my God."27 "The dear Lord will forgive me."28 "I am going to meet my God." 29 A mother with low self-esteem wrote that she had to "atone in the next world" since she had failed in this one. ${ }^{30} \mathrm{~A}$ few mentioned praying for access. "Mum, I am going to Heaven. I have been praying and I know God will take me." 31 Divine justice entered the picture because many felt aggrieved or shortchanged. An unemployed laborer wrote to his wife that "God will bless you for if not in this world he will in the next." 32 It was not God's grace but omniscience and fairness that people trusted in. A lonely widow hoped to be "in Heaven for the Lord did not mean us to live the life I have done for a long time."33 For some, Heaven had a real though unattainable presence. ${ }^{34}$ "God bless the children," wrote a widow to her sister immediately following her husband's death, "and take them to Heaven to meet their father some day for I have gone to Hell for my wickedness." 35

Visceral self-reflections cannot help but incite sympathy, wonderment, and curiosity. In respect to the latter, the repetitions of "meet in Heaven" and "God will forgive me" implied deep cultural groundings, perhaps distant from our own time. We will have more to say on that point later. What were some of the depictions of Heaven? What was the historical trajectory of their cultural grounding? What sustained or diminished them? Where did solace directed toward survivors end and personal belief begin? Indeed, who was speaking for whom? Individuals stating their right to a Heavenly reunion is a more interesting and contentious act than awarding it as solace with others in mind. Did 
moral issues attend individuals claiming this post-mortem identity for themselves? Did contemporaries contest people's assertions that they were Heaven-bound?

To investigate these questions across two centuries, we turned first to the Google Ngram Viewer, "an online search engine that charts the frequencies of any set of comma-delimited search strings using a yearly count of Ngrams found in sources printed between 1500 and 2008." "As of October 2015, the number of scanned book titles was over 25 million," approximately one-seventh of all the books published since Gutenberg. ${ }^{36}$ The Ngrams, essentially comma delimited sets of words, are matched with the text in a selected set of publications. Occurrences are then plotted on a dated x-axis to determine when words and phrases first appeared, rose in popularity, and declined. We entered "meeting in Heaven" and "God will forgive me." These two phrases were also run through three digitized newspaper collections: the British Newspaper Archive (35 million pages), Trove from the National Library of Australia (over 23 million pages and 157 million articles, 1802 to 2015), and Papers Past from the National Library of New Zealand (nearly 6 million pages, 1839-1950). Since these sources are expanding, the data should be considered indicative rather than definitive. Stories themselves are indicative too, although more compelling when backed up with quantitative evidence. ${ }^{37}$

The quantitative and qualitative information recommend departures from The Hour of Our Death, Philippe Ariès's pathbreaking history, which proposed eras to structure how Western cultures handled death and depicted an afterlife. In a millennium-long phase encompassing the Middle Ages, a clerically inspired model of death split the individual into "an immortal soul that was released by death." For a time, dualism reconciled the materialist and spiritual ideas of the mind. By the seventeenth century, this convenient arrangement had "gained almost universal acceptance." Next, around the beginning of the nineteenth century, "belief in hell had disappeared." With the removal of that danger to the dearly departed, "the next world becomes the scene of a reunion of those whom death has separated," and we find popular assumptions of "a re-creation of the affections of earth, purged of their dross, assured of eternity." 38 Ariès and others who followed - for example, Jan Bremmer, author of The Rise and Fall of the Afterlife - went further than invoking eras and asserted that cultures produced the afterlife that they deserved. The recourse to eras brilliantly over-theorized the subject to plug gaps in knowledge about how people really thought. The digitized material presented in this chapter shows a long-term trend but also more detailed patterns that shifted over shorter periods of time. Furthermore, they divulged disputes about the composition of the Beyond, theological and moral dilemmas about who should pass through Heaven's gates, and more. On the one hand, a great deal was happening that disrupts the coherence of eras, at least for modern times. On the other hand, the soul and Heaven were mentioned often enough, especially prior to 1900, to evoke a mood of the times. Ariès provided a compass, not a map.

\section{The trend, the peaks, and variations}

The Ngram Viewer and quantitative measures compiled from the digitized newspapers suggest that Heaven's ascent in the English-speaking world crested in the 1810 s and again in 1860s, coinciding with the Napoleonic Wars and, at mid-century, with the Crimean and American Civil Wars (Figure 11.1; Tables 11.1 and 11.2). Horrific death tolls give context. The estimate on military deaths in the Napoleonic Wars range from 


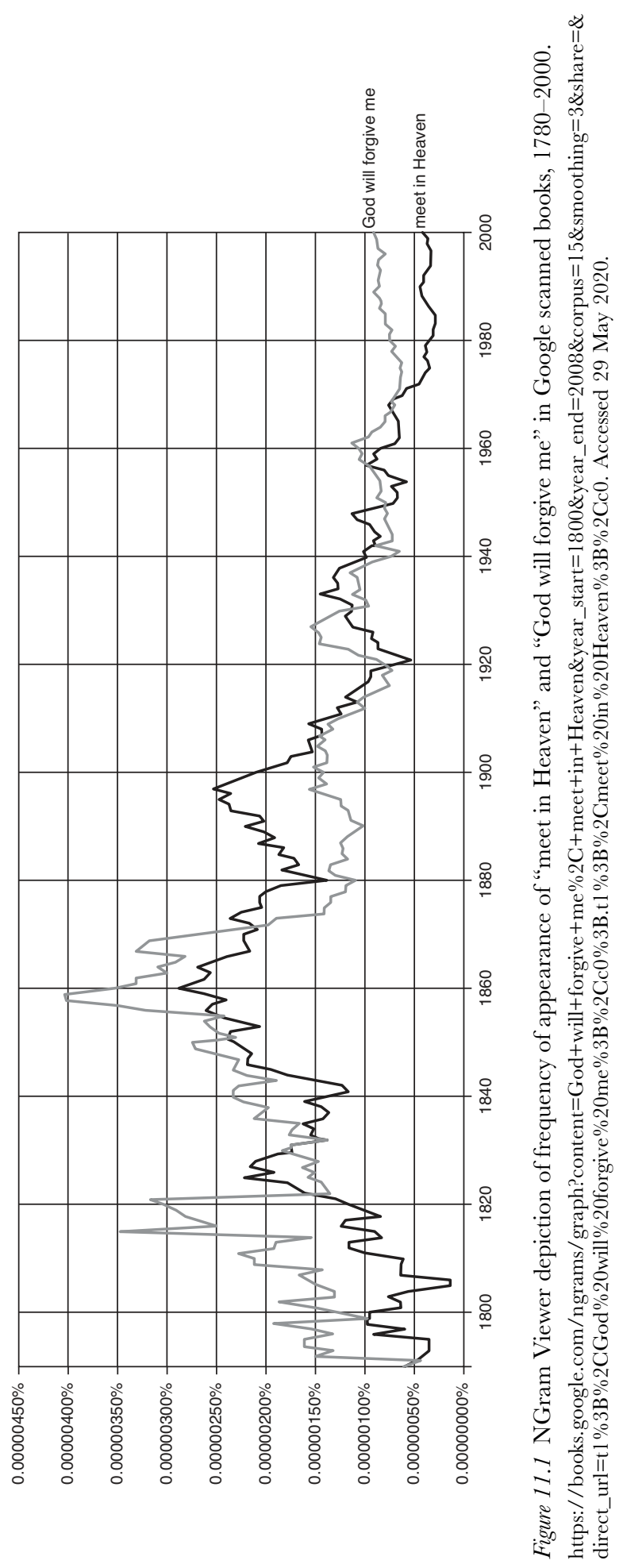


"NOW FOR THE GRAND SECRET"

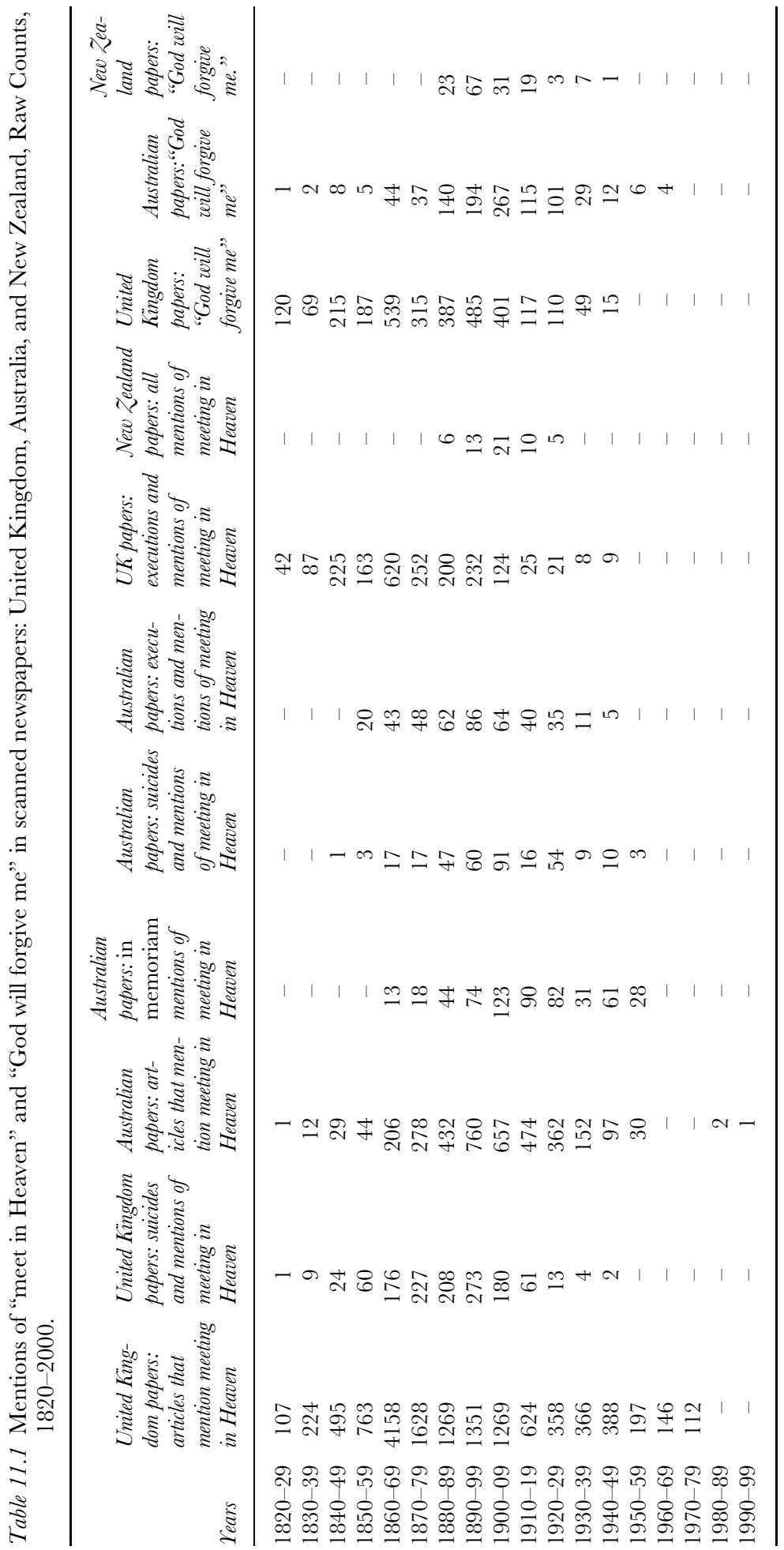


Table 11.2 Mentions of "meet in Heaven" in scanned newspapers: United Kingdom and Australia, 1820-1960. Controlled for variations in source material.

\begin{tabular}{|c|c|c|c|c|c|c|}
\hline Decade & $\begin{array}{l}\text { Count of Heav- } \\
\text { enly meetings } \\
\text { mentioned in } \\
U K \text { digitized } \\
\text { provincial } \\
\text { newespapers }\end{array}$ & $\begin{array}{l}\text { Tens of thou- } \\
\text { sands of art- } \\
\text { icles in UK } \\
\text { digitized pro- } \\
\text { vincial } \\
\text { newspapers }\end{array}$ & $\begin{array}{l}\text { Heavenly meetings } \\
\text { per } 10,000 \text { digi- } \\
\text { tized articles in } \\
\text { UK digitized pro- } \\
\text { vincial newespapers }\end{array}$ & $\begin{array}{l}\text { Count of Heav- } \\
\text { enly meetings } \\
\text { mentioned in } \\
\text { Australian digi- } \\
\text { tized } \\
\text { newspapers }\end{array}$ & $\begin{array}{l}\text { Tens of thou- } \\
\text { sands of art- } \\
\text { icles in } \\
\text { Australian } \\
\text { digitized } \\
\text { newespapers }\end{array}$ & $\begin{array}{l}\text { Heavenly meet- } \\
\text { ings per } 10,000 \\
\text { digitized articles } \\
\text { in Australian } \\
\text { digitized } \\
\text { newspapers }\end{array}$ \\
\hline $1820-29$ & 107 & 8 & 13 & 1 & 0.0 & - \\
\hline $1830-39$ & 224 & 12 & 19 & 12 & 0.1 & - \\
\hline $1840-49$ & 495 & 16 & 31 & 29 & 3.5 & 8.3 \\
\hline $1850-59$ & 763 & 22 & 34 & 44 & 8.7 & 5.1 \\
\hline $1860-69$ & 4158 & 35 & 119 & 206 & 19.0 & 10.8 \\
\hline $1870-79$ & 1628 & 36 & 45 & 278 & 32.0 & 8.9 \\
\hline $1880-89$ & 1269 & 45 & 28 & 432 & 62 & 6.8 \\
\hline 1890-99 & 1351 & 46 & 29 & 760 & 119 & 6.4 \\
\hline $1900-09$ & 1269 & 44 & 29 & 657 & 197 & 3.3 \\
\hline $1910-19$ & 624 & 30 & 20 & 474 & 281 & 1.7 \\
\hline $1920-29$ & 358 & 21 & 17 & 362 & 265 & 1.3 \\
\hline 1930-39 & 366 & 19 & 19 & 152 & 283 & 0.5 \\
\hline $1940-49$ & 388 & 20 & 19 & 97 & 181 & 0.5 \\
\hline $1950-59$ & 197 & 11 & - & 30 & 93 & 0.3 \\
\hline
\end{tabular}

2.5 million to 3.5 million, and civilian deaths from 750,000 to 3 million, producing totals ranging from $3,250,000$ to $6,500,000 .{ }^{39}$ Often neglected as a grand killer, the Crimean War was horrendous. Of an estimated 1,650,000 soldiers from all countries engaged, 900,000 died. ${ }^{40}$ Most were Russians. Of the British Empire's deaths, 2,755 fell in action and 17,580 died of disease. The America Civil War claimed an estimated 620,000 lives. ${ }^{41}$ Apropos of this conflict, Gary Scott Smith, author of Heaven in the American Imagination, referred to the novel Gates Ajar (1868) by Elizabeth Stuart Phelps. Written to give comfort to the bereaved, it depicted a Heaven where veterans conversed with Abraham Lincoln. ${ }^{42}$

Then there were the cholera pandemics of 1829-37, 1846-60, and 1863-75. ${ }^{43}$ The latter, spread with the Austro-Prussian War (1866), claimed an estimated 165,000 lives in the Austrian Empire, plus 30,000 in Belgium and 20,000 in the Netherlands. In London in June 1866, a localized outbreak claimed 5,596. In 1867, Italy lost 113,000. Outbreaks in North America in the 1870s killed some 50,000 Americans. These events punctuated a relentless grim certainty, namely infant mortality. Houlbrooke noted that "not till just before 1900 did infant mortality begin to fall consistently, gradually at first, and then, during the first decade of the new century, much more rapidly." 44 Hoped-for reunions with loved ones gave solace, and there was plenty of demand for it; solace was a feature of the popular culture invocation of Heaven but not the only motive for making a statement about a reunion.

Romantic devotional books had a natural market among the bereaved. William Branks' Heaven our Home (1861) easily led the field, followed by his Life in Heaven (1864). The multi-authored Across the River: Twelve Views of Heaven (1864) had a smaller following. From 1861 to 1869, Heaven Our Home went through many editions and appeared in 3,634 
so-called articles and 2,156 overt advertisements in the digitized British provincial newspapers. In 1869, publisher William P. Nimmo, claimed to have sold a combined total of 159,000 copies of Heaven Our Home and Life in Heaven. Edinburgh-based, Nimmo produced editions of John Milton, Robert Burns, Sir Walter Scott, and other classics, all promoted as "marvels of cheapness, elegance, and sterling worth." 45 As a Biblical romantic, Branks described "a material habitation away from earth." "Heaven" had its "position in the great ocean of space" where angels had "contemplated its scenery for nearly 6000 years." 46 Helpfully, he explained that friends would be able to recognize one another. ${ }^{47}$ Branks insisted in Life in Heaven that the Beyond was "a social heaven." Those whom we wish to associate with have been keeping tabs on our lives; new arrivals brought news, and relatives and friends could watch our lives before we arrived. ${ }^{48}$ A successful, certainly astonishing, enterprise filled needs and adroitly appealed to what Gary Smith described as "the Victorians' glorification of the home and strong desire for stability and decorum." 49

While Smith's characterization applied to publications, the ascendency of these values was incomplete. In certain distinct circumstances, when individuals stated their own fitness for life in the Beyond, their words were sometimes anti-establishment rather than conformist. Appearances of a human-centred Heaven and individual assertions of fitness for the Beyond opened diverse rifts. In The Appointed Hour: Death, Worldview, and Social Change in Brittany (1989), Ellen Badone identified the popularity of the social Heaven, writing that "the emphasis on reunion with kin after death is a product of the romantic pattern in which affectivity is channelled primarily toward members of the nuclear family." ${ }^{\text {, }}$ For devout Catholics in Brittany, this produced anxiety when family members no longer adhered to the Church. How could parents be happy in paradise without the presence of their unbelieving children? ${ }^{51}$ Questions about who is pondering the reunion make a difference. Many of the people whom we encountered facing imminent death, including the suicides that opened this chapter, were the parties imagining the meetings and listing the attendees. In a study of late-nineteenth-century Australian condolence letters, Patricia Jalland found, similar to Badone, that although reunions were "the primary consolation," deeply pious families did not make such a presumption "since for them salvation depended on divine judgement and individual faith." ${ }^{\text {52 }}$ Breton Catholics worried about the implications of family straying from the faith; Sydney Evangelicals drew a personal conclusion: "What a warning to us all. May God prepare us for our bitter end." 53

The promotional drive for Heaven Our Home biased the Ngram Viewer and newspaper data, but more than hype should be considered. Nimmo and Branks were sowing a fertile field of sentiment. ${ }^{54}$ Heaven Our Home received a boost from the death of Prince Albert in December 1861. According to an advertisement, a member of the royal household supposedly knew that Queen Victoria and Prince Albert had been reading together

a remarkable book HEAVEN OUR HOME, which had interested him very much as he lay dying of typhoid. He said to Victoria "we don't know in which state we will meet again; but we shall recognize each another and be together in eternity, I am perfectly certain." 55

A few ministers denounced the likes of Heaven Our Home as sentimental evils. "Religion would benefit were whole shiploads of such trash to be engulphed in the Atlantic." The romantics' fault was to dwell on open access to Heaven rather than the countervailing 
potential for damnation. ${ }^{56}$ Sentimental religiosity was not reputable piety. ${ }^{57}$ In the United States, the religious press condemned Phelps's novels with their Heavenly landscapes "as irreverent, immoral, and injurious." 58 Critics there and elsewhere in the English-speaking world insisted that laxity of doctrine and latitudinarianism extended the false hope of Heaven to people who were merely good rather than good as well as ardent believers. The cross-currents of opinions about the composition of the Heavenbound and the unqualified were strong.

A striking example of a contrary discourse appeared in 1899, in the assertions of English novelist Marie Corelli who brought together a number of the prevailing attitudes that suicide was a sin and, by implication, Heaven would remain closed to offenders. The gates were not ajar. "I may state at once," pronounced Corelli, "that I do not consider suicide justifiable under circumstances whatever." The "gift of freewill is a very sacred one," she went on to say,

but if we use that freewill to consummate an act of cowardice (suicide) we immediately state plainly that we care nothing for the precious gift of God which $\mathrm{He}$ gave to us in the beginning, and what the result of that contempt may be we cannot possibly imagine, save that cowardice is instinctively despised by both earth and Heaven. ${ }^{59}$

Her views may have been commonplace, a dimension of the rows over the right to go to Heaven, but her opposition was expressed with resounding clarity and a presumption about Heaven's culture.

Religious publications in the broadest sense - devotional books, songs, church magazines, and moral uplift tracts - supported ideas about the reality of a post-mortem personal identity. ${ }^{60}$ Moreover, there were assorted underpinnings from diverse popular culture sources associated with the inexpensive press. Newspapers frequently carried lurid reports of suicides and executions, mentioning that the last words included a meeting in Heaven. These sensational stories continued until newspapers began to exercise, or had forced on them, greater sensitivity and self-censorship in the 1920s. Murderers awaiting execution had long been expected to play a role in morality dramas by confessing and making declarations supposed to have salutary influences on public morals. Scenes included the condemned individual's desire to meet someone in Heaven.

Reports of shocking cases travelled the globe. Newspapers, and especially those outside the metropolitan areas, needed more copy than local events could generate. Consequently, editors looked for syndicated stories from afar. Thus, the murder of a young woman in Australia in 1913 and the attempted suicide of her assailant, who explained that "the Bible referred to a new life hereafter" and that "he wanted to go there with Miss Day," was reported in no fewer than eighteen New Zealand newspapers. Another report of a murder-suicide from Australia involved a widower murdering one son and injuring another, before taking her own life. The motive was so that they could all "go to Heaven to join their dead mother." ${ }^{, 11}$ In some cases, the Heavenly wish announced before death posed a moral worthiness problem. In November 1849, Boston businessman George Parkman, brother of noted historian Francis Parkman, disappeared. The discovery of parts of Parkman's body in the laboratory of John Webster at Harvard Medical College led to Webster's conviction and execution. The much-publicized case included protracted accounts of Webster's final minutes in September 1850. His spiritual adviser, 
Dr. Putnam prayed with him and declared that Webster's contrition "could qualify him for his departure." ${ }^{2}$ It was as if he were waiting for a Boston and Lowell train. They shook hands and Webster expressed "the hope that they should meet again in heaven."63 Account after account in the British and colonial press detailed Webster's prayers and acts of contrition. Around the same time, in a sensational British case, Mr. and Mrs. Manning, "wretched culprits," refused to confess to murder, yet, after kissing her farewell, the husband told his wife, "I hope we shall meet in Heaven."64

Such examples are illustrative of numerous comparable scenes reported throughout the century. They peaked in the 1860 s to 1890 s but kept coming. ${ }^{65}$ Convicted of murdering a policeman in Worcester in 1885, the condemned refused to confess but told his family "they should meet in heaven." 66 On and on, into the 1920s. A condemned woman wrote to her sister-in-law in 1926 that she would have "the joy of seeing me in Heaven for I am quite ready and prepared to meet my God." 67 From this point forward, such reports became rare. Attempts to restrict the British Newspaper Archive count to execution stories that mentioned the condemned party's wish to meet someone in Heaven were imperfect but suggest that from 1820 to 1940 there were about 1,800 of them; from 1850 to 1950 , the count from Trove is over 450. Trends were similar for reports of suicides. There were roughly 1,200 in British scanned newspapers from 1820 to 1950 and over 300 in Trove from 1820 to 1950 (Table 11.2). Usually, several papers repeated the same story, so these reports do not represent unique incidents. Further, the pertinent phrase appears authentic rather than a journalistic or official invention since the reports were but a small fraction of press coverage on executions and suicides.

In addition to Heavenly references at executions and before suicides, there was a wellpublicized exceptional event in the 1860s. An attempted insurrection in Ireland in 1867 led to trial coverage printed on both sides of the Atlantic and a different basis for assuming a meeting in Heaven, thanks to a just God. ${ }^{68}$ The attempted rising started in County Kerry on 14 February 1867. On 5 March, more clashes took place in Dublin, Cork City, and Limerick. The largest occurred when several hundred Fenians were attacked by the Irish Constabulary. The rebels burned down police barracks in several Tipperary towns. Twelve men were killed across the country. Throughout April 1867, trials were held and some of the accused including Thomas Burke were charged with high treason. On 1 May 1867, Burke, who was tried along with Patrick Doran, received the maximum sentence, namely death by hanging, drawing, and quartering. The sentences caused outrage in Ireland and in the United States. Two days before the scheduled execution, their sentences were commuted to life in prison. They were freed after an Amnesty campaign in 1869. When convicted and sentenced in 1867, Burke had given a much-reprinted address to the court, stating that God would forgive them and that they would meet in Heaven. God had preserved Ireland despite 700 years of English oppression. ${ }^{69}$ In these circumstances, stock phrases subverted secular authority.

Since emigration to the colonies often meant permanent separations, reference to a Heavenly meeting soothed the rupture. "Dear mother and brother," wrote a young man bound for America in 1833, "the time while son come, I hope, to meet in Heaven, when never part no more." ${ }^{70}$ A devout Methodist settler in South Australia wrote to his mother too. "If you should not come, and I never see you again, may we give our hearts to God, and meet in Heaven." ${ }^{, 1}$ Inexpensive songbooks and sheet music contributed to a culture of solace in Heavenly reunions. First published in 1861, "We'll meet in 
Table 11.3 Counts of "meeting in Heaven": poetry (1820-1940) and World War I family in Memoriam and honor roll references from UK and Australian newspapers.

\begin{tabular}{lccl}
\hline & $\begin{array}{l}\text { "Meeting in Heaven": } \\
\text { poems printed and scanned } \\
\text { UK newspapers }\end{array}$ & $\begin{array}{l}\text { "Meeting in Heaven": poems } \\
\text { printed and scanned - Austra- } \\
\text { lian newspapers }\end{array}$ & $\begin{array}{l}\text { World War I "Meet in } \\
\text { Heaven": UK newspapers, } \\
\text { family and honor roll }\end{array}$ \\
\hline $1820-29$ & 8 & - & - \\
$1830-39$ & 4 & 3 & - \\
$1840-49$ & 11 & 4 & - \\
$1850-59$ & 15 & 3 & - \\
$1860-69$ & 44 & 11 & - \\
$1870-79$ & 41 & 17 & - \\
$1880-89$ & 21 & 18 & - \\
$1890-99$ & 13 & 26 & - \\
$1900-09$ & 11 & 30 & - \\
$1910-19$ & 10 & 11 & - \\
$1920-29$ & 5 & 1 & \\
$1930-39$ & 2 & 6 & \\
\hline
\end{tabular}

Heaven, Father" depicted the deathbed scene of a boy who had received a visit from his deceased mother beckoning him away to "Heaven's unfading bowers" where "we shall all meet again."72 The author of a ponderous 335 page epic poem, "The Last Judgement," came up with a priority solution for the merely good as opposed to the good and pious. "The bodies of the righteous swift arise." 73 One way to anticipate and address the moral question was to emphasize the reward of Heaven for good conduct early in life. Lessons on following the straight and narrow began with youth. In the didactic tale "My Charlie," prepared for the American Female Guardian Society, a young lad was led astray by school chums. His mother overheard him exclaim "Where the is my cap?" After explaining the profanity, she led him to contrition "Oh, mama, I have grieved Jesus. Do you suppose God will forgive me?"74

British and Australian newspapers carried sections charitably entitled "Poetry" that from time to time published verses on the Heavenly gathering (Table 11.3). "We part once more to meet again in heaven / Where love and constancy in unison reunite." ${ }^{76}$ In "The Maiden and the Flower," men were characterized as devious; real love and flowers belonged "Above" "where love and flowers, hope faith and truth / Shall meet in Heaven and part no more."77 Another entitled "Shall We Meet in Heaven?" dismissed the "atheist's creed," because who could doubt fields of light, reunions, and angels tuning their hearts to love, especially, "when our pathway in life is strewn with thorns."78 The last line of every verse of "The Widow's Son" promised a meeting in Heaven. ${ }^{79}$ Many more poems authenticate a mid-century genre of solace literature: "Lilia, to Her Lover," "To My Mother," "A Recollection," "Consolation for Absence," "The End of it All," "Face to Face," "The Orphan Girl," "A Mother to Her Children," "The Dead Mother," "Weep Not for Her," "The Dying Girl," "Where is Rest." "W0 When Scottish newspapers celebrated Robert Burns, they cited Clarinda, his favorite inspiration, who wrote in her diary forty years after their parting - "Ae fond kiss and then we sever" - "Oh may we meet in Heaven." ${ }^{, 81}$ 
Serialized stories abounded with hope of reunions with wayward or prematurely deceased children. The heyday of the Heavenly rendezvous also saw humorous references. Among personal notices there appeared several mock romantic assignations: "shall we meet in Heaven at some future time." 82 Fooling around with his Sunday School classmates, little Johnny was rebuked by his instructor: "I think you and I will never meet in Heaven." "What did you do sir?" It circulated in at least forty Australian newspapers in the early 1890s. ${ }^{83}$ The story was told of the apprentice florist who misunderstood an order for a floral wreath. "I want 'Rest in Peace' on both sides and if there is room 'We shall meet in Heaven'." The wreath declared "Rest in Peace on Both Sides And If There Is Room We Shall Meet in Heaven." 84

Were there kernels of faith in the popular culture chaff? While it is impossible to know if every sentimental statement laden with otherworldly references originated from complete faith, let alone orthodox faith, it would be unwise to dismiss a folk trust in an afterlife with qualities meaningful to the individuals expressing the notion. ${ }^{85}$ Citing Geoffrey Gorer's Death, Grief, and Mourning in Contemporary Britain (1965), Ariès recounted how, even as late as the 1960s, elderly informants freely mentioned seeing and hearing the deceased. They maintained that Heaven was "a place where there are no worries, and where we will meet all our relatives and friends." ${ }^{86}$ A number of individuals on the gallows or preparing for suicide wanted to continue in a new location. Regardless of the depth and foundations of belief, cultural coherence was apparent across many forms of expression on the life Beyond. Whether casual or profound, the coherence was breaking down before World War I and, for once, a war failed to rally more abundant thoughts of Heavenly reunions. The trend had been drifting downward for twenty years. Moreover, references to Heaven in the suicide inquest files that we read not only tapered off but were supplanted by less specific destinations, such as "new surroundings," where someone "was going to be much more contended and happier than [he] had been for a long time." 87 The Ngram Viewer captured the rise of an ill-defined afterlife (Figure 11.1).

\section{Weakening convictions}

In the British newspapers the phrase "meeting in Heaven" peaked in the 1860s and early 1870s; however, in Australia and New Zealand, it appeared to summit before the turn of the century (Table 11.2). The data required refinement, for they could be artifacts of the number of newspapers in operation in given periods, the practice of reprinting stories, and the number of papers digitized. To control for these influences, we calculated the number of appearances of the phrase per 10,000 articles in digitized United Kingdom and Australian newspapers. It was not possible to do the same for New Zealand. After an adjustment for a quirk caused by Branks and Nimmo in the 1860s, British numbers from 1840 to 1900 averaged 35.5, and from 1920 to 1950, 18.3. In Australia, from the $1860 \mathrm{~s}$ to the $1890 \mathrm{~s}$, the number averaged 7.5, and from 1920 to 1960 , 0.6. The British newspapers carried far more Heavenly references than the Australian press, a deviation conceivably explained by revivals, church magazines, and book publishers promoting romantic devotionals. Notwithstanding the national difference, the trends were comparable: downward.

From 1875 to 1920, a materialistic or naturalistic way of looking at personhood grew in acceptance. From its peak in 1875, grieving declined slightly, as memories of wars and 
pandemics faded. The appearances of "meet in Heaven" fell to a steady peacetime level. By 1920s, the materialistic conception was firmly installed (Figure 11.1; Table 11.1). Seen as a product of the brain and nervous system, the mind was presumed to cease with the organism's death. However, the post-mortem identity had not vanished. Individuals still considered the possibility of Heaven or "the afterlife," as did a very religiously inclined 18-year-old female in 1890, whose suicide note declared, "I know Jesus loves me. I am not afraid to die; I will go with Him. We will meet in Heaven." 88 But materialistic and scientific views of the world were strengthening in the late nineteenth century. A doubting and materialistic culture had edged relentlessly onward from the 1870s into the 1910s. No single factor produced scepticism. Science, life-saving public health and falling rates of infant mortality, secular education, and World War I converged. In a less pervasive and sometimes modified form, the Beyond continued, as we have noted, occasionally rebranded in popular culture as the afterlife, which Bremmer disparaged as "rather empty, except for a few relatives." 89 This was not the social Heaven with angels and saints that had a presence among both the flamboyant romantics and the sternly pious in the 1860s.

In the decades following the Crimean War and Civil War, there was material progress and, except for small short wars and colonial conflicts, general peace. Then came the shock of World War I. Conceivably the Darwinian challenge to creation weakened the old verities, and World War I's death toll and mutilated veterans on the streets imposed strains. The total number of military and civilian casualties in World War I reached about 40 million. ${ }^{90}$ Rare but eloquent statements by veterans, found in the inquest files, offered clues for explaining the drop in the Heavenly meetings. The absence of a divine hand left an anodyne afterlife and a vacant seat of judgement. "Meet in Heaven" appearances dipped, briefly, then recovered late in the Great Depression before resuming their fall during and after World War II. War had gone from stirring faith in the nineteenth century to destabilizing it in the twentieth.

Compared to prior wars, World War I undermined some of the routine belief in God and Heaven. The presence of American books in the Google repertoire for the Ngram Viewer could have diluted the evidence of an even deeper decline in references to the next world in the British Empire. Possibly United Kingdom and Dominion sources taken alone would have presented a more dramatic picture. Nevertheless, the Ngram Viewer suggests a decline, though a modest one. World War I did not eradicate references to meeting in Heaven. The British newspapers mentioned it in family notices and honor rolls several times a month in 1916-18 (Table 11.3). The salient point, however, is the missing surge of Heaven-associated phrases that had appeared during earlier wars. Examples of a loss of trust in church and faith due to this war are legion. Charles E. Montague's Disenchantment, published in 1922, was one of the first and most caustic. He experienced the pre-war years as a paradise and then "the bottom fell out." In his late forties, he enlisted and witnessed the loss of innocence and the clergy's exhortations. The Bible and the prayer book had blinded men "to the duty of seeking war and ensuing it." With rapier-like irony, he skewered those who announced that war was character building: "even amid the horrors of peace you will find little shoots of character fed by the gentle and timely rains of plague and famine, tempest and war." "91 However, characterizations of this war included those that "providentially erased a host of memories."92 Moreover, in an ambitious exploration of what kept soldiers going, Peter E. Hodgkinson 
in "Gum Heroes" observed that a soft Christianity, shading away from theological rigor, appealed to many. Men coped though "faith, fatalism, and superstition.",93

Although World War I with its stream of death and mutilation did not precipitate a widespread and remarkable collapse in belief, neither did it bring the responses detected for earlier great wars. As well, we sensed a qualitative difference after World War I. Some returned soldiers, faced doubt with stoicism rather than endorsing an unwavering belief. As one laborer put it in 1934, "there are more soldiers dying now than when the War was on, and my time will soon be up. All men who were wounded and gassed at the War would be dead within the next ten years." 94 No mention of Heaven. Gassed in 1916, an unemployed builder wrote in 1942 that "it was too late to turn to God" and "God would not accept him."95 Another told his wife on the day of his death that "it was too late to turn to God, he was very depressed, and he thought that God would not accept him."96 It is worth considering that the war not only changed the composition of suicides by adding scores of returned soldiers who did take their own lives, a finding from our suicide research, but weakened faith and old certainties. The war fostered resentment that prayers had not been answered, that loved ones had not been spared, that God was unconcerned, and that men had done things that haunted them. References to hoped-for meetings in Heaven or the benefit of God's mercy and wisdom thinned out. In the nineteenth century it would have been unimaginable to see a note stating that "if there is a God (which I am unable to believe) may He guard and cherish you for ever and for ever." $" 97$ A concern for others, the motive of solace, lingered in this case, but without hope of a post-mortem identity and reunion.

Some still considered meeting up later but "in that sweet by and by," not explicitly in Heaven. Individuals were "going out the side door," setting out on "the long long trail" and might wind up in "new surroundings" and "perhaps meet others." 98 There was uncertainty; God may not appear at the destination to give judgement. People had to be "responsible before for [a] higher tribunal." "99 Not the omniscient Judge. Secularism made lexical inroads. A returned solider articulated the transition in 1932, when he wrote that "one's destiny is ordained. It is no use kicking. As for the sin of it [suicide], if our destiny is ordained, then there can't be any and if there is anything beyond this I will find out." Scepticism joined stoicism, and stoicism could arise in the presence of death. Alone in the bush when bitten by a venomous snake, a farmer decided to end his suffering by a shotgun blast to his chest. It was bad luck, he wrote, but given the carnage of the war "it is nothing strange." 100 Of course, the secular shift proposed here was never complete and never irreversible to so some extent. The Christian conception of Heaven has undergone an evangelical revival, new currents in old streams, as Smith explained. ${ }^{101}$

\section{Conclusion: everlasting concerns}

In the mid-twentieth century onward, popular conceptions of the Beyond languished relative to their nineteenth-century hardiness, but theologians, philosophers, historians, anthropologists, sociologist, psychologists, and grief counsellors aroused new interest in the Grand Secret. Their zealous publication activities call into question Ariès's addendum in The Hour of Our Death. In America, commercialism and technology, he argued, had penetrated the idea of dying, turning death into "the unaccepted separation." "At heart," he proposed, "we feel we are non-mortals" and therefore labor to make death 
invisible. ${ }^{102}$ Americans uniquely had the means to attempt this sleight of hand. His fascination with the American way of dying shifted attention from conceptions of the Beyond and Heavenly reunions to medical interventions to prolong life and funerial cosmetics to disguise death. ${ }^{103}$ In fact, thanks perhaps to defibrillation by Ariès and public interest in near-death-experiences, death was alive and well in the academy, the therapy industry, and to some extent in public culture. Bookshelves are filled with volumes such as Death and Bereavement (1969), Death Society and Human Experience (1977, 1981, 1986, 1991, 1995), Death: Current Perspectives (1976, 1980, 1984), Endings: A Sociology of Death and Dying (1989), First Person Mortal: Personal Narratives of Dying, Death, and Grief (1995), Death Mourning and Burial (2004), How Are We to Confront Death (2012), and What Death Means Now (2017).

The post-mortem identity and Heavenly reunions are not subjects covered in these books. Concern generally revolved around preventing premature deaths or providing a humane end to life, at least in the prosperous societies that could afford palliative care. There are serious exceptions that express a continuation of nineteenth-century ideas about an afterlife and a leading moral problem. In Death and Eternal Life (1976), theologian and philosopher John Hick proposed that "we are trying to conceive of something beyond the reach of even our most ambitious imaginings." 104 As for the materialist argument, Hicks averred that even though there had to be a correlation between brain function and mind, this association was no proof that brain function was the mind. ${ }^{105}$ Applying critical analytics and a study of world religions, he went further, proposing what life after death might comprise. The basic religious claim to "the continuance of individual consciousness," he believed, "is very likely to be true."106 This is a long way from the romantic devotional description of the Beyond; however, the mid-Victorian strain of imaginative description has not disappeared. One can order from Amazon reprints of Branks' Heaven Our Home as well as a 2016 abridged edition.

A discrete issue was revived by other academics. It turned on the unfairness of the great equalizer, because Nature paid no heed to an individual's goodness. In Immanuel Kant's assessment of morality, for example, if the materialist position was correct and no final seat of justice existed, then "obedience to the moral law may simply turn the just into fodder for the predatory unjust." ${ }^{107}$ Belief in Heaven was considered by some as a necessary but not sufficient condition for an effective defence of goodness. It was insufficient because many popular accounts of Heaven concentrated on the preservation of the current personal identity without presenting a compelling case for the "candidate's" worthiness. Confirming Kantian alarm, some individuals holding the idea of a meeting in Heaven did not seem especially deserving.

Our inquiries found a few men and women still avowing their immortal personality into the twentieth century. They were going to endure; what is more, they said that they deserved to. Some first-person assertions envisioning a life beyond death came directly from parties about to die in circumstances that presented moral complications. Their declarations about Heaven say a lot about their individualism, not always the generous valuing of all individuals, but the self-importance of the individual in question. As well, there are rare instances when low self-worth led to self-damnation and a an unpleasant Beyond. Contrary to the impression left by Ariès, hell was not completely dismantled around the early nineteenth century. Smith points out that American evangelist Billy Graham maintained in his early 1950s crusades that "hell is a lake of burning 
brimstone," a description that he later dropped in favor of the punishment of "banishment from the presence of God." 108

Details about the reputations of some persons facing an imminent end at the gallows or preparing for self-destruction recall the Kantian problem. Arguably, at the heart of human nature and the Kantian problem, there is a disposition in each person to favor himself or herself. Death can be knowable as a biological reality in the materialist sense, but the disposition of selfhood makes the subjective realization of death in its materialist form more difficult to accept than the general proposition of its inevitability. Thus, the idea of a soul found a degree of popular acceptance even in morally improbable circumstances. ${ }^{109}$

When searching for a type of immortality as a reward to address this Kantian problem, philosopher Mark Johnston remarked in Surviving Death (2010) that because "we seek premium treatment for ourselves," the supernatural is invoked to transform hope into a guarantee. ${ }^{110}$ Self-regarding individuals would want "the perpetual return of the actual life lived by one's own individual personality."111 These individuals were "just ... up themselves." 112 Johnston's response to death's inevitability as an unfair outcome to good people was that death becomes less of a loss for individuals who pursue goodness because they have already reduced an emphatically subjective selfcentredness. Then in death, they are literally "re-embodied in the onward rush of humanity."113 Some of the individuals whom we encountered in newspaper articles and documents would have been unmoved by this promise of contributing to a Hegelian-like improvement of humankind. Johnston would have been unmoved by their self-centredness. So too were a few nineteenth-century critics of unrestricted access to the Beyond.

Did the fact that some people mentioned a life after death and God's forgiveness indicate belief? Sincere hope? Cultural reflexes without commitment?

We are all waiting for an answer.

\section{Notes}

1 For elite cultural representations, see, for example, a selection from several decades: J. Hillis Miller, The Disappearance of God: Five Nineteenth-Century Writers (Cambridge, MA: Harvard University Press, 1963); Elaine Marks, Simone de Beauvoir: Encounters with Death (New Brunswick, NJ: Rutgers University Press, 1973); Kathy Charmaz, Glennys Howarth, and Allan Kellehear eds., The Unknown Country: Death in Australia and the USA (New York: St. Martin's Press, 1997); Sandra Gilbert, Death's Door: Modern Dying and the Way We Grieve (New York: W.W. Norton \& Company, 2006); Carl Watkins, The Undiscovered Country: Journeys Among the Dead (London: The Bodley Head, 2013). On a brief critique of novels and autobiographies on death, and concern about elite representations, see Houlbrooke, "Introduction," Death, Ritual, and Bereavement, 7, $17-19$.

2 Pat Jalland, Death in War and Peace: Loss and Grief in England, 1914-1970 (Oxford: Oxford University Press, 2010), 257-9.

3 Philippe Ariès, The Hour of Our Death (New York: Alfred A. Knopf, 1981); Jan N. Bremmer, The Rise and Fall of the Afterlife (London: Routledge, 2002).

4 Ralph Houlbrooke, "Introduction," in Houlbrooke ed., Death, Ritual, and Bereavement (London: Routledge, 1989), 6.

5 Ariès, The Hour of Our Death, xvii.

6 David Cannadine quoted in Houlbrooke, 5. Also see Ruth Richardson, "Why was Death So Big in Victorian Britain?," in Houlbrooke, 106, 116. 
7 A Sadly Troubled History: The Meanings of Suicide in the Modern Age (Montreal: McGill-Queen's University Press, 2009); Sorrows of the Century: Interpreting Suicide in New Zealand, 1900-2000 (Montreal: McGill-Queen's University Press, 2013). Two thousand Queensland and 10,000 New Zealand inquests were studied.

8 Our historian's temperament contrasts with the sociologist's. See the gatherings in Michael C. Kearl, Endings: A Sociology of Death and Dying (New York: Oxford University Press, 1989).

9 Queensland State Archives (QSA), JUS/N241, 1896, file 241.

10 QSA, JUS/N286), 1900, file 274.

11 QSA, JUS/N259, 1898, file 39.

12 QSA, JUS/N245, 1896, file 385.

13 QSA, JUS/N199, 1892, file 137.

14 QSA, JUS/N241, 1896, file 188.

15 QSA, JUS/N453, 1910, file 456.

16 QSA, JUS/N502, 1912, file 421.

17 Archives New Zealand (ANZ), Inquest Files (J46), 1926, file 848.

18 ANZ, J46, 1936, file 1038; file 1504; file 1571; 1938, file 941; file 1574; 1940, file 324.

19 On Heaven, see Bruce Metzger and Michael Coogan eds., The Oxford Companion to the Bible (Oxford: Oxford University Press, 1993), 270-71; John R. Knott, "Milton's Heaven,” PMLA Vol. 85, No. 3 (May 1970), 487-495.

20 ANZ, J46, 1924, file 795.

21 ANZ, J46, 1949, file 307

22 QSA, JUS/N173, 1890, file 14.

23 QSA, JUS/N510, 1912, file 619.

24 QSA, JUS/N549, 1914, file 159.

25 ANZ, J46, 1946, file 1364.

26 QSA, JUS/N308, 1902, file 285.

27 QSA, JUS/N260, 1898, file 68.

28 QSA, JUS/N173, 1890, file 14.

29 ANZ, J46, 1910, file 614.

30 QSA, JUS/N288, 1900, file 381.

31 ANZ, J46, 1950, file 862.

32 QSA, JUS/N305, 1902, file 177.

33 QSA, JUS/N491, 1912, file 151.

34 QSA, JUS/N440, 1910, file 142.

35 ANZ, J46, 1918, file 358.

36 https://en.wikipedia.org/wiki/Google_Books. Accessed 23 May 2020.

37 Regrettably, Canada and the United States have not produced similar initiatives. Currently Canada is served only by a commercial digitized platform for the Toronto Globe and Mail.

38 Ariès, The Hour of Our Death, 611.

39 https://en.wikipedia.org/wiki/Napoleonic_Wars_casualties. Accessed 12 December 2019.

40 www.findmypast.co.uk/articles/world-records/full-list-of-united-kingdom-records/armedforces-and-conflict/crimean-war-casualties-1853-1856. Accessed 12 December 2019.

41 www.battlefields.org/learn/articles/civil-war-casualties. Accessed 12 December 2019.

42 Gary Scott Smith, Heaven in the American Imagination (Oxford: Oxford University Press, 2011), 72.

43 www.history.com/topics/inventions/history-of-cholera; https://en.wikipedia.org/wiki/Choler a_outbreaks_and_pandemics. Accessed 13 December 2019.

44 Houlbrooke, "Introduction," in Death, Ritual, and Bereavement, 2.

45 New Zealand Electronic Text Collection - Te Pūhikotuhi o Aotearoa, The Pamphlet Collection of Sir Robert Stout: Volume 28, "William P. Nimmo's List of New Books."

46 Branks, Heaven Our Home, 33.

47 Branks, Heaven Our Home, 33, 197.

48 Branks, Life in Heaven (Edinburgh: William P. Nimmo, 1866), 119-34.

49 Smith, Heaven in the American Imagination, 73.

50 Ellen Badone, The Appointed Hour: Death, Worldview, and Social Change in Brittany (Berkeley, CA: University of California Press, 1989), 230. 
51 Badone, The Appointed Hour, 231.

52 Pat Jalland, Australian Ways of Death: A Social and Cultural History (Melbourne: Oxford University Press, 2002), 151.

53 Jalland, Australian Ways of Death, 151.

54 https://openlibrary.org/publishers/William_P._Nimmo; www.isfdb.org/cgi-bin/publisher.cgi? 67659. Accessed 21 December 2019.

55 Branks, Heaven Our Home, promotional insert based on an article in The Northern Whig. The story was circulating in Australia in early 1862. Trove, Queanbeyan Golden Age, 5 April 1862, 4.

56 Newspaper items were accessed through web-based facilities: Trove (Australia), Papers Past (New Zealand), and the British Newspaper Archive (UK). Trove, South Australian Weekly Chronicle, 5 May 1866, 2.

57 Trove, South Australian Weekly Chronicle, 5 May 1866, 2.

58 Smith, Heaven in the American Imagination, 73.

59 Papers Past, National Library of New Zealand, Poverty Bay Herald, 25 January 1899, 4; Taranaki Herald, 31 January 1899, 1.

60 Diana Dixon, "The Two Faces of Death: Children's Magazines and their Treatment of Death in the Nineteenth Century," in Houlbrooke, ed. Death, Ritual, and Bereavement, 141-50; Smith, Heaven in the American Imagination, 71.

61 Papers Past, National Library of New Zealand,

62 British National Archive (BNA), Liverpool Mercury, 15 September 1850, 1.

63 BNA, Perthshire Advertiser, 19 September 1850, 1; Mayo Constitution, 24 September 1850, 1; Trove, Bell's Life in Sydney and Sporting Newes, 18 January 1851, 1; Empire Sydney, 18 January 1851, 1; The Britannia Trades' Advocate, 6 February 1851, 1.

64 BNA, Nottinghamshire Guardian 22 November 1849, 4; Southeast Gazette, 20 November 1849, 3.

65 www.britishexecutions.co.uk/chronology.php. Accessed 4 January 2020.

66 BNA, The Buchan Observer and East Aberdeenshire Advertiser, 26 May 1885, 3.

67 BNA, Leeds Mercury, 25 June 1926, 5.

68 BNA, The Carlow Post, 4 May 1867, 4.

69 BNA, Newcastle Fournal, 4 May 1868, 3.

70 BNA, Liverpool Standard and General Commercial Advertiser, 9 April 1933, 315. Spelling retained.

71 Trove, South Australian Record, 11 July 1838, 2.

72 Beadle's Dime Song Book (New York: Beadle and Company, 1863), 34. The sheet music first appeared in 1861 published by Frith Pond \& Company. Both accessed through Google Books, 29 December 2019.

73 BNA, Oxford Chronicle and Reading Gazette, 13 June 1857, 6.

74 The American Female Guardian Society, The Advocate and Family Guardian vol. 30 (New York: American Female Guardian Society, 1864), 117.

75 Trove, Sydney Times, 26 March 1838, 4. Lines to "Jane, On Leaving England."

76 Trove, Sydney Times, 26 March 1838, 4. Lines to "Jane, On Leaving England."

77 BNA, The Preston Chronicle, 10 January 1852, 7.

78 Trove, The Goulburn Herald, 28 October 1848, 4.

79 Trove, The Argus (Melbourne), 27 March 1850, 4.

80 Trove, The Port Phillip Gazette, 16 December 1843, 4; The Sentinel (Sydney) 16 September 1847, 4; The Argus (Melbourne), 24 January 1854; The Armidale Express, 13 December 1856, 3.

81 BNA, Inverness Courier, 20 December 1843, 4; Dundee Evening Telegraph, 27 August 1886, 4; Newcastle Daily Chronicle, 21 July 1896, 2; Falkirk Herald, 27 January 1904, 5. For examples of solace articulated by notable figures, see Martha McMackin Garland, "Victorian Unbelief and Bereavement," in Houlbrooke ed., Death, Ritual, and Bereavement, 151-70.

82 BNA, The Scotsman, 11 December 1833, 4.

83 Trove, Search for the phrase "will never meet in Heaven."

84 Trove, Sydney Morning Herald, 14 July 1831, 1. Quoting Liverpool Mercury.

85 Several generations of my relatives and their friends held these beliefs.

86 Ariès, The Hour of Our Death, 576.

87 QSA, JUS/N1049, 1938, file 512.

88 Papers Past, Northern Advocate, 7 June 1890, 3. This episode was the subject of a paper by Carole Neill, "'Suicide at Mangawhare': Understandings of Sensationalism in Late Nineteenth 
Century New Zealand," New Zealand Historical Association Conference, Victoria University of Wellington, 30 November 2019.

89 Bremmer, The Rise and Fall, 102.

90 www.centre-robert-schuman.org/userfiles/files/REPERES $\% 20 \%$ E2 $\% 80 \% 93 \% 20$ module $\%$ 201-1-1\%20-\%20explanatory \%20notes $\% 20 \%$ E2\% 80\%93\%20World $\% 20$ War $\% 20$ I $\% 20$ casual ties\%20\%E2\%80\%93\%20EN.pdf. Accessed 12 December 2019.

91 Charles E. Montague, Disenchantment (Westport, GT: Greenwood Press, 1978; reprint of 1922 edition), 161.

92 Paul Fussell, The Great War and Modern Memory (New York: Oxford University Press, 1975), 334. Jonathan Vance elaborated on the ways in which Canadians (elites) and their government sought to gloss memories of war as evidence of heroism and cooperation that promised a bright future for the county. Vance, Death So Noble: Memory, Meaning, and the First Wold War (Vancouver: UBC Press, 1997). For a critique of the notion of a collective memory and of Vance's account in particular, see Ian McKay and Jamie Swift, The Vimy Trap: Or How We Leaned to Stop Worrying and love the War (Toronto: Between the Lines, 2016), 214-20.

93 Peter E. Hodgkinson, "Glum Heroes": Hardship, Fear, and Faith - Resilience and Coping in the British Army on the Western Front, 1914-1918 (Solihull, UK: Helion \& Company, Wolverhampton Military Studies, No. 22, 2016), 135.

94 QSA, JUS/N977, 1934, file 161

95 ANZ, J46, 1942, file 1262.

96 ANZ, J46, 1942, file 1262. Perhaps significantly, this is the only suicide note of a New Zealand WWI veteran that mentions Heaven.

97 ANZ, J46, 1940, file 296.

98 QSA, JUS/N914, 1930, file 650. JUS/N921, 1931, file 20; JUS/N1087, 1940, file 589.

99 QSA, JUS/N956, 1932, file 895.

100 QSA, JUS/N951, 1932, file 611.

101 Smith, Heaven in the American Imagination, 178.

102 On the non-mortal point, see Philippe Ariès, Western Attitudes to Death from the Middle Ages to the Present (Baltimore, MD: Johns Hopkins University Press, 1974), 105-6. For a summary of his schema, see The Hour of Our Death, 596-614. Also see Jessica Mitford, The American Way of Dying (New York: Simon \& Schuster, 1963).

103 Jessica Mitford, The American Way of Dying; Ariès, The Hour of Our Death, 596-601.

104 John Hick, Death and Eternal Life (New York: Harper \& Row Publishers, 1976), 22.

105 Hick, Death and Eternal Life, 113-15.

106 Hick, Death and Eternal Life, 11.

107 Mark Johnston, Surviving Death (Princeton, NJ and Oxford: Princeton University Press, 2010), $11,306$.

108 Smith, Heaven in the American Imagination, 174.

109 Johnston, Surviving Death, 156-61.

110 Johnston, Surviving Death, 340.

111 Johnston, Surviving Death, 352-3.

112 Johnston, Surviving Death, 352.

113 Johnston, Surviving Death, 350, 370. 


\title{
DEATH IN MODERN NORTH AMERICAN HISTORY
}

\author{
Peter $\mathcal{N}$. Stearns
}

\begin{abstract}
Rates and causes of death
Measurable patterns of death did not change greatly in North America during the first eight decades of the 19th century - though precise data emerge only from about midcentury onward. In the United States, for example, infant death rates among Americans of European origin stood at 216 per 1000 in 1860 and had dropped only to 215 two decades later. Slightly greater changes occurred in life expectancy overall - from 39.5 years at birth in 1860 to 40.5 in 1880. Clearly, however, this was not a period of major change - except, briefly and tragically, during the years of the Civil War. Largely traditional rates persisted, dominated, of course, by the high rates of infant death. ${ }^{2}$

Death experience was also colored by periodic epidemics, associated mainly with poor urban sanitation and water quality and also increasing global contacts. Typhoid was a recurrent problem. Cholera epidemics reached North America in the early 1830s Toronto faced its first outbreak in 1831 - and returned every five years or so, as global trade and immigration rose. Through most of the century, partly because of these problems, urban death rates continued to surpass those in the countryside - another traditional pattern. This disparity helped encourage suburbanization in an effort to escape the worst contagions of city life: the Cincinnati suburb called Mt. Healthy, organized during an 1830s cholera outbreak, captured the impulse quite explicitly. ${ }^{3}$
\end{abstract}

Table 12.1 Table showing the relationship between life expectancy and infant mortality from 1860 through 1990. Michael Robert Haines and Richard H. Steckel, A Population History of North America (New York: Cambridge University Press, 2000), 308.

\begin{tabular}{lll}
\hline Year & Life Expectancy & Infant Mortality per 1000 \\
\hline 1860 & 39.5 & 216 \\
1880 & 40.5 & 215 \\
1890 & 47 & 160 \\
1910 & 54 & 96 \\
1930 & 60 & 60 \\
1960 & 71 & 63 \\
1990 & 76 & 8 \\
\hline
\end{tabular}


As Table 12.1 indicates, the situation began to change dramatically from the $1880 \mathrm{~s}$ onward, thanks initially to improved public health conditions and higher living standards - even as urbanization and immigration rates continued to surge forward. Infant mortality levels, for example, dropped by over $25 \%$ in the decade of the 1880 s alone, with immediate repercussions in overall life expectancy levels. The impact of epidemics receded - the last cholera episode occurred in 1910-11, and by then the mortality results were much less severe; improved urban sanitation and the use of quarantines applied to international passenger ships accounted for the change. And the overall mortality gains continued almost uninterrupted for the next 120 years. Only the global influenza epidemic of 1919-20 briefly, though sharply, interrupted the overall trend.

\section{Americans of European origin}

Developments in Canada were broadly similar, though ultimately the improvements slightly surpassed those in the United States. In 1921 Canadian life expectancy at birth was 57 years, but by 2011 it had soared to $82 .^{4}$

This was, clearly, a quiet revolution in the incidence and ubiquity of death. Virtually all groups were affected, though important differentials were noteworthy as well. Life expectancy for women surpassed that for men, though the gap eased slightly in the later 20th century particularly thanks to improved treatments for cardiovascular problems. Gender aside, gaps also reflected overall socioeconomic differentials, as measured for example by levels of education. In the United States this meant, also, a significant separation between African Americans and both whites and Hispanics - though again the basic trends involved all groups. By the early 21 st century, African American life expectancy was about 4 years less than that for whites. ${ }^{5}$

Again in the United States, another troubling development occurred in the 25 years after 1990, as both infant mortality rates and deaths of mothers in childbirth inched upward, bucking the previous trends and also the patterns common in advanced industrial societies generally (Canada included). In the 1950s maternal mortality rates in the United States and the United Kingdom had been identical, but by 2000 a significant gap had opened. American rates doubled between 1990 and 2013, from 12 to 28 per 100,000, and similar increases applied to infant deaths - putting the United States at the bottom of the world's developed countries in these crucial categories. ${ }^{6}$

Even with these important anomalies, however, United States death rates continued to decline overall, at least until 2014, though at a more modest pace, thanks to gradual improvements in adult health. The notion of a 20th-century death revolution in North America - comparable to developments in the industrial world more generally - continues to apply. Historic changes, particularly in child mortality but impressively also in adult longevity, dramatically transformed the frequency and visibility of death. Change was particularly rapid in the half-century after 1880, but the momentum continued fairly steadily thereafter.

These same developments not only reduced the numbers of deaths annually, they also increasingly concentrated high death rates in the older age categories. In Canada, more strikingly than in the United States, the vast majority of deaths were occurring in the age group $75-87$ by the early 21 st century.

The revolution in mortality rates was matched, of course, by striking changes in the causes of death during the same period, 1880s-present. Around 1900 the leading killers 
centered primarily in the infectious disease category: influenza, tuberculosis, diphtheria headed the list. By the second half of the 20th century, the list had been dramatically transformed, as degenerative diseases - heart attacks, cancer, strokes in that order - took over the leading positions. Accidents also surged forward, thanks above all to growing reliance on the automobile. ${ }^{7}$

Priorities shifted only modestly once the basic transformation in death causes had occurred. Heart disease and cancer continued to head the list of fatal diseases from the mid-20th century into the early 21 st. (In Canada, cancer actually surpassed heart disease as a killer.) But by 2018 diabetes and Alzheimer's had moved into fifth and sixth place (and strokes had dropped back) - reflecting improvements in prevention and treatment, in the case of strokes, but also the increasing ageing of the population (Alzheimer's) and, with diabetes, the growing rates of obesity as well. The role of accidents also fluctuated, though generally holding the fourth or fifth position in overall causes of death. In the United States, fatal accident rates dropped noticeably between 1969 and 1992, thanks to improved automobile safety and regulation. But then they turned around, due in large part to falls among the growing elderly population and also accidental overdoses of opioid drugs. ${ }^{8}$

Finally - in terms of the most measurable changes - these developments were accompanied by significant alterations in the location of death, though chronologically this basic change came last on the list. Recourse to hospitals for treatment of serious disease and accidents grew steadily in the first half of the 20th century. By 1949, in the United States, $47 \%$ of all deaths were occurring in institutions - hospitals, asylums and nursing homes. By 1958 the rate had risen to 61, and by 1980 it had soared to $74 \%$ of the total - a level that then held fairly steady. Patterns in Canada were similar: in 1930 only $30 \%$ of all deaths occurred in institutions, but, by the end of the century, rates moved past $70 \%$. By this latter point a fascinating anomaly had emerged in both North American countries: while $70 \%$ of all deaths were occurring in institutions, $70 \%$ of the population were expressing a preference to die at home. But this tension, obviously, involved factors that went beyond the most measurable changes - a point to which we will return.

The basic changes themselves remain striking. The physical facts of death, in the United States and Canada, had been transformed in three ways that became so obvious and widely expected that their magnitude might be missed. Death rates had plummeted, as infants and children were removed as a leading mortality category, and, for most people, the age of expected death pushed back steadily. Concerns about the leading causes of death shifted, almost as dramatically, from contagion (including epidemics) to degeneration. And, though somewhat later in time, the characteristic location of death, or at least final declaration of death, moved from home and workplace to hospital or related institution. These developments all clustered in the decades that stretched from the late 19th century until about 2000.

\section{Gultural transformations: a 19th-century phase}

The chronology of cultural reassessments of death in North America is, at least initially, intriguingly different from that of death rates and causes. For dramatic changes in the reception and management of death began to occur from the early 19th century onward, even as the objective experience of death remained fairly constant. The disparity between new attitudes and rituals concerning death and the relative stasis in death's incidence raises important questions about cause and impact - for, almost by definition, the 
significant adjustments in approaches to death are harder to interpret than the quantitative shifts that would take shape later on.

Ultimately, of course, changes in death rates would interact with culture, bringing yet another set of transformations and adjustments in death practices - mainly from the early 20th century onward. But this intersection was itself deeply conditioned by the more ceremonial approach to death and grief that had occurred earlier on.

A number of developments can be explored. First, relative attention to death as a topic - compared to other subjects winning attention in public books - began to drop markedly. A Google Ngram - in which the relative frequency of word use is charted shows that references to death reached dramatic highs in the early to mid-18th century but then began to drop precipitously, reaching a characteristic low by 1800 that would be essentially sustained thereafter, with only a slight upward trend in the second half of the 20th century.

Death remained important, as we will see. And grief references, significantly, followed a different trajectory, sustaining a relatively high level through most of the 19th century and only dropping significantly thereafter - more clearly paralleling the incidence of death itself. The rates of reference, though undeniably hard to interpret, warrant some attention, reflecting a somewhat reduced hold of the subject of death in American Protestantism and, overall, some shift in priorities toward other subjects (see Figure 12.1).

Reassessment of death also applied to the emergence of a long debate about the death penalty, in North America as in western Europe, from the late 18th century onward. ${ }^{9}$ Concern about death as a "cruel and unusual" punishment (the term used in the 8th Amendment to the United States Constitution) would lead to recurrent experiments with new methods of execution - the electric chair, then injected toxins - into the 21 st century. (Canada, in contrast, long stuck with hanging.) Both North American countries dramatically reduced the number of crimes to which the death penalty applied during the early 19th century. And both, now concerned about exposing ordinary people to the sight of death, removed executions from public view - Canada by legislation in 1868 . Concern about the finality of the penalty itself lingered. Michigan became the first English-speaking government to abolish capital punishment outright, in 1846, and many

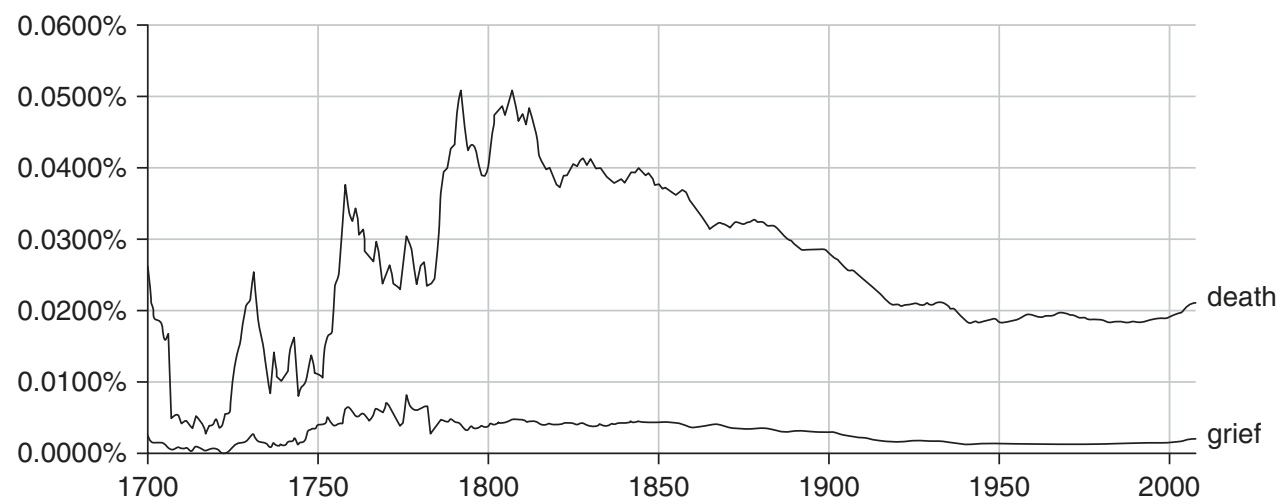

Figure 12.1 Frequency of the words "death" and "grief" in American English, 1700-2008, Google Ngram Viewer, accessed November 6, 2018. ${ }^{10}$ 
other American states moved in that direction from time to time. Canada would ban the penalty de facto in 1963, de jure in 1976, though its southern neighbor, more concerned about crime rates, wavered. Connections between death penalty discussions and larger views on death may be tenuous, but the notion of reevaluating, and possibly distancing, provides some link.

Another key change - more broadly significant, though still not easy to interpret also emerged decisively in the early 19th century, as the United States took a comparative lead in seeking a redefinition of the cemetery. As in other parts of the West, new concerns about contagion - based on the miasmic theory of disease transmission - prompted increasing efforts to relocate cemeteries, farther from urban population centers. The older tradition of situating cemeteries in the churchyard, where they would be regularly encountered, was increasingly replaced. While there was no massive effort to remove existing graveyards, new cemeteries must now be concentrated farther afield.

But, along with new space came a dramatic new look as Americans began to pioneer what became known as the garden cemetery movement. The real innovation involved making cemeteries increasingly look as much as possible like a bountiful English garden. The Boston area made the first move, following a previous change in the design of Parisian cemeteries. Several reformers there began in the 1820 s to bemoan the ugly and unhealthy state of existing facilities. Spurred by these concerns (authored by a group that included a horticulturalist but also several real estate figures), a decision was made to open to new cemetery in the then-spacious suburb of Watertown. The new (and still lovely) Mt. Auburn cemetery became the first of many projects in a number of American cities. Judge Joseph Story, opening the new facility in 1831, made its purpose quite clear. Not only were the dangers of center-city location to be avoided, but the depressing quality of traditional sites must also be transcended: their "death's heads and crossbones, scythes and hour glasses, angels with a rather diabolical expression" and characteristically gloomy inscriptions. Death should now be associated with the beauties of nature, with a sense of calmness and serenity rather than decay. ${ }^{11}$

Mt. Auburn, accordingly, was equipped with all sorts of distracting amenities, including attractive landscaping, several ponds and tidy paths. Caretakers, trained by garden enthusiasts, took meticulous care of the grounds. The Boston area was already introducing a number of attractive parks, and this new venture had much the same quality, earning high aesthetic marks. Another commentator noted,

when nature is permitted to take her course - when the dead are committed to the earth under the open sky, to become early and peacefully blended with their original dust, no unpleasant association remains. It would seem as if the forbidding and repulsive conditions which attend on decay were merged and lost in the surrounding harmonies of the creation.

Bodies would dissolve in the "embrace of nature," in the "bosom of mother earth."12

All of this meant that visits to the dead - communion with relatives - took on new aspects as well. In the first place, the visits would have to be explicitly organized; they were not part of the daily routine as churchyard sites had been. Obviously also, the visits could now have pleasant connotations, however great the grief involved. And family members could be associated in death more clearly than before, as large plots could be purchased with this in mind. A young man, learning that his father had 
bought a Mt. Auburn allotment, rejoiced that "as we have been united in life, we may not be separated in death." Judge Story insisted that the new design was not intended to distance death but rather to encourage an enlightened communion with the dead, "to hear the tones of their affection ... to listen to the voice of their wisdom" - unencumbered by needless gloom and foreboding. ${ }^{13}$

While the United States took a lead in garden cemeteries, the movement quickly spread to Canada, again with similar cultural implications. The Cataraqui Cemetery in Kingston featured abundant trees and shrubs, punctuated by natural ravines - and this site was the resting place for the nation's first prime minister. Beechwood Cemetery in Ottawa, with 65 hectares, had similar features (including 30,000 spring tulips), as did the Necropolis in Toronto, founded in 1850, embellished as well with Gothicstyle chapels.

The garden cemetery movement was further enhanced by an additional innovation: a commitment to more elaborate and individualistic monuments. Building on changes in graveside markers that had actually emerged a few decades earlier, 19th-century cemeteries filled with tombs embellished with affectionate themes: statues of cherubs or of mothers cherishing their children, expressing both a confidence in heavenly ascent and another link with strong families ties. Where finances permitted, children now might receive their own tombstones, depicting idyllic infants blissfully asleep, reflecting a widespread idea that the cemetery itself might now be seen as a "vast and exquisitely beautiful dormitory." Weeping willows, fingers pointing heavenward, butterflies emerging from cocoons were all popular motifs, obviously contrasting with the sparser embellishment and the death's-head figures that had been popular in the colonial era. ${ }^{14}$

All of this, clearly, reflected new levels of affluence in the urban middle classes. Striking, often quite large, memorials and imposing family vaults were status expressions, among other things. They stood as well as symbols of a new individualism, seeking to highlight unusual designs that stood out from the crowd. But, while not able to join in the showiest memorials, working-class families also sought to assure clear locations and markers as part of appropriate treatment of the dead; the popularity of burial insurance, with these goals in mind, easily outstripped any other kind of insurance for many decades. ${ }^{15}$

Interpreting these new styles and commitments is no easy assignment, once the status and consumerist elements are accounted for. Clearly, an effort to redefine death was involved, the less pleasant connotations deliberately countered with the combination of natural beauty, emphasis on family ties and (for those with means) individual distinction. Some observers argued that this treatment of death constituted a vital alternative to the increasingly hectic pace of ordinary life. An observer of New York's Green-Wood cemetery noted in 1838 that the cemetery was now an oasis of sorts:

A short half-hour ago, he was in the midst of a discordant Babel, he was one of the hurrying, jostling crowd, he was encompassed by the whirl and fever of artificial life. Now he stands alone in Nature's inner court - in her silent, solemn sanctuary. Her holiest influences are all around him.

The larger association with the dominant culture of Romanticism lent an air of escape and nostalgia to the treatment of death. Starkness and decay were supplanted by a sense of the dead as resting, their ascent to a peaceful afterlife assured. Cemetery names 
confirmed this pleasant imagery: Laurel Hill, Green Mount, Spring Grove. One observer claimed, in 1849, that "there is scarcely a city of note that has not its rural cemetery," and even many smaller centers joined the trend. ${ }^{16}$

Fashions did change somewhat by the later 19th century, though the notion of spaciousness and natural environment was largely preserved. Growing objections to the showiness, even discordant quality, of the big monuments encouraged somewhat more modest gravestones. Lawn cemeteries, with open meadows over rolling hills, replaced the wooded, sometimes cluttered look of the garden movement. By the 1880s a professional organization of cemetery superintendents emerged, committed to the new look, where vistas took precedence over individual gravesites. The superintendents recognized that "the requirements of the cemetery, apart from the burial of the dead, are very largely those of the park." "While there are some people who say a cemetery should be a cemetery, the great majority ... have come to believe in the idea of beauty."17

Cemeteries, in fact, became something of a big business, with a considerable price tag attached. A positive tone was part of the sales pitch. As a Kansas City professional noted, "Today cemetery making is an art, and gradually all things that suggest death, sorrow, or pain, are being eliminated." The editor of the influential Ladies Home Fournal praised the new parks for offering an alternative to what he called "grewsomenes" "there is nothing to suggest the presence of death." Death should now shed all the "many terrors with which the ignorance and superstition of older times surrounded it." "Kill off every plant and remove everything you see that suggests sadness." Correspondingly, in funeral services themselves, cemetery workers typically waited to cover the coffin, and sometimes even to lower it in the first place, until family members had departed - when a new machine, bedecked with flowers, could do the job rather impersonally. The idea was to make it seem that a "departed friend" had been gently lowered into a "curtained couch" rather than dropped into the ground. Increasing attention to coffins themselves, equipped with pillows and satin sheets, confirmed this general image - again, at some expense to the family involved. By 1870, in fact, commercial casket manufacturers were replacing local carpenters in providing the luxuries many families now expected. ${ }^{18}$

Some variety persisted, even aside from social distinctions reflecting different levels of family income. Catholic cemeteries picked up the lawn cemetery themes, but on the whole with less embellishment, in part because of the expense involved. Jewish cemeteries or cemetery sections on the whole featured simpler markers. Many Chinese Americans buried the dead in separate areas of the cemetery, hoping to save up enough money to send the bodies back to China for final interment.

Yet another innovation emerged, in what some historians have called an "American way" of death, during and after the Civil War. The war itself was exceptionally bloody, the casualty level unmistakable in a society that otherwise seemed to be bent on taming death. Over 600,000 Americans were killed in the conflict, almost half of the nation's total war dead from the Revolution until today. And while these figures did not displace the larger cultural apparatus - the lawn cemetery movement, for example, continued apace - it did prompt unprecedented concern for retrieving and honoring the fallen soldiers. Memorials to the dead sprang up in most northern cities, with individual names carefully inscribed - an innovation that would persist for subsequent conflicts. And many families themselves reached out, at considerable personal expense, to bring bodies of the fallen back home for a proper burial. ${ }^{19}$ 
This new commitment, in turn, was made possible by two further developments. Railway lines facilitated the movement of the bodies. More important, the reintroduction of the old tradition of embalming maintained the dead in conditions that would permit a later ceremony. Abraham Lincoln's body was thus embalmed after his assassination, to permit his transport back to Illinois in a fourteen-day railroad trip that allowed seven million mourners to pay their respects, his casket opened at every major stop itself a leadership ritual without precedent, that would, however, inform American practice in key situations from that point onward.

Embalming, once introduced in these special circumstances, began to catch on more widely. It might facilitate opportunities for farewells on the part of relatives who required a day or two for travel, in a geographically mobile society. It replaced the clearly fallible earlier practice of icing the body for better preservation (though icing would still be combined with embalming until about 1890). It clearly responded to health concerns about potential contagion, as against traditional practices in which the body would be kept at home until the final ceremony, wrapped in shrouds by family members themselves. It reflected as well the sometimes more crowded conditions of urban housing, where setting space aside for handling a corpse might be difficult. And it addressed one other concern, widely current in the earlier decades of the century, about the possibility of being buried alive: now, professionals would prepare the body and presumably prevent any mistakes. Most obviously, the rise of embalming represented a further step in the process initiated by the garden cemetery movement: reducing the unpleasant sensory reactions to death itself. ${ }^{20}$

For the funeral homes that now sprang up to organize not only embalming but other aspects of the final ceremony were bent on shaping more positive reactions to the dead. As one put it, praising the new combination of embalming and cosmetics, "when I come for the last time to look upon my dead, they will look to me as natural as though they were alive." By 1880 in fact the funeral association offered an annual prize of $\$ 1000$ to the member who made his subject most lifelike. Of course, the dead themselves were now dressed in normal best attire rather than shrouded. Again, new costs were involved for the families of the departed. Some professionals for a time offered a range of services, from merely "composing the features" to a more elaborate "giving the features the appearances of Christian hope and contentment."

Funeral homes themselves, reflecting one of the region's newest professions, tried to establish their own version of appropriate trappings for death. They were subdued but "as cheerful and pleasant as possible." As one observer noted, "Often the placement of the casket in the room, gracefully canopied by an attractive curtain, and banked against flowers artistically arranged, forms a picture so beautiful as to relieve the scene of death of some of its awfulness" - by now, a familiar North American theme. And, obviously, the family home itself, and particularly what once was called the parlor but now was increasingly dubbed the "living room," would be removed from any association with death. ${ }^{21}$

This whole 19th century trend, from new cemeteries to the rise of funeral homes, had obviously reduced, though did not eliminate, the role of religious officials in the death process. Ministers might still officiate at funerals, whether in the funeral home or, more commonly, with the body brought from the home to a church, but they were now only one of several kinds of professionals involved. The removal of cemeteries from the churchyard was capped now by the rise of funeral directors in organizing the body and 
at least part of the final ceremony. The shift was both confirmed and furthered by new government regulations requiring burials in approved, sanitary locations and insisting on more official confirmations of death. Much of the apparatus of death, and much of the tone in managing death arrangements, had been substantially transformed.

\section{Grief and emotion}

These various changes were accompanied by innovations in the expression, and possibly even the level, of emotional reactions. It is vital to remember that the changes in North American management of death, toward new kinds of distancing and distraction, occurred even as death rates remained stubbornly high. It is not surprising that the emotional climate involved and the rituals designed most directly to express emotion were complex.

A distinctive kind of fear increased, for several decades. Anxiety about being buried alive began to rise in the 18th century and persisted well into the 19th (on both sides of the Atlantic). A variety of stories circulated about cases of premature interment. As late as 1896 one American funeral director claimed that as many as 2\% of all "deaths" were actually cases of suspended animation, though the figures were dubious. Earlier, George Washington famously insisted that he be buried no sooner than two days after his death, in case a mistake had been made. A variety of coffins were sold with special devices - bells for warning, breathing pipes - to compensate for diagnostic errors. The frenzy is not easy to interpret, save as urban legends helped circulate and exaggerate the issue. New opportunities for reviving victims, including mouth-to-mouth resuscitation and crude defibrillation techniques, had been introduced in the 18th century, possibly encouraging a sense that death might not in fact be final. More broadly, the fear - indulged most obviously by the affluent - reflected distrust of the medical establishment even as recourse to doctors steadily increased. Correspondingly, growing confidence in the medical profession ultimately dampened this particular reaction, from the later 19 th century onward. ${ }^{22}$

More durably significant was the surge in expressions of grief, and rituals designed to express the emotion, through much of the 19th century. Grief was not, of course, a new invention, but there is no question that the emotion came to command novel levels of attention and, for some, a possibly increased intensity.

Thus some girls' schools in the United States featured needlework classes highlighting images of women draped over the coffins of dead husbands and sons. By the 1870s American girls could also buy dolls' kits with coffins and black drapery, again to practice the expression and management of grief as part of women's obligations to the family.

Correspondingly, family advice literature and family-based stories like Little Women highlighted the experience of grief in response to tragic loss. The emotion was held to be deep and virtually inevitable but with a somewhat bittersweet quality as it expressed the intensity of family bonds. A Protestant minister put the case this way, in 1882: "It may truly be said that no home ever reaches its highest blessedness and sweetness of love and its richest fullness of joy until sorrow enters its life in some way."

Children were explicitly instructed in the ubiquity and sadness of death, less now as a stern reminder of original sin than as a corollary of the deep emotions of family life. McGuffey's reader, which went through many editions as a schoolroom classic, had many 
passages emphasizing the sorrow of the death of a sibling cushioned by confidence in ascent to heaven. "I cannot see his laughing eyes, / They tell me this is death. / They say that he again will rise, / More beautiful than now." Many of these accounts also featured increasing emphasis on the expectation that family members would be blissfully reunited in heaven another interesting innovation. (And, of course, the 19th century also featured new efforts to communicate with the dead, through various kinds of séances. $)^{23}$

Letters - in a century devoted to these expressions - and diary references frequently picked up on the intensity of grief - "bitter cries of unsubmissive sorrow," as Louisa May Alcott put it. A truly massive array of popular songs picked up on the theme. The 1839 song, "Near the Lake Where Droop'd the Willow" was a major hit in concerts, inspiring many imitations for several decades, highlighting the emotions remembered for a love lost in youth. "To her grave these tears are given, ever to flow, / She's the star I missed from heaven, long time ago." Literally hundreds of songs about dying girls were published during the middle decades of the century. ${ }^{24}$

Etiquette rituals sprang up to acknowledge the grief felt on the death of a family member. Black clothing and arm bands were supplemented by black draperies around doors and windows. Manuals provided clear instruction on how a grieving family might be visited, and how the family in turn could acknowledge the attention of sympathetic friends - among other things, through black-edged cards.

Obviously, as with funeral monuments themselves, the most abundant ritual expressions of grief were available only to the middle and upper classes, but grieving letters suggest a level of emotion that applied more widely. A Schenectady father expressed his grief on a son's death: "How the world does recede ... when death enters our dwellings. All the vain things that please us the most lose their interest." And in this case the sorrow returned periodically, even years later, "with crushing effect." 25

Explaining this surge of grief is not an easy task, always remembering that the emotion was not new and that different individuals continued to react variously to the fact of death. Emotional expression partly reflected the fashions of Romanticism, which encouraged intensity and overflowing commentary. Urbanization, displacing community solidarity, placed more of the burden of emotional response and funeral attendance on extended families, which might exacerbate the experience for some. As funeral homes developed, despite their efforts to gloss over the grimness of death, practitioners might also encourage appropriate sorrow.

Two other probable prompts deserve attention as well. In the first place, emphasis on familial love was undeniably on the rise - in literature but also, insofar as we can judge, in real life. Marriages were increasingly based on declarations of mutual love through courtship, rather than parental arrangement. With lower birth rates and the decline of belief in original sin, emphasis on love for children - and particularly, maternal love surged as well. There is no reason to doubt the writers who now linked the intensity of grief to the power of love. The emotion showed particularly, of course, when death took away a child or sibling. But it also colored reactions to the death of a spouse, for example in cases of death in childbirth. One analysis suggests that men in the Southern planter class, rather insouciant about the risks of childbirth during the 18th century, became far more solicitous of the dangers their wives faced by the early 19 th. $^{26}$

Besides love, grief might also be spurred by an increased sense of guilt. The 19th century was, for the North American middle class, a century of Enlightenment-inspired optimism. Science and progress should be improving the quality of life - were improving it, 
in some clear ways. But death rates remained naggingly high. The resulting tension could promote a sense that, particularly in the case of deaths of children, responsible family members should have been more active in prevention. New reading matter, like the popular Godey's Lady's Book, repeatedly admonished mothers for their imprudent treatment of their offspring: many infant deaths, the advice-givers claimed, resulted from overfeeding or smothering a baby with excessive clothing. Rising grief may well have expressed the gap between declining fatalism - a new belief that traditional death rates should be pushed back in an enlightened society - and the facts of frequent mortality. ${ }^{27}$

Attitudes to death and grief relate to one other feature of 19th-century North American death reactions: a growing belief in the attainability of an afterlife in some kind of heaven (and a diminution of attention to hell) and a conviction that the afterlife would feature a reunion of family members (a particularly strong feature of the new religion of the Latter-Day Saints, or Mormons, but widely held more generally). These ideas, rather like aspects of the garden cemeteries, might attenuate sorrow. They also led in some cases to a sense of ongoing connections between the dead and the living. Interest in seances, in which mediums claimed to channel communications from dead family members, grew significantly, as with the efforts of Mary Todd Lincoln and her president-husband to reach their deceased son. Some of these innovations would persist in the 20th century, others, and most notably the séance movement, were gradually debunked.

Overall, the 19th century constitutes a distinctive moment in the history of death in North America. Very modest improvements in life expectancy were met with some quite dramatic changes in the ways death was viewed and the dead were treated. Death itself now inspired a new effort to distance and beautify - as in the new styles for cemeteries and, ultimately, the interest in embalming. But new levels of emotion - or, at least, a new authorization for emotional outpourings - were involved as well, and these generated their own changes in the ways children were socialized about death and in the family rituals involved. ${ }^{28}$

\section{Revisiting the culture: the 20th century}

The apparatus around death that developed in the 19th century almost inevitably had to be reassessed as awareness of the dramatic decline in death rates, particularly among children, hit home after 1900. Other factors contributed. The first half of the 20th century saw a strong movement toward greater informality in dress and manners, and this clearly influenced a reconsideration of death rituals. ${ }^{29}$ Growing emphasis on consumerism and cheerfulness played a role as well ${ }^{30}$ while the 19 th century death apparatus had a consumer element as well, the push to minimize disruptions to work and entertainment might easily overbalance some of the more elaborate practices of the 19th century.

But the big factor involved the changes in death itself. With death less common, particularly for children and birthing mothers, with growing confidence in the capacity of doctors and hospitals to push death back, a variety of popularizers displayed an impressive eagerness in trying to persuade North Americans that much 19th-century apparatus must be rethought. ${ }^{31}$

Not everything changed, to be sure. Most important, the use of funeral homes to arrange the bodies of the dead and orchestrate funerals provided no small amount of ritual continuity. ${ }^{32}$ Along with the strong role of religion in American life - compared for 
example to patterns in Europe - this traditionalism prompted a number of observers to argue that the modern disruption of death, though visible, was considerably less advanced than across the Atlantic. ${ }^{33}$ Admittedly, critics emerged to attack the considerable expenses associated with fancy caskets and funeral homes, ${ }^{34}$ but they did not clearly dent popular preferences; indeed, the investment may well have played a major role in beliefs that family obligations had been properly acquitted.

But major changes did occur. Shifts in the leading causes of death, away from infectious disease and toward degenerative ailments, encouraged a massive majority of North Americans to hope for a sudden death - ideally, in one's sleep - supplanting the more traditional belief that a good death should allow time to settle disputes and bid farewell to family members. The fact that sudden death might indeed burden loved ones emotionally yielded to the hope that death would do its work quickly, with no particular need for personal preparation.

Still more obviously, an impressive flurry of self-styled modernist commentary in the early decades of the 20th century explicitly attacked both the rituals of the past and the now-burdensome emotions that lay behind them. Magazine articles directly ridiculed 19th-century practice; now that serious illnesses could be entrusted to modern medicine with mortality concentrated in later age, there was no reason to accompany death with any particular fuss. Belief in salvation, hopes for reunion with departed relatives or, as periodic articles emphasized, the apparent joy often reported in near-death encounters all suggested a reduction in wasted emotions and needless ceremony. Death was now frequently likened to peaceful slumber, usually accompanied by "pleasurable sensations." Accounts of near-death experiences particularly highlighted "delight" and "beatific visions." "The whole idea of 'death agony' is an unscientific conception.,"35

The recalculation of death, along with the rapid decline of actual mortality rates, meant, as a number of popular articles suggested, that death could virtually "disappear" from ordinary concerns. "Perhaps the most distinctive note of the modern experience is the practical disappearance of the thought of death as an influence directly bearing on practical life." 36

With this in mind, the same popular strand urged a reconsideration of elaborate funerals, now seen as a device designed to encourage needless and wasteful grief. Grief itself came under attack: "Probably nothing is sadder in life than the thought of all the hours that are spent in grieving over what is past and irretrievable." And this was no idle judgment. Increasingly, experts of various sorts urged individuals who found it difficult to overcome grief fairly quickly to seek professional therapy, and in psychology what became known as "grief work" focused on promoting quick recovery from what was a useless and debilitating emotion. "When a woman cannot rouse herself ... from her grief there is need of the care of a physician." Correspondingly, by 1940 etiquette books themselves shifted gears. From traditional recommendations on how to interact with a grieving family, the emphasis now reversed: it was the grieving person him- or herself who risked being impolite, for intruding the emotion on others was no more than a rude imposition. Positive enjoyment of life must gain precedence over negative thoughts about death. Several women writers discussed their conversion from grieving excess to the healthier modern approach where positive self-control won out over "fruitless recollection."37

Rituals progressively changed accordingly, apart from the continued attachment to funeral homes. Mourning apparatus was progressively simplified, then discarded: no more black drapery, and even the discrete black armband faded from fashion by mid- 
century. The amount of time set aside for mourning declined as well, with employers increasingly reducing tolerance for more than a few days' absence. And all of this continued, into mid-century, to be promoted by the leading popular magazines, sometimes under dramatic headings like "America Conquers Death." or "Modern knowledge ... offers to the intelligent person today a conception of living which is a positive answer to the old death fears."38

The new approach also - though with partial inconsistency - urged reconsideration of the involvement of children with death (increasingly possible, of course, as deaths of siblings became less common). Children should not be exposed to death and grief, which might simply overwhelm them. Many urged that they be kept away from funerals. Attention to how to gloss over even the deaths of pets now won increasing attention. Even during World War II, parents were urged to avoid any reference to mortality: battles could be presented to children, if necessary, in the most matter-of-fact way, with no signs of fear or distress: with this tone, the experts hoped, "the child ... will meet many minor death references as just one more interesting phenomenon." And, of course, it was precisely in this period that the Disney company set about remaking children's fairy stories to eliminate references to death and suffering and to present uniformly happy endings. The logic of this overall recasting - the marked contrast to 19th-century socialization - was sometimes questionable: if death was not in fact a big deal, why worry about children's reactions? Quite possibly, the growing possibility of expecting children to live but a lingering nervousness about investing too much hope explained the eagerness to urge a distance: now that children could be kept from death in fact, they should also be shielded from its encounter in order not to tempt fate. Whatever the cause, the result merely added to the overall change: children who had been kept from much encounter with death might well be eager to truncate ceremony and monitor emotion when, as adults, death had to be faced. ${ }^{39}$

And all these developments pivoted around the fact that death fighting, rather than death acceptance, had gained pride of place. Growing reliance on doctors and nurses to deal with serious illness or accident, increasing familiarity with recourse to hospitals, meant increasing scope for a culture that explicitly saw death as professional failure, that was invested in maintaining life whenever possible. Reports from the 1920s past mid-century, in both the United States and Canada, urged how uncomfortable medical personnel were with death, how they sought to avoid or dismiss it. Characteristically, even dying individuals were not informed of their true condition, lest they lose hope and lest physicians be faced with emotional reactions they were not equipped to handle. And many family members, from grief or guilt, began to insist on heroic medical measures for dying relatives based on similar assumptions about the power of science to keep death at bay. ${ }^{40}$

Supplementing the recourse to medicine was increasing emphasis on good hygiene a leading theme in the public schools between the wars, supported by the soap companies - and new safety procedures, particularly to try to reduce the initially high rate of fatalities associated with the rise of the automobile, but also to reduce the hazards of the increasingly elaborate devices used in homes. Here, arguably, were some of the more positive results of the new belief that death could be pushed away, that any vestiges of traditional fatalism should be discarded. In law, similarly, juries frequently awarded large sums to families that had suffered a death due to mechanical failure or defective products: the idea that some deaths might simply be uncontrollable accidents tended to yield to a belief that deaths, at least premature deaths, were violations of the natural order and that someone or some company must be held responsible. ${ }^{41}$ 
Overall, the modernist approach to death, and the very real changes it encouraged, seemed to gain ground steadily into the 1960s. Change was qualified by some traditional religious ballast and some of the rituals that persisted from the later 19th century. A variety of ethnic subcultures also added their own take on death. Jewish tradition, for example, continued to organize several days of mourning and then annual remembrances, in ways that many found preferable to the more dismissive mainstream practices. African American funerals, called "homecoming" and often more celebratory than the average, constituted another important variant, also retaining a tradition of burying some familiar tools or household items with a dead relative to ease the passage to the next life. ${ }^{42}$

Some newer practices also emerged to deal with the roughest edges of the modernist approach. The most obvious victim of the new assumptions about death or grief was the parent whose child did in fact die - against the new normal pattern - or the spouse who lost a partner in early adulthood. These people - statistical exceptions though hardly rare - now faced an emotional culture no longer receptive to high drama and a community whose members now found it difficult to empathize with an experience most no longer shared. Heightened guilt - in a society where almost all children lived, surely parents whose offspring died must have done something wrong - could add to these burdens. Small wonder that many marriages now shattered when a child passed away, simply because the emotions were too hard to handle. But, amid these continuing challenges, some innovations did respond, from the 1940s onward. A variety of grief groups formed in cities - sometimes around churches, sometimes independently - to provide suffering individuals companionship with the only people who could clearly understand the problem: other parents or family members who had experienced premature loss. Thanatos groups and an organization called Compassionate Friends spread widely in North America, seeking to replace traditional community - now altered by the new patterns of death and modernism - with the solace of strangers who shared the burden of death in a death-fighting society. ${ }^{43}$

Additional occasions for grief also emerged during the 20th century. Miscarriages, for example, occasioned more sorrow. During the 19th century, American women had often welcomed miscarriages - "I am happy again" - given the inadequacy of birth control efforts and the growing desire to avoid too many children. Fetuses were not clearly understood in any event. But in the 20th century, with more planned conceptions and greater medical involvement, miscarriages took on more of the contours of death. ${ }^{44}$

Modernist impulses overall, particularly strong in the several decades that first reacted to the real revolution in death rates and locations, were not perhaps as unprecedented as their adepts claimed. Quite apart from the persistence of the funeral home tradition, modernists did share with their 19th-century predecessors a desire to keep a distance from the older rawness of death, to dress death up if it could not be entirely avoided. But the attack on emotion and ritual was an undeniably novel element, and some of its limitations began to be more widely recognized after mid-century.

\section{Modernism (somewhat) revisited}

Key pillars of modernism persisted through the 20th century and into the 21st. Primary reliance on medicine and death-fighting continued, amid mounting expense. Mourning remained simple, and indeed funerals themselves became even more informal. It is not 
clear that a new or "postmodern" death period was emerging in North America, but there were important shifts.

The gradual but steady rise of cremation - though slower in North America than in countries like Japan and Britain, because of persistent Christian tradition and more abundant real estate - represented a huge change in handling death while still relying on professionals for the necessary arrangements. In the United States while only $4 \%$ of the dead were cremated in 1960, the figure had climbed to $49 \%$ by 2015 ; Canadian rates were even stronger, from $6 \%$ in 1970 to $68 \%$ in 2009. Greater acceptance of the procedure by the Catholic Church (in 1963, and even more fully by 1998) obviously facilitated the trend in both countries. Cremation lent itself to more flexible ceremonies - only $8 \%$ of cremations involved no ritual at all - and certainly to more diverse treatment of the remains, with greater determination by individual families. Advocates insisted that the innovation was fully compatible with respect for the dead and expressions of grief by the affected living - but the openness to innovation was striking nevertheless. ${ }^{45}$

By the 21st century, in part because of the surge of cremation, some North Americans introduced more radical experiments in funeral ceremonies. Some rituals now involved popular music and very casual or colorful clothing; mocking signs might note the unfairness of death or highlight the individuality of the deceased. Locations might now include theaters, and emphasis shifted from burials with the body present, to lighter-hearted memorial services well after the fact. New companies sprang up to help distribute the ashes of the dead, for example around the Hawaiian islands, and older funeral homes worked to keep up with new tastes and variety, often adding event planners to their personnel. As one entrepreneur noted, "It makes a celebration of life not such a morbid affair." Some questions remained: the most dramatic innovations were not yet majority fare, and they did build on trends established earlier in the 20th century. Most important, they raised somewhat familiar issues about how grief was being handled amid the effort to emphasize the positive.

More widely, terminology continued to shift, reflecting continued efforts to smooth over potentially unpleasant realities. It was in the 1970s that the term "passing" or "passed away" began to replace reference to death or dying, for example in funeral home literature, simply in hope of distracting from undue starkness. Other efforts to conceal death suggested similar impulses. In most nursing homes, for example, dead people were removed from their rooms quietly and at night, lest the bulk of the elderly population be disturbed and despite the fact that many professed a desire to mark death with some ceremony. Organizations seeking to raise money for medical research blithely avoided the topic of death in favor of preaching medical miracles just over the horizon: thus the American Cancer Society trumpeted: "In no way do we wish to be associated with the book on death. We want to emphasize the positive aspects of cancer only." On a still grander scale, during the unending American wars of the early 21 st century, the government frequently sought to bring back the bodies of slain military personnel at night, to avoid public attention and potential grief and rebuke - of the sort that had accompanied the less managed deaths of the Vietnam war a few decades before. The modern desire to avoid death or play down its implications hardly ended. ${ }^{46}$

But the culture of the 1960s, and increasingly unavoidable recognition of some of the limits of modernism, did introduce important if sometimes unsystematic shifts in the ways death was handled, ushering in a partial third period in the North American response to modern death. Some of the changes remain incomplete, but overall the modifications do 
suggest a new interest in coming to terms with death in the contemporary context. And they are substantial enough to complicate any facile dismissal of modern culture as incapable of rising above the effort to treat death as a new taboo.

Amid strong attacks on the inadequacies of the contemporary management of death, through widely-read offerings by Elisabeth Kübler-Ross and others, new death and dying courses proliferated on college campuses in both Canada and the United States, bent on promoting a more supportive approach than unvarnished modernism had offered. A new breed of experts, echoing Kubler-Ross, urged more opportunities for individuals to express their own concerns about death and for family members to participate more fully in the process of painful adjustment and ultimate acceptance. ${ }^{47}$ And while the most obvious surge of death studies accompanied the 1960s counterculture, publications continued in a somewhat similar vein, urging clearer confrontation with death and acknowledgement of deep grief, while insisting on the variety of individual reactions; most bookstores in the 1990s still featured ten to fifteen books on the topic. ${ }^{48}$

This current, in turn, orchestrated some new adjustments in many parts of North America that went beyond the rise of more feel-good funerals. Four changes were particularly noteworthy, along with considerable continued reliance on small grief groups and psychological therapy to handle the deepest emotional reactions. First, medical personnel, though still dominant in the management of the dying process, changed their approach to some extent. Second, children began to be readmitted to the grieving process. Third, a new institution, the hospice, arose to try to recover the attributes of a good death. And finally, and hardest to interpret, new public mourning practices emerged in encountering certain kinds of highly publicized deaths.

Training of doctors and nurses was hardly revolutionized, but there were serious efforts to include greater sensitivity to the dying process. Revealingly, doctors were now willing to talk with patients and families when an illness was clearly terminal, promoting more opportunities for adjustment. At the same time, legislation increasingly promoted opportunities for individuals to sign living wills, in which people could express their wishes to avoid expensive, heroic medical measures in favor of dying with greater dignity. ${ }^{49}$ More candid approaches to death also included a rapid expansion of suicideprevention centers and a concerted effort to persuade people that accepting therapy was a valid choice. These developments are credited with stabilizing the suicide rate in North America (as in Europe) by the late 20th century, though distinctively, in the United States, suicide rates would spike upwards again after 2000.

Changes in the treatment of children are harder to measure, particularly because most children, still, encountered death only at some distance, primarily on the passing of grandparents. But the notion of keeping the young away from funerals was largely abandoned, on the assumption that some participation in grief was healthy and that opportunities to learn what death was about were preferable to avoidance. ${ }^{50}$ And many young people participated avidly in video games and other outlets that strongly featured violent death.

Unquestionably the most important single innovation in recent decades was the importation of the hospice movement and other efforts to arrange palliative care, from the 1970s onward in both Canada and the United States. Hospice treatment centered on patients whose illnesses were clearly terminal, where the focus must logically shift from therapy to compassion, with the opportunity where possible to arrange care in the home rather than an institutional setting. Maximum family involvement was encouraged, again in contrast to the forbidding atmosphere of acute care units in conventional hospitals. 
What the hospices represented, in fact, was an effort to return to many of the features of a traditional good death, modified only by the opportunities for more effective pain management. ${ }^{51}$

Finally, the growing interest in public mourning, on the deaths of certain celebrities such as pop music stars or figures like Princess Diana - and on the occasion of some mass shootings or other tragedies, signaled yet another changing behavior. Public ceremonies, even distant from the site of death, allowed strangers to express their fear and grief, often with candlelight vigils. Death sites were often marked by outpourings of flowers and - intriguingly - teddy bears contributed by concerned strangers. A new fascination with building elaborate memorials to commemorate some of the occasions, along with annual remembrances, marked the changing spirit as well. Clearly the contemporary public sought additional opportunities to express strong emotion connected with death, even as private rituals continued to be rather restrained. Dismay at the deaths of innocents and the young - as in some of the school shootings in the United States - factored in as well.

The new currents were fascinating, and unquestionably for many people they smoothed some of the roughest edges of modernism. But limitations were striking as well. Many Americans and Canadians remained blissfully unaware of the hospice movement or reluctant to abandon the recourse to death-fighting measures. Many relatives pressed still for heroic treatments, often ignoring a professed desire not to be resuscitated in cases of serious illness. In the United States, distrust of the government prevented wider policy discussions of the desirability of encouraging people to explore options with their physicians in advance of a crisis.

And the results, clearly, were a mixed bag. By the early 21 st century only $16-30 \%$ of all Canadians were involved in palliative care during their final illnesses. In the United States the figure ranged from 19 to $25 \%$. And even when hospice care was installed, it often applied only to the final day or two of life, before which time the average terminally ill patient consulted no fewer than ten different doctors during the final months. ${ }^{52}$

There was no big mystery about the kind of death most North Americans wanted, for polling made preferences clear around the turn of the century: "relationships and belonging," "having control" and "being human" were widely subscribed goals. While death has never, in human history, been arranged as people might wish, it is possible to argue that some of the gaps between wish and reality in the contemporary experience have become needlessly great. $^{53}$

Even here, however, it is important to record some continuing adjustment. In the United States in 2019, for the first time in nearly a century, more deaths were taking place at home $(31 \%)$ than in hospitals (the other leading site involved nursing homes). This new trend reflected priorities in the Medicare system, which encouraged hospitals to avoid long-term care and send terminal patients to other locations; the rise of the hospice movement (though half of home deaths did not involve hospice assistance); and the wishes of many dying individuals themselves, in what one critic called a "romanticization" of death at home. The result could be a considerable burden on families, though a new group of professions, or doulas, could provide some nonmedical assistance.

****

Dramatic changes in the nature of modern death and the complex cultural arrangements surrounding death, some dating back to the 19th century, are still being worked out in North America. The steady but very recent rise in cremation and public mourning 
suggests continued efforts at adjustment. The impact of the growing elderly sector, already visible in the rise of Alzheimer's as a major killer, has yet to be fully realized as the Baby Boom cohort passes into later age.

One other recent development must be worked into the mix, though it applies only to the United States. Over the past quarter-century, maternal mortality rates have doubled. For three years, 2016-18, against all international trends (including Canadian trends, which proceed uninterrupted), life expectancy has dropped, and even after slight recovery in 2019 remained well above averages in other industrial societies. The villains? Rising suicide rates (highest since World War II), affecting youth and middle-aged alike; the problems with opioids; uneven access to health care, with pronounced racial disparities; arguably, larger issues of economic and cultural performance. How this will work into broader modern patterns remains to be seen.

With all the problems, death may have lost some of its terror, at least until the virus pandemic of 2020. More people are able to push thoughts of death aside, in a normal year, than ever before. ${ }^{54}$ The Chapman Fear Index, a recent effort at annual sociological measurement in the United States, suggests that death stands between 16th and 20th among all expressed fears, though anxieties about terrorism and car accidents stand higher in the scale. ${ }^{55}$ A 1990 s AARP survey claimed that most older people professed not to be bothered by thoughts of death, and yet another study found that most people pondered death only rarely. Whether these are accurate assessments may be open to question, and, of course, there are no 19th-century polls available for comparison; further, the nonchalance may be undesirable. What is clear is that, first, death and its accompaniments have altered in many ways in North America over the past two centuries and that, second, the challenge of evaluating common reactions burns bright.

\section{Notes}

1 This essay excludes Mexico and Central America, where the modern history of death differs considerably from patterns in the United States and Canada.

2 Michael Robert Haines and Richard H. Steckel, A Population History of North America (New York: Cambridge University Press, 2000), 308; Richard A. Easterlin, "How Beneficent Is the Market? A Look at the Modern History of Mortality," European Review of Economic History 3 (3) (1999): 257-294.

3 Risa Barkin and Ian Gentles, "Death in Victorian Toronto, 1850-1899," Urban History Review 19 (2) (1990): 20-21; Victor W. Marshall, "Death and Dying," The Canadian Encyclopedia, February 7, 2006, last edited January 13, 2015, www.thecanadianencyclopedia.ca/en/art icle/death-and-dying (accessed November 13, 2018); Kathy Charmaz, The Social Reality of Death: Death in Contemporary America (Boston, MA: Addison-Wesley, 1980); James A. Fruehling, Sourcebook on Death and Dying (Chicago, IL: Marquis Professional Publications, 1982).

4 Katherine Arnup, Family Perspectives: Death and Dying in Canada (Ottowa: Vanier Institute of the Family, 2018).

5 Michael Robert Haines and Richard H. Steckel, A Population History of North America (New York: Cambridge University Press, 2000).

6 Jiaquan Xu, Sherry L. Murphy, Kenneth D. Kochanek, Brigham Bastian, and Elizabeth Arias, "Deaths: Final Data for 2016," National Vital Statistics Reports 67 (5) (2018): 1-76, www.cdc.gov/ nchs/data/nvsr/nvsr67/nvsr67_05.pdf (accessed November 13, 2018).

7 R. Lozano et al., "Global and Regional Mortality from 235 Causes of Death for 20 Age groups in 1990 and 2010: A Systematic Analysis for the Global Burden of Disease Study 2010," The Lancet 380 (2012): 2095-128; NCHStats, A Blog of the National Center for Health Sciences, 2018, https://nchstats.com/(accessed November 20, 2018); Geoffrey Gilbert, World Population: A Reference Handbook (Santa Barbara, CA: ABC-CLIO, 2002); John M. Last, "Public 
Health," in Robert Kastenbaum, ed., MacMillan Encyclopedia of Death and Dying (New York: MacMillan Reference USA, 2003).

8 For the United States it is also important to note the major surge of suicide as a cause of death in the early 21 st century, becoming the 7 th leading factor for men, 14 th for women, rising 30\% overall between 2000 and 2018 and reaching levels almost four times higher than rates in Canada where, as in Europe, levels continued to fall.

9 Hazel Erskine, "The Polls: Capital Punishment," Public Opinion Quarterly 34 (1970): 290-307; Barbara Norrander, "The Multi-Layered Impact of Public Opinion on Capital Punishment Implementation in the American States," Political Research Quarterly 53 (2000): 771-793; David Jacobs and Jason T. Carmichael, "The Political Sociology of the Death Penalty," American Sociological Review 67 (2002): 109-131; Amnesty International, The Death Penalty in America; Study Guide (2006); Michael Radelet and Marian Borg, "The Changing Nature of Death Penalty Debates," Annual Review of Sociology 26 (2000): 43-61; Steven Wilf, "Anatomy and Punishment in Late Eighteenth-Century New York," Journal of Social History 22 (1988): 507-530; Frank W. Anderson, A Concise History of Capital Punishment in Canada (Calgary: Frontier Publishing, 1973); Carolyn Strange, "Capital Punishment," in The Oxford Companion to Canadian History, ed. Gerald Hallowell, vol. 1 (Toronto: University of Oxford Press, 2004).

10 Google Ngram Viewer is a search application that allows one to measure the frequency of particular terms or words in the Google Books database. While in some ways problematic and obviously not a complete representation, the tool is a helpful way to assess cultural trends and changes.

11 James J. Farrell, Inventing the American Way of Death, 1820-1920 (Philadelphia, PA: Temple University Press, 1980), chapters 4 and 5. See also Lawrence R. Samuel, Death, American Style: A Cultural History of Dying in America (Plymouth, UK: Rowman \& Littlefield, 2013).

12 Farrell, Inventing the American Way of Death, 1820-1920, 87.

13 Farrell, Inventing the American Way of Death, 1820-1920, 89.

14 Nehemiah Cleveland, Greenwood Illustrated (1847), 13-14.

15 Michael K. Rosenow, Death and Dying in the Working Class, 1865-1920 (Urbana, IL: University of Illinois Press, 2015).

16 Nehemiah Cleveland, Greenwood Illustrated (1847), 13-14.

17 Andrew Downing, Landscape Illustrated (New York, 1921), 371; "Editorial," Park and Cemetery 5 (March 1895): 1; O.C. Simonds, "Review of Progress of Cemetery Design and Development," Association of American Cemetery Superintendents (AACS) 33 (1919): 59; William Falconer, "The Ideal Cemetery," AACS 26 (1912): 22; G.P. Burns, "The Application of Landscape Design to the Cemetery," AACS 31 (1917): 30; Sidney Hare, "The Cemetery Beautiful," AACS 24 (1910): 41.

18 Mrs. E.E. Hay, "Influence of Our Surroundings," AACS 14 (1900): 21; Farrell, Investing the American Way of Death, 132-133 and chapter 5.

19 Drew Gilpin Faust, This Republic of Suffering: Death and the American Civil War (New York: Vintage Books, 2008).

20 National Funeral Directors' Association (NFDA) 2 (1883): 22; W.P. Hohenschuh, The Modern Funeral: Its Management (Chicago, IL: Trade Periodical Company, 1900), 82-83; Robert Habenstein and William Lamers, The History of American Funeral Directing (Milwaukee, WI: Bulfin Printers, 1955)

21 Hohenschuh, The Modern Funeral, 17-18; A.P. Burton, “An Experience Hour," NFDA 27 (1908): 100-103; Farrell, Inventing the American Way of Death, chapter 5.

22 Joanna Bourke, Fear: A Cultural History (Emeryville, CA: Schoemaker \& Hoard, 2006); see also Chapter 20 by Bourke in Part III of this volume.

23 McGuffey's Fourth Eclectic Reader (1866); Richard Evans, "The Golden Stein," Analytical Fourth Reader (New York, 1888); Martha Finley, Elsie's Girlhood (New York, 1872), 156; Louisa May Alcott, Little Women (New York, 1868), 472, 488; Nicholas Tawa, Sweet Songs from Gentle Americans: The Parlous Song in America 1790-1860 (Bowling Green: Bowling Green University Popular Press, 1980), 134; "Literacy publications developed a near obsession with the grave," writes Lewis Sau, in "The Popular Mood of Pre-Civil War America," Contributions in American Studies, no. 46 (Westport, CT, 1980), 90. See also David Stannard, Death in America (Philadelphia, PA: 
University of Pennsylvania Press, 1975); Christy's Plantation Melodies \#2 (Philadelphia, PA, 1858); Buckley's Ethiopian Melodies (New York, 1853-1857).

24 Words by George P. Morris, music adapted from "Long Time Ago," a "blackface" song by Charles E. Horn, in William R. Ward, ed., The American Bicentennial Songbook (New York: Hansen Publications, 1975), 2, 155. In addition to songs and schoolbook references, a vast amount of popular art, including school paintings as well as samplers, was devoted to mourning themes between 1820 and the late 19th century. See Teresa M. Flanagan, Mourning on the Pejepscot (Landham, MD: University Press of America, 1992) and Steele, "Gender and Racial Politics."

25 Robert V. Wells, "Taming the 'King of Terrors': Rituals and Death in Schenectady, New York, 1844-1860," Fournal of Social History 27 (1994): 717-734.

26 Lynn Lofland, "The Social Shaping of Emotion: The Case of Grief," Symbolic Interactions 8 (1985): 171-190; Steven Seidman, Romantic Longings: Love in America, 1830-1890 (New York: Routledge, 1991); Ellen K. Rothman, Hands and Hearts: A History of Courtship in America (New York: Basic Books, 1987); E. Anthony Rotundo, "Romantic Friendship: Male Intimacy and Middle-Class Youth in the Northern United States, 1800-1900," Fournal of Social History 23 (1989): 1-25; Kenneth Lockridge and Jan Lewis, "Sally Has Been Sick: Pregnancy and Family Limitations among Virginia Gentry Women, 1780-1830," Journal of Social History 22 (1988): 5-19.

27 Peter N. Stearns, Revolutions in Sorrow: The American Experience of Death in Global Perspective (Boulder, CO: Paradigm Publishers, 2007).

28 See also the concomitant interest in near-death experiences and also in humble devices like Ouija boards that purported to allow communication with the dead. Here too, sincere conviction and charlatanism intermingled, in a set of fads that trailed off in the 20th century. See Ruth Brandon, The Spiritualists: The Passion for the Occult in the Nineteenth and Twentieth Centuries (Amherst, NY: Prometheus Books, 1984); Gary Scott Smith, Heaven in the American Imagination (New York: Oxford, 2011).

29 Cas Wouters, Informalization: Manners and Emotions since 1890 (London: SAGE, 2007).

30 Stearns, American Cool.

31 Stearns, Revolutions in Sorrow.

32 Gary Laderman, Rest in Peace: A Cultural History of Death and the Funeral Home in Twentieth-Century America (New York: Oxford University Press, 2003).

33 Philippe Ariès, The Hour of Our Death: The Classic History of Western Attitudes toward Death over the Last One Thousand Years (New York: Vintage Books, 1981).

34 Jessica Mitford, The American Way of Death Revisited (New York: Vintage Books, 1998).

35 Rev. W.R. Inge, "The Vesper Hour," Chautauqua 62 (April 1910): 260; "The Great Adventure," Outlook 103 (March 22, 1913): 605; Jane Melfield, "The Passing: An Emotional Monologue," Lippincott's Magazine 80 (September 1907): 374; "At the End of the Journey," Outlook 70 (January 25, 1902): 216; H.B. Marriott-Watson, "Some Thoughts on Pain and Death," North American Review 173 (October 1901): 540-553; "The Dying of Death," Fortnightly Review 72 (August 1899): 246-269; "A New Medical Conception of Death," Current Literature 47 (October 1909): 453; see also Elie Metchnikoff and Dr. Henry Smith, "Why Not Live Forever?," Cosmopolitan 44 (September 1910): 436-446; "Pain, Life, and Death," Living Age 281 (May 1914): 368-370; "The Conquest of Love and Death," Current Literature 53 (August 1913): 1968.

36 "The New Mien of Grief," Literary Digest 52 (February 1916): 292; see also "The Presence of Death," New Republic II (May 1917): 45-47; "Poor Death," Living Age 290 (September 1918): 360; "The Abolition of Death," Current Opinion 62 (April 1917): 270-271; "The Unseemliness of Funerals," Literary Digest 54 (April 1917): 1170; "And the Mourners Go about the Streets," Unpartizan Review 12 (July 1919): 176; Corra Harris, "Politics and Prayers in the Valley," Independent 71 (1916): 195. On World War I, see Paul Fussell, The Great War and Modern Memory (New York: Oxford University Press, 2000).

37 H.A. Dallas, "What is Death?," Living Age 332 (February 1927): 354-359; "The Tranquility of Death," Reader's Digest 56 (February 1927): 354-359; Marian Castle, "Decent Christian Burial," Forum 91 (April 1934): 243-255; M. Beatrice Blankenship, "Death is a Stranger," Atlantic Monthly 154 (December 1934): 649-657; Mabel S. Ulrich, "What of Death in 1931," Scribner's Magazine 89 (June 1931): 559-560; Milton Weldman, “America Conquers Death," American 
Mercury 10 (February 1927): 216-227; "Problems of Living," Scholastic 36 (April 1940): 31; James Averill and E.P. Nunley, "Grief as an Emotion and as a Disease; A SocialConstructionist Perspective," Journal of Social Issues 44 (1988): 79-95; J. Bowlby, Attachment and Loss, 3 vols. (Harmondsworth, 1971-1980).

38 "Agreeable Physical Aspects of Death," Current Opinion 72 (June 1922): 797; Sidney Lovett, "The Vocalism of Grief," Atlantic Monthly 130 (December 1922): 758-766; Sarah N. Cleghorn, "Changing Thoughts of Death," Atlantic Monthly (December 1923): 812.

39 D.A. Thom, Child Management (Washington, DC, 1925), 14; Daniel Anthony Fiore, "Grandma's Thought": Children and the Death Experience from the Eighteenth Century to the Present, unpublished honors paper, Pittsburgh, 1992; Peter Stearns and Timothy Haggerty, "The Role of Fear: Transitions in American Emotional Standards for Children, 1850-1950," American Historical Review 96 (1) (February 1991): 63-94; S.M. Gruenberg, ed., The Encyclopedia of Child Care and Guidance (New York, 1954), 170; Ruth Sapin, "Helping Children to Overcome Fear," Parents' Magazine 8 (August 1933): 16; see also Donald A. Laird and Eleanor C. Laird, The Strategy of Handling Children (New York, 1949), 77; C.W. Hunicutt, Answering Children's Questions (New York, 1949), 22; Harold H. Anderson, Children in the Family (New York, 1941); Tuttle Family Papers, Arthur and Elizabeth Schlesinger Library on the History of Women in America, Radcliffe College.

40 Cas Wouters, "Changing Regimes of Power and Emotion at the End of Life," Netherlands Fournal of Sociology 26 (1990): 151-155.

41 Peter N. Stearns, Anxious Parents: A History of Modern Childrearing (New York: NYU Press, 2004), 121, 126; G. Edward White, Tort Law in America: An Intellectual History (Oxford: Oxford University Press, 2003); Robert Rabin, "The Historical Development of the Fault Principle: A Reinterpretation," Georgia Law Review 15 (1980-1981): 925-961.

42 Suzanne E. Smith, To Serve the Living: Funeral Directors and the African American Way of Death (Cambridge; Belknap Press, 2010); Debra Umberson, Julie Skalamera Olson, Robert Crosnoe, Hui Liu, Tetyana Pudrovska, and Rachel Donnelly, "Death of Family Members as an Overlooked Source of Racial Disadvantage in the United States," Proceedings of the National Academies of Sciences of the United States of America 114 (5) (2017): 915-920. It is vital to remember that the higher death rates of African Americans meant that families had to deal with a measurably more frequent occurrence than was true for the population overall, amid cultural standards that often emphasize the importance of concealing public displays of emotion, except in special settings.

43 Sharon Kaufman, ... And a Time to Die: How American Hospitals Shape the End of Life (New York: Scribner, 2005); Sandra Gilbert, Death's Door: Modern Dying and the Way We Grieve (New York: W.W. Norton, 2005), 106; Christie Davis, "Dirt, Death, Decay and Dissolution: American Denial and British Avoidance," in Glennys Howarths and Peter Jupp, eds., Contemporary Issues in the Sociology of Death, Dying and Disposal (New York: Palgrave Macmillan, 1996); Donna Dickenson and Malcolm Johnson, eds., Death, Dying and Bereavement (London: Springer, 1993); see also an excellent review of post-1960 developments, John W. Riley Jr., "Dying and the Meanings of Death," Annual Review of Sociology 9 (1983): 191-216; Margaret Stroebe et al., "Broken Hearts in Broken Bonds," American Psychologist 47 (1992): 1205-1212; Wouters, "Changing Regimes of Power and Emotion at the End of Life," 151-155.

44 Shannon Withycombe, Lost: Miscarriage in Nineteenth-Century America (New Brunswick: Rutgers University Press, 2018).

45 Josh Sanburn, "The New American Way of Death," Time (June 24, 2013), http://time.com/ 645/the-new-american-way-of-death/ (accessed November 22, 2018); Douglas James Davies and Lewis H. Mates, Encyclopedia of Cremation (New York: Ashgate, 2005). Another innovation, though directly involving very few people, was the interest in cryogenics, an extreme version of the death-fighting impulse. The first case in which a body was frozen, in hopes of later medical advances that would more reliably preserve life, occurred in 1967; by 2018 several hundred people had undergone the procedure or arranged for freezing after death. Brian G. Wowk, "The Future of Death," Fournal of Critical Care 39 (6) (2014): 1111-1113. See also James Gire, "How Death Imitates Life: Cultural Influences on Conceptions of Death and Dying," Online Readings in Psychology and Culture (December 2014), https://scholarworks.gvsu.edu/orpc/vol6/ iss2/3/ (accessed December 4, 2018). 
46 Stearns, Revolutions in Sorrow.

47 Elisabeth Kübler-Ross and David Kessler, On Grief and Grieving: Finding the Meaning of Grief Through the Five Stages of Loss (New York: Scribner, 2005); Ivan Illich, Medical Nemesis: The Expropriation of Health (New York: Bantam Books, 1979).

48 Harold S. Kushner, When Bad Things Happen to Good People (New York: Schocken Books, 1981) and Conquering Fear (New York: Alfred A. Knopf, 2009); Stephen Levine, Healing Into Life and Death (New York: Anchor Books, 1987), Unattended Sorrow: Recovering from Loss and Reviving the Heart (Emmaus, PA: Rodale, 2005), and A Year to Live: How to Live This Year as If It Were Your Last (Emmaus, PA: Rodale, 2009).

49 Sharon Kaufman, ... And a Time to Die: How American Hospitals Shape the End of Life (New York: Scribner, 2005); Sandra Gilbert, Death's Door: Modern Dying and the Way We Grieve (New York: W.W. Norton, 2005), 106.

50 One other development, gaining steam steadily from the later 20th century onward, must ultimately be incorporated in evaluations of young people and death: the increasing availability of violent, graphic scenes in which death and bloodshed abounded, usually with no particular emotional valence. Violent comics and genres such as the movie Western had caused concern earlier in the 20th century, but with some limitations - as in the Hollywood convention that shooters and victims should be kept separate. But changes in the rules and the rise of special effects and video games, particularly by the 1990s. tore away restraint. Young people, particularly young men, now saw far more cases of death by violence - cinematically represented than ever before in human history.

51 Fran Smith and Sheila Himmel, Changing the Way We Die: Compassionate End of Life Care and the Hospice Movement (New York: Simon and Schuster, 2013); Dona J. Reese, Hospice Social Work (New York: Columbia University Press, 2013).

52 National Hospice and Palliative Care Organization, "National Hospice and Palliative Care Organization, 2017" report, revised April 2018, (Washington, DC, 2018).

53 George Horace Gallup, The Gallup Poll: Public Opinion 1992 (New York: Rowman and Littlefield Publishers, 1993).

54 And on this front, one other recent development: a variety of social media apps available to comfort fears and smooth adjustments to the reality of death, like the app "WeGroak," which features regular calls reminding about mortality along with wise quotes on confronting death successfully.

55 “America's Top Fears 2016: Chapman University Survey of American Fears," Chapman University, https://blogs.chapman.edu/wilkinson/2016/10/11/americas-top-fears-2016/ (accessed November 25, 2018); “America's Top Fears 2018: Chapman University Survey of American Fears," Chapman University, https://blogs.chapman.edu/wilkinson/2018/10/16/americastop-fears-2018/ (accessed November 25, 2018). 


\title{
DEATH IN MEXIGO
}

\author{
Image and reality
}

\author{
Stanley Brandes
}

\section{Images of the morbid Mexican}

Ever since my first day of fieldwork in the small town of Tzintzuntzan, Michoacán, I have been haunted by images of the morbid Mexican. ${ }^{1}$ On that fateful occasion in June 1967, one of my would-be neighbors, a potter in his mid-thirties, was struck dead by lightning. In the evening I attended the wake and, the following day, the burial. At the graveside, mourners apparently got word that I owned a camera - the only one in town in those days. They asked me to retrieve it from home to take pictures of the deceased. I was glad to comply. When actually confronted by my photographic assignment, however, I remained paralyzed. There stood fifty or sixty disconsolate men and women, staring in eager anticipation as I hovered over the open casket. I first faced the deceased from above, head on, in order to capture a good likeness. But I felt embarrassed by the directness of the angle. Despite the clear wishes of the mourners, I suspected that I was somehow intruding on the solemnity of the occasion and violating a cultural taboo.

The taboo, of course, turned out to be my own, not that of the Tzintzuntzeños. It seemed to me entirely inappropriate to photograph a cadaver, particularly in the presence of mourners. The best I could manage was to capture images from several very oblique angles. Although the pictures bore no likeness to the subject, the grieving family expressed grateful appreciation. The incident was almost enough to convince me that, yes, Mexicans do view death differently from Anglos, or at least hold different views from my own.

From what we read and hear, there exists a unique Mexican view of death. Scholars, journalists, critics, and writers from both Mexico and the US tell us so. Speaking with almost a single voice, ${ }^{2}$ they convey the impression that Mexicans develop a close relationship, not necessarily to deceased relatives, but rather to an abstract entity known as Death. It is said that Mexicans live side by side with death and are therefore able to confront death honestly and directly. They scorn death, they mock death, they are disdainful and irreverent in face of death. Whether valid or invalid, this national portrait has at least one unmistakable consequence: it furthers the cause of Mexican national identity. It delineates yet one more trait that all Mexicans share and that differentiates them from others. The portrait homogenizes the Mexican people into a single type. It portrays a pan-Mexican uniformity at the expense of variations by class, ethnicity, age, religious affiliation, and place of residence. Moreover, it is a timeless portrait, extracted from historical context and protected from the vicissitudes of politics, economics, and demographics. For these reasons, it reads suspiciously like a stereotype. It therefore needs to be scrutinized and, if necessary, challenged. 
Consider first the probable impact of abundant archaeological remains, folk arts, film, and other visual material on the creation of this stereotype. Ancient artifacts provide plenty of evidence of daily concerns, and death is almost inevitably among these. Throughout ancient Mexico, from the Mayan region in the south to the Aztec empire and its ancestors to the north, there exist diverse graphic portrayals of death and mortality. Among these portrayals, and certainly the easiest for Westerners to interpret, are representations of human skulls and skeletons. Here we can illustrate with just one striking example, the Toltec capital of Tula north of Mexico City, where dramatic indications of a palpable fascination with skulls and skeletons are clearly present. Tula flourished from the ninth until the thirteenth century A.D. The site includes the decimated remains of a tzompantli, or skull rack, which once displayed multiple rows of stone-carved skulls adorning the sides of a broad platform upon which the actual skulls of sacrificial victims were publicly exhibited. ${ }^{3}$ The tzompantli appeared during the final phases of civilization at Tula, which was destroyed around 1200 A.D. ${ }^{4}$ Chichén Itzá, almost contemporaneous with the Toltecs and located far to the southeast in the heart of Yucatan, contains a better-preserved tzompantli. Diehl says of this structure, "The platform sides are covered with grisly carved stone panels showing human skulls strung up on upright posts like beads on a necklace. These probably symbolize the real skulls which once covered the platform summit." In the Mayan area, too, Uxmal displays a tzompantli, with skulls and crossed bones.

In addition to the tzompantli, there is a freestanding wall at Tula known as the coatepan$t l i$, or Serpent Wall, which formerly enclosed the north side of the pyramid upon which stand tall rigid warrior figures, the famous atlantes. This wall, probably associated with the cult of Tlahuizcalpantecuhtli (the supreme god Quetzalcóatl in the form of Venus the Morning Star), is decorated with a series of carved stone panels, which show feathered serpents devouring human skeletons. ${ }^{6}$ The most remarkable feature of these skeletons is their lanky limbs and prominent joints, allowing for corporal flexibility; they are in fact large, stone versions of the wooden and clay toy skeletons found in markets all over Mexico around the time of the Day of the Dead. Like small toys today, these stone skeletons appear animate, with their awkwardly crossed legs and outstretched arms.

Given the diversity and complexity of skull and skeletal representations in ancient Mesoamerica, it is impossible to discount their cumulative impact on colonial and postcolonial art. The cloister at the Augustinian monastery in Malinalco, dating from 1540, contains an alcove dominated by a friar and skeleton with scythe standing next to one another. ${ }^{7}$ Consider, too, the open-air chapel at Tlalmanalco, on the road to Amecameca east of Mexico City, constructed by Franciscans in 1550-60. The chapel portrays literally dozens of skulls lining the upper part of its graceful arches, all of them alternating with diverse plants. On each of the central columns there are human figures, dressed in Renaissance garb and holding hands. The entire ornamentation at Tlalmanalco is designed to demonstrate "the Triumph of Death over humanity."

Eighteenth-century New Spain, comprising much of colonial Mexico, was similar to most of contemporaneous western Europe in that allegorical portrayals of the ages of man often included a skeletal image of death. Life was represented typically as a twosided staircase, ascending on the left side and descending on the right. Upon each stair was drawn a human figure representative of a particular age, starting at the lower lefthand side with a picture of a baby representing infancy, and ending at the bottom righthand side with a skeletal figure symbolizing death. ${ }^{9}$ Another common eighteenth-century motif was the "arbol vano" [Tree of Vanity] or "arbol del pecador" [Sinner's Tree] generally portraying a young man, the sinner, reclining or seated at the base of a tree, 
completely spent from sensual excesses. ${ }^{10}$ Invariably, there are supernatural figures surrounding the sinner, often a devil, occasionally an angel, but always a menacing skeleton, wielding an axe or a scythe.

Representations of skulls and skeletons became universally famous from reproductions of Mexican art and architecture dating from the pre-Columbian and colonial periods. But perhaps the greatest impact on world images of how Mexicans view death came in the late nineteenth century through the work of graphic artist José Guadalupe Posada. Posada's images portrayed "vivid and lively skeletons and skulls with grinning teeth, dancing, cycling, playing the guitar, plying their trades, drinking, masquerading," in addition to dozens of other comical manifestations. ${ }^{11}$ Childs and Altman observe that

everyone and everything was a likely subject of his illustrations. There are calaveras of leaders of the 1910 revolution like Francisco Madero who is depicted as a drunken peon and another of Vanegas Arroyo, Posada's publisher. There are grand ladies and gentlemen of the aristocracy and coquettish barmaids all in skeletal form. There are also scenes drawn directly from Días de los Muertos celebrations, such as the one depicting a cemetery picnic and another a seller of sugar skulls. ${ }^{12}$

Probably the most famous of Posada's images is that of the catrina, the female dandy, portrayed as a fleshless skull, topped with a fancy, wide-brimmed hat which is replete with large, billowing feathers and other decorations.

Posada's influence on Mexican art and culture is incalculable. Largely ignored by artists of his day, he was discovered and popularized through the zealous efforts of artists and writers, many of them non-Mexican. Peter Wollen recounts the birth of Posada as a recognized genius:

The painter Dr Atl, the most persistent early pioneer of modernism in Mexico, and the young French immigrant, Jean Charlot, one of the group of muralists around Diego Rivera, were the first to notice Posada's work in the new artistic context of the post-revolutionary years. Charlot, who first saw Posada prints in 1920, followed up his discovery, showed prints to other painters and wrote the first biographical and critical essays on Posada's work, in the early twenties. In time, other artistsincluding Rivera, José Clemente Orozco and David Alfaro Siqueiros-took up Posada's cause, often in hyperbolic terms, and acknowledged their debt, direct or indirect, to the humble popular print-maker. The legend was born. ${ }^{13}$

Folklorist Frances Toor promoted Posada's reputation when in 1930 she published Posada: Grabador Mexicano (Posada: Mexican Engraver). Diego Rivera, in an introduction to this book portraying 406 of Posada's engravings, called him "an engraver of genius" and "the greatest artist" among those Mexicans who have produced popular art. ${ }^{14}$ In his famous mural, "A Sunday Afternoon in Alameda Park," Rivera reproduced Posada's catrina, placing both this figure, dressed in a parody of full bourgeois costume, and a portrait of Posada himself in the center of the huge painting, where they flank Rivera himself.

Posada became nationally and internationally famous through the promotional efforts of well-known artists and writers within and outside Mexico. Posada's art became popularized, initially by struggling journalists but later and more importantly by the country's artistic elite, who read into his imagery the meaning of Mexico itself. Wollen summarizes Posada's legacy thus: 
$[\Pi \mathrm{t}$ is wrong to see Posada simply as an "influence." His name and legend were constitutive in the establishment of the Mexican renaissance; they symbolized both an alternative tradition and, crucially, a chain of succession. This particular role assigned to Posada was important both in relation to Mexicanism and in relation to Modernism. It gave credibility to claims to be part of an authentically Mexican artistic tradition, crossing both the class gap and the historic divide of the Revolution itself and, at the same time, guaranteed the modernity of the tradition by aligning it with the revival of popular imagery among the European avant-garde. $^{15}$

By the 1930s Posada and his calaveras became symbolic of Mexico. For their irreverence, the calaveras suited the revolutionary ideology of Mexico, and yet the international art community had virtually declared these satirical skulls and skeletons to constitute a kind of high art. In the terms analyzed by Liza Bakewell, Posada's work satisfied the needs of a "cultural nationalism" that started with the Mexican Revolution (1910-20) and persists to this day. ${ }^{16}$ Perhaps the most outstanding contemporary product within this tradition is the Oscar-winning now world-famous animated film Coco. Produced in 2017 by Pixar Studios, much of the film features dancing, frolicking sugar-skull skeletons. It celebrates the cross-generational unity of Mexican families, and the abiding - in this film transcendental - connection between living and deceased kin.

In addition, Posada's calaveras, through their popularization by famous artists who took up his cause, have exerted a widespread influence on Mexican street and gallery art. Childs and Altman provide several apt examples:

The wire and plaster skeleton figures of the Mexico City artist, Saúl Moreno, are on the surface not very different from toys made in Oaxaca. But Moreno's work is not produced for friends and neighbors, but is made for and consumed by art collectors. A skeleton sculpture by Moreno is more likely to be found in a Berlin art collection that on a villager's altar in Tepoztlán. Another Mexico City artist, Pedro Linares, works in papier-maché and has created Días de los Muertos objects which have no real counterpart in observances of the festival. He makes large skeleton figures which compare to the Judas figures commonly made for Holy Week. But whereas the Judas figures are made to be burned in a village fiesta, the Linares papier-maché skeletons and skulls ... have no such function and are intended for a national and in fact international art market. ${ }^{17}$

Political and social commentaries that follow the Posada tradition continue to appear in newspapers throughout Mexico around the time of the Day of the Dead. But the art that his work has spawned, through its validation by world-famous Mexican artists, now transcends Posada's immediate goals. It has become an important commercial enterprise and for this reason - in a nutshell, for the profit motive - has acquired a life of its own. Mexican skulls and skeletons sell well to the international community of tourists and collectors. Judging from the Posada reproductions drawn annually on store windows and supermarket displays, skulls and skeletons also stimulate business among Mexicans consumers. The proliferation and widespread artistic reproduction of animated skulls and skeletons have come to represent the Mexican Republic, an outstanding feature of what Claudio 
Lomnitz has termed "the nationalization of death" or, as Lomnitz elaborates, "the nationalization of a playful familiarity with and proximity to death itself." 18

These images have promoted the rise of an international image of Mexicans as a death-obsessed people. So, too, has the increasing popularity of a folk saint - La Santa Muerte, or Holy Death - venerated principally (but not exclusively) among organized criminals, urban working classes, and the poor. ${ }^{19}$ This sculptural image, endowed with skeletal face and hands and carried in procession annually, just as countless other saints are, has never been recognized by the Vatican, and yet has experienced increasing devotion throughout Mexico and even by some Catholics living in the southern United States. Claudio Lomnitz provides a telling description of this image, which is in part "entirely familiar to Catholic iconography: sickle in hand, it is the image of Death the leveller. La Santisima Muerte also has attributes of the Virgin Mary: the globe, the scapular, and the virginal entailments of the white bridal gown." ${ }^{20}$ Here is yet another iconographic figure that helps give rise to an image of the morbid Mexican.

Turning to literature, so has Octavio Paz's The Labyrinth of Solitude - an extended essay that over the past half century has become known as the most influential interpretation of Mexican national character. Labyrinth summarizes a range of popular opinions on how Mexicans relate to death. The following passage is worth citing at length:

The word death is not pronounced in New York, in Paris, in London, because it burns the lips. The Mexican, in contrast, is familiar with death, jokes about it, caresses it, sleeps with it, celebrates it; it is one of his toys and his most steadfast love. True, there is perhaps as much fear in his attitude as in that of others, but at least death is not hidden away: he looks at it face to face, with impatience, disdain, or irony.... The Mexican's indifference toward death is fostered by his indifference toward life. ... It is natural, even desirable, to die, and the sooner the better. We kill because life - our own or another's - is of no value. Life and death are inseparable, and when the former lacks meaning, the latter becomes equally meaningless. Mexican death is a mirror of Mexican life. And the Mexican shuts himself away and ignores both of them. Our contempt for death is not at odds with the cult we have made of it. ${ }^{21}$

Paz claims, in other words, that Mexicans not only blur the familiar European distinction between life and death, but they also embrace death, as if it were some sort of welcome friend. It is not too much to say that Paz believes that Mexicans actually like death, or at least live easily in its presence, while Europeans and Anglo-Americans do not. For Paz, Mexicans accept death stoically; Europeans, by contrast, cannot easily or bravely confront the prospect of dying. Certainly, my awkwardness at the Tzintzuntzan burial a half century ago seems to confirm this point of view. Implicit in Paz's analysis is an image of the macho Mexican, a person allegedly unwilling to demonstrate fear and imbued with a hard, indifferent exterior in the face of danger. Throughout The Labyrinth of Solitude, it is the isolated individual, hermetically sealed against insult and injury, who emerges as the most common personality type.

For Paz, loneliness and solitude conspire to make the Mexican unfeeling, guarded, and defended, in matters of death and life. Paz composed The Labyrinth of Solitude as a lengthy essay. It is a thought piece rather than a carefully documented scholarly treatise. Regardless of whether he accurately represented the full range of Mexican beliefs and attitudes, the impact of his forceful prose is undeniable. Its lyrical beauty and 
rhetorical power helped establish his role as authoritative spokesman for his people. Given the almost instantaneous popularity of The Labyrinth of Solitude, Paz's views on Mexican national character soon constituted a kind of intellectual orthodoxy that flourished during the 1960s and 1970s. Consider the words of several representative Mexican scholars writing at that time. Psychologist Rogelio Díaz Guerrero states that a man's virility can be proven "when he convincingly affirms or demonstrates that he is unafraid in the face of death." 22 Literary critic Juan M. Lope Blanch claims that "there is in Mexico a true obsession with death" and that "Man, the true 'macho', should fear nothing, not even death. Or at least overcome his natural fear, giving evidence of his boldness [arrojo]."23 Anthropologist Luis Alberto Vargas writes that

The Mexican of today continues to be anguished by the prospect of death, the same as all humanity, but in contrast to other peoples, he does not hide behind death, but rather lives with it, makes it the object of jokes and tries to forget it by transforming it into something familiar. ${ }^{24}$

Folklorist Gabriel Moedano says that "although the cult of death is a trait that appears among every people, there is nowhere in the world where it exists so rootedly and with so many profound manifestations as in Mexico." ${ }^{25}$ Finally, a Universidad Nacional Autónoma de México museum catalogue published in the mid-1970s states that for Mexicans death is "Triumphant or scorned, venerated or feared, but always and at every moment, walking inevitably alongside us." 26

Scholars working outside Mexico have reinforced these lugubrious impressions. Patricia Fernández Kelly sees the Mexican view of death as "bitter gaiety that philosophically recognizes ... that the definitive character of death can only be successfully confronted with gestures of indifference and scorn." 27 Barbara Brodman devotes an entire book to what she calls "the Mexican cult of death" and believes that "A much noted psychological manifestation of this cult is the Mexican's strikingly fatalistic and stoic nature." ${ }^{28}$ Judith Strupp Green writes that "the Mexican Indian seems to be on ... familiar, accepting, even humorous terms with death." 29 And Douglas Day makes the almost shocking statement that "In ways that frequently startle the foreigner, death seems to lack meaning to the Mexican. It comes easily, matters little." ${ }^{30}$ In case after case, there emerges a familiar cultural construction: Mexicans fail to distinguish life and death and seem unperturbed by death, while western Europeans are squeamish before the reality of death, repulsed by the idea of death, and differentiate markedly between life and death.

These longstanding ideas persist in the Mexican national press. Consider a report on the Day of the Dead published in the mid-1990s in Excelsior:

It cannot be denied that this festival involves the typical idiosyncratic feeling of the Mexican, which causes him to joke at death, co-exist with it, look at it straight on, an attitude opposite that of other peoples, such as Europeans, who regard death with profound respect and treat death with serenity. ${ }^{31}$

A recent photograph published in El Universal bears a caption referring to "the ludic spirit with which the Mexican confronts death." 32 A year later, a Mexican journalist reported that his compatriots address death by the pronoun $t u$, thereby establishing a familiar relationship with death. ${ }^{33}$ 
At the risk of oversimplifying, we might list the recurrent themes thus:

(1) Mexicans are obsessed with death;

(2) Mexicans fear death;

(3) Mexicans do not fear death;

(4) Mexicans are stoic in the face of death;

(5) Mexicans defy the prospect of death in order to appear manly;

(6) Mexicans are fond of, and even crave, death;

(7) Mexicans play with death;

(8) Mexicans are surrounded by and live side by side with death;

(9) Mexicans express a fatalistic acceptance of death;

(10) Mexicans perceive life and death as indivisible.

Presented in this kind of blunt, condensed form, the Mexican view of death appears highly contradictory. It is pointless to try to resolve the contradictions, however, for authors, with few exceptions, fail systematically to defend their points of view. Rather than take the list of claims at face value, as representing the Mexican state of mind, we would do well to interpret it as a construction of national character, as a projection of views about Self and Other.

\section{Intercultural variation}

It has long been recognized that people construct collective identities through differentiating their own group from others. Not all aspects of culture enter into this differentiation. ${ }^{34}$ For historical and cultural reasons, particular elements become especially salient identity markers. Applying this perspective to Mexico, we might say that over time the meaning of death and the nature of mortuary rituals have become central to the construction of what it means to be Mexican. In a multicultural context, such as that which prevails on the North American continent today, national identity is shaped by commonly accepted binary oppositions. Contrasting views of death are just one among many ways in which we distinguish Mexicans from Anglos.

And yet, a number of mortuary customs in the United States appear to challenge this point of view. It is sufficient to recall colonial New England tombstones, with their prominent skull. ${ }^{35}$ By contrast, skulls almost never appear on Mexican tombs, past or present. In the US today, the Vietnam War Memorial, with its seamless list of fallen heroes, functions as a potent national symbol. The emotional power of the memorial underscores the important role of death in this country. In fact, as Benedict Anderson says, throughout the Western world "No more arresting emblems of the modern culture of nationalism exist than cenotaphs and tombs of Unknown Soldiers"; "void as these tombs are of identifiable mortal remains or immortal souls," he continues, "they are nonetheless saturated with ghostly national imaginings." 36 Thanks to best-selling author Jessica Mitford, Americans have become aware of the enormous expense and extravagance of their funerals. The American way of death, as she calls it, belies the view that people in the United States hide from death and do everything possible to deny its existence. ${ }^{37}$ Finally, the hospice movement, growing in popularity throughout the United States, provides evidence of a resignation to and direct confrontation with the reality of death. This 
movement suggests at least as much acceptance of death as prevails in Mexico. Perhaps Mexico and the West are not so divergent after all.

There are certainly few Mexicans who actually yearn to die, who face illness and death with rigid, fatalistic stoicism, and who fail to distinguish life from death. At the same time, there are a good number of Europeans who do opt to die (suicides are statistically more common there than in Mexico) and who face illness and death stoically. Just consider the growing support in the United States for euthanasia. And the current popularity of New Age and Asian philosophies has no doubt given many Anglos the perception that death is a mere extension of life. In other words, even if we assume that the stereotypes of Mexican views of death are accurate, it is likely that numerous AngloEuropeans share these views. Scholars and critics would benefit from reading the anthropological literature on mortuary beliefs and ceremonials. Mexican and AngloEuropean perspectives on death positively pale when compared with the obsessive concerns about mortality found among the peoples of Indonesia, ${ }^{38}$ Madagascar, ${ }^{39}$ and other cultures remote from North America. ${ }^{40}$

It is clear as well that Mexicans constitute a highly complex society. In their attitudes toward death, they vary not only individually but also by class, ethnicity, and region. Just consider what Robert Laughlin says of the Tzotzil Indians in Chiapas:

Death, "the end," is not accepted impassively by the Tzotzil but is always a sudden surprise that shakes every man's uneasy security. Generally it is deemed premeditated murder. ... No matter how solicitously the lifeless body is equipped for its journey, to the afterworld the dread finality of death is a constant concern. $^{41}$

The Tzotzil, at least by this account are hardly stoic in the face of death; they neither crave death nor feel comfortable with it. Nor do their indigenous neighbors in Chiapas, the Tzeltal. Eugenio Maurer Avalos states,

In contrast with Western Christianity, Tzeltal traditional religion does not regard death as a step to a better life, but rather as a point at which "it's all over." This matter-of-fact view of death as an inevitable reality underscores the premise that true happiness is to be found in this life, here on earth. ${ }^{42}$

This author underscores this point even more forcefully when discussing Tzeltal avoidance of Extreme Unction:

The resistance of Indians and others to this custom derives from the premise that this sacrament allowed the dying person to die "with grace," or to "die well." The very idea of "dying with grace" is antithetical to Tzeltal spiritual premise that the only true life worth living is here on earth and in this life. The point of being is to "live well in harmony," not to "die well." To die is in fact the ultimate disjunction with harmony. ${ }^{43}$

It would be difficult to encounter an approach to death more at odds with the Mexican stereotype than this one. We need not invoke marginal Indian groups, however, to challenge the stereotype. James Lockhart, speaking of the Nahuas themselves during the 
colonial era, states that there is little evidence among them of a "concern with salvation and the afterlife." He continues, "The entire set of funeral rites at once defined the individual's relation to his surroundings, above all his social surroundings, and by their final seal allowed his relatives and fellow citizens to adopt a different stance toward him."44

Although cases like these demonstrate the futility of generalizations, the stereotypical portrait of Mexican attitudes toward death is in fact both accurate and inaccurate: accurate in the sense that it describes at least some of the true feelings of at least a portion of the Mexican people, inaccurate in that the portrait is a one-sided exaggeration that fails to take into account the full range of emotions that every human being experiences when confronted by death. What is lacking in the discussions of Mexican attitudes toward death is the recognition of ambivalence or uncertainty. When Brodman refers to "The Mexican's attitude toward death, his indifference toward it as well as his fascination with it," 45 she describes feelings that are virtually universal. Who, we might ask, is not sometimes indifferent towards death and at other times fascinated by it? To be sure, there are societies that have elaborated death rituals to the point where they occupy an enormous amount of time, energy, and resources. But can we be so sure that, if we could quantify Mexican investment in death-related thought and behavior, the result would differ from a similar survey carried out, say, in Italy, Japan, Kenya, Israel, or Peru?

True, Anglo-Europeans spend a good deal of time, energy, and money trying to prolong their life span, but, when they can afford it, so do Mexicans. Studies in medical anthropology bear abundant witness to the fact that Mexicans yearn to preserve health and prolong life. Mexicans, when they fall ill, are no more willing to throw in the towel than anyone else. Experience shows that when they command the resources to combat illness and stave off death, those resources will be summoned. How, then, can writer Edna Ferguson have claimed that "Mexico is probably the only country in the world where a person may look forward with some pleasure to his own death, his own funeral, his own grave"? ${ }^{46}$ Or Edward Tinker have written long ago that "the Mexican mind accepts death with philosophy, even nonchalance"? ${ }^{47}$

As for the question of whether Mexicans do or do not fear death, I know of no systematic treatment of the issue. However, logic dictates that Mexicans are as varied in their attitudes as are peoples everywhere: some fear death, others do not. Much is made in the literature of a Mexican playfulness and jocularity in the face of death. This quality indeed stands out at times, notably during the Day of the Dead. However, the symbolic meaning and psychological consequences of this ludic attitude have yet to be deciphered. It would seem to indicate a direct confrontation with death, a psychological escape from death, or some combination of the two. Surely, Mexicans are like everyone else in expressing an ambivalent response to death, a simultaneous fascination with and repugnance for it. What differs from society to society is not the existence of mixed feelings but rather the cultural means through which these feelings are projected.

\section{The origins of Mexican attitudes toward death}

If we examine the origins of Mexican attitudes toward death, authors seem to be divided into two incompatible camps. On the one hand, there exists a prevalent belief that views of death are an inheritance from the Indian past. This perspective is inherent in Paz's Labyrinth, for example, with its persistent references to ancient Aztec philosophy. Frances 
Toor, whose Treasury of Mexican Folkways introduced thousands of foreign readers to Mexican popular culture, writes that

The Mexicans, fatalists that they are, accept death uncomplainingly but also bravely. They fraternize, play, joke with death even while they weep. In their blood is the spirit of adventure of their conquerors. ... Also in them is the blood of their ancestors, who met death of their own volition for the sake of their gods. ${ }^{48}$

More recently, another commentator has stated that

The rites of the ancient Mexicans expressed a vision of death, heroic and sacrificial, as a source of renovation, fertility, and transcendence. Later, Christian sentiment which was imposed during the colonial era made of death a permanent dance of death [danza macabra]: fear and craving for the final judgment. Both concepts fused in the synthesis of the Mexican; the contemporary popular display is expressed in a syncretism in which ostensible fear has been lost and death is no longer restorative of life but rather a familiar and everyday part of life. ${ }^{49}$

Pre-columbian culture patterns, reinforced by the alleged Spanish penchant for morbidity, are usually blamed for the almost inhuman stoicism attributed to modern Mexicans.

But can personality traits be transmitted rigidly throughout millennia, despite cataclysmic upheavals in politics, economics, culture, and religion? Obviously not. The only possible explanation for such cultural tenacity is biological, the persistence of what we might call the eternally morbid Mexican. For a nation as culturally rich and historically cataclysmic as Mexico, it is impossible to accept an interpretation of national character that ignores variation through space and time. There is, however, one proposition that lends itself to critical examination. I speak of the belief that, when death is a common occurrence, a daily reality, people become callous in the face of death, possibly even morbidly attracted to death. Where survival cannot be taken for granted, people are likely to treat death with nonchalance and familiarity. Nancy Scheper-Hughes advances this thesis in her study of the Brazilian northeast, where high infant mortality rates have apparently hardened mothers against pain and outward expressions of loss. Death Without Weeping is her title, designed to describe the typical maternal reaction upon the death of their children. ${ }^{50}$ Likewise, Patricia Fernández Kelly states that, "Death is a permanent concern, a daily presence, especially in a country like Mexico in which problems ranging from the difficulty of providing medical services to the persistence of ignorance and oppression accentuate its meaning." Hence the "indifference and scorn" which she attributes to Mexicans who are faced with death. ${ }^{51}$ Here at last is a testable scenario: that is, the constant confrontation with death inures people against feelings of sadness and loss.

As a corollary to this thesis, we might hypothesize that when death becomes less prevalent, the cultural and emotional involvement with death declines as well. Certainly, Mexican statistics, at least throughout the twentieth century, show a precipitous drop in mortality rates. ${ }^{52}$ Varying between 46.6 and 48.3 per thousand per year, crude death rates were understandably highest during the Mexican Revolution (1910-20), a decade of widespread bloodshed. Aside from this anomaly, death rates have declined steadily from 34.4 in the period $1895-99$ to 12.5 in $1955-59 .{ }^{53}$ By $1975-80$, the mortality rate had 
gone as low as 7.3; in 1985-90, it was down to 5.9, and between 1990 and 1995 it declined to $5.5 .^{54}$ The decline of infant mortality rates has been equally dramatic. ${ }^{55}$ Again, excepting the revolutionary years, which experienced somewhat greater infant mortality than had existed previously, the average rates declined steadily from 226 in the late 1890 s to 78 in $1955-59$. By the early 1970 s, this figure had been reduced to 68.4 and in the late 1980s dropped dramatically to 41.3. Infant mortality rates for Mexico in 1990-95 were at an all-time low of 35.2. ${ }^{56}$

Another significant indication of mortality trends in Mexico is life expectancy at birth (itself a function of crude death rates and infant mortality rates). Here, again, Mexicans have experienced marked improvement over the course of the twentieth century. ${ }^{57}$ In 1930, overall life expectancy at birth was 36.8. By 1950, it had increased to nearly 50 . And by 1990-95, it had risen to 70.3, nearly double the figure six decades before. ${ }^{58}$ Hence, the relevant indices - aggregate mortality rates, infant mortality rates, and life expectancy at birth - all demonstrate that death has touched Mexicans to a lesser and lesser degree over the course of the past half century or more. The precipitous drop in mortality rates and corresponding increase in life expectancy over the course of the twentieth century should have had some effect on Mexican attitudes toward death. And yet, judging from the literature, attitudes have remained immutable, not only during the twentieth century but also over the course of centuries, even millennia.

Of course, they have not remained immutable, nor are they at all times and places the same. Even within the same community, people approach death in contextually specific ways. Let us return for a moment to the burial of the Tzintzuntzeño who was struck by lightning in June 1967. The wake was solemn and subdued. As I entered the home of the deceased, an occupant asked that I respectfully take off my hat. Each visitor carried a candle for the deceased. By the time I arrived, dozens of candles illuminated the casket and cadaver. The single-roomed house was crowded with mourners. Because that part of town lacked electricity, pools of candlelight fell on the crestfallen faces of the relatives, neighbors, and compadres in attendance. A villager led the mourners in reciting responsive prayers. Although there was no wailing, women wept silently, and the atmosphere was heavy with sadness. Well into the night, the victim's family served atole, which warmed us against the cold damp air and seemed to raise collective spirits temporarily. The next morning, four men carried the casket about a kilometer's distance to the cemetery. As soon as I shot a few miserable photographs, the casket was lowered into the grave. Everyone appeared tormented by the cruel fate of a healthy man in the prime of life suddenly being snatched from their midst.

The same cemetery during the Day of the Dead could not present a greater contrast. Tzintzuntzan is one of eleven towns in the Tarascan area that the government of the state of Michoacán selected in the late 1970s for touristic promotion. From that time on, November 1 and 2 has been celebrated in Tzintzuntzan and elsewhere in the Lake Patzcuaro region with an exuberance that would have been unrecognizable only a generation before. Floodlights illuminate large parts of the cemetery for the benefit of television cameras. The highway that runs through town suffers among the worst traffic jams that I have ever experienced. Middle-class visitors from Guadalajara, Mexico City, and Morelia stomp through the graveyard while munching on snacks, snapping pictures, and listening to portable radios. At considerable expense, they attend staged performances of the classic Spanish drama, "Don Juan Tenorio." For a hefty fee, they can also view a presentation of pirekuas, allegedly traditional Tarascan dances, performed on an esplanade in front of five yácatas, the famous circular bases of Tarascan temples. Citizens of Tzintzuntzan who maintain vigil over the tombs of 
their ancestors seem utterly unshaken by an atmosphere that many outsiders would consider inappropriately chaotic, noisy, even joyful. Late one night on November 1, I asked an elderly friend who was watching over his family's grave if the crowds were bothersome. "Not at all," he protested. "It's so much better now than it used to be. Estaba tan triste - It used to be so sad." In Tzintzuntzan, as anywhere in the world, the people's attitudes towards death can hardly be summarized by a few facile adjectives. Attitudes are situational. ${ }^{59}$

Despite a long, rich ethnographic tradition in Mexico, there exist very few detailed accounts of wakes and funerals. Those that do exist, however, paint a very different picture than that portrayed by national stereotypes. Consider Ralph Beals' description from the 1940s of the Tarascan village of Cherán. Following a funeral, he says,

the parents of the surviving spouse may remain very sad. If it appears they may be made ill of "sadness," the relatives visit the survivors frequently, talking to them of things which they think will alleviate their condition and giving consolation. If this does not improve matters, they seek a curer who gives medicines. ${ }^{60}$

When a Tzotzil of Chiapas dies, says George Collier, "the first order of business is the funeral." "Family members," he says, are "grief stricken." "61 William Madsen describes a typical wake for adults in Milpa Alta, just south of Mexico City:

Male visitors sit on board benches put up against the walls; the women sit on the floor on straw mats.... When thirty or forty guests have arrived, the prayermaker kneels at the head of the coffin and the dead man's widow sits at the foot of it. The prayermaker leads a rosary chant and the people say responses. ... Weeping in grief, the dead man's wife and children clap their hands loudly. As the clapping dies down, the prayermaker sings a final farewell to the dead man. Guests rush up to embrace the family and beseech them not to weep so much. ${ }^{62}$

Miguel Covarrubias observed the death of one of his friends in Juchitán. As she died, she sighed deeply, which "was regarded as a sign that the soul had left her body." rubias states,

A violent reaction shook the members of the household, strangely calm and collected before, particularly the women, her daughters and sisters, who gave vent to wild outbursts of despair and screams of "jewel of my heart!", "my little mother!" ... Soon the neighbors and distant relatives came to the house to embrace and sympathize with the mourners, as well as to deposit their alms. ${ }^{64}$

At the burial, says Covarrubias, "it is the custom for the nearest women relatives of the deceased to give a final and most violent display of despair." On the particular occasion which Covarrubias witnessed, "one of the old lady's daughters had to be restrained from throwing herself into the open grave." 65

Even this brief review is sufficient to demonstrate that all over the Mexican Republic - whether in Michoacán, Tehuantepec, or the State of Mexico-mourners react to the death of loved ones in a manner that defies national stereotypes. In none of these descriptions can we detect evidence of stoicism, humor, playfulness, and disdain in the face of death. It is true that alcoholic drink is an integral part of many wakes and that 
live music often accompanies funeral processions and burials. True, too, there exists a certain lightheartedness at funerals honoring small children - angelitos - who are said to go directly to heaven without having to pass through Purgatory. And yet, in my experience, the lightheartedness is only relative, as compared with the mood at funerals for adults.

In the 1930s, Elsie Clews Parsons described the funeral of an angelito in the Zapotec village of Mitla. The night of the death, the wake was accompanied by lively music and dancing. Parsons explains, "The theory is that unless people are gay the 'little angel' will not go directly to God." And yet, she continues,

the next afternoon I find the wake mournful enough. It is much like that of an adult except that more children are present, there are fewer candles, and the corpse lies on a table alongside the altar. ... All the children present and a few women crowd around the infant, crying. ... A few fireworks are set off behind, and a gay march is played, which to one at least makes the little procession all the sadder. ${ }^{66}$

Throughout Mexico, the mood is similar to that experienced by Parsons. Joviality and gaiety at a child's funeral exist more in people's minds, in their descriptions of events, than in their actual behavior. Those ethnographers who bother to register how mourners react emotionally to a death - be it of a child or an adult - all convey the profound, overt grief experienced by survivors. Moreover, this grief is not limited to particular ethnic groups. Tarascans, Zapotecs, Nahuas, Indians, and mestizos alike, share this fundamental human response.

Observers who insist that Mexicans have a unique relationship to death conveniently overlook the emotional texture of funerals. And yet their viewpoint is not entirely without foundation. Mexicans, in fact, sometimes do seem to disdain death, play with death, and confront it directly while still holding it at a distance. It is just that these attitudes do not predominate. Rather, they are largely confined to a particular time of year: the Day of the Dead.

The Day of the Dead is without doubt Mexico's most famous holiday. And, in my opinion, it is the popularity and commercial promotion of the Day of the Dead that is largely responsible for creating the essentialist image of a macabre Mexican. Each year, at the end of October, large numbers of foreign visitors descend upon Mexico to witness colorful - some would say carnivalesque - ritual performances and artistic displays. Decorated breads, paper cutouts, and plastic toys, most of them humorous plays on the death theme, are evident everywhere. Whimsically sculpted toys and candies made of sugar, amaranth seed dough (alfeñique), and papier-maché are prominently displayed for sale at parks, plazas, and stores all over the Republic. Taking the form of skulls, skeletons, and caskets (some with cadavers that resuscitate at the tug of a short string), these sculptures suggest an almost irreverent, even impudent, confrontation with mortality. Toys and figurines, both edible and not, are ubiquitous during the Day of the Dead. They adorn every imaginable public space, from tombstones and supermarkets to openair markets and storefront windows.

It is not only in the plastic arts, however, that the Day of the Dead demonstrates a ludic quality. Friends and relatives sometimes present one another with short poetic epitaphs, known as calaveras - "skulls." These poems mock the shortcomings of their victims. Imbued with a mixture of aggression and humor, the epitaphs almost always serve as evidence of 
close social bonds. They are a classic component of what anthropologists call a joking relationship.

During this time of year, too, newspapers and broadsides fill their pages with calaveras. Usually composed in rhymed verse, often in quatrains, these satirical epitaphs victimize public figures-politicians, athletes, literati, and movie and television personalities, among others. Formerly, the authors of these verses were always anonymous, thereby enhancing the impression that the calaveras represented a distillation of public opinionthat they were the collective voice of the Mexican people. Although most calaveras are still anonymous, newspapers in recent years have sponsored calavera contests. Readers contribute calaveras and the winners are published with a byline. One recent winner provided the ultimate calavera: a meta-calavera entitled, "A las calaveras" (To the Calaveras). ${ }^{67}$

Noviembre siempre es el mes

Ya conocido y no torio

Cuando se habla a la vez

De calacas y el tenorio.

Pues son nueskras tradictones

El escribir calaveras

Donde hay muertos de a montones

Mas nadie muere de veras.

Rescatar nuestras costumbres

Esto cumple estos fines

Hablar de cielo y de lumbres

$\Upsilon$ déjense de "jalogüines".

Es broma, lector, lectora,

No hay agravios encubiertos

$\Upsilon$ no le carguen los muertos

A quien escribe ahora.

Si acaso esta ocasión

Con tus huesos aqui asomas

Has de disculpar las bromas:

¡Calaveras, bromas son!

November is always the month

Already known and notorious

When skeletons and el tenorio

Are spoken of simultaneously.

Well, it's our tradition

To write calaveras

In which there are mountains of dead people

But really no one dies. 
To rescue our customs

That satisfies these goals

To speak of heaven and light

And leave behind "Halloweens."

It's a joke, readers,

There are no hidden insults

And let the deceased not denounce

The present writer.

For it's the case that on this occasion

With your bones showing

You have to forgive the jokes:

Calaveras, they are jokes!

\section{Maestra Hortensia Galindo Garrido \\ Colonia Lindavista (México, DF)}

The author of this brilliant epitaph captures the essence of not only calaveras as a literary form, but also the Day of the Dead as a holiday of national import. She speaks of the Day of the Dead as a famous holiday, a traditional holiday, with humorous calaveras being an integral part of the celebration. At the same time, she reminds readers that Day of the Dead traditions stand in opposition to Halloween and that they need rescuing. Calaveras, says the author, are part of "our traditions," "our customs." They are Mexican, and, moreover, a symbol of Mexican national identity. The calavera tradition in the plastic arts, of course, has enjoyed a long history, combining pre-Columbian and colonial European representations of death. But, except for the sale of toys and figurines to tourists, it is principally during the Day of the Dead that this artistic tradition flourishes. No Mexican adorns a grave with candy skulls or presents a calavera to a friend when a family member dies and is buried.

The Day of the Dead is, of course, the Mexican version of the pan-Roman Catholic celebration of All Saints and All Souls Days. The only official Church requirements for the celebration of this holiday are special Masses. Everything else that occurs from October 31 through November 2 is a folk elaboration. The Day of the Dead shares numerous elements with the same event as found throughout much of Latin America and, in fact, the Mediterranean world from which it basically derives. In Mexico, unlike elsewhere, however, the holiday has acquired a special name - the Day of the Dead, or the Days of the Dead - and with it a unique identity.

For reasons both historical and cultural, the Day of the Dead has become much more salient in Mexico than anywhere else. It has developed traits that have become intimately associated with Mexico, particularly calaveras in their literary and plastic forms as well as the pervasiveness of humor, music, and a ludic spirit. ${ }^{68}$ The Day of the Dead, in fact, has become an international symbol of Mexico, promoted by intellectuals, journalists, government officials, and even influential Mexicans. Town governments award prizes for the best altar and gravestone decoration. In these contests, the presence of so-called "foreign elements," like jack o'lanterns and witches riding on broomsticks, immediately disqualify the contestants. ${ }^{69}$ I agree entirely with anthropologist Juan Luis Ramírez Torres, who puts the matter thus: 
It is commonly said that the Mexican laughs at death, a strange saying designed for touristic appeal, but which does not correspond exactly to cultural expressions found among the Mexican people. ... Among the contemporary ways of rendering tribute to those who have preceded us in life ... the most "authentic and pure" customs ... are sought; for this reason, in schools and government offices there is installed an ofrenda striving to be the "most faithful possible" to that pursued purity. Those in charge of erecting [the ofrenda] search in libraries, interview specialists on the matter, investigate, that is, look for identities, primordial origins - the first genes - and, once this collection of material is gathered, they set up floral arrangements, pile platters with food, pour pulgue, burn copal, evoque ancestry. ${ }^{70}$

In recent years, the Archbishop of Coahuila has done his part to add to this campaign by prohibiting the celebration of Halloween, which he interprets as an entirely pagan, commercial holiday. All of these efforts, of course, attract tourists to the Day of the Dead. They present urban, middle-class Mexicans with a vision of their supposed past, they offer artisans unparalleled opportunities for profit, and they allow foreigners a lowcost window into exotic customs and beliefs.

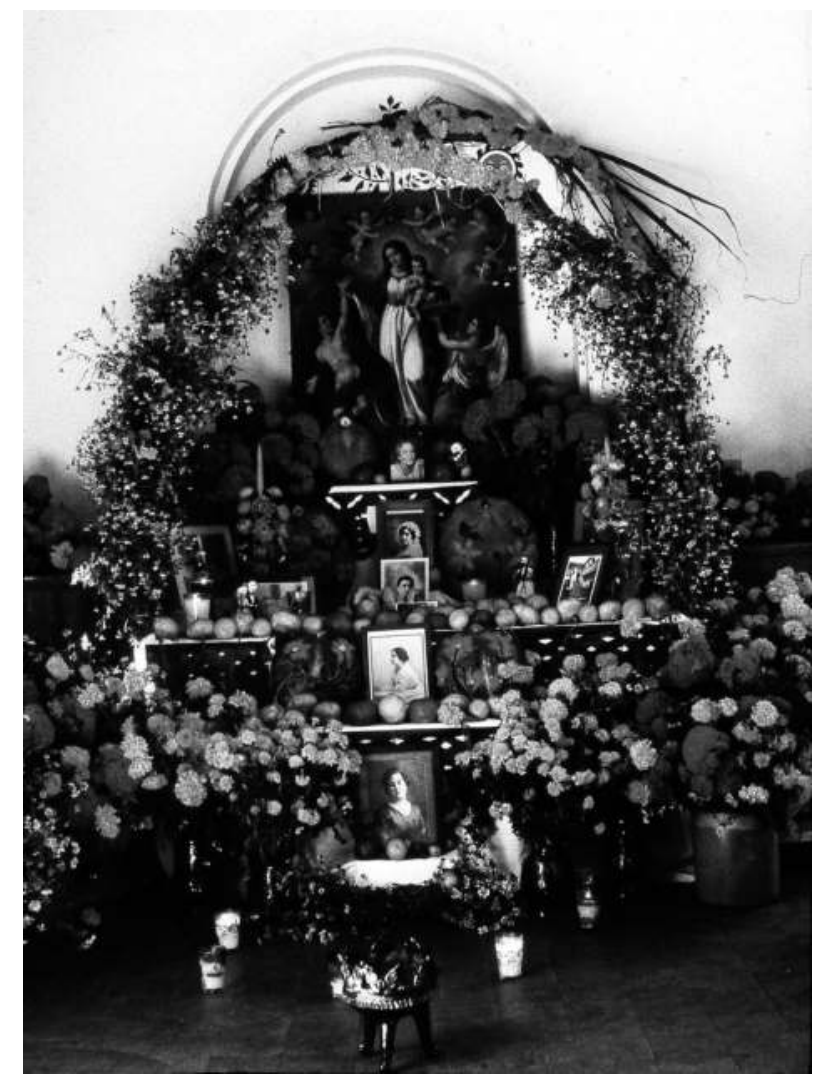

Image 13.1 Day of the Dead altar, Oaxaca.

(Photograph by the author) 
Although the popularization of the Day of the Dead has increased in velocity and intensity since 1994, when NAFTA (North American Free Trade Agreement) went into effect, the holiday began to expand much earlier. Malcolm Lowry's Under the Volcano ${ }^{71}$ in both literary and cinematic forms, probably did as much as Octavio Paz's The Labyrinth of Solitude to make this celebration famous. When the world thinks of the Mexican view of death, it thinks of the Day of the Dead. What is more, many Mexicans themselves believe that they are unique. In fact, neither the direct confrontation with mortality that I witnessed at the funeral graveside in 1967 nor the humor and banter which characterize the Day of the Dead are restricted to Mexico. Humor is a common component of death rituals cross-culturally ${ }^{72}$ and the graveside behavior of poor people in many countries reflects the extreme fragility of life that for them is a given. The image of the morbid, death-obsessed Mexican, as promoted through the Day of the Dead, has become integral to Mexican national identity. It is symbolic of Mexicanness, particularly in the increasingly complex dialogue between Mexico and the Anglo United States. Alleged Mexican attitudes towards death are cultural capital. They are among the most effective ways to create and maintain ethnic and national boundaries in an era of globalization, when boundaries are being broken as never before. They help to define Self and Other - and, as has long been true, to sell a good many candles, flowers, candy figurines, newspapers, and miniature calaveras as well.

\section{Notes}

1 This chapter represents an updated and expanded version of an article originally published in the journal Ethos, volume 31 (2003). An earlier version of this paper was delivered on April 4, 2000 at the Center for US-Mexican Studies, University of California, San Diego. I wish to thank the Center for US-Mexican Studies, University of California, San Diego for originally providing me the facilities and time necessary to analyze and write this material. Financial support for the research upon which this paper is based came from the Center for US-Mexican Studies at the University of California, San Diego; the Committee on Research, University of California, Berkeley; the National Endowment for the Humanities; the Center for Latin American Studies, University of California, Berkeley; and the John Carter Brown Library. I am grateful to these agencies for their generous assistance. All translations from Spanish are my own, except that appearing in Octavio Paz's Labyrinth of Solitude.

2 Not all observers are in accord with the predominant views. Elizabeth Carmichael and Chlöe Sayer, for example, state: "There are many who dissent from the view that the special relationship [between Mexicans and death] exists." Skeleton at the Feast: The Day of the Dead in Mexico (Austin, TX: University of Texas Press, 1992). Scholars such as Carlos Navarrete scrupulously seek to avoid being drawn into what he sees as the undisciplined morass of description and the "long list of generalizations which have been written on the theme of Death in Mexico."

3 Ross Hassig, Imperial Expansion and Political Control (Norman: University of Oklahoma Press, 1988), 206, and War and Society in Ancient Mesoamerica (Berkeley, CA: University of California Press, 1992), 112.

4 Nigel Davies, The Toltecs Under the Fall of Tula (Norman: University of Oklahoma Press, 1977).

5 Richard A. Diehl, Tula: The Toltec Capital of Ancient America (London: Thames and Hudson, 1985), 149.

6 Diehl, Tula, 64.

7 Jeanette Favrot Peterson, The Paradise Garden Murals of Malinalco: Utopia and Empire in SixteenthCentury Mexico (Austin, TX: University of Texas Press, 1993).

8 Gustavo Curiel Méndez, "Aproximación a la iconografía de un programa escatológico franciscano del siglo XVI," In Arte Funerario, vol. 1 (Louise Noelle, ed. México: Universidad Nacional Autónoma de México, 1987), 156. 
9 Museo Nacional de Arte, Juegos de Ingenio y Agudeza: la Pintura Embelmatica de Nueva Espana, (Mexico: Patronato del Museo Macional de Arte, 1994), 254-255.

10 Ibid., 256-263.

11 Peter Wollen, "Introduction," In Posada: Messenger of Mortality, (Julian Rothenstein, ed. Boston, MA: Redstone Press, 1989), 14-15.

12 Robert V. Childs and Patricia B. Altman, Vive tu Recuerdo: Living Traditions in the Mexican Days of the Dead (Los Angeles, CA: Museum of Cultural History, University of California, Los Angeles, 1982), 56.

13 Wollen, "Introduction," 14.

14 Carlos Macazaga, César Macazaga Ordoño, and Ramírez de Arellano, eds. Posada y las Calaveras Vivientes, (México: Editorial Innovación, 1977), 21.

15 Wollen, "Introduction," 16.

16 Liza Bakewell, "1985 Bellas Artes y Artes Populares: The Implications of Difference in the Mexico City Art World," in Looking High and Looking Low: Art and Culture Identity, Brenda Jo Bright and Liza Bakewell, eds. (Tucson: University of Arizona Press, 1995), 31.

17 Childs and Altman, Vive tu Recuerdo, 58-59.

18 Claudio Lomnitz, Death and the Idea of Mexico (New York: Zone, 2005), 35-41.

19 Andrew Chesnut, Devoted to Death: Santa Muerte, the Skeleton Saint (Oxford: Oxford University Press, 2012) and R.A. Kristensen, "La Santa Muerte in Mexico City: the Cult and its Ambiguities," Journal of Latin American Studies 47 (2015), 543-566.

20 Lomnitz, Death, 490.

21 Octavio Paz, The Labyrinth of Solitude: Life and Thought in Mexico, tr. Lysander Kemp (New York: Grove, 1961), 57-58.

22 Rogelio Díaz Guerrero, Estudiosde psicología del mexicano (México: F. Trillas, 1968), 15.

23 Juan M. Lope Blanch, Vocabulario mexicano relativo a la muerte (Mexico: Centro de Estudios Literarios, Universidad Nacional Autónoma de México, 1963), 8.

24 Alberto Luis G. Vargas, "La muerte vista por el mexicano de hoy," Artes de Mexico 145 (1971), 57.

25 Gabriel Moedano Navarro, "La ofrenda del Día de los Muertos," Folklore Americano (Lima: Organo del Comité Interamericano de Folklore, 1960-61), 32.

26 Anonymous, "Introducción," in La Muerte: expressions mexicanas de un enigma (México: Museo Universitario, Universidad Nacional Autónoma de México, 1974-75), 1.

27 Patricia Fernández Kelly, "Death in Mexican Folk Culture," American Quarterly 25 (1974), 533.

28 Barbara Brodman, The Mexican Cult of Death in Myth and Literature (Gainesville, FL: University Presses of Florida, 1976), 40.

29 Judith Strupp Green, Laughing Souls: The Days of the Dead in Oaxaca, Mexico (San Diego, CA: San Diego Museum of Man, 1969), 1.

30 Douglas Day, “A Day with the Dead," Natural History (1990), 67.

31 Excelsior, October 25, 1995

32 El Universal, October 31, 1995

33 Excelsior, October 27, 1996

34 Fredrik Barth, ed., Ethnic Groups and Boundaries (Boston, MA: Little Brown, 1969), 9-38, and William Graham Sumner, Folkways: A Study of the Sociological Importance of Usages, Manners, Customs, Mores, and Morals (Boston, MA: Ginn, 1906), 1-74.

35 James Deetz, In Small Things Forgotten: The Archaeology of Early American Life (Garden City, NY: Doubleday, 1977), 64-91.

36 Benedict Anderson, Imagined Communities: Reflections on the Origin and Spread of Nationalism (London: Verso, 1983), 17.

37 Jessica Mitford, The American Way of Death (New York: Simon and Schuster, 1963).

38 Robert Hertz, Death and the Left Hand (Aberdeen: Cohen and West, 1960 [1907]).

39 Maurice Bloch, Placing the Dead: Tombs, Ancestral Villages, and Kinship Organization in Madagascar (New York: Seminar Press, 1971).

40 For numerous examples and a good bibliography of the topic: see Richard Huntington and Peter Metcalf, Celebrations of Death: The Anthropology of Mortuary Ritual (Cambridge: Cambridge University Press, 1991).

41 Robert M. Laughlin, "The Tzotzil," in Handbook of Middle American Indians, vol. 7 (Evon Vogt, ed., Austin, TX: University of Texas Press, 1969), 191. 
42 Eugenio Maurer Avalos, "The Tzeltal May-Christian Synthesis," in South and Meso-American Native Spirituality: From the Cult of the Feathered Serpent to the Theology of Liberation (Gary Gossen and Miguel León-Portilla, eds., New York: Crossword, 1993).

43 Ibid., 244.

44 James Lockhart, The Nahuas After the Conquest: A Social and Cultural History of the Indians of Central Mexico, Sixteenth through Eighteenth Centuries (Stanford, NJ: Stanford University Press, 1992), 255.

45 Brodman, Cult, 39.

46 Edna Fergusson, Fiesta in Mexico (New York: Knopf, 1934), 199.

47 Edward Larocque Tinker, Corridos and Calaveras (Austin, TX: University of Texas Press, 1961), 20

48 Frances Toor, A Treasury of Mexican Folkways (New York: Crown, 1947), 236.

49 Anonymous, "Introducción," 1.

50 Nancy Scheper-Hughes, Death Without Weeping: The Violence of Everyday Life in Brazil (Berkeley, CA: University of California Press, 1992).

51 Fernández Kelly, "Death," 531.

52 José R. Narro et al., "Evolución reciente de la mortalidad en México," Comercio Exterior (1984), 636-646.

53 B.R. Mitchell, International Historical Statistics, the Americas, 1750-1988 (New York: Stockton Press, 1993), 81.

54 United Nations, Anuario Estadístico de América Latina y el Caribe (United Nations Economic Commission for Latin America and the Caribbean, 1993). All statistical figures in the text represent the average annual rates per 1,000 live births.

55 Narro et al., "Evolución," 636-646.

56 United Nations Economic Commission for Latin America and the Caribbean, (Santiago de Chile: United Nations Economic Commision for Latin America and the Caribbean, 1993), 14.

57 Narro et al., "Evolución," 636-646.

58 United Nations Economic Commission for Latin America and the Caribbean, Statistical Yearbook, 14 .

59 Stanley Brandes, "The Day of the Dead, Halloween, and the Quest for Mexican National Identity," Fournal of Amercian Folklore 111 (442) (1998), 359-380.

60 Ralph Beals, Cheran: A Sierra Tarascan Village (Washington, DC: Smithsonian Institution Institute of Social Anthropology, 1946), 208-209.

61 George Collier, Fields of the Tzotzil: The Ecological Basis of Tradition in Highland Chiapas (Austin, TX: University of Texas Press, 1975), 90.

62 William Madsen, The Virgin's Children: Life in an Aztec Village Today (Austin, TX: University of Texas Press, 1960), 209-210.

63 Miguel Covarrubias, Mexico South: The Isthmus of Tehuantepec (New York: Alfred Knopf, 1954), 391.

64 Ibid., 391.

65 Ibid., 393.

66 Elsie Clews Parsons, Mitla: Town of the Souls, (Chicago: University of Chicago Press, 1970 [1936]), 148-149.

67 Reforma, November 1, 2000.

68 Stanley Brandes, "Sugar, Colonialism, and Death: On the Origins of Mexico's Day of the Dead," Comparative Studies in Society and History 39(2) (1997), 270-299.

69 Brandes, "Day of the Dead," 359-380.

70 Juan Luis Ramírez Torres, "Ofrenda a los muertos: regeneración de la esperanza y la vida del México reciente," La Colmena: Revista de la Universidad Autonoma del Estado de Morelos 4 (1994), 43. Translation mine.

71 Malcolm Lowry, Under the Volcano (New York: Reynal and Hitchcock, 1947). This book like Labyrinth of Solitude (Paz, 1961), has been published in multiple editions and languages, up to the present day.

72 Irish wakes are perhaps the most famous examples of this phenomenon. See, for example, Seán Ó Súilleabháin, Irish Wakes and Amusements (Cork: Mercier Press, 1967). Excellent bibliography and cross-cultural treatment of humor in mortuary ritual can be found in Mahadev Apte, Humor and Laughter: An Anthropological Approach (Ithaca: Cornell Unversity Press, 1985) and Metcalf and Huntington, Celebrations of Death. 


\title{
DEATH IN MODERN JAPAN $(1800-2020)$
}

\author{
Timothy O. Benedict
}

\begin{abstract}
$* * *$
On the morning of Fune 5, 1945, an American B-29 bomber was shot down in a small village just south of Kyoto. As the wounded giant bomber circled the village, everyone ran out of their homes and glanced skyward, anxiously, wondering where the bomber would drop. All four engines were on fire and belched black smoke into the sky. The Buddhist priest who related this story to me was just a small boy at the time. Now he was much older but could still remember the ominous rumble of the bomber's engines. He explained how they sounded very different from the more familiar high-pitched scream of Japanese fighter planes. After the plane crashed down in a nearby river, the entire village rushed to the site.

Six of the American fliers who had bailed out by parachute were immediately rounded up and handed over to the local authorities. They later died in detention. The remaining five airmen's bodies were found scorched black in the wreckage except below their knees, where their skin had been protected by leather boots. They were all buried on the spot.

But their lives did not end there. This local priest's father, who managed the 450-year-old nearby temple felt compelled to make a memorial tablet (ihai) that enshrined the spirits of the American airmen. This was then placed in his temple alongside other memorial tablets of recently deceased villagers. Naturally, local parishioners were upset to see a memorial tablet of the enemy in such close proximity to their own ancestors. The priest glanced toward the temple windows and told me in a hushed tone, "I can't say this too loud, but more than a few vegetables were thrown through the temple windows because of my father's actions."

Two months later, in August, Japan surrendered, and shortly after, American soldiers came to the village to retrieve the bodies of the buried airmen. They also came to the local temple. As the priest explained:
\end{abstract}

The military officers came to hold a tribunal right here at our temple to see if any war crimes had been committed. The temple grounds were very quiet that morning. Nobody was sure what would happen. Suddenly we heard many loud voices coming from inside the temple where the tribunal was being held. My mother fearfully exclaimed, "The war has begun again!" and my sister and I ran to peek into the room. The reason for the loud voices was because my father had shown them the airmen's memorial tablet! The American officers were very impressed. They declared that nobody in the village would be held culpable for war crimes, got in their jeeps, and left. 
The now elderly priest brought out a folding chair for me to sit on in the very same room where the tribunal had taken place seventy years earlier. I ran my feet over the smooth wooden floors worn by centuries of shuffing feet. In front of me was an array of food offerings before the Buddhist altar. They were in preparation for a special autumn equinox service that was going to be held later in the evening. This was the time of year when ancestors came back to visit the living. I knew I shouldn't keep the priest too long. I asked if many others had seen the memorial tablet. Apparently a relative of the airmen had visited the temple in the 1970s and asked to view it. No foreigner had visited this small rural temple since. I leaned forward and examined the memorial tablet the priest had brought out. I could still read his father's clear English lettering carefully written horizontally in ink across the top of the tablet above the vertical Fapanese characters.

Holy Ghost

of Five unknown soldiers

super bomber $B$-29 fell

down 8:45am 5th Fune 1945

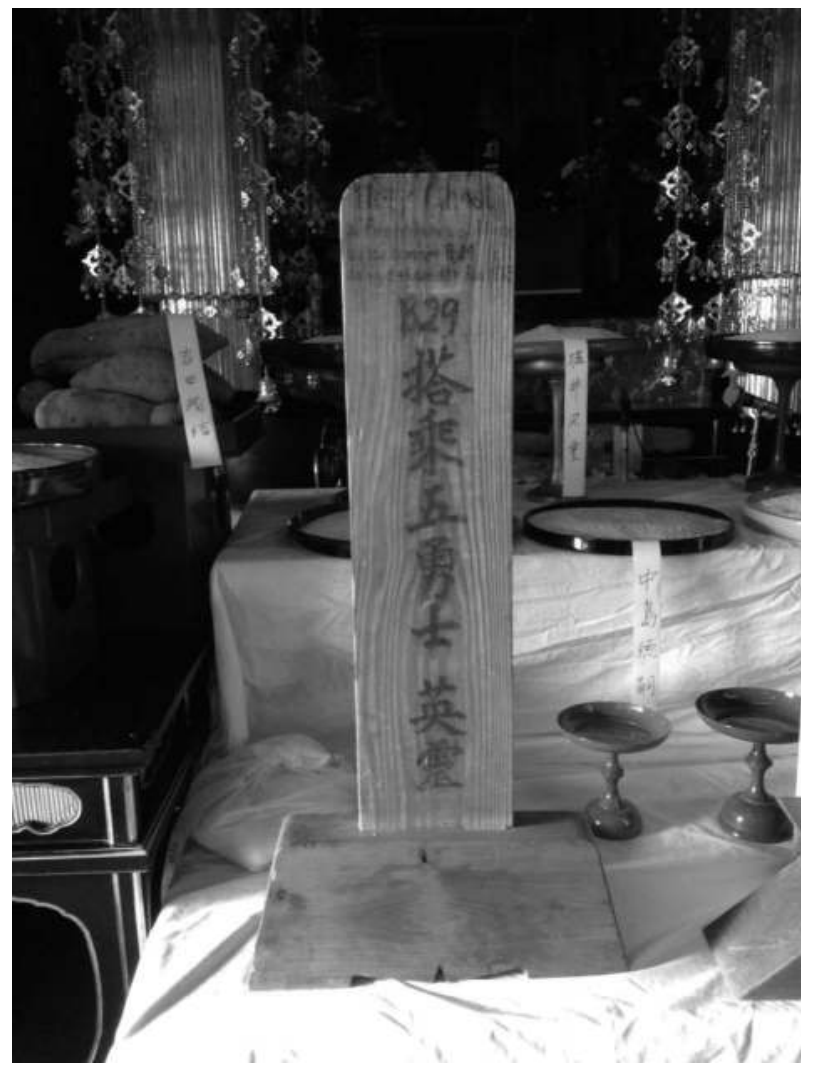

Image 14.1 Memorial tablet (ihai) for the deceased B29 Airmen.

Photo taken by author. 
When a person dies in Japan, they are said "to disappear" (nakunaru). But this not quite true. The dead in Japan do not really disappear. Rather, they stick around for a while. The deceased's ashes are first carefully placed in a Buddhist household altar where relatives can talk to him or her and make offerings of incense, favorite foods, or drinks. Even after the departed has passed on to their new life and their remains are safely interred in a grave, they still come back to visit the living - especially during the summer obon festival or spring and autumn equinox. Memorialization rites continue for years after death and bereaved relatives visit the grave periodically to wash the headstone and make offerings. Memorial tablets are placed in the household altar where they can be honored. Death anniversaries are also commemorated, typically by inviting a Buddhist priest to read a sutra in front of the household altar.

Philippe Ariès famously suggested that the removal of death from the home to the hospital helped inaugurate an era of "forbidden death" in which death was obscured from view in many modern societies. ${ }^{1}$ Although modernization has indeed helped transform the ways in which death in Japan is experienced at the bedside, in funerals, in memorialization rites, or portrayed in the popular media, in this chapter I will also show how death has stayed visible. This visibility is not limited to religious rituals. For example, death in modern Japan remains in the public eye as a demographic problem. Currently more Japanese die every year than are born. Projections estimate that by 2060, nearly 40\% of Japan's rapidly aging population will be over 65 years of age. ${ }^{2}$ This demographic crisis has made care for the elderly and dying a topic of national urgency and the subject of much discussion. In response to this crisis, the number of hospices in Japan has grown dramatically from just a handful of institutions in the mid-1980s to over four hundred such institutions today. This has, in turn, sparked a public conversation on how to best care for the elderly and dying through spiritual care or bereavement care for families.

Death in modern Japan also garners public attention as a social problem. For example, much has been made of neologisms that have emerged to describe new kinds of modern deaths in Japan. These include "death from overwork" (karōshi) and "solitary" or "lonely deaths" (kodokushi) that occur when elders die with no family members present at their side. While the actual number of such deaths in Japan is still quite low, the fear that modern Japanese society may have lost its traditional moorings and become a "bondless society" (muenshakai) causes much hand wringing in the Japanese media. This concern that modern ways of living have opened new cracks in society is driven home to many train riders in Japan for whom it is not at all rare to experience the momentary pause of a city train line due to a "human accident" - a euphemism for a suicide. Secularization, urbanization, changes in family and household structure, and economic changes have drastically transformed Japan over the last two centuries. This chapter will pay attention to how these shifts have changed the face of death in Japan as well as how certain beliefs and practices continue to persist through the renegotiation of their forms.

\section{Periodization}

At the beginning of the nineteenth century, Japan was a feudal society tightly ruled by the Tokugawa samurai clan that shunned internationalization. A century later, Japan had successfully thwarted Western colonization and become a major economic and military force in its own right in East Asia. Today, Japan is not only a leader in the global economy as one of the top producers of cars and electronics but has emerged as a cultural brand and exporter of popular culture like Super Mario, anime films, and 
conveyor-belt-sushi to consumers around the world. In order to sift through the many changes that shaped death and dying during the last two hundred years, this chapter will examine the modern history of death in Japan through the lens of four periods: early modern death (1800-1867), modern death (1868-1945), postwar death (1945-1989), and contemporary death (1989-2020). In addition, this chapter will pay special attention to the role of religion, the family, and end-of-life care.

\section{Early modern death $(1800-1867)$}

At the beginning of the nineteenth century, Japan was entering the denouement of a long era of rule by the Tokugawa warrior clan. This period, which is also called the Edo period (1603-1867), was characterized by political continuity, economic stability, a flourishing of arts and literature, and the growth of a wealthy mercantile class. This section will examine several important features of death in the early modern period and focus especially on the key role Buddhism played in managing death rites. It will explain the origin of Buddhist historical expertise in death-related rituals dating back to the medieval period as well as how, in the early modern period, the Japanese state used the Buddhist monopoly over death rites as means for social control. It will also address how Buddhist soteriological concerns shaped the structure of funerals and traditional Japanese views of the afterlife.

\section{Buddhist expertise in death}

Death rites have constituted one of the chief social and institutional roles of Japanese Buddhist clerics for a long time. In fact, even today, because death is one of the few times that a person will directly encounter a Buddhist priest, many Japanese joke that they don't really know what school of Buddhism they belong to until they are dead. Care for the dead was an integral part of the Buddhist mission from its arrival in Japan in the sixth century. In contrast, Shinto, Japan's other major religious tradition, has for the most part exempted itself from death-related matters for reasons of ritual purity. The reasons for Buddhist ascendency in matters pertaining to death in Japan can be explained by three factors: (1) compelling doctrines on the afterlife and rituals for controlling the circumstances of one's rebirth; (2) a capacity for assimilation and adaptation of elements from other traditions; and (3) the availability of Buddhist priests to serve as a class of capable religious specialists. ${ }^{3}$

In Buddhist teachings, death presents both a problem and a solution. On one hand, all of life is tinged with the suffering that comes from knowing that one will eventually die. This is the problem of impermanence. Nothing lasts forever, and death is the ultimate evidence of that. On the other hand, death in Buddhism is also an opportunity to escape the painful cycle of rebirth - known as samsära - forever, or at the very least, an occasion for rebirth into a better life. In this way death also provides a solution. The Buddhist meditator, who can recognize the law of impermanence and let go of the grasping self, can extinguish their ignorance and craving so that upon death they are released from the cycle of samsāra. This episteme forms the foundation for Buddhist teachings on death.

During the medieval period (ca. 1185-1600), Japanese Buddhists used this episteme to develop a sophisticated eschatological framework for understanding death and managing the fear of what lay beyond it. For instance, many Buddhists aspired to be reborn in a place called the Pure Land. The Pure Land, a lovely place devoid of ignorance and 
craving, guaranteed enlightenment to Buddhist practitioners, who were able to be reborn there. The key to rebirth in the Pure Land, however, was maintaining a state of right mindfulness at death. Accordingly, many medieval Buddhist priests served as ritual specialists at the deathbed, essentially working as religious midwives to ensure the dying person a successful rebirth in the Pure Land. In many cases, this successful rebirth would be confirmed through auspicious signs like unearthly fragrances, music, or appearance of clouds in the west at the time of death. ${ }^{4}$

Over time, however, the role of Buddhists priests increasingly shifted from bedside care of the dying to postmortem rituals. This allowed priests to guide the process of rebirth in a more controlled environment. For example, one way to ensure a good postmortem fate was to be ordained as a Buddhist priest. While this was traditionally done before one died, in the early modern period the ordination of the dying came to be conducted even after death. In the Zen tradition, for example, the funeral came to include an ordination for the dead in which the deceased received a Buddhist bowl, robe, and lineage chart to seal their posthumous ordination and signal their successful transformation into a Buddha. ${ }^{5}$ Although the dead could no longer audibly accept the precepts as they could when living, Zen priests interpreted the dead person's silence in a koan like fashion as representing ultimate wordless acceptance of the precepts. Such posthumous ordination rituals provided Buddhist laity with tangible assurance of salvation that demanded no rigorous Zen training before they actually died, while also serving to legitimize Buddhist religious authority. ${ }^{6}$

Although early modern funerary rituals more or less guaranteed immediate enlightenment for the dead, there were some important exceptions. Women, for example, were deemed to be in need of special salvation due to the pollution incurred by the blood produced during menstruation and childbirth. This pollution, Buddhists believed, would cause women to fall into the Blood Pool Hell. ${ }^{7}$ In order to prevent this frightening possibility, women could copy and recite the "Blood-bowl Sūtra" (ketsubonkyo) during their lifetime, and a copy of the sutra was placed in their coffin as a talisman to protect them. By the end of the early modern period, however, such practices had become less common and male and female funerary rituals became conducted in a more or less identical fashion.

\section{State support for and the structure of Buddhist funerals}

Another notable characteristic of death in the early modern period was state support for Buddhist funerals. The Buddhist monopoly over death was near total in the early modern period due to the household system (danka) instituted by the Japanese state. The original impetus for the household system arose out of the anti-Christian policies instituted by the Tokugawa government during the early seventeenth century. The outlawing of Christianity continued all the way until 1872. During this period, Japanese subjects were required to register at their local Buddhist temple to prove their anti-Christian allegiance. In this way, Buddhist temples effectively became tools for state monitoring. The state's reliance on temples as a tool for maintaining social stability benefited Buddhist temples, who, in light of mediocre income from agricultural holdings, became increasingly reliant on a stable supply of funerary rituals to supplement their financial resources. ${ }^{8}$ This arrangement later led some scholars to decry Buddhist collaboration with the state and its reliance on funerals for income during this period as "degenerate" or "funerary Buddhism." This negative depiction of early modern Buddhism continues to 
color both academic and popular depictions of Japanese Buddhism today. There are many problems with this depiction, however, such as the presupposition that "real Buddhism" should be found outside of funerary rituals. It also ignores the limited agency family members were able to exercise within the temple registration system. For example, although they were being monitored, households could also use lavish funerals at their local temple or ornate gravestones as an opportunity to assert their social standing.

\section{Structure of Japanese funerals}

The traditional Japanese Buddhist funeral can be roughly divided into four stages. ${ }^{9}$ The funeral begins with the initiatory stage which takes place shortly after the pronouncement of death. This marks the start of the deceased's transit to the next world. This first stage typically includes the bedside recitation of a "pillow sutra" by a Buddhist priest that helps ritually purify the deceased. This is followed by the wake, which takes place on the night of the death or the night after. Prior to the wake, the body was traditionally washed and beautified by family members and placed in a coffin, a task that is today often outsourced to funerary workers. The Academy Award-winning film Departures (2008) gives a close-up view of this practice as it is now conducted in the twenty-first century. During the wake, family members place incense in front of the coffin, invite a priest to give another sutra recitation, and sit around eating and drinking sake late into the night while reminiscing with relatives.

The second stage of a Japanese Buddhist funeral includes the ordination of the dead. This is necessary to secure their passage to the Pure Land or other blissful realm and often involves a ritual (not actual) shaving of the head and the sprinkling of water to signify their tonsure. A repentance text (sange) is then recited on the behalf of the deceased before the deceased are posthumously ordained and given a dharma name which is inscribed on an ancestral tablet or on a hanging scroll. This conferral of monastic status on the deceased helps to secure and confirm their safe passage out of this world.

The third stage of the funeral is centered on guiding the deceased to the other world. This practice varies by tradition and can include a funeral sermon, consecration rites, or esoteric initiation rituals. The aim of these rituals is to initiate and to ensure a safe passage to the Pure Land or the next life. After these rituals are performed, the coffin is nailed shut and transported to a crematorium in a hearse. After the body is cremated, family members will use chopsticks to pick up some of the bones to place in a small urn that will be taken home and placed on the household altar and then later be placed in their grave.

The final stage involves the transfer of merit to the deceased to make sure they make it to their destination safely. After death, it is believed that the spirit of the deceased will wander on earth for up to forty-nine days. Every seven days, the family will offer special prayers to the deceased - concluding with a special ceremony on the forty-ninth day. These memorialization rites continue on the first, third, and seventh anniversary, continuing up to the thirtythird year with the purpose of generating merit that can be transferred to the deceased in case they need some extra merit to ensure an auspicious rebirth.

\section{Viezs of the afterlife}

In early medieval Japan, the Buddhist explication of the six paths (rokud̄) provided several possibilities for rebirth based on the karma one accrued in life. This included rebirth as heavenly beings, human beings, demi-gods, animals, hungry ghosts, or hell dwellers. For example, 
ancient Japanese literature is replete with "near-death" stories that describe individuals who returned from the dead with captivating tales of reward and retribution in the afterlife that served to support this karmic cosmology. Although the ultimate goal in Buddhist teachings is to achieve enlightenment and escape the cycle of rebirth altogether, traditionally, this achievement was seen to take many lifetimes to achieve. With the popularization of Pure Land teachings in the medieval period, however, aspirations for rebirth became focused on entering a blissful realm of Amitabha Buddha's Pure Land where future enlightenment was guaranteed. The Pure Land, or $\mathcal{F} \bar{o} d o$ in Japanese, is regarded to this day as the favored destination for most mainline Japanese Buddhist denominations.

However, the vivid descriptions of the afterlife in Buddhism also lie in tension with orthodox Buddhist teachings that ultimately teach the doctrine of anatman or "no-self." From a doctrinal perspective, Buddhism holds that ultimately there exists no permanent self, so there is actually no "soul" that can be reborn. This gap between practice and doctrine is often explained by Buddhist doctrine as the distinction between "conventional" and "ultimate" truth. ${ }^{10}$ For most modern Japanese, however, these finer points of Buddhist teachings on the afterlife are often ignored or left ambiguous in daily practice. One of the Japanese verbs "to die" (ōjo suru) is synonymous with achieving Buddhahood. But the afterlife is also often vaguely described as "that [other] world" (ano yo) or more simply as "heaven" (tengoku) - a broader term that is not limited to Buddhist cosmology. Later, in contemporary Japan, the afterlife became even more ambiguous in popular culture. For example, in a film titled "After Life" (1998), the acclaimed film writer and director Hirokazu Koreeda depicts the afterlife as a kind of heaven where entrants simply relive in perpetuity their most precious and blissful memory from earth.

\section{Modern death (1868-1945)}

After several centuries of feudal rule under the Tokugawa government and isolation from foreign influence, in 1868 a new government was established, Japan's shipping ports were thrown open, and Japan embarked on an ambitious plan of modernization. The new Meiji Emperor was enthroned, the feudal system abolished, a new constitution was promulgated, and Japanese society underwent many drastic changes. One change that greatly impacted death in this period was the growth of modern medicine. Whereas in the past priests were typically called to a person's home to confirm death, in the modern period physicians increasingly took over this task and helped push the Buddhist priest more firmly into playing a strictly posthumous role. ${ }^{11}$ As industrialization and urbanization disrupted traditional patterns of daily life, the Japanese state also began forming new policies for cremation, cemeteries, and funerals. For example, with the advent of traffic jams in cities, lavish funeral processions that asserted local status were curtailed, and advances in cremation technology spawned new rituals for handling corpses. The late nineteenth century and early twentieth century decades were also characterized by a general hostility toward Buddhism by the Japanese state due to growing nationalism driven by Shinto nativists. The period even saw a failed attempt by Shinto groups to capture a share of the death market from Buddhists. Amidst all these changes, however, there were also important continuities. For instance, the householder $(i e)$ system was left intact and remained the cornerstone for the practice of funerary rituals and memorialization of ancestors. 


\section{State control and the householder system}

The householder $(i e)$ registration system was a remnant of the early modern Buddhist temple registration system which was finally abolished in 1872 . The $i e$ was a legal category that designated an extended patrilineal household. Households, which were previously required to register with temples, were released from temple control but the Meiji Civil Code in 1898 instead codified the ie into a civil registration system under which mortuary customs were homogenized. ${ }^{12}$ For instance, the law stipulated that household Buddhist altars and grave rights should be passed down from head householder to head householder - typically the eldest son. This insured that ancestors would be properly memorialized by successive generations. Interestingly, the shift from the religious temple registration system to a civil householder system did not have the effect of undermining the ties between householders and Buddhist temples. Rather, since householders felt obligated to care for the graves of their ancestors, which were located at Buddhist temples, most households continued to rely on their local Buddhist temples for funerary and memorialization services. But this system also produced some problems. For example, although the Japanese state promoted and idealized a patrilineal extended household ideal, it was just that, an ideal. As urbanization and economic development subsequently produced changes in Japanese household structures, maintaining this ideal through memorialization practices became harder to maintain. But the resiliency of this ideal remained strong and later helped produce new and innovative memorial rites that could help keep the living and the dead connected. ${ }^{13}$

\section{Cremation}

The 1870s also saw a brief experiment in outlawing cremation in Japan. Cremation had a long history in Japan dating back to the sixth century. However, although Buddhist teachings encouraged it, burial was more common until the late nineteenth century. In 1873, however, the Japanese state decided to promote burial by outlawing cremation on the grounds that it was disrespectful to the dead. Cremation was also criticized as the cause of air pollution in urban centers. This law may have also been aimed at pleasing Shinto nativists and Confucian scholars who strongly frowned on cremation. ${ }^{14}$ As the Japanese state soon realized, however, if cremation was done properly, it actually had many advantages over burial, especially in terms of saving space. As Japanese flocked to urban centers, land was at a premium. Setting aside large spaces of tax-free land for temple cemeteries also reduced the income of cities. Cremation allowed family members to be interred together in crowded areas and was more cost-efficient and sanitary. The efficiency and sanitary merits cremation offered were particularly appealing to the Japanese state since it was wholeheartedly pursuing the goal of modernizing Japanese society and discarding outdated traditions. The state thus changed course and began to effectively promote cremation. By the 1930s, cremation accounted for over half of burials in Japan and later, in the postwar period, this number rose to nearly $100 \%$.

\section{Postwar death (1945-1989)}

After the Second World War came to an end in 1945, the postwar decades ushered in a period of reconstruction and economic growth that eventually propelled Japan to become one of the world's leading economies. During this economic boom, a growing consumer culture along with changes in family and work life brought additional changes 
to death in modern Japan - especially in regard to funerary and memorial rites. This section will outline some of these developments.

\section{Commercialization of funerals}

One of the biggest changes to funerary rituals in Japan's postwar period (1945-) was the growth of funeral companies, which took on many of the tasks that had previously been handled by family members or Buddhist priests. Instead of local temples, it was now commercial companies who orchestrated funerals. This trend has remained strong to this day and has led to increased commoditization, commercialization, and professionalization of funerals in general. Although priests still play an important ritual role within funerals, their terms were now often dictated by the funeral company. For example, one priest complained: "I am asked to shorten my recitations because they need time to read telegrams. Since when are telegrams more important for the deceased than sutra recitations?"15 Whereas previously, Buddhist priests presided over the funeral, in the postwar period, many Buddhist priests were called on by funeral companies to conduct specified religious rituals rather than deal with the bereaved directly. Not unsurprisingly, the postwar period also saw the intensification of social criticism of Buddhist priests as funerary mercenaries. For example, in a memorable scene from Itami Jūzō's 1984 film The Funeral (Osōshiki), the priest arrives at the funeral in an expensive limousine. Such caricatures served to further calcify the stereotype of greedy priests who exploited the bereaved through funerals for personal gain.

\section{Ancestor veneration}

In many societies, people remember the dead. But in Japan, people care for the dead. This practice is often referred to as ancestor veneration. The souls of ancestors in Japan can be roughly divided into three categories. First are "the departed," which refers to those who died in recent memory. Second are "the ancestors," which includes those departed members of a household who have been expunged from living memory. The third and final category are "outsiders," which includes the departed souls of those with no household ties. Memorial rites are conducted for those in the first category, the recently departed, until no one remains among the living who knew the departed personally. At this point, the memorial tablet of the departed is destroyed and their soul is retired into the ranks of nameless ancestors. ${ }^{16}$

One way to care for the dead is by making offerings at the household Buddhist altar. Such altars date back to the medieval centuries but in the postwar period became more widely available and commercialized as Japanese incomes grew. The five key accessories for such an altar include a candle holder, flower vase, tea bowl, food offering bowl, and incense burner. ${ }^{17}$ While early altars could be simple in shape, in the postwar economic boom, many altars became ornate luxury items. When the altar, which looks like a two doored cabinet, is swung open, in the center is found a Buddha image and a hanging scroll or other icon depending on what Buddhist denomination the household belongs to. Memorial tablets of ancestors may also be stored in the altar. Before this altar, food and drink offerings like rice and tea or perhaps even a bowl of hot miso soup is made, incense is lit, and prayers are given. 


\section{The return of the dead}

In addition to being cared for, the dead also return to visit their relatives. The most common time when this happens is in the middle of August, during the summer obon festival. This memorialization ritual dates back to the early seventh century in Japan and is based on the "ghost festival" that originated in China. It also bears some similarities to the Day of the Dead in Mexico. The original purpose of the festival was to free the dead who might be trapped in a hungry ghost hell where they are perpetually hungry and thirsty. During this festival period, dead spirits are temporarily freed to visit the earth, and, by consuming the offerings set out by loving relatives, they can be delivered from their ghostly state and be reborn in a more auspicious heaven.

Although much of the religious import of this festival has been lost in modern Japan, the festival is still widely observed since it is commonly viewed as an important time of family gatherings and reunions. Coinciding with summer vacation, many Japanese brave heavy traffic and get on crowded trains to return to their hometowns and to connect with relatives. During the festival they may visit their family graveyard together to clean the grave and place offerings to their ancestors. Many towns also celebrate this occasion with bon dances, the lighting of fires to greet and send off their ancestors, and other local traditions. In addition to obon, ancestors can also return to the land of the living at other times of the year such as during the autumn equinox. At this time too, filial family members can make offerings at their local temple on behalf of their ancestors.

\section{The lines between life and death}

The permeable lines between the land of the dead and the living in Japan are further illustrated in debates over the ethics of brain-death diagnoses and organ donation. The first heart transplant in Japan was conducted in 1968 using a brain-dead donor. The doctor who performed the transplant, however, was later investigated and accused of being too aggressive in harvesting organs from possibly not so brain-dead donors. This incident sparked a media outcry and led to the halting of non-cadaveric donations for several decades in Japan. Finally, in 1997, the Organ Transplant Law was passed to permit transplants from brain-dead donors. However, even under this law, brain death was not formally recognized as "actual death." Instead, the law only somewhat circuitously acknowledged brain death as a "real death" after a transplant occurred. This law was then later amended in 2009 to finally recognize brain death as an actual death.

Japanese resistance to recognizing brain death and participating in organ donation remains strong for mostly cultural and religious reasons. Whereas most Western societies locate life and death in the brain, in Japan, life is more often seen as located in the "heart" (kokoro) which is diffused throughout the body. In the West, by locating life and death in the brain, "a dramatic bid can be made to postpone mortality through medical intervention," and organ transplants can be seen as a "technological fix to transcend the scandal of biological death." 18 In contrast, in Japan, there remains much resistance to organ donation due to the belief that life is not only located in the brain but is diffused throughout the body. Another factor that forestalls the spread of organ donation in Japan is the belief in ancestors who continue to participate in the everyday world. Disfiguring the body of the recently deceased is considered disrespectful and raises the possibility that such an unfilial action will hamper their journey to their next life. Consequently, 
much opposition to harvesting organs from brain dead patients persists, since the whole body is seen to be animated with life and since the ancestral soul of the departed remains connected to the world even after the body's vital signs have ceased.

As the issues surrounding brain death and ancestor veneration make clear, rather than cutting an individual off from society, death in Japan simply renegotiates the social connections between the dead and the living. The heavy sociality that imbues the lines between life and death can also be observed in the practice of memorialization of aborted fetuses (mizuko kuȳo). Although abortion is mostly uncontroversial in modern Japan, the fetuses themselves are sometimes regarded as having a liminal status. For instance, some women will choose to memorialize their aborted fetus at a temple. Although fetuses are not considered human enough to merit a full Buddhist memorial tablet (ihai), a small bodhisattva statue can be procured as a means of memorialization. One explanation for this practice is that the lines between life and death are determined by the "denseness" of social ties. ${ }^{19}$ According to this logic, fetuses slowly "densify" into human beings at birth, becoming stronger as they gain social ties during childhood, become fully human as an adult, and then slowly attenuate again at the end of life into ancestor-hood. The "density" of life is thus closely tied to the level of social connections the individual maintains with his or her household.

\section{Contemporary death $(\mathbf{1 9 8 9 - 2 0 2 0})$}

In 1989, Japan's economic bubble reached its peak. Then, within a year, the stock market lost half of its value and a series of high-profile bankruptcies sent shockwaves through the country. Land prices plunged, and by 1992 a full-blown recession began which would drag on for more than a decade. The 1990s are commonly referred to as "the lost decade" in Japan, but the 2000s were not that much better. Interest rates continued to remain below $1 \%$ for more than twenty years. This anxiety produced by Japan's economic malaise was also compounded by a growing demographic crisis. Japan's greying population was putting stress on many families and social institutions, especially in regard to the care of the elderly and dying. This next section will focus on how this demographic crisis is changing the face of death in Japan.

\section{The demographic crisis}

In 2005, the Japanese birth rate reached a new low of just 1.26 births per woman. Although this rate has since risen slightly to about 1.4, the birth rate remains well below replacement levels. Consequently, the number of Japanese aged 65 or older reached 28\% of the population in 2018 and is projected to reach nearly $40 \%$ by 2065. The aging of the Japanese population has also been accompanied by changes in the structure of Japanese households as well. Over several generations, the relative number of nuclear families has risen in Japan, while the number of three-generational households has declined. The number of single-member households has also increased. These changes are often attributed to the rise of conjugal ideals that emphasize living apart from one's parents, as well as the space constraints of urban living. For example, roughly half of the newlyweds who were born in the 1920s chose to live with one or both of their parents, compared to only $20 \%$ of newlyweds born in the 1960s. Yet, interestingly, the number for both groups converged to 30\% beyond the ten-year mark of marriage. This indicates that although Japan's postwar generation initially chose to 
live separately from their parents, such arrangements were often temporary. In many cases, the oldest son or daughter later returned to live with his or her parents for reasons related to household succession, property inheritance, and support of their elderly parents. Accordingly, the rise of nuclear families has not yet completely undermined the traditional Japanese stem family since many children will move back home to care for their parents in their hour of need. $^{20}$

However, the resilience of traditional family structures in Japan is now being tested in new ways. Simply put, even if children are willing to move back home to care for their parents, without large-scale immigration or a change in Japan's historically low birth rate, there are just not enough children to care for their elders. In the past, daughters-in-law traditionally bore the responsibility of living with and caring for their husband's parents. However, as the number of married women who choose to remain in the workforce has risen and men find it culturally difficult to take time off, this task has proved to become increasingly difficult. Moreover, if the daughter-in-law is an only child, she is likely to be responsible for the care of her own parents as well. In rural areas, where depopulation is particularly acute, the problem is compounded by a lack of job opportunities that would allow children to return home. This demographic crisis has no immediate solution in sight as Japan's birthrate remains stagnant and the possibility of large-scale immigration remains politically unpopular. Coupled with Japan's life expectancy rates, among the highest in the world, the prospect that a growing percentage of the current population will live well into their eighties and nineties presents a genuine dilemma. Who is going to care for the elderly as they approach the end of life?

\section{The growth of hospice care}

For most Japanese, the default place to die is in the hospital. This is particularly true for cancer patients. Currently, cancer accounts for nearly a third of all deaths in Japan and remains the leading cause of death among those aged 40 to 89 years. In 2015, 2.9\% of Japanese cancer deaths occurred in a nursing home, $10.4 \%$ at home, $74.3 \%$ in a hospital or clinic, and $11.6 \%$ in a hospice. Although just over a tenth of cancer deaths currently take place in a hospice, this number is trending upward. For example, a decade prior, only $5.3 \%$ of cancer deaths occurred in a hospice while $88 \%$ of such deaths took place in a hospital. ${ }^{21}$

There are many benefits to dying in a hospital. First of all, it is convenient. In fact, for Japanese who live in rural areas with no hospice nearby, dying in a hospital might be the only option. More importantly, however, dying in a hospital ensures that patients can retain access to life-extending treatments until the very end. For patients who wish to extend their life as long as possible, having full access to a range of diagnostic and treatment services is no small blessing. For instance, one patient I interviewed for a research project on end-of-life care in Japan had been battling cancer for more than a decade before finally entering a hospice. She had undergone every possible treatment and had visited countless specialists. She related that some patients liked to complain about the side effects of multiple treatments, but she had a different perspective: "I say, if you don't like it you can go home. Your cancer will just get worse." 22 Although this patient eventually embraced hospice care when she had run out of treatment options, it was clear that battling cancer in the hospital was important for her. Refusing to go gently into the night helped her cope with her 
suffering. Notably, her decision to pursue life-prolonging treatments was also made possible by Japan's National Health Insurance system which kept the costs of end of life care at reasonable levels.

However, for most Japanese patients, dying in a hospital is far from ideal. This problem was laid out clearly by Yamazaki Fumio in his 1990 bestseller, Dying in a Fapanese Hospital, which was later made into a film. In his book, Yamazaki shares the story of five patients who died horrible medicalized deaths and contrasted them with five patients who died dignified, humane deaths. He argued that modern Japanese hospitals were too focused on treating the disease at the cost of ignoring the person. Yamazaki describes an interaction between one patient and his doctor as follows:

The concern of the doctor in charge of him rapidly receded once it was seen that the treatment had little effect. When he came on his rounds, he didn't look Kinoshita in the eyes. All he did was to encourage him in a loud voice. Then he hurried away. He was interested only in the cancer, not in Kinoshita as a person. $^{23}$

Yamazaki concluded his book by calling for more hospice care where patients are treated holistically, with fewer medical interventions, with fuller and more honest prognosis disclosure, and where spiritual care is made available to all patients.

As of 2018, there are an estimated 415 hospices in Japan. ${ }^{24}$ On average, each hospice has about twenty beds, and in 2015 the average length of stay was just under thirty-three days. There are several different types of hospice in Japan. First, there are hospices that exist as a ward within a larger hospital building. These types of hospices are by far the most common and represent over $80 \%$ of Japanese hospices. Second, about $15 \%$ of hospices are located in a separate building on hospital grounds. These hospices have several advantages over those that are located inside the hospital. These include greater freedom to design the facility to make it feel more homelike, along with additional perks, such as enabling patients to smoke without falling afoul of hospital regulations. A third type of hospice, of which there is only a handful, exists completely independently from a hospital. Other forms of hospice care include palliative care teams that are assembled in a hospital to treat cancer patients who are scattered across different wards. In 2014, approximately 13\% of Japan's 7,426 hospitals had such teams in place to help treat terminally ill patients. Finally, a modest but growing number of home hospice services are provided by a range of small clinics and home-visit nursing stations that help care for Japanese cancer patients who prefer to die at home.

\section{Neze memorialization rites}

Japan's twenty-first century demographic dilemma has implications for memorialization rites as well. Without children, who will care for one's grave? This question of who will care for the dead, along with the high costs of Buddhist memorial rites and other factors, has driven some people to bypass the Buddhist establishment altogether and choose to scatter their ashes instead of being buried and continuously memorialized at a temple. Organizations like the "Grave-Free Promotion Society" advocate scattering ashes in lieu of expensive memorial rituals required at Buddhist gravesites. In postindustrial Japan, smaller households and smaller houses often leave no space for household Buddhist altars, and therefore, reciprocal interdependence between the living and their ancestors 
becomes harder to maintain. ${ }^{25}$ While ash scattering represents only a small fraction of Japanese burials, changes like this give the impression that "funerary Buddhism" might be nearing its demise. However, funerary Buddhism is not quite dead yet. Although the bonds between temples, the deceased, and the parishioners who pay to have their family members memorialized are undergoing significant challenges, they remain tenaciously strong.

For example, recognizing the demand for their services, some temples have introduced innovative strategies for memorialization, such as communal ossuaries. Whereas traditionally, graves are tended by family members who take on the responsibility of performing all the necessary memorial rites, some Japanese have requested to be buried in what are called "eternal memorial graves." These represent a relatively new form of communal ossuary, where memorialization rites are conducted by a third party (instead of by living family members) for those who are buried there. This form of burial is not only cheaper than purchasing an individual plot at a temple, it has the added benefit of ensuring that the deceased will continue to be memorialized without placing a burden of obligation on surviving family members. In some cases, this is also attractive to wives who outlive their husbands and prefer not to be buried alongside her husband's family due to resentment of her in-laws or because of an unhappy marriage.

The growing number of Japanese who are renegotiating or foregoing Buddhist memorialization rites altogether is a sign of the increasingly secular populace and disenchantment with the high prices of Buddhist funerals in a sluggish economy. Yet at the same time, this is not to suggest that the importance of maintaining bonds is attenuating. Communal ossuaries in effect provide a compromise in which the dead can rest assured that memorialization will take place without imposing a cost or burden on their children. It suggests that the bonds that bind the dead with the living are not attenuating but are just changing shape. More recently, Buddhist priests have even sought to lower the barriers for procuring funerary rites by letting customers reserve their services on internet retail sites such as Amazon.co.jp. As of 2019, one such service offering a cheap "priest for hire" had garnered 191 reviews with an average rating of three and a half stars. Their most basic funeral package cost about US $\$ 400$, and for an extra US\$300 the deceased could receive their dharma name too. In some cases, memorialization rites are becoming digitized as well. For instance, digital Buddhist altars can now be procured. ${ }^{26}$ Such experiments, though small in scope, attest to the strong demand for memorialization rites even in the twenty-first century.

\section{Death anxiety at the end of life}

Two common cultural scripts of a "good death" in Japan are a gradual aging decline into death $($ rōsul $)$ and sudden death $($ pokkur $) .{ }^{27}$ Although these two ways of dying may seem quite disparate on the surface, they share a common motivation-both stem from an anxiety about becoming a burden on others. In the case of a gradual death in old age, many elderly feel that this is an ideal way to die since it provides plenty of time to arrange their affairs and make preparations for their death. As one Japanese cancer patient I spoke with put it, 
When you have something like a stroke, even if you survive, you can't communicate your intentions. You become a great burden on your family. This is why I'm glad I have cancer. With cancer, they tell you how long you have, and during this time, you can do all kinds of things. I'm in my late sixties, and I still have strength. My wife is several years younger and still has energy and physical strength. But if this happened five to six years from now, I wouldn't be so active, and even my wife would not have been able to move the way she can now. So, I think all of this might have been the will of the unseen $\operatorname{god}(\mathrm{s})$. I am quite thankful. ${ }^{28}$

At the same time, many Japanese long for and pray for a "sudden death." This is seen as equally attractive in that it would not impose the burden of a long hospitalization on the family. ${ }^{29}$ There are even Buddhist temples that specialize in prayer requests for a sudden death.

This anxiety about becoming a burden on others at the end of life is ubiquitous in modern Japan. In fact, treating this type of anxiety has become a distinguishing feature of Japanese hospice care in general. ${ }^{30}$ Another distinguishing feature of the ways in which the Japanese deal with death anxiety and especially spiritual pain at the end of life is encapsulated in the word "shiyo ga nai" which means "it cannot be helped." As one Japanese hospice chaplain explained to me,

Japanese are very good at giving up. For example, Kübler-Ross's model [of the five stages of grief] does not fit very well with Japan. For most Japanese, the final stage is not acceptance but giving up. They all say, "It can't be helped" (shiyo ga nai)." They do not try to figure things out. Many Japanese do not think so philosophically about these things. They will just say, "c'est la vie."31

This type of resignation is not the same as avoiding thinking about death altogether, nor does it mean that many hospice patients in Japan are unwilling to engage with deep existential questions about death. Rather it indicates a tendency for many terminally ill patients to try and remain clear-eyed about the difficulty of finding definitive answers about what lies beyond death or why they must die before someone else. Another hospice chaplain noted that this idea of "resignation" (teikan) is central to Buddhism, in which it means to "see clearly" (Skt. Darśana). In fact, the Japanese word to "give up" (akirame) actually stems from this Buddhist sense of the term, "to make clear" (akiramu) which helps explain the positive tenor of the claim "Japanese are very good at giving up," since "giving up" (akirame) and "making clear" (akiramu) are etymologically linked.

\section{Suicide and new types of deaths}

The suicide rate in Japan currently remains one of the highest amongst developed economies, trailing only Russia and South Korea. In 2018, there were approximately 20,000 suicides in Japan or about 16 suicides per 100,000 members of the population. Overall, however, the suicide rate is declining. The late 1990 s and early 2000 s saw a drastic rise in the number of suicides, that peaked at 34,000 suicides a year in 2003. The relatively high rate of suicides in modern Japan can be traced to several factors. First, historically suicide was not strictly condemned. Rather, at times it was even celebrated in warrior 
culture as a way to show loyalty or accept blame. For example, when the Meiji Emperor died in 1912, one of his leading generals committed suicide as an expression of loyalty, and this was widely accepted at the time as natural. Similar suicides by military figures, as a means of accepting blame for Japan's defeat, occurred at the end of the Second World War in 1945. However, by the end of the twentieth century, such shows of loyalty had largely fallen out of date. For instance, when some former military officers committed suicide after the death of Emperor Hirohito in 1988, they were largely regarded as cultural oddities. $^{32}$

Today, over a third of suicides in Japan occur amongst persons in their forties and fifties, and especially among men. While it is difficult to accurately measure the complex causes of suicide, some of the largest factors include mental illness, economic difficulty, household problems, and work. A related phenomenon is the emergence of a new type of death called "death from overwork" (karōshi). According to Japan's Ministry of Health, Labor, and Welfare, approximately 2,000 suicides (10\%) in 2018 were work-related. Of these, an estimated 190 suicides occurred under grueling work conditions that are defined as more than eighty hours of overtime per month. Of such suicide cases, $5 \%$ were killed by logging more than one hundred hours of overtime. For example, in 2015, a 24-yearold woman jumped to her death from her work dormitory at a major advertising firm after being forced to log more than one hundred hours of overtime per month in her first year on the job. Her tragic case caused an outcry in the media, and since then the Japanese government has attempted to more strictly enforce restrictions on overwork.

Another new type of death that has received media scrutiny is known as "lonely deaths" (kodokushi). This refers to a death that occurs at home in a single-member household. In Japan, "natural deaths" are deaths that are confirmed by a medical doctor, usually in a hospital. "Unnatural deaths" on the other hand, are deaths that are discovered sometime after the fact and are recorded by a medical examiner. In Tokyo for instance, a total of 76,826 persons died in 2016. Of these deaths, 12,780 were counted as "unnatural." In addition, more than a third of these unnatural deaths occurred with the person dying alone. This number also seems to be rising; from $16.5 \%$ in 1989 to $26.3 \%$ in 2001 to $36 \%$ in $2016 .^{33}$

\section{Conclusion}

On the northeastern coast of Japan, there is an unusual telephone that can be used to dial up the dead. It is an old rotary phone that sits in a telephone booth of a quiet garden with a view of the Pacific Ocean. It is unconnected. The owner placed it there in 2010 as a means to talk to his cousin who had died that year. In the following year, however, word of this unique telephone quickly spread after a massive earthquake and tsunami decimated the northeastern coast and claimed nearly 16,000 lives on March 11, 2011. Many of the bereaved, hearing of this telephone, came to dial-up their loved ones and work through their grief. They needed to try to stay connected to the dead.

The connections between the living and the dead have been a major theme in this chapter. Despite the many cultural and social shifts in funerals, memorialization rites, graves, definitions of brain death, cultural scripts for a good death, ancestors, and end of life care over the past two centuries, one remarkable continuity is that death in modern Japan remains embedded in a culture that emphasizes the indissoluble relation between individuals and their social relations. As Margaret Lock notes, 
The idea of an autonomous, individualized self, essentially synonymous with the "person" in the West, does not sit well in Japan, where "person" is above all reproduced in the public domain, beyond the bounds of the body, as part of a network of ongoing exchange. Personhood is constructed in the space of human relationships. ${ }^{34}$

This notion of the individual-in-society remains an important theme in the study of modern death in Japan. Although modernization and demographic changes have upended Japanese society over the past two centuries, the fundamental belief that the dead do not immediately disappear from this world and should be still cared for as family members remains.

\section{Notes}

1 Philippe Ariès, The Hour of Our Death (New York: Alfred A. Knopf, 1981), translated by Helen Weaver.

2 Shakai Hoshō Kokuritsu, Jinkō Mondai Kenkyūjo. "Nihon no shōrai suikei jinkō (Heisei 24 nen ichi gatsu suiker)." January 30, 2012. www.ipss.go.jp/syoushika/tohkei/newest04/sh2401top.html. Accessed January 2, 2014.

3 Jacqueline I. Stone and Mariko Namba Walter, Death and the Afterlife in Fapanese Buddhism (Honolulu: University of Hawai'i Press, 2008), pp. 1-26.

4 Jacqueline I. Stone, "Death." In Critical Terms for the Study of Buddhism, edited by Donald S. Lopez (Chicago, IL: University of Chicago Press, 2005), p. 66.

5 William M. Bodiford, "Zen in the Art of Funerals: Ritual Salvation in Japanese Buddhism," History of Religions 32, no. 2 (1992), p. 159.

6 Ibid., p. 164.

7 Hank Glassman, "At the Crossroads of Birth and Death: The Blood Pool Hell and Postmortem Fetal Extraction." In Death and the Afterlife in Fapanese Buddhism, edited by Jacqueline I. Stone and Mariko Namba Walter (Honolulu: University of Hawai'i Press, 2008), pp. 175-206.

8 Nam-lin Hur, Death and Social Order in Tokugawa Japan: Buddhism, Anti-Christianity, and the Danka System (Cambridge, MA: Harvard University Press, 2007).

9 Mariko Namba Walter, "The Structure of Japanese Buddhist Funerals." In Death and the Afterlife in Fapanese Buddhism (Honolulu: University of Hawai'i Press, 2008), pp. 247-292.

10 George J. Tanabe, Jr., "The Orthodox Heresy of Buddhist Funerals." In Death and the Afterlife in Fapanese Buddhism, edited by Jacqueline I. Stone and Mariko Namba Walter (Honolulu: University of Hawai'i Press, 2008), pp. 325-348.

11 Andrew Bernstein, Modern Passings: Death Rites, Politics, and Social Change in Imperial Japan (Honolulu: University of Hawai'i Press, 2006), p. 42.

12 Mark Michael Rowe, Bonds of the Dead: Temples, Burial, and the Transformation of Contemporary Japanese Buddhism (Chicago, IL: Chicago University Press, 2011), p. 24.

13 Ibid., p. 24.

14 Bernstein, Modern Passings, p. 68.

15 Hikaru Suzuki, The Price of Death: The Funeral Industry in Contemporary Fapan (Stanford: Stanford University Press, 2000), p. 171.

16 David W. Plath, "Where the Family of God Is the Family: The Role of the Dead in Japanese Households," American Anthropologist 66, no. 2 (1964), p. 302.

17 John Nelson, "Household Altars in Contemporary Japan: Rectifying 'Buddhist Ancestor Worship' with Home Décor and Consumer Choice," Japanese Fournal of Religious Studies 35, no. 2 (2008), p. 314.

18 Margaret Lock, Twice Dead: Organ Transplants and the Reinvention of Death (Berkeley, CA: University of California Press, 2002), pp. 206-207. 
19 William R. LaFleur, Liquid Life: Abortion and Buddhism in Fapan (Princeton, NJ: Princeton University Press, 1992).

20 Akihiko Kato, “'Chokkei kazokusei kara fūfu kazokusei e' wa hontō ka." In Kōhōto hikaku ni yoru sengo Nihon no kazoku hendō no kenkyū, edited by Kumagai Sonoko and Ōkubo Takaji (Tokyo: Hihon kazoku shakai gakkai zenkoku kazoku chōsa iinkai, 2005), pp. 139-154. "The Japanese Family System: Change Continuity, and Regionality over the Twentieth Century." Max Planck Institute for Demographic Research (March 2013). www.demogr.mpg.de/papers/work ing/wp-2013-004.pdf. Accessed September 21, 2017.

21 Naoko Igarashi and Mitsunori Miyashita, "Dētā de miru Nihon no kanwa kea no genjō," Hosupisu kanwa kea hakusho (2017), pp. 78-107. Shōni kanwa kea no genjō to tenbō, edited by Shima Yasuo, Tsuneto Satoru, Hosokawa Toyofumi, Miyashita Mitsunori, and Yamazaki Fumio (Tokyo: Seikaisha, 2017).

22 Personal interview, March 5, 2015.

23 Fumio Yamazaki, Dying in a Fapanese Hospital, translated by Yasuko Claremont (Japan: Japan Times, 1996), p. 51.

24 Igarashi and Miyashita, "Dētā de miru Nihon", pp. 74-111.

25 Satsuki Kawano, Nature's Embrace: Fapan's Aging Urbanites and New Death Rites (Honolulu: University of Hawai'i Press, 2010), p. 13.

26 Hannah Gould, Tamara Kohn, and Martin Gibbs, "Uploading the Ancestors: Experiments with Digital Buddhist Altars in Contemporary Japan," Death Studies 43, no. 7 (2018), pp. 456-465. DOI:10.1080/07481187.2018.1544948.

27 Susan Orpett Long, Final Days: Fapanese Culture and Choice at the End of Life (Honolulu: University of Hawaici Press, 2005), pp. 54-55.

28 Personal interview, June 4, 2015.

29 Fleur Woss, "Pokkuri Temples and Aging," Religion and Society in Modern Fapan, edited by Mark R. Mullins, Susumu Shimazono, and Paul Swanson (Fremont, CA: Asian Humanities Press, 1993), pp. 191-202.

30 Timothy Benedict, "Meiwaku o kaketaku nai hosupisu kanja to supirichuaru kea," Supirichuaru kea kenkyu 3 (2019), pp. 43-53.

31 Personal interview, March 25, 2015.

32 Carl B. Becker, "Aging, Dying, and Bereavement in Contemporary Japan," Frontiers of Japanese Philosophy 4: Facing the 21st Century, edited by Wing Keung Lam and Ching Yuen Cheung (Nagoya: Nanzan Institute for Religion and Culture, 2009), p. 97.

33 Yoshimasa Kanawaku, "Koritsu (Kodoku) shi to sono jittai," Nichiidai ikaishi 14, no. 3 (2018), pp. $100-112$.

34 Lock, Twice Dead, p. 228. 


\title{
PICTURING THE DEAD IN EARLY TWENTIETH-GENTURY GHINA
}

\author{
Bodies, burial, and the photography of the Chinese \\ Red Cross Burial Corps
}

\author{
Caroline Reeves
}

\section{Introduction}

In China's Red Cross publications of the 1910s, 20s and 30s, photographs of the grisly work of China's Red Cross Burial Corps figure prominently. Chinese Red Cross workers standing with dead bodies in various stages of interment are featured in the pages of Red Cross magazines, pamphlets, and commemorative editions. The specter of the dead and their proper treatment is raised again and again visually and textually to serve as a call to national philanthropic action. An anomaly in Red Cross agendas outside of China, these signature burial activities - and their representation through photography - were part of the work of an indigenously conceived and organized Chinese Red Cross Society formed in Shanghai in 1904. Dedicated to situating China as a "modern" participant in international humanitarianism, this transnational group also insisted on including the dead in their endeavors.

This chapter interrogates the photographic images of the dead in Chinese Red Cross publications, particularly in dialogue with the publications' texts. Some scholars have mistakenly suggested that Chinese photographers did not focus on dead bodies. "Even in the twentieth century, photography [in China] never exhibited the body or the coffin of the deceased," writes Henriot. " The photography of the Chinese Red Cross shows otherwise, and these dramatic images demand voice. How can we understand this new exposure of the dead? How was it meant to be experienced by viewers, potential Red Cross members, donors? In wartime, when the sight of actual corpses was a commonplace, how were these photographic images supposed to invoke charitable action? Specific photographs will be examined to investigate what China's charitable elite intended to project with these visions of corpses and their handlers, giving interpretive depth to these images beyond their service as historical evidence. In deciphering what these images were meant to accomplish, this chapter reveals aspects of death in China on the eve of the modern era.

\section{The Ghinese context}

At the start of the twentieth century, China's natural and political landscapes were in flux. Natural disasters - floods and droughts, famines, and other catastrophes - occurred with alarming frequency. Between 1876 and 1879, nine to thirteen million Chinese died 
in the deadliest drought-induced famine in China's history. ${ }^{2}$ Within twenty years, famine hit again, and twenty years after that, China would lose another half a million people in another North China Famine. ${ }^{3}$ This dire natural situation was exacerbated by round upon round of civil violence as China's leaders and common people experimented with alternatives to the ancient dynastic system. The quasi-religious Taiping Rebellion (1850 1865) left 20-30 million Chinese dead in history's most lethal civil war. ${ }^{4}$

Even after the catastrophic Taiping Rebellion, internal unrest did not stop in China. Uprisings - religious, political, and economic - continued across the centuries' divide, leaving bodies in their wake. China's 1911 Revolution brought down the Qing Dynasty (1644-1911) but did not end the struggle for political power in China. Imperialism and geopolitics encroached on China, and these escalating external threats worsened domestic volatility. Poverty, political instability, social conflict, and increased mortality became the norm for many Chinese, of whom over 90\% were rural peasants. Corpses became a commonplace, littering China's landscape. ${ }^{5}$

Obviously, China was no stranger to death at the start of the twentieth century. In the first century of the Qing Dynasty (1644-1911), life expectancy at birth for a Chinese male was 27.2 years; for a woman, it was even lower, at 24.6 years. ${ }^{6}$ By the midnineteenth century, a man could expect to live to 34.7 years old, and by the late $1920 \mathrm{~s}$, his life expectancy had risen to 41.3 years, and women's to $36.4 .^{7}$ In a $1929-1931$ survey of rural China, the top known causes of death in China's countryside - in years of relative peace-were smallpox, typhoid, dysentery, tuberculosis, cholera, measles, and suicide. ${ }^{8}$ Violence, drought, floods, and famine worsened these numbers.

The prevalence of displaced corpses scattered around the countryside and in the streets of China's cities during these upheavals was profoundly unsettling to the Chinese. As JaHyun Kim Haboush points out in her work on seventeenth-century Korean war victims, "Discarded dead bodies ... signify the total breakdown of order, the failure of that fundamental requirement of civilized life - that the living should tend to their own dead." "In late imperial China, the imperative to bury the dead was keenly felt at all levels of the polity, from individual family units to the government itself. As J.J.M. de Groot wrote in his classic The Religious System of China, "both the body and soul require a grave for their preservation." 10 Treating the dead well with an appropriate burial was important, and not necessarily altruistic. Within the Chinese family, the dictates of ancestor worship meant that there was a direct link between the care of the dead and the well-being of the living. It was in an individual's self-interest to see their dead well cared for, because the spirits of the dead were known to "return to do harm to those who [did] not observe the proper rites. The spirits of those who die without anyone to care for them are believed to become wandering spirits who go about doing mischief, especially after sundown." "1 The content of this traditional imperative, then, was both selfish and selfless: protecting both the living and the dead.

In late imperial China, the proper manner to dispose of the dead was unquestionably burial. Confucianism emphasizes that the body is a gift from one's parents, whole and perfect, which must not be mutilated in life or death. This belief informed the convention of burying whole bodies. Even eunuchs were expected to save their castrated body parts so that they could be buried whole. Given this stricture, options such as cremation were not culturally acceptable to most Chinese. From the Song dynasty (960-1279) on, cremation, a relatively new practice for China introduced by and for Buddhists, had been increasingly prohibited in China, and the Confucian state officially mandated burial for 
the dead. ${ }^{12}$ Confucians considered cremation barbaric and unfilial. Later, in the China of the 1930s, cremation was promoted on hygienic grounds, but the practice was not widely adopted. More recently, in the late twentieth and early twenty-first century, the Communist government in the PRC and Hong Kong has insisted on cremation as a means of disposing of corpses, given the burgeoning population, the cost of land, and the desire for real-estate development sweeping Mainland China and Greater China. Nonetheless, burial remains the death rite of choice across much of Greater China.

Burial was traditionally such an important ritual for the Chinese that the imperial government became involved in its guarantee. Although ideally the deceased would find their final rest through the auspices of the family, the state was seen as the ultimate guarantor of correct burial. The state's primary responsibility was to ensure that the laobaixing-China's so-called one hundred names, its vast rural population- had the resources to take care of their departed. But bodies beyond the reach of family were also not to be ignored. Instructions to Qing dynasty magistrates, included in handbooks such as A Complete Book Concerning Happiness and Benevolence and in legal statutes, extended the state's responsibility to provide for unclaimed bodies. ${ }^{13}$

Between family and state also lay a range of options for those at risk of dying away from home or without the means for proper burial. Native-place merchant huiguan (guilds), sometimes associated with changsheng hui ("Longlife Associations," the euphemism for funerary associations), took responsibility for shipping bodies or bones to their native place, providing for proper burial, or for arranging worship for deceased members whose families could not be found to perform those duties. ${ }^{14}$ For the truly indigent, charitable benevolent societies (shantang) provided burial services. ${ }^{15}$ Government officials recognized these groups with state honors. Qing officials wrote, "When public-spirited men have given burial to a great number of corpses of the poor, or have covered up and interred considerable quantities of withered bones, then the authorities shall scrutinize the act and then reward it." 16 Burial was an important part of life in late imperial China, and resources at all levels of the polity were marshaled to make sure that no bodies were left uncovered. Thus by 1936, according to one estimate, "one-eleventh of the arable land of China [was] ... occupied by graveyards." ${ }^{\prime \prime}$ When the Chinese Red Cross emerged on the scene with its own burial corps in the early twentieth century, it was not actually doing something new; it was the way they presented what they did that was intended to be new.

\section{The Chinese Red Cross Society}

During the Russo-Japanese War (1904-1905), the first Chinese Red Cross Society was established by a group of Shanghai elites. This society, named the International Red Cross Society of Shanghai, was an indigenous Chinese innovation informed by, but not yet officially affiliated with or recognized by the Swiss-based International Committee of the Red Cross (ICRC). ${ }^{18}$ Renamed the Chinese Red Cross Society in 1911, it was recognized by the ICRC in 1912 and became an official affiliate of the international organization at that time.

The International Committee of the Red Cross had been conceived by Henri Dunant in Switzerland in 1863 as a charitable organization aimed at helping wounded soldiers on the battlefield. By the turn of the twentieth century, national Red Cross Societies linked to the international group were beginning to dot the world, spreading their goal of 
marshaling medical volunteers to dispense neutral humanitarianism on battlefields around the globe. ${ }^{19}$ Eventually turning to peacetime work as well as to medical activities during civil war, the Red Cross organization became globally known by World War I.

Shen Dunhe (Shen Zhongli), a Shanghai tea merchant well connected to China's international community, was the motivating force behind the creation of the new Red Cross Society in China. Shen had studied international law in England, ${ }^{20}$ and his knowledge of the Red Cross organization and of the neutrality provided under the Red Cross aegis and guaranteed in the Geneva Convention of 1864 informed his decision to choose the Red Cross flag for his new group. Shen used his extensive personal connections to create a joint Red Cross organization made up of prominent Westerners as well as wealthy Chinese, which ensured access to the sensitive war zones between Russian and Japanese forces, financial and medical support, and international respect and recognition. ${ }^{21}$ Qing dynasty officials (and later, Republican dignitaries) were made honorary presidents and vice presidents of the organization. ${ }^{22}$ Shen's efforts in the RussoJapanese War were extremely successful, and the new Red Cross Society, aided by government officials, Chinese gentry, and the Western medical community ultimately evacuated over 130,000 refugees from Manchuria and provided relief to over a quarter of a million people stricken by the war. ${ }^{23}$

Shen's initiative was based on an age-old charitable tradition in Chinese society. The impetus for charitable activity - for the living and the dead-was well established in Chinese culture, and philanthropy had been practiced since well before the birth of Christ. Charitable work did not suddenly arrive in the late nineteenth century with Christian missionaries, as many Westerners suggested in the early twentieth century. ${ }^{24}$ Charities in China traditionally had been formed for the burial of the dead, the care of widows and orphans, the founding of schools for indigent children, the provision of medicines and medical care for the poor, and the distribution of winter clothing to peasants and refugees, as well as for saving animals' lives, preserving sacred texts, providing life boats at dangerous river crossings, building schools, caring for ancient sites, and spreading the words of Confucius, Buddha, and the Emperor. ${ }^{25}$

China had boasted mutual-aid societies from the Han Dynasty on (206 BCE-220 CE). By the fifth century CE, voluntary associations composed of common people were formed to pool resources in the face of crises, such as an unexpected death necessitating hard-to-come-by funds for a funeral. By the Ming Dynasty (1368-1644), China's intellectual elite, a Confucian-trained oligarchy connected intellectually and practically to government service, appropriated the idea of such voluntary associations, and "resituated [them] in ... an elite milieu." ${ }^{, 26}$ Appropriating philanthropy as an expression of elite culture, scholars, officials, and merchants aspiring to social status used involvement in charitable enterprises and philanthropic societies to garner social capital and acquire merit-both religious (according to Buddhist tenets) and official (in line with Confucian conventions). ${ }^{27}$ These philanthropic institutions and the myriad societies that were formed in the next three centuries of the Qing Dynasty were encouraged by and reinforced the imperial government, but were privately run, despite the fact that they acted as an arm of the government. They provided Max Weber's informal "liturgical governance," 28 where "local elites were called upon to perform important public services on the state's behalf, at their own expense." ${ }^{29}$ Many of these charitable enterprises involved burying the unclaimed dead, a central prerogative of Chinese charitable organizations from the seventeenth century on. ${ }^{30}$ 
The Red Cross Society, taking its precedents from China's already well-established charitable tradition, styled itself as a modern innovation in China. Using age-old networking strategies, fundraising techniques, and motivational tactics, the Red Cross called on China's gentry to shoulder the burdens of people disrupted by catastrophe. None of that was new. But the organization's international ties, the bio-medicine that it dispensed in its "Western-style" hospitals, its national scope, the rhetoric it espoused, the military trope of its operations (for example, the white Western military-style uniforms adopted by founder Shen Dunhe and by subsequent directors of the Society), and its use of new technologies - including photography - to perform its functions and to advertise its cause, all bespoke a new kind of charitable association, one that its founders touted as "modern" - an important word in early twentieth-century China, pointing to a connection with a cosmopolitan world and a progressive mindset far beyond China's ancient imperial system. The Chinese Red Cross Society was meant to be one of these new kinds of social welfare institutions.

The Society's work was so popular and the need for its services so acute that rather than disbanding at the end of the Russo-Japanese war, the Red Cross in China continued its operations and continued to grow. Increasingly staffed by the Chinese themselves, replacing Western missionaries and medical men, the organization built on its initial successes to become the Red Cross Society of China. By 1924, there were 286 Red Cross chapters in 32 regions, from Sichuan to Shanghai, Jilin to Canton. ${ }^{31}$

\section{The Chinese Red Cross Burial Corps}

Although the Chinese Red Cross started its operations by focusing attention on the living, the 1911 Revolution and the resulting devastation inspired the group to embrace the dead as well. In 1911, with the outbreak of the Revolutionary War that brought down the imperial system, the Chinese Red Cross Society organized its first formal Burial Corps (yanmaidui). The Burial Corps collected corpses and body parts and buried them as appropriately as possible, providing shrouds and coffins, digging and marking graves, and noting the names of the deceased whenever possible. During its inaugural war, this new group buried over 8,000 corpses. ${ }^{32}$ These Burial Corps played a significant role in the Chinese Red Cross organization and were extremely well documented and publicized in the Society's literature, commemorated in its poetry, and vividly rendered in striking photos of the Corps' undertaking. The prominence of these Burial Corps merged two important imperatives in Republican China: the traditional imperative to bury the dead, and the more "modern" military and public health imperative to remove and "disinfect" potentially disease-producing corpses. ${ }^{33}$ The Chinese Red Cross Society played on both of these imperatives, focused equally on the living and the dead.

In the Second Revolution of 1913, the Red Cross Burial Corps continued their activities. Austrian journalist and former soldier Stephen Piero Rudinger described the work of the Burial Corps around the Jiangnan Arsenal:

Many of the bodies were partly devoured by stray starving dogs which swarmed the battlefields. The burial parties found innumerable loose bones, jaws, skulls, ribs and hipbones, torn away from the bodies by the hungry beasts, giving a terrible feature to the fights ... To collect the bones, the coolies used special [sic] constructed hooks, observing the same precautions as when finding 
a corpse, and having collected a sufficient number of [bones], they placed them in coffins, piled them up and covered them by a mound of mud. ${ }^{34}$

The Burial Corps frequently dealt with bones, rather than corpses. In China, burial of bones was particularly important, since the dry bones of the deceased would eventually represent the ancestors. The ritual reburial of bones (practiced not only in China, but in many parts of the world), purged of the polluting flesh, became the critical final interment. This custom meant that even the scattered bones were important targets of the Burial Corps' work. ${ }^{35}$ The urgency was clear. By 1914, the Burial Corps and their work were institutionalized as one of the main charges of the Society. ${ }^{36}$

In a 1912 report prepared for the Ninth International Conference of Red Cross Societies in Washington, DC, ${ }^{37}$ Shen Dunhe described the Chinese Red Cross burial parties for a foreign audience. This branch of the Chinese Red Cross's activities, so central in the Chinese field, was unknown to other national Red Cross Societies. ${ }^{38}$ Writing for a Western audience, then, Shen focused on the public health aspect of burial parties. Shen's rhetoric in his report alludes to contemporary scientific ideas that foul disease was formed by "contagions of the air," 39 an idea that dovetailed with traditional Chinese medical notions, and the ubiquitous (but incorrect) belief that dead bodies were powerful agents of disease. ${ }^{40}$ Shen's report also makes mention of Chinese donors' influence in determining the Society's decision to undertake the work of burying the dead. Donors were clearly compelled to give to the Society by the group's performance of this task, ${ }^{41}$ even though, as Shen points out, Red Cross Societies of other countries did not usually do that kind of work. Although an expression of humanitarianism specific to China, Shen framed the activities of China's Red Cross Burial Corps as congruent with modern, scientific, "Civilized" practices. ${ }^{42}$

\section{The role of images in Red Gross activities}

The Burial Corps became a permanent part of the Chinese Red Cross organization, and their work one of main foci of Red Cross photography. This photography appeared in a flurry of new publications that appeared as the new group expanded and became institutionalized after the 1911 fall of the dynasty. In its periodicals, the newly reorganized Society mobilized images to publicize and fund its activities, to attract members and donors, and to inform the world community about its work. These visual representations appeared most frequently in publications produced by the Red Cross itself, although photographs of Red Cross work also appeared in local newspapers and magazines. ${ }^{43}$ The introduction of modern printing presses imported by missionary groups in the $1870 \mathrm{~s}$, the technology of half-tone printing, and the development of the portable camera (George Eastman's Kodak, available after 1888 in the West and shortly thereafter in Asia) had made the expansion of photography increasingly possible and affordable in China. ${ }^{44}$ China's new Red Cross Society used these technologies and their resultant images to establish its own credibility as a charitable organization and to create an identity for itself as a modern and national organization. The spectacle of bodies and bones being collected for burial made particularly vivid material for Red Cross publications.

China's print culture went back to the seventh century CE, fostering the circulation of printed material, including official news gazettes, novels, histories, genealogies, letters, poetry, pornography, classical literature, and exhortatory pamphlets. Paper had been 
invented in China in the Later Han Dynasty (25-220 CE), and woodblock printing in the Sui (589-618) and Tang (618-907). ${ }^{45}$ Traditionally, print technologies had often been used in the service of philanthropy, and vivid imagery played a significant role in promoting worthy causes. In 1864 at the end of the Taiping Rebellion, Confucian scholar Yu Zhi produced a pamphlet called "A Man of Iron's Tears for Jiangnan," featuring 42 woodblock images directed at raising money for refugee relief in the Southern Yangtze area. These illustrations ranged from scenes of death, decapitation, and cannibalism to utopian images of Southeast China rebuilt and Confucian order restored. ${ }^{46} \mathrm{Yu}$ Zhi's influential work became an inspiration for future philanthropists, and in the Great North China Famine of 1876, philanthropists intentionally cribbed both the name of his pamphlet and many of his illustrative tropes for a deliberately similar charitable appeal. ${ }^{47}$ The 1878 version of Tears from Iron inspired a front-page article in the Chinese-language, Chinese circulation newspaper Shenbao, particularly for its use of images. "Why use [these images] to encourage donations for each province afflicted with flood and drought? Because, though there have been many articles written to encourage relief, only the literate can understand them."48 The power of the image was apparent to all, whether they were reading the accompanying prose or just taking in the pictures. Thus, well before the spread of photography in China, images of the dead and suffering were already in service to humanitarian goals.

Interestingly enough, despite encouragement from Japanese Red Cross advisor Ariga Nagao in 1912 to "publish images of flesh flying about"49 and China's own tradition of using dramatic images in fundraising, the new Red Cross photos did not focus on the gore and tragedy of death. Instead, the images consistently highlight Red Cross workers in action, performing to ameliorate catastrophe. Photos of Red Cross workers picking up bodies for burial feature most prominently. ${ }^{50}$ For example, in a random sample, the Red Cross Monthly Number 3 from October 1921 publishes sixteen photographs, of which five show Red Cross workers burying bodies. ${ }^{51}$ The focus in these shots is unswervingly on the actions and efficacy of the Chinese Red Cross Society's service to its constituents, alive and dead, rather than on the pathos of the scene. Red Cross uniformed personnel, in their white coats marked with Red Crosses, ${ }^{52}$ or traditionally dressed Chinese in long gowns carrying a white flag emblazoned with the Red Cross symbol, dominate the photographs. Rather than emphasizing suffering, these images stress the actors and actions being taken to remediate disaster and to relieve distress, treating the living and dead the way their audience understood they should be treated (see Image 15.1).

These photographs fit with a more general cultural call for action resounding across China. According to a growing cadre of public intellectuals voicing their opinions increasingly stridently in China's public forums, China's people needed to "wake up," to shake off their moribund natures, to embrace a new world, to become more active in determining their own fates. ${ }^{53}$ In $A$ Call to Arms (Na Han), famous writer and public intellectual Lu Xun likened China's nascent citizenry to a group of sleeping people trapped in an iron house that had just caught fire. He suggests that while waking the inhabitants in an attempt to save them from death might prove futile, those who could do so had a responsibility to try to rouse them. This "call to arms" was felt across the polity and strengthened a national commitment to social welfare provision for all of China's people. $^{54}$ The Chinese Red Cross leaders were part of this wave, and the visual humanitarianism they promoted reflected the appeal for action galvanizing Chinese citizens across the fledgling Chinese nation. 


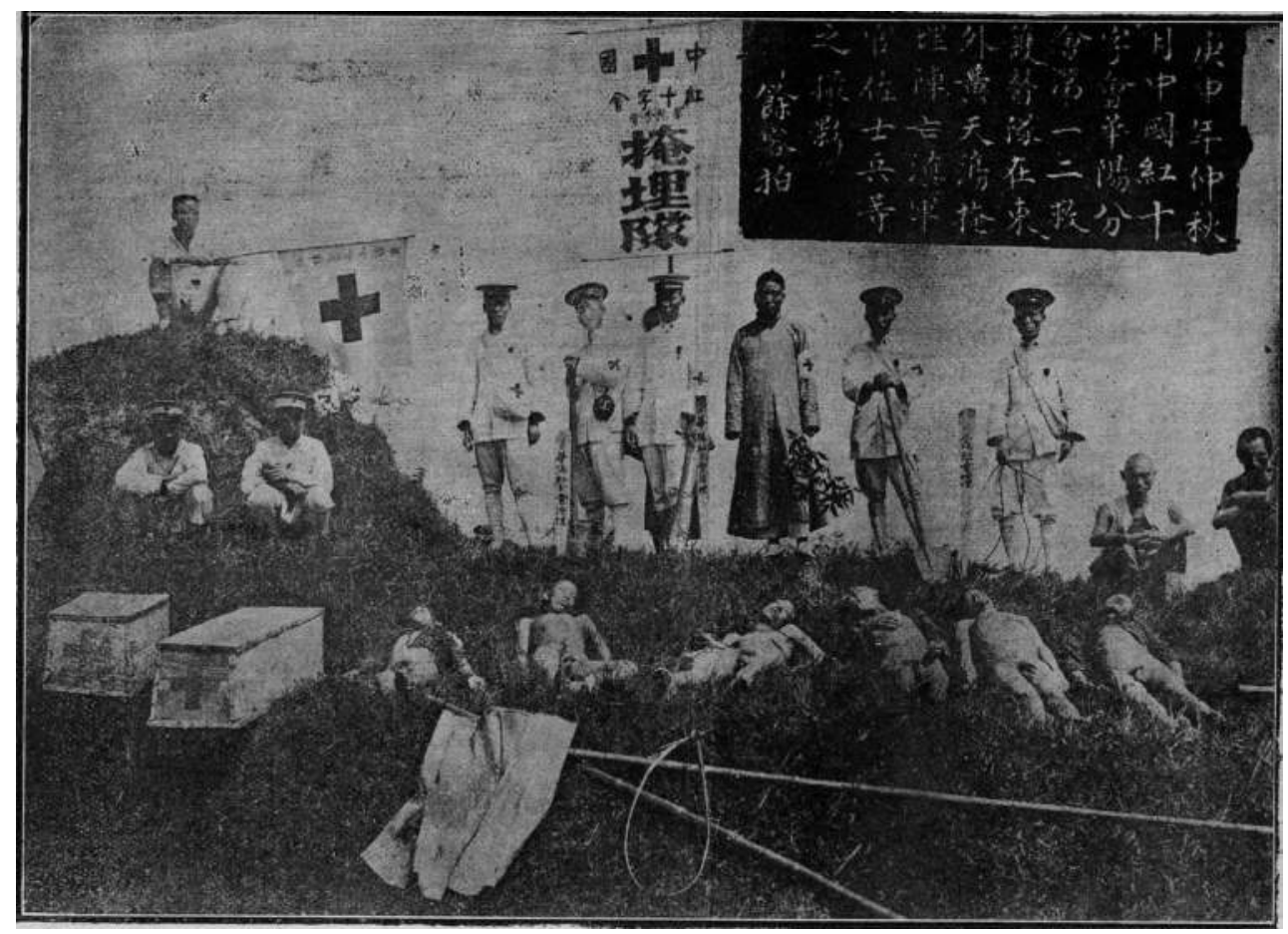

Image 15.1 Chinese Red Cross Society Burial Corps.

Thus, the directors of the Society saw images of relief work as a powerful tool for the production of nationalist feelings that would generate a concrete, participatory reaction to the Society and to relief work in general. The evocation of compassion, of sympathy, of feelings of noblesse oblige was not the primary goal of these photos, unlike the contemporary photographic repertoire of the international community. ${ }^{55}$ In the Chinese images, the viewer was to become a participant in the relief action, not just a spectator of suffering. Partaking of the image, the viewer was to identify with the active provision of aid to fellow Chinese, rather than to identify with the victims or to remain a passive audience, witnessing suffering with all the compromises inherent in that act of viewing pain. The provision of relief was a nationalistic act. Red Cross volunteers - and donorscould join in, viewing Red Cross images and then donating money to the Red Cross cause. This outspoken recognition that witnessing death and horror is not necessarily enough to move people to action - particularly not people who have been surrounded by and perhaps inured to massive death and horror-helped inform the new Chinese approach to humanitarian photography.

\section{Picturing the dead}

How did the dead figure in Chinese Red Cross publications and in the Red Cross "call to arms"? Here, I will discuss a number of photographs to develop that point. In the 1924 Twentieth Anniversary Commemorative Volume of the Chinese Red Cross Society (Zhongguo 
Hongshizihui Ershizhounian Finiance), a large section of the volume is dedicated to photographs. Taken over more than a decade, these photographs are collected from regional chapters across China. Their inclusion in the volume suggests that the directors considered these photographs to be representative of the work of the Society over the past twenty years, or as particularly efficacious in communicating the Red Cross message. Many of these photos had appeared earlier in the Red Cross Monthly magazine, published through the early 1920 s.

Photographs command their own category in the table of contents. The photography section is then further divided into three groups: Photographic Portraits, Photographs of Hospitals, and Photos of Relief Efforts. Within the third category, photos of burial work are conspicuous. For example, a triptych of photographs of the Hankou Chapter Number One Rescue Corps fills a two-page spread. The group, although nominally a rescue corps, not a burial corps, is clearly "burying dead soldiers" as it says in each caption. ${ }^{56}$ Immediately obvious to the viewer is the number of Red Cross workers on the scene and their seriousness of purpose. That not all Red Cross workers are the same is apparent from their uniforms. The volunteers wear white military-style uniforms with military caps marked with the Red Cross, but the coolies wear dark tunics, with a large red cross painted in a white circle emblazoned on the front and back. ${ }^{57}$ In all three photos, the Red Cross personnel fill three-quarters of the frame. Corpses appear explicitly only in the second photo. In the first photo, seven workers appear, four in natty white uniforms carrying white flags with the red cross clearly visible, with the coolies artfully arranged around them to set off the composition. One coolie is holding an enormous Red Cross banner. Standing, sitting, and squatting, some with arms akimbo, they pose above two burial markers planted in the newly turned earth.

The next photo is much less artful. It shows nine Red Cross coolies lined up with two white-uniformed workers bearing Red Cross flags, standing over three stretchers bearing corpses. Three half-dressed men stand closest to the bodies, two carrying poles, maybe shovels. One of these men is poking the nearest corpse with the pole. Although the corpses are in the foreground of the photo, the horizontal band of men bisecting the photo dominate the visual field. The corpses seem to be dressed or swathed in white cloth, and although their limbs are clearly visible, their faces are not. Without the caption telling us these are dead soldiers, it would not be clear who these corpses are. The Red Cross workers are explicitly poised - and posed - to do their work. Finally, the third photograph is another beautifully composed shot, with three Red Cross workers in white uniforms and flags standing around a fresh but neat burial mound, topped with a labeled grave marker. Here the corpses are no longer visible; the completed work of the Red Cross Corps is the final image.

Another photo in this section is both more subtle and more obvious. In this photo, neither the caption nor the photo contains the words or symbol of the Red Cross. The photo is simply labeled "Zhijiang Chapter Burial Accomplishments (chenji)" (Image 15.2). Posed on a hill, the photo presents ten corpses, four lined up along the base of the photo, four more heaped in the midground, and two more hogtied on poles, carried by four coolies wearing traditional coolie hats. The bodies are disheveled, half naked, and emaciated. Yet despite the predominance of the actual bodies in the photo, because of the arrangement of the corpses, the focus is on the fact that they are being moved, transported to a (presumably) proper burial, taken care of by the Zhijiang Red Cross. The focus is on the actions being taken, rather than on the pathos of the dead. 


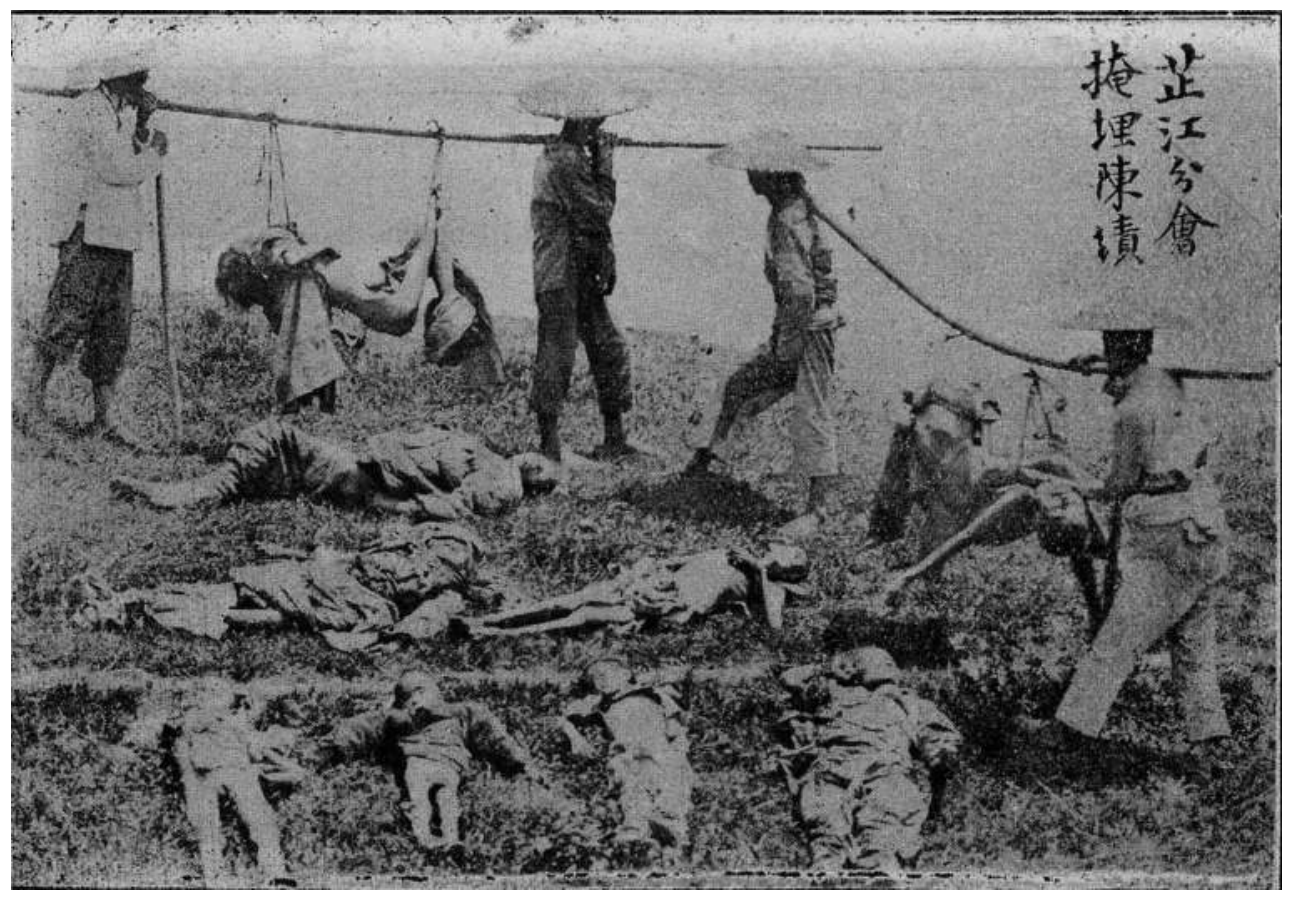

Image 15.2 Zhijiang Red Cross Chapter burying the dead.

Another photo is particularly striking. In this one, a skeleton sits up in the center of the photo, "staring" fully at the camera (Image 15.3). Whereas in most photos, the dead are not the main focus of the shot, here the skeletal dead takes front and center. Despite this centrality, however, the photographic narrative once more pulls the viewer back to the Red Cross actors in the frame and the actions they are performing. A member of the Burial Corps is touching the skeleton, patting it on the shoulder. This avuncular gesture communicates an explicit link between the Red Cross worker and the bones. Patently staged, the photograph features a Red Cross worker who is probably actually a paid coolie, rather than one of the elite volunteers. He seems to have put aside his straw peasant hat for the more formal, military cap worn by the Corps members standing safely behind the earth bulwark. Moreover, the military coat this Red Cross worker wears is less convincingly his once the observer notes that he is wearing neither pants nor shoes, which would normally match the formal uniform. But regardless of these artistic oversights, the photo makes its point. The Red Cross workers are there both for the living and for the dead, a surrogate family in extremis.

Portrayed in Red Cross photographs such as these, the Red Cross's new vision (literally) of citizens coming together as family - a national, Chinese family-brought the living and the dead into a common project of memorializing an old society and creating a new one. This photographic manipulation is not unusual in war photography. ${ }^{58}$ American Civil War photographer Alexander Gardner similarly arranged his subjects - including corpses - to convey carefully scripted messages about late nineteenth-century America. ${ }^{59}$ Yet the thrust of the Chinese photos is quite different from their American 


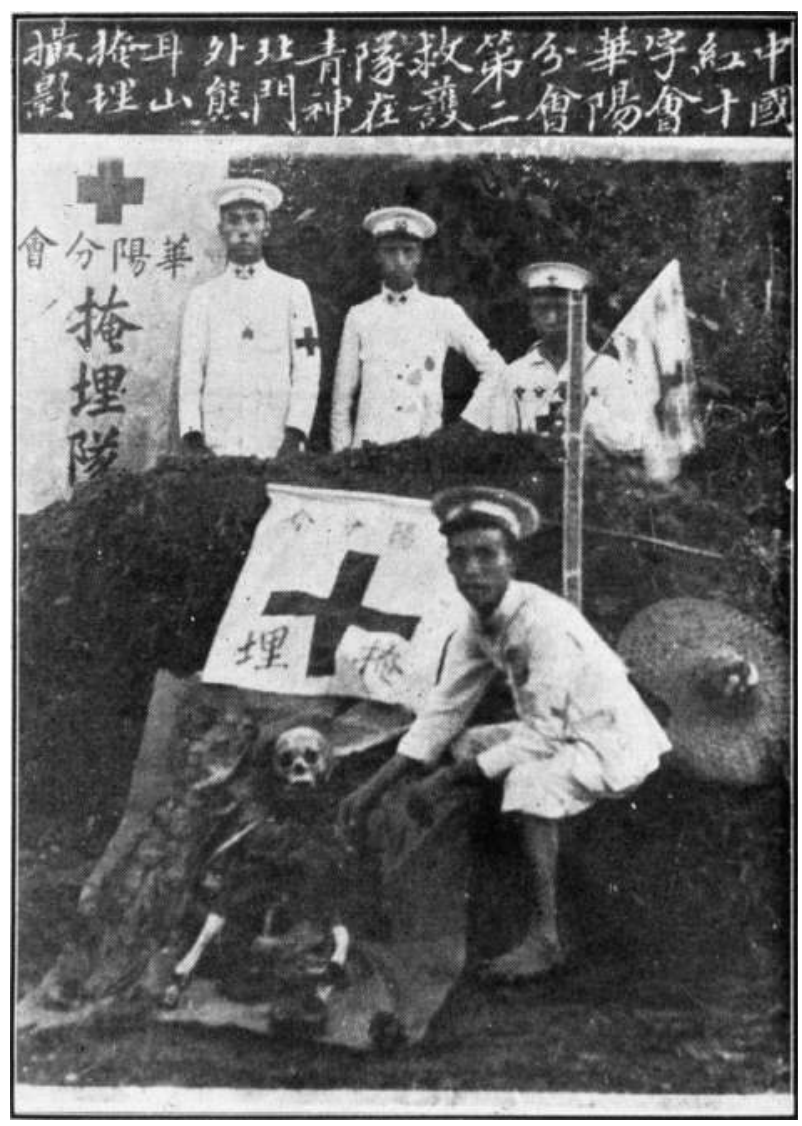

Image 15.3 Red Cross Burial Corps caring for the unclaimed dead.

counterparts. Many of Gardner's photos focus on the dead themselves and emphasize the pathos of war, the tragedy, and the waste. Unlike the American photos, the Chinese Red Cross photos of the dead do not focus on the corpses - instead, they focus on the work the living are doing for the dead.

Photographs of the Red Cross Burial Corps at work conveyed a specific message about the role and responsibilities of the Red Cross Society and of the viewers-cumdonors in times of mass mortality. That mortality was not to be dwelt upon as tragic or pathetic. Instead, it was a call to action, and the photographs - of men carrying coffins, collecting dead on poles, placing markers over newly dug graves, gathering bones to form full skeletons to be buried in ritually correct ways - were meant to inspire people to support those actions by donating money to the Society. As one of the Red Cross's first publications announces on its cover: "If you are not moved to give to the Society by this pamphlet, please give it to someone else who will." 60 These are fundraising and consciousness-raising publications, and the photos in them are meant to inspire the viewers to specific, concrete action: contributing to the cause. 
The very presence of photography also imparted another important message about the crises portrayed in Red Cross images and the Red Cross's role in them: these were modern events, featuring modern actors, whose actions were captured simultaneously with the events they were portraying, with a modern technology able to present images to an audience quickly and exactly. Visibility meant modernity, and the inclusion of a large population of viewers in the act of viewing the photos reinforced that modern vision and that vision of modernity.

\section{Chinese Red Cross images abroad}

Even beyond Chinese audiences, the usefulness of photographs in conveying the Society's accomplishments was clear to the Chinese Red Cross organizers from the start. Although the Chinese could and did present their accomplishments to the international community in English texts, photographs eliminated the mediation of language and provided the "collapsing" of distance between foreign viewers and Chinese relief work. In this case, however, rather than functioning as a fundraising strategy, the visual presentation of China's Red Cross work to foreign audiences was a "consciousness-raising" endeavor in the sense of the Chinese showing the international community its commitment to humanitarianism.

Due to the Chinese convention of writing directly on pictures (an ancient practice dating back to handscrolls, ink paintings, and continuing to photographs), the viewer today can see the discrepancy between the English captions and the Chinese captions, and thus can see distinctly where meanings were being manipulated for a foreign audience.

Apparently, the presentation of the images to Western audiences necessitated a very different tone and presentation than to Chinese audiences. Whereas Chinese photos were labeled with a straightforward set of information usually consisting of the date, the name of the Red Cross chapter and the unit involved (flood relief unit, burial unit, relief corps, medical unit helping wounded soldiers), and sometimes the action being viewed, the Westerntargeted captions were embellished, rather than translated, for the pictorials sent overseas. For example, a photo of eleven relief workers, eight in white Red Cross uniforms, two in traditional gowns, and one in a coolie's outfit, carrying a Red Cross Burial Corps placard and two Red Cross flags, standing over two corpses and a dead horse that appeared in the Twentieth Anniversary volume is labeled in Chinese: "the $22^{\text {nd }}$ of the third month of 1911 . Photo of this chapter [Xindu] on the battlefield relieving the wounded." The caption inscribed below the photograph in the Chinese Red Cross Activities Told in Pictures, re-published in English for a Western audience is, "They give up Lives; We give out Peace!!!"61

Another photograph in the English-language pictorial features a derailed train as backdrop and shows eight corpses on the ground. Two Red Cross workers, one smiling and holding a Red Cross flag, face the camera. Another worker walks with his back to the viewer towards the trains. The caption in Chinese reads: "Photo of Pingyuan Chinese Red Cross Chapter Relief Corps, in Wangyi Village, Rescuing Wounded Soldiers and Burying the Dead, 1926 [month and day unclear]." The English caption reads: "The sacred remains of the war heroes attended by our Burial Corps." The difference in language is not a stylistic difference between Chinese and English; often, Chinese Red Cross poetry about the work of the Burial Corps surpasses these English-language captions in flowery hyperbole, and poetry was not out-of-place inscribed on images in traditional China. ${ }^{62}$ But in viewing the photograph, Chinese viewers are left to their own devices without an interpretive script to devise meaning from what they are seeing. Foreigners, on the other hand, were understood to need more. 


\section{Conclusion}

Photography - from portraiture to commercial pieces - had become a commonplace in China by 1912. By the 1920s, vivid photographs of dead bodies from China's battlefields and disaster zones were animating Chinese charitable appeals and publications. How did Chinese philanthropists present the hapless victims of catastrophe whom they were trying to help, including those who had already lost their life? ${ }^{63}$

Denis Kennedy has written that "humanitarian fundraising appeals derive emotional force through their reliance on human misery." He calls this "the veritable commodification of suffering." ${ }^{64}$ Sensationalism, the fetishization of suffering, and the presentation of the needy as powerless victims were already well-established tropes in Euro-American visual humanitarianism by the early twentieth century. As in print journalism, where the mantra of the day was "if it bleeds, it reads," the spectacle of suffering seemed to be the most effective way to move Western - and Japanese - audiences to compassion and to generate funds for worthy causes. How did China's Red Cross photography of the dead fit in with this visual convention?

In Chinese humanitarian photography, the focus of the image was on the provision of aid - not on the wretchedness of the victims, alive or dead. Although there was no taboo or censorship regarding photographically (re)presenting the corpse, nonetheless, as a rule, the dead body was less interesting and less compelling to Chinese viewers than the activities surrounding it. ${ }^{65}$ The thrust of the visual message was meant to be identification with the aid providers, not with the victims of war and disaster. The time/space compression of the photograph allowed observers to participate in the burial of the dead with other Red Cross volunteers, and thus share in the performance of both the traditional good deed of burying the dead as well as the modern good deed of saving the nation. The photographic message of the Chinese Red Cross Society targeted a different set of motivational emotions than the visual humanitarianism that has become so familiar to us today. The corpse was not meant to be the galvanizing trigger to action; rather, the dramatic and meaningful work of the Red Cross Burial Corps was to be the clarion call to arms.

In all cultures, corpses occupy an unusual space between people and things. Like living human beings, or even more so than living beings, corpses should not be left lying about, even in times of major social, political, or economic upheaval. How they are treated and viewed is an extremely sensitive, and thus telling, subject. In the early twentieth century, burying unclaimed corpses became a national task-no longer a state task, but a responsibility borne by the nation and its members as a kind of surrogate family, whose fate was linked to that of the dead. Like the Red Cross volunteers themselves, these bodies became more than simply flesh and bones - they were symbols of a new national Chinese identity, a modern identity fusing both the traditional imperative of caring for the dead with the unquestionably modern dictate of participating in the nation and the wider world.

\section{Notes}

1 Christian Henriot, Scythe and the City: A Social History of Death in Shanghai (Stanford, CA: Stanford University Press, 2016), 7. Henriot contradicts his own assertion with a photograph featuring coffins he includes in his own book. Henriot, Figure 6.5, 253.

2 Kathryn Edgerton-Tarpley, Tears from Iron: Cultural Responses to Famine (Berkeley, CA: University of California Press, 2008).

3 Pierre Fuller, "North China Famine Revisited: Unsung Native Relief in the Warlord Era, 1920-1921," Modern Asian Studies 47.3 (May 2013): 820-850. 
4 Tobie Meyer-Fong, What Remains: Coming to Terms with Civil War in 19th-Century China (Stanford University Press, 2013).

5 Many foreign visitors and missionaries note this situation. Edward J. Dingle, China's Revolution 1911-1912: A Historical and Political Record of the Civil War (New York: McBride, Nast. 1912), 140; also Stephen Piero Sergius Rudinger De Rodyenko, aka St. Piero Rudinger, The Second Revolution in China, 1913: My Adventures of the Fighting Around Shanghai, the Arsenal, Woosung Forts (Shanghai: Shanghai Mercury, 1914), 13-14; Tobie Meyer-Fong also discusses the situation, Meyer-Fong, What Remains, 101-102.

6 Cameron Campbell, "Mortality Change and the Epidemiological Transition in Beijing, 1644-1990," in Ts'ui-jung Liu and James Lee, eds., Asian Population History (Oxford: Oxford University Press, 2001), 230.

7 Campbell, "Mortality Change," 230.

8 Judith Banister, China's Changing Population (Stanford, CA: Stanford University Press, 1987), 51.

9 JaHyun Kim Haboush, "Dead Bodies in the Postwar Discourse of Identity in SeventeenthCentury Korea," Journal of Asian Studies 62.2 (May 2003): 415-442.

10 J.J.M. de Groot, The Religious System of China (rpt., Taipei: Literature House, 1964), bk. 1: Disposal of the Dead, vol. 3: The Grave, 855. De Groot's work is an astonishing compendium of information on all aspects of death and burial in traditional China.

11 Mrs. J. G. Cormack, Everyday Customs in China (Edinburgh: The Moray Press, 1935), 89. De Groot discusses this phenomenon in book II, The Soul and Ancestral Worship (vols. 4 and 5). C.K. Yang also notes this connection in Religion in Chinese Society (Prospect Heights, IL: Waveland Press, 1991), 29.

12 Patricia Ebrey, "Cremation in Sung China," American Historical Review 95.2 (April 1990): 406-428.

13 Huang Liu-Hung (Djang Chu, trans. and ed.), A Complete Book Concerning Happiness and Benevolence: A Manual for Local Magistrates in Seventeenth-Century China (Tucson, AZ: University of Arizona Press, 1984) and de Groot, The Religious System, 1, III:866-934 for Qing statutes and their translations.

14 Elizabeth Sinn, "Moving Bones: Hong Kong's Role as an 'in-Between Place' in the Chinese Diaspora," in Sherman Cochran and David Strand, eds., Cities in Motion: Interior, Coast, and Diaspora in Transnational China, China Research Monograph 62 (Berkeley: Institute of East Asian Studies 2007), 247-271; Richard Belsky, "Bones of Contention: The Siming Gongsuo Riots of 1874 and 1898," Papers on Chinese History 1.1 (1992): 67-70; Bryna Goodman Native Place, City and Nation (Berkeley: University of California Press, 1995), 6-7.

15 De Groot, The Religious System, 1, III:863-866 discusses funerary societies.

16 Governor-General Wu Yingguang, Wu xuedu (A record of my studies) (Huguag Province, 1832), chap 2:1, 16, cited in de Groot, The Religious System, 1, III:866.

17 K. Chimin Wong and Wu Lien-Teh, History of Chinese Medicine, 2nd ed. (Taipei: Southern Materials Center, 1985), 762. James Ricalton, an early photographer visiting China in 1901 was overwhelmed by the implications of the huge number of graves occupying China's landscape. He wrote, "Humble graves, simple mounds covering heavy wooden coffins ... are sacredly preserved; they have been located by geomancy and that has first right, and land tenure second." James Ricalton, China through the Stereoscope: A Fourney through the Dragon Empire at the Time of the Boxer Uprising (New York: Underwood and Underwood, 1901), plate 66.

18 Caroline Reeves, "The Power of Mercy: The Chinese Red Cross Society 1900-1937," PhD dissertation (Harvard University, 1998), 96-103 tells this story.

19 For the most insightful history of the rise of the Red Cross organization, see John Hutchinson, Champions of Charity: War and the Rise of the Red Cross (Boulder, CO: Westview Press, 1996).

20 Tiaoshui Waishi, pseud. Shen Dunhe (Shanghai, 1911).

21 See Timothy Richard, Forty-five Years in China (New York: Frederick A. Stokes Co., 1916), 322; North China Herald, April 15, 1904, 798; and Shenbao, March 14, 1904 for various accounts of this event.

22 Photographs of these men became some of the first images used in fundraising appeals by the new Red Cross Society, heralding an age of (worldwide) celebrity sponsorship of charitable enterprises.

23 Shanghai International Red Cross Newes Bulletin (1938), 2. 
24 Caroline Reeves, "Lost in Translation: Local Relief Provision and Historiographical Imperialism," in New Global Studies 12.2: 277-292, DOI: https://doi.org/10.1515/ngs-2018-0033.

25 Joanna Handlin Smith, The Art of Doing Good: Charity in Late Ming China (Berkeley, CA: University of California Press, 2009).

26 Joanna Handlin Smith, The Art of Doing Good, 50.

27 Cynthia Brokaw, Ledgers of Merit and Demerit (Princeton University Press, 1991).

28 Susan Mann, Local Merchants and the Chinese Bureaucracy, 1750-1950 (Stanford, CA: Stanford University Press, 1986) develops this idea in her classic, particularly in chapter 2.

29 Mann, Local Merchants, 12-13.

30 Angela Leung says that these were "organized on a massive scale" from the 1600s on. See Angela K.C. Leung, "The Idea of Chuanran Contagion," in Leung and Furth, Health and Hygiene in Chinese East Asia (Duke, 2010), 44, particularly note 74.

31 Twentieth Anniversary Commemorative Volume of the Chinese Red Cross Society (Zhongguo Hongshizihui Ershizhounian Finiance; ZHEf) (1924), frontispiece.

32 ZHEJ, np.

33 The efficacy of this "disinfection" is unclear; it usually involves sprinkling yaoshui (Rudinger calls it "Jeyes' fluid"-ammonia?) on the bodies and at the site where the bodies were recovered and then spreading lime around the coffin or the location of burial: Rudinger. The Second Revolution, 13-14; Zhongguo Hongshizihui Tianjinfenhui Dierci Baogao (Tianjin: Zhonguo Hongshizihui Tianjinfenhui, 1914), 25.

34 Rudinger, The Second Revolution, 13-14.

35 See Katherine Verdery, The Political Lives of Dead Bodies (New York: Columbia University Press, 1999), 45, for Eastern Europe; James L. Watson, "The Structure of Chinese Funerary Rites: Elementary Forms, Ritual Sequence and the Primacy of Performance," in James L. Watson and Evelyn Rawski, Death Ritual in Late Imperial and Modern China (Berkeley: University of California Press, 1988), 113-114.

36 See Chinese Red Cross organizational charts, Hongshzihui Zazhi 1914, reprinted in Hongshizihui Yuekan 1921.

37 Red Cross Society of China, 1912 Report (Shanghai: Red Cross Society of China, 1912). English Language.

38 Information Bulletin (Geneva: League of Red Cross Societies), 6 (1927):170. The international body found it interesting enough to give it special mention in its "China" section: "A special department has been organized for giving proper burial to the dead in time of epidemics or disasters." The ICRC had not yet regularized statutes for Red Cross performance in times of civil war, which is perhaps why "war" is not mentioned here.

39 Leung, "The Idea of Chuanran Contagion"; Bridie Andrews, The Making of Modern Chinese Medicine, 1850-1960 (University of British Columbia Press, 2014), 191, 194. Shen's understanding of disease as caused by miasmas was slightly outdated for the time, although theories of "bad air" were still very much in popular circulation in China and the United States in the 1910s. Personal correspondence with Ruth Rogaski, Bridie Andrews, and Yi-li Wu, SeptemberOctober 2002.

40 Presentation on health risks of dead bodies by Médecins Sans Frontières Water Sanitation Engineer, Edith Rogenhofer, Manchester, UK March 18, 2013.

41 Unfortunately, the donors' motivations in thus earmarking their funds are not available to us.

42 For a discussion of the concept of "civilization" and the international community, particularly the international humanitarian community, in this period, see Caroline Reeves. "From Red Crosses to Golden Arches?" in Jerry Bentley, ed. Interactions: Transregional Perspectives on World History (Honolulu: University of Hawai'i Press, 2005).

43 For example, in the Dongfang Zazhi 10.2 (August 1, 1913).

44 Claire Roberts, Photography and China (London: Reaktion Books; Hong Kong: Hong Kong University Press, 2013), 53.

45 Joan Judge, Print and Politics (Stanford University Press, 1996), 17-18.

46 Meyer-Fong, What Remains, 51-63, for images and a detailed description of this pamphlet.

47 Edgerton-Tarpley, Tears from Iron, 132-141.

48 Shenbao, March 15, 1878, 1. 
49 Ariga Nagao, speech to Chinese philanthropists, Shanghai, November 19, 1912. Zhongguo Hongshizihui Zazhi 1 (May 1913).

50 Red Cross Monthly (Hongshizihui Tuekan) Number 3 (October 1921).

51 Hongshizihui Zazhi 3 (October 1921).

52 Although the photos are black and white, the red is assumed.

53 John Fitzgerald discusses this trope brilliantly in his 1996 book: John Fitzgerald, Awakening China: Politics, Culture and Class (Stanford University Press, 1996).

54 Of course, it would also give rise to the birth of various political movements, including the Chinese Communist Party.

55 Heather D. Curtis, "Depicting Distant Suffering: Evangelicals and the Politics of Pictorial Humanitarianism in the Age of American Empire," Material Religion 8.2 (2012): 154-183.

56 ZHE7, np. These photos are unfortunately not dated, but from the uniforms and locations, and the fact the group is not explicitly called a burial corps, they are probably from the 1911 Revolution.

57 These outfits and who wears what are detailed in Rudinger's description of Red Cross work in The Second Revolution, 12.

58 Alan Trachtenberg, "Albums of War: Reading Civil War Photographs," in The Nerw American Studies, Philip Fisher, ed. (Berkeley: University of California Press, 1991), 288; Francis Haskell, History and Its Images: Art and the Interpretation of the Past (New Haven: Yale University Press, 1993), 4; Peter Burke, Eyewitnessing: The Uses of Images as Historical Evidence (Ithaca NY: Cornell University Press, 2001), 21-25.

59 Alexander Gardner, Gardner's Photographic Sketchbook of the Civil War (New York: Dover, 1959); Burke 2001, 48-49; and Alan Trachtenberg, "Albums of War" for interpretations.

60 Front cover, Haoshengzhide (The Morality of Loving Life) (Shanghai, 1914).

61 The Chinese Red Cross Activities Told in Pictures, published in English by the Chinese Red Cross Central Committee, Shanghai (1929 or 1930) (stamped by American Red Cross Library, acquired in Federation Archives).

62 For a discussion about Chinese Red Cross poetry about the Burial Corps, see Caroline Reeves, "Grave Concerns," in Cochran and Strand, Cities in Motion, 48-50.

63 There is little written on indigenous Chinese photography. See Claire Roberts, Photography and China (London: Reaktion Books; Hong Kong: Hong Kong University Press, 2013); Oliver Moore suggests he has work forthcoming; Carlos Rojas, "Abandoned Cities Seen Anew: Reflections on Spatial Specificity and Temporal Transience," in Rosalind Morris, ed., Photographies East: The Camera and Its Histories in East and Southeast Asia.

64 Denis Kennedy, "Selling the Distant Other: Humanitarianism and Imagery-Ethical Dilemmas of Humanitarian Action," The Fournal of Humanitarian Assistance (February 28, 2009), 2.

65 Braester notes this phenomenon in Lu Xun's spectacle of execution. "The images known to us from contemporary executions show that the people are keenly aware that they are the object of another spectacle, namely the photograph. The cumbersome equipment of the time was especially likely to draw attention to the photographer. Some stop watching the execution and turn to look at the camera. They face it frontally and pose for it, comporting with the conventions of ceremonial photography." What Braester does not point out, however, is why the photographer is more interesting than a beheading; this is what I call the "hierarchy of novelty," and on the scale of the time, death and suffering was much lower than the camera and photographer. 


\title{
REMAKING THE HINDU PYRE \\ Cremation in India since the 1830s
}

\author{
David Arnold
}

Cremation was the most widely followed funerary practice in India in the nineteenth and twentieth centuries. It was the dominant (though by no means exclusive) means of disposing of the dead among Hindus, as well as Sikhs, Jains, and Buddhists. No precise figures exist for India as a whole, as, outside the main cities, cremation was a largely unregulated practice, but in the early twentieth century possibly as many as a million bodies were cremated annually, far more than in any other part of the world. In India, cremation was an ancient practice, uninterrupted in its observance over the millennia, whereas in the West the modern cremation movement, while harking back to classical antiquity and a remote Teutonic past, dates only from the 1870 s and involved, until after 1945, only relatively small numbers of the dead. In India, by contrast, burning funeral pyres were, by day and night, a common sight, a conspicuously visual marker of religion identity and social status, an expression of widely held religious beliefs, and a central expression of a highly complex funerary culture.

It is perhaps surprising, therefore, that despite the growth of mortuary studies in general and despite the fact that medicine, public health, and mortality in modern South Asia have attracted a detailed and extensive historical literature, the history of cremation, and of Indian funerary practices more generally, has been almost entirely ignored. ${ }^{1}$ Although anthropologists have been keenly attentive to the rites and rituals surrounding death and the disposal of the dead in India and in the South Asian diaspora, their analyses have rarely provided much historical insight and, indeed, have tended to perpetuate the idea of Indian cremation as a practice essentially unchanged until very recent times. But it should be clear from an even cursory examination of the historical evidence that those cremation practices in fact underwent significant change over the course of the modern period. It is widely assumed that cremation as historically practiced in India represents an ancient, and therefore almost immutable, tradition in singular contrast to the relatively recent rise of cremation in the West, a radical funerary innovation. In reality, cremation in India has changed substantially over the past two centuries in both its nature and its meaning, in part for reasons inherent within Indian society but also through an extended dialogue with Western death rites and mortuary practices. ${ }^{2}$

\section{Gremation and its critics}

In a conventional upper-caste Hindu cremation, the body, shrouded in cloth and decked with flowers, was carried on a bamboo bier borne by male relatives, from the place of death (usually the home of the deceased) to the nearest burning-ground or 
cremation ghat. ${ }^{3}$ Although men and women were cremated in basically the same way, among the higher castes women rarely accompanied the corpse on its final journey. At the burning ghat the body was placed on a pyre three or four feet high, consisting of wooden billets, often laid crisscross fashion and within a wooden frame, to which grass and cow-dung cakes were sometimes added, as well as unguents and oils such as camphor and ghee to give fragrance and sanctity to the process of burning the corpse. Brahmin priests recited Sanskrit verses from the Vedas, and, having circled the corpse, the pyre was lit by the eldest son or (in his absence) the closest male relative. The mourners remained until the burning of the body was well advanced: traditionally the eldest son then performed the rite (known as kapal kriya) of breaking the skull, using a bamboo stave. This symbolized the release of the spirit from the body of the deceased, freeing it of all earthly trappings and liberating it for rebirth. The task of maintaining the pyre during the several hours open-air cremation required was assigned to "untouchables," generally men from the Dom community, who were paid a fee for their work and for the fuel they supplied. Once the funerary fire had died down, fragments of unburnt bone and the ashes were collected by relatives - whether for immediate dispersal in an adjacent river or tank (reservoir) or to be taken to the Ganges at Benares (Varanasi) or upstream at Hardwar or some other sacred stream such as the Godavari at Nasik in Maharashtra. ${ }^{4}$ The dead were not normally memorialized in the Western fashion or after the manner of Muslims with tombs, mausolea, and gravestones but through a long cycle of post-mortem rites and commemorative rituals. An exception was the erection of carved stones to mark the site where "devout" Hindu widows had undergone the rite of sati-the burning or selfimmolation on the funeral pyre of their husbands. ${ }^{5}$ Further, since the early modern period and perhaps under the influence of Islam and the grand mausolea built by Muslim rulers in India, the warrior caste of Rajputs of Rajasthan in northwestern India had taken to erecting memorials, known from their domed umbrella shape as chattris, to commemorate their dead and to house their ashes.

The process of change with which this essay is concerned can be traced back to the early decades of the nineteenth century. In 1829 the East India Company administration, encouraged by Rammohun Roy, the Bengali reformer and founder of the Brahmo Samaj, formally abolished the practice of sati throughout its Indian territories. ${ }^{6}$ This prohibition was never, in fact, entirely effective in preventing sati in British India or, still more, in the semi-autonomous princely states, but it had a lasting impact on the way in which Western observers continued to associate the burning of the dead with the murderous rite of sati. Abolition also redirected critical attention away from the fate of the self-immolating widow and toward the funeral pyre itself, an object which had previously attracted little outside comment or adverse criticism - though it can be argued that modern cremation practice, especially when it involved the great and the good, came in some respects to emulate some of the attributes once ascribed to sati. ${ }^{7}$ For Hindus, cremation grounds were abhorrent, polluting places, haunted by ghosts, ghouls, and restless spirits, and redolent of Yama, god of the dead. As such they were normally situated on waste-ground or by a stream, outside the city walls or village boundary, often to the south of a settlement (the south being the direction of death), and well away from the dwelling-places of the living. The great exception to this was at Benares on the banks of the Ganges, where the burning of the dead occupied a central location on the steps 
leading down to the sacred river. Here the devout came to die and, in Hinduism's holiest city, to be cremated. ${ }^{8}$

Accustomed to Christian burial rites, many nineteenth-century Western observers were horrified and repelled by what they saw of the Hindu practice of cremation, with corpses at burning ghat - often several at a time - highly visible, some awaiting cremation, others laid on an open-air wooden pyre (see Image 16.1). Only gradually, over the course of several hours, was the corpse consumed by fire, leaving the ashes and unburnt remains to be collected or directly deposited in an adjacent stream. While touching a corpse was, in Hindu terms, a polluting act, there seemed to be no taboos about viewing the dead or witnessing the destruction of the human body through the savage action of fire. ${ }^{9}$ At a time when Christian death rites, burials, and graveyards were highly sentimentalized and romanticized in the West, and given added cultural currency through novels, memoirs, paintings, and photographs, ${ }^{10}$ Hindu cremations appeared, by contrast, to be unfeeling, callously indifferent to the physical fate of the human dead. For some visitors to Benares, the sight and smell of burning flesh, the clouds of smoke and with half-naked Doms stoking the pyres, conjured up visions of hell. One unnamed Englishwoman, who visited the city in the late 1870s, recorded her utter "horror and disgust" at the "huge cremation pyres" with their "lurid flames" and "thick, curling smoke." She felt still more sickened by "the crackling of human bones in the flames, the fetid odor ... the countless piles of swathed corpses," and the horrid appearance of the Doms, "begrimed with human soot, and ... looking more horrible than even the piled-up heaps of the dead." "From the late 1850s, photographs of the ghats at Benares communicated often highly explicit images of Hindu cremation and the open-air burning of bodies to a global public. ${ }^{12}$ Perhaps significantly, the genre of cremation photographs was one to which Indian as well Western photographers contributed, including the now celebrated Deen

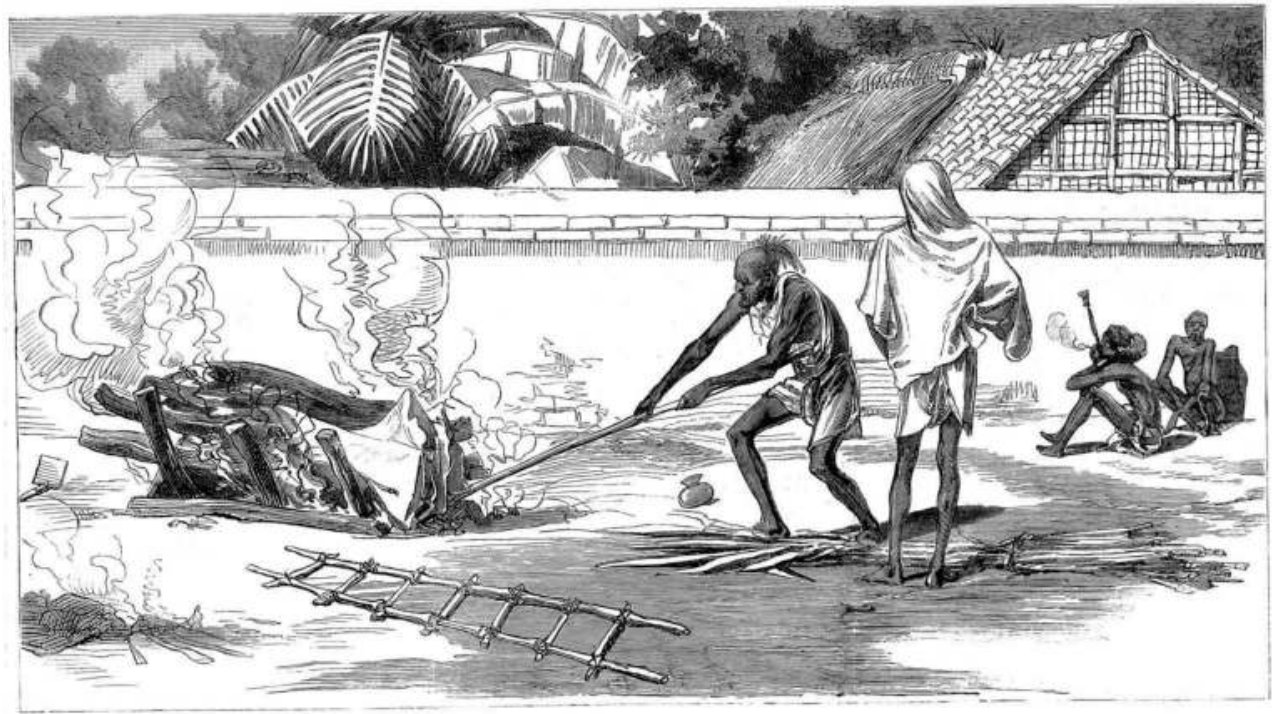

Image 16.1 A Western view of cremation in Calcutta, c. 1886.

(c) World History Archive/Alamy Stock Photo. 
Dayal. ${ }^{13}$ Unlike their Western counterparts, Indian photographs of cremation often suggested a somber and reflective mood. But a hostile representation of Hindu cremation practices as barbaric and repugnant became commonplace in many nineteenth-century travel accounts of India, especially in Christian missionary tracts, and this helped inform wider, negative representations in the West both of India and of cremation itself.

Even among advocates of cremation in Europe and North America from the 1870s, for whom the approved manner of burning the dead was for it to be conducted out of sight, in enclosed ovens, and by means of coal- or gas-fueled furnaces that could consume a corpse within minutes rather than hours, Hindu cremation was often singled out as a demonstration of how cremation should not be practiced. ${ }^{14}$ Hugo Erichsen, a pioneer of the cremation movement in the United States, thus remarked in 1889 that the "rude cremation" practiced in Calcutta was simply "far too horrible to witness."15 A few years later, a Wesleyan missionary, George W. Clutterbuck, remarked of open-air cremation in India: "With our English ideals of burial this [Hindu] method of disposing of the dead is revolting." Its "sanitary value" was undeniable, he wrote, but "one would surely prefer cremation any day," by which he meant cremation "as it is carried on at Woking [the Surrey town where the first British cremation was performed in 1885], and not as it is on the banks of the Ganges, where the bodies are exposed to view to the passers-by."16 That said, though, horror and revulsion were not the only responses. In an early expression of global necro-tourism, Hindu cremation grounds became one of the sites most visited by Western visitors in the late nineteenth and early twentieth centuries, even featuring in Thomas Cook's early India excursions. ${ }^{17}$ Others visitors found Manikarnika, the principal cremation ghat at Benares, a disappointing spectacle, while the French novelist and traveler Pierre Loti managed in his account to combine repugnance at the prevailing squalor with something close to necrophilia. ${ }^{18}$

Despite such often intense hostility, the British in India, first under the East India Company and then from 1858 under the direct rule of the British Crown, never seriously contemplated trying to outlaw Hindu cremation as they had sati. The practice was far too widespread, too culturally entrenched (especially among the higher castes), and the political risks to the British, especially after the mutiny and rebellion of 1857-58, were simply too high. ${ }^{19}$ Nor, unlike in Western countries, did such a common and ancient practice require legal sanction, with all the controversy and debate such legislation entailed elsewhere. ${ }^{20}$ But in India even modest attempts in the $1850 \mathrm{~s}$ and $1860 \mathrm{~s}$ at relocating cremation grounds away from the center of cities like Calcutta (now known as Kolkata) and Bombay (Mumbai) encountered staunch Hindu opposition. ${ }^{21}$ One of the arguments used in defense of inner-city cremation sites was that Hindu mourners had to be able to walk with the corpse to the burning ghat: relocating the place of cremation elsewhere, in the distant suburbs, would make this physically impossible. In other words, through the determination of Hindus and the accommodative policies of the British, cremation became incorporated into and institutionalized within the modern city, rather than being exiled to its periphery.

A critical moment in the modern history of Hindu cremation came in 1855 when, on the recommendation of the sanitarian A. H. Leith, the Government of Bombay proposed legislation to regulate and restrict existing cremation and burial grounds, including their relocation away from central sites in the city. This produced a strong protest from one of Bombay's leading Hindu merchants and businessmen, Jagunnath Shunkersett, who argued that the burning of Hindu bodies on Bombay's Back Bay was "an immemorial 
custom," one that the British would overturn at their peril. For Dr. Leith even to propose such a thing, Shunkersett claimed, suggested that the sanitarian was "not acquainted with the prejudices of the Hindus." The community would never tolerate the changes proposed, which were simply "not in consonance with the religion of the governed." Coming from so influential an individual, Shunkersett's remarks carried great weight with the government. Moreover, he informed them in a more conciliatory gesture that the Hindu community had already gone some way to meeting Leith's concerns by raising Rs. 25,000 for improvements to the Back Bay site, including erecting a high perimeter wall to make cremation invisible to passers-by. ${ }^{22}$ Open-air cremation, thus vigorously defended, remained a high-status ritual while burial, unlike in the West, constituted (among Hindus, Sikhs, and others) a far less prestigious, even abhorrent, funerary rite. ${ }^{23}$ Indeed, as colonial ethnography from the mid-nineteenth century onwards repeatedly showed, cremation was one of the principal attributes that differentiated the highest castes - Brahmins, Kshatriyas, and Vaishyas - from those at the very bottom of the social and ritual hierarchy. ${ }^{24}$ They were not, therefore, disposed to forego a practice so strongly redolent of their superiority. In the religious revivalism of the late nineteenth century among Hindus in India, as among Buddhists in neighboring Ceylon (Sri Lanka), cremation was an exemplary rite and one that clearly demarcated them from the Christianity of the evangelical missionaries, as well as from Muslims, Jews, and other burial-practicing religious communities.

Cremation also appealed to many colonial sanitarians as greatly superior to the unceremonious depositing of unburned bodies (as especially occurred during famines and epidemics when fuel was scarce and the labor of burning the dead too great) in rivers, in jungles, or on waste ground. ${ }^{25}$ It was thought preferable, too, to the manner in which Muslims and Christians buried their dead (in shallow graves easily raided by dogs and jackals) or the Parsis who exposed their dead in Bombay on the Towers of Silence (dokhma), to be picked clean by vultures. In the countryside and in small towns, communities were largely left to maintain their own burial grounds or cremation sites: only occasionally did the authorities intervene to close an overcrowded cemetery or to provide loans for the purchase of land for a new burial or burning ground. But in the big cities, where places for the disposal of the dead abounded (Bombay alone had 67 "places of internment, exposure, and cremation" in 1855), and where the dead seemed at times to threaten the survival of the living, a more interventionist and regulatory strategy appeared in the interests of public health to be necessary. ${ }^{26}$ It was moreover argued that, in Indian conditions, burning the dead was the most hygienic and effective means of disposing of corpses, especially of those who had died in one of the many outbreaks of cholera that afflicted the cities or (after 1896) from the bubonic plague. Consuming bodies by fire (and within 24 hours of death) was thought to break the chain of contagion and of destroying the dangerous miasmas (or microbes) that issued from overcrowded burial sites and shallow graves. ${ }^{27}$ It could perhaps be argued that colonial sanitarians were advocating a pragmatic means of disposing of Indians, a less deserving but multitudinous dead, that they themselves, given their own religious beliefs and cultural sentiments, would not personally have followed. There might be some truth in this idea of cremation as a racially discriminatory practice, but many colonial sanitarians zealously recommended the adoption of cremation in Britain as well as in India, and by the 1900s cremation was becoming fairly widely accepted among Europeans resident in India as well as among former officials and officers on their return to Britain. In this sense, India was an exemplar, even if the conventional Hindu form of out-door cremation was still regarded with dismay. 


\section{Making cremation modern}

Early attempts in the 1860 s and 1870 s to introduce more effective means of cremation (akin to those being developed in the industrial West with furnaces to aid burning and chimneys to dispel the smoke) proved unpopular among high-caste Hindus. ${ }^{28}$ Not until 1906 did Calcutta acquire a modern, enclosed crematorium, with a gasfired furnace, but this, for many years, catered for only a small number of the European dead (many expatriates retired to Britain and died there rather than in India). ${ }^{29}$ Even so, Hindu cremation had a visceral appeal to some Europeans in India as being far more spiritual, aesthetic, and empathetic than the kind of impersonal, "industrial" cremation by then being practiced in the West. ${ }^{30}$ Cremation was one of the practices adopted from the Hindu and Buddhist East by the founders of the Theosophy movement in the late nineteenth and early twentieth centuryHelena Blavatsky, Henry Steel Olcott, and Annie Besant, the first of whom was cremated at Woking crematorium in England in 1891, the latter two at Adyar on the outskirts of Madras in 1907 and 1933 respectively. Sister Nivedita (Margaret Noble), the British-born associate and disciple of one of India's most famous gurus, Swami Vivekananda, was also cremated after the Hindu manner and among her Indian friends at Darjeeling in October 1911. ${ }^{31}$ Cremation, even as practiced in India, thus appealed to those who have aptly been described as "Indians by persuasion," rather than by birth. ${ }^{32}$

However, government policy, and more especially the policy of India's municipal authorities in Bombay and Calcutta, was not one of endorsement but of reform and containment, intended to encourage the improvement of existing cremation grounds. The aim was to make them more hygienic and to confine them within walls and gates that would make them less visible (and, in theory, less offensive) to non-Hindu residents or accidental passers-by. This accommodative approach and the attention given to the materiality of cremation practices met with significant support from many influential, high-caste Hindus, like the trustees of the Sonapur cremation ghat on Queen's Road on Bombay's Back Bay. Innovations included not just the seclusion of burning grounds within gates and a high perimeter wall or fence, but also the provision of gas or electric lighting, water taps, benches and restrooms for the convenience of mourners, and a requirement that a sufficient supply of dried wood be available for the effective incineration of the body. Iron stakes were introduced to contain the logs and supports to increase the updraft beneath the wooden pyre; perforated metal screens served both to conceal the burning corpse and further dispel the smoke. At first viewed with "suspicion and alarm," these novelties were quickly accepted by Hindu mourners. ${ }^{33}$ As photographs taken at the time of the plague epidemic attest, by the late 1890s cremation grounds in urban India had begun to assume a very different appearance from those a half-a-century earlier and in their layout even to look rather more like British cemeteries. ${ }^{34}$ Cremation was becoming modernized and institutionalized but it was not therefore becoming overtly secularized. Significantly, these measures for the municipal regulation of mortality did not preclude the observance of the religious rituals that Hindus (and others) regarded as essential to their faith.

Two other developments assisted this regulatory trend. One was that the municipal authorities saw cremation as the most efficient and culturally acceptable means of disposing of the large number of the famine paupers who flocked to cities like Bombay in the 
1870 s and 1890s and died on the city streets or in police hospitals, or the multitudes of the poor who fell prey to epidemics of cholera, plague and, in 1918-19, influenza. Without relatives and friends to care for the dead, or to pay their funeral expenses, the municipality took the responsibility to dispose of their bodies (those at least who were thought to be Hindus) by means of cremation. ${ }^{35}$ In theory, Hindu cremation grounds served a further public health purpose in that they were one of the few sites where, in the absence of a more effective system of death registration, the dead could be counted and an approximate cause of death established. In reality, in the absence of a more dependable administrative agency, this means of assessing mortality was haphazard and unreliable. In addition, in order to thwart opposition and placate Hindu sentiment, colonial institutional practice in jails, the army, and elsewhere also favored cremation as the standard means by which the bodies of all Hindus should be disposed of regardless of caste, just as all Muslim and Christian convicts and soldiers were to be buried. ${ }^{36}$

State and municipal sponsorship of cremation fueled a second development. Although it was commonly assumed in the West that cremation was "all but universal" among Hindus, ${ }^{37}$ in actuality it had been a predominantly high-caste Hindu practice. The low castes and untouchables conventionally buried their dead, as did some higher status Hindu communities such as the Lingayats. The bodies of Hindu holy men and smallpox victims were also not cremated nor, conventionally, were those of individuals who had died a violent death. ${ }^{38}$ The costs of cremation were high. To be effective, it required large quantities of dry wood, normally between 400 and 800 pounds but with up to half a ton (1000 pounds) of wood being used in exceptional cases. ${ }^{39}$ Wet weather, especially the long monsoon season, might make open-air cremation difficult or require still more fuel. For the affluent and famous, substantial quantities of extremely expensive sandalwood were burned, a wood chosen for its sweet fragrance and religious associations. ${ }^{40}$ One late nineteenth-century estimate put the cost of cremation at Rupees 12-7-3, of which Rs. 7 was for fuel alone. Further costs were incurred for oil (usually ghee), camphor, flowers, and a winding sheet, as well as for the services of Brahmin priests and Dom attendants at the pyre. ${ }^{41}$ Expense was thus one factor in favoring burial over cremation, and this economic argument was often made in the nineteenth century to explain why poor Hindus buried rather than burned their dead; but inhumation was also a long-established custom among many low-caste, "untouchable," and tribal (Adivasi) communities. Low-caste burial grounds like that at Haines Road in Bombay, bleak, bare, and devoid of funerary monuments, were evidence of the persistence of Hindu burials, even in the midst of a bustling, congested, and increasingly modern city. ${ }^{42}$

However, with growing (if still modest) wealth among some sections of the lower castes and as a result of the evangelical activities of religious reform organizations like the Hindu Arya Samaj (founded in Bombay in 1875), for whom cremation was an essential rite, the burning of the dead became a practice that some of the lower castes began to adopt or aspire to, especially in the cities. The confluence of all these factors - sanitary policy, municipal practice, high-caste reformism, increased low-caste participation, and the sheer scale of mortality among urban populations, which in the case of Bombay and Calcutta, had by the early 1900s reached close to or above one million-together made Hindu cremation grounds sites of unprecedented activity. In Bombay in 1865 (one of the first years for which such statistics are available), 7,651 bodies were cremated, less than half of all Hindus who died in the city. ${ }^{43}$ By 1893 the cremation figure, for 
a substantially larger population, had risen to only 7,987, roughly a third of all deaths. In 1900 , as plague mortality in the city reached its height, 35,480 bodies were committed to fire, almost five times the number of cremations held 35 years earlier, and that despite the large number of citizens who had fled Bombay hoping to escape the epidemic. In the 1870 s and 1880s, roughly a quarter of all deaths in the city had been followed by cremation: in 1901, with 33,672 cremations, the figure reached almost 55 percent. ${ }^{44}$ Likewise, in Calcutta in 1911, a relatively healthy year, 16,809 cremations were recorded (equivalent to 65 percent of total deaths), but in 1918 and 1919, the years of the devastating influenza pandemic, the totals soared to 22,549 and 24,831 respectively, representing approximately two-thirds of all deaths in the city. By the late 1930s the total number of cremations had risen still further, to 31,876 in 1938 , close to 70 percent of all deaths in India's eastern metropolis. ${ }^{45}$

The epidemic of bubonic plague that erupted in Bombay in 1896 and ravaged much of western, northern, and central India over the following two decades presented the Indian practice of cremation with perhaps its greatest challenge in modern times. Once fears about the contagious nature of the disease had been publicly aroused, the carrying of the plague dead on open biers through the city streets to the cremation ghats created widespread alarm. ${ }^{46}$ Among some communities, mortality was so great that it was difficult to find enough family or caste members to carry or escort the bier: reports circulated of corpses being concealed within houses as a result. ${ }^{47}$ Through an exceptionally draconian piece of legislation, the Epidemic Diseases Act of 1897, the colonial authorities sought to impose new restrictions on the disposal of the dead. Arguing that the plague corpse was "a focus for infection," they required that death certificates stating the cause of death be obtained from a qualified physician before bodies could be buried or cremated. ${ }^{48}$ Such measures created intense opposition and in many places they were soon abandoned as impractical or needlessly provocative. Even so, the cremation grounds were crowded with the waiting or burning dead. At Sonapur in Bombay a hundred or more corpses were cremated - individually, not en masse - each day and throughout the night. Cremation on such a scale created a scarcity of fuel for the pyres, and the cost of wood rose accordingly. The municipal authorities in Bombay had to intervene to meet the increased expense, allocating almost Rs. 16,000 rupees for cremation firewood in 1899-1900 alone. $^{49}$

The unremitting cremations reawakened the hostility of many European residents, acutely aware of the flames and smoke and the pervading smell of incinerated bodies. The Reverend George Clutterbuck, whose hostile view of Indian cremation was cited earlier, remarked that the burning of the dead became "a hideous and nauseating spectacle. The smoke of the funeral pyre ascended day and night ... Bombay was a city of the dead." ${ }^{\circ}$ The manager of the Times of India newspaper, F. M. Coleman, voiced a similar complaint about the burning ground at Sonapur where, during the plague years, the bodies of the dead could "be plainly seen" from Queen's Road. The smoke from the funeral pyres, he continued, blew, "charged with the ashes of burnt wood and an abominable odor of charred flesh, into the eyes and nostrils of each and every passerby." ${ }^{51}$ Nonetheless, it is important to note that, despite such trenchant criticisms and despite the crisis of mortality and the disposal of the dead, open-air cremation (albeit in its modified municipal form) remained much as it had been before plague struck, underscoring its essential cultural resilience. 


\section{Gremation in the public eye}

Cremation entered India's modern social, political, and religious life in other ways, too. In discussing changing attitudes to death and the disposal of the dead in Western societies since the eighteenth century, Thomas Laqueur has referred to what he terms "the work of the dead," that is, the cultural meaning and political significance the living gave to the deceased. In his words, the "work of the dead" is a history of "how they give meaning to our lives, how they structure public places, politics, and time." Such a history is "a history of the imagination, a history of how we invest the dead ... with meaning." 52 In India, too, the socio-political function of the dead also underwent profound change. Conventionally, Hindu families expressed their grief in private, and, among the higher castes, only men accompanied the corpse to the funeral pyre. Bodies were cremated within twenty-four hours of death, allowing little time for more elaborate ceremonials. But over the course of the nineteenth century, a new sense of the public emerged in India, most obviously (but not exclusively) among the urban middle classes. The rise of the colonial public sphere and the growth of a new kind of civil society was nurtured by newspapers and the spread of print media, by the formation of non-official societies and associations, by the holding of public debates and ceremonies, and through a photography that helped to elide the distinction between what was held to be private and what merited public notice and display. Death practices evolved in line with this new sense of public identity and in ways that brought Hindu India more closely in line with funerary culture in the contemporary West. ${ }^{53}$

The death of leading Hindu men (at this stage it was almost entirely men, not women, that were so prominently commemorated) renowned for their role as politicians, religious saints, and social reformers, began to attract, at the time of their deaths and through their cremation, a degree of public attention that marked a significant departure from former, more private, observances. Early examples of this can be found in the deaths of Dayananda Sarasvati, founder of the Arya Samaj, in 1883; Keshab Chandra Sen of the Brahmo Samaj, in 1884; and the educationalist and social reformer, Iswar Chandra Vidyasagar in 1891. As in Vidyasagar's case, the body of the deceased was photographed after death (a practice recently imported from Europe and North America); the garlanded body was borne through the city streets on an open bier, watched or followed by large crowds, to the burning ghat, where large numbers of mourners gathered; patriotic speeches were made honoring the role of the deceased in Indian public life and national politics, and further photographs were then taken before the pyre was lit. ${ }^{54}$ In this way the cremation ghat (hitherto a place of avoidance and horror) and the procession leading to it, became a conspicuous display of public (rather than private) grief and a highly visible (and audible) celebration of the life and achievements of those whom Laqueur describes as "the special dead." 55

Such funerary events rapidly acquired an overtly anti-colonial significance and threatened to override the strategy of urban containment hitherto followed by colonial sanitarians and supported by the Hindu elite. Beginning in the early years of the twentieth century, national "martyrs," whose bodies had been released to families and friends following their death or execution in jail, were made the centers of explicitly anti-British demonstrations. Photographs, newspaper reports, and printed tracts further disseminated the idea of the cremation ground as a site for the commemoration of the nation's heroes and martyrs. One striking instance of this concerned the young Bengali revolutionary 
Kanai Lal Datta. Executed in Calcutta's Alipore Jail on November 8, 1908, his corpse was handed over (as was still the standard practice) to his awaiting relatives. But his body was then carried to the cremation ghat on a flower-strewn bier and followed by a thousand people, mostly students, walking barefoot through the streets. Breaking with convention, a large number of Hindu women (including many of high status) gathered at the burning ground. A funeral oration was given by Kanai Lal's uncle, nationalist songs were sung, photographs taken, and conches blown, amidst shouts of Kanai Lal ki jai ("Victory to Kanai Lal"). The cost of the sandalwood pyre was met by public subscription and a marble monument later raised in Kanai Lal's honor. The British were horrified by this turn of events and thereafter sought to prohibit such patriotic demonstrations, even to the extent of refusing to hand over a body if a similar expression of grief for the martyred dead were thought likely. ${ }^{56}$ One alternative was not to release the body at all but to cremate it within prison grounds and conceal the ashes. But the British also reciprocated in a similar manner: when loyal Indian police officers or government servants died or were assassinated, their bodies were accompanied to the cremation ground with a military or police escort, high-ranking officials stood to attention by the awaiting pyre, eulogies were delivered, a volley of shots fired, and a bugler sounded the Last Post. ${ }^{57}$

When the Hindu nationalist leader, Bal Gangadhar Tilak, died (of natural causes) in Bombay in August 1920, the provincial government, in a gesture of reconciliation it soon came to regret, allowed his body to be cremated, not in any existing cremation ground but on the open sands of Chowpatty beach in central Bombay. This effectively violated the principle of containment the municipal authorities had previously followed. Within months, the site of Tilak's cremation had become a place of veneration and for holding anti-British meetings and demonstrations. By 1933 a shrine had been erected to Tilak's memory, and demands grew for other nationalist leaders to be honored with cremation on the same, now hallowed, spot. ${ }^{58}$ When the Bengali nationalist Chittaranjan Das died in Darjeeling in June 1925, his body was brought by special train to Calcutta to be met by crowds of political dignitary mourners. From the station it was taken in procession through streets crowded with many thousands of onlookers, the bier stopping at several places associated with Das's life and work en route to the cremation ground. The place of his cremation (within an existing burning ghat) was soon marked by the erection of a memorial and became a favored place for nationalist speeches and the holding of demonstrations. ${ }^{59}$

This intense publicization and politicization of Hindu cremation was not without its negative consequences. In the 1930s and 1940s, the reclaiming of the bodies of Hindus killed during communal riots and carried in procession to cremation grounds further exacerbated tensions with Muslims during the run-up to Partition and Independence in 1947. The death and cremation of the celebrated Bengali poet and educationalist Rabindranath Tagore in August 1941 further showed the perils involved in such public demonstrations of grief. Surrounded by milling crowds and by onlookers who reportedly plucked hair from his head and beard, his body could only with difficulty be carried to the Nimtala burning ghat in Calcutta. There, too, there were wild and unruly scenes: part of the perimeter wall of the cremation ground was broken down, and some mourners, pressing in their "funerary ecstasy" dangerously close to the pyre, fainted or were crushed. Tagore's ashes were barely cold before an unseemly scramble began to collect his relics. ${ }^{60}$ The death and cremation of the great Bengali poet thus recreated some of the scenes of fervor and frenzy that had once typified the rite of sati. The cremation of 
Mohandas Gandhi in Delhi in 1948 was a much more orderly and carefully staged event, in part owing to the conspicuous involvement of the Indian armed forces; but, again, there were intense displays of public emotion that almost threatened to overwhelm the event. ${ }^{61}$

\section{Beyond India}

As India entered the era of its independence, cremation events of this kind became a frequent and established means of expressing public grief and commemorating men (and, by now, increasingly women) honored for their service to the nation. Symbolically as well as practically, cremation attained a new prominence, not just nationally but internationally, as demonstrated by Gandhi's cremation in 1948, newspaper reports and photographic images of which were rapidly relayed around the world. ${ }^{62}$ When prime minister Indira Gandhi was assassinated by her Sikh bodyguard in October 1984, her cremation in Delhi was delayed by three days to allow world leaders to attend. Attended by representatives from a hundred different nations and lasting more than four hours, the event was also transmitted via television to homes in India, through the state television channel Doordarshan, by the BBC in Britain, and, by both live coverage and cable channels in the United States. ${ }^{63}$ No cremation in history has been witnessed by so many. And yet, with its billowing black smoke and conducted against the background of arson, murder, and a vicious anti-Sikh pogrom, at its deadliest on the streets of the capital itself, the cremation seemed to capture the tense and somber mood of India at the time. ${ }^{64}$

While it might be "the special dead"-Dayananda Sarasvati, Mohandas Gandhi, Indira Gandhi - who were afforded the most public and spectacular cremations, the burning of the dead was a practice increasingly normalized across the Hindu community, indeed helping to solidify the very sense of Hindus as a community, thereby incorporating many poor and low-caste groups for whom burial might previously have been the norm. But from the mid-nineteenth century, Indian cremation also became, in another sense than that provided by newspaper and television coverage, an increasingly global issue. The Hindu, Sikh, and Jain attachment to cremation travelled outwards from India through the South Asian diaspora, especially via the migrant laborers, who from the 1830s left India to live and work in Mauritius, South Africa, Australia, the United States, Britain, Fiji, and British colonies in the Caribbean. As early as 1895 a Sikh hawker in Australia, Charam Singh, was cremated on a beach outside Melbourne. Eight years later, in 1903, in Adelaide one of the first bodies to be burned under South Australia's recent cremation act, was that of another Sikh, Bishin Singh. ${ }^{65}$ Sikh and Hindu soldiers who died abroad were also cremated, whether by their comrades or at the direction of the military authorities. Many of the dead of World War I, for example, were cremated on the battlefields of the Western Front (some posthumously exhumed for the purpose) or, as at Patcham Down outside Brighton, close to the hospital where they died from their wounds, and where a commemorative chattri was unveiled in their honor in 1921 by the Prince of Wales. ${ }^{66}$ From the 1870s members of Indian princely houses who died while traveling abroad, or students studying overseas, were also cremated, in modern crematoria rather than in the open-air Indian fashion, but not infrequently their ashes were transported back to India for dispersal in the Ganges or elsewhere. ${ }^{67}$ 
Cremation was thus doubly mobile - culturally, in its spread as a funerary practice among diasporic communities abroad and, materially, in the reverse direction, as the ashes were brought back to India for disposal. However, in the face of this deep cultural desire for funerary fire, a denial of the right to cremation was one of the "disabilities" increasingly felt by Indians abroad. ${ }^{68}$ Even where, as in Britain in 1902, cremation had been legalized, governments often remained strongly opposed to open-air cremation, seeing it as an affront to public decency and a hazard to public health. The burning of the bodies of 53 Hindu and Sikh soldiers at Patcham Down during World War I is almost the only instance in which open-air cremation has been permitted in Britain (and, even then, questions were raised as to its legality). In many cases, Indians - in Mauritius, South Africa, and the Caribbean - had either to practice covert cremation in out-of-theway places where the authorities would not intervene, or to conform to the only permitted form of cremation: in enclosed, purpose-built modern crematoria. This tension between what is seen as an essential Hindu (or Sikh) rite and what is legally permitted has been an ongoing and still largely unresolved source of personal anguish and cultural alienation among many South Asian communities abroad. ${ }^{69}$

\section{Gremation in an environmentally conscious age}

Throughout the nineteenth century and into the middle decades of the twentieth, openair, wood-pyre cremation remained the dominant mode of disposal for the Hindu dead and, for reasons previously given, was actively growing in its social constituency and political significance. To a large extent, it retains the culturally and politically hegemonic funerary practice. However, cremation on such a scale and in such a manner has increasingly been recognized as having high environmental, as well as financial, costs. The disposal of the dead must always in part be an environmental, quite as much as a religious and social, issue: where, and how, is it safest for the living to dispose of the dead? How is our idea of present, past, and future shaped by the landscapes, monuments, and relics, of the dead? Among the persuasive nineteenth-century arguments for cremation was that it was less wasteful of scarce urban space than the ever-expanding burial grounds and that it destroyed through the action of intense heat the harmful miasmas or "poisons" and the disease "germs" gradually released into soil, air, and water through the burial, decay, and decomposition of the corpse. ${ }^{70}$

However, in recent years the environmental argument has swung against open-air, wood-pyre cremation. Open-air cremation no longer seems as environmentally innocent as it once seemed. With a population now well in excess of one billion and with more than seven million Hindus dying each year, cremation in India requires enormous quantities of wood, with vast numbers of trees (put as high as 50 to 60 million a year) being felled to meet the soaring demand, some of it through illegal logging. ${ }^{71}$ Funeral pyres, it is now known, release into the atmosphere large quantities of smoke and emissions (including carbon dioxide and nitrogen oxide) that further exacerbate India's already acute pollution problem and the increasingly detrimental effect on human health. The dumping of ashes and body fragments (or even whole, un-cremated, bodies) into rivers, notably the Ganges at Varanasi, has also contributed to the growing problem of river pollution and the consequent destruction of riverine wildlife and food resources. Solutions have been slow in coming. After decades of debate and delay, Bombay city finally acquired its first electric crematorium in 1953, and many other Indian cities have since 
followed suit. ${ }^{72}$ At Varanasi the problem of human pollution from cremation ghats has been seen as part of the attempt to "clean up the Ganges." This has included the opening in 1989 of an electric crematorium at the Harischandra Ghat, which despite initial opposition, has met with substantial demand from the Hindu public and the many pilgrims the city attracts. ${ }^{73}$ Pollution is not the only issue. The price of wood for pyres has soared with increased demand and extensive deforestation, as well as the expense of other combustibles and payments for priests and attendants. Cost has thus contributed to the questioning of wood-pyre cremation. ${ }^{74}$

Although there had been little initial support in India in the nineteenth and early twentieth century for Western-style crematoria and a deep and abiding religious attachment to the openair pyre, purpose-built, gas- or electric-fired crematoria have gradually become accepted in India's major cities and even, significantly, in Muslim countries in the Middle East, where large numbers of migrant laborers now live and work. Considerable technical ingenuity is now employed in trying to find ways in which the essential features of Indian cremation can be preserved while using more efficient forms of cremation, some requiring a third as much wood as conventional open-air pyres or using alternative fuel sources like compressed natural gas (CNG). ${ }^{75}$ In 2018 Delhi had four electric and six CNG cremation furnaces in operation, though these together still only catered for a fraction of the city's dead. ${ }^{76}$ Urban Indians have begun to accept, however reluctantly, the impact of technological change on their funerary traditions, and modern electric crematoria and the consequent curtailment or displacement of conventional pyre-side rituals (like the breaking of the skull) have become a major feature in the contemporary disposal of the dead. ${ }^{77}$ These innovations and adaptations are widely seen as a very recent departure from an otherwise unmodified age-old tradition: this essay has argued that cremation in India has in fact been involved in complex processes of change - in cremation's material performance as in its socio-political significance-for many decades, extending back to the early and middle decades of the nineteenth century.

\section{Notes}

1 A notable exception is C. A. Bayly, "From Ritual to Ceremony: Death Ritual and Society in Hindu North India since 1600." In Origins of Nationality in South Asia: Patriotism and Ethical Government in the Making of Modern India, ed. C. A. Bayly (Delhi: Oxford University Press, 1998), ch. 5. For a recent survey of the demography of the period, see Tim Dyson, A Population History of India: From the First Modern People to the Present Day (Oxford: Oxford University Press, 2018).

2 For further elaboration of the material and arguments presented here, see David Arnold, Burning the Dead: India, Empire, and Cremation (forthcoming).

3 For an account of the rituals surrounding Indian cremation, see Anantanand Rambachan, "The Hindu Way of Death." In Handbook of Death and Dying, ed. Clifton D. Bryant (2 vols., Thousand Oaks, CA: Sage, 2003), 1: 640-48.

4 There are many nineteenth- and early twentieth-century ethnographic accounts of Hindu death rites and cremation practices, though these varied in detail from one caste to another and from one region to another: see, for example, Gazetteer of the Bombay Presidency, Volume XVIII, Part 1: Poona (Bombay: Government Central Press, 1885), 147-444; Gazetteer of the Bombay Presidency, Volume IX, Part 1: Gujarat Population: Hindus (Bombay: Government Central Press, 1901), 47-343; E. Thurston, "Southern India." In Customs of the World: A Popular Account of the Manners, Rotes and Ceremonies of Men and Women in All Countries, ed. Walter Hutchinson (2 vols., London: Hutchinson, 1900), 1: 475-81.

5 Strictly speaking, the term "sati" applies only to the "devoted" widow and not to the practice of self-immolation. I here follow current academic practice in using the term to refer to both the individual and the rite. 
6 On sati, its abolition, and legacies, see Lata Mani, Contentious Traditions: The Debate on Sati in Colonial India (Berkeley, CA: University of California Press, 1998); Catherine Weinberger-Thomas, Ashes of Immortality: Widow-Burning in India (Chicago, IL: University of Chicago Press, 1999).

7 For an example of the elision between cremation and sati, see E. H. Nolan, The Illustrated History of the British Empire in India and the East (2 vols, London: James S. Virtue, n.d. [1859]), 2: 37-38, 489-90.

8 The most authoritative account of cremation in modern Varanasi is Jonathan P. Parry, Death in Banaras (Cambridge: Cambridge University Press, 1994).

9 Some of the most explicit, fascinated, and repugnant descriptions of this process of corporeal disintegration were made not in the nineteenth century but by the American beat poet Allen Ginsberg in the early 1960s in Calcutta and Benares: Allen Ginsberg, Indian fournals, March 1962-May 1963 (San Francisco, CA: Dave Haselwood Books, 1970).

10 Pat Jalland, Death in the Victorian Family (Oxford: Oxford University Press, 1996); Thomas Laqueur, The Work of the Dead: A Cultural History of Mortal Remains (Princeton, NJ: Princeton University Press, 2015).

11 Anon., "Cremation in India," The Young Folk's Budget, April 19, 1879, 253.

12 In the 1860s, Samuel Bourne, one of the pioneers of European photography in India, took numerous photographs of Benares and its burning ghat: Samuel Bourne, Photographic fourneys in the Himalayas (Bath: Pagoda Tree Press, 2009), 6.

13 See his photograph of the cremation of the Maharaja of Indore in June 1886, reproduced in the London illustrated paper, The Graphic, on July 24, 1886.

14 Henry Steel Olcott, Old Diary Leaves: The Only Authentic History of the Theosophical Society, 2nd series (London: Theosophical Society, 1900), 276.

15 Hugo Erichsen, The Cremation of the Dead: From an Aesthetic, Sanitary, Religious, Historical, MedicoLegal and Economical Standpoint (Detroit: D. O. Haynes, 1887), 25.

16 George W. Clutterbuck, In India: The Land of Famine and Plague (London: The Ideal Publishing Union Ltd, 1897), 21.

17 Piers Brendon, Thomas Cook: 150 Years of Popular Tourism (London: Secker \& Warburg, 1991), 146-48.

18 Mary Frances Billington, Woman in India (1895: reprinted New Delhi: Amarko Book Agency, 1973), 2; Pierre Loti, India (5th ed., trans. George A. F. Inman, London: T. Werner Laurie, 1928), 245-54.

19 In Bombay the formal accommodation of Hindu rites and customs, including cremation, went back to the early years of East India Company control in the 1660s: James Douglas, A Book of Bombay (2 vols, Bombay: Bombay Gazette Steam Press, 1883), 1: 87.

20 As in Britain: Peter C. Jupp, From Dust to Ashes: Cremation and the British Way of Death (Basingstoke: Place Macmillan, 2006), chs. 3, 4.

21 As in Calcutta in 1864: Proceedings of an Ordinary Meeting of the Municipal Corporation of Calcutta, Held at the Town Hall on Monday, 7th March 1864 (Calcutta: Hindoo Patriot Press, 1864).

22 Correspondence Relating to a Proposed Enactment for the Regulation of Places Used for the Disposal of Corpses in the Town and Island of Bombay (Bombay: Bombay Education Society's Press, 1859), 13-14.

23 For Sikhs, who had adopted "fire-burial" from high-caste Hindu practice, the cremation in Lahore in 1839 of the ruler of Punjab, Ranjit Singh in 1839, along with his wives and concubines, and the building of a grand mausoleum over his remains, signified a renewed commitment to the practice. See John Campbell Oman, Cults, Customs and Superstitions of India (Philadelphia, PA: George W. Jacobs, 1908), ch. 5.

24 For instance, M. A. Sherring, Hindu Tribes and Castes as Represented in Benares (3 vols., Calcutta: Thacker, Spink, \& Co., 1872-81).

25 Annual Report of the Sanitary Commissioner for Bengal, 1864-65 (Calcutta: Military Orphan Press, 1865), 80.

26 Correspondence Relating to a Proposed Enactment, 25-26.

27 T. G. Hewlett, city health officer, in Annual Report of the Municipal Commissioner of Bombay, 1872 (Bombay: "Times of India" Office, 1873), 15; "The Disposal of the Dead," Indian Medical Gazette 10 (1875), 104-06; "Destruction versus Decomposition," Indian Medical Gazette 22 (1887): 175.

28 For the rise of the cremation movement in the West, see Laqueur, Work of the Dead, Part 4; Stephen Prothero, Purified by Fire: A History of Cremation in America (Berkeley, CA: University of California Press, 2001). 


\section{DAVID ARNOLD}

29 Times of India, May 17, 1906, 3.

30 See, for instance, the reflections of George Birdwood on the cremation of his friend Jagunnath Shunkersett: George C. M. Birdwood, Sva (London: Philip Lee Warner, 1895), 22.

31 Anon., Sister Nivedita: A Sketch of Her Life and Her Services to India (Madras: G. A. Natesan, n.d. [c. 1912]), 41.

32 Richard G. Fox, "East of Said." In Edward Said: A Critical Reader, ed. Michael Sprinker (Oxford: Blackwell, 1992), 153.

33 Times of India, January 13, 1899, 5.

34 See the photographs of burning grounds and cremations in "The Bombay Plague Epidemic of 1896-97: Work of the Bombay Plague Committee," an album assembled for the committee's chairman, Brigadier-General W. F. Gatacre, Wellcome Library, London.

35 Debates on this issue were most intense in Bombay in the mid- and late 1880s: see, in particular, Times of India, August 20, 1887, 5; ibid., May 9, 1888, 3.

36 George Alexander Hodge, The Bengal fail Manual (Calcutta: City Press, 1867), appendix 1, $15-16$.

37 William Eassie, Cremation of the Dead: Its History and Bearings upon Public Health (London: Smith, Elder, \& Co., 1875), 35.

38 On the continuance of untouchable burial practices in village society, see Shalini Randeira, "Carrion and Corpses: Conflict in Categorizing Untouchability in Gujarat," European Fournal of Sociology 30 (1989): 171-91.

39 Estimates of the quantity of wood required varied greatly: Birendra Nath Ghosh, A Treatise on Hygiene and Public Health (7th ed., Calcutta: Scientific Publishing, 1930), 339, gives a figure of 5 maunds, or approximately 410 pounds, but for the well-off and famous two or three times this amount might be consumed. For a figure of $320 \mathrm{~kg}$ (705 lbs) to $360 \mathrm{~kg}$ (794 lbs), see Archana Kaushik, "Can You Afford to Die? Estimates of Expenditure on Rituals and Impact on Ecology," Economic and Political Weekly, January 20, 2018.

40 For the significance attached to sandalwood, see James McHugh, Sandalwood and Carrion: Smell in Indian Religion and Culture (New York: Oxford University Press, 2012), ch. 8.

41 Eassie, Cremation of the Dead, 91.

42 See the photograph of Haines Road burial ground in the "Bombay Plague Epidemic" album.

43 Deaths in Bombay During 1865 (Bombay: Bombay Education Society's Press, 1867), iii.

44 Figures taken from the Annual Administration Report of the Municipal Commissioner, Bombay for the relevant years.

45 Figures from the Annual Administration Report of Calcutta Municipality for the relevant years.

46 Amrit Bazar Patrika (Calcutta) May 15, 1898, 4; ibid., August 28, 1898, 3.

47 Times of India, February 13, 1897, 3; ibid., May 5, 1897, 5.

48 M. E. Couchman, Account of Plague Administration in the Bombay Presidency from September 1896 till May 1897 (Bombay: Government Central Press, 1897), 49, 52, 138; [W. C. Rand], Supplement to the Account of Plague Administration in the Bombay Presidency from September 1896 till May 1897 (Bombay: Government Central Press, 1897), 6, 9. On the history of this epidemic, see David Arnold, Colonizing the Body: State Medicine and Epidemic Disease in Nineteenth-Century India (Berkeley, CA: University of California Press, 1993), ch. 5; Raj Chandavarkar, Imperial Power and Popular Politics: Class, Resistance and the State in India, c. 1850-1950 (Cambridge: Cambridge University Press, 1998), ch. 7.

49 Report of the Municipal Committee on the Plague in Bombay for the Year Ending 31st May 1900 (Bombay: "Times of India" Press, 1901), 125.

50 Clutterbuck, In India, 35-36.

51 F. M. Coleman, Typical Pictures of Indian Natives (6th ed., Bombay: "Times of India," 1899), 29.

52 Laqueur, Work of the Dead, 17.

53 On this shift to the public expression of grief and the emergence of cremation as a public spectacle, see Partha Chatterjee, "Two Poets and Death: On Civil and Political Society in the Non-Christian World." In Questions of Modernity, ed. Timothy Mitchell, (Minneapolis, MN: University of Minnesota Press, 2000), 35-48.

54 Subal Chandra Mitra, Isvar Chandra Vidyasagar: Story of His Life and Work (2nd ed., Calcutta: New Bengal Press, 1907), 268-69.

55 Laqueur, Work of the Dead, 197, 212. 
56 Times of India, November 11, 1908, 7; Durba Ghosh, Gentlemanly Terrorists: Political Violence and the Colonial State in India, 1919-1947 (Cambridge: Cambridge University Press, 2017), 71-72.

57 Times of India, January 22, 1914, 7; ibid., October 11, 1915, 10.

58 Times of India, August 2, 1923, 4; ibid, June 5, 1926, 16; ibid., August 20, 1926, 10.

59 Times of India, June 19, 1925, 9; ibid., June 16, 1927, 9; ibid., June 24, 1930, 11; ibid., June 19, 1935, 4.

60 Rajarshi Chunder, “A City in Mourning: 7 August 1941." In Calcutta: The Stormy Decades, eds. Tanika Sarkar and Sekhar Bandopadhyay (New Delhi: Social Science Press, 2015), 42-61; Alex Aronson, Brief Chronicles of the Time: Person Recollections of My Stay in Bengal, 1937-1946 (Calcutta: Writers Workshop, 1990), 88.

61 For some descriptions, see Margaret Bourke-White, Half-Way to Freedom (New York: Simon \& Schuster, 1949), 233; Larry Collins and Dominque Lapierre, Freedom at Midnight (Delhi: Vikas, 1976), 446-50.

62 Claude Cookman, "Margaret Bourke-White and Henri Cartier-Bresson: Gandhi's Funeral," History of Photography 22 (1998): 199-209.

63 Girilal Jain, "Awareness of India in U. S.," Times of India, April 9, 1985, 8.

64 Times of India, November 2, 1984, 1; ibid., November 3, 1984, 1.

65 Times of India, April 20, 1895, 6; "The First Cremation at Adelaide, Australia," The Undertakers' Fournal, undated cutting in CRE H16, Cremation Society of England, University of Durham, UK.

66 Times of India, February 24, 1921, 12; ibid., November 15, 1921, 11.

67 A rare example of an Indian ruler being cremated on an open-air pyre abroad was that of the Raja of Kolhapur in Florence in November 1870: Diary of the Late Rajah of Kolhapoor During His Visit to Europe in 1870, ed. Edward W. West (London: Smith, Elder, \& Co., 1870), appendix 4.

68 C. Kondapi, Indians Overseas, 1838-1949 (Bombay: Oxford University Press, 1951), 382-83.

69 As in Britain: Shirley Firth, Death, Dying and Bereavement in a British Hindu Community (Leuven: Peeters, 1997), ch. 4.

70 Eassie, Cremation of the Dead, ch. 4; Henry Thompson, "The Treatment of the Body After Death," Contemporary Review 23 (1874): 319-28.

71 Teo Kermeliotis, "India's Burning Issue with Emissions from Hindu Funeral Pyres," http:// edition.cnn.com/2011/09/12/world/asia/India-funeral-pyres-emissions/index.html, accessed July 26, 2017.

72 Times of India, April 3, 1953, 8.

73 Parry, Death in Banaras, 67-68; Kelley D. Alley, On the Banks of the Ganga: When Wastewater Meets a Sacred River (Ann Arbor, MI: University of Michigan Press, 2002), 93-98, 160-61.

74 For a recent estimate of costs, see Kaushik, "Can You Afford to Die?"

75 Gargi Gupta, "Saying Goodbye, the Green Way," www.dnaindia.com/india/report-sayinggoodbye-the green-way-2266024, accessed October 26, 2016.

76 Kaushik, "Can You Afford to Die?"

77 Vishwambhar Nath Prajapati and Saradindu Bhaduri, "Human Values in Disposing of the Dead: An Enquiry into Cremation Technology," fournal of Human Values 25 (2019): 58-61. 


\title{
MUSLIM BELIEFS ABOUT DEATH From classical formulations to modern applications
}

\author{
Abdulaziz Sachedina
}

The advent of new medical technologies in the last fifty years has rendered the task of defining death the most pressing issue in the field of biomedical ethics. Just as technically assisted reproduction has given rise to ethical problems in defining embryonic life and terminating it, the new modes of treatment raise serious questions of when healthcare providers should close down artificial support systems to allow nature to take its course in relieving a person from prolonged suffering. Among Muslim jurists, the answer to the question about the timing of death involves an attempt to understand the moment of imminent death in brain-dead patients. Any error of judgment in this regard could lead to morally and legally questionable decisions to end an individual's life by either actively or passively engaging in causing the death. However, formulation of a proper definition of death requires an understanding of the religious estimation of human life and an endeavor to unravel the secret of the soul (nafs) or the spirit $(r \bar{u} h)$ which, according to Muslim beliefs, is infused in the body and departs at the time of death. ${ }^{1}$

From a traditional Islamic perspective, it is obvious that the definition of death cannot be derived from medical facts or scientific investigation alone. Medical professionals can only provide an account of physiological state which he/she observes. Whether that description meets the criteria that can be treated as valid indicators of death is both a religious-ethical and legal issue. More importantly, the most critical question about the determination of the time of death among Muslims is essentially a religious problem, not simply a medical or scientific one. In the community of the faithful, it is God, the Giver of life and death, who has the knowledge of the time of death, and death occurs upon the separation of the soul from the body. Of course, this separation is not open to direct empirical observation, and this is the major source of ambiguity in the community in determining the exact moment of death. Today, the traditional view of death, which focused upon cessation of circulatory and respiratory activities as the criteria of departed spirit, has been overshadowed by the ability of the new medical technologies to intervene by maintaining a patient's heartbeat, blood pressure, respiration, and liver and kidney functions within the normal range of values. Contemporary medical science has developed highly sophisticated techniques for determining the presence or absence of vital bodily functions. More pertinently, these techniques have improved resuscitatory and supportive measures to restore at times what- if judged by the traditional definition - are life-indicating continuations of respiration and heartbeat. As we shall discuss below, this 
possibility of restoring respiration or heartbeat, even in the case of massive brain damage when there is little likelihood of an individual recovering consciousness, has given rise to the problem of validating the possibility of cerebral death. In this chapter, I will summarize problems with the definition of death and the retrieval of organs from a cadaver as contemporary issues demanding that Muslim scholars and physicians undertake a dialogue to fully understand modern biotechnology and its impact upon defining death and the relationship humans have with their body and whether they can donate any part of it for medical research and the retrieval of vital organs for transplant.

\section{Death in the scriptural texts of Islam}

Although the Qur'an contains various death themes that add significantly to our insight into the purpose of death, the concept is left undefined and always juxtaposed with creation and resurrection. God is omnipotent and controls everything that has to do with life and death. The ultimate proof of God's existence is the fact of death and the promise of resurrection, which is viewed as a new creation (khalq jadìd; Q. 17:49). And yet, it is obvious that the purpose of creation or resurrection would remain incomprehensible if death did not constitute an integral part of life and the ultimate proof that God controls it by assigning it a final destiny through creation.

The Qur'an, however, seems to be more concerned with the prevailing disbelief about life after death and the refusal of human beings to submit to a final day of reckoning. A number of passages (Q. 36:77-9) undertake to emphasize the limited nature of human sojourn on earth and remind humanity that this world is the preparation stage for the ultimate return to face the Creator. Even more significant is the emphasis the Qur'an lays on death as simply a stage before the final judgment takes place at the time of resurrection, when all the dead will be resurrected to settle their account while on earth. In this sense, death is not extinction. It signals a simple alternation of state. The Prophet's contemporaries had a hard time believing that God could bring the decomposed bodies of the dead back to life for the final judgment.

In this sense, the references to death in Muslim scripture actually serve as reminders to take one's performance in life seriously because there is an afterlife during which humans will reap the fruits of their deeds. This accountability to God was unpleasant to the ears of Meccan Arabs, who ridiculed the concept of afterlife because it morally challenged them to rise above the present moment and take on the responsibility of sharing their wealth with the less fortunate in society. Consequently, the Qur'an connected the creation to the theme of temporariness and fragility of life. All that was created will perish (Q. 55:27). Nature is the scene of constant birth, death, rebirth, and death as a divinely ordained destiny. But human beings, out of self-cultivated ignorance and arrogance generated by their ability to master and use nature to their advantage, and in spite of witnessing the phenomenon of death around them, deny the transience of their sojourn in the world. They live under an illusion that death cannot overtake them. The Qur'an, therefore, reminds humanity that death will come to all human beings, even though they may be in raised-up towers (Q. 4:78).

Accordingly, life in the Qur'an is linear in development. It has a beginning and an end. Death is one of the critical stations in this linear journey when the external physical life comes to an end, marking the beginning of the intermediate period before the final day of resurrection (yawm al-qiyāma) and recommencement of the pristine moment of 
new development when God will raise the dead as a new act of life-giving creation. The theme of resurrection combined with death forms the decisive argument for belief in God's omnipotence and omniscience in the Qur'an. Resurrection in particular marks the closing stages of individual life history that began with the ensoulment of the fetus in its mother's womb (Q. 22:5). Although the Qur'an does not define the concept of death, in one of the verses in which the description of death is provided (Q. 6:93), it affirms the existence of the soul, which must be separated from the body.

In speaking about the agonies of death suffered by the wicked, it uses the crucial term nafs, which means the entity that infuses the human body with life and that causes the body to die when it departs. In addition, in three places, the Qur'an uses both the word nafs and death (mawt) to declare that death is bound to occur for "every nafs." Every nafs (person) shall taste death, and God tests human beings with good and evil, "then unto God you shall be returned" (Q. 21:35-6; 29:57; 3:185). In other words, the Qur'an says that it is an ensouled individual who has to taste death and not just his physical existence separate from his soul. Here death is equated with the complete loss of the body's integrating capacities as signified by the presence of the soul. When the soul departs, the human body ceases to exist as an integrated human person. Death then occurs when an individual ceases to be a person. It is the termination of features that are essential to being a person capable of reasoning, remembering, feeling emotion, possessing the will to believe and disbelieve, and not simply being a living organism. In other words, by introducing the notion of "giving up one's soul," the Qur'an provides a set of criteria to determine death that has more to do with the quality of life than the activity of the central nervous system and the brain.

In another place the Qur'an uses nafs for "human being" when it declares that "God does not charge any individual (nafs) beyond his/her capacity" (Q. 2:286). Still in another instance it commands: "Do not kill one another (anfus)" (Q. 4:29). In denoting the complexity of the Arabic usage, whether in singular or plural form, the Qur'an uses the term to mean the integrating capacity that signifies personhood and not simply "soul." In the context of this chapter, then, it is accurate to interpret "every nafs shall taste death" as "every person shall face death." Later Muslim theologians' conceptualization about the soul-body dualism notwithstanding, the Qur'an, by introducing the term nafs in the context of imminent death, is speaking about some complex entity that provides an essential feature in assessing personhood. Accordingly, when death occurs, the nafs leaves the body, preventing it from maintaining its vital functions.

Nevertheless, life does not end with death. In the same way that a person does not cease to exist in sleep, so also, he does not cease to exist in death. And in the same way that a person comes back to life when waking from sleep, so too will he be revived at the great awakening on the Day of Judgment. Hence, death is merely a stage in human progression toward the End of Time. Physical death should not be feared as an evil. One should, however, worry about the agonies of spiritual death caused by living a life of moral corruption. That sinful life is not worth living.

As a logical consequence, the mystery of life and death is resolved in the Qur'an by linking it to the working of human conscience as an overall feature of the nature (fitra) with which God created human beings. As a created being endowed with an innate capacity to maintain a healthy status of human spiritual-moral existence with faith in God, human beings should serve the purposes of God by engaging in God's service ('ibada) to benefit the whole of creation. Once that necessary equilibrium between faith and work in 
life is lost, then a human being's death has set in. It is this death that should cause anxiety in human beings. Human efforts should be concerned with the revival of human conscience, which will lead to a meaningful life. Does it mean that the human body should simply be neglected? No, not at all. It basically means that human death must be viewed as secondary to the spiritual and moral quality of life.

Thus, religious evaluation of death in Muslim culture is, historically, founded upon the Qur'anic emphasis on death as an inescapable human condition that can be conquered only by God. It is only God who can deliver humanity from the condition of death for the sake of new creation, that is, the resurrection. Humans must wish to live long and pray to God for a healthy life to serve God's purposes. It is forbidden to wish for death even though the tradition encourages believers to remember and prepare for death. In this sense, life and death convey two metaphysical rather than physical states. Those who believe are ever ready to meet their Lord by detaching themselves from life and preparing to be taken by God; whereas, those who disbelieve are attached to this life and afraid to die and face God. In the case of the latter group, they are already dead in spirit.

Life in Muslim culture has a set of criteria that must be fulfilled in order for the patients, their families, and society in general to regard a person as living. These include a person's ability to make decisions and execute them through his/her own conscious and cognitive competence. In the absence of such competence, close family members or religious and lay leaders in the community assume the guardianship to protect the person's rights. The principle of equity and public good for society in Islam allow the leaders of community to act as surrogate decision makers in cases where the person, due to mental or any related incompetency, is unable to assume that responsibility.

This conceptual and cultural understanding of death in Islam is important to keep in mind as we consider the legal and ethical deliberations that were undertaken by Muslim legists in providing the criteria to determine death. Their decisions were not reached solely on the basis of what we would call normative sources like the Qur'an and the Tradition; they also considered a cultural understanding of the nature of life and death in the community. In Muslim culture, until recently when modern medical technology admitted the distinction between cessation of cerebral function, on the one hand, and cardiac and respiratory activities, on the other, the pronouncement of death was based upon the criteria provided by the vitality of the mutually dependent organic system of the human body. The terms cerebral death and brain death are neologisms in Muslim societies, which continue to see death as the cessation of vital functions in a single-organ system rather than in a part of that organ.

\section{Life and death in Islamic jurisprudence}

Evidently, in light of the lack of clear definitions in the Qur'an, there has been much speculation about the exact identification of the indicators of death in the tradition. To be sure, the task of defining death cannot be relegated to the literary sources that describe its inevitable occurrence in all human societies and the ways human cultures come to terms with it. More pertinently, as pointed out earlier, the definition of death is not merely a matter of collecting relevant data from medical facts or scientific investigation alone. The exercise involves understanding the very essence of human life that shows the interaction between an ephemeral physical substance and an eternal entity 
that departs it at the time of death. That elusive component which transforms the human body into a living being is the spirit $(r \bar{u} h)$ or the soul $(n a f s)$. This is the very entity that enters the fetus and ensouls it in the beginning of the embryonic journey to personhood, thereby effecting a change in the moral and legal status of the developing fetus. At the end of life, this entity leaves the body to return to its original source in the world of the spirits ('alam al-arwāh). Of course, both the existence of the spirit and its infusion or separation from the human organism do not lend themselves to direct empirical observation. In all religious traditions, there has been sustained interest in discovering the exact nature of this unobservable entity that enables the human body to perform all its functions through the energy that it provides in cardiac and respiratory activity - until it departs and leaves human body dead. Just as the subject of the beginning of life in Islam raises questions about the exact moment when the spirit infuses the body to determine the fetal inviolability, so at the end of life there is also the challenge of determining the moment when death has without any doubt occurred.

The exact nature of the relationship between the body and the spirit has assumed a pivotal importance in the debates that have opened up in recent decades in Mekka, Amman, and other centers about the nature of neurological death and the criteria that must be admitted to determine their validity in pronouncing an individual dead. These debates have gained considerable vigor given the robust medical tradition in many Islamic societies and the presence of a number of leading medical centers.

There are two theories concerning the relationship between the physical body and the spirit. According to one, the reality of humankind is its spiritual substance or divine element, and the body is simply an instrument that serves this spiritual substance. According to this, the spirit is not something that resides in the matter; rather, it is created by God as a source of life and linked to the body. This relationship is one of managing and having discretion over the body. ${ }^{2}$ According to Abū Ḥāmid al-Ghazālī (d. 1111) and other Sunni scholars, the spirit $(a l-r \bar{u} h)$ is the subtle divine essence (al-latîfa al-rabbāniyya), knowing, thinking and percipient, abiding in the human being - the concept that is applied sometimes to the heart (al-qalb), the soul (al-nafs), and the intellect $\left(a l^{-} a q l\right)$. The bodily parts are the tools of the spirit, which, when put to use, enable the organs to perform different tasks and enable the heart to know the true nature of things. The "heart," as al-Ghazālī explains, is merely another expression for the "spirit," which is able to learn and independently feel the emotions without any link to the bodily parts. When the body dies, the spirit is separated, and the ability of the body to function comes to a halt. ${ }^{3}$ A tradition ascribed to the sixth Shī'ite Imam, Ja'far al-Șādiq (d. 748), likens the relationship between the spirit and the body to the jewelry and the box safeguarding it, in such a way that the box would be rendered useless if the jewelry were removed from it: "Without doubt, the spirit does not intermix with the body, nor does it blend in it. However, it is like a thin veil for the body, closing in from all sides."4

Another theory regards the spirit as an attribute of life infused by God at the time of fetal ensoulment. In this sense, the spirit becomes identical with the body and the body identical with the spirit, and from their union human personhood emerges. ${ }^{5}$ The spirit as an attribute enables humans to gain knowledge and to feel the pains of sorrow as well as the pleasures of happiness. Death signifies the falling into disuse of the body because of the end of the spirit's control over the body and the end of the body's status as its tool. In the words of al-Ghazālī: "Just as the onset of an incapacitating disease may mean that the hand is no longer a tool of which use is made, so death is an incapacitating disease 
spread throughout all members." ${ }^{, 6}$ Regardless of the nature of this relationship between the body and the spirit, the spirit, which is the source of the humanness of an individual, is actually the source of the personhood that begins when the body is infused with the spirit From some Shī'ite traditions, it is possible to construe that the human being has two spirits or two lives: one, the "ancient spirit" (al-rüh al-qadīma) that has come down generation to generation from the father's loins (așläb al-rijāl) and the mother's womb (arhām al-nis $\bar{a}$ ), and second, the spirit that is known as the "spirit of life and survival" (rūh

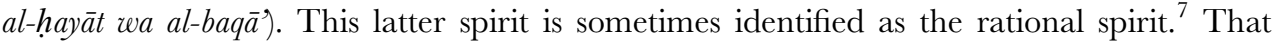
which serves as the criterion for human life is the linkage between the rational spirit and the body. Consequently, if the linkage between the knowing, thinking, percipient spirit that is, the rational spirit - and the body is severed, then cellular life alone cannot serve as evidence for human life. In this sense, death is viewed as the permanent severance of the linkage between the rational spirit and the body, ${ }^{8}$ whether that linkage has been severed as a result of the spirit being liberated from the body because of its perfection ${ }^{9}$ or as a result of an incapacitation of the body that prevents the spirit from penetrating the body, barring it from the use. ${ }^{10}$

Undeniably, whereas it is not difficult to ascertain the beginning of life, even with highly sophisticated techniques for determining the presence or absence of vital signs, it is not religiously easy to ascertain the end of life. From the religious point of view, despite the fact that it is not known when exactly the spirit departs from the body, a number of criteria have been cited in the scriptural sources that speak about the separation of the spirit from the body when death occurs. Among these signs are the deterioration and ultimate decomposition of the body, the whitening of the skin, the sweating of the forehead, discharge of fluids from the eyes, discharge of sperm, slackening of the muscles, the contortion of the eyes, and wrinkling of the lips. ${ }^{11}$ In medicine, death has been classified as two types, namely, physical and cellular death. The symptoms cited for the occurrence of each type correspond to those mentioned in the Islamic traditions. ${ }^{12}$

Life and death are ultimately existential matters. Their correlation suggests a contradiction in terms since life indicates continuation of the blessings of living, while death points to extinction. The existential aspect of death that serves a critical purpose in relieving an individual from a lengthy period of suffering through illness or other afflictions can be discerned from the following verse of the Holy Qur'an that glorifies God for having created both life and death:

Blessed be God in whose hand is the Kingdom -

God is powerful over everything -

who created death and life, so that God may try you

which of you is fairest in works.

In this passage, the act of creation is introduced as an external phenomenon, for which God is exalted, and which includes the creation of death as God's blessed activity, even though some commentators are of the opinion that since death suggests the opposite of what life indicates, there is ostensibly a contradiction in their correlation in creation. ${ }^{13}$ However, no matter whether one holds that the correlation between life and death is contradictory because death signifies the negation of existence, or that life and death are two existential matters, there is absolutely no way of conceiving them as unrelated. They 
remain linked despite the fact of their being contradictory because they are like the two irreconcilable positions for which a third possibility is cognitively inconceivable.

\section{The social-psychological dimension of death}

The focus of this chapter is to capture and describe the classical religious and juridical aspects of death in Islam that continue to influence important contemporary rulings and attitudes about death. More specifically, with the new medical discussion regarding brain death, Muslim centers of juridical studies have correctly argued that investigation needs to go beyond this to understand and deliberate on the suffering of those who are closely related to the patient. Serious illness overwhelms everyone in the family. It is undoubtedly that moment in family life that demands a collective response to handle the pain and suffering of the patient, on the one hand, and the loss and separation that will come to pass following the imminent demise of the patient, on the other. The socialpsychological dimension of death includes the way in which the family undertakes to preserve the dignity of the dead and the honor of those left behind. The preservation of the dignity of the dead involves safeguarding respect for human personhood and avoiding all decisions that would lead to desecration of the human body.

In this situation, the brain-dead people are the most vulnerable. Any procedure using newly dead patients for medical research and other humanitarian and educational purposes is subject to criticism. More critically, any suggestion of retrieving their organs at that point is repugnant because the patient can no longer protect his/ her dignity. In all Islamic communities, even when the patient has left a specific advance directive to remove his organs to help those in urgent need of transplant, the relatives cannot allow harvesting of the organs because of the invasive postmortem procedures.

In the Islamic juridical tradition on death and dying, a large number of the rulings deal with the social and psychological implications of death for those who are left behind: parents, spouse, children, and other relatives. As a matter of fact, Muslim funeral traditions appear to enable the relatives to cope with the loss of the loved one, even if it be a child, whose departure has become a major source of disruption in the normal familial life. Accordingly, the tradition deals with death on two levels: (1) at the formal level of rituals that must be performed for the dead by the family and the community; (2) at the legal level of rulings that outline the rights and duties of the immediate family members toward the dead and the survivors. Consequently, at the ritual level, the Shari'a lays down the rules about the number of days of mourning; the religious personages (the Prophet, the Companions, the Family) who should be remembered in these ceremonies; those who should bring food to the family of the dead; visitations of the graves in the cemetery; recitation of the Qur'an on the grave; and so on. Ghazāli has aptly captured the spirit and purpose of these funeral rituals to encourage people to visit and console the family members for their loss:

The properties of attending funerals include meditation, heedfulness, preparedness, and walking before the pall in humility ... The man of insight looks to the graves of others and sees his own place amongst them, and then readies himself to join them ... In general, the visitation of graves is a desirable thing, for it instills the remembrance of death and acts as an admonition. ${ }^{14}$ 
Despite all this interest in informing the right practice, distinct from the pre-Islamic period, there is a total absence of any attention to the ownership of the cadaver and whether it is within the rights of the deceased or his family to donate any organ needed by the living, or whether it is appropriate to allow postmortem dissection to determine the cause of death. The use of newly dead patients for medical research and educational purposes was far from any consideration when diagnostic evaluations were limited and certainly could not include any invasive postmortem procedures that desecrated or disgraced the dead. The Shari'a formulations dealing with the last will of testament (wasiyya) did not go beyond the external assets of the deceased and their distribution to the heirs. One does not come across a single ruling, for instance, dealing with the permissibility or prohibition of donating one's cadaver for research. The sanctity of life and the dignity of the human being were at the center of the classical rulings regarding life and death in Islam. It was inconceivable to think about the harvesting and banking of a variety of tissues and organs for future transplants. To save a life was and remains the goal of medicine. But to use human bodies to save other humans was repulsive because of the desecration that it suggested. Human bodies and spirits are regarded as God's gift and, as such, human beings do not possess absolute title over their lives or bodies. They are like stewards charged with preserving and dignifying their life by following the guidance provided in the revealed texts to lead a healthy lifestyle.

\section{Withholding or withdrawing treatment? Who decides?}

Today abortion and end of life issues have become religiously, emotionally, and politically charged topics. But while many people have challenged the abortion issue on one side or the other, not everyone will face a direct need to make that decision. However, all persons will face death. The manner in which this occurs for our loved ones and ourselves is of critical importance to each one of us. The meaning of human life is grounded in the fact that it is sacred because God is its origin and its destiny. Death does not occur except by God's leave, as the Qur'an reminds humanity. At the same time, there is recognition of the fact of diseases or trauma that cause death. Health-care providers have an obligation to do all that is possible to prevent premature death. The question that is often asked is: Is the goal of health-care professionals to maintain life at any cost or merely to provide comfort so that death may come as quickly and comfortably as possible? The question has wider implications because it evokes different and often competing ethical values in deciding the course of action or specific interventions that the situation demands. On the one hand, there is the obligation to save and prolong life; on the other, there is a call to impose limits on life-sustaining treatment as required by principle of distributive justice in resource allocations. Nevertheless, the real question is the authority that can make such decisions. Who should draw the line between the personal values and beliefs of the people and a more objective medical analysis made by healthcare providers? Should the financial burden of life-sustaining treatment ever dictate its termination?

The role of religious guidance in these and other matters related to most critical care decisions is to speak authoritatively about death and about self-imposed limits at the professional level regarding such matters. There is no doubt that medical judgments about death are usually based on probability. It is only very close to the time of death that physicians can predict the end with certainty. Hence, the Qur'an puts forward a harsh 
reminder to human beings that there are times when they need to recognize their own limits as humans and entrust nature to take its own course (Q. 39:42). Refusal to recognize the inevitability and naturalness of death leads to more aggressive life-saving interventions. At the same time, to withhold specific interventions at the most critical time leads to deliberate avoidance of responsibility in administering the appropriate treatment to save the patient's life. ${ }^{15}$

For the past three decades, people in different parts of the world have struggled to identify the proper circumstances under which life-sustaining medical treatment should be limited. The phenomenal advancements in the field of medical technology have not allowed for the development of adequate procedures and processes to regulate their introduction and use in critical-care settings. Religious and psychological factors play a major role in any decision that leads to the termination of life. Muslim scholars have debated the issue in the context of brain death and retrieval of organs. Although there seems to be a consensus among legal experts belonging to different schools of Islamic law about brain death resulting from irreversible destruction of the brain, the question that remains to be answered is a theological one connected with the location of human soul. The problem centers around determination of where exactly the human soul - which departs at the time of death - is located. The classical legal definition connects death with the traditional signs, including complete cessation of the heartbeat, whereas biological data about the function of the heart and other major activities connect life with the functions of human brain. ${ }^{16}$ This discrepancy between the religious and contemporary scientific definition of death has generated even greater challenges for family members and health professionals when making decisions about withholding life-saving medical intervention, for instance, in the treatment of cardiopulmonary arrest. Cardiopulmonary arrest is regarded as the final common pathologic-physiologic event in the dying process. In such a medical condition, failure to act immediately by undertaking CPR, which involves external chest compression and some form of artificial respiration, means certain death.

The other question in Muslim societies is the question of whether society should provide the substantial resources that aggressive treatment of long-term care patients would require. In Third World countries, where resources are limited, the issue of the proper allocation of the nation's resources takes center stage. The physician's scientific knowledge and clinical experience enable him to recognize when a life-sustaining treatment is futile. He exercises a strong paternalism that promotes the patient's (and the family's) wellbeing by limiting such treatment unilaterally and without informing the patient and/ or family. On the other side, society has an interest in limiting futile interventions to divert limited resources to more productive use within, or even outside, the health-care system.

In Islamic juridical ethics, the individual's welfare is intimately linked with his/her family and community. Accordingly, it is not the principle of autonomy that is evoked to determine a course of action in matters relating to end-of-life decisions. Whether a physician can prolong life by introducing aggressive invasive treatments without causing further harm is decided by all those connected with the patient. Yet, there are instances when the matter is referred to the religious leaders, who provide prescriptive rulings connected with terminally ill patients. In the following section we will examine some of the approaches that are in place among Muslims to help health-care providers to deal with this sensitive period in the lives of those for whom death is imminent. 


\section{Right to die?}

"How fortunate you are that you died while you were not afflicted with illness." Thus said the Prophet addressing the person whose funeral rites he was performing. Such an assessment of death without illness coming from the founder of Islam indicates the value attached to a healthy life in Muslim culture. To be sure, good health is God's blessing for which a Muslim, whenever asked: "How are you? (lit. 'How is your health?')" responds: "All praise is due to God!" However, this positive appraisal of good health might seem to suggest that illness is an evil that must be eliminated at any cost. No doubt illness is regarded as an affliction that needs to be cured by every possible legitimate means. In fact, the search for cures is founded upon unusual confidence generated by the divine promise that God has not created a disease without creating its cure. ${ }^{17}$ Hence, the purpose of medicine is to search for cures and provide the necessary care to those afflicted with disease. The primary obligation of a Muslim physician is to provide care and alleviate the suffering of a patient. Decisions about ending the life of a terminally ill patient at her/his request are beyond his moral or legal obligations. The Qur'an states its position on the matter in no uncertain terms: "it is not given to any soul to die, save by the leave of God, at an appointed time" (Q. 3:145). Moreover, "God gives life, and $\mathrm{He}$ makes to die" (Q. 3:156). And, hence, "A person dies when it is written" (Q. 3:185, 29:57, 39:42).

Death, then, comes at the appointed time, by God's permission. In the meantime, humans are faced with the suffering caused by illness. How is suffering viewed in Islam? Is it part of the divine plan to cause suffering? To what end? These general questions about the meaning and value of suffering should lead us to appraise the suffering caused by prolonged illness to an individual's personal and family life. The need to decide to end a life arises precisely at that critical point when the sick person is undergoing severe discomfort and desperation, and when all forms of advanced medical treatments have failed to restore her/his hopes of getting better.

Closely related to such a consideration on the part of the sick person is whether the unbearable circumstances caused by one's interminable illness leave existence worthwhile at all. Does such an existence, which is almost equivalent to non-existence because of an intense sense of helplessness in managing one's life, possess any value for its continuation? Beneath these concerns remain deeper questions about the quality of life that individuals and society regard as worth preserving.

\section{The question of the quality of existence}

The importance attached to the quality of life question has sometimes led Muslim scholars to evaluate suicide (in Arabic expressed as qatl al-nafs = "homicide") in ambiguous ways. On the one hand, there is unanimity in declaring the act as an irrational behavior that human beings should not commit; on the other, their interpretation of the act being committed in situations that a person is unable to cope with, indicates a factual, even condoning attitude toward suicide. There are cases reported in classical sources where some men who committed suicide under tragic circumstances highlight the ethical and legal debate surrounding the right to end one's life under compelling circumstances. 
Most of these compelling circumstances are culture-specific, that is, they are the product of cultural expectations in maintaining human relationships. In a typical Muslim cultural setting, when a person lives in very reduced circumstances and people begin to avoid her/him more and more, and her/his acquaintances no longer want to have anything to do with her/him, this would count as an undeniably cruel situation. If it goes on for a while, one might feel compelled to end one's life. Such a decision is viewed ambivalently in society. Some might praise it as a splendid thing that indicates magnanimity and a staunchness of mind for acting to free oneself from a long-drawn-out misery and from circumstances which were unbearable and brought not only isolation but great privations and a steady reduction of means. However, it is certainly difficult to justify the decision from a religious point of view. The action of the suicide leads one to escape from the dreadful situation in which the person had been into another situation, which, from the religious point of view, is considerably more frightful and of a much longer duration than that which she/he had been in. In Shari'a these and similar actions are forbidden, and even the commission of much lesser deeds is prohibited. The reason for the prohibition of suicide is that it might be committed under the influence of ideas and hallucinations, which would not have occurred to a person in full possession of her/his mental faculties. Later on, in the other world, the person who committed suicide under such circumstances would realize the baseness of her/his action and the great mistake she/he had made; but it would be too late to repair, correct, or retract what was done.

The individual should not have broken established customs, opposed entrenched opinions, and arrogated the rights of nature. Furthermore, she/he should have refrained from her/his deed since it is absolutely clear that a human being must not separate those parts and limbs that have been joined together (to form the body); for it is not she/he who has put them together, and it is not she/he who is their real owner. She/he is merely a

tenant in this temple (i.e. the human body) for Him Who made him to dwell therein and stipulated that in lieu of the payment of rent for his dwelling he take care of its upkeep and preservation, its cleaning, repair and use, in a manner which would help him in his search for happiness in both this world and the next world. ${ }^{18}$

\section{Human stewardship of the human body and suffering}

The discussion about the quality of life points to the cultural and religious attitudes regarding human existence and the control over life and death decisions when an individual is overcome by suffering. Furthermore, it underscores the view that the human being has the stewardship, not ownership, of his body. He is merely the caretaker, the real owner being God, the Creator. As a caretaker, it is his duty to take all the necessary steps to preserve it in a manner that would assist him in seeking the good in both this world and the next. In light of such a stipulation about human duty toward his earthly existence in Muslim theology, the problem of human suffering through illness assumes immediate relevance. The Qur'an provides the essential philosophy behind human suffering by pointing out that suffering is a form of test or trial to confirm a believer's spiritual station (Q. 2:153-7). Suffering is caused by the divinely ordained trial. More pertinently, it functions as an instrument in revealing God's purpose for humanity and in reminding 
it that ultimately it is to God that it belongs and to God that it will return. Accordingly, suffering from this scriptural perspective cannot be regarded as evil at all. In a wellknown tradition, the Prophet is reported to have said: "No fatigue, nor disease, nor sorrow, nor sadness, nor hurt, nor distress befalls a Muslim, even if it were the prick he received from a thorn, but that God expiates some of his sins for that." 19

In fact, in another place the Prophet has prayed: "O God, do not let Your trial be the cause of misguidance for me!" In other words, afflictions in the form of trial should not lead a person to lose hope because despair stems from a lack of trust in divine mercy. Other traditions recognize a religious purpose in illness and underline the reason for it, as for other forms of suffering, as being God's trial of the people and the cleansing effect of illness. Hence, in one tradition, the Prophet says that the patient earns merits under these trials and can attain the rank of a true believer. "When God intends to do good to somebody, He afflicts him with trials." 20

In addition to this spiritual-moral dimension, suffering serves an educational purpose. Here suffering is caused by human misconduct. It serves as a form of punishment to expiate a sin. Disbelief in God's power to heal and restore health is the major source of human desperation. It is also regarded as the source of human arrogation of the rights of God. Experiencing severe and untreatable suffering caused by illness serves as a reminder of one's being deprived of the divine blessing of good health through one's disbelief in God. As such, suffering is a means to self-purification from sinful behavior. Thus it is that when they are afflicted with illness Muslims are advised to beseech God to forgive their sins. Rather than contemplating ways to end one's life, either by refusal of life-support treatment or by requesting to die with active assistance, a Muslim is required to pray for an opportunity to have a fresh start with restored health.

\section{Human response to suffering}

This religious and spiritual valuation of suffering does not answer the critical question when one is going through illness: Should one take it upon oneself to alleviate suffering where it is not possible to endure the suffering instead?

In general, Muslims have tended to respond actively to removing the cause of suffering. The notion behind this active response is that since the human being is the cause of her/his suffering, she/he should undertake to do righteous acts to rid the world of suffering. According to the Qur'an, "surely the good deeds will drive away the acts of disobedience (which cause suffering)" (Q. 1:114). Contrary to this activist position, there is also an attitude of resignation in some quarters among Muslims who believe that since God is testing human faith and purifying it through affliction, one must endure suffering. Nevertheless, even in this passive response, the Qur'anic promise that good works alleviate divine punishment generates some endeavor to overcome suffering.

These two attitudes had their justification in Muslim creed. Muslims who believe justice to be the fundamental attribute of God also regard the human being as God's free agent, endowed with volition and ethical cognition and, hence, responsible for her/his acts. Accordingly, she/he should exercise her/his will in overcoming difficult and unbearable situations. On the other hand, Muslims who maintain omnipotence to be the essential attribute of God do not believe that the human being has the volition to act independent of divine predetermination. Hence, suffering must be endured with patience and perseverance. Life is a testing ground for humankind. Those who submit to the will 
of God shall attain prosperity. God's plans will justify and vindicate the righteous in the end.

The question that is so central to the present-day discussion, namely, the patient's "right to die" cannot be negotiated because, in the first place, life is a divine trust and cannot be terminated by any form of human intervention, and, in the second, its term has been fixed by the unalterable divine decree.

\section{End of life decisions in Muslim tradition}

The belief in God's immutable decree is also revealed in Muslim ethics, where not only is the right to die not recognized but the right to be assisted in dying, whether through "passive" or "active" means is also ruled out. It is important to clarify here that since the end-of-life decision is through divine decree, the Shari'a refuses to recognize individual right in that matter. However, it recognizes the possibility of arriving at a collective decision by those involved in providing the health care, including the attending physician and the family. The ethical principle affirmed in most unequivocal terms by the Prophet states: "No harm shall be inflicted or reciprocated in Islam" (la darar wa la dirar fil-islam). ${ }^{21}$ This is the principle that lays down the justificatory force of the ruling to avoid causing harm or harassment to the patient. It also allows for important distinctions and rules about lifesustaining treatments in terminally ill patients. The distinction upon which ethical decisions are made includes the distinction between killing (active euthanasia) and letting die (passive euthanasia). The killing/letting-die distinction often underlies distinctions between suicide and forgoing treatment or between homicide and natural death. The principle of "No harm" in some ways functions like the principle of nonmaleficence. It raises a critical moral question about the intention of health-care providers in withdrawing life-sustaining treatment: Can such a decision be regarded as a form of killing, and if so, is it assisted suicide or homicide? There is no immunity in Islamic law for the physician who unilaterally and actively decides to assist a patient to die.

\section{Pain relief treatment and withdrawal of life-sustaining treatments}

There are, however, two situations in treatment that could be interpreted as "passive" assistance in allowing a terminally ill patient to die. Pain relief treatment, which could shorten life but which is administered to relieve physical pain and psychological distress and not intended to kill, is permitted in Islamic law simply because the motive is regarded as a sufficient justification, protecting the physician against criminal or other liability in such circumstances. As long as the situation does not involve an intention to cause death, a medical intervention to provide necessary treatment for the relief of pain or other symptoms of serious physical distress is not regarded as criminal.

Similarly, in relation to withdrawing treatment - whether pursuant to a refusal of a death-delaying treatment or through the collaborative decision-making by patient, physician, and other parties involved in providing care for the patient - although there is an intention to allow the person to die when it is certain that death will result from its omission, Islamic law regards it a non-culpable act. Delaying the inevitable death of the patient through life-sustaining treatment is regarded as being against the benefit of the patient. Moreover, the principle of "juristic preference" (istihsan = "to deem something 
preferable") protects the physician by authorizing departure from the already established ruling about the prohibition of allowing death to occur in order to avoid any rigidity and unfairness in recognizing the incurable preexisting conditions of the patient. Furthermore, by authorizing the removal of life-sustaining treatment in cases where this treatment merely results in delaying death, the "juristic preference" serves the ideals of justice and public interest in a better way. In other words, enforcing the existing prohibition against allowing the patient to die could prove to be detrimental in certain situations, and a departure from it may be the only way of attaining a fair solution to a particular problem. Withdrawal of life-sustaining treatments in such cases is regarded as allowing inevitable death to take its natural course. Notwithstanding a fine line between having and not having an intention to cause the death in such omissions, Islamic law does not forbid withdrawal of futile and disproportionate treatment on the basis of the consent of the immediate family members, who act upon the professional advice of the physician in charge of the case. Some Muslim jurists have recognized the validity of a competent patient's informed refusal of treatment or "advance directives" that allow the person to die in circumstances when there is no medical reason to continue treatment. However, even in such rare cases of recognition of the patient's autonomy in Muslim culture, the law actually takes into consideration the patient's long-term treatment relationship with the physician, whose opinion, in the final analysis, serves as the grounds for turning off the respirator, for example. In this case, the death is regarded to have been caused by the person's underlying disease rather than the intentional act of turning off the machine.

The underlying principle in this ruling is that intention alone does not make an act culpable. The person's death is actually caused by the preexisting disease, when the withdrawal of the treatment is justified through the expert opinion. This can be contrasted with giving a person a lethal injection. The injection is the sole cause of the person's death and is clearly regarded as its cause in fact and in law by Muslim jurists.

To sum up the Islamic ethical-legal perspective on the "right to die" of a terminally ill patient with no hope of recovery, it is important to restate that the justificatory force of the rulings on "allowing to die" by withdrawal of life-sustaining treatments is contingent upon a well-informed consultation with the physician and other parties involved in the patient's treatment. More importantly, since Islamic legal deliberations contain and ground morality as part of the spiritual response to God in interhuman relationships, purely individual acts to terminate life, by the patient or by the assisting physician, are both regarded as acts of disobedience against God. Pain-relief treatment or the withholding or withdrawal of life-support treatment, in which there is the intention to allow the person to die when it is certain that the disease is causing untreatable suffering, are permissible as long as the structures of consultation between all the parties concerned about the wellbeing of the patient are in place. In the final analysis, besides the exceptions noted in these two situations, there are no grounds in Islam for the justifiable killing of a terminally ill person, whether through voluntary active-euthanasia or physician-assisted suicide.

Discussions of appropriate Islamic guidelines on a number of contemporary medical issues, even including organ donation, continue in many places, both within and outside Muslim-majority societies. We have seen that a well-established religious stance on death did not provide totally clear criteria on such matters as ascertaining when a life has ended. Medical breakthroughs, in turn, have created a number of new dilemmas. Islamic 
tradition continues to provide a basis for ongoing efforts to guide patients, families, and medical practitioners in making some of the critical decisions involved at the end of life.

\section{Notes}

1 The Qur'an uses the term nafs, with the meaning of personhood rather than soul.

2 In a theosophical commentary, Dawūd b. Muhammad al-Qayșarī, Sharh Qayșar̄ 'alā Fușūṣ alhikam (Tehran: Anwār al-Hudā, 1416/1994), Vol. 1:113 writes: "Know that from the point of its substance, its disengagement from substratum, and its existence the spirit (al-rüh) belongs to the world of the spirits, disengaged changing in the forms of corporeal body, linked to it, a linkage connected with governing and exercising free disposal, self-subsistent, not in need of it for its survival and for its support."

3 Abu Hammad al-Ghazālī, The Remembrance of Death and the Afterlife (Cambridge: The Islamic Text Society, 1989), pp. 122-3; see also different views of the Sunni scholars in Muhammad 'Alī al-Barr, Mawt al-qalb aw mawt al-dimāgh, (Jedda: al-Dār al-Sa'ūdīya, 1406/1986), pp. 37-45.

4 Muhammad b. al-Ḥusayn al-Ṣaffār, Bașāìr al-darajāt, p. 463

5 Muḥammad b. Rāzī, 'Umar al-Ḥusayn al-Rāzī, al-Tafsīr al-kabīr (Cairo: al-Maṭba'aa;-Baḥīya alMișrīya, 1938) Vol. 21:45.

6 al-Ghazālī, Remberance of Death, p. 123.

7 The tradition is related on the authority of the seventh Imam Mūsā al-Kāzim, who describes the embryonic development in the womb as follows: "When God desires to create a sperm drop He inspires the womb to open its entrance to let in the sperm drop ... Then God sends two maker angels to shape what God wishes to be in the womb. Hence, they intrude in the interior through the vagina until they reach the womb, where there is already in place the ancient spirit (al-rūh al-qadìma) that has come down through the loins of men (așlāb al-rijāl) and the wombs of the women (arhām al-nisa $\left.\bar{a}^{\prime}\right)$, and blow in the spirit of life (ruh al-hayajt) and survival $(a l-b a q \bar{a})$ endowing the fetus with hearing, seeing, and all other parts of the body and all that it needs from the womb, as God permits it." (Muhammad b. Ya'qūb al-Kulaynī, al-Ușūl min alKäf (Tehran: Kitāb-furūshī Islāmīya, 1392/1972), Vol. 6:13-14, 7:347). It is not far-fetched to suggest that the ancient spirit seems to be the DNA that is inherited as part of the biological parents' contribution to the child's characteristics; whereas the second spirit appears to be the innate capacity to perfect oneself by means of the abilities connected with survival.

8 Ibn Bābwayh, "Ilal al-sharāyi," (Najaf, Maktaba al-Haydarīya, 1965), Vol. 1:107-8, relates a tradition on the authority of the sixth Imam al-Șādiq which reads: "Such is the creation of a human being. He is created for this world and the next. Hence, when God combines for him the two it becomes his life on earth because he has forgone the heaven for the sake of the world. Thus when God puts separation between the two, that separation becomes death. The matter reverts to the heaven, and in this way life on earth and death in the heaven due to the separation between the spirit and the body."

9 Mulla Șadra, a Shi'ite philosopher, in his explanation of natural death says: "The basis for this is the freedom of the spirit from its inherent life and abandonment of using the bodily tools gradually until it becomes isolated by itself and frees itself totally from the body so that it can develop into the one commanding an act." Șadr al-Dīn Shīrāzī, Al-shawähid al-rubūbiyya (Tehran, Intishārāt-I Șadā va Sīmāyi Jumhūrī Islāmī-ye Iran, 1987), p. 89.

10 Hishām b. al-Hakam asked the Imam al-Ṣādiq if the spirit is other than the blood. The Imam replied: "Yes, the spirit, as I have explained to you, is the substance from the blood. When the blood becomes cold [upon death] the spirit leaves the body." See Abū Manșūr Aḥmad b. "Alī b. Abī Tāāib al-Ṭabarsī, al-Iḥtijäj (Beirut, 1966), Vol 2:97.

11 al-Barr, Mawt al-qalb aw mawt al-dimägh, pp. 71-82 covers the Sunni views on the criteria and critically evaluates the errors that have crept into the Islamic as well as medical pronouncements of death. For the Shïite views, see TTabarsī, al-Ihtijäj, Vol 2:97; Kulaynī, al-Käf̆, Vol. 3:134-5, 3:161-3; Ibn Bābwayh, "Ilal al-sharāyi", Vol. 1:309, section 261.

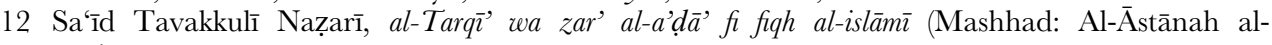
Raḍawīya al-Muqaddasa, 1422/2001), pp. 212-16. 
13 Fakhr al-Dīn al-Rāzī, Tafs̄̄r al-kab̄̄r, Vol. 30:54, writes: "It is said that life is a description for a person inasmuch as he can learn and act; the matter of death is different. Some say that it is actually a negation of this ability [to learn and to act]. We are of the opinion that this is a description of existence contrary to life and we argue that God, the Exalted says: 'He is the one who created death,' because non-existence (al-'adam) is not creatable [on its own]. This is the conclusion."

14 al-Ghazālī, The Remembrance of Death and the Afterlife, pp. 99, 108, 111.

15 In several meetings held in Mekka, Jedda, and Amman under the auspices of the Islamic Juridical Council (Majma 'al-Fiqh al-Islāmì), Muslim jurists of different schools have ruled that once the invasive treatment is intensified to save the life of a patient, it is impermissible to turn off the life-saving equipment, unless the physicians are certain about the inevitability of death. However, in the case of brain death that is caused by irreversible destruction of the brain, including loss of spontaneous respiration, the jurists have ruled that if three attending physicians attest to a totally destroyed brain that results in an unresponsive coma, apnea, and absent cephalic reflexes, and if the patient can be kept alive only through external means by a respirator, then the person is biologically dead, although legal death can be attested only when the breathing stops completely following the turning off the life-saving equipment. See the important bulletin of the organization: Al-qarar al-thani bi-sha'n mawdu' taqrir husul al-wafat wa raf ajhizat al-in'ash min j̈sm al-insan, in Majlla al-buhuth al-fiqhiyya al-mu'asira, No. 4, February, March, April, 1990, pp. 159-60.

16 In a detailed study Marg-i maghzi az didgah-i fiqh va huquq [Brain Death: From the Point of Islamic Jurisprudence and Law] (Qum: Intasharat-i daftar-i tablighat islami, 2001), Dr. Husayn Habibi, physician-cum-ethicist, has contrasted the medical-biological information with the classical juridical definition of death, and has convincingly argued about the brain being the actual location of the soul, the active principle of life endowed with consciousness. However, the majority of jurists continue to regard the complete cessation of the heartbeat as the sole criterion for legal (shar'i) death.

17 Muḥammad b. Ismā‘̄ìl, al- Bukhārī, Sahih al-Bukhari, Kitab al-Marda (Beirut: 'Ālam al-Kutub, 1986), Vol. 7, Hadith \# 582.

18 Abi al-Ḥayy al-Tawhidi, al-Muqtabaṣāt (Kuwait: Dār al-Su'ād al-Ṣabāḥ, 1992).

19 Sahih al-Bukhari, Kitab al-Marda, Hadith \# 545.

20 Sahih al-Bukhari, Kitab al-Marda, Hadith \#548.

21 The principle is regarded as the most important sources for all decisions affecting interpersonal relationships. Hence, it can be regarded as the corner stone of social ethics in Islam. In some important ways, it resembles in its terms the principle of nonmaleficence, in particular when distinctions and rules about life-sustaining treatments consider the distinction between killing and letting die, which in turn draw on the act/omission and active/passive distinctions. 


\title{
DEATH IN AFRICA
}

\section{A history c.1800 to present day}

\author{
Rebekah Lee and Megan Vaughan
}

"Death is a subject Nuer do not care to speak about," wrote E.E. Evans-Pritchard in 1949 in the opening paragraph of an article on "Burial and mortuary rites of the Nuer." What followed from these inauspicious opening remarks, however, was a description of complex burial and mortuary ceremonies. The purpose of these rites was, wrote EvansPritchard, perfectly clear: "the main intention in them is to cut the dead from the living." 1

While "Africa" often figures in the Western imaginary as a space of death (not least in the era of HIV/AIDS), at the same time, African societies are also frequently represented as being "good" at dealing with death. Africans, we are told, have "proper" funerals, not the truncated affairs so common in Europe and North America. Furthermore, they do not cut themselves off from their dead but live in relation to the world of the dead, the world of the ancestors. In Africa the living and the dead together constitute the social world. This characterization is not totally false, but the production of knowledge on death customs and beliefs in Africa has to be seen against the background of a perceived crisis in the "Western" relationship to death. In the 1970s Philippe Ariès argued that whilst mediaeval Europeans (like "primitive" peoples) accepted death as part of life, by the twentieth century they were more likely to attempt to deny it. A combination of industrialization, urbanization, and the rise of scientific medicine eventually produced a situation in which death became a private affair, and one drained of meaning. ${ }^{2}$ Against this picture of death sanitized, medicalized, and uneasily denied, African attitudes to death could be viewed with a degree of nostalgia.

But as Evans-Pritchard's very direct statements imply, if African societies evolved elaborate and complex rituals to manage death, this was because for them too death provoked fear and revulsion and posed a problem for the living. ${ }^{3}$ The ultimate purpose of mortuary customs was to allow the living to get on with living. And in order for this to be achieved, there was no shortcutting the work of mourning. ${ }^{4}$ To simplify, the dead could only find their place as ancestors rather than vengeful ghosts if their loss had been properly registered, not only by the individuals closest to them but by the social groups of which they were members.

If African societies have found effective ways of managing the universal problems posed by death, their ability to continue to do so has been called into question by contemporary developments. In a chilling account of life and death in Kinshasa, Filip de Boeck sees a society saturated with death, particularly violent death, to such a degree that the work of mourning is now meaningless. ${ }^{5}$ Civil war, genocide, and the "banalization" of violence in 
some parts of the continent produce situations in which the normal practices and processes of mourning become impossible. As in Europe after the First World War, mourning is replaced by memorialization, by the creation of museums and national memorial parks. ${ }^{6}$ Rapid urbanization in Africa and international migration have given rise to the use of new technologies of death, seemingly far removed from the burial practices described by colonial anthropologists. A burgeoning African funeral industry has grown to address these new needs. African corpses are now refrigerated and embalmed and captured on video camera. The funeral industry is big business, especially when allied to the insurance industry. To take just one example, in March 2008 the Botswanan newspaper, The Voice, reported that a local company, the Funeral Services Group, would soon be listed on the Botswana Stock Exchange. ${ }^{7}$ The Managing Director of FSG looked forward to a continued period of growth, thanks to new technologies and to a strategic partnership with the Botswana Life Insurance Limited. It is tempting to argue that the "African way of death" is going the same way as the "American way of death," but as we shall see, the reality is rather more complicated than this. 8

\section{The African way of death: death rituals analyzed}

The comparative anthropological study of death is now a large field in which the literature on African societies occupies an important place. ${ }^{9}$ It is not difficult to see why colonial anthropologists viewed the management of death as fundamental to understanding the social fabric and belief systems of "traditional" societies. Funerary and mourning practices express and shape a wide range of social relations, including the maintenance of kinship ties, the reproduction of communal values, and notions of succession and property inheritance. Because they attempt to mediate between the world of the living and the world of the dead, mortuary rituals also reveal a society's spiritual and philosophical orientation.

Although the anthropological literature is too vast to discuss here comprehensively, several elements of that scholarship are relevant to our understanding of African "ways of dying." 10 Of importance is the view that in "traditional" societies, death introduces forces of physical, spiritual, and social rupture. In order to heal these ruptures and ensure the renewal and continuity of life, two transitions must take place. The first is that the deceased must move from a state of impurity or contagion to a state of ritual purity and harmony with the spirit-world. This transition can be guided by the living, through close attention to the ritual preparation and interment of the body. Secondly, as Van Gennep has argued, societal disintegration occasioned by a death has to be repaired through its own transitional process. Through funerary and mourning rituals, survivors are re-integrated back into the community and group-solidarity preserved. ${ }^{11}$ Both transitions are related to each other, and death rituals often serve simultaneously to safely guide the deceased and the living into a beneficial and life-giving balance with each other. But this structuralist account of death rituals, whilst revealing, also disguises huge variations, and has a tendency to represent the production of social meaning around death as an unproblematic process, one devoid of emotion or dissension. ${ }^{12}$

Despite their shortcomings, colonial anthropological accounts provide an important historical record of the sometimes elaborate rites through which Africans buried and mourned their dead and of the political significance of death practices. In Audrey Richards' vivid description of the death and burial of the Bemba Paramount Chief in colonial 
Northern Rhodesia, one can read in the ritual transformation of Bemba land and of the dead body of the Paramount the hoped-for regeneration of Bemba society itself. The one-year interregnum on all land rites immediately following the Paramount's death corresponded with the year-long process of embalming the Paramount's body, until it resembled a "seed."13 This carefully orchestrated scene of ritual regeneration - a "good death" - contrasts with the markedly different case, again recorded by Richards (but not appearing in her published work), of the unnamed Bemba woman who died in childbirth and was buried at a crossroads some distance from the village. This ignominious burial meant that the woman's death could not hold the promise of regeneration.

Divisions made between good and bad deaths often reflected moral concerns over the conduct, in particular the sexual conduct, of the living. A shameful burial in part served as a warning to future generations. This was reinforced by taboos around sexual activity during periods of "contamination" of a woman's body - for example, during menses or lactation. ${ }^{14}$ An equally precise elaboration is evident in the criteria for good and bad deaths among the Anlo of the colonial Gold Coast, as shown in missionary and colonial records. Bad deaths included Anlo who died before they reproduced and those who died "in blood," which could be through war, certain diseases, or accidents. ${ }^{15}$ Furthermore, there is evidence from some African societies that deaths from suicide were regarded as particularly problematic, fearful, and polluting. ${ }^{16}$ While a good death offered the possibility of reincarnation and a welcome influence on the world of the living, a bad death brought only the specter of malevolent ancestral spirits. Indeed, a significant body of literature on witchcraft and spirit possession concerns how the "unnaturally dead" exert power over the lives of the living. ${ }^{17}$

The elaborate mortuary rituals for the Bemba Paramount Chief were essential to maintaining what Audrey Richards described as the "knife-edge" of power over the supernatural. In the context of colonial rule and the depletion of traditional authority within African polities, this "supernatural power" became all the more important to preserve. ${ }^{18}$ Certainly, in the African historical context the veneration of the dead can be seen as a potent political narrative and strategy. The public performance of funerals created a contested space within which deeper struggles over state power and communal identity could be signified. Furthermore, death and mourning rituals were by nature deeply "mnemonic" processes. ${ }^{19}$ Ritual practice could help recall a common, if highly constructed, past, and thus appeals for political reform or renewal could be framed by a mobilizing rhetoric of return. This is evident in Southern Rhodesia, where funerals and commemoration ceremonies became a vehicle through which Christianized Africans translated indigenous institutions into more flexible and politically potent markers of a new nationalist identity. These same funerals were also used by residents as an opportunity to instill notions of dignity and respect within an emergent, and distinctly urban, moral economy. ${ }^{20}$ In Northern Rhodesia, the vibrant "culture of death" forged by mineworkers on the Copperbelt enabled the formation of inter-ethnic allegiances and urban solidarities, which contested traditional authority underpinning Indirect Rule. ${ }^{21}$

In the post-colonial period, contestations over the management of death have occasioned a renewed debate over collective identity and statehood, now voiced in terms of "citizenship." In Cameroon, funerals have become an important arbiter in a new "politics of belonging," particularly for autochthonous groups. ${ }^{22}$ In South Africa, the discursive trajectory - made possible by the provisioning of ante-retrovirals - of "near death" to "new life" espoused by the Treatment Action Campaign has led its volunteers to 
a vigorous assertion of a rights-based citizenship. ${ }^{23}$ Recent debates over the memorialization of key historical figures show the susceptibility of nation-building projects to a continued "politics of death." Wale Adebanwi's sympathetic portrayal of Lt. Col. Fajuyi, and his various reincarnations as sacrificial lamb and savior of a federalist Nigeria argue not only for the plasticity of "heroic" narratives, but for a unique fusion of Christian and traditional imagery. ${ }^{24}$ Similarly, the heated discussions among ordinary Congolese sparked by the potential repatriation of the body of Mobutu Sese Seko in 2001 can be viewed as part of a larger effort to reconcile the memory of his violent rule with the imperatives of a "reborn" nation-state. ${ }^{25}$

\section{Epidemic disease and demography}

Many parents in early twentieth-century Bunyoro (Uganda) apparently did not expect their new babies to live and they named them accordingly: Kalyongera (this one will also die) and Byarufu (this child belongs to death), for example. ${ }^{26}$ As infant survival rates improved in Bunyoro, so these naming practices became less common.

There is no automatic or mechanistic relationship between demography and attitudes to death. The idea that parents in pre-modern Europe, inured to the frequent death of their infants, did not love them in life or mourn them in death, has been effectively challenged. ${ }^{27}$ Yet it is also unlikely that the religious, cultural and social practices that surround death in any society are completely free-floating and unconnected to changing demographies. The rich literature on African beliefs and practices relating to death is largely disconnected from demographic history, and this poses a major challenge for historians of death in Africa. The challenge is all the greater given the paucity of historical sources for the demographic history of the continent, which means that for many regions we have only a very general idea of demographic trends prior to the second half of the twentieth century. But the challenges do also present opportunities, as Doyle's study on Bunyoro demonstrates. "Unconventional" sources such as naming practices not only play a function for the demographic historian as indirect indicators of changing rates of infant and child mortality but are also, simultaneously, rich sources for a cultural history of childhood and mortality. In the same way, the ageing of Africa's population is likely to have some impact on attitudes towards the elderly and towards death in general. ${ }^{28}$ In some regions, the burden of care of both the elderly and the long-term sick has been increasing in recent decades. ${ }^{29}$ At the same time, in areas affected by HIV/AIDS, the elderly themselves are playing an increasing role as care-givers at a time in their lives when they might have expected to be the recipients of care. The conventional life-cycle has been disturbed and the anticipated generational order of deaths up-ended. But there is no unmediated relationship between demographic trends and cultural attitudes to death and dying. Levels of urbanization and education, taxation regimes, and (in some countries) emergent formal welfare systems are amongst the many factors which we might expect to play a role. ${ }^{30}$

The determinants of the dramatic rise in population in Africa in the twentieth century are still debated by demographic historians. Some see it as having been driven fundamentally by a fall in mortality rates; others argue that a rise in fertility rates played a critical role. ${ }^{31}$ Either way, towards the end of the century there were signs in many parts of the continent of a "transition" to lower fertility rates. ${ }^{32}$ However, in the last twenty years those areas affected by the HIV/AIDS epidemic have seen dramatic 
changes to their population profiles that have greatly complicated any picture of an orderly "demographic transition." For example, in Botswana life expectancy at birth had risen from 46 years in 1955 to 65 in 1990, but by 2005 it had dropped to $35 .{ }^{33}$ The speed with which these changes have taken place is remarkable. In particular, the implications of the HIV/AIDS epidemic in eastern and southern parts of the continent, coming on top of the earlier period of population growth and rapid urbanization, have yet to be fully worked through or understood.

There is now a very large literature on HIV/AIDS and its social and economic ramifications, including its implications for attitudes to death and practices surrounding death. ${ }^{34}$ But a great deal of the literature on AIDS is written without reference to the pre-AIDS era. ${ }^{35}$ Southern Africa, in particular, has become so saturated with presentist AIDS research, that the impression is sometimes given that history itself began with the HIV/AIDS epidemic. ${ }^{36}$ There is no doubt that there are particular features of the HIV/ AIDS epidemic which make the drawing of historical parallels hazardous. Amongst these is the critical fact that this is a long-term epidemic. This makes it very different from many other epidemic diseases, for which the duration of outbreaks is much shorter-lived. There is almost certainly something to be learned from a comparison with the social, cultural, and religious responses of African societies to other epidemic diseases, including smallpox, influenza, meningitis, plague, tuberculosis, and of course, epidemics of other sexually transmitted diseases, but the most salient comparison may, in the end, turn out to be with the effects of the slave trade. ${ }^{37}$

\section{Violence, war, and historical memory}

"Death" and "Africa" are words which, unfortunately, seem often to be twined together. In much Western media coverage "Africa" appears as a space of death: epidemic disease, famine, war, and apparently "irrational" violence dominate representations of the continent and give rise to agonized debates about how such images might be countered. This is not a new phenomenon, of course, and dates back at least to the images of the slave trade and the calls from Abolitionists to "save" the continent from darkness and death.

Many analyses of civil war in Africa aim to counter the image of a continent overwhelmed by "irrational" violence and urge us to see these conflicts as driven by the very rational motivations of desire for wealth and power. Conflict in Africa cannot be entirely reduced to competition for resources, but the map of wars on the continent is nevertheless revealing. ${ }^{38}$ If you do not want to be caught up in violent conflict, it is better not to live in a region dominated by mineral wealth. But for a minority, participation in war can be a very effective accumulation strategy. Many of Africa's conflicts are simultaneously global and local in character, involving actors ranging from agents of international criminal rings to members of local youth organizations. The state, it is clear from these conflicts, no longer has a monopoly over violence in Africa, if it ever did. ${ }^{39}$ In 1994, however, the world's attention came to be focused on a different kind of war in Africa - the genocide in Rwanda. Though competition over resources could go some way to explain Rwanda's crisis, the genocidal form which it took necessitated a much longer-historical perspective on the creation of ethnic identities and the nature of political power in Rwanda. ${ }^{40}$

War is not one phenomenon but several, and historically different parts of Africa have experienced different kinds of wars, ranging from the internal conflicts which fed into and upon the Atlantic slave trade, to the competitive warfare of political opponents in 
some nineteenth-century polities, to the wars of resistance to colonial occupation and wars of colonial liberation. In between those wars of occupation and liberation, millions of Africans were caught up in wars that were not of their making at all. Vast swathes of eastern Africa between 1914 and 1918 were devastated by a war between European powers played out on African soil; during the Second World War, African troops fought in Europe and in the jungles of Burma. ${ }^{41}$ Work on Mau Mau and decolonization of Kenya has reminded us of the colonial state's capacity for terror and violence. ${ }^{42}$ Some parts of the continent have been subject to regimes of violence from the time of the slave trade onwards. Here the haunting memory of past conflicts feeds into the experience of present-day conflict. ${ }^{43}$ Students of war and conflict in Africa grapple with the very considerable challenges of interpretation and representation in, for example, employing or eschewing psychological theories such as that of trauma to analyze these circumstances.

Two apparently quite contradictory analyses of political violence in contemporary Africa stand out in the recent literature. Some regimes of violence and terror are described as being so commonplace as to render death itself banal and emptied of meaning. ${ }^{44}$ In other cases, violence, far from being devoid of meaning, appears to be saturated with meanings of the sacred and closely associated with spirituality. ${ }^{45}$ Given the very diverse nature of African societies, it would be absurd to propose one narrative for the historical relationship between violence and political power in Africa, but clearly there is a need for more work with a long-term perspective on this issue. Much present-day violence in Africa can be understood in general terms of global forces and economic competition. Violence in Africa is no more "exotic" than violence anywhere else. On the other hand, to understand the meanings of violent death in different parts of Africa, the moral economy of violence and the line which societies draw between the legitimate and illegitimate use of violence, does require an understanding of specific histories and of cosmologies. ${ }^{46}$ Any comprehensive history of death in Africa would have to address the changing of violent death and its meanings and would have to include the nascent scholarship on suicide and road accidents, amongst other subjects alongside criminal and political violence. ${ }^{47}$

\section{Colonialism and Christianity: deathly encounters}

Like many other missionaries, Dr. David Brown, a minister of the Church of Scotland, found the funerary practices of the people amongst whom he worked disturbing and repulsive. Yet the common human experience of death also provided opportunities for the missionary. Brown was horrified by the noise and apparent disorder of "pagan" Nyakyusa burial rites, with their reference to the role of ancestral spirits in the world of the living, the role of animal sacrifice, and perhaps above all by their explicit expression of sexuality. Early Christian converts in southwest Tanganyika, as elsewhere, were asked to make a difficult choice. Banned from attending "pagan" funerals of family members, they risked creating serious social conflict and the anger of the ancestors. In exchange, Christianity claimed to have conquered death completely and held out the promise of individual salvation, but this could only be achieved by giving up those relationships with the dead that were so central to the lives of the living. Though Brown and his colleagues drew a stark contrast between "pagan" and Christian practices around death, nevertheless, the experience of death was a constant reminder of the shared human emotion of grief. When Brown's small daughter died in 1921, just a few months after arriving in Tanganyika, he and his wife were moved by the sympathy of their African neighbors, many of whom had 
been through similar losses. Over time the practice of Christian burial, whilst still a vital sign of Christian identity, became more open to a degree of negotiation. When Brown himself died, in Northern Rhodesia in 1946, his body was wrapped in a simple white cloth and buried without a coffin. His African friends were impressed. This choice of burial demonstrated, they said, that he had become a "real" African. ${ }^{48}$

Death played a central part in the conversations between missionaries and their converts. The death of Christ represented the once-and-for-all sacrifice. Death itself had been conquered, for those who had the courage to believe. In southern Africa, missionary insistence on speaking about death provoked both fascination and flight amongst their potential converts, and helped to convey a sense of the authority of the Christian god. ${ }^{49}$ For some Africans, Christian depictions of the resurrection of the dead and the second-coming of Christ held particular attraction, as these offered an innovative theological perspective on the death-process. ${ }^{50}$ Believers no longer had to fear death, nor the wrath of malevolent "shades" or ancestral spirits, and could instead await with anticipation the rising up of their dead. Conversations about death were also meditations on the very concept of the person, involving tricky linguistic and cultural translations, of the concept of the soul, for example, and of the afterlife. ${ }^{51}$ While the efficacy of these "death conversations" in converting Africans remains debatable, one enduring historical legacy of these discursive encounters is that the narrative of resurrection became, for some, incorporated into an autochthonous eschatological framework, as famously evident in the millenarial call of Nongqawuse in the Xhosa cattle-killing. ${ }^{52}$

As has been noted in other contexts, the "long conversation" between missionaries and Africans was shaped not only by philosophical concerns but also by material struggles over space, resources, and bodies. ${ }^{53}$ This is no less the case in the realm of death. In depictions of death-bed scenes, missionaries could reveal the triumphant grace of the Christian god as somehow visibly embodied on the peaceful expressions of the dying. Furthermore, the choice to bury one's kin in newly erected cemeteries under Christian rites represented a "final test" of allegiance, and for the Christian convert it was a decision potentially fraught with doubt, controversy, and dissension. ${ }^{54}$ It is evident some aspects of African beliefs in a spirit world could be incorporated into Christian practice, and others could not, though the huge variety of Christian practice in Africa makes generalizations hazardous. Whilst the "accommodation" between Christian and "traditional" practice and beliefs has been central to more recent analyses of the history of Christianity in Africa, nevertheless, the distinction between a Christian burial and a "traditional" one is still critical for many African Christians. ${ }^{55}$

Debates over death and funerary practice have also occurred in the Muslim societies of Africa. Some of these debates refer back to the origins of Islam itself. In one account of a funeral "controversy", upon the death of the leader of a rival group of munafiqun (or "hypocrites") in Medina, the Prophet Muhammed assented to praying over the dead body and clothing him in a ritual shirt. Later interpretations rebuke the Prophet for these actions on the basis of the Qu'ranic injunction against praying over the bodies of dead "disbelievers," although whether the munafiqun, who had overtly professed the Muslim faith, could be categorized as "disbelievers" was debatable. Even from its earliest beginnings, then, religious authority, textual interpretation, and claims of allegiance were contested in the practice of Muslim funerary rites. Islam's long history on the continent, particularly in East and West Africa, make any generalizations about its influence on African death cultures particularly problematic. Islamic 
burial practices were incorporated into one East African society so seamlessly that it was noted even a colonial missionary could not distinguish the Islamic features of a "native" burial ground. ${ }^{57}$ Indeed, in contemporary South Africa, recent converts to Islam have adopted a narrative of return, and view the practice of Islamic burial rites as an important way to connect with a nostalgic and more authentically "Xhosa" past. $^{58}$ Islamic reform movements have historically involved a questioning of the proper place and conduct of rituals, including funerary and mourning practices. Thus, religious revival is often accompanied by a process of cultural and social reform. We see this, for example, in the shift to a more orthodox version of Islam in the Sudan, which has had the effect of relegating women's ritual knowledge to the sphere of the "traditional" as opposed to the more pietist and privileged male world of the "scriptural." Thus marginalized, women's participation in zar spirit possession cults have become emptied of their previous ritual power. ${ }^{59}$

For European colonial rulers, African burial practices presented a complex set of dilemmas. Although in theory under the rubric of "traditional" structures that were granted some degree of autonomy under Indirect Rule in many parts of Africa, the public performance of funerary and burial rituals often invited the interference of colonial officials. In part, this was because of European fears of harmful emanations from "unsanitary" methods of corpse disposal, such as multiple or shallow graves, burials within homesteads, and practices of corpse exposure. By the 1930s, an increasingly medicalized and rationalized approach to public health had, in some parts of the continent, resulted in closer regulation of the disposal of the dead. For example, in French equatorial Africa, by the 1930s, colonial officials required the immediate burial of corpses, a regulated depth for graves, and the use of wooden coffins. ${ }^{60}$ Even in the confined spaces of death row in British-held colonial Africa, we can see the shift to "closed" and privately managed executions as driven by a larger imperative to institute a more "civilized" and sanitized approach to the exercise of imperial law. ${ }^{61}$

Undoubtedly, the development of these "sanitized" spaces of death helped to alter African ways of burying and remembering their dead. However, we need to be careful not to exaggerate the impact of the colonial state. Africans resisted colonial regulations, whether by refusing to bury their dead in demarcated cemeteries or by overtly protesting what they viewed as intrusive legislation. ${ }^{62}$ In contemporary southern Africa, as elsewhere, the continued relevance of notions of bodily contagion and the persistence of fears over the mis-use of corpses for witchcraft show that neither colonial regulation nor missionary indoctrination has successfully eradicated African beliefs around the ontological power of the (dead or dying) body. ${ }^{63}$ The historical lack of adherence among Africans to cremation, despite its widespread practice since the 1930s amongst the white and Indian populations of South Africa, may be another indication of the resilience of culturally specific notions of the body. ${ }^{64}$ A more accurate assessment of these debates would have to acknowledge the complexity and range, as well as the historical specificity, of African responses to colonial and missionary interventions.

\section{Urbanization, globalization, and new technologies of death}

I am a cut sprout, ever resprouting.

A poor man has no place in the country... 
Poor men, we are long-legged;

You know we shall die far away. ${ }^{65}$

This stanza from a sefala, or Sotho song of comradeship, speaks of the loneliness and displacement of the migrant laborer. For the many "long-legged" migrants journeying to the mines of South Africa throughout the twentieth century, it was the prospect of dying "far away" that gave a particular pathos to their situation. While the establishment of cemeteries on the mines acknowledged that mines themselves had becomes spaces of (accidental) death, it was the fact that they could now be spaces of burial that further troubled African laborers and their families. ${ }^{66}$ How would the dead be ensured safe passage into the afterlife, if not buried at "home" under the watchful eyes of the living? And how would the community attain ritual and spiritual closure without the assurances brought on by the ceremonial treatment of the body of the deceased? Throughout southern Africa, the migrant labor system imposed a necessary mathematics of distance into the delicate calibrations of social and kinship relations. African relations to death and the dying process were no less affected.

The rich scholarship on the impact of the slave trade reminds us that the more recent shaping of African mortality patterns through urbanization, labor migration, and globalization cannot be seen as entirely "new" historical processes. ${ }^{67}$ Road accidents, which are currently referred to as reaching "epidemic" proportions in some African countries, may be considered an unfortunate consequence of modern forms of motorized transport. ${ }^{68}$ However, in Sierra Leone, the phenomenon of death "on the road" was deeply associated with older forms of capitalist consumption, namely slavery. The road, like the sea that carried away enslaved Africans, became feared as a "man-eater," a voracious devourer of human flesh. ${ }^{69}$ That Sotho migrants drew on similar cannibalistic imagery to cast South African mines as a giant belly that swallowed African bodies suggests that forces of global capitalism influenced not only economies of death but African imaginaries as well. ${ }^{70}$

In the post-colonial period, the growth of regional and international networks, increased mobility (within Africa and globally), and the speed of telecommunications have contributed to a re-mapping of the ways in which people understand and daily exercise a sense of "belonging." ${ }^{\text {" }}$ Some analyses have argued that, in the context of escalating violence and political instability in many post-independence African countries, this re-mapping has been marked by a profound "un-mooring" of social ties. Particularly in the fluid and often marginalized and violent spaces of African metropolises, a dynamics of disconnection - from socio-economic, legal, and moral structures - has operated. In this space of disconnectedness, the division between life and death has become easily ruptured, and the world of the dead freely intrudes on the living. ${ }^{72}$ The use of apocalyptic timescales in Kinois music, the circulation of witchcraft rumors, and the re-emergence of the Okija shrine in Nigeria can all be understood as African attempts to re-orient themselves to this different, spectral topography. ${ }^{73}$ Narratives of liminality have also emerged out of the continuing HIV/AIDS epidemic. In rural Mpumulanga (South Africa), those with HIV/AIDS are considered the "walking dead" while in Kampala, the cyclical nature of AIDS-related illnesses has meant that AIDS sufferers live in a state of moving back and forth from the brink of death to life. ${ }^{74}$

If the problems of modernity have brokered a different relation to the spectral, the products of modernity have themselves mediated shifts in the meaning and management of death. The commoditization of funeral rites in recent decades in many parts 
of Africa has, at least materially, transformed burial and mourning practices. "Fantasy" coffins from Ga carpenters in Accra are now marketed and sold via the Internet, with one website robustly proclaiming their coffins are free-trade "antiques of the future." Purchasing (and being buried in) a coffin modeled exactly on a popular Nokia cellphone can, claims the website, "keep your line to heaven open." Africa, township-based funeral homes offer families a range of products and services, including in-house embalming, refrigerated transport of the deceased, and the use of double-decker buses and portable green "lawns" to transform dusty cemeteries into areas of comfortable reflection. ${ }^{76}$ Fueling this consumption is an equally vibrant and competitive funeral insurance industry, with both commercial and informal sector schemes offering financial security for those seeking a "dignified" funeral for themselves and their dependants. In west and southern Africa, where the commoditization process seems most apparent, social pressures to participate in increasingly elaborate and expensive funerals have sparked a lively and contentious debate about the burdensome "price" of respectability. ${ }^{77}$

Although it would be tempting to argue that commoditization has emptied the spiritual content of death rituals and marked a "great transformation" in African death cultures, there is reason to suggest this is an oversimplification. ${ }^{78}$ Firstly, colonial anthropological and missionary accounts remind us that the elaboration of funerals is not a "new" African invention. A focus on the commercialization of contemporary funerals would thus obscure older, historical dynamics at work. Furthermore, as Jane Guyer and others have stressed, monetary transactions in Africa cannot be understood in isolation away from locally derived systems of belief, sociality, and exchange. ${ }^{79}$ Commodities, therefore, need to be seen as potentially malleable mediators of local cultural values, and not simply as markers of Western capitalist consumption. ${ }^{80}$ For example, African incorporation of new technologies and communications networks, which implicitly link greater and more disparate geographical areas, has arguably increased the possibilities of spiritual engagement with the dead and the community of the deceased. For example, newly introduced mortuary technologies such as embalming and refrigerated transport of the corpse have allowed a type of reverse migration, where the dead in urban areas can be transported back to their natal homes for burial and subsequent commemoration ceremonies. ${ }^{81}$ Internet chat rooms have become, for the Congolese diaspora in the West, an important medium through which to debate death and shape mourning culture. ${ }^{82}$ Even the consumption of prescriptive drugs, in the face of certain death from AIDS, has been imbued with a particularly spiritual significance. ${ }^{83}$ Evidently, then, the forces of globalization and technological change have helped fashion alternative cultural landscapes within which Africans could re-invent their relations to death and the dying process. That these dynamics could be simultaneously "modern" and deeply "historical" attests to Africans' extensive and multi-faceted engagement with death over the course of the last two centuries. The challenge remains for historians of Africa to explore the development of this complex, and compelling, relationship.

\section{Acknowledgments}

An earlier version of this chapter appeared in the Fournal of African History as "Death and Dying in the History of Africa Since 1800," Journal of African History 49, 3 (2008): 341-59. The authors wish to acknowledge the Arts Humanities Research Council of the United 
Kingdom for funding the larger collaboration on which this chapter is based. For further information on the project, please visit: www.goldsmiths.ac.uk/deathinafrica.

\section{Notes}

1 Edward E. Evans-Pritchard, "Burial and Mortuary Rites of the Nuer," African Affairs 48, 190 (1949): 62.

2 Philippe Ariès, Western Attitudes toward Death: From the Middle Ages to the Present, trans. Patricia M. Ranum (London, 1976); Philippe Ariès, The Hour of Death, trans. Helen Weaver (London, 1981).

3 On the misrepresentations of African beliefs about death, see Louis-Vincent Thomas, La mort africaine: idéologie funéraire en Afrique noir (Paris, 1982).

4 Sigmund Freud, "Mourning and Melancholia" (1915).

5 Filip de Boeck, "The Apocalyptic Interlude: Revealing Death in Kinshasa," African Studies Review 48, 2 (2005): 11-31.

6 See Sarah Nuttall and Carli Coetzee, eds., Negotiating the Past: The Making of Memory in South Africa (Cape Town, 1998); Annie E. Coombes, History after Apartheid: Visual Culture and Public Meaning in a Democratic South Africa (Durham, NC, 2004); Ananda Breed, Performing the Nation: Genocide, Violence, Fustice, Reconciliation (Chicago, IL, 2014).

7 Zeph Kajevu, "Funeral Services Group to List on BSE," The Voice (Francistown), 4 March 2008: http://allafrica.com.stories/200803040816.html.

8 Jessica Mitford, The American Way of Death (London, 1963) and The American Way of Death Revisited (London, 1998).

9 See Arnold Van Gennep, Rites of Passage (trans London, 1960); Robert Hertz, trans. Rodney Needham and Claudia Needham, Death and the Right Hand (New York, 1960); Max Gluckman, ed., The Allocation of Responsibility (Manchester, 1972); Maurice Bloch and Jonathan Parry, eds., Death and the Regeneration of Life (Cambridge, 1982); Yvan Droz, ed., La violence et les morts: éclairages anthropologiques sur la mort et les rites funéraires (Geneva, 2003). On Africa: Simon Bockie, Death and the Invisible Powers: The World of Kongo Belief (Bloomington, 1993); J.C. Goody, Death, Property and the Ancestors (Stanford, 1962); Karen Middleton, ed., Ancestors, Power and History in Madagascar (Leiden, 1999); Maurice Bloch, Placing the Dead: Tombs, Ancestral Villages and Kinship Organisation in Madagascar (London, 1971); Marja-Liisa Swantz, Blood, Milk and Death: Body Symbols and the Power of Regeneration among the Zaramo of Tanzania (Westport, 1995); J. M. Schoffeleers, Religion and the Dramatisation of Life: Spirit Beliefs and Rituals in Southern and Central Malawi (Blantyre, 1997); Thomas, La mort africaine.

10 Zakes Mda, Ways of Dying (Cape Town, 1995).

11 Bronislaw Malinowski, "Magic, Science and Religion" (1925); Van Gennep, Rites of Passage.

12 Godfrey Wilson, trained by Malinowski, worked extensively on the symbolism of Nyakyusa burial rites, seeing them as part of a larger "system" and connecting them to other "rites of passage." Unusually, he also emphasized the emotional content of these rites. Godfrey Wilson, "Nyakyusa Conventions of Burial," Bantu Studies 13 (1939): 1-3. On the social and emotional dynamics of contemporary funerals, see Casey Golomski, Funeral Culture: AIDS, Work and Cultural Change in an African Kingdom (Bloomington, IN, 2018); Frederick Klaits, "The Widow in Blue: Blood and the Morality of Remembering in Botswana's Time of AIDS," Africa 75, 1 (2005): 26-62; Deborah Durham and Frederick Klaits, "Funerals and the Public Space of Sentiment in Botswana," Fournal of Southern African Studies 28, 4 (2002): 777-95.

13 Megan Vaughan, "Divine Kings': Sex, Death and Anthropology in Inter-War East/Central Africa," Journal of African History 49, 3 (2008): 383-401.

14 In present-day Tswana society, proscriptions on sex with widows feed into a larger AIDSinfluenced discourse about the harmful mixing of blood and bodily fluids; see Klaits, "The Widow in Blue."

15 Sandra Greene, Sacred Sites and the Colonial Encounter: A History of Meaning and Memory in Ghana (Bloomington, 2002).

16 See Megan Vaughan "Suicide in Late Colonial Africa: The Evidence of Inquests from Nyasaland," American Historical Review 115, 2 (2010): 385-404; Paul Bohannan, ed., African Homicide and Suicide (Princeton, NJ, 1960). 


\section{DEATH IN AFRICA}

17 See Peter Geschiere, The Modernity of Witchcraft: Politics and the Occult in Postcolonial Africa (Charlottesville, 1997); Luise White, Speaking with Vampires: Rumour and History in Colonial Africa (Berkeley, CA, 2000); Jean Comaroff and John L. Comaroff, eds., Modernity and Its Malcontents: Ritual Power in Postcolonial Africa (Chicago, IL, 1993); Isak Niehaus, with Eliazaar Mohlala and Kally Shokane, Witchcraft, Power and Politics: Exploring the Occult in the South African Lowveld (London, 2001); Henrietta Moore and Todd Saunders, eds., Magical Interpretations: Material Realities (London, 2001).

18 Vaughan, "Diving Kings," 385.

19 Thomas C. McCaskie, "Death and the Asantehene: A Historical Meditation," The Fournal of African History 30, 3 (1989): 417-44. See also John Parker, "The Cultural Politics of Death and Burial in Early Colonial Accra," in David Anderson and Richard Rathbone, eds., Africa's Urban Past (Oxford, 2000), 205-21.

20 Terence Ranger, "Dignifying Death: The Politics of Burial in Bulawayo," Journal of Religion in Africa 34, 1-2 (2004): 110-44. Funerals in the 1980s became a key vehicle of political activism for the African National Congress. See Garrey Michael Dennie, "The Cultural Politics of Burial in South Africa, 1884-1990" (Ph.D. thesis, Johns Hopkins University, 1996).

21 Walima T. Kalusa, "Death, Christianity and African Miners: Contesting Indirect Rule on the Zambian Copperbelt, 1935-1962," International Fournal of African Historical Studies 44, 1 (2011): 89-112; see also Walima T. Kalusa and Megan Vaughan, Death, Belief and Politics in Central Africa (Lusaka, 2013).

22 Peter Geschiere, "Funerals and Belonging: Different Patterns in South Cameroon," African Studies Review 48, 2 (2005): 45-64; David William Cohen and Elisha S. Atieno Odhiambo, Burying SM: The Politics of Knowledge and the Sociology of Power in Africa (London, 1992).

23 Steven Robins, "From 'Rights' to 'Ritual': AIDS Activism in South Africa," American Anthropologist 108, 2 (2006): 312-23; Deborah Posel, "Democracy in a Time of AIDS," Interventions 7, 3 (2005): 310-15.

24 Wale Adebanwi, "Death, National Memory and the Social Construction of Heroism," Fournal of African History 49, 3 (2008): 419-44; see also Florence Bernault, "Colonial Bones: The 2006 Burial of Savorgnan De Brazza in the Congo," African Affairs 109, 436 (2010): 367-90.

25 Bob W. White, "The Political Undead: Is It Possible to Mourn for Mobutu's Zaire?," African Studies Review 48, 2 (2005): 65-85.

26 Shane Doyle, “'The Child of Death': Personal Names and Parental Attitudes towards Mortality in Bunyoro, Western Uganda, 1900-2005," Journal of African History 49, 3 (2008): 361-82. See also Shane Doyle, Sexuality, Fertility and Mortality in East Africa, 1900-1980 (Oxford, 2013).

27 Linda Pollock, Forgotten Children: Parent-Child Relations from 1500-1900 (Cambridge, 1983).

28 Nana Apt, Ageing in Africa (Geneva, 1997); Isabella Aboderin, Intergenerational Support and Old Age in Africa (NJ, 2006).

29 Julie Livingston, Debility and the Moral Imagination in Botswana (Bloomington, IN, 2005); Julie Livingston, "Elderly Women and Concerns Over Care in Southeastern Botswana," Medical Anthropology 22, 2 (2003): 205-23.

30 Andreas Sagner, "Ageing and Social Policy in South Africa: Historical Perspectives with Particular Reference to the Eastern Cape," Journal of Southern African Studies 26, 3 (2000): 523-53.

31 John Iliffe, Africans: The History of a Continent, 2nd edition (Cambridge, 2007); John C. Caldwell, "The Social Repercussions of Colonial Rule: Demographic Aspects," in Albert Adu Boahen, ed., UNESCO General History of Africa, VII (London, 1985), 458-507; Dennis D. Cordell and Joel W. Gregory, eds., African Population and Capitalism: Historical Perspectives (London, 1987).

32 www.unicef.org/sowc03/tables/table9.html; Debby Potts and Shula Marks, "Fertility in Southern Africa: The Silent Revolution," Journal of Southern African Studies 27, 4 (2001): 189-205.

33 www.unicef.org/infobycountry/botswana_statistics.html.

34 On the latter issue see Charles Nzioka, "The Social Meanings of Death from HIV/AIDS: An African Interpretive View," Culture, Health and Sexuality 2 (2002): 1-14; Gad Kilonzo and Nora Hogan, "Traditional African Mourning Practices Are Abridged in Response to AIDS Epidemic: Implications for Mental Health," Transcultural Psychiatry 36 (1999): 259-83.

35 There are, however, notable exceptions to this generalization: John Iliffe, The African AIDS Epidemic: A History (Oxford, 2006); Felicitas Becker and Paul Wenzel Geissler, eds., Journal of 
Religion in Africa Special Issue on Religion and AIDS in Africa 37, 1 (2007); Didier Fassin, When Bodies Remember: Experiences and Politics of AIDS in South Africa (Berkeley, CA, 2007).

36 Rebekah Lee, "Art, Activism and Productive Tensions in the Next Generation of HIV/AIDS Research in South Africa," Journal of Southern African Studies 45, 1 (2019): 1-7.

37 Milton Lewis, Maryinez Lyons and Philip Setel, eds., Histories of Sexually Transmitted Diseases and HIV/AIDS in Sub-Saharan Africa (London, 1999); Terence Ranger and Paul Slack, eds., Epidemics and Ideas: Essays on the Historical Perception of Pestilence (Cambridge, 1992); Howard Phillips and David Killingray, eds., The Spanish Influenza Pandemic of 1918-19: New Perspectives (London, 2003).

38 David Keen, "A Rational Kind of Madness," Oxford Development Studies 25, 1 (1997): 67-75; William Reno, "African Weak States and Commercial Alliances," African Affairs 96 (1997): 165-88; Paul Richards, Fighting for the Rain Forest: War, Youth and Resources in Sierra Leone (Oxford, 1998); David Keen, Conflict and Collusion in Sierra Leone (Oxford, 2005); Michael Watts, "Resource Curse: Governmentality, Oil, and Power in the Niger Delta," Geopolitics 9 (2004): 50-80.

39 Achille Mbembe, "Necropolitics," Public Culture 15, 1 (2003): 11-40.

40 Mamoud Mamdani, When Victims Become Killers: Colonialism, Nativism and the Genocide in Rwanda (Oxford, 2001); Catherine Newbury, The Cohesion of Oppression: Clientship and Ethnicity in Rwanda, 1860-1960 (New York, 1988); Gerard Prunier, The Rwanda Crisis: History of a Genocide, 1959-1994 (London, 1995).

41 Edward Paice, Tip and Run: The Untold Tragedy of the Great War in Africa (London, 2007); Gregory Mann, Native Sons: West African Veterans and France in the Twentieth Century (Durham, NC, 2006); Ashley Jackson, Botswana, 1939-1945: An African Country at War (Oxford, 1995).

42 David Anderson, Histories of the Hanged: Britain's Dirty War in Kenya and the End of Empire (London, 2005); Caroline Elkins, Britain's Gulag: The Brutal End of Empire in Kenya (London, 2005).

43 Rosalind Shaw, Memories of the Slave Trade: Ritual and the Historical Imagination in Sierra Leone (Chicago, IL, 2002).

44 Achille Mbembe, "Provisional Notes on the Postcolony," Africa, 62, 1 (1992), 5-37; De Boeck, "The Apocalyptic Interlude," 17.

45 See Stephen Ellis, "The Okija Shrine: Death and Life in Nigerian Politics," Journal of African History 49, 3 (2008): 445-66; Heike Behrend, Alice Lakwena and the Holy Spirit: War in Northern Uganda, 1986-1997 (London, 1991).

46 For a longue durée approach, see David Schoenbrun, "Violence and Vulnerability in East Africa before 1800 CE," History Compass 4, 5 (2006): 741-60. On warfare and warrior traditions: Ali A. Mazrui, ed., The Warrior Tradition in Modern Africa (Leiden, 1977), 20-47; Robert S. Smith, Warfare and Diplomacy in Pre-Colonial West Africa, 2nd edition (Madison, 1989); John K. Thornton, Warfare in Atlantic Africa: 1500-1800 (London, 1999); Bethwell A. Ogot, ed., War and Society in Africa: Ten Studies (London, 1972); John Iliffe, Honour in African History (Cambridge, 2005). On the history of human sacrifice: Robin Law, "Human Sacrifice in Pre-Colonial West Africa," African Affairs 84, 1 (1985): 52-87; Clifford Williams, "Asante: Human Sacrifice or Capital Punishment? An Assessment of the Period 1807-1874," International Fournal of African Historical Studies 21, 3 (1988): 443-452. For an interpretation of "imaginary" violence, see Wyatt MacGaffey, "Aesthetics and Politics of Violence in Central Africa," Fournal of African Cultural Studies 13, 1 (2000): 63-75.

47 Mbembe, "Necropolitics," 32; Jean-Francois Bayart, Stephen Ellis and Beatrice Hibou, The Criminalisation of the State in Africa (Oxford, 1998). The inadequacy of mortality statistics for most African countries hampers any thorough analysis. See Katherine Kahn, Stephen M. Tollman, Michel Garenne and John S.S. Green, "Who Dies from What? Determining Causes of Death in South Africa's Rural Northeast," Tropical Medicine and International Health 4, 6 (1991): 433-41. On suicide in Africa: Megan Vaughan, "The Discovery of Suicide in East and Southern Africa," African Studies 71, 2 (2012); Vaughan, "Suicide in Late Colonial Africa"; Lourens Schlebusch, Suicidal Behaviour in South Africa (Durban, 2005) and Julie Livingston, "Suicide, Risk and Investment in the Heart of the African Miracle," Cultural Anthropology 24, 4 (2009): 652-80; Julie Parle, States of Mind: Searching for Mental Health in Natal and Zululand 1868-1918 (Scottsville, KZN, 2007); Fatima Meer, Race and Suicide in South Africa (London, 1976). On road accidents and fatalities in Africa: Mark Lamont "Accidents Have No Cure! Road Death as Industrial Catastrophe in Eastern Africa," African 
Studies 71, 2 (2012): 174-94; Rebekah Lee "Death in Slow Motion: Funerals, Ritual Practice and Road Danger in South Africa," African Studies 71, 2 (2012): 195-211.

48 Dr. David Brown, papers held privately; Godfrey Wilson, "Nyakyusa Conventions of Burial"; Monica Wilson, Good Company (London, 1951); Monica Wilson, Communal Rituals of the Nyakyusa (London, 1959).

49 Robert Moffat recalled of his efforts to engage ordinary Tswana in conversation, "Death and a future state are subjects they do not like to contemplate, and when they are introduced it frequently operates like an imperative order for them to depart." Isaac Schapera, ed., Apprenticeship at Kuruman. The Letters of Robert and Mary Moffat, 1820-1828 (London, 1951), 253; see also William H. Worger, "Parsing God: Conversations about the Meaning of Words and Metaphors in Nineteenth Century Southern Africa," Journal of African History 42, 3 (2001): 417-47.

50 John P.R. Wallis, ed., The Matebele Journals of Robert Moffat, 1829-1860 (Salisbury: National Archives of Rhodesia, 1976); Eugene Casalis, The Basutos, or, Twenty-Three Years in South Africa (London, 1861).

51 Hildegarde H. Fast, "In at One Ear and Out at the Other': African Response to the Wesleyan Message in Xhosaland, 1825-1835," Journal of Religion in Africa 23, 2 (1993): 147-74.

52 Jeff Peires, The Dead Will Arise: Nongqawuse and the Great Xhosa Cattle-Killing Movement of 1856-7 (Johannesburg, 1989); on African millenarianism, see Robert Edgar and Hilary Sapire, African Apocalypse (Athens, OH, 2000); De Boeck, "The Apocalyptic Interlude"; Yvan Droz and Herve Maupeu, eds., Les figures de la mort à Nairobi: une capitale sans cimètiere (Paris, 2003).

53 Jean and John L. Comaroff, Of Revelation and Revolution: Christianity, Colonialism and Consciousness in South Africa, Vol. 1 (Chicago, 1991).

54 King Moshoeshoe faced this predicament, as described by Eugene Casalis, upon the sudden death of one of his wives; Casalis, The Basutos, 89-92.

55 See Marleen de Witte, Long Live the Dead: Changing Funeral Celebrations in Asante, Ghana (Amsterdam, 2001); James L. Cox, ed., Rites of Passage in Contemporary Africa: Interaction between Christian and African Traditional Religions (Cardiff, 1998).

56 Muhammad Qasim Zaman, "Death, Funeral Processions, and the Articulation of Religious Authority in Early Islam," Studia Islamica 93 (2001), 29-30. See also Arthur S. Tritton, "Muslim Funeral Customs," Bulleting of the School of Oriental Studies 9, 3 (1938): 653-61; Jane Smith and Yvonne Haddad, The Islamic Understanding of Death and Resurrection (Albany, NY, 1981); Lila Abu-Loghod, "Islam and the Gendered Discourses of Death," International Fournal of Middle East Studies 25 (1993): 187-205. On Africa: Jose Van Santen, "We Attend but We No Longer Dance: Changes in Mafa Funeral Practices Due to Islamization," in Catherine Baroin, Daniel Barreteau, and Charlotte von Graffenreid, eds., Mort et rites funéraires dans le bassin du lac Tchad (Paris, 1995), 163-86; E. Dada Adelowo, "Death and Burial in Yoruba Qur'anic and Biblical Religion," Orita 19, 2 (1987): 104-17.

57 Felicitas Becker, "Islamic Reform and Historical Change in the Care of the Dead: Conflicts Over Funerary Practice among Tanzanian Muslims," Africa 79, 3 (2009): 417-34.

58 Islamic funerary practices were also perceived to be a welcome bulwark against the encroachment, financial and social, brought on by the demands of a costly Christian funeral; Rebekah Lee, "Conversion or Continuum?: The Spread of Islam among African Women in Cape Town," Social Dynamics 27, 2 (2001): 62-85.

59 Victoria Bernal, "Gender, Culture and Capitalism: Women and the Remaking of Islamic 'Tradition' in a Sudanese Village," Comparative Studies in Society and History 36, 1 (1994): 36-67.

60 Florence Bernault, "Body, Power and Sacrifice in Equatorial Africa," Journal of African History 47 (2006), 231. In east Africa in the 1930s, a Native Authority Ordinance was introduced which banned corpse exposure; Mark Lamont, "Decomposing Pollution: Corpses, Burials, and Affliction among the Meru of Central Kenya," in Joel Noret and Michael Jindra, eds., Funerals in Africa: Explorations of a Social Phenomenon (London, 2011), 88-108.

61 Stacey Hynd, "Killing the Condemned: The Practice and Process of Capital Punishment in British Africa, 1900-1950s," Fournal of African History 49, 3 (2008): 403-18.

62 See De Witte, Long Live the Dead; Lamont, "Decomposing Pollution"; Greene, Sacred Sites.

63 Benedict Carton, "We Are Being Made Quiet by This Annihilation': Historicizing Concepts of Bodily Pollution and Dangerous Sexuality in South Africa," International Journal of African Historical Studies 39, 1 (2006): 85-106; Benedicte Ingstad, Frank Bruun and Sheila Tlou, "AIDS 
and the Elderly Tswana: The Concept of Pollution and Consequences for AIDS Prevention," Journal of Cross-Cultural Gerontology 12, 4 (1997): 357-72; Klaits, "The Widow in Blue"; Golomski, Funeral Culture; Adam Ashcroft, Madumo: A Man Bewitched (Chicago, 2000)

64 An uneasy alliance forged between Indian nationalists, led by Mahatma Gandhi, and modernist white health officials resulted in the creation of South Africa's first crematorium, in 1918; Garrey Michael Dennie, "Flames of Race, Ashes of Death: Re-Inventing Cremation in Johannesburg, 1910-1945," Fournal of Southern African Studies 29, 1 (2003): 177-92.

65 David B. Coplan, In the Time of Cannibals: The World Music of South Africa's Basotho Migrants (Chicago, IL, 1994), 124.

66 Cemeteries were legally established on South African mines by the first decade of the twentieth century; Tshidiso Maloka, "Basotho and the Experience of Death, Dying and Mourning in the South African Mine Compounds, 1890-1940," Cahiers d'Etudes africaines 38, 1 (1998), 25. On mine-related mortality, see Elaine N. Katz, The White Death: Silicosis on the Witwatersrand Gold Mines, 1886-1910 (Johannesburg, 1994); Shula Marks, "The Silent Scourge? Silicosis, Respiratory Disease and Gold-Mining in South Africa," Journal of Ethnic and Migration Studies 32, 4 (2006): 569-89; Randall Packard, White Plague, Black Labour: The Political Economy of Health and Diseases in South Africa (Berkeley, 1989).

67 On slave mortality: Philip D. Curtin, The Atlantic Slave Trade: A Census (Madison, 1969); David Eltis, Economic Growth and the Ending of the Transatlantic Slave Trade (New York, 1989); Paul E. Lovejoy, Transformations in Slavery: A History of Slavery in Africa (Cambridge, 1983); Patrick Manning, Slavery and African Life: Occidental, Oriental and African Slave Trades (Cambridge, 1990); Joseph C. Miller, Way of Death: Merchant Capitalism and the Angolan Slave Trade, 1730-1830 (Madison, 1988). On death in the African diaspora: Vincent Brown, The Reaper's Garden: Death and Power in the World of Atlantic Slavery (Cambridge, MA, 2008); Karla F.C. Holloway, Passed On: African American Mourning Stories (Durham, NC, 2002); Joao Jose Reis, Death is a Festival: Funeral Rites and Rebellion in Nineteenth-Century Brazil (Chapel Hill, NC, 2007).

68 See James Gibbs, "The Writer and the Road: Wole Soyinka and Those Who Cause Death by Dangerous Driving," The Fournal of Modern African Studies 33, 3 (1995): 469-98; Charles Manga Fombad, "Compensation of Victims of Motor Vehicle Accidents in Botswana: An Appraisal of the MVA Fund Act," Fournal of African Law 43, 2 (1999), 151-83; Mark Lamont and Rebekah Lee, "Arrive Alive: Road Safety in Kenya and South Africa," Technology and Culture 56 (2015): 464-88.

69 Shaw, Memories of the Slave Trade, 17, 64, 231.

70 Maloka, "Basotho and the Experience of Death, Dying and Mourning," 21-3.

71 Geschiere, "Funerals and Belonging."

72 Abdou Maliq Simone, For the City Yet to Come: Changing African Life in Four Cities (Durham, NC, 2004).

73 De Boeck, "The Apocalyptic Interlude"; Ashford, Madumo; Shaw, Memories of the Slave Trade; Ellis, "The Okija Shrine."

74 Isak Niehaus, "Death before Dying: Conception of AIDS in the South African Lowveld," Fournal of Southern African Studies 33, 4 (2007): 845-60; Andrew Irving, "Ethnography, Art and Death," Journal of the Royal Anthropological Institute 13 (2008): 185-208.

75 www.eshopafrica.com/acatalog/Ga_Coffins.html.

76 However, a sense of solemnity at a burial may be marred by aggressive marketing campaigns on the funeral site itself - participants in a funeral ceremony can expect to see phone numbers of that funeral's service provider emblazoned prominently across the backs of seats and on marquee tents. Rebekah Lee, "Entrepreneurship in South Africa's Emergent Township Funeral Industry," in Ute Roschenthaler and Dorothea Schultz, eds., Cultural Entrepreneurship in Africa (London, 2016), 121-38.

77 These trends are satirized in Mda's Ways of Dying and are the subject of Rebekah Lee's film, "The Price of Death" (documentary, 2012).

78 For a version of the "transformation" thesis, see Kwame Arhin, "The Economic Implications of Transformations in Akan Funeral Rites," Africa 64, 3 (1994): 307-22

79 De Witte, Long Live the Dead, 105; Jane Guyer, Marginal Gains: Monetary Transactions in Atlantic Africa (Chicago, IL, 2004). 


\section{DEATH IN AFRICA}

80 Hikaru Suzuki, The Price of Death: The Funeral Industry in Contemporary Fapan (Palo Alto, 2000).

81 Rebekah Lee, "Death 'on the Move': Funerals, Entrepreneurs and the Rural-Urban Nexus in South Africa," Africa 81, 2 (2011): 226-47; see also Sjaak van der Geest, "Between Death and Funeral: Mortuaries and the Exploitation of Liminality in Kawhu," Africa 76, 4 (2006): 485-501.

82 White, "The Political Undead"; see Charles Tshimanga, Jeunesse, formation et société au CongoKinshasa 1890-1960 (Paris, 2001).

83 Robins, "From 'Rights' to 'Ritual”"; Golomski, Funeral Culture. 


\title{
RITUALS OF DEATH IN THE GARIBBEAN DIASPORA, $1970-$ \\ The immigrant dilemmas
}

\author{
Garrey Michael Dennie
}

On November 11, 2017 Sheryl Williams sat in the front row of seat of the Caribbean Funeral Parlor, located in Brooklyn, New York, holding back her tears as her oldest brother, Michael Williams, eulogized her mother:

In 1945, in the village of Vermont in St. Vincent and the Grenadines, Mary Sylvester, the daughter of Mr. George James and Ms. Isabella Sylvester, entered this world. And she would leave it after 72 years having lived a life of love, strength, and resilience that would be admired by those who knew her best. ${ }^{1}$

On May 23, just six months later, she would once again seat herself in the front row of the very same Caribbean Funeral Parlor and cry as once again her oldest brother Michael Williams was called upon to deliver the eulogy, this time for one of their younger brothers, Elliot Williams. The eulogy regaled the mourners with the life of their brother, who died at the relatively young age of 51 . The eulogy recalled the life that he had lived in the place of his birth, St. Vincent and the Grenadines and reminded the mourners:

Coming to the USA was both a challenge and an opportunity. Finding a new life, finding a job in Georgia, these of course tested his resolve, tested his ingenuity and he responded with his strongest virtues. In time Georgia too would become his home and now, with siblings stretched across North America, new forms of family re-unions were needed. ${ }^{2}$

The eulogist's awareness that Caribbean people's migration to the USA would propel them into new forms of family re-union is well made. But in fact, when they left their Caribbean home of St. Vincent and the Grenadines, neither Sheryl Williams, Elliot Williams, Gloria Sylvester, nor the tens of thousands of Caribbean migrants who would pour into North America from the 1970s to this present moment ${ }^{3}$ would have imagined that a Brooklyn funeral parlor would constitute a key meeting place where such reunions of families and friends would take place.

Sheryl completely understood this predicament in a way that is unique to immigrants. She herself had arrived in the USA in 1990 when she was 24 years old. Moreover, as an 
undocumented immigrant she had never returned to St. Vincent. ${ }^{4}$ Hence, at the mature age of 53 years, these funerals had indeed become a moment where she could commune with friends and family, resurrect older connections whom she would neither have seen nor heard from in a long time, and grieve in the company of Vincentians who had made America their home. ${ }^{5}$

Sitting among the mourners at both funeral ceremonies was Nathan Culzac, a close family friend, about whom more will be said later in this chapter. But as hundreds of thousands of Caribbean people sought and found permanent residence in New York, New Jersey, Miami, and Toronto, thousands would also die, and they would remain forever entombed in the graveyards of North America or, as is increasing the case, carefully preserved as ashes in the urns reserved for the bodies of the cremated. ${ }^{6}$ Nathan Culzac attended many of these funerals and provided me with critical information on the rituals of death in the Caribbean Diaspora. ${ }^{7}$

This chapter explores how Caribbean immigrants to North America have confronted the challenges of disposing of their deceased in circumstances where the medical, legal, and cultural traditions are alien to Caribbean life. It chronicles how Caribbean people in North America have sought to maintain or re-create Caribbean mortuary practices in North America. And it details how, simultaneously, within the Caribbean itself, new rituals of death have emerged that in some ways are far removed from the funerary practices idealized by Caribbean immigrants in North America. The chapter therefore argues that it is this collision between the tidal forces of an ever-changing present and the gravitational pull of an idealized past that is the peculiar feature of the rituals of death in the Caribbean diaspora.

The chapter is focused on the time period 1970 to the present. This choice of time period is governed by two interlocking factors. First, the Civil Rights Movement in the USA, culminating in the passage of the Voting Rights Act, also had as a corollary a loosening of US immigration restrictions, which resulted in Caribbean people entering the USA in greater numbers than before. The Immigration and Nationality Act of 1965 ended racial quotas in the US immigration system and in so doing opened the doors for sustained Caribbean migration to the USA. ${ }^{8}$ Second, despite the efforts of various US administrations to limit the flow of Caribbean people into the USA, these efforts have largely failed. ${ }^{9}$ Moreover, Canada has had few restrictions on entry into Canada, and Toronto has also witnessed a surge in Caribbean immigrants during this same time period. ${ }^{10}$ Hence over the past fifty years, North America has displaced the United Kingdom as the top destination of Caribbean immigrants. ${ }^{11}$

Fifty years is also a huge chunk of the average human life span. For those Caribbean immigrants who arrived in North America in the 1970s in their early twenties or thirties, they faced and continue to face an actuarial clock that is absolutely relentless. For, by the second decade of the twenty first century, if they had escaped death by illness, accident, or malice, they could not escape death by aging. Neither could their bereaved families avoid the burden of taking care of the remains of their deceased ones. Hence, whereas Caribbeanist scholarship on immigration has remained focused on the multiple ways in which Caribbean immigrants have built new lives in North America while continuing to cherish and preserve their sense of a distinct Caribbean identity, ${ }^{12}$ by the mid1990s the cemeteries, crematoria, and funeral parlors of North America had begun to tell a different story, a story of how Caribbean people have mourned their dead in North America. 
I approach this story as telling history in the anthropological mode. I therefore privilege the voices of Caribbean people obtained through personal communication and residing in my field notes as the best listening board from which we can hear Caribbean people tell this story, because no one understands the mourning rituals of Caribbean people better than Caribbean people themselves. Thus, through an examination of the broadest array of the rituals of death, including but not limited to the preparation of dead bodies for burial, the monuments for the dead, the eulogies for the dead, the songs and sounds of mourning, the processions for the dead, and above all through a careful listening to the testimonies Caribbean people offer to those of us seeking their insight on how to make sense of the history of Caribbean mourning practices, it becomes possible to explore and to bring to life the history of death rituals in the Caribbean and the Caribbean diaspora. For Caribbean immigrants came to North America seeking to validate their lives. And in their deaths they would acknowledge and celebrate that very achievement.

We can therefore enter this story with the help of a Caribbean man, Nathan Culzac, a mourner at the funerals of Elliot Williams and Gloria Sylvester. He was born in in St. Vincent in 1946 and arrived in New York in 1979 at the ripe age of 33 years old. He was therefore part of the leading edge of Vincentians who left St. Vincent in the 1970s. He arrived as a drummer with the musical band, Climax, but would leave the band to become a painter, a husband, and would father two children in New York. And over the next forty years he would be an eye witness, a participant, and an astute observer of the changing rituals of death within the Caribbean diaspora. ${ }^{13}$

Culzac offers us two critical support beams in our efforts to understand how Caribbean mourners adjusted to the experience of having to dispose of their dead in North America. First, because he arrived in New York at the age of 33, Culzac fully understood the culture of death and burial in St. Vincent, his Caribbean homeland. Clearly, the nature and scale of the challenge death in America posed to Caribbean immigrants cannot be understood absent an understanding of the culture of death and burial in the Caribbean itself. Second, however, Culzac has outlived a great number of his age mates such as Gloria Sylvester, whose funeral he attended. Indeed, by his own account, he attended more funerals than he could count. ${ }^{14}$ Hence, by chronology and geography, Culzac occupies a strategic location that allows him to speak with authority of the mortuary practices within the Caribbean and the mortuary practices within the Caribbean diaspora. In the most literal of senses, he has straddled both worlds.

\section{Traditions}

Culzac's earliest memories of a funeral in St. Vincent is that of his father. He was only 9 years old when his father died, but he vividly remembers the funeral procession. He recalls that his father's body was taken through the streets of Kingstown in a carriage hauled by a donkey while mourners walked both in front of and behind the carriage. In 1955 at the time of his father's death and funeral, the occasion was one of solemnity as men and women dressed in dark clothing attended the funeral. Children who were close family relatives also attended the funeral, but in general a funeral was a gathering for adults. At the graveside the mourners sang, the coffin was lowered into the grave, a priest led the burial service, and at the end the mourners departed the cemetery. ${ }^{15}$ 
In the 1950s most adult Vincentians would have remembered funerals in a similar manner as outlined by the childhood memories of Culzac. But they would also have filled significant gaps that escaped the attention of a child. The first of these was that the funeral was neither the first nor the last of the rituals of death in St. Vincent. In fact, despite the ubiquity of the funeral procession in the Vincentian ways of death, Vincentians understood that all funeral processions were not created equal.

Nothing better illustrates this than the funerals of the wealthiest Vincentian families. In the 1950s families such as the Hazells, the Coreas, the Punetts, and the Vieras constituted part of an elite group of families whose wealth was a legacy of plantation slavery in St. Vincent. Their families had owned slave plantations, and, although slavery had ended, more than a hundred years later, their control of the commanding heights of the Vincentian economy remained unchallenged. This was in fact true across the entire British Caribbean. Universal adult suffrage only arrived in the British Caribbean in 1951, and it would not be until the 1960s and 1970s that Britain would grant independence to its former slave colonies. ${ }^{16}$ In fact, St. Vincent and the Grenadines would not gain its independence until 1979. ${ }^{17}$

The benefits that race and class conferred on the colonial elite while alive did not stop at their deaths. For whereas poor, Black Vincentian mourners like Culzac's father used a donkey carriage to take their deceased to the Kingstown cemetery, the families of rich Vincentians used motorized hearses to lead their deceased to the cemetery. Nor did the differences end there. The coffin itself bespoke the differences between the rich Whites and poor Blacks. Rich families buried their dead in coffins made of mahogany and lined with silk and satin. Poor Vincentians used pine wood to inter their dead. Indeed, in one of the most surprising elements of the Vincentian rituals of death, families who could not afford the cost of buying a pine coffin from an undertaker would cut down a breadfruit tree and use the wood to build the coffins themselves. The breadfruit tree can grow to a great height with a big trunk. But most families used the breadfruit for food - roasting, boiling, frying. To kill a breadfruit tree for the purpose of burying your dead was a powerful indication of indigence. ${ }^{18}$ Similarly, to use a motorized hearse to convey your dead through the streets of St. Vincent was a vigorous and public statement of your wealth. Hence, although all Vincentian mourners paraded their dead through the streets of St. Vincent, the funerals differed in size, pomp, and ceremony based upon the race and class of the deceased and their families.

Culzac remembers his father being lowered into an open grave which would later be filled in by the grave workers. Most Vincentians who attended the funerals of their loved ones in 1955 would in fact have similar memories. Today, however, the great majority of these mourners would not be able to locate the graves of their loved ones. The reason is simple: in the 1950s most graves were unmarked. In fact, some families with the means to do so bought and placed headstones at the graves of their deceased. And wealthy families bought tombs which would become the resting place for generations of their family members. Those tombs and headstones record the names of their occupants, their dates of birth and death, the relatives whom they have left behind, and the great sorrow the families experienced in their passing. The public display of grief had a price as reflected in tombs of the wealthy. For the poor, whose grief was no less real, over time, as the graves sunk, the reminders of their deceased ones disappeared permanently from public view, living on only in the memories of the bereaved. ${ }^{19}$ 
Nowhere is the monumental architecture of death in St. Vincent better captured than in the churchyards of the Anglican and Methodist churches. For in these cemeteries, the intersection of race, class, and religion combined to produce a hierarchy of death that all Vincentians understood. The Anglican Church was the church of the colonial elite. Built in 1820, its proper nomenclature is the Cathedral Church of St. Georges. It stands on roughly 20,000 square feet of land in the middle of Kingstown, the capital of St. Vincent and the Grenadines and dominates the capital. ${ }^{20}$ From its inception, only the very wealthy, the very white, the very pious, or some combination thereof, could be buried within its environs. And the stones of death are everywhere memorializing white slave masters and their colonial successors who had lorded over the land of St. Vincent and the Grenadines. That time of course is no more. And the church has fallen on harder times. But in the domain of the dead it retains a power it no longer possesses in life: the visible articulation that its graveyard is a privilege only the very few would enjoy.

The Kingstown Methodist Church faces the Anglican Church on the other side of the road. It was originally built in 1780, but over the years successive hurricanes and fires have resulted in the building having to undergo extensive repairs. The church sits on land several times smaller than the Anglican Church, and its cemetery is not as imposing in size. It cannot hold the same number of graves. But this very limitation has also allowed the Methodist Church to secure for itself a high place in the hierarchy of death in St. Vincent and the Grenadines. For, as with the Anglican Church, burial within its grounds was reserved only for an elite that ticked the boxes of race, class, and piety. It may not have had the power of the Anglican Church, but it affirmed the principle that the inequalities in life replicated themselves in the rituals of death. It is certainly true that, just as is the case with the Anglican Church, time has decayed and perhaps even destroyed the idea that the Methodist Church and its cemetery should function as a preserve of privilege. The 1950s are gone forever. But time has also shown that the headstones laid down in the 1950s and before have a much longer life. Hence, these stones of memory allow us to trace and to interrogate how and why earlier generations of Vincentian mourners celebrated inequality. They also impose on us the burden of exploring how and why these ideas would lose ground. ${ }^{21}$

Paradoxically, part of the answer to this lies in the funeral procession itself. No amount of luxury, pomp, and ceremony associated with the funeral procession of the wealthy could evade the simple fact that rich or poor, black or white, we die. And we walk the same streets to the cemeteries. All Vincentians could see this. To the Vincentian poor, the visible truth that the rich and powerful were just as vulnerable to the reach of death was palpably obvious. They saw them grieve the loss of their families and friends just as they too grieved the loss of their families and friends. They understood their grief. This empathy clearly proceeded from their shared humanity. But it was also buttressed by the physicality, familiarity, and intimacy of the Vincentian culture of death.

These three elements of the culture of death in St. Vincent are of crucial import in understanding the kinds of adjustments Vincentian immigrants in North America would have to make as they faced the absolute necessity of disposing of the remains of their loved ones. Up until the 1970s, in the Vincentian culture of death, the physicality, familiarity, and intimacy of death were interlocking ideas. Preparing the body for burial was a physical act. That meant washing the body, placing it in ice to delay putrefaction, putting copper coins over the eyes, dressing the body for burial, and preparing it for viewing. But these were also deeply intimate experiences shared by 
family members and friends for whom these rituals reaffirmed the bonds they shared with the deceased and with the friends and families of the deceased. This was not treated as befitting the work of a professionalized corps such as undertakers. Rather, it was seen as a duty to family and community that was inviolate, sacrosanct, and commensurate with a sense of value that the mourners attached to the deceased and to themselves. $^{22}$

In the cemeteries of Kingstown and indeed in the cemeteries across the country, the geography of burial protected the familiarity between the living and the dead. Whether in the 1950s or today in the twenty-first century, no one can walk the streets of Kingstown without an awareness of the dead. After all, three cemeteries line Back Street, the country's busiest road. Up until the 1990s these funerals were held only on Saturdays and Sundays. Today, however, funerals can be held on any day of the week. In the 1960 s St. Vincent had a population of about 70,000 people. Today the population stands at just over 100,000. With far more people to be buried and the same number of cemeteries available, expanding the number of days for funerals made sense. But the consequence of this is that funerals in St. Vincent, which were always visible, would become much more so.

No Vincentian was immune to this leveling power of the topography of death. In an island that is only 18 miles at its longest and 14 miles at its widest, this geography militated against social isolation. In St. Vincent social power was never synonymous with social distance. Rather, social power displayed itself in intimate settings. This intimacy, however, was always capable of producing ideologies and narratives of equality. The burial of the dead in St. Vincent was a peculiar expression of this social intimacy, allowing the dead to be buried in close spaces and enabling the mourners to mingle with each other and witness and experience each other's grief. Hence, as Vincentians enacted their rituals of death year after year, decade after decade, they were embarked upon a trajectory that valorized their dead as equal in value, even if unequal in means. Hence, by the 1970 s the geography of death and the rituals of death were combining to produce shared understandings of the value of the deceased as expressed through the lived experience of the bereaved.

It is a supreme irony that, precisely at the point where Vincentian death rituals had achieved a certain level of stability, St. Vincent would experience an immigration outflow to North America that would far surpass the earlier outflow of immigrants to the United Kingdom. ${ }^{23}$ These migrants were utterly unaware that they would have to engage with a culture of death that would destabilize their own ideas of how they should dispose of their deceased. It is the collision between the rituals of death in America and the rituals of death in St. Vincent that must now command our attention.

\section{Burying the dead in North America}

That there were fundamental differences between the culture of death in America and the culture of death in St. Vincent there can be no doubt. In fact, even to speak of the culture of death in America is something of a misnomer. In a country of hundreds of millions of people stretched across an entire continent, fundamental differences exist in how different communities treat the disposal of their dead. But overwhelmingly, Caribbean people would migrate to the great cities - New York, Miami, Toronto - and it is in these urban landscapes that the Caribbean imagination of how one cares for the dead 
had to engage with new modes of thinking and new mortuary practices that were entirely unfamiliar to the ways of death as they were understood within the Caribbean.

The first challenge was the simple lack of knowledge over what to do if someone died. Medical authorities had to determine cause of death. Police might need to do the same. Certificates of death establishing who had the right to determine the final disposition of the dead body had to be obtained. Undertaking services had to be contracted. Religious observances were a must, but finding a church of similar religious beliefs was not guaranteed. Organizing the funeral and locating a cemetery were essential. And last but not least, how would this be paid for? In the home country none of these presented a problem. But in North America confusion on how to complete any one of these could bring a halt to the process of disposing of the dead. In essence, in the Caribbean, the bereaved and their families retained close control on the management of the dead body. And these were woven into the very process of grieving itself. In North America, however, until these logistical issues were understood and resolved, the grieving process was itself being placed on hold. It was a terrifying reality that no Caribbean migrant had expected to face.

In New York, the Department of State Division of Cemeteries possessed the exclusive authority to regulate the disposal of the remains of the dead. And it exercised this power through the New York State Cemetery Board. The Board consisted of the Secretary of State, the Attorney General, and the Commissioner of Health. In furtherance of its mandate the Board published a deeply detailed book that covered every aspect of the management of the dead body. Entitled, "CEMETERIES AND CREMATORIES: Laws, Rules, and Regulations of the New York State Cemetery Board," the preface declared the laws governing the use of cemeteries and crematoria was an exercise of the police powers of the state and advised readers to seek qualified legal advice to ensure that they were disposing of their dead in accordance with state law. ${ }^{24}$

The thicket of legislation surrounding the disposal of the dead in New York compelled Caribbean immigrants to seek expert help that could allow them to navigate the unfamiliar shoals of American mortuary law. And New York's funeral parlors would derive an enormous benefit from the inflow of Caribbean migrants to New York who would need their guidance to ensure that they could bury their dead within the constraints of American law. Not all Caribbean families were willing to bury their dead in New York's cemeteries. Where they could, they chose to return their loved ones to be buried at home and therefore stayed within the universe of ideas that governed Caribbean understandings of death and burial. Even here, however, they could not completely escape the reach of the American funeral directors since the movement of dead bodies within the USA and across international borders still had to conform to American law. ${ }^{25}$

As a longtime resident of Brooklyn, New York, Culzac has witnessed the mushrooming of funeral parlors in New York specifically catering to the needs of Caribbean mourners. In fact, Culzac was the person who first drew my attention to this phenomenon. He noted that when he first arrived in New York not only were there no funeral parlors advertising their services to Caribbean people but some of the funeral parlors were in danger of closing because their services were not in high demand. ${ }^{26}$ Today, however, in Brooklyn alone at least 30 funeral parlors are directly advertising their services to Caribbean people. ${ }^{27}$

These advertisements are the model of restraint and respect to Caribbean mourners. For example, The Caribe Funeral Home declares, 
We believe in personal attention and uncompromising quality. We are proud of our longstanding traditions, our serene facilities, and steadfast commitment to serving families in their deepest time of need. We are available 24 hours a day 7 days a week. We have an extensive history of earning each family's trust by creating a caring and compassionate environment every time we are called. Our licensed and well trained staff can assist you with personalized funeral and cremation services, memorials, burial as well as personalized merchandize, all of which reflect with dignity, your wishes as well as the life of your loved one. ${ }^{28}$

We need not go into any deeper analysis of this advertisement except to make three points. First, all 30 funeral parlors in Brooklyn seeking a Caribbean clientele have advertisements that broadly speaking follow the same formula of restrained earnestness as they invite the newly bereaved to make use of their services. Second, the Caribe Parlor also declares that they have been offering their services to Caribbean people for well over two decades, and this timing is consistent with Culzac's claim that the mushrooming of the funeral parlors took place as the Caribbean population exploded in Brooklyn. And third, Caribe Funeral Home also is also offering cremation services to Caribbean mourners. This last point is particularly important because it confirms that whereas in St. Vincent and several other Caribbean countries no crematoriums exist for the cremation of the dead, here in North America Caribbean immigrants were at least being asked to consider new forms of burial rituals which were alien to Caribbean ways of death.

The regime of New York law and the persuasions, convenience, and expertise of the funeral parlor industry acted as a powerful force to push Caribbean mourning practices into greater conformity with American practices. But this came at a cost and was not without resistance. Unsurprisingly, the first cost was a financial one. Today, Caribbean mourners in New York pay between $\$ 7000$ and $\$ 12,000$ in funeral costs. Lincoln Heritage Funeral Advantage observes, "These costs include viewing and burial, transporting the remains to a funeral home, a casket, embalming and other preparation. The average cost of a funeral with cremation is between $\$ 6000$ and $\$ 7000 . " 29$ Sheryl Williams paid $\$ 7000$ for the burial of her mother, and in early January 2020 she incurred a staggering cost of $\$ 14,000$ to retrieve the body of her deceased sister from Canada and inter her body in a New York cemetery. ${ }^{30}$

These kinds of expenses obviously invite a set of questions on the cultural economy of burial among immigrant communities in New York. Ultimately, spending this kind of money was a choice. Bereaved families chose to place themselves in enormous debt because they dreaded the alternative. The alternative, as they understood it, would be that they would leave the remains of their loved ones in the hands of the state for the state to dispose of as it so determined. Almost certainly that meant cremation. Sheryl Williams incurred the tremendous cost of retrieving her sister's remains from Canada because she understood that absent that intervention, the relevant Canadian authorities would cremate her remains. For Caribbean people who saw cremation as a violation of their beliefs, paying the high prices for burial services in New York granted them control of the body and the capacity to demonstrate their reverence for the deceased in a way that was consistent with their religious ideas.

Some, however, were willing to cremate their dead, even if that caused great offence to their fellow mourners. After all, cremation was several thousand dollars less expensive than burial. The funeral of Elliot Williams was one such case. Williams had 
suffered from cancer and knew he was going to die. He therefore placed his ex-wife in charge of his funeral and, to the great shock of his family, she chose to pay to cremate the body rather than have it interred in a grave. Sheryl lamented that her brother's ex-wife, Lu-Ann Williams, had decided "to burn up my brother." She therefore sought - but failed - to seize control of the body so that she could prevent the cremation. $^{31}$

It would be wrong, however, to see Caribbean mourners' use of funeral parlors in New York as merely a defensive act against the cremation of their dead or an escape hatch from potential violations of New Yok laws governing the care of the dead. Ironically, the funeral parlors also enabled Caribbean mourners to hold on to some of the funerary traditions that they had brought with them from the Caribbean. The veneration of the dead was deeply ingrained within Caribbean culture, and the funeral parlors made that possible. One of the ways they did this was by providing a place of worship within the parlors themselves. Caribbean people could not contemplate burying their dead without a full array of religious services to accompany the dead to their final resting place. In fact, several funeral parlors advertised these services. Moreover, they not only provided the place of worship. Where necessary, they provided religious leaders as well to lead the ceremony. Fundamentally, Caribbean mourners in North America insisted that the burial of the dead be treated with the deep solemnity that defined the burial of the dead in the Caribbean. ${ }^{32}$

From the late 1990s to the present, witnesses to these funerals have testified that four features continue to confer great solemnity to the moment. The first is the sermon itself. In multiple ways the pastor or priest would insist on the sacredness of the life of the deceased. Second, the mourners would sing a great number of funerary hymns whose sum effect would be to construct an atmosphere of reflection befitting the reverence accorded the dead at this moment of sadness. Like the eulogies and the sermons, these hymns proclaimed the evanescence of life, the permanence of death, and the hope of resurrection that their Christian faith promised. Third, the mourners always dressed in dark formal clothing considered appropriate for mourning purposes. Men wore jackets and ties at these funerals. Women wore dark dresses that reached below their knees and covered much of their arms. And fourth, of course, would be the tears. Essentially, the parlor provided a space for the public display of grief in a controlled setting. Left bereft of the physicality and familiar geography of death that defined the mourning moment in the Caribbean, the funeral parlor allowed the Caribbean-American community to re-create and re-capture the sense of the intimacy of death, and a sense of the communion between the dead and the living, that had prevailed in colonial St. Vincent.

However, unlike funerals in St. Vincent, the end of the funeral service in New York was not immediately followed by the interment of the body. Interment usually took place the following day with very few of the mourners present to see the body being lowered into the grave. Nor did the mourners lead a procession through the streets of Brooklyn as they would in the streets of St. Vincent. Brooklyn's streets simply could not accommodate the funeral procession. Rather, the mourners regathered either at the home of the bereaved or in more lavish circumstances, at a hired hall, to eat and drink as they shared memories of the departed one. In doing so, they transformed the funeral itself into an affirmation of the life of the dead, and the life of the living. 


\section{The funeral in modern St. Vincent, 1980-2020}

As Vincentians abroad engaged in a 50-year odyssey of constructing a mortuary culture in New York that combined Caribbean notions of the dignity of the dead within the legal and physical framework permissible within the USA, Vincentians at home were also altering, abandoning, adapting, or adopting new elements into their rituals of death. For example, by the early 1980 s, undertakers were beginning to play a more prominent role in most Vincentian burials. The Everready Undertaking Company led by Cedric Mills and family would rise to a position of dominance within the undertaking industry and offer burial services comparable to what Vincentians obtained in New York. ${ }^{33}$ This included increasingly ornate coffins, the embalming of the dead body, refrigeration services that kept the body above ground for weeks, a motorized hearse, power tools to lower the body into the grave, and, by 2015, in the most astonishment development of all, the now highly competitive undertaking industry would provide steel orchestras to accompany mourners through the streets as they danced their way to the cemeteries. ${ }^{34}$

The costs associated with these services were not minimal. Across these decades they rose steadily. By 2020 coffins at Mills undertaking company began at about \$1000 USD and could reach as high as $\$ 7000$ USD contingent on whether the coffins were imported from abroad or built in St. Vincent. Recognizing the great sums of money that could be made in the economy of death, local furniture companies also began advertising their capacity to provide less expensive and superior coffins to those on offer at the undertaking companies. Indeed, in the light of the escalating costs of Vincentian funerals, the prime minister himself advised on the necessity of the Vincentian parliament acting to rein in the costs of the Vincentian funeral. ${ }^{35}$

How precisely a government could rein in the costs of a Vincentian funeral is unclear. In fact, in 2007 the Vincentian government spent $\$ 28,000$ USD to pay for the funeral costs of Glen Jackson, a former advisor to the prime minister. The casket alone cost $\$ 14,000$ USD. $^{36}$ Much clearer are the means by which Vincentians would meet the increased costs of their funerals. The key solution was the development of a local burial society, the Marriaqua United Friendly Society, commonly referred to as Bun Pan. Founded in 1954 by Nathaniel Guy, the society required an application fee of $\$ 6.00$ and a minimum membership contribution of $\$ 20$. In 2014 the President of the Society, Theophilius Shallow offered a brief history of Bun Pan to The Vincentian newspaper. He noted that the society came into existence to ensure that poor Vincentians obtained "a proper burial." Shallow observed that in 1954 the society offered $\$ 500$ to pay for the burial of any member. By the 1980 s this had increased to $\$ 1200$. And by 2014 the society offered each member $\$ 5200$ to meet the cost of their funerals. Indeed in 2013 , the society paid for the funerals of 400 persons at the cost of $\$ 2.1$ million. ${ }^{37}$ And the society met these costs easily because of a huge dues-paying membership of 23,000 persons. Because the number of persons dying in any one year was less than 2 percent, and with new members joining every year, the society and poor Vincentians could rest assured that they could meet the costs of a "proper" burial.

At its core, the idea of "a proper burial" rested upon the deeper notion of the sacredness of the dead body, and by extension, living bodies. Vincentians therefore corralled these ideas into a funerary construct divided into two domains. The first domain had to do with how these ideas could express themselves in a religious form. Whether in the 1950 s at the time of Guy's creation of the first Vincentian burial society or in the 
twenty-first century in New York funeral parlors, Vincentian church services and eulogies to the dead, predictably and effectively, accomplished the task of placing the dead body within the shelter of Christian eschatology. Bereaved Vincentians simply would not allow their deceased to depart this earth without the religious observances deemed necessary to facilitate their passage to the afterlife.

The second domain, however, had to do with the public, performative, and visible value that should be accorded to the dead. The most important of these was the funeral itself. Hence, a "proper burial" could therefore only be assured if the funeral ceremony and the paraphernalia of burial, such as coffins, met minimum threshold standards. It therefore needs to be said that the creation of the first burial society was a fundamental critique of the existing socio-economic inequalities that in the 1950s enabled the wealthy to obtain a "proper burial" while denying the same to the less well off. What this therefore means is that from the 1950s to this current day, the Marriaqua Friendly Society and other competitors were engaged in a six-decade struggle to democratize burial in St. Vincent by ensuring that a "proper" burial was available to all Vincentians regardless of their financial means.

Through the 1970s into the beginning of the twenty-first century, Vincentians at home and abroad shared a common understanding of what constituted a "proper burial" and how this should be expressed. During that time, Vincentians returned home to attend the burials of friends and family members. And Vincentians at home also travelled to North America to attend the funerals of friends and relatives who had died overseas. Sometimes they were the chief mourners, as was the case of Culzac who returned to St. Vincent in 1998 to bury his mother. ${ }^{38}$ And the solemnity of the funerals in North America matched the solemnity of the funerals in St. Vincent. They understood and accommodated the differences in the geographies of death because they were also deeply familiar with the rituals of death which they employed to manage their grief and to send their departed into the Great Beyond.

From about 2005, however, Vincentians at home would begin to fracture the shared understanding of the meaning and display of "a proper burial." For, over the past 15 years, funerals in St. Vincent have been transformed into miniature versions of their yearly carnival. The high priests of solemnity no longer preside over these funerals. Instead, the Lords of Bacchanal are out in full as the dancing mourner replaces the grieving figure as the central image of this major shift in Vincentian mortuary practices. $^{39}$

Vincentians are very clear on when and how this transformation of the funeral procession began. Sion Hill, a community located on the hills overlooking Kingstown, is also the home to a steel orchestra which had become identified as a major carrier of the values of the community. In the early twenty-first century, when one of their community members died, the Sion Hill Steel Band members made the decision to use their steel band music to lead the procession down the hill into Kingstown and take the mourners into the cemetery. Soon, the band would be used to lead all funeral processions from Sion Hill into the Kingstown cemeteries. And the practice quickly mushroomed across the country as community after community began emulating the Sion Hill example of using steel bands to accompany funeral processions to the cemeteries. By 2017 the undertaking companies had gotten into the fray, hiring steel bands to provide the music that would lead dancing mourners through the streets of Kingstown. 
Some people, particularly older Vincentians, were aghast at what appeared to be an utter irreverence and disregard for the solemnity that had traditionally accompanied the disposal of the dead body. This was certainly the case of the oldest VincentianAmericans. For precisely at the time when the Vincentian-American community had stabilized their rituals of death centered on the use of the American funeral parlor to protect their sense of the solemnity of the dead, in the homeland itself the funeral procession had been transformed into nothing less than a carnival of music and drinking that scarcely separates these funerals from the rituals of bacchanal, dance, and wine of St. Vincent's yearly carnival festival. In sum, mourners in both St. Vincent and the USA who travelled and continue to travel to funerals in both countries became both witnesses to and participants in the collision between these competing funerary traditions.

\section{Conclusion}

We can offer some tentative conclusions on what we might refer to as the co-evolution of the rituals of death in the Caribbean and the Caribbean Diaspora. The first point is that these existed in a relationship of reciprocity that allowed Vincentians at home and abroad to shape each other's burial culture in a way that reinforced their fidelity to the idea of the reverence that must be accorded to human remains. And this process held for more than 50 years. Second, however, this fracture in the understanding of what constitutes a "proper burial" that has now occurred between the homeland and the diaspora community points to the power of the modern world to disrupt existing norms, create new traditions, and in the instance of the Vincentian rituals of death, leave in flux any confidence that we can know the final trajectory of Vincentian mortuary practices either in St. Vincent or the Caribbean-American community. Vincentians abroad had resolved their first dilemma of protecting their rituals of death against the weight of an alien American culture. Now they face a new dilemma: protecting their sense of the solemnity that must be attached to funerals against a movement in St. Vincent to treat the burial of the dead as a celebration anchored on the foundations of the Caribbean carnival culture. History offers no judgment on the outcome.

\section{Notes}

1 Sheryl Williams, Facebook message to author, September 18, 2019. Ms. Williams has kindly allowed to make use of several eulogies she sent to me as I sought to ground this paper in the voices of Caribbean mourners. I am also deeply grateful to Nathan Culzac, who is a living archive of the funerary practices of St. Vincent and the Grenadines and the VincentianAmerican community. I am also thankful to all Vincentian mourners at home and abroad whose grief I shared and who conveyed to me their understanding of their mourning rituals. Without their help this work could not have been done.

2 Sheryl Williams, Facebook communication to author, December 15, 2019.

3 In 2017 there were approximately 4.4 million Caribbean immigrants in the USA. See Jie Zong and Jeanne Batalova, "Caribbean Immigrants in the United States," February 13, 2019, at https://www.migrationpolicy.org/article/caribbean-immigrants-united-states. The authors draw their numbers from the US Census data collected in 2010 and 2017.

4 As an undocumented immigrant, Sheryl Williams risks her own safety by divulging her immigration status. I have therefore taken precautions against this risk by hiding her Facebook identity, which is different from her legal identity.

5 Sheryl Williams, Facebook communication to author, December 15, 2019. 
6 Jie Zong and Batalova, "Caribbean Immigrants."

7 I interviewed Nathan Culzac on August 15, 2019 and on November 15, 2019. These tapes have been placed in the trust of the National Archives of St. Vincent and the Grenadines.

8 For an insightful essay on this issue, see Rebekah Barber's, "How the Civil Rights Movement Opened the Door to Immigrants of Color," in Facing South, February 3, 2017.

9 Immigration is a deeply contested issue in the USA. For the past 20 years the US Congress has been mired in the fight to determine the disposition of 12 million undocumented immigrants living within its borders.

10 In 2011 the Canadian Census reported that Canada has 500,000 Caribbean people. See The Canadian Encyclopedia at www.thecanadianencyclopedia.ca/en/article/caribbean-canadians.

11 One of the most amusing Caribbean poems on immigration, "Colonization in Reverse" written by Louise Bennett reflects on the historical irony of Caribbean migration to England.

12 For a brief introduction to the subject, see the following works: Basil Wilson, "Caribbean Immigrants in New York City and the Rise of a Black Middle Class in South East Queens," Wadabagei, vol. 12, no. 1, (2011): 32-38; John Garcia, "Caribbean Migration to the Mainland: A Review of Adaptive Experiences," in The Annals of the American Academy of Political and Social Science, vol. 487 (September 1986): 114-125; Annette Mahoney, The Health and Well Being of Caribbean Migrants in the United States (Routledge, 2012).

13 Interview with Nathan Culzac.

14 Ibid.

15 Ibid.

16 For a through overview on the march toward independence in the Caribbean, see Franklin Knight, The Caribbean: The Genesis of a Fragmented Nationalism (Oxford University Press, 2011).

17 I am one of the Vincentians who on October 27, 1979 celebrated the end of colonial rule in St. Vincent.

18 Interview with Nathan Culzac.

19 I have walked all the cemeteries of Kingstown and there are many graves in a state of dilapidation.

20 The Anglican Church itself is struggling to protect its architectural heritage as can be confirmed on its web page where it is requesting donations. But it does also offer critically important information on the history of the church.

See http://stgeorgescathedralsvg.com.

21 Like the Anglican Church, the website of the Methodist Church is loaded with valuable historical information on its founding. And I have also walked its cemetery and sat in its pews. See www.kingchatmethodist.com.

22 See Hakem Smart, "Dearly Departed - Wakes and Funerals in Barbados and St. Vincent and the Grenadines," February 10, 2016. This is one of a very few publications that has some information on burial practices in St. Vincent. It is located at http://bcc.edu.bb/news/latest news. I retrieved it on January 27, 2020.

23 The creation of the St. Vincent and the Grenadines Diaspora Committee of NY is an indication of the size of the outflow of Vincentians from St. Vincent to the USA. The use of the language of the diaspora reflects the scale of the outflow that now numbers in the tens of thousands. Its webpage is www.svgdiasporacommittee.org.

24 The New York Department of State Division of Cemeteries can be found online at www.dos. ny.gov/cmty/index.html. Of course, when Caribbean migrants began arriving in the 1970s in droves, the internet did not exist.

25 The Center for Disease Control in the USA regulates the movement of human remains into and out of the USA. See its website at www.cdc.gov/importation/human-remains.html.

26 Interview with Nathaniel Culzac.

27 See "The Best 30 Caribbean Funeral Home in Brooklyn," at www.yellowpages.com/brooklynny//caribbean-funeralservices.

28 See the website, https://caribefuneral.com.

29 On the costs of funerals in New York see, "How much does a funeral cost?" at www.lhlic. com/consumer-resources/.

30 Sheryl Williams, Facebook communication to author, January 15, 2020.

31 Ibid. 


\section{DEATH RITUALS AND THE GARIBBEAN DIASPORA}

32 I have attended several Caribbean funerals in some of the funeral parlors in Brooklyn.

33 On the range of services offered by Mills Funeral service, see the advertisements in In Memoriam sections of The Searchlight and The Vincentian newspapers of St. Vincent in any week. The advertisements are published every Friday.

34 I have witnessed these dancing funerals.

35 See "PM does not mind being buried in a wicker basket," in The Famaica Observer, September 24, 2019, p. 2.

36 The Searchlight, Kingstown, March 30, 2007, p. 1.

37 The Vincentian, Kingstown, July 23, 2014, p. 2.

38 Interview with Nathan Culzac.

39 Several videos on YouTube provide visual evidence of these funerals. For example, see "Funeral Processions in St. Vincent," posted on You Tube by Eyewitness News on October 5, 2018. See as well "Funeral in Questelles, St. Vincent and the Grenadines" uploaded to You Tube on September 17, 2018. 


\section{$\because$ Taylor \& Francis Taylor \& Francis Group http://taylorandfrancis.com}




\section{PART III}

\section{SPECIAL TOPICS}

The chapters in this part suggest possibilities for deeper research into aspects of the modern history of death, including the types of sources available for more intensive analysis. Several essays fill in some gaps in regional coverage. Some, as on Australian experience in war or the design of Soviet cemeteries, offer special cases for topics also covered as well in Part I. Others, dealing, for example, with infanticide or fear of burial alive, point to distinctive cultural moments in various societies. A discussion of death in film explores an intriguing media angle over the past century, though with particular attention to the emergence of new themes in the last two decades. Many African regimes have taken to constructing elaborate tombs for past leaders, more for political credit than to honor death; but the practice raises interesting questions about modern manipulation of death and memory (going back at least to the uses of Napoleon's tomb). Several chapters treat very recent developments that may gain wider importance in the field in future - suggesting, as well, some further basic changes that may result, within the modern context, from innovations such as societal ageing. A final chapter, building on this theme, discusses potential future characteristics as the modern experience extends further in time but as regional distinctions continue to play a role.

Death has always been, and will remain, a crucial part of the human experience. But it has a rich history as well, in the sense of undergoing a host of changes in virtually every aspect - from material cause to emotional impact. Essays on special aspects of this history promote further understanding and, hopefully, encourage further work on the many topics that remain open to imaginative research.

The chapters in this part, as in the previous parts, offer opportunities to link changes and continuities in death to other historical topics, and to enrich our understanding of the past by insisting on the inclusion of death as a part of the social fabric. They offer, as well, the opportunity to consider trends in death's modern history as part of the process of understanding contemporary issues and future prospects - giving real substance to the value of historical perspective. 


\section{$\because$ Taylor \& Francis Taylor \& Francis Group http://taylorandfrancis.com}




\title{
PREMATURE BURIAL AND THE MYSTERIES OF DEATH
}

\author{
Joanna Bourke
}

In 1905, Esther Holden was nearly buried alive. Her near-fate led to major debates not only about the difficulties of diagnosing death, but also about the role of "shrouding women," male physicians, and a new, very diverse group of specialist funeral professionals. Why did the fear of being buried alive periodically excite social panics in Britain during the nineteenth century, and why did such terrors recede in the early decades of the twentieth century? Although the medicalization of death (including its removal from domestic spheres) offers important explanations for anxieties around death's finality, this chapter also argues that the mystery of death cannot be understood without paying attention to gender and class uncertainties.

29-year-old Esther Holden lived with her husband and three children (including one who was only a few months old) in a remote and "dreary little house" in Hapton, near Accrington in Lancashire. ${ }^{1}$ In January 1905, she was feeling extremely weak and believed that she was dying. When her devoted husband briefly left the room, Holden summoned up "incredible will-power" and "struggled out of bed, washed herself, and prepared for the ceremonies which followed death." "Satisfied that she was now ready to die, Holden asked her husband to "kiss the children" for her and, after bidding him "Goodbye," she "lapsed into unconsciousness" and died. ${ }^{3}$

Her husband was distraught. Her "limbs being rigid,"4 he washed her body, brushed her hair, wrapped her in a clean sheet, and "in accordance with an old Lancashire custom," covered her feet and legs with "a pair of white stockings." He then set about carrying out the necessary tasks for burial, including obtaining a death certificate from a doctor (who had been "little surprised ... to hear of her death" since she had been ailing for some days) and drawing the money owed by his wife's death insurance. As a poor tenant farmer, he was relieved the $£ 27$ insurance would help pay for her coffin and funeral coaches. ${ }^{5}$ James Waddington (the undertaker) and his assistant (who was a member of the Royal House Artillery) began measuring Holden for her coffin, but they suddenly noticed movement. When the Manchester Courier and Adviser reported what happened next, they adopted a narrative style more common to sensationalist fiction than newspaper reportage. The newspaper informed readers that Waddington "noticed a twitching of the eyes, a movement which caused his blood to run cold ... the glassy eye quivered, and then slowly, trembling, and with uncertain motion, the woman lifted her arm and clutched his hand."6 
Despite the fact that Holden's hand "still retained the coldness of the dead," Waddington immediately applied artificial respiration and friction to Holden's prostrate body. When she revived, her husband sent for some brandy from the nearby Griffin Inn in Huncoat. An elderly woman was called to act as a nurse. It was revealed that this was the third time Holden had been "laid out": she had also been left for dead as an infant and as a 15-year-old girl.

Holden's near-living burial was reported in newspapers throughout the UK. The undertaker was hailed as a hero and received letters congratulating him for "the presence of mind he displayed in a trying ordeal." Holden was pitied; she was an impoverished, near-victim of the greatest travesty that could befall anyone. Holden was inundated with gifts from people throughout the country. Blankets, "nourishment," and cash were sent to her family. ${ }^{8}$ There were so many letters and gifts that Mrs. Rhodes, the landlady of the Griffin Inn, offered to act as almoner. Less generously, it was reported that when news spread that Holden was being sent gifts, "some one had called to collect a small amount the family are owing." Holden wept when she told reporters that it was "hard trying to live in this world."9

Holden's experience generated a huge amount of interest. Curious about how it "felt" to die, people were reassured that Holden claimed to have seen "white birds ... floating above the house." ${ }^{10}$ Advice on how to avoid Holden's fate proliferated. Some maintained that, when she collapsed, Holden should have been immediately "bled." Writing from the Great Coates rectory in Lincolnshire, the Rev. J. F. Quirk recalled a similar case of live burial that had taken place in Warwickshire forty years earlier. According to his story, a "lady" had been buried wearing several valuable rings. After the burial, the clerk descended into the grave, unscrewed the coffin-lid, and proceeded to cut the fingers of the "deceased" lady, the more easily to take off the rings. To his surprise and horror, the lady slowly rose in her coffin and walked home!

Although this anecdote was about preventing grave-robbers from getting in as much as occupants of coffins getting out, the Reverend explained the revival of the "lady" as due to the "flowing of the blood" when her fingers were cut. The bleeding had "released some internal pressure." He speculated that if Holden "had been 'bled', she might have recovered sooner." 11 It was a theory premised on the increasingly discredited idea of "humors" (which were blood, phlegm, black bile, and yellow bile) and the medical value of bloodletting.

The lurid language used by journalists reporting on Holden's crisis was typical of the numerous cases of premature burial reported in newspapers in the late nineteenth and early twentieth centuries. Indeed, premature burial excited so much melodramatic interest that an unnamed "public entertainer from the North" even offered to pay Holden "£5 per week and travelling expenses if she would consent to appear in public for a few minutes nightly" in his show. ${ }^{12}$ She declined on the grounds of ill-health.

But public anxieties about premature burial were a serious business. Although the term "taphophobia" (from the Greek $\tau \dot{\alpha} \varphi o \varsigma$ or "taphos" meaning tomb and $\varphi o ́ \beta o \varsigma$ or "phobos" meaning fear) was not used in English until much later, it was a remarkably common dread. In the 1890s, psychologist G. Stanley Hall was surprised to find that alarm about the possibility of being buried alive was spontaneously mentioned by people when they were asked to describe their major fears. ${ }^{13}$ In the mid-1920s, when the Births and Deaths Registration Bill proposed safeguards against crimes relating to death (which included deliberate and non-deliberate premature burial), politicians were inundated with 
correspondence from anxious people. ${ }^{14}$ Why did this fear periodically excite panic in Britain and America during the nineteenth century, and why did such terrors eventually recede? As we shall see, there was widespread concern about the uncertainties of death. What were its "reliable signs"? Who was responsible for diagnosing death? Was this the duty of family members and neighbors, or should medical professionals view and certify that the corpse really was a corpse? Although the medicalization of death (including its removal from domestic spheres) offers important explanations for anxieties around death's finality, this chapter also argues that the mystery of death cannot be understood without paying attention to gender and class.

$$
* * *
$$

This chapter focuses on panics surrounding premature burial in nineteenth- and early twentieth-century Britain and America. However, such fears were not unique to this period of history or to these countries. In the fourteenth century, for instance, recurrent epidemics produced comparable panics. A sensationalist pamphlet entitled The Uncertainty of the Signs of Death, And Danger of Precipitate Interments and Dissections, Demonstrated (1748) was one of the first published accounts in English on the subject. ${ }^{15}$ The development of new resuscitation techniques in the eighteenth century had also led to the establishment of Humane Societies, dedicated to developing more accurate means of diagnosing "final" death. $^{16}$

But the period from the mid-nineteenth to the early twentieth centuries in Britain and America saw these fears taking on a new urgency. Without diminishing the distress that must have been experienced by near-victims like Esther Holden (and others who will be mentioned in this chapter), the alarming rhetoric generated by campaigners against premature burial was excessive. Campaigners often claimed that they had hundreds of accounts from men and women who had survived the ordeal of being buried alive. ${ }^{17}$ However, not only were most of the testimonies unverified or based on hearsay, but the same accounts were being reprinted time and again. For example, nearly a quarter of a century after Dr. Alexander Wilder (a well-regarded physician and leading member of the Theosophical Society) had addressed members of Congress about the risk of premature burial, his scaremongering statistics were still being republished in a book entitled Perils of Premature Burial (1895). Wilder had been incensed by the exhumation of corpses from cemeteries in New York in order to make way for houses and roads. He contended that six of the 1,200 corpses exhumed during the 1860s and 1870s showed signs of live burial. ${ }^{18}$ Other (guess-)estimates of the proportion of burials that took place prematurely ranged from one out of every $500(1908)^{19}$ to one in every 100,000 persons $(1911){ }^{20}$ Since most examples of premature burial were uncovered by accident, campaigners contended that, in all probability, many more poor wretches had been damned to this end. ${ }^{21}$

Fears of live burial were also fanned by horror fiction. The gothic fiction of the late eighteenth century, for example, reveled in living corpses. One example is Matthew Gregory Lewis' hugely popular novel The Monk (1796) in which an innocent young girl was buried alive and raped amid the "rotting Bones." The book continued to have an avid readership in Victorian times. ${ }^{22}$ The doyen of premature burial stories, though, was Edgar Allan Poe. His "Berenice," first published in 1835, dwelt on this theme, but it was his short story "The Premature Burial" (1850) that most excited the reading public's imagination. In it, Poe claimed that "no event is so terribly well adapted to inspire the supremeness of bodily and mental distress, as is burial before death." He described 
the unendurable oppression of the lungs - the stifling fumes of the damp earth the clinging to the death garments - the rigid embrace of the narrow house - the blackness of the absolute Night - the silence like a sea that overwhelms - the unseen but palpable presence of the Conqueror Worm ... these considerations, I say, carry into the heart, which still palpitates, a degree of appalling and intolerable horror from which the most daring imagination must recoil. We know of nothing so agonizing upon earth - we can dream of nothing half so hideous in the realms of the nethermost Hell. ${ }^{23}$

Poe's "The Premature Burial" set the tone for a proliferation of horrifying scenarios in print and film.

Journalists, too, followed in Poe's wake. The sensationalist press reveled in lurid depictions of burial horrors. The nineteenth century had seen the rise of the "yellow" (or tabloid) press, bolstered by the repeal of the newspaper tax and the introduction of cheaper printing presses. Their scaremongering had long-term effects on readers. As late as the 1970s, for example, Molly Weir recalled her childhood in the slums of Glasgow during the early twentieth century. She would tremble as she listened "to stories of coffins having been dug up and the corpses turned over on their faces, or eyes staring with terror, and clawed marks in the coffin lid where they had scrabbled to get out." ${ }^{24}$ Still others were regaled by stories of graves being opened to reveal a female corpse whose face was "gashed, her hands and arms frightfully torn, her feet drawn up in a wild endeavor to extricate herself from the horrible prison, her breasts furrowed and lacerated by finger-nails." 25 Corpses were reported to be found "inside the doors of vaults, with coffins broken open, and every indication of desperate struggles for - escape." ${ }^{26}$ In the words of The Spectator in 1895, "burning, drowning, even the most hideous mutilation under a railway train" was "as nothing compared with burying alive."27 Given that this was a period in which the "beautiful death" was lauded, a premature experience of Everyman's fate was a terrible way to die.

As these accounts suggest, sensationalist accounts of live burial followed certain conventions. Despite the fact that the most realistic scenario in actual cases of premature burials was that of Esther Holden, with whom I started this chapter - in other words, an impoverished, exhausted, and postpartum woman - sensationalized stories of premature burial typically lingered over young, beautiful, and wealthy victims. One example is that of Miss Eleanor Markham, who was described as "a young woman of respectability" who lived in Sprakers, a village in the state of New York. She had complained of heart trouble and, on 8 July 1894, died. Her relatives and friends bid their teary farewells, and the undertakers screwed down the coffin lid. However, as her body was being carried to the hearse, the pall-bearers felt movement within the coffin. When they raised the lid, they saw "poor Eleanor Markham lying on her back, her face white and contorted, and her eyes distended." "My God!," she cried in broken accents, "You are burying me alive!" Markham later contended that she

was conscious all the time you were making preparations to bury me ... . The horror of my situation is altogether beyond description. I could hear everything that was going on, even a whisper outside the door, and although I exerted all my will power, and made a supreme physical effort to cry out, I was powerless. $^{28}$ 
All her physician could say was "Hush! Child. You are all right. It is a mistake easily rectified."

$$
* * *
$$

The young and socially respectable Markham was the ideal "victim" in sensationalist journalism. In real-life situations, commentators observed that risk of premature burial was unevenly distributed: gender, class, life-circumstances, region, and religion made a difference. A disproportionate number of victims of premature burial were female. Women of a hysterical temperament, together with men exhibiting a "feminine nervous organization," were believed to be at highest risk. ${ }^{29}$ As one of the leading textbooks on premature burial advised readers, women who "take but little nourishment, and who allow their imaginations to run riot upon every gloomy subject which arrests them" should be watched attentively lest their "nervous system becomes prostrate, and they fall into a state of lethargy" that could be mistaken for death. ${ }^{30}$

Linked to such vulnerabilities were men and women who were emotional or overworked. According to a professor of physiology and psychological science at the United States College in New York, both sexes needed to guard against "any strong emotion, any unusual or protracted intellectual exertion, great physical exhaustion, unsatisfied sexual desire, or excessive indulgence," all of which could debilitate the body and lead to a cataleptic attack. He noted that men should be especially alert to disorders of the digestive system, while women should watch for diseases of the womb. Everyone had to be careful lest they indulged excessively in tobacco, cannabis, and morphine. Other dangerous activities included "overtaxing the brain, excessive bathing, mental excitement, [and] sexual aberrations," as well as "unwholesome postures in bed." 31 As an article in The Embalmers' Monthly in 1908 reasoned:

the persons most subject to one or more of the various forms of death counterfeit are to be found chiefly among those whose daily avocation necessarily exhausts the nervous force faster than the natural powers are able to recuperate it, and who have frequent recourse to stimulants or to narcotics in the hope of counteracting the feeling of depression that usually follows. ${ }^{32}$

Heaven also help residents in areas plagued by epidemics: fears of the rapid spread of infection often led to overly hasty burials, they warned. ${ }^{33}$ Even greater dangers lurked in "tropical countries and places where life is held much cheaper." 34 Poverty was certainly not healthy: hunger, economic stress, and foul air increased the likelihood of a person suffering a terrifying fate, ${ }^{35}$ in part because they reduced a person's ability to purchase the "care and attention given to people in a superior station." ${ }^{36}$ Religion, too, was a risk factor. Jewish law decreeing that the dead be interred within a few hours of death was believed to dramatically increase that community's vulnerability to this "horrible catastrophe." $"$ Anti-Papist sentiments were evident in warnings that the Roman Catholic rite of extreme unction raised "a serious impediment to the use of means for restoring animation." 38

$$
* * *
$$

Why were Victorians and early Edwardians so frightened of being buried alive? These panics were the product of a coincidence of a number of separate anxieties of the time: a fascination with physiological liminality, changing medical ideas of the body, 
insecurities about diagnosing death including who had authority to do so, the inadequacies of death certification, and distrust of medical elites.

First, the Victorians were fascinated with liminal bodily states. New technologies such as anesthesia in the 1840s had raised questions about the relationship between body and soul. The dualistic vitalism of physiological understandings of the time meant that it was impossible to think of life existing without nervous irritability. Insensibility equaled death. ${ }^{39}$ But there was also medical and popular fascination with liminal states such as catalepsy, trance, syncope, and suspended animation. In such states, people were not fully alive, but neither were they dead. Could the dead really be in a trance, as in the case of Esther Holden's scare? ${ }^{40}$ She had been susceptible to periods of insensibility since a child - a tendency that had seen her nearly buried twice before. She was proof that some people were at risk of medical conditions of "pretended death."

Second, changing medical ideas incited anxieties. There were concerns about the discarding of older ways of thinking about the body. For example, humoral medicine, which had dominated Western medical thinking for more than 2,000 years, had been jettisoned by most medical practitioners, but retained a powerful attraction in popular understandings of the body. This was what the Rev. Quirk had been alluding to in Holden's case, when he recommended "bleeding."

The introduction of more "scientific" notions about the cause of disease met with skepticism and distrust amongst lay-persons. Although William Budd and John Snow had proposed a theory of disease based on bacteria as early as 1849, and Louis Pasteur's Germ Theory and Its Applications to Medicine of 1861 maintained that diseases were caused by organisms invisible to the naked eye, many people continued to believe that disease was spread by "miasma" or noxious smells. Rapid urbanization and industrialization had resulted in the presence of over-crowded burial sites located close to urban dwellings. It was feared that miasmatic odors could cause those catatonic states that had been mistaken for death. As late as 1875, public-health promoter William Eassie contended in his book Cremation of the Dead: Its History and Bearings Upon Public Health that the "foetid air exhaled from the dead is fatal if breathed in a concentrated state." Even when "dissipated by the wind," the "miasma which escapes from coffins" was guaranteed to lower "the vital powers of the community." Wasn't the "cadaverous appearance" of gravediggers proof enough of the perilous effects of breathing the air around cemeteries, he asked? $^{41}$

Even public commentators who acknowledged the contagious properties of germs had no difficulty in simultaneously believing in miasmic influences. In 1881, for example, one influential tract on burial customs began by citing Pasteur's germ theory, noting that tiny scales of skin from scarlet-fever patients could infect healthy tissue. Yet, the author still warned readers that corpses gave off "irrespirable" gases that were fatal if breathed in concentrated form. Even if extremely faint, the author noted, the nauseating odor of a decomposing corpse could lead to a "constant depreciation of the final functions," chronic indigestion, and other debilitating illnesses, any of which could mimic death states. $^{42}$

These changing medical ideas coincided with a lack of confidence in the ability of people (including "traditional wise women" as well as male physicians) to accurately diagnose physical death. This is the third explanation for the panic over premature burial. Uncertainties about the signs of death were a perennial concern. In "The Premature 
Burial," Poe had perceptively remarked that the border between life and death was "shadowy and vague." takenly signed a death certificate for a woman who was still alive: "The moisture on the glass ... was no evidence either of life or death. No one agreed that it was a scientific test of death." ${ }^{\text {44 }}$ Even the House of Commons Committee on Death Certification admitted that death certificates were generally "misleading and untrustworthy." 45 The British Medical Fournal in 1916 conceded that death was "a process, not a sudden event, and is indistinguishable at first from a merely temporarily suspension of life."46

These worries coincided with a clash between two competing authorities: traditional "wise women" and professional physicians. This can be illustrated by turning to another case of near-premature burial, which happened four years after Esther Holden's scare. Like Holden, Mary Jane Walker's disturbing experience generated a vast amount of press coverage. Forty-year-old Walker lived with her husband, a house painter, on Cobden Street in Wolverhampton (UK) and had been "ailing for some time." On Sunday evening, 30 May 1909, she suffered a major seizure and died. ${ }^{47}$ The doctor signed the death certificate without seeing the body. Two neighbors - a Mrs. Lawrence and Mrs. Hart - were called in and, after remarking "She's gone!" they began to wash and lay-out the corpse. A visiting nurse, however, noted that Walker's body was hot, so a physician was called. Walker subsequently died, but it was clear that, when she had been measured for her coffin, she had been alive. In the coroner's court, Mrs. Lawrence detailed why she had been confident that Walker was dead. She explained that "the jaw dropped, the teeth became set, and the body gradually cooled." ${ }^{48}$ But she had been wrong. This was proof that even women who had traditionally been involved in rites associated with birth and death were fallible. Their "innate" and intimate knowledge was being challenged by male physicians, who were increasingly in league with a newly emerging profession of funeral specialists.

There was a further problem, however. Professional, male authorities were also unclear about the "signs" of death. Of course, everyone could agree that putrefaction was decisive. Despite the fact that decomposing flesh "offends our senses, shocks our memory, daunts our courage," grieving families joined with campaigners against premature burial to protest against local authorities who were increasingly insisting on the early burial of the dead. ${ }^{49}$ The kin of (suspected) deceased people sought to "hold back the interment for many days," but, they complained, local authorities "compelled" them to bury their loved ones hastily. ${ }^{50}$ This was why, in 1895, the Manchester Courier pleaded that neither inconvenience nor sanitary considerations should be regarded as more important than the need to ensure that the corpse was indeed a corpse. ${ }^{51}$ Coupled with the decline of lengthy "wakes" (which were being criticized as "primitive" and "rural" affairs) and fears that people were living through a period characterized by a great deal of "hustle," was it any wonder that many people feared "modern" approaches to death-rites?"

Other than decomposition, there was no agreement about what was a decisive proof that someone had died. Was muscular rigidity and coldness definitive, as those who initially examined both Holden and Walker believed? What about dilated pupils and the non-transparency of fingers by candlelight? Or the cessation of a heartbeat? The British Medical Association argued that it was "impossible to admit that the heart can remain for even half an hour in a state of absolute inaction in a human being, and then spontaneously recover its activity." 53 But even with the help of the stethoscope (which had been 
invented in 1815 but not routinely used until mid-century), how could a physician be 100 percent sure?

This uncertainty was exacerbated by very public disagreements about the "signs of death" being aired by physicians and the newly professionalizing undertakers and embalmers. Physicians emphasized their profession's long apprenticeship with death and the superiority of their diagnostic instruments. They insisted that death was a physiological event that could be determined objectively. Substituting properly trained, stethoscope-carrying physicians for laymen and women near the deathbed should restore confidence of patients. ${ }^{54}$ But undertakers disagreed. They even accused physicians of arrogance. In a typical rebuttal, Howard S. Eckels informed readers of The American Undertaker that doctors were "ignorant": their "absurd" faith in the value of the stethoscopes and other contraptions in ascertaining whether there was still breath left in the body left medical men vulnerable to wrongly diagnosing death. Eckels insisted that the only definitive way of determining death was by cutting an artery. If such a procedure was "properly described" to grieving relatives, he reported, families would be more confident that undertakers could be trusted with their loved ones. Undertakers like Eckels promised to ensure that "no doubt could possibly remain in the minds of the most prejudiced that there was any possibility of life remaining." 55

For members of societies concerned about premature burial, these debates about the "signs of death" were serious, but they had to be understood alongside a further problem: the lax rules around the certification of death. In both Holden's and Walker's nearburial, their physicians had signed the death certificate without actually examining the body. This was despite the fact that, in Walker's case, the physician only lived 300 yards away. The reason was that both physicians had expected the women to die and so considered it unnecessary to make the terminal diagnosis.

Certification by a physician was not a legal requirement. The Select Committee of 1893 was distressed to discover that in many instances no physician had actually viewed the corpse: they often sent an unqualified assistant to look at the body or simply appended the words "as I am informed" next to the cause of death. ${ }^{56}$ In 1909 , one-quarter of burials in Ireland went uncertified. ${ }^{57}$ As late as the mid-1920s, only 40 percent of people buried in the UK were certified on medical evidence to be actually dead. $^{58}$

This was not due to lack of governmental awareness of the problem. In 1909, for example, a Departmental Committee on the law relating to coroners found that "the present law of death certification affords every opportunity for premature burial." 59 In the mid-1920s when the Births and Deaths Registration Bill was being debated, one of its purposes was to eliminate "any element of risk or opportunity for the concealment of crime," including that of premature burial. ${ }^{60}$

Advocates of compulsory certification had to address some practical problems of implementation. Physicians (particularly those with country practices) were already overworked: it would be a formidable additional burden if they were required to physically examine every patient who died. During a debate on the Births and Deaths Registration Bill in 1926, one physician complained that "In ordinary practice the doctor was busy on his round from the time he had finished his morning surgery until the evening. He had often no time for his meals, which, as a rule, were scratch meals." He admitted that compulsory certification of death "would give greater confidence to the public" but contended that "it was impracticable" given shortages in the number of doctors. ${ }^{61}$ 
Would certification by a trained physician solve the problem, though? The final reason for the panic around premature burial was distrust of doctors. It was no coincidence that prominent people concerned with eradicating the risk of premature burial were anti-vivisectionists such as Frances Power Cobbe and Walter Hadwen. They were suspicious of medical men, believing that they prioritized their own financial, scientific, and professional advancement over that of human and non-human animals. It was self-evident to anti-vivisectionists that professionals accustomed to vivisecting animals would develop a callous attitude towards other vulnerable life forms, including human ones. ${ }^{62}$ Who was to say that medical men would not be equally pitiless with people they assumed to really be corpses?

Of course, active anti-vivisectionists were a minority in Victorian and early-Edwardian society, but their distrust of doctors was widely shared. The scandal involving William Burke and William Hare - who "snatched" the corpses of paupers and even murdered people in order to sell their bodies to anatomists like Robert Knox - was evidence that it was not wholly irrational to be wary of doctors. Although Burke and Hare operated in the 1820s, they were evil legends throughout the century. Mid- to late-nineteenth century protests against compulsory smallpox vaccination (which incited a major movement) was also regarded as proof of the callous regard physicians had for living people. ${ }^{63}$

Medical authorities attempted to counter such attacks. In 1896, for example, Scientific American informed their readers that the evidence relating to premature burial was bogus. ${ }^{64}$ Similarly, the official journal of the British Medical Association adopted a defensive tone, reminding readers that the association's failure to investigate accounts of premature burial had nothing to do with an attempt to preserve "professional reputations." They insisted that stories of live burials were improbable, non-factual, and belonged "to the domain of the novelist rather than of the scientist." ${ }^{~} 5$ The journal reviewed all the major texts on premature burial, rejecting each in turn. They accused Franz Hartmann, for example, of peddling a "farrago of nonsense." They sneered at his claim that some people could live without air or a heartbeat and that "even putrefaction of the body is not a certain sign that life has entirely departed." ${ }^{\prime 66}$ Paul Brouardel's Death and Sudden Death (1897 and 1907) was dismissed as promoting "inventions." 67 William Tebb and Edward Perry Vollum's Premature Burial: How It Can Be Prevented (1896 and 1905) was branded a "pretentious volume." Despite being 400 pages long, they claimed that Tebb and Vollum had only provided one cause of premature burial occurring in England, and that one example had occurred one and a half centuries earlier. The review concluded that "after careful perusal of the work we fail to find anything in it that is worthy of serious criticism." 68 The Association repeatedly insisted that stories of physicians wrongly diagnosing death were nothing more than "old wives' tales," often culled from "dubious American sources."69

Less reassuringly, the journal of the British Medical Association claimed in 1904 that, even if a person was prematurely buried, there would be no struggle since "a person enclosed in a coffin would die almost as quickly and just as surely as one thrown bound hand and foot into the depths of the sea." Such unlikely victims would pass "rapidly into the real sleep of death much as an animal does when placed in a lethal chamber, or a miner caught by choke-damp in a mine." 70 The journal returned to the theme later that year, insisting that if a person in a "state of trance" was mistakenly buried, it was "scarcely conceivable that the victim could awake." After all, they contended, "the unconsciousness of catalepsy would simply deepen until it became fixed in the dreamless sleep of death."71 
Caught up in this panic over premature burial, medical professionals had to respond to their patients' fears. What procedures should death professionals adopt to ensure they were not guilty of damning a person to that fate worse than (real) death? At the very least, the British Medical Association fretted that they needed to regain the confidence of patients and their kin, "without which one can do but little."72

Fool-proof procedures to ascertain death ranged from the banal to the bizarre. ${ }^{73}$ In 1925 , surgeon John Good suggested that waiting a day after death should become the norm. He admitted that

This arrangement would relieve my own feelings as to unpleasant possibilities and help to abolish the increasing dread that calls for extreme measures to establish the proof of death; otherwise a post-mortem examination should become the invariable rule in order to still all medical and social doubts and perplexities - and this I am sure the public will not submit to. ${ }^{74}$

Other suggestions involved the physician injecting ammonia into a supposed corpse, which would cause a "red blush on the skin" if the patient was still alive. ${ }^{75}$ Similarly, an injection of a dye with a yellowish-green fluorescence would turn the skin of a living person jaundice and their eyes would glow an uncanny green "like an emerald set in the orbit." "76 This technique had the advantage of not causing distressful disfiguration of an actual corpse since discoloration would only occur if the corpse was not, in fact, a corpse. $^{77}$

The Victorian craze for electricity made it inevitable that it would be used. In 1873, the British Medical Fournal proposed the use of "electro-bioscopy" or a process "showing that death has occurred by the absence of the usual farado-muscular contractions."78 They noted that in cases of lethargy, apoplexy, syncope, asphyxiation, or poisoning, electro-muscular contractions continued. Electro-bioscopy could therefore prevent premature burial. When there were a large number of casualties (as in railway accidents, epidemics, shipwrecks, or military battles), electro-bioscopy would "have the additional advantage of enabling the attendants to distinguish at once the dead from the living" and therefore allow them to triage patients in order to give attention only to those "who could still benefit by it."79 The British Medical Fournal maintained that the effectiveness of faradization had been proven by Professor Rosenthal of Vienna who happened to be present after the apparent death of a hysterical woman. Her death had been certified by "a country practitioner," who held a mirror to her mouth to observe whether moisture formed, as well as dropping "melted sealing-wax" on her skin (there was no sign of any "reflex movements"). Rosenthal was almost convinced that she was dead as well: after all, the woman's skin was "pale and cold, the pupils contracted and insensible to light, the upper and lower extremities relaxed, the heart's impulse and the radial pulse imperceptible." However, he found that her facial muscles and limbs "responded well to the faradic current." Consequently, Rosenthal diagnosed the woman as being in a trance and told her relatives that they should continue attempting to resuscitate her. He bragged that the following morning he "received a telegram, saying that the woman awoke spontaneously twelve hours after his visit, and gradually recovered her speech and movements." 80

Some of the proposed techniques were effective only because if the patient was still alive, the procedure would certainly kill them. Injecting strychnine into the "supposed 
corpse" would certainly guarantee death, if not before the injection then after it. ${ }^{81}$ The same could be said about cutting the radial artery or removing the person's head prior to burial. ${ }^{82}$ Sinking a steel needle through the "corpse's" heart was also widely promoted, "even at the risk of killing a few entranced subjects," observed one author in $1895 .{ }^{83}$ Dr. Séverin Icard, a physician at the Grande-Miséricorde children's hospital, was a fervent believer in the "flag" test: that is, a steel needle containing a small flag would be thrust into the patient's chest; if the flag waved, the heart was still beating. Unfortunately, he was accused by one family of killing their relative by using this test. Considerable pain could be caused to the "corpse" if she or he was not already dead. Burning the skin of the (alleged) deceased and watching to see if a blister formed was relative benign compared to "plunging" a "bright steel blade ... deep into the tissues" to observe whether or not oxidization occurred. ${ }^{84}$ And what if the "supposed corpse revive[d] under the treatment," fretted the British Medical Association? Would "an action for damages lie against the doctor for the stabs and burns inflicted by him?" 85

Like other forms of preventing premature burial by killing any living person, cremation was another solution. This was advanced by Henry Thompson, influential surgeon and founder of the Cremation Society of Great Britain. In 1874, he contended that it was far worse to wake up in a grave and suffocate slowly than to wake up while being cremated. "The completeness of a properly-conducted process would render death instantaneous and painless," he sagely observed, if by an "unhappy chance" a "corpse woke up in the oven." 86 Not only did cremation promise an instantaneous death in those cases where a person wasn't quite dead yet, it also ensured that people would not find themselves "buried and then living a few hours more in the grave, only to die there the most horrible of deaths known to man." 87 There was no chance of "suffering any agony whatever." 88 This was why Molly Weir recalled that "stories of people being buried alive so tormented me that I made everybody promise ... that when I died they would see to it, 'cross their hearts and hope to die', that I was well and truly cremated." ${ }^{89}$ Admittedly, its proponents insisted, "to cremate a man alive is a rather serious responsibility," but It was the best option. ${ }^{90}$

In both Britain and America at the end of the nineteenth century, Associations for the Prevention of Premature Burial also offered specific proposal to help abolish the risk. Given that they were supported by many political and social radicals - including George Greenwood (MP and animal rights advocate), Emelie Louise Lind-af-Hageby (founder of the Animal Defence and Anti-Vivisection Society), Dr. Josiah Oldfield (co-founder, with Mahatma Gandhi, of the Fruitarian Society), Dr. Anna Kingston (involved with theosophy, vegetarianism, and anti-vivisection movements), and Dr. Stenson Hooker (naturopath) - it was not surprising that they proved willing to make dramatic proposals. One of their chief aims was to improve processes by which deaths were certified. ${ }^{91}$ it was no longer acceptable for physicians to accept the statements of "quacks" or "ignorant old women" that death had taken place. ${ }^{92}$ Their members carried out experiments into suspended animation and other bodily states that feigned death. ${ }^{93}$ Since the only fail-safe way of ascertaining death was putrefaction, these associations recommended the establishment of "waiting mortuaries" in every district in the United Kingdom. These mortuaries would be 
furnished with every appliance of resuscitation, watched by qualified attendants, and in telephone communication with a medical superintendent, who shall be authorised to grant removal of the body to the cemetery only when the fact of death has been unequivocally established by the sign of decomposition. ${ }^{94}$

They lobbied medical schools to provide training on trance states, coma, and other "death counterfeits." 95

Of course, given the high levels of distrust expressed by some patients, it is little wonder that some preferred to take their own precautions. In 1904, the British Medical Association were dismayed to report that "many otherwise strong-minded persons have lived under the shadow of a great fear" of premature burial, and so have "in their wills directed payments to be made to physicians who should be willing to run the risk of homicide to prevent live burial." "96 Even as late as 1926, physicians "often saw in wills that large numbers of people thought it necessary to provide for surgical operations to be made on their reputed corpses to ensure their death."97

Who were these people? One was simply described as a distinguished literary woman who told the authors of a book on premature burial that taking things into her own hands had brought her relief. "It is a terrible subject," she informed them,

and one which has haunted me all my life insomuch as I have never made a will without inserting a clause requiring my throat to be cut before I am put underground. Of course one can have no reliance on doctors whatever, and I have myself known a case in which a very eminent [physician] insisted on a coffin being screwed down because the corpse looked so life-like and full of colour that the friends could not help indulging in hopes. ${ }^{98}$

Similarly, in 1904, anti-vivisectionists and feminist Cobbe left a note by her bedside instructing the doctor who declared her dead (fellow anti-vivisectionist Hadwen) to "perform on my body the operation of completely and thoroughly severing the arteries of the neck \& windpipe (nearly severing the head altogether) so as to render my revival in the grave absolutely impossible." 99 Lady Isabel Burton, widow of the explorer, asked for a needle to be passed through her heart. ${ }^{100}$

Self-help extended to the design of coffins. Political theorist and biologist Herbert Spencer specified that his corpse "be placed in a coffin with a loose lid easily opened from below." 101 Others were reassured by devices for observing corpses through glass walls or with coffins fitted with pipes running to the surface. Coffins with mechanical and electric methods of summoning help were also invented. For instance, in 1868, Franz Vester of Newark (New Jersey) was granted a patent for his design of a coffin complete with a tube which contained a bell and a ladder, enabling the entombed individual to climb to safety or summon help. Just three years later, Dr. Theodore A. Schroeder and Hermann Wuest of Hoboken (also of New Jersey) used a battery to create a circuit within the coffin. If the "corpse" moved, the circuit would break, causing a bell to ring and releasing fresh air into the tomb. Eleven years later, an Indianapolis man - who took the appropriate name Albert Fearnaught - proposed placing a spring in the hand of the "corpse." Any movement would cause a pin to be released, forcing a signal out of a tube which led to the surface. However, as one commentator who studied these patents concluded, the mechanisms were so complicated that there was a high chance of failure. 
Patented signals would be of little use in the large, "modern" cemeteries, with much landscaping, uneven terrain, and only the occasional patrol. Obviously, too, most patented coffins would be effective only in vault entombment, an infrequent method of burial and one that favored only the wealthiest corpses. ${ }^{102}$

$$
* * *
$$

In conclusion, fears of premature burial fell into abeyance after the 1910s. Ironically, these fears declined during the First World War, at precisely the time when live burial became all-too prevalent. In the trenches of Flanders, thousands of men were smothered to death under tons of soil during artillery bombardments. ${ }^{103}$ However, the medical profession was largely successful in debunking sensationalist accounts of this horrific fate. Movements of the coffin as it was lowered into the ground, natural decomposition and putrification, and the appetites of rodents were commonsensical explanations for what people saw when graves were opened. The prestige of medical professionals became increasingly unassailable. Even activists against vivisection and vaccines gradually gave up the fight. By the mid-twentieth century, identifying death through observing failures of the circulatory, respiratory, and nervous systems gradually became archaic, culminating in the identification of "brain death" at the end of the 1950s.

Nevertheless, the fear of premature burial did not disappear entirely. There are occasional flurries of anxiety around premature burial, often incited (as in the past) by tabloid journalists who delight in regaling their readers with stories of patients in persistence vegetative states spontaneous reviving. ${ }^{104}$ The diagnosis of "brain death," too, brought its own risks, especially when coupled with medical technologies associated with transplant surgery. "Beating-heart cadavers" revived debates about the definition of death, along with fears about the power over life and death wielded by physicians and scientists. In the counterculture of the 1960s, physicians were once again accused of treating their patients' bodies as little more than "containers of biologically useful material." The commercial demand for human organs, driven by the success of transplant surgery, caused concerns about whether the "brain death" definition was paving the way to killing patients before their time. ${ }^{105}$ This return of premature burial panics was raced: AfricanAmerican communities were believed to be at greatest risk. An editorial in Ebony in March 1968 openly referred to alarm that white doctors were experimenting on black patients. Organ transplantation was deeply unpopular amongst many black Americans because they feared that any such "gift" from a black American would encourage physicians to "hurry the death in order to complete a transplant," especially if the recipient was white. ${ }^{106}$

The panic about meeting this horrific fate also remained a potent image in popular culture. Poe's short story "The Premature Burial" remains a classic and was made into a film in 1962, directed by Roger Corman and co-directed by Francis Ford Coppola, of Godfather fame. Commercial psychological texts also continued to play on the fear of being prematurely laid to rest: examples include William S. Walsh's The Mastery of Fear (1924), Thomas J. Bonniwell's We Have to Die (1940), and Alfred Worcester's The Care of the Aged, the Dying, and the Dead (1935). ${ }^{107}$ Today, "taphophobia" is a recognized phenomenon although, as its nomenclature suggests, as a psychiatric illness. ${ }^{108}$

In Victorian and early-Edwardian Britain and America, however, fears about premature burial were an important part of many people's imaginary and social lives. As such, they shine a light on cultures of dying and death, as well as public anxieties about 
medical professionalization, authority over the dead, and rapidly shifting ideas about the body. Although this period was characterized by secularization, this was not a dominant theme in the debates. Instead, the discussions revolved around questions of the uncertainties of death for the living. What were its "signs"? Who had the authority to proclaim a person to be dead or not? In answering these questions, gender and class were central. There was a tension between the fictional representations of premature burial (often involving a well-off young woman like Miss Eleanor Markham) and its potential reality (poor women like Esther Holden, who had recently given birth, and Mary Jane Walker). The public narrative of premature burial was, therefore, one that struck at the heart of ideas about the "good death."

\section{Notes}

1 "The Accrington Escape," Birmingham Mail (21 January 1905), 3. The case was reported in dozens of newspapers. It was not unusual for a case of premature burial to gather intense interest. A similar case occurred in 1909 when Wolverhampton woman Mary Jane Walker was nearly buried alive. This case was reported in numerous newspapers, including "Strange Inquest Incident," Hull Daily Mail (7 June 1909), 6; "Strange Story at an Inquest," Mid Sussex Times (8 June 1909), 2; "Strange Story at an Inquest," Globe (3 June 1909), 10; "Jury Dispose of a Sensationalist Story," The Cornish Telegraph (10 June 1909), 3; "Town and Country Gossip," Derby Daily Telegraph (10 June 1909), 2; "Remarkable Story at an Inquest," Northern Daily Telegraph (4 June 1909), 3; "'Dead' Woman who Moved," Derry Fournal (9 June 1909), 5; "Deathbed Mystery. Jury Dispose of a Sensational Story," Gloucestershire Echo (4 June 1909), 1; "Strange Story of an Inquest," Rugby Advertiser (8 June 1909), 2; "The Test of Death," Yorkshire Post and Leeds Intelligencer (4 June 1909), 7; "Strange Story of an Inquest," Berks and Oxon Advertiser (11 June 1909), 3; "Deathbed Mystery," London Daily Newes (4 June 1909), 5.

2 "Dead Woman Lives," Manchester Courier and Adviser (21 January 1905), 17.

3 "Dead Woman Lives," Manchester Courier and Adviser (21 January 1905), 17.

4 "The Accrington Sensation," Exeter and Plymouth Gazette (18 January 1905), 2.

5 “Thrice Laid Out," Gloucestershire Chronicle (21 January 1905), 7.

6 "Dead Woman Lives," Manchester Courier and Adviser (21 January 1905), 17.

7 "The Accrington Sensation," Nottingham Evening Post (20 January 1905) and "A Sensation. Interview with the Husband," Lancashire Evening Post (19 January 1905), 3. Elsewhere the Inn was called Griffin's Head Hotel.

8 "The Accrington Sensation," Nottingham Evening Post (20 January 1905) and "A Sensation. Interview with the Husband," Lancashire Evening Post (19 January 1905), 3.

9 "Dead Woman Lives," Manchester Courier and Adviser (21 January 1905), 17.

10 Grantham Fournal (21 January 1905), 7.

11 Letter from the Rev. J. F. Quirk, "Premature Burial," London Evening Standard (20 January 1905). It was republished in the Nottingham Evening Post (20 January 1905), 4.

12 "The Accrington Escape," Birmingham Mail (21 January 1905), 3.

13 G. Stanley Hall, "A Study of Fears," The American Fournal of Psychology, 8.2 (January 1897), $152-53$.

14 "Medical Notes in Parliament," British Medical fournal, 1.3401 (6 March 1926), 450.

15 The Uncertainty of the Signs of Death, And Danger of Precipitate Interments and Dissections, Demonstrated (Dublin: George Faulkner, 1748).

16 For further discussion, see Jan Bondeson, Buried Alive. The Terrifying History of Our Most Primal Fear (London: W. W. Norton and Co., 2001) and Rodney Davies, The Lazarus Syndrome. Buried Alive and Other Horrors of the Undead (London: Robert Hale, 1999).

17 Cited in William Tebb and Colonel Edward Perry Vollum, Premature Burial and How It May be Prevented with Special Reference to Trance, Catalepsy, and Other Forms of Suspended Animation, 2nd edition, first published in 1896 (London: Swan Sonnenschein and Co., 1905), 26. 
18 Dr. Alexander Wilder, Perils of Premature Burial. A Public Address Delivered Before the Members of the Legislature, at the Capitol, Albany, New York, Fanuary 25th, 1871 (London: E. W. Allen, 1895), 10.

19 "Premature Burial," The Embalmers' Monthly, 21 (1908), 72. Estimate by Dr. John Dixwell, of Harvard University, in 1908.

20 Dr. Stenson Hooker and the Late Colonel E. P. Vollum, Premature Burial and Its Prevention, 2nd edition (Dublin: Hodges, Figgis, and Co, 1911), 3.

21 Max Levy, Why Modern Cremation Should Replace Earth-Burial (San Francisco, CA: Bacon and Co., 1885), 39.

22 Matthew Gregory Lewis, The Monk: A Romance (London: J. Bell, 1796). It was republished many times, including in 1797, 1798, 1800, 1807, 1808, 1818, 1822, 1826, 1893, 1873, 1906, and 2012.

23 Edgar Allan Poe, "The Premature Burial," in Poe, The Premature Burial and Other Tales of Horror (London: Corgi, 1966), 15.

24 Molly Weir, Best Foot Forward (London: Hutchinson and Co., 1979), 71.

25 Dr. Alexander Wilder, Perils of Premature Burial. A Public Address Delivered Before the Members of the Legislature, at the Capitol, Albany, New York, January 25th, 1871 (London: E. W. Allen, 1895), 17.

26 Ziemssen's Cyclopedia of the Practice of Medicine, vol. xix, 451-2, cited in Augustus G. Cobb, EarthBurial and Cremation. The History of Earth-Burial with Its Attendant Evils, and the Advantages Offered by Cremation (New York: G. P. Putnum's Sons, 1892), 93.

27 The Spectator, 14 September 1895, quoted in Dr. Alexander Wilder, Perils of Premature Burial. A Public Address Delivered before the Members of the Legislature, at the Capitol, Albany, New York, Fanuary 25th, 1871 (London: E. W. Allen, 1895), 24.

28 David Walsh, Premature Burial: Fact or Fiction? (London: Bailliere and Co., 1897), 18-19.

29 Dr. Henry M. Lyman, Insomnia; and Other Disorders of Sleep (Chicago, IL: W. T. Keener, 1885), 35.

30 William Tebb and Colonel Edward Perry Vollum, Premature Burial and How it May be Prevented with Special Reference to Trance, Catalepsy, and Other Forms of Suspended Animation, 2nd edition, first published in 1896 (London: Swan Sonnenschein and Co., 1905), 149.

31 Dr. Alexander Wilder, Perils of Premature Burial. A Public Address Delivered before the Members of the Legislature, at the Capitol, Albany, New York, January 25th, 1871 (London: E. W. Allen, 1895), 19-20. Also see Moore Russell Fletcher, One Thousand Persons Buried Alive by Their Best Friends. A Treatise on Suspended Animation with Directions for Restoration (Boston, MA: The Author, 1883), 5.

32 "Premature Burial," The Embalmers' Monthly, 21 (1908), 14.

33 "A Sign of Death," British Medical Journal, 2.2296 (31 December 1904), 1767.

34 "A Sign of Death," British Medical Journal, 1.2300 (28 January 1905), 214.

35 Edward P. Vollum, "Tests for Death," The American Undertaker, 2.10 (October 1901), 37 and "Premature Burial," The Embalmers' Monthly, 21 (1908), 14.

36 Sussex Daily News, 23 September 1895, quoted in Dr. Alexander Wilder, Perils of Premature Burial. A Public Address Delivered Before the Members of the Legislature, at the Capitol, Albany, New York, Fanuary 25th, 1871 (London: E. W. Allen, 1895), 26.

37 The Fewish World, 13 September 1895, cited in Dr. Alexander Wilder, Perils of Premature Burial. A Public Address Delivered before the Members of the Legislature, at the Capitol, Albany, New York, fanuary 25th, 1871 (London: E. W. Allen, 1895), 23.

38 William A. Guy and David Ferrier, Principles of Forensic Medicine, 6th edition revised (London: H. Renshaw, 1888), 214 and Professor D. Ferrier, "Death," in Quain's Dictionary of Medicine, cited in Dr. Alexander Wilder, Perils of Premature Burial. A Public Address Delivered before the Members of the Legislature, at the Capitol, Albany, New York, January 25th, 1871 (London: E. W. Allen, 1895), 7.

39 This is argued by Stephanie J. Snow, Operations without Pain: The Practice and Science of Anaesthesia in Victorian Britain (Basingstoke: Palgrave Macmillan, 2006).

40 Dr. F. William Cock, "Death Certification," British Medical Journal, 2.3369 (25 July 1925), 168.

41 William Eassie, Cremation of the Dead: Its History and Bearings Upon Public Health (London: The Author, 1875), 53.

42 Henry E. Spencer, Burial and Cremation: A Lecture (London: Hamilton, Adams, and Co., 1881), 6-8. 
43 Edgar Allan Poe, "The Premature Burial," in Poe, The Premature Burial and Other Tales of Horror (London: Corgi, 1966), 9. Also see William Tebb and Colonel Edward Perry Vollum, Premature Burial and How It May be Prevented with Special Reference to Trance, Catalepsy, and Other Forms of Suspended Animation, 2nd edition, first published in 1896 (London: Swan Sonnenschein and Co., 1905), 11.

44 "Weird Story of Corpse that Moved. Death-Bed Drama," Sheffeld Evening Telegraph (4 June 1909), 3.

45 Cited in William Tebb and Colonel Edward Perry Vollum, Premature Burial and How it May be Prevented with Special Reference to Trance, Catalepsy, and Other Forms of Suspended Animation, 2nd edition, first published in 1896 (London: Swan Sonnenschein and Co., 1905), 8. Also see "Death Certification," The Medical Times and Hospital Gazette, 31 (8 April 1903), 245 and Rev. J. Ingham Brooke, A Lecture on Burial Reform (London: J. Masters and Co., 1879), 3.

46 Citing Professor Baculo of Naples in "Apparent Death and Premature Burial in Infants," British Medical Fournal, 1.2889 (13 May 1916), 698.

47 "Weird Story of Corpse that Moved. Death-Bed Drama," Sheffield Evening Telegraph (4 June 1909), 3.

48 "Weird Story of Corpse that Moved. Death-Bed Drama," Sheffield Evening Telegraph (4 June 1909), 3.

49 Maeterlinck quoted in George Alfred Noble, Cremation: Its History and Practice (London: np, 1914), 14. Also see J. Brindley James, "Death Certification and Death Verification," British Medical Fournal, 2.2487 (29 August 1908), 567.

50 Dr. Alexander Wilder, Perils of Premature Burial. A Public Address Delivered before the Members of the Legislature, at the Capitol, Albany, New York, January 25th, 1871 (London: E. W. Allen, 1895), 17.

51 Manchester Courier, 24 September 1895, cited in Dr. Alexander Wilder, Perils of Premature Burial. A Public Address Delivered before the Members of the Legislature, at the Capitol, Albany, New York, January 25th, 1871 (London: E. W. Allen, 1895), 26.

52 John Good, "Death Certification," British Medical Journal, 2.3369 (25 July 1925), 168.

53 "The Reality of Apparent Death," British Medical Journal, 1.1799 (22 June 1895), 140.

54 J. Brindley James, "Death Certification and Death Verification," British Medical Fournal, 2.2487 (29 August 1908), 568. Also see Dr. William S. Walsh, The Mastery of Fear (New York: Dutton, 1924), 208-9.

55 H. S. Eckels, "The Danger of Burial Alive," The American Undertaker, v.2 (March 1904), 6-7.

56 J. Brindley James, "Death Certification and Death Verification," British Medical Fournal, 2.2487 (29 August 1908), 568.

57 Dr Stenson Hooker and the Late Colonel E. P. Vollum, Premature Burial and Its Prevention, 2nd edition (Dublin: Hodges, Figgis, and Co, 1911), 6.

58 "Medical Notes in Parliament," British Medical Journal, 2.3439 (4 December 1926), 1084.

59 "Medical Notes in Parliament," British Medical Fournal, 1.3401 (6 March 1926), 450.

60 "Medical Notes in Parliament," British Medical Fournal, 1.3401 (6 March 1926), 450.

61 "Medical Notes in Parliament," British Medical Journal, 2.3439 (4 December 1926), 1084. Dr. Freemantle was speaking.

62 "M. D.," The Scientist at the Bedside (London: Victoria Street Society for the Protection of Animals, c. 1882), 2-3. For an analysis, see my book What It Means to be Human (London: Virago, 2014). Also see Brayfytte, "Experimental Physiology," The Animals Guardian, 2.3 (December 1891), 39; The Vivisector (London: Middlesex Printing Works, nd); Richard BarlowKennett, Address to the Working Classes (London: Victoria Street Society for the Protection of Animals from Vivisection, n.d.), 1; Henry S. Salt, "The Rights of Animals," International fournal of Ethics, 10.2 (January 1900), 219-20; Henry J. Bigelow, A Great Surgeon on Vivisection (London: National Anti-Vivisection Society, n.d.), 2.

63 For example, see Nadja Durbach, "They Might as Well Brand Us': Working-Class Resistance to Compulsory Vaccination in Victorian England," Social History of Medicine, 10 (April 2000), 45-62.

64 For example, see J. F. Baldwin, "Premature Burial," Scientific American, 75.17 (24 October 1896), 315.

65 "The Reality of Apparent Death," British Medical Journal, 1.1799 (22 June 1895), 140. Also see "Alleged Premature Burial in Paris," British Medical Fournal, 1.1881 (16 January 1897), 160. 
66 "Premature Burial by Franz Hartmann," British Medical Fournal, 1.1835 (29 February 1896), 540.

67 "Death and Sudden Death by Dr. P. Brouardel," British Medical Fournal, 2.1911 (14 August 1897), 405.

68 "Premature Burial by William Tebb and Edward Perry Vollum," British Medical fournal, 1.1880 (9 January 1897), 87. Also see "Premature Burial: Fact or Fiction by David Walsh," British Medical Fournal, 1.1943 (26 March 1898), 828.

69 "Premature Burial," British Medical Journal, 1.2250 (12 February 1904), 386.

70 "Premature Burial," British Medical Fournal, 1.2250 (12 February 1904), 386.

71 "A Sign of Death," British Medical Fournal, 2.2296 (31 December 1904), 1767. Also see "The Fear of Premature Burial," British Medical Fournal, 1.2675 (6 April 1912), 801.

72 The editors, citing Dr. F. William Cock, "Death Certification," British Medical Fournal, 2.3369 (25 July 1925), 168.

73 The editors, citing Dr. F. William Cock, "Death Certification," British Medical Journal, 2.3369 (25 July 1925), 168.

74 John Good, "Death Certification," British Medical Fournal, 2.3369 (25 July 1925), 168.

75 J. Brindley James, "Death Certification and Death Verification," British Medical Fournal, 2.2487 (29 August 1908), 567.

76 "A Sign of Death," British Medical Fournal, 2.2296 (31 December 1904), 1767. Also see "A Sign of Death," British Medical Fournal, 1.2300 (28 January 1905), 214-15 and "A New Death Test," The Embalmers' Monthly, 18 (1905), 163. Dr. Séverin Icard was the author of La mort réelle et la mort apparente. For a review of his work, see "Le signe de la morte réelle en l'absence du médecin by Docteur Icard," British Medical Journal, 2.2545 (9 October 1909), 1074.

77 "A Sign of Death," British Medical Fournal, 2.2296 (31 December 1904), 1767.

78 "Report on Modern Medical Electric and Galvanic Instruments, and Recent Improvements in their Application: With Special Regard to the Requirements of the Medical Practitioner," British Medical Journal, 2.673 (22 November 1873), 614.

79 "Report on Modern Medical Electric and Galvanic Instruments, and Recent Improvements in their Application: With Special Regard to the Requirements of the Medical Practitioner," British Medical Fournal, 2.673 (22 November 1873), 615.

80 "Report on Modern Medical Electric and Galvanic Instruments, and Recent Improvements in their Application: With Special Regard to the Requirements of the Medical Practitioner," British Medical Journal, 2.673 (22 November 1873), 615.

81 E. E. Carpmael (of the Medical Department, Berkeley University), in the Morning Post, cited in Dr. Alexander Wilder, Perils of Premature Burial. A Public Address Delivered Before the Members of the Legislature, at the Capitol, Albany, New York, January 25th, 1871 (London: E. W. Allen, 1895), 14.

82 The editors citing Dr. F. William Cock, "Death Certification," British Medical Fournal, 2.3369 (25 July 1925), 168; Moore Russell Fletcher, One Thousand Persons Buried Alive by Their Best Friends. A Treatise on Suspended Animation with Directions for Restoration (Boston, MA: The Author, 1883), 63; Surrey Comet, 25 October 1924, in the National Archives (UK) MH74/30; the editors citing Dr. F. William Cock, "Death Certification," British Medical Fournal, 2.3369 (25 July 1925), 168.

83 The Spectator, 14 September 1895, quoted in Dr. Alexander Wilder, Perils of Premature Burial. A Public Address Delivered Before the Members of the Legislature, at the Capitol, Albany, New York, Fanuary 25th, 1871 (London: E. W. Allen, 1895), 24.

84 "Guard Against Premature Burial," Scientific American, 4.44 (21 July 1849), 349 and J. Brindley James, "Death Certification and Death Verification," British Medical Fournal, 2.2487 (29 August 1908), 567.

85 "The Prevention of Premature Burial," British Medical Fournal, 1.1949 (7 May 1898), 1218.

86 Henry Thompson, "The Treatment of the Body after Death," The Contemporary Review, 23 (December 1873), 326.

87 Augustus G. Cobb, Earth-Burial and Cremation. The History of Earth-Burial with Its Attendant Evils, and the Advantages Offered by Cremation (New York: G. P. Putnum's Sons, 1892), 93, and Ronald Sutherland Gower, Cleanliness Versus Corruption (London: Longmans Green and Co., 1910), 3. Also see Max Levy, Why Modern Cremation Should Replace Earth-Burial (San Francisco, CA: Bacon and Co., 1885), 39-40, and Dr. Alexander Wilder, Perils of Premature Burial. A Public 
Address Delivered before the Members of the Legislature, at the Capitol, Albany, New York, Fanuary 25th, 1871 (London: E. W. Allen, 1895), 16.

88 Max Levy, Why Modern Cremation Should Replace Earth-Burial (San Francisco, CA: Bacon and Co., 1885), 40.

89 Molly Weir, Best Foot Forward (London: Hutchinson and Co. 1979), 71.

90 Sussex Daily Newes, 23 September 1895, quoted in Dr. Alexander Wilder, Perils of Premature Burial. A Public Address Delivered Before the Members of the Legislature, at the Capitol, Albany, New York, Fanuary 25th, 1871 (London: E. W. Allen, 1895), 26.

91 "The Certification of Death," British Medical Fournal, 2.1876 (12 December 1896), 1726; Dr. Stenson Hooker and the Late Colonel E. P. Vollum, Premature Burial and Its Prevention, 2nd edition (Dublin: Hodges, Figgis, and Co, 1911), 3; "Premature Burials," The American Undertaker, 1.8 (August 1900), 14; "Buried Alive," Catholic World, 1866, 808; William A. Guy and David Ferrier, Principles of Forensic Medicine, 6th edition revised (London: H. Renshaw, 1888), 213; Moore Russell Fletcher, One Thousand Persons Buried Alive by Their Best Friends. A Treatise on Suspended Animation with Directions for Restoration (Boston, MA: The Author, 1883), 62; William Tebb and Colonel Edward Perry Vollum, Premature Burial and How it May be Prevented with Special Reference to Trance, Catalepsy, and Other Forms of Suspended Animation, 2nd edition, first published in 1896 (London: Swan Sonnenschein and Co., 1905), 149; Rev. J. Ingham Brooke, A Lecture on Burial Reform (London: J. Masters and Co., 1879), 3; "Death Certification," The Medical Times and Hospital Gazette, 31 (8 April 1903), 245; Edward P. Vollum, "Tests for Death," The American Undertaker, 2.10 (October 1901), 38-9.

92 The Hospital, 21 September 1895, cited in Dr. Alexander Wilder, Perils of Premature Burial. A Public Address Delivered Before the Members of the Legislature, at the Capitol, Albany, New York, Fanuary 25th, 1871 (London: E. W. Allen, 1895), 24-5.

93 See Dr. Stenson Hooker and the Late Colonel E. P. Vollum, Premature Burial and Its Prevention, 2nd edition (Dublin: Hodges, Figgis, and Co, 1911), 3.

94 William Tebb and Colonel Edward Perry Vollum, Premature Burial and How It May be Prevented with Special Reference to Trance, Catalepsy, and Other Forms of Suspended Animation, 2nd edition, first published in 1896 (London: Swan Sonnenschein and Co., 1905), 369-70. Also see "A Sign of Death," British Medical Fournal, 1.2300 (28 January 1905), 215.

95 "A Sign of Death," British Medical Fournal 1.2300 (28 January 1905), 215; Edward P. Vollum, "Tests for Death," The American Undertaker, 2.10 (October 1901), 38-9; William Tebb and Colonel Edward Perry Vollum, Premature Burial and How it May be Prevented with Special Reference to Trance, Catalepsy, and Other Forms of Suspended Animation, 2nd edition, first published in 1896 (London: Swan Sonnenschein and Co., 1905), 369.

96 “A Sign of Death," British Medical Fournal, 2.2296 (31 December 1904), 1767.

97 "Medical Notes in Parliament," British Medical Fournal, 1.3401 (6 March 1926), 450.

98 William Tebb and Colonel Edward Perry Vollum, Premature Burial and How it May be Prevented with Special Reference to Trance, Catalepsy, and Other Forms of Suspended Animation (London: Swan Sonnenschein and Co., 1896), 96.

99 Cited in Lori Williamson, Power and Protest. Frances Power Cobbe and Victorian Society (London: Rivers Oram Press, 2005), 210). Also see Beatrice E. Kidd and M. Edith Richards, Hayden of Gloucester. Man, Medicine, Martyr (London: John Murray, 1933), 146, and "Cremation," British Medical Fournal, 1.2737 (14 June 1913), 1285.

100 Mary S. Lovell, A Rage to Live: A Biography of Richard and Isabel Burton (New York: Little, Brown, 1998), 788.

101 Gited in George K. Behlmer, "Grave Doubts: Victorian Medicine, Moral Panic, and the Signs of Death," Fournal of British Studies, 42.2 (April 2003), 222.

102 All from Howard Dittrick, "Devices to Prevent Premature Burial," Fournal of the History of Medicine and Allied Sciences, 3 (1948), 161-71.

103 For examples from each of the two world wars, see Captain Ralph Smith, diary entry for 15 September 1916, in Liddell Hart Centre for Military Archives and Felix Brown, "Civilian Psychiatric Air-Raid Casualties," The Lancet (31 May 1941), 688.

104 See Rodney Davies, The Lazarus Syndrome. Buried Alive and Other Horrors of the Undead (London: Robert Hale, 1999), 108-36, and Jan Bondeson, Buried Alive. The Terrifying History of Our Most Primal Fear (London: W. W. Norton and Co., 2001). 
105 Michael Potts, Paul A. Byrne, and Richard G. Nilges, "Introduction: Beyond Brain Death," in their (eds.), Beyond Brain Death. The Case against Brain Based Criteria for Human Death (Dordrecht: Kluwer Academic, 2000), 2.

106 Editorial in Ebony, 23 (March 1968), 118.

107 Thomas J. Bonniwell, We Have to Die, 2nd printing (New York: Worthington Press, 1940), 169; William S. Walsh, The Mastery of Fear (New York: Dutton, 1924), 208-9; Alfred Worcester, The Care of the Aged, the Dying, and the Dead (London: Baillière and Co., 1935), 42.

108 For example, see Munaza Bajwa, "Prevalence and Factors Associated with Phobias among Women," Asean Fournal of Psychiatry, 15.2 (2014), 140-145, and S. Fanous and S. T. O'Keeffe, "Not Yet Dead and Buried! Three Cases of Taphophobia in Older People," European Geriatric Medicine, 6.3 (June 2015), 274-275; Oliver Turnbull and Maggie Zellner, "International Neuropsychoanalysis Society. Congress Research Presentations, Seattle 2010 and Berlin 2011," Neuropsychoanalysis, 13.2 (2011), 235-36. The psychiatric term "taphophobia" was invented much earlier: see the Italian psychiatrist Enrico Morsell in Sulla dismorfofobia e sulla tafefobia (Geneva: Medica, 1891). 


\title{
MURDERING MOTHERS AND DUTIFUL DAUGHTERS
}

\author{
Infanticide in eighteenth- and nineteenth-century \\ Mexico
}

\author{
Nora E. Jaffary
}

Few figures provoke the moral ire of our age more vigorously than does a mother suspected of killing her newborn. To take one contemporary example, in September 2019 an Ohio court convicted an eighteen-year-old cheerleader, Brooke Skylar Richardson, with gross abuse of her newborn's corpse. Richardson had confessed to burying her newborn's body in a shallow grave in her backyard immediately after miscarrying. ${ }^{1}$ Most of the reader comments on news sites and Twitter feeds covering Richardson's case were preoccupied with debates about the racial bias that many commentators argued explained her acquittal for infanticide. However, a second chain of comments denounced Richardson for violating codes of motherly conduct. Some saw her as cold-blooded and ruthless, a woman devoid of maternal emotion; others described her as a flippant debutante who valued appearing trimly in her prom dress over the health (or life) of the fetus she carried. "[W] hat a monster[;] she should get the death penalty," reads one Twitter comment. Another newsreader observed, "She took a gym selfie flaunting her flat belly just hours after smashing her newborn baby's skull. She is a self-absorbed girl without a soul who showed brutal callousness and indifference during and after the crime."2

Such characterizations of the soulless monstrosity or incomprehensible frivolity of murdering mothers surface occasionally in descriptions of alleged murdering mothers in a dramatically different historical context. Eighteen year-old Petra Sevilla was working as a wet nurse on a hacienda (large estate) outside the city of Puebla in south-central Mexico in 1835 when she was investigated for having killed her newborn moments after his birth. An anonymous writer denounced Sevilla to municipal juez de letras (judge) licenciate Miguel Tagle, recounting that bricklayers who had been repairing the comunes (open toilets) on a hacienda outside of the city had discovered the newborn's corpse. Subsequent testimony revealed Sevilla had given birth, either to a live or stillborn fetus, and thrown its body into the comunes. In arguing for Sevilla's conviction, a prosecutor in Puebla's state Supreme Court asserted that, "only a monster could take the life of someone to whom she had given life." In the 1810 infanticide trial of a plebeian indigenous woman, Angela Hernández, from the small town of San Agustin Tlautepeque in the state of Oaxaca, Mexico, the defendant told her investigating judge that although she had been jailed for the suspected murder of her newborn, "it is not credible that a mother could murder her child, especially before baptism." "Hernández's defense rested simply on the 
assertion that it was inconceivable that a mother would harm her own offspring. The curador (legal defender) of indigenous defendant María Juliana Coactle, tried in Puebla, Mexico in 1835 used a similar defense, arguing that the love a mother held for her child was so profound that only in the rarest of cases might a mother sever this bond. ${ }^{5}$

The discourse that associated murdering mothers with unnatural monstrosity, however, is not prevalent in these cases, and it appears less frequently across time. Instead, the body of eighteenth- and nineteenth-century criminal trials for women charged with the crime of infanticide in central and southern Mexico examined in this chapter reveals a distinctive portrait of how judicial authorities, peer community members, and perpetrators themselves differently conceived of the morality and repercussions of ending the lives of their newborn babies. Despite the condemnatory attitudes toward women alleged to have killed their own offspring that occasionally featured in the criminal trials of women accused in this group of trials in Mexico, the accounts are most remarkable because of the absence rather than the presence of such language. Vitriol toward murdering mothers in our own day is a reaction against the perception of women's apparent renunciation of a bond of affection and trust assumed to exist naturally between mothers and newborns. In the historic Mexican cases, however, the central preoccupation of both justices and community members when they commented on alleged baby murderers involved the evaluation of whether these women had upheld their obligations to their other family members, particularly to their parents.

This chapter examines a body of 262 criminal trials for the crimes of infanticide and abortion between 1699 and 1910, drawn from judicial archives in Central Mexico and from the southern and southeastern states of Oaxaca and the Yucatán. Judicial and popular attitudes toward defendants documented in this body of trials, as well as defendants' own views toward newborn child death, reveal that before the closing decades of the nineteenth century, infanticide was a crime that did not register dramatically among either community members or judicial officials. Rising rates of denunciation and conviction after the mid-nineteenth century indicate intensified public and judicial preoccupation with infanticide in this era, but this intensification carried distinctive connotations from attitudes toward infanticide characteristic of our own day. First, the cases from the Mexican past document little moral outrage about the lost lives of newborn infants. Second, as the following discussion elaborates, concerns with defendants' criminal or immoral behavior in the Mexican context was entwined with women's apparent failing as daughters, sisters, or wives as much as about the unnaturalness of their renunciation of their duties as mothers.

\section{Infanticide in the law}

In theory, the crime of infanticide, like that of abortion, was illegal in colonial Mexico, or as it was then called, the viceroyalty of New Spain (1535-1821), a territory whose administrative boundaries stretched from the greater Antilles, through Central America northward and westward to what is now the southwestern United States. Spanish law dictated that those convicted of infanticide should be sentenced to death. The administration of law in New Spain rested largely in the hands of the provincial magistry, alcaldes mayores (regional district judges and governors), and alcaldes (municipal judges and administrators), along with their assistants, tenientes de justicia. These first instance judges resolved disputes through the application of derecho indiano (the law of the Indies). Derecho indiano 
derived both from peninsular and New World sources, but was largely based in medieval and early modern Castilian legal codes, including, most importantly, King Alfonso X's thirteenth-century Castilian code, the Siete Partidas. ${ }^{6}$ Distinctive American contexts also influenced the formation of Spanish imperial law, particularly after 1614 when Philip III ordered that only laws formulated specifically for the Americas would govern the region. ${ }^{7}$ The state first collected royal decrees pertaining to the governance of the Indies in the 1689 Recopilación de leyes de los reynos de las Indias. The Recopilación, however, did not explicitly address the crimes of either infanticide or abortion, but the older peninsular legal codes treated both crimes severely. Infanticide carried the penalty of death by burning in medieval Spain. ${ }^{8}$ The Siete Partidas decreed that, "a pregnant woman who knowingly drinks herbs to provoke the baby's birth should be served with the same pain as for homicide [i.e. the death penalty]."9 Another Iberian royal code, the Fuero fuzgo, which Mexican judges occasionally cited even in the post-independence period, also specified that those convicted of infanticide should be sentenced to death. ${ }^{10}$

In the first five decades after Mexican independence (1821), little changed in terms of the legal codes justices used to assess the cases they tried. Formal codification of the country's penal code did not occur until after the 1871 passage of the Federal District (Mexico City's) Penal Code, a set of laws the thirty-one states of the Mexican Republic adopted more or less verbatim shortly after its passage. Before, and indeed even after codification, however, Mexican judges continued to refer to colonial law when judging criminal matters, including in cases of infanticide. Most often, they referenced the Siete Partidas in formulating their rulings. Occasionally, they cited contemporary Spanish and Mexican legal treatises, rather than codes, including Joaquín Escriche's Diccionario razonado de legislación y jurisprudencia, first published in 1837 and Febrero, ó libreria de jueces, abogados, $y$ escribanos, first published in 1834. These tracts, however, based their reasoning about infanticide on the Partidas, and continued to indicate the crime merited the death penalty. ${ }^{11}$ Nevertheless, these writers also indicated that some circumstances might dictate a modified sentence. Escriche specificed that women whose motives for committing infanticide were rooted in fear of either public infamy or the wrath of a "severe father" provoked by the revelation of a daughter's dishonorable pregnancy should be treated with "a certain degree of indulgence." 12 On the other hand, Escriche contended that those women who were,

of corrupted customs or established bad reputation (mala fama), who only commit the crime to rid themselves of an obligation, or out of antipathy for their husbands, or in order to be paid off, or for some other devious reason ... should feel the full weight of the law on their heads. ${ }^{13}$

Such may have been the case in theory. In practice, however, defendants almost without exception presented themselves as having been motivated by fear or a desire to maintain their or their family's reputations of honor, and judges accepted these interpretations of their actions.

The Federal District's 1871 penal code instituted two important reforms in judicial understandings of infanticide as it did also with respect to abortion. First, it distinguished the killing of an infant up to three days after its birth from other acts of homicide, which were normally punishable by death. In such circumstances, the 1871 code dramatically reduced the severity by which judges were to sentence convicted parties from death to 
several years' imprisonment. Second, it mandated that accused parties' efforts to preserve the public spectacle of their honor should further mitigate the severity with which judges should treat them. Defendants could establish and maintain their honor by maintaining good reputations, hiding their pregnancies and their children's births, and avoiding registering births in the civil registry, created in 1859 and mandating the public recording of all births, deaths, and marriages. The killing of children from legitimate unions was to be treated more severely than the same actions in cases of illegitimate offspring, presumably because illegitimate children could bring dishonor to women's reputations and those of their families while the births of legitimate offspring could not. ${ }^{14}$ If such circumstances were not met, however, punishment increased accordingly, and defendants might be sentenced to up to eight years' imprisonment. Articles 224 and 225 of the Code called for further reduction in sentences for those who had not reached the age of majority. The language of the 1871 Code demonstrates how central the consideration of public reputation, rather than assessing the rights of the deceased infant, was in assessing defendants' culpability.

The states of Puebla and Yucatán adopted the Federal District's code virtually verbatim. With respect to those articles treating infanticide and abortion, the 1871 Yucatán Code, like its federal corollary, did not prosecute abortion when a pregnancy or impending childbirth threatened the life of the mother. Under other circumstances, it decreed that women who intentionally aborted their pregnancies should serve prison sentences of one to four years (sentences that were slightly shorter than those decreed in the federal code), with longer sentences designated for women of ill repute, those who had not tried to hide their pregnancies, and those whose pregnancies were not the result of extramarital relations. ${ }^{15}$ Yucatán's 1871 code likewise punished infanticide, defined as the murder of an infant during the first three days of life, with between two and eight years' imprisonment, but also explicitly recognized women's incentive to commit infanticide "with the goal of hiding her dishonor" as a mitigating factor. Yucatán's code also obliged judges to consider such factors as defendants' intentions, age, social status, and degree of education when determining appropriate sentencing.

Oaxaca imposed stricter regulations on the commission of infanticide than many other states in its 1871 adoption of the federal district's code. First, Oaxaca did not make as strong a distinction as the federal code did between infanticide and other types of homicide. If the infant's death occurred during the first three days of the infant's life, Article 848 of Oaxaca's 1871 code declared the crime should be classified as a homicide and that those convicted should serve six years' imprisonment. If the crime was committed after three days, it constituted parricide, the killing of a close blood relative, and here the sentence called for "prision extraordinaria en penitenciaria" defined in Article 237 of the state Code as a substitute for the death penalty, and indicating a twenty-year penitential sentence. ${ }^{16}$ Oaxaca's code was also distinctive in that it made no references to attenuating or exacerbating factors (hiding the pregnancy, maintaining public honor) that would affect the crime's sentencing. Exceptionally, Oaxaca's code also reduced the sentence by half for women who intentionally aborted their offspring if the act occurred in the first trimester of pregnancy. ${ }^{17}$

\section{Judicial and community perceptions}

Such was the state of the changing legislation treating the crime of infanticide by the liberalizing closing decades of the nineteenth century. Strikingly, it was not until this 
same era that community members and judicial authorities more frequently denounced and prosecuted the crime. The holdings of nine archives, including the largest collection of colonial-era documents in Mexico, contain a mere twelve criminal investigations into the crimes of infanticide for the period between 1521 and $1821 .^{18}$ In viceregal Mexico, the crimes of abortion and infanticide either did not register with community members or judicial authorities, or else these groups chose not to pursue criminal investigations into their occurrence. ${ }^{19}$ A paucity of infanticide denunciation continued to characterize republican Mexico until after mid-century. This pattern is particularly clear within certain judicial districts in the state of Oaxaca, whose judicial archive houses holdings for judicial districts continuously from the colonial era and through the nineteenth century. The archives' criminal holdings for the district of Villa Alta in the north in the center of the Sierra Norte region of the state, for example, cover the period from 1650 to 1949. In that district for the 170-year period from 1650 to independence, first instance justices opened only two cases of infanticide (although local courts investigated a total of 152 homicides in this era). In contrast, in the first century after independence, local courts opened fourteen infanticide cases. In another Oaxaca judicial district, Teposcolula, whose criminal records cover the period from 1560 to 1949, judges investigated no cases of infanticide in the colonial era. However, between 1837 and 1885, courts in Teposcolula opened twelve infanticide investigations. The patterns are even more pronounced in other parts of Mexico. In Yucatán, no criminal cases treating the crime of infanticide exist for the entire colonial era, but seventy-two cases tried between 1841 and 1910; in Puebla, no cases have been uncovered for the crime in the three-hundred-year span of the colonial era, while twenty-six cases were opened between 1825 and 1872 .

Details from early nineteenth-century trials suggest that before mid-century, community residents most often deigned not to raise questions about their female peers' possible engagement in infanticide even when they were aware of pregnancies that had not ended in in felicitous births. In the first three decades after independence, only rarely did community members initiate denunciations of mothers for such crimes. For example, in the small community of Tamazulapan, Oaxaca, in 1837, one townsman initiated an infanticide investigation after discovering the body of a newborn infant in the drainage ditch of the local mill and reported the case to the community's justice of the peace. ${ }^{20}$ The justice then dispatched a municipal official, the mayor de vara of the district and his ministers to "solicit from the town any news about who could be the mother of the child." ${ }^{21}$ Later the same day, the same ministers returned to report that they had learned from "la voz pública" (public knowledge) that the mother was one María Ricarda Osorio, a seventeenyear old single woman who lived with her parents, both small cultivators.

Once apprehended, Osorio confessed to having given birth three days earlier but stated that the infant had been stillborn and declared that her only error lay in clandestinely disposing of her infant's corpse. After interviewing several witnesses, the local and regional courts both absolved Osorio citing insufficient evidence for the crime, as often happened in such cases. In Osorio's case, as in others contemporary to it, a town resident felt obliged to report the death of an infant when confronted by the discovery of the baby's corpse. However, no resident of Tamazulapan had felt compelled to denounce Osorio at the time of the birth and death of her child, which had happened three days earlier, even though the speed at which "la voz pública" identified Osorio as the party responsible for the crime indicates that these events were well known in the community. 
Similar circumstances describe other infanticide trials from the 1830s. In the 1838 infanticide trial against María de San José originating in the city of Aguascalientes, a community resident had again initiated an investigation after stumbling upon the remains of an infant in a community well. As in Osorio's trial, witnesses in this case declared they had known the defendant was pregnant, because of the "elevation of her belly," and had later seen her walking in the streets of Aguascalientes without such a substantial midriff. Still, no-one had initiated a case against San José until after the discovery of the cadaver of her infant. ${ }^{22}$ Here again, as in other contemporary cases, the court of first instance and the appellate court both absolved the defendant for lack of evidence.

Neighbors, community members, fellow laborers, and relatives began more frequently denouncing women to local courts for infanticide after mid-century. In one 1857 case from the town of Ticul in the state of Yucatán, a neighbor denounced "the bad woman," María Ix, for having killed her newborn after peering into the latter's yard one evening on her way out to get water. She observed Ix lying on the ground in a corner of her yard. On her return journey, Vázquez declared she had heard an infant crying, but when she visited Ix that evening, the latter denied that a child had cried. The subsequent day, Vázquez returned to her neighbor's house to confront Ix a second time. Ix confessed that she had miscarried but insisted she had only given birth to a "ball of blood." Suspicions unalloyed, Vázquez persisted in searching Ix's yard where she had uncovered the corpse of a newborn infant. Understanding this as confirmation that this "bad woman" had killed her infant, Vázquez went immediately to report the crime to Ticul's alcalde. ${ }^{23}$

An 1860 Mexico City case also suggests the beginning of such transformations in attitude toward infanticide in this era. In this case, several witnesses accused a neighbor of asphyxiating her child after having watched her exit her house carrying her baby but returning home later without him. ${ }^{24}$ In another Mexico City case, this one dating from 1880, a woman initiated criminal investigation against her cousin, Tomasa Escarcega, because she suspected foul play surrounding the circumstances in which Escarcega had given birth unattended to a stillborn fetus. ${ }^{25}$ In another Mexico City trial from the same year, a gendarme reported that he had initiated an investigation of one Lorenza Rodriguez for the crime of infanticide when he had overheard a woman doing her washing on a street corner remark that she pitied a "poor creature who was healthy at dusk and dead at dawn" (anocheció bueno y amaneció muerto). ${ }^{26}$

Although communities and municipal officials initiated infanticide investigations with greater frequency in the later nineteenth century, nineteenth-century magistrates, like their colonial predecessors, were often reluctant to convict women for the crime. Defendants in only three of the twelve colonial infanticide cases were found guilty, and this conviction rate of 25 percent continued to characterize nineteenth-century rates of conviction from Mexico City's Superior Tribunal. ${ }^{27}$ Conviction rates outside of the Mexican capital tended to be higher. In the body of infanticide and abortion trials from the Yucatán, conviction rates were 46 percent overall during the course of the century, although in that state, conviction rates dropped to 30 percent or lower in the last three decades of the nineteenth century. In Puebla, conviction rates were higher still, at 70 percent for existent nineteenth-century records.

Justices who were reluctant to convict defendants for these crimes, most often indicated their hesitation lay in their assessment that the evidence presented was insufficient 
to demonstrate guilt. In acquitting defendants, justices often referred to articles from the Siete Partidas that cautioned that criminal accusations must be proven with witnesses' written or oral testimony, but never only on the basis of suspicions. All accusations against an individual "must be proven and verified by evidence that is clear as day about which there is no doubt." 28 Court officials in several other trials referred to this article in the Partidas, sometimes naming it explicitly, but at other times, merely observing that the law dictated that defendants could not be convicted except on evidence that was "as clear as the light of mid-day." 29 This may have contributed to justices' comparatively lenient sentencing practices for those women convicted of infanticide. The private circumstances under which the alleged crimes occurred meant there were rarely eyewitnesses to its commission, and the high rate of neo-natal morality meant that it was always possible that babies had died of natural causes. In Puebla cases, a majority of those convicted were sentenced for four years and under; in Yucatán, especially in its closing decades, sentences were rarely over two years in length.

If justices exhibited restraint in their convictions and sentencing of those accused of infanticide, why did different attitudes exist among community members and municipal officials who denounced women for the crime in increasing numbers in the course of the nineteenth century? The explanation for the reasons behind the rising preoccupation by community members and municipal authorities alike with the sexual morality and reproductive habits of plebeian Mexican women in the course of the nineteenth century is more complex. Infanticide trials document both. In part, this novel preoccupation with scrutinizing women's sexuality may reflect a broader community preoccupation with sexuality in this period that differed from colonial attitudes. Social historians of colonial Mexico, beginning with Asunción Lavrin in her seminal 1989 essay, "Sexuality in Colonial Mexico: A Church Dilemma," have argued that a considerable gap existed between the teachings on sexuality that the colonial church hierarchy promoted and those more permissive sexual practices colonial populations actually practiced. ${ }^{30}$ Colonial society's skyrocketing rates of illegitimacy presents one obvious way that colonial populations failed to embody such Catholic teachings as the church's advocacy for exclusively procreative sex that occurred strictly within the confines of marriage. Sonya Lipsett-Rivera recently observed that colonial attitudes toward transgressive sexuality "often showed a healthy respect for or ignorance of the rules and a greater tolerance for behavior outside the norm than was reflected in the official discourse." 31 Perhaps, in the context of such research, it is safe to assume that the attitude Thomas Calvo uncovered, expressed by a single male parent, Gaspar de los Reyes, an indigenous man from Mezquitan near Guadalajara in 1708, was more typical than exceptional. De los Reyes wrote begging his brother who lived in San Luis Potosí to find his estranged daughter, Manuela: "If by chance she is lost and is no longer a maiden, she shall not lose my blessing. She is my daughter and I love her. What else can I do?"32

In the colonial period, the Hispanic elite were the principal group who had to publicly adhere to these strict sexual mores and their coincident regulation of lineage. ${ }^{33}$ In the following century, however, we see a change of attitude indicating that preoccupations about performing sexual virtue became a trait increasingly expected of plebeian women as well. Male heads of household, at least those who appeared in nineteenth-century infanticide trials, exhibited a starkly different view of the sexual transgressions of the young female members of their households from the sentiments Gaspar de los Reyes had expressed in 1708. In fact, they often judged that severe corporal punishment for their 
daughters, sisters, or nieces, and if necessary, death for these women's offspring, was a reasonable response to the discovery of the loss of the reputations for sexual honor of their female household members.

The most frequent motivation defendants in nineteenth-century infanticide trials provided to the courts to explain why they had hidden their pregnancies and childbirths was fear of punishment by their fathers, godfathers, uncles, grandfathers, brothers, or less frequently, their mothers. Such punishment, when inflicted, could be harsh indeed. María Isidora López, an indigenous woman tried in the small town of Coixtahuaca, Oaxaca, in 1849, described in her interrogation how she had given birth alone in the middle of the night outside the house of her godfather and left the baby next to some maguey plants and covered in mud since she was naked. When she returned inside, her godfather, an indio principal (head Indian) in the town, interrogated her aggressively and, deducing that she had just given birth, stripped her, tied her to a ladder, "and started to beat her with a mecate de mecapal [a leather strap]." ${ }^{34}$ A midwife who examined López testified she had been "severely whipped on the back." 35

In this case, López's godfather had taken the need for discipline into his own hands, but in the mid-nineteenth century, family members as well as municipal authorities also began denouncing those whose sexual or reproductive practices they condemned to the courts. In the same small community, just four years earlier, for example, sixty-one-yearold Fernando Mendoza, who described himself as both a laborer and a "fiscal actual del pueblo" (a lay assistant to the parish priest), began publicly confronting a young woman, Thomasa Maldonado, about her recent pregnancy. Mendoza declared that the local curate had engaged him "to keep vigil over" (velar y ver) women in his community, and so he had repeatedly demanded that Maldonado explain to him if she was or had been pregnant and by whom. He informed his town's chief constable and eventually also told a justice of the peace that he had known Maldonado was pregnant because he had observed it "with his own eyes since they were very immediate neighbors."36

Several things appear to have shifted that explain why mid-nineteenth-century Mexicans felt an obligation to scrutinize and denounce women for the crime of infanticide when they do not appear to have felt such an obligation or interest in doing so in earlier periods. First, as already suggested, these trials indicate that the sexual morality of plebeian women became a matter of increasing social surveillance over time. Kathryn Sloan's research on women's participation in (rapto) abduction practices in nineteenthcentury Oaxaca revealed a similar pattern. ${ }^{37}$ Sloan found that in the group of 212 cases she studied, young women often consented to be abducted by their suitors when their parents had objected to their suitability as marriage candidates. The women's parents were forced to accept the abductors as sons-in-law in order to restore their families' reputations for honor once their daughters had thus successfully arranged to have their virtue compromised. Significantly, the judicial maintenance of such reputations in the nineteenth century was a relative novelty among plebeian families.

Along similar lines, during her investigations of marital law suits in post-independence Costa Rica, Eugenia Rodríguez discovered in this period an intensification among plebeian families of marital and gender ideals associated previously associated with the upper class. According to Rodríguez, in the late nineteenth century, "the liberal state began to take on a more active role in the regulation and transformation of the domestic life among the popular classes according to the dominant values." ${ }^{38}$ In the group of cases she studied, plebeian women most often brought their husbands to court over charges of 
physical abuse, or for adultery, whereas plebeian men most frequently charged their wives with adultery. ${ }^{39}$ Elizabeth Dore comments that this pattern is common to Latin America more broadly, where courts "facilitated litigation over gender norms, [and] ... tended to fortify social relations that reflected the class, gender, and ethnic worldview of the elite." ${ }^{40}$ She also observes that Latin American liberal states' late nineteenth-century preoccupation with scrutinizing female sexuality was also related to the issue of inheritance. Civil codes of the 1870s, she note, "reinforced a husband's control over his wife's body for inheritance purposes ... The codes spelled out that husbands could appoint their own 'representatives' to witness their wives giving birth."41

Women's obligations to safeguard their reputations for sexual chastity, and thus their families' honorable status, is apparent in the defense arguments they presented in their trials. The most common defense they offered was that the preservation of their own sexual honor and the good reputation of their families was sufficient cause to justify the concealment of their pregnancies and births as well as the inadvertent or intentional deaths of their offspring. ${ }^{42}$ Many defendants explained they had given birth alone and in secret to hide their pregnancies from violent family members and to preserve their wider community reputations for honor. Guadalupe Martínez, tried for infanticide in Teposcolula, Oaxaca, in 1875 after townspeople uncovered the body of her newborn that she had abandoned near a pile of rocks in a public square, told the court she had given birth secretly because she was fearful of punishment from her parents or uncle. ${ }^{43}$ Her defender argued that she had hidden her pregnancy and birth because she was "fearful of the grave punishment she would suffer" if her family members discovered her state. ${ }^{44}$

Similarly, in one1864 trial from Puebla, a wealthy city resident denounced his servant, María de la Luz Díaz, for having killed her newborn baby girl by tossing her into the communes in a patio connected to his house. When the investigating judge interviewed Díaz, she explained that after having given birth, she had thrown the newborn into the comunes because she had been "confused by the shame (vergüenza) of her employers and the fear [she had] of her family." "45 Because Díaz had confessed to the crime, the first instance court trying her case convicted her for six years of service and reclusion in a Puebla hospital. Díaz's legal defender at the Supreme Court level, however, worked effectively to have her sentence reduced to only three years by arguing that, far from being understood as a "denaturalized mother," Díaz was a "simple, timid" woman who, even in the act of taking the life of her newborn, had been prompted by admirable traits: "her love of honor and the fear of her family." 46 The contemporary condemnation of women who disobeyed or deceived male family members is illustrated in the Mayan woman Paulina Uc convicted of infanticide in the city of Campeche in 1865. The fiscal (prosecutor) who assessed Uc's case in Yucatán's supreme court requested that its judges award her the harshest available sentence of ten years' imprisonment because he found the circumstances of Uc's case particularly disturbing. Her actions (alleged smothering) toward her newborn were no different from those in many other cases. Uc had deceived her husband into marrying her when she had known she was pregnant with another man's baby and then had ended her newborn's life to shield her unsuspecting husband from knowledge of the child's birth. ${ }^{47}$

For these defendants, their peers, and their judges, the social value of the preservation of women's reputations for sexual honor and their manifestation of obedience to their parents was greater than the social cost of women's engagement in a violent crime or the human cost of the lost life of the newborn baby. While court officials and 
other witnesses sometimes discussed the immorality of murdering mothers, never in these cases does a discourse about the value of the lost lives of newly born babies make an appearance in the trials.

\section{Mothers' views}

Assessing defendants' perspectives on the implications of infanticide is challenging because the sources available - defendants' testimonies in their own criminal investigations - are hardly neutral. We can never be sure if what we are seeing are the beliefs women actually held or those they wished the court to attribute to them. Notaries, further, had a tendency to standardize the language used in the trials they recorded, erasing emotion or individualism from the records. ${ }^{48}$ Nevertheless, when read carefully, these trials allow us glimpses of mothers' perspectives on the crimes with which they were charged. Occasionally, defendants expressed shame or regret during their testimonies for their actions. Petra Sevilla, the wet nurse convicted for infanticide in Puebla in 1835 whose case is discussed above, is one of the few whose trial transcripts offer a hint of a defendant's emotional state. When Sevilla was brought before the investigating judge to be interviewed, the notary recording her case included an unusual description of Sevilla's fragile state. He wrote that she

was asked why she left the house of don Gregorio Mujica without giving notice on July $17^{\text {th }}$. Without providing any response, she started to cry. Asked why she was crying instead of answering the question, she did not answer and continued to cry. ${ }^{49}$

Sevilla may have been feeling contrition over the alleged murder of her newborn child; she was most certainly intimidated by the process of the judicial investigation itself. Shortly thereafter, she recovered her voice, and in response to the judge's third request, that she should provide all the details concerning the birth and death of her child she replied, "that she had not given birth, but rather miscarried." 50 A notary described defendant María Getrudis Lucas, who was also investigated in Puebla three years after Sevilla, as responding to the judge's inquiries with a "tremulous and stammering voice." Lucas apparently also faced the court with fear and anxiety, but she, too, denied having killed her newborn and confessed only to having thrown it into a well after it had died on its own. ${ }^{51}$

More frequently, however, defendants, at least in the statements they supplied before the court officials who investigated them, described their behavior in matter-of-fact and unemotional terms. Although it is also possible that it was the notaries rather than the mothers who were dispassionate in these accounts, the existence in trial transcripts of the exceptionally emotional commentaries just described suggests that most women were stoic in the testimony they supplied. In her 1838 trial, indigenous defendant María del Carmen Camila explained to the court, without mincing her words that she had been put in jail because,

at nine in the morning on the $20^{\text {th }}$ of the month that just ended, she had given birth to a little girl in one of the alfalfa fields surrounding the mill, and she had left it there alive, covering it with some grasses until about five in the afternoon 
when, in the company of a woman called Margarita she went to see if [the baby] was still alive so she could give her to this woman who had said she would take her in if she were still alive. ${ }^{52}$

This kind of dispassionate description is typical of the confessions defendants made, whether acknowledging an act of exposure and abandonment, as in this case, or confessing to active participation in a newborn's death, as in María del Carmen Camila's 1842 trial. Del Carmen Camila testified that

Right after her birth, she put a ball (bodoque) of rags in her mouth, with the object only of stopping her from crying so the others in the house would not hear her. And after a while, she died. And shortly after she died, Francisco Luna buried her in the same house. And she did not intend to kill her, but in her ignorance, did not know she would die from just having the rags in her mouth. ${ }^{53}$

Women's presentation of their crimes suggests either an absence of a strong emotional bond with their children or an absence of the perception that defendants, as new mothers, were expected to demonstrate remorse over their deaths. Perhaps mothers and their peers adopted such an apparently unemotional response to the newborn deaths because neonatal and infant death was so common in eighteenth-century Mexico, where the rate of death rate for babies before reaching one year of life was 25 percent, and where another quarter of the population died before age ten. ${ }^{54}$

That defendants did not feel shame, or the need to exhibit shame to courts, about having exposed or more aggressively precipitated the deaths of their newborns is also suggested in the details about where and how they left their infants' bodies. Most of the accused left the corpses of the babies they had birthed on plots of land that were short distances from their dwellings. María Rosa, a woman tried in Epatlan, Puebla, in 1854, left her newborn where she had given birth to him, in the lane between her own house and her aunt's house, buildings described as "two varas" (roughly two yards) apart from one another. ${ }^{55}$ María Brigida, tried in Talcotepec, Puebla, in 1872, left the corpse of her baby in the courtyard of her own house. ${ }^{56}$ Others, who dumped bodies into communal water sources, elected very public locations to dispose of the dead. All of these women were, no doubt, desperate and exhausted when they abandoned the infants, whether living or dead. They may have felt they had no other option than to leave the bodies where they did. Nonetheless, we can reasonably assume that those who had long understood they were pregnant and had had months to contemplate the moment of childbirth and its aftermath would have realized that the newborns' corpses would soon be discovered. In such small communities, where peers and family members kept close watch over one another's movements and interactions, they must have understood they would also likely be identified as the mothers of the dead newborns. ${ }^{57}$ If such speculation is correct, then we may infer that these mothers assumed that upon discovery of the corpses, their peers, rather than reporting them to judicial authorities, would accept or ignore their actions, as they had apparently been doing for centuries prior. What began to shift in the post-independence period was not plaintiffs' behavior or their attitudes toward engaging in infanticide, nor was it a new attitude toward the value of all infants, but rather community reactions, encouraged by state expectations about public codes of conduct and the civic responsibilities of the Mexican populace. 
The absence of a discourse about the sanctity of the lives of newborns in this body of eighteenth- and nineteenth-century cases is not surprising since, before the twentieth century, neither the state, the church, nor communities in Mexico were powerful proponents of the inherent value of human life. Decades ago, John Noonan delineated the Catholic church's centuries long debate over the question of the inherent sanctity of human life and the timing of its initiation, observing that the papacy only adopted a definitive (if indirect) position on the matter in 1854, in Pius IX's proclamation of Mary's Immaculate Conception. ${ }^{58}$ Despite the papacy's 1854 pronouncement, however, as they had done in the viceregal era, Mexican representatives of the Catholic hierarchy remained virtually silent on the subject of the inherent value, or sanctity, of human life through the nineteenth century. Through the colonial era, the only types of births that were publicized or celebrated were those of the royal family or, closer to home, the births of children by the virreina, the wife of New Spain's chief governing officer. Childbirth was a fairly prosaic affair whose advent went largely unacknowledged and unannounced for most of the population in the colonial period as in the nineteenth century.

There was one important exception to the predominant invisibility of childbirth in this period: the church's registration of births through parish registers' records of newborn baptism, which, like the colonial church's historic administration of last rites and its oversight of Christian burials, the secularizing state contentiously wrested from it beginning in the late eighteenth century. ${ }^{59}$ The Mexican public, in the colonial era and long after it, steadfastly observed the primacy of marking both birth and death through the Christian rites of baptism and burial. In the eighteenth and nineteenth centuries, new mothers and other community members so valued the necessity of baptism because of its purpose in securing a soul's eternal life that they seem to have valued obtaining newborns' baptisms over ensuring babies' mortal survival. Saydi Núñez Cetina goes so far as to suggest that in seventeenth- and eighteenth-century Mexico, both the church and civil authorities affirmed that a newborn did not possess a soul until having received the sacred water of baptism. ${ }^{60}$ The church may have adopted this position in order to encourage the necessity of women's public registration of their offspring in order to discourage the occurrence of infanticide. However, it also meant that women who could not or did not want to raise their babies and disposed of them in the first few days after birth, before baptism, "did not, strictly speaking, commit a crime.",61

In eighteenth-century notices published in the Gazeta de México, one of the viceroyalty's first news periodicals that publicized, among other events, news of unusual- "monstrous" or multiple-births that had occurred in the viceroyalty, authors described any birth as "happy" or successful as long as the newborns were christened before they died. One 1793 notice, for example, which detailed the brief life of a bicorporal monster, celebrated the happy fortune (felicidad) that at least one of the heads to emerge from the womb had been baptized while still showing signs of life. ${ }^{62}$ The imperative to baptize newborns under any circumstances is also evident in secular and medical literature from the eighteenth century that encouraged midwives, or any other woman delivering babies, to be prepared to perform baptisms on them if they feared the babies would die before a priest could administer the rite. ${ }^{63}$ Mexican women clearly absorbed this message. In the trial of María del Carmen Ventura, tried for infanticide in Zacualtipan, Hidalgo in 1806, the accused testified that at the moment she had given birth alone in the middle of the night, she had seen that her baby "showed signs that he was going to die," so she "took a mouthful of water and sprayed it into his mouth saying in the name of the Father and 
the Son and the Holy Spirit.",64 The primacy of baptism is also apparent in the 1854 trial of María Rosa tried in Epatlan, Puebla, in 1854. In this case, the defendant's mother and another neighboring woman described how when they found Rosa's newborn infant, still alive in the lane outside her house, their first action, rather than to attempt to restore its health, was to carry the baby to the house of a midwife to see if she could baptize it before its death. ${ }^{65}$

Further evidence of the baptism's comparative pre-eminence over corporeal life in the early nineteenth-century infanticide trial of Francisca Torres, a woman from the small town of Chiautla, Puebla, in 1829, accused of having ingested an abortifacient to provoke a miscarriage and also with smothering her newborn baby upon its birth. The first instance judge investigating Torres admonished her when she first appeared before him, not for the crime of taking her child's life. Rather, he scolded her for having "taken medicines to abort, when she must know that the result of this would be to consign her baby to limbo, incurring a grave sin for this deed, and that she would be subjected to a penalty for this crime."

As well privileging the right of an infant's baptism, archival evidence also suggests colonial Mexicans held similar attitudes toward newborn death, where enacting the rites of a Christian burial may have mattered more than grieving the bodily loss of the newborn. One 1802 criminal complaint from Antequera (later renamed the Oaxaca City) records the plight of an indigenous woman, Marta Basque. Basque attested that her inlaws, apparently angry over the sickly state of her newborn baby, had imprisoned and tortured her, whipping her, thrusting a lit ember in her mouth, and throwing hot chili pepper in her eyes. ${ }^{67}$ When he saw the baby was on the point of death, "because no one was giving it anything to eat or drink," Basque's husband told her his family would not help her to bury the baby, and she was forced to carry the dying infant to her home town of Magdalena, a ten-hour walk south of Antequera. Basque's child died in Magdalena, but Basque did not have the money to bury him there, so she carried his body another two hours north to the regional capital of Ocotlán to beg the priest, Julian Camo, to bury her child out of Christian charity, which he did. ${ }^{68}$ The necessity of ensuring a sacred burial for her son so strongly compelled Basque that she carried her infant's body on foot on a two-day journey in order to ensure the rite's proper execution.

María Basque's sad story, like those of the other mothers of infants who died, or were killed, in eighteenth- and nineteenth-century Mexico reveals various ways in which perspectives of the crime of infanticide and its agents from this era differed significantly from our own. Current-day news consumers who follow stories of murdering mothers react strongly to these because they see such women as renouncing their duties as mothers and rejecting their natural role as protectors of newborns. Because we live in an era in which newborn babies, attributed with innocence and defenselessness, are valued as equal or even greater in importance than the lives of others, the murder of newborn babies seems particularly heinous to us now. Infanticide trial records and other documentation from eighteenth- and nineteenth-century Mexico reveal that none of these perspectives aptly describes the attitudes of judicial authorities, community members, or defendants themselves accused of murdering their newborn children in that context.

Although, in theory, the law in viceregal Mexico punished infanticide severely, in practice, colonial subjects rarely denounced women for the crime, and judicial authorities treated those who were accused of infanticide with relative leniency. This pattern continued to describe conditions in the Mexican republic after independence and up until the last 
decades of the nineteenth century, when community members denounced women to local courts with greater frequency and when magistrates, at least those in Yucuatán and Puebla, convicted them more often. Even within this context, however, the reasons for state, public, and even personal censure for the act of infanticide differ from those expressed in our own day. We see few traces in these trials of emotional attachments to the innocent symbolism of neo-natal lives and virtually no attestation of their inherent value. Instead, these trials illustrate state initiatives, largely mirrored in community attitudes, about the imperative of women's outward maintenance of sexual honor. Women's duties, as chaste and obedient daughters, trumped the consideration of their importance as new mothers.

\section{Notes}

1 The Ohio court absolved Richardson of the more serious charges of newborn murder: Brittany Shammas and Meagan Flynn, "Teen Acquitted of Killing Her Baby Says She's 'Forever Sorry." The Washington Post, September 13, 2019. www.washingtonpost.com/nation/2019/09/ 13/teen-said-she-buried-her-stillborn-baby-backyard-after-prom-she-was-just-acquitted-murder/ . Accessed October 22, 2019.

2 Twitter feed: Donna Pointer@randd6066, September 30, 2019. Replying to@ChanleyCourtTV, "Teen acquitted."

3 Archivo Histórico Judicial de Puebla, Puebla, Mexico (hereafter AHJP), Penal, Caja 595, exp. 18168, fol. 50v. In a second poblano (from Puebla) infanticide trial from 1864, the legal defender of the defendant argued that premeditated neonatal murder was a work of "monstrosity." AHJP, Penal, Caja 1177, exp. 46464, fol. 6v.

4 Archivo Histórico Judicial de Oaxaca (AHJO), Teposcolula, Penal, legajo 49, exp. 32.

5 AHJP, Penal, Caja 560, exp. 16935, fol. 39.

6 Gabriel Haslip-Viera, Crime and Punishment in Late Colonial Mexico City, 1692-1810 (Albuquerque: University of New Mexico Press, 1999), 37.

7 Charles R. Cutter, "The Administration of Law in Colonial New Mexico," Fournal of the Early Republic 18:1 (1998), 102.

8 Jane G. Landers, "Female Conflict and Its Resolution in Eighteenth-Century St. Augustine," The Americas 54:4 (1998), fn. 44, 572.

9 Gregorio Lopez, Las siete partidas del sabio rey don Alonso el nono, nueuamente glosadas, por el licenciado Gregorio Lopez .... Con su repertorio muy copioso, assi del testo como de la glossa. Vol. 7 (Valladolid: en casa de Diego Fernandez de Cordoua, 1587), Ley 8, Titulo 8, $7^{\mathrm{a}}$ Partida.

10 Joaquín Ibarra y Marín, Fuero juzgo en latin y castellano: cotejado con las mas antiguos y preciosos códices (Madrid: 1815), Ley 7, titulo 3, lib. 6. In one abortion trial originating in the small town of Chocholá, Yucatán, in 1853, for instance, the Fuero fuzgo was the first reference a first instance judge cited in judging his case. Archivo General del Estado de Yucatán (hereafter AGEY), Justicia, Penal, vol. 70, exp. 34, fol. 2.

11 Joaquín Escriche, Diccionario razonado de legislación y jurisprudencia (Paris: Librería de Rosa, 1851), 856.

12 Ibid., 857.

13 Ibid.

14 Codigo Penal Para El Distrito Federal $Y$ Territorio De La Baja-California: Sobre Delitos Del Fuero Comun, $Y$ Para Toda La Republica Sobre Delitos Contra La Federacion (Puebla: Imp. De T.F. Neve, 1876), 144, Cap. X, Art. 584.

15 Articles 474-485, Código penal del estado de Yucatán, (Mérida: Imp. Literaria dirigida por Gil Canto, 1871), 96-97.

16 Articles 237 and 848, Código criminal y penal del estado libre y soberano de Oaxaca (Oaxaca: Impreso por M. Rincon, 1871), 56, 208.

17 Article 852, Código criminal y penal del estado libre y soberano de Oaxaca, 209.

18 The archives consulted were the Tribunal Superior de Justicia del Distrito Federal (hereafter TSJDF), located in the Archivo General de la Nación (Mexico City); the Corte Suprema de Justicia; the Archivo General del Estado de Yucatán (AGEY); the Archivo Histórico Judicial de 
Puebla (AHJP); the Archivo Histórico Judicial del Estado de Oaxaca (AHJO); and the municipal archives of both Mexico City and Oaxaca City.

19 See Nora E. Jaffary, "Reconceiving Motherhood: Infanticide and Abortion in Colonial Mexico," Gournal of Family History 37:1 (2012), 3-22.

20 AHJO, Teposcolula, Criminal, legajo 58, exp. 21, fol. 2.

21 Ibid., fol. 3v.

22 Suprema Corte de Justicia, 1815-1840, Leg Av. 184, "Toca a la causa instruida a M.a San José Romo por infanticidio," fols. 9, 12.

23 AGEY, Justicia, Penal, caja 95, vol. 95, exp. 9 fol. 5v-6.

24 TSJDF, 1860 caja 341, "Toca á la causa instruida por el juez $5^{\circ}$ de lo criminal, contra Leocadia Hernández por infanticidio."

25 TSJDF 1880 caja 703, "En Averiguación de la muerte de un feto. Sospechas de aborto provocado."

26 TSJDF 1880 caja 703, "Contra Lorenza Rodríguez por infanticidio," fol. 1.

27 See Table 4, Nora E. Jaffary, Reproduction and Its Discontents in Mexico: Childbirth and Contraception form 1750 to 1905 (Chapel Hill: University of North Carolina Press, 2016), 107.

28 Las Siete Partidas Del Sabio Rey Don Alonso el Nono, ley 12, título 14, Partida 3.

29 The public defenders of Florencia May, Marcelina Ciua, and Severiana Cahum all make this argument for the absolution of their clients. AGEY, Justicia, caja 166, vol. 166 exp. 7, sn; vol. 18, exp. 6, fol. 22; vol. 24, exp. 1, sn.

30 Asunción Lavrin, "Sexuality in Colonial Mexico: A Church Dilemma," in Sexuality and Marriage in Colonial Latin America, edited by Asunción Lavrin (Lincoln: University of Nebraska Press, 1989), 47-95.

31 Sonya Lipsett-Rivera, The Origins of Macho: Men and Masculinity in Colonial Mexico (Albuquerque: University of New Mexico Press, 2019, 46.

32 Thomas Calvo, "The Warmth of the Hearth: Seventeenth-Century Guadalajara Families," in Sexuality and Marriage in Colonial Latin America, edited by Asunción Lavrin (Lincoln: University of Nebraska Press, 1989), 306.

33 Ann Twinam, Public Lives, Private Secrets: Gender, Honor, Sexuality, and Illegitimacy in Colonial Spanish America (Stanford: Stanford University Press, 1999).

34 AHJO, Teposcolula, Criminal, legajo 66, exped. 1, fol. 103.

35 Ibid., fol. $98 \mathrm{v}$.

36 AHJO, Teposcolula, Penal, 1845, legajo 64, exp. 3.

37 Kathryn A, Sloan, Runaway Daughters: Seduction, Elopement, and Honor in Nineteenth-Century Mexico (Albuquerque: University of New Mexico Press, 2008).

38 Eugenia Rodríguez S., "Civilizing Life in the Central Valley of Costa Rica, 1750-1850," in Hidden Histories of Gender and the State in Latin America, edited by Elizabeth Dore and Maxine Molyneux (Durham: Duke University Press, 2000), 91.

39 Ibid., 93, 97.

40 Elizabeth Dore, "Property, Households, and Public Regulation of Domestic Life," Diriomo, Nicaragua, "1840-1900," in Hidden Histories of Gender and the State in Latin America, edited by Elizabeth Dore and Maxine Molyneux (Durham: Duke University Press, 2000), 165.

41 Elizabeth Dore, "One Step Forward, Two Steps Back: Gender and the State in the Long Nineteenth Century," in Hidden Histories of Gender and the State in Latin America, edited by Elizabeth Dore and Maxine Molyneux (Durham: Duke University Press, 2000), 22.

42 This is an argument Kristin Ruggiero also found was common to the perceptions that both courts and defendants marshalled in her examination of late nineteenth-century infanticide trials in Argentina. Kristin Ruggiero, "Honor, Maternity, and the Disciplining of Women: Infanticide in Late Nineteenth-Century Buenos Aires," The Hispanic American Historical Review 72:3 (August 1992), 353-373.

43 AJHO, Teposcolula, Penal, legajo 105, exp.29, fol. 5.

44 Ibid., fol. 19.

45 AHJP, Penal, caja 1315, exp. 48483, fol. 8v.

46 Ibid., fol. 29v.

47 AGEY, Justicia, Penal, caja 128, vol. 128, exp. 70, fols. 3v-4. 


\section{MURDERING MOTHERS AND DUTIFUL DAUGHTERS}

48 For a discussion of notarial standardization, and the detection of idiosyncrasies in the notarial record, see Kathryn Burns, Into the Archive: Writing and Power in Colonial Peru (Durham: Duke University Press, 2010).

49 Archivo Histórico Judicial de Puebla (AHJP), Penal, caja 595, exp. 18168, fol. 15.

50 Ibid.

51 AHJP, Penal, caja 631, exp. 19361, fol. 9.

52 AHJP, Penal, caja 634, exp. 19484, fol. 8v.

53 AHJP, Penal, caja 721, exp. 22524, fol. 3.

54 Dorothy Tanck de Estrada, "Muerte precoz. Los niños en el siglo xviii," in Historia de la vida cotidiana en México III El Siglo XVIII: entre tradición y cambio, edited by Pilar Gonzalbo Aizpuru (México: El Colegio de México, Fondo de Cultura Económica, 2005), 214.

55 AHJP, Penal, caja 1014, exp. 37572, fol. 22.

56 AHJP, Penal, caja 1496, exp. 62017, fol. 3v.

57 For further discussion of community scrutiny of daily life in this period, see Sonya LipsettRivera, Gender and the Negotiation of Daily Life in Mexico, 1750-1856 (Lincoln: University of Nebraska press, 2012).

58 John T. Noonan, Jr. "Abortion and the Catholic Church: A Summary," Natural Law Forum. Paper 126, 114. https://scholarship.law.nd.edu/cgi/viewcontent.cgi? article $=1125 \&$ context $=$ nd_naturallaw_forum

59 The formalization of this process, which began with King Charles III's outlawing of the burying of the dead in 1787, was completed with the 1859 creation of the civil registry mandating the public recording of births, deaths, and marriages. For further discussion, see Pamela Voekel, Alone Before God: The Religious Origins of Modernity in Mexico (Durham: Duke University Press, 2002).

60 Saydi Núñez Cetina, "Reforma social, honor, y justicia: infanticidio y aborto en la Ciudad de México, 1920-1940," Signos Históricos 28 (julio-diciembre, 2012), 76.

61 Ibid.

62 Gazeta de México, December 30, 1793, 709-10.

63 Ignacio Segura, Avisos saludables a las parteras para el cumplimento de su obligación (Mexico City: f. de Zuñiga y Ontiveros, 1775), 4.

64 Significant portions of María del Carmen Ventura's trial are reproduced in, "María del Carmen Ventura's Criminal Trial for Infanticide (Zacualtipan, Mexico, 1806)," in Women in Colonial Latin America, 1526 to 1806: Texts and Contexts, edited by Nora E. Jaffary and Jane E Mangan (Indianapolis: Hackett Publishing Company, 2018), 250-264.

65 AHJP, Penal, caja 1014, exp. 37572, fol. 17.

66 AHJP, Penal, caja 424, exp. 12745, fol. 54v.

67 Archivo del Poder Ejecutivo de Oaxaca, Justicia, Criminal, Diligencias, caja 542, exp. 8, fol. 1.

68 In contemporary Orizaba (Veracruz), the Archbishop's arnacel (price list), indicated the cost of burying a baby was two and a half pesos. Tanck de Estrada, "Muerte precoz," 217. 


\title{
"I WISH WE GOULD HAVE SAVED HIM FOR YOU"
}

\author{
Australia's experience of death and bereavement in \\ war, 1914-1918
}

\author{
Jen Roberts
}

\begin{abstract}
"Today as we look at your photo
A soldier so brave and true

Do you know our hearts are aching

And longing, dear Jack, for you"

In Memoriam notice, $1918^{1}$
\end{abstract}

Just thirteen years after Australia became an independent nation, separate, but still economically, politically, culturally, and socially dependent on Great Britain, and still part of the British Empire, the new country found herself embroiled in the most brutal industrialized and mechanized global war in history. Of a pre-war population of just over 4 million, more than 330,000 young Australian men - all volunteers - embarked to theatres of war in Europe and the Middle East between 1914 and 1918. Over 60,000 would not return, with in excess of 166,000 wounded, gassed or taken prisoner. In short, 40 percent of the eligible white male population enlisted, and one in five of those recruits lay dead on foreign soil within four years. ${ }^{2}$ Half of those who joined up were under the age of 24 , and, by 1918, the median age of new recruits was just 20. Eighty per cent were unmarried. ${ }^{3}$ Ken Inglis has estimated that every second white Australian family was bereaved by the war, extended networks of relations who were all deeply affected by the loss of one of their own. ${ }^{4}$ L L Robson, borrowing from John Donne, wrote, if every man's death diminishes me, "then Australia was grievously diminished by the Great War.",

This chapter traces wartime bereavement from its genesis in 1914 and details the manner in which death was notified, the effects of, and reactions to, news of death and wounding, the importance of condolence letters and the reassurance provided by comrades, chaplains and nurses, and the significance of the grave or a site of burial. It further focuses on how the bereaved negotiated the public and private processes of remembering their loved ones both during and after the war, together with the uncertainty of the fate of the missing and the resulting denial and silence that could lead to prolonged and unresolved grief.

Using letters, diaries, private records, newspapers, official government documentation, and Army and medical records, this chapter argues that the Great War permanently 
both changed the way Australians negotiated the experience of death - culturally, socially, and politically - and transformed pre-war mourning behaviors in ways that are still prominent today. While specific rituals of death and displays of mourning differed according to region and class, the Australian experience of death in the nineteenth century was characterized by deathbed attendance, the funeral service, the grave, the headstone, its inscription, and the physical act of visiting the grave site to place flowers or mementoes to mark special occasions and anniversaries. ${ }^{6}$

Two realities were required to mourn within the comfort of these familiar customs: the presence of the body and the knowledge of how and where the person died. These were not available to the Australian bereaved of the Great War. Jacqueline Manuel has argued that the war rendered useless all the traditional mourning rituals that had assisted the grieving in making sense of sudden death, leaving them isolated and vulnerable as "suddenly, the established, reliable patterns of grieving [were] stripped away by war."7 The overwhelming numbers of dead meant that the unique anguish of each family could not be honored. As Jay Winter has expressed it, "the individuality of death had been buried under millions of corpses." " For Australians, the landscape that cradled the bodies of the fallen on the other side of the world was so unknown to them, as one historian put it, "their sons were missing on what may as well have been another planet." 9

Western literature has long drawn a distinction between the objective loss (bereavement), the emotional reaction to the loss (grief), and the socially defined behavior following the loss (mourning). However, Tony Walter has argued that this trinity is much less disparate than previously presumed and that studies of bereavement, grief, and mourning are central to the understanding of what binds a society together and how it reinvents and reinstates itself after tragedy. ${ }^{10}$ Pat Jalland has concurred and noted that to study death, and a society's responses to it, is to study the heart of a culture. ${ }^{11}$ Indeed, Hegel defined history itself as "the record of what man does with death". ${ }^{12}$

\section{Delivering death}

While the long-anticipated announcement of war in August 1914 saw scenes of jubilation and nationalism across Australia, the steadily growing casualty lists that began their dispatch around the country during 1915 went a long way to quelling the initial enthusiasm. By the end of 1916, a pall had descended on cities and hamlets, towns and stations. The scale of loss at Gallipoli, followed by the carnage on the Western Front and the wounded soldiers - limbless, blind and maimed - returning to Australia meant the reality of the conflict was brought home with shocking clarity. The war was no longer simply an abstract opportunity to prove Australia worthy of her place in the Empire, or a chance for adventure for the " $6 /-$ a day tourists". 13 Every family with a soldier at the war was primed for news as to the whereabouts and safety of their men. With bereavement an ever-present possibility, theirs was a perpetual anxiety, whereby every report of the dead, wounded, and missing held "for us all a horrible portentous interest"14 and "newspapers were scanned daily for names of any Australians we may have known." 15

In Australia, the clergy was the official messenger of death. ${ }^{16}$ Having accepted the government's request to convey the news of loss at the beginning of the war, many individual ministers and priests soon had cause to regret the decision. They found they could not visit the homes of their congregants "without frightening the relatives into a week's nervous sickness" and protested, "it is not fair either to the minister or to the parishioners." The 
Rector of Bulli Anglican Church informed his congregation that if bad news were to be delivered, "you may rest assured that he will not call at your house as on an ordinary visit ... you need not be frightened every time you see him.",17

Doubtless, the decision to rely on the clergy was underpinned by the expectation that the worst of news could be accompanied by some spiritual solace; however, it was not comfort that the bereaved craved, but certainty. The bald missives from the First Australian Imperial Force (AIF) were notable for what they did not say, rather than what they did. The manner in which death was first notified, and the amount of information subsequently conveyed to the families, was a significant determinant in how the bereaved would cope with their loss.

\section{Reactions to news}

The daily anticipation of loss was often as stressful as the realization of it. Bereavement most certainly began for some before any actual reports of fatalities. Describing the day her second son embarked for the front, for example, Mrs. James felt:

it seemed to me the sun had set. I went back to my lonely house, but could not go inside, nor could I seek anyone's company. I just went into the garden and dug and dug until I felt that my heart must break. ${ }^{18}$

While she attempted to keep up with her usual routine and assisted local patriotic organizations, her anxiety and loneliness threatened to engulf her. Mrs. James noted that "somehow the weeks passed ... [but] every Sunday saw my dinner untasted. That always seemed the hardest day to bear." 19 The reference to Sunday in particular reflects the central role of the Sabbath as a traditional family day with its accompanying ritual of the Sunday roast, making the absence more marked. The duration of the war, in contrast to initial hopes for a quick end to the conflict, resulted in ongoing strain. A father wrote to a friend in 1918 that he was, "wondering when this awful war will end as by the time you receive this it will be four years suffering and anxiety." 20

Some soldiers foresaw the effects of anxiety upon their families and cautioned them to be optimistic. Lieutenant Syd Duchesne was among the first contingent of the AIF to embark in October 1914, and during the six months he spent at Mena Camp in Egypt, he became increasingly concerned with reports that his mother in Sydney was not coping with his absence. He wrote to his father:

Aunt thinks I shall never return. Well, Dad, I hope you don't let Mother worry like that ... I ... wonder ... if Mother is well because by the strain of her letters, she seems to be worrying over me and that is the last thing I wish for ... PS: Mother don't worry. I am enjoying myself and am happy. ${ }^{21}$

Syd admonished his mother for fretting over "only a son", ${ }^{22}$ when many married men had left their wives and children to go to war. He advised "we must pray that they may be spared to return ... not for us single boys who have nobody depending on us." 23 Yet it was a fallacy that single men were not depended upon. Ageing parents increasingly relied on their adult sons, not only for economic or domestic benefits, but also as companion, as carer, and as potentially the provider of grandchildren and an integral part of 
the ongoing legacy of the parents as individuals. They saw their sons as carrying on the tradition of the business, the farm, the profession, the name, and the lore of the family group, long after they themselves had gone.

The untimely and violent loss of an adult son subverted these hopes and rendered the future bleak and clouded with longings and nostalgia. One father admitted, "I cannot realise it and life can never be the same to me again as all my hopes and ambitions were centred on my boy. I hope you will forgive my speaking of him." ${ }^{24}$

The distance of Australians from the battlefields, and the inverse, the distance of soldiers from their families, underpinned everyday life throughout the war. ${ }^{25}$ That correspondence was irregular, inaccurate, delayed, or liable to be lost was, if not accepted, at least an acknowledged reality. It did not make life any easier. Mrs. James despaired that "cables [were] so inadequate", ${ }^{26}$ while another mother, writing to her local member of parliament, complained "if you have a son at the front you will have an idea what it is like to wait for months for news."27

For other families, further correspondence from the authorities signaled only distress. The Meggy family of Neutral Bay had three sons away at the war, Douglas, Percy, and Albert, and a daughter, Margaret, who had enlisted as a nurse. After Albert was killed at Lone Pine in 1915 and Douglas at Pozières in 1916, the family requested the military authorities not inform Mrs Meggy if anything were to happen to the third son, Percy, and to "not publish same. My mother, having lost two sons, the shock would be too severe."28 The Meggy family were personal acquaintances of both the official historian Charles Bean and General Birdwood, and Margaret was able to petition Birdwood on behalf of her mother. He responded:

I feel that you have already made such a great sacrifice in the loss of your two others that I have decided that suitable employment be found for your remaining boy in England. ${ }^{29}$

Douglas' body was never found, and Mrs. Meggy wrote regularly into the 1920s inquiring as to whether anything had been heard of her son. In agreeing to donate her boys' letters and diaries to the newly established Australian War Memorial, she admitted to Director John Treloar in 1928, "I have had a lot of worry and sickness.",30

This experience was mirrored by the Nation family of Morphettville in South Australia who lost all three of their sons within three months in 1917. Ralph, aged 21, was killed in the line of duty on 6 March; Cliff, aged 26, on 6 May; and finally, Eric, the baby at just 18, on 10 June. Their sister wrote that their mother Emily was "prostrated after hearing of the death of her third son", 31 but it was their father on whom grief took a terrible toll. Mrs. Nation informed the authorities in 1923 that she had separated from her husband who had become "very eccentric", such that, after receiving the three telegrams with the news of his sons' deaths, he had undertaken to "never open another letter from the military or government authorities." Base Records were probably relieved to have an explanation, as their correspondence to Mr. Nation indicated they were puzzled as to why the three boys' commemorative medals remained unclaimed and other correspondence from the Army had been marked "returned to sender". 32

Mrs. Nation lived with her surviving child, a married daughter, until her death in 1946. While she survived for nearly thirty years after her sons' deaths, it is very unlikely she would ever have completely recovered from such loss. Mr. Nation definitely did not 
recover. Physically and psychologically, his health rapidly deteriorated. He was admitted to the psychiatric wing of a private hospital in Adelaide, where he died of pneumonia on 1 August $1924^{33}$

In contrast to those who struggled to cope with their loss, were others who attempted to seek solace in the fact their loved ones were now at peace, rather than survivors of grievous injury. Oliver Cumberland had been "broken up a bit" by the death of his younger brother Joe at Gallipoli, but suggested to their sister, "it might be for the best ... war is a terrible game, especially this war, and those that are killed quick are sometimes better off." "John Garibaldi Roberts wrote, "the horrors of war might have sent him back home in a condition worse than death with brain gone or so mangled that he might be a wreck only of his fine old self." 35 Another correspondent consoled a family that their son was "happy now and free from pain. If you could only see some of the poor boys who have come back without some of their limbs, you would feel that your loved one is better off." ${ }^{36}$ These interpretations of the dead as at peace and forever young were evidence of both an enduring belief among some in the honor of battlefield sacrifice and of the increasing knowledge of the horrors - both physical and mental wrought upon young men, and their families, when they returned home permanently damaged by war.

\section{So sorry - condolences and reassurance}

Letters of condolence from family, friends, and comrades demonstrated the writers were keenly aware of the devastation of bereavement, particularly among mothers. Sapper Cecil Ashdown was 19 years old when he was killed on the first night of the battle of Fromelles. His sister, serving as a nurse in France, wrote to their brother in Sydney, "Our darling baby boy's death has been one huge blow and will make such a huge gap in our lives and poor old Mother. She, I think, will be well-nigh heart broken." ${ }^{37}$ It was a common theme. Letters to parents frequently contained similar sentiments: for example, "You must be broken hearted at his loss," 38 and "How are you two poor ones to bear it all. Poor old Hector." ${ }^{39}$ In 1922, a woman asserted, "my mother died of a broken heart six months after the death of her only son."40

A soldier acknowledged, "We know that you noble women at home are suffering,"41 and another admitted, "it is hard to realize the anxious time that our people are enduring at home. Months of weary waiting and then that dread message to arrive."42 General Birdwood himself responded to a letter from Mrs. Meggy: "I am so very grieved to hear of your great trouble in the loss of your two boys, which is indeed an overwhelming blow ... I well know that nothing can make up for your loss." 43 This was undoubtedly true. However, the one imperative that could either ameliorate or exacerbate the pain of bereavement was specific and detailed information as to the cause and circumstances of death and the care that was taken of the deceased. Knowledge of what exactly had happened to their sons was the closest substitute for having been there. Those who were able to gain this closure were much more likely to cope better with the news of death than those who were left to their own imaginings.

Letters from nursing staff in the field hospitals show a keen empathy for parents' need for specific, factual detail. Matron Cecily Dale of the Bevan Military Hospital in Kent wrote to the parents of Private Dick Houghton, "it was a quiet death, all the pain ceased ... Your lad looked just like a boy asleep - absolutely at rest." ${ }^{44}$ Likewise, 
Matron Sampson reported the details of the death of Sarah Greenhalgh's younger son from pneumonia:

He had been unconscious for some time and the end was very peaceful. It had been a beautiful sunny day and his bed had been on the balcony in the afternoon, close to the sea ... Everything possible was done for him. ${ }^{45}$

Matron Reddock, on board a hospital ship moored off Lemnos, addressed the precise fears of a mother when she wrote, "he had a woman's care at the end of his young life, for there are sisters in all wards on this boat and I know they personally looked after him." "46 That the medical staff expended every energy to save their patients was also given prominence in the letters. One nurse wrote, "we would have given anything to save his life," 47 while another lamented, "you are so far away. I wish we could have saved him for you."48

Some historians have noted, correctly, that many of the letters from the front followed a formulaic pattern of skimming over the gruesome particulars of death and eulogizing the dead soldier as brave, popular, and lamented. Winter in particular has argued that these were stylized accounts containing stock messages, ${ }^{49}$ an automatic and stark response of obligation rather than a genuine expression of shared grief. Luckins also ascribes a generic nature to the letters. ${ }^{50}$ It is fair to say that the descriptions of death sometimes glossed over the ghastly nature of wounds. There was often an emphasis on death as quick or as painless and comfortable, but this was not necessarily for the benefit of the families. Very probably, the hospital staff and the men in the trenches could not have brought themselves to describe to anxious families so far away exactly what damage a machine gun or shell could do to the human body. The testimony of soldiers, though, could be blunt when dealing with more official enquiries. One witness told the Red Cross Enquiries Bureau that Private Downey "was shot first of all in the head and then a bursting bomb caught him and blew him to pieces beyond all recognition". ${ }^{1}$ Even such frank accounts, however, could not convey reality. A witness statement given to the Red Cross noted that Private Hillcoat had been "shot in the head and part of it blown away". ${ }^{52}$ A subsequent witness statement revealed the horror of this man's death: Private Hillcoat lived for almost forty-eight hours after being wounded. ${ }^{53}$

While they may have followed a common form, these letters were very powerful, both for the sender and the recipient. The level of individual detail, their length, and their poignant candor spoke to the isolation of bereavement with an extraordinary emotion that belies the stoicism said to typify the Australian soldier.

They also provide a window into the process by which men at the front transformed themselves, as Damousi has described, from "warrior to nurturer". ${ }^{44}$ In what is probably an Australian first, the letters to bereaved families at home from comrades at the front have provided historians with an opportunity to view platonic male bonding and, indeed, love, through the writing of working-class men. Men wrote, "this war has taken as victims most of my dearest friends ... I can realise how terrible your grief must be, for I loved him too"; "55 "we lost our brave little officer ... I feel a great loss keenly"; "I "I grieve with you in the loss of such a fine lad"; 57 and "I and all the rest of his friends loved him like a brother and he was more than a brother to me." ${ }^{\text {} 58}$ These letters were personal, detailed, and specific. They took time and effort to write. That the letters, and the comfort they brought, were prized by families is shown by the simple fact that they 
kept them. They were published in local newspapers, shared among neighbors, read, and re-read until the paper was shiny and brittle along the fold lines. And when the appeals began in the late 1920s, they were donated in their hundreds to the Australian War Memorial. $^{59}$

\section{Burial}

The focus of the bereaved on the grave, or its absence, demonstrates the importance of knowledge and certainty not only in accepting the death but also in seeking consolation within established cultural rituals, of which the grave was a central component. The majority of funerals in the pre-war period were held in the home and were defined by the religious rites and rituals that assisted the bereaved in making sense of death. ${ }^{60}$ For the devout, a belief in the resurrection consoled them, and they looked to reunion in the afterlife. Many also saw death as a test of their faith. ${ }^{61}$

The rituals accompanying loss were multi-layered and encompassed both the private and public. Until the increased "medicalization" of death in the post-war years, ${ }^{62}$ people died surrounded by family in the privacy of the home. The hostile Australian climate and lack of refrigeration made it imperative for the mortal remains of the deceased to be disposed of quickly, usually within forty-eight hours. Due to these constraints, many interments were private affairs attended by the immediate family. Public memorial services were held some days after the burial, especially for the more prominent members of the community. Many focused on ornate adornments and visible reminders of the deceased's piety and social standing, which is evidence that funerals performed a dual function. They both provided families with the comfort of a religious ritual and allowed them to claim their bereavement publicly.

The significance of the details of death was matched only by the need to know whether, and how, their sons had been buried. Three distinct groups of bereaved are evident in terms of knowledge of burial: those who had unequivocal information that their sons were accorded funeral rites and had a known resting place; those whose sons had not been buried at all or whose grave was not marked; and thirdly, those who had some evidence that their loved one had initially been buried, but the location was unable to be determined after the war. The men and women from the front lines who corresponded with families were intimately aware of how important details of burial were to the bereaved, and they responded accordingly, with reports such as, "I saw him put in the coffin and I placed beautiful flowers round him, mauve stock and white carnations, and I put white flowers in the middle of the Union Jack on the coffin." 63 A battalion chaplain assured another father that his son "had been buried in the military cemetery and a cross is being erected by his battalion to mark his grave. $" 64$

Mr. Freeman of Southport in Queensland was very grateful that a comrade had taken the effort to provide him with a detailed account of his son's death in 1917. Mr. Freeman felt it was,

some little consolation to us all to know that he did not suffer. We all dreaded the thought that perhaps he had been wounded and lay out in the open before death ended his sufferings. We are all pleased to know that his grave has a $\mathrm{X}$ upon it. ${ }^{65}$ 
Another mother replied to the chaplain who had written with the details of her son's burial, "There is none feel it like a mother. He was my youngest child ... I am so glad he was buried decent and that he never suffered any pain." ${ }^{66}$ Rose Venn-Brown was a young Australian working for the Red Cross in France in 1919 when she copied the names of 209 Australians buried at Abbeville Cemetery. She sent the list to her mother in Sydney asking,

Can you notify their people through the Red X that I planted forget-me-nots, primroses and daises on each of them this week for the people out there? I thought they would like to feel something had been done. I am also posting you some prints of the cemetery for any of the people who want them. They will like to know they are being looked after. ${ }^{67}$

Mrs. Venn-Brown had the list published in the Sunday Newes, and the response from grieving parents, particularly mothers, underlines the desperation to know the certain fate of their sons. Mrs. Venn-Brown was inundated with hundreds of letters, some full of gratitude, others still seeking further information. Alfred Wilson's mother wrote, "I will be very grateful if you will let me have one of the prints of my poor dear lad, God bless his Soul,"68 while Mrs. Congdon was "very thankful to receive the same [photograph of the grave] as I am far too old even to be able to go to France and see where my dear boy lies." ${ }^{69}$ Mrs. Boss wrote, "There is nothing I would cherish as the photo of my darling boy's grave, no matter what ever the price might be."70

The gratitude of those families fortunate enough to obtain details of their sons' death and burial stand in stark contrast to the desperation of those who sought some confirmation as to whether their boy had been laid to rest or at least hope for the return of his personal effects. The return of discs, bibles, diaries, watches, and wallets was important for two reasons: firstly it provided proof that the worst was indeed true, and, secondly, the small, familiar items provided a strong, almost physical connection to their sons that would be meaningless to strangers. In April 1918, Mrs. Sproule of Jamberoo on the south coast of New South Wales received a letter from another mother in Stratford, Victoria, whose son had also been killed on the Western Front in 1917. It appeared there had been a mix-up, and Mrs. Swan had received a parcel from Base Records containing letters, photographs, and a wallet belong to Stan Sproule. Mrs. Swan promised to "forward them to you and if you should have received mine, which should have a ring enclosed, I would feel deeply indebted for same."71 While Mrs. Sproule was pleased to receive Stan's mementoes, she had nothing to return to Mrs. Swan.

The Red Cross, AIF Base Records, and battalion officers were not the only avenues for enquiries. Many were directed along less official lines and often overwhelmed the recipients. Sister Wakeford was stationed at a field hospital on Lemnos and reported receiving twenty-nine letters in one mail from people asking for information, "I can imagine how they feel and do all in their power to get into touch with anyone who might have been near their dear ones. Unfortunately, I do not know any of them."72 Vera Deakin's Red Cross Missing and Enquiry Bureau fielded desperate pleas they had almost no chance of being able to answer:

I would like to know how long he lived after the wound. I would like to know the nature of the wound, what complications set in to cause death? Also did he 
suffer much and was he conscious, did he ask for his parents in any way and did he send any message ... If I am not asking too much I would like you to answer this personally please. $^{73}$

Some families faced another challenge, that of relocating a lost grave. Many makeshift cemeteries and temporary graves were destroyed when artillery fire resumed along sectors of the front that had been previously fought over. General Birdwood noted that when the line fluctuated to and fro across an already fought-over area, "men would be at once buried by the Germans with no trace left."74 The details regarding a rough grave marked out and a brief ceremony conducted hastily, perhaps at night, were related back to the families during the war by chaplains, comrades, and the Red Cross. Many families wrote to Base Records after the end of the war insisting that more be done to find lost graves. Major Lean replied to one such letter promising "an intensive search is now being made over all battlefields with a view to locating unregistered graves.",75

Lieutenant Henry Colyer of the 36th Battalion was killed in action near Villers Bretonneux in April 1918 and hastily buried along with a French officer shot by the same sniper. ${ }^{76}$ After the war, his young widow Eileen was distressed to find the Army could find no trace of Henry's makeshift grave despite assurances from a former comrade, Mr Barber, that "I, with a French sergeant and several French soldiers buried both officers side by side." standing Mrs. Colyer's need to establish the location of her husband's resting place, Barber had enclosed a rough, hand-drawn map, "which of course is not drawn to scale and I have marked with a red cross the exact spot where your late Husband, and my friend, was buried and that is about 10 yards from the corner of Hangard Wood."78

Eileen eagerly sent the sketch to the Graves Registration Unit, assuming it would enable them to pinpoint precisely the plot without delay. She received the curt reply that her treasured map had indicated an area of "over half a million square yards", which, "you will readily realise that the map references instead of assisting have only rendered more difficult the search for the grave," thus making further attempts to locate it "futile". ${ }^{79}$ In Eileen's case, the news was a double blow; two parcels containing Henry's possessions had been lost when the Barunga was torpedoed. 80 For families such as the Colyers, a "lost" grave was as devastating as it was for those who had had none in the first instance, as it undermined the comfort provided by the physical reality of a grave. As Thomas Laqueur has argued, "the nexus of name, body and place ... has deep cultural roots." 81

\section{Gone? Where?}

Many families were hampered in their emotional recovery by the doubt and uncertainty surrounding the fate of the missing. Over 25,000 of the 60,000 Australian dead had no known grave. The silence provided the opportunity for false hope to develop and conjecture to spread. Many clung to the possibility that the men could still be alive, even into the 1920 s and beyond.

A young woman whose brother had been listed as missing wrote, "I feel at times my darling brother is not killed but will return to us ... at times $I$ think that he is in a hospital or he might have lost his memory, we cannot tell." 82 Touchingly hopeful that her letter would reach someone in Europe who might recognize him, she declared, "if our darling happens to be alive, he has dark brown curly hair and blue eyes ... he is not very tall." 83 
Walter Farquharson had been killed in action on 3 May 1917 but was initially listed as missing, and conflicting reports as to his whereabouts were sent to his parents in Kiama throughout 1917. His death was finally confirmed by telegram in January 1918. However, in a letter to his father, written after the war, a comrade detailed the action at Bullecourt when Walter went missing and added, "I could not say he was killed and sincerely trust that he is still alive, although I would not entertain the slightest hope." 84 This ambiguity would not have assisted his parents to accept the fact of his death.

Private Percy Clout's mother, from Wollongong, continued to write to the Defence Department for many years seeking any further news of her son. A fellow soldier had written in 1916, "there is no doubt that he has fallen ... I am very sorry indeed to say that there is no hope of his safety, ${ }^{, 85}$ but rumours persisted that Percy had only been wounded, not killed, and "may be a mental case". ${ }^{6}$ Percy's body was never found, and the Clouts were still publishing annual In Memoriam notices until at least 1925 for the son who was "lost at Pozières". 87

\section{We will remember them}

The process of creating a public memory of private pain had begun during the war with the unveiling of honor boards and framed commemorative photographs. In July 1915 two prominent Wollongong citizens donated 2/6 and 2/- respectively toward a Wollongong District School Old Boy's Fund for a memorial tablet to be erected at the school, neither knowing that when it was finished it would record the names of both their sons. ${ }^{88}$ The Farquharsons dedicated a tablet to Walter in the Kiama Methodist Church in 1917. Beside his name was the simple inscription, "An Anzac". Another service was held in October 1918 to add the name of his younger brother, 18-year-old Frank.

Jacqueline Manuel has looked at ways in which Australian women articulated their grief through poetry and found that the poems written by grieving mothers, wives and sisters about their specific loss were "characterised by a[n] intimate register: by a deep pathos and sense of inner struggle with the personal consequences of loss and mourning". ${ }^{89}$ While some of the stylized verses used in the In Memoriam notices tended to allude to higher ideals of duty and honor, the intimacy of women's poetry often concentrated on personal vignettes of family life and childhood memories whose loss would never be consoled by the "public tropes of nationalism and noble sacrifice". 90

A memorial opened by General Sir John Monash in 1926 at the University of Melbourne bore the inscription, in Latin, "To those who served well ... their University, their Country, the Empire." 91 The trinity exalted in the proclamation did not allow the bereaved opportunity to claim public acknowledgement of their loss. Many families placed their own individual memorials to sons in their local church (as the Farquharsons had done), school or lodge, perhaps as a retort to the somewhat haughty posture of elite memorials, like the university's. Fathers were often the main instigators of need to secure public recognition of their loss. Mr. Freeman found comfort that Ned "was indeed a Son to be proud of ... We are erecting a Memorial Tablet in the Local English Church in appreciation of his self-sacrifice." 92 The opportunity to place a memorial, a stone, or a plaque within their community was one seized by many who could afford the expense. It echoed the pre-war imperative of a grave as an intrinsic part of mourning ritual and gave substance to loss in the absence of a body. 
The sense of loss did not end in 1918. As noted earlier, parents whose sons had been listed as missing, and often later declared dead, still clung to hope, writing to the Defence Department into the 1930s, as did those whose sons' graves had been lost. And the process of creating a public memory of private pain took a more substantial form after the war.

Families with known graves were given the opportunity to pay for an epitaph for the headstone at a cost of $3 \frac{1}{2}$ pence per letter, with a maximum of sixty-six letters, through the Imperial War Graves Commission. These demonstrated not only the extent of grief but also the balm of conventional Christian references or a suggestion of heroism, such as, "The supreme sacrifice for God, King and Country" 93 and "Responsive to his country's call / He gave his best, his life, his all."94 Many made particular mention of the gulf of distance: "The midnight stars / are gleaming / on a grave I cannot see" and "Tho far away you are still near." 95 In a similar vein, mothers in particular, exhorted visitors to foreign cemeteries to do that which they could not: "Will some kind hand in that far off land / Place on his grave a flower for me" 96 or to be aware of sacred ground, "Tread gently on the green grass sod / A Mother's love lies here."97

Some parents laid their grief bare and chose such sentiments as "My only darling son", 98 "My well loved laddie / Waiting for Mother", 99 and "Only a boy but died / As a man for liberty \& freedom / His Mum \& Dad." "100 Other epitaphs ranged from ambivalence to anger, such as, "Some day, some time, we'll understand"101 and "I've no darling now / I'm weeping / Baby and I you left alone."102 Some proposed inscriptions were rejected. Alfred Wilson's parents, from Sydney, having been told that their initial choice "exceeds the limit ... of 66 letters (each space counting as a letter)", ${ }^{103}$ sent their revised epitaph on Armistice Day, 1921: "Lying asleep far from home but always in the memory of Mum and Dad." 104

Length, however, was not the only reason for rejecting proposed inscriptions. The Imperial War Graves Commission (IWGG) had warned that "inappropriate" inscriptions would be returned for "modification". The most notable of these was "His Loving Parents Curse the Hun" and its amended version "With every breath we draw / We curse the Germans more," both of which were "declined". ${ }^{105}$ The meaning and legacy of the war were contested even in these last personal statements on faraway graves; some bereaved families were clearly still consumed with a furious anger; others may have been emboldened to intimacy by the permanency of the IWGC cemeteries, while others still continued to find comfort in the consolation of glorious sacrifice.

Many well-meaning relations, friends, religious advisors, and newspaper columnists exhorted the bereaved to be stoic and forbearing in their grief. This proscription was a result of a backlash against the ostentatious funerals and immodest mourning rituals of the Victorian era and the sheer scale of death wrought by the war. It also reflected an enduring cultural model of masculinity whereby overt displays of emotion were to be restrained. ${ }^{106}$ The negotiation of the experience of death had moved sharply from the public sphere to the private. And it remains thus, to this day.

Newspapers also advocated the bereaved bear their loss with quiet acceptance. An editorial written in response to the first casualty lists from Gallipoli advised they, "may rest contented in the assurance that their dear ones have died wreathed in honour". ${ }^{107}$ Another columnist saw Gallipoli as the ultimate proof that "Spartan mothers had produced Spartan sons." 108 
This model of mourning based on "stoicism, privacy and survival" 109 could lead to prolonged and unresolved chronic grief, particularly when combined with the doubt and devastation caused by the sudden, violent death of adult children a great distance from home. Australia certainly had its "cult of the dead" that persisted through the inter-war years, indicating that many families never recovered from wartime losses that they were unable to fully accept. ${ }^{110}$

Throughout the country, there remained many who were so traumatized by loss that no ritual or commemoration could provide comfort or relief, whose lives were haunted by the often silent sorrow of bereavement. ${ }^{111}$ Having been denied the comfort of pre-war mourning rituals and certain knowledge of their men's last hours or days, many found themselves living in the memory of the past, unable to reconcile or resolve their grief. It is probable that many eschewed the pomp and public display of remembrance days, preferring their own private reflection. For those who had taken Mary Gilmore's advice and would "creep into bed in the dark and weep", 112 the depth of their grief was so enormous that it caused many "constantly to live within, or even retreat from, the objective world to one sustained principally by memory or longings". 113

Justice Henry Higgins felt "my grief has condemned me to hard labour for the rest of my life."114 More than twenty years after her brother's death in battle, a daughter wrote of her father's sorrow on losing his son:

The loss of a son, an only son, is something that no other person can assess. I do not think that anyone, anyone at all, can fully know what the loss meant to my father, and increasingly as he grew older. Only the millions of other fathers who suffered in the same way can realise it. It was always in [his] heart; but he could seldom speak of it. ${ }^{115}$

The national monuments, local memorials and official days of mourning and commemoration became very public - and publicly contested - expressions of remembrance ${ }^{116}$ and reveal often uncomfortable undertones of militarism and exclusionary masculinity. Of greater interest are the more private experiences of bereavement that existed in the minds and in the homes of those for whom "[a]ll days were days of commemoration". ${ }^{117}$

Implicit in the literature, however, is a recognition that bereavement was not only about the dead. One in five of the First AIF did not return, but the remaining four in five did; however, they were not always the same men who had left Australia. What follows provides two examples. As an elderly man, Les Sproule still clearly recalled the return of his two brothers, Oliver and Hugh, from the war. Oliver, the younger of the two, arrived in Jamberoo on the south coast of New South Wales, in 1919. He looked fit and well following an extended leave in Scotland and England after being discharged, but his family were shocked when Hugh, the older brother, stepped off the train at Kiama station later in 1919. Ten-year-old Les, who had also welcomed Oliver home, rushed from school to meet his big brother:

I remember the hug he gave me and said he couldn't believe I'd grown so much in that time. I was so sad when I saw him. He was so thin and sick looking ... Hugh was suffering from diarrhoea, which later became chronic and he still had it until his death at forty-seven years on 1 October $1942 .{ }^{118}$ 
Similarly, Patsy Adam Smith recalled her mother's revulsion after visiting a brother-inlaw gassed in France, "I can't stand it. I can't go again ... No, but the smell. When he coughs ... and breathes out ... oh, I'm going to be sick." 119

If we are to engage more fully with the multi-layered and complex issues surrounding individual experiences of wartime bereavement, then the term "bereavement" itself, cannot just refer to loss as death. It must also mean the loss of what existed before, the essence of self, a potential unfilled, so clearly evident in Hugh Sproule and Patsy Adam Smith's uncle. In those terms, many Australian families who sent a man to war were bereaved. The idea of noble sacrifice in war was quickly seen for the lie that it was by those who nursed their damaged men at home or visited them in the asylums and hospitals. Many parents who had lost their sons in the trenches may have envied those whose men had survived. The parents of returned men might not have thought themselves so fortunate after helplessly watching their sons suffer through "days of agony and nights of torture". ${ }^{120}$ Many families, then, mourned, if not a life, then the loss of confidence in the future and a potential unfulfilled. The burden of caring for these men, trying to understand their experiences, continuing to love them, must have seemed unbearable, particularly for ageing parents. ${ }^{121}$

An enduring legacy of the Great War for Australian society generally was its profound role in permanently altering the social expectations that guided mourning behaviors and rituals. The war marked the end of the Victorian era of elaborate, if not ostentatious, public expressions of grief and loss. The wearing of mourning black declined substantially after 1918, and public funeral pageantry was considered an "unnecessary indulgence", ${ }^{122}$ even grotesque, in communities that had suffered substantial numbers of dead. While the funeral and burial (and increasingly, cremation, for non-Catholics) remain at the core of the formal mourning period, these are much more likely to be subdued affairs, or even private memorial services, to which only close family and friends are invited, rather than the pre-war model which attracted large crowds. In the inter-war years, particularly, the social proscription sought to inspire a public stoicism, and the overt expression of emotion was discouraged. While this has changed somewhat since the 1980s, grieving is essentially still a private matter, performed within the domestic confines of the family and home. Sympathy cards and In Memoriam notices published in the press became a popular way to claim loss and offer support to the bereaved; however, the emphasis was, and to a great extent, remains, on dignified and private sorrow, encapsulated by popular verses such as,

\section{We mourn for them in silence \\ But not with outward show \\ For they who mourn sincerely \\ Mourn silently and low. ${ }^{123}$}

For the many most directly involved, the often-silent trauma and shadow of a war without precedent and the memory of young men who had met ugly, violent, and sudden deaths so far from home permeated homes, hospitals, farms, and hamlets across the country. Some quietly endured; others broke apart. The one element that may well be common to all the bereaved of the Great War was the long-term effects of their loss. For 
some, grief became the primary motif for the remainder of their days, leading to psychological and physical collapse; for most, the memory of their sons haunted them into the inter-war years. For all of the bereaved, the war was to be a pivotal event of their lives, after which nothing would ever be the same.

\section{Notes}

1 In Memoriam notice, Sergeant Jack Buckley, South Coast Times, 25 October 1918.

2 Bill Gammage, The Broken Years: Australian Soldiers in the Great War (Ringwood: Penguin Books, 1975), xvii.

3 L L Robson, "The Origin and Character of the First AIF 1914-1918: Some Statistical Evidence", Historical Studies, 15:61 (1973), 739.

4 Ken Inglis, Sacred Places: War Memorials in the Australian Landscape (Carlton South: Melbourne University Press, 1998), 91-92.

5 L L Robson, Australia and the Great War (South Melbourne: Macmillan, 1970), 27.

6 Pat Jalland, Death in the Victorian Family (Oxford: Oxford University Press, 1996), 193.

7 Jacqueline Manuel, "We Are the Women Who Mourn Our Dead': Australian Civilian Women's Poetic Responses to the First World War," Journal of the Australian War Memorial, Issue 29 (November 1996) available at www.awm.gov.au/articles/journal/j29/ manuel.

8 Jay Winter, cited in Jalland, Death in the Victorian Family, 373.

9 Sam McSkimming, 2007 John Laffin Memorial Lecture, available at https://fffaif.files.word press.com/2007/12/microsoft-word-mcskimming-a-1st-2005.pdf, p. 6.

10 Tony Walter, On Bereavement - The Culture of Grief (Buckingham: Open University Press, 1999), 28

11 Pat Jalland, Australian Ways of Death: A Social and Cultural History 1840-1918 (South Melbourne: Oxford University Press, 2002), 1.

12 G W F Hegel, cited in Joachim Whaley (ed), Mirrors of Mortality: Studies in the Social History of Death (London: Europa Publications Ltd, 1981), 1.

13 A private's rate of pay was six shillings per day from the time of embarkation, a significant amount, given that the average working man's daily wage at home was in the vicinity of three-four shillings a day.

14 Mrs. Britomarte James, My Personal Reminiscences of the War, Mitchell Library of NSW, MSS 2871.

15 Les Sproule, These Were the Best Years, unpublished autobiography, University of Wollongong Archives, D158/2/1, Stuart Piggin Collection, 9.

16 By contrast, the class barriers so evident in the British Army persisted beyond life, where the relatives of enlisted men were sent a letter by ordinary post. Only the families of officers received telegrams, delivered by unlucky, and probably bemused, telegraph boys. See J M Winter, "Communities in Mourning," in Frans Coetzee and Marilyn Shevin-Coetzee (eds), Authority, Identity and the Social History of the Great War (Providence: Berghahn Books, 1995), 327.

17 Phillip Dowe, Rector of Bulli, cited in Stuart Piggin, Faith of Steel: A History of the Christian Churches in Illawarra, Australia (Wollongong: University of Wollongong Press, 1984), 183.

18 Mrs. Britomarte James, My Personal Reminiscences of the War.

19 Mrs. Britomarte James, My Personal Reminiscences of the War.

20 Letter dated 16 June 1918 from Mr Freeman to Mr Grigor, Australian War Memorial (hereafter AWM) PR00480 - E A Freeman.

21 Letter dated 31 January 1915 from Lieutenant Syd Duchesne to his father, private papers in the possession of the author.

22 Letter dated 31 January 1915 from Lieutenant Syd Duchesne to his father.

23 Letter dated 31 January 1915 from Lieutenant Syd Duchesne to his father.

24 Letter dated 16 June 1919 from F Goldsmith to Mrs. Venn-Brown, AWM 2DRL/0598 Venn-Brown.

25 See Bart Ziino, A Distant Grief: Australians, War Graves and the Great War (Crawley: University of Western Australia Press, 2007).

26 Mrs. Britomarte James, My Personal Reminiscences of the War. 
27 Letter dated 6 October 1915 from Mrs. M Drummond to The Hon M Charlton, Member for North Lambton, National Archives of Australia (hereafter NAA), B2455, Farr, VE 828.

28 Letter dated 27 November 1916 from Ivy Meggy to Base Records, NAA, B2455, Percy Arthur Meggy, No. 501.

29 Letter dated 3 September 1917 from W Birdwood to Mrs. S Meggy, AWM 1DRL/0495.

30 Letter dated 2 February 1932 from Mrs. S Meggy to John Treloar, in AWM 93/12/11/ 4710, Meggy Family.

31 Letter (undated) from Mrs. L G Buik to the Secretary, Defence Department, NAA, B2455, Ralph Nation.

32 Letter dated 6 August 1923 from Mrs. E Nation to Base Records, NAA, B2455, Eric Nation.

33 Death certificate, S G Nation, District of Norwood Deaths, 1924, South Australian Registry of Births, Deaths and Marriages.

34 Letter dated 26 July 1915 from Oliver Cumberland to his sister, AWM PR 86/147 Cumberland.

35 John Garibaldi Roberts, cited in Joy Damousi, The Labour of Loss: Mourning, Memory and Wartime Bereavement in Australia (Cambridge: Cambridge University Press, 1999), 59. For an excellent study on facially disfigured veterans, see Kerry Neale, Without the Faces of Men: Facially Disfigured Great War Soldiers from Britain and the Dominions, PhD Thesis, UNSW Canberra, March 1915.

36 Letter (undated) from Linda Johnston to Mrs. Carthew, cited in Noel Carthew, Voices from the Trenches: Letters to Home (Sydney: New Holland, 2002), 71.

37 Letter dated 24 August 1916 from Sister Maud Ashdown to her younger brother, AWM 2DRL/286 - C P Ashdown.

38 Letter dated 30 June 1915 from Sister Sylvia Weigall to Mrs. Petrusch, AWM PR00507 AEJ Petrusch.

39 Letter dated 8 November 1917 from Douglas Ferguson to his father, James Ferguson, on the death of elder brother Hector, AWM PR 00005 - Ferguson Brothers.

40 Joy Damousi, "Private Loss, Public Mourning: Motherhood, Memory and Grief in Australia during the Inter-war Years," Women's History Review, 8:2 (1999), 366.

41 Letter (undated) from Pte D Chopping to Mrs. Roose, on the death of her son Pet J W Roose, South Coast Times, 27 July 1917.

42 Letter dated December 1916 from George Whyte to Mrs. Higgins, AWM, 3DRL/421 Collection of letters Mervyn Bournes Higgins, 8th Light Horse Regiment.

43 Letter dated 21 August 1918 from General Birdwood to Mrs. Munro, AWM 1DRL/526 Private E C Munro MM.

44 Letter dated 11 August 1916 from Matron Cecily Dale, Commandant of Bevan Military Hospital, Sandgate, to Mrs. Houghton, Illawarra Mercury, 6 October 1916.

45 Letter dated 20 October 1918 from Matron A M Sampson to Mrs. Sarah Greenhalgh, AWM MSS1647 - Bower and Greenhalgh Families correspondence.

46 Letter dated 28 November 1915 from Matron H L Reddock to Mrs. O’Donnell, AWM 2DRL/0316 - Frederick Warren Muir.

47 Letter dated 30 June 1915 from Sister Sylvia Weigall to Mrs. Petrusch, AWM PR00507 AEJ Petrusch.

48 Letter dated 11 August 1916 from Matron Cecily Dale to Mrs. Houghton, Illawarra Mercury, 6 October 1916.

49 Jay Winter, Sites of Memory, Sites of Mourning: The Great War in European Cultural History (Cambridge: Cambridge University Press, 1995), 35.

50 Tanja Luckins, The Gates of Memory: Australian People's Experiences and Memories of Loss and the Great War (Fremantle: Curtin University Books, 2004), 48.

51 Witness Statement dated 6 June 1916, Sergeant Beale, AWM 1DRL/0428 - Australian Red Cross Society Wounded and Missing Enquiry Bureau files, 1914-18 War - 1747 Private William Downey.

52 Witness Statement dated 20 September 1917, Private Wiggins, AWM 1DRL/0428 - Australian Red Cross Society Wounded and Missing Enquiry Bureau files, 1914-18 War - 16,410 Private William George Vincent Hillcoat. 
53 Witness Statement dated 3 January 1918, Private Thatcher, AWM 1DRL/0428 - Australian Red Cross Society Wounded and Missing Enquiry Bureau files, 1914-18 War - 16,410 Private William George Vincent Hillcoat.

54 Damousi, The Labour of Loss, 25

55 Letter dated 23 January 1916 from Gunner G Lewis to Mrs. O’Donnell, AWM 2DRL/0316, Frederick Warren Muir.

56 2/Lt W M Cameron, cited in Gammage, The Broken Years, 105.

57 Letter dated 21 October 1918 from George Elliott to Maria Keat, Keat Papers, cited in John McQuilton, Rural Australia and the Great War: From Tarrawingee to Tangambalanga (Carlton South: Melbourne University Press, 2001), 57.

58 Letter dated 10 October 1917 from Private Waugh to Mrs. Webb, AWM, PR 04017 Papers of Gunner Cappur Mitchell Webb.

59 For an overview of the process by which donations to the AWM were requested, see Anne Marie Conde, "Capturing the Records of War: Collecting at the Mitchell Library and the Australian War Memorial," Australian Historical Studies, 37:125 (April 2005), 134-152.

60 Graeme M Griffin, "Defining Australian Death: Religion and the State" in Allan Kellehear (ed), Death and Dying in Australia (Melbourne: Oxford University Press, 2000), 46.

61 Jalland, Death in the Victorian Family, 41.

62 Jalland, Australian Ways of Death, 326-327.

63 Letter dated 11 August 1916 from Matron Cecily Dale to Mrs. Houghton, Illawarra Mercury, 6 October 1916.

64 Letter from Roman Catholic Chaplain Captain J Halpin to Mr and Mrs. Dobing, Illawarra Mercury, 15 June 1917.

65 Letter dated 16 June 1918 from Mr. E A Freeman to Mr. G Grigor, AWM PR00480 E A Freeman.

66 Letter from Mrs. E Brooks to chaplain, cited in Patsy Adam Smith, The Anzacs (Ringwood: Penguin Books, 1978), 175.

67 Letter dated 12 April 1919 from Rose Venn-Brown to her mother, AWM 2DRL/0598 Venn-Brown.

68 Letter dated 16 June 1919 from Mrs. E Wilson of Kogarah to Mrs. Venn-Brown, AWM 2DRL/0598 - Venn-Brown.

69 Letter dated 16 June 1919 from Mrs. B Congdon of Kogarah to Mrs. Venn-Brown, AWM 2DRL/0598 - Venn-Brown.

70 Letter dated 24 June 1919 from Mrs. C Boss of Lyndhurst to Mrs. Venn-Brown, AWM 2DRL/0598 - Venn-Brown.

71 Letter (undated) from Mrs. E J Swan to Mrs. T Sproule, Kiama Independent, 13 April 1918.

72 Correspondence dated 31 July 1915 from Sister Wakeford, Illawarra Mercury, 7 September 1915.

73 Mrs. Ellen Jones, cited in Bruce Scates, "The Unknown Sock Knitter: Voluntary Work, Emotional Labour, Bereavement and the Great War," Labour History, Issue 81 (November 2001), 41. Vera Deakin answered the letter herself.

74 Letter dated 21 April 1917 from General Birdwood to Mrs. Meggy, AWM 1DRL/0495 Meggy Family.

75 Letter dated 18 October 1921 from Major Lean to Mrs. Colyer, AWM PR 00599 - Henry Maxwell Colyer.

76 Form D16, accompanying Casualty Form - Active Service, NAA, B2455, Colyer, H M.

77 Letter dated 20 November 1921 from Laurie Barber to Mrs. Colyer, AWM PR 00599 Henry Maxwell Colyer.

78 Letter dated 20 November 1921 from Laurie Barber to Mrs. Colyer, AWM PR 00599 Henry Maxwell Colyer.

79 Letter dated 6 April 1922 from the Official Secretary, Australia House, London to Mrs. Colyer, AWM PR 00599 - Henry Maxwell Colyer.

80 Letter dated 24 February 1919 from Mrs. E Colyer to Base Records, NAA, B2455, Colyer, $\mathrm{H}$ M.

81 Thomas W Laqueur, "Names, Bodies and the Anxiety of Erasure," in John R Gillis (ed), Commemorations: The Politics of National Identity (Princeton: Princeton University Press, 1994), 127. 
82 Letter dated 19 June 1919 from Miss Hannah Fenwick to Mrs. Venn-Brown, AWM file 2DRL/0598, Venn-Brown.

83 Letter dated 19 June 1919 from Miss Hannah Fenwick to Mrs. Venn-Brown, AWM file 2DRL/0598, Venn-Brown.

84 Letter datd 30 November 1918 from J C Hepher to Mr P Farquharson, in author's possession.

85 Letter from LS Whitbread to E Clout, Wollongong Local Studies Library, file M/WHI, 1916.

86 AWM 1DRL/0428 Australian Red Cross Society, Wounded and Missing Enquiry Bureau files, 1914-1918 War - 3732, Private Edward Percy Clout.

87 South Coast Times, 8 August 1925.

88 South Coast Times, 16 July 1915.

89 Manuel, "We Are the Women Who Mourn Our Dead."

90 Manuel, "We Are the Women Who Mourn Our Dead."

91 Bart Ziino, "Claiming the dead: Great War Memorials and Their Communities," fournal of the Royal Australian Historical Society, 89:2 (2003), 158.

92 Letter dated 16 June 1918 from Mr E A Freeman to Mr G Grigor, AWM PR00480 E A Freeman.

93 Epitaph on the grave of Bombardier W H Benson, Australian Field Artillery, Killed in Action 21 November 1915, aged 25, Ari Burnu Cemetery, Anzac Cove, photograph in author's possession.

94 Epitaph on the grave of Private Frederick Stanley Barling, 17th Battalion, Killed in Action, 9 October 1915, aged 21, Beach Cemetery, Anzac Cove, photograph in author's possession.

95 McSkimming, Laffin Memorial Lecture, 4.

96 Epitaph on the grave of Sergeant W Watson, Bullecourt British Cemetery, France, photograph in author's possession.

97 Epitaph on the grave of Private John McAllister, Shrapnel Valley Cemetery, Anzac Cove, photograph in author's possession.

98 Epitaph on the grave of Sergeant F A Rawlings, 10th Australian Light Horse, KIA 7 August 1915, aged 27, Ari Burnu Cemetery, Anzac Cove, photograph in author's possession.

99 Epitaph on the grave of Private Robert Laurence Angus, 14th Battalion, KIA 19 May 1915, Aged 21, Shrapnel Valley Cemetery, Anzac Cove, photograph in author's possession.

100 Epitaph on the grave of Private H J Burton, 23 Battalion, KIA 30 November 1915, aged 18, Ari Burnu Cemetery, Anzac Cove, photograph in author's possession.

101 Epitaph on the grave of Corporal A Capps, Quinns Post Cemetery, Anzac Cove, photograph in author's possession.

102 Epitaph on the grave of Private J E Barclay, 8 Battalion, KIA 21 June 1915, aged 22, Shrapnel Valley Cemetery, Anzac Cove, photograph in author's possession. "Baby", named Jack, after his father, was born four months after his father's death. Jack Barclay served in (and survived) the Second World War.

103 Letter dated 5 November 1921 from Base Records to Mrs. E V Wilson of Kogarah, NAA Personnel files, Alfred Benjamin Wilson.

104 Letter dated 11 November 1921 from Mrs. E Wilson of Kogarah to Base Records, NAA B2455, Alfred Benjamin Wilson.

105 Minutes of the Proceedings of the 41st Meeting of the IWGG, 17 January 1922, cited in Bruce Scates, Return to Gallipoli: Walking the Battlefields of the Great War (Cambridge: Cambridge University Press, 2006), 48.

106 Jalland, Australian Ways of Death, 306.

107 South Coast Times, 7 May 1915.

108 Rutherglen Sun, 21 September 1915, cited in John McQuilton, Rural Australia and the Great War, 133.

109 Beverley Raphael, "Grief and Loss in Australian Society," in Kellehear (ed), Death and Dying in Australia, 119.

110 David Cannadine, "Death and Grief in Modern Britain," in Whaley (ed). Mirrors of Mortality, $217-232$. 
111 Manuel, "We Are the Women Who Mourn Our Dead."

112 Mary Gilmore, "These Following Men" in Mary Gilmore, The Passionate Heart (Sydney: Angus and Robertson, 1918), 2.

113 Manuel, "We Are the Women Who Mourn Our Dead."

114 Mr Justice H B Higgins, cited in Jalland, Australian Ways of Death, 321.

115 K Garvin, cited in Cannadine, "Death and Grief in Modern Britain," 216-217.

116 See, for example, John A Moses, "The Struggle for Anzac Day 1916-1930 and the Role of the Brisbane Anzac Day Commemoration Committee," Fournal of the Royal Australian Historical Society, 88:1 (June 2002), 54-75.

117 Daily Telegraph, 26 April 1920, cited in Damousi, The Labour of Loss, 34.

118 Les Sproule, Those Were the Best Years, 16.

119 Adam Smith, The Anzacs, 3.

120 Illawarra Mercury, 14 November 1924.

121 See Marina Larsson, Shattered Anzacs: Living with the Scars of War (Sydney: UNSW Press, 2009).

122 Pat Jalland, Changing Ways of Death in Twentieth Century Australia: War, Medicine and the Funeral Business (Sydney: UNSW Press, 2006), 98.

123 Jalland, Changing Ways of Death in Twentieth Century Australia, 98. 


\title{
23 \\ SOVIET GEMETERIES
}

\author{
Svetlana Malysheva
}

\section{Prologue: cemeteries before the revolution of 1917}

By the beginning of the 20th century, Russian death spaces and funerary culture preserved largely the features formed by the Christian (Orthodox) religious tradition and, to some extent, pre-Christian pagan ideas and customs. ${ }^{1}$ Despite the fact that since Peter the Great, the funeral ritual of the monarchs has become more and more secular, ${ }^{2}$ the influence of secularization and industrialization, even in urban mortuary culture - not to mention rural culture - was minimal until the revolution of 1917 and the Bolsheviks' coming into power. This was largely due to the privileged position of the Orthodox Church within the state. ${ }^{3}$ The burial of the Orthodox, who made up the majority of the population, was almost entirely within its sphere of influence. Alternatively, funerals of those of other faiths were within the purview of the representatives of the respective religions.

Cemeteries in Russia were organized according to a religious principle and were run by church parishes and communities. Tombstones in these cemeteries therefore indicated the religious affiliation of the deceased. For example, Orthodox burials included a graveyard cross or a cross on the gravestone, and often icons, the name of the deceased and the dates of birth and death (frequently only death as the time of transition into "eternal life"). ${ }^{4}$

The cemetery space was lined up hierarchically: burial places were put into seven categories based on the owner's financial and social means. The first category was the highest one and included the best and most expensive burial sites, such as a site near the church. At the other end, the burial spaces of the seventh category - meant for the poor and destitute - were usually located in abandoned, distant sites and free of charge. Since the early 1870 s, burials were split into five main categories. ${ }^{5}$ The rules of "exclusion" applied in this space as well: outside the sanctified cemetery land, beyond the surrounding fence, were buried not only suicide victims but also those who died a violent, thus "bad" death, unbaptized babies and other marginalized members of the confessional community. However, by the beginning of the 20th century, these rules were often circumvented. They relied on archaic ideas of a "good" death (peaceful, in one's bed, surrounded by loved ones, after all the necessary preparations were made), as a result of which the deceased would rest in a communal cemetery and enter the category of "ancestor", who was entitled to be honored duing the traditional days of mourning. Consequently, those who suffered a perceived "bad" death (violent or unauthorized, sudden, unprepared, without witnesses or surrounded by strangers), could not find peace and posed a danger to the living themselves, which is why they tried to bury them outside 
the community cemetery as part of a special procedure. Dying on the battlefield and being buried in a mass grave were both really demeaning for the deceased, and hence these bodies were separated from the community cemetery.

At the end of the 19th and the beginning of the 20th century, traditional notions of death and the experience of dealing with the deceased were fundamentally challenged. Bio-political utopias of "overcoming death" - inspired by achievements in science and technology - called these traditional approaches into question. Emerging ideas concerning the technical feasibility of physical immortality called conventional religious ideas about life, death and immortality into question. ${ }^{6}$ The strategy of terror and the glorification of suicide within the subculture of Russian Radicalism, coupled with a wave of youth protest suicides added up to a changed public consciousness regarding the marginal status of suicide - still condemned by the Church and the laws of the Russian Empire. ${ }^{7}$ But the deepest and most consequential impact on the changing funeral culture and the ideas about death attached to it resulted from the First World War and then the Civil War in Russia. Millions of deaths on the battlefields and the burial of the dead in mass graves contributed to the de-sacralization of death and to the minimization of the burial rites, especially with regard to their emotiveness. Even the emerging cult of fallen soldiers behind the front lines - with magnificent burial rituals, which were supposed to compensate symbolically for these practices at the front - was artificially interrupted by the revolution of $1917 .^{8}$ In any case, the war contributed to the transfer of death from the private, family and community sphere to the public sphere.

After the October Revolution of 1917, the transformation of all aspects of life in Russia deeply affected the funeral domain and culture. The solution of burial issues, the treatment of the dead and cemetery policy were in harmony with the Bolsheviks in a number of contexts related to governance and domination: the consolidation of a new ideology and the fight against religion, the political struggle against opponents of the regime and their repression, the implementation of a new social hierarchy, economic policy and austerity regimes, administrative processes of urbanization and urban planning, the assertion of new emotional regimes and norms, and so on.

\section{Management of the Soviet funeral business and cemeteries}

Up until the 1930s - what can be identified as the early period of the history of the Soviet cemeteries and funerary affairs - cemeteries partly turned out to be a field for revolutionary experimentation, an opportunity for mobilization of material resources and active economic appropriation, while preserving their former structure and order to a large extent, because the Soviet authorities had neither the means nor strength to pay sufficient attention to it. The Decree of the Soviet government (Council of People's Commissars) of December 7, 1918, "On Cemeteries and Funerals", 9 proclaimed the equality and uniformity of funerals for all citizens at no charge and, in fact, abolished the confessional and cultural homogeneity of Russian cemeteries. Theoretically, from now on anyone could be buried in any cemetery, regardless of his or her nationality or religion. The regulation and organization of funerals were secularized, and the funeral business was transferred to the local authorities, the soviets (councils). Their incompetence and helplessness in dealing with these issues, aggravated by the mass deaths during the first years of Soviet power, led to a funeral crisis; when cemeteries were overwhelmed by the number of necessary burials and failed to come up with a solution, they accumulated 
unburied corpses. ${ }^{10}$ In the years between 1922 and 1929, the period of the New Economic Policy (NEP), ${ }^{11}$ cemeteries were placed briefly under the control of religious communities. However, after the collapse of the NEP, control was re-transferred to the department of public utilities. In fact, throughout the Soviet period, control of the funeral business remained with the government bodies that managed the local economy or, in rural areas, with village councils. Until 1930, the central structure of communal services management, which included overseeing cemeteries, rested within the system of the People's Commissariat of Internal Affairs. In 1931, the apparatus for centralized management of the whole communal economy of the Russian Soviet Federative Socialist Republic (RSFSR) was created - the People's Commissariat of Communal Economy of the RSFSR (since 1946 the Ministry of Communal Economy of the RSFSR, and since 1971 the Ministry of Housing and Communal Economy of the RSFSR).

During the anti-religious campaigns of the 1920s and 1930s, old confessional cemeteries were dissolved all over the country. In the 1920s, too, monastery cemeteries were closed and dismantled in Moscow. The re-development of cities during the industrialization of the 1930s also led to there being fewer old confessional cemeteries. Usually they were overcrowded, and they now found themselves inside the city itself. Confessional cemeteries were therefore closed, and new public, "international" cemeteries were opened outside the municipal boundary. The closed confessional cemeteries made space for parks and squares, were used for industrial and road construction or were replaced by sports complexes. During these years, the authorities positioned cemeteries as an inherent element of the economic system of the country and integrated them tightly into the planned economy's operation. Annual reports of cemeteries and the Moscow Crematorium at this time were prepared on the same standard forms as the reports of any other enterprise or organization. Soviet organizations (Rudmetaltorg - Joint Stock Company for Trade in Ore, Metals and Scrap Metals in the system of the Supreme Soviet of the People's Economy of the USSR) were allowed to recover scrap metal in cemeteries, for which the gravestones were removed. Removed tombstones were to be "sold" through the People's Commissariat of Finance, to help provide for the economic needs of the country. Accordingly, tombstones were "re-used" for roads, bridges and construction or purposed for the second time as material for new gravestones. In addition, from the 1930s, cemetery workers were included in the socialist competition for the implementation of the economic plan. ${ }^{12}$

The period of the "Great Patriotic War" and the difficult years of post-war reconstruction caused the Soviet cemetery economy to deteriorate. The condition of the Soviet cemeteries generated a fair number of complaints from citizens. ${ }^{13}$ In the last few Soviet decades, the funeral economy was increasingly formalized through bureaucratic regulation by Soviet authorities, on the one hand, and saw the spontaneous development of shadow markets offering funeral services, on the other.

Most cities and especially villages did not have any special services for cemeteries during the Soviet era. Even in 1953, out of the 761 cities of the RSFSR only 129 had trusts, offices or funeral parlors. In other cities, special trusts and/or the municipal offices of cleaning, road construction or green economy ran the cemeteries. ${ }^{14}$ The authorities did not have enough energy and resources for the funeral sector, and it was therefore financed based on a residual principle with no budgetary priority. Over the last four and a half decades of Soviet power, the authorities attempted to streamline the funeral business. However, they tried to solve problems mainly by means of bureaucratic regulation, 
by issuing resolutions, rules und instructions. The most detailed regulation of burial and funeral service is the "Instruction on the Order of Funeral and Maintenance of Cemeteries in the RSFSR", approved by the Order of the Ministry of Housing and Communal Services of the RSFSR on January $12,1979 .{ }^{15}$ The instruction consisted of four sections: "funeral arrangements", "funeral order", "cemetery arrangement", and "cemetery operation and maintenance". This was the last major document of the Soviet era detailing not only the official view of the Soviet authorities on the funeral business and its organization, but also how a burial should be staged as expressed in the funeral rite. This document summed up the processes of bureaucratization concerning funerals, which had been developing for decades. Moreover, it represented the attempt to integrate various aspects of the funeral business into the economic and financial, sanitary and epidemiological, ideological and other contexts of Soviet life. However, this last Soviet impulse to modernize, regulate and formalize a clear Soviet funeral canon was ineffectual. The instructions of 1979 did not paint a real picture of the funeral business in the country but sketched an imagined desirable future. This was because the pattern of organizing and ordering cemeteries, distributing and assorting sites, setting the range of funeral attributes (coffins, burial monuments, etc.) and the quality of actual funeral services was far from what was described in the document. Until the end of the Soviet era, the disorder in the funeral business (theft, corruption in the allocation of land for burial, the lack of special vehicles and staff or of monuments and funeral equipment and resultant blackmarketeering, the violation of sanitary regulations, and sometimes a careless registration of graves) was commonplace in Soviet reality. As a result, relatives of the deceased were left to solve the problems that came with a burial by mobilizing their own forces and means or by enlisting the help of enterprises and organizations where the deceased or his/her relatives worked.

\section{Grematoriums}

Cemeteries were not the only burial spaces in Soviet Russia: already the Decree of 1918 mentioned crematoriums along with cemeteries, even though there was not a single crematory in Russia at the time. The huge problems with graves in the years after the revolution prompted Soviet authorities to raise the issue of crematorium construction once again, after discussions of cremation itself and the building of crematoriums in tsarist Russia in the 1890s were rejected by the Synod in 1909 as incompatible with the Orthodox faith. ${ }^{16}$ Mere necessity was not the only reason for the Bolsheviks' interest in cremation. They considered cremation to be an effective tool to further secularize Soviet everyday life and its important relation to death, and thus a means of creating a "new way of life" and implementing a utilitarian and pragmatic approach to all aspects of human life including death. This was meant to help break with the past and, in doing so, remove another obstacle to the goal of constructing communism. The first unsuccessful attempts to set up crematoriums in Moscow (the unfinished construction of a crematorium at the Khodynka hippodrome) and in Petrograd (a temporary cremation furnace on Vasilevsky Island was in service from December 1920 to February 1921; its capacity, though, was insufficient) did not stop proponents of the fire burial. ${ }^{17}$ In the course of the 1920s, a large number of brochures on cremation were published. The "Kommunal'noe khoziaistvo" ("Communal Economy") magazine, the main printing body of the Soviet communal services, enthusiastically described the technical novelties 
of "Western" cremation. Regularly, it published articles about the work of new crematoriums in Europe, the United States and Japan, or about the removal of cemeteries from European city centers and the creation of public parks in their place. ${ }^{18}$ On May 30, 1927, the Society for the Development and Dissemination of Cremation Ideas in the USSR (Obshchestvo razvitiia i rasprostraneniia idei krematsii v SSSR - ORRIC) was established. ${ }^{19}$ Eventually, on October 7, 1927, in the reconstructed church within Donskoy monastery's cemetery, the first official USSR crematory was put into service - the Moscow crematorium. Additional ones were to follow in the late 1920s and early 1930s in other major cities of the country, such as Leningrad and Rostov-on-Don among others. Although a crematorium was opened in Kharkov in 1934 - which was destroyed during the Second World War - these plans were not executed. There were many reasons for the failure of the cremation business. First of all, despite the active promotion of cremation, its popularity remained low among the population. Although there was quantitative growth of cremated bodies in the Moscow crematorium, this was due to the large percentage of the so-called "administrative" bodies, that is, stillborn babies, bodies that had undergone forensic medical autopsy or those that were considered "kinless"; in 1928, they accounted for $70 \%$ of all cremations, and in 1933, still, for $47 \%$. In the 1930s, however, within the circles of power, the attitude towards cremation cooled down noticeably. In fact, it remained ambivalent - manifested, on the one hand, in the practice of a solemn cremation of party and Soviet functionaries and, on the other, in the secret cremation of victims of repression. One important factor which led to the leadership's cautious attitude towards cremation, its propaganda and participation in the international cremation movement was the domestic and foreign policy context of the 1930s. During the 1920s, there was a certain delight stemming from the "technological breakthrough" in the field of human burial, both from the involvement in foreign innovation in this field and from the desire to be involved in the international movement in support of cremation and the exchange of experiences. In the 1930s, in contrast, fear of espionage and the crackdown on so-called "enemies of the people" replaced that positivity with suspicion of the political entrapments of such cooperation. Perhaps the use of cremation in Nazi-Germany, too, played a certain role in discrediting the cremation movement in the USSR. The cremation movement was narrowed down, and the ORRIC was abolished. For many years, the Moscow crematorium remained the only working crematorium in the USSR - the only exception being two crematoriums that were temporarily active during the Second World War in the besieged city of Leningrad - at the Izhora Factory (February to May 1942) and at the brick and pumice factory No. 1 (March 1942 to November 1943). Due to the dangerous epidemiological situation in the city during the siege, the crematorium was put into service as an emergency measure to fight the spread of disease. ${ }^{20}$

After the war, the construction of crematoriums froze, even though on March 18, 1943 the Council of People's Commissars of the RSFSR resolution on measures to improve the funeral business in the cities of the RSFSR in 1944 planned for the development of standard designs of simple crematoriums for the cities of republican subordination. ${ }^{21}$ In 1948/49, a crematorium in Gorky was planned, but was not built in the end. ${ }^{22}$ It is possible that, in the first years after the Second World War, cremation was connected to the memory of the mass burning of bodies in the ovens of Nazi concentration camps, which could not possibly contribute to the popularization of cremation in general. ${ }^{23}$ It was only at the end of the Soviet era, in the 1970s and 1980s, that a dozen crematoriums were built in the USSR three in Moscow (Nikolo-Arkhangelskiy, 1972; Mitinskiy, 1985; Khovanskiy, 1988), and one 
in each of the cities of Leningrad (1973), Kiev (1975), Kharkov (1977), Sverdlovsk (1982), Minsk (1986) and Odessa (1988). Also, the promotion of cremation by government authorities was resumed, mainly for economic and sanitary reasons. ${ }^{24}$ However, even in today's Russia, where there are 25 crematoriums in the cities (four of which are in Moscow), the preference for this method of burial among the population of these cities is relatively low at 15 to 20\%, with the exception of Moscow, St. Petersburg and Norilsk, where 50 to $70 \%$ of the burials are, in fact, cremations.

\section{Dualism of the Soviet culture of death and the hierarchy of burials}

Despite the equality of burials proclaimed in the 1918 Decree, the Soviet culture of death was marked by dualism. In the first two decades after the revolution, the Bolsheviks instrumentalized death and mortuary practices to build new social hierarchies. In particular, they tried to reconcile the two sides of the death culture - established in the extreme conditions of the First World War - in order to make for "peaceful conditions". The cult of those fallen during the First World War did not have enough time to take shape in Russia. Nevertheless, its elements were used to form the Bolshevik cult commemorating dead "red leaders and heroes", thus becoming a tool to distinguish the Soviet elite. The trend of reduction, secularization of rituals and emotional manifestations of grief was found in the frontline conditions of the world and civil wars. Among "ordinary citizens", it quickly came to be exploited for propagandistic purposes in order to minimize and rationalize funeral rites, to secularize this aspect of everyday life and to express a bit of cynical pragmatism against human remains and old cemeteries.

Cemeteries have played an important role in building and regulating social and political hierarchies. In the mid-1920s and the first half of the 1930s - so before the 1936 Constitution, which declared the equality of all citizens and abolished all exploitative classes in the USSR - there were different charges for burials in cemeteries and cremation. These charges depended on a person's or family's social status or class affiliation. That could mean that a burial of so-called "non-labor elements" was from 2.3 up to 20 times more expensive those of workers or employees. ${ }^{25}$

Upon coming to power, the Bolsheviks began to allocate special resting places for those considered revolutionary heroes and leaders. These special places were the Field of Mars (Marsovo Polye) in Petrograd (which had been outlined since the February Revolution of 1917 when those who fell in its course were buried with honors on the Field of Mars) and a place by the Kremlin wall on Red Square in Moscow. Gradually, as the Soviet party elite was ranked more clearly, hierarchies of its different burial places became clearer as well. A burial near the Kremlin wall (near the Mausoleum that housed the embalmed body of the founder of the Soviet state and leader of the Bolshevik Party, Vladimir Lenin, ${ }^{26}$ who died in 1924, and where Stalin's body was located in 1953-1961 before being buried on the night of October 31 to November 1, 1961 in a grave near the Kremlin wall) turned out to be the most honorable, similar to the medieval tombs ad sanctos - near churches and the graves of saints and martyrs.

Besides the geographical spot of one's resting place, the manner of burial itself was differentiated depending on the deceased's rank. Until the mid-1980s, only twelve of the most significant party and Soviet leaders were buried in individual graves near the Kremlin wall. 
The bodies of other revolutionary figures and representatives of the elite in the early years after the revolution were put in mass graves near the wall. The remains of the dead revolutionary figures were cremated in the Moscow crematorium after it was put into service in 1927; the urns were put on display in the Kremlin wall with personal memorial plaques next to them. For other outstanding representatives and scientific, artistic or other Soviet elites, the government designated privileged necropolises, for example, the Novodevichy cemetery in Moscow (1927) or, after 1970, Novokuntsevskoye, Troekurovskoye and Vagankovskoye cemeteries. In Leningrad, the Field of Mars, the "communist plot" of the cemetery of the St. Alexandr Nevskii Monastery (erected in 1919 and separated from the rest of the cemetery) and later the Volkov Cemetery's Literary Bridges became such necropolises. ${ }^{27}$

This stratification of cemeteries and burials within the capitals was transferred to the provinces and reproduced in almost all major cities of the country, where such "privileged" cemeteries or plots were built. Until the end of the 1930s, these early Soviet cemeteries had the characteristic "communist plots". Later, authorities no longer specifically designated them, but the need to provide privileged places and sections of cemeteries for burial of the Soviet elite ("places of honorary burials") remained. So from the 1930s to the 1970s, respective Soviet documents usually mention these special places, including the "Instruction on the Order of Funerals and Maintenance of Cemeteries in the

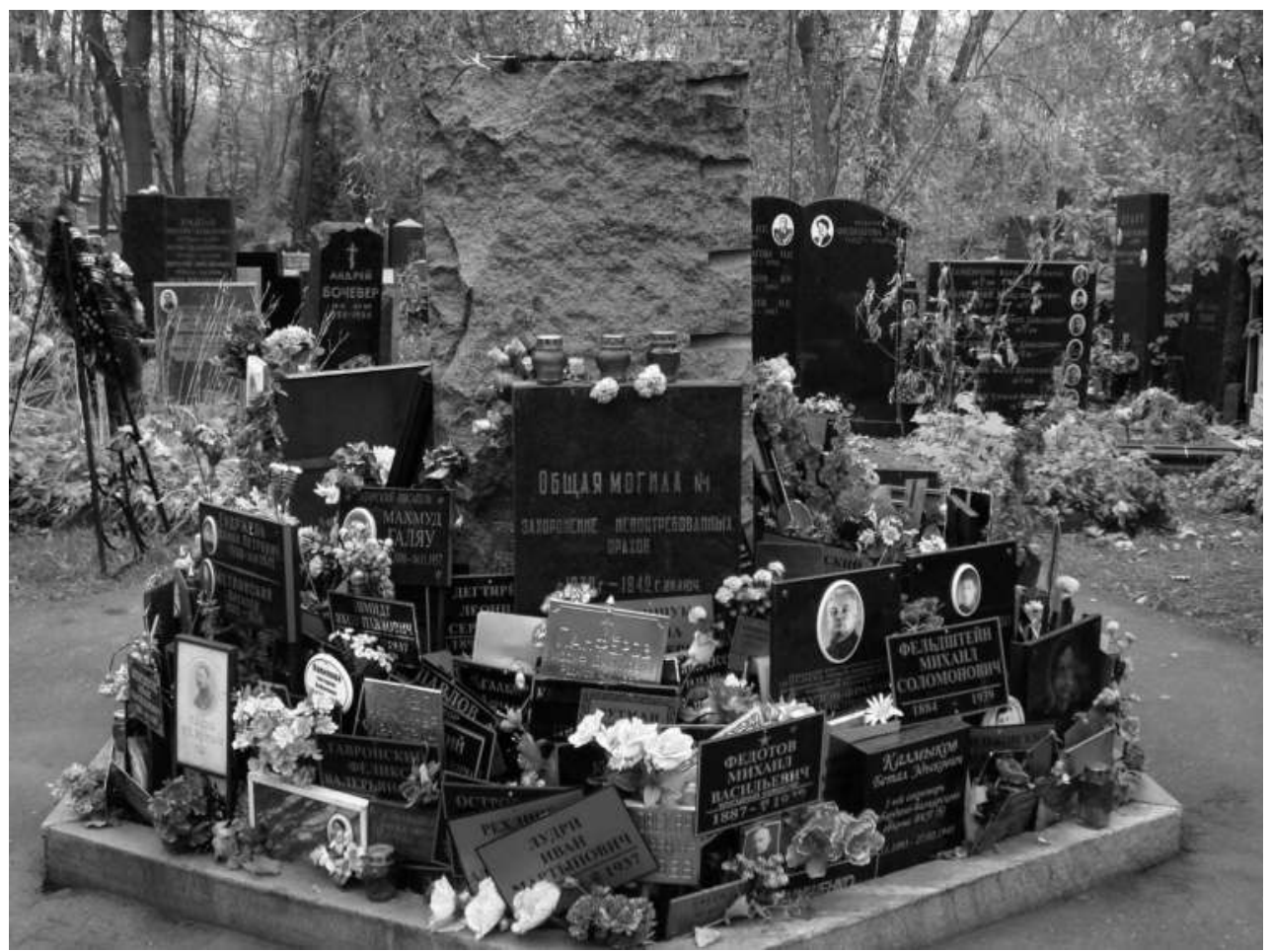

Image 23.1 A common anonymous grave of victims of reprisals with homemade signs by relatives. Don Cemetery, Moscow.

Photo by author. 
RSFSR" of 1979. The privileged burials were located at a central honorary position along the central alley, usually next to the entrance area of the cemetery and the "zone of mourning ceremonies". They hereby played a crucial role in determining the new geography of the Soviet cemeteries: instead of the pre-revolutionary cemetery churches, honorary burial spots formed the sacred core of the cemetery universe, and the proximity to this new center determined the prestige ascribed to all other tombs and graves. This new geography also differentiated according to the deceased's profession; being an important criterion for self-identification for Soviet citizens, informal "professional" sections - the military, the medical profession, actors and actresses, writers, etc. - often emerged in the course of these decades.

The proclaimed equality of burials was disavowed not only by the indicated hierarchy of cemeteries and plots but also by the practice of strict delimitation of burials of those deemed to be "outsiders" (e.g. "enemies of the people" or members of the Wehrmacht), especially during the $1920 \mathrm{~s}, 1930 \mathrm{~s}$ and $1940 \mathrm{~s} .{ }^{28}$ Burying people in mass graves was widely applied to "enemies of the people" and repressed citizens, even though it was officially banned (except in "times of national disaster") in June 1929 by the Rules of the People's Commissariat of Internal Affairs and the People's Commissariat of Health "On the Arrangement of Cemeteries and the Order of Burial". In the 1930s, the common graves of the executed and dead prisoners, mostly anonymous and secret, dotted the vast countryside. According to 2014 estimates, the number of remaining camp and prison cemeteries ranged from a few hundred to 1,000. The same study estimates the number of mass grave sites dug out in 1937 and 1938 - at the peak of the Great Terror - as between 60 and 80 sites. $^{29}$ During the Second World War, authorities had to resolve the issue of burying the bodies of fallen soldiers and officers of enemy armies. In April 1942, a number of official documents concerning the treatment of the bodies of soldiers and officers of Hitler's armies during the "cleaning of battlefields" were therefore issued. Although the bodies of enemies, as well as the bodies of fallen soldiers and officers of the Red Army, were ordered to be buried in mass graves (referred to as "brotherly graves" when talking of one's "own" dead), "enemy" graves were to be located away from populated areas. Unlike the "mass graves" of the Soviet Union's "own" dead, they had no inscriptions on them; instead, they were only marked with a number. Thus, the "enemy graves" were doomed to anonymity and, when choosing their place, "the expediency of using it in the future ... for public green spaces" was to be taken into account. ${ }^{30}$

\section{Funeral rites}

One of the ideological and practical tasks of the Bolsheviks, after their seizure of power in October 1917, was to eliminate the "old" funeral rites that were strongly connected to religion and the church. This destruction not only met the needs of the Bolsheviks' antireligious propaganda. In fact, creating a new concept of death and concomitant ritual behaviors was also an integral part of forming a novyi byt - a new lifestyle.

Funeral rituals were an important part of both the traditional religious festive and commemorative culture, therefore fiercely competing with the emerging Soviet festival and memory culture. The opponents of the former religious rites, however, were split: should there be a new colorful theatrical rite of "Red Funerals" replacing the old rites, or should the rite be simply limited to the utilitarian procedure of farewell and burial, 
devoid of any sacral quality? Should the state impose a new rite and exercise control over it or not interfere in the process of creating a new rite of worship at all? ${ }^{31}$

The "Red Funeral" is a funeral ritual that was formed before the revolution of 1917 as a non-religious, "civil" affair with neither a religious rite nor a funeral service, and without a priest and icons as well. In the 1920s, the "Red Funeral" - deemed to be of propagandistic and public importance - was promoted by the newspapers Bezbozhnik (Godless) and the Soiuz voinstvuiushchikh bezbozhnikov (Union of Militant Godless) and became somewhat widespread among both the Bolsheviks themselves and their sympathizers. However, even in urban environments, the "Red Funeral" did not find its way into the lives of ordinary citizens. Everywhere in the cities, not to mention the countryside, traditional religious funeral rites continued to dominate. In some Russian regions during the 1930s, around $93 \%$ of the dead were given religious funerals. ${ }^{32}$ On the eve of and during the Second World War, the dominance of the religious rite remained unchanged. Only in the 1960s, as part of "Khrushchev's anti-religious campaign", did Soviet ideologists make a last effort to infuse the distinctly de-ideologized Soviet rituals with just that: ideology. ${ }^{33}$ However, as for the funeral rites - as well as for other rites these attempts were unsuccessful.

In fact, the "Red Funeral" ritual for "revolutionaries", "leaders" and "heroes" turned out to be more persistent and eventually transformed into the state funerals. These "Kremlin funerals" became the foundation of the Bolshevik cult of the dead and its alternative, secular religiosity. Their canon and script combined elements of the "Red Funeral" with elements of the pre-revolutionary funeral ceremony for monarchs. Two exemplary funerals specified and defined this canon. First, the funeral of the head of the Soviet state (Chairman of the All-Russian Central Executive Committee of the Soviets of Workers' and Soldiers' Deputies; VTsIK) Iakov Sverdlov in 1919, and second, that of the founder of the Soviet state and the head of the government (Council of People's Commissars) Vladimir Lenin in 1924. After that, though with some changes, the ritual was reproduced throughout the Soviet decades.

The canon was defined by multiple elements: first, a governmental commission for the organization of the funeral was created. It designated a national mourning period und published an official announcement in the central newspapers. This announcement usually described the death of the deceased and gave information about the order of the burial. In addition, it arranged for the publication of materials bearing testimony to the mourning of the Soviet people, plus detailed reports on the funeral and official obituaries of party, Soviet and other organizations. Also, part of the canon involved the removal of the death-mask following a sketching and filming of the deceased. The deceased's brain was removed and transferred to the Brain Institute. ${ }^{34}$ The public bid farewell to the body or ashes of the deceased in the Column Hall of the House of Unions in Moscow, the Soviet castrum doloris. Then, the funeral procession to Red Square where the coffin or urn was installed on the pedestal of the Mausoleum was followed by a funeral rally with speeches by the leaders of the country and the party. Finally, the deceased was then buried with full honors - gun salutes, the playing of the national anthem and a solemn march of military units in Red Square. Naturally, the most important link to Soviet ideology in this mournful choreography was the funeral procession and its carefully considered representation of social and professional groups, which symbolized both the idea of the structure of Soviet society and, even more prominently, the hierarchy of its elites. 


\section{Mourning in the Soviet culture of death}

The policy towards grief and its manifestations in the 1920s and 1930s and the prescribed emotional regime reflected quite clearly the dualism of the Soviet instrumentalization of death. ${ }^{35}$ The attitude towards grief also differed depending on whether it was considered "private" (the grief of an ordinary person over a "simple" deceased person) or "political" (grief over leaders, heroes and other prominent members of Soviet society). The latter had an affirmative potential in asserting dominant emotional norms of life, while the authorities were mistrustful of so-called private grief because of its subversive and destructive potential.

The utilitarian and pragmatic attitude towards death in the 1920 s did not welcome private grief, since the grief of a private person and his/her mourning of personal losses did not fit into the framework of the dominant emotional regime. Because strong emotions of the "ordinary" person and their public presentation were difficult to control, they were a source of concern for the authorities. ${ }^{36}$ Even more illegitimate was the grief of a private person over his/her loved ones if they had been declared "enemies". In addition, the forms and gestures of how people dealt with grief, associated with the established rituals and with the expression of collective condolences of the community to the inner circle of the funeral party were generally deemed manifestations of "bourgeois prejudice". Despite this, in both villages and cities - which in the 1920s and 1930s saw considerable flows of migration, since the rural population fled from collectivization and drifted into the cities - the traditional practices and procedures of collective and communal treatment of "private" grief largely remained intact. For example, funeral processions were not superseded by these new practices; at least on a symbolic level, the tradition of funeral feasts was preserved, and, in the last Soviet decades, the practice of funeral feasts became widespread not only in a close circle of those grieving but also in a wider circle of colleagues of the deceased.

The deprivation of private grief in the 1920s was accompanied by the imposition of public collective "political" grief for heroes, leaders and other "significant" dead people. Those who tried to establish a new dominant emotional regime welcomed such grief. It performed two important functions necessary for the legitimization of power: creating and destroying. Creating in the sense that during public collective grief and overcoming the loss - as in any mass action - the effect of emotional contagion contributed to the solidarity of citizens. Thus, it made people aware of being part of a grieving community, which, on a broader level, was part of a common Soviet identity. These social interactions of mourning and the shared experience of loss became one of the most important communicative resources for the creation of this identity. However, political grief performed not only a creative and affirming function but also a destructive one. Grief could be converted into hatred and anger and then focused on real or imagined enemies. As a result, it helped to unify people not only against these enemies outwardly but also inwardly since it created a sense of group affiliation.

"Political" grief could merge with "private" grief, perhaps, only with respect to the fallen during the Great Patriotic War. On the one hand, it was a question of "private" losses, the grief of ordinary people over ordinary people, for which a public presentation of grief was not welcomed. On the other hand, those who became figures in the mass cult of heroes were exploited by the Soviet regime during the post-war period in order to reaffirm it. Thereby they were subject to the practice of political grief that corresponded with the dominant emotional regime. 
Combined with the weakening of state control over a person's death in the postwar decades and the peculiar emancipation of the private sphere of everyday life, the practice of collective mourning for the "little man", including its public presentation, became more and more widespread both in rural areas and in the cities. The state recognized the right for this need to mourn: the permission for a funeral procession was provided by the instructions, relatives were given a few days off to arrange the burial and funeral feasts, even soldiers were granted short leave to bury a close relative. In the late Soviet decades, the legitimization of private grief meant the return of the traditional funeral rite and the corresponding emotional expression of grief in its entirety. ${ }^{37}$

\section{Semantic and semiotic codes of the Soviet cemetery}

In the Soviet period, there were changes in the symbolic nature of the cemetery space. The pre-revolutionary tombstones marked, first and foremost, the religious affiliation of the deceased and sometimes indicated his or her social or professional status (noble title, military rank, profession, etc.). The Decree of November 11 (24), 1917 "On the Abolishment of All Classes and Civil Ranks" 38 disavowed all estates, ranks, titles, awards, coats of arms and other elements of symbolic capital that gave value to the lives of the deceased. That is why, in the early Soviet period, the practice of recording the indication of the deceased's origin, social status and class affiliation (especially the "former" classes) decreased and then almost disappeared. This absence of social identity in the burial space was compensated for by the traditional appeal to cultural identity, that is, to confessional identity and its markers. The religious affiliation of the deceased was marked by the physical form of gravestones, e.g. a simple cross for Christians (or a more expensive tomb in the form of a chapel), or by the religious symbols placed on the gravestones, for example the cross of Christians or the crescent moon of Muslims. However, due to the major anti-religious campaigns, there was some decrease in the use of religious symbols. Sometimes religious identity was also expressed in an indirect manner, through the assertion of ethnicity or the use of the regional language or script. ${ }^{39}$

At the same time, new symbols appeared in the cemetery space, most notably revolutionary and/or communist emblems and verbal markers. New, unexpected forms of monuments, for example industrial constructions as a tombstones, ${ }^{40}$ as well as revolutionary symbols on the monument (five-pointed stars, sickles and hammers, the globe with a sickle and hammer, even the state emblem of the USSR, etc.) were used to identify the deceased as a participant in the construction of and the struggle for communism.

Finally, by the 1930s, "neutral" monuments of the simplest forms, without symbols, with very little information about the deceased - the name, patronym and years of life had become commonplace. Their appearance connected not only with a cautious desire to minimize markers of identity but also with the desire to save money.

The cemetery "text" of the first two Soviet decades appeared to be contradictory. It represented both relatively "pure" examples of "old" and "new" identities and their various re-combinations. That is why some tombstones, in the form of a chapel that continued to exist, sometimes had been fitted with a five-pointed star on top, hitherto the place of the Orthodox cross. Such symbols appeared on monuments for various reasons and not always because of the deceased's commitment to the Soviet regime and communist ideology. Already in the 1920s and 1930s, the Soviet marking of gravestones 
reflected an ideological compromise, the formation of a phenomenon of "ritual formalism" that was widespread in the following Soviet decades. ${ }^{41}$

The changes in the field of monuments also reflected the incipient processes of (re)assertion, the mixing of socio-cultural differences, and the tendency to strengthen the construction of new identities. However, throughout the 1920s and 1940s, "hybrid tombstones" in the cemetery space were a quantitative minority and could not match the number of "pure" and "neutral" forms. Also, their hybridity was limited mostly to a combination of verbal elements of the revolutionary heroic/ Soviet labor biography and religious symbols. ${ }^{42}$ The stability of the ritual maintained the conservatism of consumer expectations and their aesthetic demands as well as the conservatism of tombstone manufacturers. The latter reproduced popular tombstone forms and designs. For the ensuing decades, the weak market for Soviet monuments, both official and unofficial, along with the new Soviet "stars", combined with standardized and neutral forms of monuments (obelisks, steles, rectangular pedestals) but also with "crosses" and figured pedestals or "shells", which reproduced the former shape of the chapel. ${ }^{43}$

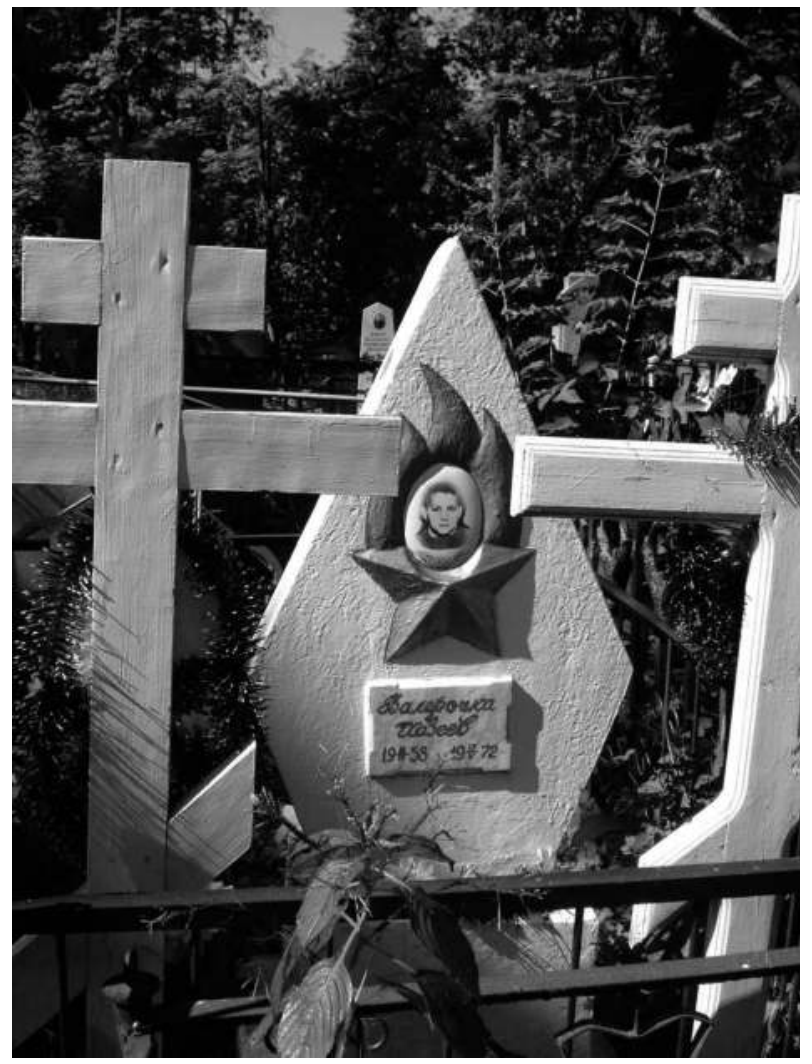

Image 23.2 The combination of Orthodox crosses and Soviet stars on the tombstones. Arsk cemetery, Kazan.

Photo by the author. 
Immediately after the Second World War, a number of documents sent signals that legitimized open confessional identification in the burial space. One way or another, in the second half of the 20th century, those religious symbols that had not disappeared from the cemetery landscape were officially countenanced. In this regard, in later Soviet decades, particularly after the 1970s, a practice developed for correcting the information on gravestones and applying a religious symbol, even years and decades after the deceased's burial. From the 1950 s to the 1980 s, a curious phenomenon of identifying family membership and providing other information on the tombstones became widespread: ${ }^{44}$ they visually testified to the interesting "symbiosis" of religious and secular symbols. ${ }^{45}$ Apparently, the appearance of such combinations did not so much evidence a "ritual formalism" but rather identified the simultaneous belonging of the deceased to the noncontradictory areas of Soviet existence, its public and private spheres. In the second half of the 20th century, the mixing of religious and secular symbols, especially verbal and symbolic designations of professions (which even largely replaced religious symbols) as identification markers became very common. The professional and labor background as well as indicators of success in his or her field, such as state awards, degrees and titles, discoveries and inventions, and other merits and achievements became an important part of the secular tombstone's text that supported the discourse of social immortality.

\section{Post-Soviet realities of the funeral sphere}

According to the calculations of independent experts,

by the beginning of the 21 st century there were at least one hundred thousand cemeteries in the country, where there were at least a few graves left to be looked after by relatives and acquaintances. Cemeteries more or less comfortable and under some administrative supervision numbered about sixty thousand. ${ }^{46}$

At the regular forum of Russian representatives of the funeral industry "Necropolis", organized by the Russian Union of Funeral Organizations and Crematoriums, which was held in Kazan in June 2019, it was said that there were 76,400 cemeteries in Russia. In addition, there are 25 crematoriums in the country (four in Moscow), one half being in private hands, and the other half part of the public purse on a municipal level. ${ }^{47}$

In the post-Soviet period, the funeral business and the culture of death in Russia as a whole experienced the contradictory impacts of new political and socio-economic realities. The funeral business, closely tied to the interests of clans of large bureaucracies, remains one of the most profitable markets in the economy, but it is also shadowy and corrupt. ${ }^{48}$ At the same time, the plans to register burial sites and regulate the state of the cemetery economy (which are long overdue) correlated with the political and ideological needs of the elites of the new Russia. In particular, they correlated with the processes of institutionalization of the political nation and the concept of a "Russian people", the elements of which are clearly differentiated by familiar ascriptive categories such as religion or ethnicity. Thus, the federal law "On Burial and Funeral Business" of January 12, 1996, which recorded the state or municipal ownership of cemeteries and the existence of historical and memorial cemeteries, at the same time divided the cemeteries "according to customs", namely "social, religious, military". It also sanctioned the creation of confessional cemeteries in the country "to bury the dead of one faith". ${ }^{49}$ The cemetery 
hierarchy, which has been preserved since Soviet times, has been corrected and in recent years has been crowned with the Federal Military Memorial Cemetery. It was founded by order of the Decree of the President of the Russian Federation on July 11, 2001, was opened in 2013 in Mytishchi near Moscow and is supposed to become the main state pantheon of the Russian elite.

Recently, the culture of death in Russia, largely influenced by the modernization of the funeral industry and the rapidly growing variety of commercial offers of funeral services, has also been subject to some adjustments. For example, the development of the funeral services market and new technologies not only meant that the digital age found its way into the funeral culture, it also partially corrected ideas of Russian citizens concerning memorial practices and ways of handling grief over their loved ones. ${ }^{50}$ Nevertheless, mass burial practices and burial culture remain to a large extent quite traditional, linked to pre-revolutionary religious customs, which have been redesigned and adapted to some of the innovations of the Soviet period.

$* * * * *$

Overall, the Soviet impact on funeral practices and material culture reflected a variety of impulses, including the tension between the desire to limit attention to and investment in death rituals and the interest in using them to enhance loyalty to the regime and its stalwarts. The result, perhaps predictably, was a real limitation on any substantial inroads on tradition, which became particularly apparent in the post-Stalinist decades. Real changes did occur, but in many ways - as in the low incidence of cremation - Russian funeral practice remains more conservative today than is the case in a number of other major regional societies.

\section{Notes}

1 On the pre-revolutionary culture of death, see T.D. Panova, Tsarstvo smerti. Pogrebal'nyi obriad Srednevekovoi Rusi XI-XVI vv. (Moscow: Radunitsa, 2004); M.O. Logunova, Pechal'nye ritualy imperatorskoi Rossii (Moscow: Tsentrpoligraf, 2011).

2 See Logunova, Pechal'nye ritualy imperatorskoi Rossii, 223-226, 254.

3 Catharine Merridale, Steinerne Nächte. Leiden und Sterben in Russland, transl. (München: Karl Blessing Verlag, 2001), 186; Encyclopedia of Cremation, ed. by Douglas J. Davies, Lewis H. Mathes (Aldershot: Ashgate, 2005), 369-370.

4 Despite all the differences in the attitude of the Russian confessions towards the tombstone and its conceptual idea, it was still mostly perceived as a symbolic gate to a better world or as an image of a permanent, "real" home. It is this context that explains the traditions of Orthodox people "eating together" with the deceased or "visiting" him/her, and the fairly common practice of putting up fences around a grave, forming a quasi-"courtyard" in front of the "house".

5 A.V. Kobak and Iu. M. Piriutko, Istoricheskie kladbishcha Sankt-Peterburga (Moscow: Tsentrpoligraf, 2009), accessed May 17, 2018, www.livelib.ru/book/44413/readpart-istoricheskie-kladbischapeterburgaaleksandr-kobak-yurij-piryutko/ 4.

6 See Irene Masing-Delic, Abolishing Death: A Salvation Myth of Russian Twentieth Century Literature (Stanford, CA: Stanford University Press, 1992); Leonid Heller and Michel Niqueux, Geschichte der Utopie in Russland, übers. Anne Hartmann, ed. Michael Hagemeister (Bietigheim-Bissingen: Edition Tertium, 2003), ch. 5 and 6; Die Neue Menschheit. Biopolitische Utopien in Russland zu Beginn des 20. Fahrhunderts, ed. Boris Groys and Michael Hagemeister in collaboration with Anne von der Heiden (Frankfurt am Main: Suhrkamp, 2005); Nikolai Krementsov, Revolutionary Experiments. The Quest for Immortality in Bolshevik Science and Fiction (Oxford: Oxford University Press, 
2014); Tatjana Petzer, "Utopie und Unsterblichkeit: Tod und Erlösung bei N. Fedorov und A. Platonov", Osteuropa 8-10 (2016): 267-282; and others.

7 See A. Geifman, Revoliutsionnyi terror v Rossiï, 1894-1917, trans. E. Dorman (Moscow: KronPress, 1997); M.B. Mogil'ner, Mifologiia "podpol'nogo cheloveka": radikal'nyi mikrokosm v Rossii nachala XX veka kak predmet semioticheskogo analiza (Moscow: Novoe literaturnoe obozrenie, 1999); O.V. Budnitskii, Terrorism v rossiiskom osvoboditel'nom dvizhenii: ideologiia, etika, psikhologiia (vtoraia polovina $X I X$ - nachalo XX v.) (Moscow: ROSSPEN, 2000); Susan K. Morrissey, Suicide and the Body Politic in Imperial Russia (Cambridge: Cambridge University Press, 2006).

8 See Aaron J. Cohen, "Oh, That!" Myth, Memory, and the First World War in the Russian Emigration and the Soviet Union, Slavic Review 62 (1) (2003): 69-86; Karen Petrone, The Great War in Russian Memory (Bloomington: Indiana University Press, 2011); Svetlana Malysheva, Bereavement and Mourning (Russian Empire), in 1914-1918-online. International Encyclopedia of the First World War, ed. by Ute Daniel, Peter Gatrell, Oliver Janz, Heather Jones, Jennifer Keene, Alan Kramer, and Bill Nasson, issued by Freie Universität Berlin (Berlin 2014), accessed August 2, 2019, http://encyclopedia.1914-1918-online.net/article/Bereavement_and_ Mourning_(Russian_Empire).

9 "Dekret Soveta Narodnykh Komissarov 'O kladbishchakh I pokhoronakh', 7 dekabria 1918 g.", in Dekrety Sovetskoi vlasti, vol. 4 (Moscow: Gospolitizdat, 1968), 163-164.

10 A. Anserov, "Etapy pokhoronnogo dela v Moskve", Kommunal'noe khoziaistvo 12 (1922): 9.

11 The New Economic Policy (NEP) was an economic policy pursued in Soviet Russia and the USSR during the 1920s. Initiated in March 1921 by the 10th Congress of the Russian Communist Party, it changed the policy of "military communism" during the civil war, which led to an economic decline throughout the country. The NEP included the introduction of private entrepreneurship and the revival of market relations, of various forms of ownership and meant the replacement of the confiscation of agricultural products with a so-called food tax in villages, the involvement of foreign capital in the form of concessions and a monetary reform.

12 See S. Malysheva, "Na miru krasna": instrumentalizatsïa smerti v Sovetskoi Rossii (Moscow: Novyi khronograf, 2019), 269-285.

13 Thus, on October 25, 1948, the famous Soviet pilot, three-time hero of the Soviet Union I.N. Kozhedub, outraged by the state of cemeteries in Podolsk and the village of Marfino in the Moscow region, wrote an angry letter to the Council of Ministers of the USSR. Kozhedub's letter was checked and a decision was made to improve the cemeteries (see Gosudarstvennyi arkhiv Rossiiskoi Federatsii (GARF), f. A-314, op. 2, d. 4794, 1. 20, 17-19, 21-24, 25). Yet, of course, only a tiny fraction of the cemeteries had such powerful advocates as did the village of Marfino, rendering their fate quite different.

14 See Malysheva, "Na miru krasna", 305-306.

15 GARF, f. A-314, op. 6, d. 508, 1. 2.

16 See A. Sokolova, “'Nel'zia, nel'zia novykh liudei khoronit' po-staromu!”, Otechestvennye zapiski 5 (56) (2013), accessed August 30, 2014, http://magazines.russ.ru/oz/2013/5/17s.html; A.N. Kashevarov, Tserkov' $i$ vlast': Russkaia Pravoslavnaia Tserkov'v pervye gody Sovetskoi vlasti (St. Petersburg: Izdatel'stvo SPbGTU, 1999), 265.

17 "Moskovskoe kommunal'noe khoziaistvo za chetyre goda revoliutsii", Kommunal'noe khoziaistvo 7 (1921): 41; S. Anserov, "Krematsiia i ee osushchestvlenie", Kommunal'noe khoziaistoo 11 (1922): 7; G. Bartel', "K predstoiashchemu otkrytiiu v Moskve pervogo krematoriia", Kommunal'noe khoziaistvo 19-20 (1926): 39.

18 "Novyi krematorii v Vene", Kommunal'noe khoziaistvo 5 (1923): 13; "Krematsiia v Berline", Kommunal'noe khoziaisto 15-16 (1923): 37; G. Bartel', "Krematsiia v SSHA", Kommunal'noe khoziaistvo 13 (1925): 14-20; G. Bartel', "Sovremennoe polozhenie krematsii v Anglii", Kommunal'noe khoziaistvo 19 (1925): 90-98; G. Bartel', "Ognennoe pogrebenie v Iaponii”, Kommunal'noe khoziaistvo 20 (1925): 61-67; "Obzor otkrytykh i reshennykh k postroike krematoriev za granitsei za 1925 g.", Kommunal'noe khoziaistvo 24 (1925): 76-79; G. Bartel', "Obzor otkrytykh i namechennykh k postroike krematoriev za 1926 g.", Kommunal'noe khoziaistvo 1-2 (1927): 92-95; "Ispol'zovanie starykh kladbishch v Parizhe", Kommunal'noe khoziaistvo 7 (1922): 22; "Ischezaiushchie kladbishcha v Berline", Kommunal'noe khoziaisto 10-11 (1922): 28-29; and others.

19 "K obrazovaniiu pervogo v SSSR krematsionnogo obshchestva", Kommunal'noe khoziaistvo 11-12 (1927): $44-47$. 
20 See Malyseva, “Na miru krasna", 251-269.

21 GARF, f. A-314, op. 1, d. 4879, 1. 1ob.

22 GARF, f. A-314, op. 2, d. 3218, 1. 5-5ob.

23 Monika Black sees the fear of cremation that spread among the Germans in the early 1950s as also associated with the memory of the mass burning in concentration camps. See Monika Blek, Smert'v Berline: ot Veimarskoi respubliki do razdelennoi Germanii, trans. V.A. Tretiakov (Moscow: Novoe literaturnoe obozrenie, 2015), 270, 273.

24 "Krematorii", in Wikipedia, accessed August 25, 2014, https://ru.wikipedia.org/wiki/ Крематорий.

25 Malysheva, "Na miru krasna", 362.

26 About the cult surrounding Lenin and the treatment of his body, see Nina Tumarkin, Lenin Lives! The Lenin Cult in Soviet Russia (Cambridge, MA; London: Harvard University Press, 1983); Benno Ennker, Die Anfänge des Leninkults in der Sorejetunion (Cologne, Weimar, Vienna: Boehlau, 1997); and others.

27 See P. Roth, "Totenkult in Russland. Politik und Religion", in Glaube in der 2. Welt, vol. 27, no. 2 (Zürich, 1999), 25-29; S. Malysheva, "The Russian Revolution and the Instrumentalization of Death", Slavic Review 76 (3) (2017): 650-651.

28 See S. Malysheva, “Bratskie mogily' i 'vrazheskie mogil'niki': simvolicheskoe oznachivanie massovykh zakhoronenii v Sovetskoi Rossii/SSSR 1920-kh - 1940-kh godov" ['Mass Graves' and the 'Burial Grounds of Enemies': The Symbolic Significance of Mass Burials in Soviet Russia/USSR, 1920s-1940s], The Soviet and Post-Soviet Review 44 (3) (2017): 233-263.

29 See "'O sostoianii mest massovykh zakhoronenii zhertv politicheskikh repressii sovetskogo vremeni na territorii Rossii'. Resul'taty issledovanii postoiannoi komissii po istoricheskoi pamiati Soveta pri Prezidente RF po razvitiiu grazhdanskogo obshchestva i pravam cheloveka", accessed February 2, 2016, http://president-sovet.ru/files/b5/72/b572093c0a8df8ed29d dabf496287 138.pdf.

30 GARF, f. A-314, op. 1, d. 4859, 1. 6.

31 A. Sokolova, “'Nel'zia, nel'zia novykh liudei khoronit' po-staromu!”, Otechestvennye zapiski 5 (56) (2013): 191-208.

32 N. Lebina, Sovetskaia povsednevnost': normy $i$ anomalii. Ot voennogo kommunizma $k$ bol'shomu stiliu (Moscow: Novoe literaturnoe obozrenie, 2015), 206.

33 V. Smolkin-Rotrok, "Problema 'obyknovennoi' sovetakoi smerti: material'noe i dukhovnoe v ateisticheskoi kosmologii”, Gosudarstvo, religiia, tserkov'v Rossii i za rubezhom 3-4 (30) (2012): 430-463.

34 The practice of transferring the brain of "outstanding people" to the Brain Laboratory (1925), later transformed into the State Institute of Brain (1928) and then to the Brain Research Institute (1981), continued until the end of the Soviet era. See Monika Spivak, Mozg otprav'te po adresu (Moscow: Astrel', CORPUS, 2010).

35 Supporters of the social-constructivist approach to the history of emotions have precisely noticed that each political regime creates its own emotional regime that determines the dominant norms of emotional life. See W. Reddy, The Navigation of Feeling: A Framework for the History of Emotions (Cambridge: Cambridge University Press, 2001), 129. Cited in B.Kh. Rosenvein, "Problemy i metody v istorii emotsii", in Gorod i gorozhane v sovetskoi Rossii 19201930-kh godov: mir emotsii i povsednevnykh praktik, ed. by A.Iu. Rozhkov (Krasnodar: Traditsiia, 2017), 48.

36 The public showing of strong emotions associated with death and its unusual nature can be considered a taboo, at least for Soviet art, according to Bettina Jungen. See Bettina Jungen, "Gestorben im Kampf um den Sieg - geopfert für das ewige Leben: Der Tod in der sowjetischen Kunst von 1917 bis 1953", in Der Tod in der Propaganda: (Sowejetunion und Volksrepublik Polen), ed. Daniel Weiss (Bern: Peter Lang, 2000), 386, 397.

37 For more information on Soviet grief, see Malysheva, "Na miru krasna", 224-246.

38 "Dekret ob unichtozhenii soslovii i grazhdanskikh chinov. 11 (24) noiabria 1917 g.", in Dekrety Sovetskoi vlasti, vol. 1 (Moscow: Gospolitizdat, 1957), 72.

39 At the New Tatar Cemetery in Kazan, the name of the deceased (and sometimes the Qur'anic Surahs) continued to be written in the Arabic script of the Tatar language associated with the 
Koran from the 1920s to the 1960s. Due to the double cultural rupture (translation of the Tatar alphabet in 1927 into Latin and in 1939 into Cyrillic), the cultural integrity of the deceased's identity and its connection with the departed and living generations of relatives and coreligionists was ensured in the 1930s-1960s by the duplication of the deceased's name and dates of life and death, first in Arabic and Latin, and later in Arabic and Cyrillic alphabets. On the Tatar tombstones, peculiarities of forms and texts until the first third of the 20th century, see D.G. Mukhametshin, Tatarskie epigraficheskie pamiatniki. Regional'nye osobennosti $i$ etnolul'turnye varianty (Kazan: Institut istorii AN RT, 2008), 62-63; G.K. Khisamieva, "Nadgrobie v kontekste natsional'noi kul'tury" (Diss., Moscow, 2007). Khisamiyeva points out that the Arabic script associated with the Qur'an was used in epitaphs until the 1960s.

40 For example, monuments on the communist plot of the cemetery of the St. Alexandr Nevskii Monastery on the graves of S. Ja. Vasilyev, S. I. Vshivtsev, A.M. Babanov, who died in 1935, and on the grave of N.V. Bakhvalov who died in 1921. See I. Tveretskii, "Kommunisticheskaia ploshadka v Aleksandro-Nevskoi Lavre. Sankt-Peterburg. Galereia", accessed April 1, 2017, http://necropol.org/223-kommunisticheskaja-pl-anl.html.

41 K. Bannikov, "Transtsendentnyi zvezdokrest", Otechestvennye zapiski 5 (56) (2013): 227.

42 Moreover, the first of them often reflected the phenomenon of "language ritualization", i.e. compliance with certain language conventions, which signaled loyalty to the authorities, and this phenomenon was mentioned by U. Schwendimann in its application to a later time. See Ueli Schwendimann, "Der Tod als Ode an das System. Textsortenmutationen als Bestandteil verbaler Propaganda - am Beispiel von Texten über den Tod", in Der Tod in der Propaganda: (Socejetunion und Volksrepublik Polen), ed. Daniel Weiss (Bern: Peter Lang, 2000): 2.

43 For example GARF, f. A-314, op. 2, d. 9633, 1. 78-79.

44 Family burials have been permitted since the 1950s in addition to the "ordinary" burials previously prescribed in the 1931 instruction.

45 Obelisks with a Soviet star and crosses made of metal fittings, monuments with a Soviet star and monuments with a crescent moon are often consistent and adjacent to one "family" fence, showing the diversity and variability of understandings and presentations of Soviet identities within the same family. And in the late Soviet and post-Soviet periods there are tombstones "family" and individual -that are applied simultaneously and symmetrically to the image of the cross and five-pointed star, or crescent moon and Soviet star. See Malysheva, "Na miru krasna", $406-414$.

46 V.N. Leksin, “Umeret' v Rossii”, Mir v Rossii 4 (2010): 124-161, accessed July 13, 2015, http://demoscope.ru/weekly/2010/0443/analit02.php.

47 I. Skriabin, "Kriminalitetu v pokhoronnyi biznes ne dali zalezt'. Kogo-to posadili, kogo-to ubili ...”, Business Online, June 19, 2019, accessed July 2, 2019, www.business-gazeta.ru/article/ 428465.

48 See, for example I. Golunov, "Kto vladeet moskovskimi kladbishchami", accessed July 10, 2019, https://meduza.io/feature/2019/07/01/kto-vladeet-moskovskimi-kladbischami.

49 Religious cemeteries could be run by local self-government bodies, which determined the procedure for their activities in coordination with religious associations. See "Federal'nyi zakon 'O pogrebenii i pokhoronnom dele' ot 12.01.1996 N 8-FZ (redaktsiia 25.05.2018)", accessed March 8, 2019, www.consultant.ru/cons/cgi/online.cgi?from=292687-0\&rnd=95B1730A 6642EA3554FCA38BC68B3E8D\&req=doc\&base $=$ LAW \&n=298701\&REFDOC $=292687 \&$ REF BASE=LAW\#20rgq2edtf9.

50 In the Russian segment of the Internet there are many virtual cemeteries. And on such sites as Ckorbim.com, "Tsifrovoe nasledie" ("Digital Heritage", https://qrmemo.ru/) you can order the paid service of applying a QR-code to the monument linked to the virtual memorial or remotely order a service to care for the "real" grave with such a code. 


\title{
DEATH IN MODERN FILM
}

\author{
Thomas Britt
}

The last quarter-century of cinema suggests that death sells at the global box office. Titanic, first released in December 1997, dramatized the real-life tragedy from 1912 and became the highest-grossing movie of all time. Science-fiction film Avatar, which was initially released in December 2009, broke Titanic's box office record with a plot about an indigenous population of a moon called Pandora being threatened with possible extinction by colonizing human forces. In 2019, Avengers: Endgame set the most recent worldwide box office record, concluding several story arcs of the comic-book movie franchise in which a villain called Thanos kills half of the population of the universe with a literal snap of his fingers. These movies, which set their respective records in direct relationship with the plots' escalating numbers of lives threatened, each grossed more than two billion dollars at the box office worldwide. ${ }^{1}$

Though these films are undeniably popular, there is no significant evidence that viewers line up to see them because the plots involve extraordinarily deadly events and conflicts. All three movies are expensive blockbusters, intended to attract audiences with state-of-the-art production values and no small degree of visual spectacle. Despite increased competition from other forms of entertainment media, blockbuster films successfully executed in a spectacular, immersive way become cinematic events ideally suited to the theatrical viewing experience. What these films do not necessarily require to enjoy them is a reflective, philosophical, or historically aware viewing perspective. It is possible to walk away from viewing any of these films merely entertained, without considering how fully death as a subject has permeated the narratives.

Some scholars have argued that there is a link between fictional film spectacle and the real threat of destruction. In 1965, Susan Sontag related the plots of science-fiction films to the mid-twentieth century anxiety/trauma that

from now on to the end of human history, every person would spend his individual life not only under the threat of individual death ... but of something almost unsupportable psychologically - collective incineration and extinction which could come any time, virtually without warning. ${ }^{2}$

The combination of fear and fascination that Sontag identifies in the appeal of science fiction recurs in broader, more recent studies of death and film, such as Daniel Sullivan and Jeff Greenberg's edited collection Death in Classic and Contemporary Film. In the editors' conclusion to the volume, called "Cinematic Death Benefits," they write, "In the confines of a film we can safely contemplate death, the ultimate tragedy, and feel our way through both familiar and foreign systems of meaning as we try to come to grips with it."3 
Other studies of death in film illustrate that these capabilities and potentialities have existed from the earliest years of film as a form of art and technology. For example, Wheeler Winston Dixon's A History of Horror explores the continuity between influential early narratives involving death (such as the Epic of Gilgamesh and Homer's Odyssey) and the death-laden gothic and supernatural literature that preceded (or coexisted with) film's emergence in the nineteenth century, many works of which were adapted into films. Michele Aaron structures her book, Death and the Moving Image: Ideology, Iconography and I, around references to early cinema, including works by groundbreaking filmmakers the Lumière brothers, Thomas Edison, and Cecil Hepworth, all in relationship to death and early cinematic techniques and subjects.

While centuries of memento mori variations in literature, philosophy, and artistic expression have reminded humans that they would someday die a physical death, modern books such as Ernest Becker's The Denial of Death (1973) have explored the fear and avoidance of that same reality. Becker's work influenced Tom Pyszczynski, Sheldon Solomon, and Jeff Greenberg's "Terror Management Theory," introduced in Worm at the Core: On the Role of Death in Life (2015) and also provided a foundation for Death in Classic and Contemporary Film. Terror management theory is a "psychological theory regarding how people manage the problem of death." ${ }^{4}$ When considering the relationship between the fact of death and the experience of watching fictional films, it could be argued that for characters within the stories involving death, and viewers consuming such stories, the reaction to accept or attempt to avoid death is a choice that guides the narrative and transcends the textual boundary separating characters from viewers.

In the twenty-first century, film and cultural critics have written regularly about the relationship between death and film. Stephen Holden of The New York Times framed his "Top 10 movies of 2007" as "Films That Look Death in the Eye":

Might they be a collective baby-boom response to looming senescence and a fraying social safety net? Or do they reflect an uneasy sense that humanity is facing end times, when global warming, terrorism, nuclear proliferation and the war in Iraq, or any combination thereof, could bring on doomsday. ${ }^{5}$

In 2015, John Patterson of The Guardian asked, "how badly do we wish to witness death at the movies?" Patterson's estimation is that films from the 1930s-1970s were too sanitized in their depictions of death and that modern films have the capacity to be as detailed and graphic as they wish but risk appearing crass or exploitative as a result. ${ }^{6}$ In truth, any given year in global cinema history has provided enough films about death to write a host of speculative articles. The digital distribution of global cinema, beginning in the 1990s on DVD and related home video formats and then online through streaming channels, has enabled critics and viewers to more easily compare and contrast treatments of death emerging from different countries and national cinemas, even as globalization reshapes the global entertainment industry toward greater homogeneity.

The present chapter examines a set of international films from the postclassical cinema era that feature choices regarding death as the most consequential narrative element. ${ }^{7}$ None of these works is a blockbuster film. Though some of them could be called entertaining, all treat death as a subject deserving of serious reflection. Readers of this volume who are interested in death as a subject, but who are not necessarily film 
specialists or enthusiasts, could benefit from considering these films as texts that contribute to understanding death issues in modern society.

Film critic and historian David Thomson summarizes a history of narrative forms to attest to the instructive outcomes of closely watching and reflecting on films:

a hundred and fifty years ago, people lived a life and referred it to books, games, and works of moral instruction. But in the time since then we have acquired this mechanism that mimics the way we attend to the world as a whole. Often enough, it supplants living, to say nothing of moral instruction. ${ }^{8}$

Richard A. Gilmore explains the value of watching and reflecting on cinema from a philosophical viewpoint, connecting the philosophies of Plato, Aristotle, and Wittgenstein, among others, to the activity of watching and thinking about movies:

If the essence of philosophy is reflection, philosophy can contribute to the completion of the experience of certain movies as we engage in philosophical reflection on them. Thinking philosophically, reflectively, about movies can contribute to the sense of depth and importance of the movies that people see, which would help to contribute to the sense of depth and importance, to the sense of meaning, in our lives as a whole ... An experience is meaningful when it comes to a kind of conclusion and we have learned something from it that we can use, something that will help us get on in the world. ${ }^{9}$

The primary evaluative framework for this analysis centers on the mechanics of the screenplays, meaning the story and plot that provide the narrative and visual schemata for the films. The films covered in this study have been selected mainly because they feature choices regarding death - acceptance or avoidance thereof - as the foremost narrative component. This is a significant shared feature that connects viewing and reflecting on these films to the role of choices in human lives: a chief concern of both philosophy and narratology. For instance, Plato's attention to making informed choices dovetails with screenwriting instructor Robert McKee's observation that "True character is revealed in the choices a human being makes under pressure - the greater the pressure, the deeper the revelation, the truer the choice to the character's essential nature." 10

In all of the films examined here, death is the pressure that demands choices, and the death-choices in all of the films reveal character. This particular grouping of films is also useful in establishing a defined period of cinematic treatment of death from various global perspectives. All are postclassical, with four films from the second half of the twentieth century, as well as one that was released globally from 2000 to 2001, bridging the turn of the century, and four films from the first quarter of the twenty-first century.

A secondary evaluative context is film form, which is the way the film has been realized by a director and other collaborators as a piece of visual storytelling. In both narrative and formal contexts, the analytical focus is on the experiences of the characters within the narrative rather than the psychological effect of the films on audiences. However, as previous studies of death and film have illustrated, any film in which the human characters encounter the inevitability of death, regardless of genre, has the potential to spill over into the viewers' attitudes toward death. The intentionally wide variety of film genres and narrative forms represented in this study is intended to illustrate how death as a subject transcends film types. 
There are three sections in the chapter, each of which examines three feature films from various places and times. The first group comprises works about the death of self and the wages of $\sin$ and/or consequences of a life lived: Japanese horror film figoku (1960), ${ }^{11}$ American romantic comedy film Defending Your Life (1991), ${ }^{12}$ and Dutch gangster film The Resurrection of a Bastard (2013). ${ }^{13}$ The second grouping is made up of various dramas about reacting to the death of loved ones: French film Ponette (1996), ${ }^{14}$ the 2018 extended cut of American experimental epic The Tree of Life (originally released in 2011), ${ }^{15}$ and American film Collateral Beauty (2016). ${ }^{16}$ The third and final set examines death as a cause and/or effect of social/familial duty and sacrifice: Belgian/French social realist drama La Promesse (1996), ${ }^{17}$ Danish musical film Dancer in the Dark (2000), ${ }^{18}$ and Japanese drama Departures (2008). ${ }^{19}$

\section{Sin, consequences, and the death of self}

Regarding the inevitability of bodily death, there is a strand of film narratives that question whether individuals have any remaining agency or choice following death. Figoku, Defending Your Life, and The Resurrection of a Bastard feature dead or death-changed individuals looking backward across death's divide to confront the consequences of their lives. In Figoku, a spoken narration follows the credits, playing simultaneously with images of bandaged, floating bodies. The narrator warns the viewer of mortality, sin, and Hell:

A handsome youth in the morning, once lured by that merciless wind, is by dusk a heap of bones. In the end, all must die. Countless people have sinned on their way towards death. Could the law even begin to punish them all? Some may slip through the law's net, but they cannot elude their own consciences. Religion dreams of a world where sinners are punished after death for sins unpunished during life. That world is Hell.

A woman's singing provides a recurring motif of death/funeral songs within the film. Against a scene set in a netherworld, she sings: "This is a tale of things not of this world / a tale of the River Sanzu / the eternal limbo of dead children," as a man shrieks in fiery torment.

This introduction is staged more like the closed world of opera or theater than film, a stylistic choice that reinforces the singer's description of "a tale of things not of this world." An additional narrative context soon follows, a university course being taught on the subject, "Concepts of Hell." This scene advances the Japanese Buddhist beliefs consistent with previously occurring elements, such as the Sanzu River, though Professor Yajima (Torahiko Nakamura) also points out to the students that "Buddhism is not alone in positing the existence of Hell. Zoroastrianism, Christianity, and Islam, among others, all share similar beliefs."

Shirō (Shigeru Amachi), a theology student, is in attendance at the lecture with his strange friend Tamura (Yōichi Numata). Tamura casually says to Shirō, "That guy from last night is dead," referring to a hit-and-run accident, one of many sins committed by the Hell-bound Shirō within the narrative. Tamura's relationship to Shirō is like that of a tempter, yet there is evidence that Tamura is more a sinning half of Shirō's self. Professor Yajima is revealed to be the future father-in-law of the engaged Shirō. As Shirō and Tamura continue to talk in the back of the lecture hall, the Professor describes Shirō's 
eventual destination: "In Japan, the most common conception is that of the 'Eight Hells' found in Buddhist sutras." Tamura tells Shirō that the professor is guilty of "a sin he wants to atone for."

Tamura is a negative force, but he is correct about Professor Yajima, whose sin of depriving another man of water during wartime joins the many sins of the film's network of characters. Shirō's beloved fiancé, Yukiko (Utako Mitsuya), is one of the only innocent characters within the narrative, and she dies while pregnant, ostensibly a casualty of Shirō's sins. In fact, the body count that escalates during the film's first hour implicates every character as a sinner and/or a victim of the sins of another person. Hence the third act of Figoku is a representation of Hell, in which Shirō encounters those he knew while alive. Various torments include being burned, bludgeoned, blinded, bound, and torn apart, all depicted in colorful, graphic detail that, while more theatrical than a realistic approach to special effects would produce, spares neither the characters nor viewers from the punishing reality of "sin without salvation." 20

Perhaps the most tragic dimension of Jigoku is the effect of Shirō's sin on his unborn child, whom the deceased Yukiko names Harumi while standing with Shirō at the River Sanzu. Yukiko reveals that she could not raise Harumi alone, so she "set her on a lotus leaf on the river." Shirō's journey into Hell is defined in part by his pursuit of Harumi, who floats along the river and ends the film stuck on an endlessly rotating wheel. Shirō reaches the wheel but never advances toward Harumi, who is stuck on the opposite crescent. In the end, Shirō's endless torment is to forever fail to rescue his innocent daughter. The freeze frame that ends their journey in Hell attests to the fixedness of Shirō's fate, which he never accepts. Shirō's desire to live, which he expresses frequently, desperately, after his death, does not transcend the judgment he faces.

Shirō's failures contribute to the tragic dimensions of death in Figoku. The films of Albert Brooks, writer/director of Defending Your Life, also revolve around failure, though to comic effect. ${ }^{21}$ In Defending Your Life, the death of protagonist Daniel Miller (Brooks) occurs much earlier within the narrative than Shirō's death in Figoku, though the screenplay and direction of Defending Your Life involve episodic flashbacks that reveal to the viewer the circumstances and especially the choices Miller made while he was alive.

Another significant distinction is that Miller's death sends him not to Hell, but to Judgment City, a fantastic post-death waystation that outwardly resembles an all-inclusive resort. Though not all the lodgings and restaurants are comparable, each person passing through Judgment City enjoys a room, clothing, ideal weather, and all they food they can eat. A recurring joke in Defending Your Life is that during one's stay in Judgment City, eating does not result in a weight gain. Thus the food, already appearing to taste heavenly, lacks any of the negative health connotations of delectable food on Earth.

Defending Your Life succeeds as a comedy, though would fail as a drama, as there are no significant dramatic stakes within the plot. Miller is in Judgment City "to defend his life" in a process overseen by attorneys and judges. If he successfully defends his choices in life, he will move forward within the universe, equipped with a higher percentage of his brain to use. However, if he fails to defend his choices in life, he will return to Earth in another body and attempt to try to live again. Thus Miller need not fear failing the process, as the worst outcome would be a redo, not even a setback.

That there is nothing to fear is precisely the point of Defending Your Life, a thoroughly humanist work. Fear provides Brooks' film with a central theme or conceit. Episodes from Miller's life are evaluated in the context of how consumed he was by fear. Miller 
and his attorney (Rip Torn) convincingly argue that there were other factors beyond fear that informed his decisions. The prosecutor (Lee Grant), however, frames Miller's past decisions about money (salary and investments), relationships and other worldly judgments as examples of his being fearful. Miller's time in Judgment City seems to provide another (damning in this case) instance of his fear, as he fails to act on the obvious potential for a romantic relationship with Julia (Meryl Streep), another visitor to Judgment City.

By focusing so consistently on fear as an impediment to moving forward within the universe, Defending Your Life subverts the thanatophobia or death anxiety that informs many film narratives about death and its unknown properties. This film specifically concerns the fears that plague life, not the fear of death. Likewise, Miller is allowed to fully participate in his post-death circumstances, including the process that will decide his fate, so his status as a recently deceased individual is an extension of his present life. His experience in Judgment City contrasts strongly with Shirō's futile heroism in Hell. For Shirō, to do the right thing after death results in no redemption because of the finality of death and Hell. Pointedly, Hell does not exist in Defending Your Life. Miller's final act, in which he risks his safety to attempt a last-minute reunion with Julia, results in his being allowed to move forward with her in the universe.

As a film narrative, Defending Your Life is written and designed to have Judgment City resemble Miller's normal living environment of Los Angeles. The restaurants, stand-up comedy stages, vehicles, and other settings are not visual or narrative metaphors for life on Earth that once was. Rather, each of these possesses a function like that on Earth. This likeness extends to key events within the plot. At the beginning of the film, Miller's death involves being hit by a bus. At the end of the film, his and Julia's successful forward movement occurs via a tram. The "courtroom" scenes in between the first and last acts motivate flashbacks that occur within the life that Miller lived. Brooks embraces a classical style of filmmaking that foregrounds the relatable human story by largely eliminating the separation between the world of the living and the world of the dead and by extending the present life into the days beyond death. Defending Your Life could be classified as a fantasy, but the film's advocacy of life without fear is centered on the observable, knowable moment, which is fitting for the film form's eternally present tense.

The Resurrection of a Bastard, adapted from 2004 graphic novel Om mekaar in Dokkum, is a gangster film that conflates past and present and collapses the borders of individual lives. The interplay of elements suits the film's source, as graphic novels allow the reader to flip back and forth in any order, for any duration, in order to consider the connections between pages. ${ }^{22}$ The primary plot is that of Ronnie (Yorick van Wageningen), a violent criminal whose past cruelty is initially belied by the tranquil, apparently clairvoyant figure we meet in the present. The secondary plot involves Eduardo (Goua Robert Grovogui), an Angolan asylum seeker with past trauma and whose connection to Ronnie is slowly revealed within the narrative.

The film begins as Ronnie rides with his associate Janus (Juda Goslinga) to Dokkum in Friesland, the Netherlands. The purpose of their visit is to find the man who shot Ronnie, who wears a neck brace indicative of his injury. In a bit of film intertextuality, Janus describes the recent change in Ronnie as the difference between Bruce Willis from Die Hard and Willis from The Sixth Sense. The Resurrection of a Bastard continues the deathchanged food fixation from Defending Your Life. Further evidence of Ronnie's appreciation 
for life and benevolent manner includes his kind compliments to cook/host Lotte (Rian Gerritsen) on trout and pomegranate, the taste of which he savors as if experiencing a new sensation.

Ronnie's newness collides with a flashback section titled, "The Old Ronnie," in which Ronnie abuses his wife, beats to death an innocent woman in front of her child, and sucks a man's eyeball out with a vacuum cleaner. The brutality on display in these scenes from Ronnie's recent past creates an uncanny relationship between the viewer and the character. To review these episodes is to attempt to reconcile the drastically different characterizations. There is no defending Ronnie's past life, but his present manner impresses as a second chance well-lived.

Also contributing to the complexity of the narrative is the secondary story of Eduardo, who at one point rides with a taxi driver to Dokkum, seeking opportunity. The film uses the occasion of their travel to introduce the history of Saint Boniface and his attempt to convert the Frisians, including the account of cutting down trees in the area. Eduardo links this to mythology from his homeland, noting that the "ghosts of the dead hang in their branches."

Death hangs over The Resurrection of a Bastard in a more direct sense, as the circumstance of Ronnie's conversion is revealed in another flashback scene. The setting is a vast party venue in which all attendees wear white. In the bathroom, a masked man who has been stalking Ronnie shoots him. The camera rises from Ronnie's body, embodying the visual perspective of a fly but equally representing his soul taking flight and reaching a higher consciousness. Following this pivotal moment are scenes that bring Ronnie's past behavior into contact with Eduardo's present agricultural job. Specifically, the young boy whose mother Ronnie killed is revealed to be the grandson of the couple for whom Eduardo works.

The continuing flashback of Ronnie's journey of transformation includes an effective but thoroughly unsubtle scene in which he wakes up from a coma, naked and crying like an infant. This new birth explains the present incarnation of Ronnie, but it also complicates the overriding question of the film: Where lies atonement for the sins of the past when the evil appears to have been exorcised from the wrongdoer? The man who shot Ronnie is the man for whom Eduardo works. He is intent on avenging his daughter's murder, and he is initially undeterred by his failed first attempt to kill Ronnie.

At present, as Ronnie grows closer to his apparent comeuppance, he develops a strong affinity for the natural world, particularly the water. When he admires a series of waterfall photos at the inn at which he's staying, Lotte says, "the mystery of life is hidden in the visible and tangible." Her dialogue is an expression of Ronnie's envelopment by the mystery of life. A subsequent scene involves Ronnie stripping naked on a beach at night and going into the sea, utterly exposing himself to the bullet of the vengeful father on the beach. Ronnie, once paranoid and haunted by the prospect of death, has been freed by near-death and reborn into an acceptance of whatever fate he will meet.

The concluding events of The Resurrection of a Bastard are mysterious and inconclusive regarding life and death. Ronnie and Eduardo join together, apparently saving the life of the man who would kill Ronnie. Next, an undefined period of time after said fateful night, they exist side by side on the branch of a tree. Darkly comic to the end, the closing dialogue of the film is, "In the land of the Frisians a tree is not a safe place." Here sit Ronnie and Eduardo, otherworldly observers, converted by trauma and sharing uncommon perception for the hidden mystery of life. 


\section{After life: the deaths of loved ones}

In the next set of films being examined, the death of a loved one threatens to cloud and deaden the lives of those left behind. Ponette, The Tree of Life, and Collateral Beauty are stories of faith in the unseen and of the struggle to accept loss. The question underlying each of these films is how to move on with life when those persons who gave life meaning are dead?

Ponette is a unique film within the history of the medium. Though there have been many wrenching cinematic variations on loss and grieving, never before or since Ponette has the entire narrative of a feature film about death been identified exclusively with the perspective of a four-year-old child. The plot for the film could be summarized concisely thus: Ponette (Victoire Thivisol) survives a car accident that took the life of her mother (Marie Trintignant), the driver. The young girl spends the following days and weeks striving to bring her mother back while conversing with adults and other children about the meaning of death.

The cinematography of Ponette primarily focuses on the yearning face and body of the protagonist. Adults with whom Ponette interacts are only briefly the visual or narrative focus. The dialogue-driven film is observational and naturalistic by design, as the filmmaker "Doillon carefully constructed his screenplay by establishing a workshop for fourand five-year-olds selected during a comprehensive audition process" and then "worked for six months with the children" that were cast in the film, "acting out scenarios and creating sketches, asking their opinions on a variety of subjects and adapting his script from their dialogue."23

While Doillon's writing and direction processes and Thivisol's performance are groundbreaking in a number of ways, the film's narrative journey substantiates long-standing research about how children understand death. One relevant study preceding the making of Ponette is Mark W. Speece and Sandor B. Brent's "Children's Understanding of Death: A Review of Three Components of a Death Concept," which focuses on "irreversibility, nonfunctionality, and universality" as "three of the most widely studied components of the 'mature' concept of death." 24 A tearful discussion early in the film, between Ponette and her father (Xavier Beauvois) involves universality, as the father makes his daughter promise that she will never die. This is a promise she cannot keep, but her age prevents her from understanding the universality of death. The bulk of Ponette's narrative, which develops along with the child's questions and reasoning regarding death, is concerned with Ponette's inability to understand irreversibility and nonfunctionality.

While staying with young cousins that are around her age, Ponette talks with the other children about death, burial, and the afterlife. Evidence of their failure to understand nonfunctionality includes theories about the need for a pillow in the coffin and the duration of the sleep such a pillow would aid. Ponette's hope that her mother could still function in a living way, if only just to sleep as the living do, blossoms quickly into a belief that she reunites with her mother at night, during which time her mother lives in a sky of castles and colors. These dreams fade quickly, though Ponette avoids believing that her mother's death is irreversible.

The most sophisticated aspect of Doillon's script is how the child's reasoning responds to and complicates adults' explanations. When Ponette's Aunt Claire (Claire Nebout) tells her about Jesus Christ's resurrection, Ponette holds hopefully to this fact as evidence of imminent irreversibility for her mother: If Jesus came back to see his friends, she reasons, her mother will certainly come back for a loving daughter. Aunt Claire later tells Ponette 
that her mother can hear and love her but cannot come back, arguing for functionality but against irreversibility. The theological matters of Ponette grow more complex as she converses with other adults and children about God, Jesus, and heaven, in relationship to her dead mother. Yet there is a recurring impression that until Ponette receives an answer in the form of her returned, able mother, all such theorizing and reasoning by young and old will only prompt more questions.

It is this blend of uncertainty and potentiality that gives Ponette credibility within the context of available research on how children understand death. Speece and Brent observe that "(a) the majority of children achieve at least some understanding of all three components ... and (b) all three components are understood at about the same timebetween age 5 and 7."25 As a four-year-old, Ponette is presently on the brink of a fuller understanding, so the plot is premised on an especially pivotal phase for her understanding of death. Yet her desire to reunite with her mother (and, frankly, a lack of any totalizing theory of death put forth by adults or children) outweighs whatever acceptance might accrue as a result of the theories she is exposed to.

Ponette concludes with a miracle. Alone in the cemetery, Ponette appears to be utterly broken. She digs in the dirt, crying, "Mommy. I'm here," attempting to physically unearth her mother. Her mother's return, in "flesh and blood," ends the agony of Ponette's yearning, as well as the audience's identification with that agony. Some critics who otherwise praised Ponette cited the mother's return as the only flaw of the film. For McGavin, "the moment destroys the emotional intensity and honesty Doillon has worked so hard to sustain." 26 Roger Ebert wrote, "In the real world, when mothers die they don't come back. Ponette has just about dealt with that when the movie sneaks in a happy ending. She'll never learn that way." 27 These critics arguably err by applying an adult's too-sure grasp of irreversibility and nonfunctionality to a narrative that is inextricably identified with a four-year-old child's view of death. By the film's climax, Ponette has encountered these components of death but has not accepted them as wholly true. Thus it is possible for her mother to return and for Ponette to cherish that return as the most consequential piece of evidence for how life and death work. Her mother's miraculous return ends the child's grief. As the film ends, Ponette shares with her father her returned mother's one instruction: "to learn to be happy."

The Tree of Life shares similar narrative content with Ponette, including the death of a family member as an inciting incident for the plot and corresponding grief as a potentially insurmountable obstacle. The film is also structured around dialogue with God and prayers to God and family members. There are themes of abstraction coming into focus for young characters, in this case nature and grace, as embodied by parents and other characters. Furthermore, the film ends with a vision of restored bodies reuniting on the shores of eternity.

Despite these similarities, the formal execution of The Tree of Life contrasts so fully with Ponette that the two films have not previously been examined for their shared qualities within film studies. Whereas Ponette is a film of modest scale, intimately tied to the perspective of a single young child, The Tree of Life is an epic, experimental film that probes many different characters' psychologies and spans vast amounts of space and time. The initial 2011 theatrical cut of the film and the 2018 extended cut have both been discussed at length in cinematic and philosophical terms. The film's unconventional narrative design and multiple character perspectives contribute to the multiplicity of possible readings. 
Based in part on autobiographical events, the film tells the story of the O'Brien family of Waco, Texas: Mrs. O'Brien (Jessica Chastian), Mr. O'Brien (Brad Pitt), and their sons Young Jack (Hunter McCracken), Steve (Tye Sheridan), and R.L. (Laramie Eppler). Though much of the film's narrative takes place in the 1950s, the inciting incident occurs in the 1960s when Mrs. O'Brien learns of R.L.'s death at age 19. Thus a majority of viewers' engagement with this film and family occurs with the knowledge that a son/ brother's death will disrupt their lives. Scenes from R.L.'s childhood are imbued with dramatic irony, by the inevitability of his death. Scenes that take place in the days and decades after his death are haunted by his absence.

Malick's original, unpublished screenplay for The Tree of Life differs in many ways from the finished film. Certain passages from the text provide evidence that R.L.'s death and his mother's response to it form the central narrative arc, even more significant than the evident battle between nature and grace that takes place within son Jack. Mrs. O'Brien's grief consumes her in the days following R.L.'s death. No words of comfort offered by friends and family lessen the effect of the loss. Then, as the screenplay describes, "Friends come by less. She does not mind. Her child is more present to her in death than, really, they in life. Good night, my child. She shall not see him. Till heaven and earth have passed away." 28

The events that follow this point in Mrs. O'Brien's life include one of the most extraordinary flashbacks in cinema history, covering "the growth of the cosmos, the great epic of evolution, from the Big Bang through the long ages of geological time, down to the present day." 29 Next the film depicts daily life for the O'Brien family in the 1950s, as the sons grow up and encounter the contrast between nature and grace, depicted by their father and mother, respectively. The ongoing effects of all these events on adult Jack (played by Sean Penn), adrift in a hypermodern landscape, constitute the other major portion and period of the narrative.

In the film's final minutes, Malick depicts "the end of the voyage of life" as the O'Briens and other families reunite on the shore of eternity. Mrs. O'Brien at last "embraces her lost son," R.L., and "Her grief is cured. Her doubts are ended." 30 All of the film's conflicts, some ancient, some modern, and some futuristic, are subsumed into the arc of Mrs. O'Brien's grief and its movement: from her inability to accept R.L.'s death to her realization of life/love beyond death. Such transcendence, such exposure to a superior reality, is Malick's ultimate subject in The Tree of Life. Reflecting on the whole film, the text that opens both cuts provides a significant clue. Before any visual storytelling takes place, appearing on screen are these words from Scripture, an account of God's response to Job: "Where were you when I laid the earth's foundation ... while the morning stars sang together and all the sons of God shouted for joy?"31 This concept, the preeminence of God, provides a context for the film's human problems and projects.

Collateral Beauty, the final film in this section, departs from the spiritual concerns of Ponette and The Tree of Life, situating a father's grief within a capitalistic, commercial sphere. Compared to those films, in which surviving family members eventually move beyond grief and towards joy, Collateral Beauty is explicitly concerned with material matters. The protagonist is Howard (Will Smith); introduced as a charismatic advertising executive whose approach to selling products involves tapping into "three abstractions" of love, time, and death. These are effective components of advertising, he says, because they "connect every single human being on Earth" through longing for love, wishing for more time, and fearing death. This introduction, which invokes the immaterial world as 
a means to profit, sets Howard up as a man who will soon need to rethink why and how such ideas are attractive to him as an executive.

Howard's six-year-old daughter dies from cancer. The shift this event creates within his characterization is nearly instant, in cinematic terms, as a number of seconds convey the years and months passing. Color diminishes from the frame and Howard's demeanor turns grave. For half a year following his daughter's death, Howard has been barely functional. He and his wife have separated, and he fails to communicate or make decisions at work. Grief has negatively affected the success of Howard's firm. His three partners, who want to sell the firm and their shares in it, cannot do so because he is the major shareholder. Their plan is to have him declared not mentally fit to be in his position at the firm and to force the financial resolution they desire.

Yet even as money is the motive for Howard's colleagues, their methods lead them back to the very same abstractions that serve as the major themes of the plot. Unwilling or unable to face the death of his daughter, Howard has been mailing letters to Love, Time, and Death, a behavior that could be used as evidence of his mental instability. The trio of shareholders hire actors to portray each of these abstractions to visit Howard, encounters they will document in order to substantiate his fragile mental state. In the movie's too-tidy narrative construction, each of Howard's co-workers also benefits in a particular way from their encounters with Love, Time, and Death, who are finally revealed to be spirits/nonhuman entities and not actors, after all.

The structure of Collateral Beauty is highly evocative of Charles Dickens' A Christmas Carol, with its three spirits visiting a rich man who must learn to see beyond money. As if to reinforce the comparison, Collateral Beauty is also set at Christmastime. The seeming irony of using such a structure is that A Christmas Carol serves, in part, to argue against basing one's life decisions on finances, especially when self-interest precludes charity and helping the less fortunate. Collateral Beauty, on the other hand, begins and ends with acts of selling in a story world for which selling ideas and products produces only good. Howard's co-workers' scheme to make him sign over the company corresponds with his realization that there is beauty within life, both despite and within his daughter's death. His marriage is also restored.

Collateral Beauty's reputation is as a critical and commercial disappointment, yet the film is not nearly as incoherent or improbable as the harshest critics claim, because the theme of goodwill is a consistent through-line for the film's setting and events and its connection to A Christmas Carol. Dickens' novella is frequently described as a narrative concerning goodwill. The placement of Collateral Beauty within a story world of advertising credibly motivates goodwill as a theme, as advertising exists in order "to build an intangible capital asset called goodwill." 32 So while it is true that Collateral Beauty uses the death of a child as an occasion for wealth creation and goodwill, this is perfectly consistent with the philosophy the film sells viewers, in a line directly spoken by Death (Helen Mirren): "Nothing's ever really dead if you look at it right."

\section{Social and familial dimensions of death}

The commercial/philosophical mutualism of Collateral Beauty could be perceived as concentrating too readily on what can be gained from tragic circumstances. The third and final set of films explores death in relationship to social and familial responsibilities. $\mathrm{La}$ 
Promesse, Dancer in the Dark, and Departures are narratives about the costs endured and the freedoms found through sacrificing one's position in the world for the benefit of another.

La Promesse, a social realist drama, is set in Seraing, Belgium. Though the story and action are carefully scripted and choreographed, the visual style of the film resembles an observational documentary, a quality associated with the filmmakers' previous work in nonfiction filmmaking. Like Ponette, La Promesse features an encounter between youth and death, which serves as the inciting incident for the plot. However, in this film the young character is a teenage boy, Igor (Jeremie Renier), who is old enough to understand death and who is also old enough to feel the weight of his sins and the sins of his father. In this sense, La Promesse also connects to the first grouping of films about the consequences for a life lived.

Viewers' introduction to Igor is his theft of a billfold from a woman in his community. After pocketing the money, he buries the wallet underground, as if to hide the evidence of his transgression. Such digging in the ground is a frequently occurring image in American and European films about death. Ponette from Ponette and Young Jack from The Tree of Life are also depicted in variations on using their hands to contend with soil, a physical activity and a substance linked within Scripture to mortality and transgression. ${ }^{33}$

The stolen wallet foreshadows a much more significant cover-up. Igor's dishonest father Roger (Olivier Gourmet), a contractor and landlord, illegally houses undocumented individuals and families. Hamidou (Rasmane Ouedraogo), a Burkinabé immigrant, works for Roger and lives in one of Roger's buildings with his wife Assita (Assita Ouedraogo) and baby son. Hamidou is injured when he falls from a scaffold after Igor alerts the workers to arriving building inspectors. With his final words, Hamidou says to Igor, "My wife, my child, look after them. Say you will." Igor replies, "I promise," providing the films' title.

Up until the point of Hamidou's accident, Igor has been reluctantly complicit in his father's exploitation of immigrants. There have been signs that he is ashamed of this complicity, as when Igor hides to avoid seeing a group of men he helped his father betray, rounded up by police. But Hamidou's request and Igor's promise create a dramatic turning point in Igor's maturation. Roger's refusal to help Igor treat Hamidou's wounds or take him to a hospital further reveals the difference between father and son: Roger is willing to let Hamidou die if that means Roger will avoid facing responsibility for his illegal activities. Roger throws Hamidou's body away with construction debris.

The rest of La Promesse illustrates Igor's response to Hamidou's death and final request. Igor tries to offer money, heat, and other tangible sources of survival to Assita, who is persistent in wanting to know what happened to her husband. Igor sees in Assita maternal, spiritual dimensions that are wholly lacking in his family life. Yet, even as he is drawn to those aspects of her that are mysterious to him, he lacks the ability to confess what he knows: that Hamidou is dead. The Dardenne brothers use their observational camera style to create tension around potential moments of confession, such as a scene in which Igor hugs Assita and cries or another moment before he holds her baby. Such potential falls away when Igor is unable to speak out.

However, Igor keeping his promise to the deceased Hamidou involves more than fessing up. Hence there are increasingly consequential acts of care that he bestows on Assita, including helping her to safety and care when she and her baby become sick. Additionally, he makes other sacrifices, such as giving up his teenage hobbies and selling the ring 
his father bought him. Igor's character arc is one in which keeping his promise to a dead man he barely knew produces a sense of morality and responsibility that was previously nascent at best. By growing out of Roger's shadow, Igor becomes capable of honor.

When Igor's confession finally arrives, the moment surprises because the shot is in motion, lacking the tense observational tableaux of earlier scenes with Assita. "Hamidou's dead," he says to Assita, rather than lead her on into the fruitless search for her husband, a search he has enabled to this point. These are the last words of the film. Assita turns around, wordlessly. She and Igor look at one another, she walks away, and then he follows in her direction.

There is no falling action to conclude the drama of La Promesse, reinforcing the film's relationship to social realism and observational documentary. Igor's divulgence is not necessarily the final act of keeping a promise. Yet the information he shares, equips Assita to move forward as the wife of a dead man and the mother to that dead man's living son. She is now able to act on her concern for Hamidou's body and soul in the light of what is true. Igor's decision to tell the truth is evidence of his growing understanding of death, notably another component shared by the living that co-exists alongside the irreversibility, nonfunctionality, and universality of the dead: "The belief that we should be true to the dead," which "goes deep in the human heart." 34

Dancer in the Dark is the third installment of Lars von Trier's Golden Heart Trilogy, following Breaking the Waves (1996) and Idioterne (1998). In these films, wife and/or mother characters at the center of the narratives behave in sacrificial, arguably irrational ways that are connected to brokenness, loss, and death. Breaking the Waves and Idioterne are divisive films because of the extent to which their protagonists descend into humiliation. In Dancer in the Dark, however, the heroine's escapism and purity offer a way to transcend death and its finality.

Selma (Björk) is an immigrant from Czechoslovakia, living in Washington with her son Gene (Vladica Kostic). Selma, whose vision has degenerated to near-blindness, works at a factory and also cards hairpins, all to earn money for an operation that will save Gene's vision when he turns thirteen years of age. Providing this medical intervention for Gene has motivated Selma's journey to America and is the reason for Selma's daily exhaustion. This motivation also leads directly to Selma's various points of no return later in the drama.

Dancer in the Dark is a musical film, shot on digital video. Some musical numbers were shot with one hundred cameras rolling all at once to maximize visual coverage of the song and dance. This approach to capturing footage, which was novel at the time of the film's production, and is still rare within image acquisition for feature films, corresponds to the function of musicals within the plot. In real life, Selma cannot see. In musical fantasy, sight (one hundred cameras) is ubiquitous. Though the first proper musical number occurs forty minutes into the film, von Trier includes musicals as a key feature of Selma's life. She rehearses for a local musical production, attends film musicals with her friend Kathy (Catherine Deneuve), and most significantly, explains her attachment to musicals to her neighbor, police officer Bill (David Morse). She explains that the game she plays when life gets hard is to "start dreaming and it all becomes music," an escape tactic the film goes on to visualize several times in Selma's hard life. Tellingly, Selma says that as a girl she would leave musicals just after the penultimate song so that "the film would just go on forever." Her avoidance of the final song of a musical is part of Dancer in the Dark's exploration of death avoidance. 
As a period piece, a musical about musicals, that is shot on video instead of film, and utilizes nontraditional aural/visual coverage of the song and dance numbers, Dancer in the Dark is a postmodern musical whose innovations exemplify Jane Feuer's description of postclassical Hollywood musicals: "The materials with which musical entertainment builds change across time, but the process of nostalgia for the old and mystification of the new never changes." Within the context of these formal-aesthetic death concepts (the final song of a movie, the cyclically undead form of the movie musical) enters a significant plot development involving death. Bill is in debt and steals all the money Selma has saved for Gene's surgery. When Selma tries to get her money back from him, Bill attacks her, holding his gun. The gun goes off and Bill is shot, then he says to Selma, "Kill me, just kill me ... be my friend, show some mercy ... If you want the money, you're gonna have to kill me." Victimized and distraught, Selma kills Bill, honoring his request and retrieving her money. Though the death scene is jarringly violent, a musical number immediately follows, in which Selma revives Bill, they dance, and he forgives her. In this musical version of her life, Selma is not punished for doing what she needed to do to save her son's sight.

Selma's fantasy only sustains her for so long. Throughout her arrest, trial, death sentence, and stays of execution, Selma follows her heart, knowing that her sacrifice is for Gene's benefit. Her walk to the gallows, aided by a merciful guard, turns into an unbearable display of sorrow. A last-second intervention occurs when Kathy hands Selma Gene's glasses and tells her she was right to listen to her heart. This is a mere kindness, not a clemency. Nevertheless, Selma calms and sings to Gene that there is nothing to fear. Right after she sings, "It's the next to last song," Selma is suddenly, violently hanged. The film closes with the text, "They say it's the last song. They don't know us, you see. It's only the last song if we let it be." Thus Dancer in the Dark concludes with Selma's ultimate sacrifice, that of her life for her son, while simultaneously reiterating her belief in cheating death by refusing to acknowledge the last song. Viewers who have watched the film to its end confront the irreconcilable duality of that act and potential.

Departures is the final film discussed in this chapter; suitable as a conclusion since the film is relevant to both the social/familial responsibilities surrounding death and also comprehensive in its array of attitudes towards death. The central character of Departures is Daigo (Masahiro Motoki), a cellist in Tokyo whose "orchestra is dissolved" and who chooses to move back to his hometown of Yamagata to reset his life with wife Mika (Ryôko Hirosue). They move into the home at which his deceased mother lived.

Responding to a job advertisement involving "departures," Daigo presumes he is interviewing for a position related to travel. The position, however, concerns the departed. The job, which Daigo accepts in light of few options, is to assist boss Sasaki (Tsutomu Yamazaki) with "casketing" and the rite of encoffinment. In this film's depiction of modern Japan, the encoffinment process that was traditionally carried out by the family of the deceased is now completed by professionals such as Sasaki. The point remains, to provide a peaceful departure. The rite of encoffinment entails using cotton to shape the visage of the deceased, washing and clothing the body, and applying cosmetics. ${ }^{36}$ Daigo, who has never seen a dead body before, soon offers his own body to the procedure, humorously serving as a corpse stand-in for a casketing procedure training video created by Sasaki. Daigo commits to his job and interacts with corpses from a variety of death states, eventually moving beyond assisting Sasaki and taking the lead in preparing bodies for their peaceful departure. 
In one sense, Departures is the story of Daigo humbling himself and accepting a deathrelated job he never anticipated in order to provide for himself and his wife. By doing so, Daigo suffers the indignity of smelling like death and becoming a pariah in his own home. When Mika finally finds out what he does, she cannot accept such a "filthy" profession and leaves him for a significant portion of the plot's duration. More generally, Departures attests to the rite of encoffinment as a stabilizing process across a range of different reactions to death.

The families of the dead, with whom the viewers of Departures identify, as the encoffinment rite is being performed for them as it is for us, exhibit a wide range of emotions and behaviors from joy and acceptance to sorrow and rage. The high body count of Departures is not a spectacular element of the film because the regularity of death is Daigo's everyday labor. The universality of death makes his job secure.

Nor does physical death mark the end of the departed, but rather a gate to the spirit's ongoing life, as a crematorium worker says in the film. Atsushi Asai, Miki Fukuyama, and Yasunori Kobayashi observe that

the encoffinment specialist removes the repugnance and fears from the ambivalent emotions of love and hatred that the family members hold for the deceased, and having thus left the family members filled solely with love, then, the deceased is able to embark on the journey to the afterlife ${ }^{37}$

By the end of the film, Daigo sees how being true to the dead likewise results in restoration for the living. His marital discord is healed, and he experiences an unlikely final encounter with his estranged, now-deceased father.

The role Daigo plays in his community is a necessary one. As his boss Sasaki is growing older and moving toward his own death, Daigo will provide continuity for the death ritual in Yamagata. Though he sacrifices his intended career path and, for a time, his marriage and dignity among friends, Daigo persists in service to the departed and those who survive them. Much scholarly writing about death, including chapters in this volume, explores the public and private dimensions of death and how, since the nineteenth century, death is more likely to occur in institutions than in homes. Yet there is a paradox in this shift toward death in public: "Sociologists and social historians call this the emergence of 'hidden' or 'invisible' death," as the dying is "masked behind the closed doors of the hospital and dressed up in the parlors of the mortician.",38

The films covered in this chapter all feature characters who find that encountering death is not limited to an individual, private experience. In every case, the acceptance or avoidance of death contains a relational aspect. For the sinners, there remains the question of how to answer to those to whom the sinner has done wrong. For the bereaved, there remain survivors whose interests exist in some kind of balance with the memory of the dead. For those who sacrifice their interests and lives for others, social and familial responsibilities are the substance that motivates selflessness.

This common relational thread of international postclassical death narratives in film illustrates the relationship between story structure and social structure that writing theorists Craig Batty and Zara Waldeback observe, particularly in films that counter the "selfcentered" modern take on the "Hero's Journey." In such films, "all life is connected, human beings interweave, time is not linear and small moments create a bigger picture." 39 One or more of those components is present in each of the films examined. 
These works suggest that remaining open to the perspectives, rights, and needs of others (living and dead) is a means by which the individual self, who cannot conquer death alone, moves from avoidance to acceptance of mortality.

This evolution of thought within screenplay narratology has a precedent within contemporary philosophy, as Emmanuel Levinas thought "most about the death of the other person" rather than the death of self. ${ }^{40}$ Richard A. Cohen summarizes Levinas's thinking about death thus:

precisely when care for the other's death takes precedence over care for one's own - all the way to the extreme point of "dying for" the other ... the human subject achieves its true humanity, and hence the proper height of a morally and socially responsible selfhood. ${ }^{41}$

In this philosophical tradition, moral development is directly linked to a deepening awareness of how one might do unto others regarding mortality.

It must be noted that the works in this chapter have limited audiences compared with crowd-pleasing blockbusters that break records at the worldwide box-office. However, the nine films explored here have value as cultural indicators, as they will continue to represent the conditions of their portrayed places and periods. For example, La Promesse, Dancer in the Dark, and The Resurrection of a Bastard share little to nothing concerning film genre or style. Yet they are connected through their depictions of immigrant or asylum-seeker experiences, respectively of a family from Burkina Faso living in Belgium, a single mother from Czechoslovakia living in North America, and a young man from Angola living in the Netherlands. The specificity of these depictions might have limited audience appeal within a commercial context, but their inclusion in film narratives is valuable for preserving a diversity of documented/dramatized life experiences within a branch of arts and commerce (filmmaking) that is becoming increasingly homogeneous within twenty-first century globalization.

As David Thomson, Richard A Gilmore, and others have observed, watching movies in a reflective and philosophical manner affects the way human beings view their own place in the world, including their relationships to others. For anyone seeking to better understand issues in society, it is worthwhile to construct a musée imaginaire of films from different places and periods. This chapter is an example of such a collection, with works that are united by death-choices as a subject; the inevitable subject for every living person.

\section{Notes}

1 "Top Lifetime Grosses," Box Office Mojo, accessed December 12, 2019, www.boxofficemojo. com/chart/top_lifetime_gross/?area=XWW.

2 Susan Sontag, "The Imagination of Disaster," Commentary, October 1965, www.commentaryma gazine.com/articles/the-imagination-of-disaster/.

3 Daniel Sullivan and Jeff Greenberg, "Conclusion: Cinematic Death Benefits," in Death in Classic and Contemporary Film, eds. Daniel Sullivan and Jeff Greenberg (New York: Palgrave Macmillan, 2013), 243.

4 Jeff Greenberg and Alisabeth Ayars, "A Terror Management Analysis of Films from Four Genres," in Death in Classic and Contemporary Film, eds. Daniel Sullivan and Jeff Greenberg (New York: Palgrave Macmillan, 2013), 19.

5 Stephen Holden, "Films That Look Death in the Eye," The New York Times, December 23, 2007, www.nytimes.com/2007/12/23/movies/23hold.html. 
6 John Patterson, "How Hollywood has Learned to Embrace the Realities of Death in Film," The Guardian, June 9, 2015, www.theguardian.com/film/2015/jun/09/hollywood-death-filmfault-in-our-stars.

7 Postclassical refers to American cinema from 1960 onward, following the period memorably examined by David Bordwell, Janet Staiger, and Kristin Thompson in The Classical Hollywood Cinema: Film Style and Mode of Production to 1960. Bordwell later covered post-1960 Hollywood in The Way Hollywood Tells It. Other publications specifically about the post-classical period include Barry Langford's Post-Classical Hollywood and Eleftheria Thanouli's globally focused Post-Classical Cinema: An International Poetics of Film Narration.

8 David Thomson, How to Watch a Movie (New York: Vintage, 2017), 5.

9 Richard A. Gilmore, Doing Philosophy at the Movies (Albany: State University of New York Press, 2005), 8, 12.

10 Robert McKee, Story (New York: Harper-Collins Publishers, 1997), 101.

11 Figoku, directed by Nobuo Nakagawa (1960; New York, NY: The Criterion Collection, 2006), DVD.

12 Defending Your Life, directed by Albert Brooks (1991; Burbank, CA: Warner Brothers, 2010), DVD.

13 The Resurrection of a Bastard, directed by Guido Van Driel (2013; Toronto, Ontario: Syndicado, 2019), Blu-ray.

14 Ponette, directed by Jacques Doillon (1996; New York, NY: Fox Lorber Home Video, 1998), DVD.

15 The Tree of Life, directed by Terrence Malick (2011; New York, NY: The Criterion Collection, 2018), Blu-ray.

16 Collateral Beauty, directed by David Frankel (2016; Burbank, CA: Warner Brothers, 2017), Bluray.

17 La Promesse, directed by Jean-Pierre Dardenne and Luc Dardenne (1996; New York, NY: The Criterion Collection, 2012), Blu-ray.

18 Dancer in the Dark, directed by Lars von Trier (2000; Burbank, CA: New Line Home Entertainment, 2001), DVD.

19 Departures, directed by Yojiro Takita (2008; Port Washington, NY; E1 Entertainment, 2009), DVD.

20 Chuck Stephens, "Hell on Earth," in Nobuo Nakagawa, Figoku, The Criterion Collection, 2006.

21 Christian Long, "When Success is Failure," Senses of Cinema, Issue 80, August 2016.

22 Robert Lewis (graphic novel illustrator) in discussion with the author, May 2019.

23 Patrick Z. McGavin, "Small Wonder," Chicago Reader, June 26, 1997, www.chicagoreader.com/ chicago/small-wonder/Content?oid=893775.

24 Mark W. Speece and Sandor B. Brent. "Children's Understanding of Death: A Review of Three Components of a Death Concept," Child Development Vol. 55, No. 5 (Oct., 1984), 1671.

25 Mark W. Speece and Sandor B. Brent. "Children's Understanding of Death: A Review of Three Components of a Death Concept," Child Development Vol. 55, No. 5 (Oct., 1984), 1679.

26 Patrick Z. McGavin, "Small Wonder," Chicago Reader, June 26, 1997, www.chicagoreader.com/ chicago/small-wonder/Content?oid=893775.

27 Roger Ebert, "Ponette," June 27, 1997, www.rogerebert.com/reviews/ponette-1997.

28 Terrence Malick, The Tree of Life screenplay (2007), 7.

29 Terrence Malick, The Tree of Life screenplay (2007), 13.

30 Terrence Malick, The Tree of Life screenplay (2007), 122-123.

31 Job 38:4,7 NIV.

32 Nariman K. Dhalla, "Assessing the Long-Term Value of Advertising," Harvard Business Review, January 1978, https://hbr.org/1978/01/assessing-the-long-term-value-of-advertising.

33 Genesis 3:17-19 NIV.

34 Raimond Gaita, The Philosopher's Dog (Abingdon: Routledge Classics, 2017), 70.

35 Jane Feuer, The Hollywood Musical (Bloomington: Indiana University Press, 1993), 92.

36 Atsushi Asai, Miki Fukuyama, and Yasunori Kobayashi, "Contemporary Japanese View of Life and Death as Depicted in the Film Departures (Okuribito)," Journal of Medical Ethics; Medical Humanities Vol. 36 (2010), 34, doi: 10.1136/jmh.2009.002543. 
37 Atsushi Asai, Miki Fukuyama, and Yasunori Kobayashi, "Contemporary Japanese View of Life and Death as Depicted in the Film Departures (Okuribito)," Fournal of Medical Ethics; Medical Humanities Vol. 36 (2010), 34, doi: 10.1136/jmh.2009.002543.

38 William R. Wood, "Capital Punishment," in Encyclopedia of Death \& Human Experience: 1, eds. Clifton D. Bryant and Dennis L. Peck (Newbury Park: SAGE, 2009), 143.

39 Craig Batty and Zara Waldeback, Writing for the Screen: Creative and Critical Approaches (London: Red Globe Press, 2019), 180-181.

40 Richard A. Cohen, "Levinas: Thinking Least about Death: Contra Heidegger," International Fournal for Philosophy of Religion Vol. 60, No. $1 / 3$ (2006), 25, www.jstor.org/stable/27646177.

41 Richard A. Cohen, "Levinas: Thinking Least about Death: Contra Heidegger," International fournal for Philosophy of Religion Vol. 60, No. 1/3 (2006), 25-26, www.jstor.org/stable/27646177. 


\title{
OF PRESIDENTIAL MAUSOLEUMS AND POLITIGS IN NEO-LIBERAL ZAMBIA, 2008 TO $2018^{1}$
}

\author{
Walima T. Kalusa and Kelvin Chanda
}

\section{Introduction}

On 3 June 2011, a heated debate erupted in the House of Parliament in Lusaka, Zambia, after an opposition lawmaker under the Patriotic Front (PF) party challenged the government of the ruling Movement for Multi-Party Democracy (MMD) to disclose the name of the company to which it had awarded a contract to build a mausoleum for Zambia's third Republican President Levy Mwanawasa, who had died in office three years earlier. ${ }^{2}$ The Member of Parliament further demanded to know how much money the firm would be paid for undertaking this project and when the project itself would end. In response, the then Deputy Minister of Works and Supply in the MMD government announced that it had single-sourced a Chinese company, Messrs Yangts Jiang Enterprises Limited, to construct the presidential mausoleum, adding that the firm would be paid K2,876,147,959.20 (equivalent to $\$ 204,179,000)^{3}{ }^{3}$

This revelation provoked immediate outrage in and outside parliament. Some MPs not only from PF but other opposition political parties lampooned the government for ignoring legal tender procedure in awarding the contract, for single-sourcing a foreign company, and for giving it such a lucrative contract. Others blasted MMD authorities for spending such a colossal sum of money on constructing a mausoleum for a dead leader. Still, other contrarians openly accused the authorities of corruption in awarding the contract, an accusation that was reportedly shared by Michael Chilufya Sata, the PF founder and soon-to-be Republican President himself. ${ }^{4}$ This accusation reinforced the shrill complaints of many ordinary Zambian citizens who joined the chorus of protest against what came to be infamously known as the "MMD mausoleum saga."

But the government turned a deaf ear to these protests. Over the rising din of complaints, its officials continued to fund the construction of the costly mausoleum until they were dethroned from office through losing the presidential and parliamentary polls that brought Michael Sata and his party to power three months after the controversy erupted. Yet the change of government marked no end to the acrimonious mausoleum debate, nor to the Mwanawasa mausoleum project in Lusaka. In an ironic twist, between late 2011 and 2016, the PF government not only paid for the completion of the construction of the mausoleum for the dead president but also erected two entirely new mausoleums close to his, much to the chagrin of the now defeated MMD leaders. Of the two mausoleums, one was for the immediate predecessor of Levy Mwanawasa and Zambia's second 
Republican president, Frederick Chiluba, who had died barely three months before the electoral triumph of the PF. The other one, more paradoxically, was for Michael Sata, who, himself, died as a sitting head of state toward the end of 2014. Each of these mausoleums reportedly gobbled more money to build than Mwanawasa's. ${ }^{6}$ Intriguingly, however, not a single official in the new regime objected to the erection of the additional mausoleums. Ample oral and documented evidence indeed suggests that, once in power, the former opposition leaders enthusiastically designed the construction of mausoleums for other fallen and living presidents, irrespective of their political affiliation. ${ }^{7}$ Conversely, the MMD, now as an opposition party, spared no effort in denouncing PF rulers as little more than hypocrites who had ignited a controversy over the Levy Mwanawasa mausoleum but constructed even more expensive mausoleums once in office. ${ }^{8}$

The controversy over presidential mausoleums in Zambia is neither new nor peculiar to the country - though the establishment of mausoleums was unprecedented in Zambia itself. Worldwide, conflicts surrounding mausoleums and the interment of dead politicians in them are legion. The pervasiveness and intensity of these controversies have captured the imagination of many scholars working on societies far removed in time and space. ${ }^{9}$ In their quest to unearth the roots of the contentiousness of mausoleums and of other political symbols, these academics have explored, inter alia, the political significance of dead politicians and royals and their burial sites for the construction, survival, and fall of political regimes. Elizabeth Self, for one, aptly demonstrates that successive monarchs in seventh-century Japan built grandiose mausoleums for deceased royals to boost the surviving rulers' own political stature and to tighten their grip on power. ${ }^{10}$ Similarly, a leading authority on the association between politics and mausoleums in the former Soviet Union argues that the construction and burial of Lenin in the now world-famous mausoleum in 1920s Moscow was a political maneuver central to the constitution of Soviet communism as a political force. The expert insists that, in burying Lenin in the mausoleum, Soviet authorities hoped to roll back the tide of suffocating opposition to their leadership from their political foes. Lenin's burial in the mausoleum was, therefore, a political act. This act was designed to turn his final resting place into a source of legitimate authority indispensable to warding off political competition and to sustaining popular enthusiasm for communist rule. ${ }^{11}$

Benjamin Forest and Juliet Johnson have more recently built upon this perspective. They have adroitly shown that the Lenin mausoleum continued to occupy the center-stage in Russian politics in spite of the collapse of the Soviet state in the 1990s. According to Forest and Johnson, some Russian politicians appropriated the mausoleum to as much bolster their own political influence as to undermine that of their rivals and to recreate postSoviet national identity atop the ruins of the collapse of the communist project. ${ }^{12}$

Unlike in other parts of the world where scholarship on the politics of mausoleums is rich and vast, studies of a similar of nature on Africa societies are few and far between. A notable exception in this regard, however, is a small body of studies inspired recently by the seminal work of Kathryn Verdery on the political value of the dead body in postcommunist Eastern Europe. ${ }^{13}$ This nascent scholarship is perhaps best exemplified by the separate studies of Megan Vaughan and Reuben Chirambo. Both of these works focus upon the mausoleum that President Bingu wa Mutarika of Malawi built for the late dictator President Kamuzu Banda in Lilongwe, the country's capital city, in $2006 .^{14}$ Vaughan shows that the construction of the mausoleum for Banda can best be understood in the context of the waning political fortune of wa Mutarika himself because of 
his failure to arrest corruption, eradicate poverty, or create employment. By building and interring President Banda in the mausoleum, Bingu wa Mutarika, the social historian concludes, hoped to regain political popularity especially in the central region of Malawi, where Banda allegedly had enjoyed unrivalled popular support before his demise. ${ }^{15}$

Chirambo shares these perspectives. His greater concern, however, is to illuminate the contentious debate that the burial of the late strongman in the mausoleum sparked across Malawi. The Malawian scholar demonstrates that President Bingu wa Mutarika along with his supporters passionately defended the construction of the impressive mausoleum and the burial therein of President Banda as a befitting tribute to the founder of the Malawian nation. However, Chirambo shows Malawians who had suffered persecution at the hands of the dictatorial regime of President Banda, contemptuously dismissed his mausoleum as no more than a monument to a blood-thirsty tyrant. To these critics, the mausoleum symbolized Banda's reign of terror and was an apt reminder of a ruthless dictator who prolonged his power through killing, incarcerating, and forcing into exile his political rivals, real or imaginary.

Collectively, then, this academic discourse attests to the fact that mausoleums are a significant form of symbolic capital, despite the novelty of their surge in contemporary Africa. Like the corpses of politicians interred in them, they are a potent resource whose symbolic value ${ }^{16}$ political rulers have historically harnessed since antiquity to construct political meaning, constitute power, and secure legitimacy and compliance from pliant citizens. ${ }^{17}$ But mausoleums are by nature as ambiguous as they are polyvalent. Thus, they tend to possess multiple, if not conflicting, layers of meaning, sometimes linking to other practices and emotional responses to the death of a leader. In this sense, mausoleums are not incapable of generating diverse reactions from different groups of people and at different points in time. ${ }^{18}$ It is unsurprising, therefore, that contrarians seeking to de-legitimize existing regimes focus some of their political aspirations on the target of mausoleums. ${ }^{19}$

This chapter draws on these valuable insights to comprehend why presidential mausoleums in Zambia's Third Republic became politically useful and yet a contested terrain in the decade from 2008 to 2018 . To this end, it explores the political struggles and challenges that confronted the ruling elites who dethroned the one-party state in the country in the early 1990s. It further examines the political value of the mausoleums in the authorities' efforts to overcome those challenges and thus gain popular support and legitimacy. Largely drawing on personal observations and oral interviews and the few official documents on mausoleums available to the historian, the paper suggests that the genesis of the controversial mausoleum debate was rooted in the intense power struggles within the Zambian political class and the consequent polarization of national unity that closely followed on the heels of the country's return to multi-party politics in 1991. In the face of enervating inter- and intra-party struggles for power and mounting popular disenchantment with the neo-liberal policies of the MMD and PF regimes that worsened living conditions during the period under review, political authorities built and interred dead presidents in mausoleums in order to forge a symbolic site. There, PF and MMD rulers hoped to reverse the forces that undermined their political legitimacy, their authority, and their influence. But efforts to gain popular political mileage on the symbolic terrain of mausoleums proved to be a shot in the dark. For, like their counterparts in Bingu wa Mutarika's Malawi, ordinary Zambians read conflicting meanings in the presidential mausoleums, meanings that were evidently at variance with those of the governing elites. 
Ordinary people thus demonstrated their ability to constrain the political agenda of the powerful. For some, this involved using the mausoleum as a framework for a more traditional emotional response to the death of a leader, though this was not the only option.

For analytical purposes, this paper is divided into three main sections. The first section outlines the broad context within which the presidential mausoleums were conceived and nurtured in Zambia. It does so through exploring the social, economic, and political challenges that confronted the architects of the Third Republic in the country, challenges that undermined their political legitimacy, making democratic rule questionable. The second section examines the processes and procedures through which officials in both MMD and PF regimes endeavored to reverse these forces of de-legitimation on the symbolic front of mausoleums. Particularly, the section describes the ways in which the authorities architecturally designed the mausoleums and, more importantly, the meanings they infused in them to win public support for their power and influence. The section insists that in designing, building, and inscribing such meanings into the mausoleums, MMD and PF politicians sought to sanitize and appropriate the legacies of fallen presidents while taking advantage of the fact of their death. In the same vein, they also hoped to restore national unity and thus appear fit to rule, at least in the eyes of the general populace. Yet, as this paper later argues, efforts to mobilize public political endorsement on the symbolic site of mausoleums did not go uncontested. Through a cursory glance at popular reactions to and understandings of presidential mausoleums in Lusaka, the third and final section of the paper illuminates the diverse and, often conflicting, ways the governed in Zambia appropriated presidential mausoleums. It demonstrates that ordinary men and women in the country perceived and deployed the presidential mausoleums in ways that did not always neatly mesh with the expectations of their rulers. The section thus underscores the ability of the ruled to constrain the power of the political class in Zambia to mould the meanings and uses that ordinary citizens imbued into the final resting space of their late rulers. ${ }^{20}$

\section{Political context: new rulers, old problems, and politics in Zambia's Third Republic}

The contestation over the mausoleums in question may perhaps best be understood against a brief background of economic and political challenges with the resultant intraand inter-party struggles for power that accompanied the restoration of multi-party politics in Africa generally and Zambia particularly after $1990 .^{21}$ The Zambian economy had by the end of that decade virtually collapsed. As keen observers of economic conditions have rightly noted, this partly stemmed from the decline in copper prices on the world market from the 1970s onward and partly from the gross mismanagement of the economy by the authoritarian regime dominated by President Kenneth Kaunda, whose United National Independence Party (UNIP) had led Zambia to independence in 1964 and imposed upon it one-party dictatorship in $1972 .{ }^{22}$ These economic and political woes were aggravated by the socialist policies under which the regime both created stateowned companies and nationalized the economy in the late 1960s and early 1970s. Such drastic policies had a telling impact on the economy. By the late 1980s and early 1990s, most industries in Zambia, including mining, agriculture, and manufacturing, had collapsed. This had devastating socio-economic consequences, ranging from high unemployment and a huge foreign debt to social decay, crime, cholera epidemics, and a crippling 
HIV/AIDS pandemic. Equally, the provision of social services such as education and health had virtually ground to a standstill.

Things on the political landscape were scarcely any better. As the UNIP totalitarian government proved incapable of tackling deepening social and economic difficulties in the country, and thus lost its credibility and legitimacy in the public eye, it became increasingly intolerant of any form of political dissent. UNIP leaders spared no effort to suppress political opposition through detaining and imprisoning their political competitors or co-opting them into influential positions in the party and/or its government. Their government therefore had by the inception of the 1980s and 1990s unsurprisingly become synonymous with abuse of human rights and suppression of freedoms of political expression and association.

It was under this political climate that towards the end of the 1980s some members of the ruling class in Zambia overcame their fear of intra-class conflict and launched a successful bid for the reintroduction of multi-party democracy, a topic on which much ink has now been spilt. ${ }^{23}$ At the forefront of this campaign, out of which the MMD was born in 1990 and the UNIP eventually removed from power in 1991, were Frederick Chiluba, a former Chairman of the Zambia Congress of Trade Unions (ZCTU); Levy Mwanwasa, a successful lawyer; and Michael Sata, one of President Kaunda's ministers, who ditched his boss and joined the MMD. ${ }^{24}$ From the outset, these leaders promised the electorate to right the wrongs of "Mr Cholera" (as the UNIP government came to be contemptuously branded in many MMD circles) through replacing the one-party state with a more democratic, accountable, and transparent government and implementing neo-liberal economic policies. Backed by Western lenders, donors, the IMF, and the World Bank, these policies were to boost the private sector and foreign investment, thereby creating employment, eradicating poverty, and providing a better life for all.

The MMD leaders equally promised to liberalize the political space, champion freedoms of expression and association, and put an end to the abuse of human rights rampant during the Kaunda regime. Egged on by the IMF and the World Bank, which made reintroduction of neo-liberal economic and political liberalization the pre-condition for giving aid to Africa in the 1990s, and supported by ZCTU, businessmen, and the general public fed up with UNIP dictatorship, the MMD trounced UNIP in the presidential and parliamentary elections of 1991. The defeat of the UNIP and the creation of the MMD government marked the end of the one-party era and the beginning of Zambia's Third Republic. Chiluba became Republican president with Mwanawasa as his vice-president. Sata subsequently held a number of ministerial portfolios within the government before becoming the party's National Secretary in December 1995 and later Minister without Portfolio, a position he held until he split away from the MMD in 2001.

It is indisputable that the restoration of multi-party democracy brought with it some positive changes in Zambia, not least of which were the birth of a lively, free print media and freedoms of expression and association unseen during the UNIP days. ${ }^{25}$ However, removing UNIP from office proved much easier than fulfilling the lofty promises MMD authorities made to voters prior to assuming power. As students of post-UNIP economy have noted, the neo-liberal reforms that the MMD regime (and later the PF government) implemented deepened rather than resolved the economic crisis the new government inherited from its predecessor. Its implementation of IMF- and World Bank-inspired structural adjustment programs, for instance, created massive retrenchments of workers both in the public and private sectors. And, as the much-expected inflow of foreign 
investment into the economy hardly materialized, unemployment and poverty soared, further aggravating the already deplorable living conditions of the majority of Zambians. More importantly for this paper, the liberalization of political space by the MMD heightened political and electoral competition and tension, whose intensity and associated inter- and intra-party violence, was unheard of during the one-party state when Kaunda's rivals were simply silenced through either co-optation or dismissal. Struggles for power in turn led to the formation of numerous political parties in the country. For example, by 1996, there were no fewer than thirty-five political parties in Zambia locked in combat for power against the MMD and with each other. Notable among the parties that challenged the MMD was the defeated UNIP, which continued to muster not a small following countrywide, partly because of the failure of MMD to make good its electoral promises and partly because UNIP organs such as wards and branches continued to operate smoothly after its loss of power.

The MMD also faced stiff internal struggles for power, resulting in its balkanization. Parties that split away from the MMD in turn competed against it for power. Notable among such parties was the Zambia Democratic Congress (ZADECO) and the National Party formed by the former MMD ministers who were dismissed by President Chiluba. Another source of threat to the MMD regime emanated from the Lima Party established by officials who had resigned from the government amidst accusations and counteraccusations of corruption, drug trafficking, and inefficiency among some cabinet ministers. The rival political parties not only weakened the MMD's grip on power but, since some of them were little more than regional or ethnic associations, they also wreaked havoc on national unity and on the cohesion of the Zambian political class, thereby posing no minor threat of inter-ethnic strife and violence in the country. ${ }^{26}$ Unable to contain this opposition, the MMD government, like the UNIP regime before it, resorted to draconian measures to silence its political opponents. In 1996, for example, it detained one of the founders of ZADECO as well as former president Kaunda over unproven allegations that they had masterminded a coup attempt.

But the greatest challenge to the MMD's grip on power emerged in 2001 when Michael Sata broke away from the party. Unhappy that President Chiluba had side-lined him and chosen Levy Mwanawasa as his successor, Sata founded the Patriotic Front in the same year. A shrewd political operator, the charismatic leader soon turned to his advantage the high levels of unemployment, poverty, and corruption in the MMD government to successfully de-campaign the ruling party and to popularize his own. The PF thus soon secured a substantial following especially in the politically important Zambian Copperbelt as well among Sata's fellow Bemba-speaking people in Luapula and the Northern Provinces. The PF, therefore, soon became a formidable opposition party. ${ }^{27} \mathrm{By}$ the time President Mwanawasa passed away in August 2008, it was indisputably the largest opposition party in the country that posed a threat of defeating the MMD in the presidential by-election occasioned by the president's death. Although Sata lost the byelection, his political party managed to oust the MMD from power in the presidential and general polls held barely three years later and formed the next government.

But the PF government, which pursued neo-liberal policies akin to those of its predecessor, did not escape the socio-economic and political doldrums that had led to the demise of the MMD regime. The slow recovery of the economy coupled with a crippling international debt precluded eradication of high levels of unemployment and poverty. It also prevented PF rulers from fulfilling their promises to "put more money" in people's 
pockets or improve living conditions. ${ }^{28}$ To make matters worse, corruption, authoritarianism, and a back-breaking power struggle crept into the PF government itself, especially after Sata's death in October $2014 .^{29}$ This wreaked havoc upon internal unity in the governing party, forcing some key PF leaders such as the former vice-president Guy Scot and Defence Minister Godfrey Bwalya Mwamba to abandon the party. The intraparty struggles for power further led to the expulsion in 2017 of the outspoken Minister of Information and Broadcasting Chishimba Kambwili, who, in response, formed the National Democratic Congress (NDC) to challenge the PF. ${ }^{30}$ Political competition between the ruling party and other opposition parties equally swelled. This state of affairs sometimes degenerated into bloody, if not fatal, inter-party clashes. Most notable were clashes between cadres of the PF and those of the United National Development Party (UPND), which became the most formidable contender for power in the aftermath of PF's electoral triumph in 2011. Similar clashes between PF and NDC supporters have more recently made headlines in the print media. In the face of this struggle for power and of the consequent fragmentation of the political class and national unity, consolidating democratic rule in Zambia remained a pipe dream.

\section{Making presidential mausoleums and their meaning}

Julius O. Ihonvbere has perceptively observed that the failure of the architects of Zambia's Third Republic to eradicate poverty and corruption, to contain divisive politics, to enhance national unity, and to consolidate democratic rule spawned popular resentment and even "authoritarian nostalgia" for the Kaunda era of a one-party state, when corruption and struggles for power were comparatively minimal. ${ }^{31}$ This situation, Ihonvbere continues, sparked a political crisis that in turn "set in motion a process of de-legitimation" inimical to the very survival and stability of democratic governance in neo-liberal Zambia. ${ }^{32}$ Worldwide, socio-economic and political forces that endanger the survival of regimes trigger an urgent search for political symbols by which incumbent rulers seek to reverse such forces and (re)gain popular enthusiasm for their authority. ${ }^{33}$ The first major step in this direction following the return of multi-party democracy in Zambia took place a few days after the burial on 3 September 2008 of President Mwanawasa, when the MMD government declared his burial place at the pristine Embassy Park in Lusaka as a national presidential burial site. The process of taking new advantage of the death of a leader was underway. The choice of Embassy Park was motivated by the fact that the site was located in the capital city's upscale area with good roads and thus easily accessible to local and foreign visitors. ${ }^{34}$

In another equally important step, MMD authorities began to raise money for the construction of what was initially officially known as "a National Monument" over the grave of the late president, obliging the Cabinet Office to spearhead this project. ${ }^{35} \mathrm{In}$ a move that not only informed the building of the proposed monument but also mausoleums erected later for deceased presidents Chiluba and Sata by the PF regime, the government directed that the monument for Mwanawasa was to be designed and built in such a manner that it "properly housed" his grave and also "serve[d] as a symbol of honour of his values and legacy." "The monument was to inspire "those who [had] remained to emulate" the deceased leader through "upholding his values, achievements, beliefs and legacy" that the MMD government deemed essential to "fostering peace, unity and national development."37 
From the outset, then, MMD functionaries had a clear understanding of the political value and uses of the posthumous monument they wanted to build for Levy Patrick Mwanawasa. The authorities were, however, less certain as to what of type monument would best preserve his legacy and, simultaneously, protect their own political interests. For one thing, Levy Mwanawasa was the first Zambian president to die in office. The authorities and the country as a whole, therefore, possessed neither a legal text nor a history of presidential burials that the leaders could draw on to plan, design, or build the intended monument. Indeed, in the early days after his death, there was much confusion in government circles over whether the proposed monument for the late president should be a mausoleum or what an official described as a coliseum. ${ }^{38}$ For another thing, the proposal to erect a monument for the dead president was a sensitive issue in a country devoid of an intra-mural burial culture. This situation risked sparking resentment from the Mwanawasa family, the general public, and rival political parties, thereby fueling the already hostile debate that PF leaders had begun in parliament.

The need to circumvent or at least minimize this opposition had two important ramifications. First, the political elites in the MMD government (and later their counterparts in the $\mathrm{PF}$ regime) thoroughly monopolized the mausoleum-making process, confining the planning and designing of presidential mausoleums exclusively to government ministries and organs. Second, the authorities decided that only ideals, beliefs, and attributes of the dead president that fostered national development and unity and were thus beyond public reproach or ridicule were to be infused into his mausoleum. In this way, they hoped that his mausoleum as well as its meanings could be insulated from rejection by the Mwanawasa family or abhorred and destroyed by any disaffected parties in future. ${ }^{39}$ One of the consequences of this decision was that the political class in Zambia carved out no space for popular involvement in the creation of the presidential symbols for dead leaders. Apart from hindering the public from participating in the creation of such symbols, this decision also prevented the print media from documenting how mausoleums in the country were planned, designed, and publicly debated. This situation was fundamentally at odds with the worldwide practice whereby political rulers actively invite their subjects to contribute ideas, proposals, sketches, and drawings toward the making of national symbols. ${ }^{40}$

To overcome the dearth of information on construction of presidential national symbols in Zambia, the MMD government set up a four-man committee in the Ministry of Works and Supply (MoWS) in 2008. It obliged the committee to visit African countries with presidential burial sites to ascertain how mausoleums for dead presidents "were constructed" and managed. ${ }^{41}$ Between 21 September and 1 October of that year, members of the committee visited the mausoleums of the late presidents Kamuzu Banda in Malawi, Jomo Kenyatta in Kenya, Augustino Neto in Angola, and Laurent Kabila in the Democratic Republic of Congo. Apparently, the officials were most impressed by the mausoleums of Banda and Kabila, both of which memorialize their legacies, accomplishments, and greatness. ${ }^{42}$ It is no wonder, then, that upon their return to Zambia, the committee members advised the government to erect a mausoleum "befitting the greatness" of Levy Mwanawasa and reflecting the important role he had played in fostering economic development and national unity. To this end, the committee recommended that "The design and concept of [his] mausoleum depict his life, belief and contribution to the wellbeing of the [Zambian] nation.",43

Consistent with these recommendations, the government assembled a research team of officials from the National Museums Board (NMB) and the National Heritage and 
Conservation Commission (NHCG) to document Mwanawasa's life experiences, values, beliefs and, above all, his achievements. ${ }^{44}$ The research team's report was then forwarded to the National Arts Council (NAG) mandated by the government to make a sketch of a mausoleum whose features were to represent the values and accomplishments of Mwanawasa recorded in the research team's report. It was on the basis of the NAC's sketch that experts in the Department of Building in MoWS produced an architectural design of the mausoleum for the late politician. Needless to say, this design firmly embodied what was considered his accomplishments and legacy. In 2011, the MMD government approved the design. It further single-sourced the Chinese firm to start constructing the mausoleum, sparking the acrimonious debate with which this paper began.

The Mwanawasa mausoleum rests on four huge boot-like pillars. These pillars celebrate his neo-liberal policies by which he reportedly successfully confronted four major challenges that dogged Zambia during his presidency: corruption, low productivity in agriculture and mining, slow growth of the economy as a whole, and, lastly, poverty. Engraved on the tombstone inside the mausoleum to symbolize that President Mwanawasa successfully redressed all these socio-economic woes is a biblical scripture based on 2 Timothy 4:7 which reads as follows: "I have fought a good fight. I have finished my course. I have kept the faith." The notion that he had been an accomplished lawyer who before turning to politics had championed the rule of law, social justice, and human rights is encoded in the mausoleum's roof, which, with its supporting structure, resembles an attorney's wig. His mausoleum as a whole is shaped like an African stool, reflecting that Levy Mwanawasa died as a sitting president. ${ }^{45}$ Lastly, the five wide steps leading to the entrance of the mausoleum represent the full term of office the president severed as Republican president from 2001 to 2006. Three tapered steps reflect his incomplete term of office.

In September 2011, with the construction of Mwanawasa's mausoleum still underway, the MMD government was overwhelmingly defeated and removed from office in

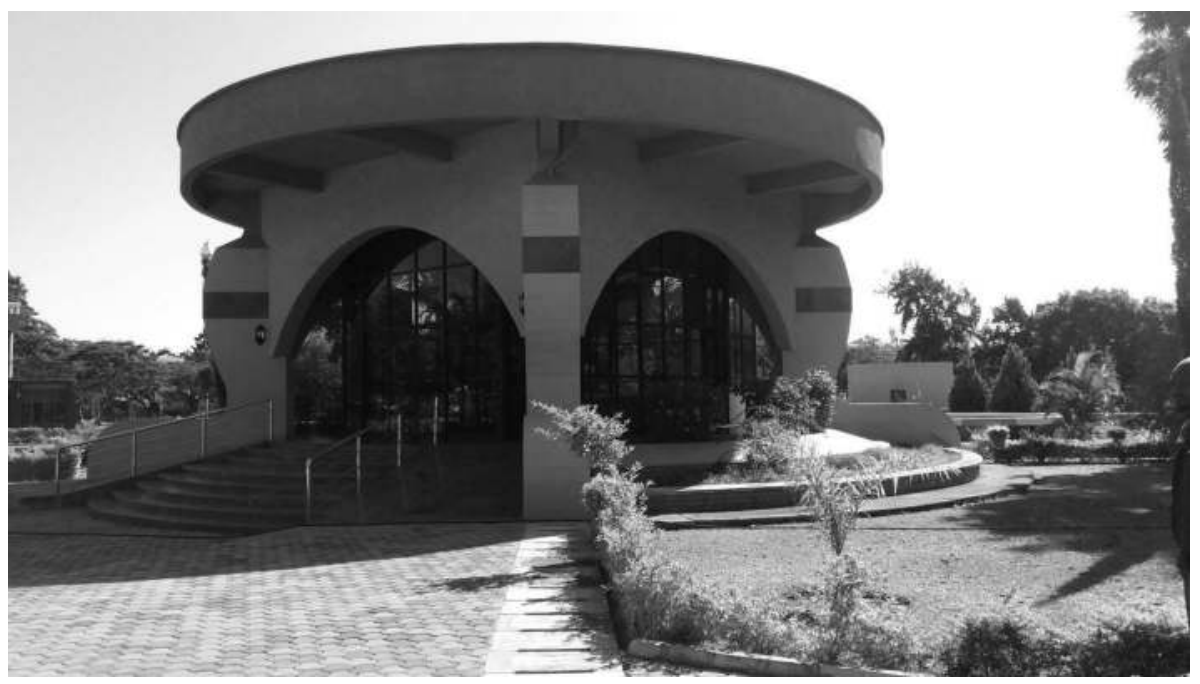

Image 25.1 President Levy Patrick Mwanawasa's Mausoleum

Source: Courtesy of National Heritage Commission 
presidential and general elections won by Michael Sata and his Patriotic Front. In a move that astonished many Zambians and also infuriated defeated MMD leaders, the PF government, whose functionaries had sparked the "mausoleum saga" before assuming power, mobilized more funds to complete the mausoleum. They also, more ironically, adopted the same mausoleum-making process and procedure of the MMD, deploying them to erect a new mausoleum for another MMD politician, President Frederick Chiluba, who had died only a few months before his party lost power to the PF.

In December 2011, the newly established government formed its own research committee with personnel from the National Museums Board (NMB), MoWS, NHCC, and the Ministry of Chiefs and Tradition Affairs to shepherd the planning and designing of Chiluba's mausoleum under the supervision of the Cabinet Office. ${ }^{46}$ Like the earlier research team, some officials of the new research team documented the life history of the dead president, illuminating his experiences, religious beliefs, and achievements. Others reportedly travelled to Ghana, Tanzania, and China. There, they collected more information on presidential burial sites. ${ }^{47}$ The religious beliefs, the sartorial habits, and the legacy of President Chiluba as well as information gleaned from burial sites abroad constituted the basis of the architectural design and meanings of the mausoleum that the PF regime began to build for him close to Mwanawasa's in 2012.

His mausoleum is built as a round chapel, the roof of which has a miniature polygonal house topped with a crucifix (see Image 25.2). Embedded in the ceiling above the president's tombstone inside the mausoleum is another crucifix illuminated by bright lights. The chapel-like mausoleum and its crucifixes respectively embody Frederick Chiluba's declaration of Zambia as a Christian nation and his unwavering commitment to upholding the Christian faith after his ascent to power in 1991. On the other hand, the polygonal house on top of the roof of the mausoleum symbolizes his Presidential Housing Initiative, an ambitious project under which the president and his government had constructed houses in the country's capital city, ostensibly to empower ordinary people. Supporting the mausoleum outside are ten strong pillars denoting the ten years Chiluba ruled the country. Finally, his penchant for wearing expensive, monogrammed attire, a practice that came to be fondly known as "New Culture" during his reign, is equally celebrated through a red pavement shaped like a necktie at the entrance of the mausoleum! $!^{48}$

While the building of the mausoleum for the deceased charismatic leader was going on, President Michael Sata died in October 2014. It may be recalled that the PF founder had been party to the saga over the mausoleum of President Levy Mwanwasa in 2011. Soon after Sata's own death, however, the PF government that he had established initiated high-level talks about erecting his mausoleum. Demonstrating the immense importance it attached to constructing this mausoleum, the talks involved only directors from NMB and NHCG as well as six permanent secretaries from different ministries. The talks culminated in the formation of a Technical Committee in December $2014 .^{49}$ Chaired by NMB's director and directly responsible to the Secretary to the Cabinet, the committee was mandated to manage the process of designing and building Sata's mausoleum. ${ }^{50}$ Two years later, the PF government turned the Technical Committee into a high-powered Standing Committee of permanent secretaries only, with several subcommittees responsible for, among other things, coordinating, overseeing, and building mausoleums for incumbent and former presidents. ${ }^{51}$ 


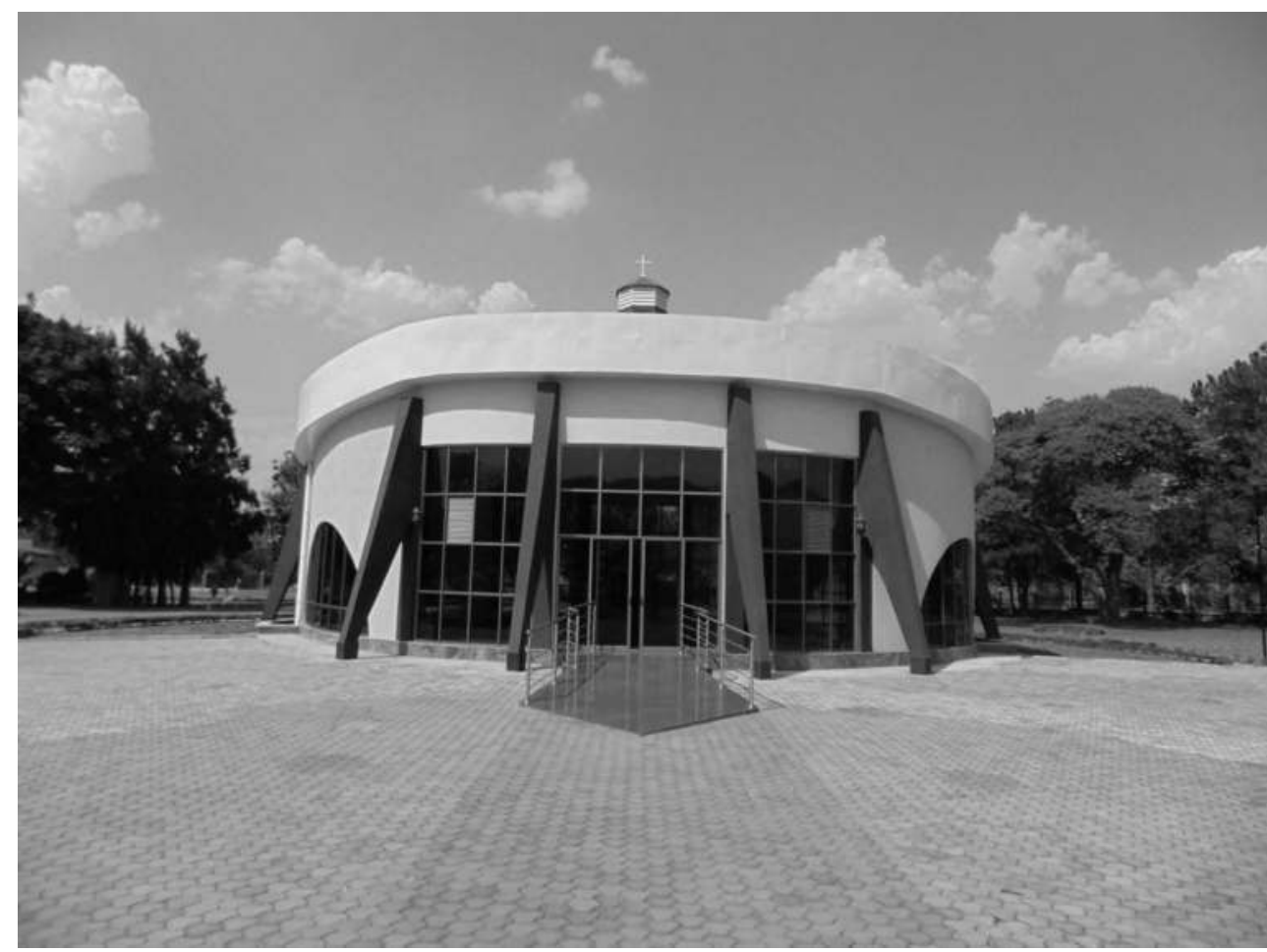

Image 25.2 President Frederick Titus Chiluba's mausoleum.

Source: Courtesy of National Heritage Commission.

In designing the mausoleum of Michael Chilufya Sata, the Technical Committee established in 2014 was guided particularly by the widely held belief in PF quarters that the maverick politician had been "a great, wise statesman," a staunch Catholic, a passionate advocate of the rights of the voiceless, and a relentless supporter of national development and unity. ${ }^{52}$ In conformity with this belief, the government urged the committee to design an elaborate, sophisticated mausoleum befitting his status. ${ }^{53}$ The committee complied. Between 2014 and 2015, it carried out very thorough research on the life of the dead president. The committee also designed what undoubtedly is the most sophisticated mausoleum at the Embassy Park, infused it with statesman-like attributes of the late leader. This was notwithstanding the ridicule from his political rivals in the MMD and other opposition parties, who dismissed "King Cobra," as Michael Sata was known to his political opponents, as having been a been little more than a war-monger in life and presumably, therefore, undeserving of such honor in death. ${ }^{54}$

The only one-storey building with lower and upper sections (see Image 25.3) at the presidential burial site, Michael Sata's mausoleum is said to resemble the palace of King Solomon of the Old Testament. This symbolizes that, in life, the Zambian ruler had been a wise leader comparable to his biblical counterpart. Leading to the lower section of the building are ten steps, the last of which marks the tenth province of Zambia that Sata created during his tenure as the country's fourth Republican president. On the 
other hand, the twenty-one steps that lead to the building's upper section recall the equal number of districts he similarly established in tandem with his government's policy of decentralization, intended to improve its effectiveness and efficiency in delivery of services to the governed. ${ }^{55}$ The names of the districts are engraved on the steps. Inscribed on ten pillars inside the mausoleum in both Hebrew and English are the biblical Ten Commandments by which the president had sworn to rule the country. The sophistication of his mausoleum as a whole denotes his robust infrastructural programs, which had involved building universities, schools, hospitals, and roads in order to enhance the provision of public social service, create employment, and combat penury.

This general description of the architectural designs of presidential mausoleums with their meanings points to the conscious efforts of the political elites who built them to forge in the country's seat of power a site replete with symbolic capital, a not uncommon practice of politicians worldwide. ${ }^{56}$ The infusion by the Zambian rulers of scriptures and Christian imageries into the mausoleums is indicative of their intention to sacralize the burial space of the fathers of the Third Republic in Zambia, thus turning the mausoleums in which they are interred into an arena of patriotic worship for those who visited them. There can be no doubt, moreover, that in suffusing these buildings with the merits, religious beliefs, and neo-liberal measures of Sata, Mwanawasa, and Chiluba,

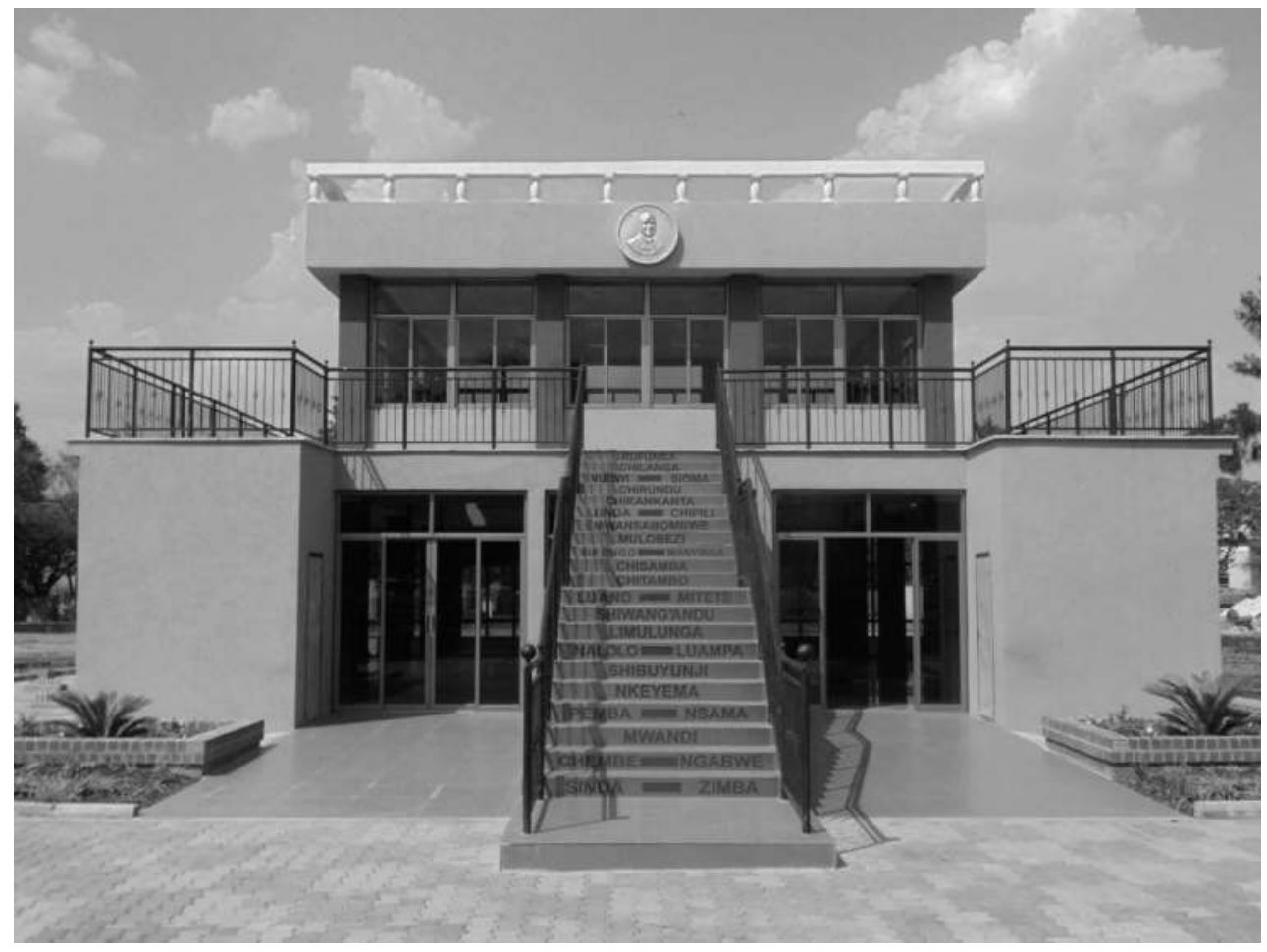

Image 25.3 President Michael Chilufya Sata's mausoleum.

Source: Courtesy of the National Heritage Commission. 
while simultaneously ignoring their shortcomings, both MMD and PF authorities strove to popularize them, at least in the eyes of the public. The dead rulers could thus enter popular consciousness or memory as heroic figures who in life had liberated the country from one-party dictatorship and, more crucially, endeavored to overcome the socioeconomic and political malaise that arose or deepened under their watch. In this sense, the mausoleums at Embassy Park were a means of exalting the three dead politicians or in the memorable phrase of some scholars, an instrument of "political communication" by which political rulers in Zambia tried to encourage the ruled to admire and to emulate the qualities of their deceased presidents. ${ }^{57}$ This, by extension, would secure for the creators of the mausoleums popular support and legitimacy. The presidents interred in the mausoleums at Embassy Park would, therefore, continue to do political work from their mausoleums for surviving leaders. ${ }^{58}$

If political actors in both parties deployed mausoleums for a similar purpose, why, then, was there such a feud over them? A close examination of this conflict indicates that neither party was opposed per se to the construction of mausoleums. It is instructive that when PF members of parliament ignited the mausoleum controversy in 2011, for example, they were not much concerned with the fact that the MMD government had already begun building a mausoleum for President Levy Mwanawasa. Rather, they questioned the modus operandi of the then ruling party regarding the mausoleum. The MPs took the government to task over the irregular manner by which it had handled the mausoleum contract, for awarding it to a foreign firm, and for paying the company a colossal sum of money. But at no time did the contrarians seek to put to an end to the mausoleum project for Mwanawasa. The observation that PF leaders were more concerned with the modality of building the mausoleum than opposed to its construction applies to the MMD functionaries, too. It is true that MMD politicians assailed some aspects of the making of mausoleums by the PF government. They, for instance, scoffed at the infusion into Sata's mausoleum of qualities that portrayed him as a great statesman and Christian. It should be emphasized, however, that no MMD leader raised a finger as such against the erection of mausoleums by the PF government. In this context, the hostility between the MMD and PF over mausoleums must be, with the benefit of hindsight, construed as a symbolic contest in which each political party sought to monopolize their building to gain symbolic profit that presidential mausoleums signified.

\section{Do mausoleums have political meaning?}

The strategy of using the sanitized legacies of the dead rulers buried at the Embassy Park was premised on the assumption that visitors to mausoleums in Lusaka would comprehend their architectural designs and meanings in ways that neatly resonated with the expectations of the ruling class. But, as Graeme Gill astutely demonstrated recently, people's perceptions and understandings of political symbols or their meanings are seldom compatible with those of the rulers who produce them. ${ }^{59}$ Gill's observation is vindicated by the varied, and often conflicting, attitudes, and reactions expressed over the mausoleums by informants I interviewed intermittently in Lusaka between 2013 and 2017.

Reminiscent of African culture of funerals and mourning, some family members of dead presidents visited the burial site not just to mourn and pray together at the entrance of the mausoleum of their departed relation but also to seek his intervention in their 
daily lives. "You left [i.e. died] too early, Father (Mwafwile bwangu tata), and now the family is falling apart," wept a female relative of the late President Frederick Chiluba at his mausoleum in early 2016 in an apparent reference to family disputes over his property after his death. "Who will now restore peace in the family without you?" the woman sobbed uncontrollably as she addressed the dead president as if he was still alive. ${ }^{60}$ This touching outpouring of grief together with beseeching the dead politician for succor was not restricted to Chiluba's relative. Many mourners, related or unrelated to other politicians interred at Embassy Park often emotionally broke down or addressed the late leaders at the park to help them, while others asked them to save the country from its deepening economic challenges, including drought, crime, and the ever-escalating cost of fuel and food. ${ }^{61}$

These reactions to dead rulers in Lusaka were scarcely the consequence of the expectations or bidding of the ruling political elites. Rather, as David Gordon lucidly shows in his study of the link between the living and the mystical Other, such responses mainly issued from the deep-rooted culture of death in Africa in general and Zambia in particular, where the living have historically perceived the dead as ancestors capable of bestowing blessings upon their surviving kith and kin. ${ }^{62}$ In this regard, relatives and non-relatives alike may have perceived the deceased presidents as recent ancestors capable of helping them to overcome their family disputes and other social and economic challenges.

This perspective resonates well with other different reactions and attitudes that many informants exhibited toward the mausoleums during interviews at the presidential burial site. One of the respondents was a final-year student from a nearby college whom I found drawing Michael Sata's mausoleum in mid-2015. Drawing the mausoleum, the young student told me, was an assignment he was required to do in order to complete his studies leading to earning his college certificate. When I asked him what he thought of the political value of Mwanawasa's mausoleum, the artist looked at me with surprise in his eyes and countered with his own question: "Do mausoleums have any political meaning?" His concluding remarks were more telling. "If they do," he said with finality in reference to all the presidential mausoleums at the burial site, "that's none of my business!"63 The student's message was unmistakable; he was more interested in using the mausoleums to secure college education than in exploring their symbolic political implications.

Another revealing response to the presidential mausoleums emanated from a wife and a husband who visited the burial site and took pictures of each other as research for this study neared completion. They had travelled, the couple said, all the way from the Zambian Copperbelt to see the mausoleums because they had heard rumors that the PF government, which they both supported, had spent millions of dollars on building the mausoleums. "What a waste of money!" the wife, who was primary school teacher, exclaimed. But she was not yet finished. "How could the government," she lamented, "spend so much money on buildings for the dead when there are no desks in our schools, no drugs in our hospitals, teachers are paid slave wages, and the majority of Zambians live on less than $\$ 2$ per day?" Without belaboring the point, this couple visited the mausoleums out of curiosity and desire to confirm the wastefulness of their own government, not to appreciate the political mysteries of mausoleums or to touch base with traditions about the dead.

For other informants, the aesthetic significance of the mausoleums was the most appealing reason for visiting Embassy Park. In the words of one, they visited the presidential burial site "to admire the beautiful houses in which our presidents are buried." 64 
But perhaps the most revealing reactions to the presidential mausoleums were expressed by scores of supporters of various opposition parties. Obviously aware that the mausoleums at Embassy Park were/are intended to buttress the political influence of leaders in power, die-hard supporters of the MMD remarked that they used to patronize the Mwanawasa mausoleum after it was erected but lost interest in the burial site as a whole after the PF came to power and built Sata's mausoleum there. "We visited mausoleum of Patrick Mwanawasa regularly when it was built," said the spokesperson of a group of MMD cadres I encountered at their party secretariat in Lusaka in October 2018. But "Why should we go there today," the spokesman continued, "when people who stabbed Mwanawasa in the back are now buried shoulder to shoulder with him?"65 This bitter response was undoubtedly triggered by the frosty relationship and consequent rancor that had existed between President Levy Mwanawasa and Michael Sata until their reconciliation a few weeks before the former passed away.

Admittedly, the various reactions toward the presidential mausoleums in Lusaka are scattered, and it would take further analysis to deal with factors like age, gender, and level of education that underpin people's perceptions toward mortuary monuments as political symbols. ${ }^{66}$ However, the varied nature of these responses illustrates the diverse meanings and attitudes that ordinary citizens in Zambia invest into the mausoleums. Far from being dictated by the political rulers who created the mausoleums, the responses were motivated by visitors' own needs, interests, and considerations. These reactions suggest the creative way in which the governed understood and used the mausoleums, ranging from seeking deceased politicians' intercession in family matters to fulfilling a college assignment, stimulating aesthetic admiration, or questioning the priorities of incumbent rulers. This is a vivid testimony to the ability of the governed to constrain politicians' ambition to shape the meanings and uses their subjects read into monuments to the dead or other political symbols.

If the responses and interest of the ordinary citizens in presidential mausoleums proved fluid, those of incumbent political leaders in the country seem to have remained unshaken. The incumbent Zambian President Edgar Lungu, who succeeded Michael Sata in 2015, periodically visits the presidential burial site to lay wreaths of beautiful flowers at the mausoleums of Chiluba, Mwanawasa, and Sata - often in the company of his ministers and/or visiting foreign dignitaries. Placing flowers at the graves of the dead is certainly a Western cultural import, but there is hardly anything "modern" in the homage that President Lungu pays to his departed predecessors. ${ }^{67}$ There is incontrovertible evidence that, in times of socio-economic and political disorder, "traditional" rulers in nineteenth- and twentieth-century Africa offered libations and other sacrifices at sacred locations where their forbearers were buried. ${ }^{68}$ This political ritual, still widespread in the continent today, was meant to elicit deceased leaders' succor or blessings perceived as crucial to the smooth working of the African society, its political stability, its fertility of the land, and the fecundity of its people. ${ }^{69}$

\section{Conclusion}

Confronted with what one academic aptly describes as a "crisis of legitimation" sparked by the failure of their neo-liberal policies to redress poverty, corruption, and political fragmentation, the creators of the Third Republic in Zambia sought to reverse this crisis on the symbolic terrain. ${ }^{70}$ They, therefore, established mausoleums in Lusaka and 
endowed them with the reported accomplishments and legacies of the late presidents Michael Sata, Levy Mwanawasa, and Frederick Chiluba, who before their death had played an important role in the restoration of democratic politics in Zambia in the early 1990s. The leaders hoped that, in so doing, they would create a symbolic site in the capital city, where citizens would come to admire and emulate the legacies of the leaders interred in the mausoleums. In this manner, citizens would acquiesce in the rule of the political class that built the mausoleums. This chapter takes this narrative to task. It insists that ordinary men and women who visited the presidential mausoleums in Lusaka between 2008 and 2018 were often motivated by factors and considerations that were frequently at variance with those of the ruling class. This attests to the truism that rulers and the ruled rarely pursue similar political goals, even on the symbolic terrain.

\section{Notes}

1 Part of the material in this paper derives from the wider "Death in Africa" project generously funded by the Arts and Humanities Council of the UK in which I participated at Cambridge University from 2008 to 2010. I am most grateful to AHRC and to my fellow collaborators on the project: Professor Megan Vaughan and Drs Rebekah Lee and Mark Lamont. I also wish to thank Professor Motlatsi Thabane for his comments on the paper.

2 For a detailed study of President Mwanawasa's death and funeral as well as their political implications in Zambia, see Walima T. Kalusa, "The Politics of the Corpse: President Levy Mwanawasa's Death, Funeral and Political Contestation in Post-Colonial Zambia," Journal of Southern African Studies 43, 6 (2017), pp. 1137-1155.

3 www.parliament.gov.zm debates, 3 June 2011. Accessed on 9 January 2019.

4 Interview with Mzandabwe Mbewe, Patriotic Front [hereinafter PF] Ward Secretary, Lusaka, 15 July 2013.

5 Interview with Bernard Michael Mutale, PF cadre, Lusaka, 15 July 2013.

6 Personal communication with Monica Chanda, 18 January 2019.

7 See Government of the Republic of Zambia [hereinafter [GRZ]/Cabinet Office [hereinafter CO] 75/3/4, Roland Msiska to Collins Chipote, Executive Director, National Heritage Conservation Commission [hereinafter CC], 14 April 2016, which shows that the PF government has designed mausoleums for former presidents Kenneth Kaunda, who led Zambia to independence in 1964 and ruled up to 1991 on the ticket of the United National Independence Party (UNIP), and Rupiah Banda who succeeded Mwanawasa on a PF ticket. Both Kaunda and Banda are still alive.

8 Interview with Andrew Mambepa Chisanga, MMD cadre, Lusaka, 12 November 2016.

9 Examples of such scholars include Andre Demshuk, "A Mausoleum for Bach? Holy Relics and Urban Planning in Early Communist Leipzig, 1945-1950," History and Memory 28, 2 (2016), pp. 47-88; Reuben Makayiko Chirambo, "'A Monument to a Tyrant,' or Reconstructed Nationalist Memories of the Father and Founder of the Malawi Nation, Dr. H. K. Banda," Africa Today 56, 4 (2010), pp. 2-21. For studies that address similar concerns but from the point of view of other political symbols, see Robin Jeffrey, "What the Statues Tell: The Politics of Choosing Symbols in Trivandrum," Pacific Affairs 53, 3 (1980), pp. 484-502; Wale Adebanwi, "The Cult of Awo: The Political Life of a Dead Leader," Fournal of Modern African Studies 46, 3 (2008), pp. 335-360; David Esposito, "The Politics of Death: State Funerals as Rites of Reconciliation in Porfirian Mexico, 1876-1889," Americas 62, 1 (2005), pp. 65-94.

10 Elizabeth Self, "Fit for a Shogun's Wife: The Two Seventeenth-Century Mausoleums for Sũgen-in," Japan Review 31 (2017), pp. 41-67.

11 Nina Tumarkin, Lenin Lives! The Lenin Cult in Soviet Russia (Cambridge, MA: Harvard University Press, 1983); and "Political Ritual and the Cult of Lenin," Human Rights Quarterly 5, 2 (1983), pp. 203-206. See also Graeme Gill, "Lenin Lives': Or Does He? Symbols and the Transition from Socialism," Europe-Asia Studies 60, 2 (2008), pp. 173-196. 
12 For an arresting analysis of this issue, see Forest and Juliet Johnson, "Unravelling the Thread of History: Soviet-Era Benjamin Monuments and Post-Soviet National Identity in Moscow," Annals of the Association of American Geographers 92, 3 (2002), pp. 524-547.

13 Kathryn Verdery, The Political Life of Dead Bodies: Reburial and Post-Socialist Change (New York: Columbia University Press, 1999). Works inspired by this study include, Meghan Vaughan, "Big Houses for the Dead: Burying Presidents Banda and Bingu wa Mutarika of Malawi," in Walima T. Kalusa and Megan Vaughan (eds.), Death, Belief and Politics in Central Africa (Lusaka: Lembani Trust, 2013), Chapter IX; Wale Adebanwi, "The Cult of Awo: The Political Life of a Dead Leader," Fournal of Modern African Studies 46, 3 (2008), pp. 335-360.

14 Vaughan, "Big Houses for the Dead"; Chirambo, "Monument to a Tyrant."

15 Vaughan, "Big Houses for the Dead," Chapter IX.

16 For fascinating studies of the political importance of symbolic images, see Harald Wydra, "The Power of Symbols - Communism and Beyond," International fournal of Politics, Culture, and Society 25, $1 / 3$ (2012), pp. 49-69; Verdery, Political Life of Dead Bodies; Mona Ozouf, Festival and the French Revolution, translated by Alan Sheridan (Cambridge, MA: Harvard University Press, 1988); David I. Kertzer, Ritual, Politics and Power (New Haven: Yale University Press, 1988).

17 For a study of the political uses of mausoleums in antiquity, see "Patrick Kragelund, Galba's 'Pietas,' Nero's Victims and the Mausoleum of Augustus," Historia: Zeitschrift für Alte Geschichte 47, 2 (1998), pp. 152-173.

18 Graeme Gill, "Lenin Lives': Or Does He? Symbols and the Transition from Socialism," Europe-Asia Studies 60, 2 (2008), pp. 173-196.

19 See Gill, "Symbols and Transition"; Forest and Johnson, "Unravelling History."

20 My approach here is inspired by Forest and Johnson, "Soviet-Era Monuments."

21 This section derives the bulk of its material from Julius O. Ihonvbere, "From Movement to Government: The Movement for Multi-Party Democracy and the Crisis of Democratic Consolidation in Zambia Author," Canadian Fournal of African Studies 29, 1 (1995). See also his "The Crisis of Democratic Consolidation in Zambia," Civilisations 43, 2 (1996), pp. 83-109).

22 See for example, Andrew Sardanis, Zambia, The First 50 Tears: Reflections of an Eyewitness (London and New York: I.B. Tauris, 2014); Nic Cheeseman and Marja Hinfelaar, "Parties, Platforms, and Political Mobilization: The Zambian Presidential Election of 2008," African Affairs, 109, 434 (2009), pp. 51-76.

23 Examples of studies that address this issue include Carol Graham, "Zambia's Democratic Transition: The Beginning of the End of the One-Party State in Africa?" Brookings Review 10, 2 (1992), pp. 40-41; and Miles Larmer, Rethinking African Politics: History of Opposition in Zambia (Farnham: Ashgate, 2011).

24 I attended a mammoth rally in 1991 in Lusaka at which Sata announced that he would to join the MMD.

25 See http://news.bbc.co.uk2hi/africa/1715419/stm Accessed on 24 September 2019.

26 See Owen B. Sichone, "The Sacred and the Obscene: Personal Notes on Political Ritual, Poverty and Democracy in Zambia," in Jonathan Hyslop (ed.), African Democracy in the Era of Globalization (Johannesburg: Witwatersrand University, 1999), p. 155.

27 For a fascinating study on the political rise of Michaela Sata, see Sishuwa Sishuwa, "The Making of an African Populist: Explaining the Rise of Michael Sata, 2001-2006" (MSc dissertation, University of Oxford, 2011).

28 In the period leading up to the 2011 presidential and parliamentary election, the PF campaign slogans were "Creating Jobs" and "Putting Money in Your Pocket." Personal observation.

29 Sishuwa Sishuwa, "Zambia: Lungu's Authoritarianism Now Turns to Opponents Within," African Arguments, 26 July 2019.

30 See Sishuwa Sishuwa, "He is Their Leader: Why Edgar Lungu Cannot Stop the Violent Behaviour of PF Cadres," Lusaka Times, 2 May 2019.

31 Ihonvbere, "From Movement to Government."

32 Ihonvbere, "From Movement to Government," p. 2.

33 See Gill, "Symbols and Transition."

34 NHCG/Con/101/05/9, D.C. Chikumbi, Executive Director to Mukuka L.N. Zimba, Permanent Secretary, 29 September 2008. See attachment to this letter, p. 2. 
35 GRZ/Ministry of Works and Supply [hereinafter MoWS], "Report on Case Studies on Mausoleums; Malawi, Kenya, Angola and the Democratic Republic of Congo from 21st September to 1 October 2008" (Unpublished manuscript, Lusaka 2008).

36 Ibid, p.1.

37 GRZ, "A Brief Life and Political History of Michael Chilufya Sata, 1937-2015," Report prepared by the Sub-Committee on Research on the Preparation of the Late President Chilufya Sata's Mausoleum, unpublished, 2015.

38 NHCG/Con/101/05/9, D.C. Chikumbi, Executive Director to Mukuka L.N. Zimba, Permanent Secretary, 29 September 2008.

39 Interview with Dominic Mwanza, MMD functionary, Lusaka, 18 June 2018; see also GRZ, "Brief Life and Political History," p. 3.

40 See for example, Jigjid Boldbaatar and Caroline Humphrey, "The Process of Creation of National Symbols and Their Adoption in the 1992 Constitution of Mongolia," Inner Asia 9, 1 (2007), pp. 3-22.

41 GRZ/MoWS, "Report on Case Studies on Mausoleums: Malawi, Kenya, Angola and the Democratic Republic of Congo: From 21st September to 1 October 2008,” Draft Report, 2008.

42 GRZ/MoWS, "Report on Mausoleum."

43 GRZ/MoWS, "Report on Mausoleum," p. 14.

44 GRZ/MoWS, "Report on Mausoleum," p. 14

45 NHCC, "Embassy Park: Presidential Burial Site," unpublished report, 2018, pp. 7-9.

46 See GRZ/CO,75/3/2, Roland Msiska, Secretary to the Cabinet to Permanent Secretaries in Ministries of Chiefs and Traditional Affairs, Home Affairs, Information and Broadcasting, Tourism and Arts, Transport, Works and Supply.

47 Telephone conversation with Friday Mufuzi, 27 October 2016.

48 For a fascinating analysis of the political implications of "New Culture," see Karen Tranberg Hansen, Salaula: The World of Secondhand Clothing in Zambia (Chicago and London: University of Chicago Press, 2000), pp. 92-96.

49 See GRZ/CO/75/3/2, Ronald Msiska, Secretary to the Cabinet to Permanent Secretaries, 5 December 2014.

50 Ibid.

51 GRZ/CO, 75/3/4, Roland Msiska to Collins Chipote, Executive Director, NHCC, 14 April 2016.

52 GRZ, "Brief Life and Political History," p. 24.

53 Ibid.

54 Interview with Henry Habeenzu, opposition leader, Lusaka, 27 March 2015.

55 GRZ, "Brief History," p. 24.

56 Examples of this scholarship include Avner Ben-Amos, Funerals, Politics, and Memory in Modern France, 1789-1996 (Oxford and New York: Oxford University Press, 2000); Sean Wilentz (ed.), Rites of Power: Symbolism, Ritual and Politics Since the Middle Ages (Philadelphia: University of Pennsylvania Press 1985).

57 Jeffrey, "Politics Choosing Symbols," pp. 496-498; for similar arguments, see Wale Adebanwi, "The Cult of Awo: The Political Life of a Dead Leader," Journal of Modern African Studies 46, 3 (2008), pp. 335-360; N. Turmarkin, "Political Ritual and the Cult of Lenin," Human Rights Quarterly 5, 2 (1983), pp. 203-206.

58 My insights here stem from Wale Adebanwi, "Death, National Memory and the Social Construction of Heroism," Journal of African History 49, 3 (2008), pp. 419-444; see also his "Cult of Awo."

59 Gill, "Symbols and Transition."

60 Personal observation.

61 Personal observation.

62 Gordon, Invisible Agents.

63 Interview with Joshua Kalenga, Art Student, Embassy Park, Lusaka, 25 August 2014.

64 Interview with Mary Zulu, visitor to the burial site, Lusaka, 2 August 2017.

65 Interview with MMD supporters, Lusaka, 30 October 2018. Similar sentiments were expressed in interviews with Bernard Zulu, cadre of the United National Development Party (UNDP), Lusaka, 19 October 2018; Veronica Mwangala, 19 October 2019; and Michael Mulenga, MMD cadre, Lusaka, 20 October 2019. 


\section{MAUSOLEUMS AND POLITICS IN ZAMBIA}

66 For an interesting study that takes this approach, see Gill, "Symbols and Transition."

67 This observation is informed by Jeffrey, "Politics of Choosing Symbols," pp. 496-498.

68 For a useful collection of anthropological papers that explore such issues, see Maurice Bloch, "The Ritual of the Royal Bath in Madagascar: The Dissolution of Death, Birth and Fertility into Authority," in David Cannadine and Simon Price, (eds.), Rituals of Royalty: Power and Ceremonial in Traditional Societies (Cambridge: Cambridge University Press, 1987).

69 For a detailed exploration of this issue, see David Gordon, Invisible Agents: Spirits in Central African History (Athens: Ohio University Press, 2011).

70 I borrow the quotation from N. Turmarkin, "Political Ritual and the Cult of Lenin," Human Rights Quarterly 5, 2 (1983), pp. 203-206. My observations here are shaped by Wale Adebanwi, "The Cult of Awo: The Political Life of a Dead Leader," Fournal of Modern African Studies 46, 3 (2008), pp. 335-360; 


\title{
GELEBRATING GREATION AND GOMMEMORATING LIFE
}

\author{
Ritualizing pet death in the U.S. and Japan
}

\author{
Barbara R. Ambros
}

At the 77th General Convention of the Episcopal Church in Indianapolis in 2012, the House of Deputies Committee on Prayer Book, Liturgy and Church Music presented a new liturgy for "Service at the Loss of a Beloved Animal." The proposal was adopted, and the ritual is now available in an expanded version among the various liturgical resources of the Episcopal Church alongside miscellaneous prayers for companion and service animals. ${ }^{1}$ Since then at least 45 Episcopal churches in the U.S. have established pet memorial gardens on church grounds.

In Tokyo in February 2019, Zōjōji, the head temple of the Pure Land School, staged a debate on the topic of whether pets could enter Amitabha Buddha's Pure Land. The two speakers, Adachi Shun'ei (a researcher at the Pure Land temple Chion'in's Institute for the Study of Religious Doctrine) and Hayashida Kōjun (a professor at the Buddhist Taishō University), both agreed that pets could be reborn in Amitabha's Pure Land eventually, but Adachi argued that they first had to be reborn as humans before they could enter the Pure Land because they could not intone the name of Amitabha Buddha, whereas Hayashida contended that they could be reborn in the Pure Land in the best form the owners wished for immediately after a memorial service was conducted and merit had been transferred to them. ${ }^{2}$ Such pet memorial services have become commonplace across most major Buddhist denominations in Japan over the past 40 years, and there are about 170 Buddhist temple precincts with pet cemeteries today.

The demand for posthumous religious rituals for companion animals has grown because many people in the U.S. and in Japan, as elsewhere in the industrialized and post-industrial world, regard their companion animals as family members, who after all share their everyday living spaces. Therefore, many pet owners have also become willing to spend more on their animal companions. The U.S. and Japan have two of the largest and steadily expanding pet-industry markets, with $\$ 75$ billion (2019) and $¥ 1,536$ billion (2018) of revenue respectively. ${ }^{3}$ This context of increased spending and thinking about companion animals in kinship terms, combined with the needs of urban pet ownership, have fueled the demand for pet funeral services and even religious mortuary rites that mirror mortuary practices for humans. While in the U.S. the connections between pet mortuary practices and institutionalized religion have generally been ad hoc and have only become more established - and therefore more visible - over the past decade, Japanese Buddhist institutions have played influential roles in the Japanese pet funeral practices 
for about four decades. In both cases, such commemorative rituals demonstrate that companion animals have taken their place in kinship relations and are to some degree included as subjects in religious communities, but, due to persistent ritual and spatial differences from human mortuary practices, pet mortuary rites ultimately tend to re-affirm ontological differences between humans and other animals.

\section{Pet death and religion in the U.S.}

Before exploring the emerging connections between pet death and religion in the U.S. and Japan, it is helpful to briefly outline the necrogeography of pet cemeteries and crematoria, beginning with the U.S. context. It is difficult to determine the exact number of pet cemeteries and pet crematories. Most pet crematoria and pet cemetery directories are compiled by industry associations, whose membership is voluntary and thus not exhaustive. Small pet cemeteries that do not see a need for broad advertising or do not offer cremation services may not be included in industry directories. Nonetheless, the available data shed light on the regional distribution of pet cemeteries and crematoria, allowing us to draw conclusions about regional variations and national trends.

Pet mortuary rites and burial spaces in the U.S. developed along with modern pet keeping in the context of industrialization and the rise of the middle class. As Katherine Grier explains, ceremonial pet funerals are documented beginning in the 1800s. Mortuary observances for pets appropriated human mortuary rituals to mark the passing of their family pets with rites of mourning, commemorative sentimental poetry, posthumous portraiture, burial on family property, and headstones. By the mid-1800s, some pet burial sites had grown into sizable pet graveyards on the properties of private homes. Around the turn of the twentieth century, the first modern, commercial pet cemeteries began to emerge in the northeastern U.S., beginning with Hartsdale Pet Cemetery, which was founded in 1896 and incorporated in 1914. This expansive pet cemetery has been in continuous operation to this day and has about 80,000 occupants. Similarly, the 11 -acre Francisvale Pet Cemetery, now operated in conjunction with an animal shelter, was founded in Philadelphia, PA, in 1908 and has 2,000 occupants. ${ }^{4}$ The 27-acre Pine Ridge Pet Cemetery in Dedham, MA, run by the Animal Rescue League of Boston, was founded in 1907. The cemetery now has almost 17,000 occupants but has recently stopped selling burial lots and offering cremations. ${ }^{5}$

Pet cemeteries, once only the privilege of the socio-economic elite, became more widespread beginning in the 1970 s and 1980s. According to industry estimates, there were about 200-300 pet funeral services in the U.S. by the mid 1970s. The growth in pet cemeteries and pet funeral services has not only been attributed to new conceptualizations of cross-species kinship relationships but also to increasing urbanization. ${ }^{6}$ Indeed, pet funeral services and pet cemeteries are more common in more densely populated areas of the U.S., but since per-household pet ownership tends to be higher in rural and suburban areas than in dense urban centers, ${ }^{7}$ the highest concentration of such services is found in areas that are in a sweet spot in terms of pet ownership numbers, availability of space, and, and demand created by urbanization. My 2019 survey of three directories of pet mortuary services in the U.S. yielded over 700 pet funeral businesses including pet cemeteries, crematories, and funeral and memorial centers. Of these, 430 were explicitly listed as pet cemeteries, many of which also offered cremation services. ${ }^{8}$ The vast majority (almost 500 pet funeral services including nearly 300 pet cemeteries) were found in 
the eastern U.S. The South Atlantic region had the largest number of pet funeral services (157) while the Middle Atlantic region, despite occupying a much smaller territory, had the largest number of actual pet cemeteries (91); it was after all the region where pet cemeteries first emerged in the U.S. Pennsylvania was the state with the most pet funeral services and pet cemeteries, at 77 and 57 respectively. Florida and Ohio, with 46 and 45 pet funeral services respectively, are in distant second and third places in that category, while Texas, which has 37 pet funeral services, is in second place with in terms of actual pet cemeteries (28) followed by Florida and Ohio (24 each). New York (24 and 22) and California (27 and 18) both have a sizable number of pet funeral services and pet cemeteries.

U.S. pet cemeteries have received some scholarly and media attention over the past few decades. They have been showcased not only as sites of historical heritage - such as Hartsdale Pet Cemetery-but also as sentimental sites of excess, especially in media reports. ${ }^{9}$ Yet the vast majority of pets in the U.S. are not interred at pet cemeteries but are cremated, which opens up a wide array of possibilities beyond interment in a pet cemetery, such as keeping the cremains at home or scattering the ashes. According to the 2012 and 2013 annual surveys of the Pet Loss Professionals Alliance, a subsidiary of the International Cemetery, Cremation and Funeral Association, 99 percent of pets in the U.S. were cremated while only 1 percent were buried in a pet cemetery. These figures translated into an estimated 1,840,965 cremated pets and 20,811 buried pets in 2012 and an estimated 1,463,456 cremated pets and 14,774 buried pets in 2013. Of the pets cremated in 2012, 49 percent were cremated in group cremations, which mixes the cremains of several animals, and 28 percent in partitioned cremations, in which several pets share the cremation chamber but the cremains are kept separate. Only 23 percent were private cremations in which a single animal is cremated in an individual cremation chamber. Of the pets cremated in 2013, 63 percent were group cremations and 7 percent were partitioned cremations, whereas only 30 percent were private cremations. ${ }^{10}$ This means that 70-75 percent of pets are cremated in a different and (more energy and cost effective) fashion than humans, who are usually cremated in private cremations. Nonetheless, close to half the businesses surveyed in 2012 and 2013 also operated cremation services for humans. ${ }^{11}$

Cremation has facilitated opportunities for joint human and pet burials, but not just in the sense of secretly mingling the processed cremains of humans with that of their companion animals, as observed by Douglas Davies in the UK two decades ago. ${ }^{12}$ Some human cemeteries in the U.S. now permit the interment of pets or at least pet cremains; conversely, some pet cemeteries, especially those deeded in perpetuity, allow the interment of human cremains. In addition, some human cemeteries are paired with a pet cemetery or feature a pet section even though they do not allow the actual burial of pets in human plots. The website of the Green Pet-Burial Society, for instance, lists 21 U.S. cemeteries or pet cemeteries that allow inter-species interment and another 15 pet cemeteries adjacent to or pet sections of human cemeteries. ${ }^{13}$

Despite the development of a robust pet funeral industry, connections with institutionalized religion - so prevalent in the case of human death-remain relatively rare, or rather they are difficult to trace because they often occur in an ad hoc manner as individual religious professionals may choose to accommodate pet owners who request memorial rituals for their deceased companion animals. From mainstream doctrinal perspectives, pet funerals - let alone pet cemeteries on the grounds of religious 
institution or within confessional human cemeteries - tend to present theological challenges. As Michael Schaffer argues in his One Nation under Dog, companion animals are commonly excluded from mainstream conceptualizations of the afterlife and from receiving religious burial rites:

Airports and workplaces may be pet-friendly, the hereafter is not. At least not in most major religions, which don't simply ignore the old chestnut that all dogs go to heaven, but go one step farther, declaring paradise off-limits to animals. People who work with pet grief say religious teaching that animals lack souls, and thus a means of accessing the afterlife, is the largest stumbling block people face when dealing with their animal's death ... The vacuum is one reason poems like "The Rainbow Bridge" and memorial sites like ilovedmypets.com and nontraditional funerals by nontraditional officiants ... are so popular: For bereaved pet people, the institutions that would support them at other moments of grief are unavailable. ${ }^{14}$

This spiritual void has in part been filled by the relatively new vocation of pet chaplains, who emerged in the first two decades of the twenty-first century and often draw on an array of religious traditions and pet-loss discourses. One of the earliest examples is Robert Gierka in Raleigh, NC. Originally of a Catholic background but now a member of a Baptist congregation, Gierka received training to serve as hospital chaplain before he decided to become a volunteer non-denominational pet chaplain at the Veterinary School of the North Carolina State University. However, after he received media attention in the local newspaper in 2006, the university no longer welcomed his services. Reports of him praying with pet owners raised concerns over the separation of church and state at the state university. Gierka then began to run a pet-loss support group at the Wake County SPCA in Raleigh. ${ }^{15}$ He has also developed a training program in veterinary pastoral care and founded the Association for Veterinary Pastoral Education after completing a doctorate at NC State's School of Education. ${ }^{16}$ In conjunction with his petloss support group, Gierka has occasionally held memorial observances of his own design. During one such event that I attended, participants wrote a few words for their deceased pets on small pieces of joss paper, which they then incinerated in a bowl provided by Gierka. Participants also released balloons outside following the ritual indoors. During another year, Gierka held a remembrance gathering at a local funeral home, where members of his group gathered and shared memories of their pets. The gathering including a wide variety of participants, including a local pet communicator who received communications from living and dead animals in the form of poetry and demonstrated her abilities during the session. ${ }^{17}$

While the interment of animal bodies may not be a possibility at many Christian and Jewish religious institutions, pet cemeteries offer some possibilities for physical proximity and the incorporation of religious symbolism and rituals. The Friends of Noah pet cemetery in Frazer, PA, is located across the street from the Haym Salomon Memorial Park, a Jewish cemetery, and both are operated by the same Jewish owner who explained that pet owners were comforted by the arrangement. ${ }^{18}$ For over a decade, a retired Episcopal priest, Rev. David L. James has served as the chaplain at Hartsdale Pet Cemetery and conducted pet funerals on demand, a service used only by a small number of cemetery clients. ${ }^{19}$ Similarly, Reform Rabbi Andrea Frank, who is based in Westchester County 
and specializes in wedding ceremonies, also has also offered pet funerals, including at Hartsdale Pet Cemetery. ${ }^{20}$

As Stanley Brandes has observed, sometimes religious identities are inscribed onto pet graves at pet cemeteries. His survey of Hartsdale Pet Cemetery showed that many headstones erected from the 1980s onward were engraved with the Christian crosses or the Star of David. In addition, many of the Jewish graves were also lined with pebbles and some also had yahrzeit candles. ${ }^{21}$ In my fieldwork, I witnessed similar practices at Pine Ridge Pet Cemetery in Dedham, MA, and San Diego Pet Memorial Park, CA, where a significant number of the headstones from the early 1990s onward were engraved with crosses or the Star of David. At Pine Ridge, some of the Jewish headstones were also lined with pebbles. In addition, one of the headstones at Pine Ridge Pet Cemetery had an inscription in Chinese characters that expressed the wish for the pet to be reborn in the Western Pure Land of Amitabha Buddha. Both Brandes and Auster concluded from surveys of Hartsdale Pet Cemetery and pet cemetery in Lancaster, PA, that these developments coincided with inscriptions that used kinship terms for the pets and the increased use of human names for pets, which is also substantiated by my surveys of Pine Ridge Pet Cemetery and San Diego Pet Memorial Park. ${ }^{22}$

Over the past two decades, however, some of the more progressive-leaning religious organizations, especially the Episcopal Church, Reform and Reconstructionist Judaism, and Buddhist Churches of America, have begun to establish liturgical resources and physical spaces for pet memorials, but these developments are not captured in industry data owing to their non-commercial nature. As mentioned above, the leadership of the Episcopal Church adopted a liturgy for commemorating beloved animals at its General Convention in 2012. The adoption of this liturgy has been part of a larger development within the church to commemorate companion animals. A survey of church websites reveals that there are currently at least 45 Episcopal churches that maintain a pet cemetery - usually termed "Pet Memorial Garden" or "St. Francis Pet Memorial Garden"on church grounds alongside human cemeteries. Like commercial pet cemeteries, these Episcopal pet memorial gardens are concentrated in the eastern United States, and the largest number are found in the south (26), especially in Florida (9) and in Georgia (6). However, many of the pet memorial gardens are located either in densely urbanized or very rural areas that do not have other pet cemeteries nearby, but since these pet memorial gardens accept only cremated remains, pet cremation is obviously available in these areas.

There are also considerable variations among Episcopal pet memorial gardens. Some of the pet memorial gardens are intended solely for members of the congregation while others are open to the general public, depending on whether the church considers the memorial garden as a worship/spiritual care opportunity for its own members or as a form of outreach to the larger community. Some were established at the initiative of the rector while others were established through the initiative of the congregation or even as eagle scout projects. Some only accept the unmarked interment of pet cremains whereas others allow for varying degrees of markers - from handcrafted, inscribed rocks to more formal memorial markers. Some offer interment at no cost while others charge a modest fee to cover the cost of the marker and the maintenance costs of the pet memorial garden. In rare cases, memorials for pets serve as fundraising opportunities for the church. Some churches allow pet owners to inter the remains on their own and may even provide a brochure with a funeral liturgy for this purpose, while at others the rector 
may conduct a funeral or remembrance service on demand. In addition to the churches with pet memorial gardens, there are a handful of Episcopal churches that publish liturgies for pet funerals and memorial rituals on their websites or whose rectors conduct such rituals regularly upon request.

The vast majority of these pet cemeteries at Episcopal churches were founded in the past five to ten years. In 2007, I was able to find only two pet cemeteries on the grounds of Episcopal churches in the New York metropolitan area: one at St. Andrew's in New Providence, NJ, and one at St. Andrew's Episcopal Church in Richmondtown, NY. There may have been a few more, but the congregations did not yet publicize their establishment of a pet memorial garden at the time.

The pet cemetery at St. Andrew's in New Providence, NJ, is likely the oldest of its kind. According to the church staff, the pet cemetery was founded shortly after the church was founded by Rev. Shreve, an avid dog lover, in the early 1960s, and the entries in the memorial book, which lists the interred pets, started in 1973. The pet cemetery is located beside the church building. It consists of a small, paved patio with a statue of St. Francis flanked by two dog statues holding baskets in their mouths and a second smaller statue of St. Francis. The larger statue and the dogs were donated by a wealthy woman from Elizabeth, NJ. There are no individual markers, and the cremains are interred directly into the ground without urns. The human cemetery for parishioners, located in front of the church, does not have personal grave markers either, and human cremains are also interred directly into the ground without urns. In the case of humans, however, individual copper memorial plaques are placed on the outside church wall next to the cemetery. According to the church staff, some people ended up seeking out other pet cemeteries when they found out they could not have individual markers for their pets. Pet owners can, however, record their memories of their interred animal companions in a memorial book kept at the church. In 2007, there were only two or three interments every year. Some of the pet owners belonged to the congregation, others did not. When I interviewed the rector in 2007, she was reluctant to disclose details about the funeral services she conducted for pets because such services were not (yet) officially approved by the church. She only noted that she kept them simple by reciting a prayer, such as the Prayer of St. Francis and/or the Lord's Prayer, for the family and their loss rather than for the dead animal. She explained that funeral rituals marked moments of transition and gave meaning to change and that they helped people who were experiencing loss and allowed them to transform a moment of loss into something sacred. ${ }^{23}$

The pet cemetery at St. Andrew's Episcopal Church in Richmondtown, NY, is located in the rear of the human cemetery, next to the rectory, and is called "Cemetery for All God's Creatures" (see Image 26.1). As the Rev. Delaney explained, this helped avoid negative associations with the term "pet cemetery," which tends to remind people of Stephen King's horror novel, Pet Sematary. Rev. Delaney established the Cemetery for All God's Creatures in 2005 to share the gifts of St. Andrew's with the community. He was not aware at the time that this would be the first pet cemetery in New York City. Delaney was inspired by his experience as a bereavement counselor at a hospital. A woman had approached him one day to tell him that she was grieving more for her dog than for her mother, who had died a while ago after a long illness. The Cemetery for All God's Creatures features a seated statue of St. Francis with several animals flanked by two memorial benches and simple, parallel double rows of pavers to mark the plots, each of 


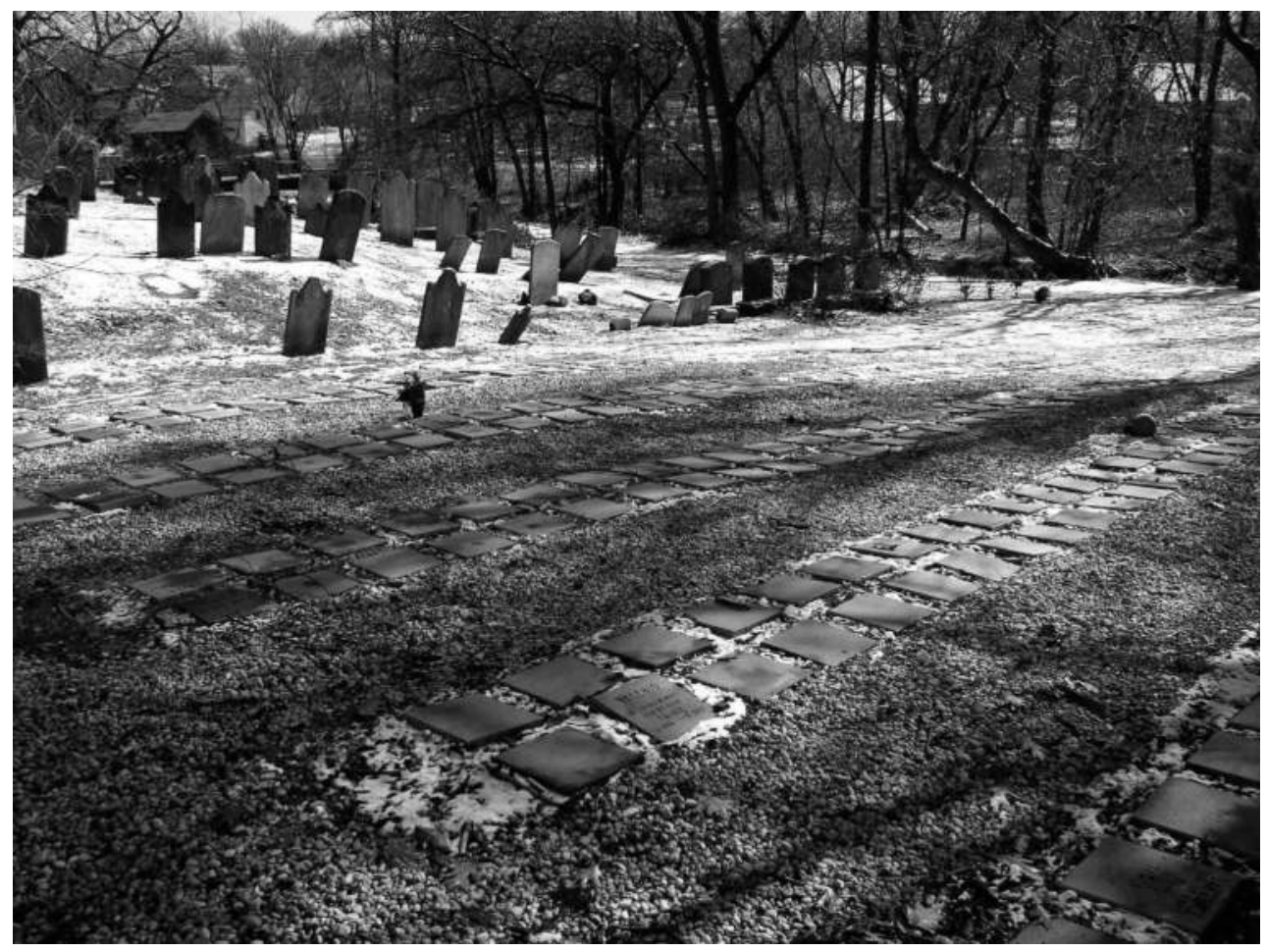

Image 26.1 The Cemetery for All God's Creatures at St. Andrew's in Richmondtown, NY, is located adjacent to the church's historical human cemetery

Photograph by the author

which can hold up to four animals (see Image 26.2). The rest of the cemetery is covered with gravel. All interred animals have to be cremated as stipulated by local law. The pet cemetery's proximity to the human cemetery on the grounds of a historic church is attractive to pet owners who might worry about pet cemeteries that are not deeded in perpetuity. $^{24}$

As Rev. Michael Delaney explained in an interview in 2007, pet funeral liturgies demand a different focus since the idea of the resurrection stressed in human burial services would be inappropriate in the case of pets. Well ahead of the officially adopted liturgy for pets, Rev. Delaney opted for using selections from Anthony F. Chiffolo and Rayner W Hess, Jr.'s eclectic We Thank You God, for These: Blessings and Prayers (2003), which draws on not only Christian prayers and biblical sources but also poetry and Jewish, Muslim, and Hindu prayers. Hesse is an Episcopal priest, and both Chiffolo and Hesse have lived in Hartsdale, NY, near Hartsdale pet cemetery. ${ }^{25}$ The performance of funeral rituals for people of varying faiths at Hartsdale Pet Cemetery provided the inspiration for the book. ${ }^{26}$ According to Rev. Delaney, its eclectic nature makes Chiffolo and Hesse's book useful in actual application since many of the pet owners who have their pets buried at the pet cemetery at his church are not members of his congregation or Episcopal but of a variety of religious backgrounds. 


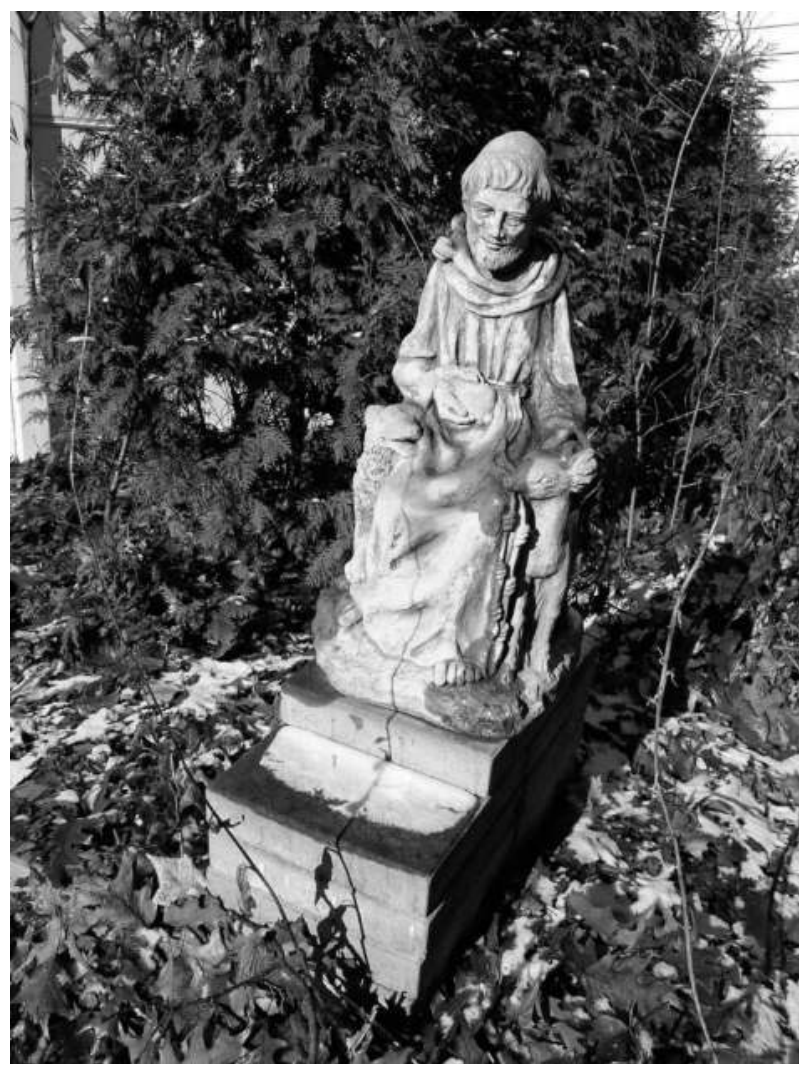

Image 26.2 Statue of St. Francis in the Cemetery for All God's Creatures Photograph by the author

Rev. Delaney stressed that pet funerals were a way of honoring God's creation. During funerals, the facility manager handles the actual interment of the urns, but Rev. Delaney performed a simple ritual for the companion animals upon request. According to my interview with him in 2007, he did a reading from Job and said the Prayer of St. Francis, had pet owners cast flowers into the grave and told them that this would be the seed for new life that needed to be nourished. Many people would add the pet's favorite blanket or toy, even if there was little space. Especially when the owners were Episcopalian, Lutheran, or Catholic, he made the blessing of the grave with water a prominent part of the ceremony; if they were not, he deemphasized this element. He had even conducted a full funeral service for a dog, which he described as very well attended, better than some human funerals at his church. Rev. Delaney admitted that at first, he thought having a full funeral service for a dog was odd, but when he saw the sentiment among the attendees, he changed his mind. In addition to requesting pet funerals, many people visited the pet graves once a year, but others came more often. Visitors brought flowers and American flags to decorate the graves, which I also witnessed when I visited Pine Ridge Pet Cemetery in Dedham, MA, around Memorial Day. 
Given the progressive orientation and the strong liturgical focus of the denomination, as well as its recent emphasis on stewardship of creation, it is not surprising that Episcopal churches have been exceptionally open to holding pet funerals and establishing pet memorial gardens on church grounds. The church has been able to do so by shifting the focus away from the posthumous salvation to a celebration of God's creation. According to the Episcopal Book of Common Prayer, human burial services (and by extension burial on church grounds) are framed around the concept of the resurrection, eternal life, and salvation in heaven. ${ }^{27}$ By contrast, Episcopal liturgies for pet funerals and remembrances emphasize consolation for loss and grief, gratitude and enduring love toward the animal, and God's love of creation. The "Service at the Loss of a Beloved Animal" officially adopted at the 2012 General Convention opens with "Canticle 12: A Song of Creation" and an invocation, both of which praise God's creation, followed by multiple animalrelated reading options from the Old and New Testament. While none of these passages includes the story of creation in Genesis, the central portion of the ritual that directly commemorates the deceased animal focuses on God as the creator of all life, God's love for his creation, and God's charge for humanity to be good stewards of his creation by acknowledging the human responsibility for proving care for animals. The service includes phrasing that addresses the remorse that the pet owner may feel for not having provided sufficient care or for having had to euthanize their pets to end their suffering and offers prayers for both the bereaved owners and the deceased pets. The burial service ends with a hopeful, celebratory segment that returns to praising God and his creation and stresses the interconnectedness of all life gifted by God. ${ }^{28}$ In this way, this liturgy provides consolation for the bereaved and an opportunity to celebrate God's creation rather than promising posthumous salvation for the animals. In surveying churches with pet memorial gardens, I discovered that some use this liturgy for their funeral and remembrance services, though they may alter it to suit their needs. One rector noted that he preferred to use the story of creation in Genesis as a reading, even though it is not included in the options provided in the official version.

Other Episcopal churches may prefer liturgies that are of their own design and are shorter and less complex. King of Peace Episcopal Church in Kingland, GA, for instance, provides a link for a burial service of a pet that was created by Rev. Frank Logue, who formerly served at the church. The Episcipal Diocese of Newark, NJ, also provides a link to this liturgy. Rev. Logue's liturgy begins with an acknowledgement of the grief felt at the loss of a pet and then transitions to the love of God's creation, and ends on the expression of gratitude for the companionship the pet provided for its human family and a wish for love and peace. ${ }^{29}$ In this way, Rev. Logue's liturgy guides the mourners from grief for the pet toward loving remembrance in order to facilitate healing. Some of the Episcopal churches that I surveyed had composed independent liturgies that likewise focused on creation.

In addition to liturgy, the ability to draw on traditions around St. Francis and the prevalence of blessings of the animals has provided Episcopal churches with a conceptual framework that could easily be adapted to the commemoration of pets. Even before commemorative rites for pets became sanctioned by the denomination, Episcopal churches had also been leaders in promoting annual Blessings of the Animals around the feast day of St. Francis, which has now become common in many other Christian denominations as well. ${ }^{30}$ This was a connection that several churches with pet memorial gardens emphasized as well, and these congregations usually also celebrated the Annual Blessing 
of the Animals. Some of the Episcopal churches that have pet memorial gardens integrate these spaces into the ritual life of their congregation not only through private and communal burial and memorial services, but also by holding their annual Blessing of the Animals in their pet memorial gardens. Moreover, pet memorial gardens at Episcopal churches often incorporate St. Francis of Assisi as the patron saint of animals - not only in name and through rituals and prayer but also through the use of statuary. For instance, this was the case at St. Francis Pet Cemetery at St. Andrew's in New Providence, NJ, and in Richmondtown, NY, and the rectors at the two churches often recited the prayer of St. Francis during pet funerals.

The demand for pet cemeteries in religiously affiliated human cemeteries and the performance of pet funeral rituals have also led to debates in Jewish communities, particularly in the Reform movement but occasionally also in Conservative, Modern Orthodox, and Reconstructionist communities. Generally, Jewish communities have been resistant to pet burials in Jewish cemeteries or to allowing the appropriation of human memorial rituals for pet commemoration. For instance, according to a 1998 news article in the Chicago Tribune, plans to develop a pet cemetery on the grounds of Jewish Graceland, a historic Jewish cemetery on Chicago's North Side, led to vehement objections from local Jewish leaders. Proponents of the pet cemetery argued that the pet cemetery would generate funds for the maintenance of the human cemetery and that, since most existing pet cemeteries in the Chicago area were located in the suburbs, there was high demand for a pet cemetery near the urban center. Conservative and Orthodox Jewish leaders, however, countered that a pet cemetery would dishonor the human dead by defiling the human cemetery. ${ }^{31}$ Likewise, a Reform responsum by the Central Conference of American Rabbis rejected the burial of pets in Jewish cemeteries, arguing that even though there was no clear prohibition against the burial of pets in a human cemetery, this would have been unthinkable, especially because dogs in particular were traditionally regarded with contempt and because cemeteries were holy grounds. ${ }^{32}$ Similarly, a 1984 responsum by Reform Rabbi Walter Jacob rejected the recitation of the Kaddish for deceased pets, arguing that there is no precedent for this in the Jewish tradition (which considered dogs as defiled and had a moderately more positive view of cats). The performance of the Kaddish for a pet would demean the tradition and alienate other mourners whose human loved ones would end up being commemorated alongside the non-human dead. ${ }^{33}$

Despite the opposition of Jewish leaders against the memorialization of pets according to established Jewish ritual practices, some rabbis have found innovative ways to accommodate Jewish pet owners. While these rabbis have also argued against the use of human mourning rituals for pets, they have created entirely new rituals. For instance, Rabbi Janet Offel, the Director of Consulting and Transition Management at the Union for Reform Judaism and formerly the rabbi of Temple Kol Tikvah in Woodland Hills, CA, advocates for creating new ritual to mark the loss of a pet rather using standard mourning and memorial liturgies used for humans in order to maintain clear boundaries between pets and avoid the denigration of "responsibilities, benefits and privileges that come with being human."34 For this ritual, Rabbi Offel adapted a ritual created by Ruth Chodrow, Jewish veterinarian, that was published in Central Conference of American Rabbis Fournal in 1998. ${ }^{35}$ Conservative Rabbi Irwin Kula and feminist Jewish studies scholar Vanessa L. Ochs also provide a ritual for "Saying Good-bye to a Beloved Pet," acknowledging that the loss of a pet is real and needs to be mourned even though it is not the same as losing a human. Instead, they consider 
the parting from a deceased companion animal an opportunity to "reaffirm the infinite value of life and of love." 36 The Reconstructionist website Ritual Well provides a variety of resources from Reconstructionist, Reform, and Conservative rabbis and Jewish intellectuals for grieving and commemorating companion animals, but there is a clear consensus that traditional rituals for human death should not be used for pets. ${ }^{37}$ For scriptural sources, the above-mentioned rituals draw on the story of creation in Genesis 1:20-25 and the story of Noah in Genesis 8 and 9, as well as a variety of Psalms, to show that God's consideration also extends to animals. Like the focus on the creation in Episcopal pet funeral liturgies, these strategies open up theological possibilities for the inclusion of animals in the community of religious subjects. And yet, despite the gestures of inclusion, there are persistent concerns about ritually inscribing clear distinctions between human and non-human animals.

In his analysis of modern Jewish rituals for pets, Aubrey Glazer raises doubts as to what extent such new rituals have had any impact on Jewish communities. ${ }^{38}$ It is indeed difficult to track whether pet owners actually use these new rituals, but recently a few synagogues have begun memorializing pets even though they still do not permit the actual interment of pet remains. For instance, the Reform Temple Beth Emet in Cooper City, FL, has a pet memorial garden where congregants can place a memorial stone in remembrance of their companion animal. ${ }^{39}$ Congregation Kol Ami Reform Synagogue in West Hollywood, CA, has a pet memorial tree in its courtyard that allows congregants to dedicate leaf-shaped plaques of three sizes. ${ }^{40}$ Temple Israel, a Conservative congregation in Norfolk, VA, features a Pet Remembrance Board that references the Rainbow Bridge, a device widely used by secular pet loss professionals to help pet owners deal with their grief. ${ }^{41}$ Another example is the Modern Orthodox Moses Montefiore Anshe Emunah Greengate Jewish Center in Pikesville, MD, which features a pet memorial board titled "Hashem's Little Angels," where members of the congregation can install plaques with their deceased pet's name. Rather than drawing on scriptural sources, the board incorporates the Rainbow Bridge poem. ${ }^{42}$ Rabbi Yerachmiel Shapiro was willing to accommodate a request from a congregant who lost two cats, but he insisted on clearly distinguishing the memorial board for pets from that for human congregants through the use of different materials, a distinctive design, and placement in a separate location. ${ }^{43}$ While the board had a largely positive reception in the Jewish community in Baltimore, two local Orthodox rabbis were critical of the memorial board for pets, arguing that animals should not be equated to humans. ${ }^{44}$

A number of Buddhist temples affiliated with Buddhist Churches of America (and by extension with the Hongwanji branch of Jōdo Shin Buddhism in Japan) hold annual memorial services for companion animals, but whereas the liturgical focus in similar rituals in Christian and Jewish communities is on animals as God's creation, these Buddhist communities focus on the commemoration of life. Even though the temples belong to the same denomination, the timing and framing of the ritual differ from temple to temple. The Venice Hongwanji Buddhist Temple in Culver, CA, and the Buddhist Temple of San Diego, CA, both celebrate "All Life Forms Memorial Service," which comprise humans, companion animals, and even plants, but the former does so in June in conjunction with the Festival of Dead (Obon) while the latter does so in late October. As a flier for the 2019 service at the Buddhist Temple of San Diego explains, 
This year, we will have a board posted where you can put a photo. Please feel free to bring any photo to celebrate the life of the one you've lost. It can be your pet, a tree or your partner in life. ${ }^{45}$

Meanwhile, the Midwest Buddhist Temple in Chicago celebrated its 2018 pet memorial service in August, and the San Jose Buddhist Church Betsuin held its 2019 pet memorial service in early November, whereas the New York Buddhist Church held its 2019 pet memorial service in conjunction with Nirvana Day in early February, explaining that paintings of the Buddha's passing usually depict him as being surrounded by all kinds of living beings, including animals. ${ }^{46}$ There is no clear denominational consensus about when to conduct such rituals; however, none of these Buddhist communities appear to perform pet funerals or cremations even though this is common at Buddhist temples in Japan. I should note, however, that among the various Buddhist denominations Jōdo Shin Buddhist temples are the least likely to be involved in pet funerals and cremations.

\section{Pet death and religion in Japan}

Japan serves as a useful point of comparison with the recent developments in the U.S. According the Japan Pet Food Association, there were about 8,903,000 dogs and 9,649,000 cats in Japan in 2018, far exceeding the number of children under the age of 15 in Japan's greying society. ${ }^{47}$ Japan also has an exceptionally high number of pet funeral services and pet cemeteries, and the links between pet cemeteries and Buddhist temples are well-established. Data from the first decade of the millennium suggest that there were about 600-900 pet cemeteries and a total of 6000-8000 businesses related to the pet mortuary industry, which includes not only pet cemeteries and pet crematories but also stonemasons and producers of various kinds of accoutrements. ${ }^{48}$ One relatively comprehensive directory of Japanese pet cemeteries, the Japanese Animal Funeral and Cemetery Association's Petto sōgi reien netto, lists 1035 pet funeral providers and pet cemeteries as of 2019, though a search on the internet suggests that there are more pet cemeteries and funeral services unaffiliated with the site. Of the 1035 businesses listed on Petto sōgi reien netto, 739 have pet cemeteries or pet columbaria. With 326 pet funeral services and pet cemeteries, the Kantō region has the highest number in Japan, but this is not surprising since that this includes the densely populated Tokyo-Yokohama metro region. Other regions with large numbers of pet mortuary services and pet cemeteries are the Kansai region including Ōsaka (157), Kyūshū (130), and the Tōkai region including Nagoya (108). ${ }^{49}$ While some of the larger pet cemeteries are located in the suburbs, many pet cemeteries with columbaria and collective graves are also found in densely populated urban centers.

Cremation vans are a ubiquitous feature of the Japanese pet funeral industry. Of the 320 businesses on Petto sōgi reien netto that operate mobile cremation vans, many do not have pet cemeteries or columbaria but only provide cremation and funeral services. Cremation vans are convenient in both urban and rural areas as they provide companies with flexibility and the ability to cover a large territory. Pet owners also appreciate the convenience of having pets cremated near their home. One such business in Hokkaidō, where winters are harsh with much snowfall, reported that the demand for mobile cremations was particularly high in the winter months. ${ }^{50}$ Despite the convenience, anecdotally attested to by acquaintances who had used these services, I also encountered 
negative views of cremation vans during my fieldwork. Some compared the mobile crematoria to trucks selling roasted sweet potatoes, implying that they are undignified. Occasionally, some regarded the mobility of the crematoria as spiritually harmful. More frequently, the crematoria were regarded as environmental and traffic nuisances and potentially shady businesses. ${ }^{51}$

Another distinctive feature is the high degree of involvement of religious institutions, particularly Buddhist temples, in the pet mortuary industry. Historically, Buddhist temples have conducted funerals and maintained cemeteries for Japan's human population. During the Edo period (1603-1868), Buddhist temples virtually had a monopoly on funeral and memorial rites. While there are examples of Buddhist clerics conducting funeral and memorial rites for animals in the Edo period, the development of pet cemeteries on temple grounds - and secular pet cemeteries - became prevalent only in the twentieth century, particularly from the 1980s onward as urban pet ownership in Japan increased and pet owners began to regards their companion animals as members of the family. ${ }^{52}$

Of the 1035 pet mortuary businesses on Petto sogi reien netto, 170 are located on the grounds of Buddhist temples of many different denominations. This means that they are either operated by a Buddhist temple or the Buddhist temple rents out space to a pet funeral business while only providing ritual care for the dead. In addition, some pet funeral services without interment facilities have relationships with Buddhist temples with pet cemeteries or columbaria. ${ }^{53}$ Moreover, even though this is often not explicitly stated in the Petto sōgi reien netto's directory, many secular pet cemeteries have contractual relationships with Buddhist clerics at a nearby temple, meaning that a Buddhist cleric will hold rituals at the pet cemetery on important annual holidays such as the spring and autumn equinoxes (higan) and the Festival of the Dead (obon) in July or August, as well as monthly collective memorial services. ${ }^{54}$

Buddhist clerics tend to use standard liturgical formats for mortuary services rather than creating significantly different rituals for pets. Funerals (sōgl) and memorial rites $(k u y \overrightarrow{0})$ for pets typically follow a simplified Buddhist format, including encoffining, the chanting of sutras, offering of incense, merit transfers, cremation, and interment in a pet cemetery or pet columbarium, though interment may be delayed longer than in the case of the human dead. After the funeral, daily commemoration in the home as well as on death anniversaries and Buddhist holidays associated with such rites are also common, but death anniversaries are generally not observed as long as in the case of the human dead, which may last for two generations. During commemorative rituals, grave offerings of food, flowers, candles, and other material objects are common, as is the use of ritual accoutrements with Buddhist funeral practices such as memorial tablets, tôba (wooden slats inscribed with the name of the deceased typically placed at gravesites), etc. Memorial observances are usually directed at physical representations of the deceased pet, from urns holding the cremains to memorial tablets with the pet's name (though no posthumous name is used as would be the case with the human dead) and photographs. However, pet funeral rites tend to be intimate affairs that only involve close family rather than the large-scale affairs that might comprise extended family, acquaintances, and coworkers in the case of human funerals. ${ }^{55}$

While Buddhist temples do not usually handle human cremations, many Buddhist temples with pet cemeteries also have pet crematoria on their grounds though the day-to-day operation of the crematory may be subcontracted to a commercial company. At both Buddhist temples and at commercial crematoria, pet cremations are staggered in price, 
depending on whether they involve collective cremations, individual cremations, or attended cremations. Only the latter type approximates the cremation of the human dead, during which the chief mourners are present as the deceased is placed in the crematorium, wait until the cremation is finished, and then transfer the unprocessed cremated remains in the urn. During attended cremations, this is typically performed by two chief mourners who have to coordinate picking up the cremated bones with chopsticks and placing them into the urn. If the owners are not present, this action will be performed by the crematory staff. After the cremation, the owners have the option of taking the cremains home or interring them in a collective ossuary, on a columbarium shelf, or in an individual plot at a pet cemetery (see Images 26.3 and 26.4). When pet owners no longer pay maintenance fees for columbaria spaces, the cremains are transferred to the collective ossuary. ${ }^{56}$ During my fieldwork, crematory staff often took considerable pride in keeping the cremated bones as intact as possible by carefully regulating the temperature of the crematory chamber and in presenting the cremated remains to the bereaved. The top-most bone of the spine, called "nodo botoke" (literally, "throat Buddha") in Japanese, frequently receives special attention in both human and companion animal cremations. When turned on its side, the bone is shaped like a robed cleric with hands folded in prayer. Yokota Harumasa, the abbot of Chōfukuji, a Sōtō Zen temple in rural Niigata Prefecture, used the bone to illustrate the universality of Buddha nature in all living beings. He noted that this bone looked the same

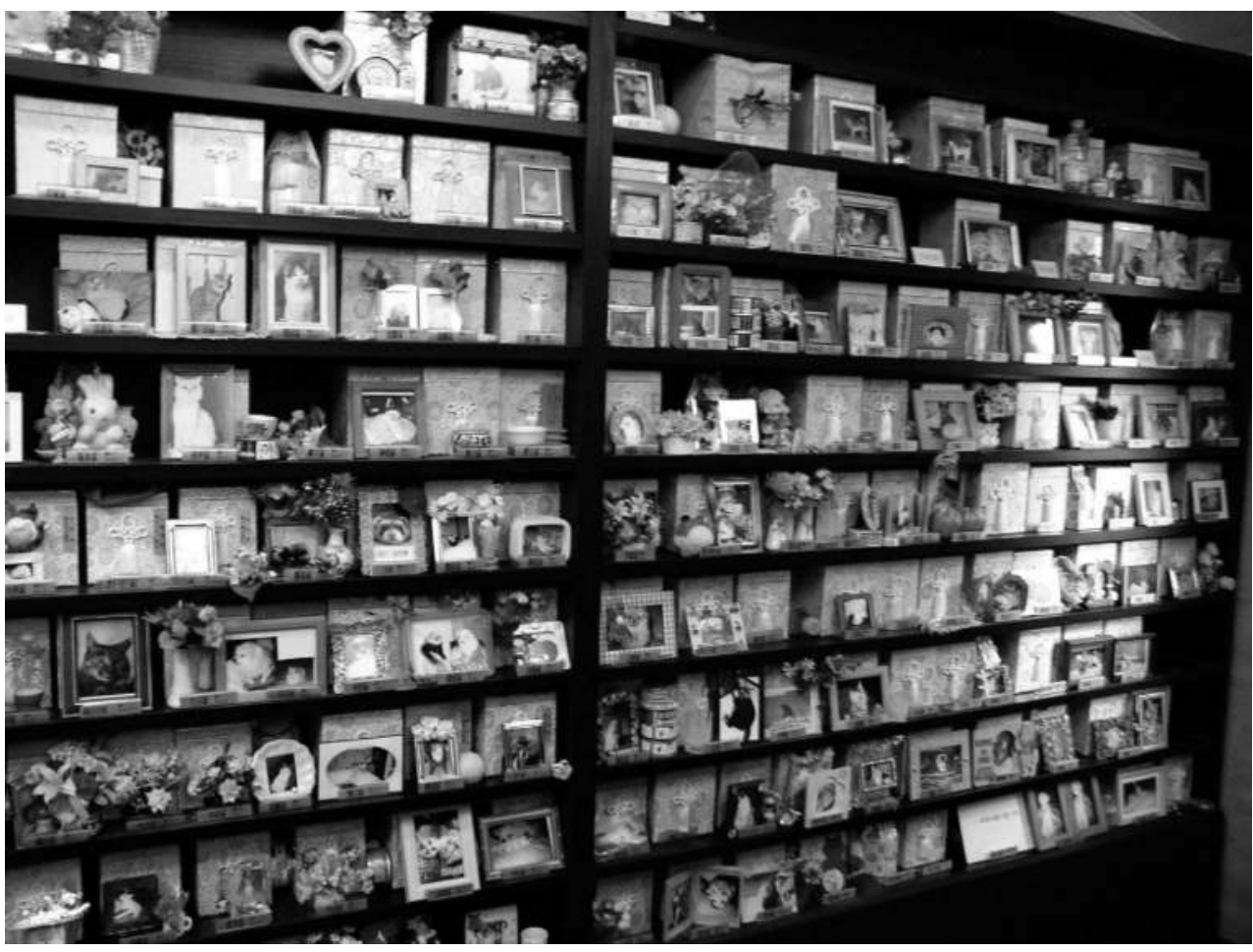

Image 26.3 Pet columbarium at the Rengeji Tetsugakudō Animal Cemetery in Tokyo, Japan Photograph by the author 


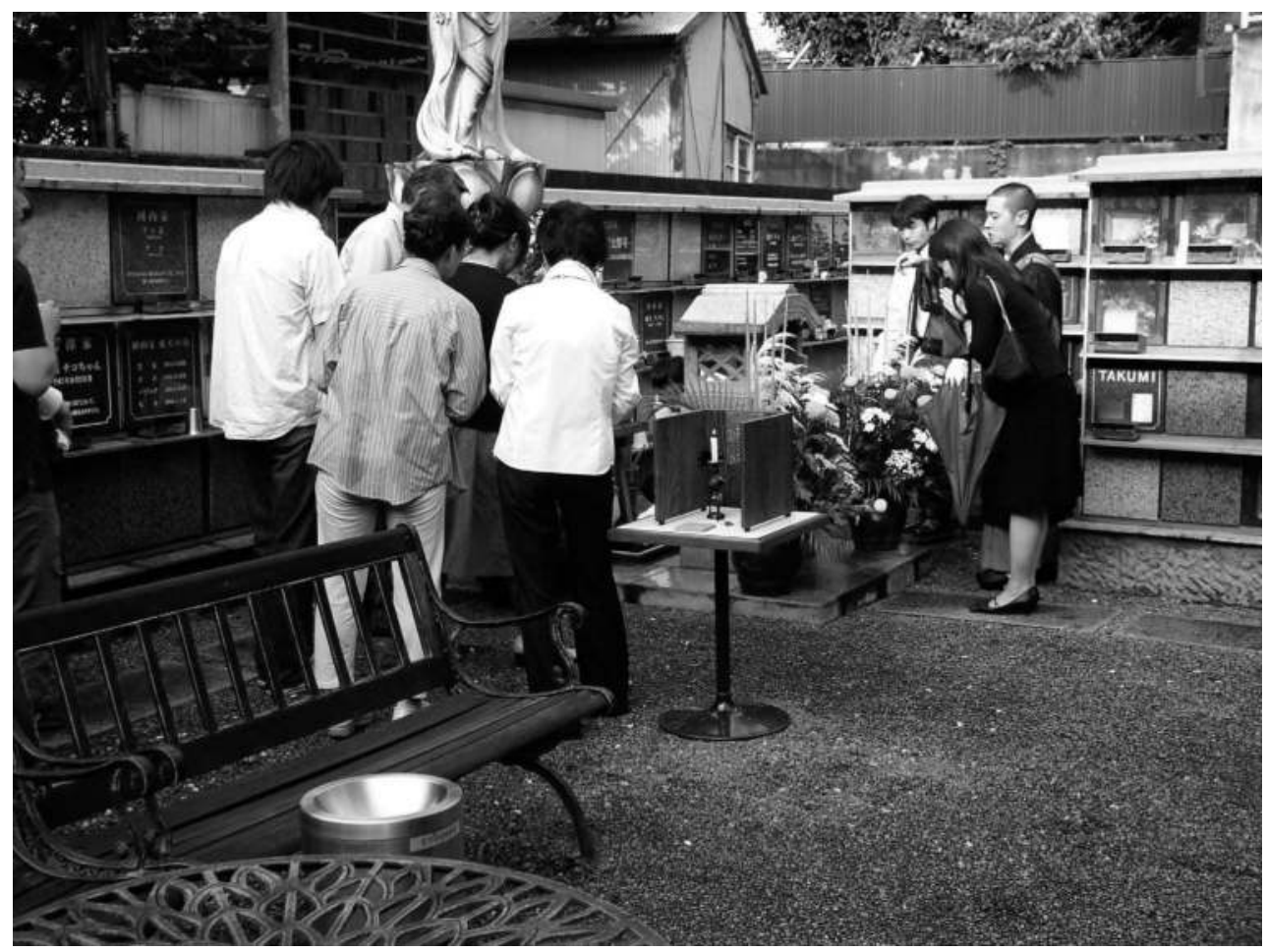

Image 26.4 Interment service at the Rengeji Tetsugakudō Animal Cemetery in Tokyo, Japan Photograph by the author

in all mammals even though their other bones might look different from species to species. It looked exactly the same in a hamster, a cat, a dog, and a human. The only thing that was different was the size. Yet the size was not a reflection of the being's potential for Buddhahood; otherwise, he quipped, elephants would have greater potential than humans. ${ }^{57}$

Pet owners in Japan take comfort in burying their pets at Buddhist temples, because not only will the pets receive some form of posthumous attention in perpetuity but it is also less likely that the cemetery will go out of operation. Another benefit is that if the owners are also parishioners at the temple, they will eventually be buried near their pets. As in the U.S., human cemeteries in Japan, including a few at Buddhist temples, now also allow joint species interment, and according to cemetery operators, the so-called "with-pet" sections are often very popular, indeed so popular that the "with-pet" plots of one such cemetery in the Tokyo area, Machida Izumi Jōen, which began offering this option about fifteen years ago, have already sold out. Likewise, pets are often memorialized alongside human ancestors in the home. Despite the growing popularity of such boundary-crossing practices, there has also been resistance to joint species interment or joint memorialization in the home altar as fellow cemetery users or Buddhist clerics aimed to inscribe firmer boundaries between humans and non-human animals and to delineate who is a legitimate member of the family qualified to be treated as an ancestor. ${ }^{58}$ 
As most contemporary Japanese tend to identify as non-religious and/or do not have rigidly exclusive affiliations with a single religious organization but participate in religious observances of various kinds as the occasion or the life-cycle ritual demands, most pet owners do not object to Buddhist funeral rituals for their pets or to rituals conducted in the style of a Buddhist denomination different from their customary family affiliation. Only the listing of two pet funeral business in Petto sōgi reien netto's directory (one in Gifu and one in Ibaraki) explicitly mention that the services of a Buddhist cleric, a Shinto priest, or, in one case, a Christian minister are available on demand. Moreover, only two pet funeral services (the Shinbutsu Shinko Shrine in Okayama City, Okayama, and the Ontake Shrine in Sapporo, Hokkaidō) are operated by a Shinto shrine rather than a Buddhist temple. Of these two, only the Ontake Shrine in Sapporo provides the full array of services including a pet cemetery and crematory. ${ }^{59}$

The absence of pet cemeteries and relative scarcity of pet memorial observances at Shinto shrines is due to ritual taboos against death as well as historical precedent. Shinto shrines have not commonly operated cemeteries even though modern law does allow any religious organization to operate cemeteries. There are, however, examples of Shinto shrines conducting spirit pacification rituals for the deceased, including some non-pet animals. For instance, the Yasukuni Shrine in Tokyo, which is dedicated to the human war dead, also features appeasement rites (ireisai) to military animals - dogs, horses, and pigeons, while the Namiyoke Inari Shrine in front of the Tsukiji fish market has multiple memorial markers to animals sold at the market. The posthumous ritualization of these animals, however, does not involve any physical remains. ${ }^{60}$ There are now also a number of shrines that have started catering to pet owners by providing other life-cycle rituals, such as the Ichigaya Kamegaoka Shrine in Tokyo, which has offered special amulets and blessings for pets since 2009, allowing visitors to bring their pets to mark the New Year or their third, fifth, and seventh birthdays as one would with a human child. ${ }^{61}$ The first of its kind in Japan, the Megushi Shrine in Chiba Prefecture, which was founded in 2009 and also opened a branch in Yokohama in 2018, even specializes exclusively in offering rituals for pets. ${ }^{62}$

While pet mortuary rites and pet cemeteries are still criticized as sites of excess, they also have been met with a high degree of social acceptance in Japan. As Elizabeth Kenney puts it, "in the Japanese religious setting, with its relaxed mixture of possible afterlives for humans and animals (reincarnation, heaven, wraiths), the courteous treatment of animal corpses (and perhaps more importantly animal spirits) is 'common sense'."63 From doctrinal perspectives, neither Buddhism nor Shinto draws an impermeable line between humans and other animals even though the relationship between human and non-human animals has traditionally been conceived of in hierarchical terms. In Buddhism, animals were traditionally regarded as living beings with a potential for salvation - even if this potential was diminished in comparison to humans since animals were considered a lesser, spiritually hindered path of reincarnation. ${ }^{64}$ As such, animals have long been considered as worthy of ritual posthumous attention, and there were few doctrinal impediments to conducting mortuary rites for animals though they were usually not buried in the same spaces. Whereas Episcopal liturgies for pets in the U.S. skirt the issue of the pets' posthumous salvation by focusing on creation, Buddhist funeral and memorial rites liturgically center around the reading of Buddhist scriptures and a merit transfer, ostensibly to ensure the posthumous salvation of the deceased living being. This is true also for pet mortuary rites. The climax of Japanese pet mortuary 
rituals at Buddhist temples is usually the reading of scriptures and a merit transfer during which the pet owners offer incense before the altar. The merit of the recitation is transferred to the companion animals, often by name. The pet names and the wishes articulated during the merit transfer may be the only indication that the ritual is for pets. Shinto animal memorial rites, by contrast, are conceived of as spirit pacification rituals to appease the spirits of animals, particularly those who died untimely deaths due to human agency. This aspect is often also implied in the performance of Buddhist rituals as the expression "to become a Buddha" usually means "to appease the spirit of the deceased," though Buddhist clerics performing funerals and memorial rites tend to conceptualize the purpose of such services in these terms. Instead, they may see the rituals as comforting the bereaved. ${ }^{65}$

These doctrinal points may be implied in the rituals, but they are rarely articulated openly and even when they are there is no clear consensus. The above-mentioned debate between Adachi Toshihide and Hayashida Kōjun on whether pets could be reborn directly into Amitabha's Pure Land began at an event held at Bukkyō University in Kyoto in September 2016 and has continued to the present day but has not been resolved conclusively. ${ }^{66}$ In my fieldwork, I encountered a wide range of positions among Pure Land clerics, including even some who were agnostic on the issue of an afterlife as a whole. Buddhist clerics providing animal mortuary rituals have received no guidance from their denominational headquarters on doctrinal issues or scriptural resources on animals but have to find answers and devise rituals, sermons, and merit transfers by themselves. Buddhist clerics may mention that all animals are living beings that have Buddha nature or that pets may be reborn near their former owners in the future, but pet owners and ritual specialists conducting funerals and memorial services for pets no longer find traditional views of animals as inhabitants of the realm of beasts, that is, as a lesser form of reincarnation, or the doctrinal position that animals cannot be reborn into the Pure Land in their current corporeal form particularly compelling. Likewise, notions of dead pets as vengeful spirits, common in popular self-help literature until the late 1990s, also no longer seem particularly popular. Instead, deceased pets are now often depicted as angels and are said to have gone to heaven rather than conceptualized as reborn as a lower life form or as potentially haunting their owners. In most cases, pet funeral providers are more likely to remain silent on doctrinal issues or to resort to the psychologized language of pet loss (including concepts such as the Rainbow Bridge) and the need to express one's love for and gratitude toward pets as family members. ${ }^{67}$

\section{Conclusion}

Pet cemeteries, and even more so pet cremation, have become common in the industrialized and postindustrial world. As companion animals have been accorded the status of family members and have been increasingly included in human living spaces, they have been given ceremonial and religious rites of commemoration in death, and joint-species burials have also become an important trend in both the U.S. and Japan. From doctrinal perspectives, thinking about companion animals either as God's creation in Christian and Jewish contexts and as living beings with the potential for posthumous salvation in Buddhist contexts has made the development of religious mortuary practices possible. While many religious organizations in the U.S. are still excluding animals from commemorative spaces and funeral rites, some progressive groups have begun to develop 
funeral and memorial practices, from commemorative liturgies and prayers to pet memorial gardens and memorial boards on the grounds of religious centers; but these practices still remain relatively small-scale and marginal. By contrast in Japan, pet cemeteries at Buddhist temples have become common across most Buddhist sects, so much so that they are similar in scope to commercial pet funeral services. They are included in industry directories, and, from a legal perspective, the proceeds from pet cremations, funerals, and columbaria/burial plot rental fees have been designated as taxable income from unrelated business. In other words, they are not legally recognized as tax-exempt religious activities. ${ }^{68}$ Ultimately, both in the U.S. and in Japan, the religious commemoration of pets remains fraught with contention as arguments for the inclusion or exclusion of companion animals from ritual practices and mortuary spaces make tangible the shifting distinctions between human and non-human animals. The ongoing debate adds an important ingredient to the many changes attached to the valuation of death in contemporary society.

\section{Notes}

1 The Archives of the Episcopal Church, Fournal of the 77th General Convention of the Protestant Episcopal Church in the United States of America Otherwise Known as The Episcopal Church 2012, (2016), 719-721. www.episcopalarchives.org/sites/default/files/publications/2012_GC_Jour nal.pdf (accessed December 22, 2019); The Episcopal Church, "Service at the Loss of a Beloved Animal," Resources - Liturgy \&O Music: Other Resources https://episcopalchurch.org/ files/service_at_the_loss_of_a_beloved_animal.pdf (accessed December 22, 2019); The Episcopal Church, "Prayers Suitable for Use in Church or for Other Gatherings," Resources_Liturgy ${ }^{\circ}$ Music: Other Resources https://episcopalchurch.org/files/prayers_suita ble_for_use_in_church_or_other_gatherings_for_beloved_animals.pdf (accessed December 22, 2019).

2 Hikari Maruyama, "When RIP Literally Means Being Buried with Your Beloved Pet," The Asahi Shimbun (April 30, 2019) www.asahi.com/ajw/articles/AJ201904300004.html (accessed December 24, 2019); see also Hidenori Ukai, Petto to sōshiki: Nihonjin no kuyōshin wo saguru (Tokyo: Asahi Shinbun Shuppan, 2018), pp. 32-38.

3 American Pet Products Association, "Historical Totals (1996-2019)" www.americanpetpro ducts.org/Uploads/MarketResearchandData/PetIndustryMarketSize2019.pdf) (accessed December 20, 2019); "Pet Industry Market Size \& Ownership Statistics" www.americanpetpro ducts.org/press_industrytrends.asp (accessed December 20, 2019); Yano Research Institute, "Pet Related Business in Japan: Key Research Findings 2018" (May 24) www.yanoresearch. com/en/press-release/show/press_id/1892 (accessed December 20, 2019).

4 Katherine Grier, Pets in America: A History (Chapel Hill: University of North Carolina Press, 2006), pp. 105-111; see also Hartsdale Pet Cemetery, "History," https://petcem.com/history/ (accessed December 22, 2019); Francisvale Home for Smaller Animals, "Memorial Grounds," www.francisvalehome.org/memorial-services/(accessed December 25, 2019).

5 Animal Rescue League of Boston, "Pine Ridge Pet Cemetery," www.arlboston.org/services/ pine-ridge-pet-cemetery/ (accessed December 22, 2019).

6 Stanley Brandes, "The Meaning of American Pet Cemetery Gravestones," Ethnology 48, no. 2 (2009), 99-118;

Alex Franklin, Animals and Modern Cultures: A Sociology of Human-Animal Relations in Modernity (London: Sage Publications, 1999), pp. 93-94; David Witt, "Pet Burial in the United States," Clifton Bryant, ed. Handbook of Death and Dying (Thousand Oaks, CA: Sage Publications, 2003); Carol Auster, Content Analysis: Using Pet Cemetery Memorial Plaques to Examine Possible Changes in Conceptions of Family (London: Sage Publications, 2018).

7 Andrew N. Rowan, "Companion Animal Statistics in the U.S.A.," Demography and Statistics for Companion Animal Populations Collection 7 (2018), https://animalstudiesrepository.org/demsca pop/7 (accessed December 18, 2019). 
8 International Cemetery, Cremation \& Funeral Association, "Pet Loss Professionals Alliance," https://iccfa.com/membership/plpa/ (accessed December 20, 2019); Association for Pet Loss and Bereavement, "Pet Cemeteries and Crematories," https://aplb.org/support/aftercare.html (accessed December 20, 2019); Everlife Memorials, "Pet Cemetery Directory," www.everlifeme morials.com/v/pet-loss/pet-cemetery-directory.htm (accessed December 20, 2019). The latter is a pet funeral business based in Texas.

9 Grier, Pets in America, 105-111; Brandes, The Meaning; and Hilda Kean, "Human and Animal Space in Historic 'Pet' Cemeteries in London, New York and Paris," Jay Johnston and Fiona Probyn-Rapsey, eds. Animal Death (Sydney: Sydney University Press, 2013), pp. 21-42.

10 Pet Loss Profession Association, ed., "PLPA Documents and Forms: Annual Pet Loss Profession Survey Summaries," International Cemetery, Cremation and Funeral Association (2013), http://s3. amazonaws.com/iccfa-media/2017/08/PLPA-2013AnnualPetLossProfessionSurvey.pdf (accessed December 22, 2019); Pet Loss Profession Association, ed., "PLPA Documents and Forms: Annual Pet Loss Profession Survey Summaries," International Cemetery, Cremation and Funeral Association (2012), http://s3.amazonaws.com/iccfa-media/2017/08/PLPA-2012survey summary.pdf (accessed December 22, 2019).

11 Ibid.

12 Douglas Davies, Death, Ritual and Belief: The Rhetoric of Funerary Rites (London: Continuum, 2002), pp. 192-193.

13 Green Pet-Burial Society. "Whole-Family Cemeteries," https://greenpetburial.org/providers/ whole-family-cemeteries/ (accessed December 22, 2019).

14 Michael Schaffer, One Nation under Dog (New York: Henry Holt and Company, 2009), p. 245.

15 Yonat Shimron, "Chaplain Helps the Grieving Confront the Loss of a Pet," News \& Observer (January 30, 2006), www.newsobserver.com.libproxy.lib.unc.edu (accessed December 21, 2019); "Chaplains Ease the Loss of Four-Legged Family," NPR (2006), www.npr.org/templates/story/ story.php?storyId=5290121 (accessed December 21, 2019); "The Pet Chaplain," Chronicle of Higher Education 53, no. 4 (2006), 52.

16 Pet Chaplain: Pet Loss Training and Support. www.petchaplain.com (accessed December 23, 2019).

17 A similar training program, pet loss counseling sessions, and pet memorial services are also offered by Interfaith Minister Karen Kobrin Cohen, who is based in Rockledge, FL. See Animal Chaplain Services https://animalchaplain.info (accessed December 26, 2019); for other examples of pet chaplains, see Matthew Abrams, "YYou Have a Church for Cats?' The Service of an Animal Chaplain," Tricycle (July 16, 2019), https://tricycle.org/trikedaily/animal-chap lain/ (accessed December 26, 2019); Association for Pet Loss and Bereavement, "Pet Chaplains," www.aplb.org/support/chaplains.html (accessed December 26, 2019).

In addition to pet or animal chaplains, there are also sourcebooks published in the first decade of the millennium that draw on resources from a variety of religious and literary traditions to create memorial prayers and services. See for instance, Lynn L. Caruso, ed., Blessing the Animals: Prayers and Ceremonies to Celebrate God's Creatures, Wild and Tame (Woodstock, VT: Skylight Paths, 2006); Diana L. Guerrero, Blessing of the Animals: A Guide to Prayers \& Ceremonies Celebrating Pets \& Other Creatures (New York: Sterling Publishing, 2007); Carol J. Adams, vegan feminist activist and Presbyterian, follows a more focused Christian format. Carol J. Adams, Prayers for Animals (New York: Continuum, 2004) and God Listens When You're Sad: Prayers When Your Animal Friend Is Sick or Dies (Cleveland: Pilgrim, 2005). It is difficult to judge to what extent these books have been used widely.

18 Adam Reinherz, "When Pets Die, Jewish Owners Have Options," The Pittsburgh Jewish Chronicle (March 29, 2017), https://jewishchronicle.timesofisrael.com/when-a-beloved-pet-dies-jewishowners-have-options/ (accessed December 20, 2019).

19 Brandes, The Meaning, 111.

20 Rabbi Andrea Frank, L’Or V'Dor. "Pet Funerals for Pet Owners.” https://jewish-weddingrabbi.com/pet-funeral.html/ (accessed December 23, 2019).

21 Brandes, The Meaning, 109-110.

22 Brandes, The Meaning, 102-105; Auster, Content Analysis.

23 Personal communication, December 6, 2007. 
24 Personal communication, December 6, 2007; Nicole Seiferth, "A Resting Place for All God's Creatures," The Episcopal New Yorker (May/June 2005), http://eny.dioceseny.org/Archives/ 0505/petCemetery0505.html (accessed May 11, 2007).

25 Personal communication, December 6, 2007; Anthony F. Chiffolo and Rayner W. Hesse, Jr., We Thank You, God, for These: Blessings and Prayers for Family Pets (New York: Paulist Press, 2003).

26 Ibid., 2-3.

27 (Online) Book of Common Prayer. "Pastoral Offices: Burial I," The Church Hymnal Corporation, New York, 469-489, www.bcponline.org/PastoralOffices/BurialI.html (accessed December 23, 2019); (Online) Book of Common Prayer, "Pastoral Offices: Burial II," The Church Hymnal Corporation, New York, 491-505, www.bcponline.org/PastoralOffices/BurialII.html (accessed December 23, 2019).

28 The Episcopal Church, "Service at the Loss."

29 Frank Logue, "A Liturgy for the Burial of a Pet," King of Peace Episcopal Church: Resources http:// kingofpeace.org/resources/petfuneralliturgy.htm (accessed December 21, 2019); The Episcopal Church, "Frank S. Logue: Biography," https://episcopalchurch.org/library/author/franks-logue (accessed December 23, 2019); Episcopal Diocese of Newark, "Liturgy for the Burial of a Pet," https://dioceseofnewark.org/liturgical/liturgy-burial-pet (accessed December 23, 2019).

30 Laura Hobgood-Oster, Holy Dogs and Asses: Animals in the Christian Tradition (Urbana and Chicago: University of Illinois Press, 2008).

31 Jodi S. Cohen, "Jewish Leaders Protest Pet-Burial Plan at Cemetery," Chicago Tribune (October 14, 1998), www.chicagotribune.com/news/ct-xpm-1998-10-14-9810140212-story.html (accessed December 20, 2019).

32 Central Conference of American Rabbis, "Burial of a Pet Animal," http://ccarnet.org/ responsa/curr-165-169/ (accessed December 23, 2019).

33 Walter Jacob, ed., Contemporary American Reform Responsa (New York: Central Conference of American Rabbis, 1987), p. 210.

34 Janet Offel, "When a Beloved Pet Dies," Kalsman Institute of Judaism and Health, Institute of Religion, Hebrew Union College: Writings, http://kalsman.huc.edu/articles/Offel_WhenABelovedPet Dies.pdf (accessed December 23, 2019).

35 Ruth E. Chodrow, "A Jewish Response to Pet Loss," CCAR Fournal: A Reform Fewish Quarterly (Fall 1998), 23-27; see also Aubrey L. Glazer, "Taking the Circumcised Dog by the Throat: A Critical Review of Contemporary Rituals for Dogs in America," Phillip Isaac AckermanLieberman and Rakefet Zalashik, ed. A Jew's Best Friend? The Image of the Dog Throughout Jewish History (Eastbourne, UK: Sussex Academic Press, 2013), pp. 231-250.

36 Irwin Kula and Vanessa L. Ochs, eds., The Book of Jewish Sacred Practices: CLAL's Guide to Everyday $\mathcal{E}^{2}$ Holiday Rituals $\mathbb{E}$ Blessings (Woodstock: Jewish Lights Publishing, 2001), pp. 172-173.

37 Joshua Snyder, "Creating a Ritual for the Loss of a Companion Animal," Ritual Well: Tradition \& Innovation www.ritualwell.org/ritual/creating-ritual-loss-companion-animal (accessed December 23, 2019); Susan Schein, "Burial Service for an Animal Companion," Ritual Well: Tradition \& Innovation www.ritualwell.org/ritual/burial-service-animal-companion (accessed December 23, 2019); Reena Friedman, "Saying Good-bye to a Beloved Pet," Ritual Well: Tradition E Innovation www.ritualwell.org/ritual/saying-good-bye-beloved-pet (accessed December 23, 2019); Alden Solovy,"Passing of a Beloved Pet," Ritual Well: Tradition \& Innovation www.ritualwell.org/ritual/ passing-beloved-pet (accessed December 23, 2019); and Rona Shapiro, "Grieving the Loss of a Pet," Ritual Well: Tradition \& Innovation www.ritualwell.org/ritual/grieving-loss-pet (accessed December 23, 2019).

38 Glazer, Taking the Circumcised Dog, 236. Indeed, this is difficult to judge whether such rituals are performed widely. The only citation of Chodrow's service at a synagogue was posted in July 2009 by Emanuel Synagogue in West Hartford, CT. See Emanuel Synagogue in West Hartford. "Mourning a Pet: A Jewish Response to Pet Loss," The Emanuel Synagogue: Helpful Tips on living a more Fewish life! Perform Daily and Weekly Rituals, www.emanuelsynagogue.org/ourjewish-world/how-to-what-if/ (accessed December 23, 2019).

39 Temple Beth Emet. "Dedication \& Memorial Opportunities: Pet Memorial," www.templebethe met.org/giving/dedication-memorial-opportunities (accessed December 23, 2019).

40 Congregation Kol Ami. "Pet Memorial Tree: Remember Your Beloved Pet," www.kol-ami. org/PetMemorial (accessed December 23, 2019). 
41 Temple Israel. "Plaques: Pet Remembrance Board-Honor the Memories of our Cherished Pets," https://templeisraelva.org/support-donate/plaques (accessed December 23, 2019).

42 Moses Montefiore Anshe Emunah Greengate Jewish Center. "Dedication Opportunities," www.mmae.org/dedication.html (accessed December 23, 2019).

43 Reinherz, "When Pets Die."

44 Arthur Hirsch, "At Pikesville Synagogue, Memorial Honors All God's Creatures," Baltimore Sun (November 29, 2013), www.baltimoresun.com/maryland/baltimore-county/bs-md-co-memor ial-pets-20131125-story.html (accessed December 23, 2019).

45 Buddhist Temple of San Diego, "All Life Forms Memorial Service is October 27, 2019," www.buddhisttemplesandiego.org/all-the-latest/2019-10-27-all-life-forms (accessed December 24, 2019).

46 Midwest Buddhist Temple, "Pet Memorial Service August 18," https://mbtchicago.org/petmemorial-8-18/ (accessed December 24, 2019).

47 Hisashi Ishiyama, "Goaisatsu," Japan Pet Food Association https://petfood.or.jp/outline/greeting/ index.html (accessed December 27, 2019).

48 Barbara Ambros, Bones of Contention: Animals and Religion in Contemporary Japan (Honolulu: University of Hawai'i Press, 2012) 6.; Kunio Sakurai, "Petto kuyō kazei shobun torikeshi soshō hanketsu to shūkyō handan kijun." Shükyōhō 25 (2006), 99-122.

49 Petto sōgi reien netto. www.petsougi.net (accessed December 25, 2019).

50 Ibid.

51 Ambros, Bones of Contention, 131.

52 Ambros, Bones of Contention, 2-6; Elmer Veldkamp, "The Emergence of 'Pets as Family' and the Socio-Historical Development of Pet Funerals in Japan,” Anthrozoös 22, no. 4 (2009), 333-346.

53 Petto sōgi reien netto.

54 Ambros, Bones of Contention, 6. My fieldwork focused on the Kantō, Kinki, and Tōkai regions, where the largest numbers of pet cemeteries are found, as well as some selected sites in Ishikawa and Nagano Prefectures.

55 Elizabeth Kenney, "Pet Funerals and Animal Graves in Japan," Mortality 9, no. 1 (2004), 42-60; Ambros, Bones of Contention, 10.

56 See for instance, Ambros, Bones of Contention, 96.

57 Personal communication, June 28, 2007.

58 Ambros, Bones of Contention, 132-138, 142-153; Maruyama, "When RIP."

59 Sapporo Ontake Jinja Fuzoku Petto Reien Yasuragi no Oka. www.satu-petyasuragi.com (accessed December 24, 2019).

60 Ambros, Bones of Contention.

61 Kamegaoka Hachimangū Ichigaya, "Petto omamori annai," www.ichigayahachiman.or.jp/pet/ pet_omamori.html (accessed December 24, 2019).

62 Megushi Jinja, http://megushijinjya.com (accessed December 24, 2019).

63 Kenney, Pet Funerals, 45.

64 Ambros, Bones of Contention, 35-42; and "Animals in Japanese Buddhism: The Third Path of Existence," Religion Compass 8, no. 8 (2014), 251-263.

65 Ambros, Bones of Contention, 109,163; Kenney, Pet Funerals, 59.

66 Ukai, Petto to sōshiki, 32-38; Hidenori Ukai, "Can Dogs Be Reborn in Paradise? Japan's Buddhist Funerals for Pets," Nippon.com (February 5, 2019), www.nippon.com/en/in-depth/d00460/candogs-be-reborn-in-paradise-japan's-buddhist-funerals-for-pets.html (accessed December 24, 2019); Maruyama, When RIP.

67 Ambros, Bones of Contention, 159-183; Kenney, Pet Funerals, 58-59.

68 Ambros, Bones of Contention, 94-104. 


\title{
HOSPICE
}

\author{
A way to die
}

\author{
Lucy Bregman
}

\section{Introduction: Hospice as the answer to dying in the modern era?}

"No, I don't want hospice. Hospice is for dying people!" exclaimed my aunt. She died of cancer a few days later. This quote encapsulates the paradox of hospice: a philosophy and method of medical treatment aimed to alleviate the pain and isolation of the dying, but nevertheless tainted with the aura of death, and therefore to many persons utterly unacceptable. When a newspaper referred to the founding hospice center as "St. Christopher's Hospice for the Dying," Dame Cecily Saunders vigorously protested. It was never named "for the Dying," and she wanted to make clear that hospice was a program for patients who could live fully until they died. ${ }^{1}$ A similar spirit of repugnance was voiced by a representative of a Jewish organization in Hungary: "Hospice spirituality is against Jewish spirituality, because the Jewish philosophy is for life and not for death." While the last speaker changed his views and eventually supported hospice and accepted funds for it, the link with death is pervasive, across regions and cultures, and when all is said and done, accurate. Hospice began as a philosophy and method of medical treatment aimed specifically at terminally ill cancer patients. It would not be included in a volume explicitly devoted to death were it not for this link. However, the major transition was to substitute the term "palliative care," and eventually to make palliative medicine a recognized medical specialty. As a branch of medicine, this is no longer restricted to the dying, and is, whenever possible, fully integrated into the main systems of medical care. Thus the isolation of the dying, and the stigma of death, are to some degree mitigated. But that is not how hospice began, nor how it finds a place in a history of death, together with suicide and euthanasia.

This chapter deals exclusively with the modern hospice movement. That can be dated to 1967, when St. Christopher's Hospice in London opened. Dr. Saunders began her work in 1959 at a hospital for the dying run by Roman Catholic nuns. It is true that in pre-modern times, pilgrims were sheltered in monasteries and other welcoming houses that cared for the sick and dying in Europe. ${ }^{3}$ These were run by religious orders or by pious laypersons. But there is no direct continuous link between such efforts and those of Saunders and other pioneers of modern hospice.

Modern hospice has two aspects. One echoes the older ideal of compassionate care for the dying, focused not on the disease but on the patient as a person, and on the patient's family. The second is the introduction of twentieth-century pharmacology, 
methods of pain control that were the mark of Saunders' success from the beginning. It is this that has translated into "palliative care," a term devised by Dr. Balfour Mount of Canada in the 1970s. Without control over physical pain, the goal of peaceful dying and living fully until death would be impossible. To accomplish this for patients, specific practices of pain relief were necessary. Saunders found that since part of the experience of pain is anticipation of future, returning pain, it is better to give patients pain medicine before their pain actually starts up again. In sharp contrast to the philosophy that patients "must earn their morphine," (i.e. by first experiencing severe pain), she loaded them up on strong pain-blocking medication before. Then, they gradually needed lower doses of medicine as the anxiety component of pain was eliminated. This strategy works. It is described in great detail in chapter 3 of her co-authored book with Mary Baines, Living with Dying: The Management of Terminal Disease, ${ }^{4}$ and plays a large part in the case narratives of Ira Byock's Dying Well: Peace and Possibilities at the End of Life. ${ }^{5}$ Another way to state this is that, in direct contrast to an image of "natural death," hospice dying is medical tinkering, tinkering that is very high-tech when it comes to pharmacology and other methods of pain-control.

Yet Saunders taught that pain, as "total pain," included more than physical pain. Psychological, social and spiritual pain were just as real, and the intent of hospice was to treat all of these together. This is why a strictly medical history of the hospice movement would be only half the story. Nevertheless, the greatest single step for hospice worldwide has been the development, in Singapore in the 1990s, of easy-to-use, inexpensive oral morphine. This potentially solves the problem of affordable and effective pain control for all patients everywhere. Emphasize "potentially": although it is technically possible to eliminate almost all physical pain associated with diseases such as cancer or AIDS, there is a long way to go before politics, public awareness, medical services and funding make this a reality.

Because this book centers on death, we will focus our attention on that aspect of hospice. What philosophy of dying and death spurred the movement from its origins, and how did this continue and change as hospice moved beyond urban United Kingdom? What obstacles did it meet, at home and among persons such as my aunt, and elsewhere in the world? We will not be reviewing hospice programs and initiatives everywhere, but select a few locations: the United Kingdom, the United States, Russia, Japan and Uganda. Hospice clearly began in one kind of setting, amid a medical system geared to cure and deliver aggressive treatment of cancer in a hospital. It was intentionally brought into new places to face wildly different health settings and challenges. Intentionally, because the story was of exceptionally charismatic individuals motivated to plant seeds, raise funds to start programs and create models ("beacons") 6 that would inspire others to develop similar hospice programs elsewhere. ${ }^{7}$ As the movement matures and becomes part of regular medical care, so the distinctive role for such inspirational leaders may wane. But that has definitely not happened yet in many parts of the world.

\section{Why hospice? The problems of dying in Western hospitals}

Although, historically, hospice began in London, we will start from Switzerland. There, in 1971, an American named Edgar Snow was dying of cancer. His treatment was the best a highly technological medical system could offer, and money was not an issue. But Snow was never told his diagnosis, he was left snubbed by a range of doctors, and he 
and his wife Lois were both enraged by the inhumane attitude of doctors and staff. Hospitals were not for the dying, pain was ignored, and the Snows were desperate. Lois was run ragged attempting to care for her very sick husband at home. And then The Chinese Came, the subtitle of her book A Death with Dignity. Edgar Snow was an expert on modern Communist China, and a personal friend of Chairman Mao. So when the Chinese leader learned that Snow was sick, a team of Chinese physicians and support staff was sent to help. For Lois, the Chinese provided security, respite and medical expertise. They were not really completely successful in controlling her dying husband's pain, but they were compassionate, attentive and non-hierarchical. As was part of Chinese Communist culture, they solved issues by group discussions. "We shared questions, answers, knowledge, limitations ... the sharing brought us together and did much to make the inevitable easier to accept." ${ }^{\prime \prime}$ In complete contrast with their treatment by the hospital staff, the Snows found they were given dignity and respect. Edgar's death was therefore with dignity; not that it was joyous, but Lois believed that she and the Chinese had done right by him. She wrote her story to promote a better way to die, unknowingly promoting the same values lauded by those who experienced hospice care at St. Christopher's and other hospices. What makes this story so striking as testimony to the problem of hospital dying is that the values of Mao's Chinese state could not have been more different from those of devoutly Christian Saunders. Eventually, by the way, Lois Snow became an outspoken critic of the Chinese regime and so was barred from visiting a memorial monument to her dead husband in China. ${ }^{9}$

The problems of hospital dying went far beyond snobbish uncommunicative doctors. The entire institutional structure was aimed to cure with the most efficiency, and with the very latest technology. Inventions such as ventilators and inaugurations of intensive care units were relatively recent when Snow's husband fell ill. Had they been in America, her story would have been the same; her complaints were echoed by endless authors of similar narratives, personal testimonies to the human experience of dying in a high-tech medicalized environment. The patients interviewed by Dr. Elisabeth Kübler-Ross in the late 1960s shared this sense of extreme isolation, loss of control and human anguish. The interview transcripts in On Death and Dying reveal how dying was equated with failure. ${ }^{10}$ Once there was no more chance of curing the patient, that person was abandoned and avoided by the staff or, in some cases, given futile and uncomfortable medical treatments because doctors felt compelled to do something rather than lose the battle vs. death. ${ }^{11}$ The hospital's model of death was of a military defeat, for the doctor and, of course, for the patient. And like the Snows, Kübler-Ross sought an alternative, in her case drawn from a mix of psychoanalysis and humanistic psychology. Death was, as her title for an edited anthology proclaimed, The Final Stage of Growth. ${ }^{12}$ Her famous "five stages of dying" offered hope that patients could emotionally come to accept their deaths and end their lives in peace.

If we trace this language and imagery back before the era of I.C.U.s and really advanced technology, we can find it in a 1936 work by Cabot and Dicks, The Art of Ministering to the Sick. Here, the hospital is already an alien environment, dominated by specialists' expertise and frightening to patients. ${ }^{13}$ Patients were not yet hooked up to machines, but their perspective as human beings was eclipsed; the two authors aimed to restore this, and became patient advocates rather than religious specialists. So it is most likely the hospital as institution, rather than the specific medical techniques or treatments, that created the ethos Snow, Kübler-Ross and others complained about so bitterly. As 
Sharon Kaufman's fine book And a Time to Die depicts it in an anthropological study of hospitals in America more recently, the essential problems remain the same. ${ }^{14}$ Hospitals are now places where patients and families must make decisions they were not permitted to make back in the era of Kübler-Ross or Snow, but they are still not where most of us wish to spend our last days on earth.

Indeed, running through all modern discussions of death, hospitals and hospice is the plaintive wish of the sick to die at home. That is what most of us want. In Japan, it is voiced as "to die on tatami," the sleeping mat of one's home floor. To some, the issue around hospice is to create a possible home death-place or to construct a special space as home-like as possible. The Western model of dying, however, makes hospital care the best care from a technological/medical perspective, and so although the percentages fluctuate, a majority of us will still be likely to die in hospitals or nursing homes. Families wanting to do the best for a dying member will insist on hospital care, especially because some of the care-taking tasks are too complex for non-medical, untrained persons to perform.

Dying at home, with dignity, surrounded by family and friends: this ideal haunted Kübler-Ross, as she contrasted death in her childhood Swiss village with what she found at Billings Hospital in Chicago. Its nostalgia became intertwined with hospice as an "alternative," a counter-cultural expression of discontent with medical expertise in the United States. In the 1970s, it was often described by its advocates as "low-tech, hightouch." But in the writings of Cecily Saunders there is no nostalgia for the good old days. Saunders knew that the problem of physical pain was a scientifically solvable one. "Total pain" was more complex, but the psychological and other aspects of pain could not be dealt with until the physical pain was treated successfully. Significantly, too, there is no use in Saunders' letters of the imagery of "natural death." Indeed, Saunders herself was much less romantic about death than American enthusiasm for "natural" and "good death" implied. When writing about the death of a colleague, she could state: "Death is an outrage" and "Death is all right," in the same paragraph. And yet, she followed this by remarking "One does turn medicine upside down when you care for the dying, and strange things happen when you do this." 15

How did hospice turn medicine upside down? Not by totally challenging medicalized death, but by focusing on the patient as person, whose dying condition did not destroy or determine their identity. Of the many statements of hospice principles, produced as part of the "movement," all emphasize compassionate, holistic care, the team of carers and the goal of peaceful, pain-free living until death. The simplest of these declarations comes from Merriman's Hospice Africa Uganda, expressing "the hospice ethos":

1. The patient and families are the center of care.

2. We will respect and care for each other.

3. We will respect and share with our networking organizations. ${ }^{16}$

Here, "networking organizations" reaches out to others including government, NGOs and funding sources, as well as healthcarers elsewhere who wish to learn how to do hospice work.

Hospice turned things upside down in two other ways at least, right from the beginning. In the Western medical disease model, the patient is an individual organism, whose social and familial environment is ignored for purposes of diagnosis and treatment. The cancer is in one person's body, whatever their social milieu. Not so in hospice, where the 
family is included under the umbrella of care. For this reason, bereavement counseling is intrinsic to the philosophy, just as is spiritual care. This commitment to the patient-asfamily-member is particularly illustrated in the book that helped to introduce hospice to Americans: A Way to Die, by Victor and Rosemary Zorza. Their daughter Jane, age 25, dying of cancer in great pain, was admitted to a hospice in Oxford. The Zorzas could not have been easy parents of a patient to deal with, but their story shows how their concerns, unfinished emotional issues and practical worries for their daughter were all taken seriously. They were permitted to stay at the hospice to be with their daughter. Jane was only there for nine days before her death, but for the Zorzas it meant that the story of her illness could be one with a good ending, of peace and possibilities at the end of life (to borrow Byock's language). Jane herself seems to have experienced this, full of peace and gratitude toward those who cared for her, as well as for family and friends. ${ }^{17}$

The other reversal, a hallmark of hospice in America even more than in U.K., was that hospice was a place where people could be open about cancer and dying. At the time Saunders founded St. Christopher's, it was normal for patients never to be told directly that they would die from their illness. They may not even have been told their diagnosis of cancer, since that was in their minds the equivalent of a death sentence. But hospice was different; people knew they were dying, even if the full name of the place did not include "for the dying." Jane knew, the Zorza parents knew, and everyone on the staff knew that they knew. This "open awareness" was extremely rare in 1975. Most patients probably figured out the truth, but the Zorzas' account shows how much more easily communication flowed when this was no longer an issue at all. As we will see, this practice of "truth-telling" continues to be a violation of previous medical and cultural norms elsewhere, such as in Japan and Russia.

It is misleading to think of hospice as a revolutionary movement, intent on dismantling the hospital system and Western scientific medicine. It is clear that Saunders always hoped otherwise. "The work of the hospice should be integrated with general medical practice, forming a complementary resource and service." 18 It was recognized by the National Health Service of the U.K. by 1980, an important milestone everywhere for hospices in relation to whatever national health policy and funding exists. Once part of the normal framework for healthcare, such as being covered by Medicare in the U.S. from 1982, it could gain the trust of patients and the public. And when the transition to palliative medicine progressed, this could gain the respect of physicians practicing other medical specialties. As Cathy Siebold's fine history The Hospice Movement: Easing Death's Pains interprets it, this step of accommodation led to a certain amount of fragmentation, because some hospice advocates in the U.S.A. still clung to the counter-cultural mystique of the early days. ${ }^{19}$ But overwhelmingly, acceptance of hospice internationally has been marked by integration into national systems and policies for healthcare.

Another way in which hospice did not seek to overturn traditional medicine centers in its stance against euthanasia. By the time Saunders began her work, there was an active Euthanasia Society in the U.K., whose goal was to legalize voluntary euthanasia, direct ending of patients' lives by their physicians. Their argument - and this continues to be used by euthanasia advocates down to the present - was that patients should not have to endure intolerable pain as they slowly died. Saunders agreed; patients should not have to die in pain, and she believed, as we saw, that pain was no longer a necessary and unavoidable part of dying. What she disagreed with was that euthanasia, direct killing, was the only way to achieve this goal. She early on initiated conversation with 
Euthanasia Society leaders, ${ }^{20}$ and these communications were apparently respectful and appreciated. Hospice, in short, did not want to yield all control to patients, and has consistently defined itself as an alternative to active euthanasia. However, when the phrase "death with dignity" is used, it has often been linked to euthanasia advocacy, or to the practice of physician-assisted dying (as in the Oregon Death with Dignity Law). Confusion over these terms exists in Japan as well; ${ }^{21}$ in spite of the popularity of the slogan "death with dignity," hospice as palliative holistic care seems now to avoid it due to this confusion.

\section{Hospice and the United Kingdom}

Cecily Saunders' contribution was enormous, and her energy and activism meant that she traveled and gave hundreds of public lectures and educational training sessions in many countries. Thus, the importance of hospice in the U.K. is not only for the U.K., but for many other parts of the world. When healthcarers from Eastern Europe or Central Asia wished to learn about palliative care, they most often learned from trained personnel visiting from the U.K. Many partnerships with impoverished and struggling hospitals and clinics were established in this way. Sometimes the links are more personal; Charles Dickens' great-grandchild, with a Hungarian relation on her mother's side, visited Hungary and helped to support the Hungarian Hospice Foundation. ${ }^{22}$ So any story of hospice worldwide really does center on the U.K. The Princess Diana Foundation is another example of funding for hospice projects dealing with AIDS in Africa in particular.

What made hospice acceptable in the U.K. in the later part of the twentieth century and today? One feature of the National Health Service is that it has more control over patients' treatment options than Americans especially are accustomed to. There is much less frequent use of medically futile end-of-life aggressive treatments, and patients' families do not get to tell doctors "Do everything," when the medical consensus is that nothing curative can be done. ${ }^{23}$ Therefore, space for an alternative medical path, treatment for a goal of comfort at the end of life, is more acceptable than in United States. Hospice, then, is less likely to be linked with "defeat" or "no hope," regardless of individuals" psychological struggles with dying, simply because national policies do not permit what Americans expect.

Nevertheless, Saunders experienced opposition from many doctors, and some of this has persisted elsewhere. Do patients really want to die, to relinquish all hope for a cure? Will the regime of steady opiates for pain relief really work as she recommended and endorsed, or will problems of addiction appear? Although, if we are thinking of cases such as Jane Zorza, whose nine days of hospice care gave her freedom from pain, this appears to be an unreal concern, it may not be. America's current opioid addiction crisis was not caused by use of morphine for dying hospice patients, but it shows how hard it has been to keep such drugs for a select, restricted group to receive them.

Originally, back in 1967, St. Christopher's accepted adult cancer patients whose disease had progressed to where they needed inpatient care. Home care was not, or no longer, an option for them. But hospice could include outpatients, those still living at home, and eventually, one other group not part of the original plan: children. In 1982, Sr. Frances Dominica, an Anglican nun, founded Helen House, a pediatric hospice. But, as Dominica explained, a children's hospice is different in function from one for adults. 
It is primarily for "respite care," to give families a break from continuous home-caregiving for an invalid child. ${ }^{24}$ This at least is the case in the U.K., where a terminally ill child is an anomaly. As with Saunders as world-leader in the hospice movement, so U.K. has led the way in establishing pediatric hospice services elsewhere. For example, the AIDS epidemic affected large numbers of Romanian children, and pediatric hospices were established to care for them, thanks in part to the influence and funding of the Ellenor Foundation, a British-based organization established by hospice-advocate Graham Perolls. ${ }^{25}$ It is interesting that these connections do not follow the paths of British colonial rule; they are made possible because individuals with motivation and access to funding worked hard with local healthcare leaders. In this, the third of the basic hospice principles enunciated in the Ugandan document is evident.

\section{Hospice in the United States}

We have already mentioned the extremely popular work of Kübler-Ross in making death and dying a topic for public discussion and education. ${ }^{26}$ It is likely that this development made Saunders' simultaneous inauguration of hospice as public and wellreceived as it was. For what marks the early decades of hospice in America was its link with an ideology of "post-Vietnam," one in which critique of military-industrial power and technological mindsets made hospice seem far more an alternative to conventional hospital treatment than it eventually turned out to be. But it did seem radical. An appropriate beginning is to listen to the words of retired Navy officer Gene Cote, at a meeting of the community in Branford, CT, in 1976. Many local persons did not want a place for dying patients near their homes; it was gruesome and morbid. Then Cote stood up and spoke: "I am one of those terminally ill cancer patients the hospice is taking care of." ${ }^{, 27}$ He told how hospice had helped him, and the community was so impressed by his honesty and assurance that they voted to let the hospice facility be built. Although Siebold believes the demand for hospice in America came from healthcare professionals and not from patients, ${ }^{28}$ in this instance we have a dramatic instance of the patient's own voice pleading for the hospice philosophy.

Siebold, writing the history of American hospice in 1992, believed the movement had two ultimately conflicting aims: the de-medicalizing of death - the restoration of a humanistic approach that focused on patient experience - and the medicalizing trend via analgesic drug therapy. The latter trend won out, aided by the argument that hospice home-care would be cheaper than hospitalization, and by 1982 with the acceptance of hospice care by Medicare, the idea of hospice as a direct alternative to hospital was doomed. Whether or not the economic argument is empirically accurate, it has proved irresistible to governments. ${ }^{29}$ However, in one sense American hospice remains an alternative: to be eligible for hospice reimbursement through Medicare, one must explicitly forego pursuit of aggressive, curative treatments. This either/or situation remains built into the funding system, and so shapes patients' expectations that hospice equates with "giving up."

American hospice became home-care services, primarily, rather than the inpatient self-standing British model of St. Christopher's. This happened not just because homecare was allegedly cheaper, but because of a quasi-nostalgic ideal, held by patients, that dying at home was better, more peaceful and familiar. Hospice's popularity here seems tied to this ideal. Ira Byock's extremely readable Dying Well tells stories of his patients in 
Montana whose families rallied to provide such care, often heroically, when the doctor provided the anti-pain medication. "Typically, as a hospice patient nears death, the medical details become almost automatic and attention focuses on the personal nature of this final transition, what the patient and family are going through emotionally and spiritually." 30 This in a home-setting minimizes medicalization and reinforces Byock's own ideal that families care for their members and grow as human beings when giving to each other.

Of the fundamental needs of persons as they die, only the need to control physical symptoms is uniquely medical. Their more basic needs are broader than the scope of medicine ... They need companionship and need others to recognize their continued existence. ${ }^{31}$

Unfortunately, this idealization of home-care and family mutual service is not often approached, let alone met. A hospice nurse told me of a dying patient's husband who stole her pain medications and sold them on the street. In other cases, family dynamics make Byock's hopes seem ludicrous. Diane Rubin's Caring: A Daughter's Story ends with the decisive statements about hospice and her mother's death:

Hospice helped us graciously.

Hospice was a godsend.

And still we were in hell. ${ }^{32}$

Even within a culture that by that time was willing to speak openly about death and dying, and provide government support for hospice, some families faced with home-care for a dying member could not fulfill the promise that Saunders' original vision offered.

It is important to hear voices of this sort, at the risk of ending the section on hospice in the United States on a discouraging note. The exorbitant idealism that launched hospice in the early 1970s and still runs through Byock's book gives the illusion that hospice ought to be the solution to dying in America. The institution has helped. The percentage of potential "hospice eligible" patients served has increased from $27 \%$ several years ago to $48 \%$ today, although many of these enter the program less than two weeks before death. Nor should we ignore the unequal distribution of hospice users. Due to historical mistrust of the healthcare system, many African-Americans believe anything less than "Do everything" promotes neglect, and hospice's relinquishment of aggressive treatments certainly carries the appearance of doing less than what could possibly be done.

\section{Hospice in Japan}

The spread of the hospice philosophy and practical methods of treatment for the terminally ill has been remarkable. The sense of mission and advocacy that characterized Saunders also fueled the efforts of many others to bring hospice to places where healthcare and cultural expectations were different from those in London or New Haven. One place where this is evident is Japan, a high-tech country with an advanced Western-style healthcare system, and where an aging population faces a relative lack of nursing homes, in comparison to the U.S.A. Traditionally Japanese were cared for by members of their ei, the extended household, but there are far fewer relatives around 
in a heavily urbanized environment of tiny apartments. So although Japanese people still wish to die "on the tatami," in their homes, this is rarely the case. In 2014, only $8 \%$ of all cancer deaths took place at home. ${ }^{33}$ Only $9 \%$ of such patients died in hospice or palliative home-care programs. So although hospice came to Japan early, its acceptance has been slow.

The first hospice program was brought to Japan from England in 1975 and began at Seirei Mikataba Hospice. ${ }^{34}$ The first recognized program was at Yodogawa Christian Hospital, started in 1981. These facts are significant: hospice retains a sense of foreignness and is written in Katakana, the Japanese script for foreign words and phrases. A Christian hospital is not a coincidence either; although Christians constitute about $2 \%$ of the Japanese population, they have promoted an ethic of altruism for strangers that was not indigenous to Japan. So, hospice in Japan was associated with Christianity, and there were debates over whether it was even compatible with Buddhism. However, "palliative" as in "palliative medicine," is written in Kanji, the Chinese-derived ideographic writing that will make it fully Japanese. When hospice moved to becoming palliative medicine, recognized as a separate medical specialty, the association with foreignness was lessened.

Not until 2007-2008 was a large-scale evaluation done on both hospice and palliative home-care programs, and the second phase of the Japan Hope Study was done in 2010. By 2014, there was a third study, to evaluate the process, structure and outcome of these programs. "Outcomes," for the first time, included the success of bereavement services offered by the programs to family. The results of these surveys showed an elderly population where wives cared for dying husbands; $35 \%$ of the patients were older than 80 , and $32 \%$ between 70 and 79 . It is no surprise that one of the main reasons to discontinue home-care was poor health on the part of the care-giver. ${ }^{35}$ Nevertheless, a large majority who used these services found them helpful. In another survey of inpatient care, Morita et al. reported that $47 \%$ were satisfied and $41 \%$ very satisfied. ${ }^{36}$ So, given the number of dying persons in Japan, and the generally positive feedback about hospice and palliative home-care, why is the percentage of persons using these so low? And what accounts for the slow growth of hospice, even though it has been reimbursed by the government healthcare system since 1989?

The answer touches on both the Japanese medical system, as it developed in the twentieth century, and some much older Japanese values. Japan embraced Western science and medicine early, almost as soon as the country was opened up to foreigners in 1868 . But what they embraced was a paternalistic model of medicine, including a prohibition against informing patients of a terminal prognosis. In the case of cancer, this also meant that patients were rarely given their diagnosis; the word "cancer" was never spoken. "Truth-telling" remains an issue; even by the 1990s approximately $30-50 \%$ of patients had not been told they had cancer. ${ }^{37}$ To invite them to accept only palliative rather than curative care is therefore extremely unlikely. Moreover, although hospitalized dying was definitely a twentieth-century Japanese development, it was perceived as the best care, in spite of patients' own preferences to die in a home setting. Home-care was believed to shorten patients' lives. These concerns relate to cancer in particular, as that was the disease for which modern hospice care was developed. When AIDS came to Japan, the situation was far worse as to open discussion, truth-telling and public awareness.

What lies behind the issue of truth-telling about diagnosis and prognosis is something that goes back further and runs deeper than medical practice alone. First, it is simply 
bad luck to mention death, to deal with death directly, to hold conversations about death. People do not normally do this, and the medical system has responded to this pattern of avoidance. But also, there is great value put on indirect communication, ishindenshin; adults learn how to interpret gestures and demeanor so that it is not, for a mature Japanese person, considered necessary to be told anything to your face, least of all bad news. The justification for this is omoiyari, consideration for the feelings of others, and the desire to protect a vulnerable family member from harm. Together with medical paternalism, trust in authority figures, this makes the lack of "truth-telling" less like a violation of patients' right to know and more of a stance of protection and care. The flip side of indirect communication is that patients are expected to know - without being told - that they are nearing death. ${ }^{38}$

Another traditional Japanese value is "filial piety," the obligation of the younger generation to care for their elders, motivated by a sense of on, unrepayable debt to those who gave them life. These days, Glass suggests, it is better understood as sekentei, concern with one's appearance to others. ${ }^{39}$ Families want to do the best, or what they believe to be best, for their sick members, and want others to know this. To send an elderly relative to the hospital for care is viewed as good behavior, while putting them in a nursing home or accepting hospice care is not.

Although studies have been done comparing the Japanese with Western concepts of a "good death," it is not clear how distinct these patterns are. Americans and Japanese both want good relations with their families, to avoid burdening these families and, of course, to die at home. They want to be free from pain and have positive relations with the medical staff. While the stereotypes accentuate differences, the reality is that both groups would hope for a peaceful, pain-free death, surrounded by family. No one wants to "linger," to undergo prolonged dying that would create stress and a burden for the family. ${ }^{41}$ The long-lived Japanese Emperor Hirohito died a very lingering death in 1989, and Japanese people looking back on this regretted the disruption of national life it caused and hoped fervently that this would not be their fate.

\section{Hospice in Russia}

Hospice was a movement, an agenda. It was spread intentionally, by individuals and organizations that hoped to alleviate the suffering of dying persons. They promoted hospice as A Way to Die, better than what conventional hospital treatment in Western advanced hospitals offered. But as hospice was intentionally brought into new environments, it encountered medical systems, governments and patient expectations wildly different from those Saunders' work assumed. It is here that we introduce the most pervasive problem for hospice and palliative care found outside prosperous, technologically advanced countries: difficulty in obtaining cheap, effective drugs for pain relief. The search for cheap morphine is a story connected to that of hospice, since the hospice philosophy of pain control demands available, sufficient and effective control over physical pain. Rajagopal, Mazza and Lipman focused on the obstacles to this, with the subtitle of their book $A$ Global Challenge. ${ }^{42}$ The problem is not just a lack of funds or healthcare personnel untrained for palliative care. Governments do not want opiates to be given out, even to the dying. Therefore, the laws surrounding their availability to physicians in hospitals are too strict to make hospice-style pain control possible. Physicians themselves are often very suspicious of addictive drugs and so are reluctant to provide them to patients 
in severe pain. And there are Western drug companies whose policies for a long time were to sell newer and more expensive drugs while withholding older and cheaper ones that were just as effective. ${ }^{43}$ While the last problem seems to have been overcome, many of the countries included in Clark and Wright's survey would be too poor to be able to establish palliative care that was effective.

From the perspective of hospice and palliative-care advocates, these obstacles are needless, unjustified and, by now, in violation of a basic, universal human right, established by the World Health Organization: that humans have the right to be free of physical pain. Yes, that is a declared human right, affirmed by statements on palliative care as a matter of public health policy not just individual private healthcare decisions. In a way, this outlook and its promotion by WHO represents the triumph of hospice's medical face. Pain control as a human right, now that the medications make this realistic, is technically possible and ought to be a goal of every national health policy. WHO's definition of palliative care includes this wording:

Palliative care is an approach that improves the quality of life of patients and their families facing the problems associated with life-threatening illness, through the prevention and relief of suffering by means of ... treatment of pain and other problems, physical, psychosocial, and spiritual. ${ }^{44}$

Here, it is palliative care as an emergent medical specialty that is being charted, but the inclusion of types of pain in addition to physical pain shows the influence of hospice. The inclusion of "families" also expresses the values of the movement, which never wished the patient to be treated as an isolated organism. Alas, to read case histories and the survey data of Clark and Wright is to recognize how far from actual reality this vision remains. People still die in pain.

The U.S.S.R., as it became Russia plus a number of independent republics, offered one setting where miserable conditions applied. Those nations which had been under Soviet influence, such as Hungary and Romania, mostly shared them. A comprehensive survey written by David Clark and Michael Wright, published in 2003 tells the story up until that date. Their Transitions in End of Life Care: Hospice and Related Developments in Eastern Europe and Central Asia is organized country by country, with a focus on five "beacons," programs that have worked and could be models for others in the region. ${ }^{45}$

What almost all of these countries have in common is relative poverty compared to the West, and a healthcare system dominated by values of the Soviet regime. "In essence, healthcare systems were structured in relation to one over-riding function: the production, reproduction and maintenance of the labor force." 46 They were medically dominated, focused on physical problems and hierarchical rather than team-based. It was actually illegal for a doctor to tell the patient of a cancer diagnosis, ${ }^{47}$ and patients did not question medical authority. Within such a system, care for the dying and their families would be a non-priority even under the best of circumstances. At the time when Clark and Wright surveyed the programs, the countries had suffered from the disintegration of the Soviet Union economically and, in the case of the Balkan countries and some in Central Asia, from wars. There were in addition particular horrors such as the consequences of the Chernobyl nuclear disaster, which led to a large rise in cancer cases in both Ukraine and near-by Belarus. Among the many statistics collected by Clark and Wright, one marked the entire region: a drop in average life-expectancy for both men 
and women. For Russia, the male life-expectancy dropped by 7 years to 57.3. It has since rebounded to 70 years, still lower than that for all other industrialized nations.

Within a model of medicine so dismal, Victor Zorza entered the picture. After he and his wife Rosemary experienced the benefits of hospice for their daughter Jane, he became a public advocate for the movement. Because, as a refugee child escaping from the Nazis in World War II, he had found help and shelter in Russia, he remained grateful to the Russian people. His adult career as a journalist was as an expert in Soviet policy and things Russian. Therefore, he determined to bring the hospice idea to Russia, to establish a program in end-of-life care that expressed the values of hospice in Saunders' model. This was an incredibly difficult task. Zorza was a powerful, charismatic figure who had support and funding from the U.K. He worked with Russian psychiatrist Andrei Gnezdilov to do this. Zorza and Gnezdilov and others joined together to establish Lakhta Hospice in the city that was re-named St. Petersburg, which had served as a "window on the West" in its past. Their efforts were celebrated in 2003, the 300th anniversary of the city and the thirteenth year of the hospice. The reports on this, from Westerners who contributed to the event, reveal the obstacles as well as successes. Russian doctors' salaries were very low, the dedication of the staff was matched by the problems of physical maintenance and obstruction by government policies and practices. This was inpatient hospice care for those whose families could not care for them at home or who had no families. A series of essays by Western (British and American) visitors report these serious problems, while lauding the motivation and commitment of the hospice staff. $^{48}$ One giant step forward was when palliative medicine was recognized as a genuine medical specialty in Russia; this is how doctors committed to hospice gained respect from their colleagues.

While most of the print treatments of hospice and palliative care focus on the emergence of the latter as a medical discipline, there is another side to hospice also present in the Russian setting. Perhaps because of the dire lack of funds and medical resources, those who worked in the St. Petersburg hospice and elsewhere in Russia could discover, or rediscover, the dimensions of Saunders' vision that were holistic and humanistic. Psychiatrist Gnezdilov, in a speech delivered in Exeter, U.K, affirmed this. Years of service in hospice

has made me aware of the importance of spirituality in the world ... It is in the delight expressed in the unobtrusive heroism of simple people in the face of death. It has revealed the existence of an inner life, in which suffering may be transformed into a positive experience for the individual and into a treasure of the human spirit. ${ }^{49}$

Although several observers mention that there is a strong taboo against speaking of death in Russia, a voice such as Gnezdilov's seems to reach back into an older Russian understanding of suffering. Described over 100 years ago by British traveler Stephen Graham, a "Religion of Suffering" makes physical pain a positive purification for those anticipating death. It is not that physical pain is in itself a value, but it is somehow a gateway into the transcendent, a "treasure of the human spirit," to use Gnezdilov's language. ${ }^{50} \mathrm{We}$ may say that although the Soviet system, including its healthcare, valued persons only as laborers for the state, an older cultural value of a spirituality of suffering persisted, and hospice could somehow give it a place. 


\section{Hospice in Uganda}

Hospice was originally created as a complement to acute medical care in a Western hospital. But when it was brought intentionally to Uganda and from there to elsewhere in sub-Saharan Africa, its versatility became apparent. In Uganda in the 1990s, it is no exaggeration to say that it was almost the only healthcare possibility, and not an alternative, for $50 \%$ of Ugandans died without having been seen by any health worker. About $95 \%$ of cancer patients who did see some medical treatment, were too far gone in their disease to be given anything beyond palliative care, if this was available. Therefore, although it is stated by Clark, Wright, Hunt and Lynch that "Palliative care should be integrated with and not separated from the mainstream of medical practice and health and social care," especially in rural areas where there was no "mainstream" with which to integrate.

Hospice Africa Uganda was begun by yet another charismatic and dedicated figure, Dr. Anne Merriman. An ex-nun and devout Roman Catholic, Dr. Merriman had worked in Singapore and Nigeria as a medical missionary before she dedicated herself to bringing hospice care to impoverished nations in Africa. She lined up a team and sponsors, received a grant to explore which African country was best to establish as a model, and settled on Uganda. Although that country had experienced a horrific dictatorship and civil war, by the 1990s it was relatively stable. Here, as in Russia and so many other places, the availability of affordable and effective anti-pain drugs was a major problem. The big advance, one that made Merriman's and her staff's work possible, was the synthesizing of cheap oral morphine in Singapore early in the 1990s. This was sent in powdered form, mixed with water and dyed green; then it could be given to patients in their homes. It made pain control possible for patients in the advanced stages of cancer. By the 1990s, AIDS had infected a huge proportion of the adult population, and, before antiretrovirus (ARV) drugs, there was no other option for these patients except palliative care. Disease conditions such as Burkett's lymphoma, appeared in children with weakened immune systems.

Even this brief introductory description does not come close to describing the issues faced by those who work in Uganda. To say that hospice cares for patients in their homes gives the false impression that the setting resembles domestic conditions in the United States. In the newsletter and 2018 Annual Report from Rays of Hope Hospice Jinja, situated in a rural and neglected area of Uganda, we see that people's houses are sometimes the size of garden sheds. There is not enough room for a sick person to lie down inside, and so the house must be expanded or rebuilt in order to provide any protection for patients. ${ }^{52}$ Because patients are adults in the prime years for economic productivity, food and other basic support must be given to their families, along with the oral morphine for the sick persons themselves. Children, many of them AIDS orphans, can be helped with tuition money to continue to attend school. Hospice, in short, continues with its goal to provide holistic care, but in this setting holistic means something all-encompassing. These problems are in addition to those of every remote rural area: difficult transportation conditions, lack of education about services even when these are available and a population forgotten by the urban centers of the country.

The case stories that fill Merriman's book, The Audacity to Love: The Story of Hospice Africa, ${ }^{53}$ reveal a different side of global hospice than that portrayed in The Oxford Textbook of Palliative Medicine. ${ }^{54}$ There, the story of progress was the establishment of a medical specialty, a goal that made absolutely no sense for Uganda. In Merriman's work, a major aim 
was to have the Ugandan government permit nurses and non-physicians to distribute morphine within hospice auspices. The government also explicitly included hospice or palliative care as one of its health priorities, a central step advocated by WHO. Rather than duplicate the Western pattern of increased specialization in medicine, Hospice Africa Uganda and its offshoots make basic, simple and affordable pain relief the center of its work. Even after the introduction of ARVs, this remains the situation. Uganda is one of the few African nations where the rate of AIDS has declined, but the model of care depicted in a recent hospice newsletter from Hospice Jinja sounds very similar to that of Merriman's book, written in 2010.

For what emerges in this setting is the importance of three basics in offering hospice as a treatment for the terminally ill: cheap, accessible drugs, i.e. morphine; government policy that explicitly supports hospice and allows for a flexible distribution of these drugs; and education. These three are illustrated as a triangle in Rajagopal and Twycross. ${ }^{55}$ Education means training sessions for health professionals in how to effectively manage pain - i.e. how to deliver palliative care using the principles developed by Saunders at the start of hospice. But it also means communicating knowledge to the public regarding the diseases and available treatments. In a setting where so few persons received any medical care in the early phases of cancer, and where the technology to treat cancers aggressively is not realistically available, communicating what is available becomes crucial.

Merriman's work would not have been possible without international support. She seems to have had a genius for rounding up sponsors and donors. For example, the Princess Diana Foundation gave money to help AIDS children. The Irish Hospice Foundation gave financial and medical help and published Merriman's book. As in the case of Russia, a large number of medical personnel from the U.K. visited or taught in Uganda. The plight of Africans suffering from AIDS captured world attention, although as Merriman wryly pointed out, money was available for condoms but not for palliative care! Uganda now serves as a model, a "beacon" for other countries with scarce resources. But there are nevertheless still obstacles beyond those already named. A large proportion of doctors wind up in the government bureaucracy rather than actually treating patients. And, as in other impoverished settings in a global environment, many professionally trained persons leave to find more stable and secure positions in more prosperous countries. ${ }^{56}$ This "brain drain" exists internally, as enterprising and younger persons leave rural areas to work toward better lives in cities. This reality far outweighs the willingness of self-selected dedicated individuals from the West, such as Merriman, to spend their lives working in Africa. Thus, although inequality of healthcare may always have been a reality, it is starker and more extreme now than ever before, even with abundant sponsors and support from the prosperous nations.

\section{Hospice "ethos": bereavement, spirituality and religion}

We conclude with a glimpse of what a medicalized history of hospice transitioned into palliative medicine omits. These least-medicalized facets are bereavement care for families and the relief of "spiritual pain." While the former has emerged as a legitimate psychological specialty, with degrees and certificates available in American universities and the emergence of "grief counselor" as a profession, it has also been the site of battles over funding. If grieving is a disease, it is a health condition worthy of insurance 
reimbursements. If it is a normal, expectable part of living with loss, then its medical status is questionable.

Colin Murray Parkes, who joined Saunders' staff at St. Christopher's early on, has been the world leader in researching Bereavement: Studies of Grief in Adult Life ${ }^{57}$ and has continued to advise governments on how to provide services to populations struck by largescale disasters such as the 2004 tsunami in Indonesia. But Parkes and others, while expert social scientists, are not medical specialists. Their work should be an intrinsic part of hospice, for the hospice "ethos" states that "Patients and families are the center of care," and this should continue after the patient's death. ${ }^{58}$ The stress of caring for a dying member who "lingers" does not mean that the death will bring relief. Instead, many narratives of others' fatal illnesses, such as the Zorzas wrote, were done as part of the work of grieving, to re-process the meanings of events and reconstruct the dead person in memories that will now cohere, however intensely unpleasant. This entire topic of bereavement restores the close connection between hospice and death, which an emphasis on palliative care as medicine attenuates.

The case of "spiritual pain," and the roles for spiritual and/or religious care, is even further from a medical textbook emphasis. Partly, this is because of the varied permitted roles for religion in national health care, and these in turn reflect the religious and cultural environments we have discussed. However, all agree that hospice originated in the historical context of Christianity, where long before modern scientific medicine, monks and nuns cared for the sick, strangers and the dying. Yet the link between hospice and Christianity is stronger and more personal than this general historical one. Saunders was a devout Christian, whose sense of personal calling to alleviate "total pain" of dying patients shines through her letters. Moreover, an early statement in the document "Aims and Basis" for hospice shows this dimension of the program directly. It states that "Dying people must find peace and be found by God, without being subjected to special pressures," and "Such service must be group work, led by the Holy Spirit, perhaps in unexpected ways." 59 Among the unexpected ways, we may say retrospectively, was that "religion" was no longer in any way a requirement for work in hospice by the time Jane Zorza entered it.

For Saunders, hospice was a mission, a vocation, a personal calling from God. The same is certainly true in Anne Merriman's case. An ex-nun, she continues to be a practicing Roman Catholic and clearly believes hospice work to be a special calling. To work with patients none of whom will recover, and who can be expected to die quite soon, takes a special sense of compassion and what Merriman labels The Audacity to Love. This includes a profound respect for the humanity of all and a deep commitment to the value of humans beyond our contribution to the workforce and the national economy. It may be that organized religious bodies, such as religious orders of nuns, are the ones already equipped to express and live through such a vision. The hospice in St. Petersburg was private and began in what was still an officially atheist state. But, very shortly, an order of nuns, the Sisters of Mercy, began to care for "hospice orphans," those children whose mothers had died in Lakhta Hospice. This was to avoid sending the children to state-run orphanages, where the death rate was notoriously high. ${ }^{60}$

While in the U.S.A. this or any link with religion is muted, the program usually includes a chaplain on the team, and "spiritual care" is listed as one of its services. Since the term "spiritual" is more flexible and fuzzy than "religion," all sorts of arrangements can be worked out as to who provides such care. A social worker, rather than a clergy person or 
trained chaplain, may be considered adequate. Moreover, there is great concern that any spiritual care does not take the form of "undue pressure," or direct proselytizing of patients, an issue raised regularly in the American context today. In very sharp contrast, the hospice team who visit patients' homes to deliver morphine and other supplies in Uganda always pray with their patients and the families. This is apparently expected and welcomed. When the family is Muslim, the (Christian) team pray without using the name of Jesus. ${ }^{61}$ A case history reveals how spontaneously such active religious practice is mingled with care we would divide between medical, social work and community support. ${ }^{62}$ As Merriman explains, all Africans share a value, ubuntu, which is "holistic" in the most expanded sense. Ubuntu expresses the sense of real worth, the sense of community, the sense of God and the "sense of neatly combining the good in this-worldly life with thegood-in-the-life hereafter." 63 The hospice approach draws upon this pervasive vision and has developed a practical agenda for hospice which can fit the limited resources of a country such as Uganda.

But can hospice be done from within a religious tradition other than Christianity? To look at Byock's account of dying Americans in Montana, that is a non-question, because the secularity of the program, of its basic tasks, is simply taken for granted. A few of the patients are religious, but that is a private resource for them, not integrated into what the hospice program itself provides. Nevertheless, he ended Dying Well with a recommendation for meditation or other spiritual practice that will enhance one's capacity to cope with pain and loss. ${ }^{64} \mathrm{He}$ is careful to be generic here. Meanwhile American converts to Buddhism pioneered unique hospice programs that try to apply Buddhist understandings of self, death and ultimate reality to the inpatient care of the dying. In the field of death education for professionals, the Upaya Being with Dying program, run by Roshi Joan Halifax, is a way in which those already working in the field of hospice and palliative care can deepen their vision and understanding. ${ }^{65}$

To conclude, when hospice is totally assimilated into palliative medicine, its link with death is weakened. In this, it would become acceptable to my dying aunt, and she did receive "comfort care" at the very end of her life. Nevertheless, there has always been more to hospice than this, perhaps voiced best by Russian hospice pioneer Gnedzilov. What Saunders began not only provides relief from physical pain, but opens up what he found to be "a treasure of the human spirit." Perhaps that comes close to the fullness of ubuntu, connection to the world, people and life here, and the good of life hereafter. This is and should remain intrinsic to the ethos of hospice.

\section{Notes}

1 Cecily Saunders, Cecily Saunders, Founder of the Hospice Movement: Selected Letters 1959-1999. Oxford: Oxford University Press, 2003.

2 Quoted in David Clark and Michael Wright, Transitions at the End of Life Care: Hospice and Related Developments in Eastern Europe and Central Asia. Buckingham, UK: Open University Press, 2003.

3 Sandol Stoddard, The Hospice Movement: A Better Way of Caring for the Dying. Briarcliff Manor, NY: Stein and Day, 1978, 12-36.

4 Cecily Saunders and Mary Baines, Living with Dying: The Management of Terminal Disease. Oxford: Oxford University Press, 1983, 21-36.

5 Ira Byock, Dying Well: Peace and Possibilities at the End of Life. New York: Riverhead Books, 1997.

6 Clark and Wright, Transitions, ch.4.

7 Anne Merriman, The Audacity to Love: The Story of Hospice Africa. Dublin: Irish Hospice Foundation, 2010, 51ff. 
8 Lois Snow, A Death With Dignity: When the Chinese Came. New York: Random House, 1974, 104.

9 "Obituary for Lois Wheeler Snow," www.nytimes.com/2018/04/11.

10 Elisabeth Kübler-Ross, On Death and Dying. New York: Macmillan, 1968.

11 Ibid., 119.

12 Elisabeth Kübler-Ross, ed. Death: The Final Stage of Growth. Englewood Cliffs, NJ: Prentice-Hall, 1975.

13 Richard C. Cabot and Russell L. Dicks, The Art of Ministering to the Sick. New York: Macmillan, 1936, 45ff.

14 Sharon Kaufman, And a Time to Die: How American Hospitals Shape the End of Life. New York: Scribner, 2005.

15 Saunders, Letters, 145.

16 Merriman, Audacity to Love, 146.

17 Victor and Rosemary Zorza, A Way to Die. New York: Alfred Knopf, 1980, 239.

18 Saunders, Letters, 130.

19 Cathy Siebold, The Hospice Movement: Easing Death's Pains. New York: Twayne Publishers, 1992. 139ff.

20 Saunders, Letters, 18

21 Nao Okishira et al., "Japan Hospice and Palliave Care Study: Views about Legalization of Death with Dignity and Euthanasia Among the Bereaved whose Family Members Died at PCUs," American Fournal of Hospice and Palliative Medicine 26 2:98-104, 2009.

22 Clark and Wright, Transitions, 65.

23 Siebold, The Hospice Movement: Easing Death's Pains, 70.

24 Mother Frances Dominica, "The Role of the Hospice for the Dying Child," Fournal of Hospital Medicine 38 4:334 63, 1987.

25 Clark and Wright, Transitions, 94-100.

26 Lucy Bregman, "Kübler-Ross and the Revisioning of Death as Loss: Religious Appropriation and Responses," Journal of Pastoral Care and Counseling 73 1:4-8, 2019.

27 Stoddard, The Hospice Movement, 149.

28 Siebold, The Hospice Movement: Easing Death's Pains, 77.

29 Ibid., 177.

30 Byock, Dying Well, 181.

31 Ibid., 247.

32 Diane Rubin, Caring: A Daughter' Story. New York: Holt, Rinehart and Winston, 1982, 197

33 "The Japan Hospice and Palliative Care Evaluation Study 3,"American Journal of Hospice and Palliative Medicine 34 7:654-64, Aug. 2017.

34 Ibid.

35 Makoto Kobayakawa et al., "Family Caregivers Require Mental Health Specialists for End-ofLife Psychosocial Problems at Home: A Nationwide Survey in Japan," Psycho-Oncology 25, 6: 641-47, June 2016.

36 Morita et al., "Family Satisfactions with Palliative Care in Japan," Palliative Medicine 16 3:18593, 2002.

37 Shinzue Suzuki, Jane Kirshling and Inoue Iku, "Hospice Care in Japan," American Fournal of Hospice and Palliative Medicine 10 4:35-40, July 1993.

38 Ibid.

39 Anne P. Glass et al., "A Cross-Cultural Comparison of Hospice Development in Japan, South Korea and Taiwan," Journal of Cross-Cultural Gerontology 25:1-19, 2010.

40 Mitsunori et al., "Good Death Inventory: A Measure for Evaluating Good Death from the Bereaved Family Member's Perspective," Journal of Pain and Symptom Management 35 5:486-98, May 2008.

41 Susan Orpett Long, "Cultural Scripts for a Good Death in Japan and the United States: Similarities and Differences," Social Sciences and Medicine 58:913-28, 2004.

42 M.R. Rajagopal, David Mazza and Arthur Lipman, eds., Pain and Palliative Care in the Developing World and Marginalized Populations. New York: Haworth Medical Press, 2003.

43 Jan Stjernwärd, "Foreward: Instituting Palliative Care in Developing Countries - An Urgently Needed and Achievable Goal," in Rajagopal, Pain and Palliative Care in the Developing World and Marginalized Populations, xixff. 


\section{LUCY B REGMAN}

44 David Clark, "International Progress in Creating Palliative Medicine as a Specialized Discipline," in Geoffrey Hanks et al., eds., Oxford Textbook of Palliative Medicine, Fourth Edition. Oxford: Oxford University Press, 2010, 10.

45 Clark and Wright, Transitions, 174-268.

46 Ibid., 247

47 Ibid., 153.

48 Michael Siggs et al., "A Celebration of Hospice and Palliative Care in St. Petersburg, Russia," Progress in Palliative Care 12 1:24-26, 2004. Charlene Thurston, "To Russia with Hospice," Palliative and Supportive Care of Nantucket, Fall, 1999. John Ely, "Report on IAHPG Traveling Fellowship to St. Petersburg, Russia," Hospice.com/newsletter2005/Feb.05/Russia. Irene Gilliland, "Hospice in Russia," Fournal of Hospice and Palliative Nursing 2 2:73-75 April-June 2000.

49 Quoted in Michael Wright and David Clark, "Hospice Care in Russia," Progress in Palliative Care 12 1:29.

50 Stephen Graham, The Way of Martha and the Way of Mary. New York: Macmillan Co., 1915, 149.

51 David Clark et al., "Hospice and Palliative Care Development in Africa: a Multi-Method Review of Services and Experiences," Fournal of Pain and Symptom Management 33 6:698-710, June 2007.

52 "Newsletter" and "2018 Annual Report" from Rays of Hope Hospice Jinja. www.raysofhope hospicejinja.org. I am indebted to Ms. Cathy Kreutter for these sources.

53 Merriman, Audacity to Love, for example, ch. 13 "A Day in the Life of a Hospice Nurse in Kampala," by Rose Kiwanuka and Martha Rabwoni, 116-123.

54 Hanks et al., Oxford Textbook of Palliative Medicine.

55 M.R. Rajagopal and Robert Twycross, "Providing Palliative Care in Resource-poor Countries," in Hanks et al., 23-30.

56 Merriman, Audacity to Love, 182ff.

57 Colin Murray Parkes, Bereavement: Studies of Grief in Adult Life. New York: International Universities Press, 1972.

58 Merriman, Audacity to Love, 146.

59 Saunders, Letters, 68.

60 Siggs, "A Celebration of Hospice and Palliative Care in St. Petersburg, Russia,"24-26.

61 Merriman, Audacity to Love, 123.

62 Ibid., 120.

63 Ibid., 131.

64 Byock, Dying Well, 233ff.

65 Upaya Zen Center, Being with Dying Project. www.upaya.org/being-with-dying. 


\title{
"A PROFOUND SHIFT IN POLIGY" \\ The history of assisted suicide
}

\author{
Ian Dowbiggin
}

On February 8, 2019, The Guardian, the provincial newspaper of Prince Edward Island, Canada's smallest province, carried a front-page story that would have been unimaginable a decade earlier. The story described how employees lined up outside the home of Neil Harpham, who ran a local taxicab business, to give him a heartfelt send-off only hours before his medically assisted death. The 74-year-old Harpham had been suffering from cancer, which left him exhausted much of the time. As he told The Guardian reporter, "I'm just so glad I don't have to wake up to this shit anymore."

The story was noteworthy not just because medically assisted death was news in Canada. It was also hard to miss The Guardian's celebratory tone. The Guardian's coverage showed how, in the twenty-first century, media reporting of human-interest stories involving medically assisted death frequently (if unwittingly) suggested that someone such as Harpham who killed himself did the right thing.

By exercising his right to choose the time, place and manner of his death, Harpham took advantage of the 2015 unanimous decision of Canada's Supreme Court to strike down the section in the country's Criminal Code which had banned assisted suicide. What made this decision even more remarkable is that the ruling overturned the Court's 1993 decision upholding the ban.

The landmark Canadian ruling on assisted suicide was one of the latest in a series of stunning events dating back to the last years of the twentieth century. There is little evidence that between classical antiquity and the late twentieth century assisted suicide has ever been viewed as anything other than a poor moral choice. Yet, as of 2019, other countries around the world - including Germany, Belgium, the Netherlands, Switzerland as well as seven U.S. states and the District of Columbia - permitted physician-assisted suicide (PAS). Additionally, national surveys report large majorities in favor of $\mathrm{PAS}^{2}$ In the annals of modern history, the twenty-first-century moral permissiveness surrounding PAS marks a revolution with significant social, cultural, political, economic, ethical and medical dimensions.

What accounts for this turn of events? The swift rise in the acceptability of PAS is inseparable from the history of euthanasia in general. The history of euthanasia, a word taken from the Greek word for "good death," is the story about the various means of hastening death through medical or non-medical methods. As a form of medical assistance-in-dying in which the patient administers a drug prescribed by a licensed physician, PAS is considered to be a type of euthanasia alongside physician-administered lethal injection, voluntary or involuntary, or the withdrawal of medical technology keeping a patient alive, with or without consent. As we shall see, in the 1980s, proponents of 
euthanasia in general began advocating the legalization of PAS as an overdue reform in various countries. The twentieth-century increases in life expectancy rates, combined with the mounting incidence in chronic diseases such as cancer, heart disease, dementia and acquired immunodeficiency syndrome (AIDS), engendered support for laws permitting different methods of euthanasia, including PAS. Simply put, as societies have aged, people have increasingly tolerated PAS.

Yet while disease patterns and trends in mortality, aging and medical practice played a role in the liberalization of official attitudes and public opinion toward medical-aid-indying, they alone cannot account for the fortunes of PAS since the turn of the twentyfirst century. The fact that PAS enjoys considerable public support in many Western countries in the twenty-first century is linked to other historical currents, such as the post-1960s emergence of the "post-modern patient" who combines unprecedentedly high sensitivity about his or her own health with a mounting mistrust of organized medicine. ${ }^{3}$ The other factor which has paved the way for the acceptance of PAS is the growth of the so-called right-to-die movement which in the last half-century has urged courts and legislatures in different countries to legalize euthanasia practices. ${ }^{4}$

In other words, the recent status of PAS in many Western, industrialized countries represents a significant change in policy, public opinion and medical practice. As the twenty-first century unfolds, the history of assisted suicide may look promising to its proponents but it continues to be contingent on events shaped by the conflicts among groups with widely dissimilar value systems. Much of this history can be reduced to battles in the courtrooms and legislatures of various political jurisdictions. These conflicts also involve exercises in public relations as activists on either side of the debate over the moral righteousness of assisted suicide seek to win over hearts and minds without appearing to impose their values. The morality, legality and practicality of assisted suicide might appear at first glance a simple matter, but if history teaches anything about assisted suicide it is that it is anything but straightforward. ${ }^{5}$

\section{Suicide and assisted suicide in history}

The historical background to assisted suicide is both similar and dissimilar to the history of suicide itself. Opinion polls did not exist before the twentieth century, so it is difficult to know exactly how much public support there was in favor of assisted suicide. Yet, over the last few centuries, mounting permissiveness about suicide has not coincided with a similar acceptance of assisted suicide. If anything, as punishments for and condemnation of suicide abated in the modern era, disapproval of assisted suicide not only remained robust but actually strengthened until the last decades of the twentieth century.

What is known is that all the major global faith traditions strongly condemned the taking of life through suicide or mercy killing as methods to escape suffering. In medieval Christendom, suicide was treated as a grave sin and governments decreed punishments for committing the act. Canon and civil law upheld the medieval consensus that selfmurder was a terrible crime.

Exceptions to suicide existed, of course, as in the early centuries when some Christians seemingly welcomed martyrdom, in the practice of sati, in which a Hindu widow climbed onto her deceased husband's funeral pyre, or with modern-day Islamist suicide bombers. However, Hinduism heavily censures self-murder, as does Sikhism. Buddhism also forbids suicide because it violates the first of five fundamental precepts: "kill not any living thing." 
This includes voluntary death as an acceptable means of escaping sufferings that are due to former deeds. In Confucianism, persons who commit suicide rob their ancestors of veneration and service due to them and demonstrate ingratitude to parents for the gift of life.

As for Judaism, the Central Conference of American Rabbis declared in 1972:

The Jewish idea of the sanctity of human life and the supreme value of the individual soul would suffer incalculable harm if, contrary to the moral law, men were at liberty to determine the conditions under which they might put an end to their own lives and the lives of other men.

Judaism teaches that choosing death is an affront to God that demonstrates contempt for the gift of life. Even Reform Judaism, the most permissive of all branches of Judaism, believes assisting suicide is incompatible with its teachings. ${ }^{6}$

Islam, arriving in the seventh century A.D., did not challenge Judaic teaching about suicide. Those who kill themselves, the Koran teaches, are denied paradise and sentenced to hell. In Islam, as in Christianity, suicide was both a sin and crime for centuries until the modern era.

Despite these strict religious condemnations of suicide, suicide has been a topic of interest for numerous scholars, poets, playwrights and novelists down to the twentieth century. The word "suicide" itself was coined in 1643 by English physician Thomas Browne (1605-1682). Theologians denounced suicide, but readers and theatre-goers were fascinated with its powerful dramatic overtones. Voltaire, Montesquieu and David Hume were just a few of the eighteenth-century thinkers who wrote about suicide shorn of its religious overtones. Goethe's The Sorrows of Young Werther (1774), whose central character committed suicide over unrequited love, was a best-seller in its day.

Yet, if some law-makers and opinion-makers - notably in the eighteenth century - tried to normalize suicide, their reasons for doing so were rarely consistent. ${ }^{7}$ Some Enlightenment philosophes hailed suicide as an example of rational individual choice, while others defined it as an involuntary act due to a distortion of the natural laws governing body and mind. This latter theory - the "medicalization" thesis - was expressed by an Englishman in 1793: "the commission, therefore, of suicide, must, necessarily, be always an act of insanity."8

Studies of suicide records from previous centuries show that sickness or disability was an important motivation behind suicide. One historian has even proposed that suicide be viewed as an "illness strategy," a form of "self-euthanasia" to end suffering. This was particularly the case with syphilitics who, because they suffered from a painful, disfiguring and socially shameful disease for which there were only futile and debilitating treatments, were frequently driven to despair. That people committed suicide in the past due to their battles with sickness is hardly surprising, but there was no contemporary evidence that assisting suicidal individuals to kill themselves was believed to be anything other than a serious moral mistake. ${ }^{9}$

Similarly, in the 1500-1800 period in Europe, popular viewpoints endorsed the idea of hastening the deaths of people, say, mortally wounded in battle or hanging from the gallows. Yet such traditions only affected people who were dying and writhing in agony, not persons who wished to kill themselves due to illness. And they were linked to widespread beliefs that helping others die faster released souls that were sometimes reluctant to leave the body. ${ }^{10}$ The notion of assisted suicide, as defined in the twenty-first century, was alien to common attitudes prior to the nineteenth century. 


\section{IAN DOWBIGGIN}

Thus, by the nineteenth century, few thinkers wanted to restore the medieval punishments for killing oneself, and many felt that suicides were more to be pitied than vilified. In 1790, revolutionary France became the first country to decriminalize suicide, and the rest of the European continent followed suit in later years. At the same time, commonlaw punishments against suicide were mitigated or repealed outright. Nonetheless, educated opinion remained unsympathetic to justifications for taking one's own life to escape the physical or emotional pain of disability, disease or impending death. As attitudes toward suicide became increasingly tolerant in some Western countries, there was no corresponding toleration for assisting suicide.

\section{The nineteenth century}

Indeed, as the decriminalization of suicide gathered momentum, opposition to assisted suicide from courts and governments became more pronounced, notably in the United States, where the earliest statute to outlaw assisted suicide was New York State's in 1828. In the words of Larson and Amundsen:

By 1900 most American states had statutes or clear juridical precedent establishing assisted suicide as a crime. During that era no court apparently even considered a claim that such a statute might violate the federal constitution, much less hold that it did so. ${ }^{11}$

Legal and legislative developments matched the sentiments of physicians about end-of-life care. Prior to the last years of the nineteenth century, the term "euthanasia" usually referred to "a calm and easy death," during which physicians would do their best to make dying patients as comfortable as possible by ensuring that the bedroom was well ventilated and not too bright, noisy or hot to upset the patient. ${ }^{12}$ Should a physician conspire with patients to help them die, a German physician argued in 1806, "the consequences would be unforeseeable and the doctor could well become the most dangerous person in the state." ${ }^{\prime 3}$ In 1847, the American Medical Association stated that the physician

should be the minister of hope and comfort to the sick, that by such cordials to the drooping spirit, he may soothe the bed of death ... and counteract the depressing influence of those maladies which often disturb the tranquility of the most resigned in their last moments.

A physician's moral support and concern for a patient's well-being, as well as a judicious use of opium-laced remedies, were the keys to "an easy, calm and collected death."14 Narcotics should be used to ease pain, not hasten death. In 1904, the Fournal of the American Medical Association editorialized: "We may excuse the person who commits suicide to avoid inevitable torture or dishonor, but we can not justify in any way the physician who deliberately cuts short his patient's existence under any conditions whatever." ${ }^{15}$

In 1896, a California judge voiced the American consensus on the topic when he wrote: "I think it would be a crime for anyone to advise or encourage another to commit suicide." ${ }^{16}$ In 1920, the Michigan Supreme Court upheld an earlier ruling sentencing a Michigan man to life imprisonment for preparing a poisonous beverage for his dying 
wife. The court ruled: "the real criminal act charged here is not suicide, but the administering of poison."17

Against this historical background, a handful of writers, such as the colorful U.S. freethinker Robert Ingersoll (1833-1899), defended people killing themselves because they were wracked by incessant pain. In 1891, the German-born Felix Adler (1851-1933), founder of the Ethical Culture movement, wrote that if someone was hopelessly ill and in great pain, attending physicians might be free to administer "a cup of relief." But Adler never made it clear whether the doctor or the patient was supposed to administer the poison. ${ }^{18}$ Support for PAS remained a decidedly minority position well into the twentieth century.

\section{The black stork}

Besides moral and medical objections to assisted suicide, another major reason for PAS's marginal status in the early twentieth century was that the few justifications for legal euthanasia were couched in social Darwinist terms. PAS implies a measure of patient autonomy and the notion that an individual's wishes should be paramount when considering the advisability of assisted suicide. Yet, as Darwinist theories in evolutionary biology were increasingly applied to social policy in the early 1900s, the supposed needs of national health and welfare eclipsed those of individuals. Even pro-euthanasia opinionmakers were uninterested in assisted suicide as policy or practice.

The trend toward applying evolutionary biology to social policy mainly began in 1859, when British biologist Charles Darwin (1809-1882) published The Origin of Species in which he argued that throughout natural history non-human species were modified because the fittest individuals survived the struggle for existence over limited food resources. In his 1871 The Descent of Man, Darwin explicitly extended his analysis of species change throughout natural history to the evolution of human beings themselves. Darwin worried about the effects of civilized society and its charitable institutions - hospitals, asylums and organized medicine - on the process of natural selection. The "unfit" - the weak and improvident - were protected from the laws of natural selection, he wrote, and were thus free to reproduce their own kind. Meanwhile, the so-called "fit" classes of sober, thrifty, lawabiding and tax-paying men and women tended to have small families. ${ }^{19}$

Darwin's influence spread far and wide in the late 1800s and into the 1900s. To selfproclaimed Darwinists such as the German biologist Ernst Haeckel (1834-1919), a widely read popularizer of Darwin, "the theory of evolution finally makes clear that the eternal, iron laws of nature are also valid in the organic and moral world." ${ }^{20}$ Robert Ingersoll called the 1800s "Darwin's century.",21

Darwin's ideas dove-tailed with those of his cousin, Francis Galton (1822-1911). Beginning in the 1860s, Galton, a brilliant explorer, anthropologist and mathematician, advocated raising the birth rates of society's best and brightest. In 1883, he coined the term "eugenics" to refer to "positive" methods aimed at persuading the fit classes to have large families. "Negative" eugenics, by contrast, consisted of policies such as involuntary sterilization that would prevent the "unfit" classes from breeding.

In the name of eugenics, early twentieth-century governments around the world ordered the forcible sterilization of people with a range of mental and physical disabilities, including schizophrenia, epilepsy, deafness and alcoholism. The most notorious of eugenic sterilization laws was Nazi Germany's 1933 statute; by 1939, roughly 400,000 German men and 
women had been sterilized. Meanwhile, eugenic organizations sprang up in Europe, Canada, the United States, Latin America and Japan. Colleges, universities and public schools taught the principles of eugenics. In the first half of the twentieth century, eugenics caught on like few other ideas in modern history. ${ }^{22}$

The cumulative impact of eugenics and social Darwinism on death and dying was evident during the 1915 controversy over Chicago surgeon Harry Haiselden (1870-1919). Haiselden advised a couple whose son had been born with severe disabilities that their baby should be denied life-saving surgery. The news of the baby's death soon thereafter was splashed across the headlines of the nation's newspapers and magazines. When asked by a reporter for the reason behind his decision not to operate, Haiselden said: "Eugenics? Of course it's eugenics." 23 Lawyer Clarence Darrow was asked his opinion of Haiselden's actions. He answered: "Chloroform unfit children. Show them the same mercy that is shown beasts no longer fit to live." 24

Haiselden's eugenic-inspired attitudes reflected changing attitudes toward life, death and dying. ${ }^{25}$ A new definition of euthanasia, heavily influenced by eugenic and social Darwinist considerations, emerged in the late nineteenth century. Some opinion-makers began voicing approval of euthanasia in terms of mercy-killing by physicians. Attention focused on the doctor's freedom to hasten the death of people who from a social or biological perspective had out-lived their usefulness. The emphasis was on removing any legal punishments for physicians who killed their patients, rather than making it easier for patients to request medically assisted death. As late as 1952, a Connecticut euthanasia activist accused her colleagues of "neglect[ing] to emphasize the desire for release on the part of the sufferer" in favor of the legal right of physicians to put people with disabilities out of their misery. ${ }^{26}$ Thus, by the early twentieth century, "most people [in the United States] no longer argued about producing euthanasia but about committing it," in the words of historian Peter Filene. ${ }^{27}$

\section{The Euthanasia movement}

By the 1930s, support for physician-administered euthanasia enjoyed only sporadic backing and was still a minority viewpoint restricted to certain elite, educated circles. Nonetheless, small groups of like-minded reformers founded organizations in Great Britain and the United States that sought to spearhead a movement in favor of enacting laws to permit physician mercy-killing in the two countries. The concept of assisted suicide was alien to the thinking of these early euthanasia reformers.

In 1935, the Voluntary Euthanasia Legalisation Society (VELS) was founded in Great Britain. In the United States, the Euthanasia Society of America (ESA) was launched in 1938. Both the VELS and ESA were dedicated to persuading law-makers to enact statutes permitting mercy-killing by physicians with the consent of the patient. The main aim was to free physicians from prosecution for ending the lives of their patients. However, attempts to do so in Britain's Parliament and at the state level in America over the next half century ended in failure. Over the same time period, U.S. court cases involving mercy-killing often resulted in acquittals, but to the chagrin of the ESA, judges repeatedly rejected any legal justifications for "one person taking the life of another to save that other from the suffering, actual or anticipated, of any disease or malady." 28 If courts selectively refused to enforce laws against mercy-killing or assisted suicide, it "reflected a pragmatic approach to the law, not a repudiation of it."29 
Neither the VELS nor ESA was a grass-roots organization. Both groups were successful in recruiting some prominent physicians, scientists, educators, journalists and clergymen into their ranks, but their memberships remained small. ${ }^{30}$ The most common denominator was membership in eugenic and birth-control organizations. ${ }^{31}$ The eugenic background of VELS and ESA members meant many hoped for the eventual enactment of involuntary euthanasia laws, notably targeting children with disabilities and adults suffering from mental or emotional diseases. But leading figures in the two groups strategized that the best way to win such laws was to first convince legislators to pass euthanasia statutes based on patient consent. Once the principle of voluntary euthanasia for incurable and painful diseases was established, a VELS member reasoned in 1935, "it would be extended to other cases." 32

VELS founder C. Killick Millard believed that World War I had had "a loosening and disintegrating effect upon ... the orthodox "morality" surrounding euthanasia. ${ }^{33}$ But whatever impetus World War I had given to the euthanasia movement, World War II had the opposite effect. In the fall of 1939, Nazi dictator Adolf Hitler signed a secret authorization protecting Germany's doctors and nurses from prosecution for murdering people with mental and physical disabilities, notably chronically ill, hospitalized patients and institutionalized infants and toddlers. By the end of World War II, at the hands of the Germans, roughly 200,000 men, women and children had been killed by starvation, poison, gassing or shooting. ${ }^{34}$

The full news about Nazi medical murder surfaced in 1946-1947 during the so-called "doctors' trial" that was part of the Nuremberg trials of German war criminals. Twentythree of the defendants in the doctors' trial (all but three of them physicians) were charged with murdering sick or injured persons or conducting illicit human experiments. ${ }^{35}$

In the postwar era, the ESA and VELS found it difficult to escape comparisons between their agendas and Nazism. ${ }^{36}$ The stigma of Nazism attached to the word "euthanasia" lingered into the 1960s. By that point, the euthanasia movement was moribund. The prospects of law-makers passing mercy-killing legislation any time soon were dim. The opposition of the Roman Catholic Church to euthanasia proved crucial in U.S. political jurisdictions where Catholic voters tended to follow the teachings of their church, such as New York State, New Jersey and Connecticut where ESA-sponsored petitions failed in the 1950s. Even where Roman Catholics were comparatively few in number, such as England, euthanasia bills proposed by the VELS were soundly defeated. Medical opposition was just as strong. In 1950, the World Medical Association, the British Medical Association and the American Medical Association issued clear-cut condemnations of legalized euthanasia.

Yet, just when the euthanasia movement's fortunes appeared to be at a low ebb, fastmoving events and trends were occurring in the 1960s and beyond that would hearten euthanasia activists and help pave the way for the acceptance of assisted suicide.

\section{A new way of dying}

By mid-century, unprecedented demographic factors were starting to affect end-of-life health care. Prior to World War I, most people in industrialized countries could expect to die either from accidents or infectious or contagious diseases such as tuberculosis, influenza, pneumonia or cholera, which typically devastated urban populations. Yet, as the twentieth century unfolded, improvements in public sanitation, personal hygiene, housing, overall diet, occupational safety and access to medical care meant that people 


\section{IAN DOWBIGGIN}

tended to live longer lives than earlier generations. Chronic illnesses such as cancer, diabetes, heart disease and stroke increasingly accounted for most mortality. As age expectancy rose and mortality rates declined, most industrialized countries witnessed "the graying of society." By the mid-1900s, more and more people could expect to die late in life in hospitals from incurable and debilitating conditions that left patients at the mercy of intrusive and paternalistic medical attention.

Initially, modern medicine's unprecedented power to prolong life was viewed as a success story, bolstering the reputation of organized medicine. ${ }^{37}$ The mid-century consensus - notably in the United States - was that physicians, researchers, hospitals and medical schools should run their own affairs as they saw fit; and the more medicine society received the healthier it was. ${ }^{38}$ Clinicians tended to take an aggressive, "can do" attitude toward treatment, especially when it came to surgery. ${ }^{39}$

This high estimation of medical science and the medical profession began to decline in the 1960s, fed by growing qualms about modern medicine's capacity to prolong the lives of dying patients. In 1957, an American woman described her husband's death at the hands of hospital staff as "a new way of dying ... the slow passage via modern medicine" featuring surgeries, oxygenation, intubations and multiple medications. Though vowing to "put a stop" to his "torture," the wife was powerless to end the many staff attempts to extend her husband's life. ${ }^{40}$ The invention and use of respirators, intravenous feeding, organ transplantation and kidney-dialysis machines led euthanasia activists to write that: "[d]ying is rendered obscene by technology." 41

Two important events testified to the mounting challenges of end-of-life care. The first was the founding of palliative care in the 1950s, thanks to the efforts of Cicely Saunders (1928-2005), a U.K.-born physician and former nurse. In 1967, Saunders founded the first modern hospice in London. She argued that the distress and suffering of the vast majority of dying patients could be relieved through proper pain management and better overall end-oflife care. Saunders's efforts paved the way for "palliative care," the term coined in 1974 by Canadian oncologist Balfour Mount, who also pioneered the principles and practices used to address the spiritual and physical problems of dying patients. ${ }^{42}$ Saunders, Mount and their allies viewed palliative care as a bulwark against the legalization of mercy-killing, but their criticism of the ways that medical personnel typically dealt with dying patients often reinforced the growing backlash against modern medicine's efforts to prolong life.

Then, in 1969, Luis Kutner (1908-1993), who co-founded Amnesty International in 1961, introduced the Living Will, a legal document specifying in advance what kind of treatment dying patients wanted. The living will was an idea whose time had come. By 1978, three million living wills had been distributed. By the end of the century, most U.S. states had enacted legislation to make living wills legally binding. Medical ethicists have often disagreed about the effectiveness and implications of the living will, but in the short term it bolstered the notion of patient freedom to choose end-of-life options. ${ }^{43}$

\section{The Quinlan effect}

The "obscenity" of technology preventing a person from dying became international news due to the plight of Karen Ann Quinlan who, one night in 1975, fell into a coma in a New Jersey roadside bar. Three months later, she was still in a coma when her parents tried to have her disconnected from the respirator that had been keeping her alive. But the New Jersey hospital where she was staying opposed their wishes. The ensuing legal wrangle 
between the Quinlans and the hospital mesmerized millions around the world, a sign of the growing power of the media to sensationalize stories like Karen Ann's.

On March 31, 1976, the New Jersey Supreme Court ruled in the Quinlans' favor but Karen Ann lived for another nine years without re-gaining consciousness. Her misfortune was not about assisted suicide, but it reinforced the trends in public opinion which focused on individuals' freedom from a paternalistic medical profession that seemingly blocked patients' wishes. "In re Quinlan was the Brown v. Board of Education of the right-to-die movement," according to historian Peter Filene. The New Jersey Supreme Court's ruling that Karen Ann enjoyed a right to privacy against intrusion by doctors and the state implied that she possessed a personal "right to die." The notion that individuals could determine the time, place and method of their deaths with the assistance of a physician who prescribed lethal medicine seemed less objectionable after Karen Ann Quinlan's accident. ${ }^{44}$

Even before Karen Ann Quinlan became a household name, signs of support for PAS were cropping up in the 1970s. Prior to that decade, euthanasia activists had urged the legalization of active euthanasia as an argument against suicide. ${ }^{45}$ In 1971, the Mayo Clinic physician and popular medical advice columnist Walter Alvarez wrote that a 60-year-old man dying from esophageal cancer deserved "a legal right to commit suicide," and that doctors like Alvarez should have "a legal right to get him the drug for which he had begged." But Alvarez's desire to change the law to permit PAS was not based on any appeal to patient autonomy or individual choice; he insisted: "this problem of voluntary or obviously logical euthanasia can and should be left in the hands of us physicians." Voicing his utilitarian motives, Alvarez stated that law reform governing death and dying "might save the country a few billion dollars a year." ${ }^{46}$ It took the media coverage of Karen Ann Quinlan to shift rationales for PAS from social and professional considerations to personal autonomy.

In his assertion of physicians' professional autonomy and the putative cost savings to society to justify PAS, Alvarez was a voice from the movement's past. In the 1970s, the pendulum was swinging in favor of patients' rights. In 1972, the American Hospital Association approved a Patients' Bill of Rights, including the right to informed consent and to considerate and respectful care. The same year, the American Medical Association decreed that patients needed to agree with treatment options offered by their physicians and recommended that patient wishes "be respected, insofar as possible." ${ }^{47}$ In the words of historian David Rothman, in the 1970s, "outsiders, not doctors, defined the moral codes that were to guide physician behavior." 48

These unprecedented steps, alongside events like the legal wrangle over Karen Ann Quinlan, reflected the shifting terms of doctor-patient relations. In the 1970s, it was evident that a "post-modern" patient was emerging. Post-modern patients typically were distrustful of organized medicine, refused to accept what doctors said as the gospel truth and instead educated themselves about health issues. Adopting a consumerist ethos, typical post-modern patients insisted that they were active participants in the decisions that governed health care. If patients decided life was no longer worth living, the role of physicians was not to prevent this from happening but to validate patient choice. $^{49}$

\section{Hemlock and Holland}

No one played a bigger role in paving the way for the acceptance of assisted suicide than British journalist Derek Humphry (b. 1930). In 1978, Humphry leapt to notoriety when he described how he had helped his terminally ill first wife, Jean, commit suicide. In 
1980, he and his second wife, Ann Wickett, started the Hemlock Society in his Santa Monica, California, garage. Hemlock quickly grew into what the ESA and VELS decidedly were not: a grass-roots, West Coast organization with tens of thousands of members. Ninety-nine per cent of its members were white (65 per cent were women) and tended to come from families with above-average incomes. The vast majority were welleducated and reported that they were in good health. ${ }^{50}$

In the 1980s, Humphry became a best-selling author and media star preaching the need for governments to legalize PAS. Initially - and controversially-Humphry published information on non-violent suicide methods which could not be botched and would not upset relatives. Hemlock's newsletter, the Hemlock Quarterly, called Humphry's advice a "nuts and bolts" approach to assisted suicide. Humphry's books surged to the top of the best-seller lists. ${ }^{51}$

Humphry and Hemlock spawned several imitators around the world who published information about how to commit suicide, including the Scotland-based group EXIT and Australia's Philip Nitschke. Another sign that pro-assisted suicide sentiment was growing internationally occurred in 1976 when delegates representing organizations from five countries-Australia, Great Britain, Japan, the Netherlands and the United Statesmet in Tokyo. Two years later, ten delegations from eight countries met in San Francisco. In 1980, twenty-seven organizations from eighteen nations met in England to form the World Federation of Right-to-Die Societies (WFRTDS). There was no mistaking the pro-PAS attitudes of the early WFRTDS: some delegates had already helped others to die, including a Swedish journalist and the UK's Nicholas Reed, who was charged with assisting eight suicides. At the WFRTDS 1984 meeting in Nice, France, various speakers joined Hemlock in openly endorsing assisted suicide.

Back in the United States, pro-PAS organizers were busy gathering the necessary signatures to get PAS initiatives on state ballots in Washington State (1991) and California (1992). Also, in 1991, in the New England Fournal of Medicine, Timothy Quill, a Rochester, New York, physician told the story of how he had prescribed barbiturates to a leukemia patient - named "Diane" - so she could commit suicide. ${ }^{52}$ A grand jury refused to indict him, and a state medical board ruled that "no charge of misconduct was warranted." Quill's public backing of PAS, though highly controversial, won the cause many converts.

Then, in 1994, 51 per cent of Oregon voters approved a pro-PAS ballot measure making Oregon the first American state in history to legalize PAS. Oregon's vote on PAS, according to one euthanasia advocate, looked to be a "turning-point for the death with dignity movement." 53 Oregon's PAS law was challenged in the courts, so state voters went back to the polls in 1997, and this time close to 60 per cent cast their ballots for assisted suicide.

The ascendency of Hemlock and the WFRTDS, as well as events in Oregon, were matched by developments in the Netherlands. In the wake of the 1960s countercultural revolution, during which the country exhibited increasing toleration of abortion, pornography, prostitution and illicit drug use, a Dutch court in 1973 effectively struck down the nation's statute banning mercy-killing. That same year, the Dutch Society for Voluntary Euthanasia was formed. For the rest of the century, Dutch courts refused to punish any doctor who had helped a patient die or who had administered a lethal injection. Meanwhile, euthanasia deaths at the hands of physicians numbered in the thousands, many of which went unreported. Then, in 2002, the Netherlands became the first country to legalize both PAS and doctor-administered death. Belgium (2002) and Luxembourg (2008) followed suit. 


\section{A screeching halt}

Mounting international awareness about PAS in the 1980s took on additional significance as, at the same time, AIDS swiftly became the leading killer of men in North America and Europe between the ages of 25 and 44, notably homosexual men. As researchers scrambled to find a cure, AIDS typically killed its victims slowly and in great pain, often with disfiguring skin lesions. It was difficult to imagine anything more undignified than a death from AIDS. ${ }^{54}$

Studies showed that there was a kind of PAS "underground" in urban gay communities throughout the developed world, notably in San Francisco, Toronto and Sydney, Australia. Physicians who treated AIDS sufferers performed PAS more than any other medical specialty. Media accounts multiplied of gay men who formed support groups to help each other commit suicide. When Hemlock's membership doubled between 1988 and 1990, it was partly due to an influx of AIDS patients, presumably attracted to the group's willingness to spread information about suicide methods. ${ }^{55}$

The coming of AIDS helped to establish a network of doctors who had few qualms about prescribing narcotics to AIDS patients and writing death certificates that covered up their actions. One notable example was Canadian Maurice Genereux, who, in 1998, became the first physician in North America to be convicted of assisted suicide. Genereux's sentence of two years in jail less a day was upheld on appeal a year later. ${ }^{56}$ By the time he entered a plea in a Toronto courtroom, Genereux's face had been splashed across the pages of numerous leading newspapers.

The AIDS epidemic set the stage for a turbulent decade in the history of PAS. Initially, it appeared that the momentum from the 1980s would lead to major changes to laws governing assisted suicide. However, the momentum behind the campaign in the United Kingdom, Canada and the United States to legalize assisted suicide came to "a screeching halt" in the 1990s, in the words of one U.S. legal expert. ${ }^{57}$ In 1991, Derek Humphry's second wife Ann Wickett, stricken with cancer, left a suicide note which blamed him for her suicide, a gesture that PAS opponents did their best to publicize. ${ }^{58}$ By 1997, Hemlock's membership had halved to $22,000 .^{59}$

PAS advocacy continued to meet stiff legal and legislative resistance in the 1990s. England's 1961 Suicide Act had decriminalized taking one's own life, but also applied a sentence of up to fourteen years for someone who assisted the suicide of another person. ${ }^{60}$ In 1994, the U.K. House of Lords Select Committee on Medical Ethics refused to recommend a change in the law banning assisted suicide. ${ }^{61}$ That same year, the New York State Task Force on Life and the Law came to roughly the same conclusion.

In 1972, Canada had decriminalized attempted suicide, but its Criminal Code ban on assisted suicide remained. In 1991, Sue Rodriguez, a British Columbia woman, was diagnosed with amyotrophic lateral sclerosis (Lou Gehrig's disease) - and given between two and five years to live. A lawsuit on her behalf challenged Canada's Criminal Code ban on assisted suicide. Two years later, however, Canada's Supreme Court ruled that the country's law against assisted suicide did not violate her constitutional rights. Rodriguez found a mystery doctor who helped her die in February, 1994, at the age of 44, but in 1995, Canada's Senate Committee on Euthanasia and Assisted Suicide voted not to change the status quo on assisted dying.

Even where assisted suicide was not prohibited, as in Scotland and Sweden, prior to the 1980s, anyone carrying out such an action would have probably been arrested for murder 
or manslaughter. Additionally, in the last thirty years of the twentieth century, eight American states passed new statutes outlawing assisted suicide, and eleven other states revised their own statutes. ${ }^{62}$ By 1999, thirty-seven states had laws explicitly criminalizing assisting a suicide, and seven states and the District of Columbia criminalized assisted suicide through the common law. U.S. courts, even when recognizing the right of patients to refuse medical treatment, "explicitly distinguished that right from a right to commit suicide, and ... consistently affirmed the state's interest in preventing individuals from taking their own lives. ${ }^{\prime 63}$ In 1997, the U.S. Supreme Court ruled unanimously against a constitutional right to PAS, noting that "[i]n almost every State - indeed, in almost every western democracy - it is a crime to assist a suicide" and that bans on assisted suicide were "deeply rooted" in American history. The U.S. Supreme Court ruled that there was no right to assistance in suicide protected by the Due Process clause of the American constitution (although individual states were free to enact their own PAS legislation). ${ }^{64}$

In the 1990s, PAS as a moral cause also became popularly associated with gay rights advocacy in the United States. In 1997, one California physician noted that "the gay community has pushed the envelope as far as it can possibly go on assisted suicide." Some PAS proponents openly expressed their worries that the issue was being increasingly identified with an underground practice of politicized gay men whose lifestyles had made them sick in the first place. "The problem with the AIDS folks," a U.S. PAS activist commented, "is that they bring this whole other issue about how they came to this point physically ... All I'm saying is that the opponents of helping people die seize on that." ${ }^{, 65}$ In the public eye, young gay men with AIDS hoarding and then ingesting lethal drugs to kill themselves proved to be less sympathetic than grief-stricken individuals such as Karen Ann Quinlan's parents.

However, nothing discredited the PAS cause like the actions of Jack Kevorkian (1928-2011), born in Pontiac, Michigan. Nicknamed "Doctor Death" by the media, between 1990 and 1997, Kevorkian brazenly defied Michigan's law banning assisted suicide by helping more than forty-five people die. Between 1994 and 1997, Kevorkian was tried four times for assisting suicide. Three times he was acquitted and a fourth resulted in a mistrial. Most of Kevorkian's patients were women and were not in the terminal stages of their illnesses. As autopsies showed, some were not even physically sick.

Then, on November 22, 1998, twenty-two million viewers of the CBS television show 60 Minutes tuned in to watch Kevorkian on videotape inject lethal chemicals into 52-yearold Thomas Youk, diagnosed with Lou Gehrig's Disease. In 1999, a Michigan jury found the unrepentant Kevorkian guilty of second-degree murder, and he was sentenced to ten years in prison. Kevorkian's in-your-face campaign for PAS was disastrous from a public relations perspective: by the late 1990s, when Americans thought about the issue, they saw his gaunt features and harrowing eyes. Ballot-initiative defeats in Michigan (1998) and Maine (2000) were confirmation that the fall-out from Kevorkian's antics to legalize PAS in the United States was significant.

\section{The Schiavo effect}

Thus, by the end of the 1990s, events may have been moving fast in the Netherlands, but what had looked like a promising start for the American PAS movement in Oregon had seemingly ground to a halt. In 2000, U.S. voters elected as president the openly prolife George W. Bush. ${ }^{66}$ In the first decade of the 2000s, polls showed that a majority of 
Americans found PAS to be morally unacceptable, a reversal of trends in the late 1900s. ${ }^{67}$ Derek Humphry claimed in 2006 that "the right-to-die movement in America is just paddling in the water ... It's not getting anywhere." 68

Yet, Humphry would soon be proven wrong, thanks to two events which involved Bush's presidency. Bush's pro-life politics became particularly polarizing when the White House and Oregon faced off over the latter's PAS law. In 2006, the U.S. Supreme Court ruled that the White House had over-stepped its authority in trying to use governmental power to control the prescription of narcotics in Oregon. The spectacle of Washington overturning state laws and intervening in end-of-life decisions which most Americans believed should be left to patients, their families and their doctors not only offended many Oregonians, it likely affected the preferences of voters in neighboring Washington State who, in 2008, approved a ballot initiative virtually identical to Oregon's PAS law by a 58-42 margin, making Washington the second American state to legalize PAS. ${ }^{69}$

A series of events having nothing to do with assisted suicide also influenced the results of Washington's Ballot Initiative. In 1990, Terri Schiavo, then 27-years old, had a cardiac arrest at her home in St. Petersburg, Florida, and until her death survived in a persistent vegetative state in nursing homes with constant care. In 1998, her husband petitioned the court to have her feeding tube removed, but Terri's parents objected, and after the courts ruled against them, her parents went to the Florida state legislature, which passed a law giving Florida's governor (and the U.S. president's pro-life brother) Jeb Bush the authority to reinstate the feeding tube. However, the Florida Supreme Court ruled the legislation unconstitutional, and, when the U.S. Supreme Court refused to hear an appeal, the case went back to the state legislature, whose Senate declined to approve a bill restoring the feeding tube. Then, in what Time magazine called "a political circus," the U.S. Congress for the first time in its history convened a special emergency session during the 2005 Easter recess to pass a bill giving Terri Schiavo's parents a final avenue of appeal. President Bush flew back from vacation to Washington, D.C., to sign the bill. ${ }^{70}$ Nonetheless, the courts ultimately rejected legal attempts to reinstate Terri's feeding tube and she died on March 31, 2005.

U.S. press reaction to the Schiavo family tragedy was consistently critical of the Bush White House and likely helped to rejuvenate the PAS movement in the United States and elsewhere. Polls which had shown in 2003 that a majority of Americans morally disapproved of PAS reported that ten years later roughly 55 per cent approved. The spectacle of heavyhanded federal government intrusion in a family matter alongside images of radical pro-life protestors such as Randall Terry gave new life to the movement. Terry emerged as a spokesman for Terri Schiavo's parents and loudly proclaimed his views that women should

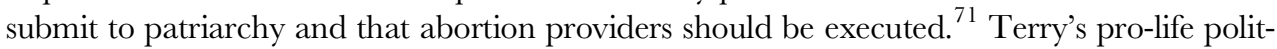
ics could not have been more different from Jack Kevorkian's views, but each in his own courting of the media and rigid self-righteousness was a public relations fiasco for his side.

In 2009, the Montana Supreme Court side-stepped the question of whether or not PAS was a right guaranteed under the state constitution but ruled that state law protected physicians who helped their patients die. The pendulum, at least in the United States, seemed to be swinging again, this time in favor of assisted suicide.

\section{Conclusion}

As the saying goes, legalized assisted suicide has gone from being unthinkable to debatable to justifiable and (in some localities today) to unexceptional. To use another 
metaphor, the yardsticks marking acceptance of assisted suicide have moved a long way in a short time period. In retrospect, this recent development was due to several historical reasons, including the aging of society and the rising incidence of chronic, "lifestyle" diseases. These conditions had not changed dramatically by the third decade of the twenty-first century. Some defenders of assisted suicide have predicted that the future will feature "death on demand" - including an end-of-life "poison pill" available to anyone who finds life unbearable. ${ }^{72}$

However, in the twenty-first century, there is nothing inevitable about the future of assisted suicide. Its history over the last half century is not a simple, triumphalist, linear story. It is important to remember that PAS is of recent vintage: it only emerged as an important issue in the last two decades of the twentieth century. Legal PAS is limited to a small list of countries in Europe and North and South America. In the words of the 1994 New York State Task Force on Life and the Laws, legalizing assisted suicide represents "a profound shift in public policy and professional standards." ${ }^{\text {"73 }}$ Three years later, the Justices of the U.S. Supreme Court agreed that permitting assisted suicide signaled an abrupt turn in American history. The historical evidence for other nations around the world suggests the same conclusion: legal PAS is a practice with shallow roots in history.

There is another factor to consider: societies that at the same time devote enormous resources to the prevention of suicide and provide the legal and clinical means for people to end their own lives not only cultivate a form of cognitive dissonance. Legalizing PAS also likely leads to a result that most citizens would abhor: an increase in the number of suicides. ${ }^{74}$ As some political jurisdictions expand the categories of people-notably teenagers - who qualify for PAS, it means that governments are aiding and abetting suicides, no matter what the law calls them and no matter what safeguards are enacted.

Moreover, the cultural and political wars over PAS are hardly a rout: in the ten years prior to 2019, nine American states actually tightened their laws against assisted suicide. Consensus-building in favor of PAS faces stiff challenges going forward: increasingly PAS shows signs of becoming a politically partisan issue in some countries; for example, the U.S. Democratic Party officially endorses PAS, and a Gallup poll in 2007 showed that about 60 per cent of Democrats (and 39 per cent of Republicans) found PAS "morally acceptable." 75 In America, the future of PAS will likely depend on political battles pitting "blue" states against "red" states.

By 2020, there were signs that media attitudes might be changing about the potential abuses and lax safeguards surrounding PAS laws, notably in Belgium and the Netherlands. In Oregon, as of 2019, 1,500 people have died under its 1997 PAS law. However, in 2015, in the Netherlands, 4.5 per cent of all deaths were due to either PAS or lethal injection. A case of assisted suicide from Belgium in 2012 brought the complexities surrounding such decisions into sharp focus. ${ }^{76}$ The slogan "death with dignity" hid the fact that, in these countries, people with psychiatric diseases - or people simply "tired of life" - were eligible under the law for "medical aid-in-dying." Dutch woman with mental health issues died by drinking poison prescribed by her doctor. $^{78}$ Increasingly, worries also spread about elderly individuals feeling pressured to accept a "duty to die" in a day and age when governments find it difficult to pay for escalating health-care costs. ${ }^{79}$

In the end analysis, death and dying are intensely private matters for individuals, families and friends who resist seeming efforts on the part of governments or interested parties to politicize difficult decisions that people make about loved ones. Most people agree 
in theory that individuals have rights, but in practice decisions about assisted suicide- as with other forms of euthanasia - are rarely as clear-cut as they appear at first glance. Questions loom: Who decides at the bedside? Families, friends, individuals, health-care personnel or government? More often than not, we die not alone but "in the arms of others," as historian Peter Filene argues.

The issue has also proved to be divisive among health-care professionals. Many worry that the legalization of PAS may change the practice of medicine itself. In 2019, both the American Medical Association and the World Medical Association re-affirmed their long-standing opposition to PAS. The AMA stated that PAS was incompatible with the physician's role as healer and would entail societal risks. ${ }^{80}$ The unsettling stories coming out of Belgium and the Netherlands may also spark a backlash against assisted suicide, much as the antics of Jack Kevorkian slowed the momentum of the PAS movement in the 1990s. If so, it will only underscore the conclusion that the future of assisted suicide depends heavily on the frequently unexpected twists and turns of history.

\section{Notes}

1 Jim Day, "His Time to Go: Well-known P.E.I. Businessman Ends 'Wonderful' Life on His Own Terms," Charlottetown Guardian, February 8, 2019. www.theguardian.pe.ca/news/local/his-timeto-go-well-known-pei-businessman-ends-wonderful-life-on-his-own-terms-282972/. The coverage of Harpham's death by the Canadian Broadcasting Corporation (CBC) was similarly laudatory. www.cbc.ca/news/canada/prince-edward-island/pei-assisted-death-neil-harpham-1.5012899

2 In 2016, for instance, eight in ten Canadians told pollsters that they supported the Supreme Court ruling on PAS. " 8 in $10(80 \%)$ Canadians Support Advance Consent to PhysicianAssisted Dying," www.ipsos.com/en-ca/news-polls/eight-ten-80-canadians-support-advance-con sent-physician-assisted-dying

3 Edward Shorter, Bedside Manners: The Troubled History of Doctors and Patients. New York: Viking Penguin, 1985, pp. 211-240.

4 As of 2020, there were only two book-length studies of the movement, one set in the United States and the other in Britain. See Ian Dowbiggin, A Merciful End: The Euthanasia Movement in Modern America. New York and Oxford: Oxford University Press, 2003; N.D.A. Kemp, "Merciful Release": The History of the British Euthanasia Movement. Manchester: Manchester University Press, 2002. For an international perspective, see Ian Dowbiggin, Life, Death, God and Medicine: A Concise History of Euthanasia. Lanham, MD: Rowman and Littlefield, 2007.

5 Peter G. Filene, In the Arms of Others: A Cultural History of the Right-to-Die. Chicago: Ivan R. Dee, 1998.

6 Edward J. Larson and Darrel W. Amundsen, A Different Death: Euthanasia and the Christian Tradition. Downers Grive, IL: InterVarsity Press, 1998, p. 54.

7 Lester G. Crocker, "The Discussion of Suicide in the Eighteenth Century," Fournal of the History of Ideas, 13, 1952: 72.

8 Kevin Siena, "Suicide as an Illness Strategy in the Long Eighteenth Century," in John Weaver and David Wright (eds), Histories of Suicide: International Perspectives on Self-Destruction in the Modern World. Toronto, Buffalo and London: University of Toronto Press, 2009, p. 54.

9 Siena, "Suicide as an Illness Strategy in the Long Eighteenth Century," p. 65.

10 Stolberg argues that for this and other reasons such practices "clearly did not mean the same to contemporaries as performing 'active euthanasia' does to us today." Michael Stolberg, "Active Euthanasia in Pre-Modern Society, 1500-1800: Learned Debates and Popular Practices," Social History of Medicine, 20, 2007: 205-221, p. 216.

11 Larson and Amundsen, A Different Death, p. 206.

12 This definition was the Englishman William Munk's. See William Munk, Euthanasia. Or, Medical Treatment in Aid of an Easy Death. New York: Arno Press, 1997, reprint of 1887 edition, p. 5.

13 Michael Burleigh, Death and Deliverance: "Euthanasia" in Germany, 1900-1945. Cambridge: Cambridge University Press, 1994, p. 12.

14 Quoted in Filene, In the Arms of Others, pp. 3-4. 


\section{IA N D OWB I G G IN}

15 Shai J. Lavi, The Modern Art of Dying: A History of Euthanasia in the United States. Princeton and Oxford: Princeton University Press, 2005, p. 85.

16 William H. Beatty, "A Man's Life Is His Own," San Francisco Call, August 2, 1896, p. 17. Cited in Kevin Yuill, "An Extravagant Sympathy with Wretchedness: Tolerance of Suicide in the American Press in the 1890s," unpublished paper, p. 3. Cited with permission by the author.

17 George J. Annas, "At Law: Killing Machines," The Hastings Center Report, 21, \#2, 1991: 33-35, p. 34.

18 This was the only time Adler spoke out on suicide. The responses in the press to his comments were overwhelmingly negative. Twenty-seven years later, Adler cited both traditional objections to suicide to relieve intense sufferings and pro-suicide arguments, so that it was difficult to know what he truly believed on the topic. Stephen Louis Kuepper, "Euthanasia in America, 1890-1960: The Controversy, the Movement and the Law," Rutgers University: Unpublished Ph.D. dissertation, 1981, pp. 31-32, 39, 52. Later, numerous members of U.S. Ethical Culture groups supported the legalization of medical mercy-killing. See Dowbiggin, A Concise History of Euthanasia, pp. 59-60.

19 For Darwin's thoughts on "Natural Selection as Affecting Civilized Nations," see Charles Darwin, The Descent of Man and Selection in Relation to Sex. 2 Volumes. New York: D. Appleton and Company, 1871, Vol. 1, pp. 161-173.

20 Richard Weikart, From Darwin to Hitler: Evolutionary Ethics, Eugenics and Racism in Germany. New York: Palgrave Macmillan, 2004, p. 25. The Englishman Karl Pearson, the first occupant of the Galton Chair of Eugenics at University College London, argued in 1900 that "the doctrine of evolution applies to the history of nations" and taught that "a community of men is as subject as a community of ants or as a herd of buffaloes to the laws which rule all organic nature." Karl Pearson, "National Life from the Standpoint of Science (1900)," http://media. bloomsbury.com/rep/files/Primary\%20Source \%2013.1\%20-\%20Pearson.pdf

21 Ingersoll wrote that a man in agony "being slowly devoured by cancer" was "of no use to himself" and was a "burden to himself and to others, useless in every way." It was only "rational" for such a man to kill himself. Yet there is no evidence that Ingersoll's ideas enjoyed any public backing. Eva Ingersoll Wakefield, ed., The Letters of Robert G. Ingersoll. New York Philosophical Library, 1951, pp. 698-704.

22 Dowbiggin, A Concise History of Euthanasia, pp. 54-55.

23 Martin Pernick, The Black Stork: Eugenics and the Death of "Defective" Babies in American Medicine and Motion Pictures since 1915. New York and Oxford: Oxford University Press, 1996, p. 41.

24 Pernick, The Black Stork, p. 92.

25 In 1938, a U.S. physician, advocating the mercy-killing of "the congenitally mindless" and "incurably sick who wish to die," wrote: "The saving of a life puts a halo on the head of the physician but may add a load to the back of society. Medical science tends to repeal the law which has been the mainstay of evolution - the survival of the fit. On account of public health measures and publicly dispensed medical care a high infant death rate no longer offsets a high birth rate in those families in which congenital mental and physical defects are common." William G. Lennox, "Should They Live? Certain Economic Aspects of Medicine," American Scholar, 7, Autumn 1938: 454-66, p. 456.

26 "Report for the Connecticut Committee by M.H. Clark, Chairman," January 14, 1952, Euthanasia Society of America archives (ESA), Box C-3. These records, once housed in Baltimore, MD, Lewis Associates, are now unaccountably lost.

27 Filene, In the Arms of Others, p. 4.

28 Kuepper, "Euthanasia in America," p. 187.

29 Larsen and Amundsen, A Different Death, p. 162.

30 In 1955, the VELS counted only 226 "paying or life members." Kemp, "Merciful Release," p. 151.

31 N.D.A. Kemp estimates that twelve per cent of the VELS membership belonged to Britain's Eugenics Society. Kemp, "Merciful Release," p. 88.

32 Kemp, "Merciful Release," p. 110.

33 Kemp, "Merciful Release," p. 90.

34 See Burleigh, Death and Deliverance.

35 Dowbiggin, A Concise History of Euthanasia, pp. 77-80.

36 One ESA member admitted in 1952 that the group was desperate to "dissociate our cause in the mind of the general public from the horrors of Hitler's euthanasia." Eleanor Dwight Jones, 
"To Members of Our Advisory Council," ca. 1952, ESA, Box C-1; Mildred H. Clark to Mrs. Edwards, January 17, 1952, ESA, Box C-3.

37 John C. Burnham, “American Medicine’s Golden Age: What Happened to It?” Science, 215, 1982: 1474-1479.

38 Paul Starr, The Social Transformation of American Medicine. New York: Basic, 1982, p. 379.

39 Charles B. Inlander, Lowell S. Levin, and Ed Weiner, Medicine on Trial: The Appalling Story of Medical Ineptitude and the Arrogance that Overlooks It. New York: Pantheon, 1988, pp. 113-114.

40 M.L. Tina Stevens, "What Quinlan Can Tell Kevorkian about the Right to Die," The Humanist, March-April 1997: 10-11.

41 O. Ruth Russell, Freedom to Die: Moral and Legal Aspects of Euthanasia. Revised edition. New York: Humanities Press, 1977, p. 15. Another example of frantic medical attempts to stave off death is the 1975 United Press Agency Report on the efforts of Spanish dictator Francisco Franco's doctors to keep him alive. Roy Porter, The Greatest Benefit to Mankind: A Medical History of Humanity. New York and London: W.W. Norton, 1997, pp. 700-701.

42 Milton J. Lewis, Medicine and the Care of Dying: A Modern History. Oxford and New York: Oxford University Press, 2007, p. 123, pp. 154-155.

43 Udo Benzenhofer and Gisela Hack-Molitor, Luis Kutner and the Development of the Advance Directive (Living Will). Wetzlar, Germany: GWAB-Verlag, 2009.

44 Filene, In the Arms of Others, pp. 76-95, p. 73.

45 Julius Kaunitz, "Legalizing Voluntary Euthanasia," The Ethical Outlook, 43, \#4, JulyAugust 1957: 129.

46 Walter C. Alvarez, "Death with Dignity," The Humanist, September-October 1971, pp. 13-14. Alvarez added that he would happily grant the right to assisted suicide to "psychotic" patients who "were almost ruining the lives of others."

47 Starr, The Social Transformation of American Medicine, p. 379.

48 David J. Rothman, Strangers at the Bedside: A History of How Law and Bioethics Transformed Medical Decision-Making. New York: Basic, 1991, p. 4.

49 For more on the "post-modern patient," see Shorter, Bedside Manners, pp. 211-240.

50 Valery Garrett, "The Last Civil Right? Euthanasia Policy and Politics in the United States, 1938-1991," University of California, Santa Barbara: Unpublished Ph.D. Dissertation, 1998, pp. 171-172. See also Anne Fadiman, "Death News: Requiem for the Hemlock Quarterly," Harper's Magazine, April 1994: 74-82. p. 74.

51 "Final Exit Book Ready to Order," Hemlock Quarterly, 42, January 1991, p. 2.

52 Timothy E. Quill, "Death and Dignity: A Case of Individualized Decision Making," New England Fournal of Medicine, 694, 1991: 691-694. See also Timothy E. Quill, Caring for Patients at the End of Life: Facing an Uncertain Future Together. Oxford and New York: Oxford University Press, 2001.

53 Dowbiggin, A Merciful End, p. 174.

54 See Siena, who made the connection between AIDS and syphilis. Siena, "Suicide as an Illness Strategy in the Long Eighteenth Century," pp. 64-65.

55 Fadiman, "Death News," p. 82; "Changing the Rules of Dying," U.S. News and World Report, July 9, 1990, p. 22.

56 Genereux, one of the few Toronto physicians willing to minister to AIDS patients in the city's "gay village," had already been convicted (though never disciplined) by the Ontario College of Physicians and Surgeons for fondling six different male patients. Dowbiggin, A Concise History of Euthanasia, pp. 1-4.

57 Yale Kamisar, "The Rise and Fall of the 'Right' to Assisted Suicide," in Kathleen Foley and Herbert Hendin, eds., The Case Against Assisted Suicide: For the Right to End-of-Life Care. Baltimore and London: The Johns Hopkins University Press, 2002, p. 75.

58 See Rita Marker, Deadly Compassion: The Death of Ann Humphry Wickett and the Truth about Euthanasia. New York: William Morrow and Co., 1995.

59 Derek Humphry and Mary Clement, Freedom to Die: People, Politics, and the Right-to-Die Movement. New York: St. Martin's Griffin 2000, p. 121.

60 Kemp, "Merciful Release," p. 178.

61 "Medical Ethics: Select Committee Report," May 9, 1994. https://api.parliament.uk/historichansard/lords/1994/may/09/medical-ethics-select-committee-report 


\section{A N D OWB I G G I N}

62 In 1902 and 1908, the Texas Court of Appeals overturned two prior murder convictions of assisted suicide. Yet, neither case involved a terminally ill person. In any event, in 1973, Texas banned aiding or abetting a suicide. See H. Tristram Engelhardt, Jr., and Edmund L. Erde, "Euthanasia in Texas: A Little Known Experiment," Hospital Physician, 9, 1976: 30-31.

63 The New York State Task Force on Life and the Law, When Death Is Sought: Assisted Suicide and Euthanasia in the Medical Context. May 1994, pp. 69-70.

64 Washington et al. v. Glucksberg et al. (1997) www.law.cornell.edu/supct/html/96-1 10.ZS.html

65 Filene, In the Arms of Others, pp. 187-188. See also Steve Friess, "Uneasy Alliance," The Advocate, March 5, 1996, pp. 46-48; David France, "This Doctor Wants to Help You Die," New York Magazine, January 13, 1997, pp. 25-29.

66 Bush's press secretary told the nation that Bush was "fully committed to building a ... culture of life that is built on valuing life at all stages." "Supreme Court Removes Obstacle to Assisted Suicide Laws," New York Times, January 17, 2006. www.nytimes.com/2006/01/17/politics/poli ticsspecial 1/supreme-court-removes-obstacle-to-assistedsuicide.html?mtrref=www.google. com\&gwh=28C8FC0F63D2549AEED6D1 14008E0C57\&gwt=pay

67 Julie Ray, "The Legality vs. Morality of Life and Death," Gallup, July 8, 2003. https://news. gallup.com/poll/8794/legality-vs-morality-life-death.aspx

68 Oralandar Brand-Williams, "Assisted Suicide Advocate Jack Kevorkian Dies," The Detroit News, June 3, 2011. http://nhne-pulse.org/assisted-suicide-advocate-jack-kevorkian-dies/

69 For the Bush administration's handling of this and the Terri Schiavo issue, see Ian Dowbiggin, "From Sander to Schiavo: Morality, Partisan Politics and America's Culture War over Euthanasia, 1950-2010" Fournal of Policy History, 25, 2013: 21-40, especially pp. 32-36.

70 Daniel Eisenberg, "Lessons of the Schiavo Battle," Time, March 27, 2005 http://content.time. com/time/magazine/article/09171104246000.html. See also Kathy L. Cerminara, "Collateral Damage: The Aftermath of the Political Culture Wars in Schiavo," Western New England Law Review, 29, 2007: 279-308.

71 David T. Courtwright, No Right Turn: Conservative Politics in a Liberal America. Cambridge: Harvard University Press, 2010, pp. 194-195.

72 "Death on Demand: Has Euthanasia Gone Too Far?" The Guardian, January 18, 2019. www. theguardian.com/news/2019/jan/18/death-on-demand-has-euthanasia-gone-too-far-nether lands-assisted-dying

73 When Death Is Sought, p. 119.

74 That might explain why some assisted suicide activists try to convince listeners that they are not talking about assisted suicide by using euphemisms such as "medical aid-in-dying" or simply assisted "self-deliverance." Wesley Smith, "Death Plays the Name Game," Wall Street Fournal, August 15, 2004. www.weeklystandard.com/wesley-j-smith/death-plays-the-name-game

75 "The Quick Guide to American Political Parties' Stances on Euthanasia," www.isidewith.com/ political-parties/issues/social/euthanasia-2

76 Rachel Aviv, "Letter from Belgium: The Death Treatment," The New Yorker, June 15, 2015 www.newyorker.com/magazine/2015/06/22/the-death-treatment

77 Scott Kim, "How Dutch Law Became a Little Too Comfortable with Euthanasia: The Story of a 17-Year-Old's Assisted Death Wasn't Real-But It Could Have Been," The Atlantic, June 8, 2019. www.theatlantic.com/ideas/archive/2019/06/noa-pothoven-and-dutch-euthan asia-system/591262/

78 "The Troubled 29-Year-Old Helped to Die by Dutch Doctors," BBC News, August 9, 2018. www.bbc.com/news/stories-45117163

79 In 2000, Derek Humphry and Mary Clement argued that PAS was a method of "cost containment" that might save financially strapped health-care systems. See Humphry and Clement, Freedom to Die, p. 348.

80 The American Medical Association, "Ethics: Physician-Assisted Suicide," www.ama-assn.org/ delivering-care/ethics/physician-assisted-suicide 


\title{
FUTURE TRAJEGTORIES OF DEATH \\ Issues and possibilities
}

\author{
Cortney Hughes Rinker
}

In 2010, President Barak Obama and his administration in the United States implemented a policy as part of the Affordable Care Act (ACA) that would allow physicians to be reimbursed by Medicare ${ }^{1}$ for discussing end-of-life care with patients:

the government will pay doctors who advise patients on options for end-of-life care, which may include advance directives to forgo aggressive life-sustaining treatment ... The new rule says Medicare will cover "voluntary advance care planning," to discuss end-of-life treatment, as part of the annual visit. ${ }^{2}$

This came on the heels of the political and public outcry in 2009, when the ACA was still being crafted, over so-called "death panels," a term coined by 2008 U.S. Vice Presidential nominee Sarah Palin (the former governor of Alaska). Palin claimed these "death panels" would be comprised of bureaucrats who make a "subjective judgement of [someone's] 'level of productivity' in society"3 that would inform the care at the end of life a person receives, meaning that individuals who are not deemed worthy to live because they would not be contributing members to society would be encouraged to forego certain procedures and treatments. Palin was specifically referencing her son who was born with Down syndrome. Those against the policy, including Palin, claimed that the "Obama administration was bringing back a procedure that could be used to justify the premature withdrawal of life-sustaining treatment with severe illnesses and disabilities."4 The controversy over "death panels," which Obama officially denied would exist under the new health care bill, caused it to be removed from the proposed legislation that would restructure the health care system. However, the Obama administration was able to enact the new rule starting January 1, 2011. The rule allows for advance care planning discussion to be part of a yearly visit to the doctor.

To achieve this, officials and proponents cited academic research that demonstrates conversations about end-of-life care between physicians and their patients guarantee that people's wishes are honored when it comes to death and that this can enhance patient autonomy and independence. ${ }^{5}$ Literature has shown that in reality, in the United States, end-of-life conversations between medical providers and patients do not happen as soon or as frequently as they should, leaving patients unaware of their options and families unsure of what treatments loved ones would have or have not wanted in their final days. ${ }^{6}$ One concern put forward as part of the debate surrounding "death panels" was that some patients would be encouraged not to choose to have life-sustaining treatments 
performed depending on the whims or prejudices of the physician or health care provider, but others in favor of allowing end-of-life care discussions to be reimbursed reiterated that everyone can choose the care and procedures they do or do not wantmeaning they could opt to have all curative treatments administered during their final days if they so desired.

The debates about end-of-life care and death are not unique to the United States. In the United Kingdom, end-of-life care has been a priority for the government and policymakers, and there have been moves to try to improve its quality as well as reduce the cost. A paper released by the Institute for Public Policy Research has noted that discussions about end-of-life care between patients and providers is a key area of interest: "The government, NHS [National Health Service] organisations and Health Education England should consider ways to encourage the health and care workforce to have meaningful conversations about death and dying with those at the end of life, their families and carers." 7 The Queensland Government in Australia ${ }^{8}$ made a formal policy statement that includes end-of-life discussions between patients and their providers:

Ideally, conversations about life-sustaining measures should occur as early as possible in the context of the broader advance care planning. The emphasis in decision-making for patients at the end of life is on patient-centred care and supported decision-making. This means involving patients in discussions about their end-of-life preferences and values as early as possible to minimise the need to determine their wishes through substitute decision-maker/s when no one knows what they would have wanted. ${ }^{9}$

The primary goal is to invite patients and their families to have conversations with their health care providers about the end of life so that care provided during the final days of life is in line with what the patients would have wanted for themselves. In Queensland, as in the United States and other countries, the focus of policy and guidelines is the patient and respecting his or her wishes and values during the final stages of care; protocols and procedures are put in place so that surrogate decision makers do not need to be consulted in cases where the patients are incapacitated, and if these decision-makers must step in, they are also aware of what care the patients would have wanted to receive.

In 2002, a euthanasia law was passed in the Netherlands, which "codified a legal option for doctors, whose primary duties - to preserve life and to relieve suffering - were thought to conflict in the case of certain anguished patients." ${ }^{10}$ Prior to this, there had been a series of court cases that ruled in favor of Dutch doctors who had enabled the deaths of their patients. Euthanasia in the Netherlands

legally covers cases in which medical professionals administer lethal injection and those in which doctors provide drugs that patients ingest to end their life. The Dutch system gives deference to doctors' expertise; it respects the relationship between an individual doctor and a patient; and it recognizes that mental illness can be painful and debilitating. ${ }^{11}$

Anthropologist Frances Norwood in her ethnographic study of euthanasia in the Netherlands found that even though the law exists, it was not used as often as people 
anticipated, for the process of encouraging patients just to talk about illness and suffering, their wishes for end-of-life care, and their control over death and their final days actually provided a form of healing for them, and thus made death (i.e., euthanasia) unnecessary, or at least delayed it considerably. Norwood argues that euthanasia has served much more as a conversation starter about care and about life and death than as a regular medical practice.

The landmark Dutch euthanasia law gained international attention in particular in 2019 after the case of Noa Pothoven, a 17-year-old who was reported to be euthanized, particularly in English language media. Pothoven had experienced multiple sexual assaults; had anorexia, depression, and post-traumatic stress disorder (PTSD); and had been admitted to the hospital on numerous occasions. Despite the media coverage, her death did not in fact meet the definition of euthanasia by the Royal Dutch Medical Association, as she died from the voluntary refusal of foods and liquids over an extended period of time. ${ }^{12}$ One of the questions that came out of Pothoven's case was whether or not she was too young to even make decisions about her death and euthanasia, as she did request euthanasia from a clinic when she was 16 years old, but was denied since her parents were not aware. ${ }^{13}$ The report of her death in the media "has predictably been seized upon by staunch opponents of all forms of assisted dying," as it raises deep-seated ethical, moral, and cultural questions about life and death, as well as concerns over the legality of medically assisted dying and euthanasia in different countries. ${ }^{14}$

Often death is associated with old age, but Pothoven's death reminds us that end-oflife care is not only for those who are elderly - and it is important to recognize, as anthropologists and other social scientists have already, that "aging," "older," and "elderly" are culturally and socially relative terms. ${ }^{15}$ Death can come at any age. The Russian writer, Leo Tolstoy, wrote how friend of the judge Ivan Illych, Pytor Ivanovich, had mused, "Three days of terrible suffering, followed by death. That could happen to me at any moment ... and for a moment he became frightened" 16 when he spoke about the judge's death in the famous novella The Death of Ivan Illyich. Pytor's fear came from the realization that death, and the suffering leading up to it, could take place at any moment and possibly even without warning. Nancy Scheper-Hughes argues that Americans are insecure and uncertain about death, and her comments might apply to some other modern societies. ${ }^{17}$ As in Tolstoy's novella, Americans often do not want to talk about the end of life and are fearful of what their final days may entail. Historically death was once seen as "ordinary and expected, part of everyday life," but with the coming of industrialization in the nineteenth century and the establishment of capitalism and later neoliberalism, it has become unseen or "banished' from polite society and denied."18 Scheper-Hughes proposes, "As death was professionalized and removed from the home, new anxieties arose. Americans began to worry about end-of-life decisions made by medical staff in emergency rooms, intensive care units, and even hospices. How dead is dead?" 19 This shift in how death is viewed and experienced in American society stems above all from the development of medical interventions and technologies whose main purpose is to prevent death, along with changes in where death was actually taking place, most often now in medical facilities rather than the home. Scheper-Hughes notes that people fear "where and how they will live out their final days." 20 She traces this anxiety to several discursive and institutional shifts that occurred in the United States, including capitalism, the wider availability of health care and medical technologies, and the framing of death as a failure within the health care system. ${ }^{21}$ 
So far, this chapter has laid out some of the more recent, twenty-first century developments and trends when it comes to death and end-of-life care in countries such as the United States, the Netherlands, the United Kingdom, and Australia, with particular attention to shifts in policy, to areas of improvement as identified by governments and agencies, and to changes in how death takes place and is conceptualized. Arguably, the developments also reflect the rapid aging of the relevant populations, which suggests further contextual changes in the coming decades, as we will discuss below. The remaining sections of this chapter will work to build on these developments along with the historical experiences that were presented in the different chapters of this volume to speculate on what death may look like in the foreseeable future within a more global context. I acknowledge that death is culturally, socially, and structurally shaped and that people's experiences will vary, so there is no way to capture all that may happen to death in the rest of the twenty-first century and beyond. But it is possible to think through some of the larger questions that impact different parts of the world, such as the tension between the limitations of the human body and technological interventions in death; rapidly aging societies and how this demographic trend impacts how, when, and where death occurs; the intersection of biomedicine with traditional or indigenous healing systems; and how the differentiation between the living and non-living (and even human and non-human) is being altered due to medical advancements and the spread of scientific thinking about and medicalization of death. As a whole, this volume has considered death from multiple historical perspectives, and this final chapter will give a small glimpse into the future given worldwide trends. It is not meant to be an exhaustive review or to recall all the nuances of death around the globe but rather is intended to raise additional inquiries about the end of life that can be pursued in the future.

This conclusion is speculative, as we are not really sure what the future will bring. However, to craft my hypotheses, I will draw on empirical data, ethnographic research mainly from anthropology, government and organizational reports, and news coverage from reputable sources that describe and analyze death in various cultural contexts. In addition, I will draw on my own research ${ }^{22}$ on end-of-life care in the Washington, D.C., metropolitan area and on chronic and terminal illness and pain management in Morocco. ${ }^{23}$ Compared to many of the other chapters in this volume, the literature I engage with comes from the more recent past (mostly the 1990s to today). Even though the accounts that I use in my analysis and speculations come from diverse cultures and geographic locations, they all revolve around the larger questions about death that have already been posed: medical technology, biomedicine and traditional healing, aging populations, scientific framing and medicalization of death, and the living and non-living. In writing this chapter, I also draw inspiration from scholarship in feminist anthropology and specifically that of Lila Abu-Lughod, who calls for "narrative ethnographies of the particular." 24 In using this technique, I use certain accounts and experiences as a lens through which I analyze death and end-of-life care and make predictions about what may happen in the future. Abu-Lughod writes, "The effects of extralocal and long-term processes are only manifested locally and specifically, produced in the actions of individuals living their particular lives, inscribed in their bodies and their words." ${ }^{25}$ I suggest that by taking these "particulars" as a main object of inquiry, it is possible to investigate how larger global trends surrounding death are impacting the way that it takes place in specific contexts. But I also want to add a cautionary note here about what I see as a possible limitation of this chapter. I do not view these trends as only flowing from the 
Global North to Global South or from the West to the East, as some of the scholarship in anthropology, the social sciences, and the humanities has framed discussions of globalization. ${ }^{26}$ I try to avoid making this assumption in what I present here and highlight the spaces in which these global flows are multi-directional, which empirically is true, as a number of case studies suggests, but I recognize that this fact may not always come through as explicitly as might be desirable in the remaining sections. Nevertheless, I am hopeful that this conclusion will raise further inquiries about death that can be taken up more fully in the future.

\section{Aging populations, disease burden, and pain at death}

Overall, the world population is aging dramatically. In many countries, people are living longer than they have ever before, and this combines with a falling birth rate. The United Nations observes, "The world's population is ageing: older persons are increasing in number and make up a growing share of the population in virtually every country, with implications for nearly all sectors of society." 27 Commonly countries in the "developed world," such as Japan, South Korea, and Europe, are considered to have the fastest aging populations. Adults over the age of 65 also constitute a fast-growing population in the United States, but "the U.S. isn't experiencing the same gray wave that many other developed nations are. At least one-in-five people in Japan, Germany, and Italy are already 65 or older, and most other European countries are close behind." 28 (China is also on the list of aging populations.) An aging population is the result of two main drivers: fertility and longevity. ${ }^{29}$ The interaction between the two means that a country's population determines the age structure of a population. ${ }^{30}$ A decreasing fertility rate coupled with a low mortality rate - as seen in Japan, Italy, and other countries in Europe for instance - means that the population will have a higher average age. Italy sought to counter the sagging birth rate, which reached a record low in 1992 at 1.2 births per woman, with a 2003 "baby-bonus" law that provide monetary bonuses for Italian heterosexual couples who gave birth to or adopted a second child between 2003 and $2004 .{ }^{31}$ Critics of the law noted that the policy incorporated debatable social and cultural norms about gender, the family, and sexuality in designating who could access the incentives, as individuals and couples who were immigrants or refugees or who did not identify as heterosexual were excluded.

But the main point about this and similar remedial policies is that they have had little effect: 29\% of Italy's population is over 60 years old, ranking just above Japan $(33 \%)$ and just behind Germany $(28 \%))^{32}$ Japan has a particularly interesting set of gerontological concerns given crises such as the Great East Japan Earthquake, tsunami, and the Fukushima nuclear disaster in 2011, which may suggest broader concerns about how global ageing may interact with increasingly frequent environmental stressors:

This earthquake revealed positive aspects of the Japanese society: older adults' wisdom and resilience for survival and coping, active social and labor participation at old ages, and strengths of social relationships. However, the disaster highlighted challenges that Japan is facing, especially rebuilding disaster area communities and addressing population aging in urban communities where Japanese traditional qualities are fading. ${ }^{33}$ 
Social isolation of older adults in Japan (including a growing problem with individuals who die alone and often unnoticed), and the use of technology to address this, has been featured in the academic scholarship as well as the popular media. ${ }^{34}$ Future projections for 2050 show that other places in East Asia, including China, Korea, Taiwan, and Hong Kong, will join the list of being some of the oldest populations in the world. ${ }^{35}$

Even though population structures in Europe, Asia, and North America are changing most rapidly, it is vital not to overlook the fact that several populations in the "developing world" 36 are also ageing at a faster rate than ever before: "The developing regions are home to a growing share of the world's older population ... Projections indicate that in 2050, 79 per cent of the world's population aged 60 or over will be living in the developing regions." ${ }^{37}$ The Middle East and North Africa are currently experiencing the same demographic transition that has already occurred in places such as Europe, the United States, and Japan. After having substantial population growth between 1950 and 2000, due to a decrease in mortality rates and better health care systems, this region is seeing slower population growth, lower fertility rates, and people living longer lives - all of which adds up to more rapid ageing. The Middle East and North Africa still feature a large youth population due to the higher-than-average fertility rates that prevailed until recently and a later transition to slower population growth, which raises governmental concerns about education and job opportunities for the future generations. However, by 2050, the number of older adults will exceed the number of youth in Algeria, Bahrain, Kuwait, Lebanon, Libya, Morocco, Oman, Qatar, and Tunisia. ${ }^{38}$ What makes this situation more convoluted is the fact that older adults typically experience more complex health conditions than children, adolescents, and younger adults, which raises policy questions about the adequacy and availability of health care. In some countries in the Middle East and North Africa, non-communicable diseases (NCDs) have become more prominent, which include cancers, diabetes, and cardiovascular and respiratory diseases: "Once dominant infectious diseases are now being replaced by chronic non-communicable diseases, which comprise $47 \%$ of the region's burden of disease, and anticipated to rise to $60 \%$ by $2020 .{ }^{\prime 39}$ NCDs are now leading causes of morbidity and mortality in the region, which can be correlated with the demographic transition that it is experiencing - and the challenge is all the greater given the novelty of this disease pattern.

Similarly, individuals in Sub-Saharan Africa ${ }^{40}$ are also living longer lives, leading to some potentially complex health issues in the upcoming decades:

The Region continues to face a burden of persistent infectious diseases, while the prevalence of risk factors for chronic diseases is also on the increase. Estimated at 43 million in 2010, the population of elderly people in sub-Saharan Africa is projected to reach 67 million by 2025 and 163 million by $2050 .^{41}$

Traditionally, elders in Sub-Saharan Africa are viewed with respect. They are seen as having wisdom and having gained tremendous amounts of knowledge over their lifetimes. Erdmute Alber, in her ethnographic research on ageing in West Africa, found that older adults must now more commonly prepare for "retirement," as they can no longer rely on their children or families to take care of them during older age. She writes, "People in Benin who are envisaging old age as retirement talk about the desire of living without the help of others during the phases of their life after they quit working." 42 Alber connects the development of the idea of "retirement" to the rise of the middle class in West 
Africa, which has in part been created out of global flows of capital and labor, with some of her participants' grown children living and working in places like France and sending money home. Family still holds importance and still provides a network of caregivers for the elderly when they need it, but "development and modernization are closely connected with social and economic changes that can weaken traditional social values and networks that provide care and support in later life." 43

Furthermore, palliative care, or the treatment of pain and symptoms, is not yet widely available in the Middle East and North Africa, nor is it firmly established in Sub-Saharan Africa. This means that patients who have cancer or other terminal illnesses may experience physical and emotional suffering due to pain or discomfort over the course of their disease and during their final stages of life. In my own interviews with physicians and individuals on care for patients with terminal illnesses in Morocco, I discovered that pain management was one of the top concerns for both parties, as there are strict regulations surrounding the prescription of opioids - so much so that physicians are actually prohibited from prescribing them for their patients - and palliative care was not incorporated into the medical school curriculum, which is regulated by the state, until 2015, which means that physicians who received their degree before this year did not receive instruction on how to control pain and symptoms. In the United States, physicians can be board-certified in palliative medicine, and in Europe, they may be trained in palliative care as a specialty, but in Morocco, it is not a medical specialization (most of those physicians in Morocco who advocate palliative care or who step in to provide some comfort measures, I have found, are anesthesiologists or oncologists). Human Rights Watch published a report that emphasizes the need for better palliative care in the country: "Every year, more than 150,000 Moroccans face advanced - and often incurablechronic illnesses ... Over 62,000 Moroccans, including 6,000 children, suffering from these illnesses will experience debilitating symptoms such as pain, breathlessness, and depression.",44 Morocco has implemented national health care policies that emphasize the importance of establishing high-quality palliative care that is accessible to all who need it, but this is recent and has not been fully operationalized. Likewise, in countries in Sub-Saharan Africa, palliative care is generally underdeveloped: "Palliative care is a relatively new discipline in Africa and its development is hampered by the fact that the concept of pain management is not integrated into healthcare systems." 45 In some health care systems in Africa, there is a fear of opioids, or what may be termed "opiophobia," and I found this among some physicians in Morocco as well, which means some providers will offer "restricted treatment, leaving patients in unnecessary pain at the end of life."46 One cardiac surgeon I interviewed in Morocco noted that some of his colleagues at the hospital will send their patients home with Tylenol, when they really could use prescription pain medication.

The link between the world's ageing population and palliative care is crucial because as people live longer, they tend to experience more complex health problems that invite a greater amount of medical intervention. For example, in the United States, the Centers for Medicare and Medicaid Services (CMS) estimate that about one-quarter of its annual spending is on the $5 \%$ of beneficiaries who die. ${ }^{47}$ Moreover, older adults are usually the ones to develop NCDs, many of which, like certain cancers, can be accompanied by bouts or extended periods of severe pain and many other symptoms, like fatigue or breathing difficulty. If the "developing" world will experience growth in the number of older adults in the upcoming decades, and palliative care is not well-established-although it is a recognized need in many countries - it is fair to surmise that the end of life in regions like the Middle East and North Africa and in Sub-Saharan Africa could be physically 
painful and even traumatic for some. I recognize that the causes of weakened health care systems in these regions may not be completely internal to the countries themselves, and may have to do with the restructuring or decline of the system through development, global neoliberalization, and international economic policies. ${ }^{48}$ Some countries in the "developing world" have relatively young populations as compared to those in the "developed" world, but this should not distract from the fact that their populations are ageing faster than before. There is likely to be increased strain on health care systems to keep up with the needs of both older adults and younger generations at the same time. On the other hand, as populations age at an extremely fast pace, as we have seen in "developed" countries such as Japan and Italy, there is great concern that the older adults will face more isolation and perhaps even die alone, given that they do not have as much familial and social support as in the past due to changing norms, with more elderly living alone owing to the separate responsibilities placed on their children who are in the workforce or caring for their own families. Rapid urbanization in parts of Africa and in countries like India can generate other problems for intergenerational contact.

\section{The medicalization of death: scientific framing and the advancement of medical technologies}

Elizabeth J. Emerick writes of the views and treatment of the dead body in the United States:

The corpse, in the American way, is the repository of fears about death including the medical, theological, and social failures to prolong life. Perceptions of aging, dying, and decaying are vivid because the corpse physically changes, beyond that, the corpse becomes a focal point for the fears Americans have about these changes. ${ }^{49}$

Emerick recognizes that these perceptions are culturally and historically constructed and suggests that we look to the development of the West and Judeo-Christian beliefs to understand where this fear of death and the dead body comes from. But the question of the relationship of religion and death is complex, providing a vital topic - for present and near-future alike - in many societies around the world. A study by a research team with members from Oxford, Coventry, Royal Holloway, Melbourne, and Otago Universities and Gordon College found that, in 100 articles published between 1961 and 2014 that included data on 26,000 people from around the world,

higher levels of religiosity were weakly linked with lower levels of death anxiety. The effects were similar whether they looked at religious beliefs such as belief in God, and an afterlife, or religious behavior like going to church and praying ... 18\% of the studies found that religious people were more afraid of death than non-religious people; and over half the research showed no link at all between the fear of death and religiosity. ${ }^{50}$

Commonly it is believed that those who are more religious will be less afraid of death, but these researchers found that those who are non-religious as well as those who are religious feel the same about death-more accepting and less anxious than might be imagined. 
In my own research on end-of-life care among diverse Muslim patients, families, and physicians in the Washington, D.C., area, I found that many of my participants also talked about Islam encouraging believers to be prepared for death. Similarly, imams, Muslim chaplains, ${ }^{51}$ and Islamic scholars and academics from the region and other parts of the country stated in our interviews that death is to be viewed as a natural part of life by Muslims, as everyone will die at some point; no one can escape death, but it is determined by God. Death should not be feared and should not create anxiety for the person dying nor for their families. The Qur'an reads,

Every soul shall taste death. You shall receive your rewards only on the Day of Resurrection. Whoever is spared the Fire and is admitted to Paradise will surely triumph; for the life of this world is nothing but a fleeting vanity.

$$
(3: 185)^{52}
$$

Even with this pattern emerging from my data, I observed Muslim family members arguing against having their loved one discharged from the hospital because they felt they would be unable to care for them at home. I also observed Muslim family members demand that the hospital team perform life-saving measures to the point that the providers questioned whether or not these procedures went against clinical ethics and even their own morals. (To note, I observed these requests among patients and families who identified as other religions, who did not specify their religious affiliation, or who identified as non-religious too.) Again, with varying rates of secularization, but with increasing reliance on doctors and hospitals, the religious variable may not be as decisive as is sometimes imagined (and scholars have shown that religiosity is particularly hard to define).

Death has become medicalized, particularly in the "developed" world where biomedicine is the dominant health care paradigm and disease and health are viewed scientifically, but also in the "developing" world where biomedicine and scientific views of death are becoming ever more pervasive:

as an example of medicalization, death may represent the ultimate exemplar of how a human condition comes to be defined and treated as a medical problem. We acknowledge that the causes of death are multiple and often, even usually, medical. Common knowledge, however, dictates that everyone's fate is death, medical causes or otherwise. ${ }^{53}$

There is cultural and geographic variation when it comes to the medicalization of death because medicalization is not "a fully formed social phenomenon, but instead is a social meaning embedded within other social meanings and contexts, all of which are historically evolving." ${ }^{54}$ Moreover, anthropologists have masterfully argued that health care systems are themselves culturally constructed.

Across Latin America, research has shown that death has become medicalized. Murguía, Ordorika, and Lendo write, "Latin America has been affected by the phenomena of medicalization, as shown by the historiography of medicine." Furthermore, they note,

One of the reasons why medicalization has spread throughout Latin America has been the marginalization of alternative methods of treating ailments ... In this process, the role of the State has been crucial ... we should not ignore the 
cultural dimension of the phenomenon, in contemporary societies science has an epistemic authority traditionally associated with progress. ${ }^{55}$

What scholars have demonstrated is that with the medicalization of death, and given the fact that biomedicine and scientific thinking are more often than not associated with development and modernity, death becomes viewed as a failure within health care and by the community or society in general. Sharon Kaufman notes that the medicalization of death (along, often, with changing attitudes toward pain) creates a virtual obligation to medically intervene in the dying process, especially given the advancement in technology and the numerous life-saving and life-sustaining measures that are available in many countries. Death can no longer happen naturally or be dictated by solely the human body. ${ }^{56}$ In her ethnographic research at a hospital in the United States, Kaufman finds that there is a regular tendency 'to emphasize discrete entities in opposition-'palliation' versus 'treatment,' 'technology' versus 'care,' 'control' versus 'powerlessness,' 'choice' versus 'inevitability." "'57 Even though the concepts in these pairs are framed as being in opposition to one another, Kaufman's work exposes the fact that over the course of the dying experience within a medical setting in the United States, the boundaries between the two concepts in each pair, and among the pairs themselves, are actually blurred and even non-existent, or the relationships among them changes over time and space.

Biomedicine has become the hegemonic health model in many regions of the world; however, biomedicine often interacts and becomes entangled with other systems of healing and treatment present in local communities. Biomedicine does not come without its faults, and scholars have illustrated the broader societal and political implications that come with its hegemonic status, particularly when it comes to how illness and death are viewed and approached. In Ghana, a study revealed that members of Dagomba, an ethnic group in the northern part of the country, were apprehensive about accepting injections because they believed that it was possible to inject jogu, which "translates as 'bush-illness' (and by Ghanaian English-speakers is often referred to as 'anthrax,' though it is not)." 58 No evidence of people dying specifically from injections was found, but rather, "The anxiety surrounding jogu injections and hospital-medicine refers to circumstances where people with painful boils, that may have been caused by a variety of infections, have reported too late and therefore subsequently died in the hospital (the stronghold of biomedicine)."59 This caused Dagomba to fear biomedicine and the hospital, since these were seen not as sources not of healing but rather of more frequent death. The author of the study reminds us that it is imperative to not just consider all the positives that come with biomedicine, but to also analyze its failures and how it is culturally and historically situated.

One question that remains, then, concerns the limits of the human body in light of the biomedical framing of death and the use of medical interventions and technologies within the dying process, sometimes to the extent that death is not allowed to arrive naturally but is artificially kept at bay for some period of time. One of the key changes in death in modern history, now in many parts of the world, results from the fact that when death is medicalized, it becomes seen as a failure since there is an expectation that medical interventions can either reverse, stop, or delay the dying process. When natural death is superseded by medical interventions, the definition of "living" is called into question. (And, it goes without saying, that there are questions surrounding the availability of and access to these types of technologies and medical procedures, which are not spread evenly across the globe.) 
Medicalization intertwines with other social changes, impacting different categories of the elderly, even aside from regional differences in resources. Rose Keimig develops the concept of "chronic living" through her ethnographic research in nursing homes in southwest China. She argues that those elderly individuals who are residents of the nursing homes do not receive curative treatments, even though they may be able to access them, because their bodies are too frail or these types of measures are seen as futile; but at the same time, they do not receive palliative care, or comfort care, which makes their everyday experience uncomfortable and painful. Keimig attributes their liminal state to changing norms in society, with older adults today not living with their children as was common in the past, combined with a rapidly ageing population and the wider availability of biomedical interventions that have staved off death for these residents prior to or during earlier parts of their stay in the nursing homes. ${ }^{60}$

The hegemonic nature of biomedicine and subsequently the medicalization of death lead us to ask what "natural death" actually means, and this will continue to be a question in the future as biomedicine continues to spread around the world, albeit in distinctive ways within local communities given cultural contexts and other types of healing practices and beliefs that are present. Death now invites specificity and planning - just as in countries such as the United States women must specify that they want a "natural" birth free of painkillers and medical interventions unless they are absolutely necessary. ${ }^{61}$ Death was once capricious, as even seen in Tolstoy's novella quoted earlier in this chapter, and in some ways there is hope for capriciousness as patients may express preferences for quick rather than lingering deaths in their own futures. Now, however, with medical interventions - such as breathing machines, feeding tubes, monitors, and surgeries - death becomes much more "choreographed." ${ }^{62}$ Dr. Kathryn Mannix, a specialist in palliative care in the UK, writes, "More than half a million people in the UK die each year and almost all of them from a condition that gives at least some warning that death is approaching.",63 When this warning comes is when medical interventions are usually deployed, which may actually cause the patient more harm than good, given they cannot ultimately reverse the dying process. With medicalization, death is no longer just a natural event in people's lives but rather it is much more calculated and also more fully determined by outside individuals, including health care providers, family members, and even the government. Given that new discoveries are made almost daily - from new machines to new medications to new instruments for surgeries or types of surgical procedures - it seems probable that the distinction between "natural" death and a medicalized death will fade in the future, and it will become more difficult to define what a "natural" death actually is within different cultural contexts. Keimig discovered in her research in China that some nursing-home residents expressed a desire to just die, but, even though their bodies were feeble and deteriorating, that was not possible. Their death was delayed due to medical interventions and services, putting them into the state of "chronic living." Medical anthropologist Margaret Lock notes, "Biological death is recognized in society and in law by the standards of medical science ... a dominant approach in the 'modern' world argues that nature, including the human body in life and death, functions according to scientific laws." 64 This implies that there is "an end of recognizable life."65 But, I would argue that the end of natural life was much clearer prior to the medicalization of death and the plethora of technologies and scientific discoveries that have occurred over the past century. With the medicalization of death and the increased salience of biomedicine, this "biological death" will become much more muddled in the future. 


\section{Death, the body, and the living and non-living, human and non-human}

I remember one day walking into an older Muslim patient's room at the hospital where I was conducting my fieldwork on end-of-life care in the Washington, D.C., region in 2015. The patient was conscious and able to speak relatively well, albeit softly and slowly. His son sat in the chair next to his bed. The patient was connected to numerous machines, including a heart monitor. The physician who I shadowed during that visit pointed to the monitor and said as he turned to me, "Do you see those lines? That is his pacemaker, his heart is not beating on its own anymore." Without the pacemaker provoking and regulating the man's heartbeat, the man would not have been able to survive. After being in and out of the hospital a few times over the course of three months, he passed away in the hospital while connected to the numerous monitors and machines that were keeping him alive. I was looking at a human lying in the hospital bed on that day I first visited him, but I have to question, was I really looking at just a human given that a device was controlling a vital bodily process - the beating of his heart? Was he really "alive" in the way that "being alive" is typically defined?

With the advancement of medicine and technology, there is not such a distinction between the living and non-living or even the human and non-human. Donna Haraway in her work on science, technology, and feminism writes of the "cyborg," or a

cybernetic organism, a hybrid of machine and organism, a creature of social reality as well as a creature of fiction ... Contemporary science fiction is full of cyborgs - creatures simultaneously animal and machine, who populate worlds ambiguously natural and crafted. Modern medicine is also full of cyborgs, of couplings between organism and machine. ${ }^{66}$

Research at the University of Oxford has found that placing electrodes in specific parts of the brain can help alleviate chronic and debilitating pain for those who have had a stroke, which allows the patients to avoid taking high doses of pain medication. The person can control the electrical current depending on their pain level. ${ }^{67}$ With these technologies and devices, the line between living and non-living, or human and non-human, is murky and raises ethical and legal questions about life and death, particularly with regards to when we are to consider someone as dead. Kelly Joyce and Laura Mamo talk about "graying the cyborg," or how "within biomedicine, the aged body is increasingly constructed as a set of age-related diseases as well as a site for continual restoration and improvement," ${ }^{, 68}$ most often through the use of technologies, like a pacemaker, to keep the body functional and "alive."

Again, however, there is cultural variety even around shared medical trends. Lock, in her ethnographic study on brain death and organ transplants in North America and Japan, found differences between how brain death is defined culturally, politically, and medically in the two countries, which has resulted in different approaches and laws surrounding organ donations and transplants. She writes that the "debate about technologically manipulated death is elaborated in radically different forms in the scientifically sophisticated spaces of Japan and North America.",69 Historically she documents "the first respirators were developed in the 1960s, making it possible to sustain brain-dead but otherwise alive human bodies for anything from a number of hours to, very occasionally, 
several weeks (and recently for much longer periods of time), ${ }^{, 70}$ which made defining death in "technologically advanced" countries to be of the utmost importance. In some cases, organs cannot be harvested until a person is declared clinically dead, and then there is a limited time frame in which they must be removed in order to remain viable, but she questions what being dead actually means in two different cultural contexts when a person's brain is no longer functioning but their bodies are able to live on, usually dependent on machines to do the work of organs and other internal processes. Lock draws on Haraway's concept of the cyborg in her analysis of brain death in North America and Japan: "A comparative ethnography of technoscience ... must immediately confront the question of why in specific locales certain cyborgs raise little concern while in others they create havoc." "71 This contrast comes from Lock's findings on brain death: in North America, brain death is legitimate death and has been institutionalized, but in Japan, given that death is much more of a "social event," it raises greater ethical and legal concerns and has prompted pushback against scientifically defining death. (In North America, Lock notes that when certain criteria are filled or boxes are checked, then the person is declared clinically dead, and they are removed from any machines keeping them alive.)

But the binary of living and non-living is not unique to just death. Scholarship has also taken up this question in regards to certain diseases that will either eventually lead to death or will make the ageing or dying process more complicated. An example of this is dementia. Late-stage dementia can put a person into a coma and potentially lead to death. Some dementia symptoms may be relieved or even treated or reversed with proper medication and care. Christopher Johnson, Jordan Kelch, and Roxanna Johnson observe, "Industrialized societies offer a proliferation of scary dementia stories and labels that are reflected in mainstream media, fictional books and literature." ${ }^{, 72}$ They suggest that anxiety and fear about the disease stems from the medicalization of dementia combined with the "social construction of PLWD [people living with dementia] as 'zombies." 73 Dementia or Alzheimer patients can as a result be dehumanized and their agency taken away owing to the connotations surrounding the zombie. Sharon Kaufman found that sometimes a person with dementia is viewed as "as good as dead" because dementia becomes the "rationale for facilitating death," "74 partly because of its medicalization. It is seen as a condition that can be medically treated but ultimately it cannot, as to date there is no cure. Medication and treatment can only slow down its progress or delay its more severe onset. Janelle Taylor argues that even though those with dementia may not be able to remember someone's name or specific details about their lives due to the cognitive decline associated with the disease, that does not mean they "cannot 'recognize' or 'care' about other people." 75 This is a hallmark of a zombie, which refers to a person raised from the dead who lacks the awareness, reflexes, and control that a human being possesses: "Zombie lore may give us a lot of variety, but one thing every zombie scenario has in common is the reanimation of the body after death. The body's movements are slave to a brain that is no longer in control." 76

A zombie invasion is most commonly seen as a part of science fiction, and on television and in movies, but some question whether it is so far-fetched when human bodies are able to go on after the brain stops working ("brain death") or after extensive cognitive decline, as in the case of dementia. ${ }^{77}$ I suggest that the zombie can represent what may actually be happening to us - human beings - with the advancement of science and technology. The technological and medical innovations that have occurred in the more 
recent past, and that will continue to be developed in the future, make the figure of the zombie even more relevant, I argue, as it also calls into question what it means to be living, alive, or human and what it means to be dead, non-living, or non-human.

\section{Concluding remarks}

In the conclusion to this volume on the global history of death, the primary goal has been to generate meaningful more questions about what death will look like or how it will take place in the future - from thinking about palliative care and pain during death, to what "natural" death is, to whether or not zombies are really just part of our imaginations. Empirical and ethnographic evidence from the last few decades, and from various places in the world, can be deployed in order to offer some speculations about the future trajectories of death. As I noted at the outset, I am cognizant of the fact that death is not universal in terms of how, when, and where it happens. These factors are culturally and geographically situated. Furthermore, while everyone will die, as a biological fact, even biology is culturally constructed and locally specific. Humans in every part of the word are all biologically and physiologically similar. But, Margaret Lock "suggests that it is important to think in terms of 'local biologies,' which reflect the very different social and physical conditions of [people's] lives from one society to another." So even a life event that we will all experience - death - will not physically be the same for everyone. I do not think there are any easy or straight-forward answers to the question: What will happen to death in the future more globally? For instance, given that the failures or unintended consequences of biomedicine have been recognized by various communities in "developed" and "developing" regions alike, it is possible that in future people will try to shake loose from the medicalization model and that death will return to its more "natural" state. Alternatively, of course, again, biomedicine may become even more salient around the globe and more integral to everyday life, causing death to be seen even more as a medical problem that must be treated and reversed. Rather than suggesting any definitive answers, this chapter has relied on on-the-ground research that is culturally situated to propose some quandaries about death within different domains: the limitations of the human body, demographic trends and rapidly ageing populations, technological advancements and medical innovations, and the division of the human/non-human and living/non-living. A key goal is to propose future lines of inquiry about death that students and scholars from across the disciplines will take up and explore further. Death is ultimately an unavoidable topic, and recent trends in many parts of the world suggest that it is likely to be discussed more frequently in future.

\section{Notes}

1 Medicare is the federal government program in the United States that provides health care and insurance to individuals over 65 years of age, some younger individuals who have disabilities, and those with End-Stage Renal Disease (ESRD) who require dialysis or a transplant.

2 Robert Pear, "Obama Returns to End-of-Life Plan that Caused Stir," New York Times, December 25, 2010, www.nytimes.com/2010/12/26/us/politics/26death.html.

3 Don Gonyea, "From the Start, Obama Struggled with Fallout from a Kind of Fake News," NPR, January 10, 2017, www.npr.org/2017/01/10/509164679/from-the-start-obama-strug gled-with-fallout-from-a-kind-of-fake-news.

4 Pear, Obama Returns to End-of-Life Plan. 
5 Pear (2015) cites this work as being influential in the Obama administration's arguments: Stacy Fischer, Sung-Joon Min, and Jean Junter, "Advance Directive Discussions Do Not Lead to Death," Journal of the American Geriatrics Society 58, no. 2 (2010): 400.

6 Sharon Kaufman, And a Time to Die: How American Hospitals Shape the End of Life (Chicago: University of Chicago Press, 2006).

7 Jack Hunter and Martina Orlovic, End of Life Care in England: A Briefing Paper (London: Institute for Public Policy Research, 2018).

8 Queensland is located in the northeast of Australia.

9 Queensland Health, End-of-Life Care: Guidelines for Decision-making about Withholding and Withdrawing Life-Sustaining Measures from Adult Patients (Queensland: State of Queensland, 2018).

10 Scott Kim, "How Dutch Law Got a Little too Comfortable with Euthanasia," The Atlantic, June 8, 2019, www.theatlantic.com/ideas/archive/2019/06/noa-pothoven-and-dutch-euthan asia-system/591262/.

11 Kim, How Dutch Law Got a Little too Comfortable with Euthanasia.

12 Melanie Newman, "Noa Pothoven Wasn't Euthanized-But It Was What She Wanted," BMf (2019): 365, https://doi.org/10.1136/bmj.14142.

13 Ibid.

14 Ibid.

15 Cati Coe, "Imagining Institutional Care, Practicing Domestic Care: Inscriptions around Aging in Southern Ghana," Anthropology E Aging 39, no. 1 (2018): 18-32.

16 Leo Tolstoy, The Death of Ivan Ilyich and Confession (New York: Liveright Publishing Corporation, 2014), 36.

17 Nancy Scheper-Hughes, "Death and Dying in Anxious America," in The Insecure American: How We Got Here and What We Should Do About It, ed. Hugh Gusterson and Catherine Besteman (Berkeley: University of California Press, 2009), 317-344.

18 Nancy Scheper-Hughes, "Death and Dying in Anxious America," 2009, 320.

19 Ibid.

20 Ibid.

21 See Atul Gawande, Better: A Surgeon's Notes on Performance (New York: Picador, 2007).

22 My research was supported College of Humanities and Social Sciences and the Ali Vural Ak Center for Global Islamic Studies at George Mason University.

23 Cortney Hughes Rinker, "Islam, Medicine, and Practice: The Manifestation of Islamic Moral Values in Everyday Aspects of the U.S. Health Care System," in Treating the Body in Medicine and Religion: Fewish, Christian, and Islamic Perspectives (New York: Routledge, 2019), 137-153.

24 Lila Abu-Lughod, "Writing against Culture," in Recapturing Anthropology: Working in the Present, ed. Richard G. Fox (Santa Fe: School of American Research, 1991), 153,

25 Ibid., 150.

26 Erica Vogel, "Predestined Migrations: Undocumented Peruvians in South Korean Churches," City E Society 26, no. 3 (2014): 331-351.

27 United Nations, World Population Ageing: 2017 Highlights (New York: United Nations, 2017$), 2$.

28 Gretchen Livingston, "Americans are Aging, but Not as Fast as People in Germany, Italy and Japan," Pew Research Center, May 21, 2015, www.pewresearch.org/fact-tank/2015/05/21/ameri cans-are-aging-but-not-as-fast-as-people-in-germany-italy-and-japan/.

29 Annamaria Simonazzi, and Fiorenza Deriu, "Ageing and Employment in the Mediterranean: Old and New" in Ageing in the Mediterranean, ed. Joseph Troisi and Hans-Joachim von Kondratowitz (Bristol, UK: Policy Press, 2013), 102.

30 Joseph Troisi, "Demographic Outlook of Ageing in the Mediterranean 1950-2050" in Ageing in the Mediterranean, ed. Joseph Troisi and Hans-Joachim von Kondratowitz (Bristol, UK: Policy Press, 2013), 33-56.

31 Elizabeth L. Krause and Milena Marchesi, "Fertility Politics as 'Social Viagra': Reproducing Boundaries, Social Cohesion, and Modernity in Italy," American Anthropologist, 109, no. 2 (2007): 350-362.

32 United Nations, World Population Ageing: 2017 Highlights, 2017.

33 Naoko Muramatsu and Hiroko Akiyama, "Japan: Super-Aging Society Preparing for the Future," The Gerontologist 51, no. 4 (2011): 426. 
34 Malcom Foster and Kim Kyung-Hoon, "Ageing Japan: Robots' Role in Future of Elder Care," Reuters, March 28, 2018, https://widerimage.reuters.com/story/ageing-japan-robotsrole-in-future-of-elder-care.

35 United Nations, World Population Ageing: 2017 Highlights, 2017.

36 I am following the United Nations when using the terms "developed" and "developing" to refer to countries and regions in this chapter. Here "developed" refers to North America, Europe, Australia, New Zealand, and Japan. My usage of the term follows the UN. I recognize that these terms are constructed and have long political and colonial histories but am not able to detail these histories here due to space. See Arturo Escobar, Encountering Development: The Making and Unmaking of the Third World (Princeton: Princeton University Press, 1995).

37 United Nations, World Population Ageing: 2017 Highlights, 2017, 4.

38 Shereen Hussein and Mohamed Ismail, "Ageing and Elderly Care in the Arab Region: Policy Challenges and Opportunities," Ageing International, 42 (2017): 274-289.

39 R.R. Hajjar, T. Atil, Z. Al-Mandhari, M. Oudrhiri, L. Balducci, and M. Silberman, "Prevalence of Aging Population in the Middle East and Its Implications on Cancer Incidence and Care," Annals of Oncology, 23, Supplement 7 (2013): 12.

40 I have included North Africa, which includes Morocco, Algeria, Tunisia, Libya, and Egypt, with the Middle East. I have followed conventions that separate this region from Sub-Saharan Africa given social and political histories.

41 World Health Organization. "Aging," www.afro.who.int/health-topics/ageing.

42 Erdmute Alber, "Préparer la Retraite: New Age-Inscriptions in West African Middle Classes," Anthropology Ë Aging, 39, no. 1 (2018): 66.

43 Barney Cohen and Jane Menken, ed. Aging in Sub-Saharan Africa: Recommendations for Furthering Research (Washington, D.C.: The National Academies Press, 2006), 1.

44 Human Rights Watch, "Pain Tears Me Apart' Challenges and Progress in Ensuring the Right to Palliative Care in Morocco," February 4, 2016, www.hrw.org/report/2016/02/04/paintears-me-apart/challenges-and-progress-ensuring-right-palliative-care-morocco.

45 Christian R. Ntizimira, Jean Luc Nkurikiyimfura, Olive Mukeshimana, Scholastique Ngizwenayo, Diane Mukasahaha, and Clare Clancy, "Palliative Care in Africa: A Global Challenge." Ecancermedical Science, 8, no. 493 (2014): 2.

46 Ibid., 2.

47 Helen Adamopoulos, "The Cost and Quality Conundrum of American End-of-Life Care," The Medicare NewsGroup, June 3, 2013, www.medicarenewsgroup.com/context/understanding-medi care-blog/understanding-medicare-blog/2013/06/03/the-cost-and-quality-conundrum-of-ameri can-end-of-life-care.

48 For more on this topic, see Adia Benton and Kim Yi Dionne, "5 Things You Should Read before Saying the IMF is Blameless in the 2014 Ebola Outbreak," The Washington Post, January 5, 2015, www.washingtonpost.com/news/monkey-cage/wp/2015/01/05/5-things-youshould-read-before-saying-the-imf-is-blameless-in-the-2014-ebola-outbreak/.

49 Elizabeth J. Emerick, "Death and the Corpse: An Analysis of the Treatment of Death and Dead Bodies in Contemporary American Society," Anthropology of Consciousness, 11, no. 1-2 (2000): 34.

50 "Study into Who Is Least Afraid of Death," University of Oxford News and Events, March 17, 2017, www.ox.ac.uk/news/2017-03-24-study-who-least-afraid-death.

51 All of my participants who identified as Muslim identified as Sunni, the branch of Islam that includes the majority of the world's Muslims.

52 N.J. Dawood, trans. The Koran (London: Penguin Classics, 2006).

53 John Z. Sadler, Fabrice Jotterand, Simon Craddock Lee, and Stephen Inrig, "Can Medicalization Be Good? Situating Medicalization within Bioethics," Theoretical Medicine and Bioethics, 30 (2009): 418 .

54 Ibid., 413.

55 Adriana Murguía, Teresa Ordorika, and León F. Lendo, "The Study of Processes of Medicalization in Latin America," História, Ciências, Saúde-Manguinhos, 23, no. 3 (2016), http://dx.doi. org/10.1590/S0104-59702016005000009.

56 The Centers for Disease Control and Prevention (CDC) states, "Terms such as senescence, infirmity, old age, and advanced age have little value for public health or medical research." 
These terms should be avoided on a death certificate. When "old age" is reported on a death certificate, additional information about the cause of death is required. If there are multiple problems that could have led to death, the certifying physician should pick the one that most likely led to death. See www.cdc.gov/nchs/data/dvs/blue_form.pdf under the section "Common problems in death certification."

57 Sharon Kaufman, "Intensive Care, Old Age, and the Problem of Death in America," The Gerontologist, 38, no. 6 (1998): 716.

58 Bernhard Bierlich, "Injections and the Fear of Death: An Essay on the Limits of Biomedicine among the Dagomba of Northern Ghana," Social Science \& Medicine, 50 (2000): 706.

59 Ibid.

60 Rose Keimig, "Chronic Living and Delayed Death in Chinese Eldercare Institutions," Anthropology \& Aging, 41, no. 1 (2020): 17-30.

61 The American College of Obstetricians and Gynecologists (ACOG) released the opinion, "Approaches to Limit Intervention During Labor and Birth" in 2017, www.acog.org/ClinicalGuidance-and-Publications/Committee-Opinions/Committee-on-Obstetric-Practice/ Approaches-to-Limit-Intervention-During-Labor-and-Birth?IsMobileSet=false.

62 Mara Buchbinder, "Choreographing Death: A Social Phenomenology of Medical Aid-in-Dying in the United States," Medical Anthropology Quarterly, 32, no. 4 (2018): 481-497.

63 Kathryn Mannix, "Radical Ideas: Dying Isn't as Bad as You Think," Science Focus, January 3, 2019, www.sciencefocus.com/the-human-body/dying-isnt-as-bad-as-you-think/.

64 Margaret Lock, Twice Dead: Organ Transplants and the Reinvention of Death (Berkeley: University of California Press, 2001): 32-33, original emphasis.

65 Ibid., 38.

66 Donna Haraway, Simians, Cyborgs, and Women: The Reinvention of Women (New York: Routledge, 1991): 149-150.

67 James Hughes, Cyborg Citizen: Why Democratic Societies Must Respond to The Redesigned Human of the Future (Cambridge, MA: Westview Press, 2004).

68 Kelly Joyce and Laura Mamo, "Graying the Cyborg: New Directions in Feminist Analyses of Aging, Science, and Technology," in Age Matters: Realigning Feminist Thinking, ed. Toni M. Calasanti and Kathleen F. Slevin (New York: Routledge, 2006): 99-100.

69 Margaret Lock, "Death in Technological Time: Locating the End in Meaningful Life," Medical Anthropology Quarterly, 10, no. 4 (1996): 575.

70 Ibid., 577.

71 Ibid., 578 .

72 Christopher Johnson, Jordan Kelch, and Roxanna Johnson, "Dementia at the End of Life and Family Partners: A Symbolic Interactionist Perspective on Communication," Behavioral Sciences, 7, no. 42 (2017): 1.

73 Ibid., 2.

74 Sharon Kaufman, And a Time to Die: How American Hospitals Shape the End of Life (Chicago: University of Chicago Press, 2006): 23.

75 Janelle Taylor, "On Recognition, Caring, and Dementia," Medical Anthropology Quarterly, 22, no. 4 (2008): 318.

76 Sabrina Stierwalt, "Real-Life Zombies," Scientific American, September 20, 2019, www.scientifica merican.com/article/real-life-zombies/.

77 The concept or word "zombie" has a long history that traces to Haitian Creole traditions that developed out of African religious practices. See Lakshmi Gandhi. "Zoinks! Tracing the History of 'Zombie' from Haiti to the CDC," NPR, December 15, 2013, www.npr.org/sections/ codeswitch/2013/12/13/250844800/zoinks-tracing-the-history-of-zombie-from-haiti-to-the-cdc. 


\section{INDEX}

Page numbers in bold type refer to information in tables. Italic type indicates figures and images. an 'n' following a page number indicates a note.

1984 (Orwell) 103

Aaron, Michele 424

Abdulmutallab, Umar Farouk 97

abortion: Bonger 80; Japan 264; maternal deaths 63; Mexico 373, 374, 375, 376, 377; moral statistics 87

Abu Aisha, Dareen 100

Abu Eid, Raqiya 102

Abu-Lughod, Lila 520

accidental death $167,215,225,328$

Achenwall, Gottfried 82, 83

Adler, Felix 503

Africa: biomedicine and hospitals 3, 526; capital punishment 117, 327; Christianity and colonialism 325-326, 327; data 21; death rituals 321-322; demographic transition 323-324, 522; good and bad deaths 322;

Hindu cremation 298, 299; historiography

13; HIV/AIDs crisis 25, 46, 320, 323-324, $328,329,445$, 486, 494; homicide 65, 69, 70; hospice in Uganda 482, 484, 493-494, 496; Islam 326-327; Kamuzu Banda's mausoleum 442-443; malaria 38; palliative care 523-524; plague compassion 58; political meaning 322-323, 443, 447-448, 449, 451-455, 456; smallpox 30, 37, 324; urbanization and technologies of death 321 , 327-329; violence and war 320-321, 324-325, 328; see also presidential mausoleums (Zambia)

African Americans: funerals 13, 226; homicides 69, 70, 71; hospices 488; life expectancy 214; organ transplants 365 ; victims of smallpox violence 53-54

African Union 117

Afterlife 260 afterlife: animals 460, 463, 464, 475, 476; baptism 383; Buddhism in Japan 257-258, 259-260, 426, 427, 475; Christian missionaries in Africa 326; family reunion 222, 223, 224, 394, 428, 430, 431, 432; see also Heavenassociated phrases (study) in film 260, 426-427, 430, 432; Muslim scripture 305; North American death reactions (nineteenth century) 222, 223; self-regard (twentieth century) 208-209; suicide terrorism 92, 99, 101; Tzeltal tradition 242

ageing populations 521-524; "chronic living"

(Chinese nursing homes) 527; developing world 323, 522-524; and the history of assisted suicide 500, 506; Italy's "babybonus" law 521; Japan 9, 14, 36, 256, 264-265, 269, 488-489, 521-522, 524; and non-communicable diseases 14, 22, 230, 500, 506, 522, 523

AIDS/HIV pandemic see HIV/AIDS pandemic al-Akhras, Ayat 100

Alber, Erdmute 522

Alcott, Louisa May 221, 222

al-Ghazālī, Abū Hāmid 308-309, 310

al-Sādiq, Ja'far 308

Altman, Patricia B. 237, 238

Alvarez, Walter 507

Alzheimer's disease 14, 215, 230, 529

American Civil War 9, 12, 136, 149n7, 197, 200, 213, 219, 281

Amundsen, Darrel W. 502

Anderson, Benedict 241

Andreev, Leonid 95

animal death 10; see also pet death

animal rights movements 167,363

Anlo people 322

antibiotics 27-28, 36, 37, 38 


\section{IN D E X}

anti-vivisectionists $361,363,364,365$

Antonine Plague (165-180 CE) 47

Ariès, Philippe: on beliefs about an afterlife 197, 205, 207-208; on contemporary deficiencies 11; death eras/mentalities 159-160, 168, 184, 194-195, 197, 256, 320; death of 169; and the death taboo ("forbidden death") 158-161, 168, 256, 320; and Jacobsen's 'spectacular death' 168

(The) Art of Ministering to the Sick 483

artificial life support machines 3, 304-305, 506-507, 527, 528-529

Ashdown, Cecil 392

Asia Pacific Forum of National Human Rights Institutions 118

assisted suicide see euthanasia; physician-assisted suicide (PAS)

Athens, Plague of 46-47

Augustine of Hippo 78-79

Australia: assisted suicide 508; end-of-life-care

518; Heaven-associated phrases (study) 195, 197, 199, 200, 201, 202, 203, 204, 205; homicides 63, 64, 69; Indian cremation 298; lasting trauma (First World War) 399, 400-401; mortality revolution 24; transformation of pre-war mourning behaviors 389, 400; see also family bereavement (Australia, First World War)

Austria 65

Austrian Empire 109, 187, 188, 200

Avalos, Eugenio Maurer 242

Avatar 423

Avengers: Endgame 423

average age at death 22, 23

B-29 Bomber crash (Japan, 1945) 254-255

Badone, Ellen 201

Baehrel, René 45

Baines, Mary 482

Banda, Kamuzu, mausoleum of 442-443

Barbados 115

Basque, Marta 384

Batty, Craig 437

Beals, Ralph 246

Beccaria, Ceasare 80, 111

Becker, Ernest 157-158, 161, 162, 424

Belarus 114, 491

Belgium 139, 200, 434, 438, 499, 508, 512, 513

Bell, Richard 86

Bely, Andrei 94

Bemba people 321-322

Benares 289-290, 291

Bentham, Jeremy 111

Beres, Louis René 102

Berridge, Kate 164-165, 166, 171
Besant, Annie 293

Bianchi movement 48

Birchwood, General W. 391, 392, 396

birth rates 24, 222, 264, 265, 503, 521; see also fertility rates

Births and Deaths Registration Bill (United Kingdom, 1926) 354, 360

Black Death (1348) 46, 47-48

Black, Donald 68

Black Lives Matter movement 70

Blavatsky, Helena 293

body snatching 49, 361

Boehm, Christopher 109

Bogdanov, Aleksandr 103

Bogrov, Dmitrii 98

Bonger, Willem Adriaan 80-81

Boss, C. 395

Botswana 25, 321, 324

brain death 2-3, 263-264, 304, 305, 307, 308, 312, 365, 528-529

Brandes, Stanley 464

Branks, William 200-201, 205, 208

Brazil 69, 112, 188, 244

Breaking the Waves (film) 435

Bremmer, Jan N. 197, 206

Brent, Sandor B. 430, 431

Breshko-Breshkovskaia, Ekaterina 99

Brilliant, Dora 96, 97, 102

Britain see United Kingdom

Brittain, Vera 141

Brodman, Barbara 240, 243

Brown, David 325-326

Browne, Thomas 80, 501

Brown, Peter 186

Buddhism 11, 13; in China 273, 275; death in modern Japan 254-255, 256, 257-260, 261, 262, 264, 266-267, 268; hospice programs (US) 496; in India 288; Figoku (film) 426, 427; pet death 460, 464, 470-471; Sri Lanka 292; suicide 500-501

Buddhist Churches of America 464, 470-471

Buddhist Temple of San Diego 470-471

Budd, William 358

Bunyoro 323

burial: Africa 320, 321-322, 325, 326, 327, 328, 329; Australian war dead 388, 394-395, 396, 397, 398; Caribbean tradition of 343-344, 347; China 273-274; Chinese Red Cross Society, burial corps 272, 274, 276-284; churchyards 123, 124-127, 180; Hinduism 292, 294; suicides 79, 80, 125; war dead 138; see also cemeteries; funerals; premature burial (nineteenth- and early twentieth-century Britain and America)

Burke, Thomas 203

Burke, William 361 
Burrows, George 84

Bush, George W. 510

Byock, Ira 482, 487-488, 496

Cadet de Vaux, Antoine 180

Caillaux, Henriette 66

Caillot, Antoine 131

Caldwell, Jack 40

Calmette, Gaston 66

Cameroon 322

Campo Santo cemetery 188, 189

Camus, Albert 97, 114

Canada: assisted suicide 499, 509; capital punishment 115, 216, 217; Caribbean diaspora $337,341,343$; cremation 227; end-oflife care 225, 228-229; epidemics 54-55, 213; eugenic organizations 504; garden cemetery movement 218; gun ownership 71; life expectancy 214, 230; location of death 215; non-communicable diseases 215; see also North America

cancer: antibiotics enabling surgery 36; assisted suicide 499, 500, 507, 509; charities 227; Collateral Beauty 433; and early life disease 35; end-of-life care 156, 164, 165, 523; (see also hospices); following Chernobyl 491; Japan 265-266, 267-268, 489; Middle East and

North Africa 522, 523; mortality patterns, historical shifts 1, 22, 27, 33, 215, 506, 522; openness about 156, 227, 266, 485, 489; smoking 27; Uganda 493

capital punishment see death penalty

Cardenas, Ramírez 116

care and nursing homes: China 527; death out of view 5, 162, 227; Japan 265, 488, 490; location of death $5,215,229,265,484$; memorial rituals 168

Caribbean countries: capital punishment 115; diaspora 336-338, 340, 341-345, 346, 347; homicides 69; Indian cremation 298, 299; modern funerals 345-347; mortality revolution 24; traditional funerals 338-341, 342, 343-344

Casanova, Giacomo 80

Catherine II (of Tuscany) 109

Catholicism see Roman Catholicism

cemeteries 2, 8, 11, 12, 19, 20; for the

Caribbean diaspora (in New York) 342, 343; churchyard decline and cemetery reform 126-127, 130-131, 138, 180-181, 186-189, 217; colonial Africa 326, 327; compared to churchyards 123, 124-125; Field of Mars 411, 412; garden cemetery movement 123 , 127, 128, 130-131, 217-219, 220, 223; "heterotopias" 123-124; lawn-park cemeteries (US) 132-133, 219; Mount Auburn Cemetery
123, 131, 132, 181, 216, 217; Père Lachaise

Cemetery 123, 127-129, 130, 131, 133, 138, 181, 187; for pets $461-462,463,464-467$, 469, 471-476, 477; Russia 406, 407-409, 411-413, 416-418, 419; St. Vincent 338, 339, 340, 341, 345, 346; social cohesion 131-132; suicides 79; Victorian death culture 7, 159; see also military cemeteries; tombstones "Cemetery for All God's Creatures" (New York City) 465-467

Cenotaph 140

cerebral death 2-3, 263, 304, 305, 307, 308, $312,365,528-529$

changsheng hui ("Longlife Associations, China) 274

Charlot, Jean 237

Chechen terrorists 99, 100

Chernov, Viktor 94

Chiffolo, Anthony F. 466

children: at Caribbean funerals 338; death taboo 155, 161, 165; eugenics 504, 505; exposure to death and grief $5,156,161,170$, 221-222, 223, 225, 228; film exploring children's understanding of death (Ponette) 430-431; grief and guilt for the death of a child 5, 223, 226, 244, 247; Heavenly reunions 200, 201, 204, 205; hospices 486-487; infanticide 63, 64, 372-385; infectious diseases $21,27,28,31,32,34,35-36$, 38; life expectancy measures 22, 23, 23; literature 5; malaria 38; Muslim funeral traditions 310; suicide terrorism 100-101, 102; vaccination 3, 28, 37, 55; see also infant mortality

Childs, Robert V. 237, 238

Chiluba, Frederick Titus 441-442, 442-443, 445, 446, 447, 450, 454, 455, 456

China 21, 26, 40; ageing population 521, 522; ancestor worship 273; capital punishment 118, 287n65; charitable traditions 275; Chinese Red Cross Society burial corps 272, 274, 276-284; civil violence 56, 273; disease $38,45,56,273$; displaced corpses 273; economic growth 40; famine 28, 272-273, 278; funerary practices 273-274; "ghost festival" 263; homicides 65; nursing homes 527; registering deaths 21; Revolution (1911) 273, 276; support for Edgar Snow 483; Taiping Rebellion (1850-1865) 273

"Chinamen" 54

Chinese Americans 219

Chinese Red Cross Society 272, 274, 276; burial corps 272, 274, 276-284; Chinese context 272-274

Chirambo, Reuben 442, 443

Chodrow, Ruth 469 
cholera: Africa 444; China 273; cholera riots $48-53,56,57,59$; and the decline in infection-related mortality 27, 32, 34, 35, 505; and frequency of Heaven-associated phrases 200; India 48, 292, 294; rates and causes of death (North America) 213, 214

Christianity: Armistice Day ceremonies 146; Caribbean funerals 340, 342, 343, 344, 345-346; churchyards 123, 124-127, 180; and colonialism (Africa) 325-326, 327; death discourse 11, 12, 157, 158, 159, 160; Episcopal churches 460, 464-469, 470, 475; folk religion and expressed belief in Heaven and God's forgiveness see Heaven-associated phrases (study); and the hospice movement 481, 489, 495-496; Orthodox 146, 406, 409, 416, 417; see also Roman Catholicism; secularization

Christmas Carol, A (Dickens) 433

Church of the Transfiguration of the Savior 142-143

churchyards 123, 124-127, 180

Cimetière des Saints Innocents 125, 126-127, 186

civilizing process (Norbert Elias) 66-67, 161

Civil War (American) 9, 12, 136, 149n7, 197, 200, 213, 219, 281

Clark, David 491, 493

Clout, Percy 397

Clutterbuck, George W. 291, 295

Coactle, María Juliana 373

Cobbe, Frances Power 361, 364

Coco (film) 238

coffins: Africa 326, 327, 329; Buddhist funeral rites 258, 259; Caribbean funerals 339, 345, 346; in case of premature burial 6, 221, 364-365; Chinese Red Cross Burial Corps activities 276, 277, 282; coffin clubs (new Zealand) 15; commercialization 168, 219, 329, 345; funeral reform (Holy Roman Empire, 1784) 187; Japanese encoffinment traditions 259, 436-437, 472; Soviet regulation of funerals 409

Congregation Kol Ami reform Synagogue (West Hollywood, CA) 470

Gohen, Richard A. 438

Coleman, F. M. 295

Collateral Beauty (film) 426, 430, 432-433

Collier, George 246

Collier, Richard 46

Colyer, Eileen 396

Colyer, Henry 396

Commonwealth War Graves Commission (formerly Imperial War Graves

Commission) 138

Comte, Auguste 83, 86, 87
Condorcet, Jean-Antoine-Nicolas de Caritat, marquis de 178, 179

Confucianism 261, 273-274, 275, 278, 501

Congdon, B. 395

Cook, James 153

Corbin, Alain 126

Corelli, Marie 202

Cornado, Gerardo Murillo (Dr Atl) 237

Costa Rica 40, 115, 379

Covarrubias, Miguel 246

Covid- 19 crisis $15-16,230$

cremation 2, 15, 160; Britain 227, 291, 292, 298, 299, 363; Caribbean diaspora (North America) 343-344; China 273-274; India 288-298, 299-300; Indians abroad 298-299; Japan 259, 260, 261; for pets 461, 462, 464, 465, 466, 471-473, 475, 476, 477; Russia 409-411, 412, 418; to prevent premature burial 363; United States of America 227, 229, 291

Crimean War (1853-1856) 3, 136, 197, 200

crimes of passion 64, 66

Croft, H. J. 132

Crosby, Alfred 45-46

cryonics 9, 14

Culzac, Nathan 337, 338, 339, 342, 343, 346

Cumberland, Oliver 392

cyborgs 528, 529

Dagomba people 526

Dale, Cecily 392

Dancer in the Dark (film) 426, 434, 435-436, 438

dark/thano tourism 15, 129, 131, 166, 167, 291

Darrow, Clarence 504

Darwin, Charles 503

Datta, Kandysai Lal 297

Dayal, Deen 291

Day of the Dead festival 167, 236, 237, 238, 240, 245-246, 247-251, 263

Day, Douglas 240

Deakin, Vera 395

Death of Ivan Illyich, The (Tolstoy) 519, 527

death penalty: Africa 117, 327; Asian states 118; Canada 216; China 118, 287n65; for cholera rioters 54; in Dancer in the Dark 436; Enlightenment thinkers 110-112; European regions 113, 114-115; and gender 66; Heaven-associated phrases used by those facing execution $\mathbf{1 9 9}, 202-203,209$; history of homicide 68; human rights 109, 110, 112, 113-114, 115, 116, 117, 118; for infanticide 373, 374-375; newspaper reports of 202-203; Organization of American States (OAS) 115-117; for plague spreaders 48; post-war debate 112-113; prehistoric times 109-110; public executions 9, 112, 216, 287n65, 327; 
religious sanction 109, 110; terrorists 96, 102; United Nations 109, 113-114, 115, 118; United States of America 112, 115, 116-117, 216-217

death rates see mortality patterns

death taboo see taboo

death tourism 15; cemeteries 129, 131; see also dark/thano tourism

de Boeck, Filip 320

Defending Your Life (film) 426, 427-428

de Groot, J.J.M. 273

Delaney, Michael 465, 466, 467

Del Carmen Camila, María 381-382

De los Reyes, Gaspar 378

Delumeau, Jean 12

dementia 14, 22, 500, 529

demographic transition see ageing populations

Departures (film) 259, 426, 434, 436-437

diarrhoeal diseases 32, 34

Díaz, María de la Luz 380

Dickens, Charles 112, 433, 486

Diderot, Denis 177-178, 179, 185

digitalization 15, 267, 419, 463

disease: antibiotics 27-28, 36, 37, 38; battle deaths 11, 30; DDT to control insect carriers 38; declining mortality (England, 1870-1940) 27, 31-36; epidemiological transitions framework 27; eradication of and Enlightenment immortality 177, 178; famine-related mortality 29-30; miasmic theory 126-127, 128, 217, 292, 299, 358; and personal wealth 31; traditional remedies (Taiwanese people) 3; urban populations $21,28,31,41,213,217$, 358; vaccination $3,28,30,37,40,53,361$; see also epidemics; non-communicable diseases

Disney films 225

Dixon, Wheeler Winston 424

Dominica, Mother Frances 486

Donne, John 80

Doran, Patrick 203

Dore, Elizabeth 380

Dostoevsky, Fyodor 84, 85

Downey, William 393

Dr Atl 237

Dreifuss, Ruth 114

Duchesne, Syd 390

Dulaure, Jacques-Antoine 129

Durkheim, Émile 77, 85, 86, 87

Eassie, William 358

Easterlin, Richard 21, 40

East Germany 148

Eckels, Howard S. 360

economics: Covid-19 pandemic 15; life expectancy 28, 38, 39, 40

Egyptian cemeteries 130, 188 elderly people see ageing populations

Elias, Norbert 66, 67, 161-162

embalming 2, 9, 220, 223, 321, 322, $329,343,345$

Emerick, Elizabeth J. 524

end-of-life care: "death panels" (US) 517;

discussion with patients (North America and

UK) 225, 228, 517-518; and Muslim beliefs about death 304-305, 307, 308, 311-312, 313, 316-318, 525; see also hospices; palliative care

Enlightenment thought 1,9-10, 175; capital punishment 110-112; churchyard decline and cemetery reform 126-127, 130-131, 180-181, 186-189; immortality 8-9, 177-178, 183, 407; North American surge in the expression of grief 222-223; remembrance 183-185; secularization of death 12, 180, 184, 185, 186, 188, 407; suicide 77, 78, 80, 181, 501; as a watershed moment 179 epidemics: ancient Greece and Rome 46-47; Black Death (1348) 46, 47-48; cholera riots 48-53, 56, 57, 59; Covid-19 pandemic 15-16, 230; and expressions of Heavenly reunion 200, 206; famine-related mortality 29-30; historiography of death 11; India 37, 48, 55, 56-57, 292, 294, 295; Influenza pandemic (1914-1918) 45-46, 57-58; plague protests 56-57; risk of premature burial 357; smallpox violence $53-55,56,57,58,59$; urban populations 21, 28, 31, 213; yellow fever 52, 58-59; see also HIV/AIDS pandemic

Episcopal churches 460, 464 469, 470, 475 epitaphs 129, 398, 422n39

Erichsen, Hugo 291

ESA see Euthanasia Society of America (ESA)

Escarcega, Tomasa 377

Escriche, Joaquín 374

Esquirol, Jean-Étienne Dominique 81, 83, 84

Estonia 148-149

eugenics 503-504, 505

European Convention on Human Rights 112

European Union 114, 115, 149

euthanasia: case of Karen Ann Quinlan 506-507; eugenic and social Darwinist influences 503-504, 505; euthanasia organizations and the effects of world war 504-505; and the hospice movement 485-486, 506; nineteenth century criminalization 502-503; twentieth century demographic transitions 500,

505-506; twenty-first century permissiveness 64, 242, 499-500, 511, 512, 518-519; see also physician assisted suicide (PAS)

Euthanasia Society of America (ESA) 504, 505

Evans-Pritchard, E.E. 320 


\section{IN D E X}

executions see death penalty

Ezerskaia, Lidiia 99

family bereavement (Australia, First World War): casualty statistics 388 ; condolence letters from field hospitals and comrades 388, 392-394; cultural significance of 389, 400; graves/sites of burial 388, 394-397, 398; long-term effects of loss 399, 400-401; manner of death notification 388, 389-390; public and private commemoration 388, 397-399; reaction to news 388, 390-392

famine: Africa 324; China 28, 272-273, 278; dwindling impact of 28-30,31,37, 40; and Hindu cremation 292, 293; Ireland 28, 182; and suicide 79

faradization 362

Farquharson, Frank 397

Farquharson, Walter 397

Federal Military Memorial Cemetery (Russian Federation) 419

Federovna, Elizaveta (Grand Duchess) 142

Ferguson, Edna 243

Fernández Kelly, Patricia 240, 244

fertility rates 24, 38, 40, 323, 521, 522; see also birth rates

Feuer, Jane 436

Field of Mars 411, 412

Filene, Peter 504, 507, 513

films: Afterlife 260; after life and the deaths of loved ones 260, 426-427, 430-433; blockbusters 423; Coco 238; death of self and the wages of $\sin 426-429,437$; Departures 259, 426, 434, 436-437; Disney 225; Gothic Aesthetic 166, 424; Nazi propaganda/Triumph of the Will (Triumph des Willens) 145; postmodern cult of death 167; Premature Burial, The (1962) 365; social and familial dimensions of death 426, 433-438; studies/criticism of 423-424, 425; war 136

First World War (1914-1918): in Africa 325; casualty figures 137, 388; cremation of Hindu and Sikh soldiers 298, 299; euthanasia movement 505; expressions about God and Heaven 204, 206-207; Freud's writings on 154, 155; German fascism 140, 145; Italian fascism 144; male adult smoking patterns 27; military cemeteries $138,141,142-143,144$, 394, 395, 396, 398; premature burial, fears of 365; and Russian funeral culture 146, 407; Tombs of the Unknown Soldier 139, 142; and Victorian death culture 398, 400; war memorials 139-140, 141-144, 145, 146, 397-398; see also family bereavement (Australia, First World War) folk religion see Heaven-associated phrases (study)

Forest, Benjamin 442

Foscolo, Ugo 188

Foucault, Michel 123, 124, 166, 172

France: churchyard closure, dechristianization of death and the cemetery revolution 126-127, 127-128, 130, 179, 180, 181, 186-187, 188-189; Cimetière des Saints Innocents 125, 126-127, 186; eighteenth century population growth 182; gun ownership 71; homicides 64, 65, 68, 82; Louis XVI 111, 177; Paris Catacombs 132; Père Lachaise Cemetery 123, 127-129, 130, 131, 133, 138, 181, 187; suicides $79,81,82,83,84,86,502$; war dead 137, 139, 145, 147-148

Francisvale Pet Cemetery (Philadelphia, PA) 461

Franklin, Benjamin 178, 179

Fraternal Cemetery (Moscow City) 142-143

Freeman, Ned 394, 397

Freud, Sigmund 153-154, 154-155, 160, 166

Friends of Noah pet cemetery (Frazer, PA) 463

Frumkina, Fruma 99

Fumio, Yamazaki 266

funeral homes and undertaking services: academic interest in 11, 12; Caribbean funerals $336,341,342-343,344,345$; expense of and commercialization of death $168,220,224$, 262, 343, 345; Japan 262, 436-437; North American history of death 12, 220-221, 222, 223-224, 226, 227; pets 463; premature burial 353,354, 360; secularization of death 180, 220-221; South Africa 329; and traditional gender roles 189

funeral processions: Abraham Lincoln 220; Caribbean funerals 338, 339, 340, 344, 346-347; Edward VII 156; Hindu funerals 288-289, 291, 296, 297; Japan 260; and the Mexican culture of death 247; Soviet Russia 146, 414, 415, 416; Taiwan 15

funerals: Africa 321-322, 325-326, 327, 329; African American 13, 226; Caribbean diaspora $336-338,340,341-345,346,347$; as a celebration of life 227 ; commercialization of 160, 162, 168, 241, 262, 329; and ethnicity (North America) 219, 226; geographical mobility, impact of 3,15 ; historiography 13 ; Islamic traditions 310; Japan 258, 259, 260, 262, 266-267, 436-437; minimalistic practices 160; modern Caribbean funerals 345-347; and the Morbid Mexican stereotype 235, 245, 246-247; playing down the unpleasantness of death 219, 220, 226; postmodern 13, 226-227; post-Soviet era 418-419; post-war 
Japan 262; Shari'a law 310; Soviet Russia 146, 407-409, 411 , 413-414, 415, 416; state funerals 146, 156, 406, 414; traditional Caribbean funerals 338-341, 342, 343-344; see also funeral homes and undertaking services; funeral processions

funeral strippers 15

Funeral, The (film) 262

Galton, Francis 503

Galvani, Luigi 178

Gandhi, Indira 298

Gandhi, Mohandas 298, 363

García, Leal 116

garden cemetery movement $123,127,128$, 130-131, 217-219, 220, 223

Gardner, Alexander 281, 282

Gates Ajar (Phelps) 200, 202

"gay panic defense" 64

gender: diagnosing physical death and fear of premature burial 358-359; expression and management of grief 221, 246; Great Influenza 58; Hindu cremation ceremonies 289, 296, 297, 298; infanticide (Mexico) 372-385; Islam in Africa 327; Japanese funerary rites 258; life expectancy (North America) 214; marital law suits (post-independence Costa Rica) 379-380; mortality patterns 9; premature burial 357; professionalization of funerary services 189 ; resistance to cemetery reform 188-189; suicide 77-78; suicide terrorists 99-100; victims of violence (Africa) 65-66; women killers 66

Genereux, Maurice 509

genocide $78,320,324$

Germany: ageing population 521; assisted suicide 499, 502; epidemics 52, 58, 59; global cemetery revolution 131,188 ; homicides 65 ; military cemeteries 148; Nazis 103-104, 144, $145,146,147,148,410,503-504$, 505; suicide 83; war casualties 137; war memorials 138, 140, 144; Weimar Republic 145

Ghana 526

Gierka, Robert 463

Gill, Graeme 453

Gilman, Sander 45

Gilmore, Robert A. 425, 438

Ginzburg, Carlo 45

Gioia, Melchiorre 87

Giotto 79

Gnezdilov, Andrei 492

Godwin, William 177, 178, 179, 181-184, 185

Goebel, Stefan 143-144

Goethe, Johann Wolfgang von 184, 185-186, 189, 501 "good death": Africa 322; euthanasia 499; hospices 228, 229, 485; Japan 267-268, 269, 490; and premature burial 366; quick death $4-5,224$; Soviet cemeteries 406

Good, John 362

Google Ngram Viewer 16n10, 197, 198, 199, 201, 205, 206, 216

Gorer, Geoffrey 12, 155-157, 161, 165, 166, 205

Gorkii, Maksim 94

Gothic Aesthetic 166, 424

Gots, Mikhail 97

Graham, Billy 208-209

Graham, James 178

Graham, Stephen 492

Graunt, John 82

graves see burial; cemeteries; mass graves; tombstones

Great East Japan Earthquake (2011) 521

Great Influenza see Influenza pandemic (19141918)

Great Leap Forward famine (China, 1959-1961) 28

Great War see First World War (1914-1918)

Great Western Railway War Memorial 143

Greenberg, Jeff 423, 424

Greenhalgh, Sarah 393

Green, Judith Strupp 240

Gregory the Great (Pope) 124

grief: children's exposure to 5,156,161, 170, 221-222, 223, 225, 228; in film 430-431, 432, 433; and gender 221, 246; and guilt for the death of a child 5, 223, 226, 244, 247; historiography 13 ; pet death 463,468 ; private and political (Soviet Russia) 146, 415-416; and the stereotype of the morbid Mexican 246-247; taboo 5, 154, 156-157, 224; telephone to dial up the dead (Japan) 269; therapeutic management (twentieth century) 5 , 224, 228, 494; see also family bereavement (Australia, First World War); mourning rituals Gruner, Christian 80

Guerrero, Rogelio Díaz 240

Guerry, André-Michel 81, 82, 84

Guizot, François 111

Gulliver's Travels (Swift) 177

gun violence 70,71

Gurr, Ted 64

Guyana 115

Guy, Nathaniel 345

Haboush, JaHyun Kim 273

Hadwen, Walter 361, 364

Haeckel, Ernst 503

Haiselden, Harry 504 


\section{IN D E X}

Hall, G. Stanley 354

Halloween 166, 167, 249, 250

Hanawalt, Barbara 66-67

Harari, Yuval 110

Haraway, Donna 528, 529

Harding, Vanessa 125, 180

Hare, William 361

Harpham, Neil 499

Hartsdale pet Cemetery 461, 462, 463, 464, 466

Harumasa, Yokota 473-474

Hazani, Moshe 98, 102

headstones see tombstones

Heaven-associated phrases (study): during war and epidemic 197, 198, 199, 200, 205, 206-207; materialistic argument 206, 208, 209; methodology 194-195, 197; midtwentieth century onwards 207-209; personal notices (newspapers) 205; poetry (newspapers) 204, 204; serialized stories (newspapers) 205; songbooks and sheet music 203-204; used by colonial emigrants 203; used by those facing death (by suicide or execution) 195-196, 202-203, 205, 206, 207; Victorian novels 200-202, 205

Heaven Our Home (Branks) 200-201, 208

Hegel, G.W.F. 82, 87, 152, 389

Hell 196, 197, 208-209, 223, 426, 427, 427-428, 501

Hemlock Society 508, 509

Henriot, Christian 272

Hermann, Karl Theodor 84

Hernández, Angela 372

Hertz, Robert 136, 137

Hesse, Jr., Raynor W. 466

Hick, John 208

Higgins, Henry 399

Hillcoat, Vincent 393

Hindenburg, Paul von 140

Hinduism: burial 292, 294; cholera riots 57; cremation (beyond India) 298-299; cremation (India) 288-298, 299-300; pet funerals 466; reincarnation 11; suicide 500

Hitler, Adolf 145, 505

HIV/AIDS pandemic: Africa 25, 46, 320, 323-324, 328, 329, 445, 486, 494; Japan 489; pediatric hospices (Romania) 486; and physician-aided suicide 509; socio-physiological reactions 45, 46, 54, 59; tuberculosis resurgence 37

Hodgkinson, Peter E. 206-207

Holden, Esther 353-354, 355, 356, 358, 359, 360, 366

Holden, Stephen 424

Holy Innocents Cemetery see Cimetière des Saints Innocents homicide 19; American exception 63, 64, 66 , 68, 69-72; capital punishment 68, 112 , 202-203; counting issues 63; as a declining phenomenon 64-65, 66-69; externalized pressures of modernity 85; final words of murderer John Webster 202-203; and gender 65-66; as a measure of violent societies 64; newspaper reports of murder-suicides 202; for organ harvesting 365; state role 63-64, 67-69, 72-73; to avoid premature burial 362-363, 364; without punishment 64; see also infanticide; suicide terrorism

Hong Kong 65, 274, 522

honour killings $64,67,68-69,70-71,72$

Hospice Jinja 493, 494

hospices: answer to dying in the premodern era 481-482; Canada 229; and euthanasia 485-486, 506; family care 484-485, 488, 491, 494, 495; Japan 256, 265, 266, 268, 482, 486, 488-490; and post/neo modern death discourse 168-169; problems of dying in Western hospitals 482-486; Russia 490-492, 495; spiritual care 482, 485, 488, 491, 492, 494, 495-496; Uganda 482, 484, 493-494, 496; United Kingdom 481, 485, 486-487, 506; United States of America 228-229, 241, 485, 486, 487-488, 495-496

hospitals: and biomedicine (Africa) 3, 526; condolence letters from nursing staff (Australia, First World War) 388, 392-394; and the hospice movement 482-486; location of death 2, $5,160,161,162,215,256,260,265-266$, 484, 519; targets of epidemic violence 48, 49, $50,51,52,55,56,58,59$

Houghton, Dick 392

Houlbrooke, Ralph Anthony 194, 200

huiguan (guilds, China) 274

humanization of death 168, 227-228; see also palliative care

Hume, David 77, 185, 501

Hume, John 80

Humphry, Derek 507-508, 509, 511

Hungarian Hospice Foundation 486

Hungary 50, 182, 481, 486, 491

Icard, Séverin 363

Ichigaya Kamegaoka Shrine (Tokyo) 475

ideological extremism see suicide terrorism

Idioterne (film) 435

Idris, Wafa 99-100

Ihonvbere, Julius O. 447

Illich, Ivan 12, 162

Imhof, Arthur Erwin 179

immortality: death denial 155, 160, 161, 305; Enlightenment thought 177-178, 183; suicide terrorism 102-104; see also afterlife 
immunization 3, 28, 30, 37, 40, 53, 361

Imperial War Graves Commission (subsequently called Commonwealth War Graves

Commission) 138

India: death registration 21 ; epidemics 37,48 , 55, 56-57, 292, 294, 295; famine victims 294-295; Hindu cremation 288-298, 299-300; homicides 65, 69; life expectancy $26,37,40$

Indonesia 69, 242, 495

infanticide 63, 64, 372-385

infant mortality: Africa 323; exposure of children to death and grief 156; Heavenassociated phrases (study) 200, 206; infectious diseases 21, 28, 34, 35, 37, 55; maternal expressions of grief 244; Mexico 245, 382; North America 213, 213, 215; tabooist discourses of death 160

Influenza pandemic (1914-1918) 16, 31, 45-46, 57-58, 295

Ingersoll, Robert 503

Inglis, Ken 388

Innocents see Cimetière des Saints Innocents

International Committee of the Red Cross 274, 275,277

International Federation for Human Rights 118 inter-species interment 462, 474

Ireland 28, 63, 67, 77, 84, 182, 203

Islam: in Africa 326-327; assisted suicide 316; capital punishment 110; death discourse 11; defining death 3, 304-305, 307, 308-309, 312, 317; end-of-life care 304-305, 307, 308, 311-312, 313, 316-318, 525; Hell 426; monuments 289; mourning rituals 310 ; Muslim prayers for pets (at the "Cemetery for All God's Creatures") 466; Muslims in India 57, 297; organ donation 305, 310, 311, 312 , 317; purpose of suffering 314-315, 316; Qur'an 305-307, 309, 310, 311-312, 313, $314,315,326,525$; right to die 307,313 , 316, 317; in Soviet Russia 416; start of life 304, 306, 308, 309; suicide 313-314, 501; suicide terrorism 93-94, 95, 97, 98, 99-100, 101, 102, 104, 500; Ugandan hospices 496

Israel 71, 97, 100, 101, 102

Italy: ageing population 521, 524; Arena Chapel (Padua) 79; cemetery revolution 180 , 181, 188; emotional response to epidemics 48; epidemics 47, 49, 50-51, 52-53, 56, 57, 59, 200; fascism 144; homicides 65; patterns of mortality 36 ; suicide $85-86$

Ix, María 377

Jacobsen, Michael Hviid 167-169

Jacob, Walter 469

Facques le fataliste (Diderot) 185
Jalland, Patricia 194, 201, 389

Jamaica 37, 40

James, David L. 463

Japan: ageing populations 9, 14, 36, 256, 264-265, 269, 488-489, 521-522, 524; assisted suicide 508; B-29 Bomber crash 254-255; brain death 3, 263-264, 528, 529; capital punishment 118; cholera riots 48, 51-52; contemporary death (1989-2020) 14, 256, 264-269, 521-522, 524; death in modern film 259, 426, 426-427, 428, 434, 436-437; early modern death (1800-1867) 24, 257-260; eugenic organizations 504; homicides 65; hospices 256, 265, 266, 268 , 482, 486, 488-490; mausoleums 442; modern death (1868-1945) 260-261; patterns of mortality 24, 36; pet death 460, 471-476, 477; postwar death (1945-1989) 261-264; suicide 81, 256, 268-269

Jarrah, Ziad 98

Järta, Hans 82

Jenner, Edward 30

Jigoku (film) 426-427, 428

jihadists 93-94, 95, 97, 98, 99-100, 101, $102,104,500$

Johnson, Christopher 529

Johnson, Juliet 442

Johnson, Roxanna 529

Johnston, Mark 209

joint species interment 462, 474

Joseph II (of Austria) 109, 181, 187

Joyce, Kelly 528

Judaism: anti-Semitic epidemic violence 46, 47, 48, 50; capital punishment 110 ; and the hospice movement 481; North America 219, 226; Palestinian suicide terrorism 101; pet death 463, 464, 466, 469-470, 476; risk of premature burial 357 ; suicide 86,501

Justinian, Plague of (541 CE) 47

Jūzō, Itami 262

Kaliaev, Ivan 96

Kambwili, Chishimba 447

Kamo (Simon Ter-Petrosian) 97

Kant, Immanuel 111, 208, 209

Kaufman, Sharon 484, 526, 529

Kaunda, Kenneth 444, 445, 446, 447, 456n7

Keimig, Rose 527

Kelch, Jordan 529

Kennedy, Denis 284

Kenney, Elizabeth 475

Kensal Green Cemetery 123, 132, 181

Kenya 325, 448

Kevorkian, Jack 510, 513

Khamour, Thouria 100

Khapaeva, Dina 165-167, 171 
King of Peace Episcopal Church (Kingland, GA) 468

Kingstown Methodist Church (St. Vincent) 340

Konopliannikova, Zinaida 102

Korea 29, 65, 268, 273, 521, 522

Koreeda, Hirokazu 260

Krasin, Leonid 103

"Kremlin funerals" 414

Kübler-Ross, Elisabeth 12, 228, 268, 483, 484, 487

Kudriavtsev, Evgenii 96

Kula, Irwin 469

Kutner, Luis 506

Labyrinth of Solitude, The (Paz) 239-240, 243, 251

Lakhta Hospice 492

Laqueur, Thomas W. 125, 127, 137, 138, 140, 179, 189, 296, 396

Larson, Edward J. 502

Latin America: capital punishment 112, 115; cemetery reform (Brazil) 188; economic performance 40; epidemic violence 51,55; eugenic organizations 504; global mortality patterns 24; homicides 69; infant mortality (Brazil) 244; lawsuits and gender norms 379-380; malaria 38; medicalization of death 525-526; see also Mexico

Laughlin, Robert M. 242

Lavrin, Asunción 378

lawn-park cemeteries 132-133, 219

Lebedintsev, Vsevolod 102

Leites, Nathan 102

Leith, A. H. 291, 292

Lemkin, Raphael 78

Lendo, León F. 525-526

Lenin, Vladimir 103, 146, 411, 414, 442

Leopold II (of Tuscany) 109

Lesy, Michael 13

Levinas, Emmanuel 438

Lewis, Matthew Gregory 355

Liadov, Martin 103

life expectancy: and average age at death 22-23; economic growth 21, 24, 38-40; epidemiological transitions framework 27; famine 28-30, 31, 37, 40, 273; global patterns (overview) 24-27; HIV/AIDs 25, 37, 324; and the hospice movement (Russia) 491-492; impact on death discourse 5, 156, 159, 160, 161; infection-related mortality (England, 1870-1940) 27, 31-36; Japan's ageing population 265; measurement 1, 21, 24; Mexico 245; non-communicable disease, treatment and prevention 22, 27, 36, 214; North America 213, 214, 215, 223, 230; personal wealth 31; post-communist Russia 27; smoking patterns (UK and US) 27; society's appetite for physician-assisted suicide 500; survivorship curves (England and Wales) 22-23, 23; treating and preventing infectious disease 27-28, 30-31, 36-38 505; urbanization 21, $28,31,41$

Life in Heaven (Branks) 200, 201

life support machines 3, 304-305, 506-507, 527, 528-529

Linares, Pedro 238

Lincoln, Abraham 220, 223

Lincoln, Mary Todd 223

Linden, Blanche M. G. 126

Lipsett-Rivera, Sonya 378

literature: 1984 (Orwell) 103; Chinese Red Cross poetry 276, 277, 283; Death of Ivan Illyich, The (Tolstoy) 519, 527; Enlightenment reformulations of death 177, 184, 185-186, 187, 188, 189; Heaven-associated phrases 200-202, 204, 205; The Labyrinth of Solitude (Paz) 239-240, 243, 251; poetry written by grieving families (Australia) 397; premature burial in Gothic fiction 355-356, 358-359, 365; Russian extremism 95; suicide as a literary trope (Dostoevsky) 84; war poetry 140; women's poetic responses to the First World War 397

Livi, Carlo 85

living wills 228, 506

Livy (Roman historian) 47

Locke, John 110-111

Lockhart, James 242-243

Lock, Margaret 269-270, 528, 529, 530

Logue, Frank 468

Lombroso, Cesare 85, 87, 98

Lomnitz, Claudio 238-239

Lope Blanch, Juan M. 240

López, María Isidora 379

Louis XVI (of France) 111, 177

Lucas, María Getrudis 381

Lunacharskii, Anatolii 103

Lur'e, Rashel' 96

Luxembourg 508

Lu Xun 278, 287n65

McGavin, Patrick Z. 431

McGuffey's reader 221-222

Machida Izumi Jōen 474

McKee, Robert 425

McManners, John 178

Madsen, William 246

Maison-Alfort war memorial 145

malaria 27, 29, 31, 38

Malawi 442-443, 448

Malaysia 118

Maldonado, Thomasa 379

Malinalco monastery 236 
Malthus, Thomas 29, 178, 183

Mamo, Laura 528

Manuel, Jacqueline 389, 397

Maria da Fonte rebellion 187, 189

Markham, Eleanor 356-357, 366

Marriaqua United Friendly Society 345, 346

Mars, Field of 411, 412

Martineau, Harriet 84

Martínez, Guadalupe 380

martyrdom: Bolshevik revolution 146; colonial India 296, 297; ideological extremism 97, 98 , 99, 100, 101, 102, 104; Sati 500; and volunteers during the Influenza pandemic (19141918) 46, 58

Masaryk, Tomáš Garrigue 81, 85

mass graves 126, 138, 144, 148, 407, 412, 413

maternal mortality 9, 24, 36, 214, 222, 230

mausoleums: Indian chattri 289; of Kamuzu Banda (Malawi) 442-443; Lenin 103, 146, 41 1, 442; worldwide controversy 442; see also presidential mausoleums (Zambia)

measles 31, 37, 273

Medellín, José Ernesto 116

medicalization of death: artificial life support technology 3, 304-305, 506-507, 527, 528-529; brain death 2-3, 263-264, 304-305, 307, 308, 312, 365, 528-529; chronic living 527; death anxiety (US) 519; death as the failure to prolong life 225,483 , $484,506,524,526$; desire to avoid 'heroic' treatment 228, 229; dying in hospital 2, 5, 160, 161, 162, 215, 256, 260, 265-266, 484, 519; epidemic violence against medical personnel and hospitals 48, 49, 50, 51, 52, 55, $56,57,58,59$; and fear of premature burial 220, 221, 353, 355, 357-358, 359-360, 361-362, 365, 366; and the hospice movement 168-169, 228-229, 265-266, 482-485, 487, 489, 490; marginalization of alternative treatment 525 ; postmodern humanization $163,168-169,530$; and quality of life 6,527 , 529; secularization $160,180,320$; suicidology 77,80 ; taboo/death denial 160, 162, 168, 437, 519; Western origins 8; see also physicianassisted suicide (PAS)

Medicare 229, 485, 487, 517, 523

Meggy family 391, 392

Megushi Shrine (Chiba) 475

Mendoza, Fernando 379

Menin Gate Memorial 139-140

mentalities (Ariès's death mentalities) 159-160, 168, 184, 194-195, 197, 256, 320

merchant huiguan (guilds, China) 274

Merriman, Anne 493-494, 495, 496

Merriman's Hospice (Uganda) 484, 493

Meslier, Jean 185
Métayer, Christine 125

Mexico: academic interest 8, 13; archaeological artifacts 236; baptism 383-384; divine protection 15; funerals 235, 245, 246; homicide 69; infanticide $372-385$; mortality rates 244-245; popular culture and the stereotype of the morbid Mexican 235-241, 245-246, 247-251; pre-Columbian culture patterns 236, 243-244; stoicism in the face of death 239, 240, 241, 242, 244, 246; and US death customs 241, 242

miasmic theory 126-127, 128, 217, 292, 299, 358

Michel-Louis-Étienne, Count Regnault de Saint Jean d'Angély 129

Middle East and North Africa 522, 523-524; see also Egyptian cemeteries

Midwest Buddhist Temple (Chicago) 471

migrants: Caribbean diaspora 336-338, 340, 341-345, 346, 347; and disease 30, 49, 54; ethnic subcultures (US) 226; European population maintenance 28; exporting Hindu cremation 298; Italy's "baby-bonus" 521; labor migration (Africa) 328, 329; in modern film 434, 435, 438

military cemeteries: American Civil War 9; First World War 138, 141, 142-143, 144, 394, 395, 396, 398; Italian fascism 144; Second World War 147-148

military death see war dead

Millard, C. Killick 505

miscarriage 226, 372, 377, 381, 384

Mitford, Jessica 162, 241

Moedano, Gabriel 240

Monash, John 397

Monkkonen, Erich 71

Monk, The (Lewis) 355

Montague, Charles E. 206

monuments: chattris 289, 298; Islam 289; museums and national memorial parks (Africa) 321; public mourning 229, 297; war memorials 138, 139-140, 141-144, 145, 146, 147, 219, 241, 298, 397-398; see also mausoleums; tombstones

moral statistics $78,82-83,87$

Moreno, Saúl 238

Morocco 522, 523

Morselli, Enrico 81, 85, 87

mortality patterns: and average age at death 22-23; demographic history 11; economic growth 21, 24, 38-40; epidemiological transitions framework 27; famine 28-30 31, 37, 40, 273; global patterns (overview) 24-27; HIV/AIDs 25, 37, 324; and the hospice movement (Russia) 491-492; impact on death discourse 5, 156, 159, 160, 161; 


\section{N D E X}

infection-related mortality (England, 18701940) 27, 31-36; Japan's ageing population 265; measurement 1, 21, 24; Mexico 244-245; non-communicable disease, treatment and prevention 22, 27, 36; North America 213-214, 215, 223, 230; personal wealth 31; post-communist Russia 27; smoking patterns (UK and US) 27; society's appetite for physician-assisted suicide 500; survivorship curves (England and Wales) 2223 23; treating and preventing infectious disease 27-28, 30-31, 36-38 505; urbanization $21,28,31,41$

Moscow 411-412

Moscow City Fraternal Cemetery 142-143

Moses Montefiore Anshe Emunah Greengate Jewish Center (Pikesville, MD) 470

Mosse, George 137, 141, 142, 144, 145

Mount Auburn Cemetery 123, 131, 132, 181, 216, 217

Mount, Balfour 482, 506

mourning rituals: Africa 320, 321-322, 329, 453-454; churchyards 125; cultural imperialism 130, 188; Egypt 130; instruction of children 221-222; Islam 310, 327; Laqueur's "bounded community of the dead" 125; medicalization 5; migrant groups (US) 226, 336-338, 342-345, 346, 347; privatization, avoidance of excess emotional display and the death taboo 5, 154, 156-157, 160, 164, 165, 224-225, 226, 398-399; re-ritualization of death (twenty-first century) 166, 168; significance of a grave $388,389,394-396$, 397-398; war dead 137, 141, 142, 389, 394-396; see also funeral processions; funerals; pet death; public mourning; Victorian death culture

movies see films

Muhammed (Prophet) 326

Mumbai plague riots 56

Munich War Memorial 144

murder see homicide

Murguía, Adriana 525-526

Mutarika, Bingu wa 442-443

Mwanawasa, Levi Patrick 441, 442, 445, 446, 447-450, 452, 456

Myanmar 118

mycoremediation 133

Najar, Fatma 99

Namibia 25

Namiyoke Inari Shrine (Tsukiji) 475

Naples 52-53

Napoléon Bonaparte (Napoléon I) 130, 136, 140, 181, 188, 351
Napoleonic Wars (1803-1815) 84, 136, 138, 140, 197, 200

National Health Service (NHS, UK) 485, 486, 518

National Human Rights Commission of Myanmar 118

Nation family 391-392

Native Americans 69

Nazis 103-104, 144, 145, 146, 147, 148, 410, 503-504, 505

necronominalism 137, 138, 142, 143

Nelkin, Dorothy 45

Netherlands: assisted suicide 499, 508, 512, 513, 518-519, 520; burial practices 180; cholera pandemics 200; homicides 63, 65, 67; Psychiatric Juridical Society of Amsterdam 80-81; The Resurrection of a Bastard (film) 426, 428-429, 438

Neue Wache 140

New Economic Policy (NEP, Soviet Russia) 408, 420n 1

New Tatar Cemetery (Kazan) 422n39

New York Buddhist Church 471

New Zealand: coffin clubs 15; "gay panic defense" 64; Heaven-associated phrases (study) 195, 197, 199, 202, 205; mortality patterns 24

Ngram Viewer 16n10, 197, 198, 199, 201, 205, 206, 216

NHS see National Health Service (NHS, UK)

Niebuhr, Barthold Georg 45

Nigeria 323, 328, 493

Nightingale, Florence 2

Nimmo, William P. 201, 205

Nitschke, Philip 508

Noble, Margaret (Sister Nivedita) 293

non-communicable diseases 1 ; ageing populations 14, 22, 230, 500, 506, 522, 523; dementia $14,22,500,529$; and early-life infections 35 ; epidemiological transition $1,22,27,33$, 215, 224, 505-506, 522; and euthanasia laws 500 ; treatment and prevention raising life expectancy 22, 27, 36; see also cancer

Nongqawuse people 326

Noonan, John 383

North America: brain death and organ harvesting study (Lock, 2001) 528-529; Caribbean diaspora 336-338, 340, 341-345, 346, 347; cholera (1870s) 200; cremation 227, 229; cultural transformations (nineteenth century) 215-221; cultural transformations (twentieth century) 216, 223-226; emotional expression of grief 221-223, 224; fear of death 230; HIV/AIDs crisis 509; rates and causes of death 213-215; twenty-first century 
226-230; see also Canada; Mexico; United

States of America

Northern Rhodesia 322, 326

North Korea 29

Norwood, Frances 518-519

Nuer people 320

Nuremberg trials 505

nursing homes see care and nursing homes

Nyakyusa burial rites 325

OAS see Organization of American States (OAS)

Oaxaca 238, 250, 372, 373, 375, 376, 379, 380

Obama, Barack 517

obon festival 263

Ochs, Vanessa L. 469

Odoevskii, A. I. 92

Oettingen, Alexander von 85, 87

Offel, Janet 469

Olcott, Henry Steel 293

Omran, A. R. 27, 28

"One Law of Sociology" 77, 83, 86

Ordorika, Teresa 525-526

organ donation: artificial prolonging of life 506; brain-death definition of death 3, 365, 528-529; enabling medical advances 3, 36; Islam 305, 310, 311, 312, 317; Japan 263-264

Organization of American States (OAS) 115-117

Orozco, José Clemente 237

Orthodox Christianity 146, 406, 409, 416, 417

Orwell, George 103

Osorio, María Ricarda 376, 377

Ostuni cholera riots 52

Paine, Thomas 111

Palestinian suicide terrorists 97, 99-100, 101,102

Palin, Sarah 517

palliative care 13, 164, 168-169, 208, 506, 523, 527, 530; see also hospices

pandemics: cholera 200; Covid-19 15-16, 230;

Influenza/Spanish Flu (1914-1918) 16, 31, 45-46, 57-58, 295; Plague of Justinian (541

CE) 47; yellow fever 52; see also HIV/AIDS pandemic

Parkes, Colin Murray 495

Parkman, George 202

Parsis 292

Parsons, Elsie Clews 247

PAS see physician-assisted suicide (PAS)

Pasteur, Louis 358

Patchham Down, Hindu cremation 298, 299

Patterson, John 424

Paz, Octavio 239-240, 243, 251 pediatric hospices 486-487

penicillin 36; see also antibiotics

Pennant, Thomas 180

Père Lachaise Cemetery 123, 127-129, 130, 131, 133, 138, 181, 187

Perovskaia, Sof'ia 99

Perry, Luke 133

pet death: Buddhism (Japan) 460, 471-476,

477; Buddhist Churches of America 464, 470-471; commercial pet cemeteries (US) 461-462, 463, 464; Episcopal church 460, 464 469, 470, 475; exposure of children to grief 225; general attitude to death 10; Judaism 463, 464, 466, 469-470; Shinto 475, 476

Petrone, Karen 143

Phelps, Elizabeth 200, 202

Philip III (of Spain) 374

Philippines 118

photography: American Civil War 281; at Tzintzuntzeños funeral 235; Hindu cremations 290-291, 293, 296; humanitarian photography (Chinese Red Cross Society) 272, 277, 278, 279-284; Victorian-era fashion 296 physician-assisted suicide (PAS) 486, 512-513; case of Karen Ann Quinlan 506-507; HIV/ AIDS pandemic 509, 510; legislative resistance (1990s) 509-510; nineteenth century criminalization 502-503; pro-PAS successes (1980s) 507-508, 509; twenty-first century permissiveness 499-500, 511, 512, 518-519; see also euthanasia

Pine Ridge Pet Cemetery (Dedham, MA) 461, 464, 467

Pinker, Steven 10, 118

plague: adoption of mortality statistics 82 ; demographic history 11 ; India 37, 292, 294, 295; mortality decline 28, 30, 31, 37, 182; socio-physiological reactions to $15,46-47$, 56-57

Plague of Athens 46-47

Plague of Justinian (541 CE) 47

Poe, Edgar Allan 355-356, 358-359, 365

Pokotilov, Aleksei 96, 98

Poland 141-142

policing 68, 70, 72

polio vaccinations 3,37

Ponette (film) 426, 430-431, 432, 434

Porter, Roy 45, 180

Porter, Theodore 87

Portugal 112, 187, 188, 189

Posada, José 237-238

postmodern revivalism 6, 13, 163-169, 171, $172,226-230,500,507$

post-Soviet era 27, 418-419, 442

Pothoven, Noa 519

Pound, Ezra 95 
premature burial (nineteenth- and early twentieth-century Britain and America): class and gender 357, 365, 366; Eleanor Markham 356-357, 366; Esther Holden 353-354, 355, 356, 358, 359, 360, 366; fictional depiction 355-356, 358-359, 365, 366; May Jane Walker 359, 360, 366; mitigating procedures 6, 220, 221, 362-365; sensationalist journalism 221, 354, 356, 357, 359, 365; taphophobia 354, 465; Victorian and Edwardian context 221,357-361

Premature Burial, The (film, 1962) 365

"Premature Burial, The" (Poe) 355-356, 358-359, 365

presidential mausoleums (Zambia): making and meaning 441-442, 443, 447-453, 455-456; political context $443,444-447$; responses of ordinary Zambians 443-444, 453-455, 456

Preston, Samuel (Preston Curves) 35, 38, 39

Priestley, Joseph 178

Princess Diana Foundation 486, 494

Priscillian of Avila 110

processions see funeral processions

Procopius of Caesarea (Greek scholar) 47

Promesse, La 426, 434-435, 438

Prost, Antoine 137, 141, 145, 146

Protestants: suicide 77, 83, 84

Prussia 80, 180, 181

psychiatry: suicide 77

Psychomachia (Prudentius) 79

public mourning: celebrities, royals and notables 13, 156, 165, 228, 229, 296-298, 441-442, 443-444, 447-456; public display of the urns of revolutionary figures (Soviet Russia) 412; Russian mortuary culture 407; Soviet Russia 146, 414, 415; war dead 141, 142, 143, 146, 394, 397-398, 399, 400; see also funeral processions

Puchkov, Sergei 142

Puebla State $372,373,375,376,377,378,380$, 381, 382, 384, 385

Pure Land 257-258, 259, 260, 460, 464, 476

Qarafa 130

Qing Dynasty (1644-1911) 273, 274, 275

Quetelet, Adolphe 81, 82-83

Quill, Timothy 508

Quinlan, Karen Ann 506-507

Qur'an 305-307, 309, 310, 311-312, 313, 314, $315,326,525$

Radicati, Alberto 179, 185

Ragon, Michel 132

Rapoport, Leibish 101-102
RCCP see Royal Commission on Capital Punishment (RCGP)

Reagan, Ronald 148

Red Cross societies 274, 275, 277, 393, 395, 396; see also Chinese Red Cross Society

Reddock, H. L. 393

"Red funerals" 146, 413-414

Reed, Nicholas 508

Reform Judaism 464, 469, 470, 501

reincarnation 11, 322, 460, 464, 475, 476

religion: Armistice Day ceremonies 146; capital punishment 110, 113; Caribbean funerals 340, 342, 343, 344, 345-346; churchyards 123, 124-127, 180; and death anxiety 12 , 181,524 ; in death discourse $11,12,157,158$, 159, 160, 161, 166, 168; Episcopal churches and pet death 460, 464-469, 470, 475; and the hospice movement 481, 489, 495-496; life after death 11; Orthodox Christianity 146, 406, 409, 416, 417, 469, 470; plague protests 57; and premature burial 357; Shinto 257, 260, 261, 475, 476; Sikhs 288, 292, 298, 299, 500; spiritual care in hospices 495-496; see also Buddhism; Heaven-associated phrases (study); Hinduism; Islam; Judaism; Roman Catholicism; Remizov, Aleksei 94

Rengeji Tetsugakudo Animal Cemetery (Tokyo) 473, 474

Resurrection of a Bastard, The 426, 428-429, 438

revivalist discourse 163-169

Rhodesia 322, 326

Riashi, Reem 100

Richards, Audrey 321-322

Richardson, Brooke Skylar 372

Richarson, Ruth 180

Riley, James 40

Rio de Janeiro 55

riots: cholera riots $48-53,56,57,59$; plague protests 56-57; smallpox violence 53-55, 56, $57,58,59$

Rivera, Diego 237

Roberts, John Garibaldi 392

Robson, L. L. 388

Rodríguez, Eugenia 379

Rodriguez, Lorenza 377

Rodriguez, Sue 509

Roman Catholicism: belief in the afterlife 196, 201; capital punishment 113; cemeteries 127, 180, 186, 188, 219; and cremation 227; death anxiety 12,181 ; epidemic riots 49,54 ; euthanasia 505; historiography 12 ; and infanticide (Mexico) 378, 383; and the Mexican cult of the dead 239, 249; and premature burial 357 ; suicide $77,78-79,80,83,84,85$, 86, 196 
Roman Empire 47, 78-79, 110, 124

Romania 487, 491

Rosa, María 382, 384

Rosenberg, Charles 52

Rosenthal, Professor (of Vienna) 362

Roth, Randolph 64-65, 68-69, 71

Rousseau, Jean-Jacques 111, 183, 184

Royal Commission on Capital Punishment (RCCP) 112-113

royal deaths 156, 165, 229, 406

Roy, Rammohun 289

Rubin, Diane 488

Rücker, Joachim 114

Rudinger, Stephen Piero 276-277

rural populations $10,28,29,31,40$

Russia see Russian Empire; Russian Federation; Soviet Russia

Russian Aircraft bombings (2004) 99

Russian Empire: cholera riots 48, 49, 50, 56, 57; confessional cemeteries 406, 408, 416; historical dislocation 92, 93-94, 95, 100; suicide 84, 85, 86, 95, 96-97, 268; suicide terrorism 95-99, 100-101, 102-103; war dead 137, 142-143, 407

Russian Federation 27, 69, 99, 418-419, 490-492, 495

Russian Orthodox religion 146, 406, 409, 416, 417

Russia, Soviet see Soviet Russia

Russo-Japanese War (1904-1905) 274-275, 276

Rwandan genocide (1994) 324

Sa'adi, Kahira 99

St. Andrew's Episcopal church (New Providence, NJ) 465, 469

St. Andrew's Episcopal church (Richmondtown, NY) 465

St. Christopher's Hospice 481, 483, 485, 486, 495

St. Georges Cathedral (St. Vincent) 340

St. Leon: A Tale of the Sixteenth Century (Godwin) 183

Sampson, A. M. 393

San Jose Buddhist Church Betsuin 471

San José, María de 377

Santa Muerte 239

Sarasvati, Dayananda 296, 298

Sardo, Ranieri 48

Sassoon, Siegfried 140

Sata, Michael Chilufya 441, 442, 445, 446, 447, 450-452, 453, 455, 456

sati $289,291,297,500$

Saunders, Cecily: and hospices outside the UK 486, 487, 488, 490, 492, 494; pain control 482,484 ; on the purpose of hospices 481 ,
485-486, 496, 506; religion of 483, 495;

St. Christopher's Hospice 481, 485

Savinkov, Boris 98

Sazonov, Sergei 97

scarlet fever 31, 36, 358

Schaffer, Michael 463

Scheper-Hughes, Nancy 244, 519

Schiavo, Terri 511

school shootings 70

Scotland 49, 63, 67, 77, 180, 508, 509

seances 223

Second World War (1939-1945): African troops 325; civilian deaths 147, 149n3; euthanasia movement 505; expressions about God and Heaven 206, 207; Japan 254-255, 269; scope and scale of the commemoration 146-148; Soviet Union 410, 413, 414

secularization: cemetery reform 138, 186; Enlightenment thought 12, 179, 180, 184, 185, 186, 188, 407; Japan 256, 267; and the medicalization of death 160, 180, 320; religious officiation of funerals 2, 220; Russian funeral culture 406, 407, 408, 409, 411, 414, $416,417,418$; suicide $77,78,80,88$

Segni cholera riots 51,59

Seirei Mikataba Hospice 489

Self, Elizabeth 442

Sellin, Thorsten 113

Sen, Amartyr 29

Sen, Keshab Chandra 296

September 11 attacks 98

Sevilla, Petra 372, 381

Shapiro, Yerachmiel 470

Sharpe, James 64

Sheehan, James 141

Shelley, Percy 124

Shen Dunhe (Zhongli) 275, 276, 277

Sherlock, William 182

Shinto 257, 260, 261, 475, 476

Shkol'nik, Mariia 96

Shneidman, Edwin S. 81

Shunkersett, Jagunnath 291, 292

Siebold, Cathy 485, 487

Sierra Leone 3, 328

Sikhs 288, 292, 298, 299, 500

Simpson, Michael A. 170

Singapore 65, 118, 482, 493

Slavin, Phil 29

Sloan, Kathryn 379

Slotkin, Richard 70

smallpox: Africa 30,37, 324; anti-vaccination protests 361; China 273; and Hindu cremation 294; mortality decline 27, 29, 30-31, 37; smallpox violence 53-55, 56, 57, 58, 59

Smith, Gary Scott 200, 201, 207, 208 


\section{IN D E X}

Smith, Richard 29

Snow, Edgar 482-483

Snow, John 358

Snow, Lois 483

social Darwinism 78, 86, 87, 503, 504

social isolation: for disease control 16,30,32;

"lonely deaths" (kodokushi, Japan) 14, 256, $269,522,524$

socio-physiological reactions (to epidemics): ancient Greece and Rome 46-47; Black Death (1348) 46, 47-48; cholera riots 48-53, 56, 57, 59; HIV/AIDs 45, 46, 54, 59; and human nature 45-46, 59; Influenza pandemic (1914-1918) 45-46, 57-58; plague protests 56-57; smallpox violence 53-55, 56, 57, 58, 59 ; yellow fever $52,58-59$

Soman, Alfred 64

Sombart, Werner 87

Sontag, Susan 45, 423

Sorrowes of Young Werther, The (Goethe) 184, 501 souls: African missionaries 326; ancestor veneration 262, 264, 273; animals 463; baptism 383; Buddhism 260; cinematic representation 429; Enlightenment thought on immortality 183; in Islam 304, 306, 308, 312, 313, 525; Mexican attitudes to death 246; popular belief in 194, 196, 197, 209; suicide and assisted suicide 501; Victorian fascination with liminal states 358

South Africa 25, 58, 65, 298, 299, 322-323, 327, 328, 329

Southern Rhodesia 322

South Korea 65, 268, 521

Soviet Russia: cemeteries 407-409, 411-413, 416-418, 419; cemeteries pre-revolution 406-407; cremation 409-411, 412; and First World war dead 142, 143, 407; hospices 491; private and political grief 146, 415-416;

"Red funerals" 413-414; siege of Leningrad (1941-1943) 30; suicide terrorism 97, 102-103

Soviet Union: life expectancy 27; war memorials 148-149, 408, 415; see also Soviet Russia

Spain 46, 65, 110, 115, 374

Spanish flu see Influenza pandemic (1914-1918)

spectacular death 168-169

Speece, Mark W. 430, 431

Spierenburg, Pieter 68, 70

Spiridonova, Mariia 99

Sproule, Hugh 399, 400

Sproule, Les 399

Sproule, Oliver 399

Sproule, Stan 395

Sri Lanka 40, 292, 676

Stalin, Josef 148, 411

Stannard, David E. 12
Stassen, Glenn 110

Stone, Lawrence 74n12, 182

St. Petersburg 492

strokes 22, 215, 268, 506, 528

Sture, Lidiia 98

Sub-Saharan Africa 21, 25, 69, 493, 522, 523-524

suicide: Africa 322; Buddhism 500-501; by homicide 66; China 273; Confucianism 501; criminality of $77,79,80,81,502$; disposal of corpses 79, 80, 125, 406; in Enlightenment thought 77, 78, 80, 181, 501; following the First World War 207; gun-related deaths (US) 70, 71; Heaven-associated phrases (suicide notes) 195-196, 202, 203, 205, 206, 207; Hinduism 500; Islam 313-314, 501; Japan 81, 256, 268-269; Judaism 86, 501; medicalization trends 3-4; prevention 4, 78, 88; Roman Catholicism 77, 78-79, 80, 83, 84, 85, 86, 196; Russian Empire 84, 85, 86, 95, 96-97, 268; suicide revolts 79-80; suicidology 77-88; see also physician-assisted suicide (PAS); suicide terrorism

suicide terrorism 19; children and adolescents 100-101, 102; gender 99-100; historical dislocation 92, 93-94, 95, 100; Muslims 93-94, 95, 97, 98, 99-100, 101, 102, 104, 500; repudiation of death 102-103; Russian Empire 95-99, 100-101, 102-103, 407; search for immortality $102-104$

Sulfa drugs 36

Sullivan, Daniel 423

survivorship curves 22,23

Sverdlov, Iakov 414

Swaziland 25

Sweden 24, 30, 31, 66, 82, 509

Swift, Jonathan 177

Switzerland 65, 71, 274, 482, 499

taboo: care and nursing homes 5, 162, 227; expression of grief $5,154,156-157,224$; Hindu cremation 290; indeterminacy and ambivalence 169-172; medicalization of death $160,162,168,437,519$; photographing the dead (Mexico) 235; postmodern shift away from (revivalist discourse) 6, 13, 163-169, 171, 172, 226-230, 500, 507; Shintoism 475 ; suicide as 3 ; tabooist discourse 6 , $7,12,152,153,154-162,165,171,172$; as

Western culture 6, 7, 8, 12, 13, 169, 241

Tagore, Rabindranath 297

Taiping Rebellion (1850-1865) 273, 278

Taiwan 3, 15, 522

Tannenberg Memorial 140

Taylor, Janelle 529

Teitgen, Henri-Pierre 112 
Temple Beth Emet (Cooper City, CA) 470

Temple Israel (Norfolk, VA) 470

Templewood, Samuel John Gurney Hoare, 1st Viscount 113

Ter-Petrosian, Simon (Kamo) 97

terrorism see suicide terrorism

Terror Management Theory (TMT) 158, 424

Terry, Randall 511

thano/dark tourism 15, 129, 131, 166, 167, 291

Thiry, Paul-Henri, Baron d'Holbach 179, 183, 185

Thompson, Henry 363

Thomson, David 425, 438

Thucydides 46-47

Tilak, Bal Gangadhar 297

Tinker, Edward 243

Titanic (film) 423

Tlalmanalco open-air chapel 236

TMT see Terror Management Theory (TMT)

Tolstoy, Leo 519, 527

tombstones: Caribbean graves 339, 340; commercialization of death 168; garden cemeteries $128,129,131,218$; lawn park cemeteries 133; and the morbid Mexican stereotype 241; outlawing (Holy Roman Emperor) 187; Père Lachaise Cemetery 128, 129, 131; pet graves 461, 464; Russia 406, 408, 416, 417, 418; war dead 138, 139, 148, 397, 398, 407; Zambia's presidents 449, 450

Tombs of the Unknown Soldier 139, 142, 241

Toor, Frances 237, 243-244

Torres, Francisca 384

Torres, Juan Luis Ramírez 249-250

Tree of Life, The 426, 430, 431-432, 434

Treptower Park cemetery 148

Trinidad and Tobago 115

Triumph of the Will (Triumph des Willens, dir.

Riefenstahl) 145

Trotsky, Leon 103

tuberculosis: Africa 324; China 273; England and Wales 27, 31, 32, 33, 34, 35, 36; European populations 36 ; global populations 37 ; United States of America 215

Tula 236

typhoid 29, 32, 34, 35, 37, 201, 213, 273

typhus $27,29,31,79$

Tzeltal people 242

Tzintzuntzeños people 235, 245-246

tzompantli 236

Tzotzil people 242, 246

Uc, Paulina 380

Uganda 323, 482, 484, 493-494, 496

Ukraine 50, 491

undertakers see funeral homes and undertaking services
United Kingdom: assisted suicide 504-505, 508, 509; capital punishment 112-113; Caribbean diaspora 337, 341; Caribbean independence 339; cemeteries (England) 123, 131, 132, 181, 182; cholera and smallpox violence 48-49, 51, 55; colonial India and Hindu cremation 291-292, 293, 294, 295, 296-297; cremation 227, 291, 292, 298, 299, 363; end-of-life-care 481, 485, 486-487, 506, 518; famine-related mortality 29-30; First World War casualties 139; Heaven-associated phrases (study) 195, 197, 199, 200, 201, 203, 204, 205, 206; homicides $63,64-65,66,67$; infection-related mortality 21, 27, 30, 31-37, 200; life expectancy (statistics for England and Wales) 22-24, 25; maternal mortality 214; Patchham Down, Hindu cremation 298, 299; police shootings (figures for England and Wales) 70; smoking and cancer 27; suicide 77, 79, 81, 84, 86, 501, 509; urbanization 28, 31; war memorials 139, 140, 143, 144; see also premature burial (nineteenth- and early twentieth-century Britain and America)

United States of America: African American funerals 13, 226; ageing population 230, 521; American Civil War 9, 12, 136, 149n7, 197, 200, 213, 219, 281; anti-child immunisation 3; assisted suicide 242, 499, 502-503, 504, 505, 506-507, 508, 509, 510-511, 512; capital punishment 112, 115, 116-117, 216-217; Caribbean diaspora 336-338, 340, 341-345, 346, 347; cemeteries 123, 130-131, 132-133, 181, 216, 217-219; college courses 228; commercial exploitation of funerals 160 , 162, 241; cremation 227, 229, 291; cryonics 9; death anxiety $230,519,524$; end-of-life care 228-229, 241, 485, 486, 487-488, 495-496, 517-518, 523; epidemics 52, 53-54, 56, 59, 200; eugenic organizations 504; expressions about God and Heaven 202-203, 207-208; French war graves 147-148; Great Influenza 58; HIV/AIDS crisis 46, 54, 509; homicides $63,64,66,68$, 69-72; Indian cremation 298; and the Mexican cult of death 241, 242; mortality patterns 9, 27, 36, 38, 39, 213, 214, 215, 230; Mount Auburn Cemetery 123, 131, 132, 181, 216, 217; pet death 460, 461-471, 475; religious decline 12; suburbanization 213; suicide 4, 10, 86, 231n8; war monuments 241; yellow fever relief 52, 58-59; see also North America; premature burial (nineteenth- and early twentieth-century Britain and America)

Universal Declaration of Human Rights (1948) 109, 113

Unknown Soldier, Tombs of 139, 142, 241 
urbanization: death denial 320; disease transmission 21, 28, 31, 34, 35, 358; historical dislocation 93; intergenerational contact 524; Japan 256, 260, 261; life expectancy 21, 28, 31,41 ; and mourning ritual 222; pet funeral services 461; and technologies of death (Africa) 321, 327-329

USSR see Soviet Union

vaccination $3,28,30,37,40,53,361$

Van Gennep, Arnold 321

Vargas, Alberto Luis G. 240

Vaughan, Megan 442-443

VELS see Voluntary Euthanasia Legalisation Society (VELS)

Venezuela 40,69, 112, 115

Venice Hongwanji Buddhist Temple (Culver, CA) 470

Venn-Brown, Rose 395

Ventura, María del Carmen 383-384

Verbicaro cholera riots 50-51

Verdery, Kathryn 442

Versailles, Treaty of 140, 144, 145

Victorian death culture: comfort or exploitation debate 195; and the contemporary culture of the dead 166; cultural and regional specificity 7; as 'death of the other' 159; and First World War 398, 400; sentimental religiosity 201-202; and tabooist discourse 6, 7, 155-156, 159, 165

Vidyasagar, Iswar Chandra 296

Vietnam 40, 118

Vietnam War (1955-1975) 227, 241

Villani, Matteo 48

vivisectionism 49, 311; see also anti-vivisectionists

Vnorovskii, Boris 98

Voluntary Euthanasia Legalisation Society (VELS) 504, 505

Vovelle, Michel 12, 170, 179

Waddington, James 353, 354

Wagner, Adolf 83, 85, 87

waiting mortuaries 363-364

Wakeford, Sister 395

Waldeback, Zara 437

Walker, Mary Jane 359, 360, 366

Walter, Tony 154, 163-164, 168, 389

war dead 19-20, 136-137; Africa 325; American Civil War 9, 12, 136, 149n7, 197, 200, 213, 219, 281; B-29 Bomber crash Japan, 1945) 254-255; burial of 138, 142, 219; casualty figures $11,137,146-147,200,219,388$; civilian deaths 136, 147, 149n3; commemoration ceremonies 146, 148, 394; enemy graves (Soviet Russia) 413; fascist instrumentalization of 140, 144, 145; Hindu and Sikh soldiers 294, 298, 299; lasting trauma (Australia, First World War) 399, 400-401; monuments 138, 139-140, 141-144, 145, 146, 147, 219, 241, 298, 397-398; necronominalism 137, 138, 142, 143; premature burial in the trenches 365 ; and public opinion on euthanasia 505; recovering the body 2, 3, 9, 12, 219-220, 227; Russian funeral culture 146, 407, 414; of the Second World War (1939-1945)

146-148, 149n3; Soviet burial and cremation 410, 413; Taiping Rebellion (1850-1865)

273; Unknown Soldier 139, 142, 241; wartime expressions about God and Heaven 197, 200, 204, 205, 206-207; see also family bereavement (Australia, First World War); military cemeteries

Warner, Lloyd 132

Washington, George 6, 221

waterborne diseases 32, 34-35

"We Are Seven" (Wordsworth) 189

Weber, Max 67, 87, 275

Webster, John 202-203

WeCroak app 15

Weir, Molly 356, 363

WFRTDS see World Federation of Right-to-Die Societies (WFRTDS)

WHO see World Health Organization (WHO)

Wickett, Ann 508, 509

Wilder, Alexander 335

Williams, Elliot 336, 338, 343-344

Williams, Sheryl 336, 343, 344

Wilson family 395,398

Winter, Jay 140, 141, 147, 149n6, 389, 393

Wolfe, Tom 95

Wollen, Peter 237-238

Wollstonecraft Godwin, Mary 124, 183

Woods, Robert 31

Wordsworth, William 189

World Coalition Against the Death Penalty 118

World Federation of Right-to-Die Societies (WFRTDS) 508

World Health Organization (WHO) 3, 35, 37, 78, 491, 494

World War I see First World War (1914-1918)

World War II see Second World War (19391945)

Wright, Elizabethada 132

Wright, Michael 491, 493

Xhosa people 326,327

Yasukuni Shrine 475

yellow fever $37,38,52,58-59$ 
IN D E X

Yodogawa Christian Hospital 489

Yucatán State 236, 373, 375, 376, 377, 378 Yu Zhi 278

Zambia 25; see also presidential mausoleums (Zambia)
Zen tradition 258

Zhijiang Red Cross Chapter 280, 281

Zinsser, Hans 11

Zorza, Jane 485, 486, 495

Zorza, Rosemary 485, 492, 495

Zorza, Victor 485, 492, 495 